\title{
Final Program \\ Forty Sixth Annual Meeting \\ International Neuropsychological Society
}

February 14-17, 2018

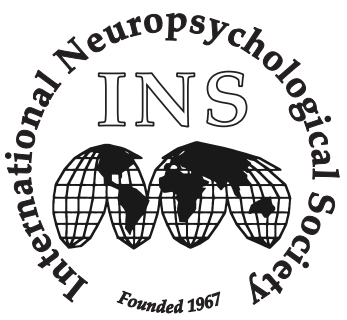

Washington, DC, USA

\section{PROGRAM CHANGES \& AUTHOR REVISIONS}

The following abstracts were rescheduled after being accepted for presentation in Washington:

WEDNESDAY, FEBRUARY 14, 2018

2:30-3:45 PM

33. SABBAH, LE

36. SHAKED, D

4:00-5:15 PM

60. HOFFMAN, $\mathrm{S}$

10:45 AM-12:00 PM

36. SHIELDS, BJ
Poster Session 1. Aging

Cognitive Structure, Cognitive Engagement and Adaptive Functioning: The Aging, Demographics, and Memory Study (ADAMS) (L.E. Sabbah \&. M. Suvak)

Revision Details: This abstract was rescheduled from where it currently appears in these proceedings

(as \#33 in Poster Session 1) and was instead presented as \#90 in Poster Session 4.

Telomere Length and Cognitive Function: Differential Relations across Sociodemographic Groups (D. Shaked, D.K. Leibel, N.P. Weng, H. Liu, M.K. Evans, A.B. Zonderman \& S.R. Waldstein)

Revision Details: This abstract was rescheduled from where it currently appears in these proceedings (as \#36 in Poster Session 1) and was instead presented as \#89 in Poster Session 4.

\section{THURSDAY, FEBRUARY 15, 2018}

\section{Poster Session 6. Acquired Brain Injury}

Examination of Symptom Over-Reporting and Self-Reported Pain and Depression in Iraq and Afghanistan Veterans with Mild Traumatic Brain Injury (R.S. Vasudevan, M.S. Herbert, S.M. Jurick, N.E. DeFord, A.V. Keller, S.N. Hoffman, M. Lee, M. Sanderson-Cimino \& A.J. Jak)

Revision Details: This abstract was rescheduled from where it currently appears in these proceedings (as \#60 in Poster Session 6) and was instead presented as \#46 in Poster Session 3.

\section{SATURDAY, FEBRUARY 17, 2018}

Poster Session 11. Executive Function, Language, and Memory

Impact of Early Neurologic Insult on Executive Functioning Development in Preschool Children (B.J. Shields \& A. Heffelfinger)

Revision Details: This abstract was rescheduled from where it currently appears in these proceedings (as \#36 in Poster Session 11) and was instead presented as \#92 in Poster Session 5. 


\title{
WITHDRAWN ABSTRACTS
}

The following abstracts were withdrawn by their authors after they were accepted, and were not presented at the Forty Sixth Annual Meeting in Washington:

\section{THURSDAY, FEBRUARY 15, 2018}

\author{
8:00-9:15 AM \\ 12. KAHRILAS, IJ \\ 45. LØVSTAD, M
}

\author{
4:00-5:15 PM \\ 32. KEY-DELYRIA, S \\ 43. PERNA, R \\ 65. WENDEL, C
}

\author{
10:15-11:30 AM \\ 74. SEESE, $\mathrm{S}$
}

\author{
1:45-3:00 PM \\ 48. ANAND, D \\ 3:30-4:45 PM \\ 27. SCHARAGA. EA
}

9:00-10:15 AM

2. CORNETT, $\mathrm{K}$

10:45 AM-12:00 PM

6. CIPOLOTTI, L
Poster Session 2. Neuropsychiatry, Forensics, and Inclusion and Diversity Positive Affectivity Distinctly Influences Negative Interpretation Bias in Individuals with Remitted Depression (I.J. Kahrilas, D. Dickson, C. Lee \& R. Silton)

Relationships Between Subjective Cognitive Complaints and Emotional Functioning, Post Traumatic Stress Symptoms, Fatigue, Sleep and Pain, as well as Neuropsychological Test Performance in Hospitalized Victims of Terror (M. Løvstad, G. Månum, K. Wisløff-Aase, I. Larsen, J. Ræder, J. Stanghelle \& A. Schanke)

\section{Poster Session 5. Child Medical and Neuropsychiatry}

Flexibility Capacity: Differences Between Young and Older People in Nesplora Aquarium Test (A. Aierbe, M. González, M. Moreno, M. Mejías \& M. Redondo) Nesplora Aquarium: Utility of the Tool to Identify People with Attention-Deficit Hyperactivity Disorder (A. Aierbe, M. Redondo, M. González, M. Moreno \& M. Mejías)

\section{Poster Session 6. Acquired Brain Injury}

Using Cognitive Profiles in Individuals With and Without Mild Closed-Head Injury to Predict Sentence Comprehension Performance (S. Key-DeLyria \& C.M. Mosteller) Case Study: Disorder of Consciousness to full Functional Recovery (R. Perna)

LONG-Term Outcome After Stroke: What Contributes to Participation? (C. Wendel, E. Schenk zu Schweinsberg \& A. Bateman)

\section{FRIDAY, FEBRUARY 16, 2018}

\section{Poster Session 7. Assessment Across the Lifespan}

A Multimodal Research Battery for Assessing Executive Function Skills in Autistic Teens (S. Seese, M.F. Skapek, M. Powers, D. Gohari, D. Limon, L. Anthony, L. Kenworthy \& J.F. Strang)

\section{Poster Session 8. Adult Neurological}

Living under the Shadow of Chronic Illness: Coping and Conflicts in Multiple Sclerosis (D. Anand, S. Awasthy \& R. Anand)

\section{Poster Session 9. Adult Medical}

Cognitive Sequelae of Neurofibromatosis Type 1: A Progressive Look (E.A. Scharaga \& A. Chang)

\section{SATURDAY, FEBRUARY 17, 2018}

\author{
Poster Session 10. Cognitive Neuroscience, Neuromodulation, and \\ Rehabilitation \\ Efficacy of Interdisciplinary Post-Acute Rehabilitation for Impairments in Attention and Processing \\ Speed in Traumatic Brain Injury (K. Cornett)
}

Poster Session 11. Executive Function, Language, and Memory Inhibition, Estimation and Response Generation: Evidence from Patients with Prefrontal Cortex Lesions (L. Cipolotti, E. Chan, S. Gharooni, S.E. MacPherson, B. Spano, T. Shallice, J. Duncan \&. P. Nachev) 


\section{Final Program \\ Forty Sixth Annual Meeting \\ International Neuropsychological Society}

February 14-17, 2018

Washington DC, USA

9:00 AM-12:00 PM

1. FLETCHER, JM

9:00 AM-12:00 PM

1. JOHNSTON, MV

9:00 AM-12:00 PМ

1. NADEAU, SE

12:00-1:00 PM

1:00-4:00 PM

1. JAK, AJ

1:00-4:00 PM

1. LUNA, B

\section{WEDNESDAY, FEBRUARY 14, 2018}

CE Workshop 1. Test and Treat or Treat and Test? Scientific and Ethical Issues in Neuropsychological Approaches to Learning Disabilities Presenter: Jack M. Fletcher

Maryland A-C

Test and Treat or Treat and Test? Scientific and Ethical Issues in Neuropsychological Approaches to Learning Disabilities

CE Workshop 2. Role of Neuroplasticity in Outcomes from Perinatal Brain Injuries

Presenters: Michael V. Johnston, Gwendolyn J. Gerner, Joanna Burton Virginia A-C

Role of Neuroplasticity in Outcomes from Perinatal Brain Injuries

CE Workshop 3. Language Foundations in Population Encoding Networks and Implications for Aphasia Therapy

Presenters: Stephen E. Nadeau, Irene Minkina, Lisa Edmonds

Delaware A-B

Language Foundations in Population Encoding Networks and Implications for Aphasia Therapy

Lunch (On Own)

Conference-Wide

CE Workshop 4. Assessment and Treatment of Postconcussive Symptoms in Veterans: Rethinking the Role of Concussion

Presenter: Amy J. Jak

Virginia A-C

Assessment and Treatment of Postconcussive Symptoms in Veterans: Rethinking the Role of Concussion

CE Workshop 5. How Adolescence Specializes The Brain to Establish Adult Modes of Operation

Presenter: Beatriz Luna

Maryland A-C

How Adolescence Specializes The Brain to Establish Adult Modes of Operation 
1:00-4:00 PM

1. JACOBSON, L

2:30-4:00 PM

2:30-3:45 PM

1. BAJAJ, S

2. BOOTS, EA

3. CAMPBELL, LM

4. DIVERS, $\mathrm{R}$

5. EMMERT, NA

6. EVANGELISTA, ND

7. FISCHER, EM

8. GETZ, S

9. GOGNIAT, M

10. GRACIAN, EI

11. HALPIN, A

12. HAM, L

13. MUKAI, K

14. SOL. K

15. KAUZOR, K

16. KELLY, DA

17. KRAAL, AZ

18. MATCHANOVA, A

19. MARGOLIS, SA

20. MARGOLIS, SA

21. MAYE, JE

22. MCVEIGH, KS

23. MENDOZA. L

24. MOLDEN. J

25. NIERMEYER, MA

26. ORD, AS

27. OSUNA, JR

28. PASSLER, JS

29. POLSINELLI, A

30. ROBINSON, TL

31. RODRIGUEZ, RA

32. ROTBLATT, LJ
CE Workshop 6. Ethical Practice in a Learning Healthcare System: Tomatoes, Technology, and the Marriage of Clinical Practice and Research Presenters: Lisa Jacobson, T A. Zabel, Alison Pritchard Ballroom Salon 3

Ethical practice in a learning healthcare system: Tomatoes, technology, and the marriage of clinical practice and research

Workshop, Presented by the INS Student Liaison Committee: The Role of Neuropsychology in an İntegrated Healthcare Setting

Presenter: Neil H. Pliskin

Delaware A-B

\section{Poster Session 1. Aging Exhibit Hall B North (Exhibition Level)}

\section{Aging}

Preservation of limbic network structure in healthy young adults

Preclinical Profiles of Memory Versus Executive Function Weakness as Related to Cognition, Stroke Risk, and White Matter Integrity in Older Adults

Cognitive Performance and Alzheimer's Disease Biomarkers in SuperAging and Normal Older Adults Unique Effects of Aging on Everyday Action Performance in Older Adults Compared to Younger Adults Performing a Dual Task

An Examination of Neuropsychological Functions and Depression as Predictors of Health and Safety Abilities in Older Adults with Dementia

Endothelin-1 and Cerebral Blood Flow Interact to Affect Memory Performance in Cognitively Normal Older Adults

Vascular Hemodynamics Relate to Cognitive Performance in Older Adults

Low Emotional Reserve as a Risk Factor for the Frailty Syndrome

Relation between Lutein. Zeaxanthin, and Brain Volume in Older Adults

Non-Uniform Age-Related Differences and Neuropsychological Correlates of Medication Management Ability in Cognitively Normal Adults

Maine Understanding Sensory Integration \& Cognition (MUSIC) Project: Feasibility of a music training intervention for cognition in older adults

Simulating Cognitive Aging with a Dual Task in the Virtual Kitchen

Lower Memory Performance in Males with Presence of Multiple Risk Factors for Dementia Neighborhood Quality and Cognition Among Diverse Older Adults: An Analysis of the Health and Retirement Study

Test Performance of MCI Patients With and Without Caregivers as Compared to AD and NC

Influence of Family History and APOE $\varepsilon 4$ Allele on Five Year Longitudinal Memory Performance in Cognitively Intact Elders

Religiosity, Religious Attendance, and Cognition among Racially/Ethnically Diverse Older Adults

The Effect of Cognitive Aging and Resource Limitations on Everyday Task Completion and Interleaving

Cognitive Decline Predicts Perceived Loneliness in Non-Demented Older Adults at Risk of Alzheimer's Disease

Sedative/Anticholinergic Drug Burden Predicts Worse Memory Acquisition in Racially/Ethnically Diverse Older Adults with Type 2 Diabetes

Divergent Production, Executive Function, and Daily Functioning in Older Adults

Associations between social isolation and cognitive function in older adults

The effect of acculturation on verbal fluency among older adults

Effects of Stereotype Threat and Diagnosis Threat Manipulations on Older Adults

Neuropsychological Test Performance

The Relationship Between Executive Functioning and Gait Speed Among Older Adults: Does Measurement Matter?

Can Alcohol and Tobacco Use Predict Neurocognitive Functioning in Older Adults?

The Benefits of Moderate-Intensity Physical Activity on Cognition in Older Adults

The Relationship of Cognitive Decline and Impairment to the AD8 and Activities of Living in a General Population

Multi-method measurement of cognitive ability in healthy older adults

Associations Between Body Composition Measures and Cognitive Reserve in Older Adults Birthplace, Acculturation Level, and Cognitive Trajectory among Hispanic Older Adults

The Effects of Hypertension and Polypharmacy on Changes in Reasoning Abilities over 10 Years in the ACTIVE Trial 


\author{
33. SABBAH, LE \\ 34. SALONER, R \\ 35. SEELYE, A \\ 36. SHAKED, D \\ 37. STAFFARONI, AM \\ 38. STEFFEN-ALLEN, F \\ 39. STEPHENS, B \\ 40. STICKEL, A \\ 41. SUMIDA, C \\ 42. SUN-SUSLOW, N \\ 43. SUNDERARAMAN, P \\ 44. SZAJER, J \\ 45. TAM. JW \\ 46. TAYLOR, L \\ 47. TOMASINO, DF \\ 48. TURNER, SM \\ 49. VAN ETTEN, EJ \\ 50. WALL, KM \\ 51. WIGGINS, ME \\ 52. SHVARTSUR, A
}

53. KARR, JE

54. HESSEN, E

55. COnstatinou, M

56. HESSEN, E

57. LETTNER, S

58. HOKKANEN, L

4:30-4:45 PM

4:45-5:45 PM

1. KOPELMAN, M
Cognitive Structure, Cognitive Engagement and Adaptive Functioning: The Aging, Demographics, and Memory Study (ADAMS)

Associations of Dopamine-Related Genes with Executive Functioning in Functionally Normal Older Adults

Age-Related Declines in Cognitive Control and Verbal Learning in Veterans with Bipolar Disorder Telomere Length and Cognitive Function: Differential Relations across Sociodemographic Groups The Longitudinal Trajectory of the Default Mode Network Varies with Age and Predicts Changes in Episodic Memory

Predicting Age-Related Decline on the Trail Making Test: An MRI Study of White Matter Integrity Is Caregiver Burden Influenced by Relationship Type and Race in a Veteran Population?

Body mass index and percent body fat differentially predict memory in young old and older old adults

Medication Management in Community-Dwelling Older-Adults: Correlates Between

Performance-Based, Self-Report and Neuropsychological Measures

The Association Between Indicators of Physical Frailty Syndrome and Cognitive Function

Correlates of Financial Risk Taking in Non-Demented Older Adults

The Effect of Worry on Memory for Odors in Apolipoprotein E \&4 Carriers

Baseline Cognition and Everyday Functioning in Older Adults with Subject Cognitive Complaints from a Randomized Clinical Trial

Utility of the Repeatable Battery for the Assessment of Neuropsychological Status (RBANS) with a Geriatric Veteran Population: A Comparison of Standard and Demographic Regression Based Norms Informant Report of Cognitive Functioning in Geriatric Depression: Correlates with Objective Cognitive Tests and Structural Imaging

Translational Cognitive Training Paradigm Elicits Persistent Functional Connectivity Changes in Aged Animals

Age-Related Differences on a New Memory Test for "Who, When, and Where" Begin in Middle Age Biomarkers and Neuropsychological Function in Mild Cognitive Impairment (MCI): Salivary Dehydroepiandrosterone (DHEA-S) and Overall Cognition

Volume and Region of Leukoaraiosis Relative to Cognition in Non-Demented Older Adults Longitudinal Assessment of Metamemory in Mild Cognitive Impairment and Alzheimer's Disease

\section{Concussion}

Acute Symptoms Predict Return to School Following Concussion in Student Athletes

\section{Career Development/Education/Training}

Symposium on core competencies in Clinical Neuropsychology training across the world and the current status of Clinical Neuropsychology in thirty-one European countries - Results from a review conducted in collaboration with INS and FESN and from a survey by the European Federation of Psychological Associations (EFPA) on Clinical Neuropsychology in Europe

The Role of Clinical Neuropsychologists in Health Care- Results of a Survey on 31 European Countries

Core competencies in clinical neuropsychology training across the world

The Legal Status and Licensure of Clinical Neuropsychologists - Results of a Survey on 31 European Countries

Training Models in Clinical Neuropsychology - Results of a Survey on 31 European Countries

Program Welcome

Program Committee Chair: Shawn M. McClintock

Ballroom Salon 2-3

\author{
Plenary A (INS Presidential Address). Anomalies of Autobiographical \\ Memory \\ INS President: Michael Kopelman \\ Ballroom Salon 2-3
}

Anomalies of Autobiographical Memory 
5:45-6:30 PM

6:30-7:30 PM

7:20-8:50 AM

1. GUR, RC

7:20-8:50 AM

1. RAO, SM

8:00-9:15 AM

1. ARIAS, F

2. NGUYEN-LOUIE, T

3. PACHECO-COLON, IM

4. PATT, VM

5. WACLAWIK, K

6. SCOTT, TM

7. CRAUN, E

8. PRITCHETT, A

9. ALKOZEI, A

10. DENO, M

11. EASTER, RE

12. KAHRHAS, IJ WITHORAWN

13. OLARU, M

14. TAIWO, Z

15. BIDDLE, C

16. GAASEDELEN, O

17. GUZMAN, D
INS Awards Ceremony

Awards Committee Chair: Roy P.C. Kessels

Ballroom Salon 2-3

INS Welcome Reception

Ballroom Salon 1

THURSDAY, FEBRUARY 15, 2018

CE Workshop 7. "Precision Neuropsychology": Neuropsychological Assessment in the "Precision Medicine" Revolution

Presenter: Ruben C. Gur

Ballroom Salon 1

Introduction to "Precision Neuropsychology": Background and initial results of making neuropsychological assessment participate in the "Precision Medicine" revolution

CE Workshop 8. Role of Computerized Screening in Healthcare Teams: Why Computerized Testing is NOT the Death of Neuropsychology Presenter: Stephen M. Rao Maryland A-C

Role of Computerized Screening in Healthcare Teams: Why Computerized Testing is NOT the Death of Neuropsychology

Poster Session 2. Neuropsychiatry, Forensics, and Inclusion and Diversity Exhibit Hall B North (Exhibition Level)

\section{Addiction/Dependence}

Executive Functioning, Characterological Facets of Inhibitory Control, and Participation in Risky Behaviors in Adults Receiving Opioid Agonist Treatment

Earlier Alcohol Use Onset Predicts Poorer Neuropsychological Functioning in Young Adults Adolescent Cannabis Use Trajectories and Motivation: A Longitudinal Analysis

Effect of Combined HIV Infection and Methamphetamine Dependence on Spatial Working Memory Cognitive Impairment in Marginally Housed Youth: Associations with Substance Use and Viral Infection

\section{Career Development/Education/Training}

Diversifying Neuropsychology from the Bottom Up: Outcomes from a Student Pipeline Workshop

\section{Drug/Toxin-Related Disorders}

Alcohol Use Disorder Symptoms, Neurocognitive Functioning, and Criminal Behavior of Jail Inmates Effects of Prenatal Cannabis and Tobacco Exposure on Offspring Stress Hormones

\section{Emotion Regulation}

A Positive Cognitive Style Mediates the Relationship Between Trait Gratitude and Depressive Symptoms

The Effect of Physiological Response to Acute Stress on Emotional Attentional Bias Among Japanese Adolescents

Effects of age, education, and mood state on facial emotion processing in bipolar disorder

Positive Affeetivity Distinetly Influenees Negative Interpretation Bias in Individuals with Remitted

Depression

The Relationship of Self-Esteem and Cognitive Styles in the Development of Depression Individual Differences in Working Memory Associated with Empathy-Related Frontal Lobe BOLD Activity

\section{Forensic Neuropsychology}

Exploring the California Verbal Learning Test- $2^{\text {nd }}$ Edition Short Form Forced Choice Scores as a Potential Measure of Performance Validity Among Older Adults Referred for Memory Complaints Utility of the Symptom Validity Scale and Response Bias Scale in Predicting PVT Failure in a Veteran Sample

Prevalence and Prediction of PVT Failure in a Research Sample of MS Patients 


\author{
18. KLIPFEL, KM \\ 19. KLIPFEL, KM \\ 20. LAU, L \\ 21. MULLIGAN, R \\ 22. PIPER, LE \\ 23. REGINELLA, VM \\ 24. ROLIN, S \\ 25. STIKA, MM \\ 26. VARGA, CM \\ 27. VOGT, E
}

28. DAY. L

29. FORTE, M

30. MEJIA, A

31. RATTO, A

32. RIEGER, RE

33. SALAZAR. R

34. WEIZENBAUM, E

35. WERRY, AE

\author{
36. BABIONE, JM \\ 37. BODZY, M \\ 38. CERNY, BM \\ 39. COBIA, DJ
}

40. CZEPIELEWSKI, LS

41. SANGUTNETTI CZEPIELEWSKI, L Delayed sleep phase and impaired verbal memory of patients with Bipolar Disorder

42. DEV, SI

43. ESTEVIS, E

44. FERNANDES, P

45. LOVSTAD, M WITHORAWN

46. NAKHLA, MZ

47. PETERMAN, J

48. PIERS, RJ

49. SO, RP

50. HOPYAN, T

51. RENSBERGER. J

52. AGHVINIAN, M

53. ANDERSEN, T

54. AXELROD, J
The Relationship Between IQ and Effort Among Worker's Compensation and Fitness-for-Duty Examinees: A Replication of Findings in Criminal Defendants

Cross-Validation of TOMMe10 Among Criminal Defendants and Compensation-Seekers

Accuracy of TOMM Supplementary Indices Among Test Coached Simulators

Classification Accuracy of the Word Memory Test Genuine Memory Impairment Index

Development of a Combined Visual Spatial Performance Validity Test Using Logistic Regression

Relationship of Trauma \& Depression in Litigants \& Non-Litigants Following Concussion

Examination of the Classification Accuracy of the Word Choice Test with Stand Alone Performance

Validity Measures

The Relationship Between IQ and Effort in Worker's Compensation and Fitness-for-Duty Examinees:

Replication of WTAR-WAIS-III Findings with the TOPF and WAIS-IV

Examining the Utility of the RBANS Hartford Consistency Index in an Adult Outpatient Sample

CPT 3 Promising as an Embedded PVT for Pediatrics

\section{Inclusion and Diversity/Multiculturalism}

A Special Group Study of the Wechsler Intelligence Scale for Children, Fifth Edition: Deaf Children Who Utilize American Sign Language

Cross-cultural Analysis of the WAIS-IV Advanced Clinical Solutions (ACS): Cultural Identity and

Performance on Social Perception Subscales

Digit Span Performance in Russian and American Rural Populations: Cross-Cultural and Cross-

Linguistic Considerations

Differential Effects of Dual Language Exposure on Language and Nonverbal Problem-Solving in Children with ASD and ADHD

Baby, Let's Play Together: Maternal Behaviors and Child Development in Spanish and English Speaking Toddlers Born Preterm

Cross-Cultural Differences in Neuropsychological Performance: A Stereotype Threat Investigation Cross-Cultural Differences in Neuropsychological Performance: The Contributions of Acculturation and Academic Self-Identification

Influence of Demographic Variables on Measures of Attention and Working Memory for Spanish Speaking Hispanic and Matched White Older Adults

\section{Neuropsychiatry}

Initial-Trial Learning is Associated with Severity of PTSD Symptoms in OEF/OIF/OND Veterans Neurocognitive Functioning in Inpatient Children Following Childhood Maltreatment and the Role of PTSD

Impairments in Affective Flexibility May Improve When Depression Remits

Subcortical Features of Dorsolateral Prefrontal Circuitry in Neuropsychologically Near-Normal Schizophrenia

Intellectual Functioning and Not Cognitive Performance Predicts Functional Outcomes in Bipolar Disorder

Impulsivity and cerebral blood flow in bipolar disorder

Patterns of Verbal Memory Performance in Depressed Inpatients

Effects of Selective Serotonin and Serotonergic-Noradrenergic Reuptake Inhibitors on

Neuropsychological Performances in the United States

Stress Symptoms, Fatigure, Sleep and Pain, as well as Netropsychologieal Test Performance in Hospitalized Vietims of Terror

Gender Differences in Social Cognition in Bipolar Disorder with Possible Links to Inflammation Differential Effects of Increased BMI on Working Memory Performance in Individuals with Bipolar Disorder Compared to Unipolar Depression and Controls

Depressive Symptoms and Cognitive Decline in Younger- and Middle-Aged Adults: Cross-Sectional Analyses from the Framingham Heart Study

Characterizing the Neuropsychological Profiles of Those With and Without a History of Childhood Trauma Among a Sample of Psychiatric Inpatient Adolescents

\section{Other}

Neurocognitive Effects of Drug-Induced Changes for Choice Reaction Time in Recreational Polydrug Users

Hypothalamic pituitary adrenal axis functioning and PTSD symptoms in Army National Guard Special Forces

\section{Schizophrenia}

Social Functioning, Empathy, and Working Memory in Schizotypy

Altered Neuromagnetic Evoked Responses Related to Visual Habituation in Schizophrenia Clinical phenotypes in psychosis: Relationship between catechol-O-methyltransferase with positive and negative symptoms 


\author{
55. BUCHHOLZ, A \\ 56. CRAWFORD, J \\ 57. HENRICKS, AM \\ 58. JACKSON, A \\ 59. SHAAFI KABIRI, N \\ 60. MATSUI, M \\ 61. MOLL, A
}

62. PRILUCK, JL

9:00-10:30 АM

1. MOSCOVITCH, M

2. NADEL, L

3. WINOCUR, G

4. MCANDREWS, M

5. MOSCOVITCH, M

9:00-10:30 АM

1. GREEN, $\mathrm{R}$

2. COLELLA, B

3. TERPSTRA, A

4. VASQUEZ, B

9:00-10:30 AM

1. WILKINSON-SMITH, A

2. HARDER, L

3. HOWARTH, RA

4. WILKINSON-SMITH, A

9:00-10:30 AM

1. STIVER, J

2. MUNIZ, MC

3. ALIOTO, AG

4. CHAN, M

5. KARR, JE

6. MAHONEY, EJ

7. VOGT, E
Clarifying the Effect of Interference on Task Switching Latency in Schizophrenia The Contributions of Olfaction to the Neuropsychological Assessment of Schizophrenia Patients Comorbid Cannabis use Disorder is not Associated with Improved Cognition in Patients with Schizophrenia

Semantic Processing On Verbal Fluency Tasks In Schizophrenia Spectrum Disorder Intra-Individual Variability and its Relations with Cognitive Functioning in Healthy Volunteers and Subjects with Schizophrenia

Subjective and Objective Cognitive Impairment in Schizophrenia

Endothelial Nitric Oxide Synthase (eNOS), Metabolic Syndrome, and Cognition in SchizophreniaSpectrum Disorders

\section{Executive Functions/Frontal Lobes}

Verbal Fluency for Emotion Following Traumatic Brain Injury

Invited Symposium 1. Multiple Trace Theory (MTT) at 20: A Review of Theoretical, Empirical and Clinical Developments, and Future Directions Chair: Morris Moscovitch

Presenters: Lynn Nadel, Gordon Winocur, Mary Pat McAndrews,

Morris Moscovitch

Ballroom Salon 2-3

Multiple Trace Theory at 20: A review of theoretical, empirical and clinical developments, and future directions

MTT: Origins and Predictions

Trace Transformation Theory and its Implications

Clinical Implications of Multiple Trace and Trace Transformation Theories

MTT and TTT: Limitations and Future Directions

\section{Symposium 1. Remote and Progressive Effects of Brain Injury: Novel} Findings and Novel Treatment Approaches

Chair \& Discussant: Robin Green

Presenters: Brenda Colella, Alexander Terpstra, Brandon Vasquez

Ballroom Salon 1

Remote and Progressive Effects of Brain Injury: Novel Findings and Novel Treatment Approaches Neural, cognitive and mood decline in the chronic stages of moderate-severe TBI: Implications for treatment

Remote Effects of Multiple Concussions in Retired Professional Athletes

Variability Increases in the Chronic Stages of Moderate-Severe TBI

\author{
Symposium 2. Neuropsychology of Pediatric Neuroimmunological \\ Diseases \\ Chair, Presenter, \& Discussant: Alison Wilkinson-Smith \\ Presenters: Lana Harder, Robyn A. Howarth \\ Maryland A-C \\ Neuropsychology of Pediatric Neuroimmunological Diseases \\ Neuropsychology of Pediatric Multiple Sclerosis and Related Disorders \\ Neuropsychology of Anti-NMDA Receptor Encephalitis \\ Neuropsychology of Antibody-Negative and Presumed Autoimmune Encephalitis
}

\section{Paper Session 1. Assessment \\ Moderator: Justin B. Miller \\ Virginia A-C}

The Rapid Naming Task: Pilot Data on a Novel Naming Paradigm for Older Adults

The Relationship Between Performance Errors and Amyloid Burden in Clinically Normal Older Adults White matter correlates of the UCSF Brain Health Assessment

Differential Item Functioning of the Everyday Cognition (ECog) Scales in Relation to Racial/Ethnic

Groups

The Other Side of the Bell Curve: Multivariate Base Rates of High Scores in Executive Function

Assessment

Identifying New Targets for Detecting Memory Malingering: Insights from a Combined fMRI and Eye

Tracking Investigation

Child and Adolescent Validity Cut-Scores for the Victoria Symptom Validity Test and Reliable Digit

Span-Revised 
9:30-10:45 AM

1. ASKEN, BM

2. BITAN, Z

3. BURNS, AR

4. BURNS, AR

5. CAPOZZOLI, MC

6. COLBERT, AM

7. COOK. NE

8. DUNCAN B

9. KIRKWOOD, M

10. KIRKWOOD, M

11. FIELDS, L

12. FONG, AK

13. GALLAGHER, V

14. GERRALD, L

15. GHILAIN, C

16. GUTY, E

17. HOFFMAN, SN

18. HOUCK, ZM

19. JOSEPH, KR

20. JURICK, SM

21. JURICK, SM

22. KELLER, AV

23. LEVAN, AJ

24. LIMA, E

25. MALLECK, M

26. MANDERINO, LM

27. MORENO, CC

28. O'NEILL, JA

29. PETERSON, J

30. PETRAUSKAS, VM

31. RABINOWITZ, AR

32. REGINELLA, VM

33. REYNOLDS, M

34. RICHARDSON, RJ

35. RITCHIE, KA

36. SCAVONE, A

37. SCHLEICHER-DILKS, S

38. SHAPIRO, E

39. SVINGOS, AM

40. TAYLOR, B

\section{Poster Session 3. Concussion Exhibit Hall B North (Exhibition Level)}

\section{Concussion}

Baseline Serum Biomarker Concentrations: Association with Clinical Measures and Brain Trauma History

Establishing A Reference Database For Collegiate Athletes Using The Elite Balance Protocol Reliability and validity of the Concussion Learning Assessment and School Survey (CLASS): A measure assessing academic problems following concussion

Neuropsychological Assessment as a Function of Time since Injury Following Pediatric Concussion Predictors of Post-Concussion Symptom Invalidity Using the Validity-10 in Treatment-Seeking Adults With Persistent Complaints After Mild Traumatic Brain Injury

Comparing Subjective and Objective Cognitive Functioning in Youth with Persistent Postconcussive Symptoms

Athletes with ADHD Take Longer to Return to School but not to Sports Following Concussion An Analysis of Performance on Measures of Performance Validity and Self-Report of Concussion Symptoms

Parent and Child Agreement on a Postconcussive Symptom Measure: Baseline Data from Competitive Youth Soccer Players

Symptom Outcomes Following Concussion from a Prospective Study of Competitive Youth Soccer Players

Number of Concussions Not Associated with Neuropsychological Performance in Retired NFL Players Functional NeuroCognitive Imaging: Effective Identification for PCS

The Effects of Sex and Hormonal Contraception on Outcomes Following Collegiate Sports-Related Concussion

The Relationship Between Preexisting Conditions and Recovery Patterns in Pediatric Concussion Patients

ImPACT-Pediatric versus ImPACT: Pediatric Sample Differences in Baseline Performance

Executive Functioning in College Students with Chronic Postconcussion Symptoms

The Role of Pain Catastrophizing in Cognitive Functioning Among Veterans with History of Mild Traumatic Brain Injury

Exploratory Analysis of the Relationship Between Symptom Clusters and Neurocognitive Functioning in a Concussed Sample Presenting to a Specialty Clinic

National Institute of Neurological Disorders and Stroke, National Institutes of Health and Department of Defense Common Data Language for Clinical Research Studies: Sports-Related Concussion Common Data Element Recommendations

Association Between Multiple Concussions and Return to School and Sports in High School and College Athletes

Can Trauma-Focused Treatment Improve Poor Neuropsychological Performance Validity in Veterans with PTSD and History of mTBI?

The Interactive Effects of Traumatic Brain Injury Burden and Cognitive Functioning on Suicidal Ideation in Veterans

Working Memory Functioning Following Pediatric Concussion

Using Tests of Validity to Predict Performance on Neurospcyhological Exams

The Effect of Menstrual Cycle Phase and Birth Control Pill Usage on Post-Concussive Symptom

Reporting in Non-Concussed Adults

Self-Reported Constancy in Sports Is Correlated with Previous Concussions Resulting in Loss of Consciousness

Stability of baseline serum biomarker concentrations in female collegiate athletes

The Relationship Between the Sport Concussion Assessment Tool $-3^{\text {rd }}$ Edition (SCAT-3) and

Neuropsychological Functioning in Adolescents with Concussion

The ImPACT and performance validity: Examination of standard and experimental validity indicators

Concussion Knowledge and Self-Reported Attitudes in Middle School and High School Athletes Financial Incentives Influence ImPACT Validity Indicator Measures but not Cognitive Composite Scores

Concussion with Loss of Consciousness \& Depression

Comparing Perceptions of Mild Traumatic Brain Injury to Orthopedic Injury

Sex Differences and Post-Concussion Symptom Reporting in a Large Clinical Sample

Premorbid intellectual functioning impacts baseline concussion screening performance

Performance Validity at Baseline Testing on the ImPACT in Young Athletes with ADHD

Examining Performance on Cognitive, Effort, and Psychological Measures Among Individuals With Concussion, Controls, and Coached Dissimulators

Impact of Mild Traumatic Brain Injury on PTSD Treatment Outcomes

Micro-RNA Upregulation Following Sport-Related Concussion in Collegiate Athletes

Brief Cognitive Testing in the Acute Stage of Concussion: Does it Increase Symptoms? 
41. UY, PJ

42. VARGAS, G

43. WERHANE, ML

44. ZUCCATO, BG

45. COHEN, J

10:30-10:45 AM

10:45-11:45 АМ

1. MILLER, EK

11:45 AM-1:15 PM

1. KANE, RL

2. KANE, RL

3. VINCENT, A

4. KAY, C

5. SEATON, K

6. WILLIAMS, T

11:45 AM-1:15 PM

1. MADORE, $\mathrm{M}$

2. MACDONALD, B

3. SALINAS, C

4. VERNEY, SP

11:45 AM-1:15 PM

1. NGUYEN, DV

2. ZIMMERMAN, ME

3. KRESGE, HA

4. SEMMEL, E

5. PARKER, HA

6. JENKINS, LM
Symptom Endorsement Validity on the Sports Concussion Assessment Tool 3 (SCAT3)

Neuropsychological Test Performance in Young Children After Concussion

Apolipoprotein E- $\varepsilon 4$ Genotype and Pulse Pressure Interact to Affect Cortical Thickness in Brain Regions Vulnerable to Neurodegeneration in Veterans with Mild Traumatic Brain Injury Latent Structure of Post-Concussion Symptoms in Athletes at Baseline

\section{Neuroimaging}

Adults with Higher Cardiorespiratory Fitness Show Evidence for Greater Neural Efficiency During the Stroop Task

AM Coffee Break

Exhibit Halls B North (Exhibition Level)

Plenary B. Rule + Rhythms = Cognition

Presenter: Earl K. Miller

Ballroom Salon 2-3

Rule + Rhythms = Cognition

Invited Symposium 2. Neurocognitive Function in Aerospace and Aeronautics

Chair: Robert L. Kane

Presenters: Robert L. Kane, Andrea Vincent, Gary Kay, Kimberly Seaton,

Thomas Williams

Ballroom Salon 2-3

Neurocognitive Functioning in Space and Aviation

Development of the Spaceflight Cognitive Assessment Program

Neurocognitive Health During Space Flight

Aviation Clinical Neuropsychology

Cognitive Assessment in Long-Duration Space Flight

WinSCAT Test-Retest Reliability in Senior Military Leaders

\section{Symposium 3. Increasing Minority Engagement in the Field of \\ Neuropsychology \\ Chair: Michelle Madore \\ Presenters: Beatriz MacDonald, Christine Salinas, Steven P. Verney, Justina Avila \\ Ballroom Salon 1}

Increasing Minority Engagement in the Field of Neuropsychology

What Happens Beyond Active Recruitment and Enrollment?

State of the Art in Cultural Neuropsychology Mentoring \& Training: Results from the Hispanic

Neuropsychological Society Mentoring Program and AACN Relevance 2050 Survey of postdoctoral programs in the U.S

Engaging in Difficult Dialogues towards Multicultural Knowledge, Attitudes, and Skills in

Neuropsychology: Peer-to-Peer Discussions and Interactions

\section{Paper Session 2. Adult Medical Disorders \\ Moderator: Cady K. Block \\ Maryland A-C}

Post-Shunting Cognitive Outcomes in Normal Pressure Hydrocephalus Patients

Neuropsychological Function and Nocturnal Blue Light Exposure in Insomnia: A Pilot Randomized Controlled Study

Subclinical Compromise in Cardiac Strain Relates to Worse Neuropsychological Performances in

Older Adults: The Vanderbilt Memory \& Aging Project

Cerebellar Volume and Executive Function in Congenital Heart Disease

Evaluating the Moderating Role of Medical Comorbidity, Pain, and Depression on the Relationship

Between Sleep Biomarkers and Cognitive Functioning in Obstructive Sleep Apnea

Reduced Local Connectivity of the Medial Prefrontal Cortex Predicted by Slower Executive

Functioning in Middle Aged Adults at Risk for Cardiovascular Disease 
11:45 AM-1:15 PM

1. ZAKRZEWSKI, JJ

2. TOOMEY, R

3. SALMINEN, L

4. KEILP, JC

5. GORLYN, M

6. JAYAKAR, R

\section{2:00-1:15 PM}

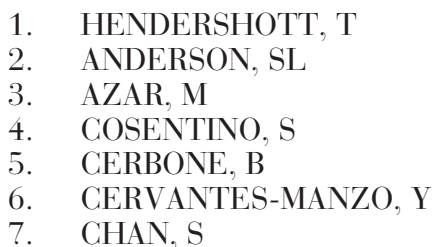

8. JOYCE, J

9. CHAPMAN, S

10. MEINERDING, ME

11. CHIRLES, T

12. CHOUDHURY, TK

13. CLARK, LR

14. CRIVELLI. I

15. D'ERRICO, I

16. DRZEWIECKI, MA

17. DUTT. S

18. CLARENS, M

19. FLOWERS, AT

20. GRAVES, LV

21. HACKETT, K

22. BRICKMAN, AM

23. HALLIDAY, D

24. EBNER, M

25. KORTHAUER, LE

26. LIN SS

27. MALONE, C

28. MAXA, KM

29. MCINTOSH, EC

30. MILLER, AK

31. NAKHLA, MZ

32. NANCE, C

33. PUGH. EA

34. RINDGE, M

35. SCHAFFERT, J

\section{Paper Session 3. Mood and Anxiety Disorders Moderator: Sara Weisenbach Virginia A-C}

Effect of Treatment on Neurocognitive Function in a Treatment Seeking Sample of Individuals with Hoarding Disorder

Neurocognitive deficits in remitted vs. current depression: Findings from the Vietnam Era Twin Study of Aging (VETSA)

Hippocampal Subregion Abnormalities in Current and Lifetime PTSD: International Analysis from the PGC-ENIGMA PTSD Working Group

Neuropsychological Dysfunction in Planned and Unplanned Suicide Attempts

Neurocognitive Impairment in Depression is Distinct from Ratings of Mood and Cognitive Problems Association Between Amygdala Volume and Social Anxiety Symptom Severity: A Multi-Method Study

\section{Poster Session 4. MCI and Dementia \\ Exhibit Hall B North (Exhibition Level)}

\section{Dementia (Alzheimer's Disease)}

Informant and Self-Reports of Navigation Abilities in Preclinical Alzheimer's Disease

Depression's Influence on Cognitive Functioning in Older Adults who Develop Alzheimer's Dementia Processing Speed best Classifies Autopsy Confirmed Alzheimer's disease versus AD with Lewy Bodies Awareness in Alzheimer's Disease: Self and Other-Evaluation

Predictive Utility and Longitudinal Correlates of Phonemic Cueing Benefit in Alzheimer's Disease Factors Affecting Older Adults' Fear of Developing Alzheimer's Disease

Does Statin Use Mitigate Risk of Conversion to Alzheimer's Disease? The Association between Statin Use and Incidence of Alzheimer's Disease by Gender and Race

Age-Anchored Subjective Cognitive Decline is Sensitive to Short-Term Memory Binding

Cross Domain Self-Monitoring in Anosognosia for Memory Loss in Alzheimer's Disease

Retrospective Subjective Cognitive Decline relates to Essentialist Beliefs and Subjective Age

Functional Connectivity Patterns Associated with Aging, Physical Activity, and Genetic Risk for Alzheimer's Disease

Statistical Model of Dynamic Markers of the Alzheimer's Pathological Cascade

A Comparison of Subjective Cognitive Decline Across Cohorts: The Wisconsin Registry for

Alzheimer's Prevention and The Vanderbilt Memory \& Aging Project

Algorithm for the Detection of Risky Driving in Subjects with Mild Dementia

Probability of First Recall in Asymptomatic Older Adults with CSF Biomarkers of Alzheimer's Disease

Correlates of Hot and Cold Cognition in a Neurodegenerative Population

Hippocampal Hyperperfusion is Associated with Worse Memory and Executive Function in Older Adults

Neuropsychological Biomarkers in Mild Cognitive Impairment due to Alzheimer's Disease

Differences in Caregiver Burden Amongst Individuals With Various Forms of Cognitive Impairment

Novel Recognition Discriminability in Alzheimer's and Huntington's Disease: New Insights from the CVLT-3

Perceptions of Childhood and Current Memory Abilities in Adults Presenting to a Memory Clinic Cognitive Complaints Among Older Adults are Associated Selectively with Entorhinal Cortex Thickness

Intraindividual Dispersion Profiles for Identifying the Cognitively Impaired

Statin Use and Risk of Cognitive Decline in the ADNI Cohort

Response Time Associated with Stimulus Uncertainty but Not Response Uncertainty Discriminates Alzheimer's Disease from Healthy Aging

Inferring Brain Change from Reliable Change Indices: AVLT and Brain Volume

False Memory, Response Bias, and Executive Functioning, in Alzheimer's Disease

Sedentary Behavior Associates with Vessel Pulsatility in the Brains of Adults at Risk for Alzheimer's

Disease

Untreated Hyperglycemia Accelerates Cognitive and Functional Decline in Older Adults

Relationship Between Cerebrospinal Fluid Biomarkers of Alzheimer's Disease and Neuropsychological Functioning

The Differences Between Semantic and Episodic Memory Predicting Shopping Skills in Alzheimer's disease and Mild Cognitive Impairment

Pathology of Rapid Cognitive Decline

Effects of Normative Adjustments on Test Characteristics of the Montreal Cognitive Assessment

Predictors of Conversion from Mild Cognitive Impairment to Alzheimer's Dementia Confirmed by Autopsy: A Pilot Study

Traumatic Brain Injury History and Survival After Dementia Onset in Autopsy-Confirmed

Alzheimer's Disease 


\author{
36. SHOUEL, HL \\ 37. STASENKO, A \\ 38. STOJANOVIC, M \\ 39. SUHR, J \\ 40. SUNDERMANN, EE \\ 41. UNGRADY, MB \\ 42. VANDEBUNTE, AM \\ 43. VENKATESAN, UM \\ 44. VILA-CASTELAR, C \\ 45. WANK, AA \\ 46. WEISSBERGER, G
}

47. WEISSBERGER, G

48. BOEVE, A

49. BULL, TP

50. COHEN, C

51. DALLEY, BC

52. DALLEY, BC

53. DEL BENE, $V$

54. DEL BENE, V

55. DEL BENE, V

56. DEVITO, AN

57. ECONOMOU, A

58. EDMONDS, EC

59. EMRANI, S

60. EPPIG, J

61. EVANS, SA

62. GONZALES, M

63. HSU, Y

64. LOPEZ, M

65. CHAR, D

66. KREMEN, WS

67. LAGEMAN, SK

68. LEBOWITZ, BK

69. MACAULEY,

70. COOK MAHER, A

71. MIS, R

72. NORMAN, AL

73. ROCHETTE, AD

74. SMITH, EE

75. STEWARD, K

76. SZELES, DM
Social Support and Cognition in White and African American Older Adults at Risk for Alzheimer's Disease

Sensitivity of the Multilingual Naming Test (MINT) to Level of Cognitive Impairment Across the

Alzheimer's Disease (AD) Spectrum

Exercise Engagement and Longitudinal Change in Alzheimer's Disease Biomarkers

Cognitive Outcomes in Caregivers of Individuals with Dementia

Sex-based norms in clinical tests of verbal memory might improve diagnostic accuracy in Alzheimer's disease

Predicting Lexical Dropout in Progressive Aphasia via Eye Tracking

The Effect of Depression on the Cognition of those with Alzheimer's Disease

Disentangling Deficits in Semantic Integration and Retrieval in Aging and Alzheimer's Disease

Attention predicts long-term cognitive, functional and behavioral response to donepezil in

Alzheimer's disease

Disrupted Retrieval Fluency for Remote Autobiographical Events in Cognitively Healthy

Apolipoprotein E e4 Positive Individuals

The Impact of Education on Neuropsychological Differences Detected in Early Preclinical Alzheimer's Disease: A Meta-Analysis

\section{MCI (Mild Cognitive Impairment)}

Intra-individual Variability in Reaction Time Performance Predicts BOLD Activation During a Working Memory fMRI Task in Healthy Older Adults and Older Adults with Mild Cognitive Impairment

MCI and Depression Increases Fall Risk Through Slowed Gait

Investigating Driving Performance in Older Adults with Mild Cognitive Impairment Amnestic Single-Domain and Multiple-Domain

Age of Acquisition and Typicality in Semantic Fluency and Mild Cognitive Impairment

Validating premorbid RBANS scores in older adults with amnestic MCI

Predicting premorbid RBANS scores in older adults

Trail-Making Test Performances of Patients with Amnestic and Non-Amnestic MCI

Financial capacity deficits in patients with amnestic and non-amnestic forms of mild cognitive impairment

Verbal memory decline on the California Verbal Learning Test-II (CVLT-II) in APOE 4 gene carriers: A replication study

Examining Differences in Neuropsychiatric Symptom Prevalence by Empirically Derived Mild Cognitive Impairment Subtype

Predictors of Accidents in Patients with Mild Cognitive Impairment, Mild Alzheimer's Disease and Healthy Controls in Simulated Driving

Improved Staging of "Early" and "Late" MCI Using a Neuropsychological Approach

Visual versus Verbal Serial List Learning in Mild Cognitive Impairment: A Comparison Between the BVMT-R and the CVLT-MS

Considerable Cognitive Heterogeneity in 'Amnesic Mild Cognitive Impairment' in ADNI: A

Longitudinal Latent Profile Analysis

Initial Visit Predictors of Diagnostic Discrepancy Between Clinician and Test-Based Diagnoses of

Mild Cognitive Impairment

The Association Between Chronic Depressive Symptomatology and Cerebrospinal Fluid Biomarkers in Mild Cognitive Impairment

Prospective Memory Performance and Microstructural Correlates in Subjective Cognitive Decline and Mild Cognitive Impairment

Differences in Time to Conversion Between Subtypes of Amnestic Mild Cognitive Impairments Subtypes of Amnestic Mild Cognitive Impairments and Functional Status

Improving the Diagnosis of MCI and the Potential for Early Identification of Alzheimer's Disease Risk Clinical Trial Results Comparing a Neurocognitive Intervention to Supportive Therapy in Individuals with Parkinson's Disease

White/Gray Matter Correlates of Math Fluency and Error Analysis in Community-Based Older Adults Dual-Task Gait Assessment in a Clinical Sample: Implications for Improved Detection of Pre-Clinical Dementia

Racial Differences in Clinician-Test Diagnostic Discrepancy of Mild Cognitive Impairment with Executive Deficits

Heterogeneity of Informant-Reported Functional Performance in Mild Cognitive Impairment:

A Latent Class Analysis of the Functional Activities Questionnaire

Mild Cognitive Impairment: 5-Year Growth Trajectories

Mild Cognitive Impairment Category Predicts Weight Loss Following Bariatric Surgery

A Comparison of Three Neuropsychological Instruments in Detecting Mild Cognitive Impairment (MCI) in African Americans

Relationship between BDNF Val66Met Polymorphism and Processing Speed in Older Adults with Amnestic MCI and Probable Dementia

Dual-Task Gait Performance in Normal Aging and Mild Cognitive Impairment Subtypes 
77. TIMPANO SPORTIELLO, MR

78. TIMPANO SPORTIELLO, MR

79. VAN PATTEN, $R$

80. WASSERMAN, VJ

81. WEAKLEY, A

82. HEWITT, KC

83. HEWITT, KC

84. CARMICHAEL, AM

85. CHANEY, G

86. CHERAN, G

87. SABSEVITZ, DS

88. WESBECHER, K

\section{1:15-2:15 PM}

2:15-3:15 PM

1. BUXBAUM, LJ

2:15-3:45 PM

1. SHAPIRO, D

2. WARSCHAUSKY, S

3. SHAPIRO, D

4. WADE, SL

5. HURWITZ, A

6. TAYLOR, H

\section{2:15-3:45 PM}

1. LAMAR, M

2. PRICE, CC

3. RENTZ, DM

4. AU, R

5. GERSHON, $\mathrm{R}$

6. KING, J
Comparison Between Free and Cued Selective Reminding Test and Wechsler Memory Scale-IV Edition: A Descriptive Study on Amnesic Mild Cognitive Impairment

Visual Reproduction of the Wechsler Memory Scale-Fourth Edition as a Sensitive and Specific test for Amnestic Mild Cognitive Impairment Diagnosis

Enhanced clinical utility of the Modified Mini Mental State Examination compared to the MMSE in older adults with mild cognitive impairment

Visual Serial List Learning in Mild Cognitive Impairment: An Analysis of Learning Curves and Errors of Commission

Naturalistic Assessment of Task Interruption in Individuals with Mild Cognitive Impairment Application of Virtual Reality-Based Neuropsychological Assessments within Mild Cognitive Impairment

\section{Dementia (Non-AD)}

A Neuropsychological Case Study of Dementia with Lewy Bodies Autobiographical Memory in Huntington's Disease

The Neuropsychological Profile of Abnormal Feeding Behavior in Behavioral Variant Frontotemporal Dementia

Structural Brain Changes in Preclinical Tauopathic Behavioral-Variant Frontotemporal Dementia (bvFTD)

Early Episodic Memory Loss in Semantic Dementia: A Phenotypic Variant?

Postoperative Cognitive Dysfunction Following Total Knee Arthroplasty: A Case Study

\section{Lunch (On Own)}

Conference-Wide

Benton / Mid-Career Awardee Presentation: Objects and Actions in the Healthy and Damaged Brain Award Recipient: Laurel J. Buxbaum Delaware A-B

Tools in the Mind: Objects and Actions in the Healthy and Damaged Brain

Symposium 4. Technological advances in pediatric neuropsychological assessment and intervention Chair \& Presenter: Danielle Shapiro

Discussant: H. Gerry Taylor

Presenters: Seth Warschausky, Shari L. Wade, Andrew Hurwitz Ballroom Salon 2-3

Technological advances in pediatric neuropsychological assessment and intervention Challenges in utilizing technology for accessible testing

\#BrainInjury: Using social media analysis as a naturalistic measure of change associated with acquired brain injury

Telehealth interventions for youth with acquired brain injury: Lessons learned

Sensors as a social intervention: Understanding how schools utilize head impact sensors

Discussion of issues raised and future directions

Symposium 5. The Present and Future of Technology in Neuropsychological Assessment: What is Feasible (and Fundable) Today, Tomorrow and in Years to Come

Chair: Melissa Lamar

Discussants: Richard Gershon, David Libon, Daniel A. Nation, Dana Penney, Jonathan King, Wendy Nilsen, Nina Silverberg, Molly Wagster

Presenters: Catherine C. Price, Dorene Rentz, Rhoda Au Ballroom Salon 1

The Present and Future of Technology in Neuropsychological Assessment: What is Feasible (and Fundable) Today, Tomorrow and in Years to Come

Digital Pen Technology

iPad and Digital Based Assessments in Clinic and Home Environments

e-Cog and e-Home Technologies: Wearable Activity Monitoring and Assessment Technology Round Table Discussion 1: Clinical \& Research Neuropsychologists

Round Table Discussion 2: NIA/NIH and NSF Stakeholders 
2:15-3:45 PM

1. WADE, SL

2. SCHORR, EM

3. MILLER, C

4. BUSSELL, CA

5. WILMOTH, K

6. RASKIN, S

2:15-3:45 PM

1. COLVIN, LE

2. SPERLING, SA

3. DHIMA, K

4. BROWN, DS

5. HIZEL, L

6. MORAN, EE

7. COSTABILE, T

2:30-3:45 PM

\section{Paper Session 4. Concussion and Traumatic Brain Injury Moderator: Nyaz Didehbani Maryland A- $\dot{\mathrm{C}}$}

Behavior Problems following Childhood TBI: The Role of Sex, Age, and Time Since Injury The Relationship Between Pre-Injury Internalizing Symptoms and Post-Concussive Symptoms in Children with Mild Traumatic Brain Injury and Orthopedic Injury

Detecting ADHD symptoms in young athletes using the ImPACT

Effects of Media Sensationalization on Cognitive Performance and Post Concussive Symptoms Psychological and Injury-Related Factors as Predictors of Prolonged Return to Play in Adolescents following Sports-Related Concussion

Time Perception and Prospective Memory in People with Brain Injury

\section{Paper Session 5. Parkinson Disease and Movement Disorders Moderator: Dawn Schiehser Virginia A-C}

Exploring the association between psychological characteristics and metacognition among adults with essential tremor

Non-Motor Outcomes and Quality of Life After-Focused Ultrasound Thalamotomy in TremorDominant Parkinson Disease

A Preliminary Examination of Parkinson's Disease Subtypes and Associated Differences in Cognitive and Motor Symptom Trajectories

Cognitive Correlates of Apathy in Parkinson's Disease

Digestive health in Parkinson's disease relates to fatigue, pain, and affective dysfunction but not cognition

Cognitive and Motor Functioning in Heterozygous Non-manifesting Glucocerbrosidaise (GBA) Carriers

Emotion Recognition and Psychological Comorbidity in Friedreich's Ataxia

\section{Poster Session 5. Child Medical and Neuropsychiatry Exhibit Hall B North (Exhibition Level)}

\section{ADHD/Attentional Functions}

\begin{tabular}{ll} 
4. & AERBE, A \\
2. & AERBE, A \\
3. & ALI, J \\
4. & ALI, S \\
5. & ANGERS, K \\
6. & BAILEY, BA \\
7. & BORRANI, J \\
8. & BROOKER, B \\
9. & COOK, C \\
10. & DE LA GARZA, V \\
11. & ELLIS, A \\
12. & GALLEGOS, $\mathrm{C}$ \\
13. & HAMILTON, SK \\
14. & HIDALGO, GM \\
15. & IRWIN, LN \\
16. & KATZ, LA \\
17. & KIM, K \\
18. & KORHONEN, N \\
19. & LEE, GJ \\
20. & MARCELLE, ET \\
21. & MILLER, C \\
22. & MURO, A \\
\hline
\end{tabular}

WHAHORAWN Ftexibility Gapacity. Differenees Between Young and Otter People in Nesplora Aquarium Test WITHORAWN Nesplora Aquarium: Utility of the Tool to Identify People with Attention-Defieit Hyperaetivity Đisorder

Attention, Executive Function, and Transition to Adult Care Among Youth with Sickle Cell Disease Intra-Individual Variability in Children is Related to Parent Ratings of Attention, but not Teacher Ratings

Indices of Self-Report Validity in Attention-Deficit/Hyperactivity Disorder (ADHD) Symptomatology and Its Relationship to Self-Reported Functional Impairment

The Contribution of Executive Dysfunction to Social Self-Concept in Youth with ADHD

Analysis of ADHD and Conduct Disorder Symptoms, Attention and Executive Functions in Juvenile Delinquents

Differential Associations of Inattention and Impulsivity Across Modes of Neuroenhancement Behavior Relationship of Performance Validity Test Failure to Self-Reported Functional Impairment in Attention-Deficit/Hyperactivity Disorder (ADHD)

Analysis of Components of Attention Under low Stimulation

Frontal Alpha Asymmetry Predicts Inhibitory Processing in Youth with Attention Deficit/

Hyperactivity Disorder

Effects of practice on the attentional blink

Experiential Avoidance and Attention Among Stressed Graduate Students

Preschool ADHD but not Neuropsychological Functioning Is Associated with Elevated School-Age BMI

Is There a Functional Relationship Between Set Shifting Demands and Hyperactivity in Children With and Without Attention-Deficit/Hyperactivity Disorder?

Inhibitory Control in Children with Pediatric Bipolar Disorder and Attention-Deficit/Hyperactivity Disorder

Sluggish Cognitive Tempo and Executive Functioning in Children With Learning Difficulties Childhood ADHD Symptoms and Intellectual Functioning in Adulthood

The Effects of Treatment Expectancy on Subjective and Neuropsychological Outcomes in Neurofeedback (NFB) for Attention-Deficit/Hyperactivity Disorder (ADHD): A Pilot Study Hypertensive Pregnancy Predicts Improved Working Memory Functioning in ADHD Mindfulness-based Interventions as Complementary Therapy for Attention Problems Analysis of the components of attention in early and late adolescents 
23. NITTA, ME

24. OSTOJIC. D

25. ROSCH, KS

26. RICHARDSON, RJ

27. ROECKNER, AR

28. ROITSCH. J

29. ROSCH. KS

30. SULLIVAN, E

31. SWEENEY, KL

32. TADROUS-FURNANZ, SK

33. TAMEZ, S

34. VAN DESSEL, J

35. WALLACE, ER

36. GONZALES, EC

37. GONZALES, EC

38. CUREWITZ, AM

39. HEITZER, A

40. HOOD, A

41. HOOD, A

42. ISAAC, L

43. KАMATH, NN

44. MATTES, A

45. NORRIS, T

46. PIERCY, J

47. PLIEGO, JA

48. POMMY. J

49. RIEGER, RE

50. SKILLICORN, K

51. SVINGOS, AM

52. THOMPSON J

53. TURNER, EM

54. THOMASON, MM

55. THOMASON, MM

56. BRADBURY, KR

57. BRADSTREET. LE

58. CASEY, JE

59. DAI. Y

60. DIAMOND, BJ

61. DIAZ-ORTIZ, LM

62. EVANS, L

63. FLORES, A

64. КОСН. S
Performance and Symptom Validity does not Impact the two Dimensional Structures of Barkley's Adult ADHD Rating Scale-IV Self-Report Measures

Using Ecological Momentary Assessments to Evaluate Extant Measures of Mind Wandering

Predicting Adolescent Risk-Taking in Attention-Deficit/Hyperactivity Disorder: A Longitudinal Study

Errors on a Graphomotor Tracking Task as a Predictor of Pediatric ADHD

Comparison of Motor Cortex Physiology During Response Inhibition in Children with ADHD vs.

Controls

Predictors of Comfort Level in Speech-Language Pathologists (SLPs) Working with Students with

Attention Deficit Hyperactivity Disorder (ADHD)

Reduced Value-Driven Attentional Capture Among Children with ADHD Compared to Typically

Developing Controls

Assessing the Relationship between PTSD Symptoms and Attention in a Trauma-Impacted Female Sample

Finger Tapping Speed in Preschool Predicts ADHD Status and Symptom Trajectory

Can Cogmed have Far-Transfer Effects on Reading for some Children with Emerging Needs?

Changes of Components of Attention in Children from 4 to 7 Years of age

The Amygdala in Attention-Deficit/Hyperactivity Disorder: Structural and Functional Correlates of Delay Aversion

Attention-Deficit/Hyperactivity Disorder Malingering Detection in College Students: A Meta-Analysis of Performance and Symptom Validity Tests

Socioeconomic Status and Neuropsychological Performance in College Students with and without ADHD Symptoms

\section{Medical/Neurological Disorders/Other (Child)}

Relationship Between Brain Structures and Working Memory in Preschoolers Born Preterm Emotional functioning in children with neurofibromatosis type 1 and Noonan syndrome The Fetoplacental Ratio and Neuropsychological Functioning of Preschool-Age Children Born Prematurely

Cognitive Developmental Trajectories in Children with Sickle Cell Disease

IQ and Grade Retention Predict Poor Executive Functioning for Children with Sickle Cell Disease

Sex-Specific Processing Speed Recovery in Pediatric Brain Tumor Survivors

Ethnicity, SES, and Adaptive Function as Predictors of Health-Related Quality of Life Among

Children and Adolescents with Spina Bifida Myelomeningocele

Retinopathy of Prematurity and Neuropsychological Functioning at Preschool-Age

Subtypes of Attention Deficits in Survivors of Acute Lymphoblastic Leukemia

Arterial Cord pH and Neuropsychological Functioning of Preschool-Age Children Born Prematurely

Group Differences in Neurocognitive Profiles for Children Post Heart Transplant

Cortical Structure Associated with Executive Functions in Children Born Preterm

Socioeconomic Status, Family Stress, White Matter, and Language Abilities in Preschoolers Born

Preterm

Anti-NMDA Receptor Encephalitis: Serial Assessment of Bilingual 5 year old Female

Pediatric Anti-N-Methyl-D-Aspartate Receptor Encephalitis: Aftermath of a Brain on Fire

Cognitive Outcome in Individuals with Childhood Extracorporeal Membrane Oxygenation: A Case

Series

Dual Cases of Non-Verbal Learning Disorder Following Twin to Twin Transfusion Syndrome:

Coincidence or Causality?

Cognitive Effects of Subdural Empyema in an Adolescent Female

\section{Autism Spectrum Disorders}

Neurocognitive Phenotyping Distinguishes Schaaf-Yang Syndrome from Prader-Willi Syndrome Behavioral Comparison of High-Risk and Low-Risk Toddlers Identified with the Modified Checklist for Autism in Toddlers, Revised with Follow-Up

Relationships Between Autism Traits, Psychosis Traits, and Mentalizing in a Clinical-Community Sample

Differentiating Nonverbal Learning Disorder and Higher Functioning Autism

Training Albanian Parents of Children with Autism Spectrum Disorder via Video

Fearful Faces, Processing Speed and Autism Spectrum Disorder: Indexing Mild Autism with EEG Spectral Correlates

Hippotherapy and Molecular Markers in a Sample of Male Children with High Functioning Autism Disorder.

Neurological and Behavioral Functioning in Infants at High- and Low-Risk for Autism Spectrum Disorder

Relationship between Resting State Alpha Motor Network Synchrony and Restricted and Repetitive

Behaviors in Children with ASD

Measures of Social Perception as Predictors of Clinician-Observed and Parent-Rated Autism Symptomology 

65. LEE, CM
66. MEYERING, K
67. MILLER, LE
68. PANFILI, DP
69. POWERS, MD
70. RAU, S
71. SEGHATOL-ESLAMI, V
72. SHADA, K
73. SKAPEK, MF

74. PULSIPHER, DT

75. ALLAIRE, T

76. BECKER, $\mathrm{N}$

77. CIRINO, PT

78. FERNANDES, M

79. GUERIN. JM

80. GUERIN. JM

81. HARDY, LM

82. KIVISTO, LR

83. MACDONALD, K

84. OSSOWSKI, A

85. RIGGALL, E

86. VADNAIS, S

87. WINTER, R

88. JONES, EF

89. KIM, S

90. SCHOFIELD, H

91. YАMАMOTO, А

3:45-4:00 PM

4:00-5:00 PM

1. VAN TOL, M
Autistic Characteristics and Developmental Trajectories among Individuals with Fragile X Syndrome and Non-Syndromic Autism Spectrum Disorder

Web-Based Cognitive Assessments in Individuals with Autism Spectrum Disorder and their Immediate Family Members

The Relationship Between Socio-Emotional and Cognitive Development in Infants and Toddlers at Risk for a Developmental Disorder

Using Machine Learning on Resting State and DTI to Classify ASD

Linguistic Diversity in Neurodevelopmental Disorders: Dual Language Exposure Relates to Cognitive Flexibility in Children with ASD but Not ADHD

Comparing the Diagnostic Discriminability of the ADHD Rating Scale and Achenbach Checklists to Capture Comorbid Attention-Deficit/ Hyperactivity Disorder Symptoms in Autism Spectrum Disorder Functional Connectivity of the Fusiform Face Area with the Social Brain during Action Perception in Autism

Executive Function in ASD, ADHD, Comorbid ASD/ADHD and Typically Developing Children Assessed Using Parent Rating Scales and Task-Based Performance Measures

Anxiety Relates to Interference Associated with Planning and Flexibility Weaknesses in Youth with ASD

\section{Epilepsy/Seizures}

The Contribution of Executing Functioning to Memory Performance in Pediatric Focal and Generalized Epilepsy

\section{Learning Disabilities/Academic Skills}

A Cluster-Analytic Approach to Examining Links Between Psychodiagnostic Profiles and Neuropsychological Task Performance Among an Outpatient Clinical Sample

Double Disadvantage in Reading Performance: Socioeconomic Status amplify the Effects of Learning Disabilities on Reading Performance

Contributions of Executive Function to Reading

Exploring associations among verbal language knowledge, social anxiety, and nonverbal processing in children

Direct and Indirect Investment of Fluid Intelligence in Word Reading in Children Evaluated for Attention-Deficit/Hyperactivity Disorder

Connecting Statistical Learning with Reading: The Importance of Task-Driven Attention and Key

Mediating Variables

Neuropsychological Correlates of Bilingualism in a Sample of Children with Neurodevelopmental Disorders

What Factors Affect Children's Perceptions of Their Assistive Technology and Assistive Technology Training?

Longitudinal overlap of reading and math achievement and their predictors

Effects of ADHD Symptoms and Executive Function Impairments on Response to Reading Intervention

Deficits in Implicit Sequence Learning in Children with Dyslexia with and without Specific Language Impairment and Response to Reading Intervention

Right Inferior Frontal Gyrus Volume Predicts Math Calculation in Children with Reading Disability Working Memory Related to Decoding Gains Across Reading Intervention in Children with Developmental Dyslexia

\section{Other}

Improvements in Parent Self-Efficacy Ratings Following Pediatric Neuropsychological Assessment Gait Variability of Children According to Walking Path Width and Motor Development Level Tailoring Feedback Sessions for Young Adults with Cognitive Difficulties: Case Examples From Oncology

Cognitive Impairment in a Sample of Precariously Housed Youth

PM Coffee Break

Exhibit Halls B North (Exhibition Level)

Early Career Awardee Presentation: Neurocognitive Mechanisms Underlying Development, Course and Treatment of Major Depressive Disorder

Award Recipient: Marie-Jose van Tol

Delaware A-B

Neurocognitive Mechanisms Underlying Development, Course and Treatment of Major Depressive Disorder 
4:00-5:30 PM

\author{
1. LEVIN, HS \\ 2. BELANGER, H \\ 3. TATE, DF \\ 4. WALKER, W \\ 5. WILDE, EA
}

4:00-5:30 PM

1. MASH, LE

2. CASTELLUCCIO, B

3. ADUEN, PA

4. NGUYEN, T

5. TAN. A

6. VAN DESSEL, J

\section{4:00-5:30 PM}

1. ALTENDAHL, M

2. SANDERSON-CIMINO, M

3. CAVANAGH, L

4. RHODES, E

5. MELROSE, $\mathrm{R}$

6. MACPHERSON, SE

4:00-5:30 PM

1. $\mathrm{AU}, \mathrm{R}$

2. KAMALYAN, L

3. YOU, MY

4. SOLDAN, A

5. COLON, JM

6. SOL, K

4:00-5:15 PM

1. BABAKHANYAN. I
Symposium 6. Chronic Effects of Mild Traumatic Brain Injury in Veterans and Service Memvers on Cognition, Vestibular Function, Motor Performance, and Brain Imaging: A Biopsychological Model

Chair: Harvey S. Levin

Discussant: Tracy Nolen

Presenters: Heather Belanger, David F. Tate, William Walker,

Elisabeth A. Wilde

Ballroom Salon 2-3

Chronic Effects of Mild Traumatic Brain Injury in Veterans and Service Members on Cognition, Vestibular Function, Motor Performance, and Brain Imaging: A Biopsychological Model

Chronic Effects of mTBI in Veterans and Service Members on Cognition: A Biopsychological Model Predicting Concussion History Using Diffusion Imaging in Veterans: Results from the Chronic Effects of Neurotrauma Consortium (CENC)

The Relationship Between Historical Mild Traumatic Brain Injury and Balance Performance: A Chronic Effects of Neurotrauma Consortium (Cenc) Multi-Center Observational Study Interim Analysis

Preliminary Findings on Motor Functioning in the Chronic Effects of Neurotrauma Consortium

\section{Paper Session 6. Pediatric Neuropsychiatry \\ Moderator: Lana Harder \\ Ballroom Salon 1}

Atypical Relationship Between Static and Dynamic Functional Connectivity in Autism Spectrum Disorders

Neural Mechanisms of Generalization for Language Learning in Autism Spectrum Disorder Neurocognitive Predictors of Motor Vehicle Crashes: A Prospective Cohort Study Language Functioning at 13 Years and its Developmental Trajectory from 2 Years in Very Preterm Children

Neurocognitive and Psychosocial Profiles of Pediatric Multiple Sclerosis and Transverse Myelitis Distinctive Neural Response Towards Certain and Conditional Monetary Loss Anticipation in Adolescents

Paper Session 7. Cognitive Reserve

Moderator: Rosemary Fama Maryland A-C

REM Sleep Associated with White Matter Integrity in Cognitively Healthy, Older Adults Evaluation of Phenotypic and Genetic Indices of Cognitive Reserve in Middle Age

The impact of current leisure activities on cognitive and brain reserve among community-dwelling adults

Factor Structure of Grit in Older Adults: Consistency and Perseverance in Successful Aging Compensatory Scaffolding of Prefrontal Cortex in Cognitive Aging

The Impact of Age, Aetiology and Cognitive Reserve on the Cognitive Performance of Frontal and Posterior Patients

\section{Paper Session 8. Neurocognitive Trajectory and Aging} Moderator: Kamini Krishnan Virginia A-C

Midlife Metabolic Syndrome and Late-Life Cognitive Trajectories in the Framingham Heart Study Effect of Lifetime Occupation Level on Cognitive Trajectory among Diverse Older Adults Subjective Cognitive Symptoms Predict Cognitive and Structural Brain Aging Trajectories in Otherwise Healthy Older Adults

Self-reported lifestyle activity and relationship to longitudinal cognitive change Relationship of African Ancestry with Cognitive Trajectory among Older Latino/as Psychosocial Predictors of Memory Decline in a Diverse Longitudinal Sample of Older Adults

Poster Session 6. Acquired Brain Injury Exhibit Hall B North (Exhibition Level)

Acquired Brain Injury (TBI/CerebrovasculaInjury \& Disease - Adult)

NIH Toolbox Emotion Battery: Application of Summary Scores to Spinal Cord Injury, Traumatic Brain Injury, and Stroke 
2. BABAKHANYAN, I

3. BEDARD, M

4. BELKONEN, $\mathrm{S}$

5. GIAMMITTORIO, A

6. BRAY. MJ

7. BRENNER, EK

8. BROWN. ST

9. CASTOR, N

10. CASTOR, N

11. CONNORS, EJ

12. CROCKER, LD

13. DAILEY, NS

14. DOCKLER, LM

15. EPSTEIN, E

16. FEDIO, AA

17. FRODSHAM, K

18. GARCIA, K

19. GROSSNER. EC

20. GROSSNER, EC

21. HAMMOND, J

22. HAMMOND, J

23. HAN, T

24. HANKS, R

25. HOLIDAY, KA

26. HROMAS, G

27. IVERSON, GL

28. JANULEWICZ, PA

29. KANSER, R

30. KAUP, AR

31. KAUP, AR

32. KEY-DFIYPIA S

33. KILLGORE, WD

34. LA CORTE, V

35. LANGAN, LM

36. LIPPA, SM

37. LIPPA, SM

38. LOPEZ, W

39. MARRA, DE

40. MERRITT, VC

41. MEYER, J
Cognitive Changes Following Intensive Outpatient Program for Mild Traumatic Brain Injury Within a Military Cohort

Long-term prospective memory impairment following mild traumatic brain injury with loss of consciousness

Symptom Report in Rural OEF/OIF/OND Veterans with TBI and PTSD

Detection of Symptom Over-Reporting on the Neurobehavioral Symptom Inventory in OEF/OIF/ OND Veterans with Mild TBI

Homelessness and the Neuropsychiatric Sequelae of Traumatic Brain Injury: A Scoping Review Cognitive Reserve is Associated with Connectivity in Moderate to Severe Traumatic Brain Injury The Role of Neuropsychology in the Relationship Between Acquired Brain and Spinal Cord Injury The influence of education level on cognitive recovery in people with brain damage

Resilience and education level in brain damaged people: does it impact on cognitive performances? The Influence of Executive Function on two Types of Verbal Learning Among Individuals with a Traumatic Brain Injury

Do TBI Injury Characteristics Affect PTSD Treatment in Veterans with PTSD and a History of TBI?

Neural Correlates of Aggression in the Chronic and Post-Acute Stages of Recovery from Mild

Traumatic Brain Injury: A Diffusion Tensor Imaging Study

Effect of Cognitive Deficit on Emotional Functioning following ABI

Differences between Male and Female Iraq and Afghanistan Veterans in Behavioral Outcomes with co-occurring Traumatic Brain Injury and Posttraumatic Stress Disorder

Importance of Health Professionals Informing Brain-Injured Patients about Functional Changes

Predicting Rehabilitation Outcomes Using Day-of-Injury Computed Tomography (CT): A

Comparison Between Marshall and Rotterdam CT Scoring Criteria

White Matter Integrity and Memory Impairment in TBI

Frontal Network Connectivity Predicts Metacognitive Accuracy Following Moderate to Severe

Traumatic Brain Injury

Racial and Ethnic Differences in Discharge Destination for Individuals with Traumatic Brain Injury in Pennsylvania

Promoting Helmet Use on the Ski Hill: Trends in Helmet Use Among Skiers and Snowboarders After Implementation of a Policy to Mandate Employee Helmet Use

Using the Transtheoretical Model of Behavior Change to Promote Bicycle Helmet Use: An Analysis Based on Stage of Change and Other Features

Relationship Between Cognitive Complaints, Mood, Life Satisfaction, and Participation in a

Traumatic Brain Injury Sample Presenting for Outpatient Rehabilitation

Prediction of Cognitive Outcomes from Diffusion Tensor Imaging in Traumatic Brain Injury

Hippocampal Volume Independently Predicts Subjective Memory Complaints in Mild Traumatic

Brain Injury

The effect of ocular convergence insufficiency following traumatic brain injury on performance

during a computerized neuropsychological screener

A Two-Week Intensive Clinical Program for Veterans with Traumatic Brain Injury and Psychological Health Problems

Self- Reported Mild Traumatic Brain Injury (mTBI) and Toxicant Exposures During the 1991 Gulf

War and Gulf War Illness: The Boston Gulf War Illness Consortium

Utility of WAIS-IV Digit Span Indices as Measures of Performance Validity in Complicated-Mild to Severe Traumatic Brain Injury

Subjective Cognitive Complaints among Older Veterans with Traumatic Brain Injury are Related to PTSD Symptoms

Feasibility of Mobile Cognitive Intervention among Older Veterans with Traumatic Brain Injury:

Preliminary Findings from the BRAVE Training Study

WHTHBRAWN Using Gognitive Profites in Individuals With and Without Mild Closed-Head Injury to Prediet Sentenee Gomprehension Performanee

Blue Light Therapy Accelerates Brain and Cognitive Recovery from Mild Traumatic Brain Injury Neuropsychological rehabilitation for prospective memory deficits in traumatic brain injury: a new ecological treatment in virtual reality

Refining Test Batteries Appropriate for Patients with Craniocerebral Gunshot Wounds Undergoing Pre-Surgical Assesements

The Relationship between Plasma Amyloid and Neurocognition Following Mild Traumatic Brain Injury: A Longitudinal Analysis

The Relationship between Plasma Tau and Amyloid and Diffusion Tensor Imaging Following Mild Traumatic Brain Injury

Memory Process Deficits in Retired Professional Football Players

Re-analyzation of Belanger and Colleagues' (2005) mTBI Meta-Analysis via Meta-Regression APOE- 44 Genotype Modifies the Relationship Between TBI History and Neuropsychological

Performance in Military Veterans

Prevalence of Subconcussive Head Trauma, Traumatic Brain Injury, and Hypoxic Brain Injury in Victims of Intimate Partner Violence and Potential Long Lasting Effects on Cognitive Functioning 
42. MULLEN, CM 43. PFRNA R Traumatic Brain Injury

44. POLEJAEVA, E

45. ALKOZEI, A

46. ALKOZEI, A

47. ROWLAND, J

48. SALINAS, SL

49. SORG, SF

50. STROM, J

51. SUNDERARAMAN, P

52. TERRY, DP

53. TERRY, DP

54. TIBBS, J

55. TREBLE-BARNA, A

56. TUPLER, LA

57. UCHANI, SD

58. UKUEBERUWA, DM

59. VACCARIELLO, E

60. HOFFMAN, S

61. WALKER, A

62. WALSH, MJ

63. WARE, AL

64. WEICHSELBAUM, A

\section{WENDEL, G}

66. WRIGHT, MJ

67. ZANE, KL

68. ZANE, KL

69. DYKSTRA, JB

70. ESCHLER, BD

71. GOODRICH-HUNSAKER, NJ

72. HAIGHT, A

73. HASHEMIAN, $\mathrm{N}$

74. HASSARA, K

75. HUANG, W

76. KOEHL, LM

77. LACE, JW

78. LACE, JW Traumatic Brain Injury Injury (CABI) Concussion Program

Traumatic Brain Injury Brain Injury

Traumatic Brain Injury A Meta-Analysis in Adolescents Tumor Brain Injury
Subjective Cognitive Complaints and Emotional Symptoms in Veterans with Polytrauma and Mild

WITHDRAWN Gase Study: Disorder of Gonseiotrness to full Funetional Reeovery

Relation between apathy, depression, and symptoms of Posttraumatic Stress Disorder in mild

Grey Matter Volumetric Differences with Increasing Numbers of Previous Mild Traumatic Brain Injuries: A Voxel-Based Morphometric Study

Increased Cerebellar Grey Matter in the Presence of Decreased Subjective Sleep Quality Following Mild Traumatic Brain Injury

Changes in Resting-State Network Topology Associated with the Development of Posttraumatic

Stress Disorder Following Deployment Acquired Traumatic Brain Injury

The Effects of Polytrauma on Neuropsychological Performance: Veterans with Mild Traumatic Brain

The Relationship Between Subjective and Objective Memory Performance, PTSD Status and Injury Severity in Veterans with History of Mild Traumatic Brain Injury

Associations Between Time Since Injury and Cognitive Recovery Across Mechanism of Traumatic

Brain Injury: Preliminary Evidence for Protracted Cognitive Recovery in Blast-Related Neurotrauma

The Influence of Impulsivity on Financial Capacity in Individuals with Chronic Acquired Brain Injury

Pre-Injury Migraine History as a Risk Factor for Prolonged Return to School and Sports Following

Perceived Injustice is Associated with Worse Outcome from Mild Traumatic Brain Injury

Exploring the Role of Alcohol and Substance Use on Neuropsychological Status During Post-Acute

Rehabilitation in Survivors of Traumatic Brain Injury

Epigenetic Changes in the Brain-Derived Neurotrophic Factor (BDNF) Gene Following Traumatic Brain Injury: Preliminary Findings

Impairments in Crystallized and Fluid Intelligence in Veterans with History of Traumatic Brain Injury: Influence Through Effects of Severity and Multiplicity of Trauma on Depression and Posttraumatic Stress Disorder

Adverse Childhood Experiences and Neuropsychological Outcomes Among Adults with Moderate to Severe Traumatic Brain Injury: Preliminary Results

Baseline Personality and Psychopathology Symptom Reporting of Veterans within a TBI/PTSD

Examining the Cognitive Proficiency Index in Rehabilitation Patients

Examination of Symptom Over-Reporting and Self-Reported Pain and Depression in Iraq and

Afghanistan Veterans with Mild Traumatic Brain Injury

Use of Performance Validity Tests in Acute TBI

Regional Gray Matter Volumetric Differences Predict Fatigue Symptoms in Veterans with Mild

High-Definition Fiber Tracking Study of the Executive Control Network in Blast-Related Traumatic

Revealing Subtle Cognitive-Linguistic Differences in Adults with Mild Traumatic Brain Injury

Through Discourse Analysis

WHTHORAWN-LONG-Term Outeome After Stroke: What Contributes to Partieipation?

Semantic Clustering Partially Mediates Encoding Deficits in Traumatic Brain Injury

An Exploration of Neuropsychological Status and Diffusion Tensor Imaging Findings in Adults with Mild to Severe Chronic Traumatic Brain Injury

An Examination of Neuropsychological Performance in Adults with Mild to Severe Chronic

\section{Acquired Brain Injury (TBI/Cerebrovascular Injury \& Disease - Child)}

Adolescent maladaptive illness beliefs predict prolonged concussion recovery

Internalizing and Externalizing Symptomatology Following Pediatric Traumatic Brain Injury:

Integrity of the corpus callosum predicts outcomes of pediatric mild traumatic brain injury Exploring the relationship between social and empathetic performance in pediatric TBI Effects of Premorbid Attention-Deficit/Hyperactivity Disorder (ADHD) on Postconcussion Symptoms

Language Outcomes in Children with Arterial Ischemic Stroke

Neurocognitive Functions and Psychosocial Adaptions In Survived Children With Posterior Fossa

Irritability and Aggression as Indicators of Concussion Symptoms in a Pediatric Population

Convergent and Divergent Validity between ImPACT and CPT-3 in Adolescents with Mild Traumatic

Relationships between Parent- and Self-Reported Executive Dysfunction in Adolescents with Mild

Traumatic Brain Injury 


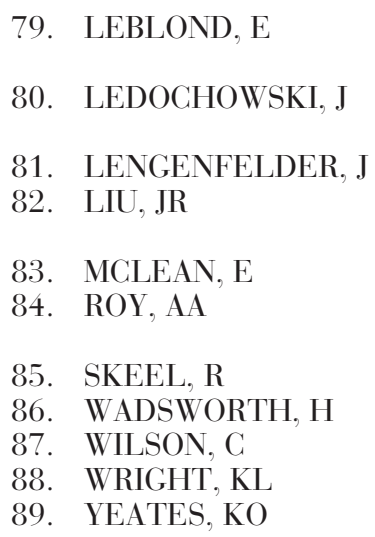

90. ETTENHOFER, M 91. ETTENHOFER, M

92. ENGLE, J

93. HERSHAW, J

5:30-6:30 PM

1. NICOLELIS, M

7:00-9:00 PM

7:20-8:50 АM

1. WILLIAMSON, JB

7:20-8:50 AM

1. COHEN, RA

8:15-9:00 AM

9:00-10:00 AM

1. LISANBY, SH
Neurocognitive Outcomes of Methylphenidate Treatment for Secondary Attention Deficit Hyperactivity Disorder Following Pediatric Traumatic Brain Injury

Mental Health Outcomes in Children with Unilateral Basal Ganglia Arterial Ischemic Stroke and Secondary Dystonia

Attention After Pediatric Brain Injury: Objective Assessment Versus Parent Report

Association Between Depressive Symptoms in Post-Concussional Children and Adolescents and

Missed School Days Following Concussion

Empathy in Pediatric Traumatic Brain Injury (TBI)

Use of the Cognitive and Linguistic Scale (CALS) and the Functional Independence Measure for Children (WeeFIM) in a Pediatric Brain Injury Population

Survey of Professionals' Recommendations for Rest following Concussion

Concussion Symptom Reporting in Male and Female Adolescent Ethnic Minority Athletes Associations of Attention Problems Following Early Childhood Pediatric Traumatic Brain Injury White Matter Hyperintensities in Pediatric TBI: Comparison of Imaging Methods

Headache Long After Pediatric Concussion: Severity and Relation to Subjective and Objective Cognitive Functioning

\section{Acquired Brain Injury (TBI/Cerebrovascular Injury \& Disease - Adult)}

Multimodal Neuropsychological Assessment in Chronic Traumatic Brain Injury: The Fusion Project Saccadic Evidence for Reduced Cognitive Efficiency in Chronic Traumatic Brain Injury Subjective Mental Workload and Motor Impairment in Traumatic Brain Injury

Identification of Measurable Cognitive Processing Differences in Chronic TBI Using Pupillometry

Plenary C. Linking Brains to Machines: From Basic Science to Neurological Neurorehabilitation

Presenter: Miguel Nicolelis

Ballroom Salon 2-3

Linking Brains to Machines: From Basic Science to Neurological Neurorehabilitation

Student Social, Hosted by the INS Student Liaison Committee To Be Announced

\section{FRIDAY, FEBRUARY 16, 2018}

CE Workshop 9. Vulnerability to Post Traumatic Distress Disorder After Traumatic Brain Injury; Chronic Stress and Accelerated Aging Presenter: John B. Williamson Ballroom Salon 1

Vulnerability to post traumatic distress disorder after traumatic brain injury; chronic stress and accelerated aging

CE Workshop 10. Cognitive and Brain Aging: Neurodegeneration, Cumulative Risk and Etiological Factors, or Epigenetic Phenomena? Presenter: Ron A. Cohen Maryland A-C

Cognitive and brain aging: Neurodegeneration, cumulative risk and etiological factors, or epigenetic phenomena?

INS Business Meeting (Business \& Bakeries) INS President: Michael Kopelman Ballroom Salon 2-3

Plenary D. Where Neuromodulation and Neuropsychology Meet Promoting Plasticity to Enhance Brain Health Presenter: Sarah H. Lisanby Ballroom Salon 2-3

Where Neuromodulation and Neuropsychology Meet - Promoting Plasticity to Enhance Brain Health 
10:00-10:15 AM

10:15-11:45 AM

1. THAMES, A

2. RIVERA MINDT, M

3. BYRD, D

4. MANLY, JJ

5. JUDD, T

10:15-11:45 AM

1. KENWORTHY, L

2. KENWORTHY, L

3. HARDY, KK

4. WADE, SL

5. STRANG, JF

10:15-11:45 AM

1. BRICKMAN, AM

2. JACK JR, C

3. BRICKMAN, AM

4. BONDI, MW

5. MANLY, JJ

\section{0:15-11:45 AM}

1. FAMA, R

2. GONZALEZ, R

3. WADE, NE

4. SCOTT, J

5. OBERMEIT, LC

6. LEVINE, TA
AM Coffee Break

Exhibit Halls B North (Exhibition Level)

Invited Symposium 3. Challenging the Status Quo: Future Directions in Cultural Neuropsychology

Chair: April Thames

Presenters: Monica Rivera Mindt, Desiree Byrd, Jennifer J. Manly, Tedd Judd Ballroom Salon 2-3

Challenging the status quo: Future directions in Cultural Neuropsychology

Integration of Cultural and Linguistic Competency into Neuropsychology Training

Cross cultural neuropsychological research: Developments and forecasts

Advocacy in Cultural Neuropsychology

Global Neuropsychology Priorities

Symposium 7. Bringing Neuropsychology to the People: Transforming Our Knowledge of Executive Function (EF) Phenotypes into Effective Community-based Interventions for Children

Chair \& Presenter: Lauren Kenworthy

Discussant: Hilde Geurts

Presenters: Kristina K. Hardy, Shari L. Wade, John Strang Ballroom Salon 1

Bringing Neuropsychology to the People: Transforming Our Knowledge of Executive Function (EF) Phenotypes into Effective Community-Based Interventions for Children

Evidence for Cross-Diagnosis Efficacy of School-Based Unstuck and On Target Executive Function Intervention in Low Income Communities

Applying Contingency-Based Behavioral Management Strategies to Treat Cognitive Inflexibility in Children with ADHD and ASD: Development and Efficacy of a School-Based Intervention in LowIncome Communities

Epilepsy Journey: An E-Health Intervention to Improve Executive Functioning in Adolescents with Epilepsy

Improving Autistic Adolescent Executive Function Skills: Development and Evaluation of the On

Target for Life School Curriculum

Symposium 8. Toward a biomarker-only diagnosis of Alzheimer's disease Chair \& Presenter: Adam M. Brickman

Presenters: Clifford Jack Jr, Adam M. Brickman, Mark W. Bondi, Jennifer J. Manly

Maryland A-C

Toward a biomarker-only diagnosis of Alzheimer's disease

Implications of a biologically based definition of Alzheimer's disease

Diagnosing Alzheimer's disease with biology not behavior: Are we there yet?

Improving neuropsychological characterization of the preclinical period of Alzheimer's disease

Will research on disparities in Alzheimer's Disease benefit from a biomarker-focused classification framework?

\section{Paper Session 9. Alcohol and Substance Abuse}

Moderator: Michael Kirkwood

Virginia A-C

Neural Correlates of Selective Cognitive and Motor Deficits in Alcoholism

Reward Sensitivity and Motivation among Adolescent Cannabis Users

Aerobic Fitness and Marijuana Use Interact to Predict Neuropsychological Functioning in Emerging Adults

A Meta-Analysis of Cannabis and Cognitive Functioning in Adolescents and Young Adults

The Impact of Methamphetamine Dependence, HIV, and Frontal Systems Dysfunctions on the Relationship Between Sexual Risk Intentions and Behaviors

Early Inhibitory Control and Spatial Working Memory Abilities of Children Prenatally Exposed to Methadone 
10:15-11:45 AM

10:15-11:30 AM

1. AHRENS, AP

2. AITA, SL

3. ARMSTRONG. G

4. ARMSTRONG, G

5. AVILA, J

6. BEACH, J

7. BERG, J

8. BERNSTEIN, J

9. BLOCH, A

10. BOLCEKOVA, E

11. BRUNETTE, AM

12. CAMPBELL, ME

13. CARRIÓN, CI

14. CHAN, M

15. DAVIS, J

16. DONDERS, J

17. FEIRSEN, N

18. FEIRSEN, N

19. FORRESTER, RA

20. MULLER-OEHRING, EM

21. GAVRON, L

22. GRABYAN, JM

23. GRETH, Z

24. HAM, L

25. HOCHBERGER, WC

26. HURTUBISE, J

27. JACKSON, GM

28. JENNETTE, K

29. KEITH, C

30. KOSMIDIS, MH

31. KRISHNAN, K

32. MARTINDALE, SL

33. MARTINEZ, $\mathrm{F}$

34. MBURU, T

35. MESSA, I

36. MESSA, I
Panel Discussion. Presented by the INS Student Liaison Committee: Technology and New Approaches in Cognitive Rehabilitation Presenters: Jonathan Evans, Bruce Luber, Sarah S. Morimoto, Matthew Kurtz Delaware A-B

\section{Poster Session 7. Assessment Across the Lifespan Exhibit Hall B North (Exhibition Level)}

\section{Assessment/Psychometrics/Methods (Adult)}

Deconstructing the Relationship Between Computerized Neurocognitive Assessment Tools (NCATs) and Mild Traumatic Brain Injury (mTBI) Symptomatology

An Examination of the Construct Validity of CNS Vital Signs Using EFA

A Computerized Version of the Color Trails Test

Generating Neuropsychological Assessment Profiles Using Latent Class Analysis

Investigating Measurement Invariance of English and Spanish Versions of the National Alzheimer's Coordinating Centers Uniform Dataset Neuropsychological Battery in Hispanics

Pain Catastrophizing Predicted by Emotional Distress and Cognitive Function in Chronic Pain Sample

Comparing the Boston Naming Test and the Neuropsychological Assessment Battery Naming Subtest in an Outpatient Memory Disorders Clinic

Evaluating the Construct Validity of the King-Devick Test in a Psychological Outpatient Clinical Sample

A New Vision for Neuropsychological Assessment: Unlocking the Gate to Rehabilitation for Individuals with Brain Damage

Interference Scores In Trail Making Test And Prague Stroop Test Do Not Seem As Valid Discriminative Measures In Patients With Neurodegenerative Diseases

Is Episodic Future Thinking Important for Instrumental Activities of Daily Living? A Study in Neurological Patients and Healthy Older Adults

A Comparison of Canadian and American Normative Scores on the Wechsler Adult Intelligence ScaleFourth Edition in a Traumatic Brain Injury Population

Alertness Maintenance Tasks Improve Driving Behaviors for Younger and Older Adults

Associations Between the Everyday Cognition (ECog) Scales and Neuropsychological Performance Among Diverse Racial/Ethnic Groups

Estimating Premorbid Ability in Rehabilitation Patients Using the Test of Premorbid Functioning and Wide Range Achievement Test-4

SCREENING FOR DEPRESSION AFTER TRAUMATIC BRAIN INJURY

The Attention, Memory, and Frontal Abilities Screening Test (AMFAST): A Normative Study in Children \& Adults

The Attention, Memory, and Frontal Abilities Screening Test (AMFAST): A Clinical Efficacy Study in Children and Adults

Independent Components of Visual-Spatial Perception are Factorially Related to Specific

Dimensional Components of Intelligence and Spoken Language

Characterizing procedural learning and memory with perceptual-motor sequence tasks

Elevations on the MMPI-2-RF Neurological Complaints and Cognitive Complaints Scales and

Performance on Effort Measures on Neuropsychological Testing in Veterans

Performance on the First Trial of the Word Memory Test Predicts Failure on Later Trials

Abbreviated Validity Testing: Differential Performance Between Litigating and Non-Litigating

Clinical Outpatients

Using Wearables to Measure Pauses During Everyday Activities

Augmenting Brief Cognitive Screens with Assessment of Judgement: Integration of The Test of

Practical Judgement (TOP-J) and the Montreal Cognitive Assessment (MoCA)

Performance Validity Indicators Embedded Within the D-KEFS Trails

Construct Validity of Montreal Cognitive Assessment Index Scores in Cognitively Normal Adults

The Influence of Binge Drinking Behavior on Verbal Learning and Memory Strategy in Young Adults Relationships Among Self-Reported Sleep, Somatic Complaints, and Cognitive Performance in a Mixed Clinical Sample of Older Adults

Neuropsychological Outcome and Cognitive Reserve Following Cardiac Surgery Employing

Cardiopulmonary Bypass

Computerized Assessment of Cognitive Function in Older Adults

Symptom Burden and Cognitive Outcomes in Iraq and Afghanistan Veterans: The Role of Validity

A Role for Neuropsychology in Preventive Healthcare: Executive Ability Predicts Physical Injuries in Healthy Young Adults

Underutilized Psychometric Correlates of Executive Function in TBI

Use of the WCST and WCST-64 in the Assessment of First Episode Psychosis

Base Rates of Invalid Responding in First-Episode Psychosis 


\begin{tabular}{|c|c|}
\hline $\begin{array}{l}37 . \\
38 . \\
39 .\end{array}$ & $\begin{array}{l}\text { MIDDLETON, K } \\
\text { MILLER, JB } \\
\text { MONTGOMERY, V }\end{array}$ \\
\hline $\begin{array}{l}40 . \\
41 . \\
42 .\end{array}$ & $\begin{array}{l}\text { NDUKWE, N } \\
\text { OJEDA, N } \\
\text { PAULSON, J }\end{array}$ \\
\hline 3. & PETERSON, J \\
\hline $\begin{array}{l}44 . \\
45 . \\
46 . \\
47\end{array}$ & $\begin{array}{l}\text { QUINN, C } \\
\text { RHYNER, KT } \\
\text { ROYE, S } \\
\text { SAGAR, S }\end{array}$ \\
\hline 48. & GÁNDARA MARTÍNEZ, M \\
\hline $\begin{array}{l}49 . \\
50\end{array}$ & $\begin{array}{l}\text { SOBER, J } \\
\text { TAYLOR, S }\end{array}$ \\
\hline $\begin{array}{l}51 . \\
52 .\end{array}$ & $\begin{array}{l}\text { TOLLE, KA } \\
\text { TRIFILIO, E }\end{array}$ \\
\hline $\begin{array}{l}53 . \\
54 .\end{array}$ & $\begin{array}{l}\text { UDALA, MR } \\
\text { VALENTINE, TR }\end{array}$ \\
\hline $\begin{array}{l}55 . \\
56 .\end{array}$ & $\begin{array}{l}\text { VERMILION, B } \\
\text { WEBBER, TA }\end{array}$ \\
\hline-7 & WEITZNER, D \\
\hline $\begin{array}{l}58 . \\
59 . \\
60 . \\
61 .\end{array}$ & $\begin{array}{l}\text { WONG, CG } \\
\text { WOOD, AE } \\
\text { YESIAN, R } \\
\text { YOCHIM, B } \\
\text { ZUCGATO, BG }\end{array}$ \\
\hline
\end{tabular}

63. AUSTIN, CA

64. BARTLETT, AN

65. DEASLEY, $\mathrm{S}$

66. GONZALEZ. I

67. GREEN, R

68. GREIF, SM

69. HENNRICK, H

70. LAJINESS-O'NEILL, R

71. MOORE, MW

72. NOONAN, N

73. ROLIN, S

74. SEESE. S

75. SWICK, C

76. TEAGUE, AM

77. THOMAS, C

78. TURNER, EM

79. KISELICA, A
Validation of a Modified Reitan-Wolfson Screening Test Using MRI Findings in a TBI Population Performance validity tests and associations with hippocampal volume in neurodegenerative disease Preliminary evaluation of demographic adjusted MoCA scores and functional competence in postacute rehabilitation

Quantifying a Relationship Between Somatic Concerns and Frequency of Exercising

Estimation of cognitive performance based on the premorbid intelligence in Parkinson's disease

Replication of the Embedded Performance Validity Index within the Memory Module of the Neuropsychological Assessment Battery (NAB)

Use of the Victoria Symptom Validity Test to evaluate performance validity in baseline concussion testing

Development and Preliminary Evaluation of an Alternate form of the Test of Practical Judgment Equivalence of HVLT-R Forms in a Veteran Population

The Compensatory ADHD Behaviors Scale (CABS): Development and Initial Validation

The Development of the Hyperactivity/Inattention Trait Scale for the Detection of Feigned AttentionDeficit/Hyperactivity Disorder: A Preliminary Examination

Investigating the Relationship of Word Reading Tests and Verbal/Nonverbal Intelligence Measures in Spanish in a Mexican Sample

Measurement Invariance of a Verbal Fluency Task for the Oldest Old

Examination of the Repeatable Battery for the Assessment of Neuropsychological Status Performance Validity Index in a Chronic Pain Sample

The Implications of Different Cutoff Scores for the Montreal Cognitive Assessment (MoCA)

Neuroanatomical Correlates of an Alternative Story Memory Test in Older Adults: The Left Trumps the Right

Cognitive Functioning and Psychopathology in a Traumatic Brain Injury Sample

Clinical Predictors of Subjective Cognitive Functioning Among Medically Healthy Younger Adult

Neuropsychology Patients: An MMPI-2-RF Study

See what I'm Saying: Speechreading Facilitates Accuracy on List-learning

Utility of Various WAIS-IV Digit Span Indices for Predicting Noncredible Performance in Older

Veterans With and Without Cognitive Impairment

The Relationship Between Self-Reported Executive Dysfunction and Self-Reported Everyday

Functioning in Older Adults

Verbal Memory Performance Under Real and Simulated Hearing Loss Conditions

Rey-Osterrieth Figure Copy Size and Cognitive Functioning in Veterans

Third Party Observers and the California Verbal Learning Test-2: A Preliminary Analysis

Performance on the Verbal Naming Test in Individuals with Left Hemisphere Stroke

Early Bird Fails the PVT? The Effects of Timing Artifacts on Performance Validity Tests

\section{Assessment/Psychometrics/Methods (Child)}

Multifactor Predictors of Parent Satisfaction with Neuropsychological Assessment

The Association of the Grooved Pegboard Test to Everyday Motor Skills

Screening and Identification of Developmental Coordination Disorder in Children With and Without ADHD

Comprehensive Pediatric Neuropsychological Clinical Battery in Spanish: A Case Study

Developmental Trends of Neuropsychological Function in Mexican Youth using The Cambridge

Neuropsychological Test Automated Battery (CANTAB)

Criterion Validity of the Memory Validity Profile in a Diagnostically Heterogeneous Clinical Pediatric Sample

Significant Discrepancy Between Digit-Span Forward and Backward Impacts Relationship Between

Working Memory and Executive Functioning

Development and Validation of PediaTrac: A Web-Based Tool to Screen and Track Developing Infants

Role of Socioeconomic Status in the Relationship Between Parent and Performance-based Measures of Executive Function in Children with ADHD

Children in an Inpatient Setting Perform with Significant Weakness on Several Common

Neuropsychological Measures When Compared to an Outpatient Sample

Utility of the Memory Validity Profile in Detecting Performance Invalidity in a Pediatric Sample

WITHORAWN A Multimodal Researeh Battery for Assessing Exeentive Funetion Skills in Autistie Teens

Criterion Validation of the Social-Emotional/Communication Domain of PediaTrac ${ }^{\mathrm{TM}}$, an Infant

Screening Tool

Test of Memory Malingering (TOMM) performance and utility of trial 1 score in a pediatric sample The effect of home location on performance on a phone-based memory task in rural Zambia Identification of Embedded Effort Measures Using the Children's Memory Scale

\section{Other}

What is the Clinical and Economic Value of a Neuropsychological Assessment? A Systematic Review 
11:45 AM-12:45 PM

1. PITEL, A

12:45-1:45 PM

1:45-3:15 PM

1. FRANKE, B

2. RIBASES, M

3. BRALTEN, J

4. FARAONE, S

5. FRANKE, B

\section{1:45-3:15 PM}

1. HAMPSTEAD, BM

2. RECKOW, J

3. RAHMAN-FILIPIAK, AM

4. WOODS, AJ

5. HAMPSTEAD, BM

\section{1:45-3:15 PM}

1. RYMAN, S

2. CRAWFORD, Ј

3. KARPOUZIAN, T

4. ALDEN, EC

5. CALLAHAN, BL

6. NGUYEN, TT

\section{1:45-3:15 PM}

1. THOMAS, KR

2. WEIGAND, AJ

3. NATION, DA

4. VAKIL, E

5. OSBORN, KE

6. SHANY-UR, T

7. DEVAUGHN, S
Plenary E. Neuropsychology and Neuroimaging of Alcohol Use Disorder With and Without Korsakoff Syndrome: A Better Understanding for a Better Treatment

Presenter: Anne Lise Pitel

Ballroom Salon 2-3

Neuropsychology and neuroimaging of Alcohol Use Disorder with and without Korsakoff syndrome: a better understanding for a better treatment

Lunch (On Own)

Conference-Wide

Invited Symposium 4. From Genes to Behavior in ADHD - How is the Brain Involved?

Chair: Barbara Franke

Presenters: Marta Ribases, Janita Bralten, Stephen V Faraone, Barbara Franke Ballroom Salon 2-3

Attention-deficit/hyperactivity disorder (ADHD): emerging insights into a category at the extreme of a population continuum

Genetics of ADHD across the lifespan

Categories at the extreme of population distribution of behaviour - the real nature of neurodevelopmental disorders?

Adult onset ADHD - what is the evidence really?

From genes to behaviour in ADHD - how is the brain involved?

Symposium 9. Transcranial direct current stimulation in older adults Chair \& Presenter: Benjamin M. Hampstead Presenters: Jaclyn Reckow, Annalise Rahman-Filipiak, Adam Woods Ballroom Salon 1

Transcranial direct current stimulation in older adults

Tolerability and blinding of $2 \mathrm{~mA}$ versus $3 \mathrm{~mA}$ in cognitively diverse older adults receiving HD-tDCS Why did a single-session of $3 \mathrm{~mA}$ HD-tDCS have no effect on object location memory or working memory in older adults? A methodological evaluation

Functional neural mechanisms of tDCS-related working memory improvement in older adults Neurophysiological effects of high-definition transcranial direct current stimulation in older controls and patients with mild cognitive impairment

\section{Paper Session 10. Bipolar Disorder and Schizophrenia Moderator: Jimmy Choi Maryland A-C}

Impaired Theta Power and Connectivity During Proactive Cognitive Control in Schizophrenia Facial Affect Recognition and Memory Impairment in Schizophrenia

Reduced Task-Evoked Pupillary Pesponse on an Executive Control Task in Individuals across the Psychosis Spectrum and Relatives with Elevated Psychosis Spectrum Personality Traits

Verbal working memory and hippocampal surface anatomy in high and low functioning schizophrenia Early Signs of Dementia in Bipolar Disorder

Apolipoprotein E (APOE) Genotype is Associated with Poorer Executive Function in Bipolar Disorder

\section{Paper Session 11. MCI and Dementia \\ Moderator: Heidi C. Rossetti \\ Virginia A-C}

Using Process Scores to Identify Subtle Cognitive Decline and Predict Progression to MCI Inaccuracy of Subjective Cognitive Complaints in the Diagnosis of Mild Cognitive Impairment Circulating Progenitor Cells Correlate with Memory, Posterior Cortical Thickness and Hippocampal Perfusion

Facial Expressions Yielding Context Memory Effect: The Additive Contribution of Eye Movements Verbal Memory Performance Across Preclinical Alzheimer's Disease (AD) and Suspected Non-AD Pathophysiology Cerebrospinal Fluid Biomarker Profiles

Neuroanatomy of Recognizing Realistic versus Static Emotions in Neurodegenerative Diseases

The Differential Cognitive and Neural Substrates of Episodic Memory Performance in Semantic

Variant Primary Progressive Aphasia and Alzheimer's Disease 


\section{1:45-3:00 PM}

1. MILLER, JS

2. STEFANATOS, G

3. ALVERSON, WA

4. ALVERSON, WA

5. BUSCH, RM

6. EHRLICH, TJ

7. ELVERMAN, KH

8. FANTO, EJ

9. FIUMEDORA, MM

10. GRANT, A

11. KATZ, N

12. KHURSHID, K

13. LOBLEIN, H

14. LUCCHETTI, A

15. MAIMAN, M

16. MAIMAN, M

17. MAIMAN, M

18. MCCLEARY, KR

19. ONO, K

20. PINJALA, M

21. QUASNEY, EE

22. REALE-CALDWELL, A

23. RESCH, Z

24. SABSEVITZ, DS

25. SALINAS, SL

26. SCHRAEGLE, W

27. SLUGH, M

28. SRNKA, KD

29. STEFANATOS, AK

30. BANERJEE, NS

31. CHANEY, G

32. CROWLEY, SJ

33. HARRIS-RAINS, K

34. HENDERSHOTT, T

35. LARUM. A

36. LOPEZ, FV

37. PLUTM, CF

38. PULASKI, S

39. RAEIN, KL

40. ROSEN, SA

41. SACCÀ, F

\section{Poster Session 8. Adult Neurological Exhibit Hall B North (Exhibition Level)}

\section{Behavioral Neurology/Cerebral Lateralization/Callosal Studies}

Adaptive Behavior in Agenesis of the Corpus Callosum: Self and Informant Reports

Fused Dichotic Word test: A comparison of standard vs computer-mediated adminstration

\section{Epilepsy/Seizures}

Interrelations Among Cognition, Symptom Validity, Depression, and Self-Reported Disability WHODAS 2.0 and Symptom Validity in an Inpatient Seizure Disorder Sample

ILAE Hippocampal Sclerosis Subtype is Related to Verbal Memory Performance in Temporal Lobe Epilepsy

The Role of Executive Functioning in Quality of Life for Adults with Treatment-Resistant Temporal Lobe Epilepsy

A Cluster Analytic Approach to Exploring Cognitive Phenotypes in Temporal Lobe Epilepsy

The relationship of language and explict memory in pediatric epilepsy

Self-Report and Objective Memory Deficits in Temporal Lobe Epilepsy

Memory and Executive Functions in Children with Frontal and Temporal Lobe Seizures

Neurocognitive Functioning Pre and Post Surgery in a Male Adult with Epilepsy Secondary to Gun

Shot Wound: A Case Study

Olfactory Dysfunction in Epilepsy

The Role of Stigma in Depression and Anxiety in Youth with Epilepsy

Using Epilepsy Risk Factors to Predict IQ in Pediatric Patients

Does Executive Dysfunction Predict Behavioral Problems in Children with Focal Epilepsy?

Does Working Memory Predict Math and Reading Skills in Children with Epilepsy?

Observed sex differences with the Repeatable Battery for the Assessment of Neuropsychological

Status (RBANS) in adult epilepsy patients

The Safety and Efficacy of Propofol as a Replacement for Amobarbital in Intracarotid Wada Testing of Pre-surgical Epilepsy Patients

Psychosocial Comorbidities in Children and Adolescents with New Onset Seizures

Relationship of Cognitive Functioning to Quality of Life in Patients with Epilepsy

Wada Language Lateralization Index (LI) and Wada Memory Asymmetry (WMA) Scores as

Predictors of Verbal Memory Decline After Left Anterior Temporal Lobectomy (L-ATL)

Predictive Ability of Verbal Memory Tests in Post-Operative Epilepsy Patients

5-year Longitudinal Change in Attentional Vigilance in Childhood Epilepsy

Does Memory Decline Really Matter Following Epilepsy Surgery? Impact of Memory Decline on

Perceived Cognitive Functioning in a Sample of Left Anterior Temporal Lobectomy (ATL) Patients

With Well Controlled Seizures

Gender Differences on a Test of Spatial Memory Among Patients with Epilepsy

Neuropsychological Functioning in Youth with Temporal Lobe Epilepsy With or Without

Hippocampal Sclerosis: An Unexamined Cognitive Phenotype?

Examining Cutoff Values of Elevated BDI and BAI Scores in Relation to Validity Scales on the MMPI-2-RF in Adults with Epilepsy

Intraindividual Variability Mediates Five Year Longitudinal Relationship With Age of Diagnosis, IQ. and Academic Achievement

Neuropsychological Outcomes of Adolescents Following Hemisperectomy: A Multiple Case Study

\section{Movement and Movement Disorders}

Age-related Differences in the Association Between Cardiometabolic Risk Factors and Cognition in Parkinson's Disease

Clinical Utility of Neuropsychological and Olfactory Measures in the Differential Diagnosis of

Parkinson's Disease

Cognitive Phenotypes in Early Idiopathic Parkinson's Disease

Title: The Unique Contribution of Motor, Cognitive, and Mood Symptoms to Functional Impairment in Parkinson's Disease

Cognitive and Motor Processes Differentially Affect Visuospatial Performance in HIV Infection and Parkinson's Disease

Non-Motor Symptoms in Men and Women with Parkinson's Disease: Impact on Quality of Life Medication Management in Parkinson's Disease: Novel Error Analysis Reveals Those with MCI Indicated Taking Fewer Pills than Instructed on a Performance-Based Task

Are Apathy and Fatigue Distinct Symptoms in Parkinson's Disease?

Cognitive Impairment and its Relationship to Perceived Quality of Life in Parkinson's Disease Patient and Care Partner's Priorities for Cognitive Treatment Outcomes in Parkinson's Disease The Verbal Series Attention Test (VSAT) as a Measure of Executive Functioning in Parkinson's Disease

Brain Functional Changes and Cognitive Dysfunction in Friedreich's Ataxia 
$\begin{array}{ll}\text { 42. } & \text { SALAZAR, R } \\ \text { 43. } & \text { SARNO, M } \\ \text { 44. } & \text { SCOTT, BM } \\ 45 . & \text { SPLIT, M } \\ 46 . & \text { THORN, J } \\ 47 . & \text { VANDEBUNTE, AM }\end{array}$
Relation of Stigma Perception to Clinical and Demographic Characteristics of Parkinson's Disease Is Age of Onset Related to Cognition and Mood in Essential Tremor?

Mixed Linear Modeling of Changes in Functional Independence in De Novo Parkinson's Disease Motor Subtypes

Memory for Positive and Negative Faces and Words in Parkinson's Disease

Associations Between Hallucinations and Sleep Quality in Nondemented Patients with Parkinson's Disease

The Effect of Depression on the Cognition of those with Parkinson's Disease

\section{Multiple Sclerosis/ALS/Demyelinating Disorders}

48. ANAND, D WHTHOAWN Living under the Shadow of Ghronie Illness: Goping and Gonfliets in Muttiple Selerosis

49. BARLOW-KRELINA, E

50. CHAPMAN, K

51. COSTABILE, T

52. CRIVELLI, L

53. DE SOMMA. E

54. DI BENEDETTO, MG

55. HAGUE, C

56. HAKE, $\mathrm{P}$

57. HOFFMEISTER, J

58. LEBKUECHER, A

59. MEDINA, LD

60. NEUFELD, $\mathrm{R}$

61. ZICCARDI, $\mathrm{S}$

62. RANDOLPH, J

63. RAPHAIL, A

64. SANCHEZ, O

65. SCHMIDT, ST

66. SPADONI, G

67. SPIROU, A

68. TIRRI, DJ

69. EVANS, J

70. HANSEN, J

71. JAYWANT, A

72. JOHNSON, N

73. KESSELS, RP

74. LEHOCKEY, KA

75. RAMÍREZ, D

76. HIZEL, L

77. SAADATPOUR, L
Examining Executive Dysfunction as a Contributor to Verbal Reasoning Deficits in Youth and Young Adults with Pediatric-Onset Multiple Sclerosis

Fitness Outcomes After Starting a New Exercise Routine in Persons with Multiple Sclerosis: The Role of Cognition

Exploring Mental Imagery in Relapsing Remitting Multiple Sclerosis

Inhibitory Control Dysfunction in Early Multiple Sclerosis

Disrupted cognitive development following acute demyelinating syndromes: A longitudinal study

Can't Stress it Enough: Examining the Role of Personality, Coping, and Percieved Stress in Multiple Sclerosis

Comparing fatigue in pediatric multiple sclerosis and transvers myelitis

Sensory Processing in Multiple Sclerosis and its Relation to Processing Speed

Reliability and Validity of the Perceived Deficits Questionnaire in Multiple Sclerosis

Say What?: The Role of Language and Articulatory Speed in Verbal Fluency of Patients with Progressive Multiple Sclerosis

Disease Modifying Treatments in Multiple Sclerosis and Their Differential Impact on Cognition The Effect of Cognitive Load on Visuomotor Integration in Youth with Multiple Sclerosis Switching And Clustering Performance In Verbal Fluency Tasks In A Group Of Multiple Sclerosis Patients Without Cognitive Impairment: Preliminary Investigation With Clinical Implications Subgroup Analysis of Individuals with MS Showing Increased Resilience and Cognitive Reserve Examining the Impact of Cognitive and Motor Impairment on Visual Perception in Individuals with Multiple Sclerosis

Minnesota Multiphasic Personality Inventory-2 Restructured Form (MMPI-2 RF) Profiles in Veterans with Multiple Sclerosis

Social Cognition Impairments in Multiple Sclerosis are Associated with Poor Community Integration Amyotrophic Lateral Sclerosis (ALS) and Primary Lateral Sclerosis (PLS): the Differences in Cognitive Functioning and in Emotional Recognition Giulia Spadoni ${ }^{2}$, Maria Luisa Presenti ${ }^{2}$, Marco Timpano and Stefania Tocchini ${ }^{2}{ }^{1}$ Public Health Authority ASL Nordovest della Toscana ${ }^{2}$ University of Pisa

Aversive Motivation is Associated with Greater Learning and Caudate Volume in MS

Examining the Relationship between Resilience, Anxiety, Depression, and Social Integration in MS

\section{Stroke/Vascular Cognitive Impairment}

A Pilot Single-Blind Randomised Controlled Trial of Mindful Music Listening to Enhance Cognitive Recovery and Mood After Stroke: Feasibility, Acceptability and Effect Sizes

In the Eye of the Beholder: Investigation of Attentional Patterns in Neglect through Eye Tracking

The Clinical Utility of a 30-Minute Cognitive Screening Battery in Acute Inpatient Stroke Rehabilitation

Racial differences in rates of return to work post-stroke

Accelerated Long-Term Forgetting in Patients with TIA or Minor Stroke: A More Sensitive Measure for Detecting Vascular Cognitive Impairment?

Patients Participating in Inpatient Cardiac Rehabilitation and Stroke Programs are Similarly Impaired on a Brief Cognitive Screening

Decline in Semantic Verbal Fluency is Associated with Cerebrovascular Risk Factors

\section{Visuospatial Functions/Neglect/Agnosia}

Post clockface latency as an indicator of executive functioning in Parkinson's disease

A Case of Posterior Cortical Atrophy with Vertical Neglect 
3:15-3:30 PM

3:30-5:00 PM

$\begin{array}{ll}\text { 1. } & \text { CULLUM, M } \\ \text { 2. } & \text { CULLUM, M } \\ \text { 3. } & \text { CULLUM, M } \\ \text { 4. } & \text { HARDER, L } \\ \text { 5. } & \text { VARGAS, B }\end{array}$

3:30-5:00 PM

1. BERNIER, RA

2. VENKATESAN, UM

3. HAJ-HASSAN, S

4. CLARK, AL

5. BRAY, MJ

6. VANUK, JR

3:30-5:00 PM

1. HAN, D

2. LA CORTE, V

3. GRILLI, MD

4. GUZMAN, VA

5. GAYNOR, LS

6. VESPERMAN, CJ

\section{3:30-5:00 PM}

1. GEVA, R

2. MCKINNEY, TL

3. OKABE, $\mathrm{H}$

4. WOODARD, JL

5. SKILES, MA

6. RIZVI, B

7. WILLIAMS, VJ
PM Coffee Break

Exhibit Halls B North (Exhibition Level)

\section{Symposium 10. Teleneuropsychology Coming of Age in Clinical and Research Applications \\ Chair: Munro Cullum \\ Discussant: Robert L. Kane \\ Presenters: Lana Harder, Bert Vargas \\ Ballroom Salon 2-3}

Teleneuropsychology Coming of Age in Clinical and Research Applications

Practical and Ethical Issues in Teleneuropsychology

Evidence-Based Teleneuropsychology in Adults

Feasibility, Validity, and Patient Satisfaction of Teleneuropsychology in Pediatric Populations

Teleconcussion Assessment

\section{Paper Session 12. Traumatic Brain Injury \\ Moderator: Maria T. Schultheis \\ Ballroom Salon 1}

Diminishing returns in network plasticity: thresholds for hyperconnectivity as a predictor of cognition after moderate and severe TBI

Functional Connectivity within Lateral Posterior Parietal Cortex and its Alteration in Traumatic Brain Injury

The RBANS Effort Scale and Effort Index: Base Rates in an Inpatient Rehabilitation Setting

Blast Exposure is Associated with Anterior Cortical Thinning in Veterans with Mild Traumatic Brain Injury

Shifting Perspectives on Post-Traumatic Brain Injury Psychosis: Evidence for a Novel

Neurodegenerative Hypothesis

Short-Wavelength Light Therapy as a Way of Improving Sleep, Cognition, and Amygdala-Prefrontal Connectivity Following a Mild Traumatic Brain Injury

\section{Paper Session 13. Alzheimer's Disease \\ Moderator: Duke Han \\ Maryland A-C}

Detectable Neuropsychological Differences in Early Preclinical Alzheimer's Disease: A Meta-Analysis Patterns of cognitive prospection impairment in early Alzheimer's disease and semantic dementia Evidence of Reduced Autobiographical Memory Richness in Individuals at Increased Genetic Risk of Alzheimer's Disease

Is the Alzheimer's disease-associated Increase in Regional White Matter Hyperintensity Volume due to Wallerian Degeneration?

Translational object discrimination task and associated imaging variables improve prediction of early cognitive impairment related to AD

Cardiorespiratory Capacity Attenuates Age-Associated Aggregation of White Matter Hyperintensities: Findings from the Wisconsin Registry for Alzheimer's Prevention

\section{Paper Session 14. Neurophysiology and Neuroimaging Markers Moderator: Scott A. Langenecker Virginia A-C}

Electrophysiological Changes and Attention Correlates Following Preterm Birth The Moderating role of Uncertainty in Fluid Intelligence and Neural Activity EEG Correlates of Mental Exertion During Sustained Reaction Time Performance Unique and Shared Contributions of Imaging Biomarkers to Prediction of Cognitive Functioning The Impact of FreeSurfer Version on Volumetric Analysis

Fiber Tract-Defined Regional White Matter Hyperintensities and Memory

Increased Task-related fMRI Activation During a Hyperinsulinemic-Euglycemic Clamp Procedure 
3:30-4:45 PM

1. AILION, A

2. CLARK, BE

3. ASHFORD, J

4. DASHER, NA

5. DUDA, TA

6. EASTMAN, JA

7. EDELSTEIN, $\mathrm{K}$

8. EVANS, CL

9. FOX, ME

10. KAUTIAINEN, RJ

11. OSWALD, K

12. MZAYEK, Y

13. TARKENTON, T

14. THOMASON, MM

15. VAN DYK, K

16. VANNORSDALL, TD

17. VANNORSDALL, TD

18. WIER, R

19. WILLARD, VW

20. FEE, RJ

21. FOY, A

22. GODFREY, M

23. KHANG, T

24. LAURENT, R

25. NAYLON, K

26. POWELL, AA

27. SGHARAGA, E

28. SEMERJIAN, C

29. YUND, BD

\author{
30. DEVLIN, KN \\ 31. HARDY, DJ \\ 32. KORDOVSKI, VM \\ 33. LEVITCH, CF \\ 34. MAHMOOD, $\mathrm{Z}$ \\ 35. MICHEL, S \\ 36. PAOLILLO, E \\ 37. SCOTT, J
}

\section{Poster Session 9. Adult Medical Exhibit Hall B North (Exhibition Level)}

\section{Cancer}

Double Dissociation of Auditory Attention and Visual Scanning in Long Term Survivors of Childhood Cerebellar Tumor: A Deterministic Tractography Study of the Cerebellar-Frontal and the Superior Longitudinal Fasciculus Pathways

Cancer Patients' Mental Health Concerns, Cognitive Abilities, and Spiritual Well-Being After Chemotherapy

Predicting Cognitive Performance in Children Treated for Brain Tumors During Infancy: Preliminary Findings from a Prospective, Longitudinal Trial

Neurocognitive Outcomes in Patients Undergoing Hematopoietic Cell Transplantation (HCT) 100

Days Post-Operatively

Reliable Change in Pediatric Brain Tumor

Cancer-Related Cognitive Impairment and Cognitive Function in Older Adults With a Pre-Cancer Baseline Examination

Cancer-Related Cognitive Dysfunction in Young Adults (YA): A Prospective, Longitudinal Trial Executive Functioning Following Proton Radiation Therapy for Pediatric Brain Tumor

The Neurological Predictor Scale as a Predictive Measure of Motor Outcomes in Long-Term Survivors of Childhood Brain Tumors

Neurological Predictor Scale Predicts Academic Achievement Outcomes in Long-Term Survivors of Childhood Brain Tumors

Motor functioning in pediatric Acute Lymphoblastic Leukemia survivors

Improved Processing of Diffusion Tensor Imaging Data Increases Sensitivity to Detect Brain White

Matter Changes in Breast Cancer Patients

School Attitude Associates with Academic Peformance Better than Neurocognitive Variables in Pediatric Leukemia Survivors

Circumscribed Memory Impairment Following Proton Beam Radiation Therapy for Hypothalamic

Tumor in an Adolescent Female

The Effects of Cancer Treatment on Cognitive Functioning in Early Breast Cancer Survivorship

The Safety Profile of a Genu Avoidance Approach to Whole-Brain Radiotherapy to Prevent Cognitive Decline

Cognitive Functioning in Cancer Survivors: Analyses of the Women's Health and Aging Study II White Matter Integrity in the Inferior Fronto-Occipital Fasciculus Mediates Behavioral Regulation and Internalizing Symptoms in Pediatric Brain Tumor Survivors

Executive Functioning in School-Aged Survivors of Retinoblastoma

\section{Genetics/Genetic Disorders}

Dystrophin gene mutation location and academic achievement in the dystrophinopathies Predictors of Social Skills in Children with RASopathies: A Comparison of Noonan Syndrome and Neurofibromatosis Type 1

Identifying Autism Diagnostic Interview- Revised Algorithm Items that Significantly Distinguish Autism Spectrum Disorder and Down Syndrome

Neuropsychological Profile of 4 Biological Siblings with Myotonic Dystrophy Type 1

Neuropsychological Impairment in a Patient with Metachromatic Leukodystrophy

Fibromuscular Dysplasia Linked to Adverse Neurological and Neuropsychological Outcomes

Neurodevelopmental Varaibility in Social Skills Associated with Phelan-McDermid Syndrome

N Gognitive Sequelae of Netrofibromatosis Type 1: A Progressive Look

Comparing Executive Functioning and Social Problems in Pediatric Neurofibromatosis Patients with and without comorbid Attention-Deficit/Hyperactivity Disorder

Executive Functioning Profiles in Children with NF1 Using Lab-Based and Functional Measures: Influence of ADHD?

\section{HIV/AIDS/Infectious Disease}

Functional Outcomes Among Latent Subtypes of HIV-Associated Neurocognitive Disorder (HAND) Disentangling Measures of Information Processing Speed: A Meta-Analytic Illustration with HIV/ AIDS

The Effects of Aging and HIV Disease on Alternating Semantic Verbal Fluency

Neuropsychological Assessment and Management of an Individual with Listeria Encephalitis in the Acute Inpatient Rehabilitation Setting

The Effects of Poor Sleep on Cognition Are Greater Among HIV+ versus HIV-individuals

Neurocognitive and Neuropsychiatric Change Due to HIV and Neurosyphilis May Worsen Personality Pathology: A Case Study

Depressive Symptoms Are Associated with Cognitive Decline in HIV/AIDS

Evaluation of a Brief Computerized Neurocognitive Battery for the Detection of HIV-Associated Neurocognitive Disorders (HAND) 


\author{
38. SHEPPARD, DP \\ 39. SUMMERS, A \\ 40. TIERNEY, SM \\ 41. TURESON, K \\ 42. WILSON, MJ
}

43. BELLONE, J

44. BELSER-EHRLICH, J

45. BLOCH, A

46. CHOWDHRY, S

47. CLAWSON, A

48. COTHRAN, TP

49. DEMIAN, M

50. DUCGA, EL

51. FIGUEROA, $\mathrm{P}$

52. HARRISON, C

53. ISLAM, RM

54. KALSCHEUR, E

55. KOSMIDIS, MH

56. LAGEMAN, SK

57. MATCHANOVA, A

58. MERZ, Z

59. PANOS, AH

60. PRICE, JS

61. ROGERS, S

62. SHAH, M

63. SUNDARAM. S

64. VICKERS, KL

65. WEBER, E

66. WYMAN-CHICK, KA

67. ZUNDEL, CG

68. ALKOZEI, A

69. SANBORN, V

70. ALKOZEI, A

\section{5:00-6:00 PM}

1. VERFAELLIE, M
Intraindividual Neurocognitive Variability is Associated with Lower Physical Quality of Life in West Nile Virus Infected Individuals

The Relationship Between Sociocultural Factors \& Decision-Making in HIV+ Latinx Adults

Additive Effects of HIV and Aging on the Unified Parkinson's Disease Rating Scale: Associations with Everyday Functioning and Quality of Life

The Impact of Sociocultural Factors on Prospective Memory Function in HIV+ Latinx Adults Methods for Determining Neurocognitive Risk Among Veterans with HIV/AIDS in an Interdisciplinary VA Infectious Disease Clinic

\section{Medical/Neurological Disorders/Other (Adult)}

Feasibility and Initial Results of Inspiratory Muscle Training on Cognition and Functional Capacity in Older Veterans with Heart Failure

Neurocognitive-Affective Dysfunction in Dandy Walker Malformation Involving the Cerebellum: A Case Study

Characterizing Implicit Sequence Learning Deficits Following Spinal Cord Injury Using an

Oculomotor Serial Reaction Time Task among Individuals with Tetraplegia

Reduced White Matter Volume in Metabolic Syndrome

Cognitive Profile and Rehabilitation in Susac's Syndrome: A Case Study

Prediction of Cognitive Change in Normal Pressure Hydrocephalus Following External Lumbar Drain Lower Health Literacy Levels are Associated with a Worse Clinical Profile in Renal Transplant Recipients

Neuropsychological assessment following critical illness for the prediction of long-term functional outcomes

Self-Perception of Social Norms and Empathy in Adults After Childhood Right Hemispherectomy Adult Behavior and Executive Functioning Following Childhood Hemispherectomy: Self-Report Unilateral Motor-Symptom Onset in Parkinson's Disease related to Facial Emotional Expression: Right-Hemisphere, Valence, and Motoric Direction Hypotheses

Subjective Cognitive Complaints in Patients with Fibromyalgia: Objective Results Compared to Those of a Neurological Sample

Neuropsychological Effects of Pesticide Use Among Elderly Farmers

Training Leaders of Neurodegenerative Disease Support Groups: Development of a Support Group Well-Being Measure

Post-Operative Cognitive Function after Cardiac Surgery: A Meta-Analytic Review

Examination of the Construct Validity of the Repeatable Battery for the Assessment of

Neuropsychological Status Language Index in a Mixed Neurological Sample

Adult Adaptive Functioning Following Childhood Hemispherectomy

Neuropsychological Assessment Informed Intervention during Liver Transplantation Evaluation: A

Case Report

Poor Punctuality Reflects Cognitive Impairment in Neurology Patients

'Brain on Fire'- Autoimmune Encephalitis: A Neuropsychological Case Study

Impaired Speed of Information Processing in Older Adults with HIV: A Comparison with Parkinson's Disease

A Quantitative Review of Neuropsychological Function in Lyme Disease: Current Status and Future Directions

Learning and Memory Profile of Individuals with Spinal Cord Injury

Relationship Between Verbal Fluency Performance and Side of Motor Symptoms in Older Adults with Early-Stage De-Novo Parkinson Disease

Rates of Medical Conditions: Do Gulf War Veterans Differ from the General Population?

\section{Other}

Men, but not Women, Show a Decrease in Implicit Preferences for Low-Calorie Food After 3 Weeks of Chronic Sleep Restriction

Cognitive Impairment is Associated with Poorer Quality of Life in Persons with Severe Obesity

Chronic Sleep Restriction Increases Implicit Racial Biases and Affects Actual Decision-Making About People

Plenary F (Birch Memorial Lecture). Medial Temporal Lobe Amnesia:

Past, Present, and Future

Presenter: Mieke Verfaellie

Ballroom Salon 2-3

Medial Temporal Lobe Amnesia: Past, Present, and Future 


\section{SATURDAY, FEBRUARY 17, 2018}

7:20-8:50 AM

1. MARQUINE, M

7:20-8:50 AM

1. FERNANDEZ, AL

9:00-10:30 AM

1. CRAM. L

2. VAN BAVEL. J

3. HAAS, I

4. CROCKET, M

5. CRAM, L

9:00-10:30 AM

1. HERMANN, B

2. MEYERAND, M

3. PRABHAKARAN, V

4. HERMANN, B

5. NAIR. VA

9:00-10:30 AM

1. JAPARDI, $\mathrm{K}$

2. KNUDSEN, K

3. MOORE, $\mathrm{E}$

4. WALZAK, LC

5. SEELYE, A

6. BETTCHER, BM

9:00-10:30 AM

1. O' SHEA, DM

2. FOX, E
CE Workshop 11. Neuropsychological Research in Hispanics/Latinos Living in the United States: The Case of HIV

Presenter: Maria Marquine

Ballroom Salon 1

Neuropsychological research in Hispanics/Latinos living in the United States: The case of HIV

CE Workshop 12. Cross-Cultural Neuropsychological Assessment: Challenges and Solutions

Presenters: Alberto L. Fernandez, Jonathan Evans

Maryland A-C

Cross-cultural neuropsychological assessment: challenges and solutions

Invited Symposium 5. Neuropolitics: Getting 'Under the Hood' of Political Attitudes, Identities and Behaviours in a Turbulent World

Chair: Laura Cram

Presenters: Jay Van Bavel, Ingrid Haas, Molly Crocket, Laura Cram

Ballroom Salon 2-3

Neuropolitics: Getting 'Under the Hood' of Political Attitudes, Identities and Behaviours in a

Turbulent World

The Partisan Brain: A Value-Based Model of Political Belief

Political Ideology Moderates Neural Evaluation of Incongruent Policy Positions

Moral Outrage in the Digital Age

Trust, Fairness and Social Belonging: The Neuropolitics of Legitimacy

Symposium 11. Investigating Temporal Lobe Epilepsy with High Resolution Magnetic Resonance Imaging and Connectome Approaches Chairs \& Presenters: Mary Meyerand, Bruce Hermann

Presenters: Veena A. Nair, Vivek Prabhakaran

Ballroom Salon 1

Investigating Temporal Lobe Epilepsy with High Resolution Magnetic Resonance Imaging and

Connectome Approaches

The Epilepsy Connectome Project

Application of Machine Learning to Identify and Characterize Temporal Lobe Epilepsy

Neurobiological and Cognitive Correlates of Processing Speed in Temporal Lobe Epilepsy

Epilepsy Connectome Project (ECP) - Gender Differences in Temporal Lobe Epilepsy Patients

Paper Session 15. Aging

Moderator: Vonetta M. Dotson

Maryland A-C

The Big-C Project: Distinct Language Processes Responsible for Divergent and Convergent Thinking in Big-C Individuals

The Big-C Music Project: Positive Psychopathology and Task Positive Brain Networks

Increased Left Ventricular Mass Index is Associated with Compromised White Matter Microstructure

Among Older Adults: The Vanderbilt Memory \& Aging Project

Theory of Mind in Aging: A Pilot Study Investigating Associations with Risky Decision-Making and

Fraud Susceptibility

Cognitive Correlates of Unobtrusively Monitored Medication Adherence in Older Adults

Increases in a Pro-Inflammatory Chemokine Are Related to Decreases In Episodic Memory Over Time

Paper Session 16. Sleep, Stress, and Exercise

Moderator: Justin E. Karr

Virginia A-C

Discrepancies between crystalized and fluid ability are associated with frequency of social and cognitive engagement in community dwelling adults

Baseline Sleep Efficiency Predicts rate of Parahippocampal Volume Loss in Cognitively Normal Older Adults 
3. TAIWO, Z

4. CARBINE, KA

5. CASALETTO, KB

9:00-10:15 АМ

1. CHIPMAN, K

$\begin{array}{ll}\text { 2. } & \text { CORNETT, K WHAHORAW } \\ \text { 3. } & \text { DE WIT, L } \\ \text { 4. } & \text { DIGIOVANNI, C } \\ \text { 5. } & \text { FARRIS, P } \\ \text { 6. } & \text { FISHER, EL } \\ \text { 7. JEAN, K } \\ \text { 8. } \\ \text { 9ANGAL, PC } \\ \text { 9. NOVAKOVIC-AGOPIAN, T } \\ \text { 10. OJEDA, N } \\ \text { 11. SADEGHI, M } \\ \text { 12. SHOJI, K } \\ \text { 13. SIMON, SS }\end{array}$

14. PASCOE. L
15. GRAVANO, J
16. HAWKSHEAD, BE
17. KILLGORE, WD
18. KILLGORE, WD
19. KING, M
20. MCLAREN, ME
21. MUSTAFA, N
22. RUBINOW, D
23. SMITH. HA
24. TAGHDIMI, M
25. TAGHDIMI, M
26. URGESI. C
27. WATSON, EM
28. TSAPANOU, A
29. TSAPANOU, A
30. ALEKSONIS, HA

Sleep Fragmentation is Related to Altered Structural Brain Volume among Older Adults Free of Dementia: The Vanderbilt Memory \& Aging Project

Neural Indices of Food-Related Inhibitory Control: The Influence of a Morning or Evening Exercise Program

Perceived stress potentiates age-related cytokine markers of macrophage inflammation in cognitively normal older adults

\section{Poster Session 10. Cognitive Neuroscience, Neuromodulation, and Rehabilitation Exhibit Hall B North (Exhibition Level)}

\section{Cognitive Intervention/Rehabilitation}

Using Goal Management to Support Transfer of Memory Training to Everyday Life in Mild Cognitive Impairment

-Effieaey of Interdiseiplinary Post-Aeute Rehabilitation for Impairments in Attention and Proeessing Speedin Trammatie Brain Fnjury

Cognitive Functioning and Self-Efficacy as Predictors of Memory Support System Training

Responsiveness in Mild Cognitive Impairment

Traumatic Brain Injury (TBI) Facebook Support Groups: How Online Supportive Exchanges Relate to Subjective Well-Being as a Function of TBI Sequelae

Work-Family Conflict and Cognition Among Older Adults Working Full-Time

Improvements in Adaptive Functioning among Children with Medical Disorders Following Cognitive Remediation Program: An item-level analysis

The Effect of a Dual Driving/Distraction Task Game on Visuospatial Skills in Older Adults

Mindfulness and Cognitive Training Interventions in Older Adults Improve Performance in Cognitive Control

Training in Attention Regulation Applied to Participant Defined Goals for Veterans with PTSD and mild TBI

Social Cognition and Functional Outcome after Cognitive Rehabilitation in Neuropsychiatric

Pathology, Neurodegenerative Diseases and Healthy Elderly Population

Understanding Engagement and Experience with Computerized Cognitive Training in Youth with ADHID

Application of Cognitive Stimulation Therapy for Dementia in a Veteran Population

Special Interest Group - Brazil (SIG-BRA) on Neuropsychological Rehabilitation: Who works with NR in Brazil? Towards to professionals profile

\section{Connectomics}

Whole-Brain Structural Connectivity Relates to Intrinsic Motivation in Children born Extremely Preterm: Insights Using Graph Theory

\section{Cognitive Neuroscience}

Associations Between Resting-State Connectivity and Cognition in Mild Traumatic Brain Injury and Posttraumatic Stress Disorder

Hormonal Effects on Cognition in Oral Contraceptive Users and Naturally-Cycling Women

Default Mode Activation and the Ability to Resist Sleep Deprivation

Personality Traits Predict the Ability to Sustain Executive Function Abilities During Sleep Deprivation Neural Correlates of Improvements in Personality and Behavior Following a Neurological Event:

A Neuropsychological Approach

Frontal Structural Neural Correlates of Processing Speed Performance in Older Adults

Cognitive Profile Prior to Deep Brain Stimulation Surgery: A Case Study on Adult-Onset

Leukodystrophy with Essential Tremor

Working Memory of Highly Self-Critical Men is Impaired by Failure

An assessment of cognitive control differences among individuals with psychopathology: Congruency sequence effects in depression, generalized anxiety, and OCD

Schizotypy, Attachment Styles, and Attentional Biases Toward Substance Related Stimuli in Young Adults

Relationship between PTSD Symptom Severity and Cognitive Functioning

Dissociated Alterations of Self-Body Schema and Others' Body Holistic Perception in Children with

Supratentorial and Infratentorial Tumor

Poor Sleepers Have Delayed Behavioral Responses to Positive Sleep Imagery

Subjective cognitive complaints and sleep problems in the elderly

\section{Neuroimaging}

Sleep medication and brain morphology in the elderly

Using Diffusion Tensor Imaging to Assess Associations of White Matter Integrity and Memory in

Pediatric Brain Tumor Survivors 
31. ALFINI, AJ

32. ALKOZEI, A

33. ARRINGTON, C

34. BEDNARZ, H

35. BOUTZOUKAS, EM

36. EBRAHIMI. CT

37. GARCIA-EGAN, PM

38. GATES, T

39. GULLETT, JM

40. JOSEPH, A

41. KIM, J

42. LANGENECKER, SA

43. LINDBERGH, CA

44. SALINAS, SL

45. SIDEMAN, N

46. TANNER, JJ

47. TART-ZELVIN, A

48. TRAN, S

49. YUAN, J

50. ZANE, KL

51. HOLLAND, AK

52. JUAN, R

53. LEHOCKEY, KA

54. MODERSITZKI, E

55. PIMENTEL-RODRÍGUEZ, LF

56. REYNOLDS, J

57. SCAVONE. A

58. VACGARIELLO, E

59. WINTERS, AR

60. BRYANT, AM

61. EDWARDS, M

62. GARCIA, SL

63. MECHANIC-HAMILTON, D

64. WILLIAMS, M

65. WILLIAMS, M

66. TART-ZELVIN, A

67. OSIPOWICZ, K

68. ELINE, EE

69. GENOVA, HM

70. RIZZO, A

71. ANDERSON, VA

72. MCDONALD, S

73. GENOVA, HM
Effects of Acute Exercise on Conflict-Dependent Functional Activation in the Aging Brain A Voxel-Based Morphometric Analysis of Resilience to Vigilant Attention Impairment during Sleep Deprivation

Examining Individual Differences in Reading and Attention Networks Using an Oddball Paradigm fMRI Task

Relationship between cerebellar functional connectivity and symptoms of attention-deficit/ hyperactivity disorder

Verbal Working Memory Contributes to Atypical Language Dominance in Pediatric Epilepsy As Shown by fMRI

An Investigation of the Various Domains of Gifted Children Intelligence: Emotional vs. Exceptional Intellectual Intelligence

Cerebellar morphometry abnormalities associated with early life stress

What is the Magnitude of Structural Brain Changes in Virally-Suppressed HIV-Infected Persons?

White Matter Disease in Amnestic Mild Cognitive Impairment: Contributions to White Matter Integrity

Functional Connectivity in TBI Patients with Comorbid PTSD or Depression

Neurocognitive Function is Predicted by Striatal Dopamine D2/D3 Receptor Availability in

Medication-Free Major Depressive Disorder Patients

Recent Binge Drinking Dampens Response to Anticipation of Potential Monetary Loss

White Matter Hyperintensities Relate to Functional Ability in Individuals with Mild Cognitive Impairment and Dementia

Dissociable Patterns of Neural Activity for Semantically and Arbitrarily Related Word Pairs

Task Specific Network Refinement: Multiple Cognitive Tasks Prune from a Broader Resting State Network

Reliability and Validity of White Matter Cerebrovascular Disease Segmentation from T1

Cognitive Strategy Use Reveals Neural Efficiency on a Working Memory Task

Functional Connectivity of Attention Nodes at Midlife

$\mathrm{HbO}_{2}$ Variability and Gait Performance in Older Adults

Diffusion Tensor Imaging Findings in Adults with Mild to Severe Chronic Traumatic Brain Injury

\section{Neurophysiology/EEG/ERP}

Evidence for Reduced Left Frontal Inhibitory Control in the Absorption Phase Relative to the Cephalic Phase of Food Intake

Relationship Between Task-Specific QEEG Peak Frequency Values and Rey-Osterrieth Complex Figure Performance

The Relationship Between Electrophysiology, Behavioral Activation, and Continuous Positive Airway Pressure Nonadherence in a Sample of Adult Patients with Obstructive Sleep Apnea

Neural Indices of Food-related Inhibitory Control and Attention: Accurate Prediction of Dietary Intake?

Effects of Sleep Deprivation on Attention and EEG Delta Power

Changes in Cerebral Laterality in the Cephalic and Absorption Phases of Food Ingestion as a Function of BMI Classification: Neurophysiological Evidence of Reduced Cognitive Control in Overweight Men

Mindfulness Induction and Parasympathetic Tone

Behavioral and EEG Correlates of Mental Effort and Mind-wandering

Behavioral Inhibition Subscales Relate to Resting Frontal Asymmetry and Sleep

\section{Neurostimulation/Neuromodulation}

Does Surgery Side Matter? Verbal Fluency Decrements Following Deep Brain Stimulation for Treatment of Parkinson's Disease and Essential Tremor

White Matter Integrity Before and After Repetitive Transcranial Magnetic Stimulation in Individuals with Comorbid PTSD and Depression

Variable behavioral and neurophysiological response to HD-tDCS in Primary Progressive Aphasia: Preliminary data from a case series

Pilot M.I.N.D.S. Study: Modulating Intellect with Noninvasive DC Stimulation

\section{Cognitive Neuroscience}

New Methods to study Cognitive Development using Diffusion Tensor Imaging Reducing Error in DTI: Methods to Combine Fractional Anisotropy and Mean Diffusion

Brain Development and Language Mediation of Behavior Control >

Examining Sex Differences in Language and Brain Development

Applying New DTI Measures to Autism Spectrum Disorder

Social Cognition: Innovative Approaches to its Examination and Assessment

Can Automated Conversational Virtual Human Interviewers Elicit More Self-Disclosure About

Clinical Symptoms?

Evaluating social competency using PEERS with paediatric clinical populations

Development of the Complex Assessment of Audio-Visual Emotions (CAVEAT)

Utilization of Neuroimaging to Examine Social Cognition in Traumatic Brain Injury 


\author{
74. CHIOU, KS \\ 75. GIOVANNETTI, T \\ 76. COSENTINO, $\mathrm{S}$ \\ 77. CHIOU, KS \\ 78. LARSON, M \\ 79. DOCKREE, P
}

10:30-10:45 AM

10:45 AM-12:15 PM

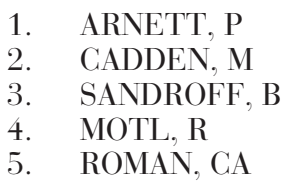

10:45 AM-12:15 PM

1. SHARKEY, CM

2. VAN DER WILLIK, K

3. TONNING OLSSON, I

4. BANERJEE, $\mathrm{P}$

10:45 AM-12:15 PM

1. SULLIVAN, KL

2. HOKKANEN, L

3. ALKOZEI, A

4. BEATTIE, JF

5. KUHN, T

6. WOODWARD, LJ

10:45 AM-12:15 PM

1. MAHON, S

2. LEAFFER, EB

3. YEW, B

\section{Executive Functions/Frontal Lobes}

Dynamic Evaluations of Self: New Insights to Understanding the Mutli-Dimensionality of SelfAwareness

Everyday Error Monitoring in Older Adults

Measuring Metacognition to Improve the Utility of Subjective Cognitive Decline

Investigation of Retrospective Monitoring and Functional Living Skills After Traumatic Brain Injury Neuroimaging Approaches to Understanding Deficits in Awareness and Relationships with Illness Severity and Daily Functioning

Assays of Metacognition Across Different Patient Populations: Implications for Understanding Impaired Self-Awareness

\section{AM Coffee Break}

Exhibit Halls B North (Exhibition Level)

\section{Symposium 12. Health-Promoting Activities as Protective Factors Underlying Disease Outcomes in MS \\ Chair \& Discussant: Peter Arnett \\ Presenters: Margaret Cadden, Brian Sandroff, Robert Motl, Cristina A.F. \\ Roman \\ Ballroom Salon 2-3}

Health-Promoting Activities as Protective Factors Underlying Disease Outcomes in MS Cognitive Reserve, Depression, and Cognition in Multiple Sclerosis

Exercise and Cognition in Persons with Multiple Sclerosis

Exercise and Its Benefits in Persons with Multiple Sclerosis

Exercise and Structural Connectivity in Multiple Sclerosis: A Graph Theoretical Perspective

\section{Paper Session 17. Cancer Across the Lifespan \\ Moderator: Jeffrey S. Wefel}

Ballroom Salon 1

Relations Between Observer- and Performance-Based Outcomes in Pediatric Cancer Survivors: Differences as a Function of Disease and Informant

Mild Cognitive Impairment and Dementia Show Contrasting Associations with Risk of Cancer Neurocognitive Outcomes in Long-Term Survivors of Childhood Wilms Tumor

Anesthesia Exposure, Neurocognitive Function, and Neuroimaging Outcomes in Long-Term

Survivors of Childhood Acute Lymphoblastic Leukemia

\section{Paper Session 18. Memory Function \\ Moderator: Amanda Gooding \\ Maryland A-C}

Intraindividual Variability in Neuropsychological Test Performance Is Associated With Time-Based Prospective Memory in Older Adults

Prospective Memory - an Evaluation of the PROPS Test and the Impact of Low Birth-Weight and Other Perinatal Risks in Adulthood

Exposure to Blue Wavelength Light During Memory Consolidation Improves Long-Delay Verbal Memory Performance

Hippocampal Dentation in Healthy Children and Adolescents: Associations with Age and Verbal Episodic Memory

Early Intellectual Giftedness is Associated with White Matter Integrity in Regions Subserving Episodic Memory

Visuospatial Working Memory Impairments in Very Preterm Survivors Persist from Childhood to Adulthood

Paper Session 19. Cerebrovascular Issues and Epilepsy

Moderator: Michael Williams

Virginia A-C

Long-Term neuropsychological impairment in 4 year stroke survivors

Distinct Neuropsychological Profile and Associated Neurochemical Changes in Patients with Mitochondrial Encephalomyopathy, Lactic Acidosis, and Stroke-Like Episodes (MELAS) Increased Cerebrovascular Resistance Correlates With Worse Cognitive Performance in Older Adults Exhibiting Normal Cerebral Blood Flow 
4. REYES, A

5. BLACKMON, K

6. COULEHAN, $\mathrm{K}$

10:45 AM-12:00 PM

1. AINGER, TJ

2. AL-KHARAFI. HT

3. BAIRD, AD

4. BERL, $M$

5. BLAIR, MA

6. СТРОНОТТI I WHTHORAW

7. CLARK, SV

8. CORDEAUX, C

9. COLBY, KA

10. COLBY, KA

11. CRAUN, E

12. DOWNES, M

13. DOYLE, L

14. ENEVA, K

15. EVERETT, LS

16. FISCHER, M

17. RICHARDSON, R

18. HUBER, RS

19. KAIS, LA

20. LABODE, VM

21. MASSONI, SG

22. LEGARRETA, M

23. MIETCHEN, JJ

24. MORGAN, B

25. NGUYEN, CM

26. NICHOLS, A

27. NIXON. KH

28. ORR, BC

29. PAGAN, CR

30. POLNASZEK, KL

31. POMMY, J

32. REYNOLDS, BW

33. RYU, K

34. SANTOS. OA

35. PENNINGTON, CR

36. SHIELDS, BJ

37. SKEEL, $R$

38. TESSIER, J

39. VANDEN BUSSCHE, A

40. WELSH. M

41. WELSH, M

42. XU, J

43. ZIEMNIK, RE
Does bilingualism increase cognitive reserve in patients with temporal lobe epilepsy?

Temporal lobe gray-white blurring and Wada memory impairment in MRI-negative temporal lobe epilepsy

Decline in Activities of Daily Living in Epilepsy and MND: Risk Factor for Future Dementia?

\section{Poster Session 11. Executive Function, Language, and Memory Exhibit Hall B North (Exhibition Level)}

\section{Executive Functions/Frontal Lobes}

Enhancing The Understanding of Executive Functioning of Individuals with Schizophrenia The Neurobehavioral Examination: Exploring the Differences Between Controls, Alzheimer's Disease and Parkinson's Disease Groups

Getting Things Done: Coaching Undergraduates with Attentional Complaints

Data Driven Profiles of Executive Function Distinguish Psychiatric Behaviors in Pediatric Populations Neuropsychological Correlates of Adaptive Risk-Taking in Adolescence

\section{tesions}

Cerebellar Contributions to Inhibitory Control in the Stop Signal Task: A Systematic Review Trauma and Executive Functioning: Predictors of Length of Stay in Inpatient Children Executive Dysfunction in Eating Disorders: A Meta-Analytic Comparison of Anorexia Nervosa and Bulimia Nervosa

Executive Functioning Deficits in Children and Adults with Anorexia Nervosa: A Meta-Analysis PTSD symptoms, traumatic experiences, and their relationship with neurocognitive functioning among inmates

Executive Development in Preschool Children with Sickle Cell Disease: Shifting the Focus from Disease to Environment in Future Research

Impact of Comorbid Oppositional Behavior on Executive Function Among Youth with Histories of Heavy Prenatal Alcohol Exposure

Executive Functioning in Women with Binge-eating Disorder Across Weight Groups

Examining the Influence of Post-Deployment Family Support on Executive Functioning in Post-9/11 African-American Veterans

Self-Reported Executive Functioning Deficits in TLE Relate to Both Working and Episodic Memory Another Ineffective Performance-Based Task of Executive Functioning: Where Do We Go From Here? Executive Functioning and Anterior Cingulate Volume in Adolescent Bipolar Disorder

The Influence of Affect on Interference Processing in Blocked and Mixed Presentations of a Stroop Color-Word Task

Impulsivity as a Moderator of the Relationship Between Stressful Life Events and Binge Eating Exposure to family violence and executive functions: a pilot study

Impulsivity and Aggression: The Relationship to Suicidal Behaviors in US Veterans

Moderating Variables of Sleep Disordered Breathing and Executive Functioning: A Meta-Analysis The Relationship of Craving and Negative Affect to Impulsive Decision-Making During Heightened mood Conditions

The Contribution of Executive Functioning to Performance on the Texas Functional Living Scale

A Cluster Analytic Approach to Defining Executive Dysfunction Subtypes

Learning and Executive Functioning in Humanitarian Aid Workers During Times of Overwhelming Stress

Is Verbal Fluency a Language Measure?

Culture and English Fluency on Measures of Executive Functioning in Ethnically Diverse Groups Inhibitory Control and Psychological Symptoms Influence Pain Symptomatology in Emerging Adults Executive Function in the Context of Intimate Partner Violence

Gambler's Ruin: Negative Affect Guides Pursuit of Punishing Choices on the IGT

Relationship Between Naming Ability And Other Neuropsychological Functions

Investigating the Effect of Bilingualism and Nonverbal Intelligence on Executive Functions

Exploratory Factor Analysis of Neuropsychological Test Data Suggests a Four Factor Model of Executive Functioning in an Undergraduate Sample

Impact of Early Neurologic Insult on Executive Functioning Development in Preschool Children Understanding the Relationship Between Working Memory, Impulsivity, and Aggression Sleep and Executive Functioning in Post-9/11 Combat Veterans With and Without Posttraumatic Stress Disorder

Cognitive Underpinnings of Frontolimbic Dysfunction in PTSD and Impact on Quality of Life Child Maltreatment and Hot Executive Function as Predictors of College GPA

Trauma-specific Symptoms and General Mental Health Status each Predicts Hot Executive Function Performance in a College Sample

Predicting Performance in the Alternate Uses Task

Office-based measures of medication management as predictors of real-world medication management 


\author{
44. CROOK, CL \\ 45. DEKHTYAR, M \\ 46. MCCULLAGH, J \\ 47. OLIVIER, TW \\ 48. RAYMER, AM \\ 49. ANDERSON, JR \\ 50. BEZDICEK, O \\ 51. CARVALHO, JO \\ 52. DION, C \\ 53. GRILLI, MD \\ 54. JACOBSON, B \\ 55. KALSCHEUR, E \\ 56. PRIMOSCH, M \\ 57. MCFARLAND, CP \\ 58. MEMEL, MB \\ 59. PIOLINO, P \\ 60. REITER, K \\ 61. STEWART, BT \\ 62. STRANG, J \\ 63. WILKISON, C \\ 64. YEUNG, RC \\ 65. OTERO, TL
}

\section{2:15-1:15 PM}

1. RAMACHANDRAN, V

1:15-2:15 PM

\section{Language and Speech Functions/Aphasia}

Sleep and Language Functioning in Young Adulthood

Cognitive Control and its Relationship with Cognition and Language in Monolingual and Bilingual Aphasia

Relationships Between Cognitive, Language, and Central Auditory Processing Assessments in Children

Impact of Ototoxicity on Reading-Related Skills in Patients Treated for Pediatric Medulloblastoma Influence of Noise and Visual Information on Auditory Processing in Aphasia

\section{Memory Functions}

Variability in Actigraphic Sleep Duration Predicts Word List Recall Performance: A Bayesian Variability Analysis

The learning/encoding versus retrieval deficit hypothesis in Parkinson disease revisited

Does Perceived Control over Memory Affect Performance?

Sex Differences in the Contribution of Physical Activity to Verbal Learning and Memory in Older Adults

The Fractionation of Personal Semantic Memory: Evidence from Two Individuals with Anterior Lateral Temporal Lobe Lesions

The Effect of Subsyndromal Symptomatic Depression on Verbal Learning and Memory

Duration of Fame and Extent of Semantic Knowledge of Famous Names in Cognitively Intact Older Adults

An investigation of the effects of depressive-rumination on prospective memory

What if time isn't on my side?: Depressive symptoms and psychological distance from past and future events

Visual Integration of Objects and Scenes Results in Age-Specific Changes in Hippocampal and Parahippocampal Contributions to Successful Memory Retrieval

Does Virtual Reality Have a Future for the Study of Episodic Memory in Clinical Neuropsychology? Developmental amnesia: A case series of adults with congenital heart disease

An Investigation of the Relations Between Stress and Prospective Memory

May I Have Your Keys? Prospective Memory and Remembering to Remember

Omission Neglect as Insensitivity to Missing Information: Effect of Episodic Simulation and

Relationship with Neuropsychological Measures

Enhanced Memory for Threat Distractors in High Social Anxiety

\section{Assessment/Psychometrics/Methods (Child)}

Virtual Reality as a Method of Phenotyping Neurocognitive Function in Children and Youth

Plenary G (Kaplan Memorial Lecture). Disembodied Minds and Embodied Brains

Presenter: Vilayanur Ramachandran

Ballroom Salon 2-3

Disembodied Minds and Embodied Brains

\section{Kaplan Lecture Luncheon \\ Ballroom Salon 1}


https://doi.org/10.1017/S1355617718000528 Published online by Cambridge University Press 


\title{
Abstracts Presented at the Forty Sixth Annual Meeting International Neuropsychological Society
}

\author{
February 14-17, 2018 \\ Washington DC, USA
}

WEDNESDAY MORNING, FEBRUARY 14, 2018

\begin{abstract}
CE Workshop 1. Test and Treat or Treat and Test? Scientific and Ethical Issues in Neuropsychological Approaches to Learning Disabilities
\end{abstract}

\author{
Presenter: Jack M. Fletcher \\ 9:00 a.m.-12:00 p.m.
}

\begin{abstract}
J.M. FLETCHER. Test and Treat or Treat and Test? Scientific and Ethical Issues in Neuropsychological Approaches to Learning Disabilities.

Neuropsychologists are commonly called upon to evaluate and treat children with highly prevalent learning disabilities (LDs). But the role of the neuropsychologist is not well-defined and not always aligned with contemporary scientific research. In a recent 2017 exchange in Archives of Clinical Neuropsychology, Fletcher and Miciak and Schneider and Kaufman (2017) argued over the relative merits of the assessment of cognitive processes associated with LDs, with Schneider and Kaufman concluding that "the existing evidence base that demonstrates the value of comprehensive cognitive assessments for this purpose is not nearly as strong as it needs to be." This workshop outlines the views of Fletcher and Miciak, who argued for reduced emphasis on cognitive processes in favor of comprehensive evaluations of academic performance and comorbid conditions in an effort to develop well-defined intervention plans for children with these disorders. The current status of research on LDs will be discussed, integrating cognitive, neurobiological, and educational perspectives. The implications of this research for assessment and intervention will be reviewed with an emphasis on the ethical importance of aligning practice with research. Effective interventions for LDs will be discussed with the goal of collecting assessment data that will allow the neuropsychologist to prescribe evidence-based interventions as part of a comprehensive evaluation. By knowing the nature of the academic disabilities and associated disorders (e.g., ADHD, anxiety), the neuropsychologist can provide evaluations that target effective intervention based on a thorough understanding of the current evidence-base on LDs. Learning Objectives

1. Deeper understanding of contemporary approaches to neuropsychological assessment and intervention of learning disabilities

2. Discuss current scientific research on learning disabilities, including cognitive, neurobiological, and educational perspectives

3. Appreciate potential ethical issues that may emerge when clinical practice is not aligned with research

4. Prescribe effective interventions for children with LDs and comorbid conditions
\end{abstract}

Correspondence: Jack M. Fletcher, PhD, Psychology, U of Houston, 1703 Hazard St, Houston, TX 77019, United States. E-mail: jack.fletcher@ times.uh.edu

\section{CE Workshop 2. Role of Neuroplasticity in Outcomes from Perinatal Brain Injuries}

Presenters: Michael V. Johnston, Gwendolyn J. Gerner, Joanna Burton

$$
\text { 9:00 a.m.-12:00 p.m. }
$$

M.V. JOHNSTON, G.J. GERNER \& J. BURTON. Role of Neuroplasticity in Outcomes from Perinatal Brain Injuries.

Brain plasticity is enhanced in the developing brain and strongly influences the outcome from brain injuries in fetuses and neonates. To provide a clinical framework for understanding the influence of plasticity in these injuries, it is convenient to classify plasticity into four major types: 1) adaptive plasticity, which is shaped by exeperiences such as language development and learning to play a musical instrument; 2) impaired plasticity due to diverse types of genetic and environmental influences; 3) excessive or dysfunctional plasticity such as phantom pain after loss of a limb or dystonia due to repetitive movements; 4) plasticity as the "Achilles Heel" in which heightened plasticity during development creates vulnerability to over-stimulation of specific excitatory neurotransmitter circuits in the brain.

The expected audience for this symposium will be neuropsychologists interested in brain development, injury and plasticity, and no past experience with brain imaging or neonatal intensive caee is required. There are three learning objectives:

1) Understand the major types of brain plasticity during development and recognize their role in the outcome of a variety of insults to the fetal or neonatal brain;

2) Recognize the major cognitive and behavioral outcomes of injuries to the fetal or neonatal brain as assessed by brain magnetic resonance imaging and ultrasound brain imaging as well as standardized behavioral and neurological testing in the newborn intensive care unit (NICU); 3) Recognized the major cognitive and behavioral outcomes of injuries to the fetal or neonatal brain as assessed by brain imaging and neuropsychological testing in toddlers who have graduated from the NICU. Correspondence: Michael V. Johnston, MD, Neurology and Pediatrics, Kennedy Krieger Institute/Johns Hopkins School of Medicine, 707 North Broadway, Baltimore, MD 21205, United States. E-mail: johnston@ kennedykrieger.org 


\section{CE Workshop 3. Language Foundations in Population Encoding Networks and Implications for Aphasia Therapy}

\section{Presenters: Stephen E. Nadeau, Irene Minkina, Lisa Edmonds}

$$
\text { 9:00 a.m.-12:00 p.m. }
$$

S.E. NADEAU, I. MINKINA \& L. EDMONDS. Language Foundations in Population Encoding Networks and Implications for Aphasia Therapy.

One of the great challenges of cognitive neuroscience has been accounting for cognition in terms of the activity of 100 billion neurons — lipid bilayer encased electrochemical entities that are interconnected on a vast scale by synapses. We now know that representations in the brain are population-encoded, reflecting patterns of activity of millions or billions of neurons. Forty years of research in the field of parallel distributed processing have substantially defined the unique capabilities conferred by networks supporting population encoded representations (e.g., content addressable memory, working memory, representation of frequency and age of acquisition effects, the power of regularities wired into network connectivity, and graceful degradation) and the constraints they impose. Any domain of cognitive function can be understood on this basis but language provides particularly broad and granular insight into how the neurodynamics operate. This course will begin with an introduction to cerebral foundations of language function viewed through the lens of population encoding. We will then consider the implications for aphasia therapy with a particular focus on mechanisms of generalization: the extension of the benefits of aphasia therapy to untreated exemplars and everyday verbal communication. The last hour of the session will be devoted to two specific therapies for anomia in aphasia, phonomotor and semantic, that intrinsically generalize because they build on regularities in acquired knowledge wired into neural connectivity. The target audience will be participants with intermediate or advanced knowledge of language function.

Correspondence: Stephen E. Nadeau, MD, Neurology, University of Florida College of Medicine, Research-151, Malcom Randall VA Medical Center, 1601 SW Archer Road, Gainesville, FL 32608, United States. E-mail: stephen.nadeau@neurology.ufl.edu

\section{Lunch (On Own) \\ 12:00-1:00 p.m.}

\section{WEDNESDAY AFTERNOON, FEBRUARY 14, 2018}

\section{CE Workshop 4. Assessment and Treatment of Postconcussive Symptoms in Veterans: Rethinking the Role of Concussion}

\author{
Presenter: Amy J. Jak
}

$$
\text { 1:00-4:00 p.m. }
$$

\footnotetext{
A.J. JAK. Assessment and Treatment of Postconcussive Symptoms in Veterans: Rethinking the Role of Concussion.

Protracted recovery from trauma is notable in Veterans; those with a history of concussion endorse more severe PTSD and neurobehavioral symptoms than those without history of concussion. Polytrauma presentations with comorbid mental health conditions complicate recovery from both physical and psychological disorders, magnify cognitive complaints, and contribute to poor functional outcomes. Cognitive complaints in the post-acute period after concussion are nonspecific symptoms, however, and arise from numerous, often non-neurological, sources. Neuropsychological assessment and intervention, therefore, needs to take into account the multitude of factors perpetuating cognitive symptoms, including misattribution of symptoms, pain, sleep, mental health conditions, history of TBI, and/or other medical conditions. This workshop will delineate the unique challenges of assessment and rehabilitation of Veterans with persistent postconcussive symptoms and will describe treatment options for this population, including novel treatments targeting common comorbidities. The workshop will highlight the importance of reducing emotional distress in Veterans as key to attenuating neurobehavioral symptom reporting and cognitive complaints but will also discuss cases where concussion history may more prominently figure into the assessment and treatment picture. Though the workshop focus will be on the Veteran population, the topics covered will also have applicability to civilians with persistent postconcussive symptoms.

Upon conclusion of this course, learners will be able to:

1. Describe the neuropsychological presentation of treatment-seeking Veterans with history of concussion and persistent cognitive complaints. 2. Recognize both neurological and non-neurological factors impacting functioning in this population
}

3. Identify treatment options for individuals with cognitive complaints in the post-acute period following concussion Correspondence: Amy J. Jak, Ph.D., VA Healthcare System, San Diego/ UCSD, 3350 La Jolla Village Dr., 151B, San Diego, CA 92161, United States.E-mail:ajak@ucsd.edu

\section{CE Workshop 5. How Adolescence Specializes The} Brain to Establish Adult Modes of Operation

\author{
Presenter: Beatriz Luna
}

1:00-4:00 p.m.

\section{B. LUNA. How Adolescence Specializes The Brain to Establish} Adult Modes of Operation.

Adolescence is a unique period of development when there is an adaptive increase in exploration underlied by sensation seeking that can lead to risk-taking behavior undermining survival. This is also a time of risk for the emergence of psychiatric disorders. Identifying the specific brain processes that are undergoing plastic change through adolescence can inform mechanisms underlying vulnerability to mental illness but also provide opportunities to influence developmental trajectories. The workshop will begin by reviewing the evidence on the specific changes that occur in normative adolescent brain maturation followed by multi-modal neuroimaging evidence identifying functional brain changes supporting cognitive development. Next, we will examine how neurodevelopment can become impaired in psychopathology including future next steps for correcting trajectories. This workshop aims to build from basic knowledge to an intermediate and advanced understanding of adolescent brain development. Specifically, evidence will be presented describing changes in brain structure, from the molecular to systems levels followed by evidence examining brain connectivity and changes in brain systems that support cognitive maturation. A model will be presented that proposes that foundational aspects of neurocognition, including the prefrontal cortex, are on-line by adolescence. However, the engagement of these neurocognitive systems stabilizes into adulthood 
as affective (reward and emotion) systems attenuate and processes that support the integration of experience are prioritized. Vulnerabilities for impaired development in psychopathology will build from the understanding of normative development and variability in trajectories that can lead to impaired outcomes. As a result of participation in this course, the learner will achieve the following objectives: 1. An understanding of the changes that occur in brain physiology specific to the adolescent period, 2. Gain insight into how cognition is supported by the brain processes available in adolescence, and 3. Have increased awareness of how development can lead to impairment such as mental illness. Correspondence: Beatriz Luna, PhD, Psychiatry, University of Pittsburgh, 121 Meyran Avenue, Pittsburgh, PA 15213, United States. E-mail:lunab@upmc.edu

CE Workshop 6. Ethical Practice in a Learning Healthcare System: Tomatoes, Technology, and the Marriage of Clinical Practice and Research

\section{Presenters: Lisa Jacobson, T A. Zabel, Alison Pritchard \\ 1:00-4:00 p.m.}

L. JACOBSON, T.A. ZABEL \& A. PRITCHARD. Ethical practice in a learning healthcare system: Tomatoes, technology, and the marriage of clinical practice and research.

As a result of technological advances, healthcare systems are now, more than ever before, positioned to utilize the information generated by patient care in the service of improving and personalizing patient care. A Learning Healthcare System (LHS) framework is discussed for making sense of the intersection of clinical care and research and guiding ethical practice of neuropsychology. Under this framework, the commitments and responsibilities of the patient and the healthcare provider/system are delineated, and both practical and ethical implications of thorough integration of research and practice will be considered. To demonstrate this framework, specific strategies and applications of technology to both clinical practice and research will be presented, including "lessons learned" through the presenters' own DIY experience and development of clinical triage algorithms to improve efficiency and precision of neuropsychological practice. The ethical considerations of implementing such changes in practice will be reviewed, including unique clinician responsibilities and challenges associated with patient information acquired via LHS technologies and screening methodologies. Finally, methods for disseminating quality improvement information gained through analysis of patient care will be discussed, both for clinician guidance within the LHS as well as outside of it (e.g., national organizations, other health care systems).

Following participation in the course, the learner will: 1) become familiar with the concept of a Learning Healthcare System, 2) describe applications of technology to clinical and research practice, and $3)$ identify ethical challenges and considerations of incorporating technology into practice within a Learning Healthcare System. Correspondence: Lisa Jacobson, Ph.D., Neuropsychology, Kennedy Krieger Institutel Johns Hopkins School of Medicine, 1750 E. Fairmount Ave, Dept. of Neuropsychology, Baltimore, MD 21231, United States. E-mail:jacobson@kennedykrieger.org

\section{Workshop, Presented by the INS Student Liaison Committee: The Role of Neuropsychology in an Integrated Healthcare Setting}

\author{
Presenter: Neil H. Pliskin
}

2:30-4:00 p.m.

Poster Session 1. Aging

2:30-3:45 p.m.

\section{Aging}

S. BAJAJ, N.S. DAILEY, A. ALKOZEI, J.R. VANUK \& W.D. KILLGORE. Preservation of limbic network structure in healthy young adults.

Objective: Aging has widespread effects on cells, molecules, cognition, and brain structure. There is extensive research showing significant negative impacts of age on cognitive abilities and structural characteristics. However, it still remains unclear how multiple structural measures of the brain, such as cortical thickness (CT), cortical surface area (CSA), and cortical volume $(\mathrm{CV})$ respond to healthy aging. Furthermore, how these changes may differ across different functional networks has not yet been explored.

Participants and Methods: Neuroanatomical data were collected from 56 healthy young adults between 18-45 years of age (mean age $=$ $30.8 \pm 8.1$ years, 27 females). FreeSurfer was used to preprocess the data and further parcellate the whole brain into seven functional networks, including the visual network, somatomotor network, dorsal-attention network, ventral-attention network, limbic network, fronto-parietal network, and default-mode network. CT, CSA, and CV for each network was calculated separately for each hemisphere and correlated with age. Results: Apart from the limbic network, which was unrelated to age, CT was negatively correlated with age for all the functional networks for each hemisphere $(p<0.05)$. There was no significant correlation between age and CSA $(p>0.05)$, but age and CV showed negative significant correlations for all the functional networks for each hemisphere $(\mathrm{p}<0.05)$. All reported correlations were corrected for multiple comparisons.

Conclusions: The limbic functional network appears to be the most preserved and the least susceptible to age-related morphometric changes in healthy young adults. Our findings also suggest that among three cortical measures (CT, CSA, and CV), CT is a more reliable and sensitive measure to determine age-related structural changes in young adults. We conclude that the detailed age-related analysis of structural characteristics could help to better understand the relationships between healthy aging and brain tissue loss.

Correspondence: Sahil Bajaj, Psychiatry, University of Arizona, 1501 N. Campbell Ave, Tucson, AZ 85724, United States. E-mail: sbajaj1@ psychiatry.arizona.edu

E.A. BOOTS, C. DION, N. RAJENDRAN, A. KARSTENS, J. COHEN, O. AJILORE \& M. LAMAR. Preclinical Profiles of Memory Versus Executive Function Weakness as Related to Cognition, Stroke Risk, and White Matter Integrity in Older Adults.

Objective: Prior work has shown there are relatively greater memory (MEM) deficits in Alzheimer's disease and relatively greater executive function (EF) deficits in vascular dementia. Our objective was to examine whether these differences, identified preclinically, are associated with other predictors of pathological aging in older adults.

Participants and Methods: 111 non-demented/non-depressed adults (mean age $=67.8,50.5 \%$ male) underwent cognitive and medical testing and MRI. Groups were made using composite z-scores for MEM 
(CVLT-II Trials 1-5, long delay free recall \& recognition) and EF (Trail Making Test Parts A\&B, Letter-Number Sequencing); scores below the median were deemed 'weaknesses'. MANCOVAs adjusting for age, sex, and predicted VIQ assessed whether MEM and EF weakness associated with individual tests of language [FAS, Animals, Semantic Association Index (AI), Boston Naming Test (BNT)] and visuospatial [Judgement of Line Orientation (JLO), Matrix Reasoning (MR)] function. ANCOVAs investigated Framingham 10-Year Stroke Risk (Fram-10) and T1/ T2-FLAIR quantified white matter hyperintensities (WMH).

Results: MEM (FAS p=.01; Animals p=.002) and EF (FAS p<.001; AI $\mathrm{p}=.07$ ) weakness separately associated with poorer language function. There was a MEM*EF interaction for BNT only $(p=.05)$; those with MEM and EF weakness performed worse. EF weakness associated with worse visuospatial function (JLO \& MR both $\mathrm{p}<.001$ ); MEM weakness trended significant (JLO p=.07); there was no interaction. Only EF weakness associated with higher Fram-10 $(p=.04)$ and greater WMH $(\mathrm{p}=.09)$.

Conclusions: Weakness in either EF or MEM associated with lower letter fluency performance. In contrast, distinct semantic association, visuospatial difficulties, stroke risk and $\mathrm{WMH}$ were seen with EF weakness, while MEM weakness corresponded to additional difficulty with semantic retrieval only. Preclinical profiles of cognitive weakness in EF or MEM signal other alterations that when combined may be predictive of differential profiles of pathological aging.

Correspondence: Elizabeth A. Boots, B.S., Psychology, University of Illinois at Chicago, Behavioral Sciences Building Room 1080, 1007 W Harrison St, Chicago, IL 60607, United States.E-mail: eboots2@ uic.edu

L.M. CAMPBELL, J. EPPIG, K.R. THOMAS, E.C. EDMONDS \& M.W. BONDI. Cognitive Performance and Alzheimer's Disease Biomarkers in SuperAging and Normal Older Adults.

Objective: Prior research has investigated 'SuperAgers' (SA), defined as older adults with superior delayed recall on verbal memory tests. The current study compared SAs and cognitively normal older adults on cerebrospinal fluid (CSF) biomarkers associated with Alzheimer's disease (AD).

Participants and Methods: Participants were from the Alzheimer's Disease Neuroimaging Initiative. All were $\geq 80$ years old and cognitively normal based on neuropsychological performance. They were classified as SAs if they: 1) had a raw score $\geq 9$ on RAVLT delayed-recall (i.e., average normative performance for 50-60 year olds) and 2) performed within $1 \mathrm{SD}$ on tests of language and executive functioning. Twenty-six SAs were matched on age, education, and sex to 26 normal controls (NC). All participants underwent APOE genotyping and a neuropsychological assessment including scales of premorbid IQ, depression, and vascular risk. A subsample had a lumbar puncture to assess CSF levels of $A \beta_{1-42}$, tau, and $p-\operatorname{tau}_{181}$.

Results: There were no differences in demographics, depression, vascular risk, or APOE $\varepsilon 4$ status (all $p s>0.30$ ) between SAs and NCs. SAs performed significantly better on the BNT, animal fluency, Logical Memory I \& II, and the ANART (all $p s<0.04$ ). However, in a subsample with CSF data (SA: $n=14$, NC: $n=16$ ), there were no differences between groups on CSF biomarkers of $\mathrm{A} \beta_{1-42}$, tau, $\mathrm{p}$-tau ${ }_{181}$, or the $\mathrm{p}$-tau ${ }_{181} / \mathrm{A} \beta_{1-42}$ ratio (all $p s>0.22$ ).

Conclusions: SAs performed better than NCs on multiple tests of language and memory, but were not significantly different on AD biological markers (i.e., APOE genotype, CSF, vascular risk). The finding that the SA group performed better on a measure of premorbid intelligence supports the possibility that superior memory in older adults may reflect high cognitive reserve rather than neurobiological differences. Future research should examine longitudinal neurocognitive outcomes of these groups and further explore other measures related to cognitive reserve.
Correspondence: Laura M. Campbell, Joint Doctoral Program in Clinical Psychology, SDSU/UCSD, 3350 La Jolla Village Drive, 151B - Attn: Laura Campbell, San Diego, CA 92161, United States. E-mail: lauracampbell166@gmail.com

R. DIVERS, K. HOWARD, L. HAM, A. MATCHANOVA, S.S. RYCROFT \& T. GIOVANNETTI. Unique Effects of Aging on Everyday Action Performance in Older Adults Compared to Younger Adults Performing a Dual Task.

Objective: Mild functional difficulties are reported by older adults but remain poorly understood. We examined micro-errors (i.e., inefficient actions) during the completion of everyday tasks, and investigated the hypothesis that older adults would show the same number and type of micro-errors as young adults in a dual task condition designed to reduce cognitive resources.

Participants and Methods: 25 healthy older adults performed an everyday task (prepare lunch) with target and distractor objects on a tabletop. 21 young adults performed the same lunch task while performing the Oral Trail Making Test. Micro-errors were coded from video, tallied, and characterized on three different levels: 1) timing beginning, middle, or ending segment of the task; between or within sub-tasks 2) monitoring - the extent to which the participant made contact with an incorrect object reflected the severity of a monitoring failure (i.e., reach no touch - mild failure; extra action- severe failure) and 3 ) object properties -target object, distractor object). Nonparametric analyses were used.

Results: Young adults in the dual task condition made as many total micro-errors as older adults without the dual task, but the pattern of micro-errors differed significantly. Older adults made significantly more micro-errors during the final segment of the task $(Z=-1.73$, $\mathrm{p}<.01)$, between subtasks $(\mathrm{Z}=-2.14, \mathrm{p}<.01)$, to unrelated distractors $(\mathrm{Z}=-2.60, \mathrm{p}<.01)$, and reflecting egregious monitoring failures (e.g., extra actions; $\mathrm{Z}=-1.75, \mathrm{p}<.01)$. Younger adults with the dual task made significantly more micro-errors within a subtask $(Z=2.38, p<.01)$, at the beginning task segment $(\mathrm{Z}=2.24, \mathrm{p}<.01)$, and reflecting rapid error monitoring (e.g. reach no touch; $\mathrm{Z}=2.06, \mathrm{p}<.01$ ).

Conclusions: Everyday action difficulties across clinical populations may differ in severity as well as type. Difficulties due to cognitive aging cannot be simply explained by limited cognitive resources but may be due to reduced error monitoring and increased interference.

Correspondence: Ross Divers, Psychology, Temple Univeristy, Temple University, Psychology Department, 1701 N 13th St., Philadelphia, PA 19122, United States. E-mail: ross.divers@temple.edu

N.A. EMMERT, A. ARBUCKLE, L. SCHWARZ, J.S. VANDER WAL \&. J. GFELLER. An Examination of Neuropsychological Functions and Depression as Predictors of Health and Safety Abilities in Older Adults with Dementia.

Objective: The Independent Living Scales Health and Safety (ILS HS) scale is commonly utilized by neuropsychologists when evaluating older adults' functional abilities. However, there is minimal research on its validity in dementia populations, and specifically, its relationship to neuropsychological test performance. Therefore, this study investigated relationships between the ILS HS scale and measures of cognition and depression, to examine the criterion-related validity of commonly utilized neuropsychological measures, and the cognitive abilities that underlie health and safety abilities in older adults.

Participants and Methods: The current study utilized archival data from a mixed clinical sample $(N=142)$ of older adults diagnosed with various types of dementia (e.g., Alzheimer's disease), who were administered the WTAR, RBANS, TMT, Stroop Color-Word Test, WCST, ILS HS scale, and the GDS as part of a larger neuropsychological evaluation at an outpatient service.

Results: Correlational analyses revealed significant, positive relationships between the ILS HS scale and most measures of cognitive functioning $(p ' s<.01)$. Multiple regression analyses demonstrated that up to 
$39 \%$ of the variance in ILS HS performance was attributed to measures of global cognition, immediate verbal recall, processing speed, cognitive flexibility, working memory, and verbal fluency after controlling for age, education, and estimated premorbid intelligence.

Conclusions: Results from the current study suggest that older adults' ability to function in health and safety situations requires a wide range of cognitive abilities. Our results also indicate that the neuropsychological tests used in the current study have significant ecological validity. Performance on these measures can help guide clinicians who need to make decisions about an older adult's ability to carry out every day functional skills related to their health and safety.

Correspondence: Natalie A. Emmert, M.S., Psychology, Saint Louis University, 3700 Lindell Blvd., St. Louis, MO 63108, United States. E-mail:emmertna@slu.edu

N.D.EVANGELISTA, M.L. WERHANE, A.L.CLARK, V.C. MERRITT, M. TRAN, J.C. CHALMERS, M.W. BONDI, L. DELANO-WOOD \& K.J. BANGEN. Endothelin-1 and Cerebral Blood Flow Interact to Affect Memory Performance in Cognitively Normal Older Adults. Objective: Although alterations in endolthelin-1 (ET-1)—a peptide associated with endothelial dysfunction and disruption of the blood brain barrier (BBB) - and changes in cerebral blood flow (CBF) have been independently linked to cognitive impairment in older adults, it remains unclear how these variables may interact to affect cognition. We therefore sought to clarify associations between ET-1, CBF, and verbal memory function in well-characterized cognitively normal older adults. Participants and Methods: Twenty-three cognitively normal older adults (age: $M=74$ years; $S D=5.41$ ) underwent neuropsychological assessment, a blood draw, and magnetic resonance imaging (MRI). The California Verbal Learning Test $2^{\text {nd }}$ Edition (CVLT-II) was used to assess memory. Blood samples were analyzed for mean serum ET-1 concentration $(\mathrm{pg} / \mathrm{mL})$, and arterial spin labeling (ASL) MRI was used to quantify $\mathrm{CBF}$ in medial temporal regions of interest subserving memory functions.

Results: Multiple linear regression models adjusted for age, education, blood pressure, and antihypertensive medication use revealed a significant ET-1 x CBF interaction on memory performance in fusiform and left parahippocampal gyri $\left(p^{\prime} s<.05\right)$. Specifically, greater CBF was associated with better memory performance in older adults with elevated ET-1, while no such association was observed in individuals with lower ET-1.

Conclusions: Our results demonstrate that, among older adults with elevated ET-1 levels, higher CBF supports better memory performance. Findings further suggest that, in at-risk older adults, the brain may compensate for BBB breakdown through elevated cerebral perfusion, particularly in areas known to be affected early in the Alzheimer's disease process. Importantly, our results suggest that CBF and its associations with cognition may serve as a reliable marker of brain function in cognitively normal older adults at risk for cognitive impairment at a stage when interventions are likely to be beneficial.

Correspondence: Nicole D. Evangelista, B.S., VA San Diego Health Care System, Veterans Medical Research Foundation, 3350 La Jolla Village Dr., San Diego, CA 92161, United States.E-mail:nevangel@ucsd.edu

E.M. FISCHER, B. SACHS, T.M. HUGHES, K. SINK, L.D. BAKER \& S. CRAFT. Vascular Hemodynamics Relate to Cognitive Performance in Older Adults.

Objective: Blood pressure (BP) is associated with Alzheimer's dementia $(\mathrm{AD})$ and related pathology. We examine the pulse wave hemodynamics that underlie BP and their associations with cognitive performance among older adults with normal cognitive function, mild cognitive impairment (MCI), and AD.

Participants and Methods: In 2013-2016, participants of the Wake Forest Alzheimer's Disease Prevention Participant Repository underwent detailed clinical and neurocognitive exams [Mini-Mental Status Examination (MMSE), WMS-III Logical Memory subtest (LM), Digit
Span, Category Fluency, Trail Making Test A\&B (TMT), W AIS-R Digit Symbol Coding subtest (DSC), and Boston Naming Test (BNT-30), Rey Auditory Verbal Learning Test (AVLT), Brief Visual Memory TestRevised (BVMT-R), and COWAT]. Cognitive status was adjudicated by a consensus composed of neuropsychologists and geriatricians. Vascular hemodynamics of carotid-femoral pulse wave velocity (cfPWV) and wave reflection magnitude were measured by the SpygmoCor XCEL device. We examined the relationship between the mean standardized vascular measures and cognitive scores adjusted for gender, age, and education level.

Results: Among the 97 ADPPR participants $[$ mean (SD) age $=67(8)$, 68 female, 16(2) years of education, 19\% with MCI and $7 \%$ with $\mathrm{AD}$ ], wave reflection magnitude was associated with $\mathrm{LM}$ immediate recall $[$ beta $(\mathrm{SE})=-0.29(0.1), p=0.01], \mathrm{LM}$ delayed recall $[$ beta $(\mathrm{SE})$ $=-0.25(0.1), p=0.02]$, Category Fluency (animals) $[$ beta $(\mathrm{SE})=$ $-0.22(0.1), p=0.03]$, TMT A [beta $(\mathrm{SE})=0.3(0.1), p=0.01]$, and DSC $[\operatorname{beta}(\mathrm{SE})=-0.25(0.1), p=0.02]$. There was no significant relationship found between CfPWV or BP to cognitive performance on all tests.

Conclusions: Wave reflection measuring of peripheral arterial resistance was associated with performance on LM immediate and delayed recall, Category Fluency (animals), TMT A, and DSC.

Correspondence: Eric M. Fischer, MA, Internal Medicine - Gerontology and Geriatrics Medicine, Wake Forest Baptist Health, 2015 Elizabeth Avenue, Apt 5, Winston Salem, NC 27103, United States. E-mail: ericmwfischer@gmail.com

S. GETZ, K.F. MCINERNEY, N. SUN-SUSLOW, N.S. BANERJEE, A. BURE-REYES, M. SARNO \& B.E. LEVIN. Low Emotional Reserve as a Risk Factor for the Frailty Syndrome.

Objective: Frailty is highly prevalent, occurring in $25 \%$ of elderly individuals, up to $50 \%$ of people over 85 , and associated with a significant increase in the risk of disability, extended long-term care, and death. The relationship between frailty and cognition is bidirectional. Few studies have examined the role of emotional factors in frailty. This investigation explores the impact of emotional reserve on the frailty syndrome.

Participants and Methods: 204 participants (ages 50 to 91) completed the Adverse Childhood Experiences (ACE) questionnaire, Beck Anxiety Inventory (BAI), Beck Depression Inventory (BDI-II), and a full frailty assessment based on Fried criteria. Participants were grouped by frailty phenotype (non-frail or pre-frail \& frail) and categorized according to level of emotional reserve. Participants were also grouped into High (HER) vs. Low emotional reserve (LER) based on ACE, BAI, and BDI-II scores. A binary logistic regression was performed. Age and education were included as covariates in the model.

Results: Of the 204 participants, 143 met criteria for frail and pre-frail and 104 were classified as having LER. The logistic regression model was statistically significant, $\left(X^{2}=12.85, \mathrm{p}<.001\right)$, explaining $21.0 \%$ (Nagelkerke $\mathrm{R}^{2}$ ) of the variance in frailty phenotype and correctly classifying $77.0 \%$ of cases. LER participants were 4.84 times more likely to be frail. In the LER group, weak grip strength $(80.8 \%)$ was the most frequently met frailty criteria, followed by fatigue (43.3\%). In the HER group, $58.7 \%$ had weak grip strength and all other frailty criteria fell below $7 \%$.

Conclusions: Results from a binary logistic regression analysis indicate that LER is a significant predictor (risk factor) for frailty. This conclusion is further supported by the lack of any significant change in model significance after controlling for age and education. These data suggests that history of adverse childhood events and emotional factors like depression and anxiety are both important correlates of frailty later in life.

Correspondence: Sarah Getz, Ph.D., Neurology, University of Miami, 3164 Virginia St, Miami, FL 33133, United States. E-mail:sjg144@ med.miami.edu 
M. GOGNIAT, C. MEWBORN, T. ROBINSON, K. JEAN, C. LINDBERGH, J. REYNOLDS, L. RENZI-HAMMOND, B. HAMMOND \& L. MILLER. Relation between Lutein, Zeaxanthin, and Brain Volume in Older Adults.

Objective: Recent evidence suggests that prolonged consumption of lutein and zeaxanthin (L\&Z), nutrients found in many colorful vegetables, may be neuroprotective. Thus, the purpose of this study was to determine the relation between L\&Z and brain volume in healthy older adults.

Participants and Methods: Participants were 52 older adults $(\mathrm{M}=71.8$ years; $\mathrm{SD}=6.05)$ enrolled in a larger study examining the effects of L\&Z on cognitive outcomes. Accumulation of L\&Z was measured via macular pigment optical density (MPOD), a validated proxy for neural levels of lutein and zeaxanthin. Structural magnetic resonance imaging (MRI) brain scans were collected and used to determine total gray and white matter volume and volume, as well as of a priori regions of interest (ROIs) that were chosen because they are areas of primary age-related decline: prefrontal cortex (orbitofrontal and dorsolateral areas), hippocampus, and anterior cingulate cortex. Scans were processed using Freesurfer and measures were corrected for intracranial volume prior to use in statistical analysis. Linear regressions were used to determine the relationship between MPOD and each ROI controlling for age.

Results: When controlling for age, MPOD significantly predicted orbitofrontal cortex volume $\left(\Delta \mathrm{R}^{2}=.075, \mathrm{~F}(1,51)=4.164, p=.046\right)$. MPOD did not significantly predict total gray and white matter volume or volume in the dorsolateral prefrontal cortex, hippocampus, or anterior cingulate cortex.

Conclusions: These results suggest that greater consumption of L\&Z may be related to brain volume, particularly in the orbitofrontal cortex, which is an area vulnerable to age-related decline even in healthy older adults. This is a novel finding linking consumption of specific nutrients to brain volume that will need replication and confirmation in the context of a larger sample and longitudinal design.

Correspondence: Marissa Gogniat, Clinical Psychology, University of Georgia, 935 Baxter Street \#8, Athens, GA 30606, United States. E-mail:mag53440@uga.edu

E.I. GRACIAN, D. KAZAKOV, Z. RESCH, M.B. AUSTIFF, B. YANG, D.C. OSMON \& K.E. MOSACK. Non-Uniform Age-Related Differences and Neuropsychological Correlates of Medication Management Ability in Cognitively Normal Adults.

Objective: Normal age-related cognitive deficits can impair medication management that could contribute to unintentional medication noncompliance. For instance, executive function (EF) and memory deficits are significantly related to problems with medication management in aging populations. However, age-related cognitive impairment is not homogenous; previous investigators dichotomized cognitively normal older adults into impaired-older (IO) and unimpaired-older (UO) subsets, and found that IO adults were disproportionately deficient in memory abilities that could be predictive of forthcoming Alzheimer's disease. However, researchers have not investigated (1) whether IO adults are also disproportionately deficient on the Medication Management Ability Assessment (MMAA) than UO adults and young adults, or (2) compared $\mathrm{EF}$ and memory contributions to MMAA scores in cognitively normal young and older adults.

Participants and Methods: Thirty young adults (Men $\mathrm{n}=8$; Mean Age $=21$ years, $\mathrm{SD}=2.7$ ) and 29 older adults $($ Men $\mathrm{n}=2$; Mean Age $=73$ years, $\mathrm{SD}=8.1$ ) completed measures of $\mathrm{EF}$, memory, and the MMAA. The MMAA is a role-play in which participants were given instructions for taking fake medications and then were asked to inform the researcher how and when they will take pills. Older adults were classified as IO $(n=14)$ or UO $(n=15)$ based on performance on a verbal memory test; IO adults did not perform within the normal range.

Results: IO adults earned lower MMAA scores than UO adults (Cohen's $d=0.81)$ and young adults $(d=0.87 ; p=.004)$. Regression analyses revealed that better performance on memory measures predicted higher scores on the MMAA $(\mathrm{p}=.003)$; neither age $(p=.55)$ nor performance on EF measures were related to MMAA scores $(p=.84)$.

Conclusions: We found disproportionate MMAA deficits in IO adults. Further, memory measures predicted MMAA scores over EF measures and age. Forthcoming work should determine if MMAA deficits in IO adults are predictive of major neurocognitive disorders and medication noncompliance.

Correspondence: Enrique I. Gracian, MA, Psychology, University of Wisconsin, Milwaukee, 1869 N. Cambridge Ave, Apt 207, Milwaukee, WI 53202, United States.E-mail: gracian2@uwm.edu

R.K. MACAULAY, A. HALPIN, P. EDELMAN, J. CHAMPAGNE \& J.S. BROWN. Maine Understanding Sensory Integration \& Cognition (MUSIC) Project: Feasibility of a music training intervention for cognition in older adults.

Objective: Research suggests that learning to play a musical instrument increases neural plasticity, as it involves coordinating motor sequences with planned actions that require higher cognitive resources. Notably, evidence also suggests that music training associates with better working memory and executive function in older adults; however, this work is primarily correlational studies comparing musicians to non-musicians. The present study presents feasibility data from the Maine Understanding Sensory Integration \& Cognition (MUSIC) Project. MUSIC investigates learning to play a musical recorder in an economic group format as a cognitive intervention for older adults.

Participants and Methods: Ten naïve to music literacy older adults $\left(\mathrm{M}_{\mathrm{age}}=69\right.$ years $)$ recruited from two independent living community dwelling sites participated in the pilot study. The National Alzheimer's Disease Coordinating Center's (NACC) neuropsychological test battery evaluated cognitive function at baseline and post-intervention. For the Trails Making Test (TMT), a difference score was created (Trail B minus Trail A) to adjust for the influence of motor speed on Trail B. There were twelve weekly music learning sessions lead by a trained music instructor. Results: Repeated-measure ANOVAs revealed a trend towards global improvements in cognition on the Montreal Cognitive Assessment test. $p=.08$. Executive functioning as measured by the TMT difference score significantly improved, once age was investigated as a covariate, $p=.045$. Delayed visual memory and phonemic fluency scores were significantly higher following the intervention, $p s<.05$. No significant differences in attention/processing speed, working memory, episodic memory, or language were found, all $p s>.10$.

Conclusions: Preliminary findings indicate improvements in global cognition and measures associated with frontal lobe function following a music learning intervention. Future studies intend to use an active control group to assess for practice effects on the neuropsychological tests.

Correspondence: Amy Halpin, M.S., Orono, ME 04469, United States. E-mail: halpin.amy@gmail.com

L. HAM, R. DIVERS, E. ROLL, S.S. RYCROFT, J. HULSWIT, A. MATCHANOVA, K. HOWARD, T. YAMAGUCHI \&. T. GIOVANNETTI. Simulating Cognitive Aging with a Dual Task in the Virtual Kitchen.

Objective: Functional abilities in older adults are associated with many outcomes, but remain poorly understood in neuropsychology. Thus, efficient and standardized methods for assessing mild functional difficulties are needed. This study used a novel virtual reality (VR) task to assess functional performance in young and older adults, and investigated if a Dual Task condition would simulate the effects of cognitive aging. Participants and Methods: 14 community-dwelling older adults and 21 healthy young adults completed the Virtual Kitchen (VK), a non-immersive VR task in which participants manipulate virtual objects on a touch screen interface. All participants completed two everyday tasks (breakfast, lunch) in the Standard condition. Young adults also completed the Dual Task condition (VK + Oral Trail Making Test (TMT)) to induce resource limitations. Videos were coded for completion time and error 
types reflecting different levels of error monitoring (e.g. reaching toward inaccurate object=rapid error monitoring; touching/using inaccurate object=slow error monitoring). Analyses compared older adult Standard VK to young adult Dual VK to determine whether resource limitations simulated cognitive aging.

Results: Compared to young adults in the Dual, older adults required more time to complete the Standard $(p<.01)$, but did not differ in total errors $(p=10)$. Further, older adults made more errors due to slow error monitoring (e.g. moving ( $p<.01)$, touching ( $p=.024)$ inaccurate objects). In young adults, there was a significant positive relation between errors on the Dual and errors on the TMT $(r=.59)$.

Conclusions: The VK is sensitive to functional difficulties associated with cognitive aging and resource limitations, though these difficulties present differently in performance. Some difficulties in older adults may be due to the novelty of VR: however, VR findings corroborate results from real objects. Both outcomes suggest mild functional difficulties in older adults may be due to slowed error monitoring. Correspondence: Lillian Ham, Psychology, Temple University, 1701 N. 13th Street, Weiss Hall, Sth floor, Philadelphia, PA 19122, United States.E-mail: lillian.ham@temple.edu

C.E. HARRISON, K.J. MILLER, L. ERCOLI, G. SMALL \& P. SIDDARTH. Lower Memory Performance in Males with Presence of Multiple Risk Factors for Dementia.

Objective: Research has shown that incidence of memory decline is greater in males than in females, particularly for those in their $60 \mathrm{~s}$. However, the effects of multiple risk factors for dementia on memory performance in older individuals are not fully understood. This cross-sectional study examined how various risk factors for dementia impact the verbal memory performance of older men and women.

Participants and Methods: Two hundred and fifty-one non-demented older adults (161 women. 90 men: age: 62.0 (8.2) vears, education: 16.5 (2.8) years; MMSE: 29.0 (1.3)) with demographic, genetic and vascular risk data, completed Buschke Selective Reminding Task. Risk factors examined included presence of APOE-4, family history, high body mass index, and high cholesterol. Participants were classified as lower risk (0 or 1 risk factor) or higher risk (2 or more risk factors). General linear models were used to investigate memory performance in males and females in lower and higher risk groups, controlling for age, education and MMSE scores.

Results: Verbal encoding (Buschke Total and Consistent Long-Term Retrieval. CLTR) was significantly lower for males in the higher risk group (Total: 96.2 (15.3); CLTR: 44.5 (29.5)) compared to males in lower risk group (104.1 (21.5); 65.7 (38.1), $p=.02,002)$, females in the lower risk (104.8 (21.6); 65.3 (39.4), $\mathrm{p}=.003, .0005)$ and higher risk groups (105.5 (16.7); 64.1 (32.9), p = .0001). Similarly, delayed memory scores were significantly reduced among males with higher risk $(6.9(3.2))$, when compared to all other groups (8.5 (3.4) - 8.9 (2.8), $\mathrm{p}=.01-.0001)$.

Conclusions: Results suggest that pattern of memory decline is greater in older men with two or more dementia risk factors when compared to females and males at lower risk. Ultimately this mav lead to better characterization and early detection of memory changes, particularly in older adult males with multiple risk factors.

Correspondence: Cory E. Harrison, PhD, Neuropsychology, Pacific Neuroscience Medical Group, 515 W Foothill Blvd, Unit A, Monrovia, CA 91016, United States. E-mail: cburns12@apu.edu

A.C. HENCE, A. RAPP, K. SOL, A.Z. KRAAL, P. FARRIS \& L.B. ZAHODNE. Neighborhood Quality and Cognition Among Diverse Older Adults: An Analysis of the Health and Retirement Study.

Objective:

Objective: Previous research suggests that poor neighborhood quality is associated with worse cognitive aging, but most studies have focused on a single, objective measure of regional socioeconomic status (SES) obtained from U.S. census data. It is less clear whether perceived physical conditions of neighborhoods and social relationships formed between neighbors influence cognitive aging. This study investigated two subjective measures of neighborhood quality: neighborhood physical disorder (NPD) and neighborhood social cohesion (NSC). We hypothesized that (1) NSC and NPD would be positively and negatively associated with memory performance, respectively; and (2) the association between NPD and memory would be particularly strong among racial/ethnic minorities, based on the theory of compound disadvantage.

Participants and Methods: Participants and Methods: Data from 4,200 White, African American, and Hispanic adults aged 65+ in the Health and Retirement Study in 2006 were used in this cross-sectional study. Linear regression quantified the independent associations between neighborhood factors and verbal episodic memory composite scores, controlling for sociodemographics, socioeconomic status, chronic disease burden, and depressive symptoms.

Results: Results: NPD, but not NSC, was significantly associated with worse memory performance. This association was not significantly different across racial/ethnic groups.

Conclusions: Conclusion: These results suggest that the physical quality of neighborhoods may influence the cognitive health of older adults, regardless of race/ethnicity. Given the impact that physical neighborhood quality may have on the cognitive function of older adults, future work should explore whether policies designed to improve neighborhood safety, physical structures, and economic revitalization will improve the long term cognitive health in older adults.

Correspondence: Alyssia C. Hence, Eastern Michigan University, 1000 N. Huron River Dr. , 305, B, Ypsilanti, MI 48197, United States. E-mail: ahence@emich.edu

K. KAUZOR, A.T. FLOWERS, D. BANUELOS, K. GONZALEZ, L. APOSTOLOVA, E. WOO \& J. RAZANI. Test Performance of MCI Patients With and Without Caregivers as Compared to AD and NC. Objective: Individuals with dementia commonly require caregiver assistance for their activities of daily living, and there is recent evidence that some individuals with MCI require assistance. At this point, it is unclear as to whether those MCI with caregivers (MCIcg) are significantly more impaired in their ADLs as compared to those who do not utilize a caregiver (MCIno cg), and that is what the present study examined.

Participants and Methods: As part of an ongoing, longitudinal study, 173 total participants (AD: $n=61$; MCIcg: $n=19$; MCIno cg: $n=34$; NC: $\mathrm{n}=59$ ) were recruited from the greater Los Angeles area. Participants were administered functional ADL and neuropsychological (NP) tests. The Direct Assessment of Functional Status (DAFS) was used as the functional measure and the following subscales were included in the analysis: Time orientation, communication, transportation, financial and shopping. For the NP evaluation, the following tests were used: Rey-O copy/ 3 min. delay, BNT, CVLT saving score, WCST errors, FAS total words, and the MMSE.

Results: A MANCOVA, controlling for education, evaluated group differences on the DAFS outcomes and overall significance was found, Wilks' Lambda $F(39,412.36)=4.08, p<.001$. Specifically, MCIcg performed significantly worse than the MCIno cg group on communication and shopping tasks, and AD performed worse than all groups on most DAFS tasks. ANCOVAs evaluating group differences in performance across the NP measures revealed that only the MMSE revealed differences between the two MCI groups. ANCOVAs also indicated similar patterns of performance between the MCIcg and AD groups on both the Rey-O measures.

Conclusions: Significant differentiation in daily functioning of MCI is important for treatment planning as this indicates that even at every mild stages of cognitive impairment as subset of individuals require assistance

Correspondence: Kaitlyn Kauzor, M.A., Psychology, California State University, Northridge, 28716 Kathleen Ave., Santa Clarita, CA 91390, United States.E-mail: kaitlyn.kauzor.943@my.csun.edu 
D.A. KELLY, M. ABRAHAM, M. SEIDENBERG, J.L. WOODARD, K.A. NIELSON, J. SMITH, S. DURGERIAN \& S.M. RAO. Influence of Family History and APOE $\varepsilon 4$ Allele on Five Year Longitudinal Memory Performance in Cognitively Intact Elders.

Objective: Family history (FH) of Alzheimer's Disease (AD) and the APOE $\varepsilon 4$ allele are considered risk factors for development of AD. Few studies to date have examined the independent and combined influence of these two risk factors on memory, particularly in a longitudinal design. The purpose of this study was to examine the combined and individual influence of FH and the APOE e4 allele on the trajectory of memory performance in cognitively intact elders over five years.

Participants and Methods: 115 participants (ages 65-85) were divided into four groups based on the presence/absence of family history and the APOE $\varepsilon 4$ allele: low risk (no FH or $\varepsilon 4$ allele, $\mathrm{n}=35$ ), $\mathrm{FH}$ only $(\mathrm{n}=34), \varepsilon 4$ only $(\mathrm{n}=15)$, and high risk (both FH and $\varepsilon 4$ allele, $\mathrm{n}=31$ ). Individuals were considered cognitively intact at baseline based on scores on the Mini Mental State Exam, Dementia Rating Scale-2, and Rey Auditory Verbal Learning Test (RAVLT). Linear Mixed Effects (LME) longitudinal analyses were conducted to model the effect of the $\varepsilon 4$ allele and FH on RAVLT indices over three intervals (baseline, 18 months, 5 years). To control for multiple comparisons, False Discovery Rate correction procedures were applied to all LME analyses. Results: At baseline, the $\varepsilon 4$ only group (Mean z-score $=-0.08$ ) had significantly lower scores on RAVLT delayed recall $(p=0.03)$ than the low risk group (Mean z-score $=0.40$ ). Over five years, only the high risk group showed a significantly greater decline than the low risk group on immediate (low risk $=-0.01$; high risk $=-0.13 ; p=0.01$ ) and delayed (low risk $=-0.01$; high risk $=-0.12 ; p=0.01)$ recall.

Conclusions: These findings underscore the importance of additional investigation of the contribution of family history and the $\varepsilon 4$ allele for prediction of cognitive decline in older healthy adults.

Correspondence: Dana A. Kelly, Psychology, Rosalind Franklin University of Medicine and Science, 1130 S. Michigan Ave., Apt 2308, Chicago, IL 60605, United States. E-mail: dana.kelly@my.rfums.org

\section{A.Z. KRAAL, K. SOL \& L.B. ZAHODNE. Religiosity, Religious Attendance, and Cognition among Racially/Ethnically Diverse Older Adults.}

Objective: Religiosity (religious belief) and religious attendance have been inconsistently associated with cognition, and few studies have assessed their unique relationships with cognition within the same model. Most previous research on religious involvement and cognition has not focused on older adults, included diverse samples, or accounted for physical and mental health. This study examined racial/ethnic differences in religion variables and tested their independent associations with episodic memory in a racially/ethnically diverse sample of older adults. We hypothesized that 1) Blacks and Hispanics would report more religiosity and religious attendance than Whites, and 2) each religion variable would be positively associated with memory in the whole sample.

Participants and Methods: Cross-sectional data from 17,399 White, Black, or Hispanic adults aged 50+ from the 2004 Health and Retirement Study were used. Religiosity was assessed by a four-item scale and religious attendance by a single frequency question. ANOVA with post-hoc tests examined racial/ethnic differences in religious involvement. Linear regression quantified independent associations between religion variables and verbal episodic memory composite scores, controlling for sociodemographics, depressive symptoms, and chronic health conditions. Fisher's r-to-z transformation compared the magnitude of associations between religion variables and cognition.

Results: Blacks and Hispanics reported more religiosity and religious attendance than Whites, and there was a trend for greater religiosity among Blacks compared with Hispanics. Both religion variables were positively and similarly associated with memory.

Conclusions: Findings replicate previous work demonstrating racial/ ethnic differences in religiosity and religious attendance and suggest that both aspects of religious involvement may be beneficial in cognitive aging. Future research should examine pathways by which religious involvement could attenuate racial/ethnic disparities in cognitive aging. Correspondence: A. Z. Kraal, University of Michigan, 530 Church ST., Ann Arbor, MI 48109, United States.E-mail:azkraal@med.umich.edu

A. MATCHANOVA, L. HAM, R. DIVERS, E. ROLL, S.S. RYCROFT, J. HULSWIT, K. HOWARD \& T. GIOVANNETTI. The Effect of Cognitive Aging and Resource Limitations on Everyday Task Completion and Interleaving.

Objective: Dementia dramatically reduces the accomplishment of everyday tasks. Detailed performance analyses have shown that relative to younger adults, healthy older adults are slower and more error-prone when performing everyday tasks. However, everyday task accomplishment has not been investigated with comparable detail. We investigated the hypotheses that older adults would complete fewer steps and interleave more steps than younger adults in everyday tasks and that younger adults would complete fewer steps and interleave more steps in the Dual Task vs. standard condition.

Participants and Methods: 21 healthy young adults and 14 nondemented older adults completed two everyday tasks (breakfast, lunch) while being video-recorded. Young adults also completed the everyday tasks under a Dual Task condition to examine performance under resource limitations. Two coders reviewed videos for the completion of 88 task micro-steps (e.g., add jelly, close jelly jar, etc.) and for instances when micro-steps were interleaved (e.g., pick up lid to jelly jar, stir coffee, add lid to jelly jar).

Results: On average, participants completed 61.94 (SD=6.75) microsteps and interleaved $4.18(\mathrm{SD}=3.53)$ micro-steps when performing the everyday tasks, and there was no difference between younger and older adults in the number of micro-steps that were accomplished $(\mathrm{d}=.36, \mathrm{p}=.20)$ or interleaved $(\mathrm{d}=.27, \mathrm{p}=.26)$. Dual Task vs. the standard condition comparisons among young adults also showed no difference in the number of micro-steps accomplished $(\mathrm{M}=59.62 \mathrm{vs}$. 60.76 ) or interleaved ( $\mathrm{M}=3.61$ vs. $3.33 ; \mathrm{d}<.14, \mathrm{p}>.45$, for both).

Conclusions: Cognitive aging and resource limitations increase subtle errors and inefficient behaviors in everyday tasks, but unlike dementia, do not affect the accomplishment or order of essential and non-essential task steps. Implications for understanding the nature of functional difficulties in cognitive aging and dementia will be discussed.

Correspondence: Asal Matchanova, Psychology, Temple University, 1701 N. 13th Street Room 857, Philadelphia, PA 19122, United States. E-mail:matchanova.asal@gmail.com

S.A. MARGOLIS, B. DUDA, G.O. REYNOLDS, C.B. DENBY, K.M. POLL, M.M. GILMORE, G. TREMONT \& B. OTT. Cognitive Decline Predicts Perceived Loneliness in Non-Demented Older Adults at Risk of Alzheimer's Disease.

Objective: Loneliness may be an early consequence of cognitive decline in older adults. Yet, the extent to which subtle cognitive change is linked to feelings of social disconnectedness remains understudied. To date, no research has specifically addressed this topic in an Alzheimer's disease (AD) prevention registry. This longitudinal study examined whether decline in cognitive screening test performance is related to greater loneliness in non-demented older adults drawn from the Rhode Island Alzheimer Prevention Registry.

Participants and Methods: Participants ( $\mathrm{n}=66 ; 78 \%$ female; age $=65 \pm 8$ years) were at increased risk of $\mathrm{AD}(77 \%$ had family history of $\mathrm{AD}$; 19/43 [44\%] APOE-tested participants were $\varepsilon 4+$ ). Perceived loneliness was rated at the most recent follow-up (Three Item Loneliness Scale: $\alpha=.78$; higher values reflect more loneliness) and regressed on a Minnesota Cognitive Acuity Screen (MCAS) change score (i.e., baseline Total Score - most recent Total Score), controlling for age and time between assessments. Mean time to follow-up was $3.8 \pm 1$ years (range $=1-5$ years). Results: All baseline MCAS Total Scores were in the non-impaired range, whereas $6 \%$ declined to the mild cognitive impairment (MCI) range by most recent follow-up. Over one-third of participants (36\%) 
displayed cognitive decline (MCAS change score $>0$ ) and reported some degree of loneliness $(38 \%)$ by follow-up. Regression analysis $\left(\mathrm{R}^{2}=.09\right.$, $\mathrm{p}=.03)$ showed that MCAS decline $(\beta=.28, \mathrm{p}=.03)$ predicted more loneliness independent of age $(\beta=.11, p=.38)$ and time between assessments $(\beta=.10, p=.45)$.

Conclusions: This longitudinal study found that subtle declines in cognition predicted greater perceived loneliness in non-demented individuals at risk of $\mathrm{AD}$. As baseline ratings of loneliness were not collected, future studies should test whether cognitive decline leads to increases in loneliness over time, and if individuals at heightened risk of AD reporting more loneliness convert to MCI and dementia at faster rates. Correspondence: Seth A. Margolis, Ph.D., Psychiatry/Neurology, Rhode Island Hospital, Alpert Medical School of Brown University, Rhode Island Hospital, Ambulatory Patient Center 593 Eddy Street, Providence, RI 02903, United States.E-mail: seth.a.margolis@gmail.com

S.A. MARGOLIS, M.Z. SEARS, C. SOLON, L.A. DAIELLO, L. NAKHUTINA, C. HOOGENDOORN \& J.S. GONZALEZ. Sedative/ Anticholinergic Drug Burden Predicts Worse Memory Acquisition in Racially/Ethnically Diverse Older Adults with Type 2 Diabetes. Objective: Sedative/anticholinergic drug use, measured by the Drug Burden Index (DBI), has been linked to cognitive impairment in older adults. Yet, studies on the association of DBI and neuropsychological domains are lacking, especially in underserved groups at risk of cognitive deficit. We elucidated cross-sectional relationships between the DBI and the Repeatable Battery for the Assessment of Neuropsychological Status (RBANS) in a diverse type 2 diabetes sample. Based on results of a prior study, we anticipated that higher DBIs would negatively affect memory at older ages.

Participants and Methods: Participants were 105 adults with type 2 diabetes (age $=57 \pm 9$ years, $65 \%$ female, $65 \%$ African American, $28 \%$ Hispanic/Latino, $\mathrm{Hb}_{\mathrm{A} 1 \mathrm{c}}=7.8 \pm 1.8$ ). As the DBI was positively skewed, Spearman correlations assessed its bivariate relationships with RBANS Index scores. Adjusting for comorbid illnesses, glucose control $\left(\mathrm{Hb}_{\mathrm{A} 1 \mathrm{c}}\right)$, and education, OLS regression tested the moderating effect of age on significantly associated DBI-RBANS relationships at mean \pm 1 standard deviations of age. Non-parametric bootstrap confidence intervals were run based on 10,000 draws with replacement.

Results: Nearly a third of participants (31\%) endorsed current sedative/anticholinergic use. Mean DBI was $0.39 \pm 0.76$ (range $=0.00-4.17$ ). RBANS Index scores ranged from mildly impaired to average. DBI scores were negatively correlated with Immediate Memory $\left(r_{\mathrm{s}}=-.26\right.$, $\mathrm{p}=.008$ ) but no other RBANS Indices (p's $\geq .103$ ). There was a significant $\mathrm{DBI}^{*}$ Age interaction $(\mathrm{b}=-.68,95 \% \mathrm{CI}=-1.32,-0.49, \mathrm{p}=.035)$; the negative effect of DBI on Immediate Memory was only significant for those $\geq 57$-years-old.

Conclusions: Sedative/anticholinergic drug use was prevalent in these diverse adults with type 2 diabetes. Adjusting for a priori covariates, greater drug burden was associated with worse memory acquisition but only among older adults. Prospective studies should examine these relationships over time and consider whether inflammation and cerebrovascular disease (common in diabetes) act as mediators.

Correspondence: Seth A. Margolis, Ph.D., Psychiatry/Neurology, Rhode Island Hospital, Alpert Medical School of Brown University, Rhode Island Hospital, Ambulatory Patient Center 593 Eddy Street, Providence, RI 02903, United States. E-mail: seth.a.margolis@gmail.com

\section{J.E. MAYE, A. GOLDMAN, K.R. THOMAS \& M. MARSISKE. Divergent Production, Executive Function, and Daily Functioning in Older Adults.}

Objective: Divergent thinking, the creative process related to exploring and generating possible solutions (Guilford, 1956), has received relatively little attention in studies of problem solving and functional independence in late life. Using novel, age-relevant, open-ended problem solving tasks, this study examined elders' divergent production abilities alongside self-reports of everyday function and neuropsychological measures including executive functioning (EF). The study sought to investigate relationships between EF and Everyday Function and to determine whether divergent processes also relate to Everyday Function, accounting for EF.

Participants and Methods: Elders (N=91; Age 80.1 +/- 8.7; Edu. 15.94 +/- 2.77, MoCA 24.1 +/- 3.6) completed cognitive tasks of memory, language, and EF. Self-reported measures of daily living (FAQ, OARS) allowed an overall composite of Everyday Function. Dimensions of divergent production extracted from responses to open-ended problems included Fluency (\# of safe/ effective solutions), Flexibility (\# of solution styles), and Originality. Hierarchical regressions were used to examine relationships between these three dimensions, Everyday Function, and neuropsychological performance including EF.

Results: Initial correlations suggested that components of EF (i.e. COWA, Trails B) aligned with problem solving Fluency $(\mathrm{p}<.05)$. In hierarchical regressions, three blocks of predictors [1) demographics: $\left.\mathrm{R}^{2}=.189 ; 2\right)$ memory \& language- $\left.\left.\mathrm{R}^{2}=.334 ; 3\right) \mathrm{EF}^{2}=.430\right]$ explained variance in Everyday Function ( $\mathrm{p}<.001)$, and each block significantly improved the prior model (Sig F change= .001). A final Fluency block did not improve the model (F change=.098). Flexibility and Originality were not related to EF or Everyday Function.

Conclusions: Although no dimension of divergent production added significantly to current understanding of Everyday Function, findings support the idea that EF relates to Everyday Function in later life and could potentially be used to help identify functionally at-risk elders.

Correspondence: Jacqueline E. Maye, M.S., Clinical Psychology, University of Florida, 1225 Center Drive, Room 3151, Gainesville, FL 32611, United States. E-mail: jmaye@phhp.ufl.edu

K.S. MCVEIGH, S.A. MOSELEY, A. POLSINELLI \& E.L. GLISKY. Associations between social isolation and cognitive function in older adults.

Objective: A reported lack of social connection is associated with poorer cognitive function in older adults. The aim of this study was to further examine this relation, specifically to determine whether self-reported isolation (i.e., a subjective measure) or observed isolation measured with a naturalistic observation tool (i.e., an objective measure), is a better predictor of cognitive function.

Participants and Methods: As part of a larger study, 51 older adults (age range=65-87, M=76.75) completed two measures of social isolation and cognitive testing. We measured self-reported isolation with number of high-contact roles from the Social Network Index (SNI) and observed social isolation with the percentage of time spent alone, as assessed by the Electronically Activated Recorder (EAR; audio-records snippets of participants' daily lives over four days). Cognitive testing included measures of executive function, memory, and processing speed. We ran three separate standard multiple regressions, controlling for age and education, to determine the associations between the two measures of social isolation and cognitive function.

Results: The overall model explained a marginally significant amount of variance in executive function, $F(4,46)=2.33, p=.07, R^{2}=.17,95 \% \mathrm{CI}$ $[.00, .30]$. Observed social isolation was the only significant predictor of executive function $(\beta=-.33, p=.033)$. Neither measure of social isolation significantly predicted processing speed or memory.

Conclusions: Results of the study found that greater time spent alone, as observed with the EAR, was associated with poorer executive function. Although isolation is commonly measured using self-report, this research suggests that objective observational measures may be better predictors of certain cognitive domains in older populations.

Correspondence: Katelyn S. McVeigh, Psychology, University of Arizona, $2246 S$ Heritage Drive, Gilbert, AZ 85295, United States. E-mail: kkmcveigh@email.arizona.edu 
L. MENDOZA. The effect of acculturation on verbal fluency among older adults.

Objective: The purpose of this study was to examine the effects of acculturation on commonly used measures of verbal fluency, memory, and learning.

Participants and Methods: Archival data was used. The sample consisted of 142 Hispanic Cognitively normal [CN; $\mathrm{n}=70$; mean age $=70.44(\mathrm{SD}=6.15)]$, mild cognitive impairment [MCI; $\mathrm{n}=27$; mean age $=76.19(\mathrm{SD}=6.17)]$, and dementia $[\mathrm{D} ; \mathrm{n}=45$, mean age $=76.98$ $(\mathrm{SD}=6.96)]$ individuals. Median split was used to create groups of high and low acculturation level using The Short Acculturation Scale for Hispanics (SAHS). Verbal fluency (COWAT-FAS), category fluency, Boston naming test (BNT), Hopkins Verbal Learning (HVLT), and Semantic Interference Test (SIT) were examined. Data was analyzed with a series of ANCOVAs covary-ing for age, education, and then with MMSE

Results: Among the MCI participants, there was a significant difference between acculturation groups in the Category Fluency test: $[F(1,21)$ $=5.88, p<.024]$ after controlling for age and education. When the analyses were done controlling for MMSE score as well, there were no significant differences in any of the groups.

Conclusions: Although the results were initially significant, the effect size was small $(\mathrm{n}=.20)$.

This indicates other confounding variables, may play a role in this disparity. When MMSE was included as a co-variate, there were no significant results, indicating that cognitive decline may moderate the effect of acculturation on verbal fluency performance among older adults. The small sample size, as well as the overall restricted range of acculturation reported by the subjects may have contributed to current results. Further studies should examine larger samples.

Correspondence: Lisandra Mendoza, Clinical PsychologyNeuropsychology Concentration, Psy.D., Carlos Albizu University, 8665 SW161CT, Miami,FL33193,United States.E-mail: lizzmendoza1987@ gmail.com

\section{J. MOLDEN \& M. MAXFIELD. Effects of Stereotype Threat and Diagnosis Threat Manipulations on Older Adults' Neuropsychological Test Performance.}

Objective: Effective assessment requires understanding how sociocultural context may affect functioning. For older adult patients, this includes awareness of age-based stereotypes. This study examined whether older adults' neuropsychological test performance might be affected by age-based stereotype threat (ABST) and dementia-related diagnosis threat (DRDT). We hypothesized cognitive functioning would be negatively impacted to a similar degree in both threat conditions as compared to a neutral control condition.

Participants and Methods: An older adult sample ( $N=101$, mean age $=72.38)$ was randomly assigned to one of three conditions. Threat inductions were based on existing diagnosis threat studies (Suhr \& Gunstad, 2002). ABST instructions emphasized societal assumptions of age-related cognitive decline, DRDT instructions underscored the diagnostic nature of the tests to be completed, and control instructions made no mention of age or dementia-related information. After reading instructions, participants completed a short battery of neuropsychological tests.

Results: When univariate and multivariate outliers were removed, one-way MANOVA did not suggest a significant effect of threat manipulations on the 10 cognitive outcome dependent variables, Wilks' $\wedge=.74, F(20,168)=1.40, p=.13$, partial $\eta^{2}=.14$. Results of a one-way ANOVA assessing reliable digit span (RDS) as a measure of effort reflected a marginally significant effect of condition on reliable digit span, $F(2,98)=3.07, p=.05$, partial $\eta^{2}=.06$; RDS in the DRDT group $(M=8.97, S D=1.57)$ was lower than Control $(M=9.97$, $S D=1.73), p=.02$.

Conclusions: Results did not support previous ABST research and suggested that diagnosis threat may not generalize to a cognitively intact older adult sample (though DRDT may mildly influence effort).
More research on how these constructs may operate in clinical samples (e.g., an MCI population, or those self-referred for memory testing) would be valuable.

Correspondence: Joie Molden, University of Colorado Colorado Springs, 1067 Nolan Lane, Cordova, TN 38018, United States.E-mail:jmolden@ uccs.edu

M.A. NIERMEYER, J.L. TAGGART-NERIA, C. BARRON, R. HEYREND, A. RAY, A. GILLESPIE, R.E. ZIEMNIK \& Y. SUCHY. The Relationship Between Executive Functioning and Gait Speed Among Older Adults: Does Measurement Matter?

Objective: In the literature examining the connection between EF and mobility among older adults there is considerable variability in the way researchers have operationalized EF, as well as the extent to which they have accounted for component processes (CPs) of EF (e.g., processing speed). Demnitz et al.'s (2016) meta-analysis showed that both processing speed and EF were significantly related to gait, and that EF composite measures and the Stroop test tended to show the strongest effects. The current study investigated the role CPs play in the association between EF and gait speed, and compared methods of operationalizing EF and its CPs in a single sample.

Participants and Methods: 55 older adults completed measures of gait speed during both single- and dual-task (serial subtraction) conditions, as well as measures of EF and its CPs using subtests from the DelisKaplan Executive Function System. Three commonly used methods of operationalizing EF and CPs were computed: (1) the Trail-Making Test (TMT), (2) the Stroop Test, and (3) composite measures derived from $8 \mathrm{EF}$ and $6 \mathrm{CPs}$ subtests.

Results: Hierarchical regressions showed that the composite measures and the TMT revealed similar findings. Using these two methods singletask gait speed showed a trend level association with CPs beyond age ( $p$-values<.07), but EF did not account for additional variance in gait speed beyond CPs ( $p$-values>.30). In contrast gait speed during the dual-task was related to EF beyond CPs and age ( $p$-values<.017). The Stroop subtests did not account for variance in gait speed for either condition (single or dual; $p$-values $>.10$ ).

Conclusions: These results demonstrate the importance of controlling for CPs. If CPs are not adequately controlled for, there is the possibility that researchers may confound CPs with EF. The results also suggest that researchers should take caution when relying on a single $\mathrm{EF}$ measure when testing the relationships between $\mathrm{EF}$ and mobility. Correspondence: Madison A. Niermeyer, MS, Clinical Psychology Department, University of Utah, Department Of Psychology University Of Utah380 S 1530 E Beh S 502, Salt Lake City, UT 84112, United States.E-mail:madison.niermeyer@psych.utah.edu

A.S. ORD, U. ALEXANDER, E. SHULL, J. LINDO, K. MORALES, D. GRAHAM, T. WOLTERSTORFF, J. PHILLIPS, K. BHATTACHARJEE, A. MELCHIORRE \& S. SAUTTER. Can Alcohol and Tobacco Use Predict Neurocognitive Functioning in Older Adults?

Objective: Previously published research has consistently linked negative effects of substance use (including alcohol and tobacco use among other substances) with concerns related to memory as well as attention and concentration. However, very few studies have examined these effects in older adults. The present study aimed to address this gap in literature and examined the differences in neurocognitive functioning in older adults, based on participants' self-reported use of alcohol and tobacco.

Participants and Methods: Participants were 453 adults (ages 56 to 89) who were seen for a neuropsychological evaluation at an outpatient neuropsychological clinic in Virginia. Average age of participants included in this study was $74.28(S D=8.43)$ with an average MMSE score of $27(S D=5.08)$ and average education level of 14 years $(S D=3.87)$. 
Results: A $2 \times 2$ ANCOVA was conducted to examine differences in neurocognitive functioning (as measured by the RBANS Total Scale Score) based on participants' self-reported use of alcohol and tobacco (each participant either endorsed or denied the use of each substance). Age was included as a covariate. Results indicated significant main effects for both alcohol use $(F[1,449]=3.98, p=.047)$ and tobacco use $(F[1,449]=6.09, p=.014)$. Participants who reported using tobacco had significantly lower RBANS Total Scale scores $(M=83.28$; $S D=11.17)$ as compared to those who denied using tobacco $(M=89.77$; $S D=8.11)$. Similar results were observed for those who reported using alcohol $(M=85.74 ; S D=9.24)$ as compared to those who denied alcohol use $(M=89.53 ; S D=7.13)$.

Conclusions: Overall, results of the study revealed that participants who reported using both alcohol and tobacco had the lowest total RBANS scores $(M=82.41)$, which were significantly lower as compared to all other groups, whereas participants who denied using both alcohol and tobacco received the highest scores on the RBANS $(M=90.89)$. Clinical implications of these findings will be further discussed in this presentation.

Correspondence: Anna S. Ord, Psy.D., Psychology \& Counseling, Regent University, 3304 Eamon Court, Apt. 103, Virginia Beach, VA 23452, United States. E-mail: shirokova.anna@gmail.com

J.R. OSUNA, Z.Z. ZLATAR, C.C. HAYS, L.M. CAMPBELL \& C.E. WIERENGA. The Benefits of Moderate-Intensity Physical Activity on Cognition in Older Adults.

Objective: Physical activity (PA) has been implicated as a potential protective factor for age-related cognitive decline. Moderate to vigorous physical activity (MVPA) has been associated with higher cerebral blood flow $(\mathrm{CBF})$ and better cognitive function in older adults, however, research examining the associations between objectively-measured physical activity and its impact on cognition is lacking. To address this gap, we examined the association between sedentary time, MVPA, and moderate-intensity physical activity (MIPA) and cognition in older adults.

Participants and Methods: 52 cognitively normal older adults underwent activity monitoring using tri-axial accelerometry and completed comprehensive neuropsychological testing. Sedentary time was defined as 0-99 accelerometer counts per minute (CPM), MIPA as 1041-1951 CPM and MVPA as $>=1952$ CPM. Linear regressions and correlations were performed to investigate the associations between all physical activity levels and executive and memory functions, while adjusting for age.

Results: MIPA significantly predicted executive function above and beyond the effects of age, MVPA, and sedentary time, such that higher MIPA was associated with better scores on a composite measure of executive function. Results also indicated (trend) that higher sedentary time was associated with poorer performance on an autobiographical memory task (recent famous face naming).

Conclusions: Our findings indicate that MIPA may support better executive functions in cognitively healthy older adults, while sedentary time may negatively affect autobiographical memory for recent famous faces. These results suggest that behavioral interventions may focus on increasing physical activity and reducing sedentary time to bolster cognition in aging. Future research should investigate the independent contributions of different levels of physical activity intensities and sedentary time on cognition.

Correspondence: Jessica R. Osuna, Bachelor's of Arts, Research Services/ Psychiatry, Veterans Medical Research Foundation/University of California, San Diego, 9500 Gilman Drive, La Jolla, CA 92093, United States.E-mail:jrosuna@ucsd.edu
J.S. PASSLER, R.E. KENNEDY, M. CROWE, O.J. ClAY, V.J. HOWARD \& V.G. WADLEY. The Relationship of Cognitive Decline and Impairment to the AD8 and Activities of Living in a General Population.

Objective: The AD8 is a sensitive and specific screening measure of mild dementia, although less research exists pertaining to its use as a self-report measure and in non-clinical samples. This study examined the relationship of incident cognitive impairment and cognitive decline over a 10-year span to self-reported dementia symptoms and functional impairment.

Participants and Methods: Participants included 14,453 adults from the national, population-based, longitudinal, and prospective REasons for Geographic and Racial Differences in Stroke (REGARDS) sample. A validated cutoff of $\geq 2$ symptoms endorsed on the AD8 was used for dementia status. Functional status was determined by 7 instrumental activities of daily living (IADL) items that were coded as independent vs. having difficulty/needing assistance. Incident cognitive impairment was defined via change from intact to impaired status in Six-Item Screener (SIS) score, and cognitive decline was defined by trajectories computed for animal fluency (AF), letter F fluency (LF), Consortium to Establish a Registry for Alzheimer's Disease (CERAD) word list learning (WLL), and CERAD word list delayed recall (WLD). Logistic regression models controlled for demographic variables, health behaviors, and vascular risk factors and disease at baseline, and depressive symptoms at 10-year follow-up (i.e., 10-item Center for Epidemiological Studies Depression Scale).

Results: Incident cognitive impairment significantly increased the odds of self-reporting dementia and IADL impairment by $96 \%$ and $59 \%$, respectively. A one-word decline in WLL significantly increased the odds of self-reporting dementia $(27 \%)$ and IADL impairment (16\%). Overall, stroke history, incident stroke, and incident cognitive impairment showed the largest effect sizes for increased odds of dementia symptoms and functional impairment.

Conclusions: The findings of this study elucidate the relationship of cognitive change over time to self-reported dementia symptoms and functional impairment in a large, population-based sample.

Correspondence: Jesse S. Passler, M.A., Psychology, University of Alabama Birmingham, 1222 W DALLAS ST, Houston, TX 77019, United States. E-mail:jpassler@uab.edu

\section{A. POLSINELLI \& S.A. MOSELEY. Multi-method measurement of cognitive ability in healthy older adults.}

Objective: New technologies offer an exciting opportunity to go beyond the traditional self-report and lab-based measurement of cognition to explore these abilities in vivo-- in real life, as they happen. However, it is unclear to what degree in vivo behavioral measures of cognition may be associated with lab-based and self-report measurements, which is important for establishing their validity. Thus, we aimed to explore how one such in vivo methodology was associated with validated lab-based and self-report measures.

Participants and Methods: As part of a larger study, 60 older adults (age range $=65-90, \mathrm{M}=77.25)$ completed lab-based, objective measures of executive functioning - including shifting, updating, and inhibition - and self-report measures of cognitive ability and errors. They then wore the Electronically Activated Recorder (EAR), which recorded short audio clips of participants' daily lives over four days. A trained rater coded the audio files for instances of cognitive errors in daily life, which we used as our measure of observed daily cognitive ability. We ran three separate Pearson bivariate correlations to explore the associations among the multi-method measures of cognitive ability. Results: Observed daily cognitive ability was significantly associated with the composite measure of objective executive functioning, $r=.33$, $p=.01$. Although in the predicted direction, observed daily cognitive ability was not significantly associated with the subjective self-report composite, $r=-.19, p=.16$. The objective executive functioning composite and subjective self-report composite were significantly associated, $r=.39, p=.002$. 
Conclusions: Results suggest that observed daily cognitive behavior can be a useful indicator of objective cognitive ability in healthy older adults but is less related to an individual's subjective experience of their cognitive ability. This preliminary study supports continued use of daily observational methodologies to study cognitive ability.

Correspondence: angelina polsinelli, MA, Psychology, University of Arizona, 207 - 708 e 26th ave, Vancouver, BC V5V2H8, Canada. E-mail: angelina.polsinelli@gmail.com

T.L. ROBINSON, M. GOGNIAT, R.E. SALYER, C.X. TORRES, K. JEAN, C. MEWBORN \& L. MILLER. Associations Between Body Composition Measures and Cognitive Reserve in Older Adults.

Objective: Research on physical health in older adults and its relation with cognitive function has sought to determine which measures of physical integrity are most predictive of cognitive function and functional independence. The purpose of the current study was to determine which of several physical health measures is most related to a proxy measure of cognitive reserve in a sample of healthy older adults.

Participants and Methods: Participants included 26 communitydwelling older adults, ages 65-87 $(M=74, \mathrm{SD}=6)$, recruited from an ongoing study of cognitive aging. Participants were $38 \%$ male and $62 \%$ female. The average educational attainment in the sample was 16.4 years, and the average Body Mass Index (BMI) was 26.6 (SD $=4.9$ ). Cognitive reserve was determined using the Wechsler Test of Adult Reading (WTAR) - Demographics Predicted Full Scale IQ (FSIQ). Measures of physical integrity included BMI $\left(\mathrm{kg} / \mathrm{m}^{2}\right)$, umbilical waist circumference, and ratio of lean mass to overall weight and percent fat mass measured using Dual-energy $\mathrm{X}$-ray absorptiometry (DXA). Pearson bivariate correlations were run to determine the relation between each measure and WTAR-Demographics Predicted FSIQ. Results: The average BMI of the sample was in the overweight range. The average predicted FSIQ was in the high average range $(M=111$, $\mathrm{SD}=10.1)$. BMI and umbilical waist circumference were not significantly related to the measure of cognitive reserve. Cognitive reserve was significantly related to percent lean mass $(r=.442, p<.05)$ and percent fat mass $(\mathrm{r}=-.439, p<.05)$.

Conclusions: Our results suggest that greater lean mass and less fat mass compared to overall weight were related to greater predicted cognitive reserve. These results support the use of more specific body composition measures to evaluate physical health in older adults as it relates to cognitive function. Further study may be needed to evaluate these associations in a more functionally heterogeneous sample.

Correspondence: Talia L. Robinson, Psychology, University of Georgia, 1688 Barnett Shoals Rd, Apt 3, Athens, GA 30605, United States. E-mail: tlr13043@uga.edu

R.A. RODRIGUEZ, A.M. BRICKMAN, R.A. LANTIGUA, N. SCHUPF, R. MAYEUX \& J.J. MANLY. Birthplace, Acculturation Level, and Cognitive Trajectory among Hispanic Older Adults.

Objective: Dominican-born Hispanic elders have historically experienced poor quality of education compared to Cubans and Puerto Ricans. We hypothesized that independent of acculturation level, birthplace in the Dominican Republic would be associated with poor cognitive trajectory, and explained by educational experience.

Participants and Methods: Participants included 2,614 self-identified Hispanics from the Washington Heights Inwood and Columbia Aging Project, aged 65 or older ( $67 \%$ female). Participants were compared by birthplace: Puerto Rico $(N=314)$, the Dominican Republic $(N=1628)$, Cuba $(N=375)$, Mexico, Central and South America $(N=235)$, and countries outside of Latin America $(N=62)$. All participants completed a neuropsychological battery at baseline and 18-24 month follow-up visits. Acculturation level was measured via self-reported English proficiency, proportion of life in the US, and WRAT-3 English Reading Recognition scores. Linear mixed models tested the effects of birthplace, acculturation, and education on cognitive trajectory, taking into account sex, age, and overall health burden.
Results: Dominicans obtained lower initial cognitive scores than other Hispanic subgroups ( $\mathrm{p}<.01$ for all domains). Dominicans had less rapid decline in language and speed, but there was no independent effect of birthplace on change in memory and visuospatial skill. The effect of Dominican birthplace on memory was accounted for by acculturation. Years of school accounted for the relationship between Dominican heritage and language and visuospatial trajectory. Even after accounting for all covariates, educational quality and quantity, and acculturation, Dominicans had lower baseline scores and less rapid decline on measures of speed.

Conclusions: Examination of social and biological mechanisms underlying differences in cognitive trajectory across Hispanic subgroups may reveal protective factors and opportunities for interventions aimed at maintaining cognitive function with age.

Correspondence: Ramon A. Rodriguez, Columbia University, 50 Haven Ave, Box B273, New York City, NY 10032, United States. E-mail: rar2196@cumc.columbia.edu

L.J. ROTBLATT, A. HORGAS \& M. MARSISKE. The Effects of Hypertension and Polypharmacy on Changes in Reasoning Abilities over 10 Years in the ACTIVE Trial.

Objective: While the negative effects of hypertension (HTN) on cognition have been well documented, there is limited research examining the effects of HTN treatment and polypharmacy (total medication use) on cognition over time. Thus, this study aimed to investigate whether changes in HTN status over time were predictive of changes in reasoning abilities, and whether concurrent antihypertensive medication use and polypharmacy had additional effects.

Participants and Methods: Participants were 2802 older adults, aged $65-95$ (75.9\% female), free of frank dementia (MMSE $>23)$ who participated in the NIH ACTIVE Trial, a multi-site, longitudinal study examining the effects of cognitive training. In addition to a reasoning composite (of Letter Sets, Letter Series, and Word Series tests), participants completed medication audits (yielding count of total and HTN medications) and systolic HTN assessments (BP>140 mm/Hg) at 1-, 2-, 3-, 5-, and 10-years after baseline. Data were analyzed using a multilevel modeling approach.

Results: On average there was annual decline in reasoning abilities. In models adjusting for age, education, gender, retest, and cognitive training assignment, persons with HTN showed lower average reasoning, and greater decline was seen in those who had higher average polypharmacy, as well as on those occasions where polypharmacy and hypertension were greater $(\mathrm{p}<.05)$.

Conclusions: Over ten years, polypharmacy (probably as an indicator of overall comorbidity or iatrogenic medication effects) and hypertension were risk factors for lower reasoning and greater decline, and there was no evidence of a moderating effect of hypertension medication use. Thus, these results suggest preventative treatment to avoid disease is likely to have greater impact on reasoning than treatment later in the course of disease progression.

Correspondence: Lindsay J. Rotblatt, Clinical and Health Psychology, University of Florida, 1225 Center Drive, Gainesville, FL 32603, United States.E-mail:lrotblatt@ufl.edu

L.E. SABBAH \& M. SUVAK. Cognitive Structure, Cognitive Engagement and Adaptive Functioning: The Aging, Demographics, and Memory Study (ADAMS).

\section{Objective:}

A confirmatory factor analysis (CFA) revealed that a bi-factor model with a general cognitive factor and two specific factors (Language and Memory) best fit data from the Aging, Demographics, and Memory Study (ADAMS). This study examined how these cognitive domains predicted change in cognitive engagement and adaptive functioning. Participants and Methods: The ADAMS, the first population-based study of cognitive impairment in the USA, was part of the Health and Retirement Study. Adults 70 years and older completed 
neuropsychological testing and structured interviews supplemented with informant questionnaires. Experts categorized participants as: Exhibiting normal cognitive functioning, cognitively impaired, not demented (CIND), or demented. Data was collected over four time points between 2000 and 2009. Ten items from the informant questionnaire assessed cognitive engagement and the Blessed Dementia Scale (BDS) was used to assess adaptive functioning.

\section{Results:}

Latent growth curve analyses (LGCA) indicated that on average cognitive engagement tended to decline over time and adaptive functioning showed an accelerated pattern of worsening. The general and language factors predicted Time 1 cognitive engagement. No cognitive factor predicted change in cognitive engagement. Examining this relationship across diagnostic groups at time 1 (Normal vs. CIND) revealed that the language factor was no longer significantly associated with cognitive engagement at time 1 for the CIND group. For adaptive functioning, only the memory factor predicted change in the BDS in the CIND (i.e., worse memory predicted larger increases in adaptive problems), while none of the factors predicted change over time in the healthy group. Conclusions: Initial cognitive performance predicted initial status in cognitive engagement but not change over time. In addition, memory performance predicted decline in adaptive functioning for individuals with CIND, but not healthy controls.

Correspondence: Liorah E. Sabbah, M.A., Psychology, Suffolk University, 75 Saint alphonsus street, apt 708, Boston, MA 02120, United States. E-mail: liorah.sabbah@gmail.com

R. SALONER, J. YOKOYAMA, G. COPPOLA, A. KARYDAS, E. FOX, M.Y. YOU \& J. KRAMER. Associations of Dopamine-Related Genes with Executive Functioning in Functionally Normal Older Adults. Objective: Although prior research has identified dopamine-related genetic contributions to cognition in clinical populations (e.g., schizophrenia, Parkinson's), less is known about the relationship of genetic variation affecting dopamine bioavailability to cognition in normal aging. In this study, we studied several genetic variants in dopamine pathways and examined their relationships to cognition in functionally intact older adults. We hypothesized that executive functioning would be worse in normal adults with genotypes associated with reduced dopamine bioavailability in frontal regions.

Participants and Methods: 389 older adults (mean age=69.1) recruited from the UCSF Hillblom Aging Network underwent neuropsychological assessment and genotyping. Participants were grouped according to genotype for DRD2 (CT/TT; $\mathrm{n}=131$ vs. CC; $\mathrm{n}=258$ ) or $C O M T$ Val158Met (MetMet; n=98 vs. MetVal; n=188 vs. ValVal; n=103). A subset of participants was also genotyped for DRD4 (7-repeat carriers; $\mathrm{n}=49$ vs. non-7-repeat carriers; $\mathrm{n}=155$ ). Global executive functioning and memory composite scores were computed for analyses.

Results: Controlling for age, education, and sex, ANCOVA yielded significant group differences by genotype in executive functioning for DRD2 $(\mathrm{F}(1,384)=5.15, \mathrm{p}<0.05)$ and DRD4 $(\mathrm{F}(1,199)=4.24, \mathrm{p}<0.05)$ but not COMT Val158Met $(\mathrm{F}(2,383)=.668, \mathrm{p}>0.05)$. There were no significant group differences in memory performance.

Conclusions: We provide evidence that genetic variation related to reductions in dopamine receptor density (DRD2) and potency (DRD4) may negatively affect executive functioning in aging adults. Results support prior findings that dopamine genes associated with frontal networks impact executive functioning. Future work aimed at elucidating the interactions between these genetic contributions to dopaminergic activity and the neurobiological processes underlying executive functioning in aging may improve the identification and targeted treatment of individuals at-risk for cognitive decline. Correspondence: Rowan Saloner, Neurology, University of California, San Francisco, 120 El Capitan Drive, San Rafael, CA 94903, United States.E-mail: rowansaloner@gmail.com
A. SEELYE, S. UROSEVIC, B. DOANE, S.A. MOSELEY, A. ALIOTO, C.L. CLASON \& W. VANVOORST. Age-Related Declines in Cognitive Control and Verbal Learning in Veterans with Bipolar Disorder.

Objective: Bipolar disorder (BD) is associated with broad executive functioning (EF) deficits. Less is known about the nature and specificity of deficits in cognitive control (CC) processes, a subset of EF that are implicated in core BD etiology, or attentional aspects of EF such as verbal learning (VL) among older adults with BD. This study investigated age-related changes in CC and VL in Veterans with BD and healthy controls (HC) ages 27 to 94 .

Participants and Methods: Participants were 13 adults with BD $(\mathrm{M}$ age $=54.2, \mathrm{SD}=19.9 ; 85 \%$ male $)$ and $28 \mathrm{HC}$ adults $(\mathrm{M}$ age $=69.4$, $\mathrm{SD}=8.3 ; 93 \%$ male) who completed a clinical neuropsychological evaluation. Stroop Color-Word and TMT-B assessed CC processes of response inhibition and set-shifting. CVLT-II Acquisition assessed VL. Two-way ANOVAs examined the effects of diagnostic group (DG) and age ( $<64$ vs. $>64)$ on test performance.

Results: There was a significant main effect of age on Stroop and TMT-B; older adults performed significantly worse than younger adults on both measures $(p$ 's $<.003)$. There was also a significant main effect of DG on TMT-B and CVLT-II Acquisition; BD performed more poorly than HC $(p$ 's <.006). The interaction between DG and age was significant on Stroop $(p=.05)$; older adults with BD $(M=22.8, S E=3.1)$ performed significantly worse than both younger BD adults $(M=37.4$, $S E=2.8)$ and older HC adults $(M=33.3, S E=1.6)$. The age by DG interaction approached significance for CVLT-II Acquisition with the same pattern of results $(p=.06)$, though it was not significant for TMT-B $(p=.159)$.

Conclusions:

$\mathrm{BD}$ adults showed steeper age-related decline on response inhibition (Stroop) but not set-shifting (TMT-B), suggesting that BD poses a risk for greater aging decline in particular CC processes. Results extend prior studies showing deficits in CC processes and VL in younger adult BP and highlight the need for longitudinal studies with larger samples to assess trajectories of these declines in BD older adults.

Correspondence: Adriana Seelye, Minneapolis VA Healthcare System, VA Medical Center, One Veterans Drive, Minneapolis, MN 55446, United States.E-mail:seelyea@ohsu.edu

D. SHAKED, D.K. LEIBEL, N.P. WENG, H. LIU, M.K. EVANS, A.B. ZONDERMAN \& S.R. WALDSTEIN. Telomere Length and Cognitive Function: Differential Relations across Sociodemographic Groups.

Objective: Shorter telomere length (TL) has been linked with all-cause mortality and several age-related chronic diseases. However, the evidence for relations between TL and cognition is equivocal. The present study investigates whether TL is uniquely related to specific cognitive domains, and if these relations vary across sociodemographic groups.

Participants and Methods: Participants were 337 socioeconomically diverse, African American and White urban-dwelling adults $(M$ age $=$ 47; $51 \%$ White; $49 \%$ female; $48 \%$ below poverty) from the Healthy Aging in Neighborhoods of Diversity across the Life Span (HANDLS) study. Cognitive function was derived from standardized tests, and TL was assayed from peripheral blood mononuclear cells using quantitative polymerase chain reaction. Multiple linear regression analyses, adjusted for age and sex, examined the interactive relations of TL, poverty status, and race on the following tests: Trail Making Test (TMT) parts A and B, Digit Span Forward (DSF) and Backward (DSB), animal naming, and the California Verbal Learning Test-II.

Results: Results revealed significant interactions of TL, race, and/or poverty status $(p$ 's $<.05)$ such that White individuals living in poverty with shorter TL had the lowest levels of performance on DSF and DSB. Also, individuals below poverty with shorter TL performed most poorly on TMT part B. 
Conclusions: These results demonstrate that TL may be differentially associated with simple and complex attention/executive function among Whites with household income below poverty. The unique social position occupied by urban Whites below poverty, characterized by concurrent racial privilege and socioeconomic disadvantage, might make this group disproportionately susceptible to the effects of TL on cognition, at least in part, via medical comorbidities, systemic inflammation, and neurobiological pathways. Clarification of the various biological indicators involved in these sociodemographic health disparities may suggest methods for altering trajectories of cognitive aging.

Correspondence: Danielle Shaked, MA, Psychology, University of Maryland, Baltimore County, 1244 Battery Ave., Baltimore, MD 21230, United States.E-mail: dshaked1@umbc.edu

A.M. STAFFARONI, J.A. BROWN, K.B. CASALETTO, F. ELAHI, J. DENG, P. MUMFORD, J. NEUHAUS, R. SALONER, H. ROSEN, W.W. SEELEY \& J. KRAMER. The Longitudinal Trajectory of the Default Mode Network Varies with Age and Predicts Changes in Episodic Memory.

Objective: The default mode network (DMN) is implicated in episodic memory performance and selectively vulnerable to early Alzheimer's disease (AD) pathology. Cross-sectional studies have indicated that within-DMN functional connectivity $(\mathrm{FC})$ reconfigures with increasing age and the spread of AD pathology. We investigated the longitudinal trajectory of DMN FC and its relationship with cognition in cognitively normal older adults.

Participants and Methods: Participants with at least three task-free fMRI scans were eligible for inclusion. After excluding subjects with excessive head motion, we retained 93 cognitively normal older adults with 315 scans (avg:3.4; range:2-7). The DMN was parcellated using the Brainnetome Atlas, and mean connectivity within DMN subregions was calculated controlling for nuisance covariates (i.e., physiological and motion). Linear mixed effects models with random slopes and intercepts were employed, entering age (M:69,SD:7), education (M:18,SD:2), gender (F:47), DMN gray matter volume, TIV and APOE status (E4:26) as covariates. Cognitive composites were created for memory, executive functions, working memory and processing speed.

Results: The interaction between age and time was significant $(\mathrm{b}=-.001, \mathrm{p}=.022)$. Plotting the fitted values suggested a $\mathrm{U}$-shaped curvature, with increasing DMN FC in middle-age subjects and decreasing DMN FC in older subjects. FC change positively correlated with change in episodic memory $(b=.78, p=.015)$, even with hippocampal volume as an additional covariate. DMN FC did not correlate with change in any other cognitive domains (ps>.23).

Conclusions: In cognitively normal older adults, longitudinal change in DMN, but not atrophy, correlates with changes in memory. Consistent with prior cross-sectional studies, we demonstrated increased connectivity in middle-aged adults that predates network breakdown in older adulthood. DMN trajectories may be a sensitive marker of early agerelated changes, such as protein aggregation, even among clinically normal older adults.

Correspondence: Adam M. Staffaroni, Ph.D., Neurology, University of California, San Francisco, University of California, San Francisco, 675 Nelson Rising Lane, Suite 190, San Francisco, CA 94158, United States. E-mail: adam.staffaroni@ucsf.edu

F. STEFFEN-ALLEN, K.J. MANNING, R. KAPLAN, D. WAKEFIELD, N. MOSCUFO, C.R. GUTTMANN \& L. WOLFSON. Predicting AgeRelated Decline on the Trail Making Test: An MRI Study of White Matter Integrity.

Objective: Normal aging is associated with changes in white matter (WM) integrity including obvious lesions (white matter hyperintensities or WMH) as well as microstructural changes in normal appearing WM measured with diffusion tensor imaging (DTI). The relationship between these two parameters and cognition in older adults over time is unclear. We examined how change in different indicators of WM integrity predicts change on the Trail Making Test (TMT) in a normal aging sample.

Participants and Methods: Healthy adults (no major medical conditions or cognitive impairment [MMSE <24]; aged 75-89) underwent cognitive evaluation and magnetic resonance imaging at three timepoints, spaced 2 years apart $\left(\mathrm{N}_{\text {baseline }}=85 ; \mathrm{N}_{2}\right.$ Yrs $\left.=64 ; \mathrm{N}_{4 \mathrm{Yrs}}=34\right)$. We included the Wechsler Test of Adult Reading (WTAR) to control for the effect of baseline IQ on cognitive change. We used multilevel modeling to predict change on the TMT using global values of WMH and DTI measures of fractional anisotropy (FA) and mean diffusivity (MD) at each time point, as well as and baseline age, WTAR score, and gender. Results: Increases in MD predicted slowing on TMT A ( $\beta=-314.4$, $S E=100.1, p=0.002)$, while increases in WMH predicted slowing on TMT B $(\beta=-40.7, S E=9.2, p<0.001)$. No other associations were found and FA did not predict change on either subtest $(p>0.05)$. Moreover, individuals with higher baseline WTAR scores showed less slowing over time on TMT A than individuals with lower WTAR scores. $(\beta=-0.17, S E=0.09, p=0.049)$.

Conclusions: In this study, WM integrity predicted slower speed of processing in normal aging similar to previous studies. However, these results suggest WMHs and DTI indices may predict different measures of cognitive decline. Further, our results suggest that higher IQ, an index of cognitive reserve, may protect against age-related decline in processing speed.

Correspondence: Faith Steffen-Allen, B.A., Psychology, University of Connecticut, 85 Squaw Hollow Road, Ashford, CT 06278, United States.E-mail:faith.steffen@uconn.edu

B. STEPHENS, J. JACOBSON, T.J. ARENTSEN, E. CROUSE, L. GAVRON \& B.L. ROPER. Is Caregiver Burden Influenced by Relationship Type and Race in a Veteran Population?

\section{Objective:}

Functional decline and neuropsychiatric behaviors are most predictive of caregiver burden (CB) followed by sociodemographic variables with older, female, co-resident spouses of care-recipients reporting higher $\mathrm{CB}$ (Kim et al, 2012), and Caucasians reporting higher CB than African Americans (Haley et al., 1996, Hinrichsen et al., 1992, Haley et.al, 2003). Our aim was to determine if severity of reported CB differed based on relationship type and care-recipient race in a cognitively-impaired veteran population.

Participants and Methods: Veteran patients with cognitive and functional decline ( $n=129$; Age $>65$ years) completed neuropsychological evaluation at a VA medical center memory clinic, and CB was measured in caregivers by the Zarit Burden Interview-Rev (ZBI-R). The patient group was $69.8 \%$ Caucasian and $30.2 \%$ African American, with mean age of 81.3 ( $\mathrm{SD}=7.4)$ and most diagnosed with some form of dementia. Caregivers were either spouses $(62.0 \%)$ or children $(38.0 \%)$.

Results: Using a two-factor ANOVA, both patient race $(\mathrm{F}[1,125]=5.21$, $\left.\mathrm{p}<0.5, \eta^{2}=.04\right)$ and spouse/child relationship $(\mathrm{F}(1,125)=7.73$, $p<0.01, \eta^{2}=.06$ showed main effects on CB. Spouses reported higher CB relative to children, and caregivers of African American patients showed lower CB than Caucasians.

Conclusions: CB differed based on type of relationship and carerecipient race. Results are consistent with previous research in which Caucasian spouses reported higher $\mathrm{CB}$ and provide additional information relative to caregivers of cognitively-impaired veterans. Findings will instruct further investigation aimed at identifying caregiver assessment and intervention needs, which may vary according to sociodemographic differences.

Correspondence: Bethanie Stephens, Psy.D., Neuropsychology, Memphis VAMC, 45 North Arcadian Circle, 201, Memphis, TN 38103, United States.E-mail: bstephens2008@my:fit.edu 
A. STICKEL, A. MEYER \& L. RYAN. Body mass index and percent body fat differentially predict memory in young old and older old adults.

Objective: Higher body mass index (BMI) in middle age is associated with increased risk for dementia in later life. However, investigations of BMI and cognitive functioning in older adults have had mixed results. One reason for the inconsistency is that BMI may be a less accurate measure of adiposity in older adults compared to more direct measures of adiposity such as percent body fat (PBF) measured via impedance testing. The present study compared relationships between two measures of adiposity (BMI and PBF) and cognition among older adults.

Participants and Methods: Two groups of older adults (young-old: ages 60 - 68 years, $n=33$; older-old: 69 - 80 years, $n=31$ ) were tested. Groups were matched on education, gender, apolipoprotein e4 status, and systolic blood pressure. General linear models tested the interaction between age group and adiposity measures, controlling for gender and education, on three cognitive composite measures-- short delay memory, long delay memory, and executive functioning.

Results: Higher BMI was associated with poorer short delay memory among older-old adults but was unrelated to performance in young-old adults. Surprisingly, however, higher PBF was associated with better short delay memory in young-old adults but did not predict performance in the older-old group; this pattern was also observed for long delay memory. Executive functioning was not predicted by the interactions between either measure of adiposity and age. Neither the main effect of BMI or PBF independently predicted performance on any measure. Conclusions: Taken together, two measures of adiposity provided a unique, age-dependent influence on short delay memory, highlighting the importance of instrument selection in cognitive aging research. PBF may be a better predictor of long delay memory than BMI, but only among young-old individuals. Neither adiposity measure predicted executive functions, suggesting more complex relationships between executive functions and cardiovascular risk factors with increasing age. Correspondence: Ariana Stickel, Psychology, University of Arizona, 1804 N Tucson Blvd, Tucson, AZ 85716, United States. E-mail: astickel@email.arizona.edu

C. SUMIDA, A. WEAKLEY, L. CHUDOBA, M. BOEGE \& M. SCHMITTER-EDGECOMBE. Medication Management in Community-Dwelling Older-Adults: Correlates Between Performance-Based, Self-Report and Neuropsychological Measures.

Objective: Although self-report and performance-based measures are commonly used as proxy measures of everyday functioning, little is known about the consistency between these measures when assessing medication management. The present study examined the relationship between two performance-based tasks and self-report of medication management. We also examined neuro correlates of these measures.

Participants and Methods: The Medication Management Abilities Assessment (MMAA), the medication subtests of the Revised Observed Tasks of Daily Living (OTDL-R), and three self-report medication management items were collected from community dwelling older adults $\left(\mathrm{N}=41, M_{\text {age }}=73.0, S D_{\text {age }}=9.53 ; M_{\text {edu }}=15.3, S D_{\text {edu }}=2.7\right)$. Self-report items were coded on a 5-point Likert scale and included: "How often do you 1) have someone help organize your medications 2 ) rely on others to remind you to take your medications, and 3) ask someone if you have taken your pills?" Participants were also administered neuropsychological tests of processing speed, executive functioning, memory and working memory.

Results: Spearman's rank correlations between the performance-based, self-report and neuropsychological tests were examined. Poorer performance on the OTDL-R was associated with of the self-report items of needing help remembering when $\left(r_{s}=-.31, p<.05\right)$ and if medications had been taken $(r=-.39, p<.05)$. The MMAA was not significantly related to the OTDL-R, nor to the self-report items. In terms of neurocorrelates, poorer OTDL-R performance was significantly related to poorer working memory $\left(r_{s}=0.42, p<.05\right)$. Lower performance on the
MMAA, however, was significantly related to slower processing speed $\left(r_{s}=-0.42, p<.05\right)$.

Conclusions: The results indicate that the MMAA, OTDL-R and selfreport items may be measuring different aspects of medication management and cognitive functioning. Future work is needed to develop an ecologically valid, gold standard measure of medication management. Correspondence: Catherine Sumida, M.A., Psychology, Washington State University, 1630 NE Valley Rd., L104, Pullman, WA 99163, United States.E-mail: catherinesumida@gmail.com

N. SUN-SUSLOW, A. DACOSTA, N.S. BANERJEE, M. SARNO, A. BURE-REYES, S. GETZ, K.F. MCINERNEY \& B.E. LEVIN. The Association Between Indicators of Physical Frailty Syndrome and Cognitive Function.

Objective: Frailty syndrome is the accumulation of specific age-related indicators and is associated with cognitive decline. The link between frailty and specific cognitive domains is poorly understood and findings on this relationship are inconsistent. To further develop our understanding of this widely diagnosed syndrome, a clinical research study was undertaken to evaluate the link between frailty symptoms and cognitive functioning using in-depth neuropsychological assessments. Participants and Methods: The 214 participants $\left(M_{\text {age }}=62.2\right.$, $\mathrm{SD}_{\mathrm{age}}=9.9$ ) underwent a frailty assessment based on Fried criteria (grip strength, walking speed, fatigue, activity level, weight loss) and were administered a comprehensive neuropsychological battery. Regression models were used to estimate the associations among indicators of frailty and six cognitive domains.

Results: Compared to non-frail participants, those who were frail were: less educated, prescribed more medications, and more depressed (all $p<.002)$. The number of frailty symptoms were negatively correlated with verbal functioning $\left(\beta=-2.83, R^{2}=.148, F(2,211)=17.950\right)$, verbal memory $\left(\beta=-.171, R^{2}=.227, F(4,209)=15.029\right)$, visual memory $\left(\beta=-.162, R^{2}=.190, F(4,209)=11.992\right)$, and visuospatial functioning $\left(\beta=-.258, R^{2}=.166, F(3,210)=13.677\right)$. Slow gait was associated with verbal memory $\left(\beta=-1.66, R^{2}=.229, F(4,209)=15.558\right.$; all $\left.p<.001\right)$.

Conclusions: Frailty in mid and later life is associated with risk factors that include reduced education, increased medication usage. and increased depression. Number of symptoms is negatively associated with verbal functioning, verbal and visual memory, and select visuospatial skills. In particular, walking speed is linked to verbal memory. The findings show that while increased frailty is associated with reduced cognitive performance, slower walking speed may play a particularly important role with regard to memory. These findings support previous research showing slow walking speed is linked to poorer cognitive outcome relative to those who walk at a normal pace.

Correspondence: Ni Sun-Suslow, M.S., Psychology, University of Miami, 5665 Ponce de Leon Blvd., Coral Gables, FL 33124-0751, United States.E-mail:n.sun1@umiami.edu

P. SUNDERARAMAN, S. CHAPMAN, K. EDWARDS, R.E. ABRAHAM, B.L. DEFEIS, C. HALE, B. IDNAY, M. AZAR, D. WEISS, E. HUEY \& S. COSENTINO. Correlates of Financial Risk Taking in Non-Demented Older Adults.

Objective: Financial risk taking (FRT) can compromise real-life financial decisions such as partaking in excessive stock trading. Research suggests that older adults (OAs) are generally more risk averse than young adults. However, OAs are also vulnerable to making unwise financial decisions and are particularly prone to financial scams. Few studies have comprehensively examined the factors that increase FRT in OAs. The current study sought to investigate the extent to which FRT is associated with a range of factors including demographics, subjective and objective cognition, affect, and attitudes towards aging.

Participants and Methods: The sample included 36 healthy older adults (neuropsychological scores > - 1.5 SD; 61\% female, 80\% Caucasian) with an average age of $70.03(\mathrm{SD}=8.07)$ and $16.86(\mathrm{SD}=2.52)$ years of education. Financial items from the Domain Specific Risk-Taking 
(DOSPERT) Scale were used to measure FRT. To measure subjective cognition, OAs judged themselves against similarly aged peers on 20 cognitive abilities using a 7 -point likert scale. Objective cognition was assessed using experimental tasks sensitive to preclinical Alzheimer's disease (AD) including a selective attention task, Short Term Memory Binding Task, and the Face Name Associative Memory Exam. Various self-report tools were used to measure affect and attitudes about aging. Results: Mean FRT was 1.89 (SD=.70, range 1 to 3.67), with higher scores indicating higher FRT. FRT was inversely correlated with age group identification $(r=-.35, p=.04)$. No other correlations were significant.

Conclusions: In OAs, FRT increases as identification with same-aged people (in this case other older adults) becomes weaker. In other words, as OAs identify more with younger age groups, they are more willing to take financial risks. In contrast, neither subjective nor objective cognitive performance related to FRT. Assessing social attitudes towards aging may help identify OAs' propensity to take financial risks and hence subsequently make compromised decisions.

Correspondence: Preeti Sunderaraman, PhD, Columbia University Medical Center, 630 West, 168th Street, New York, NY 10032, United States.E-mail: preetis.ps@gmail.com

\section{J. SZAJER, E. BOWER \& C. MURPHY. The Effect of Worry on} Memory for Odors in Apolipoprotein E $\varepsilon 4$ Carriers.

Objective: Increased levels of worry and possession of the $\varepsilon 4$ allele of the Apolipoprotein E (ApoE) gene are associated with increased risk for Alzheimer's disease (AD). Odor memory has been shown to vary as a function of age and ApoE genotype, and olfactory tests have utility in the pre-clinical identification of AD. Worry has been shown to affect verbal memory performance; however, the influence of worry on odor memory is unknown. This study aimed to assess the influence of worry, age, and ApoE- $\varepsilon 4$ carrier status on odor memory.

Participants and Methods: Participants included 53 young and 45 older adults. Forty-four percent were $\varepsilon 4$-carriers. Worry was evaluated using the Penn State Worry Questionnaire (PSWQ). Participants with neurocognitive or psychiatric diagnoses were excluded. Odor memory was assessed using the California Odor Learning Test (COLT), an olfactory analogue to the California Verbal Learning Test. Olfactory threshold was assessed using a forced choice, step-down method, and controlled for.

Results: Results of a multivariate analysis of co-variance (MANCOVA) indicated only worry level had a significant effect on long delay free recall memory scores such that increasing scores on the PSWQ were associated with better recall, across age and $\varepsilon 4$ status $(p=.01)$. However, a significant interaction between $\varepsilon$ 4-carrier status and PSWQ was found for short and long delay cued recall performance $(p=.016)$. Among $\varepsilon 4$-negative adults, cued recall performance improved as self-reported worry increased. Among $\varepsilon$ 4-carriers, however, cued recall performance declined at higher worry levels.

Conclusions: Findings demonstrate that across age, self-reported worry level influences odor memory, and in cued recall, this effect varies as a function of ApoE- $\varepsilon 4$ carrier status. Worry is a modifiable predictor of cognitive decline and risk for dementia in aging. Future studies on the effects of treatments aimed at reducing worry (e.g., cognitive behavioral therapies for anxiety) on changes in cognitive functioning are warranted. Correspondence: Jacquelyn Szajer, Clinical Psychology, Psychology/ Psychiatry, San Diego State University/UC San Diego, 750 State Street Unit 413, San Diego, CA 92101, United States.E-mail: jszajer@gmail. com

J.W. TAM, D. ClarK, H. XU, C. CALLAHAN, D. MILLER, L. MOSER \& F. UNVERZAGT. Baseline Cognition and Everyday Functioning in Older Adults with Subject Cognitive Complaints from a Randomized Clinical Trial.

Objective: Change in cognition can affect performance of daily activities. However, less is known about the relationship between cognition and everyday functioning in older adults (OAs) with subjective cognitive complaints (SCC). We evaluated the role of cognition, health status, and mood in everyday functioning in OAs with SCC.

Participants and Methods: OAs age 65 years and older with SCC (i.e., self-report of memory being "Somewhat worse" or "Much worse" as compared to 10 years ago on a 5-point Likert scale from "Much better" to "Much worse") were recruited for a randomized clinical trial of the efficacy of cognitive and physical training on cognition through senior agencies, geriatric clinics, community centers, and advertisement. $931 \mathrm{OAs}$ were screened and 103 agreed to participate (mean age $=75.3$ yrs; $83 \%$ female; $74 \%$ white; mean education $=14.4$ yrs $)$. Cognition was measured by a composite score of list learning (Rey AVLT sum recall), psychomotor speed (WAIS-IV Symbol Search), and executive function (Trail Making Test Part B, Stroop Color-Word, and Controlled Oral Word Association). Self-report of health was obtained with the SF-36 and number of chronic conditions (i.e., diabetes, hypertension, myocardial infarction, stroke, transient ischemic attack, chronic obstructive pulmonary disease, and cancer), depressive symptoms with the CES-D, and ability to perform instrumental activities of daily living (IADLs) with the MDS-Home Care. Multiple linear regression models examined the role of cognition, age, sex, race, education, occupation, mood, and health to performance of IADLs.

Results: Lower CES-D $(\beta=0.10, p=0.03)$, excellent self-rated health $(\beta=-2.32, p=0.03)$ as compared to fair/poor health, and higher cognitive composite score $(\beta=-1.45, p=0.01)$ were found to be significantly related to lower level of difficulty with IADLs.

Conclusions: Objective cognitive ability, depression, and self-reported health independently contribute to perceived functional deficits in OAs with SCC.

Correspondence: Joyce W. Tam, M.A., Washington State University, PO BOX 644820, Pullman,WA 99164,United States.E-mail:jwt140@ gmail.com

L. TAYLOR, A. VAILLANCOURT \& V. ZIZAK. Utility of the Repeatable Battery for the Assessment of Neuropsychological Status (RBANS) with a Geriatric Veteran Population: A Comparison of Standard and Demographic Regression Based Norms.

Objective: To examine potential with-in performance differences of geriatric Veterans on the RBANS utilizing the standardization norms and a set of demographically based regression norms established by Duff and Ramezani (2015).

Participants and Methods: This study included archival data from geriatric veterans $(\mathrm{N}=22$; mean age $=78)$ referred to the VA Long Beach Healthcare System neuropsychology outpatient clinic. All Veterans underwent a clinical interview and were administered a neuropsychological evaluation, which included the RBANS. Veterans' raw test scores on the RBANS were scored using both the separate normative data sets. The RBANS indices assessed included attention, visuospatial/constructional, language, as well as, immediate and delayed memory

Results: A paired samples t-test was used to examine the differences in index scores between the two sets of norms. A Bonferroni correction for multiple comparisons was conducted, modified $p<.0027$. As expected the analyses yielded statistically significant differences for most indices (all p's $=.000$ ) except the visuospatial/constructional index (NS, p =. 094). The standardization norms were found to be more stringent, yielding significantly lower/ more impaired performance scores compared to the regression based norms.

Conclusions: Findings emphasize the importance for incorporating more demographically based norms that likely will lead to enhanced diagnostic accuracy. Duff and Ramezani norms may be more representative of the older adult patients with multiple medical conditions and account better for demographic variables. Non-verbal tasks may be less affected by demographic differences. Future studies geared to examine which set of norms improve neurocognitive diagnostic accuracy are of interest. Incorporating more specific demographics may also better capture patients' performance and will be discussed. 
Correspondence: Leanne Taylor, M.S., Clinical Psychology, Florida Institute of Technology, 55 W Church St, apt 1614, Orlando, FL 32801, United States.E-mail: leanne2010@my.fit.edu

D.F. TOMASINO, K.J. MANNING, D.C. STEFFENS \& G. POTTER. Informant Report of Cognitive Functioningin Geriatric Depression: Correlates with Objective Cognitive Tests and Structural Imaging. Objective: The Dementia Severity Rating Scale (DSRS) is an informant questionnaire assessing patient impairment in 11 cognitive-functional domains. Cognitively normal older adults (CN) are generally described as non-impaired on DSRS (i.e., score 0-2/51) whereas higher scores are sensitive to a diagnosis of dementia. We hypothesized that nondemented older adults with major depression (MD) would be rated higher on DSRS compared to CN. Secondary analyses investigated cognitive and structural imaging correlates of DSRS in this cohort.

Participants and Methods: Participants $\geq 60$ years were enrolled in the Neurocognitive Outcomes of Depression in the Elderly Study at Duke University. Dementia and other neuropsychiatric illnesses were exclusion criteria. The current sample was comprised of $57 \mathrm{MD}$ and $35 \mathrm{CN}$ for whom there was a completed DSRS. Participants underwent testing including the Consortium to Establish a Registry for Alzheimer's Disease (CERAD) battery and common cognitive tests. A subset of $20 \mathrm{CN}$ and $35 \mathrm{MD}$ underwent a 3T structural MRI that provided a global measure of white and gray matter lesions and bilateral hippocampal volumes. Results: Differences between MD $($ mean $=4.77 \pm 4.60)$ and $\mathrm{CN}$ $($ mean $=1.05 \pm 2.02)$ were observed on DSRS after controlling for age/education/gender $\left(F\left({ }_{1,83}\right)=15.38, p<.001\right)$. DSRS significantly correlated with multiple measures of cognitive function in MD (e.g., CERAD $\rho=-.41 p=.002$, Trails $\mathrm{A} \rho=.35 p=.06$ ) but not CN. DSRS scores correlated with total lesion load in MD $(\rho=.30 p=.03)$ but not $\mathrm{CN}(\rho=-.08 p=.94)$. No association was found with hippocampal volumes. DSRS did not correlate with psychiatrist ratings of depression severity in $\mathrm{MD}(\rho=.15 p=.30)$.

Conclusions: DSRS correlates with cognitive tests and measures of brain integrity in MD. Clinical evaluations of MD may benefit from this quick, easy to use scale. Further investigation is needed to understand if DSRS can predict cognitive decline in MD.

Correspondence: Debra F. Tomasino, Undergraduate Student, Department of Psychiatry, UConn Health Center, 263 Farmington Avenue, MC2103, Farmington, CT 06030, United States. E-mail: debra. tomasino@uconn.edu

S.M. TURNER, L.M. COLON-PEREZ, K.N. LUBKE, S.N. BURKE \& M. FEBO. Translational Cognitive Training Paradigm Elicits Persistent Functional Connectivity Changes in Aged Animals.

Objective: Changes in large-scale neural connectivity are a hallmark of brain aging that have been linked to cognitive decline. Recent behavioral models for probing the integrity of inter-regional communication along with functional MRI offer a unique opportunity to study connectivity in conjunction with quantification of the neurobiological mechanisms that underlie alterations in large-scale network communication. In this study, we determined how cognitive training on an object-place paired association (OPPA) task, which requires interactions between prefrontal, medial temporal and subcortical structures, altered functional connectivity.

Participants and Methods: Resting state fMRI was collected in a 11.1 Tesla Bruker system from young $(4 \mathrm{mo}, \mathrm{n}=5)$ and aged $(24 \mathrm{mo}$, $\mathrm{n}=5$ ) Fischer 344 x Brown Norway rats during three sessions: 1) before cognitive training, 2) after two weeks of training on the OPPA task in which both young and aged rats were not performing above an $80 \%$ criterion, and 3) after four weeks of OPPA training in which the young rats, but not old, were performing at criterion. Anatomical scans for image overlay and reference-to-atlas registration were collected using a fast spin echo sequence. Time series fMRI signals were extracted from each region of interest (ROI) based on the atlas-guided seed location (150 total areas). The networks were quantified with the graph theory metrics of node strength (sum of edge weights), and rich club (highly interconnected subnetwork commonly termed as hubs).

Results: Young rats did not show differences in global node strengths or rich club between the three sessions. The aged rats, however, had increased node strength connectivity and rich club values after the first training session that persisted following the second training session in the anterior cingulate and striatum.

Conclusions: This suggests that in aged, but not young rats, learning leads to persistent changes in the underlying architecture of resting networks, which may reflect dedifferentiation for compensation.

Correspondence: Sean M. Turner, BS Psychology, Neuroscience, University of Florida, 320 SE 3rd ST, B18, Gainesville, FL 32601, United States.E-mail: seanmturn@gmail.com

E.J. VAN ETTEN, C. SUMIDA, L.V. GRAVES, H.M. HOLDEN, F.V. LOPEZ \& P. GILBERT. Age-Related Differences on a New Memory Test for "Who, When, and Where" Begin in Middle Age.

Objective: Age-related deficits in episodic memory (EM) have been well documented in older adults. However, few studies have assessed this construct in middle-aged adults. The objective of the present crosssectional study was to examine age-related differences across the adult lifespan using a new EM test assessing memory for "who, when, and where" in addition to associations among these elements.

Participants and Methods: Young (ages 18-25), middle-aged (ages 40-55), and older adults (ages 65+) were administered our EM test. During the test, the participant was asked to remember a sequence of pictures of different faces paired with different places. After viewing the sequence, the participant was asked to pair each face with the correct place and put the face-place pairs in the correct sequence.

Results: Young adults remembered significantly more face-place pairs in the correct sequence than middle-aged $(p<.05)$ and older adults $(p<.05)$. However, there were no significant differences between middle-aged and older adults in the number of face-place pairs in correct sequence. Furthermore, young adults remembered significantly more face-place pairs irrespective of sequence than older adults $(p<.05)$. However, there were no significant differences between young and middle-aged adults or between middle-aged and older adults in the number of correct face-place pairs irrespective of sequence.

Conclusions: Using a rapidly administered test that integrates various aspects of everyday EM, we found evidence for age-related differences in test performance beginning in middle age. Given that young and middle-aged adults did not differ in memory for face-place pairs irrespective of sequence, these age-related associative memory differences are not due solely to impaired memory for the individual elements in the associations.

Correspondence: Emily J. Van Etten, Psychology, University of Arizona, 8732 Pacheco Avenue, Westminster, CA 92683, United States. E-mail: emilyjoyvanetten@gmail.com

K.M. WALL, J. STARK, A. SCHILLACI, H. CHRISTIAN, A. KALRA- LALL, M. VANBRAKLE, M. MICHEL, M. MALONEY, N. BARCELOS, B. COHEN \& C. ANDERSON- HANLEY. Biomarkers and Neuropsychological Function in Mild Cognitive Impairment (MCI): Salivary Dehydroepiandrosterone (DHEA-S) and Overall Cognition.

Objective: Neuropathology in later life can lead to debilitating cognitive decline, such as in dementia of the Alzheimer's type. Research continues to explore the possible underlying mechanisms in hopes of finding effective interventions to prevent or ameliorate cognitive decline. A variety of biomarkers have been implicated in the pathway linking complex biological changes of later life with cognitive decline. This crosssectional study examined salivary Dehydroepiandrosterone (DHEA-S), cortisol, and Insulin-like Growth Factor 1 (IGF-1) in a sample of older adults to evaluate possible relationships with cognition, as has been reported previously (Johansson et al., 2011 \& de Menezes et al., 2016). 
Participants and Methods: The study consisted of 25 older adults with an average age of 79.0 years $(S D=6.2$ years $)$. Participants each provided a saliva sample and took several neuropsychological tests including, Montreal Cognitive Assessment (MoCA), Alzheimer's Disease Assessment Scale (ADAS) wordlists, Flanker, Trails A and B, Stroop and digit span. Saliva samples were analyzed using enzyme-linked immunosorbent assays (ELISA) and data was corrected using Bicinchoninic Acid protein concentration assays.

Results: A significant positive correlation was found between DHEA-S and overall cognition, as measured by the MoCA, $r=0.637(p=0.003)$ when accounting for sex and age.

Conclusions: This correlation is supported by the idea of DHEA-S positively correlating with Mild Cognitive Impairment (Johansson et al., 2011) and increased global cognition (de Menezes, 2016). Results provide preliminary evidence that DHEA-S may be a biochemical marker indicating the role of neuroendocrine processes behind cognitive decline, such as in Mild Cognitive Impairment. Future research should focus on using a larger cohort to replicate and extend this pilot study. Correspondence: Kathryn M. Wall, Union College, 212 Church St, Amston,CT 06231,United States.E-mail:wallk@union.edu

M.E. WIGGINS, N.A. SCHWAB, B. BRUMBACK, J.J. TANNER \& C.C. PRICE. Volume and Region of Leukoaraiosis Relative to Cognition in Non-Demented Older Adults.

Objective: Leukoaraiosis (LA), or imaging based white matter hyperintensities, occurs in $\sim 15$ to $65 \%$ of adults. This study was conducted to provide a normative reference on location, typical percentage, and cognitive thresholds associated with LA in non-demented individuals. Hypothesis: The majority of LA distribution will occur in the frontal lobe, and individuals with greater LA load would have lower scores on processing speed and working memory tasks, but not episodic memory. Participants and Methods: All participants completed isovoxel T1 and FLAIR sequences (Siemens Verio 3T), and a comprehensive neuropsychological protocol. Inclusion criteria: $>55$ years of age and a score $\geq 30$ on the Telephone Interview for Cognitive Status (TICS). Regional LA was segmented into lobe and axial region (periventricular- $5 \mathrm{~mm}$ around ventricles; subcortical- $5 \mathrm{~mm}$ beneath the cortex; deep- all other white matter). We controlled LA for white matter (WM) and total intracranial volume (TICV).

Results: From a set of 160 participants, 5 did not meet inclusion criteria. The final set included 155 participants (age: 69.23 \pm 5.82 ; education: $16.03 \pm 2.72$ : TICS: $38.10 \pm 3.44$ : male:female: $70: 85$ ). Total LA for this group ranged from 0.02 to $11.85 \%$ of the white matter (mean $0.97 \% \pm 1.44 \%$ ). LA was dominant in frontal, parietal, and periventricular regions. Only frontal and periventricular regions negatively associated with processing speed and working memory $(r$ 's $=-0.17$ 0.19 , p<.05; threshold for mild impairment was observed when LA was $>0.5 \%$ of the white matter).

Conclusions: Non-demented older adults on average have LA in approximately $1 \%$ of the white matter with this largely confined to the frontal, parietal, and periventricular regions and associating with reduced processing speed and working memory. The study provides normative references for future neurodegenerative regional LA group comparisons.

Correspondence: Margaret E. Wiggins, Clinical and Health Psychology, University of Florida, 1225 Center Drive, Gainesville, FL 32603, United States.E-mail: ellewiggins@ufl.edu

A. SHVARTSUR, H. LEE, F. CHANG, M.J. WRIGHT, L. APOSTOLOVA \& E. WOO. Longitudinal Assessment of Metamemory in Mild Cognitive Impairment and Alzheimer's Disease.

Objective: Metamemory, the awareness and self-monitoring of one's cognition, was evaluated in healthy cognition, mild cognitive impairment (MCI), and Alzheimer's disease (AD) at baseline and at one-year follow up.
Participants and Methods: Participants were 77 controls, 108 MCI subjects, and 44 AD subjects. Participants were provided a description of each neuropsychological test and made predictions about how they would perform. Then, they were administered the test and made postdictions. Metamemory accuracy was the signed difference between predictions/postdictions and performance.

Results: Mixed model ANCOVAs were conducted to determine the impact of diagnosis and prediction type (prediction vs postdiction) on metamemory, covarying for age and education, separately for each test and time point. There was an effect of diagnosis for CVLT-II Delayed Free Recall, in which controls and the MCI group were more accurate than the AD group. For Rey-O 3-minute recall, controls were more accurate than the MCI group, who did not differ from the AD group. There was an interaction of diagnosis by prediction type for Visual Reproduction I; at baseline, both the controls and the MCI groups' postdictions were more accurate than predictions. The AD groups predictions were more accurate than postdictions. At follow-up, the AD groups' predictions were again more accurate than postdictions, while the other groups showed no change in accuracy. For the same interaction for CVLT Trial 5 at baseline, the AD group's postdictions were more accurate than predictions, and there was no change in accuracy for the other groups. For follow-up, the controls' and AD groups' postdictions were more accurate than predictions, with no change in accuracy for the MCI group. The 3-way interaction of diagnosis, prediction type, and time was not significant.

Conclusions: Metamemory accuracy in older adults is dependent on test type and cognitive status but is generally stable over one year.

Correspondence: Ellen Woo, UCLA, 710 Westwood Blvd, Suite 3250, Los Angeles, CA 90095, United States.E-mail:ewoo@mednet.ucla.edu

\section{Concussion}

J.E. KARR, D.P. TERRY, B. MAXWELL, R. MANNIX, R. ZAFONTE, P.D. BERKNER \& G.L. IVERSON. Acute Symptoms Predict Return to School Following Concussion in Student Athletes.

Objective: There are very few studies on predictors of return to school following concussion with little evidence to help clinicians and educators identify students at risk for delayed return to school. We examined whether acute symptoms predicted days to return to school in high school and college athletes

Participants and Methods: Participants were 265 high school $\left(M_{\text {Age }}=16.22\right.$ years; $44.2 \%$ female $)$ and 404 college athletes $\left(M_{\text {Age }}=20.29\right.$ years; $42.6 \%$ female) who sustained a sports-related concussion and had symptoms assessed within 3 days of injury ( $M d n=1$ day) by an athletic trainer via a mobile assessment device. The total symptom score was used as a predictor in a series of bivariate logistic regression analyses with outcomes of return to school at day 7, 10, or 14 days post concussion.

Results: Among high school students, 64\% returned to school by 7 days, $78 \%$ by 10 days, and $86 \%$ by 14 days post-injury. Symptoms significantly predicted return to school at 7 days $(\beta=.020, p=.012$, Odds Ratio $[O R]=1.021$ [95\% CI: $1.004,1.037])$ and 10 days $(\beta=.021$, $p=.016, O R=1.021[1.004,1.039])$, but not at 14 days $(\beta=.018, p=.08$, $O R=1.018[0.998,1.038])$. Among college students, $71 \%$ returned to school by 7 days, $83.9 \%$ by 10 days, and $90.6 \%$ by 14 days post injury. Symptoms significantly predicted return to school among college students at 7 days $(\beta=.048, p<.001, O R=1.049[95 \% C I: 1.034,1.065])$, 10 days $(\beta=.047, p<.001, O R=1.049[1.031,1.066])$, and 14 days $(\beta=.041, p<.001, O R=1.042[1.022,1.062])$.

Conclusions: Greater acute symptoms after concussion predicted greater time out of school in both high school and collegiate athletes. Correspondence: Justin E. Karr, M.Sc., Psychology, University of Victoria, P.O. Box 1700 STN CSC, Victoria, BC V8W 2Y2, Canada. E-mail: jkarr@uvic.ca 


\section{Career Development/Education/Training}

E. HESSEN. Symposium on core competencies in Clinical Neuropsychology training across the world and the current status of Clinical Neuropsychology in thirty-one European countries - Results from a review conducted in collaboration with INS and FESN and from a survey by the European Federation of Psychological Associations (EFPA) on Clinical Neuropsychology in Europe.

Symposium abstract

After signing a Memorandum of Understanding between the INS and the Federation of European Societies of Neuropsychology (FESN) in 2016, a working group with representatives from both bodies met, aiming to review core competencies in Clinical Neuropsychology training across the world. A review of standards for post-graduate training programs in Clinical Neuropsychology from countries with well-established specialties in clinical neuropsychology was conducted, resulting in a set of suggested core competencies that likely will apply to clinical neuropsychologists regardless of regional and cultural context. Also in 2016, the EFPA Task Force on Clinical Neuropsychology conducted another survey on the status of Clinical Neuropsychology in Europe. Thirty-one European countries responded to the EFPA survey. The aim was to assess the status of Clinical Neuropsychology in Europe with the possible future intention of developing common European standards. The suggested core competencies in Clinical Neuropsychology is relevant for developing of common European and international standards in training of clinical neuropsychologists. Results from the review and the survey will be presented and discussed.

Correspondence: Erik Hessen, Ph.D., Psychology, University of Oslo, Krags vei 13, Oslo 0783, Norway. E-mail:dr.hessen@gmail.com

M. CONSTATINOU, B. PERSSON, F. BARBOSA \& E. KASTEN. The Role of Clinical Neuropsychologists in Health Care- Results of a Survey on 31 European Countries.

Objective: In Europe the legislation and training of neuropsychologists is quite variable, but practices are supposedly similar. The European Federation of Psychologists' Associations (EFPA) appointed a Task Force on Clinical Neuropsychology in 2015 to collect information on the current situation on the specialization training within clinical neuropsychology and on the legal and professional status of clinical neuropsychologists within the profession of psychologists in Europe.

Participants and Methods: A survey was distributed to all the national Psychological Associations and to all the national Neuropsychological Societies in Europe in September 2016 using an online platform, and 31 countries in Europe submitted responses.

Results: In $84 \%$ of the countries full neuropsychological assessments are performed by clinical neuropsychologists with a psychologist's background. Cognitive screening (Mini Mental State examinations, short sport concussion testing etc.) is widely performed also by psychologists of other fields and other professionals such as neurologists, psychiatrists, geriatric doctors or different rehabilitation professionals. In 54\% of the countries clinical neuropsychologists may give DSM or ICD diagnoses independently. In $83 \%$ of the countries they are independent in conducting rehabilitation and treatment. Clinical neuropsychologists work most often within adult neuropsychology, geriatric and paediatric fields being second and forensic and educational fields being less common. The work mostly involves assessment, rehabilitation, or consultation. In the public sector services are widely offered in hospitals, health centers and rehabilitation centers in all countries.

Conclusions: The health care needs appear similar in the European countries and neuropsychologists are well situated within the system. Discussion on the European level on the common issues will benefit the field.

Correspondence: Marios Constatinou,Cyprus.E-mail:marios0769@ hotmail.com
E. HESSEN, L. HOKKANEN, J.L. PONSFORD, A. WATTS, M. VAN ZANDVOORT \& K.Y. HAALAND. Core competencies in clinical neuropsychology training across the world.

Objective. The aim of this work was to review main competency requirements from training models in countries with well-established specialties in clinical neuropsychology, and extract core competencies that likely will apply to clinical neuropsychologists regardless of regional and cultural context.

Participants and Methods. We conducted a review of standards for post-graduate training programs in Clinical Neuropsychology from countries in Europe, Australia and North America based on existing literature, presentations at international conferences in 2015, and from the description of the training models from national psychological or neuropsychological associations.

Results. Despite differences in training models, the programs reviewed share similar core competencies considered necessary for a specialty in clinical neuropsychology: 1. In-depth knowledge of general psychology (post-graduate level), including ethical and legal standards. 2. Expert knowledge about clinically relevant brain-behavioral relationships. 3 . Comprehensive knowledge about related clinical disciplines. 4. In-depth knowledge about and competency in neuropsychological assessment, including decision-making and diagnostic competency according to current classification of diseases. 5. Communication competency of neuropsychological findings and test results to relevant and diverse audiences. 6. Knowledge about and competency in psychological and neuropsychological intervention, including treatment and rehabilitation. Conclusion. All the models have undergone years of testing and development in accordance with requirements of health care systems in different parts of the world. Despite differences, the common core competency requirements across different regions of the world, suggest generalizability of these competencies.

Correspondence: Erik Hessen, Ph.D., Psychology, University of Oslo, Krags vei 13, Oslo 0783, Norway. E-mail:dr.hessen@gmail.com

S. LETTNER \& E. HESSEN. The Legal Status and Licensure of Clinical Neuropsychologists - Results of a Survey on 31 European Countries.

Objective: In European countries, the legislation mostly appears clear on who is a psychologist but the regulation of neuropsychologists differs. The European Federation of Psychologists' Associations (EFPA) appointed a Task Force on Clinical Neuropsychology in 2015 to collect information on the current situation on the specialization training within clinical neuropsychology and on the legal and professional status of clinical neuropsychologists within the profession of psychologists in Europe. Participants and Methods: A survey was distributed to all the national Psychological Associations and to all the national Neuropsychological Societies in Europe in September 2016 using an online platform, and 31 countries in Europe submitted responses. The survey included questions about the regulatory legislation of psychologists, clinical psychologists and neuropsychologists in each country. The questions focused separately on the regulation of the title, practice and education of these professions.

Results: While 'psychologist' is a protected title by law in $83 \%$ of the countries, 'clinical neuropsychologist' is in $17 \%$. In $50 \%$ of the countries, the ministry of health is the regulatory body. The level of regulation of psychologist, clinical psychologist and clinical neuropsychologist lessens as per this order. In countries where practice of either clinical psychology or clinical neuropsychology was regulated, the law mostly forbids other professions besides psychologists practicing in the field of Clinical Neuropsychology. The few exceptions include e.g. physicians, speech therapists and other health professions in some countries. Conclusions: The legislative regulation of clinical neuropsychology is rare and builds upon the regulation of psychologists and clinical psychologist. Over-regulation can be a concern but without proper legal regulation, quality assuring standards of the profession are at risk. Correspondence: Sandra Lettner. E-mail: Sandra.Lettner@bhs.at 
L. HOKKANEN, S. MONDINI, N. VARANKO \& L. HARPER. Training Models in Clinical Neuropsychology - Results of a Survey on 31 European Countries.

Objective: The level of training in clinical neuropsychology across Europe currently appears quite variable. The legislation is mostly clear on who is a psychologist but the regulation of neuropsychologists differs. The European Federation of Psychologists' Associations (EFPA) appointed a Task Force on Clinical Neuropsychology in 2015 to collect information on the current situation on the specialization training within clinical neuropsychology and on the legal and professional status of clinical neuropsychologists within the profession of psychologists in Europe. Participants and Methods: A survey was distributed to all the national Psychological Associations and to all the national Neuropsychological Societies in Europe in September 2016 using an online platform, and 31 countries in Europe submitted responses.

Results: The academic degree required to practice clinical neuropsychology is mostly the same as required for clinical psychologists: Doctorate in the UK and Ireland, Master's or below in other countries. The total length of degree studies varied from 3 to 10 (mean $5.6 \pm 2.3$ ) years. If there was a commonly agreed model of specializing in clinical neuropsychology, it mostly involved a pre-planned training program (lengths ranging from 1 to 5 years) or a selection of individual courses. After universities, the second most common organizers of specialist education were the national neuropsychological societies followed by national psychological associations. A practical training period was required for specialization in $73 \%$ of the countries, the length ranging from 18 hours to 5 years. In $31 \%$ of the countries, there was no commonly agreed model on how to specialize in clinical neuropsychology.

Conclusions: The results shed light on the heterogeneity of neuropsychology training models within Europe. This raises concerns on patient protection. Based on these results EFPA has in 2017 appointed a Standing Committee to work towards a more common framework of training.

Correspondence: Laura Hokkanen, PhD, Department of Psychology, University of Helsinki, P.O.Box 21, Helsinki 00014, Finland. E-mail:laura.hokkanen@helsinki.fi

\section{Program Welcome}

\section{Program Committee Chair: Shawn M. McClintock}

$$
\text { 4:30-4:45 p.m. }
$$

\section{Plenary A (INS Presidential Address). Anomalies of Autobiographical Memory}

\section{INS President: Michael Kopelman}

$$
\text { 4:45-5:45 p.m. }
$$

\section{KOPELMAN. Anomalies of Autobiographical Memory.}

Autobiographical memory alludes to personal recollection of past episodes and incidents. In clinical disorders, neurological or psychological, it can be affected in a variety of ways. This presentation will highlight examples, illustrated throughout by clinical case-examples and video-clips (and even the author's own minor brush with history), and it will review current explanatory theories.

Retrograde amnesia (RA) is a fascinating phenomenon, referring to loss of memories for autobiographical episodes and/or personal semantic facts which occurred before the onset of a brain disease or injury. The severity of RA is only loosely associated with the severity of anterograde amnesia, suggesting different underlying mechanisms. There are various theories of how and why RA occurs, and it will be (politely!) suggested that they all have their limitations.
Spontaneous confabulation refers to the unprovoked flow of erroneous memories, seen in some neurological patients, now thought to relate to damage in the ventro-medial and orbito-frontal regions of the frontal lobes. A recent study will be presented which tested between alternative theories of confabulation, finding damage to autobiographical memory and executive systems to be most critical to the 'rise and fall' of confabulation.

Psychogenic amnesia intrigues the media! It can be 'global' or 'situation-specific'. A recently published study of 53 cases has highlighted 4 different types of syndrome (or subgroups), and case-examples will again be given. The study emphasised the psychosocial circumstances in which the amnesia occurs, and demonstrated different patterns of autobiographical memory loss across the subgroups, and with differential patterns of outcome.

Commonalities, controversies, and outstanding issues across these 'anomalies' of autobiographical memory will be spotlighted. I won't promise to stick to the conventional wisdom, but I'll try not to tread on too many toes!

Learning objectives:

1. Understand about theories of retrograde amnesia and their limitations. 2. Understand about theories of confabulation and their limitations.

3. Understand about theories of psychogenic amnesia and their limitations.

Correspondence: Michael Kopelman, Institute of Psychiatry, Psychology and Neuroscience, King's College London, Academic Neuropsychiatry Kings College London, St Thomas's Hospital, London SE1 7EH, United Kingdom.E-mail: michael.kopelman@kcl.ac.uk 
WEDNESDAY EVENING, FEBRUARY 14, 2018

\section{INS Awards Ceremony}

Awards Committee Chair: Roy P.C. Kessels

5:45-6:30 p.m.

INS Welcome Reception

6:30-7:30 p.m.

THURSDAY MORNING, FEBRUARY 15, 2018

\section{CE Workshop 7. "Precision Neuropsychology": Neuropsychological Assessment in the "Precision Medicine" Revolution}

Presenter: Ruben C. Gur

7:20-8:50 a.m.

R.C. GUR. Introduction to "Precision Neuropsychology": Background and initial results of making neuropsychological assessment participate in the "Precision Medicine" revolution.

Neuropsychologists have pioneered advances in cognitive neuroscience that capitalize on structural and functional neuroimaging methods, yet their clinical tools and training are still based predominantly on laborious testing procedure validated with outdated "clinical-pathological" correlations methodology. Furthermore, clinical expertise is still couched in categorical diagnostic nomenclature that does not cut at the biological joints. Medicine is undergoing transformation from symptom-based to mechanism-based diagnosis where genomic data are integrated with "biomarkers" to generate individualized detection, prevention and intervention. This approach requires large-scale studies and clinical investigators and practitioners who can integrate their toolkits with multimodal brain behavior parameters. The workshop will describe an approach for deploying fast and effective neurocognitive assessments that are firmly rooted in modern neuroscience and permit precise statements linking behavioral domains to brain systems across diagnostic categories. The workshop will illustrate how a future neuropsychologist can go beyond psychological tests summarized with vague and tentative statements on involvement of brain systems. Neuropsychologists can lead the design and integration of multimodal neuroimaging data and be important parts of the multidisciplinary team crafting mechanism-based diagnoses. We will show how the approach can be applied in multiple settings ranging from neuropsychiatric clinics to educational to military and even space and analog conditions. Objectives: 1. Appreciate the transformation of medicine from symptom- to mechanism-based diagnosis. 2. Understand limitations of current testing tools for "Precision Medicine". 3. Learn an approach that has generated appropriate tools and procedures for "Precision Neuropsychology" by integrating behavioral measures with multimodal brain parameters. 4. Consider implications for training and clinical practice.

Correspondence: Ruben C. Gur, PhD, Psychiatry, Neurology, Radiology, Neuroscience, University of Pennsylvania, 10th Fl., Gates Pavilion/HUP, 3400 Spruce Street, Philadelphia, PA 19104, United States. E-mail: gur@mail.med.upenn.edu

\section{CE Workshop 8. Role of Computerized Screening in Healthcare Teams: Why Computerized Testing is NOT the Death of Neuropsychology}

\author{
Presenter: Stephen M. Rao \\ 7:20-8:50 a.m.
}

S.M. RAO. Role of Computerized Screening in Healthcare Teams: Why Computerized Testing is NOT the Death of Neuropsychology.

Today, many neuropsychologists view computerized testing as a threat to their clinical practice. The argument centers on the notion that physician referrals for neuropsychological assessments will be replaced by computerized neuropsychological examinations that provide meaningful clinical interpretations. All that a medical practice would require is the purchase of a computer/tablet and appropriate software. No doubt, in some settings, this concern is valid. However, in this workshop I argue that computerized testing has the capability of expanding clinical neuropsychological services to a broader range of patients in a wider range of clinical settings. This argument is based on the premise that an extraordinarily large number of patients who require neuropsychological services are simply not identified by current healthcare practices because, unlike blood pressure or weight, cognition is rarely measured during routine medical visits. With the growing interest in integrated care and a shift to a population health based reimbursement model in the US, there will be an increased need for valid, reliable cognitive screening measures that can be incorporated seamlessly into a standard medical visit with minimal disruption of service delivery flow or need for additional personnel. I will outline our experiences with the development, validation and implementation of self-administered iPad-based neuropsychological screening assessment using two illustrative examples involving primary care and a large hospital-based multiple sclerosis clinic.

Objectives:

1. Explain how integrating self-administered computerized cognitive screening tests into large volume clinical practices can improve health care with little cost to the health care system.

2. Descibe how the integration of computerized screening into clinical practice will create unique opportunities for neuropsychologists in a medical environment with a growing emphasis on population health. Correspondence: Stephen M. Rao, PhD, Schey Center for Cognitive Neuroimaging, Cleveland Clinic, Neurological Institute, 9500 Euclid Avenue / U10, Cleveland, OH 44195, United States.E-mail: raos2@ ccf.org. 


\section{Poster Session 2. Neuropsychiatry, Forensies, and Inclusion and Diversity}

\author{
8:00-9:15 a.m.
}

\section{Addiction/Dependence}

\section{F. ARIAS, J. ARNSTEN, A. MANGALONZO, T.M. SCOTT, P. OLSEN \& M. RIVERA MINDT. Executive Functioning, Characterological Facets of Inhibitory Control, and Participation in Risky Behaviors in Adults Receiving Opioid Agonist Treatment.}

Objective: Opioid use disorders (OUD) represent a US public heath epidemic. Opioid agonist treatment (OAT) with methadone or buprenorphine reduces craving, illicit drug use, \& criminality in adults with OUD (Mattick et al., 2009, 2014). However, participation in risky behaviors (risky sexual practices [RSP] \& injection drug use [IDU]) \& treatment non-adherence remain major issues. Given the dearth of research exploring patient-specific characteristics that diminish health outcomes, this study examined whether significant associations exist between executive functioning (EF) \& characterological facets of inhibitory control (CFIC) with RSP \& IDU in adults receiving OAT

Participants and Methods: This cross-sectional study recruited 131 adults receiving OAT $(M$ age $=45.9 \pm 10.6$ yrs; $M$ education $=11.3$ ( $\pm 2.2 \mathrm{yrs} ; 65 \%$ male; $60 \%$ Latino). EF was assessed using the Iowa Gambling Task (IGT), Stroop, Trail Making Test -B (TMT-B), \& the WCST-64. EF scores were analyzed using demographically corrected T-scores. CFIC were assessed using a standardized Likert-type scale, the Urgency, Premeditation, Perseverance, Sensation Seeking, \& Positive Urgency scale (UPPS-P). RSP \& IDU (within past year) were assessed using a standardized Likert-type scale, the Risk Assessment Battery $(\mathrm{RAB})$. Raw scores were analyzed for the UPPS-P \& RAB.

Results: A series of regressions revealed that lower IGT score \& greater Positive Urgency were associated with increased IDU $\left(X^{2}(6)=18.14\right.$, $p<0.01)$. Also, TMT-B scores explained a significant portion of the variance in $\operatorname{RSP}(F(6,107)=2.85, p<0.05)$. Younger age \& male gender were associated with greater IDU \& RSP.

Conclusions: Decision-making, cognitive flexibility, Positive Urgency, age, \& gender are important to assess in adults with OUD. In OAT, patient-specific characteristics may help to identify adults who may need additional support/psychoeducation. Future research would benefit from examining an expanded definition of risky behaviors (including risky spending \& socializing) within a larger \& more diverse sample. Correspondence: Franchesca Arias, PhD, Psychology Department, Fordham University, 1700 Southwest 16th Court, Unit A22, Gainesville, FL 32608, United States. E-mail: arias.franchesca@gmail.com

T. NGUYEN-LOUIE, N. CASTRO \& S. TAPERT. Earlier Alcohol Use Onset Predicts Poorer Neuropsychological Functioning in Young Adults.

Objective: Over 20\% of U.S. high school students begin drinking before age 14. These individuals are four times more likely to develop psychosocial and psychiatric difficulties than those who began drinking after turning 20. However, little is known about how alcohol use onset age influences brain development.

Participants and Methods: This study prospectively examined the effects of alcohol use onset age on neurocognitive functioning in adolescent drinkers $(\mathrm{N}=215)$. Youth were administered a neuropsychological (NP) battery before substance use initiation ( $\mathrm{M}=13.6$ years, $\mathrm{SD}=0.8)$, and on average 6.8 years later $(\mathrm{M}=20.2$ years, $\mathrm{SD}=1.5)$. Hierarchical linear regressions examined if earlier ages of onset for first and weekly alcohol use adversely influenced neurocognition, above and beyond baseline neurocognition, substance use severity, and familial and social environment factors.

Results: As hypothesized, an earlier age of first drinking onset predicted poorer performance in psychomotor speed and visual attention $(p s<.05$, $N=215)$ and an earlier age of weekly drinking onset predicted poorer cognitive inhibition and working memory, above and beyond effects of baseline NP performance, drinking duration, and past-year marijuana use $(p \leq<.05, N=127)$. First and weekly drinking onset age were not linked to verbal learning and memory or visuospatial performances.

Conclusions: This is the first study to assess the association between age of adolescent drinking onset and neurocognitive performance using a comprehensive test battery. Results suggest that early onset of drinking increases risk for alcohol-related neurocognitive vulnerabilities, and that initiation of any or weekly alcohol use at younger ages may be risk factors for poorer subsequent NP functioning. Findings have important implications for prevention programming. Further studies are needed to replicate these preliminary findings and better understand mediating processes and moderating conditions.

Correspondence: Tam Nguyen-Louie, M.S., Psychiatry, SDSU/UCSD, 8950 villa la jolla dr., Suite C213, La Jolla, CA 92037, United States. E-mail:ttn045@ucsd.edu

I.M. PACHECO-COLON, S.W. HAWES \& R. GONZALEZ. Adolescent Cannabis Use Trajectories and Motivation: A Longitudinal Analysis.

Objective: Reduced motivation is often noted as a consequence of cannabis use (CU). Prior work has been cross-sectional and yielded mixed findings. This study examines changes in adolescents' motivation over time as a function of different $\mathrm{CU}$ trajectories, predicting that adolescents with escalating CU will show a decrease in motivation and those with stable $\mathrm{CU}$ will show no change.

Participants and Methods: Participants were 401 healthy adolescents at risk for escalation in CU aged 14-17 at baseline who completed 5 bi-annual visits over a 2 -year period. Past 6-month frequency of substance use was assessed at all visits. Motivation was measured through the Apathy Evaluation Scale (AES) at visits 1, 3, and 5. We used growth mixture modeling to identify CU trajectories. Growth curves of motivation were then calculated for all participants $(\mathrm{n}=362)$, and for each CU trajectory class.

Results: A 3-class model of adolescent CU was identified as providing the best fit, and consisted of the following groups: "Escalating" $(\mathrm{n}=62)$, with initially moderate levels of CU that increased significantly over time; "Low" ( $\mathrm{n}=304)$, with consistently low levels of CU; and "High" ( $\mathrm{n}=35)$, with consistently high levels of CU.

The overall unconditional growth curve of the AES score had a significant positive mean estimated intercept $(x=28.37, p<.001)$, and a negative slope $(x=-.50, p=.05)$, indicating that motivation slightly increased over time. For all CU trajectory classes, mean intercepts of the AES were significant and positive (Escalating: $x=28.89$; Low: $x=28.37$; High: $x=29.60 ; p \mathrm{~s}<.001)$, and mean slopes were negative and nonsignificant (escalating: $x=-.68$; low: $x=-.42$; high: $x=-1.35 ; p \mathrm{~s}>.05)$. There were no significant between-class differences, $p \mathrm{~s}>.05$. These results remained after controlling for alcohol and nicotine use, and depression.

Conclusions: Our results suggest no differences in motivation between observed CU trajectories, and therefore do not support a link between $\mathrm{CU}$ and reduced motivation among adolescents.

Correspondence: Ileana M. Pacheco-Colon, Psychology, Florida International University, 5091 NW 7th St, Unit 1011, Miami, FL 33126, United States.E-mail: ipach008@fiu.edu

V.M. PATT, D. KREITNER, E. WEBER, B. HENRY, M. CHERNER, G.G. BROWN, M.A. GEYER, W. PERRY, A. MINASSIAN \& I. GRANT. Effect of Combined HIV Infection and Methamphetamine Dependence on Spatial Working Memory.

Objective: Working Memory (WM) - the active brain system that provides temporary storage and manipulation of information - involves brain circuitries closely overlapping those affected by methamphetamine (Meth) neurotoxicity, especially the dopamine-abundant prefrontal cortex. Meth use is frequent among HIV-infected individuals, with results from human studies and animal models suggesting increased 
risk of cognitive impairment in combined HIV infection and Meth use. This project examines the combined effects of HIV infection and Meth dependence on spatial WM, a key aspect of WM dealing with information pertaining to orientation and location in space.

Participants and Methods: Participants were recruited through the NIDA-funded Translational Methamphetamine AIDS Research Center (TMARC). They included individuals with a documented history of Meth dependence $(n=28)$, a diagnosis of HIV infection $(n=23)$, combined Meth dependence and HIV infection $(n=27)$, and neuro-medically healthy individuals $(\mathrm{n}=30)$. A computerized spatial span paradigm was constructed based on the Corsi Block Task, requiring remembering a series of targets changing from white to gray in a specific order.

Results: Average performance was found to decline with age $(F=27.3$, $\left.p<.001, \eta^{2}=.21\right)$, but was not impacted by education $(F=1.8, p=.181$, $\eta^{2}=.02$ ). After accounting for age and education, there was a significant negative effect of Meth $\left(F=10.2, p=.002, \eta^{2}=.09\right)$, but no significant effect of HIV $\left(F=0.1, p=.762, \eta^{2}=.001\right)$ and no interaction of Meth and $\mathrm{HIV}\left(F=1.4, p=.236, \eta^{2}=.01\right)$.

Conclusions: Spatial WM constitutes a fluid neurocognitive ability that declines with age but is independent from educational background. HIV infection in the era of antiretroviral therapy (ART) does not appear to affect spatial WM abilities as measured by this instrument. On the other hand, impaired spatial WM may constitute a distinguishing feature of Meth dependence. Cognitive interventions targeting spatial WM may thus be useful to improve functional levels in Meth-dependent individuals.

Correspondence: Virginie M. Patt, Ph.D., MedStar National Rehabilitation Hospital, 628 N. Illinois St., Arlington, VA 22205, United States.E-mail:vpatt@ucsd.edu

K. WACLAWIK, K. GICAS, C. GIESBRECHT, A.A. JONES, N.Y. WANG, T.A. O'CONNOR, H. BAITZ, F. VILA-RODRIGUEZ, O. LEONOVA, S. MATHIAS, A. BARR, R.R. PROCYSHYN, D. LANG, G.N. SMITH, G.W. MACEWAN, W. PANENKA, A. YAMAMOTO, W.G. HONER \& A.E. THORNTON. Cognitive Impairment in Marginally Housed Youth: Associations with Substance Use and Viral Infection.

Objective: Marginal housing is associated with increased rates of various health risks, including substance use and viral infections, which are known to compromise cognition. Youth are recognized as one of the fastest-growing and most vulnerable sectors of the homeless population. There is limited research on the cognitive impact of multimorbidity in vulnerable populations such as the marginally housed. The present study aimed to characterize cognitive functioning and identify health-related correlates of cognition in a sample of marginally housed youth. Participants and Methods: Youth $\left(N=89 ; M_{\text {a ge }}=25.01\right.$; range 20-29 years) were recruited from marginal housing sites and assessed for substance dependence and viral infections. Cognitive measures included verbal memory, attention, executive functions, and premorbid IQ. Candidate predictor variables included substance dependence and hepatitis $\mathrm{C}(\mathrm{HCV})$ and were correlated with cognition.

Results: Rates of substance dependence (88\%) and mental illness (99\%) were extremely high. Rates of HIV (3\%) and HCV infection (32\%) were lower than in other marginally housed samples with a broader age range. While $96 \%$ of participants had estimated premorbid IQ in the average or above average range, $33 \%$ were cognitively impaired ( $\leq 2$ standard deviations below the normative mean on at least two domains). Poorer performance on verbal memory was associated with cocaine dependence, poorer inhibition with alcohol dependence, and poorer attention and visual scanning with heroin dependence and injection drug use $(r$ range $=-.24$ to -.56 , all $p<.05)$. Poorer sustained attention was associated with HCV infection $(r=-.31)$.

Conclusions: Marginally housed youth represent a unique subpopulation whose health risks differ from older marginally housed samples. Despite premorbid IQ estimates in the average range, rates of cognitive impairment were elevated in the sample. HCV infection and specific substance use dependencies and characteristics were associated with deficits on select cognitive domains.

Correspondence: Kristina Waclawik, MA, Psychology, Simon Fraser University, 8888 University Dr, Burnaby, BC V5A 1S6, Canada. E-mail: kwaclawi@sfu.ca

\section{Career Development/Education/Training}

T.M. SCOTT, A. REYES, A. SUMMERS, R. VOGEL, K. TURESON, K. DUMAS, C.E. LOVE \& O.A. SANTOS. Diversifying Neuropsychology from the Bottom Up: Outcomes from a Student Pipeline Workshop.

Objective: By 2050, over half of the U.S. will identify as a racial/ ethnic minority. Unless appropriate steps are taken to increase the representation of diverse providers in neuropsychology (NP), a barrier will continue to exist in delivering culturally competent NP services. The American Academy of Clinical Neuropsychology (AACN) Relevance 2050 (R2050) Student Pipeline Subcommittee (SPS) presented a workshop at the 2017 AACN Annual Meeting to brainstorm ways to increase the pipeline of diverse students in NP.

Participants and Methods: Seven attendees, including neuropsychologists, postdoctoral fellows, and graduate students, were introduced to the purpose, goals, and initiatives of R2050 and SPS. Administration of real-time, text message-based polls were used to identify attendees' cultural competencies and training experiences, as well as to facilitate a brainstorming session.

Results: All participants, regardless of current level of training or practice, self-identified as "somewhat competent" in providing crosscultural NP services and indicated increasing cultural competency as an ongoing professional goal. Attendees reported that "greater exposure to working with diverse clientele" and "mentoring from a member of an underrepresented group" would have been most helpful earlier in their career to increase cultural competency. The following themes emerged from the brainstorming session: 1) introducing trainees to NP earlier; 2) connecting trainees to mentors; 3 ) increasing training resources; and 4) making curricular changes to NP programs.

Conclusions: Our workshop generated ideas for increasing the pipeline in NP for underrepresented students using an innovative and interactive text message-based polling service. Interorganizational efforts in NP should focus on collaboration to increase access to mentors from underrepresented groups and generating/disseminating diversity-related resources for current and future neuropsychologists.

Correspondence: Travis M. Scott, MA, Psychology, Fordham University, 139 Payson Ave, New York, NY 10034, United States. E-mail: tshivleyscott@fordham.edu

\section{Drug/Toxin-Related Disorders}

E. CRAUN, S. KAPLAN, M.M. WONG \& N. AUBUCHON-ENDSLEY. Alcohol Use Disorder Symptoms, Neurocognitive Functioning, and Criminal Behavior of Jail Inmates.

Objective: Inmates have higher rates of Alcohol Use Disorder (AUD) than the general public (Substance Abuse \& Mental Health Services Administration, 2011). Alcoholism has been associated with both neurocognitive deficits and greater frequency of criminal behavior (Smith et al., 1992; Rourke \& Grant, 2009). The purpose of this study was to assess the relationship between alcohol use disorder symptomatology and (i) neurocognitive deficits and (ii) frequency of criminal behavior in a sample of jailed individuals.

Participants and Methods: Two hundred and fifty (58.4\% women, aged 18-71) inmates were randomly selected from two U.S Northwestern jails and completed a diagnostic screening interview and brief neurocognitive battery. The MINI International Neuropsychiatric Interview (MINI) was used to quantify the number of alcohol use disorder 
symptoms endorsed. The Trails Test and Color-Word Interference Test (CWI) of the Delis-Kaplan Executive Functioning System (D-KEFS) were used to capture cognitive functioning. For the Trails Test, contrast scores comparing switching trial performance to combined numberletter sequencing trial performance (resulting in a measure of cognitive flexibility) was used. Frequency of convicted felonies and misdemeanors were confirmed using the state repository system.

Results: Linear regression models revealed that alcohol use symptoms significantly predicted contrast score of the Trails Test $(b=-0.98$ $(0.05), p<0.05)$ and inhibition/switching of the CWI $(b=-0.124(0.05)$, $p<0.05)$, such that more endorsed symptoms of AUD were associated with poorer performance on measures of cognitive flexibility and inhibition. AUD symptomatology also significantly predicted more frequent convicted criminal behavior $(b=0.39(0.19), p<0.05)$.

Conclusions: The results converge with previous findings reporting associations with alcohol use and cognitive deficits and alcohol use and criminal behavior. Implication of these findings on the development and treatment of criminal behavior will be discussed.

Correspondence: Elizabeth Craun, Master's of Science, Idaho State University, 921 S 8th Ave, Pocatello, ID 83209, United States. E-mail: craueliz@isu.edu

\section{A. PRITCHETT \& Y. NOMURA. Effects of Prenatal Cannabis and Tobacco Exposure on Offspring Stress Hormones.}

Objective: Cannabis and tobacco are often used by women during pregnancy, yet their effects on the offspring's developing stress response system are still largely unknown. CB1 receptors, targets for the binding of cannabinoids, are operational at early time points in fetal development, and are expressed in key limbic brain structures. Longitudinal studies have associated prenatal cannabis exposure with increased fearfulness, and mood disturbances (Faden, 2000; Gray, 2005). Prenatal tobacco exposure has also been associated with negative emotionality (Schuetze, 2007). Despite the growing evidence for offspring emotional dysfunction associated with prenatal cannabis and tobacco exposure, there remains a gap in knowledge linking prenatal exposure to early deviations in stress hormone concentrations.

Participants and Methods: The Stress in Pregnancy Study (SIPS) screened pregnant women at two urban hospital sites. Hormone assays were performed on hair samples of 159 infants. Of these mothers, 34 had self-reported cannabis and/or tobacco use during pregnancy.

Results: Cannabis but not tobacco exposure was associated with significantly higher levels of infant cortisol $(p=0.03)$ compared to unexposed offspring. There was a significant interaction between cannabis and tobacco exposure, such that tobacco users who also used cannabis during their pregnancy had offspring with significantly higher levels of dehydroepiandrosterone (DHEA) $(\mathrm{p}<0.0001)$. DHEA plays an important role in the neuroregulation of the stress response system. Significance was largely unchanged after covarying for child gender and race, and socioeconomic factors of the mother.

Conclusions: Infants who display higher stress hormone levels and an inability to self-regulate, are at an increased risk for developing future mood disorders. This study reports hormone alterations in substance exposed offspring that may underlie limbic dysfunction in infancy and beyond.

Correspondence: Alexandra Pritchett, B.S. , Psychology, Queens College, 57 Hausman St, Brooklyn, NY 11222, United States. E-mail: lexi.pritchett@gmail.com

\section{Emotion Regulation}

A. ALKOZEI, R. SMITH \& W.D. KILLGORE. A Positive Cognitive Style Mediates the Relationship Between Trait Gratitude and Depressive Symptoms.

Objective: Gratitude is associated with greater psychological wellbeing, and lower depressive symptoms. One mechanism that may explain this relationship is a difference in cognitive style, such that individuals with higher levels of gratitude may interpret neutral events in a positive rather than negative manner. Here, we tested the hypothesis that high levels of gratitude are associated with a positive cognitive style, and that such a positive cognitive style mediates the relationship between trait gratitude and depressive symptoms.

Participants and Methods: Eighty-eight non-clinical individuals (41 females, Mean age=19.1, SD=1.9) completed the Gratitude Resentment and Appreciation Test, as a measure of trait gratitude, the Sentence Completion Test for Depression and the Ambiguous Scenarios Test for Depression, as measures of cognitive style, and the Beck Depression Inventory as a measure of depressive symptoms. To explore the hypothesis that a positive interpretation bias mediates the relationship between gratitude and depressive symptoms, mediation analyses using Hayes' PROCESS tool in SPSS were conducted.

Results: Positive cognitive style, as indexed by thinking about oneself, the world and other people in a more positive way, and assuming positive rather than negative endings to open-ended scenarios, partially mediated the relationship between trait gratitude and depressive symptoms $(b=-.04,95 \% C I[-.08,-.02])$.

Conclusions: The findings suggest that high levels of gratitude are associated with lower depressive symptoms partially because these individuals employ a positive thinking style. Gratitude interventions may therefore be effective in increasing wellbeing and reducing depression, because they require individuals to practice a positive thinking style, which in turn contributes to increased happiness and decreased negative affect. However, future longitudinal research is necessary to disentangle the causal relationships between these variables in greater detail. Correspondence: Anna Alkozei, Ph.D., Department of Psychiatry, University of Arizona Medical Center, University of Arizona Medical Center, Department of Psychiatry, 1501 N Campbell Ave, Tucson, AZ 85724, United States.E-mail: aalkozei@psychiatry:arizona.edu

M. DENO, T. TACHIKAWA, S. IZAWA, K.K. FUJISAWA, K. HANITA $\&$ M. TANAKA. The Effect of Physiological Response to Acute Stress on Emotional Attentional Bias Among Japanese Adolescents.

Objective: Evidence has demonstrated the effect of physiological response to psychosocial acute stress on emotional attentional bias, but this effect has not been investigated among Japanese adolescents. Previous studies did not account for baseline attentional function. Here. we examined the association between cortisol responses and attentional bias toward emotional facial expression before and after acute stress among Japanese adolescents.

Participants and Methods: Twenty healthy girls (10-16 years old) completed the Trier Social Stress Test for Children (TSST-C). We used data from one randomly selected individual from pairs of monozygotic female twins. Saliva samples were collected for evaluating cortisol levels 25 and 5 minutes before and $0,15,30$, and 45 minutes after administration of the TSST-C. Participants also engaged in the dot-probe task for emotional facial expression (angry, happy, and sad) before and after the TSST-C.

Results: Cortisol levels measured 0 and 15 minutes after administration of the TSST-C were higher than those measured 25 and 5 minutes before and 30 and 45 minutes after the TSST-C. Although attentional bias toward angry faces after the TSST-C was correlated with cortisol levels 15 minutes after acute stress $\left(\beta=-.488, p<.05, \Delta R^{2}=.188\right)$, the discrepancies in attentional bias between before and after the TSST-C were not significantly influenced by cortisol levels $\left(\beta=-.098 \mathrm{~ns}, \Delta R^{2}\right.$ $=.112$ ).

Conclusions: The significant association between physiological response and emotional attentional bias after acute stress among Japanese adolescents supports previous research. However, considering the baseline emotional attentional function, the attentional bias after acute stress was not significantly related to physiological response, which may suggest that cognitive responsiveness to psychosocial acute stress might not be influenced by physiological responses to stress. 
Correspondence: Minako Deno, The University of Tokyo, 7-3-1 Hongo, Bunkyo-ku, Tokyo 113-0033, Japan. E-mail: denominako@gmail.com

R.E. EASTER, S.A. LANGENECKER, P. BABU, S. ASSARI, B. MUNSON, D. MARSHALL, J. PETERMAN, M. MCINNIS \& K. RYAN. Effects of age, education, and mood state on facial emotion processing in bipolar disorder.

Objective: Prior research has found age and education effects on emotion processing in controls and inconsistent results on mood state effects in bipolar disorder (BD). This study investigates differences in facial emotion processing in age, education, and mood state groups in a BD sample.

Participants and Methods: 466 individuals with BD completed assessments of depression and mania and the Facial Emotion Perception Test - a computerized test of facial perception of happiness, sadness, anger, and fear. Age groups were defined as 20-29, 30-44, and 44-60 years. Education was defined as $<16$ or $>16$ years. Depressive, euthymic, and manic mood states were defined by mood assessment cutoffs.

Results: Analyses of variance found a main effect of age for overall accuracy, reaction time, and fear and anger accuracies.

A two-way interaction between education and mood state was found for overall accuracy, sadness accuracy, and reaction time, with the higher educated performing 1) better when euthymic than the less educated, 2) worse when depressed, and 3) not differently when manic.

A three-way interaction between age, education, and mood state was found in anger and happiness accuracies. When euthymic, education did not significantly impact performance except in the oldest group, where the higher educated performed better. When manic, the less educated were more accurate in the youngest group, higher educated were better in the middle-aged group, and finally education level was not significantly different in the oldest group. When depressed, age and education did not interact to affect performance.

Conclusions: In older adults with BD, age is a significant factor for poorer facial emotion processing. Education appears to affect emotion perception differently depending if a person is euthymic or depressed. Finally, age, education, and mania interact to impact perception of anger and happiness. This study suggests that treatment of emotion processing difficulties should be targeted based on age and a combination of education and mood state.

Correspondence: Rebecca E. Easter, University of Michigan, 2101 Commonwealth Blvd, Ann Arbor, MI 48105, United States. E-mail: reaste4@uic.edu

WITHDRAWN. I.J. KAHRILAS, D. DICKSON, C. LEE \& R. SILTON. Positive Affectivity Distinctly Influences Negative Interpretation Bias in Individuals with Remitted Depression.

Objective: Gognitive models of depression have predominantly studied the role of negative affectivity (NA; e.g., depressed mood) on eognitive processes that contribute to the development and maintenance of tepressive symptoms. Howerer, tow positive affectivity (PA) may bea signiffieant risk factor for reocenrrence of depressive episfdes for individuals with a history of depression. The present sfund luated the Hypothesis that fow PA is associated with inte s5 Hreogtive interpreeompared to those without a history tepressiotr.

Participants and Methods: Pfity o fo-students (36 females), ages

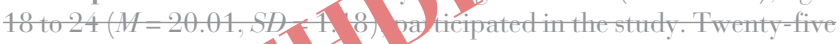
partieipants met y. Hia or Tast major depressive episode (MDE, i.e., remitted fer esst t), atrd 25 participants did not meet eriteria for a current o part WE. Affectivity was assessed using the Hoot and Anxicty of Pum Questiommaire. Psychiatrie history was obtained via the MINI diagnostic elinieal interview. Interpretation bias mnder cognitive load and no-load conditions were assessed experimentally using the Serambled Sentenec Test.

Results: Regression analyses showed that low PA significantly predicted inereased negative interpretation bias under high eognitive load $(p<.05)$, even after aceotmting for the effeets of $\mathrm{NA}$ and anxiety. This effect was signifieantly larger for individuals with a lifetime history of depression, compared to those who had never been depressed $(p<.05)$. Regardless of depression history, $\mathrm{NA}$ did not signifieantly predict interpretation bias after aceounting for PA and anxiety.

Gonelusions: Low PA may serve as a minique risk factor for megative interpretation brias, partientarly for individuals with a history of eperession. Futtre basie and translational researeh should eonsider the distinet role of low PA on dysfunetional eognitive processes that contribute to tepressiont.

Gorrespondence: Ian J. Kahritas, Psychology, Loyola University Chicago, 293 Columbia Aventue, Park Ridge, It 60068, United States. E-mait.HKG996s@gmait.com

M. OLARU, C. WESTBROOK \& R.J. DAVIDSON. The Relationship of Self-Esteem and Cognitive Styles in the Development of Depression.

Objective: An fMRI study was used to measure the neural and cognitive mechanisms involved in the development of depression in relation to self-esteem utilizing a diathesis-stress model. Self-esteem is a factor which may serve as a moderator for the formation of depression. Although prior research has shown that self-esteem can buffer the risk conferred by a maladaptive cognitive style, this has not yet been assessed in a neuroimaging context. The unique variance of each factor within the cognitive mechanism was also measured for comparison.

Participants and Methods: The participants were undergraduate students enrolled in the Introductory Psychology course at the University of Wisconsin Madison. They were recruited in two groups, highrisk versus low-risk of developing depression, as determined by their scores on the Cognitive Styles Questionnaire. Self-esteem scores were measured with the Self Esteem Questionnaire. Neural activity was assessed following the acute stress of a midterm exam using fMRI and a working-memory task. For the working-memory task, participants were (1) shown either a control, negative, or exam-related set of words, (2) given a rehearsal period to memorize the words in a specific sequence, and then (3) instructed to recall a specific word within the sequence.

Results: Self-esteem and cognitive style factors self-worth and stability independently predicted differences in brain activity. For self-esteem, there was bilateral activation of the precentral gyrus when the exam-related words were first shown. For the cognitive style subscales, selfworth and stability had activation of the upper and lower precuneus cortex respectively during the rehearsal period of the task.

Conclusions: Self esteem may the initial factor implicated in response to a trigger of a stressful life event. Furthermore, the relationship of Self Esteem seems to be independent of Cognitive Styles, raising more questions about the potential interactions of these two factors.

Correspondence: Maria Olaru, Neurobiology, Neuroscience, University of Wisconsin-Madison, 1090 South Van Ness Ave., San Francisco, CA 94110, United States. E-mail: molaru95@gmail.com

Z. TAIWO, G. MIRABITO, M. BEZDEK \& S.N. LIGHT. Individual Differences in Working Memory Associated with Empathy-Related Frontal Lobe BOLD Activity.

Objective: Empathy deficits are common across clinical populations. Neuroscience models of empathy propose the role of executive function in empathic processing, however, the normative relationship between executive function and empathy is unclear. This study relates executive function and frontal correlates of empathic happiness and empathic concern (vicarious positive \& negative emotion, respectively).

Participants and Methods: 20 healthy adults (21.7 years, $63 \%$ female) completed neuropsychological testing assessing inhibition, working memory \& cognitive flexibility; and an fMRI-based empathy paradigm consisting of empathic happiness, empathic concern and neutral emotion eliciting video clips. Whole brain analyses were conducted to examine overlapping frontal brain activity across emotions. Linear 
regressions related executive function to frontal brain activity during the empathy paradigm.

Results: fMRI analyses (cluster-corrected $p<0.001$ ) revealed significant clusters of activity in the middle frontal gyrus (MFG, BA 10) during empathic happiness and concern eliciting video clips. Better working memory was associated with greater activity in the MFG (BA 10) during empathic concern $\left(R^{2}=.25, \beta=.50, p=0.03\right.$, BCa $\left.95 \% \mathrm{CI}=.02-.15\right)$ and empathic happiness $\left(R^{2}=.29, \beta=.54, p=0.09\right.$, BCa 95\% CI= .01-.15). Inhibition and cognitive flexibility were unrelated to task-based frontal activity

Conclusions: Findings show that in a normative sample working memory relates to empathic processing. Working memory may play a role in the regulation and control of other-oriented emotion regulation and control. Further investigation in clinical samples is warranted to establish whether deficits in working memory explain maladaptive empathic responding across clinical populations.

Correspondence: Zinat Taiwo, Georgia State University, Department of Psychology, 140 Decatur Street, Atlanta, GA 30303, United States. E-mail:ztaiwo1@student.gsu.edu

\section{Forensic Neuropsychology}

C. BIDDLE, K. YAMOUT \& R. FAZIO. Exploring the California Verbal Learning Test-2 ${ }^{\text {nd }}$ Edition Short Form Forced Choice Scores as a Potential Measure of Performance Validity Among Older Adults Referred for Memory Complaints.

Objective: To explore the potential usefulness of the California Verbal Learning Test- $2{ }^{\text {nd }}$ Edition Short Form's Forced Choice Trial as a Performance Validity Test (PVT) with an older adult sample.

Participants and Methods: 240 examinees age $60+$ referred for outpatient memory evaluation. Inclusion criteria were that the individual was diagnosed with Major Neurocognitive Disorder (MND), Mild Neurocognitive Disorder (Mild Cognitive Impairment; MCI) per DSM-5 diagnostic criteria (APA, 2013) or No Cognitive Impairment Patients with potential medicolegal issues were excluded. The full CVLT-2 SF was administered as part of a clinical neuropsychological assessment.

Descriptive statistics were calculated for the sample. Distributions of CVLT-2 SF FC scores and false-positive classification rates were completed in addition to examining demographic and clinical variables that may have contributed to the classification outcome.

Results: Most (87.6\%) examinees obtained perfect (9/9) scores on Forced Choice Test (FCT). We calculated false positive rates for different cut-off scores on the FCT and found that setting $6 / 9$ as a cut-off criterion for "poor validity" would miss-classify ("false positive error") none of the Normal Cognition group, only $1(0.8 \%)$ of the MCI group, and only $3(4.5 \%)$ of the MND group. All these false positive rates fall below the $10 \%$ false positive rate that is typically recommended for Performance Validity Tests (PVT's).

Conclusions: At a cutoff criterion of 3 errors on the FCT (i.e., score $\leq 6$ out of 9 ), the false positive rate for miss-classification of poor validity was below $10 \%$ even for examinees with MND. This FCT therefore carries significant potential to be considered as a Performance Validity Test for individuals being evaluated for dementia.

Correspondence: Christine Biddle, CPC, $810312 T H$ AVE NW, Bradenton,FL 34209, United States.E-mail: cbiddle@carterpsych.com

O. GAASEDELEN, S.K. HAMILTON, B. ARDOLF, J. WESTHAFER \& A. ZARTMAN. Utility of the Symptom Validity Scale and Response Bias Scale in Predicting PVT Failure in a Veteran Sample. Objective: The Symptom Validity Scale (FBS-r) and Response Bias Scale (RBS) are commonly used MMPI-2-RF symptom validity tests; however, research examining their validity in predicting performance validity test (PVT) scores in veteran samples remains limited. The current study presents validation evidence for the use of FBS-r and RBS to predict PVT failure in a veteran sample. The study also examined potential differential predictive variables, including age, gender, education, and race.

Participants and Methods: Participants consisted of 493 consecutive neuropsychological referrals at a VA hospital (Mean age $=47.7$, $\mathrm{SD}=12.8$; Mean education $=13.5, \mathrm{SD}=2.4 ; 16.8 \%$ Female; $9.7 \%$ Hispanic; $21.2 \%$ African American) who completed at least one freestanding PVT and the MMPI-2-RF. A logistic regression model was fit where RBS and FBS-r simultaneously predicted PVT failure, where PVT failure was defined as scoring below established cut-offs on the Test of Memory Malingering and the Word Memory Test. Additionally, linear models were fit to examine any effects of differential prediction for RBS and FBS-r across age, education, gender, and race.

Results: Results from the simultaneous logistic model indicated that RBS significantly predicted PVT failure $(\beta=0.04, p<.001)$, while FBS-r did not $(\beta=.004 ; p=.63)$. Women tended to score significantly higher on the FBS-r $(\beta=4.1, p=.03)$, while no significant differences in gender were observed for the RBS $(\beta=1.5, p=.56)$. Higher levels of education were significantly associated with lower RBS scores $(\beta=-2.1, p=.02)$, but not FBS-r scores $(\beta=-1.0, p=.11)$. Additionally, no significant effects of differential prediction were found for age or race. Conclusions: Findings suggest RBS is a superior predictor of PVT failure compared to FBS-r. Clinicians are also encouraged to consider the effects of gender and education in their interpretations of these scales to avoid potentially misleading conclusions.

Correspondence: Owen Gaasedelen, University of Iowa, 312 Samoa Ct, Iowa City, IA 52246, United States. E-mail: owen-gaasedelen@ uiowa.edu

D. GUZMAN, M.R. BASSO, L. LAU, D. WHITESIDE \& D. COMBS. Prevalence and Prediction of PVT Failure in a Research Sample of MS Patients.

Objective: In clinical settings significant numbers of examinees exaggerate neuropsychological dysfunction (Mittenberg et al., 2002). Little research exists concerning the prevalence of exaggeration in research settings or in people with multiple sclerosis (MS). Bigler (2014) stated that performance validity test (PVT) failure is more frequent when symptoms such as fatigue and pain are prevalent, and these symptoms occur often in MS. The current study investigated the prevalence of invalid performance among people with MS in a research context, and the impact of fatigue, depression, and pain upon PVTs.

Participants and Methods: 98 MS patients MS and 49 controls were recruited as part of an $\mathrm{NIH}$-funded study concerning neuropsychological function. They were notified that their test results would be de-identified and embargoed from clinical records or providers. All completed a neuropsychological battery and measures of symptom severity, and the Word Memory Test (WMT), CVLT-II Forced Choice, and Reliable Digit Span (RDS) served as PVTs.

Results: Patients were classified as impaired or unimpaired based on the neuropsychological battery (30 impaired and 68 unimpaired). Failure of two or more PVTs was used to classify non-credible performance. Although 13 patients failed the WMT, only nine failed two or more PVTs, whereas one control subject failed two or more PVTs. PVTs failed to correlate with depression, fatigue, pain, or motor impairment. In a logistic regression, no variable predicted PVT failure status.

Conclusions: Using a conservative criterion, few MS patients or control subjects in a research setting failed two or more PVTs, but approximately $13.2 \%$ of MS patients and $4 \%$ of control subjects failed the WMT. Non-credible effort is a slight but viable threat to internal validity of MS research. Moreover, PVT performance is not associated with MS-related symptoms of depression, fatigue, pain, or motor impairment. Thus, PVT failure likely reflects poor effort uniquely.

Correspondence: Daniel Guzman, PhD, The University of Tulsa, 800 South Tucker Drive, Tulsa, OK 74104, United States.E-mail:dag773@ utulsa.edu 
K.M. KLIPFEL, M.M. STIKA \& D.S. GOLDSTEIN. The Relationship Between IQ and Effort Among Worker's Compensation and Fitness-for-Duty Examinees: A Replication of Findings in Criminal Defendants.

Objective: Our clinic previously examined the relationship between IQ and effort among criminal defendants (Matthews et al., 2013), revealing WAIS-III FSIQs are unreliable when effort is in question. The current investigation replicated these analyses (examining IQ and its relationship to performance on commonly used stand-alone and embedded effort measures) in samples of worker's compensation claimants and fitness-for-duty examinees, whose base rates of suboptimal effort vary significantly.

Participants and Methods: A large sample of consecutively referred worker's compensation claimants and fitness-for-duty examinees underwent comprehensive effort and neurocognitive assessment. Based on Slick et al. (1999), three effort groups were established, reflecting base rates of malingering, suboptimal effort and good effort. In addition to obtaining a performance-based predicted WAIS-III FSIQ using the traditional combination of WTAR reading score and demographics (p-FSIQ), a demographics-only predicted WAIS-III FSIQ (d-FSIQ) was calculated using race, gender, age and education in order to provide an IQ estimate free from contamination by suboptimal effort. Analyses included 1) correlations between actual FSIQ/p-FSIQ/d-FSIQ and individual effort measures; 2) correlations between actual FSIQ/p-FSIQ/dFSIQ and effort group; and 3) ANOVA comparing actual FSIQ, p-FSIQ and d-FSIQ across effort group.

Results: The manner in which effort impacts actual vs. proxy measures of IQ across samples with varying base rates of suboptimal effort is discussed.

Conclusions: Results contribute to the expanding literature on the relationship between effort and intellectual functioning, and call attention to the effects of performance contamination. Prior investigations concluding low IQ contributes to poor performance on effort measures are called into question. Future directions include determining at what IQ level intellectual disability may begin to meaningfully impact performance on effort measures, and replication with WAIS-IV/TOPF measures.

Correspondence: Kristen M. Klipfel, M.S., Psychology, Rosalind Franklin University of Medicine and Science, 2144 E. Republic Rd., Apt. A203, Springfield, MO 65804, United States.E-mail: kmklipfel@gmail.com

K.M. KLIPFEL, K.P. NITSCH \& D.S. GOLDSTEIN. CrossValidation of TOMMe10 Among Criminal Defendants and Compensation-Seekers.

Objective: The current investigation aimed to expand on Denning's (2012) finding of improved accuracy of the Test of Memory Malingering (TOMM) in predicting suboptimal effort using errors within the first 10 items of Trial 1 (TOMMe10). In addition to extending findings from a veteran sample to criminal defendants and compensation-seeking claimants, we used traditional Slick et al. (1999) criteria to determine effort groups, aiming to cross-validate Denning's findings in effort groups classified solely by invalid performance on the Medical Symptom Validity Test. Finally, we aimed to demonstrate typical TOMMe10 error patterns among controls.

Participants and Methods: Samples of consecutively referred criminal defendants and compensation-seeking claimants (worker's compensation, pension disability and personal injury claimants) underwent comprehensive effort and neurocognitive assessment. Consecutively referred fitness-for-duty and public safety pre-hire psychological screening examinees similarly underwent comprehensive assessment and comprised two natural control groups, as effort was deemed optimal in all cases. Examinees were classified into groups reflecting Optimal Effort vs. Suboptimal Effort (combined definite malingering and other suboptimal effort) based on Slick et al. Optimal cutoff scores for TOMMe10. TOMM Trial 1 and TOMM Trial 2 were determined using receiver operating characteristic (ROC) analyses, maintaining specificity at $\geq .90$.
Results: The relative sensitivity and specificity of optimal cutoff scores for TOMMe10, TOMM 1 and TOMM 2 is provided for criminal and compensation-seeking samples, and TOMMe10 patterns in these two groups are illustrated relative to controls. The improved accuracy of TOMMe10 over traditional TOMM 1 and 2 cutoff scores is discussed.

Conclusions: Results contribute to the identification of more efficient and accurate methods of assessing effort using the TOMM, as well as to an embedded effort measure that may be less vulnerable to manipulation.

Correspondence: Kristen M. Klipfel, M.S., Psychology, Rosalind Franklin University of Medicine and Science, 2144 E. Republic Rd., Apt. A203, Springfield, MO 65804, United States. E-mail:kmklipfel@gmail.com

\section{LAU, M.R. BASSO, R. MULLIGAN, D. WHITESIDE \& D. COMBS. Accuracy of TOMM Supplementary Indices Among Test Coached Simulators. \\ Objective:}

Sensitivity and specificity of the TOMM and WMT in detecting non-credible effort are relatively well established. To elaborate upon its utility, investigators have generated indices from the TOMM which examine the errors on the first 10 items of Trial 1 (TOMMe10) and the consistency of recall across its three recall trials, namely the Albany Consistency Index (ACI) and Invalid Forgetting Frequency Index (IFFI). The TOMMe10 and consistency indices seem to possess acceptable classification accuracy, with some indications that the latter manifest better sensitivity than the TOMM recall trials (Buddin et al., 2014). Yet, little research has compared their classification accuracy to TOMM and WMT recall scores. In this experiment, neurologically normal participants were coached to feign mild TBI symptoms and evade detection by performance validity tests.

Participants and Methods: Participants were neurologically-normal university students who were assigned to one of three simulation groups (each $n=20$ ). The groups received either superficial information about symptoms of head injury, comprehensive information concerning symptoms of head injury, or comprehensive symptom information and strategies to evade detection by PVTs. A control group $(n=20)$ was instructed to provide their optimal effort. The TOMM and WMT were administered.

Results: The TOMM ACI and IFFI indices achieved the best classification accuracy, with $91-95 \%$ of simulators being identified. The TOMM and WMT Recall Tests successfully identified $78-88 \%$ and $83-93 \%$ of simulators, respectively. The TOMMe10 yielded the least effective detection $(66 \%)$. With increasing levels of provided information, the sensitivity of the measures greatly diminished.

Conclusions: Compared to the TOMMe10 and TOMM and WMT recall scores, The TOMM consistency indices possessed the greatest utility in classifying examinees simulating symptoms of head injury. Such findings should provide clinicians with confidence to employ these measures when evaluating performance validity.

Correspondence: Lily Lau, PhD, Psychology, University of Tulsa, 226 S Richmond Ave., Tulsa, OK 74112, United States. E-mail: lily-lau@ utulsa.edu

R. MULLIGAN, M.R. BASSO, L. LAU, D. WHITESIDE \& D. COMBS. Classification Accuracy of the Word Memory Test Genuine Memory Impairment Index.

Objective: The Word Memory Test (WMT; Green, 2003) assesses non-credible effort. To mitigate risk of false positives, Green (2003) derived the Genuine Memory Impairment Profile (GMIP), and asserted that severely impaired people are most likely to manifest this profile. The present study evaluated the classification accuracy of the GMIP in a group of healthy individuals coached to simulate mild traumatic brain injury (mTBI) related memory impairment on the WMT.

Participants and Methods: Eighty healthy individuals participated. One group was provided superficial information concerning mTBI symptoms (naïve simulators), another was provided extensive information 
concerning symptoms (sophisticated simulators), and a third group was provided extensive symptom information and tactics to evade detection by PVTs (test-coached). An honest responding group was directed to give their best performance. All participants were given a neuropsychological battery that included the WMT.

Results: GMIP profiles were calculated according to Green (2003). All of the control group was correctly identified as having normal memory. Within the test coached group. 90\% exceeded WMT cutoffs, but only $5 \%$ manifested the GMIP profile. Among the sophisticated and naive groups $95 \%$ and $100 \%$ exceeded WMT cutoffs for invalid performance, respectively. Of these, $25 \%$ and $60 \%$ manifested the GMIP profile, respectively.

Conclusions: Nearly all of the simulators were accurately classified as exerting non-credible effort, and test-coaching diminished sensitivity only modestly. Nonetheless, occurrence of the GMIP profile was markedly different between groups. The test-coached group was least likely to show the GMIP profile (5\%), whereas the naïve simulators were most likely to display the GMIP $(60 \%)$. This implies that the GMIP is apt to falsely identify individuals as having genuine memory impairment, especially if a naïve or unsophisticated effort to exert poor effort is made. Use of the GMIP should be scrutinized carefully and used cautiously. Correspondence: Ryan Mulligan, Dept. of Psych/University of Tulsa, 800 South Tucker Drive, Tulsa, OK 74105, United States. E-mail: Ryanmulligan@utulsa.edu

\section{L.E. PIPER, D. WHITESIDE, K.J. CARAHER \& M.R. BASSO. Development of a Combined Visual Spatial Performance Validity Test Using Logistic Regression.}

Objective: Although research on embedded performance validity tests (PVTs) has increased in recent years, little research has been conducted on embedded PVTs in visual spatial measures. One recent study (Whiteside et al., 2011) examined the Judgment of Line Orientation (JLO), Benton Facial Recognition Test (FRT), Hooper Visual Organization Test (VOT), and Rey Complex Figure Test Copy (RCFT-C) and Recognition (RVFT-R) as possible embedded PVTs. In addition to the individual measures, Whiteside et al. evaluated the classification accuracy of a PVT created by adding the scores of the individual measures together (called the Visual Spatial Measure (VSM)). This variable had better overall classification accuracy and sensitivity than the individual measures. More recently, logistic regression has used to combine individual embedded PVTs, with evidence for improved classification accuracy. This study utilized the original Whiteside et al (2011) data to develop an embedded PVT derived from a logistic regression of the original study variables (L-VSM).

Participants and Methods: Methods were the same as in the original study, with 421 consecutive neuropsychological patients who completed the study measures. The participants were divided into PVT-Pass ( $\mathrm{N}=415)$ and PVT-Fail ( $\mathrm{N}=30)$ groups based on TOMM and/or California Verbal Learning Test-II Forced Choice (CVLT-FC) failure.

Results: Results indicated that the logistically derived PVT had a slightly higher overall AUC (.83 for L-VSM compared to .81 for the VSM). Further, L-VSM had a higher sensitivity (47\% compared to $40 \%$ for the VSM) when the specificity of the cut-score was $90 \%$. Both combined variables had significantly higher sensitivity than the individual measures reported in the Whiteside et al. paper.

Conclusions: The results support the use of this logistically derived PVT, and the logistic regression methodology provides improved sensitivity over arithmetically combined PVTs. which in turn supports for this combined PVT.

Correspondence: Lauren E. Piper, IL, United States.E-mail: lpiper129@ gmail.com

V.M. REGINELLA \& J.D. PETRICK. Relationship of Trauma \& Depression in Litigants \& Non-Litigants Following Concussion.

Objective: The objective of this study was to examine the relationship between depression and severity of neurological injury in people in litigation and non-litigation. The recovery following mild traumatic brain injury (mTBI) or concussion and the neuropsychological sequella is controversial, especially when there is pending litigation.

Participants and Methods: 34 participants were divided into 2 groups, "litigation" ( $\mathrm{n}=17)$ and "non-litigation" ( $\mathrm{n}=17)$. All participants presented with a history of mTBI or Post-Concussion Syndrome (PCS). Males and females in the age range of 17-66 years of age with years of education ranging from 8.9 to 16.9 were represented in this sample. Injuries were due to falls, assaults, motor vehicle accidents (MVA), or sports-related activities.

Results: Analyses were performed post-hoc using $t$-tests with an alpha level of 0.05. Variables included the index scores from the Wechsler Adult Intelligence Scale-IV (WAIS-IV), Beck Depression Inventory-II (BDI-II), California Verbal Learning Test-II (CVLT-II), and the Controlled Oral Word Association Test (COWAT). A significant difference was found on the COWAT, but no differences were found for the other measures.

Conclusions: The findings in this study contradict much of what the literature in forensic neuropsychology currently maintains, particularly in cases involving mTBI, suggesting that there are differences in neuropsychological assessment performance between individuals in litigation and not in litigation (Lees-Haley \& Brown, 1993; Millis, 1994; Reynolds, 1998; Moore \& Donders, 2004; Green et al., 2009; Mittenberg et al., 2010). Reasons for this finding may be due to small sample sizes, etiology of depression (i.e. organic vs. acquired), and demographic criteria. Further research should emphasize expanding on the dimensions of the current study, in respect to sample size and number and nature of neuropsychological measures utilized.

Correspondence: Vincent M. Reginella, M.S. , Neuropsychology, James D. Petrick, PhD. Neuropsychological Consultation \& Rehabilitation, 606 Golden Oaks Lane, Pittsburgh, PA 15237, United States. E-mail: vmr5492@gmail.com

S. ROLIN, A. WILLIAMS, S. SANTISTEVAN-ANDREWS \& J. KING. Examination of the Classification Accuracy of the Word Choice Test with Stand Alone Performance Validity Measures.

Objective: Examination of concordance and accuracy of the Word Choice test (WC) from the Advanced Clinical Solutions (ACS; Wechsler Adult Intelligence Scale-Fourth Edition) with the Word Memory Test (WMT), Medical Symptom Validity Test (MSVT) and Nonverbal Medical Symptom Validity Test (NV-MSVT) in distinguishing validity groups in a mixed clinical population.

Participants and Methods: The sample ( $\mathrm{N}=63)$ was $46 \%$ female and $54 \%$ Caucasian with average age and education of $42.5(\mathrm{SD}=14.2)$ and $13.6(\mathrm{SD}=2.9)$ years. Participants underwent outpatient neuropsychological evaluation for TBI (62\%), ADHD (19\%), neurologic $(16 \%)$ and psychiatric conditions (3\%). Participants completed a flexible battery of neuropsychological tests with WC, WMT, MSVT, NV-MSVT, and embedded PVTs.

Results: Participants completed an average of $5(\mathrm{SD}=0.7$, range 3-6) embedded PVTs; $16 \%$ of the sample failed two or more embedded PVTs. Criterion PVTs failure rates were as follows: WMT (47\%), MSVT $(27 \%)$, and NV-MSVT $(24 \%)$. Thirty-five percent of the sample failed one or more criterion PVTs. Logistic regression models were fitted using dichotomous criterion PVTs the outcome and embedded PVTs and WC as predictors. A model using embedded PVTs was preferred over a constant-only model, $\chi^{2}(1)=8.3, p=.004$, showed overall classification of $75 \%$ and area under the receiver operating characteristic curve (AUC) of .68. A second model WC and embedded PVTs was also preferred over a constant-only model, $\chi^{2}(2)=15.2, p<.001$, showed overall classification of $73 \%$ and AUC of .83. Using dichotomous pass/ fail cutoffs on measures of interest, agreement between WC and WMT was $63 \%$, Kappa $=.24, \mathrm{p}=.03$; agreement between $\mathrm{WC}$ and MSVT was $84 \%$, Kappa $=.59, \mathrm{p}<.001 ;$ agreement between $\mathrm{WC}$ and $\mathrm{NV}$-MSVT was $84 \%$, Kappa $=.56, p=.003$. 
Conclusions: WC demonstrated incremental validity over embedded PVTs in predicting outcomes on criterion measures. WC showed greater agreement with MSVT and NV-MSVT than WMT.

Correspondence: Summer Rolin, Psy.D., Physical Medicine \& Rehabilitation, University of Utah, 1155 East 2100 South, 832, Salt Lake City, UT 84106, United States. E-mail: sumrolin@gmail.com

M.M. STIKA, K.M. KLIPFEL \& D.S. GOLDSTEIN. The Relationship Between IQ and Effort in Worker's Compensation and Fitness-forDuty Examinees: Replication of WTAR-WAIS-III Findings with the TOPF and WAIS-IV.

Objective: We previously demonstrated that WAIS-III FSIQs are significantly correlated with performance on effort measures among criminal defendants, and are therefore unreliable when effort is suboptimal (Matthews et al., 2013). In contrast, this same investigation revealed that WTAR-derived IQ proxy measures free from performance-based input (i.e., those using demographic variables alone) are not significantly correlated with effort measure performance, and do not differ significantly from WAIS-III FSIQs when effort is optimal. We have since replicated these findings in samples of worker's compensation and fitness-for-duty examinees, calling into question and highlighting the circularity of conclusions from prior investigations that low-IQ examinees fail effort tests when such investigations failed to control for effort itself. The current investigation aimed to replicate these findings using WAIS-IV and TOPF-derived IQ proxy measures.

Participants and Methods: A sample of consecutively referred worker's compensation claimants and fitness-for-duty examinees underwent comprehensive effort and neurocognitive assessment. Based on Slick et al. (1999), three effort groups were established, reflecting base rates of malingering, suboptimal effort and good effort. In addition to obtaining a performance-based predicted WAIS-IV FSIQ using the TOPF reading score (p-FSIQ), a demographics-only predicted FSIQ (d-FSIQ) was calculated to provide an IQ estimate free from contamination by suboptimal effort. Analyses included 1) correlations between actual FSIQ/p-FSIQ/d-FSIQ and all effort measures; 2 ) correlations between actual FSIQ/p-FSIQ/d-FSIQ and effort group; and 3) ANOVA comparing actual FSIQ, p-FSIQ and d-FSIQ by effort group.

Results: Comparisons between WAIS-IV/TOPF and WAIS-III/WTAR data are discussed, as are failure rates on effort measures by effort group, p-FSIQ and d-FSIQ.

Conclusions: Results demonstrate the impact of performance contamination on IQ proxy measures and elucidate the relationship between effort and IQ.

Correspondence: Monica M. Stika, PhD, Edward Hines, Jr. VA Hospital, 5000 S. 5th Avenue, Building 228, Room 3012, Hines, IL 60141, United States.E-mail:monica.stika@gmail.com

\section{C.M. VARGA, V. WATOREK, S. MING \& C.P. PECK. Examining the Utility of the RBANS Hartford Consistency Index in an Adult Outpatient Sample.}

Objective: Varga et al. (2017) recently presented a study on the initial validation of the Hartford Consistency Index (HCI), a newly developed embedded effort measure for the Repeatable Battery for the Assessment of Neuropsychological Status (RBANS). In that study, an HCI cutoff of $>6$ resulted in $88 \%$ accurate classification of dementia referrals as putting forth sufficient effort, while $37 \%$ of the invalid sample were also accurately identified. In that particular sample, the HCI outperformed the RBANS Effort Index (EI). The purpose of the current study was to examine the utility of the HCI in an adult outpatient sample.

Participants and Methods: For this study, we used Varga et al.'s Invalid Profiles (IP) group, a sample consisting of individuals $(n=19)$ under the age of 65 who produced a failing score on the Test of Memory Malingering or Word Memory Test. Our Valid Profiles (VP) group consisted of non-demented adults $(n=40)$ under the age of 66 who passed all validity measures administered. There were significantly more males in the IP group $(p=.04)$; however, no statistically significant group differences emerged on demographic variables of age $(p=.42)$ or education $(p=.59)$

Results: Using an HCI cutoff of $>6$ correctly classified $90 \%$ of the VP group, and accurately identified $37 \%$ of the IP group. In comparison, the EI cutoff of $>3$ correctly classified $93 \%$ of our VP group, but only accurately identified $26 \%$ of the IP group.

Conclusions: In this adult sample, the HCI provided more robust classification rates than the $>3$ cutoff of the RBANS EI. As such, the HCI may also be useful for detecting poor effort in a younger sample.

Correspondence: Claire M. Varga, IL, United States. E-mail: clairevarga@gmail.com

E. VOGT, S. PARDEJ, A. HEFFELFINGER \& J. KOOP. CPT 3 Promising as an Embedded PVT for Pediatrics.

Objective: This project aimed to evaluate the effectiveness of the Conner's Continuous Performance Test - Third Edition (CPT 3) scales as embedded performance validity tests (PVTs) with pediatrics. Previous adult literature has identified CPT-II error rate and reaction time scores as beneficial for validity indication.

Participants and Methods: Retrospective chart review of children and adolescent $\left[N=125, M_{\text {age }}=13.06(2.55)\right.$, range 7.10 to 16.12$]$ neuropsychological evaluations was conducted. Children and adolescents with complete Victoria Symptom Validity Test (VSVT), CPT 3, and Reliable Digit Span (RDS) scores were considered for inclusion $\left[N=96, M_{\text {age }}=13.37(2.39)\right.$, range 8.11 to 16.12$]$. The Likely Invalid group $\left[n=7, M_{\text {age }}=12.21(2.89)\right.$, range 9.04 to 16.09$]$ included those with performance below cut-offs on both VSVT and RDS. Whereas, children and adolescents with passing VSVT and RDS scores comprised the Likely Valid group $\left[n=67, M_{\text {age }}=13.87\right.$ (2.21), range 8.11 to 16.12]. Those with scores below cut-offs on either VSVT or RDS were excluded $(n=22)$.

Results: Receiver operator curve (ROC) analyses revealed that multiple CPT 3 scales displayed acceptable discrimination as indicated by area under the curve (AUC) values (Detectability .79, Omissions .70, Perseverations .76, HRT SD .72, and Variability .76). Sensitivity, specificity, and likelihood ratios were generated at multiple cut-off points for each scale. Maximization of specificity generally resulted in low sensitivity. Conclusions: CPT 3 error rate and reaction time statistics were generally consistent with previous adult literature. Detectability (d'), a signal detection scale, could be promising as a novel PVT. Due to low sensitivity, embedded CPT 3 scales may be useful as additional validity indicators in conjunction with stand-alone measures. Additional investigation with healthy controls, simulators, and other clinical groups is necessary to ensure differentiation of cognition and performance validity.

Correspondence: Elisabeth Vogt, MS, Psychology, Marquette University, 2928 N 84th Street, Milwaukee, WI 53222, United States. E-mail: elisabeth.vogt@marquette.edu

\section{Inclusion and Diversity/Multiculturalism}

L. DAY, E.B. ADAMS COSTA \& T. BRIDGETT. A Special Group Study of the Wechsler Intelligence Scale for Children, Fifth Edition: Deaf Children Who Utilize American Sign Language.

Objective: Some of the limitations of data on cognitive functioning in deaf children are that sample sizes are not well described and likely to be widely heterogeneous with regard to key variables. The present study collected data on deaf children's performance on the Wechsler Intelligence Scale for Children, Fifth Edition (WISC-V) ensuring accessible test administration and controlling for variables known to lead to variability in outcomes.

Participants and Methods: The sample consisted of deaf children who utilize American Sign Language (ASL) as their primary communication modality and do not have additional clinical diagnoses. A matched control sample was drawn from the WISC-V standardization sample. 
Both samples were administered the WISC-V; the deaf sample was administered the WISC-V in ASL.

Results: The majority of the deaf sample consisted of children whose home and school language was ASL and who were identified as deaf by two months of age. The mean composite scores for the Verbal Comprehension Index (VCI) and Full Scale IQ (FSIQ) were significantly different between the deaf and control groups. There were no significant differences on the other primary index scores or the Nonverbal Index (NVI). The VCI differences showed large effect sizes, and the Working Memory Index and FSIQ differences showed moderate effect sizes. Among the primary subtests, the deaf group means on Vocabulary and Digit Span were significantly lower than the matched control group and showed large effects.

Conclusions: The deaf sample in this study was largely a group of children who benefited from early intervention and early language access; and the results highlight important aspects of this group's cognitive functioning. The benefits of a well-defined sample can help to clarify the impact that other variables have on cognitive functioning, such as language access and co-morbid conditions, and suggests that factors beyond hearing loss itself likely contribute to variability in outcomes. Correspondence: Lori Day, PhD, Psychology, Gallaudet University, 800 Florida Ave, NE, HMB S422, Washington, DC 20002, United States. E-mail:lori.day@gallaudet.edu

M. FORTE, A. WOODHULL, K. O'DONOVAN \& P. NESTOR. Crosscultural Analysis of the WAIS-IV Advanced Clinical Solutions (ACS): Cultural Identity and Performance on Social Perception Subscales.

Objective: The goal of the current study was to examine the Social Perception subtest of the Advanced Clinical Solutions for WAIS-IV (ACS; Pearson, 2009) and to preliminarily explore the cultural sensitivity of each component of the subtest by looking at ethnic identity as a potential moderator of performance on different subscales of the task. Participants and Methods: A $2 \times 3$ ANOVA with one between-subjects factor of ethnic group (Asian/White) and one within-subject factor of sub-test (Affect Naming, Prosody Face Matching, Prosody-Pairs Matching) was run. The study's sample included 131 college students who self-identified as ethnically White $(n=75)$ or Asian $(n=56)$.

Results: Analyses revealed a significant main effect for ethnicity, $[\mathrm{F}(1,126)=16.08, p<.01, \eta 2 p=.96]$, with White participants $(M=10.35, S D=2.75)$ outperforming Asian participants $(M=8.92$, $S D=2.80), t(126)=-2.87, p<.01, d=.52$. There was a significant main effect for sub-tests $[\mathrm{F}(1,126)=10.27, p<.01, \eta 2 p=.08]$. Participants scored highest on Prosody Matching $(M=10.08, S D=2.61)$ and Prosody Pairs $(M=10.29, S D=2.87)$ and performed significantly lower on Affect Naming $(M=9.18, S D=2.86)$. A two-way interaction for ethnic group by subtest was significant, $[\mathrm{F}(1,126)=5.76, p=.01$, $\eta 2 p=.05]$. Subsequent t-tests showed that White participants $(M=10.82, S D=2.44)$ outperformed Asian participants $(M=9.16$, $\mathrm{SD}=2.53)$ on the subscale of Prosody-Face matching, $t(129)=-3.80$, $p<.01, d=-.67$. Participants who identified as White $(M=11.35$, $S D=2.47$ ) also obtained significantly higher scores on Prosody-Pair matching than those who identified as Asian $(M=8.98, S D=2.87)$, $t(118)=-5.05, p<.01, d=-.89)$.

Conclusions: Our analyses revealed a cultural effect for performance on the two ACS-SP subscales that involved complex emotion recognition and identifying higher order meaning from facial and vocal expression of emotions.

Correspondence: Mayte Forte, B.A. , Neurology, University of Miami Miller School of Medicine, 1120 NW 14th Street, Miami, FL 33136, United States. E-mail: Mayte.Forte001@umb.edu
Z. MELIKYAN, H. KUWABARA, S. RUBINO, A. MEJIA, I. NAGORSKAYA \& A.E. PUENTE. Digit Span Performance in Russian and American Rural Populations: Cross-Cultural and Cross-Linguistic Considerations.

Objective: To date very little cross-cultural neuropsychology research has been done comparing populations of Russia and the United States. We compare performance of healthy rural adults from Russia and the United States on the Digit Span Test.

Participants and Methods: Healthy adults who lived their entire lives (except college years, if any) in rural areas (population<50,000, no regular commute to big cities/cultural/ university centers) in Russia (native Russian speakers) and the United States (native English speakers) participated in the study. Russian ( $N=49$; ages $18-76 ; 42 \%$ male), and American ( $N=41$; ages $19-75 ; 39 \%$ male) participants with education $\leq$ Master's level were tested in their native languages.

Digit Span Forward Test (DSFT) and Digit Span Backwards Test (DSBT) (from WAIS-IV) were administered as part of a comprehensive 2-hour battery. Since there are no norms for the Russian DSFT and DSBT, total raw scores were analyzed. t-test was used.

Results: The American group performed significantly ( $t=-3.66$, $p=.0005)$ better than the Russian group on the DSFT. Differences in DSBT did not reach significance, but the American group showed a tendency $(t=-1.65, p=.10)$ to perform better.

Conclusions: Difference in performance between Russian and American groups could be due to several factors. First, in Russian language words to name numbers are longer than in English therefore acoustic processing is longer in Russian. And since DSFT mostly relies on acoustic processing and reproduction, whereas DSBT mostly requires mental transformation and its representation, differences are more significant in the former test. Second, lack of prior exposure to standardized tests (e.g., academic tests, etc.) in Russian, but not in American group, may negatively affect performance on Digit Span standardized test. These linguistic and cultural discrepancies can contribute to differences in test's performance and warrant separate norms for Russian and American groups.

Correspondence: Zara Melikyan, PhD, MSU, Hewitt Hall University of California, Irvine, CA 92697, United States. E-mail: zmelikyan@ gmail.com

A. RATTO, L. ANTHONY, M.D. POWERS, A.D. VERBALIS, S. SEESE, J. SAFER-LICHTENSTEIN \& L. KENWORTHY. Differential Effects of Dual Language Exposure on Language and Nonverbal Problem-Solving in Children with ASD and ADHD.

Objective: Dual language exposure (DLE) has been linked to reduced vocabulary and improved executive function. Little is known about the effect of DLE in autism spectrum disorder (ASD) and Attention-Deficit/ Hyperactivity Disorder (ADHD), in which these skills are vulnerable. Based on findings in neurotypical samples, we hypothesized that DLE would be associated with lower vocabulary and higher visual problem-solving skills in a sample of children with neurodevelopmental disorders.

Participants and Methods: Participants were112 ( $\mathrm{n}=46$ DLE) children with ASD $(n=40)$ or ADHD $(n=72)$ ages 8-11 years, enrolled in Title I schools. All participants were tested with the Wechsler Abbreviated Scales of Intelligence-II (WASI-II) in English and had an FSIQ $>70$. Scores on the Vocabulary and Block Design subtests were analyzed. Income and parent education were significantly higher among single-language exposed children, $t(110)=5.51, p<.0001 ; t(110)=5.81$, $p<.0001$, and those with ASD, $t(110)=2.05, p=.04 ; t(110)=2.59$, $p=.01$.

Results: Two-way ANCOVAs were conducted to examine the effects of DLE and diagnosis on WASI-II scores, with household income and parent education as covariates. There was a significant main effect of DLE on Block Design, $F(1,106)=4.24, p=.04$, with DLE children receiving higher scores; income was also a significant predictor, $F(1,106)=3.91, \mathrm{p}=.05$. For Vocabulary, the main effect of diagnosis, $F(1,106)=3.56, \mathrm{p}=.06$ and the interaction effect, $F(1,106)=3.63, \mathrm{p}=.06$, 
approached significance, such that among children with ADHD only, DLE was associated with lower scores; parent education was also a significant predictor, $F(1,106)=5.18, p=.03$.

Conclusions: After controlling for socioeconomic status, DLE was associated with advantages in visual problem-solving among children with ASD and ADHD, and reduced vocabulary among children with ADHD only. DLE may provide protective benefits for problem-solving skills in ASD and ADHD. The significant language impairments conferred by ASD may attenuate the negative impact of DLE on vocabulary. Correspondence: Allison Ratto, Children's National Medical Center, 15245 Shady Grove Rd., Suite 350, Rockville, MD 20850, United States. E-mail:aratto@childrensnational.org

R.E. RIEGER, N.C. MOSS, P. COULOMBE, S. ERICKSON \& J. LOWE. Baby, Let's Play Together: Maternal Behaviors and Child Development in Spanish and English Speaking Toddlers Born Preterm.

Objective: Children born preterm are at risk for difficulties with cognition, language, and executive functioning. Parental communication and behaviors during play provide a rich context for child development. Cultural differences, including primary language, may help to explain differences in maternal communication among diverse populations. The purpose of this study was to examine English- and Spanish-speaking maternal behaviors during play, child cognitive, language, and executive functioning, and to investigate the association between maternal behaviors and child abilities.

Participants and Methods: 49 preterm ( $<32$ weeks) and 50 term toddlers ( $>36$ weeks) and their mothers were video recorded during play. Maternal behaviors were assessed using the Dyadic Parent-Child Interaction Coding System. Toddler cognitive and language skills were evaluated with the Bayley-III, and executive functioning skills were evaluated with the A-not-B and Snack Delay tasks.

Results: Differential associations were found between maternal behaviors and developmental outcomes based on prematurity, primary language, and child sex. Mothers of preterm toddlers displayed more information and reflective statements than mothers of term toddlers. Spanish-speaking mothers displayed fewer encouraging behaviors, and more structuring, information questions and reflective statements than English-speaking mothers. The association between information questions, reflective statements, and executive functioning differed for boys and girls. Toddlers born preterm scored higher on the language scale when their mothers used more structuring.

Conclusions: Preterm toddlers whose mothers used structured play had better attention, which may improve self-regulation and language. Parent training programs may be helpful for children born preterm: providing strategies for structuring during play and using more reflective and information statements. Programs should be tailored to the sociocultural context of the family.

Correspondence: Rebecca E. Rieger, PhD, Psychology, University of New Mexico, 1510 A Gold Ave SE, Albuquerque, NM 87106, United States. E-mail:rerieger@unm.edu

R. SALAZAR, M. MANIERI, E. WEIZENBAUM, J. NARDI, A. CRONIN-GOLOMB \& M. RICHARDSON. Cross-Cultural Differences in Neuropsychological Performance: A Stereotype Threat Investigation.

Objective: Considerable debate exists around the extent to which stereotype threat impairs neuropsychological performance. The investigation of stereotype threat in neurological samples may be limited by small samples and variability in neuropsychological profiles. The present study assessed the impact of stereotype threat on neuropsychological performance in a large sample of healthy White and Latino/a undergraduate students.

Participants and Methods: Eighty-nine White and 85 Latino/a undergraduates completed eight subtest of the Wechsler Adult Intelligence Scale-Fourth Edition (WAIS-IV). The experimental group (44 W, 43 L) received test instructions highlighting potential cognitive disparities between Latino/a and White students; these instructions were omitted for the control group ( $45 \mathrm{~W}, 42 \mathrm{~L}$ ). The WAIS-IV Full Scale Intelligence Quotient (FSIQ) was computed along with indices of verbal comprehension (VCI), perceptual reasoning (PRI), working memory (WMI), and processing speed (PSI). The State-Trait Anxiety Inventory (STAI) short-form and State-Trait Motivation Scales (STMS) were subjective indices of anxiety and motivation. Prior to the experimental manipulation, measures of academic achievement and word reading (Wide Range Achievement Test 4 [WRAT-4], Wechsler Test of Adult Reading [WTAR]) were administered.

Results: There was a main effect of group (Latino<White) for WAIS-IV FSIQ, VCI, PRI, WMI, WRAT-IV, and WTAR ( $p<.01)$, without a significant main effect of condition or group-by-condition interaction. The STAI and STMS yielded no differences between groups or conditions.

Conclusions: The predicted impact of stereotype threat was not evident on neuropsychological or subjective measures. Instead, Latino/a participants underperformed relative to White participants, suggesting an impact of cultural factors on standard neuropsychological assessments. These cross-cultural differences in a neurologically healthy, high-functioning sample emphasize the importance of culturally sensitive clinical practices.

Correspondence: Robert Salazar, M.A., Boston University, 648 Beacon St, 2nd Fl, Boston, MA 02215, United States.E-mail: rdsalaz@bu.edu

E. WEIZENBAUM, R. SALAZAR, M. MANIERI, A. CRONINGOLOMB \& M. RICHARDSON. Cross-Cultural Differences in Neuropsychological Performance: The Contributions of Acculturation and Academic Self-Identification.

Objective: The conceptualization of neurocognitive function requires consideration of how culture impacts performance on standardized neuropsychological assessments. This study assessed the impact of culture on a sample of healthy undergraduates, with specific focus on how performance across cognitive domains varies, and how acculturation and academic self-identification (identifying with academic achievement) relate to cultural-group differences.

Participants and Methods: We assessed 85 Latino/a and 89 white Non-Latino/a students with the Wechsler Test of Adult Reading (WTAR), Wide Range Achievement Test-IV (WRAT-IV), and indices of the Wechsler Adult Intelligence Scale-IV (WAIS-IV): verbal comprehension (VCI), perceptual reasoning (PRI), working memory (WMI), processing speed (PSI), and Full Scale Intelligence Quotient (FSIQ). All scores were converted to standardized composite scores. Pearson correlations were used to examine the relation between cognition, indices of acculturation (Abbreviated Multidimensional Acculturation Scale [AMAS] and time lived in US), and academic self-identification.

Results: Latino/a participants had poorer scores on WTAR, WRAT-IV reading, and WAIS-IV FSIQ, VCI, WMI, and PRI than Whites $(\mathrm{p}<.01)$. For the Latino/a group, less time lived in US related to poorer WTAR, WRAT-IV, WMI $(p<.01)$, and FSIQ $(p=.03)$; greater self-identified second-language proficiency (AMAS native language subscale) related to poorer WTAR and WRAT-IV $(p<.05)$. The positive correlation between academic self-identification and cognition was significant for Latino participants only: FSIQ $(p=.02)$ and PSI $(p<.001)$.

Conclusions: Healthy Latino/a undergraduates had poorer scores across multiple domains of standard neuropsychological assessments. These scores correlated with word reading, acculturation, and academic self-identification. Future directions should consider the relations between culture, language, and performance on traditional neuropsychological tests that do not consider factors of culture and identity. Correspondence: Emma Weizenbaum, Clinical Psychology PhD, Psychological and Brain Sciences, Boston University, 648 Beacon St, Rm 210, Boston, MA 02215, United States. E-mail: eweizen@bu.edu 
A.E. WERRY \& M. DANIEL. Influence of Demographic Variables on Measures of Attention and Working Memory for Spanish Speaking Hispanic and Matched White Older Adults.

Objective: While the impact of cultural factors on tests of attention and working memory (WM) is documented in literature, there is little data on this influence for older adults of different ethnic groups. Determine the impact of demographic variables on attention and WM in neurologically healthy older adults of different ethnicities.

Participants and Methods: Subjects from the National Alzheimer's Coordinating Center were selected if they were not diagnosed with dementia within 5 years $($ mean $/ \mathrm{SD}$ : age $=74.2 / 6.5$; education $=$ 12.6/3.7). Groups of self-identified White Non-Hispanic $(n=161)$ or Hispanic $(n=161)$ individuals were matched for age, education, and gender. Neuropsychological tests were administered in Spanish for the Hispanic subjects and English for Whites. Dependent variables for a series of multiple regression analyses included: Digit Span Forward Total and Longest Span (DSFT \& DSFL) and Backward Total and Longest Span (DSBT \& DSBL), Trail Making Test A and B, and Digit Symbol (DS). Independent variables were age, years of education, and gender.

Results: Education was a statistically significant predictor for all attention and WM measures in both groups. Education was a statistically stronger predictor for Trail Making Test A and B in the Hispanic group. While age was a significant predictor for more tests in the Hispanic group, it was a statistically stronger predictor in the Hispanic group only for DSFL. Gender was a statistically significant predictor for DS in the White group, and a statistically stronger predictor than for Hispanics. Conclusions: These results indicate that predictive relationships of demographic variables and attention / WM tests are more similar than different for Spanish speaking Hispanic and matched White older adults. Correspondence: Amy E. Werry, Pacific University, 18376 Tall Pines Drive, Fort Worth, TX 75703, United States. E-mail: amywerry@ pacificu.edu

\section{Neuropsychiatry}

J.M. BABIONE, A. OSBORN, L. EVERETT, I. HASSAN, M. TAN, C. SCHROTH, A. KENNEDY, E. PROESCHER, J. GREENSTEIN, D.M. AASE \&. K.L. PHAN. Initial-Trial Learning is Associated with Severity of PTSD Symptoms in OEF/OIF/OND Veterans.

Objective: Individuals with PTSD often show cognitive deficits, with moderate effect sizes in verbal learning, processing speed, and attention/ working memory having been most evident (Scott et al.. 2015). While learning is commonly assessed via repeated exposure of material, the initial trial of these tasks hold important ecological validity, as immediate encoding abilities are essential to daily functioning. We examined the relationship between PTSD symptoms and performance on the initial trial of two learning tasks.

Participants and Methods: Ninety-six OEF/OIF/OND veterans were selected from a larger sample of participants. This select sample excluded those who received a score of $<100 \%$ on an embedded measure of performance validity and those who reported having experienced loss of consciousness $>30$ minutes following a head injury. PTSD diagnosis and symptom severity was obtained using the CAPS-IV, while Trial-1 of the CVLT-II and Trial-1 of the BVMT-R were used to assess encoding abilities.

Results: Bivariate correlations showed that total PTSD severity was negatively correlated with Trial-1 of the CVLT-II $(p=-.26)$. CAPS-IV PTSD clusters were also negatively correlated with Trial- 1 of the CVLT-II (i.e., re-experiencing, $p=-.29$; avoidance, $p=-.24$; hyperarousal, $p=-.23)$. Total CAPS-IV and the symptom clusters were not associated with Trial-5 of the CVLT-II, indicating that over repeated trials the initial relationship had diminished. The CAPS-IV hyperarousal symptom cluster was negatively associated with BVMT-R Trial-1 performance $(p=-.21)$, while total CAPS-IV and the additional symptom clusters were not.
Conclusions: These findings suggest that PTSD symptoms may especially impact the initial acquisition of verbal material, as compared to information presented in the visual modality. Additional research is needed to identify variables that contribute to the relationships observed here and to better understand differential verbal and visual memory abilities often reported in the PTSD literature.

Correspondence: Joseph M. Babione, Jesse Brown VA Medical Center, Mental Health Service Line, 5136 S. Blackstone Ave. Apt. 207, Chicago, IL 60615, United States. E-mail: josephbabione@gmail.com

M. BODZY, N. NOONAN, C. CORDEAUX, J. STUDENY, B.C. KAVANAUGH, J. DUPONT-FRECHETTE \& K.A. HOLLER. Neurocognitive Functioning in Inpatient Children Following Childhood Maltreatment and the Role of PTSD.

Objective: Childhood maltreatment and post-traumatic stress disorder (PTSD) impact neurodevelopment and neurocognitive performance in areas such as attention, memory, executive function (EF), language, and visual-motor integration. Few studies examine the role of maltreatment and PTSD in child neurocognitive performance. This study offers a unique opportunity to examine differences related to maltreatment and PTSD on neurocognitive measures in a child inpatient population. We expect children with comorbid maltreatment and PTSD to exhibit the greatest levels of impairment.

Participants and Methods: Children 6 to 12 years old (IQ $>=70$ ) with $(\mathrm{n}=122)$ and without $(\mathrm{n}=176)$ maltreatment history and children with $(n=126)$ and without $(n=46)$ comorbid PTSD were compared on measures of cognition, language, memory, visual-motor, EF, and attention. T-tests were used to examine group differences on neurocognitive measures.

Results: Even after controlling for IQ, children with maltreatment histories had significantly lower IQ's than children without, despite both group means falling within the average range. Children with maltreatment histories had greater impairment in semantic fluency (animals) relative to those without. Children with comorbid maltreatment and PTSD demonstrated greater impairment in simple verbal memory (sentence repetition) and inhibition/interference (Stroop color-word) than peers without PTSD $(p<.05)$.

Conclusions: Children with maltreatment histories exhibited lower IQ's and deficits in semantic fluency; those with maltreatment and PTSD exhibited EF deficits related to response inhibition/interference and verbal memory, likely related to simple attention. Maltreated children, especially those with PTSD, exhibited greater impairment than those without PTSD or maltreatment histories. These findings highlight the neurocognitive impact of maltreatment and PTSD in children and stress the importance of neuropsychological assessment in maltreated children. Correspondence: Mary Bodzy, PhD, Child and adolescent inpatient, Bradley Hospital, 165 Black Rock Dr, Hingham, MA 02043, United States.E-mail:akac25@yahoo.com

B.M. CERNY, J.P. STANGE, L.R. KLING \& S.A. LANGENECKER. Impairments in Affective Flexibility May Improve When Depression Remits.

Objective: Individuals with active major depressive disorder (MDD) previously have shown impairments in cognitive flexibility, particularly for negatively valenced stimuli. We evaluated whether such impairments in affective flexibility would remain present during the remitted state of MDD (rMDD), potentially representing trait- or scar-like effects of illness.

Participants and Methods: 35 healthy controls (HCs) and 93 individuals with rMDD (mean age $23 ; 69 \%$ women). Participants completed the Emotion Card Sort Test (ECST) and the Emotion Words Test (EWT). The ECST is designed to resemble the Wisconsin Card Sorting Test, with the addition of words valenced to elicit emotional responses from participants. The EWT presents emotionally valenced words, one at a time, with delayed recall and recognition. Each task includes negative, neutral, and positive words. 
Results: On the ECST, a 2 group (HC, rMDD) x 3 condition (negative, neutral, positive) ANOVA did not yield a significant group $\mathrm{x}$ condition interaction or a significant main effect of group on performance. However, there was a significant main effect of condition; across groups, individuals exhibited greater perseverative errors for negative relative to positive stimuli (negative vs. neutral, and neutral vs. positive, did not differ significantly). On the EWT, the group $\mathrm{x}$ condition interaction was not significant for recall. but was significant for recognition trials: however, HC and rMDD did not differ on performance for any of the 3 word types. For recall and recognition, the main effects of group were not significant, but there were significant main effects of condition in the same directions (negative > positive $>$ neutral; all pairwise comparisons significant).

Conclusions: In contrast with active MDD, across two affective flexibility tasks, individuals with rMDD did not demonstrate negative affective biases relative to HCs, providing converging evidence that some cognitive biases may improve during remission from MDD.

Correspondence: Brian M. Cerny, Psychiatry, University of Illinois at Chicago, 1963 W Winona, Chicago, IL 60640, United States. E-mail: bcerny@psych.uic.edu

D.J. COBIA, M.J. SMITH, J.G. CSERNANSKY \& L. WANG. Subcortical Features of Dorsolateral Prefrontal Circuitry in Neuropsychologically Near-Normal Schizophrenia.

Objective: Schizophrenia is a severe psychiatric illness typically characterized by impaired neuropsychological functioning; however, some individuals are known to perform in the near-normal range. Studies of brain structure and function in this group continues to expand in attempts to identify mechanisms supporting cognition. The aim of this study was to characterize shape features in brain structures involved in frontal networks functions. It was hypothesized that the "near-normal" group would demonstrate structural integrity similar to healthy individuals, which would relate to executive functions.

Participants and Methods: Schizophrenia subjects were clustered into impaired $(\mathrm{NPI}=34)$ and near-normal $(\mathrm{NPNN}=45)$ subgroups using a k-means algorithm; matched healthy comparison subjects were also included $(\mathrm{COM}=56)$. Neuropsychological functioning was assessed through a comprehensive battery, MR scanning included T1-weighted images. Estimates of caudate (CD), globus pallidus (GP) and regions of the middle frontal gyrus (MFG) were derived using surface-based pipelines. MANOVA and Pearson models tested group differences and relationships with neuropsychological performance.

Results: Results revealed significant shape abnormalities between groups in the $\mathrm{L}(\wedge=2.1, p=0.005)$ and $\mathrm{R}(\wedge=1.7, p=0.034) \mathrm{GP}$, but not CD. Significant negative correlations between GP shape with $\mathrm{L}$ $(r=-0.53$ to $-0.52, p<0.001)$ and $\mathrm{R}(r=-0.46$ to $0.40, p<0.02)$ caudal MFG volumes were observed in both NPNN and NPI. However. GP shape was related to cognitive flexibility (Wisconsin Card Sorting Test perseverative errors) only in NPNN ( $r=0.32$ to $0.30, p<0.05)$.

Conclusions: Of all subcortical regions in the DLPFC loop, only GP morphology varied among NPNN and NPI, and related to MFG integrity. Relationships with cognition and GP occurred in NPNN. Findings provide additional support for the neurobiological heterogeneity in cognitively derived schizophrenia subtypes.

Correspondence: Derin J. Cobia, PhD, Psychology, Brigham Young University, 1036 SWKT, Brigham Young University, Provo, UT 84602, United States. E-mail: derin_cobia@byu.edu

L.S. CZEPIELEWSKI, J. BUCKER, F. LIMA, F.D. RABELO DA PONTE, D. SANTOS MARTINS, M. SOUSA, C. PETRY, A. ROSA \& C. GAMA. Intellectual Functioning and Not Cognitive Performance Predicts Functional Outcomes in Bipolar Disorder.

Objective: Bipolar disorder (BD) is associated with increased impairments in psychosocial and occupational functioning. Depressive symptoms and cognitive performance are described to be important predictors, however the role of intellectual functioning (IQ) it is still unclear. Therefore, we aimed to investigate the influence of intellectual performance to functional outcome.

Participants and Methods: Participants were 66 outpatients with confirmed diagnosis of BD by Structured Clinical Interview for DSM-IV (SCID) and 52 healthy volunteers without current, past history or first-degree family history of psychiatric disorder. Inclusion criteria for patients were euthymia for at least one month before assessment with Hamilton Depression Rating Scale and Young Mania Rating Scale $\leq 7$, receiving psychiatric psychiatric medication for more than three months. All participants signed informed consent. Procedures were approved by Ethics Committee. The cognitive and intellectual functioning assessment was administered by experienced clinical psychologists. Psychosocial functional outcome was measured using the Functioning Assessment Short Test (FAST).

Results: For the prediction of FAST, the linear regression model that included cognitive composite, IQ and current symptoms as predictors, controlling for age and years of education, was significant in BD $\left(F(6,59)=3.994, p=.002\right.$, Adj $\left.R^{2}=.217\right)$, with a main effects of IQ $(B=-.420, t=-2.379, p=.021)$ and depressive symptoms $(B=3.82$, $\mathrm{t}=3.332, \mathrm{p}=.001)$, but not of cognition $(B=.220, \mathrm{t}=1.278, \mathrm{p}=.206)$ or manic symptoms $(B=.063, \mathrm{t}=.546, \mathrm{p}=.587)$. In HC, the model was not significant $(\mathrm{F}(4,46)=2.512, \mathrm{p}=.054)$.

Conclusions: Therefore, in individuals with BD during euthymia, higher intellectual functioning and reduced depressive symptoms, but not cognitive performance, are predictors of better psychosocial functioning. These results may explain the difficulty of full recovery after episodes for functional domains in BD.

Correspondence: Letícia S. Czepielewski, Universidade Federal do Rio Grande do Sul (UFRGS), Rua Ramiro Barcelos 2350 - Centro de Pesquisas, Porto Alegre 90035-903, Brazil.E-mail: czepielewski@gmail. com

F.D. RABELO DA PONTE, L. SANGUINETTI CZEPIELEWSKI, D. SANTOS MARTINS \& M. KUNZ. Delayed sleep phase and impaired verbal memory of patients with Bipolar Disorder.

Objective: Our aim was to examine the relationship between mid-sleep time and verbal memory in patients with Bipolar Disorder (BD), controlling for intellectual functioning (IQ). Mid-sleep time is the half-way point between sleep-onset and sleep-end.

Participants and Methods: Participants were 40 outpatients from Bipolar Disorder Program from Hospital de Clínicas de Porto Alegre and 40 healthy volunteers. Experienced psychiatrists carried the diagnosis of BD confirmed by Structured Clinical Interview for DSM-IV. Inclusion criteria for outpatients were euthymic patients for, at least, one month before assessment with Hamilton Depression Rating Scale and Young Mania Rating Scale $\leq 7$, age between 18 and 65 years old, without changes in medication for more than three months. Exclusion criteria for outpatients were night shift workers, comorbid intellectual disability (IQ < 69), and severe medical comorbidity. All patients were receiving psychiatric medication. Healthy controls were between 18 and 65 years old, psychiatric medication free volunteers, who had no current, past history or first-degree family history of psychiatric disorder, dementia or intellectual disability and could not be night shift workers. Every participant provided written informed consent. All procedures were approved by Ethical Committee. The neuropsychological assessment was administered by psychologists. The verbal memory assessment was carried out with Hopkins Verbal Learning Test and the sleep assessment was performed with Munich ChronoType Questionnaire.

Results: After adjustment for IQ, later mid-sleep time was a predictor of poor performance on verbal memory task in BD patients (IQ: $B=0.19, \beta=0.46, p=0,02$. mid-sleep: $B=0,0037, \beta=0,28, p=0,04$. $\left.\mathrm{R}^{2}=0,34, \mathrm{~F}=9,6, \mathrm{p}=0.001\right)$. While in healthy subjects, mid-sleep time was not a predictor of verbal memory (IQ: $\mathrm{B}=0,13, \beta=0,43, \mathrm{p}=0,01$. Mid-sleep: 0,002, $\left.\beta=0,24, p=0,1 . R^{2}=0,1, F=4, p=0,02\right)$.

Conclusions: This finding suggests that delayed sleep phase may be linked to poorer verbal memory in patients with BD. 
Correspondence: Leticia Sanguinetti Czepielewski, Porto Alegre 90035007, Brazil. E-mail: leticia.czepielewski@fadergs.edu.br

\section{S.I. DEV, A.N. SUTHERLAND \& L.T. EYLER. Impulsivity and cerebral blood flow in bipolar disorder.}

Objective: Impulsivity is a common feature of bipolar disorder (BD) and is linked to altered brain structure and function in frontal and temporal regions among those with and without psychiatric illness. Studies investigating cerebral blood flow (CBF) in BD have noted both hyper- and hypo-perfusion in BD compared to healthy comparison (HC) groups, yet little is known about relationships between CBF and impulsivity. The objective of this exploratory analysis is to determine associations between self-reported levels of current impulsivity and resting $\mathrm{CBF}$ in frontal and temporal regions of interest (ROIs) in BD and HC participants.

Participants and Methods: 39 euthymic BD and 45 age-and-education comparable HC participants were administered the Conners Adult ADHD Rating Scale (CAARS) and underwent Multiphase Pseudocontinuous Arterial Spin Labeling MRI. CBF values within 10 frontal and temporal ROIs were extracted using Freesurfer parcellations. Independent $\mathrm{t}$-tests determined group differences in age, education, impulsivity, and CBF. Group-wise bivariate correlations evaluated relationships between self-report impulsivity and CBF.

Results: There were no group differences in age, education, or in regional $\mathrm{CBF}$. BD patients reported greater symptoms consistent with impulsivity/mood lability (IM) and impulsivity/hyperactivity (IH) subscales of the CAARS. Among the BD group, greater scores on IH subscale were associated with higher levels of CBF in left pre-central and parahippocampal gyri. In contrast, HCs with higher scores on IM subscale demonstrated lower CBF in left superior frontal, hippocampal, middle temporal, and bilateral superior temporal regions.

Conclusions: Results confirm previous reports of greater impulsivity in $\mathrm{BD}$, suggest that levels of regional $\mathrm{CBF}$ at rest may play a role in maintaining these behaviors in both $\mathrm{BD}$ and HCs, and indicate that this relationship is different in BD compared to HC. More targeted investigations are required to further disentangle contributions of cerebrovascular health in impulsivity.

Correspondence: Sheena I. Dev, M.S., Clinical Psychology, SDSU/UCSD Joint Doctoral Program, 3350 La Jolla Village Drive, Building 13, MC 151B, San Diego, CA 92161, United States. E-mail: sdev25@gmail.com

E. ESTEVIS, S. KIM, M. ROSSETTI, M.R. BASSO \& R. PURDIE. Patterns of Verbal Memory Performance in Depressed Inpatients. Objective: Impairments in neurocognitive functioning has been found to be a core feature in major depression. For example, impaired verbal memory has been shown to be a frequent occurrence often among depressed individuals, especially in inpatients. However, these impairments have been observed inconsistently across studies. In addition, the majority of studies that have examined memory functioning in depression have not included a measure of performance validity to assess for effort. This highlights a potential limitation in the literature given the current trend in neuropsychology to incorporate measures of performance validity in the evaluation process. The present study examined patterns of verbal memory performance and performance validity in a group of depressed inpatients and healthy-controls.

Participants and Methods: 18 unipolar depressed inpatients and 21 healthy-controls were administered a structured diagnostic interview, CMDI, the CVLT-2, WMS-IV Logical Memory I and II, and the Word Memory Test (WMT).

Results: One-way ANOVAs were utilized to examine performance on the CVLT-2 and WMS-IV indices between the two groups. Results showed that the two groups differed significantly on WMS-IV Logical Memory I and II $(\mathrm{p}<.05)$, but not on the Recognition Trial. The two groups also differed on the following CVLT-2 indices ( $p \leq .01)$ : Trial 5 , Total Score, Short and Long Delayed Recall With and Without Cueing, and Total Recognition Hits. These differences occurred despite having displayed no evidence of poor effort on the WMT.

Conclusions: The current study revealed that depressed inpatients experience considerable differences in memory performance in comparison to healthy-controls. Specifically, depressed inpatients exhibited a frontal-subcortical memory profile on a measure of contextualized memory and broad impairments on a non-contextual memory measure. These findings suggest that depressed inpatients are apt to experience declines in verbal memory functioning in the absence of suboptimal effort.

Correspondence: Eduardo Estevis, M.A., Clinical Psychology, James A. Haley VA Medical Center, 8801 Hunters Lake Dr. \#123, Tampa, FL 33647,United States. E-mail: eduardo-estevis@utulsa.edu

P. FERNANDES \& D. REAM. Effects of Selective Serotonin and Serotonergic-Noradrenergic Reuptake Inhibitors on Neuropsychological Performances in the United States.

Objective: To examine the effects of selective serotonin reuptake inhibitors (SSRIs) and serotonergic-noradrenergic reuptake inhibitors (SNRIs) on neuropsychological performance on memory tasks, verbal and semantic fluency and perceptual processing speed.

Participants and Methods: Our study consisted of $n=2935$ subjects [age $(M=69.48, S D=6.78)$ using the National Health and Nutrition Examination Survey (NHANES); a program operated by the Centers for Disease Control and Prevention designed to assess the health and nutritional status of the Unites States civilian population. Inclusion criteria consisted of subjects aged 20 and over from 2011 to 2014. Exclusion criteria consisted of marijuana and cocaine users. Control group consisted of $n=2598$ subjects [age $(M=69.44, S D=6.77)$ ], SSRI group consisted of $n=273$ subjects [age $(M=70.01, S D=6.81)$ ], and SNRI group consisted of $n=64$ subjects [age $(M=68.77, S D=6.99)$ ]. Three conditions were analyzed: 1) Control group without SSRIs or SNRIs, 2) SSRIs only, and 3) SNRIs only. To evaluate cognitive performance, subjects were administered the word learning and recall modules from the Consortium to Establish a Registry for Alzheimer's disease (CERAD), Animal Fluency, and Digit Coding.

Results: A chi-square showed a significant difference in race $\chi^{2}(4, N=2935)=43.89, \mathrm{p}<.05$ and gender $\chi^{2}(2, N=2935)=44.64$, $\mathrm{p}<0.05$. Study groups were mostly Non-Hispanic White and female. Also, the presence of sleep disorder was significant, $\chi^{2} \quad(2, N=2935)$ $=64.76, \mathrm{p}<0.05 .31 \%$ of SNRI subjects, $23 \%$ of SSRI subjects and $10 \%$ of control subjects revealed difficulty sleeping. A one-way ANOVA revealed no significant effects between groups ( $p>0.05$ ); moreover, Bonferroni post-hoc analyses were not significant.

Conclusions: SSRIs and SNRIs show no significant relationship on neuropsychological performances in our sample that is representative of the United States population.

Correspondence: Paulo Andre Fernandes, Doctor of Pharmacy, 3940 W. Broward Blvd., APT 106, Fort Lauderdale, FL 33312, United States. E-mail: pauloandref@yahoo.com

WITHDRAWN. M. LØVSTAD, G. MÅNUM, K. WISLØFF-AASE, I. LARSEN, J. RADER, J. STANGHELLE \& A. SCHANKE. Relationships Between Subjective Cognitive Complaints and Emotional Functioning, Post Traumatic Stress Symptoms, Fatigue, Sleep and Pain, as well as Neuropsychological Test Performance in Hospitalized Victims of Terror.

Objeetive: On July $22^{\text {ntd }} 2011,46$ people were hospitalized due to physs= ieal injuries following two terror attacks in Osto. This study explores the relationship betweensulbjective cognitive complaints ande entrotional funetioning;, post tratmatie stress symptoms, fatigue, steep and pain, as well as netropsychologieal performanee.

Partieipants and Methods. Thirty of the 46 hospitalized persons

were assessed 2.5-3 years after the incident, and the twenty-six with no brain injury are included here. Fourteen were severely injured (New fnjury Severity Seore $\geq 15$ ). Two items related to stbjeetive experience of 


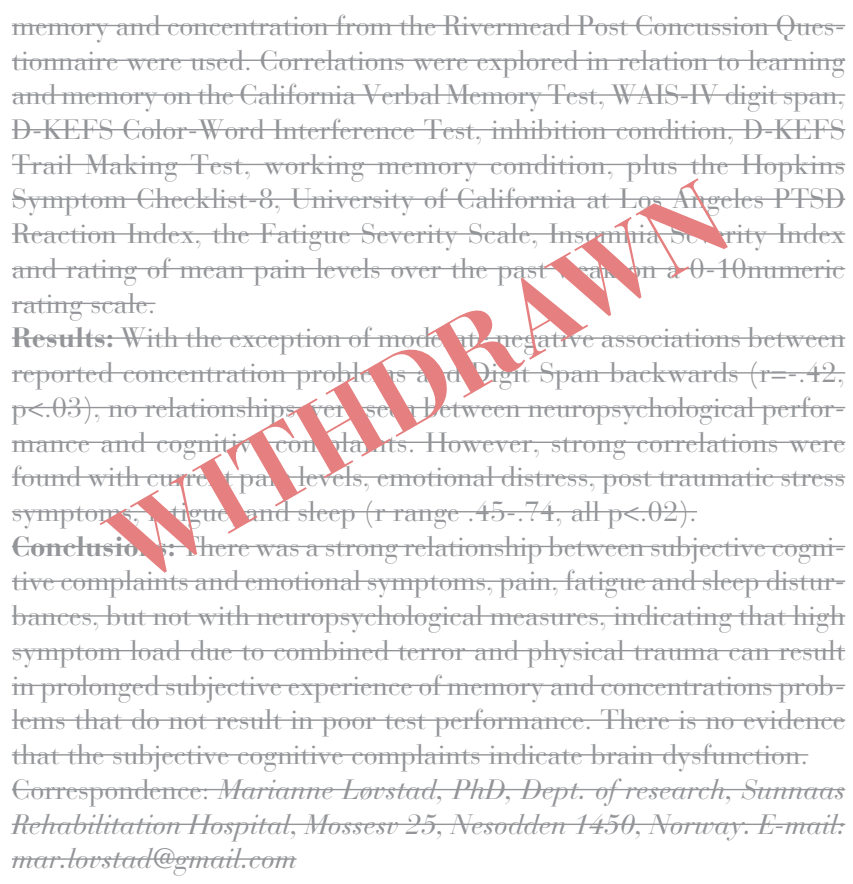

M.Z. NAKHLA, C. KAUFMANN, H. YOON, A.N. SUTHERLANDOWENS \& L.T. EYLER. Gender Differences in Social Cognition in Bipolar Disorder with Possible Links to Inflammation.

Objective: Patients with bipolar disorder (BD) experience cognitive deficits, with a growing body of literature demonstrating that inflammation may play a role. Despite evidence suggesting that inflammation differs by gender in the normative population, gender differences in both cognition and inflammation in BD is an understudied area. The purpose of this study was to explore any gender differences in cognition of $\mathrm{BD}$ patients, and whether this relationship was possibly linked to inflammatory differences.

Participants and Methods: 21 BD patients and 52 Healthy Controls (HC) were administered the MATRICS Consensus Cognitive Battery, a test assessing a wide range of cognitive domains. Blood samples were taken to assess pro-inflammatory cytokine levels. ANOVAs with follow-up t-tests were performed to assess differences between BD and HC on cognitive domains, gender effects, and possible group differences in the nature of gender effects. Pearson correlations tested the role of inflammation in performance on gender-related cognitive domains.

Results: Groups were comparable on age and gender composition. $\mathrm{HC}$ performed significantly better than $\mathrm{BD}$ on a majority of cognitive domains. Across groups, women had higher scores than men in processing speed, attention/vigilance, and working memory. In the social cognition domain, BD performed worse than HC ( $p=.003)$, and $\mathrm{BD}$ women performed significantly better than BD men but there were no gender effects in the HC group (gender $\mathrm{x}$ group $p=.04$ ). Higher interleukin (IL)-6 levels were related to poorer social cognition across groups $(p<0.009)$, but as there were no gender differences in IL-6 levels within the BD group, inflammation did not explain the gender effect on social cognition in $\mathrm{BD}$

Conclusions: Preliminary findings of this study suggest that gender should be taken into account when examining social cognitive deficits in BD patients. Moreover, these findings highlight that inflammation and its relationship to gender differences in BD cognition is an area that warrants future exploration.

Correspondence: Marina Z. Nakhla, B.A., Psychology, California State University, Northridge, 18111 Nordhoff St, Northridge, CA 91330 , United States. E-mail: marina.nakhla.304@my.csun.edu
J. PETERMAN, D. MARSHALL, S.A. LANGENECKER, K. ANGERS, R.E. EASTER, P. BABU, M. MCINNIS \& K. RYAN. Differential Effects of Increased BMI on Working Memory Performance in Individuals with Bipolar Disorder Compared to Unipolar Depression and Controls.

Objective: Individuals with bipolar disorder (BD) and unipolar depression (MDD) experience a higher rate of obesity than the general population, putting them at risk for poorer functional outcome. Whether obese individuals with BD or MDD also exhibit greater neurocognitive deficits compared to their healthy weight peers is less clear. We examined the interaction of BMI and cognitive functioning in a large sample of BD in comparison to a psychiatric control group, MDD individuals, and healthy age-matched controls.

Participants and Methods: Participants with BD ( $\mathrm{n}=199)$, MDD $(\mathrm{n}=81)$, and healthy controls (HC; $\mathrm{n}=112$ ) (age range 18 to 40 ) performed a computerized spatial working memory (SWM) task that included three graded increases in target identification. Errors within each level (e.g. 4, 6, 8) were recorded. Participants were subdivided by BMI classification into six diagnostic-BMI subgroups (Normal Weight-BD, Overweight/Obese-BD, Normal Weight-MDD, Overweight/ Obese-MDD, Normal Weight-HC, and Overweight/Obese-HC). Performance on the SWM task was indexed by number of errors produced in each difficulty level and the subgroups were compared in a repeated-measures ANOVA

Results: The number of errors, across all groups, increased with increasing task difficulty $(\mathrm{F}(2,384)=18.28 ; \mathrm{p}<0.0001)$. There was an interaction between number of errors and diagnostic-BMI group $(F(10,770)=3.19 ; p=0.05)$. Post-hoc analyses indicated that while the Normal Weight-BD group did not differ in performance from the MDD and HC groups, the Overweight/Obese-BD group performed significantly worse than the HC groups.

Conclusions: Overweight/Obese-BD individuals significantly differ in working memory functioning compared to HC individuals. Thus it appears that psychiatric illness in the context of increased metabolic burden is associated with greater neurocognitive deficits.

Correspondence: Joel Peterman, Ph.D., Psychiatry, Michigan Medicine, 2101 Commonwealth Blvd, Ann Arbor, MI 48105, United States. E-mail: jpeterma@med.umich.edu

R.J. PIERS, Y. LIU, T. ANG, R. AU \& W.Q. QIU. Depressive Symptoms and Cognitive Decline in Younger- and Middle-Aged Adults: Cross-Sectional Analyses from the Framingham Heart Study.

Objective: The association between depression and risk of dementia in older adults is well-documented. However, few studies have looked systematically at the association between depression and cognition in younger-to-middle age adults.

Participants and Methods: Framingham Heart Study Offspring cohort participants were invited to participate in Exam 7 between 1999 and 2005. Participants were screened for depressive symptoms using the Center for Epidemiologic Studies - Depression questionnaire (CES-D). Presence of depressive symptoms was set at CES-D $>16$. Within 5 years of the initial CES-D, participants were administered a comprehension neuropsychological (NP) evaluation, which included tests of verbal and visual memory, learning, attention, executive function, abstract reasoning, and visuospatial ability. The association between depressive symptoms and cognition was investigated using analysis of covariance, adjusted for age, sex, education, time between CES-D and NP, and antidepressant medication use.

Results: One thousand eighty-eight younger- and middle-aged participants met criteria for the study (mean age $=53.41 \pm 4.71$, age range $=34-59,55.70 \%$ female, $46.5 \%$ college graduate, MMSE $=29.19 \pm 1.10)$. Depressive symptoms were present in $9.83 \%$ of the sample and $11.59 \%$ reported antidepressant use. Some neuropsychological tests were $\log$ transformed and reflected to account for skewness in the data. After adjustment for covariates, depression was 
associated with lower scores on Logical Memory: Immediate Recall (Estimate \pm standard error $[\mathrm{SE}]=0.82 \pm 0.32, \mathrm{p}=0.01$ ), Hooper Visual Organization Test (Estimate $\pm \mathrm{SE}=-0.13 \pm 0.05, \mathrm{p}=0.01$ ), and Visual Reproductions: Immediate Recall (Estimate $\pm \mathrm{SE}=-0.07 \pm 0.03, \mathrm{p}=0.02$ ). Conclusions: Depressive symptoms in younger-to-middle age adulthood may impact encoding and visuoperceptual function. Future research should explore the association between depression and cognition longitudinally, and determine whether there are concomitant neuroimaging biomarkers.

Correspondence: Ryan J. Piers, Clinical Psychology, Psychological and Brain Sciences, Boston University, 19 Harvard Avenue, Brookline, MA 02446, United States. E-mail: rpiers@bu.edu

R.P. SO, R. RANGAMANNAR \& K.A. HOLLER. Characterizing the Neuropsychological Profiles of Those With and Without a History of Childhood Trauma Among a Sample of Psychiatric Inpatient Adolescents.

Objective: Despite the growing evidence for the link between trauma and psychosis, few studies have addressed the influence of early trauma on neurocognitive functioning among individuals with psychotic symptoms, particularly within a psychiatric inpatient population. This study aimed to characterize the neuropsychological profiles of inpatient adolescents who experienced psychosis-like symptoms and to compare these profiles between those with and without a history of childhood trauma.

Participants and Methods: A retrospective medical chart review was conducted on 40 adolescents (history of trauma $n=23$ ), who endorsed experiences of hallucinations and delusions during the initial psychiatric evaluation. Trauma was defined as self-reported experience of physical, emotional, and/or sexual abuse.

Results: There were no age $(M=15.5$ years; $t(38)=.276, p=.784)$ or IQ $(M=92.5 ; t(38)=-.530, p=.599)$ differences between the adolescents with and those without a history of early trauma. Preliminary results revealed no group differences on measures of language (BNT), verbal memory (WRAML), visual-motor (Rey Copy Trial), processing speed (TMT-A), working memory (Digit Span), verbal fluency (COWAT), planning (WCST Categories), cognitive flexibility (TMT-B, WCST Perseverative Errors), and interference control (WCST Failure to Maintain Set) ( $p$ values ranged from .094 to .972). Compared to those without a history of trauma, adolescents with a history were found to make more perseverative errors on the CPT $(t(31)=2.148, p=.040$, Cohen's $d=.771)$ and name fewer items correctly in the color-word condition of the Stroop Test $(t(37)=-2.801, p=.008$, Cohen's $d=-.921)$.

Conclusions: Among a sample of psychiatric inpatient adolescents, those with a history of trauma performed more poorly on measures of impulsivity and inhibition than those without a history of trauma. Future research exploring the link between childhood trauma and the development of psychosis may explore the potential moderating role of neurocognitive deficits, such as executive dysfunction.

Correspondence: Rachel P. So, M.A., Psychological Sciences, University of Connecticut, 406 Babbidge Road, Unit 1020, Storrs, CT 06269 , United States.E-mail: rachel.so@uconn.edu

\section{Other}

T. HOPYAN \& B. SETNIK. Neurocognitive Effects of Drug-Induced Changes for Choice Reaction Time in Recreational Polydrug Users. Objective: To investigate the neurocognitive effects on a test of psychomotor performance in recreational drug users following the oral administration of sedative-hynoptics (alprazolam, $1.5 \mathrm{mg}$ and $3.0 \mathrm{mg}$; zolpidem, $15 \mathrm{mg}, 30 \mathrm{mg})$; ethanol $(1.0 \mathrm{~g} / \mathrm{kg})$; a dissociative anaesthetic (oral ketamine, $100 \mathrm{mg}$ ); and a cannabinoid (dronabinol, 40 $\mathrm{mg}$ and160 $\mathrm{mg})$.

Participants and Methods: Neurocognitive performance was measured using the choice reaction time (CRT) task, including data from 4 singledose, randomized, double-blind, placebo-controlled, crossover studies.
Participants were a total of 95 individuals with a history of recreational polydrug use who were experienced with the drug class of interest (ages 18-55 years). CRT is a classic test of psychomotor performance; electronic data for reaction times were captured through validated proprietary software (Scheduled Measurement System - SMS). Peak effect $\left(\mathrm{E}_{\max }\right)$ was calculated for CRT reaction time outcomes across different drugs compared to placebo.

Results: Analysis of variance showed $\mathrm{E}_{\max }$ values were significantly larger for all doses of alprazolam, zolpidem, and ethanol $(p<0.0001)$ against placebo. No significant differences in $\mathrm{E}_{\max }$ for CRT outcomes were observed for ketamine and dronabinol $(p>0.05)$.

Conclusions: Outcomes on CRT vary according to drug class. As expected, impaired reaction times (i.e., significant increase) on the CRT task were associated with administration of CNS depressants (alprazolam, ethanol, and zolpidem). Oral administration of ketamine was not associated with significant decreases in reaction time on the CRT task. Dronabinol drug administration had no effect on psychomotor performance, suggesting that either it does not impair reaction time or users of this drug may have developed palpable tolerance to the acute disruptive cognitive effects. Findings also suggest that performance on CRT is an effective measure for capturing neurocognitive impairment of CNS depressant drugs.

Correspondence: Talar Hopyan, PhD, C.Psych, INC Research, 720 King Street West, Toronto, ON M5V 2T3, Canada.E-mail: thopyan@ gmail.com

J. RENSBERGER, N.M. HENNIG, E. AN, A.A. TURK NOLTY, S. AMANO \& J. BUCKWALTER. Hypothalamic pituitary adrenal axis functioning and PTSD symptoms in Army National Guard Special Forces.

Objective: Dehydroepiandrosterone (DHEA) and dehydroepiandrosterone-sulfate (DHEA-S) have been shown to counter deleterious effects of long-term exposure to cortisol from chronic or traumatic stress and bolster resilience to stress. This antiglucocorticoid effect has been evidenced in levels of DHEA, DHEA-S, and the ratio of DHEA to cortisol. However, hypothalamic pituitary adrenal (HPA) axis disruption in the case of PTSD can lead to an attenuation of cortisol levels and a dampened ratio of DHEA to cortisol. We explored the connection between levels of stress hormones and symptoms of posttraumatic stress disorder (PTSD) in cases of subthreshold PTSD. It was hypothesized that stronger levels of DHEA, DHEA-S, and the ratio of DHEA to cortisol would be associated with fewer symptoms of PTSD. It was also hypothesized that greater levels of cortisol would be associated with increased symptoms of PTSD.

Participants and Methods: Twenty-three male members of the Army National Guard Special Forces who did not meet DSM-5 diagnostic criteria for PTSD completed the PTSD Checklist-Military Version. Levels of DHEA and DHEA-S were obtained through blood samples while cortisol was obtained through saliva.

Results: After controlling for age, we found a negative association between symptoms of PTSD and levels of DHEA, $r(21)=-0.50$, $p=.018$, and between symptoms of PTSD and DHEA-S, $r(21)=-0.53$, $p=.011$. However, there were no significant associations between levels of cortisol or the ratio of DHEA to cortisol and symptoms of PTSD.

Conclusions: Levels of DHEA and DHEA-S appear to predict a more adaptive response to traumatic experiences. This relationship may suggest a role for DHEA and DHEA-S supplementation and modulation in protecting against PTSD. Further, it may provide support for utilizing these hormones as biomarkers for resilience against developing symptoms of PTSD following traumatic experiences, which could inform recruitment and training of members of the armed forces.

Correspondence: Jared Rensberger, Fuller Graduate School of Psychology, 135 N. Oakland Ave, Pasadena, CA 91101, United States. E-mail: jaredrensberger@fuller.edu 


\section{Schizophrenia}

\section{AGHVINIAN \& M. SERGI. Social Functioning, Empathy, and Working Memory in Schizotypy.}

Objective: Schizophrenia is a mental illness involving hallucinations, delusions, and marked cognitive dysfunction (Messias et al., 2007). Individuals displaying sub-clinical symptoms are characterized by the construct of schizotypy (Meehl, 1962). Individuals with schizotypy display impairments in maintaining social roles, empathy, and working memory, leading to deficits in functional outcome (Henry et al., 2007:Cohen and Mohr, 2015). The present study explores the relationship between schizotypy status and impairments of working memory, social functioning, and empathy.

Participants and Methods: Schizotypy status was determined by scores on the Schizotypal Personality Questionaire-Brief. Persons with high $(\mathrm{N}=48)$ and low $(\mathrm{N}=13)$ schizotypy completed a battery of cognitive tests of working memory and questionnaires examining social functioning and empathy, including the Social Adjustment Scale (SAS), Interpersonal Reactivity Index (IRI), Empathy Quotient (EQ), MOS-Social Support Survey (MOS-SSS), and Social Functioning Scale (SFS). Results: Preliminary results showed persons with high schizotypy demonstrated impaired empathy on the Fantasy subscale of the IRI $(\mathrm{t}(58)=-2.67, \mathrm{p}=.01)$ and on the Perspective Taking subscale of the IRI $(t(58)=-2.09, p=.04)$. Persons with high schizotypy performed less well on the Family subscale of the SAS $(\mathrm{t}(59)=-2.77, \mathrm{p}=.01)$ and on the Social/Leisure subscale of the SAS $(t(59)=-3.86, p=<.01)$. There were no significant group differences on tasks of working memory between the high and low schizotypy group.

Conclusions: Results indicated that persons with high schizotypy were impaired on measures of social functioning and empathy. Interestingly, individuals with high and low schizotypy did not differ on tasks of working memory. This is congruent with past studies reporting mixed findings regarding impairments in working memory for persons with schizotypy (Cannon et al., 1994; Lenzenwegger and Gold, 2000). Future studies should examine social skills training strategies that can ameliorate these deficits.

Correspondence: Maral Aghvinian, M.A., Psychology, California State University, Northridge, 18111 Nordhoff St., Northridge, CA 91330,

United States.E-mail:maghvinian@gmail.com

T. ANDERSEN, S. BOWYER, K. MCFARLANE, S. TARABEN, B. WEILAND, N. BOUTROS, J. MORAN, N. TEPLEY \& R. LAJINESSO'NEILL. Altered Neuromagnetic Evoked Responses Related to Visual Habituation in Schizophrenia.

Objective: Schizophrenia (SCH) is characterized by positive symptoms (PS) that may include delusions/hallucinations and negative symptoms (NS) such as disorganized thought. Literature suggests aberrant gating is associated with impairment in cognition. Most have evaluated auditory gating, and less is known about visual gating. This study investigated relationships between event-related potentials (ERPs) during a visual paired-flash paradigm and positive/negative symptoms in individuals with deficit (D) and nondeficit SCH (N) compared to controls (C).

Participants and Methods: Nine D (Age: $\mathrm{M}=42.2 ; \mathrm{SD}=11.7)$, $9 \mathrm{~N}$ (Age: $\mathrm{M}=41.1 ; \mathrm{SD}=11.6)$ and 9 age and gender matched $\mathrm{C}$ (Age:M=41.5;SD:9.5) underwent magnetoencephalography while attending to a visual paired-flash paradigm. P50 and P100 latencies/ amplitudes and gating ratio (GR) were calculated. D and $\mathrm{N}$ groups completed the Positive and Negative Syndrome Scale and SCID for DSM-IV TR. Kendall-tau correlations were computed to explore relationships between PS/NS and ERPs.

Results: D had significantly higher P50 amplitude (M=1.2nanoAmp-Meters;SD=.7) than $\mathrm{C}(\mathrm{M}=.5 \mathrm{nAmp}-\mathrm{M} ; \mathrm{SD}=.02)$. D had significantly higher P50 latency ( $\mathrm{M}=.5 \mathrm{~ms} ; \mathrm{SD}=.02)$ than $\mathrm{N}(\mathrm{M}=.4 ; \mathrm{SD}=.01)$. There were no significant differences among groups in S1 amplitude/ latency or GR. There were no significant differences in S2 amplitude. $\mathrm{N}$ had significantly higher $\mathrm{S} 2$ latency $(\mathrm{M}=.61 \mathrm{~ms} ; \mathrm{SD}=.010)$ than $\mathrm{C}$
$(\mathrm{M}=.63 \mathrm{~ms} ; \mathrm{SD}=.012)$. There were no significant correlations between PS/NS and ERPs.

Conclusions: Consistent with literature, D group had difficulties with P50 suppression compared with C, suggestive of information overload. Despite no significant difference in GR, difference in S2 latency between $\mathrm{N}$ and $\mathrm{C}$ indicate some impairment in sensory gating. Future research on the relationship between ERPs and symptoms may need to evaluate more specific subscales of PANSS. Results suggest suboptimal coordination of neuronal responses may be a mechanism to impairments in $\mathrm{SCH}$ and provide valuable diagnostic information before emergence of behavioral symptoms.

Correspondence: Tiffany Andersen, Psychology, Eastern Michigan University, 525 N Adams St, Apt 2, Ypsilanti, MI 48197, United States. E-mail:tander33@emich.edu

J. AXELROD, T. RHOADS, H. PATTON \& S. HILL. Clinical phenotypes in psychosis: Relationship between catechol-Omethyltransferase with positive and negative symptoms.

Objective: Schizophrenia is a mental disorder that is clinically characterized by positive and negative symptoms. There has been a shift in focus from symptom-based diagnosis to a biological or genetic model to capture the phenotype of psychotic disorders. This study assessed the relationship between catechol-O-methyltransferase (COMT) genotype and positive and negative symptomatology in the psychotic spectrum disorders. COMT has been linked to psychotic disorders because of the long hypothesized role of dopamine (DA) in schizophrenia. Individuals homozygotic for the Val allele have an approximate 4-fold increase in enzymatic activity compared to Met carriers, which results in increased metabolism of DA, while the Met allele codes for a low-activity variant of the COMT enzyme. It was hypothesized that Met carriers would have more negative symptoms and the Val allele would be associated with more positive symptoms.

Participants and Methods: This project utilized participants ( $n=1157)$ from the Bipolar-Schizophrenia Network of Intermediate Phenotypes (BSNIP) database diagnosed with a psychotic disorder. Symptoms were assessed via the Positive and Negative Syndrome Scale (PANSS). Given the significant portion of low scores on the positive and negative scales reflecting the absence of certain symptoms, generalized linear models using zero-inflated negative binomial (ZINB) regressions were used.

Results: Contrary to expectations, the present findings indicate that there were no significant differences related to COMT genotype in terms of positive and negative symptoms.

Conclusions: The polarizing effect of COMT genotype may not have been captured due to study limitations, including patient antipsychotic medication stabilization and assessment taking place over the course of only one week. Future studies can explore this relationship by examining clinical symptoms in first episode psychotic individuals who are not medicated.

Correspondence: Jenna Axelrod, Psychology, Rosalind Franklin University of Medicine and Science, 3859 n Greenview, Chicago, IL 60613, United States. E-mail: jenna.axelrod@my.rfums.org.

A. BUCHHOLZ, J. AXELROD, T. RHOADS, M. ABRAHAM, S. KEEDY \& S. HILL. Clarifying the Effect of Interference on Task Switching Latency in Schizophrenia.

Objective: To identify potential endophenotypic markers of schizophrenia (SZ) spectrum disorders by examining the effects of diagnosis, task switching, and interference on response time and accuracy.

Participants and Methods: Participants were 39 individuals with SZ spectrum disorders and 61 healthy controls (HC). Participants completed an experimental task-switching paradigm, which also contained interference. Performance on switching versus non-switching and interference versus no interference trials was compared.

Results: ANOVA showed that the SZ group was significantly less accurate overall but not slower than healthy controls. The costs of task switching were similar for both groups. There was a differential effect 
of interference in terms of latency in which the SZ group responded significantly faster than HCs in the context of interference.

Conclusions: Cognitive dysfunction in SZ may not be due to poor taskswitching per se, but to processes embedded within task-switching, such as interference. How individuals with SZ compare to HC in terms of response latency may be dependent, at least in part, upon such contextualized effects. This group difference may have utility as an endophenotype for SZ.

Correspondence: Alison Buchholz, Doctor of Philosophy, Johns Hopkins University School of Medicine, 3506 Green Bay Road, Apt. 104A, North Chicago, IL 60064, United States. E-mail: alison. buchholz@my.rfums.org

J.L. CRAWFORD, S. DUBOIS, D.J. SCHRETLEN, A. SAWA \& V. KAMATH. The Contributions of Olfaction to the Neuropsychological Assessment of Schizophrenia Patients.

Objective: Persons with schizophrenia (SCZ) show impairments of odor identification and odor discrimination. Whether these reflect overall cognitive dysfunction or a unique form of disease morbidity remains unclear. Here we attempt to elucidate the relationship between cognitive and olfactory dysfunction in SCZ.

Participants and Methods: Adults with first-episode SCZ (n=63; $76 \%$ male; $22+4$ years old) and healthy adults ( $\mathrm{n}=63 ; 67 \%$ male; $24+4$ years old) completed tests of odor identification and discrimination (Sniffin' Sticks), and 9 other cognitive tests (Grooved Pegboard, Perceptual Comparison Speed, Digit Span, Brief Test of Attention, Modified Wisconsin Card Sorting Test, Calibrated Ideational Fluency Assessment, Hopkins Verbal Learning Test, and Brief Visuospatial Memory Test). Pearson correlation was used to examine relationships between olfactory and cognitive variables. Stepwise multiple regression was used to find which cognitive variables best predict olfactory functioning, and stepwise linear discriminant analysis was used to test whether olfactory measures help discriminate SCZ from healthy adults.

Results: In healthy adults, olfactory measures showed almost no relationship with cognitive performance. In persons with SCZ, odor discrimination correlated with executive functioning (M-WCST categories and perseverative errors). Odor identification correlated with multiple cognitive measures (GPT, DSp, BTA, HVLT-R learning and recall). Among patients, odor discrimination was best predicted by M-WCST perseverative errors and letter word fluency, and odor Identification was best predicted by GPT speed. Finally, stepwise LDA revealed that GPT speed, letter word fluency, and odor discrimination each help distinguish SCZ from healthy adults.

Conclusions: Odor identification and discrimination share attributes with other cognitive processes, but still measure unique qualities in persons with first-episode schizophrenia.

Correspondence: Jeffrey L. Crawford, Bachelor of Arts in Psychology, Medical Psychiatry, Johns Hopkins University, 3307 Pinole Valley Road, Baltimore, MD 21231, United States. E-mail: jcrawf29@jhmi.edu

A.M. HENRICKS, A.S. FISCHER, R.M. ROTH, M.F. BRUNETTE \& A.I. GREEN. Comorbid Cannabis use Disorder is not Associated with Improved Cognition in Patients with Schizophrenia.

Objective: Up to $42 \%$ of patients with schizophrenia (SCZ) also have a co-occurring cannabis use disorder (CUD), which is associated with a more severe course of illness in these patients. However, some studies suggest that patients with SCZ and CUD (dual diagnosis) outperform patients with SCZ alone on many cognitive tasks, while others report no effects of comorbid cannabis use on cognition. The present study thus compared patients with dual diagnosis to those having CUD alone or SCZ alone to better understand the impact of cannabis use on cognition in patients with SCZ.

Participants and Methods: Healthy comparison (HC) subjects $(\mathrm{n}=22)$, individuals with CUD $(n=27)$, patients with SCZ $(n=9)$, and patients with dual diagnosis $(n=24)$ participated in the study, and were required to be abstinent from cannabis, alcohol, and illicit drugs for at least four days prior to neuropsychological testing. The neuropsychological battery included assessments of processing speed (Symbol Digit Modalities Test; SDMT) and working memory (Letter Number Sequencing; LNS). A univariate ANOVA was used to analyze differences in cognitive performance across groups, followed by post-hoc analysis using Tukey's HSD for multiple comparisons.

Results: There were no significant differences between groups for age, gender, handedness, or ethnicity (all p's $>0.05$ ), but HC had significantly more years of education than all other groups (all p's $<0.01$ ). When controlling for years of education, HC performed better on the SDMT and LNS than the SCZ and dual diagnosis groups (all p's<0.05).

Conclusions: Processing speed and working memory were impaired in patients with SCZ and those with dual diagnosis relative HC subjects. Contrary to some reports, we did not observe any difference in cognitive functioning between the two SCZ groups. Furthermore, patients with CUD only did not differ from HC subjects. Thus, these findings do not indicate that cannabis has protective effects on cognition in patients with SCZ. Correspondence: Angela M. Henricks, Ph.D., Psychiatry, Dartmouth College, PO Box 601, Grantham, NH 03753, United States. E-mail: angela.m.henricks@dartmouth.edu

A. JACKSON, L. PINEAUlT, L. BUChanaN, L. FlashMAN \& R. ROTH. Semantic Processing On Verbal Fluency Tasks In Schizophrenia Spectrum Disorder.

Objective: Individuals with schizophrenia spectrum disorders (SSD) exhibit abnormalities in semantic processing. They also demonstrate worse performance on verbal fluency tasks, particularly category fluency, as compared to healthy comparison subjects (HC). A novel approach to investigating semantic processing abnormalities is analysis of semantic neighborhood density (SND). SND is based on a co-occurrence model of semantic organization, whereby words that commonly occur together in everyday language are located near each other in semantic space. SND is a variable that represents the proximity of such neighbors to a target word. In the present study, we used analysis of SND on verbal fluency tasks in persons with SSD to further characterize semantic processing abnormalities.

Participants and Methods: Participants included 41 SSD and 32 HC. Performance on D-KEFS letter and category fluency tasks was scored for total correct raw score and mean SND. Mean SND included numerical values for each word that was averaged across tasks.

Results: The SSD group generated words from sparser semantic neighborhoods during letter fluency and from denser semantic neighborhoods during category fluency. SND was not associated with raw scores. Symptomology was associated with SND on the category fluency task, whereby increased symptoms were associated with the production of words from sparser semantic neighborhoods.

Conclusions: SSD was associated with abnormal semantic processing consistent with difficulty inhibiting semantic neighbors. They produced words from less dense neighborhoods when the inhibition demand was greater (letter fluency) and from denser neighborhoods when the same demand was more limited (category fluency). This difficulty would cause greater spreading of activation in semantic space, which may explain some symptoms of SSD (e.g., loose associations).

Correspondence: Andrea Jackson, Dartmouth-Hitchcock Medical Center, 1 Medical Center Drive, Lebanon, NH 03756, United States. E-mail:jackson.anniem@gmail.com

N. SHAAFI KABIRI, N. MENDEZ-LOZANO, A. COTE, B. DUPEE, J. BHANGU \& K. THOMAS. Intra-Individual Variability and its Relations with Cognitive Functioning in Healthy Volunteers and Subjects with Schizophrenia.

Objective: Stop Signal Task (SST) trial-by-trial fluctuations in reaction-time (RT) were used to measure Intra-individual Variability (IIV) to (1) Explore IIV in healthy volunteers (HV) and subjects with schizophrenia (SZ); (2) Evaluate the relationship between IIV with other cognitive tasks. 
Participants and Methods: Thirty-one HV and thirty SZ adults completed SST, MATRICS Cognitive Consensus Battery (MCCB), Attention Network Task (ANT), and Change Localization (CL) twice ( $14 \pm 2$ days). IIV was measured by calculating intra-individual standard deviation (iSD), intra-individual coefficient of variance (iCV), and modeling RTs as an Ex-Gaussian distribution (mu, sigma, tau). Pearson's correlations were calculated for visit 1 .

Results: $H V$ results: Improved only in visit 2 iSD and sigma (all $\mathrm{p}<.05)$. iSD, iCV, and tau showed good test-retest reliability (all ICC>.6, all $\mathrm{p}<.001$ ); mu and sigma showed fair test-retest reliability (all ICC $>.4$, all p<.05). IIV correlations with MCCB: iSD with Continuous Performance Task-Identical Pairs 4D (r=.52, $\mathrm{p}<.01)$, tau with reasoning and problem solving $(\mathrm{r}=-.52, \mathrm{p}<.01)$, and tau with overall composite score $(\mathrm{r}=-.47$, $\mathrm{p}<.05)$. IIV correlations with ANT: iSD with Grand Mean Effect (GME) $(\mathrm{r}=.43, \mathrm{p}<.05)$, tau with GME $(\mathrm{r}=.43, \mathrm{p}<.05)$, and mu with Alerting Effect $(r=-.47, p<.01)$. In CL, mu showed correlation with Working Memory Capacity $(\mathrm{r}=.37, \mathrm{p}<.05)$.

SZ Results: iSD showed good test-retest reliability (ICC=.7, p<.001); $\mathrm{iCV}, \mathrm{mu}$, sigma, and tau showed fair test-retest reliability (all ICC $>.5$, all $\mathrm{p}<.01)$. IIV correlation with ANT task: iSD correlated with GME $(\mathrm{r}=.39, \mathrm{p}<.05)$.

No other associations were observed.

Conclusions: In SZ, smaller IIV was associated with better performance only in attention (GME). In HV, smaller IIV was associated with better performance in multiple areas of cognition (reasoning and problem solving, overall MCCB composite score, attention, and working memory). The Ex-Gaussian approach, iSD, and iCV showed fair to good test-retest reliability.

Correspondence: Nina Shaafi Kabiri, Anatomy and Neurobiology, Boston University School of Medicine, 650 Albany Street, Unit 140, Boston, MA 02118, United States.E-mail:nskabiri@bu.edu

M. MATSUI, K. EBINA, Y. INADA, S. NAKADA, E. KADOMOTO, Y. HIGUCHI \& M. SUZUKI. Subjective and Objective Cognitive Impairment in Schizophrenia.

Objective: The presence of cognitive impairment in patients with schizophrenia has been recognized. The purpose of this study was to examine whether subjective evaluation and objective assessment of cognitive function of schizophrenia patients were consistent or not. In addition, we compared subjective evaluation by patients with that of their family.

Participants and Methods: Participants were 45 patients who met ICD-10 criteria for schizophrenia. The average age was $35.1 \pm 9.4$ (SD) years old, the average duration of education was $14.9 \pm 4.0(\mathrm{SD})$ years, the mean duration of illness was $11.6 \pm 8.4(\mathrm{SD})$ years, the mean onset age was $23.5 \pm 6.6(\mathrm{SD})$ years old, and every premorbid IQ measured using the Japanese Adult Reading Test (JART) was over 90. We conducted the Schizophrenia Cognition Rating Scale (SCoRS), which allow to evaluate subjective cognitive functions on daily life, individually for patients and families, and examined everyday life skills, processing speed, memory function, language function, attention function using neuropsychological tests as objective evaluation.

Results: We analyzed the relationship between the subjective evaluation by the patients themselves and their objective evaluation of the cognitive function, so that there was no relationship between subjective evaluation by patients and objective evaluation in all the items. As a result of comparing the evaluated values of the patients themselves and their family, the total score was significantly higher for the patients themselves than for the family rating.

Conclusions: Schizophrenia patients are expected to have insufficient insight into cognitive function and it may be difficult to correctly evaluate their cognitive dysfunction in some cases. It was also suggested that patients with schizophrenia tend to evaluate their own cognitive impairment more heavily than their families.
Correspondence: Mie Matsui, PhD, Clinical Cognitive Neuroscience, Kanazawa University, Kakuma-machi, Kanazawa 9201192, Japan. E-mail:miematsui@staff.kanazawa-u.ac.jp

A. MOLL, A.Z. KRAAL, N. ARVANITIS, R. DOUGHERTY, T. GROVE, K. BURGHARDT \& V. ELLINGROD. Endothelial Nitric Oxide Synthase (eNOS), Metabolic Syndrome, and Cognition in Schizophrenia-Spectrum Disorders.

Objective: Poor cognition is prevalent in schizophrenia and associated with metabolic syndrome (MetS), but mechanisms underlying this relationship remain unclear. The $\mathrm{C}$ allele of the $e N O S$ gene $\mathrm{T}^{-786} \mathrm{C}$ polymorphism has been associated with increased risk of MetS, and preliminary animal research has shown that eNOS inactivation worsens cognition. However, the association between eNOS-786, MetS, and cognition in schizophrenia remains unexplored. Thus, to clarify the mechanism underlying the MetS-cognition association, this study examined the effects of eNOS $\mathrm{T}^{-786} \mathrm{C}$ and MetS on cognition in schizophrenia-spectrum disorders.

Participants and Methods: Cross-sectional data from 139 (mean age: $44.2 \pm 11.5$ years; $42.4 \%$ female; $41.7 \%$ White) community-dwelling participants diagnosed with a schizophrenia-spectrum disorder were analyzed. Assessments included: genotype of eNOS $\mathrm{T}^{-786} \mathrm{C}$, MetS screening using established guidelines, and cognition (Brief Assessment of Cognition in Schizophrenia; BACS) transformed into z-scores. Groups were formed according to eNOS $\mathrm{T}^{-786} \mathrm{C}$ carrier status and MetS diagnosis. Forty-four $(31.7 \%)$ participants were eNOS C-carriers and $70(50.4 \%)$ were diagnosed with MetS.

Results: Factorial Analysis of Covariance (covariates: age, education, and race) showed group differences in global cognition as indexed by BACS total z-scores. Specifically, C-carriers without MetS performed notably better in global cognition compared to the other groups, and were the only group with above average scores. Similar findings were found in domains of 1) semantic fluency and 2) attention and speed of processing.

Conclusions: These preliminary results suggest that genetic variation in eNOS may partially explain the association between MetS and cognition. Specifically, presence of MetS may remove the potentially beneficial effect of the $\mathrm{C}$ allele in buffering against mechanisms contributing to the poor cognitive function typically demonstrated in schizophrenia. Correspondence: Allison Moll, University of Michigan, 1211 Prospect St, 1211 Prospect St, Ann Arbor, MI 48104, United States. E-mail: acmoll@umich.edu

\section{Executive Functions/Frontal Lobes}

J.L. PRILUCK, M. HAMZA, P. FEDIO \& A.A. FEDIO. Verbal Fluency for Emotion Following Traumatic Brain Injury.

Objective: The purpose of this study was to extend assessment of verbal fluency to the emotional domain by studying generation of positive, negative, and neutral words compared with traditional clinical measures of verbal fluency in individuals with brain injury.

Participants and Methods: Seventeen individuals with moderate/ severe TBI (10M/7F, mean age: 35; 4 years post injury; 16 with documented/self-reported frontal lobe damage) completed verbal fluency tasks (CFL; Animals), Neurobehavioral Functioning Inventory (NFI), and an experimental measure requiring generation of positive, negative, or neutral words, each within $60 \mathrm{sec}$.

Results: Sample mean for CFL $(32.9 \pm 12)$ was approximately one standard deviation below published norms $(38.1 \pm 7.9)$, and Animals sample mean was consistent with published norms. CFL fluency correlated moderately with Animal fluency $(r=.46)$ and strongly with Negative fluency ( $(r=.85)$, but not significantly with Positive fluency $(r=.27)$. Negative $(r=.69)$ and Positive $(r=.55)$ fluency each correlated with Animal fluency. Patients' self-rating of neurobehavioral functioning 
was within the average range with females scoring higher than males on complaints of memory/attention problems. Independent samples t-tests revealed that females generated more neutral $(p<.02)$ and negative $(p<.09)$ words than did males. There were no gender differences for Positive fluency, Animal fluency, or CFL, although females generated more CFL words than did males.

Conclusions: Moderate/severe TBI patients with frontal lobe involvement demonstrated the expected pattern of impaired phonemic fluency relative to semantic fluency based on published norms. Negative fluency correlated strongly with phonemic fluency and moderately with semantic fluency. Moreover, only Negative and Neutral word fluency yielded a gender bias in the expected direction benefiting females, who typically are more verbally expressive than males. Findings suggest the benefit of further study of emotion word generation as a component of verbal fluency.

Correspondence: Jacob L. Priluck, Master of Clinical Psychology, Clinical Psychology, American School of Professional Psychology at Argosy University, 2311 Hanway Road, Baltimore, MD 21209. United States.E-mail: jlpriluck@gmail.com

\section{Invited Symposium 1. Multiple Trace Theory (MTT) at 20: A Review of Theoretical, Empirical and Clinical Developments, and Future Directions}

\author{
Chair: Morris Moscovitch \\ Presenters: Lynn Nadel, Gordon Winocur, \\ Mary Pat McAndrews, Morris Moscovitch
}

9:00-10:30 a.m.

M. MOSCOVITCH, L. NADEL, G. WINOCUR \& M. MCANDREWS. Multiple Trace Theory at 20: A review of theoretical, empirical and clinical developments, and future directions.

Memory is not static, but can change with time and experience, from context-specific detailed (episodic) memories dependent on the hippocampus and related structures, to context-general gist-like or schematic memories that are dependent on extra-hippocamapal, neocortical structures. Multiple Trace Theory (MTT) and Trace Transformation Theory (TTT) were proposed to account for the neural organization that underlies the retention and retrieval of both types of memory from acquisition to retention over long intervals. Our working hypothesis is that changes in memory representation are accompanied by corresponding changes in neural organization. In this symposium we review the theoretical, empirical and clinical contributions made by these theories, note some of their limitations, and consider future directions.

Correspondence: Morris Moscovitch, PhD, Psychology, University of Toronto/Rotman Research Institute, 100 Saint George Street, Toronto, ON M5S 3G3, Canada.E-mail:momos@psych.utoronto.ca

\section{NADEL. MTT: Origins and Predictions.}

Multiple Trace Theory (MTT) was proposed as an alternative to the standard model of memory consolidation, which holds as its central premise that both forms of explicit memory (episodic and semantic) are initially dependent on the hippocampus, but eventually become established in their original forms in other brain regions. MTT, by contrast, proposed a critical distinction between episodic and semantic memory, with the former remaining dependent on the hippocampus for as long as the episode memory in question was detailed and vivid. In this presentation I will review the origins and central premises of MTT, and research that addressed its core postulates, including (1) the distinct fates of episodic and semantic memories; (2) the critical role played in episode memories of the (typically spatial) context in which the episode transpired; and (3) the idea that retrieving an episode memory can lead to alterations in the memory that reflect the current retrieval context.
Correspondence: Lynn Nadel, PhD, Department of Psychology, AZ, United States.E-mail:nadel@email.arizona.edu

G. WINOCUR. Trace Transformation Theory and its Implications. As an alternative to the standard theory of memory consolidation, trace transformation theory (TTT) builds on multiple trace theory (MTT) by specifying the relationship between episodic and semantic memory. In line with MTT, TTT posits that a memory, when it initially forms, is episodic, context-specific, and hippocampus-dependent for as long as it is retained. However, with time and experience, it undergoes a transformation process that supports the development, in extrahippocampal structures, of a semantic (schematic) version that retains essential features of the original memory (gist), but few of its contextual details. The two types of memory co-exist with a dynamic interplay between them that enables either to be dominant depending on demands at retrieval. Evidence in support of TTT from the animal and human literatures will be discussed.

Correspondence: Gordon Winocur. E-mail: gwinocur@research. baycrest.org

M. MCANDREWS. Clinical Implications of Multiple Trace and Trace Transformation Theories.

Multiple Trace and Trace Transformation Theories have changed dramatically the way we appreciate episodic, and particularly autobiographical, memory deficits associated with medial temporal lobe damage. When patients attempt to recover personal memories. gist information is often retained but the vivid perceptual details that contribute to 're-experiencing' autobiographical memories are inaccessible. In patients with temporal lobe epilepsy (TLE), this is true even with limited damage to one hippocampus and even in the context of relatively normal episodic memory on neuropsychological tests, which is important in reconciling the individual's experience of memory dysfunction with the more benign 'objective' data. Building on the implications of MTT and TTT for brain networks has enabled us to devise fMRI connectivity measures that aid in prediction of memory decline following surgery for TLE. As these theories have also evolved to help understand how hippocampally-mediated memory processes influence other cognitive domains, such as future thinking and problemsolving, we can better prepare patients for those types of outcomes from surgery in TLE or disease progression in aMCI/Alzheimer's Disease. Although the rehabilitative potential of these theories has not been explored extensively, in at least one instance the link between autobiographical memory and hippocampus has led to a novel program of brain stimulation research for Alzheimer's Disease. In this talk I will elaborate on the past and imagine the future clinical implications of these influential theories.

Correspondence: Mary Pat McAndrews. E-mail: mcandrws@playfair. utoronto.ca

\section{MOSCOVITCH. MTT and TTT: Limitations and Future Directions.}

MTT and TTT provide a good account of the neural correlates of episodic/ autobiographical memory retention and retrieval from acquisition to retention over long intervals. In this presentation, I will discuss some of the limitations of these theories, how they may be addressed, and what implication these theories have for understanding other functions that rely on episodic memory, such as problem solving, imagination and language, all of which can become impaired following damage to the medial temporal lobe.

Correspondence: Morris Moscovitch, PhD, Psychology, University of Toronto/Rotman Research Institute, 100 Saint George Street, Toronto, ON M5S 3G3, Canada.E-mail:momos@psych.utoronto.ca 


\section{Symposium 1. Remote and Progressive Effects of Brain Injury: Novel Findings and Novel Treatment Approaches}

\section{Chair \& Discussant: Robin Green}

\section{Presenters: Brenda Colella, Alexander Terpstra, Brandon Vasquez}

9:00-10:30 a.m.

R. GREEN, B. COLELLA, A. TERPSTRA \& B. VASQUEZ. Remote and Progressive Effects of Brain Injury: Novel Findings and Novel Treatment Approaches.

The proposed symposium will build on our group's research into the chronic and degenerative effects of traumatic brain injury (TBI), focusing on two related populations: retired Canadian Football League players with a history of multiple concussions, and adults with moderate-severe TBI. The first presenter (Brenda Colella) will provide an overview and update on findings from the Toronto TBI Recovery Study, our longitudinal program of research that has revealed neural, cognitive and mood declines in chronic TBI, as well as novel treatment targets. The second presenter (Alex Terpstra) will move onto our more recent explorations of novel treatment targets in his examination of inconsistency of responding (or "variability"), which is an index of executive control. Variability was the most salient cognitive characteristic distinguishing retired athletes with high vs. low concussion histories, and it mediated the relationship between concussion history and manifestation of neuropsychiatric deficits. Thus, treating variability might avert psychiatric dysfunction in this population. The third speaker (Brandon Vasquez), will discuss his more novel approach to the examination of variability in moderate-severe TBI (using ex-gaussian parameters). He reveals, (i) a deeper understanding of elevated variability in TBI, and (ii) that variability worsens in the chronic stages of TBI. He also discusses promising treatments for variability. The Discussant (Robin Green), will synthesize all of the findings presented, and describe how they are being implemented in our new centre for chronic TBI. The centre, which permits scaleability through the use of telehealth, permits patients in local and remote areas to receive assessment and treatment through research participation. We see such dual-purpose, telehealth enterprises as the future of neurorehabilitation. We will end with a panel discussion with the audience (led by RG) to discuss the future of neurorehabilitation for chronic TBI.

Correspondence: Robin Green, PhD CPsych, Research, Toronto Rehab, Toronto Rehab, 550 University Avenue, Toronto, ON M5A2G2, Canada. E-mail:Robin.Green@uhn.ca

B. COLELLA, M. BAYLEY, J. GLAZER, A. TERPSTRA \& R. GREEN. Neural, cognitive and mood decline in the chronic stages of moderate-severe TBI: Implications for treatment.

Objective: To review research by our group into the chronic and degenerative effects of TBI and discuss clinical implications.

Participants and Methods: 160 patients with moderate-severe TBI took part in a longitudinal, recovery study with assessments undertaken at 2, 5, 12 and 24+ months post-injury. Investigations included (i) quantitative MRI, including diffusion tensor imaging (at 5, 12 and 24+ months only), (ii) comprehensive, performance-based clinical neuropsychological assessment and (iii) self-report neuropsychiatric tests at all time points. Repeated measures ANCOVAs, reliable change index (RCI), and mixed effects modelling were employed to analyze results. Pearson correlation was used to examine cognitive environmental enrichment as a predictor of hippocampal atrophy. Bivariate latent difference score modelling was employed to examine anxiety as a predictor of hippocampal atrophy.

Results: Neuroimaging revealed atrophy (whole brain, hippocampi and corpus callosum) and loss of white matter integrity (fornix) from 5 to 12 to $24+$ months, with only modest impact of age and years of education on trajectories. RCI analyses showed an increasing number of patients declining across time on two or more cognitive tests. The proportion of patients with moderate or greater symptoms of depression and anxiety was found to double from 2 months to two years post-injury. Reduced cognitive environmental enrichment and increased anxiety were associated with increased hippocampal atrophy.

Conclusions: Clear deterioration across time on neuroimaging, cognitive and mood outcomes reveal a critical need to treat patients in the chronic stages of injury. Post-injury, treatable risk-factors for decline include anxiety and insufficient cognitive stimulation. Ongoing identification of post-injury risk-factors that can be targeted for treatment is needed.

Correspondence: Brenda Colella, Toronto, ON, Canada. E-mail: brenda.colella@uhn.ca

A. TERPSTRA, T.A. GIRARD, B. COLELLA \& R. GREEN. Remote Effects of Multiple Concussions in Retired Professional Athletes. Objective: To characterize the remote neuropsychiatric and cognitive effects of multiple concussions on high-functioning retired professional athletes to identify novel neurorehabilitation targets

Participants and Methods: Fifty retired Canadian Football League players were administered a comprehensive, performance-based clinical neuropsychological assessment and comprehensive neuropsychiatric assessment, a combination of self-report measures (the Personality Assessment Inventory) and structured clinical interview (the Mini International Neuropsychiatric Inventory), and a comprehensive neuropsychological battery that included executive control measures (response inhibition and intra-individual variability [IIV]).). 30 controls casematched for age and years of education underwent the same measures. Results: (1) Retired athletes scored significantly higher than controls on the Mania and Aggression scales of the PAI, and significantly worse on an index of executive control. (2) Retired players in a high-concussion sub-group (with 4 or more concussions; mean=6.48) showed significantly elevated Depression, Mania and executive dyscontrol (i.e., response inhibition and IIV) as compared to low-concussion participants (3 or fewer concussions; mean=1.84). (3) IIV was found to mediate the relationships of concussion exposure with Mania and Aggression scores. Conclusions: Higher concussion exposure was associated with acquired elevations in mania, aggression, and depression symptoms, and executive dyscontrol. Results raise the hypothesis that executive control (indexed by IIV), mediates the relationship between concussion exposure and neuropsychiatric symptoms, whereby the neuropsychiatric symptoms manifest following high concussion exposure only when executive dyscontrol is present. The findings suggest that executive dyscontrol should be further researched as a potential treatment target for neuropsychiatric symptoms, which cause notable distress in this population. Correspondence: Alexander Terpstra. E-mail: alexander.terpstra@ gmail.com

B. VASQUEZ, B. COLELLA, J. TOMASZCZYK \& R. GREEN. Variability Increases in the Chronic Stages of Moderate-Severe TBI.

Objective: There is growing evidence that early cognitive recovery is followed by later declines in the chronic stages of injury. We examined longitudinal recovery of an index of executive control functioning, behavioural inconsistency, which can be measured by the extent of response variability across trials of a reaction time (RT) task. Persisting impairments in variability have been observed in TBI patients even after other cognitive impairments have resolved. We used ex-Gaussian analysis to examine the components of variability. Participants and Methods: A secondary analysis of data from 70 patients with moderate-severe TBI was undertaken. The patients had completed a go/no-go RT task at 2, 5, 12 and 24+ months post-injury. Ex-Gaussian parameters (mu, sigma and tau) as well as mean, intra-individual coefficient of variation 
[ICV], and intra-individual standard deviation [ISD]) were analyzed with repeated measures MANOVA. We also examined extremely fast responses (i.e., less than $150 \mathrm{msec}$ ) as an index of impulsivity. Results. Early recovery from 2 to 5 to 12 months post-injury was observed on a number of indices (ICV, ISD and tau [i.e., extremely slow responses]), but followed by later decline. The frequency of extremely fast responses showed the same pattern of post-recovery decline. Age accentuated this quadratic pattern, which was particularly notable in extremely fast and slow responses. Conclusions. The study confirms that variability is a highly sensitive index of impairment in TBI. Inconsistency - an index of reduced executive control - is a common complaint in moderate-severe TBI. Encouragingly, several interventions show promise for reducing inconsistency (e.g., meta-cognitive strategies).

Correspondence: Brandon Vasquez.E-mail: brandon.vasquez@uhn.ca

\section{Symposium 2. Neuropsychology of Pediatric Neuroimmunological Diseases}

\section{Chair, Presenter, \& Discussant: Alison Wilkinson-Smith}

\section{Presenters: Lana Harder, Robyn A. Howarth}

\section{9:00-10:30 a.m.}

\section{A. WILKINSON-SMITH, L. HARDER \& R.A. HOWARTH. Neuropsychology of Pediatric Neuroimmunological Diseases.}

Pediatric neuroimmunology is a rapidly changing field. Neuroimmunological diseases often involve complex patterns of symptoms that include neurological, psychiatric, cognitive, and psychosocial features. Neuropsychologists play a vital role in characterizing and monitoring symptoms, particularly in response to treatments and also provide the medical team with a richer picture of disease status than they would be able to access through parent report and laboratory data alone. Importantly, neuropsychologists contribute to the growing body of scientific knowledge to promote understanding of these conditions to inform treatment planning. When managing diseases for which treatment guidelines are evolving or nonexistent, neuropsychology's contribution is often invaluable. On an individual level, neuropsychologists describe and document how symptoms and treatments impact a patient's day-to-day life and advocate for their needs in various settings, and develop tailored treatment plans that can improve outcomes in a variety of domains.

In this symposium, we will present relevant information regarding several neuroimmunological diseases that affect pediatric populations. We will start with diseases about which relatively more is known, followed by conditions that are less well understood, but which nonetheless require a thoughtful plan for management. We will review empirical data where applicable, including original research findings, and provide examples from case studies to illustrate important points. Throughout this integrated presentation, we will emphasize the need for pediatric neuropsychologists to play a role in coordinated multidisciplinary care in order to promote the best outcomes for these patients. We will also define key areas for future directions of research.

Correspondence: Alison Wilkinson-Smith, PhD, Children's Medical Center Dallas, 6300 Harry Hines, Suite 900, Dallas, TX 75235, United States.E-mail: alison.wilkinson-smith@childrens.com

\section{HARDER. Neuropsychology of Pediatric Multiple Sclerosis and Related Disorders.}

Pediatric central nervous system (CNS) disorders, impacting the brain, spinal cord, and/or optic nerve, present patients with an array of challenges impacting cognitive performance. Additionally, patients regularly experience a host of symptoms including those related to internalizing mood symptoms and fatigue. Perhaps the most well-known of these conditions is multiple sclerosis (MS), a chronic inflammatory disease of the brain and spinal cord. Neuropsychological outcomes in adult MS is well documented. Over the last decade, significant literature describing pediatric MS and associated neuropsychological outcomes has emerged. It has been established that approximately one-third of these patients experience cognitive impairment, though a clear profile has yet to be established. While less is known about cognitive functioning among patients with Acute Disseminated Encephalomyelitis (ADEM), Transverse Myelitis (TM), and Neuromyelitis Optica (NMO), emerging literature suggests patients with these conditions are also at risk for neuropsychological deficits. Research on these populations, focused on our center's original research, will be presented and will include descriptions of neuropsychological and psychosocial outcomes, including educational outcomes, as well as imaging findings from a pilot study on transverse myelitis.

Correspondence: Lana Harder, PhD, Psychiatry and Neurology, Children's Medical Center Dallas/UT Southwestern, 1935 Medical District Drive, CH 9.01, Dallas, TX 75235, United States. E-mail: lana. harder@childrens.com

\section{R.A. HOWARTH. Neuropsychology of Anti-NMDA Receptor Encephalitis.}

Anti-NMDA receptor encephalitis is a recently described auto-immune disorder with a predictable clinical course. Early symptoms include headache, fever, and nausea followed by memory difficulties, psychiatric symptoms, and/or personality changes. Psychiatric symptoms may present as acute psychosis, anxiety, hallucinations, and/or irritability, which may result in psychiatric hospitalization. Seizures, altered mental status, movement disorders, mutism, catatonia, insomnia, and/or autonomic instability are also common. Diagnosis is confirmed through testing of the cerebrospinal fluid (CSF) and/or serum for the presence of anti-NMDA receptor antibodies. Neuroimaging is often unremarkable, although EEG findings may be abnormal. Contrary to the adult literature, pediatric patients are less likely to have an ovarian teratoma. Treatments include first-line immunotherapy (steroids, IVIG, PLEX), second-line immunotherapy (rituximab, cyclophosphamide), and/or medications for symptom management.

The clinical presentation of patients with anti-NMDA receptor encephalitis is associated with decline in motor functioning, independence with activities of daily living (ADL's), and cognitive-linguistic skills. As such, individuals may be referred for intensive rehabilitation services with medical management and monitoring up to several years following diagnosis. Neuropsychologists are often an integral part of the treatment team and responsible for assessing and monitoring changes in cognitive functioning across the disease course. Persistent cognitive impairments have been documented in the adult literature, including deficits in attention, executive functioning, memory, and language. However, little is known about the early functional outcomes and trajectory of neurocognitive recovery in pediatric patients with anti-NMDA receptor encephalitis. Results of original research on the outcomes associated with anti-NMDA receptor encephalitis in a pediatric sample will be presented. Correspondence: Robyn A. Howarth, Ph.D. , Neuropsychology, Children's Healthcare of Atlanta, Children's Healthcare of Atlanta, Neuropsychology, 5455 Meridian Mark Rd. NE Suite 180, Atlanta, GA 30342, United States. E-mail: Robyn.Howarth@choa.org

\section{A. WILKINSON-SMITH. Neuropsychology of Antibody-Negative and Presumed Autoimmune Encephalitis.}

Neurologists and neuroimmunologists often encounter patients with symptoms that appear to represent some kind of autoimmune encephalitis, but for which a definitive diagnosis cannot be identified. These patients can be extremely challenging to manage, for a variety of reasons. There may be a struggle to differentiate these patients from those experiencing primary psychiatric disorders, including functional neurological disease, and there are certainly patients who experience both. The sparse literature addressing this population recommends a systematic approach to diagnosis and treatment (e.g., Graus et al., 
2016), which we will review. We will discuss the unique challenges of applying these diagnostic methods within a pediatric population, and we will describe the role of neuropsychology in addressing these challenges. We will present an overview of data from our clinic showing the significant variability in neuropsychological functioning in these patients, as well as highlighting some common concerns, particularly regarding emotional/behavioral and memory functioning. We will also present longitudinal data from a case study that emphasizes the importance of neuropsychology within the multidisciplinary team.

Correspondence: Alison Wilkinson-Smith, PhD, Children's Medical Center Dallas, 6300 Harry Hines, Suite 900, Dallas, TX 75235, United States. E-mail: alison.wilkinson-smith@childrens.com

\section{Paper Session 1. Assessment}

\section{Moderator: Justin B. Miller}

\section{9:00-10:30 a.m.}

J. STIVER, S. WALTERS, E. FOX, M.Y. YOU, M. ALTENDAHL, R. SALONER, A.M. STAFFARONI, M. GORNO-TEMPINI \& J. KRAMER. The Rapid Naming Task: Pilot Data on a Novel Naming Paradigm for Older Adults.

Objective: Word-finding difficulty is a primary cognitive complaint among older adults. Classic measures of confrontation naming, however, were designed to assess aphasia; they tend to be negatively skewed in older normals and are insensitive to subtle change. To address the longstanding need for a more sensitive naming test, we developed the Rapid Naming Task (RNT), a one-minute, computerized, speeded naming test. Participants and Methods: Cognitively intact older adults $(n=80$; mean age $=75.4$ ) were administered the RNT, abbreviated Boston Naming Test (aBNT), Stroop Color Naming (CN), WRAT-4 reading, and phonemic fluency. On the RNT, examinees are instructed to name as many visually presented object drawings as possible within one minute; the time limit for each item is five seconds. A subset of subjects $(n=50)$ was given the Everyday Cognition Self-Report questionnaire to assess for word-finding complaints.

Results: Mean RNT score was 34.5 ( $S D=5.5$; range 22-44). Scores were normally distributed and strongly associated with age $(r=-.51, p<.001)$. Linear regression, controlling for age, sex, and education, showed that RNT performance was associated with subjective language complaints $(\beta=-.33, p<.05)$. Even after also controlling for the aBNT, non-semantic rapid naming $(\mathrm{CN})$, reading, and phonemic fluency, the RNT remained a predictor of word-finding complaints $(p<.05)$, uniquely adding an additional $12 \%$ of the variance.

Conclusions: Results provide evidence of the RNT's association with subjective real-world language difficulties in healthy older adults. This relationship with self-reported complaints remains significant even after adjusting for non-semantic naming speed, reading, verbal fluency, and aBNT performance, suggesting that the RNT is assessing aspects of word finding not tapped by these measures. In addition, RNT scores are normally distributed and negatively correlated with age. This study represents a key initial step in the development of a psychometrically robust test capturing subtle age-associated language changes.

Correspondence: Jordan Stiver, Fordham University, 441 E. Fordham Road, New York, NY 10458, United States.E-mail: jstiver@fordham.edu

M.C. MUNIZ, A. LAMBERT, Y.T. QUIROZ, E. MCCORD, J. MARTINEZ, K. JOHNSON, R. SPERLING, D.M. RENTZ \& K.V. PAPP. The Relationship Between Performance Errors and Amyloid Burden in Clinically Normal Older Adults.

Objective: Alzheimer's disease (AD) dementia is associated with not only decrements in summary cognitive metrics, but also increases in performance errors. It is unclear whether increases in performance errors are detectable earlier in the AD trajectory. Thus, our aim was to examine the relationship between errors on neuropsychological tasks and amyloid deposition on Positron Emission Tomography (PET) both cross-sectionally and longitudinally in clinically normal (CN) older adults.

Participants and Methods: $284 \mathrm{CN}$ older adults in the Harvard Aging Brain Study (mean age $=75.9,60 \%$ female, mean education $=15.89$, mean follow-up $=4$ years) underwent annual evaluations including Semantic (CAT) and Phonemic (FAS) fluency tasks, Trail Making Test (TMT), and the Selective Reminding Test (SRT). Intrusions and perseverative errors were collected for CAT, FAS, and SRT and ordering and set-shifting errors were recorded for TMT. Amyloid $\left(11^{\mathrm{C}}\right.$ PiB-PET) was measured in an aggregate of cortical regions. Linear mixed models controlling for age, sex, and education were used to assess performance differences, including errors, in relation to amyloid deposition at baseline and longitudinally.

Results: High amyloid levels were associated with decline in CAT $(p<0.001)$, SRT total $(p<0.001)$, and both TMT A $(p=0.006)$ and B $(p=0.006)$, but not FAS. Participants made more errors (both intrusions and perseverations) on verbal fluency tasks regardless of amyloid status over time. Higher amyloid was associated with an increase in commitment of SRT intrusions $(p<0.001)$ and TMT sequencing errors $(p=0.003)$ but not other errors.

Conclusions: The more robust relationships between high amyloid and cognitive decline on overall performance versus errors committed suggests that error counts on traditional neuropsychological tests may not add robust predictive information about cognitive decline in preclinical AD. However, further exploration as to why SRT intrusions errors and TMT sequencing errors are particularly burdened by amyloid status is warranted.

Correspondence: Martha C. Muniz, Master of Arts, Neurology, MGH, 358 Commonwealth Avenue, \#11, Boston, MA 02115, United States. E-mail:mmuniz2@partners.org

A.G. ALIOTO, S. ERLHOFF, K.B. CASALETTO, P. MUMFORD, J. KRAMER, K.H. RANKIN \& K.L. POSSIN. White matter correlates of the UCSF Brain Health Assessment.

Objective: The UCSF Brain Health Assessment (BHA) is a 10-minute tablet-based assessment for the detection and diagnosis of neurocognitive disorders. The neuroanatomic validity of the 3 new tests of memory (Favorites), executive functions / speed (Match), and visuospatial skills (Line Orientation) has been supported using regional gray matter volumes. We aimed to further evaluate their validity using diffusion tensor imaging (DTI) of specific white matter (WM) tracts.

Participants and Methods: Individuals diagnosed with a neurodegenerative disease $(\mathrm{N}=32$, age $=67+/-9.2)$ or as neurologically healthy $(\mathrm{N}=48$, age $=74+/-8.1)$ participated. WM integrity was determined by mean fractional anisotropy (FA) from 27 major WM tracts throughout the brain. WM tracts were summed bilaterally for Favorites and Match analyses, but were investigated separately by hemisphere for Line Orientation because we hypothesized stronger $\mathrm{R}$ hemisphere associations. To determine which WM tracts uniquely contributed to performance on each subtest, we performed stepwise regressions with backwards elimination including all WM tracts with significant pairwise associations. Age and sex were included as covariates.

Results: In the regressions, $\mathrm{FA}$ of the fornix $(\mathrm{B}=.35 ; p=.001)$ and of the superior cerebellar peduncle $(\mathrm{B}=.33 ; p=.001)$ each uniquely predicted Favorites, FA of the corpus callosum $(\mathrm{B}=.5 ; p=<.001)$ uniquely predicted Match, and FA of the $\mathrm{R}$ anterior corona radiata $(\mathrm{B}=-.29$; $p=.003)$ predicted Line Orientation.

Conclusions: We found expected relationships between the BHA subtests and white matter integrity. Memory performances were associated with WM integrity in the medial temporal lobe, speeded executive functions with callosal hemispheric connections, and visuospatial abilities with $\mathrm{R}$ hemisphere tracts underlying dorsal stream frontal-parietal regions. Results are consistent with previous validity evidence with regional gray matter, and reinforce the specific roles of white matter tracts in cognitive functions. 
Correspondence: Andrea G. Alioto, Neurology, University of California San Francisco, 247 San Carlos Avenue, Redwood City, CA 94061, United States.E-mail: aalioto@paloaltou.edu

T. FILSHTEIN, D. MUNGAS \& S. FARIAS. Differential Item Functioning of the Everyday Cognition (ECog) Scales in Relation to Racial/Ethnic Groups.

Objective: The Everyday Cognition (ECog) measure has been shown to aid in predicting incident cognitive impairment and dementia, and in tracking disease progression. We have not previously evaluated whether ECog measurement differs across ethnic/racial groups. This study examined measurement invariance across non-Hispanic Whites (NHW), Blacks (BL), and Hispanics (HISP).

Participants and Methods: Participants were 1,177 NHW, 243 BL, and 216 HISP older adults. ECog measures of six daily function domains (Divided Attention (DA), Language (LAN), Memory (MEM), Organization (ORG), Planning (PLAN), and Visuospatial (VSP)) were used. Differential item functioning (DIF) for each domain was evaluated separately for BL and HISP compared to NHW. An iterative multiple group confirmatory factor analysis of ordinal item scores was used to identify items with DIF and to adjust scale scores for DIF. DIF adjusted scores were compared with unadjusted scores to evaluate the impact of item level DIF on scale level scores.

Results: Varying levels, patterns, and impacts of DIF were found across domains and groups. The impact of DIF was relatively small and DIF effects on scores generally were less than one standard error of measurement. For BL, MEM and VSP domains had the most pronounced DIF. MEM impairment was underestimated in BL when DIF was not addressed: VSP scores were biased by DIF but showed no systematic pattern. For HISP, DA and VSP demonstrated the largest amount of DIF. DA impairment in HISP was increasingly overestimated as level of DA impairment increased. VSP impairment was underestimated at lower levels of impairment and overestimated at higher levels. All scales had DIF free items that enabled linking of scores across groups.

Conclusions: Differing patterns of DIF were observed across BL and HISP compared to NWH and across ECog domains. While DIF effects on domain scale scores were generally small, adjusting for DIF or modifying DIF influenced items would improve measurement precision in the minority groups.

Correspondence: Teresa Filshtein, PhD, Epidemiology and Biostatistics, University of California, San Francisco, 1047 Miller Drive, Davis, CA 95616, United States. E-mail: teresa.filshtein@ucsf.edu

J.E. KARR, G.L. IVERSON, J.A. HOLDNACK \& M.A. GARCIABARRERA. The Other Side of the Bell Curve: Multivariate Base Rates of High Scores in Executive Function Assessment.

Objective: Past researchers have examined the frequency at which healthy patients obtain one or more $(1+)$ low scores on neuropsychological test batteries, identifying five psychometric principles: (1) low scores are common, with their frequency contingent on (2) the low score cutoff used, (3) the number of tests administered/interpreted, and (4) the demographic characteristics (e.g., education) and (5) intelligence of patients (Brooks et al., 2013). The current study aimed to identify if these principles applied to high scores as well, using the Delis-Kaplan Executive Function System (D-KEFS).

Participants and Methods: Multivariate base rates of high scores ( $\geq 75 \mathrm{th}, 84 \mathrm{th}, 91 \mathrm{st}, 95 \mathrm{th}, 98 \mathrm{th}$ percentile) were derived for a threetest, four-test, and full D-KEFS battery, using the adult portion of the normative sample (ages 16-89; $N=1,050$ ) stratified by education and intelligence. The full D-KEFS battery provides 16 total achievement scores (primary indicators of executive function).

Results: High scores occurred commonly for all batteries. For the three-test battery, $24.1 \%$ and $12.4 \%$ had $1+$ scores $\geq 95$ th percentile and $\geq 98$ th percentile, respectively. High scores occurred more often for longer batteries: $61.6 \%, 72.9 \%$, and $87.8 \%$ obtained $1+$ scores $\geq 84$ th percentile for the three-test, four-test, and full batteries, respectively.
The frequency of high scores increased with more education and higher intelligence.

Conclusions: The psychometric principles of low scores frequencies also applied to high D-KEFS scores, where high scores were common, and contingent on the cutoff used, the number of tests administered/ interpreted, and the education/intelligence of examinees. Knowing the base rates of high scores might help clinicians and researchers differentiate a single isolated high test score from a true strength in cognitive functioning. Moreover, it is possible that an algorithm that combines multivariate base rates in both tails of the distribution might improve the identification of acquired cognitive deficits in high functioning people. Correspondence: Justin E. Karr, M.Sc., Psychology, University of Victoria, P.O. Box 1700 STN CSC, Victoria, BC V8W 2Y2, Canada. E-mail:jkarr@uvic.ca

E.J. MAHONEY, A.E. NICKEL \& D.E. HANNULA. Identifying New Targets for Detecting Memory Malingering: Insights from a Combined fMRI and Eye Tracking Investigation.

Objective: The current investigation explored questions about the effects of malingered memory performance on eye-movement behavior and neural activity.

Participants and Methods: Forty healthy participants encoded several scene-face pairs and were subsequently tested with 3-face displays preceded by studied scene cues. The associate of the cue was either present or not, and on each trial participants were asked to make a present/absent judgment. Half of the participants (controls $n=20$ ) were instructed to perform optimally while the remainders were told to simulate memory impairment (simulators $n=20$ ). Eye tracking and fMRI data were acquired throughout the experiment.

Results: Simulators performed at chance levels on present/absent decisions, while controls significantly outperformed simulators. However, participants from both groups spent a disproportionate amount of time looking at associates of the scene cue within $500-750 \mathrm{~ms}$ of the onset of the 3-face display. Results from fMRI analyses showed that for both groups, hippocampal activity during the scene cue predicted this early disproportionate viewing of associates. Activity in other regions differentiated the two groups. Specifically, simulators showed greater activation than controls in the right angular gyrus, which could reflect simulators' reorientation of attention away from associates as they attempted to conceal their knowledge. In addition, simulators, but not controls, had greater anterior cingulate cortex (ACC) activity for incorrect than correct trials, a result that likely reflects conflict between accurate memory retrieval and compliance with malingering instructions.

Conclusions: The results here suggest novel targets for the detection of memory malingering to be investigated in future work. Early eve movement effects, hippocampal activity during memory cues, and ACC and angular gyrus activation during test displays may reflect the complex interplay of memory retrieval and cognitive control processes required to succeed at feigning memory impairment.

Correspondence: Elaine J. Mahoney, University of Wisconsin Milwaukee, 2510 N Murray Ave, Milwaukee, WI 53211, United States. E-mail:mahoneye@uwm.edu

E. VOGT, K.A. RITCHIE, D.E. MARRA, M.E. NITTA, J. KOOP \& J. HOELZLE. Child and Adolescent Validity Cut-Scores for the Victoria Symptom Validity Test and Reliable Digit Span-Revised. Objective: This project aimed to evaluate the Victoria Symptom Validity Test (VSVT) and embedded Digit Span validity measures for children and adolescents with and without clinical conditions.

Participants and Methods: Children and adolescents $\left[N=191, M_{\text {age }}\right.$ $=12.19(2.80)$ range 7.10 to 16.03$]$ that were evaluated in a medical center $(n=125)$ or recruited from the community $(n=66)$ completed the VSVT and WISC-V Digit Span in addition to other neuropsychological measures. Community-recruited children with pre-morbid diagnoses $\left[n=23, M_{\text {age }} 10.25(2.55)\right]$ and medical center patients $\left[n=23, M_{\text {age }}\right.$ $13.06(2.55)]$ completed measures according to manual instructions, 
whereas, some community-recruited children were randomly instructed to put forth best effort $\left[n=23, M_{\text {age }} 9.82(2.10)\right]$, or simulate cognitive impairment $\left[n=20, M_{\text {age }} 11.72(2.62)\right]$.

Results: Receiver operator curve (ROC) analyses and area under the curve (AUC) discrimination values were similar for embedded measures and the stand-alone validity test for both control (VSVT: Easy .76, Difficult .75, Total .75, DS ACSS .81, RDS .76, RDS-R .79) and clinical participants (VSVT: Easy .67, Difficult .65, VSVT .66, DS ACSS .66, RDS .71, RDS-R .70). Recommended cut-scores were determined to optimize specificity for the control sample (VSVT: Easy $\leq 21$, Difficult $\leq 19$, Total $\leq 34$, DS ACSS $\leq 7, \operatorname{RDS} \leq 6$, RDS-R $\leq 10)$ and clinical samples (VSVT: Easy $\leq 19$, Difficult $\leq 13$, Total $\leq 31$, DS ACSS $\leq 4$, RDS $\leq 5$, RDS-R $\leq 8)$.

Conclusions: This is the first study to recommend empirically derived VSVT and RDS-R cut-off scores for a pediatric sample. Suggested VSVT cut-off scores for both control and clinical groups are above the manual recommended invalid range. Cut-off scores for RDS and DS ACSS are lower for this developmental, medical, and neurological clinical sample than previous empirical scores from pediatric mTBI samples. Additional findings and clinical implications will be presented.

Correspondence: Elisabeth Vogt, MS, Psychology, Marquette University, 2928 N 84th Street, Milwaukee, WI 53222, United States. E-mail: elisabeth.vogt@marquette.edu

\section{Poster Session 3. Concussion}

\section{9:30-10:45 a.m.}

\section{Concussion}

B.M. ASKEN, R.M. BAUER, Z.M. HOUCK, A.M. SVINGOS, C.C. MORENO, G. HROMAS \& J.R. CLUGSTON. Baseline Serum Biomarker Concentrations: Association with Clinical Measures and Brain Trauma History.

Objective: Fluid biomarkers may provide objective markers of the physiological effects of brain injury, such as sport-related concussion, as well as cumulative exposure to repetitive brain trauma common in collision sports. We evaluated the effect of concussion history and years playing collision sports on baseline serum biomarker concentrations, as well as associations with baseline clinical outcomes.

Participants and Methods: We analyzed baseline serum concentrations of UCH-L1, GFAP, S100B, A $\beta-42$, total tau, MAP2, and CNPase collected via venipuncture from male $(n=156)$ and female $(n=105)$ University of Florida varsity athletes $($ mean \pm SD age at baseline $18.8 \pm 1.2$ years). Regression analyses evaluated biomarker concentration $(\mathrm{pg} / \mathrm{mL})$ associations based on concussion history, cumulative years playing collision sports (diving, football, ice hockey, soccer, and wrestling), and composite scores obtained from the Immediate Post-concussion Assessment and Cognitive Testing (ImPACT Verbal Memory, Visual Memory, Visual Motor Speed, and Reaction Time).

Results: We found that more cumulative years playing collision sports was associated with significantly lower serum S100B ( $\beta=-.161, p=.007)$. There were no other significant associations between concussion history or cumulative years playing collision sport and any baseline serum biomarker concentrations. Lower baseline GFAP concentration was associated with better Visual Memory $(\beta=.144, p=.038)$ but worse Visual Motor Speed $(\beta=-.156, p=.033)$. No other associations between biomarker concentration and clinical outcomes were observed.

Conclusions: Serum biomarker concentrations revealed no indications of detrimental effects of concussion history or years playing collision sports. Sporadically observed associations between biomarker concentrations and cognitive scores were weak and inconsistent, and were likely spurious statistical findings.
Correspondence: Breton M. Asken, MS, ATC, Clinical and Health Psychology, University of Florida, Dept. of Clinical and Health Psychology, P.O. Box 100165, Gainesville, FL 32610, United States. E-mail: basken8@phhp.ufl.edu

Z. BITAN, M. SOLOMITO, A. HACKETT \& S. RASKIN. Establishing A Reference Database For Collegiate Athletes Using The Elite Balance Protocol.

Objective: There is a need for a clinical tool to track recovery after concussion. Static balance alone has not proven to be sensitive. Therefore, a dual task (cognitive load and balance) was investigated. Thus, testing patients in a dual task condition is more applicable to a real world setting. This study aimed to establish a reference database that can be used to compare this Elite Balance Protocal (EBP) data collected from concussed athletes to this reference data.

Participants and Methods: 128 healthy collegiate athletes were included. Cognitive load was The Sport Concussion Assessment Tool 3 (SCAT3), immediate memory. Nintendo Wii Balance Board tracked the location of center of pressure in anterior-posterior and medial-lateral directions. MATLAB collected and plotted the $(x, y)$ coordinates of center of pressure. The sway area was calculated by fitting an ellipse, to the maximums of the plotted center of pressure data. The order of tasks was: eyes open-double leg stance; eyes open-single leg stance; eyes closed-double leg stand; eyes closed-single leg stance; eyes closed-double leg stance-cognitive load; eyes closed-single leg stance-cognitive load. Results: One-way ANOVA revealed no statistically significant difference among the double leg stance conditions. However, there was statistically significant difference in single leg conditions, such that the greatest sway was seen with eyes closed and no cognitive load, followed by eyes closed and cognitive load, followed by eyes open $(p<.05)$.

Conclusions: The results indicated an overall trend for decreased sway area when subjects performed the dual task balance compared to single task balance conditions. This is consistent with other studies that have noted an improvement in balance when a dual task was administered. Therefore, using the collected reference data it will possible to compare EBP data collected from concussed athletes to the reference data collected in this study; thus allowing for a clinical tool to assess and track the recovery of a concussion.

Correspondence: Zachary Bitan, BS, Hartford, CT 06106, United States.E-mail: zachary.bitan.2017@trincoll.edu

A.R. BURNS, T.R. SNEDDEN \& G.A. GIOIA. Reliability and validity of the Concussion Learning Assessment and School Survey (CLASS): A measure assessing academic problems following concussion.

Objective: To demonstrate the psychometric properties of a measure used to assess perceived academic problems following concussion.

Participants and Methods: 103 children (ages 10-18, M=14.61; 57\% female) and 122 parents (of children ages 5-18, M=13.59; $57 \%$ female) presented for an initial visit at a concussion clinic within 31 days of the injury and 47 children (mean age $=14.72,55 \%$ female) and 58 parents (mean age $=13.90,53 \%$ female) returned for a follow-up visit within 60 days of injury. The CLASS assesses the degree of perceived academic problems following concussion (e.g., headaches interfering with classwork; 14 items), the level of concern about the injury impacting academic performance (1 item), and the degree of worsened problems in particular subjects (e.g., math; 5 items). Patients and parents also completed the post-concussion symptom inventory and a monitoring version of the BRIEF; scores were adjusted for pre-injury ratings.

Results: Four academic problem items were highly correlated due to overlapping content (e.g., headaches during classwork/homework) and were dropped. Factor analyses revealed that a one factor solution best explained the ten remaining academic problems. Analyses revealed that degree of worsened problems in particular subjects could be also be explained by a single factor. Internal consistency $(\alpha=.83-.95)$ and test retest reliability $(\mathrm{r}=.29-.58)$ were adequate across subscales and 
reporters. Perceived academic problems was associated with symptom severity ( $\mathrm{r}=.62 / .78$; parent/child) and executive dysfunction ( $\mathrm{r}=.47 / .77)$ as were degree of worsened problems in particular subjects $(r=.58 / .70$ and $r=.54 / .71$, respectively) and level of concern regarding academic functioning $(r=.55 / .58$ and $r=.46 / .66$, respectively).

Conclusions: Findings demonstrate adequate reliability and construct validity of the CLASS. The CLASS can assist clinicians in the management of concussion by targeting specific areas of need within the school setting (e.g., academic accommodations or emotional supports). Correspondence: Alison R. Burns, PhD, Neuropsychology, Children's National Health System , 15245 Shady Grove Road, Suite 350, Rockville,MD 20850,United States. E-mail: ali.r.burns@gmail.com

\section{A.R. BURNS, A. DEL CASTILLO, J. KATZ, S. ROBERTS, A. KRAVITZ, C.G. VAUGHAN \& G.A. GIOIA. Neuropsychological Assessment as a Function of Time since Injury Following Pediatric Concussion.}

Objective: Examine differences in the results of neuropsychological assessment as a function of time since injury following pediatric concussion.

Participants and Methods: 121 youth seen in a specialty concussion clinic $(55 \%$ female, mean age $=13.71)$ were administered the WISC-V or WAIS-IV working memory (WMI) and processing speed (PSI) indices. A separate group of 307 participants ( $41 \%$ female, mean age $=14.81)$ were administered the Immediate Post-Concussion Assessment and Cognitive Testing (ImPACT). Individuals were split into two groups based on the number of days since injury (1-10 days and 11-20 days). Parents completed the Post Concussion Symptom Inventory (PCSI) and scores were adjusted for retrospective pre-injury ratings.

Results: Overall, youth seen within 3 weeks of injury demonstrated average cognitive performance (although some individuals fell 1.5 SDs or more below the mean; $7 \%$ WMI, $10 \%$ PSI, $17 \%$ ImPACT verbal memory, 19\% ImPACT visual memory, 15\% ImPACT processing speed, $26 \%$ ImPACT reaction time). No group level differences in test performance were found as a function of time since injury. Moreover, time since injury was not associated with most performance-based measures of cognitive functioning after controlling for GPA, age, gender, and symptom burden. Greater deficits in ImPACT processing speed and reaction time was associated with longer time since injury, although time since injury predicted only two percent of the total variance. In contrast, symptom burden was associated with worse performance in processing speed (WISC-V/WAIS-IV and ImPACT), reaction time (ImPACT), and verbal memory (ImPACT).

Conclusions: Cognitive test performance following pediatric concussion was age appropriate for most and time since injury was not a robust predictor of performance. However, up to $26 \%$ did perform poorly on testing. Cognitive performance was associated with parent-reported symptom burden. Impairment on cognitive testing may indicate the need for close clinician monitoring and greater supports within the academic setting.

Correspondence: Alison R. Burns, PhD, Neuropsychology, Children's National Health System, 15245 Shady Grove Road, Suite 350, Rockville,MD 20850,United States.E-mail: ali.r.burns@gmail.com

M.C. CAPOZZOLI, L.S. TERRYBERRY-SPOHR, R.H. RISS, D.A. HOPE \& K. BAXTER. Predictors of Post-Concussion Symptom Invalidity Using the Validity-10 in Treatment-Seeking Adults With Persistent Complaints After Mild Traumatic Brain Injury.

Objective: Interpretation of reported post-concussion symptoms (PCS) is strengthened by considering symptom validity, as PCS are non-specific and overlap with other conditions, such as depression, anxiety, and post-traumatic stress disorder (King et al., 2012). The Validity-10 scale (Vanderploeg et al., 2014) was designed to identify possible symptom invalidity within a measure of PCS. As clinical studies of the Validity-10 have been limited to military populations, the aim of this study was to elucidate factors that relate to potential symptom invalidity in a treatment-seeking population in a community-based rehabilitation hospital. Participants and Methods: Adults with persistent PCS $(N=37)$ presenting for multidisciplinary rehabilitation completed the Neurobehavioral Symptom Inventory, a neurocognitive test battery, and self-report measures of depression and anxiety symptoms. Information was also collected regarding active injury-related litigation, receipt of workers' compensation, and history of treatment for psychological or substance use problems.

Results: Validity-10 failure was significantly predicted in a multiple regression analysis $\left(\mathrm{R}^{2}=.352, F(6,31)=2.807, p=.027\right)$ by the presence of active injury-related litigation $(\beta=.451, p=.010)$ and failure of neurocognitive performance validity measures $(\beta=.317, p=.039)$. Non-significant relationships were demonstrated between Validity-10 failure and receipt of workers' compensation, history of psychological or substance use treatment, and self-reported emotional symptoms.

Conclusions: In this small treatment-seeking sample of adults with persistent PCS, symptom invalidity was predicted by active injuryrelated litigation and neurocognitive performance invalidity. Results were partially consistent with the extant literature, as emotional symptoms did not relate to symptom validity. Heterogeneity of emotional selfreport measures used may have contributed to non-significant results. Correspondence: Michelle C. Capozzoli, M.A., Psychology, University of Nebraska-Lincoln, 2656 English Oaks Cir, Charlottesville, VA 229118268, United States. E-mail: mcapozzoli@gmail.com

A.M. COLBERT, R.L. PETERSON, A.K. CONNERY, D.A. BAKER \&. M. KIRKWOOD. Comparing Subjective and Objective Cognitive Functioning in Youth with Persistent Postconcussive Symptoms. Objective: Most children recover quickly after a concussion, but a minority experience persistent postconcussive symptoms (PPCS), including subjective cognitive complaints (e.g., memory). Little is known about the relationship between subjective cognitive problems and objective memory performance in youth with PPCS

Participants and Methods: Youth referred clinically due to concerns about PPCS following a mild head injury $(\mathrm{n}=71$; ages $8-17$ ) as well as a comparison sample of uninjured youth $(\mathrm{n}=247$; ages $7-17)$ were included. Subjective cognitive symptoms were evaluated in both samples using the Health and Behavior Inventory (HBI) and compared. PPCS youth who failed performance validity tests were excluded from additional analyses (remaining $n=61$ ). Objective memory performance was evaluated in the PPCS sample using the California Verbal Learning Test (CVLT; Children's or Second Edition) and compared to published norms.

Results: Youth and parents in the PPCS sample reported more cognitive symptoms than the comparison sample $(p<.001 ; d=1.42-2.41)$. However, within the PPCS sample, objective memory performance was similar to CVLT norms for learning and recall. Only CVLT recognition discriminability was significantly worse than the norming sample $(p=.007 ; d=-.4)$. Subjective cognitive symptoms were not related to CVLT performance or to injury-related or demographic variables among youth with PPCS.

Conclusions: Objective memory performance was largely intact in a sample of youth with PPCS reporting high levels of subjective cognitive complaints. Moreover, objective memory performance was not related to subjective cognitive symptom report. The PPCS sample did have mild weakness in recognition in the context of intact learning and recall, which may be indicative of attentional variability or subthreshold suboptimal effort. Results suggest neuropsychological assessment can provide reassurance regarding cognitive recovery after concussion in the context of high subjective symptom reporting.

Correspondence: Alison M. Colbert, PhD, Rehabilitation, Children's Hospital Colorado, 8501 E Alameda Ave, 1438, Denver, CO 80230, United States.E-mail: alison.mh.colbert@gmail.com 
N.E. COOK, D.P. TERRY, R. MANNIX, R.ZAFONTE, P.D. BERKNER \& G.L. IVERSON. Athletes with ADHD Take Longer to Return to School but not to Sports Following Concussion.

Objective: To examine prospectively whether high school and collegiate student athletes with self-reported Attention-Deficit/Hyperactivity Disorder (ADHD) take longer to return to academics and athletics following a sport-related concussion compared to athletes without ADHD.

Participants and Methods: Participants were 1,157 high school and college athletes ( $43 \%$ female; mean age $=18.5, S D=2.4)$ who sustained a concussion; 111 athletes (8.8\%) reported having ADHD. Athletic trainers used a web-based injury surveillance system (Head Injury Tracker) to record information related to concussion recovery. Due to non-normal distributions, nonparametric Mann-Whitney U tests were conducted to compare athletes with and without ADHD on days to return to school and athletics. Chi-square tests examined the proportion of athletes with and without ADHD who had not returned to athletics/ academics at 7-, 10-, 14-, and 21-days post injury (i.e., they had prolonged recovery at each of these time points).

Results: Athletes with ADHD took a median of 6 days to return to school and 14 days to return to sports. Athletes without ADHD took a median of 5 days to return to school and 14 days to return to sports. Athletes with ADHD took significantly longer to return to school $(U=44,074.0$, $p=.009)$ but did not take longer to return to sports $(U=40,685.0, p=.72)$. More specifically, athletes with ADHD were significantly more likely to remain out of school at 7 days $\left(42.9 \%\right.$ vs. $\left.31.8 \% ; \chi^{2}(1)=4.98, p=.03\right)$ and 10 days $\left(28.6 \%\right.$ vs. $\left.19.1 \% ; \chi^{2}(1)=5.07, p=.02\right)$ post injury. There were no statistically significant group differences in recovery rates when examining return to athletics.

Conclusions: High school and collegiate athletes with ADHD may be at risk for longer return to school within the first two weeks following injury but not at risk for longer return to sports following concussion. Correspondence: Nathan E. Cook, Ph.D., Sports Concussion Program, MassGeneral Hospital for Children, 101 Merrimac Street, Boston, MA 02114, United States. E-mail: necook@mgh.harvard.edu

\section{B. DUNCAN, J. KIEFEL \& K. ONO. An Analysis of Performance on Measures of Performance Validity and Self-Report of Concussion Symptoms.}

Objective: This study examines if there is a relationship between number of reported post-concussive symptoms and performance on measures of effort in a sample of children/adolescents with concussion. Participants and Methods: This study consists of 48 children who sustained a concussion within 0-3 months of testing. Participants were administered the CNS Vital Signs (CNSVS) and Green's Medical Symptom Validity Test (MSVT). The CNSVS, a quick and portable computerized battery with embedded validity indicators, is emerging to show clinical utility in pediatric neuropsychology. The MSVT has proven to be an important tool in examining concussion in pediatric patients with failure on the MSVT linked to report of greater post-concussive symptoms.

Results: Participants were categorized into four groups based on whether they passed/failed either of two validity measures (i.e., MSVT and the CNSVS-Neurocognition Index Validity Indicator). One-way ANOVAs were conducted to determine if there was a significant difference between the four groups and the number of concussion symptoms reported utilizing the Acute Concussion Evaluation symptom checklist. Statistics reveal a significant effect for self-report of total physical symptoms $[\mathrm{F}(3,32)=4.401, \mathrm{p}=.011]$, and overall report of concussion symptoms $[\mathrm{F}(3,33)=3.121, \mathrm{p}=.039]$, such that those who failed the MSVT but passed the CNS indicators reported more physical symptoms as well as symptoms overall.

Conclusions: Individuals who failed the MSVT/passed the CNSVS indicator or failed both reported more physical symptoms. Overall report of symptoms was also significant and more symptoms were reported by individuals who failed the MSVT only or failed both performance validity measures. These findings are consistent with existing literature and emphasize the importance of using performance validity measures in the assessment of pediatric concussion. The study also highlights the importance of using both stand-alone and embedded performance validity tests.

Correspondence: Brittany Duncan, MA, Neuropsychology, Children's Healthcare of Atlanta, 7075 Derby Court, Cumming, GA 30040, United States. E-mail: brit021491@gmail.com

M.M. CROSSLAND, R.L. PETERSON, D.S. MASON \& M. KIRK WOOD. Parent and Child Agreement on a Postconcussive Symptom Measure: Baseline Data from Competitive Youth Soccer Players.

Objective: The literature has shown significant differences between parent and child scores on symptom reports covering a variety of different disorders and psychiatric conditions. Yet only a small body of research has investigated parent and child agreement on postconcussive symptom measures, and none of these studies looked specifically at baseline data. The current study compared parent and child symptom reports at baseline and possible influencing factors, such as age and gender.

Participants and Methods: 332 youth (145 males; mean age $=11.9$ years; age range $=7.75$ to 17.9 ) participating in a local competitive soccer club and their parents completed the Health and Behavior Inventory (HBI) before the start of the season. 46 of the participants were 14 years of age or older and categorized as adolescents.

Results: Regarding agreement between parents and children, symptom reports from the two raters were moderately to strongly correlated in the sample as a whole $(r=0.63)$. Children showed stronger agreement with their parents compared to adolescents $(r=0.68$ versus $r=0.32$; $p<0.001)$. No differences in agreement were found by gender. Regarding level of symptomatology reported, youth reported more symptoms compared to their parents $(p=0.0048)$. A greater mean difference between reporters was seen for somatic symptoms compared to cognitive symptoms $(p=0.013)$ and for adolescent report compared to children 13 years and younger $(p<0.001)$.

Conclusions: In line with previous findings, parents tended to report fewer symptoms than youth, especially for somatic symptoms. An important novel finding was that age was related to parent/youth agreement, with adolescents showing greater discrepancies from their parents than children. These findings support the need for both self and parent report when assessing postconcussive symptoms in youth, especially during adolescence when there is a heightened risk of concussive injury.

Correspondence: Maria M. Crossland, B.S., Rehabilitation, Children's Hospital Colorado, 1033 Yale Circle, Boulder, CO 80305, United States. E-mail:maria.crossland@childrenscolorado.org.

M.M. CROSSLAND, R.L. PETERSON, D.S. MASON \& M. KIRK WOOD. Symptom Outcomes Following Concussion from a Prospective Study of Competitive Youth Soccer Players.

Objective: Previous research has established the natural history of sport-related concussion in adolescent and adult athletes, but much less methodologically rigorous research has included younger athletes. The current prospective cohort study examined the trajectory of postconcussive symptoms in concussed competitive youth soccer players compared to both orthopedic injury and uninjured controls.

Participants and Methods: Youth participating in a local competitive soccer club and their parents completed the Health and Behavior Inventory (HBI) to assess baseline symptoms before the start of the season. 23 athletes were enrolled after sustaining concussions. These athletes were matched on age, gender, and baseline symptom level to 23 uninjured controls. A second control group of 23 athletes with orthopedic injuries was also recruited. The total sample included 69 youth (36 males; mean age $=13.2$ years; age range $=9.3$ to 17.1$)$. Symptoms were rated by youth and their parents at 6 time points after enrollment (24-48 hours, and $4,7,10,30$, and 90 days post injury). 
Results: The pattern of results was similar for child and parent report. No differences in baseline symptoms were found across the three groups $(p>.2)$. Compared to both control groups, the concussion group showed a large increase in symptoms at 24-48 hours and 4 days post injury ( $p$ from .008 to .001). Symptoms decreased through 7 days post injury, returning to baseline within approximately 10 days of injury. Female gender (according to both parent and youth report) and age younger than 14 years (according to parent report) were associated with slightly longer recovery times.

Conclusions: Overall, symptom recovery after youth -sport- related concussion followed a similar pattern to what has been documented in adolescent and young adult athletes. However, the initial postinjury symptom spike may last slightly longer in female athletes and preteens, and full resolution of symptoms may also take on average a few days longer.

Correspondence: Maria M. Crossland, B.S., Rehabilitation, Children's Hospital Colorado, 1033 Yale Circle, Boulder, CO 80305, United States. E-mail:maria.crossland@childrenscolorado.org

L. FIELDS, N. DIDEHBANI, J. HART \& M. CULLUM. Number of Concussions Not Associated with Neuropsychological Performance in Retired NFL Players.

Objective: A history of multiple concussions has been postulated to represent a risk factor for neuropsychological dysfunction later in life. Whereas retired NFL players have been reported to have elevated rates of mild cognitive impairment and depression, the potential relationship between concussion history and neuropsychological status remains poorly understood. This study was designed to examine whether the number of prior concussions was associated with neuropsychological status among retired NFL players.

Participants and Methods: We recruited 35 retired NFL players over age 50 (range $=52-78$. Mean $=65)$ from a longitudinal aging study. Number of concussions was gathered by interview and ranged from 1 to $17(\mathrm{M}=5.83)$. Cognitive measures included Boston Naming Test (BNT), Semantic Object Retrieval Test (SORT), Controlled Oral Word Association Test (COWAT), Category Fluency, California Verbal Learning Test - Second Edition (CVLT-II), Rey-Osterrieth Complex Figure, Trails A \& B, WAIS-IV Digit Span and Coding, and Beck Depression Inventory - II. Partial correlations controlling for age (corrected for multiple comparisons) examined the relationship between number of concussions, cognitive scores, and depressive symptoms.

Results: Partial correlations revealed that the number of prior reported concussions was unrelated to any of the neuropsychological test scores or number of depressive symptoms. Partial correlation $r$ values ranged from -0.17 to 0.31 ( $p>0.05)$.

Conclusions: The findings from this pilot study do not support an association between number of prior concussions and neuropsychological function or depressive symptoms among retired NFL players, though more research in this complex area is needed.

Correspondence: Lindy Fields, PhD, Psychiatry, UT Southwestern, 6333 Forest Park, Suite BLA.110, Dallas, TX 75235, United States. E-mail: lindy.fields@utsouthwestern.edu

K. NEUBERGER, M. JOHNSON, A.K. FONG \& M.D. ALLEN. Functional NeuroCognitive Imaging: Effective Identification for PCS.

Objective: Current research suggests that the pathological dysregulation of Neurovascular Coupling (NVC) caused by mild traumatic brain injury (mTBI) is the predominant source of chronic post-concussion syndrome (PCS) symptomology. FMRI has sometimes been dismissed as an appropriate imaging tool to identify mTBI pathology on the grounds that it is not a direct measure of neuronal activity. However, because $\mathrm{fMRI}$ is an effective and reliable means of measuring changes in NVC, it is precisely the right tool to assess PCS. Understanding the common physiology that underlies both the fMRI signal and neurological dysfunctions observed in PCS builds the foundation for developing this imaging technique into a powerful diagnostic, treatment planning, and assessment tool.

Participants and Methods: 476 concussed patients participated in fNCI scanning and Enhanced Performance In Cognition (EPIC) treatment. NVC data was collected before and after treatment and reported as Severity Index Score (SIS). Data was then compared to a normativebased atlas to provide statistical contextualization. Initial subjective (PCSS) and objective (SIS) findings were used to develop individualized. targeted, sustained, and cyclical week-long Enhanced Performance in Cognition (EPIC) therapy incorporating cognitive, occupational, and neuromuscular modalities.

Results: PCSS and SIS changes were assessed and calculated in percent change from pre- to post-treatment. PCSS scores showed a mean improvement of 65.7 percent, $\sigma=23.3$. Objective SIS findings showed a mean improvement of 76.5 percent, $\sigma=23.3$. Longitudinal reassessment of patients show maintained SIS improvement as measured in average of 7.6 months post-treatment.

Conclusions: FNCI provides reliable measurement of NVC dysregulation allowing for identification of concussion pathology. Additionally, fNCI derived SIS scores direct tailored EPIC therapy to restore NVC and subsequently resolve chronic PCS resulting from mTBI.

Correspondence: Alina K. Fong, UT, United States.E-mail: alinafong@ yahoo.com

V. GALLAGHER, N. KRAMER, K. ABBOTT, J. ALEXANDER, T. LINDLEY, H. BREITER, A. HERROLD, J. MJAANES \& J. REILLY. The Effects of Sex and Hormonal Contraception on Outcomes Following Collegiate Sports-Related Concussion.

Objective: The objective of this study was to examine the effects of sex and hormonal contraception (HC) use (e.g. oral contraceptive pill and NuvaRing) on outcomes following concussion among collegiate varsity athletes. We hypothesized females would have longer length of recovery (LOR) and that peak symptom severity would be associated with longer LOR in both males and females. Among females, we hypothesized non-HC users would have longer length of recovery and higher peak symptom severity compared to HC users.

Participants and Methods: 91 collegiate student-athletes were included in this study ( 41 males, 50 females; 24 HC-users, 25 non-HC users). Demographic, injury, and recovery information was abstracted via retrospective review of treatment records. Length of recovery was defined as days between injury and clearance for full return to play by team physician. The student-athletes' peak symptom severity score (SCAT-2 or -3) observed within the first five days of injury was used in analyses.

Results: Males had significantly shorter LOR compared to females $(F(1,87)=4.572, \mathrm{p}<.05, d=0.50)$ but comparable symptom severity scores. Svmptom severity was strongly related to LOR for males $(r=.467$. $\mathrm{p}<.01)$ but not for females $(r=-.003, \mathrm{p}>.05)$. Among females, non-HC females demonstrated significantly higher symptom severity compared to HC females $(F(1,47)=5.142 p<.05, d=.70)$. There were no significant differences between HC and non-HC females on length of recovery.

Conclusions: This study provides evidence for differential concussion outcomes based on sex and hormonal contraceptive use among collegiate athletes.

Correspondence: Virginia Gallagher, MS, Psychiatry \& Behavioral Sciences, Northwestern University Feinberg School of Medicine, 100 W Chestnut St., Apt 2505, Chicago, IL 60610, United States. E-mail: virginia.k.terwilliger@gmail.com

L. GERRALD, A. REOTT, A. GOODMAN, S. LEVIN ALLEN \& R. CLAIR. The Relationship Between Preexisting Conditions and Recovery Patterns in Pediatric Concussion Patients.

Objective: Pediatric concussion patients have been shown to demonstrate varying recovery patterns. The objective of this study is to analyze the recovery patterns of patients with and without a history of preexisting conditions (i.e. mood disorder, 
504 plans, Individualized education plan, \& migraines) based on self reported post concussion symptom scales.

Participants and Methods: 65 pediatric patients age 10 to 18 in grades 4 through college treated for concussion at a regional sports medicine clinic. Researchers reviewed de-identified patient records provided by the clinic and extracted data including educational, physical, and mental health history. Patients were considered "recovered" when they either demonstrated a post concussion symptoms scale of $<1$ or were cleared by the physician who indicated the any symptoms reported were back to baseline (i.e. pre-injury ratings).

Results: Differences in recovery patterns were seen between those patients with pre-existing conditions (i.e. mood disorders, school accommodations, prior concussions) compared to those with no history. Recovery times were changed based on the mechanism of injury (i.e. type of sport or accident). Recovery times of patients with pre-existing conditions were, on average, greater and demonstrated different characteristics than those with no history.

Conclusions: This study provides a descriptions of the profile of those with and without pre-existing conditions in terms of their recovery profile. Pediatric and adolescent concussion patients with a history concussion, mood disorder, school accommodations (e.g. learning disabilities), or migraines have different recovery patterns compared to those without pre-existing issues. Mechanism of injury also change the length of recovery. The review of these recovery patterns are provided in an effort to help inform the practice of practitioners in similar clinic settings.

Correspondence: Leland Gerrald, School Psychology, Psychology, Philadelphia College of Osteopathic Medicine, $809 \mathrm{~S} 18 T H \mathrm{ST}$, PHILADELPHIA, PA 19146-1917, United States. E-mail: lelandge@ pcom.edu

C. GHILAIN, D. MURDAUGH, K. ONO \& T. BURNS. ImPACTPediatric versus ImPACT: Pediatric Sample Differences in Baseline Performance.

Objective: Immediate Post-Concussive Assessment and Cognitive Testing, Pediatric Version (ImPACT-Ped) is a computer-based assessment measuring neurocognitive functioning post-concussion in children ages 5-11, and is a downward extension of the ImPACT (ages 12-59). Prior to the release of ImPACT-Ped, the ImPACT was often used with children ages 8-11. Therefore, this study assessed whether differences in performance exist between the ImPACT-Ped and ImPACT across analogous tests, specifically the word list delay, picture memory delay, and reaction time tests.

Participants and Methods: 477 children between 8-11 years who were identified as participants in high impact sports (e.g., football, soccer), completed baseline assessments from the ImPACT-Ped ( $\mathrm{n}=259,224$ males) and ImPACT ( $\mathrm{n}=218,79$ males) batteries. Percentage correct scores and reaction time (in seconds) were used to compare performance between analogous tests from the two batteries.

Results: One Way Analysis of Variance (ANOVA) were conducted to assess group differences in baseline neurocognitive performance. Significant between group differences were found across all measures. Specifically, children recalled significantly more words on word list $F(1,475)=288.55, \mathrm{p}<.001$ and had faster response time $F(1,475)=17.02, \mathrm{p}<.001$ on the ImPACT. In contrast, children recalled significantly more pictures on the ImPACT-Ped $F(1,475)=162.64$, $\mathrm{p}<.001$.

Conclusions: Results, as expected, suggest differences in performance on the ImPACT-Ped as compared to the ImPACT across word list delay, picture memory delay, and reaction time tests. These results highlight the differences in performance across measures in two similar samples of non-concussed children. This study provides support for the use of developmentally appropriate measures for assessing neurocognitive functioning at baseline. The variability among tests regardless of the ImPACT version used emphasizes the need for individualized baseline testing for young children in high-impact sports.
Correspondence: Christine Ghilain, Ph.D., Neuropsychology, Children's Healthcare of Atlanta, 1547 Clifton Road NE, Atlanta, GA 30322, United States.E-mail: christine.ghilain@choa.org

E. GUTY, J. MEYER \& P. ARNETT. Executive Functioning in College Students with Chronic Postconcussion Symptoms.

Objective: Research has demonstrated the relationship between overall symptom reporting following a concussion and general cognitive performance, but less is known about how symptom indices following a concussion may be related to domain-specific cognitive functioning. The goal of this study was to explore the relationship between symptom reporting and executive functioning (EF) in individuals with chronic postconcussion symptoms.

Participants and Methods: 42 college-aged participants were recruited for history of sports-related head injury and reported symptoms from 6 months to 3 years following injury. Symptoms were assessed using self-report scores from the Post-Concussion Symptom Scale. Based on a published factor analysis (Merritt \& Arnett, 2014), four clusters of symptoms were included: Physical, Sleep, Affective, and Cognitive. Although headache did not load onto any of the four factors, it was also examined, given its prevalence and importance as a post-concussion symptom. EF was operationalized as the composite score of seven neuropsychological tests of EF.

Results: Performance on the EF composite was significantly correlated with overall symptom reporting, with higher symptom scores related to worse performance, $r(40)=-.353, p=.026$. EF performance was also significantly correlated with headache symptoms, $r(40)=-.416, p=.008$, and cognitive symptoms, $r(40)=-.439, p=.005$. There was no significant relationship of physical, sleep or affective symptoms with EF performance.

Conclusions: Cognitive symptoms and headache were significantly associated with poorer performance on a broad-based composite of $\mathrm{EF}$ in individuals with long-term chronic concussion symptoms. These results highlight the potential additional role of more specific symptom factors. Causal relationships are unclear, but if post-concussion headaches cause EF problems in concussed athletes, successful treatment of such headaches may lead to an improvement in executive functioning. Correspondence: Erin Guty, Pennsylvania State University, 850 Toftrees Ave., Apt. 515, State College, PA 16803, United States. E-mail: erin. guty@gmail.com

S.N. HOFFMAN, M.S. HERBERT, L.D. CROCKER, N.E. DEFORD, A.V. KELLER, S.M. JURICK, M. SANDERSON-CIMINO, M. LEE, R.S. VASUDEVAN \& A.J. JAK. The Role of Pain Catastrophizing in Cognitive Functioning Among Veterans with History of Mild Traumatic Brain Injury.

Objective: Cognitive difficulties and chronic pain are highly prevalent among Veterans with a history of mild traumatic brain injury (mTBI). Moreover, those with mTBI history report chronic pain as a significant problem at a higher rate than those with moderate or severe TBIs. Pain catastrophizing, the exaggerated appraisal of the negative components of an actual or anticipated pain experience, has been associated with several adverse clinical outcomes, including cognitive deficits. However, relationships between pain catastrophizing and cognitive functioning in Iraq/Afghanistan Veterans with a history of mTBI has not been well characterized thus far.

Participants and Methods: 35 Iraq/Afghanistan Veterans in the post-acute phase following mTBI with valid neuropsychological testing completed questionnaires assessing TBI injury variables, pain catastrophizing, quality and intensity of pain, depression, and PTSD symptoms. Executive functioning, processing speed, learning/memory, and attention composites were created and entered as dependent variables into separate linear regressions to examine relationships with pain catastrophizing. 
Results: Pain catastrophizing was a significant predictor of executive functioning, even when controlling for TBI injury variables, gender, pain intensity, depression, and PTSD symptom severity $(\beta=-.020$; $p=.037)$. Pain catastrophizing was not a significant predictor of processing speed, learning/memory, or attention.

Conclusions: Pain catastrophizing is associated with worse executive functioning in Veterans with mTBI, even after controlling for TBI injury variables, gender, pain intensity, and psychiatric symptoms. This finding suggests that understanding not just severity of pain but one's interpretation of pain has implications for cognitive functioning. Treatments designed to alter how individuals cope with pain may also improve executive functioning.

Correspondence: Samantha N. Hoffman, Physiology and Neuroscience, B.S., Psychology, VA San Diego Healthcare System, Veterans Medical Research Foundation, 3350 La Jolla Village Drive, Bldg. 13, 3rd Floor MC151B, San Diego, CA 92161, United States.E-mail:snh.hoffman@ gmail.com

\section{Z.M. HOUCK, B.M. ASKEN, M. CUNEO \& R.M. BAUER. Exploratory Analysis of the Relationship Between Symptom Clusters and Neurocognitive Functioning in a Concussed Sample Presenting to a Specialty Clinic.}

Objective: To assess the relationship between neurocognitive functioning and concussion symptom clusters - cognitive/fatigue, vestibular, ocular motor, anxiety/mood, and post-traumatic migraine.

Participants and Methods: Data were obtained from 67 participants that presented to an interdisciplinary concussion clinic. Participants were excluded due to invalid neurocognitive data $(\mathrm{N}=6)$, missing neurocognitive ( $\mathrm{N}=5)$ and symptom data $(\mathrm{N}=14)$, and moderate/severe head injury $(\mathrm{N}=6)$. The final sample consisted of 36 participants (median age $=37.0$ years; median days since injury $=72$ ). The Sport Concussion Assessment Tool - 3rd edition symptom evaluation (S3SE) was used to obtain post-concussion symptoms. Symptoms were grouped into the clusters described above, which were based on a clinical categorization of concussion subtypes offered by Collins (2014). Neurocognitive functioning was measured using CNS Vital Signs multiple test domain scores - neurocognitive index, memory composite, psychomotor speed, complex attention, and cognitive flexibility. Pearson bivariate correlations were used to assess the relationship between neurocognitive functioning and symptom clusters.

Results: Higher cognitive/fatigue ( $\mathrm{r}=-.489, \mathrm{p}=.002)$, ocular motor $(\mathrm{r}=-.379, \mathrm{p}=.023)$, and anxiety/mood symptoms $(\mathrm{r}=-.401, \mathrm{p}=.019)$ were associated with lower psychomotor speed scores. Higher vestibular symptoms were associated with a lower neurocognitive index ( $r=-.461$, $\mathrm{p}=.005)$, psychomotor speed ( $\mathrm{r}=-.515, \mathrm{p}=.001)$, and cognitive flexibility scores $(\mathrm{r}=-.346, \mathrm{p}=.039)$.

Conclusions: Higher levels of symptoms, regardless of cluster, negatively affect speeded neurocognitive performance. However, vestibular dysfunction following concussion may result in additional executive functioning deficits.

Correspondence: Zachary M. Houck, MS, Clinical and Health Psychology, University of Florida, 1257 sw 9 th rd, 306, Gainesville, FL 32601, United States. E-mail: zhouck@phhp.ufl.edu

K.R. JOSEPH, C. MOY, S. ALA'I, J. ESTERLITZ, K. GAY \& P. BELLGOWAN. National Institute of Neurological Disorders and Stroke, National Institutes of Health and Department of Defense Common Data Language for Clinical Research Studies: SportsRelated Concussion Common Data Element Recommendations. Objective: The National Institute of Neurological Disorders and Stroke(NINDS), National Institutes of Health(NIH) and Department of Defense(DOD) initiated the development of Sports-Related Concussion(SRC) Common Data Elements(CDEs) in 2016 as part of the NINDS project to further develop data standards for all funded clinical neuroscience research. The purpose of CDEs is to educate new clinical investigators, increase efficiency and effectiveness of clinical research studies and treatment, increase data quality, facilitate data sharing, significantly reduce study start-up time, and more effectively aggregate information into significant metadata results.

Participants and Methods: In 2016 a working group(WG) was developed, composed of 34 experts from around the world with varied fields of related expertise. The WG divided into three Subgroups to review SRC during specific periods relative to time of injury: Acute $(<72 \mathrm{hrs}$ post concussion), Sub-Acute(3 days -3 months post concussion) and Persistent/Chronic(3 months and greater post-concussion). These three subgroups met regularly to review existing CDE domains, selecting CRFs and data elements from national registries and funded research studies. After this internal review period, the CDE recommendations were made available on the NINDS CDE website for public review and comments. Results: In June 2017, SRC CDEs Version 1.0 were posted on the NINDS CDE website. Recommendations include Core and Supplemental - Highly Recommended CDEs for cognitive measures and symptom checklists, and also, other outcomes/endpoints and sample CRFs for typical domains in clinical research.

Conclusions: The NINDS CDEs are an evolving resource, requiring updates as research advances. Due to lack of validation and empirical study of many SRC-specific measures, future updates to these CDEs are important. The NINDS encourages the clinical research community to use SRC CDEs as they are a valuable starting point for researchers to facilitate streamlining and sharing data.

Correspondence: Kristen R. Joseph, MA, The Emmes Corporation, 401 N. Washington Street, Suite 700, Rockville, MD 20850, United States. E-mail:kjoseph@emmes.com

S.M. JURICK, D.P. TERRY, N.E. COOK, B. MAXWELL, R. MANNIX, R. ZAFONTE, P.D. BERKNER \& G.L. IVERSON. Association Between Multiple Concussions and Return to School and Sports in High School and College Athletes.

Objective: The evidence regarding whether a personal history of concussion predicts or modifies clinical recovery from a future concussion is mixed and inconclusive. We examined whether having a pre-injury history of one or more concussions was associated with a prolonged return to school or athletics.

Participants and Methods: In this prospective cohort study, athletic trainers monitored 1,265 high school and college athletes ( $43 \%$ female) who sustained a concussion using a web-based surveillance system that collected information about their recovery. Athletes were divided into four groups based on number of self-reported prior concussions: 0, 1, 2 , or $3+$. The nonparametric Kruskal-Wallis tests were used to compare days to return to school/athletics across groups due to non-normal distributions.

Results: The total sample stratified by concussion history was as follows: $0=57.7 \% \quad(n=730), 1=24.3 \% \quad(n=306), 2=9.7 \% \quad(n=123)$, and 3 or more $=8.4 \%(n=106)$. There was no significant difference in days to return to academics between the groups, $X^{2}(3)=4.562, p=.207$. With regard to days to return to athletics, the groups did significantly differ, $X^{2}(3)=8.043, p=.045$. Follow-up contrasts using the KomogrovSmirnov test showed the group with 3 or more prior concussions took longer to return to athletics compared to the group with no prior concussions $(Z=2.080,<.001)$ and 1 prior concussion $(Z=1.734, p=.005)$.

Conclusions: Athletes with multiple prior concussions may be at risk for a protracted recovery after their next concussion, specifically with regard to returning to sports.

Correspondence: Sarah M. Jurick, B.A., Psychiatry, University of California San Diego, 9500 Gilman Drive, Mail Code 0603, La Jolla, CA 92037-0603, United States. E-mail:smjurick@gmail.com 
S.M. JURICK, L.D. CROCKER, A.V. KELLER, S.N. HOFFMAN, K.R. THOMAS, B. BOYD, C. RODGERS, E.W. TWAMLEY \& A.J. JAK. Can Trauma-Focused Treatment Improve Poor Neuropsychological Performance Validity in Veterans with PTSD and History of mTBI?

Objective: Operation Enduring Freedom/Operation Iraqi Freedom (OEF/OIF) Veterans with history of mild to moderate traumatic brain injury (mTBI) and post-traumatic stress disorder (PTSD) have high rates of performance validity test (PVTs) failures. We sought to determine whether Veterans with invalid PVTs benefit from psychotherapy and if psychotherapy can improve PVT performance.

Participants and Methods: OEF/OIF Veterans ( $\mathrm{N}=100)$ with PTSD, history of mTBI, and cognitive complaints underwent comprehensive neuropsychological testing as part of a randomized controlled trial of cognitive processing therapy (CPT), and CPT plus cognitive rehabilitation (SMART-CPT). Participants were tested at baseline, posttreatment, and three months posttreatment. Performance below standard cutoffs on either Test of Memory Malingering (TOMM) trials 2, retention, or California Verbal Learning Test-Second Edition (CVLT-II) forced choice condition was considered invalid, whereas performance above cutoff on all measures was considered valid.

Results: Using generalized estimating equation (GEE), invalid PVT rates decreased from baseline ( $27 \%$ invalid) to post-treatment $(12.2 \%$; $\mathrm{b}=1.235, p=.001)$, and at three months posttreatment $(5.7 \% ; \mathrm{b}=.415$; $p=.031$ ), but did not differ by treatment condition ( $\mathrm{ps}>.05)$. This finding was also significant using multilevel modeling (MLM) with TOMM trial 2 as the outcome $(b=.04, t(108.68)=2.012, p=.047)$. MLM and repeated-measures ANOVA showed no differences in validity groups' decreases in neurobehavioral, PTSD, and depressive symptoms $(p \mathrm{~s}>.05)$.

Conclusions: Those with invalid PVTs were better able to effortfully engage in assessment resulting in higher rates of valid PVTs following treatment. PVT performance at baseline was not associated with treatment response. Veterans with invalid PVTs should not be excluded from trauma-focused treatment, and may benefit from neuropsychological assessment after, rather than before, trauma-focused treatment.

Correspondence: Sarah M. Jurick, B.A., Psychiatry, University of California San Diego, 9500 Gilman Drive, Mail Code 0603, La Jolla, CA 92037-0603, United States.E-mail: smjurick@gmail.com

A.V. KELLER, L.D. CROCKER, S.M. JURICK, M. SANDERSONCIMINO, S.N. HOFFMAN, V.C. MERRITT, E.W. TWAMLEY \& A.J. JAK. The Interactive Effects of Traumatic Brain Injury Burden and Cognitive Functioning on Suicidal Ideation in Veterans.

Objective: A history of multiple traumatic brain injuries (TBIs) is associated with increased risk of suicidality in Iraq/Afghanistan-era military personnel and Veterans. There is some evidence that cognitive dysfunction is also associated with suicidality; however, it is unclear whether there is an interactive relationship between TBI burden and cognitive functioning on suicidality.

Participants and Methods: $71 \mathrm{Iraq} /$ Afghanistan-era Veterans with PTSD and a history of mild to moderate TBI completed neuropsychological assessment and psychological symptom measures at baseline as part of a treatment study. Participants were divided into those who endorsed current suicidal ideation (SI; $n=21)$ and those who did not $(n=50)$. Composites of attention/processing speed (PS), learning/memory, and executive functioning were created and entered into separate logistic regressions to examine possible interactive effects with TBI burden (1-2 TBIs vs. 3 or more TBIs) on suicidal ideation. All regressions included psychological distress and postconcussive symptoms (PCS) as covariates.

Results: There were no main effects for TBI burden or any of the cognitive composites. However, the interaction between attention/PS and TBI burden was associated with suicidal ideation over and above psychological distress and PCS. Specifically, for Veterans with greater TBI burden (3 or more), poorer attention/PS conferred additional risk of suicidal ideation. Interactions between TBI burden and learning/memory and executive functioning were not significant.

Conclusions: Neuropsychological performance may help inform risk for suicidal ideation in Veterans with a history of TBI. Veterans with greater TBI burden who experience suicidal ideation may have more difficulty engaging in cognitive tasks or attention/PS deficits may increase risk for SI. In addition to standard mental health interventions to address SI, strategies to improve attention/PS (e.g.., cognitive training or cognitive rehabilitation) may be of benefit in Veterans with greater TBI burden. Correspondence: Amber V. Keller, Bachelor of Arts in Psychology, Research, VA San Diego Healthcare System, 3350 La Jolla Village Drive , Building 13 MC151B, San Diego, CA 92161, United States. E-mail: ambervkeller@gmail.com

A.J. LEVAN, C.G. VAUGHAN, A.R. BURNS, J.B. NEWMAN, A. KRAVITZ, G. HILLS, T. SCHABERG, M.R. WIRTZ \& G.A. GIOIA. Working Memory Functioning Following Pediatric Concussion.

Objective: To examine working memory in youth following concussion. Participants and Methods: 433 youth aged 6-19 (mean 13.6 \pm 2.5 , $52 \%$ male) presented for an initial visit within 31 days from injury $($ mean $=13.7 \pm 6.9)$ and returned for a second visit an average of $29.4 \pm 11.3$ days later. $68 \%$ were seen for sport-related concussion, $14 \%$ sustained LOC, and $31 \%$ reported amnesia. Youth and/or their parents completed the working memory scale of the BRIEF2 monitor form, adjusted for retrospective report of pre-injury problems, at both visits. A subsample $(\mathrm{n}=64)$ completed the working memory $(\mathrm{WM})$ index on either the WISC-V or WAIS-IV. The Children's Exertion Effects Rating Scale (ChEERS), a self-report measure assessing worsening of post-concussive symptoms following mental exertion, was also completed by youth following cognitive assessment. Changes in WM over time, comparison across measures of WM, and factors associated with improvements in $\mathrm{WM}$ were examined.

Results: Parent $(\mathrm{F}=8.9, p=.003,=.021)$ and child $(\mathrm{F}=9.2, p=.003$, $=.025$ ) report of WM improved between first and second visit, controlling for variation in time between visits. WISC-V/WAIS-IV WM performance correlated significantly with BRIEF2 WM scale $(\mathrm{r}=-.331, p=.007)$ for child, but not parent. Regression analyses revealed cognitive exertional effects were a significant predictor of ratings of WM problems at second visit for child ( $\left.p=.02, \mathrm{R}^{2}=.052\right)$ and parent $\left(p=.02, \mathrm{R}^{2}=.042\right)$, while WM performance, symptom burden, and time were not $(p>.05)$.

Conclusions: This study examines changes in working memory in youth following concussion over the course of recovery. Although cognitive performance and ratings of working memory problems are associated, improvements in working memory symptoms at second visit are predicted by cognitive exertion, highlighting the value of multimodal assessment following pediatric concussion.

Correspondence: Ashley J. Levan, PhD, Neuropsychology, Children's National Medical Center, 44 Maryland Ave, Apt 312, Rockville, MD 20850, United States.E-mail: alevan2@childrensnational.org

E. LIMA, J.R.M. LIU, A. PATEL, K. HARTLINE, N. PAWLENKO \& A. HERRERA-HAMILTON. Using Tests of Validity to Predict Performance on Neurospcyhological Exams.

Objective: Our study sought to examine whether validity tests predict lower overall performance on neuropsychological tests, for which limited published data exists.

Participants and Methods: Children and adolescents $(\mathrm{N}=118)$ (age 4.3-19.6; $\mathrm{M}=13.68 ; \mathrm{SD}=3.5)$. Participants included more male $(53 \%)$ than female $(47 \%)$, and were from a diverse cultural population $(58 \%$ Caucasian, 28\% Latino, 8\% African American, 4\% Asian American, $2 \%$ Native American). All participants were diagnosed with concussion and were receiving post-concussion treatment at Children's Hospital Los Angeles. All patients were examined at an initial exam and for neuropsychological testing due to persistent symptoms. Method: All participants were given Green's Medical Symptom Validity Test (MSVT) and Verbal 
Fluency subtest of DKEFS as part of their neuropsychological exam. All analyses were conducted in SPSS 21.0 (IBM, 2012).

Results: A correlation was conducted to assess whether Green's MSVT and DKEFS verbal fluency scores were correlated. Results indicated a positive correlation between MSVT free recall $(\mathrm{r}=.274, p<.05)$ and total score on the verbal fluency subtest of DKEFS. Additionally, a Simple Linear Regression was conducted. Results indicated that performance on the free recall of the MSVT, a validity measure, explained a significant amount of the variance in performance on a measure of verbal fluency $\left(R^{2}=.075\right)$. Specifically, as performance on the MSVT increases by one point, performance on DKEFS verbal fluency increases by $.274,95 \%$ CI $[.169,2.734],(p<.05)$.

Conclusions: Results of the current study support the hypothesis that tests of validity explain a portion of the variance on performance of neuropsychological exams. Further research in the predictive nature of measures of validity on all aspects of neuropsychological exams is supported. Further, discussions surrounding the consideration of utilizing validity testing as standard protocol may bolster performance and/or findings on neuropsychological tests.

Correspondence: Evan Lima, Psychology, Loma Linda University, 9095 La Paz Road, Atascadero, CA 93422, United States. E-mail: evanjohnlima@gmail.com

\section{MALLECK, K. MILNE \& C. ABEARE. The Effect of Menstrual Cycle Phase and Birth Control Pill Usage on Post-Concussive Symptom Reporting in Non-Concussed Adults.}

Objective: Many symptoms associated with concussion are also associated with symptoms related the menstrual cycle, such as headache, nausea, and fatigue. This study sought to investigate the relationship between these symptoms at different points in the menstrual cycle to determine if it is appropriate to compare baseline symptom reports obtained in one menstrual phase with post-concussive symptom reports from another.

Participants and Methods: A measure of post-concussive symptoms (PCSS) and a measure of mood and stress symptoms (DASS) were given to 44 females, 23 of which were using birth control pills, and 34 males. Females were also given a menstrual symptom questionnaire (DSRS). Participants completed the questionnaires on two occasions, two weeks apart. Females completed the questionnaires once during the follicular phase of their menstrual cycle and once during the luteal phase.

Results: PCSS and DSRS scores in females were significantly correlated at both time points. There were no differences between females on birth control pills, females not taking birth control pills, and males on the PCSS or any DASS subscales. Symptoms on the DASS Anxiety subscale, DASS Stress subscale, and PCSS were found to decrease over time. PCSS scores at time 1 and time 2 were correlated in males and in females who were not taking birth control pills, but not in females taking birth control pills.

Conclusions: The absence of an increase in the number and severity of symptoms experienced by females during the luteal phase, regardless of birth control status, suggests that there may be no need to consider menstrual phase at baseline when conducting post-injury evaluations. However, given that PCSS scores of females who take birth control pills were not correlated over time, the particular symptoms they experience may vary even if overall number and severity do not.

Correspondence: Malayna Malleck, Psychology, University of Windsor, 3770 Vaughan Street, Windsor, ON N9C1Z5, Canada.E-mail:malleck@ uwindsor.ca

\section{L.M. MANDERINO \& J. GUNSTAD. Self-Reported Constancy in Sports Is Correlated with Previous Concussions Resulting in Loss of Consciousness.}

Objective: Long-term outcomes of sports-related concussion (SRC) remain an important area of research. While some evidence suggests that a history of SRC increases risk for adverse psychological outcomes, the prevalence and predictors of such outcomes remain unknown. The present study examines possible associations between SRC history and self- reported mental toughness, a construct related to effective coping with stress in athletic settings.

Participants and Methods: A total of 162 NCAA student-athletes answered questions regarding SRC history, including total number of SRCs sustained and numbers of SRCs resulting in loss of consciousness (LoC), confusion, or posttraumatic amnesia (PTA), during preseason neuropsychological testing. Participants also completed the Sports Mental Toughness Questionnaire. Pearson correlations examined associations between SRC history, overall mental toughness, and three mental toughness subscales: confidence, constancy, and control.

Results: Correlations indicated that number of SRCs sustained was not associated with mental toughness or its subscales ( $\mathrm{r}$ range -0.08 to -0.10 ; all $\mathrm{p}>.05)$. There were no significant relationships between numbers of SRCs resulting in confusion or PTA with mental toughness ( $\mathrm{r}$ range -0.05 to -0.14; all p>.05). However, number of SRCs resulting in LoC was significantly correlated with scores on the constancy subscale, such that more previous SRCs with $\mathrm{LoC}$ was associated with lower self-reported constancy $(\mathrm{r}=-0.158, \mathrm{p}=0.045)$.

Conclusions: Few relationships were found between SRC history and mental toughness in sports. However, number of SRCs resulting in LoC was negatively correlated with constancy, or an individual's persistence through adversity and commitment to goal achievement. This may reflect that certain injuries are associated with changes in an individual's perceptions of his ability to cope with stressors. Replication and future research is necessary to clarify causality and whether perceived mental toughness is related to objective indicators of performance.

Correspondence: Lisa M. Manderino, Kent State University, 600 Hilltop Drive, Kent, OH 44242, United States. E-mail: Imanderi@kent.edu

C.C. MORENO, B.M. ASKEN, R.M. BAUER, Z.M. HOUCK, A.M. SVINGOS, G. HROMAS \& J.R. CLUGSTON. Stability of baseline serum biomarker concentrations in female collegiate athletes.

Objective: Fluid biomarkers potentially offer a means of objectively assessing brain injury status and have gained significant momentum in the area of sport-related concussion, specifically. Evidence to-date is mixed regarding their sensitivity and specificity, likely due in part to the absence of reliability data. This study reports the test-retest stability and reliable change indices for a panel of commonly studied serum biomarkers.

Participants and Methods: We analyzed baseline serum concentrations of ubiquitin carboxy-hydrolase L1 (UCH-L1), S100 calcium-binding protein B (S100B), amyloid beta peptide 42 (A $\beta 42)$, and total tau (T-Tau) collected via venipuncture from 32 female collegiate athletes $($ mean \pm SD age $=19.0 \pm 0.9$ years $)$ at two time points $(\mathrm{T} 1$ and $\mathrm{T} 2$, range 5-11 months, mean $\pm \mathrm{SD} 7.6 \pm 2.1$ ). No athlete sustained a concussion between time points. Athletes participated in lacrosse $(n=13)$ and soccer ( $n=19)$. Stability coefficients (Pearson correlations), quantification of change (paired samples t-test), and reliable change estimates were calculated for each biomarker.

Results: All biomarkers demonstrated low stability coefficients (r): UCH-L1=-.021, S100B=.328, A $\beta 42=.337$, and T-Tau=.380). $\mathrm{A} \beta 42$ significantly increased from T1 to T2 (mean elevation=2.39 $\mathrm{pg} / \mathrm{mL}$, $\mathrm{t}(31)=-3.388, p=.002)$. No other markers significantly increased or decreased between T1 and T2 ( $p>.05$ for all comparisons). Elevations required for statistically reliable changes based on a $95 \%$ confidence interval of the standard error of the difference between T1 and T2 for each biomarker are as follows $(\mathrm{pg} / \mathrm{mL})$ : UCH-L1=379.6, $\mathrm{S} 100 \mathrm{~B}=37.5$, $\mathrm{A} \beta 42=7.82$, and $\mathrm{T}-\mathrm{Tau}=1.66$.

Conclusions: The studied biomarkers demonstrated poor reliability across a test-retest interval of 5-11 months. Accordingly, reliable change estimates indicate that substantial elevation from baseline would be required for a post-concussion concentration to be considered statistically reliable. 
Correspondence: Charles C. Moreno, BA, Clinical and Health Psychology, University of Florida, 4455 SW 34th St, Apt QQ226, Gainesville, FL 32608, United States.E-mail: charles.moreno@ufl.edu

J.A. O'NEILL, S.C. TERRY, L. SARRETT, D.C. SCHWEBEL, T.A. NOVACK, J.M. JOHNSTON, O.J. CLAY, D. FERGUSON, S. GOULD, M.H. HALE, J. ORTEGA, R.E. COLBERG \& L.E. DREER. The Relationship Between the Sport Concussion Assessment Tool $-3^{\text {rd }}$ Edition (SCAT-3) and Neuropsychological Functioning in Adolescents with Concussion.

Objective: Concussions are estimated to affect over 1.9 million youths in the United States each year. Return to activity (RTA; e.g., return to play and return to school) decisions following concussions are based on the assessment of several factors including symptom resolution and neuropsychological functioning. The Sport Concussion Assessment Tool - Third Edition [SCAT-3] was developed as an assessment tool to aid in symptom detection and RTA decisions. Prior research has demonstrated a relationship between the SCAT-2 and measures of attention and verbal learning, but no studies have examined this relationship using the SCAT-3. The objective of this study was to examine relations between SCAT-3 scales (symptom severity and Standardized Assessment of Concussion [SAC]) and measures of attention, verbal learning, and executive functioning in concussed adolescents.

Participants and Methods: Participants included 42 adolescents (52\% male, $74 \%$ Caucasian) with a diagnosis of concussion. The sample ranged in age from 13-18 years $(M=15.2, S D=1.5)$ and mean days since injury was $7.9(S D=3.7)$. Individuals completed a SCAT-3 assessment along with a memory task (California Verbal Learning Test [CVLT]), computerized measure of attention (Conner's Continuous Performance Test $-3^{\text {rd }}$ Edition [CPT]), and paper-and-pencil measures of processing speed and executive functioning (Trail Making Test [TMT] and Symbol Digit Modalities Test [SDMT]).

Results: In multiple regression analysis, symptom severity was associated with CVLT learning slope $(\beta=.34, p<.05)$, performance on TMT-A $(\beta=-.42, p<.05)$, and performance on SDMT $-W(\beta=-.45, p<.01)$. SAC scores were associated with CVLT response bias $(\beta=.38, p<.05)$ and CPT omissions $(\beta=-.31, p<.05)$.

Conclusions: The SCAT-3 is a brief sideline assessment tool that relates to neuropsychological status in the areas of learning, processing speed, and attention and may serve as a useful guide in RTA decisions.

Correspondence: Jilian A. O'Neill, M.A., Psychology, University of Alabama at Birmingham, 306 McGowen St., Apt. 1313, Houston, TX 77006, United States.E-mail: jaoneill@uab.edu

J. PETERSON, C. BOLTON, J. LOWE, D. BARNHART, C. KIBBLE, B. PYYKKONEN \& K. KUTNAR. The ImPACT and performance validity: Examination of standard and experimental validity indicators.

Objective: Performance validity indicators are embedded in the Immediate Post-Concussion Assessment and Cognitive Testing (ImPACT) battery, but only two studies to date have investigated their effectiveness at identifying intentionally poor performance. The present study evaluated the ImPACT's standard validity indicators, examined experimental validity indicators described in the literature, and extended previous research by applying these indicators to the same sample to allow comparisons.

Participants and Methods: 48 undergraduates completed the ImPACT in a simulation design that included full-effort, uncoached, and coached groups. Uncoached and coached participants were both instructed to complete the measures as if they were concussed so as to artificially deflate their baseline scores, but coached participants were warned that the test could recognize individuals exaggerating poor performance.

Results: Chi square analyses demonstrated that all indicators identified participants intentionally performing poorly at rates better than chance. The ImPACT's five standard validity indicators identified $65.6 \%$ of the participants who were were instructed to perform poorly. This rate is consistent with previous findings. Both the experimental Word Memory Correct Distractors score (sensitivity $=.97$, specificity $=.94$ ) and a combination of nine indices (sensitivity $=.84$, specificity $=1.00$ ) were more effective than the five standard validity indicators at identifying participants who were instructed to perform poorly.

Conclusions: Either including four additional indicators along with the ImPACT's current five indicators or using one of the experimental indicators, the Word Memory Correct Distractors score, could improve the ImPACT's ability to identify invalid performance.

Correspondence: Jesse Peterson, Wheaton College, 2302 Whitegate Dr., Apt. 3A, Columbia, MO 65202, United States. E-mail: jesse. peterson.12@my.wheaton.edu

\section{V.M. PETRAUSKAS \& B.M. MERKER. Concussion Knowledge and Self-Reported Attitudes in Middle School and High School Athletes.}

Objective: Four million children participate in sports, with $1.6-3.8$ million affected by concussions yearly. Unreported concussions are concerning in children since they are more susceptible to negative consequences of concussion given differences in brain physiology (Zhang et al., 2016). Athletes' limited knowledge regarding concussions may predict premature return to play. Prior research has focused on college or high school level athletes. We examined knowledge and self-reported attitudes towards reporting concussions among both middle and high school athletes.

Participants and Methods: Athletes ( $\mathrm{n}=81$, ages $11-18, M=14.89$. $S D=1.53 ; 44$ Males 37 Females) completed a survey about education, knowledge, and self-reported attitudes and behaviors regarding concussion reporting. Linear regression evaluated association of age, gender, and type of sport played with knowledge and self-reported attitudes. Source of previous concussion education was examined as a factor affecting knowledge.

Results: Average percent correct for knowledge-based questions was $54.57 \%$. Only $53.1 \%$ of athletes would immediately report experiencing symptoms. There was no relationship between age, gender, or type of sport played and overall knowledge. There was an association between receiving concussion education from a physician and correctly knowing that imaging studies do not show physical damage to the brain following concussion, $\chi^{2}(1)=4.865, p=0.04$.

Conclusions: Misconceptions about concussions exist, and many athletes would not report a concussion. No personal factors were associated with improved concussion knowledge. Source of education was not predictive of improved overall knowledge. Results highlight the need for continued dissemination of concussion education programs.

Correspondence: Vilija M. Petrauskas, PhD, Neuropsychology, Henry Ford Health System, 1 Ford Place, Detroit, MI 48202, United States. E-mail:vpetrau1@hfhs.org

A.R. RABINOWITZ, V.C. MERRITT, E. GUTY, J. MEYER, L. GREENBERG \& P. ARNETT. Financial Incentives Influence ImPACT Validity Indicator Measures but not Cognitive Composite Scores.

Objective: Interpreting changes in cognitive performance across baseline and post-concussion evaluations is complicated by poor understanding of how incentives, such as return-to-play, may influence performance. This study examines the influence of an anticipated financial incentive on two sets of outcomes from the ImPACT computerized concussion battery: 1) Cognitive Composite scores (CCs), meant to serve as measures of cognitive function, and 2) Validity Indicator indices (VIs), proposed as indicators of test invalidity.

Participants and Methods: 82 uninjured college students, randomized into two groups, completed a concussion assessment battery including ImPACT. The Control group received standard administration instructions. The Incentive group was told they would receive $\$ 20$ if their scores ranked in the top third of students who had completed the test. 
Examiners were blinded to condition. No financial incentives were provided, and all participants were debriefed at the end of the session. Results: Independent samples t-tests revealed no significant differences between Incentive and Control groups on any of the CCs-Verbal Memory: $\mathrm{t}(79)=1.29, \mathrm{p}=.20$; Visual Memory: $\mathrm{t}(79), \mathrm{p}=.24$; Visual Motor Speed: $\mathrm{t}(79)=.34, \mathrm{p}=.75$; Reaction Time: $\mathrm{t}(79)=.79$; $\mathrm{p}=.43$. MannWhitney $U$ Tests revealed significant differences between groups all five VIs-Impulse Control Composite: $\mathrm{U}=604, \mathrm{p}=.03$; Word Memory Learning \% Correct: $\mathrm{U}=1,025, \mathrm{p}=.045$; Design Memory Learning \% Correct: $\mathrm{U}=1,097, \mathrm{p}=.02$; Xs \& Os Interference Total Incorrect: $\mathrm{U}=598$, $\mathrm{p}=.02$; Three Letters Total Letters Correct: $\mathrm{U}=1,058.0, \mathrm{p}=.02$.

Conclusions: Expectation of financial incentive did not significantly influence the ImPACT's standard metrics of cognitive function (CCs). However, the Incentive group exhibited significantly better performance on each of the validity indicator indices (VIs). These results suggest that ImPACT CCs are robust to incentive-related changes in effort, and provide support for the VI indices as measures of effort towards testing. Correspondence: Amanda R. Rabinowitz, Ph.D., University of Pennsylvania, 2944 Cambridge St., Philadelphia, PA 19130, United States. E-mail: rabinowitz.a@gmail.com

\section{V.M. REGINELLA \& J.D. PETRICK. Concussion with Loss of Consciousness \& Depression.}

Objective: The objective of this study was to investigate the relationship between the presence of loss of consciousness (LOC) following a concussion and self-reported depression. The recovery following mild traumatic brain injury (mTBI) or concussion and the neuropsychological sequella are controversial, especially when there is pending litigation. Previous research (Iverson, 2005; Iverson, 2006; Lange et al., 2011) has found that the majority of symptoms commonly attributed to PostConcussion Syndrome (PCS) are actually due to underlying depression. Participants and Methods: 38 participants were divided into 2 groups; "LOC" (n=19) and "No LOC" (n=19). All participants presented with a history of $m$ TBI or PCS. Males and females in the age range of 17-66 years of age with years of education ranging from 8.9 to 16.9 years were represented in this sample. Injuries were due to falls, assaults, motor vehicle accidents (MVA), or sports-related activities.

Results: Data collection and analyses were performed post-hoc using $t$-tests with an alpha level of 0.05 . A significant difference was found between the two groups on the Beck Depression Inventory-II (BDI-II), suggesting that concussion without LOC is associated with increased depression.

Conclusions: The findings of this study theoretically support the existing body of literature regarding concussion-related symptoms and depression. It is possible that the severity of neurological injury is negatively correlated with depression (i.e increasing severity of injury is associated with decreasing severity of depression). Results from this study should also be considered contextually, as sample size and a lack of control for length of LOC could also factor into said results. Further research could expand upon the relationship between length of LOC and self-reported depression.

Correspondence: Vincent M. Reginella, M.S., Neuropsychology, James D. Petrick, PhD. Neuropsychological Consultation \& Rehabilitation, 606 Golden Oaks Lane, Pittsburgh, PA 15237, United States. E-mail: vmr5492@gmail.com

M. REYNOLDS, S. HALL, E. CARROLL, P. BEAN \& B.E. CLARK. Comparing Perceptions of Mild Traumatic Brain Injury to Orthopedic Injury.

Objective: The terms mild traumatic brain injury (mTBI) or concussion may evoke different expectations. These expectations are important because previous researchers have shown that expected symptoms at the time of injury were the best predictors of actual symptoms post-injury. The current study compared perceptions of brain injuries to orthopedic injuries and the impact of familiarity with brain injury terminology.
Participants and Methods: Participants $(n=153)$ read a vignette depicting a person sustaining either an mTBI or an orthopedic injury in a motor vehicle accident. Groups were told that they had sustained a "concussion," "mTBI," "no diagnosis because their concussion symptoms had resolved," or that they had sustained a broken leg. Participants reported expectations regarding symptoms, consequences, self-efficacy, injury recovery, length of recovery, positive and negative changes in life perspective, and undesirability of the injury.

Results: There was a main effect of type of injury on expected symptoms, $H(3), 30.62, p<.001$. Brain injuries, either mTBI, concussion, or no diagnosis, were viewed as more symptomatic than an orthopedic injury. Participants also thought that an mTBI would result in more positive changes in life perspective compared to an orthopedic injury, $F(3,150)=5.00, p=.002$. Participants were more familiar with the term "concussion" relative to "mTBI." Those with more familiarity with the terminology thought they would have more ability to control the symptoms of the brain injury and would have fewer life consequences compared to those with less familiarity.

Conclusions: Brain injuries are viewed as more serious conditions compared to orthopedic injuries - even concussive events that are asymptomatic and resolve quickly are viewed as worse than orthopedic injuries. Those more familiar with brain injury terminology think they can better control symptoms and believe it will impact their lives less than those who are less familiar with the terminology.

Correspondence: Meredith Reynolds, MA, Psychology, University of Montana, 32 Campus Dr., SB 137, Missoula, MT 59812, United States. E-mail:meredith.reynolds@umontana.edu

\section{R.J. RICHARDSON, M. MALLECK, B.G. ZUCCATO \& C. ABEARE. Sex Differences and Post-Concussion Symptom Reporting in a Large Clinical Sample.}

Objective: Previous studies have demonstrated inconsistent results regarding post-concussion symptom reporting at baseline and postinjury among males and females. Much of the research related to symptom reporting has been conducted on males, yet it is unclear whether these results generalize to females. Other studies have found that females more frequently endorse post-concussive symptoms compared to males both at baseline and post-injury, whereas others have found no sex differences. The present study seeks to add to our knowledge of sex differences in symptom reporting by investigating the base rates of individual post-concussion symptoms at baseline and post-injury among males and females.

Participants and Methods: Male and female athletes were assessed at baseline $(\mathrm{N}=10,604)$ and post-injury $(\mathrm{N}=1,388)$ at a large American medical center. Participants completed the Post-Concussion Symptom Scale (PCSS), a 22-item questionnaire asking participants to rate the presence and severity of symptoms commonly reported following concussion at baseline and following injury.

Results: A significant sex difference was found at baseline such that females $(M d n=3.00)$ reported more post-concussive symptoms than males $(M d n=1.00)$. Similarly, post-injury, females $(M d n=6.00)$ also reported more symptoms than males $(M d n=4.00)$. Despite these significant results, effect sizes were small in both cases. Base rates for specific symptom endorsement at baseline and post-concussion are reported. Conclusions: Given the large size of this sample, the data support the conclusion that females report more symptoms than males both at baseline and following concussion. This suggests that sex differences should be accounted for when assessing concussive symptoms.

Correspondence: Robin J. Richardson, B.A. Hons., Psychology, University of Windsor, 401 Sunset Ave, Department of Psychology, Windsor, ON N9B3P4, Canada.E-mail: richar1a@uwindsor.ca 
K.A. RITCHIE, J. HOELZLE, L.D. NELSON \& M.A. MCCREA. Premorbid intellectual functioning impacts baseline concussion screening performance.

Objective: Intellectual ability influences neuropsychological test performance. This study investigates the rate at which healthy individuals of below average, average, and above average intellectual functioning obtain impaired scores on the Standardized Assessment of Concussion (SAC: McCrea et al., 2000) and Immediate Post-Concussion and Cognitive Testing (ImPACT, 2013).

Participants and Methods: An archival sample of pre-season, baseline test data from healthy amateur athletes $[N=1988, M$ age $=17.82(1.90)]$ was utilized that included the SAC, ImPACT, and the Wechsler Test of Adult Reading (WTAR; Wechsler, 2001). Participants were stratified into groups based on WTAR performance. Chi-square tests for independence determined whether rates of obtaining one or more impaired score (i.e. scores below the $5^{\text {th }}$ percentile) varied among groups.

Results: Pearson Chi-square tests indicated a significant association between estimated IQ group and observed frequency of impaired scores on both the SAC $\left[\chi^{2}=36.38, p<.05\right.$, Cramer's $\left.V=0.14\right]$ and ImPACT $\left[\chi^{2}=25.63, p<.05\right.$, Cramer's $\left.V=0.14\right]$. On the SAC, participants with below average estimated IQ obtained impaired scores at a higher rate than anticipated, while those with above average estimated IQ obtained impaired scores at a lower rate than would be expected. On ImPACT, participants with average estimated IQ obtained impaired scores at a higher rate than would be expected, while individuals with above average estimated IQ obtained impaired scores at a significantly lower rate than anticipated.

Conclusions: Although concussion screening instruments are designed to detect cognitive impairment due to injury, this study suggests that intellectual ability meaningfully influences performance on these instruments at baseline. These findings are relevant to consider when using these instruments for clinical decision-making as intellectual ability may influence the likelihood of obtaining impaired scores, rather than the injury characteristics.

Correspondence: Kathryn A. Ritchie, B.A., Psychology, Marquette University, 1930 N. Prospect Ave, \#51, Milwaukee, WI 53202, United States.E-mail:kathryn.ritchie@marquette.edu

\section{A. SCAVONE, B.G. ZUCCATO, C. MILLER, L.A. ERDODI, B.M. MERKER \& C. ABEARE. Performance Validity at Baseline Testing on the ImPACT in Young Athletes with ADHD.}

Objective: Baseline testing among young athletes is widely used for decision-making about return-to-play following concussion. Invalid performance on these assessments is of particular concern given that baseline scores are used as a point of comparison for post-injury performance. This study examined whether young athletes with attention-deficit hyperactivity disorder (ADHD) were more likely to demonstrate invalid performance on baseline testing than their non-ADHD peers. In addition, given gender differences in ADHD status, we examined the association between gender and failure rate on published embedded validity indicators (EVI) on the ImPACT

Participants and Methods: In a large sample of youth athletes ( $\mathrm{N}=11,262 ; 51.2 \%$ male; mean age: 14.82 years) collected between 2010 and 2016 at a Midwestern urban medical center, 7.97\% reported a history of ADHD.

Results: Failure rates on EVIs were significantly higher among those with ADHD compared to those without on the ImPACT (relative risk: 1.24-1.70). To further explore predictors of invalid performance, ADHD status and gender were entered into a logistic regression, with EVI failure as the dependent variable. Athletes with ADHD had significantly higher rates of invalid responding $(p<.001$; odds ratio $(\mathrm{OR})=1.73$; relative risk: 1.25), with invalid responding greater among males $(p=.015$; $\mathrm{OR}=1.12$; relative risk: 1.08$)$. However, the gender $\mathrm{x} A \mathrm{DHD}$ interaction was not significant $(p=.171)$.

Conclusions: These findings suggest that clinicians working with injured young athletes should be aware that athletes with ADHD, as well as males, have a higher rate of invalid cognitive baseline performance.
Lastly, it is unclear whether the elevated EVI failure reflects false positives, genuinely poorer performance due to ADHD, or a true increase in the rate of invalid performance associated with ADHD.

Correspondence: Antonette Scavone, Psychology, University of Windsor, 805-166 University Ave W, Windsor, ON N9A5N9, Canada. E-mail: scavone@uwindsor.ca

S. SCHLEICHER-DILKS, W. GARMOE, K. BRADY, K. LARSON, P. LUM \& A. DROMERICK. Examining Performance on Cognitive, Effort, and Psychological Measures Among Individuals With Concussion, Controls, and Coached Dissimulators.

Objective: There were four aims of this study: 1) determine if there were significant differences in performance on cognitive measures, performance validity test (PVTs), and symptom inventories between adults with concussion and controls; 2) describe the performance of dissimulators on these measures; 3 ) determine the frequency of PVT failure after concussion; 4) assess symptoms in individuals with concussion after 30 days.

Participants and Methods: The 115 participants consisted of 50 individuals with a diagnosis concussion within the past 9 days $(M=6, S D=2)$, 50 healthy controls, and 15 subjects coached to dissimulate. ANOVAs and t-tests were used for analyses and effect sizes were calculated.

Results: There were significant differences between concussion participants and controls on the PHQ-9 $t(99)=5.35, p<.001$, Rivermead Post-Concussion Symptom Questionnaire $t(99)=9.98, p<.001$, BAI $t(99)=4.88, p<.001$, and PCL-5 $t(99)=4.77, p<.001$. Dissimulators performed significantly worse than controls and those with concussion on all measures. Only one concussion participant failed a single PVT. After 30 days, individuals with concussion reported a significant decrease in symptoms on the Rivermead $t(39)=6.96, p<.001$.

Conclusions: No significant differences were found on neuropsychological measures between controls and concussion subjects, suggesting generally intact cognitive abilities following mild head injury, even with the presence of highly symptomatic individuals in the concussion group. Along with the fact that only dissimulators failed PVTs, this suggests that individuals who fail PVTs after mild head injury are not putting forth their best effort. Compared to objective cognitive difficulties, somatic and emotional symptoms predominate the clinical picture after concussion. Despite the presence of emotional difficulty following concussion being a risk factor for complicated recovery, many individuals experienced a significant reduction in symptoms one month post-injury.

Correspondence: Sara Ann Schleicher-Dilks, PhD, Psychology, MedStar National Rehabilitation Hospital, 340 Taylor St NE, Apt 43P, Washington DC, DC 20017, United States.E-mail: ssdilks@gmail.com

E. SHAPIRO, C. BASS, C. SCHRAUFNAGEL, P. JONES, J. HART, M. CULLUM \& N. DIDEHBANI. Impact of Mild Traumatic Brain Injury on PTSD Treatment Outcomes.

Objective: Previous research suggests a history of both mild traumatic brain injury (mTBI) and Posttraumatic Stress Disorder (PTSD) may complicate treatment recovery from PTSD. The objective of this study was to examine the impact of a history of mTBI on treatment response following combined Cognitive Processing Therapy (CPT) and repetitive transcranial magnetic stimulation (rTMS) in a sample of veterans with PTSD. Participants and Methods: Subjects were part of a randomized controlled trial of rTMS to enhance CPT for PTSD. Repeated measures ANOVAs examined differences between subjects with a history of mTBI $(n=49)$ and those without TBI history (nonTBI n=54) on PTSD symptom severity, depression, and psychosocial/occupational/ educational daily functional impairment ratings pre- and post- treatment.

Results: Pre-treatment levels of PTSD symptom severity, depression, and daily functional impairment ratings were not significantly different between the mTBI and nonTBI groups. Post-treatment levels of PTSD symptom severity and depression were not significantly different between the mTBI and nonTBI groups. However, there was a significant 
post-treatment difference between groups found in ratings of daily functional impairment $F(1,51)=5.31, \mathrm{p}=.025$. Although both groups showed improved daily functioning post-treatment, the nonTBI group improved significantly more than the mTBI group.

Conclusions: A history of mTBI in veterans with PTSD was associated with less improvement in daily functioning post rTMS/CPT compared to those without a history of mTBI. Thus, implementation of interventions and support in the domains of psychosocial/occupational/educational daily functioning in veterans with both PTSD and TBI may be beneficial to maximize treatment gains.

Correspondence: Emma Shapiro, Ph.D., Neuropsychology, North Texas VA Health Care System, 4500 S. Lancaster Rd., Dallas, TX 75216, United States.E-mail: emmamshapiro@gmail.com

A.M. SVINGOS, B.M. ASKEN, Z.M. HOUCK, G. HROMAS, C.C. MORENO, S.C. HEATON, R.M. BAUER \& J.R. CLUGSTON. Micro-RNA Upregulation Following Sport-Related Concussion in Collegiate Athletes.

Objective: Accurate diagnosis of concussion in athletes would significantly benefit from objective diagnostic markers. Micro-RNA(miRNA) are small, highly conserved, non-encoding RNA sequences that may serve as reliable and valid concussion biomarkers given their ability to cross the blood brain barrier and stability across environmental conditions. Here, we examine pre- to post-concussion differences in miRNA regulation.

Participants and Methods: Peripheral blood samples were obtained from 27 collegiate athletes (female $n=16$ ) at baseline (mean age $=19.4 \pm 0.9$ years) and following concussion (mean time after injury $=6.4 \pm 7.3$ hours). Sample size varied slightly per biomarker. Serum samples were processed using quantitative PCR and cycle thresholds were transformed to yield relative quantifications of a panel of miRNA biomarkers: miRNA let-7a-5p, miRNA 153-3p, miRNA 133a-3p, miRNA 19a-3p, miRNA 210-3p, miRNA 223-3p, miRNA 26a-5p, miRNA 423-3p. Wilcoxon signed-rank test was used to assess significance of pre-post injury changes in miRNA regulation.

Results: Significant upregulation was observed for miRNA 223-3p $(p=.029)$, miRNA let-7a-5p $(p=.046)$, and miRNA 153-3p $(p=.029)$ following concussion relative to baseline. Considering individual-level changes yielded the following sensitivity to injury detection: miRNA $223-3 p-69.6 \%$, miRNA let-7a-5p - 65.2\%, and miRNA $153-3 p-$ $59.3 \%$. No other miRNA biomarker significantly increased relative quantifications from pre- to post-injury.

Conclusions: Micro-RNA may objectively signal a concussion in collegiate athletes and could aid in current clinical-based diagnostic and treatment decisions. The present study was limited in that it did not include a control group, making it impossible to compute receiver operating characteristics beyond sensitivity. Future studies must replicate observed findings in a larger sample that will allow for consideration of demographic and historical factors (e.g., prior head injury) as they could relate to miRNA expression.

Correspondence: Adrian M. Svingos, M.S., Clinical and Health Psychology, University of Florida, P.O. Box 100185, Gainesville, FL 32610, United States. E-mail: adrianmchambers@phhp.ufl.edu

\section{B.G.ZUCCATO, I. CHILD, B. TAYLOR, B.M. MERKER \& C. ABEARE. Brief Cognitive Testing in the Acute Stage of Concussion: Does it Increase Symptoms?}

Objective: Current recommendations for best practice suggest a brief period of cognitive and physical rest following a mild traumatic brain injury (mTBI; concussion). It is also common practice for athletes suspected of having sustained a concussion to undergo brief neurocognitive testing. Previous research has shown that the cognitive exertion from this testing has the potential to exacerbate symptoms if tests are completed too soon after concussion. The purpose of the current study was to determine whether symptoms increased after cognitive testing in the total sample, at 3 days, and 7 days post-concussion.
Participants and Methods: Data were a consecutive case series collected at a prominent southeastern Michigan hospital for clinical purposes. Participants were 347 (54.8\% male) athletes ranging from 10 to 24 years of age $\left(M_{\text {age }}=16.078\right)$. Participants completed a baseline and post-concussion assessment as well as a pre- and post-cognitive testing symptom checklist. Participants who completed both baseline and post-concussion testing, both pre- and post-cognitive testing symptom scales and passed embedded validity indicators were included in this study.

Results: There was no significant difference between pre- and post-cognitive testing symptom scores in the total sample $(\mathrm{n}=347)$, at 7 days post injury $(n=61)$, or at 3 days post-injury $(n=16)$. Furthermore, there were no significant correlations between symptom change and a composite concussion severity variable, time since injury, or number of previous concussions.

Conclusions: The current study did not yield significant symptom change from pre- to post-cognitive testing, nor was there a significant correlation between severity and change. These findings are contrary to those from previous work and suggest that the completion of neurocognitive testing following concussion is likely not detrimental to the recovery process.

Correspondence: Brian Taylor, University of Windsor, 401 Sunset Ave, Windsor, ON N9B 3P4, Canada.E-mail:taylo125@uwindsor.ca

P.J. UY, Y. LEE, A. CHILDS, Y. MATSUZAWA, J. RICKER, W. BARR \& W.S. MACALLISTER. Symptom Endorsement Validity on the Sports Concussion Assessment Tool 3 (SCAT3).

Objective: To investigate the associations between self-report validity of the Symptom Evaluation section on the Sports Concussion Assessment Tool 3 (SCAT3) with the Minnesota Multiphasic Personality Inventory-2-Restructured Form (MMPI-2-RF) validity scales.

Participants and Methods: Participants included 83 individuals diagnosed with concussions/uncomplicated mild traumatic brain injuries (mTBI) who completed neuropsychological assessments at an urban medical center. The study design involved a retrospective analysis of the data. The battery included various neuropsychological measures and included the MMPI-2-RF and SCAT3.

Results: Higher MMPI-2-RF FBS-r significantly predicted higher SCAT3 Total Symptoms, $\beta=.429, t(3.472)=0.137, p=.001$ and the overall model fit was $R^{2}=.594, F(5,77)=8.401, p<.0001$. Higher MMPI-2-RF FBS-r also significantly predicted higher SCAT3 Symptom Severity, $\beta=.342, t(2.804)=0.602, p<.010$ and model fit was $R^{2}=.607, F(5,77)=8.994, p<.0001$.

Conclusions: The MMPI-2-RF FBS-r scale was associated with Severity and Total Score on the Symptom Evaluation section of the SCAT3. Individuals with concussions endorse a variety of symptoms and the results suggest that endorsement validity should be considered when using the SCAT3. The present study also shows potential for the SCAT3 as a useful tool to identify symptom magnification among individuals diagnosed with concussions in the general population.

Correspondence: Philip J. Uy, Ph.D., ABPP, ABN, Rehabilitation Medicine, NYU Langone Health/Rusk Rehabilitation, 200 E. 30th Street, Apt.1K, New York, NY 10016,United States.E-mail: philipjuy@gmail. com

G. VARGAS, J. CROWLEY \& B. BODT. Neuropsychological Test Performance in Young Children After Concussion.

Objective: There are very few studies on young children's recovery from concussion. Judging this group's recovery can be difficult as they are less reliable symptom reporters and the ImPACT is only valid for ages 10 and up. Recent studies have found that children with more symptom burden overall and certain symptoms at presentation have longer recoveries. This is the first known study to investigate neuropsychological test performance after concussion in young children and how this relates to symptomatology and recovery. 
Participants and Methods: Children were seen in an outpatient concussion clinic at a tertiary care center. Data was obtained for 1986 to 9-year-olds via retrospective chart review of the first visit with a neuropsychologist. At the visit, parents completed a Post-Concussion Scale and parent and child (if able) were asked to rate the child's recovery out of $100 \%$ relative to their baseline functioning. Depending on age, the child completed the Symbol Digit Modalities Test, Trail Making Test Parts A\&B, the Visuomotor Precision subtest from the NEPSY-II, and the Code transmission subtest from the TEA-Ch.

Results: Symptomatology at visit was significantly related to performance on Code transmission and Visuomotor precision. Parent and child's ratings of recovery were significantly related to performance on Visuomotor precision and Trails B, and parent rating also to Trails A. Only performance on Trails B was significantly related to duration of symptoms after visit.

Conclusions: More symptomatic children performed more poorly on tests of sustained attention, speeded visuomotor control, and speeded set shifting. Only one test was significantly related to symptom duration after visit. Symptomatology and rated recovery at visit were stronger predictors of symptom duration than test performance. Neuropsychological tests appear to be a useful tool in helping to judge the current state of concussed younger children but it remains to be seen whether they can help predict which children will have longer recoveries.

Correspondence: Gray Vargas, Ph.D., Nemours/A.I. duPont Hospital for Children, 3 Julie Lane, Newark, DE 19711, United States. E-mail: grayvargas@gmail.com

M.L. WERHANE, K. LAPIRA, A.L. CLARK, N.D. EVANGELISTA, S.F. SORG, M.W. BONDI, D. SCHIEHSER \& L. DELANO-WOOD. Apolipoprotein E- $\varepsilon 4$ Genotype and Pulse Pressure Interact to Affect Cortical Thickness in Brain Regions Vulnerable to Neurodegeneration in Veterans with Mild Traumatic Brain Injury. Objective: Although the APOE- $\varepsilon 4$ allele has been repeatedly linked to poor cognitive and brain outcomes in mild traumatic brain injury (mTBI) samples, it remains unclear how it might interact with vascular risk factors in the expression of pathological brain changes in the aftermath of neurotrauma. Thus, we explored the independent and interactive effects of elevated vascular risk and APOE- $\varepsilon 4$ status on cortical thinning in brain regions vulnerable to neurodegenerative changes in Veterans with history of mTBI.

Participants and Methods: 48 Veterans (mean age $=32$ years) with history of mTBI underwent structural magnetic resonance imaging (MRI), APOE genotyping, and blood pressure (BP) assessment. Pulse pressure (PP), an index of arterial stiffening, was derived from blood pressure (BP) values (systolic - diastolic BP). Multiple regression was used to assess whether PP and genetic status predicted cortical thickness in FreeSurfer-derived regions of interest (ROIs).

Results: Multiple regression, adjusting for age and PTSD severity, showed that APOE- $\varepsilon 4$ status significantly modified the relationship between PP and cortical thickness in the medial orbitofrontal $(p=.023)$ and precuneus $(p=.045)$ regions. Specifically, for $\varepsilon 4$ carriers, lower PP values were associated with greater cortical thinning in these regions. No such relationship was observed in non- $\varepsilon 4$ carriers.

Conclusions: Our results revealed that, in our sample of relatively young Veterans with a history of mTBI, PP and APOE- $\varepsilon 4$ status interact to affect cortical thickness in regions known to be affected early in the context of neurodegenerative disorders such as Alzheimer's disease. Specifically, even after adjusting for age and PTSD symptom severity, APOE- 44 carriers with lower PP demonstrated reduced cortical thickness in orbitofrontal and precuneus regions. These results add to a growing literature highlighting the complexity of vascular and genetic implications on AD pathology following neurotrauma.

Correspondence: Madeleine L. Werhane, PhD, SDSU/UC San Diego Joint Doctoral Program in Clinical Psychology, 4930 Del Mar Ave, 106, San Diego, CA 92107, United States. E-mail: mlwerhane@gmail.com
B.G.ZUCCATO, T.L. MCAULEY, R.J. RICHARDSON, B.M. MERKER \& C. ABEARE. Latent Structure of Post-Concussion Symptoms in Athletes at Baseline.

Objective: The purpose of the present study was to examine the latent structure of the 22-item Post-Concussion Symptom Scale (PCSS) in healthy athletes at baseline. An ancillary objective was to investigate differences in factor scores by age and gender.

Participants and Methods: Archival clinical data were used in this study. Participants were 10,604 healthy athletes, aged 10 to 24 $(M=14.484, S D=1.78)$. Participants whose performance on the cognitive measures was deemed invalid by the ImPACT embedded validity indicators were excluded. Principal axis exploratory factor analysis (EFA) using oblimin rotation was conducted. Factor scores were then computed based on the factors delineated by the EFA.

Results: The data yielded four factors: a four-item cognitive factor, fiveitem somatic factor, a three-item sleep factor, and a three-item affective factor. Seven items did not load on any factor. There were significant differences by age and gender, although effect sizes were very small.

Conclusions: The factor structure for the baseline data supports the structure yielded by other studies, despite methodological differences. Although significant differences between ages and genders were found, small effect sizes limit their practical significance.

Correspondence: Brandon G. Zuccato, B.A., Psychology, University of Windsor, 401 Sunset Ave., Windsor, ON N9B 3P4, Canada. E-mail: zuccatob@uwindsor.ca

\section{Neuroimaging}

J. COHEN, X. XIE, B. BROWN, G. GROVE, M.E. WOLLAM, J.C. WATT \& K.I. ERICKSON. Adults with Higher Cardiorespiratory Fitness Show Evidence for Greater Neural Efficiency During the Stroop Task.

Objective: Higher cardiorespiratory fitness (CRF) levels are associated with greater neural efficiency in older adults and children, particularly in frontal brain regions. However, few studies have examined this relationship in young adults to understand whether these associations exist across the lifespan. We examined whether higher CRF in a young adult sample was (a) associated with elevated performance on the Stroop task, which elicits frontal lobe activity, and (b) with more efficient brain activity.

Participants and Methods: CRF was measured by maximal oxygen consumption $\left(\mathrm{VO}_{2 \max }\right)$ in 50 young adults $(\mathrm{m}$ age $=25.22+5.17 ; 44 \%$ male). Functional Magnetic Resonance Imaging (fMRI) data was collected during the performance of a color-word Stroop task with three conditions: incongruent, congruent, and neutral. Multiple regression analyses, controlling for sex, were used to examine associations between CRF and Stroop performance, as well as brain activation patterns during incongruent and congruent conditions.

Results: Comparing activation during incongruent and congruent conditions revealed bilateral clusters in the medial prefrontal cortex, superior parietal cortex, and right caudate nucleus that were negatively associated with CRF. Consistent with our predictions on neural efficiency, smaller differences in activation between conditions was associated with higher CRF (all $\mathrm{r}(49)<-.423$, all $\mathrm{p}<.002$ ). CRF was not significantly related to behavioral measures.

Conclusions: Higher CRF was associated with increased neural efficiency in younger adults in regions that are often implicated in exercise and fitness, including the prefrontal cortex. These associations have not previously been demonstrated in younger adults and provide evidence that higher CRF might be related to increased neural efficiency across the lifespan. The non-significant associations with behavior may reflect limited variability in task performance and suggest that brain activity is a more sensitive metric related to CRF.

Correspondence: Jamie Cohen, Psychology, University of Pittsburgh, 210 S. Bouquet Street, 3211 Sennott Square, Pittsburgh, PA 15260, United States.E-mail: jcohe7@gmail.com 
AM Coffee Break

10:30-10:45 a.m.

\author{
Plenary B. Rule + Rhythms = Cognition \\ Presenter: Earl K. Miller \\ 10:45-11:45 a.m.
}

\section{E.K. MILLER. Rule + Rhythms = Cognition.}

Working memory, the "blackboard" of consciousness, is at the very center of cognition. By choosing what we hold in mind, we free ourselves from reflective reactions to the environment. For decades, it has been thought that sustained spiking activity was the neural correlate of working memory. It was an attractive idea: If neural spiking underlies thoughts, perceptions, etc. then sustained spiking should hold those things in mind. However, mounting evidence from our lab and others has revealed something more complex. Underlying working memory are brief bursts of spiking activity, not sustained activity per se, and interplay between beta (20-35 Hz) and gamma (45-100 Hz) rhythms, (Lundqvist et al., 2016). This sparse activity and rhythmic interplay may allow the brain to juggle multiple stimuli that are simultaneously held in working memory.

Further, the interplay between beta and gamma rhythms may underlie the volitional control of working memory. We found evidence that gamma rhythms, which are linked to the bottom-up stimulus information held in working memory, are regulated by the beta rhythms. By contrast, top-down information, like learned rules, is expressed in beta. When sensory information needs to be cleared out of working memory, beta increases and gamma decreases, and there is a suppression of spiking carrying bottom-up stimulus information. This beta-gamma interplay also occurs as the animals read information out of working memory. In fact, when the animals made errors, the patterns of beta and gamma bursting gave insight into why errors were made.

In short, these data suggest a new model of working memory in which interplay between these different rhythms underlies the volitional control that turns on and off the faucet of working memory storage.

As a result of participation in this lecture, the learner will have a deeper understanding of working memory, its neural substrates, and the mechanisms that may underlie volitional control.

Correspondence: Earl K. Miller, PhD, MIT, 77 Massachusetts Ave, 466241, Cambridge, MA 02139, United States.E-mail: ekmiller@gmail. com

\section{Invited Symposium 2. Neurocognitive Function in Aerospace and Aeronautics}

\section{Chair: Robert L. Kane}

\section{Presenters: Robert L. Kane, Andrea Vincent, Gary Kay, Kimberly Seaton, Thomas Williams}

\section{1:45 a.m.-1:15 p.m.}

R.L. KANE, K. SEATON, T. WILLIAMS, A. VINCENT \& G. KAY. Neurocognitive Functioning in Space and Aviation.

This symposium presents an overview of the role neuropsychology plays in the US space program and in the medical certification of aviators. The Space Flight Cognitive Assessment Tool for Windows (WinSCAT) has been implemented on every expedition to the International Space Station. Preflight baselines are obtained and the test battery is administered on orbit to maintain proficiency. It can be used as needed to assess astronauts' cognitive status in response to adverse events. Neuropsychological assessment also plays an important role in civil and military aviation. In this symposium, Dr. Robert Kane will present the history of the NASA WinSCAT program and review considerations for implementing this capability. Dr. Kimberly Seaton from Johnson Space Center (JSC) will review the implementation of the WinSCAT program. The NASA-JSC protocol for cognitive assessment will be described along with lessons learned and future directions. From the beginning of the WinSCAT program there has been an emphasis on refining decision rules used to identify nominal vs. off-nominal test performance. Dr. Andrea Vincent from the University of Oklahoma Cognitive Science Research Center will present an analysis using space and ground-based data pertaining to the refinement of decision rules. Dr. Thomas Williams from the NASA Behavioral Health and Performance Laboratory will present data from recent psychometric and criterion validation studies that also contributed to the development of astronaut related norms. Dr. Gary Kay from Cognitive Research Corporation will provide an overview of the contribution of neuropsychologists to the FAA medical certification process when issues arise related to injury, medical and neurological disorders, substance abuse, and ADHD. His presentation will provide an overview of the FAA requirements for neuropsychological assessment of aviators, an introduction to the CogScreen test battery, and a description of training opportunities for this specialty area.

Correspondence: Robert L. Kane, PhD, Cognitive Consults and Technology LLC, 3604 Whitehaven Pkwy NW, Washington, DC 20007, United States. E-mail: rlkane@mac.com

\section{R.L. KANE. Development of the Spaceflight Cognitive Assessment Program.}

Development of the Space Flight Cognitive Assessment Tool began in August 1996 under the direction of Christopher Flynn, MD with the goal of implementing an objective method for monitoring the neurocognitive status of space crews. Anticipated risks included toxic exposure, radiation, anoxia, decompression, injury, illness, and medication effects. To address the need to monitor cognitive status and to develop a capability for in-flight assessment, NASA formed an integrated product team (IPT). The IPT set out requirements for an in-flight cognitive assessment tool and carried out an analysis of alternatives (AOA) of available options. Dr. Kane will review the initial requirements and processes that led to the implementation of the Spaceflight Cognitive Assessment Tool and the subsequent adaptation of the initial tool to a Windows platform. He will discuss the technical, operational, and validation considerations that drove the development of the test system and continue to guide medical and operational considerations going forward.

Correspondence: Robert L. Kane, PhD, Cognitive Consults and Technology LLC, 3604 Whitehaven Pkwy NW, Washington, DC 20007, United States.E-mail: rlkane@mac.com

\section{A. VINCENT. Neurocognitive Health During Space Flight.}

Space missions expose astronauts to an environment that may negatively affect performance. The Spaceflight Cognitive Assessment Tool (WinSCAT) was designed to routinely monitor the neurocognitive status of astronauts during spaceflight. WinSCAT serves as the current standard for measuring astronaut cognition in space. Using data collected over a number of space missions, we examined profiles in WinSCAT performance and evaluated various approaches for identifying offnominal performance. Results will inform efforts to validate or refine existing guidelines.

Correspondence: Andrea Vincent. E-mail: avincent@ou.edu

\section{G. KAY. Aviation Clinical Neuropsychology.}

Aviation clinical neuropsychology is the application of neuropsychology in the context of civil and military aviation. Aviation neuropsychologists are called upon to determine if an aviator has neurocognitive deficits which would be considered aeromedically significant. This information plays a key role in determination of the medical certification of pilots with a range of conditions including: recovery from substance abuse/ dependence, head trauma, HIV seropositivity, history of diagnosis or 
treatment for ADHD, and for those being treated with SSRI antidepressant medication. This presentation will provide an overview of the FAA requirements for neuropsychological assessment of aviators, an introduction to the CogScreen test battery, and a description of training opportunities for this specialty area.

Correspondence: Gary Kay. E-mail: gkay@cogres.com

K. SEATON. Cognitive Assessment in Long-Duration Space Flight. The Spaceflight Cognitive Assessment Tool for Windows (WinSCAT) was developed for medical operations at NASA's Johnson Space Center and is an application that assesses cognitive functioning through a battery of five neurocognitive tests. The purpose of WinSCAT is to provide crewmembers and flight surgeons with an objective assessment of cognitive functions under the following conditions: exposure to toxins or other noxious stimuli, decompression sickness, following an unexpected traumatic event or medical condition, periodically throughout the mission, and at the request of the crew surgeon, commander, or Chief Medical Officer (CMO) when neurological deficit is suspected. WinSCAT is a medical requirement for long-duration US, JAXA, ESA, and CSA astronauts and has been implemented with all expeditions to date on the International Space Station (ISS). 2011 updates to WinSCAT included incorporating a moving rather than fixed baseline, stricter interpretation rules, and network capability to support a 6 -person crew. The WinSCAT software can be completed with or without instructions. Crew members can review results onboard, without assistance from the BHP Section specialists, using a color-coded scheme. A test score that is flagged red indicates a percentage drop from an individual's baseline. If two or more test results are flagged red, the crewmember is instructed to contact the Crew Surgeon. The ability for crewmembers to have an objective measure of cognitive functioning will be increasingly important as mission durations increase to one year and longer. Self-assessments with immediate feedback will be essential with increased crew autonomy and communication delay. Immediate objective feedback can provide crewmembers an alert to implement in-flight countermeasures to reduce the risk of errors or degraded performance. The NASA-JSC protocol for cognitive assessment will be described, and lessons learned as well as forward plans and considerations will be discussed.

Correspondence: Kimberly Seaton.E-mail:kimberly.seaton@nasa.gov

T. WILLIAMS, J. SCHNEIDERMAN, J. TISSON, K. SEATON, L. LANDON, W.B. VESSEY, R. STANLEY \& C.J. KUSMIESZ. WinSCAT Test-Retest Reliability in Senior Military Leaders.

The importance of ensuring the integrity of the cognitive systems to ensure sustained, high-level cognitive performance in spaceflight of any duration cannot be overemphasized. In addition, there is also a need ensure any measures of neurobehavioral functioning offer sensitive, specific, and valid methods to assess the individual variability in cognitive performance, in the differential sensitivity to sources of that variation (e.g., gender, age, environmental factors) as well as within both the basic functions (e.g., processing speed, attention) and more complex functions (e.g., executive reasoning, emotional processing). WinSCAT (SPACEFLIGHT Cognitive Assessment Tool for Windows) is currently used as a clinical tool onboard the International Space Station (ISS) to monitor the neurocognitive status of astronauts if an accident occurs (e.g.., injury, decompression accidents, fitness for duty evaluation), robust and validated norms specific to the astronaut corps for this measure are lacking. The presentation provides WinSCAT normative data using a population of senior military officers with histories of high demands similar to those experienced by astronauts (e.g., deployments, isolation, extreme environments). Individuals attending senior service colleges are among the most elite of leaders in the military and serve as a valuable surrogate for astronauts (i.e., 6 of 8 astronauts in the 2013 class were military officers, with 219 of the 330 current and former astronauts having military experience). We provide an evaluation of WinSCAT's criterion validation by examining its test-retest reliability (across 3 months) and sensitivity to performance-related individual differences (e.g., heart rate variability and ratings of mental effort during testing).

Correspondence: Thomas Williams. E-mail: thomas.j.williams-1 @nasa.gov

\section{Symposium 3. Increasing Minority Engagement in the Field of Neuropsychology}

\section{Chair: Michelle Madore}

\section{Presenters: Beatriz MacDonald, Christine Salinas, Steven P. Verney, Justina Avila}

\author{
11:45 a.m.-1:15 p.m.
}

\section{MADORE, B. MACDONALD, C. SALINAS, S.P. VERNEY \& J. AVILA. Increasing Minority Engagement in the Field of Neuropsychology.}

Professional development guidelines of clinical neuropsychologists often indicate training in cultural differences and diversity should be included in the training of individuals in our field (APA Multicultural Guidelines, 2003). While cultural competence as a neuropsychologist is ethical and necessary, less information is available regarding increasing the diversity of neuropsychologists beyond actively recruiting and enrolling individuals from diverse backgrounds into our training programs. Even less is available regarding the retention of diverse individuals for future success or how to manage difficult dialogues regarding sensitive cultural issues. Consistent with the mission of the Society for Clinical Neuropsychology's Ethnic Minority Affair's Sub-Committee, this symposium will focus on providing practical strategies and information on how to increase minority engagement in our field. The strategies discussed will be applicable to a wide range of diversity issues in a variety of settings. The aim of this symposium is to provide guidance on how one could implement aspects of the multicultural guidelines.

Drs. Beatriz MacDonald, Christine M. Salinas, Steven P. Verney, and Justine Avila, MA will be the persons who will discuss strategies focusing on how to best cultivate an environment of inclusivity from the lenses of recruitment, retention, mentorship, and peer-to-peer interactions. Correspondence: Michelle Madore, PhD, Psychiatry, VA Palo Alto Health Care System, 3801 Miranda Ave, MC 151-Y, Palo Alto, CA 94304, United States. E-mail:madoremr@gmail.com

\section{B. MACDONALD. What Happens Beyond Active Recruitment and Enrollment?}

Neuropsychological training programs often aspire to increase their numbers of staff, faculty, and students from diverse backgrounds. The American Psychological Association recently revised this 'aspiration' and requires it as a 'standard' for accreditation in the effort to better integrate the silos of diversity. In order to better care for our increasingly diverse population, we as a field need to value our own diversity. Diversity standards need to be more than just active recruitment and increases in enrollment. Once recruited, faculty and students from diverse backgrounds often find the transition to training programs difficult, confusing, and culturally isolating. Inclusive excellence is the acknowledgement that a program's success is dependent on how well it values, engages and includes the rich diversity of its members and their valuable contributions. Programs need to create engaging environments that foster this inclusive excellence at each phase, from the program's website to the graduation ceremony. Some programs find their excellence through writing a 'diversity newsletter', developing reading and research groups to engage in dialogue, or revising the content of coursework. Others by modeling cultural openness, acknowledging cultural knowledge, and practicing both cultural competence and humility. Cultivating inclusive excellence begins with defining what diversity signifies to the program as a whole, which in turn provides a 
blueprint of achievable goals and standards. These goals serve as a guide to develop ongoing assessments of the program. Ultimately all of these initiatives will be fruitless without champions and supporters. At the end of this talk, participants will be better able to be these champions, with achievable goals and examples of mechanisms to create their own inclusive excellence.

Correspondence: Beatriz MacDonald.E-mail:bmacdon@post.harvard.edu

C.SALINAS. State of the Art in Cultural NeuropsychologyMentoring \& Training: Results from the Hispanic Neuropsychological Society Mentoring Program and AACN Relevance 2050 Survey of postdoctoral programs in the U.S.

The seismic U.S. demographic shift presents unique practice challenges and enormous opportunities for the field of neuropsychology to better develop a pipeline of professionals who can deliver culturally responsive evaluations and interventions to meet the needs of our patient population. Within this context, multicultural issues have become a central emphasis of recent inter-organizational efforts, Summits, national conventions, and APA policies. Hence, it is critical to understand current practices in training postdoctoral fellows in cultural issues in neuropsychology in order to identify gaps, strengths and resources needed to translate expert opinions that have been discussed into practical recommendations. In the current study, we surveyed trainees, supervising clinical neuropsychologists, and Training Directors from APPCN and non-APPCN postdoctoral training programs regarding their multicultural assessment training, clinical practices, didactics and perspectives. For the purposes of this study, we explored various diversity factors in training but had an emphasis on ethnicity and bilingualism.

In this talk, we will also briefly describe the need to diversify the field plus mentor neuropsychologists from diverse backgrounds. We will briefly define/describe models of mentoring guided by the literature and APA. We will describe the evidence base for mentoring in assisting with recruitment, retention, and advancement in various fields, including psychology/neuropsychology. We will describe our methods/procedures of recruiting and pairing 54 individuals from HNS, pre- and post- survey data findings plus discuss the strengths, limitations, and implications of our results for trainees, professionals, and the field.

Correspondence: Christine Salinas. E-mail: drchristinesalinas @gmail.com

S.P. VERNEY \& J. AVILA. Engaging in Difficult Dialogues towards Multicultural Knowledge, Attitudes, and Skills in Neuropsychology: Peer-to-Peer Discussions and Interactions.

Neuropsychology training programs and more generally, psychology departments, often struggle with implementing the APA multicultural guidelines (2003) to infuse diversity concepts throughout psychology training curricula. Many faculty may not have been adequately trained to do so and/or may also struggle with multicultural tenets. Multicultural courses and courses that strive to integrate multicultural concepts are the most challenging of graduate training courses as they balance intellectual issues with emotional processing. Both students and faculty often experience significant apprehension and reluctance to multicultural training and minimization of course material and those with multicultural expertise. The purpose of this presentation is to offer our experiences as a doctoral student and faculty member in a clinical psychology program in creating meaningful peer-to-peer discussions and interactions towards increasing multicultural knowledge, attitudes, and skills within the department.

Qualitative experiences will be used to offer methods for overcoming many of the challenges racial/ethnic minority students and faculty face, including 1) building community, 2) opening the dialogue with peers, 3) continuing the dialogue, and 4) changing the climate. Experiences from a student perspective will be related to developing a professional identity grounded in social justice, finding a like-minded community in an academic setting, opening the dialogue with faculty and peers about multicultural topics, and developing relationships with majority group faculty. From a faculty perspective, experiences shared will involve building campus communities and organizations that provide multicultural resources for students and faculty, initiating dialogue with peers from different ideological backgrounds, fostering multicultural identity development through continued dialogue, and changing the climate through campus community involvement.

Correspondence: Steven P.Verney, NM, United States.E-mail:sverney@ unm.edu

\section{Paper Session 2. Adult Medical Disorders}

$$
\text { Moderator: Cady K. Block }
$$

11:45 a.m.-1:15 p.m.

D.V. NGUYEN, A. LEE, P. MALLOY, I. PIRYATINSKY, P. KLINGE \& S. SALLOWAY. Post-Shunting Cognitive Outcomes in Normal Pressure Hydrocephalus Patients.

Objective: Idiopathic normal pressure hydrocephalus (NPH) is characterized by impairment in gait, continence, and cognition. Ventriculoperitoneal shunting may improve these functions, but evidence for cognitive recovery is mixed, particularly in the executive domain and among patients with comorbid Alzheimer's disease (AD). This study examined shunt outcomes for NPH patients with a focus on executive function (EF) and comorbid AD

Participants and Methods: We recruited 38 NPH clinic patients, who on average were 75 years old and had 13 years of education. Participants completed a standard cognitive battery, gait exam, neurologic exam, and caregiver questionnaire at baseline and after 3 and/or 12 months of shunting. Amyloid PET imaging and cerebrospinal fluid (CSF) assays were performed to assess AD pathology. Twelve patients showed AD comorbidity based on these biomarkers (amyloid PET standard uptake value ratio $>1.1$ or CSF total tau to $\mathrm{A} \beta 42$ ratio $>1)$. Mixed ANOVA models were used to examine post-shunt outcomes and interaction effect with AD comorbidity.

Results: Regardless of AD status, patients showed significantly improved gait and continence based on clinician rating, as well as improved global cognition and fine motor dexterity based on testing at 3 and 12 months post-shunt. Results for EF were variable and moderated by AD comorbidity. Post-shunt EF improvement by family rating (Frontal System Behavior Scale, FrSBe) was found only in the AD group. Better inhibition on Stroop tests was identified in all patients, but was more pronounced in the AD group. No significant changes were present in attention, speed, language, visuospatial skills, or memory.

Conclusions: Findings suggest that AD comorbidity may contribute to the mixed EF shunt outcome in prior NPH studies. This study was unique in its inclusion of family rating of executive function (FrSBe), which captured significant changes post-shunt. AD comorbidity and family rating should be considered in future NPH shunt studies and clinical evaluation of shunt candidates.

Correspondence: Diem-Khanh V. Nguyen, Brown University, 69 Brown Street, Box 5804, Providence, RI 02912, United States. E-mail: diemkhanh_nguyen@brown.edu

M.E. ZIMMERMAN, B.M. KIM, C. HALE, A.M. BRICKMAN \& A. SCHECHTER. Neuropsychological Function and Nocturnal Blue Light Exposure in Insomnia: A Pilot Randomized Controlled Study.

Objective: Insomnia svmptoms are associated with neuropsychological dysfunction. Increasing evidence points to the role of nocturnal light exposure in poor sleep, particularly blue light emitted through smartphones/computers. This study aimed to test experimentally whether blocking nocturnal blue light exposure improves cognition in individuals with insomnia svmptoms. 
Participants and Methods: The study utilized a randomized, placebo-controlled, crossover design. Participants were randomly assigned to a 1-week intervention with amber glasses (to block blue light) or clear glasses (control) and then switched conditions after a 4-week wash-out period. Neuropsychological function was evaluated with the NIH Toolbox at baseline (BL), following the 1st intervention week, and following the 2nd intervention week. Within-subjects general linear models contrasted neuropsychological test performance following the amber glasses and clear glasses treatment conditions with BL performance.

Results: Fourteen participants (mean(sd): age $=46.5(11.4)$; education=15.9(1.8)) with insomnia symptoms were included. Compared with baseline, individuals performed better on the List Sorting Task, a test of executive functioning, after 1-week treatment with amber glasses $(p=0.002)$, but similarly after one week treatment with the clear glasses $(p=0.419$, omnibus effect $F=5.16, p=0.014)$. A similar pattern emerged with the Pattern Comparison Task, another test of executive functioning $(\mathrm{F}=7.65, \mathrm{p}=.002)$. Assessment of premorbid ability indicated that treatment with amber glasses "normalized" performance on each test from about 1 standard deviation below expected performance to expected performance.

Conclusions: Utilizing a randomized, placebo-controlled design, we demonstrated improvement in executive function with a blue light blocking intervention among individuals with insomnia symptoms. Future studies will examine potential biological mechanisms.

Correspondence: Molly E. Zimmerman, PhD, Psychology, Fordham Unviersity, 441 E Fordham Rd, Dealy Hall Room 226, Bronx, NY 10458, United States. E-mail: mzimmerman7@fordham.edu

H.A. KRESGE, D. LIU, F.E. CAMBRONERO, K. GIFFORD, T.M. SHONE, K.E. OSBORN, J.G. TERRY, S. NAIR, E. MOORE, K.R. PECHMAN, M.A. WAGENER, J.E. BOGNER, S.L. LAMBROS, C. SEABOLT, J. THOMPSON, L. WALLJASPER, T.J. HOHMAN, S.P. BELL, T.J. WANG, J.J. CARR \& A.L. JEFFERSON. Subclinical Compromise in Cardiac Strain Relates to Worse Neuropsychological Performances in Older Adults: The Vanderbilt Memory \& Aging Project.

Objective: Global longitudinal strain (GLS), the total shortening of the myocardium during the cardiac cycle, has emerged as a more reliable myocardial function measure than left ventricular ejection fraction (LVEF). Because GLS is unaffected by pre-load and diastolic abnormalities, it accurately reflects cardiac contractility even when LVEF is normal. This study examines subclinical cardiac dysfunction, assessed by GLS and LVEF, and neuropsychological performance among older adults.

Participants and Methods: Vanderbilt Memory \& Aging Project participants free of clinical dementia, stroke, and heart failure $(n=318$, $73 \pm 7$ years, $58 \%$ male) completed neuropsychological assessment and cardiac magnetic resonance to quantify GLS and LVEF. Linear regression models separately related GLS and LVEF to neuropsychological performances, adjusting for age, sex, race/ethnicity, education, Framingham Stroke Risk Profile, cognitive diagnosis, and $A P O E-\varepsilon 4$ status. Models were repeated with a cardiac $\mathrm{x}$ cognitive diagnosis interaction term.

Results: Compromised GLS (reflected by higher values) related to worse Boston Naming Test $(\beta=-0.07, p=0.04)$, California Verbal Learning Test-II Delayed Recall $(\beta=-0.11, p=0.007)$, Biber Figure Learning Test (BFLT) Immediate Recall $(\beta=-0.83, p=0.03)$, and BFLT Delayed Recall performances $(\beta=-0.22, p=0.03)$. LVEF did not relate to worse performance on any measure ( $\mathrm{p}$-values $>0.13)$. No diagnostic interactions were observed.

Conclusions: Our study results are among the first to suggest impaired GLS, but not LVEF, relates to worse episodic memory and language performances among older adults free of clinical dementia and stroke. Subclinical cardiac dysfunction may be related to cognitive health in late life even when LVEF remains normal. Results add to a growing body of evidence that GLS is a more sensitive and preferred method for quantifying subclinical changes in cardiac function.
Funding: IIRG-08-88733, R01-AG034962, K24-AG046373, K23-AG045966, K23-AG048347, K12-HD043483, K01-AG049164, R24-HL085343, UL1-TR000445

Correspondence: Hailey A. Kresge, Nashville, TN, United States. E-mail: hailey.a.kresge@vanderbilt.edu

E. SEMMEL, V.M. DOTSON, T. BURNS, W. MAHLE \& T.Z. KING. Cerebellar Volume and Executive Function in Congenital Heart Disease.

Objective: This study investigates cerebellar $(\mathrm{CB})$ involvement in executive function (EF) in young adults with congenital heart disease (CHD). We expect that CHD patients will have smaller CB volumes and perform more poorly on measures of EF. Based on previous research, we expect a functional dissociation between posterior and anterior areas of the $\mathrm{CB}$ such that posterior lobules are related to cognition and anterior areas to motor function. We also expect that the $\mathrm{CB}$ will contribute to $\mathrm{EF}$ beyond processing speed alone.

Participants and Methods: 22 participants with CHD and 22 matched controls were assessed with Verbal Fluency (VF), Trail Making (TMT), and Color-Word Interference (CWI) on the D-KEFS; WASI Matrix Reasoning (MR); BRIEF; Oral Symbol Digit Modalities Test; and Grooved Pegboard (GP). CB volumes were calculated with the SUIT toolbox for SPM8. $t$-tests assessed group differences. Pearson's correlations and Fisher's Z Test assessed relationships between the CB and outcomes. Linear regression tested whether CB volume predicts EF above and beyond processing speed.

Results: Group differences emerged for the posterior CB, CWI Inhibition, MR, BRIEF GEC, and GP, all lower in CHD ( $d=.64-1.01)$. CWI Inhibition and GP were significantly correlated with the posterior CB but not the anterior $\mathrm{CB}$, while MR was correlated with both. The strengths of the correlations with CWI Inhibition and Inhibition-Switching were significantly higher with the posterior $\mathrm{CB}$ compared to the anterior. Posterior CB volume significantly accounted for variance above and beyond processing speed in predicting CWI Inhibition and MR.

Conclusions: Our results support previous findings of cognitive and motor deficits in CHD. Although we found differences in correlation strength, we were unable to demonstrate a double dissociation between anterior and posterior CB. Our findings demonstrate that the posterior CB does, however, contribute to EF skills above and beyond processing speed. Implications for future research and methodology will be discussed.

Correspondence: Eric Semmel, B.A., Psychology, Georgia State University, 140 Decatur Street NE, Department of Psychology, Atlanta, GA 30303, United States. E-mail: esemmel15@gmail.com

H.A. PARKER, C.M. CONSIDINE, J. BRIGGS, E. QUASNEY, E.R. LARSON, H. SMITH, S. SHOLLENBARGER \& C. ABEARE. Evaluating the Moderating Role of Medical Comorbidity, Pain, and Depression on the Relationship Between Sleep Biomarkers and Cognitive Functioning in Obstructive Sleep Apnea.

Objective: Obstructive Sleep Apnea (OSA) is associated with cognitive impairment but the relationships between specific biomarkers (e.g., hypoxia, sleep fragmentation) and cognitive domains remain unclear. Moreover, research is limited examining the potential influence of common health comorbidities, which was the focus of this study. Participants and Methods: Adults with suspected OSA ( $\mathrm{N}=61 ; 52 \%$ male; $\mathrm{M}$ age $=52$ ) underwent neuropsychological evaluation prior to polysomnography (PSG) to assess processing speed, attention, vigilance, memory, executive functioning, and motor dexterity. Apneic syndrome severity, hypoxic strain, and sleep architecture were assessed with PSG. Depression, pain, and medical comorbidity were assessed via questionnaires.

Results: A winnowing approach identified 10 potential moderation models comprised of correlated PSG, comorbidity, and cognitive performance variables. Age was a covariate. Regression analyses identified two moderation models: (1) Average Oxygen Saturation (O2Sat) and 
Depression predicted Memory, $F(4,52)=10.39, p<.001, R^{2}=.31$. There were no main effects, but the interaction between O2Sat and Depression was significant, $b=.04, t(52)=3.57, p=.001, \Delta R^{2}=0.11$. The strongest relationship between low O2Sat and low Memory was in the context of high Depression. (2) Total Sleep Time (TST) and Medical Comorbidity $(\mathrm{MC})$ predicted Processing Speed, $F(4,56)=7.49, p<.001, R^{2}=.45$. There was a main effect of TST, $b=0.23, t(56)=2.43, p=.018$, and the interaction between TST and MC was significant, $b=-0.02, t(56)=-2.04$, $p=.047, \Delta R^{2}=0.04$. At low levels of MC, higher TST corresponded with faster Processing Speed, but this relationship diminished at high levels of MC.

Conclusions: This study contributed to ongoing efforts to associate PSG biomarkers with specific cognitive functions. This study also revealed that depression and medical comorbidity uniquely influence this pathophysiological relationship, which may aid clinical management of cognitive morbidity in OSA.

Correspondence: Hillary A. Parker, PhD, Clement J. Zablocki VAMC, 2430 N. 60th ST, Milwaukee, WI 53210, United States. E-mail: greeneha@gmail.com

L.M. JENKINS, C. GARNER, C. DUQUE, S. KURIAN, L. WANG \& F. SOROND. Reduced Local Connectivity of the Medial Prefrontal Cortex Predicted by Slower Executive Functioning in Middle Aged Adults at Risk for Cardiovascular Disease.

Objective: The longitudinal influence of risk factors such as hypertension on local brain network connectivity is not well understood. We aimed to identify whether individuals at increased risk for cardiovascular disorders (CVD) have reduced resting state local functional connectivity of neural networks associated with executive functioning, and whether this is related to longitudinal measures of hypertension.

Participants and Methods: Fifty-five adults (17 female; 32 white) aged 55.64 years from the longitudinal Coronary Artery Risk Development in Young Adults (CARDIA) study participated. Systolic and diastolic average real variability (SARV, DARV) were calculated as the difference between successive blood pressure measures conducted at 8 visits over 30 years. Participants completed the Trail Making Test part $\mathrm{B}$, and a 4-minute resting state fMRI scan. We calculated regional homogeneity (ReHo), which indicates the similarity of the time series of a given voxel to those of its nearest neighbours.

Results: Multiple regression analysis predicting ReHo from time to complete Trails B (TTB), covarying age, sex and race found greater TTB predicted reduced ReHo in the bilateral medial prefrontal cortex $(\mathrm{mPFC})$, left frontal pole and right dorsolateral prefrontal cortex (DLPFC). Greater TTB was also significantly associated with increased ReHo in the left frontal pole more ventrally, and the orbitofrontal cortex (OFC). Reduced ReHo in the mPFC that was significantly associated with slower TTB was significantly correlated with DARV.

Conclusions: Local functional connectivity of the DLPFC, involved in cognitive control, and the mPFC, an important part of the default mode network, is reduced in middle-aged individuals who are slower on a measure of executive functioning. Increased OFC ReHo be compensatory. The significant correlation between increased DARV and reduced ReHo in the mPFC suggests that BP variability may be a risk factor for executive functioning deficits involving the mPFC.

Correspondence: Lisanne M. Jenkins, PhD, Psychiatry and Behavioral Sciences, Northwestern University, 710 N Lakeshore Drive, Suite 1315, Chicago, IL 60611, United States.E-mail: lisanne.jenkins@ northwestern.edu

\section{Paper Session 3. Mood and Anxiety Disorders}

\author{
Moderator: Sara Weisenbach \\ 11:45 a.m.-1:15 p.m.
}

J.J. ZAKRZEWSKI, S. MACKIN, C. CHOU, S.Y. UHM, L. BAIN, S.J. STARK, M. GAUSE, O.R. VIGIL, J. FRANKLIN, M. SALAZAR, J. PLUMADORE, L.C. SMITH, K. KOMAIKO, G. HOWELL, E. VEGA, J. CHAN, M. ECKFIELD, J.Y. TSOH, K. DELUCCHI \& C.A. MATHEWS. Effect of Treatment on Neurocognitive Function in a Treatment Seeking Sample of Individuals with Hoarding Disorder.

Objective: Hoarding disorder (HD) is a highly impairing psychiatric disorder that affects $2-4 \%$ of adults. HD prevalence increases linearly with age, reaching $>6 \%$ among adults over age 55 . In addition to functional impairment from hoarding symptoms, individuals with HD have been shown to have neuropsychological deficits in visual memory, visual detection, and visual categorization. In this study, we aim to determine the relationship between neurocognitive $(\mathrm{NC})$ functioning and HD-specific behavioral treatments, including the impact of $\mathrm{NC}$ functioning on treatment outcomes, and the impact of treatment on NC functioning. Participants and Methods: After phone screening using standard HD diagnostic scales, 323 individuals were randomized for behavioral treatment of HD in San Francisco. Treatment consisted of 20 weeks of peer or clinician lead group CBT. 242 participants completed pre- and post neuropsychological testing covering $8 \mathrm{NC}$ domains. The effect of neurocognition on treatment outcome was examined using regressions with change in hoarding severity as the outcome measure. Paired t-tests were conducted on scale scores of performances pre- and post treatment for NC tests across all domains.

Results: Participants were older $(60 \pm 10.4)$ and majority female $(77 \%)$. There was no significant effect of NC domains on treatment outcome as measured by improvement in HD severity score. There was significant $(p<.05)$ improvement in verbal learning \& memory, abstract reasoning, decision making, and planning pre- to post-treatment. Information processing speed, visual learning \& memory, visuospatial processing, attention/working memory showed highly significant levels of improvement $(\mathrm{p} \leq 0.001)$.

Conclusions: Targeted treatment for hoarding disorder significantly improved neuropsychological functioning in six of the eight domains examined. These improvements occurred without targeted treatment in these domains, but as an effect of treatment focused on the reduction of hoarding symptomology.

Correspondence: Jessica J. Zakrzewski, Clinical and Health Psychology, University of Florida, 20 SE 9th Street, Gainesville, FL 32601, United States.E-mail: jesszakr@ufl.edu

R. TOOMEY, C.E. FRANZ, R. MCKENZIE, M.S. PANIZZON, W.S. KREMEN \& M.J. LYONS. Neurocognitive deficits in remitted vs. current depression: Findings from the Vietnam Era Twin Study of Aging (VETSA).

Objective: Neurocognitive deficits in depression are well established. More recent work has examined whether these deficits persist beyond active episodes. Using depression data from examinations at three time points to determine group status of current vs remitted vs. never depressed, we investigated the neurocognitive functioning in these groups in late middle-aged men from the Vietnam Era Twin Study of Aging (VETSA).

Participants and Methods: Depression was measured at three time points by diagnostic interview or self-report questionnaire. Depression status groups were: Never depressed $(n=736)$, remitted depression $(\mathrm{n}=166)$, and current depression $(\mathrm{n}=136)$. In mixed effects models, we tested the effect of depression status on cognition. All models were adjusted for age and premorbid cognitive ability (Armed Forces Qualification Test at mean age of 20). 
Results: The currently depressed group performed significantly worse than the never depressed group on short-term/working memory, abstract reasoning, verbal fluency, Trails switching, and processing speed. Persons with remitted depression performed significantly worse than those never depressed on processing speed. Persons with remitted depression performed significantly better than those currently depressed only on abstract reasoning. Depression status was not significant overall or for any pairs in visual spatial functioning, episodic memory, category fluency switching, and executive inhibition.

Conclusions: Our results confirm recent literature suggesting that cognitive impairments in depression are not merely secondary to low mood and that there may be a disassociation between trait-like and state-like cognitive deficits in depression. Cognition in those with remitted depression lies mid-way between those never and currently depressed. Future research in depression may benefit from examination of those with persisting cognitive deficits between episodes.

Correspondence: Rosemary Toomey, PhD, Psychological and Brain Sciences, Boston University, 648 Beacon Street, Room 214, Boston, MA 02215, United States.E-mail: toomey@bu.edu

L. SALMINEN, D. VELTMAN, S. KOCH, M. VAN ZUIDEN, M. OLFF, D.J. STEIN, S. KOOPOWITZ, J. IPSER, K.J. RESSLER, J.S. STEVENS, T. JOVANOVIC, S.J. VAN ROOIJ, S.J. VAN DER WERFF, L.A. LEBOIS, M.L. KAUFMAN, S.A. GRUBER, S.B. HILL, J.D. WOLFF, S.G. DISNER, S. SEEDAT, L. VAN DEN HEUVEL, S. DU PLESSIS, R. BRYANT, M. KORGAONKAR, T.G. VAN ERP, N. SOICHIRO, M. HOLLIFIED, I. LIBERZON, X. WANG, A. KING, E. GEUZE, K.A. MCLAUGHLIN, M. PEVERILL, P. SAEMANN, C.G. ABDALLAH, I. HARPAZ-ROTEM, I. LEVY, K.M. WROCKLAGE, C. HASWELL, N. JAHANSHAD, P.M. THOMPSON \& R.A. MOREY. Hippocampal Subregion Abnormalities in Current and Lifetime PTSD: International Analysis from the PGC-ENIGMA PTSD Working Group.

Objective: Impaired fear learning and memory are core features of post traumatic stress disorder (PTSD) that are subserved by the hippocampus. Some, but not all, studies report smaller hippocampal volumes in PTSD patients compared to controls, and this inconsistency may reflect underlying differences in hippocampal subregions across cohorts. We tested this in an international sample of patients and controls in the PGC-ENIGMA PTSD Working Group.

Participants and Methods: Hippocampal subregion volumes were identically computed in 15 cohorts worldwide (732 PTSD: 1.212 controls) using a harmonized protocol. We ran multiple regressions to examine group differences in subregion volumes according to current PTSD (C-PTSD) in the full sample, lifetime PTSD (L-PTSD) and C-PTSD in a subsample with lifetime data $(N=1196)$, and PTSD status after covarying for child trauma $(N=821)$. Covariates for age, sex, cohort, and military vs. civilian status were included in all analyses. Results: C-PTSD (vs. controls) was associated with smaller volumes in the bilateral hippocampal tail $(p ' s<.001$; left $d=-.15$, right $d=-.16)$ after false discovery rate (FDR) corrections. In subsample analyses, L-PTSD (vs. controls) was associated with larger CA3 volumes bilaterally ( $p$ 's<.001; left $d=.31$, right $d=.21$ ), and smaller volumes in the right presubiculum $(p<.001, d=-.22)$. C-PTSD (vs. controls) was associated with smaller left CA3 volumes $(p<.001, d=-.28)$. Subregion associations with PTSD status were not detected after covarying for child trauma, and whole hippocampal volume was not associated with PTSD after FDR corrections.

Conclusions: Results suggest that subregion volumes are more closely associated with PTSD than whole hippocampal volume, and that L-PTSD and C-PTSD are uniquely associated with structural differences in the CA3 - an important structure for memory encoding and retrieval. We are recruiting additional cohorts to conduct highly powered analyses of hippocampal subregions and key variables related to PTSD (depression, alcohol use, child trauma).
Correspondence: Lauren Salminen, PhD, Imaging Genetics Center, University of Southern California, 4676 Admiralty Way, Marina del Rey, CA 90292, United States. E-mail: lauren.salminen@loni.usc.edu

J.G. KEILP, M. GORLYN, A. BURKE, B. STANLEY, M.A. OQUENDO \& J.J. MANN. Neuropsychological Dysfunction in Planned and Unplanned Suicide Attempts.

Objective: Executive dysfunction in the context of depression has long been thought to play a role in the risk for suicidal behavior. However, certain subtypes of suicidal behavior appear to be accompanied by good executive performance, which may be manifest in the planning of their attempts (Keilp et al., 2014). Other types of neuropsychological dysfunction - in cognitive control and memory - appear to be more common to all attempters (Keilp et al., 2013).

Participants and Methods: This study examined the broader neuropsychological characteristics of planned vs. unplanned suicide attempts in a sample of depressed, unmedicated past suicide attempters $(n=149)$, unmedicated depressed non-attempters $(n=202)$, and healthy volunteers $(n=142)$, with planning defined as contemplation of attempt for more vs. less than 3 hours. Clinical differences between subgroups defined in this way had previously been established (Chaudhury, 2016).

Results: Planned attempters $(\mathrm{n}=65)$ were more similar neuropsychologically to non-attempters and only differed from them and healthy volunteers on measures of cognitive control (Stroop Interference: $\mathrm{F}[3,480]=3.39, \mathrm{p}=.018$ ) and memory (Buschke SRT: $\mathrm{F}[3,481]=8.04$, $\mathrm{p}<.001)$. Unplanned (impulsive) attempters $(\mathrm{n}=84)$, by contrast showed a wider arrav of deficits relative to other groups, with the worst reaction time (Choice RT: F[3,445]=7.99, p<.001), memory (Buschke SRT: $\mathrm{F}[3,481]=8.04, \mathrm{p}<.001$ ), language fluency (Phonemic Fluency F-A-S: $\mathrm{F}[3,488]=3.96, \mathrm{p}=.008$ ), and executive performance (Wisconsin Card Sort Categories: $F[3,460]=2.86, p=.037$; Wisconsin Card Sort Errors: $\mathrm{F}[3,462]=2.90, \mathrm{p}=.035)$ of all groups.

Conclusions: Executive dysfunction and a variety of other neuropsychological deficits appear to be most pronounced in impulsive attempters. Planned attempters were relatively more intact, but show characteristic deficits in cognitive control and memory. Neuropsychological performance, then, appears related to both overall risk for suicide attempt, as well as the manner in which this behavior is implemented. Correspondence: John G. Keilp, Ph.D., Psychiatry/MIND, Columbia University/NYSPI, Box 42, 1051 Riverside Drive, New York, NY 10032, United States.E-mail:jgk13@cumc.columbia.edu

M. GORLYN, J.G. KEILP, A. BURKE, M.A. OQUENDO \& J.J. MANN. Neurocognitive Impairment in Depression is Distinct from Ratings of Mood and Cognitive Problems.

Objective: Cognitive impairment is a core symptom of Major Depressive Disorder (MDD). Patients commonly report memory and concentration difficulties, but these problems are not often a focus of treatment or formally assessed. Neuropsychological studies have detected mild to moderate deficits in depressed samples, but findings have been inconsistent when examining the relationship between depressive symptoms and cognitive problems. Our prior work demonstrated a lack of association between objective cognitive impairment and depression severity. Here, we investigated whether patient self-report of cognitive difficulties reflected the deficits seen on neuropsychological testing.

Participants and Methods: Medication-free patients with current MDD $(n=206)$ and healthy volunteers $(n=121)$ were compared on a battery of neuropsychological tests. Correlations were computed between test scores, self-report of cognitive problems (Cognitive Failures Questionnaire) and symptom severity scores (Profile of Mood States [POMS], Hamilton Depression Rating Scale and Beck Depression Inventory) in the patient sample.

Results: MDD patients had mild neurocognitive deficits in multiple domains relative to non-patients. None of these measures correlated at a level greater than .20 $(\mathrm{p}<.01)$ with total or factor scores from depression or cognitive problem rating scales. Cognitive complaints were 
not correlated with depression measures, and association with POMS $(\mathrm{r}=.4, \mathrm{p}<.01)$ was driven by the relationship with the Confusion factor. Conclusions: MDD patients' neurocognitive deficits were only weakly associated with depression severity and subjective cognitive complaints. Impaired performance on objective neuropsychological measures is distinct from mood disturbance as it is currently measured, and does not correspond with patient reports of disruption in everyday functioning. Neurocognitive deficits are an independent component of depression and may provide clinical and prognostic information beyond that obtained via symptom and behavioral ratings.

Correspondence: Marianne Gorlyn, Columbia University/NYSPI, 1051 Riverside Drive, Box 42, New York, NY 10032, United States. E-mail: mgorlyn@gmail.com

R. JAYAKAR, E. TONE, B. CROSSON, J.A. TURNER, P.L. ANDERSON, K.L. PHAN \& H. KLUMPP. Association Between Amygdala Volume and Social Anxiety Symptom Severity: A MultiMethod Study.

Objective: Functional neuroimaging has consistently shown a robust association between atypical amygdala activation and social anxiety (SA). However, there are disparities in the small structural imaging literature on the amygdala and SA, which may be a function of the varying methods used to obtain volumes. In the present study, we compared amygdala volumes generated based on Freesurfer's boundaries to those generated based on manually corrected boundaries. We also examined whether differences in volume measurement, as generated with each of the two segmentation techniques, impacted estimates of the association between amygdala volume and SA symptom severity. Participants and Methods: Participants $(N=76)$ comprised adults from one of three archival projects (Georgia State University; University of Illinois Chicago; University of Michigan). All structural brain images were T1-weighted and of high quality. Image analysis was conducted using Freesurfer V.5.3.0. The Liebowitz Social Anxiety Scale-SelfReport served as a measure of SA.

Results: Participants' SA scores ranged from $0-108(M=54.59 \pm 33.34)$. Two paired-samples $t$-tests (left, right), determined a priori, were conducted to evaluate the impact of manual boundary correction on amygdala volume measurements. Freesurfer's boundaries consistently produced larger amygdala volumes than manually corrected boundaries. However, planned hierarchical multiple regressions showed that there was no significant association between amygdala volume and SA, regardless of the volumetric technique used.

Conclusions: The present findings strongly suggest that volumetric measurement of the amygdala is not helpful for understanding variability in SA symptom severity; manual correction does not provide added benefit over the use of Freesurfer with regard to predicting SA symptoms in a sample of neurotypical adults. Our study calls into question numerous aspects of existing volumetric studies of the neural correlates of SA.

Correspondence: Reema Jayakar, M.A., Psychology, Georgia State University, 2003370 Avenue, Unit 182, Langley, BC V2Y3A2, Canada. E-mail: reemajayakar@gmail.com

\section{Poster Session 4. MCI and Dementia 12:00-1:15 p.m.}

\section{Dementia (Alzheimer's Disease)}

S. ALLISON, T. RODEBAUGH, A. FAGAN, J. MORRIS \& D. HEAD. Informant and Self-Reports of Navigation Abilities in Preclinical Alzheimer's Disease.

Objective: Recent research suggests that performance on a virtual reality spatial navigation task is sensitive to preclinical Alzheimer's disease (AD). However, the clinical utility of the task as a screening measure could be limited by the long administration time and specialized software requirements. Prior studies suggest that brief informant and self-report questionnaires are effective screening tools for earlystage symptomatic $\mathrm{AD}$. Thus, such questionnaires regarding navigation abilities may represent effective screening tools for preclinical AD. The purpose of this study was to determine if informant and self-reports of changes in spatial navigation abilities are reliable and sensitive to preclinical AD.

Participants and Methods: The psychometric properties (i.e., internal consistency, test-retest stability, and diagnostic accuracy) of informant and self-report questionnaires regarding changes in navigation abilities over the past several years were examined in a sample of 91 clinically normal (CN) individuals. Thirty of the $91 \mathrm{CN}$ individuals had a lumbar puncture for the purpose of CSF collection.

Results: The self-report (Cronbach's alpha=.965) and informant report (Cronbach's alpha=.957) were within the excellent range for internal consistency. The test-retest stability of the self-report was in the good range (intraclass correlation coefficient $($ ICC $)=.838$ ), whereas the test-retest stability of the informant report was in the acceptable range $(\mathrm{ICC}=.723)$. In terms of diagnostic accuracy, $\mathrm{CSF} A \beta_{42}$, but not tau, was associated with self-reported changes in navigation abilities (87\% sensitivity; $67 \%$ specificity). In contrast, CSF A $\beta_{42}$ and tau were not significantly associated with informant reports of changes in navigation abilities.

Conclusions: These findings suggest that a self-report questionnaire regarding changes in spatial navigation abilities represents a reliable tool sensitive to the earliest stages of AD.

Correspondence: Samantha Allison, PhD, University of WisconsinMadison, 5113 Hazelcrest Drive, Madison, WI 53704, United States. E-mail: samanthazilka@gmail.com

S.L. ANDERSON \& M. DANIEL. Depression's Influence on Cognitive Functioning in Older Adults who Develop Alzheimer's Dementia.

Objective: Past research suggests depression can affect executive functioning (EF) and processing speed (PS). Few studies have examined this influence specifically in healthy older adults who later develop Alzheimer's dementia (AD). The objective was to determine the impact of depression on EF and PS in older adults who eventually develop AD.

Participants and Methods: Subjects were 193 individuals from the National Alzheimer's Coordinating Center (mean/SD: age $=75.11 / 6.22$; education $=16.03 / 2.96)$ that developed $\mathrm{AD}$ within 5 years after initial evaluation. A series of multiple regression analyses were done. Dependent variables were measures of PS: Digit Symbol, Trail Making Test A: and EF: Trail Making Test B, Digit Span Backwards, Animal Fluency, and Vegetable Fluency. Predictor variables were scores on the Geriatric Depression Scale-Short Form (GDS) and clinical diagnosis of depression. Covariates included imaging evidence of cerebrovascular lesions (e.g., one or more strategic infarcts and/or pervasive white matter ischemia), current diagnoses of diabetes or hypertension, and current use of antidepressant medication.

Results: After accounting for cerebrovascular risk factors and antidepressant use, neither the GDS nor a clinical diagnosis of depression were statistically significant predictors of scores on any EF or PS measures. Conclusions: These findings are similar to individuals that do not develop $\mathrm{AD}$ within five years of initial evaluation and suggest that below average scores on PS and EF tests for senior patients in the general population are not related to mood disorder and likely represent genuine cognitive deficits.

Correspondence: Sarah L. Anderson, Neuropsychology, Pacific University, 706 River Hill Dr, Greenville, NC 27858, United States. E-mail: sanderson@pacificu.edu 
M. AZAR, S. CHAPMAN, Y. GU, S. LAWLESS, M.E. MEINERDING, Y. STERN \& S. COSENTINO. Processing Speed best Classifies Autopsy Confirmed Alzheimer's disease versus AD with Lewy Bodies.

Objective: Clinical differentiation between pure Alzheimer's disease(AD) and $\mathrm{AD}$ with Lewy Body disease(LBD) is relatively imprecise. Accurate diagnosis has implications for treatment and enrollment in research studies. The current study examined the ability of neuropsychological tests to discriminate between autopsy confirmed $A D$ vs $A D+L B D$. Results have the potential to aid differential diagnosis, prognosis and medical decision-making

Participants and Methods: 53 participants who underwent autopsy and received pathological diagnoses of $\mathrm{AD}$ or $\mathrm{AD}+\mathrm{LBD}$ were drawn from the Predictors Study. T-tests examined differences in word recall(Hopkins Verbal Learning Test), naming(Boston Naming Test), processing speed and executive function(Trails A\&B) and verbal fluency (CFL and category), for postmortem diagnosis of pure $\operatorname{AD}(n=31)$ vs $\operatorname{AD}+\operatorname{LBD}(n=22)$. Logistic regression examined best predictor of pathology

Results: The two groups did not differ in age, education, or gender. Independent samples t-tests indicated that the pure AD group had better processing speed(Trails A $M=84.44 \mathrm{sec}, S D=47.99)$ than mixed $\operatorname{group}(M=132.19, S D=69.91) \mathrm{t}(39)=-2.60, p=0.01$; there were no group differences in the other tests. Binary logistic regression indicated that in the overall model, including processing speed, naming, word recall, and verbal fluency, only processing speed predicted group membership $(b=0.017, \mathrm{SE}=2.44$, odds ratio=1.017, $p=0.049,95 \%$ $\mathrm{CI}[1.0,1.034])$. Odds of belonging in AD+LBD group increases $1.7 \%$ for every additional second to complete Trails A. The model correctly classified $68.4 \%$ of participants $(73.9 \% \mathrm{AD}, 60 \% \mathrm{AD}+\mathrm{LBD})$ which is an improvement from the null model $(60 \%)$, explaining a relatively moderate proportion of the variance in mixed pathology, Cox and Snell $\mathrm{R}^{2}=0.23$, Nagelkerke $\mathrm{R}^{2}=0.31$

Conclusions: Processing speed assists in discrimination between pure $\mathrm{AD}$ vs. AD+LBD pathology. Future research should further explore neuropsychological performance in relation to differential diagnoses with larger and demographically diverse group of individuals Correspondence: Martina Azar, B.S., Psychology, Drexel University, 119 Tioga Street, New York, NY 10032,United States.E-mail:ma523@ cornell.edu

E. BERTRAND, S. COSENTINO, M.C. N. DOURADO, J. LAKS, R.G. MORRIS, J. LANDEIRA-FERNANDEZ \& D.C. MOGRABI. Awareness in Alzheimer's Disease: Self and Other-Evaluation.

Objective: Impaired awareness of cognitive changes is common in Alzheimer's disease (AD) and has important clinical implications, such as impaired decision making capacities and increased caregiver burden. Evidence from several neurologic populations shows that differential compromise of self- versus other-evaluation is an important feature of disordered awareness; however, the extent to which this is true in $\mathrm{AD}$ is unclear. This study examined the extent to which disordered self-awareness in $\mathrm{AD}$ is specific to the self.

Participants and Methods: 22 patients with mild to moderate AD and 22 healthy older adults participated in the present study. Two experiments, one with reaction time (RT) tasks and one with memory tasks, were carried out. Each task had two separate conditions, success and failure, in which performance was titrated to a specific level. Following each task condition, participants were asked to estimate their own performance, as well as the performance of the other person based on observation. Awareness of self and other performance was measured by comparing participant estimations of performance with actual performance.

Results: For both the RT and memory tasks, the results indicate a main effect of condition, such that both groups overestimated performance in the failure condition and underestimated performance in the success condition. There was also a main effect of self versus other, with participants overestimating another's performance more than their own.
Additionally, for both conditions of the memory task, AD patients tended to overestimate their own performance more than control subjects did. Conclusions: Results suggests that overall, both $\mathrm{AD}$ and healthy older adults present the same pattern when asked to evaluate self- and otherperformance, with a bias leading to attribution of better performance to another. However, in the domain of memory, AD patients' overestimation of their performance in both conditions is in line with previous studies highlighting a global metamemory deficit in AD.

Correspondence: Elodie Bertrand, PhD, Psychology, UNIGRANRIO / PUC-Rio, Rua Marquês de São Vicente 225, Edifício Cardeal Leme, $2^{\circ}$ Andar - Sala 201, Rio de Janeiro 22451-900, Brazil. E-mail: elodie. bertrand1@gmail.com

B. CERBONE \& P.J. MASSMAN. Predictive Utility and Longitudinal Correlates of Phonemic Cueing Benefit in Alzheimer's Disease.

Objective: Previous cross-sectional findings on phonemic cueing benefit in Alzheimer's disease (AD) patients' naming performance have indicated significant relationships with dementia severity and some verbal semantic measures. We examined whether phonemic cueing benefit on the Boston Naming Test (BNT) in AD patients predicted rate of cognitive decline, and whether declines in phonemic cueing and naming performance were related to changes in dementia severity, semantic measures, and non-semantic measures.

Participants and Methods: 241 individuals with mild to moderate probable AD were assessed at baseline and at 2-year follow-up. A Phonemic Cue Index (PCI) was calculated to determine the extent of benefit from BNT phonemic cueing. Dementia severity was measured using the MMSE, CDR-SB, and ADAS-Cog total score.

Results: Multiple regression (with predictors of baseline dementia severity, premorbid IQ, age, PCI, and BNT Total score) showed that older age, lower premorbid IQ, and lower dementia severity predicted lower dementia severity at follow-up $(p$ 's $<.05)$. Partial correlations were significant $(p$ 's $<.01)$ between change scores in PCI and change scores in BNT-Total $(r=.36)$, W AIS Similarities $(r=.20)$, Logical Memory (LM; $r=.18)$, MMSE $(r=.29)$, and ADAS-Cog total score $(r=.27)$. However, once the BNT Total change score was added to the analyses, only LM remained marginally significant $(r=.16$, $p=.017)$. Partial correlations with BNT Total change scores were significant with change scores in dementia severity measures $(r ' s=.42$ - .53), WAIS Information $(r=.30)$ and Similarities $(r=.33)$, semantic and phonemic fluency $(r ' s=.17$ and .21$)$, and the Verbal Series Attention Test $(r=.24)$.

Conclusions: Spontaneous naming and phonemic cueing benefit did not enhance prediction of rate of cognitive decline. While declines in both measures were associated with changes in dementia severity and select semantic measures, their patterns of associations with declines in other cognitive measures were somewhat distinct.

Correspondence: Brittany Cerbone, M.A., Psychology, University of Houston, 4100 Southwest Freeway, Apt 459, Houston, TX 77027, United States.E-mail: brittany.cerbone@gmail.com

S. FRENCH, Y. CERVANTES-MANZO \& V. LLAMAS. Factors Affecting Older Adults' Fear of Developing Alzheimer's Disease.

Objective: There are currently more than 5 million Americans living with Alzheimer's disease (AD) and it is projected that by the year 2050 . the number could rise as high as 16 million. Given this phenomenon, apprehension about developing the neurodegenerative disease (anticipatory dementia or fear of developing AD) has become a topic of study in the past decade. Nonetheless, most studies focusing on anticipatory dementia have used a sample of individuals younger than age 65 and have used a single-item questionnaire to explore their apprehension. Participants and Methods: The current study utilized 50 community dwelling, non-institutionalized, older adults (24 males, 26 females) ages 65 to $84(M=72.24, S D=5.37)$ to examine anticipatory dementia and its relationship with subjective and objective memory, family history of $\mathrm{AD}$, and knowledge of $\mathrm{AD}$. Fear of developing $\mathrm{AD}$ was assessed using 
a new 30-item, psychometrically sound instrument titled the Fear of Alzheimer's Disease Scale (FADS).

Results: Results of the study revealed that (1) in-line with existing research, subjective memory complaints were positively associated with fear of developing AD, (2) family history of AD, knowledge of AD, and objective memory were not significantly correlated with fear of developing $\mathrm{AD}$, (3) subjective memory was the only significant predictor of fear of developing $\mathrm{AD}$; neither family history of $\mathrm{AD}$, knowledge of $\mathrm{AD}$, nor objective memory predicted fear of developing the disease, and (4) there was no significant relationship between subjective and objective memory.

Conclusions: The results of this study broaden our understanding of how variables such as subjective and objective memory, family history of $\mathrm{AD}$, and knowledge of $\mathrm{AD}$ relate to anticipatory dementia. Additionally, findings will aid clinicians in determining potential contributors to their patients' fear of developing AD; providing psychoeducation regarding the relationship between the aforementioned variables, may help assuage patients' fear of developing AD.

Correspondence: Yurivia Cervantes-Manzo, Loma Linda VAMC, 11201

Benton Street, Loma Linda, CA 92357, United States. E-mail: yurivia. cervantes-manzo@va.gov

S. CHAN, K. DENNISON, J. CONLEY \& M. DANIEL. Does Statin Use Mitigate Risk of Conversion to Alzheimer's Disease? The Association between Statin Use and Incidence of Alzheimer's Disease by Gender and Race.

Objective: Although findings are mixed, studies show promise that statin use may delay or prevent an onset of Alzheimer's disease (AD). This study analyzed the effect of statin use on conversion to AD.

Participants and Methods: Participants were selected from the National Alzheimer's Coordinating Center (NACC) database that did not have a diagnosis of $\mathrm{AD}$ at their first visit $(n=3,907 ; \mathrm{M} / \mathrm{SD}$ : age $=84.93 / 7.11$; education $=15.59 / 6.33 .51 .7 \%$ were women . Ethnicity: Caucasian= 89.4\%, African American $=7.8 \%$, Asian $/$ Pacific Islander $=2.4 \%$, Native American= $0.3 \%$. Race: $6.1 \%$ Hispanic. $16.8 \%$ $(n=658)$ of participants were diagnosed with $\mathrm{AD}$ from $1-5$ years after initial evaluation. Cox proportional hazards models were used to analyze the risk of $\mathrm{AD}$ diagnosis related to age, sex, race, and statin use.

Results: After controlling for age, gender, race, and ethnicity, statin use was not significantly associated with decreased risk of conversion to $\mathrm{AD}(\mathrm{HR}=.96, p=.65)$. The interaction effects between statin use and age $(\mathrm{HR}=1.00, p=.72)$, sex $(\mathrm{HR}=1.17, p=.34)$, race $(p=.48)$, and ethnicity $(\mathrm{HR}=1.25, p=.64)$ likewise did not result in significant change in $\mathrm{AD}$ conversion risk.

Conclusions: In contrast to previous research, statin use was not associated with reduced risk of conversion to AD. Interactions between statin use and race or gender were non-significant. Study limitations include: Data was not available regarding vascular health, medications, or other health conditions; inclusion of these variables may have yielded different results. We also lacked data on prior statin use, creating a potential confound regarding the level of exposure on conversion outcome.

Correspondence: Sara Chan, PsyD, School of Graduate Psychology, Pacific University, 190 SE 8th Ave., Hillsboro, OR 97123, United States. E-mail: sarachan@pacificu.edu

S. CHAPMAN, M. AZAR, P. SUNDERARAMAN, L.E. COLVIN, M.E. MEINERDING, R.E. ABRAHAM, B. IDNAY, J.L. JOYCE, E. HUEY \& S. COSENTINO. Age-Anchored Subjective Cognitive Decline is Sensitive to Short-Term Memory Binding.

Objective: Subjective Cognitive Decline (SCD) is receiving increasing interest as a potential marker of pre-clinical $\mathrm{AD}$. The way we measure SCD can influence its frequency and association with objective cognition. We examined the extent to which cognitively healthy older adults endorse SCD in general (standard framework), as compared to 5 years ago (retrospective), and as compared to others their age (age-anchored). Responses were acquired both dichotomously and ordinally. We assessed the associations of SCD, when measured in these different ways, with cognitive tasks shown to be sensitive to preclinical AD.

Participants and Methods: The sample included 36 healthy older adults (neuropsychological scores $>-1.5 \mathrm{SD} ; 61 \%$ female, $80 \%$ Caucasian) with an average age of $70.03(\mathrm{SD}=8.07)$ and $16.86(\mathrm{SD}=2.52)$ years of education. All participants completed the three SCD questionnaires, cognitive tasks (short-term memory (STM) binding, selective attention and face-name associative memory), and a mood inventory in a counterbalanced order. Two repeated measures ANOVAs compared frequency of SCD in each administration / response condition. Correlational analysis assessed the relationship between SCD and cognitive measures.

Results: Older adults endorsed less SCD in the age-anchored than standard framework for ordinal responses, and less than both standard and retrospective formats for dichotomous responses. STM binding was strongly associated with dichotomous age-anchored SCD $(r=-.44$; $p=.007)$. Face-name associative memory was associated with all the ordinally measured SCD $(\mathrm{r}>.35 ; p<.05)$. When controlling for depression and age, only STM binding and age-anchored SCD were related $(\mathrm{r}=-.389 ; p=.02)$.

Conclusions: Age-anchored SCD is most sensitive to variability in STM binding, a cognitive ability shown to deteriorate in preclinical $\mathrm{AD}$. These results provide guidance on how best to assess SCD in order to detect subtle cognitive changes in older adults who appear clinically normal on standard neuropsychological evaluation.

Correspondence: Silvia Chapman, PhD, Psychology, University of London, Cognitive Neuroscience Division, Taub Institute for Research on Alzheimer's disease and the Aging Brain., PH18 room 323, New York, NY 10032,United States. E-mail:ps201sc@gold.ac.uk

S. CHAPMAN, L.E. COLVIN, M. VUORRE, G. COCCHINI, J. METCALFE \& S. COSENTINO. Cross Domain Self-Monitoring in Anosognosia for Memory Loss in Alzheimer's Disease.

Objective: Unawareness for memory loss, or anosognosia, is a common feature of Alzheimer's disease (AD). Recent theories have proposed that anosognosia may reflect specific deficits in self-monitoring. Though anosognosia for memory loss has been shown to relate to memory monitoring, it is not clear if self-monitoring deficits exist in other domains. We examined this question by analyzing the relationship between anosognosia for memory loss, memory monitoring, and action monitoring.

Participants and Methods: 35 participants with mild to moderate AD were included in this study. The sample was $68.5 \%$ female, $>91 \%$ Caucasian, with a mean age of $77.7(\mathrm{SD}=9.4)$ and $16.3(\mathrm{SD}=2.9)$ years of education. All participants were assessed for anosognosia via a clinical interview and completed: 1 ) a metamemory task (dependent variable is the gamma statistic representing the association between predictions for performance and memory accuracy); and 2) an action monitoring task (computerized agency task used to determine whether individuals know when they are in control of task output). Cognitive and psychological measures assessed memory, executive functioning and depression. Correlational and regression analyses examined the relationship between our three measures of self-awareness and known correlates.

Results: As expected, memory monitoring was a predictor of clinically rated awareness $(\mathrm{B}=3.77, \mathrm{SE}=1.79, p=.03)$. Agency was not related to clinically rated awareness but was predictive of memory monitoring (B $=.23, \mathrm{SE}=.10, p=.008)$. Cognition and depression associated differently with each aspect of self-awareness.

Conclusions: The results are interpreted within current hierarchical models of unawareness where awareness is characterized both by level and domain. Findings suggest that measures of awareness are associated within a level of awareness (i.e., global or local) or within a domain (i.e., memory); and global awareness (i.e., anosognosia) is influenced by a lower, local level of awareness (self monitoring) within the same domain. 
Correspondence: Silvia Chapman, PhD, Psychology, University of London, Cognitive Neuroscience Division, Taub Institute for Research on Alzheimer's disease and the Aging Brain., PH18 room 323, New York, NY 10032, United States.E-mail:ps201sc@gold.ac.uk

T. CHIRLES \& J. SMITH. Functional Connectivity Patterns Associated with Aging, Physical Activity, and Genetic Risk for Alzheimer's Disease.

Objective: Leisure time physical activity (PA) protects cognitive function in healthy older adults and in adults with the APOE- $\varepsilon 4$ allele, a genetic risk for Alzheimer's Disease (AD). The connectivity of the posterior cingulate cortex (PCG) and the medial prefrontal cortex (mPFC) in the Default Mode Network (DMN) has been shown to be disrupted in $\mathrm{AD}$ progression and aging. The purpose of this study is to investigate differences in the aging trajectories of this connection depending on PA level and genetic risk for AD prior to changes in cognition.

Participants and Methods: Resting state fMRI scans were collected in 69 healthy adults (22-51 years) as well as saliva for APOE genotyping, and responses to the Paffenbarger Physical Activity Questionnaire. Multiple linear regression models were used to analyze the aging trajectory depending on PA level (High PA >1500 kcal per week), and APOE- $\varepsilon 4$ status.

Results: The model investigating the interaction of APOE and PA level reached significance $[\mathrm{F}=3.743, \mathrm{p}=0.001]$ for the long-range connection in the left hemisphere. The Low PA non-carriers had a positive slope indicating increased connectivity with age while carriers and non-carriers in the High PA category had horizontal aging trajectories.

Conclusions: The differences in projected trajectories of the connectivity are potentially reflective of the compensatory time-course in our participants based on interactions of PA level and APOE status. PA is associated with cognitive reserve (CR), a term describing the protection and adaptation of cognitive processes through neural efficiency and compensation mechanisms, and it is possible the Low PA non-carriers exhibited compensatory increases in connectivity of the left mPFC-PCC earlier than High PA study participants due to lower levels of CR. The promising findings that rs-fMRI can be used as an early detection of brain changes sensitive to PA levels and APOE- $\varepsilon 4$ status are critical to the research and treatment of $\mathrm{AD}$.

Correspondence: Theresa Chirles, University of Maryland, College Park, 1101 North Calvert St., Apt. 614, Baltimore, MD 21202, United States. E-mail:tsmith27@umd.edu

S. BALSIS, L. GERACI, J. BENGE, D.A. LOWE, T.K. CHOUDHURY, R. TIRSO \& R.S. DOODY. Statistical Model of Dynamic Markers of the Alzheimer's Pathological Cascade.

Objective: Alzheimer's disease (AD) is a progressive disease reflected in markers across assessment modalities, including neuroimaging, cognitive testing, and evaluation of adaptive function. Identifying a single continuum of decline across assessment modalities in a single sample is statistically challenging because of the multivariate nature of the data. To address this challenge, we implemented advanced statistical analyses designed specifically to model complex data across a single continuum. Participants and Methods: We analyzed data from the Alzheimer's Disease Neuroimaging Initiative (ADNI; N = 1056), focusing on indicators from the assessments of MRI volume, FDG-PET metabolic activity, cognitive performance, and adaptive function. Item response theory was used to identify the continuum of decline. Then, through a process of statistical scaling, indicators across all modalities were linked to that continuum and analyzed.

Results: Findings revealed that measures of MRI volume, FDG-PET metabolic activity, and adaptive function added measurement precision beyond that provided by cognitive measures, particularly in the relatively mild range of disease severity. More specifically, MRI volume and FDG-PET metabolic activity become compromised in the very mild range of severity, followed by cognitive performance and finally adaptive function.
Conclusions: Our statistically derived models of the AD pathological cascade are consistent with existing theoretical models.

Correspondence: Tabina K. Choudhury, BS, Psychology, Texas A\&M University, Milner Hall, 425 Ross Street, College Station, TX 77843, United States.E-mail: choudhtk@tamu.edu

L.R. CLARK, K. GIFFORD, D. LIU, C. CARLSSON, S.P. BELL, L. ACOSTA, K. BLENNOW, H. ZETTERBERG, S.C. JOHNSON \& A.L. JEFFERSON. A Comparison of Subjective Cognitive Decline Across Cohorts: The Wisconsin Registry for Alzheimer's Prevention and The Vanderbilt Memory \& Aging Project.

Objective: Subjective cognitive decline (SCD) may signify early Alzheimer's disease (AD) but heterogeneity exists. We related SCD to cerebrospinal fluid (CSF) markers of $\mathrm{AD}$ in two cohorts enriched for $\mathrm{AD}$ risk, the Wisconsin Registry for Alzheimer's Prevention (WRAP) and the Vanderbilt Memory \& Aging Project (VMAP).

Participants and Methods: WRAP included late middle-aged cognitively healthy adults ( $\mathrm{n}=142,62 \pm 6$ years) and VMAP participants were free of clinical dementia and stroke $(\mathrm{n}=155,72 \pm 6$ years $)$. All completed the Memory Functioning Questionnaire (MFQ), depressed mood inventory, and a lumbar puncture. Multiple linear regression analyses related MFQ total score (higher score=greater SCD) and CSF metrics (AB42, total tau, hyperphosphorylated tau ( $\mathrm{p}$-tau) $(\mathrm{pg} / \mathrm{mL}))$ covarying for age, depressed mood, and $A P O E$ genotype. Secondary models assessed for interactions with age, depressive symptoms, and objective memory performance.

Results: The VMAP cohort was significantly older and reported more memory complaints $(\mathrm{MFQ}$ total $=163 \pm 51$ ) than the WRAP cohort $(118 \pm 30)$. In WRAP, no significant associations between MFQ and CSF biomarkers were observed ( $\mathrm{p}$-values $>$.75). Conversely, in VMAP, higher MFQ score related to lower $\mathrm{AB} 42(\mathrm{~B}=-0.05, \mathrm{p}=0.01)$ and higher total tau $(B=0.05, p=0.01)$ but not to $p$-tau $(p>0.05)$. Regardless of cohort, interactions between CSF biomarkers and age, memory performance, or depressive symptoms were non-significant ( $p$-values > .18). However, post-hoc analyses of primary models restricting the VMAP sample to age $<70(n=62)$ revealed null associations between MFQ and CSF biomarkers ( $p$-values $>0.08$ ).

Conclusions: Inconsistent associations between SCD and AD biomarkers across two cohorts were observed. Age may partially drive this discrepancy as relationships were only observed within the older cohort. These discordant findings and additional potential moderating factors including education, family history of $\mathrm{AD}$, and SCD assessment method are discussed.

Correspondence: Lindsay R. Clark, PhD, Medicine, University of Wisconsin-Madison, 600 Highland Ave, J5/1 Mezzanine, Madison, WI 53792, United States. E-mail: lrclark@medicine.wisc.edu

L. CRIVELLI, M.F. FAREZ, J. RUSSO, M. BONETTO, C. PRADO, J. CAMPOS, G. COHEN, P. CHREM MÉNDEZ, L.R. SABE \& R.F. ALLEGRI. Algorithm for the Detection of Risky Driving in Subjects with Mild Dementia.

Objective: As life expectancy increases, there is a growth in proportion of elderly people who want to continue driving. While it has been established that patients with mild dementia are higher-risk drivers, it is also true that a large proportion of them can drive safely. The objective of this study is to identify the cognitive tests that best predict driving skills in elderly subjects.

Participants and Methods: 28 drivers with mild dementia and 28 healthy elderly underwent an extensive cognitive assessment and a driving assessment that included driving a car in a controlled circuit. A multivariable logistic regression model was built in a forward fashion including all variables significant in univariate analysis. A score system was developed and points were assigned to each variable based on the magnitude of its regression coefficient. A total driving risk score was then calculated for each individual as the sum of points for each variable. 
Results: Drivers with dementia made more mistakes in the on-road test and had slower responses in the subtests of brake reaction of the simulator. Age, Digit Symbol Modalities Test (DSMT) and Boston Naming test (BNT) were the variables than best predicted performance on the driving test and were included in the logistic regression model. A cut off score of 1.5 was established in order to determine if subjects were apt to continue driving. Each of the three variables (age, SDMT, Boston naming test) sums a maximum of one point. No single variable is enough to add up to the cutoff score, but a good performance in two of the three variables can reach the threshold.

Conclusions: An algorithm including three variables (age, DSMT and BNT) could be used as a screening tool for the prediction of risky driving in elderly subjects. This study supports the importance of cognitive assessment as a useful tool for deciding whether patients with dementia can safely operate a motor vehicle.

Correspondence: Lucía Crivelli, Institute for Neurological Research Dr. Raúl Carrea (FLENI), Montañeses 2325, Buenos Aires C1428AQK, Argentina.E-mail: lcrivelli@fleni.org.ar

L. D'ERRICO, M.A. GLENN, D.A. WOLK, D. MECHANICHAMILTON \& S.T. MOELTER. Probability of First Recall in Asymptomatic Older Adults with CSF Biomarkers of Alzheimer's Disease.

Objective: We hypothesized that memory indices based on temporal order effects would reveal differences in healthy adults at risk for Alzheimer's Disease (AD). This is an extension of work previously presented, now with 20 additional participants and a new method for calculating probability of first recall (PFR).

Participants and Methods: Participants were healthy adults ( $N=52)$ who were part of the normal control cohort evaluated at The Penn Alzheimer's Disease Center (ADC). A subset of participants $(n=20)$ demonstrated at least one CSF biomarker (low amyloid beta (AB), and/ or an increased tau or ptau ratio over $\mathrm{AB}$ ), and $\mathrm{CDR}=0$. The remaining participants $(n=32)$ were demographically matched to the positive biomarker group. Participants completed standardized MRI, CSF, and cognitive assessments including the 10-item CERAD list learning (CWL). CWL data was taken at the time closest to the CSF measurement. Conditional response probability as a function of lag (Lag CRP) and PFR weighted difference scores were calculated. The PFR difference score ranged from +1 to -1 , with more positive values indicating first recall from earlier list items.

Results: There were no group differences in total CWL recall. Lag CRP measures were not different between the CSF normal $(M=0.14 \pm .05)$ and abnormal $(\mathrm{M}=.15 \pm .05)$ groups, $p=.49$. The PFR index, however, revealed group differences with normal CSF participants showing a tendency to initiate recall with earlier list items $(\mathrm{M}=-.003 \pm .53)$ than participants with abnormal CSF $(\mathrm{M}=-.321 \pm .43), t(50)=2.25, p=.03$, Cohen's $D=.66$.

Conclusions: These results extend our prior findings using a larger sample and revised metric for calculating group PFR differences. Assessment of temporal recall may demonstrate improved sensitivity to subtle biological dysfunction associated with $\mathrm{AD}$. We contend that CSF abnormal participants have greater episodic memory inefficiency and rely more heavily on short term memory thus switch to recall of later list items to initiate recall.

Correspondence: Lisa D'Errico, The University of the Sciences, 46 roberts drive,Westampton, NJ08060,United States.E-mail: lderrico@ mail.usciences.edu

\footnotetext{
M.A. DRZEWIECKI \& R. FOLTZ. Correlates of Hot and Cold Cognition in a Neurodegenerative Population.

Objective: Executive functions (EFs) are a complex set of top-down processes. These functions are utilized on a regular basis and when deficits of EFs occur it typically leads to limitations of functionally ability. Many neurodegenerative conditions will lead to impairments of EFs and dementia is a disorder where these deficits are commonly seen.
}

especially as the disease progresses. Depression is a highly comorbid disorder with dementia and it was hypothesized that depression and executive functioning have a correlation in a dementia population.

Participants and Methods: A retrospective design was utilized to examine an outpatient population of 170 patients from the University of Chicago Medical Center. The Geriatric Depression Scale (GDS) was used to assess depression. Clock Drawing Command, Trail Making Part $\mathrm{B}$, and the Wisconsin Card Sorting Task were used to assess executive functions. Individuals with impaired scores on the Mini-Mental State Exam were utilized in the dementia group. The design assessed the entire sample as well as a dementia group.

Results: In the overall sample a correlation was identified between the GDS and Trails B $(r=0.17)$. In the dementia group a stronger correlation was identified between the GDS and Trails B ( $r=0.39)$, as well as the WCST $(r=0.33)$. Trails B was further analyzed to determine if predictive validity was present with the GDS. Predictive validity was identified in both the overall sample and the dementia group. There was a stronger finding in the dementia group where GDS scores accounted for $15 \%$ of the explained variability in Trails B scores compared to $3 \%$ variance in the overall sample.

Conclusions: Results suggest that different domains of executive functioning are related to depression, as identified on the GDS. When compared to the dementia group the level of relatedness increased. This study contributes to the literature the connection between emotional and executive functioning, as well as important treatment implications. Correspondence: Michelle A. Drzewiecki, Clinical Psychology, The Chicago School of Professional Psychology, 1022 Park Ave, Chesterton, IN 46304, United States.E-mail: maa2588@ego.thechicagoschool.edu

S. DUTT, B. YEW, J.K. HO, E.C. MCINTOSH, A.E. BLANKEN, J.Y. JANG, K.J. BANGEN, A. GAUBERT \& D.A. NATION. Hippocampal Hyperperfusion is Associated with Worse Memory and Executive Function in Older Adults.

Objective: Our aim was to examine the associations between hippocampal perfusion and sensitive tests of memory and executive function in order to evaluate the potential role of hippocampal hyperperfusion as an early risk indicator for cognitive decline.

Participants and Methods: 41 older adults (mean age $=71, \mathrm{SD}=7.1$ ) free of neurological disease, stroke, and dementia were recruited from the community to undergo brain magnetic resonance imaging (MRI) scans and neuropsychological testing, including Rey Auditory Verbal Learning Test (RAVLT) and Trail Making Test. Cerebral perfusion was measured using pulsed arterial spin labeling. Pearson correlations and multiple linear regressions examined relationships between hippocampal perfusion and cognition, with age, sex and education as covariates.

Results: Higher bilateral hippocampal perfusion correlated with worse performance on the RAVLT as indicated by a standardized score across the 5 learning trials. Greater bilateral hippocampal perfusion also correlated with increased Trails B errors across the entire sample, as well as with increased time-to-complete Trails B both across the entire sample and within the cognitively normal participant subsample $(\mathrm{n}=$ 33). Multiple linear regressions confirmed that higher left hippocampal perfusion significantly correlated with time-to-complete Trails B.

Conclusions: Hippocampal hyperperfusion is associated with worse performance on leading indicators of memory and executive function in older adults, suggesting that early hyperperfusion of the hippocampus may be indicative of increased risk for cognitive decline.

Correspondence: Shubir Dutt, Psychology, University of Southern California, 3620 South McClintock Ave, SGM 440, Los Angeles, CA 90089, United States.E-mail: shubirdu@usc.edu 
M. CLARENS, P. CHREM, L. CRIVELLI, M. MARTIN, I. CALANDRI, J. RUSSO, J. CAMPOS \& R.F. ALLEGRI. Neuropsychological Biomarkers in Mild Cognitive Impairment due to Alzheimer's Disease.

Objective: The importance of early diagnosis in dementia has focused on Alzheimer's disease (AD) biomarkers in mild cognitive impairment (MCI) in order to predict conversion to Dementia. Neuropsychological biomarkers are still poorly studied in relation to the physiopathology of $\mathrm{AD}$ and are an easy inexpensive option to incorporate into diagnostic tests. The aim of this study is to identify which cognitive tests more accurately predict the underlying pathophysiology of $\mathrm{AD}$ and thus be able to predict better the conversion of MCI to AD.

Participants and Methods: We studied 141 patients with MCI with an extensive neuropsychological battery (based on the Uniform Data Set (UDS-2) of the Alzheimer's Disease Centers program of the National Institute of Aging), laboratory and brain MRI. The pathophysiology was studied by the measurement of the $\mathrm{A} \beta 42$ and tau proteins in Cerebrospinal Fluid (CSF) and/or a brain Positron Emission Tomography (PET) with amyloid labeling with Pittsburg Compound B (Pib).

Results: Patients with MCI were classified according to the presence or absence of amyloid (PET with PiB and/or A $\beta 42$ in CSF). The following memory tests differed significantly between groups: logical memory test $(\mathrm{p}<.05)$ and its delayed recall $(\mathrm{p}<.05)$, total Rey Auditory Verbal Learning Test (RAVLT) $(p<.001)$ and its delayed recall $(p<.05)$ as well as in the first trial $(p<.05)$. In RAVLT, intrusions and false positives in recognition as well $(p<.05)$. Furthermore, visual memory through delay recall of complex Rey Figure $(p<0.05)$ showed significant differences. The executive-attention tests did not yield significant results.

Conclusions: The results obtained conclude that memory tests predict more accurately the presence of underlying pathology of AD in MCI. Therefore, the phases related to these memory tests should be studied in greater depth. These neuropsychological biomarkers should be used across all Latin America dementia centres, for the correct diagnosis of MCI due to $\mathrm{AD}$, as this is the first study to be carried in this population. Correspondence: Maria Florencia Clarens, Cognitive Neurology, FLENI, Montañeses 2325,Buenos Aires 1428,Argentina.E-mail: fclarens@ fleni.org.ar

\section{A.T. FLOWERS, S. MICHEL, K. KAUZOR \& J. RAZANI. Differences in Caregiver Burden Amongst Individuals With Various Forms of Cognitive Impairment.}

Objective: Alzheimer's disease is the most common form of dementia, accounting for $60-80 \%$ of all cases documented. Caregiver burden has been researched extensively amongst this population. However, minimal research has been conducted on the impact of caregiver burden amongst individuals with Mild Cognitive Impairment (MCI) as well as other forms of dementia. The purpose of this study will be to analyze differences between caregiver burden amongst individuals with MCI and three types of dementia.

Participants and Methods: A total of 93 individuals with caregivers comprised four groups: Alzheimer's disease (AD) (48), Frontotemporal Dementia (FTD) (10), Vascular Dementia (VD) (15), and MCI (20) recruited from medical centers in the Los Angeles area. All caregivers were administered The Caregiver Burden Inventory (CBI), The Brief Symptom Inventory (BSI), The Beck Depression Inventory (BDI), and The Neuropsychiatric Inventory (NPIQ) as apart of a larger test battery. Results: Several MANCOVAs were conducted, with education and age as covariates. Analysis revealed significant differences between all four groups. Post hoc analysis revealed that caregivers of individuals with FTD reported higher levels of burden and psychological distress in comparison to AD, Vascular Dementia, and MCI groups on the Neuropsychiatric Severity total score. Results also revealed significant differences between groups reported severity of burden associated with amount of time allocated to caring for individuals with dementia. Caregivers of individuals with Vascular dementia reported higher levels of burden associated with time spent caring for these individuals in comparison to other groups.
Conclusions: These findings revealed that levels of distress may differ amongst caregivers of individuals with various forms of cognitive impairment and severity of caregiver burden and psychological distress was related to years of caregiving. These findings have important implications for treatment planning.

Correspondence: Amina T. Flowers, Clinical Psychology, Psychology, California State University, Northridge, 5454 Brynhurst Avenue, Los Angeles, CA 90043, United States. E-mail:flowersamina@gmail.com

L.V. GRAVES, H.M. HOLDEN, E.J. VAN ETTEN, L. DELANOWOOD, M.W. BONDI, D. SALMON, J. COREY-BLOOM, D. DELIS \&. P. GILBERT. Novel Recognition Discriminability in Alzheimer's and Huntington's Disease: New Insights from the CVLT-3.

Objective: The California Verbal Learning Test-2 (CVLT-2) includes an index of Novel Recognition Discriminability (NRD) on the Yes/No Recognition trial that captures the ability to distinguish novel distractors from List A targets. Novel distractors include: (1) prototypical words semantically related to targets and (2) words semantically unrelated to targets. A potential limitation of this measure is that half of the distractors are prototypical members of the categories on List A, and thus have strong semantic associations with targets. This study therefore assessed performances in Alzheimer's (AD) and Huntington's disease (HD) on two new CVLT-3 NRD sub-measures that parse the degree of semantic association between novel distractors and targets: (1) NRD-prototypical (hits vs. prototypical novel distractors only) and (2) NRD-unrelated (hits vs. unrelated novel distractors only).

Participants and Methods: Individuals with AD and HD were characterized as mild or moderate in dementia severity based on Dementia Rating Scale-2 scores: 100-119 = moderate $(\mathrm{mod}), 120+=$ mild. The study sample consisted of $27 \mathrm{AD}$-mod, $25 \mathrm{AD}$-mild, $16 \mathrm{HD}$-mod, and 39 HD-mild individuals. CVLT-3 normative data were applied to archival CVLT-2 raw scores to generate scaled scores on CVLT-3 NRD-prototypical and NRD-unrelated indices.

Results: Multivariate analysis of variance tests revealed a significant effect of group on the NRD-unrelated $(p<.05)$, but not on the NRD-prototypical index ( $p>.05)$. Tukey's post-hoc pairwise comparisons revealed that the $\mathrm{HD}$-mild group outperformed the $\mathrm{AD}$-mod group on the NRD-unrelated index; no other group differences were observed. Conclusions: The present results provide evidence that, upon word list learning, the novelty of prototypical novel distractors is influenced by strong semantic associations with targets. Moreover, findings highlight the utility of two new CVLT-3 sub-measures of NRD-prototypical and NRD-unrelated in elucidating nuances in the nature and degree of recognition memory impairment in $\mathrm{AD}$ and $\mathrm{HDD}$.

Correspondence: Lisa V. Graves, SDSU/UCSD JDP in Clinical Psychology, 6363 Alvarado Court, Suite 103, San Diego, CA 92120 , United States.E-mail:lvgraves@mail.ucsd.edu

K. HACKETT, R. MIS, E.E. CAESAR, J. MELÉNDEZ-CABRERO, C.A. GANZER, A. SEIFAN, L. MOSCONI, R. KRIKORIAN, T. GIOVANNETTI \& R.S. ISAACSON. Perceptions of Childhood and Current Memory Abilities in Adults Presenting to a Memory Clinic.

Objective: Little is known regarding older adults' perceptions of their memory abilities in youth. We investigated perceptions of cognitive abilities in childhood versus adulthood and whether lower retrospective, childhood ratings predicted cognitive abilities in late life.

Participants and Methods: 256 nondemented adults (M age $62.9 \pm$ 14.4; M education $16.6 \pm 2.8$ ) from the Weill Cornell Memory Disorders/Alzheimer's Prevention Clinic who were diagnosed as Cognitively Normal, Subjective Cognitive Decline, amnestic MCI, or non-amnestic MCI rated themselves against their same-aged peers during "childhood" and "nowadays" in memory, attention, and decision making. Ratings were analyzed for change over time using paired-sample t-tests. Linear regressions adjusting for age and education examined childhood ratings 
and difference scores (childhood vs. now) as predictors of current neuropsychological test performance.

Results: Participants endorsed significantly lower contemporaneous ("nowadays") ratings than retrospective, childhood ratings in all cognitive domains (all p's <.05), most notably in memory where the difference was significant within each diagnostic group. Childhood ratings of memory, but not difference scores (childhood vs. now), predicted scores on a delayed recall list learning task $(\beta=.18, p<.01)$. By contrast, childhood ratings of decision making only marginally predicted set switching scores ( $\mathrm{p}=.06$ ), and childhood ratings of attention did not predict attentional task scores.

Conclusions: Adults presenting to a memory clinic consistently rated their memory abilities as decreasing from childhood to present, relative to their peers. The degree of perceived decline in memory was not associated with current memory performance; however, individuals with lower childhood memory ratings obtained significantly lower scores on memory tests than those with higher childhood memory ratings. Thus, memory, perceived to be the greatest cognitive concern in this population, may be selectively vulnerable from a young age.

Correspondence: Katherine Hackett, BA, Neurology, Weill Cornell Medicine, 1811 Chestnut Street, \#705, Philadelphia, PA 19103, United States.E-mail:tug90253@temple.edu

C. HALE, B. RIZVI, M. BUDGE, A. NARKHEDE, B.S. LAST, E. EBRAHIMI, L. HONIG, S. COSENTINO \& A.M. BRICKMAN. Cognitive Complaints Among Older Adults are Associated Selectively with Entorhinal Cortex Thickness.

Objective: Older adults frequently perceive changes in cognitive abilities in the absence of objective clinical evidence of neuropsychological dysfunction. It is unclear whether this subjective decline is a harbinger of a neurodegenerative condition, such as Alzheimer's disease (AD), or whether it represents normative age-related changes. Since the earliest pathology in $\mathrm{AD}$ is observed in the entorhinal cortex, we evaluated the relationship between perceived difficulties in cognition and entorhinal cortical thickness among older adults without dementia.

Participants and Methods: Fifty-three adults (mean age $=69.2+/$ 7.3; 25 women) were included in the analyses. Participants received MRI scans and as part of a comprehensive neuropsychological battery were assessed with the Neuro-Quality of Life measure on cognitive function. The computerized questionnaire uses an adaptive approach to evaluate self-perceived difficulties in cognitive abilities (memory, attention, executive abilities) or in the application of these abilities to daily life. Regional cortical thickness was derived separately for each hemisphere with FreeSurfer. We examined the relationship between entorhinal cortex thickness and cognitive complaints and determined whether this relationship was specific by examining cortical thickness in other key cortical regions.

Results: There was a selective relationship between left hemisphere entorhinal cortical thickness and cognitive complaints $(\beta=6.14$, $p=0.045$ ), such that higher thickness was associated with fewer cognitive complaints. There was no association between cortical thickness in other areas in the left hemisphere $(p=0.29)$, right hemisphere $(p=0.73)$, or right entorhinal cortex $(p=0.75)$ and cognitive complaints.

Conclusions: Self-perceived cognitive complaints may reflect early neurodegenerative changes in the entorhinal cortex in the absence of observable symptomatology. Hemispheric differences are consistent with previous reports that implicate dominant hemisphere atrophy in subjective cognition and early AD.

Correspondence: Christiane Hale, M.S., Taub Institute, Columbia University, 622 W 168th Street, PH18/333, New York, NY 10032, United States.E-mail: ch3190@cumc.columbia.edu
D. HALLidAY, R. STAWSKI, E. CERINO, K. GREWAL \& S. MACDONALD. Intraindividual Dispersion Profiles for Identifying the Cognitively Impaired.

Objective: Increased intraindividual variability (IIV) in function has been linked to various age-related outcomes including cognitive decline and dementia. Most studies have operationalized IIV as fluctuations across trials (eg, response latencies) for a single task, with comparatively few studies examining variability across multiple tasks for a given individual. In the present study, we derive a multivariable operationalization of dispersion across a broad profile of neuropsychological measures, and use this index to predict risk of cognitive impairment.

Participants and Methods: Participants $(n=65)$ were community-dwelling older adults aged $65+$ years $(M=74.1, \mathrm{SD}=6.0)$ participating in a cross-sectional investigation of risk factors for amnestic mild cognitive impairment (a-MCI) and probable Alzheimer's Disease (AD). Healthy controls $(n=36)$ performed $>$ than -1 SD across all cognitive domains in the absence of memory complaints or functional impairments. The a-MCI group $(\mathrm{n}=22)$ had self- or informant-reported memory complaints and scored $\leq-1$ SD below the mean on at least one memory task while performing > than -1 SD on all other cognitive domains, in the absence of functional impairments. The AD group ( $\mathrm{n}=7$ ) performed $\leq-2 \mathrm{SD}$ in two cognitive domains (including memory) with impairments in functioning. Measures spanned a range of cognitive domains

(episodic memory, executive function, language), with the derived dispersion estimates reflecting variability across an individual's profile relative to the group average.

Results: Multinomial logistic regression models examined the risk of being classified as a-MCI or AD as a function of increased dispersion. Greater dispersion was associated with an increased likelihood $(\mathrm{OR}=1.74, \mathrm{p}<.01)$ of being classified with $\mathrm{AD}$.

Conclusions: As a measure of IIV, dispersion across neuropsychological profiles holds promise for the early detection of cognitive impairment. Correspondence: Drew Halliday, Psychology, University of Victoria, 3800 Finnerty Rd, Victoria, BC V8P 5C2, Canada. E-mail: drewh@ uvic.ca

E.C. KEMP, S. RAMANAN, M. EBNER, T. GODEK, J. MCDONALD, E.A. PUGH, C.H. VAN DYCK \& A.P. MECCA. Statin Use and Risk of Cognitive Decline in the ADNI Cohort.

Objective: To investigate the association between statin use and cognitive decline, as well as risk for diagnostic conversion, in cognitively normal (CN) individuals, individuals with mild cognitive impairment (MCI), and individuals with Dementia due to Alzheimer's disease (AD-dementia).

Participants and Methods: 1735 adults 48 to 91 years-old with early MCI (EMCI), late MCI (LMCI), AD-dementia, or CN status at baseline were followed for up to 24 months in a multi-center longitudinal cohort study (Alzheimer's Disease Neuroimaging Initiative). Cognition was measured with a composite executive function score, a composite memory score, and a global score (Alzheimer's Disease Assessment Scale-cognitive sub-scale). Conversion to a more impaired diagnostic group was determined by clinician assessment. Statin users at baseline were compared to non-statin users using linear repeated measures mixed effects models for continuous measures and cox proportional hazards models for conversion to a more advanced diagnostic category.

Results: Statin use was not significantly associated with decline in longitudinal cognitive measures for CN, LMCI, or AD participants. EMCI participants on a statin did show a significantly slower rate of decline on the memory composite (parameter estimate $=0.006, p=0.019$ ), but no other cognitive measure. Statin use was not significantly associated with increased risk of diagnostic conversion in any diagnostic group.

Conclusions: This study thoroughly investigated the effect of statin use on cognitive decline using several outcomes. These findings add further support against claims that statin use may contribute to cognitive decline. 
Correspondence: Emily C. Kemp, B.S., Psychiatry, Yale University, 107 Nicoll Street, First Floor, New Haven, CT 06511, United States. E-mail: emily.kemp@yale.edu

L.E. KORTHAUER, D. SALMON, E.K. FESTA, D. GALASKO \& W.C. HEINDEL. Response Time Associated with Stimulus Uncertainty but Not Response Uncertainty Discriminates Alzheimer's Disease from Healthy Aging.

Objective: The Hick-Hyman law states that response time (RT) increases linearly with information uncertainty. Two additive components, stimulus uncertainty and response uncertainty, contribute to this phenomenon. Prior research has demonstrated that representation of stimulus uncertainty is mediated by the cognitive control network (CCN), which is disrupted in Alzheimer's disease (AD). Thus, we predicted that manipulating stimulus uncertainty, but not response uncertainty, would differentiate AD from healthy aging.

Participants and Methods: Participants were 24 AD patients $(14$ males; $M$ age $=71.6 ;$ DRS $=114.2)$ and 26 elderly controls ( 7 males; $M$ age $=76.9 ;$ DRS =140.1). Participants completed one of two cardsorting tasks in which either stimulus uncertainty (i.e., the probability of each stimulus type with a fixed number of sorting piles) or response uncertainty (i.e., the number of sorting piles with a fixed probability of each stimulus type) was manipulated.

Results: In the response uncertainty condition, there was a linear increase in RT $(p<.001)$ as uncertainty load (in bits) increased. AD patients were marginally slower than EC $(p=.056)$, but there was no group by uncertainty interaction. In the stimulus uncertainty condition, higher uncertainty was associated with a linear increase in RT $(p<.001)$, and there was a main effect of group $(p=.005)$, with slower RTs for AD patients than EC. A group by uncertainty interaction $(p=.017)$ further revealed that $\mathrm{AD}$ patients had steeper slopes (i.e., greater slowing under high uncertainty) than EC $(p=.007)$.

Conclusions: EC and AD patients did not differ in their sensitivity to increasing burden of response uncertainty, though $\mathrm{AD}$ patients were slower to respond overall. However, consistent with presumed disruptions to the CCN in AD, patients had significantly slower RT at higher levels of stimulus uncertainty. This differential sensitivity to stimulus uncertainty may be a potential cognitive biomarker to discriminate between healthy and pathological aging.

Correspondence: Laura E. Korthauer, M.A., Psychology, University of Wisconsin-Milwaukee, 2441 E. Hartford Ave., Milwaukee, WI 53212, United States.E-mail: korthau2@uwm.edu

\section{S.S.-H. LIN \& B.E. GAVETT. Inferring Brain Change from Reliable Change Indices: AVLT and Brain Volume.}

Objective: Changes in cognitive test scores should be reflective of changes in the brain. The current study investigates whether reliable change on a memory test is indicative of brain atrophy in older adults. Participants and Methods: Data were obtained from the Alzheimer's Disease Neuroimaging Initiative. Participants completed the Auditory Verbal Learning Test (AVLT) and had brain volume measurements taken using magnetic resonance imaging at two time points one year apart. Brain volumetric data, including whole brain, hippocampus, entorhinal cortex, ventricles, and middle temporal gyrus, were extracted. Two groups of elders were selected from the larger data set: one group $(n=42)$ showing reliable cognitive decline on the AVLT, aged 71.98 $(S D=8.67)$, and a control group $(n=42)$, aged $72.59(S D=7.11)$, that did not exhibit reliable change, selected using a genetic algorithm to closely match on demographic variables, baseline AVLT scores, and baseline clinical diagnosis. Reliable change on the AVLT was determined using a standardized regression-based formula that accounts for the reliability of the test, demographic variables, and the retest interval. Results: A series of Bayesian $t$-tests revealed that elders who exhibited reliable cognitive decline on AVLT showed more hippocampal atrophy than the control group, $b=-202.44,95 \%$ CI [-325.10, -76.19]; Cohen's $d=-0.86$. In contrast, whole brain $(b=-2384.47 ; d=-0.17)$, entorhinal $(b=-244.12 ; d=-0.45)$, middle temporal gyrus $(b=-111.08 ; d=-0.22)$, and ventricular $(b=1254.77 ; d=0.32)$ changes did not differ as saliently between the two groups.

Conclusions: After statistically matching for potential confounds, reliable change on the AVLT was strongly related to hippocampal atrophy, but less strongly linked to atrophy in other brain regions. Reliable change indices may provide some insight into brain volume changes; more research is needed with other tests and brain volume measurements.

Correspondence: Shayne S.-H. Lin, B.S., Psychology, University of Colorado Colorado Springs, 1420 Austin Bluffs Pkwy, Colorado Springs, CO 80907, United States. E-mail: slin@uccs.edu

C. MALONE, K. TURK, M. TAT, N. HEYWORTH \& A. BUDSON. False Memory, Response Bias, and Executive Functioning, in Alzheimer's Disease.

Objective: Patients with Alzheimer's disease (AD) are predisposed to false memories, when compared to healthy controls, and this is thought to result from impairment in both memory abilities and the ability to monitor and inhibit memory decisions. Cognitive strategies to improve memory have taken on emphasized importance in the context of ineffective pharmacological interventions to enhance cognition in AD. In the present study, we examine the effectiveness of Conservative Responding and Item-Specific Encoding, alone and in combination, to reduce false memory in a categorized word list paradigm.

Participants and Methods: 16 healthy older controls, 16 patients diagnosed with MCI due to $\mathrm{AD}$, and 16 patients with $\mathrm{AD}$ were tested during four study sessions where they were presented with a computer memory test and neuropsychological measures. Groups were matched according to age, education, and gender.

Results: Between group analyses revealed significant differences across session for Hit rate $(\mathrm{F}(12.679), \mathrm{p}<0.000005)$, Related Lure rate $(\mathrm{F}(8.902), \mathrm{p}<0.001)$, and Unrelated New rate $(\mathrm{F}(6.444), \mathrm{p}<0.005)$. Within subject effects analyses revealed significant differences across sessions for Hit rate $(\mathrm{F}(24.136), \mathrm{p}<0.0000001)$, Related Lure rate $(\mathrm{F}(9.815), \mathrm{p}<0.00001)$, and Unrelated New rate $(\mathrm{F}(7.337), 0<0.001)$. Cohen's d estimates of effect sizes and correlations between computer memory test performance and neuropsychological measures were conducted.

Conclusions: The strategies were effective for each group though to differing degrees suggesting that intact executive functions are necessary but not sufficient to benefit from the use of cognitive strategies. Evidence suggests a positive relationship between levels of executive functioning and effective use of cognitive strategies and a negative relationship between executive functioning and level of false memory. The use of cognitive strategies may provide a clinically significant reduction of false memory among patients with AD.

Correspondence: Christopher Malone, PsyD, Clinical Psychology, William James College, 199 Winthrop Rd, Apt 11, Brookline, MA 02445, United States.E-mail: christopher_malone@williamjames.edu

K.M. MAXA, S. BERMAN, L. RIVERA-RIVERA, L. LAW, R. DOUGHERTY, C. MITCHELL, D. EDWARDS, R. KOSCIK, C. GALLAGHER, C. CARLSSON, B. BENDLIN, S. ASTHANA, M. SAGER, B. HERMANN, O. WIEBEN, S.C. JOHNSON, D. COOK \& O.C. OKONKWO. Sedentary Behavior Associates with Vessel Pulsatility in the Brains of Adults at Risk for Alzheimer's Disease. Objective: There is increasing evidence of a vascular component in the pathogenesis of Alzheimer's disease (AD). One important measure of cerebrovascular health is pulsatility index (PI). Increased PI indicates greater vessel stiffness and has been associated with reduced mean blood flow in the intracranial vasculature. Sedentary behavior (SB) has emerged as an independent risk factor for vascular conditions, such as cardiovascular disease. However, its relation to vascular indices in the brain, such as PI, has not yet been fully explored. Therefore, the aim 
of this study was to examine the association between SB and pulsatility characteristics of cerebral vessels of the Circle of Willis.

Participants and Methods: Thirty-six cognitively healthy, late-middle-aged adults (age $=64.03 \pm 4.80,50 \%$ male) from the $W$ isconsin Registry for Alzheimer's Prevention participated in this study. Participants underwent 4D flow MRI brain imaging to measure PI in the following cerebral vessels: internal carotid artery (ICA), middle cerebral artery, posterior cerebral artery, basilar artery, transverse sinus, and superior sagittal sinus. Participants also wore an accelerometer (ActiGraph GT3X+) for one week to record free-living physical activity, which yielded a measure of SB. Multiple linear regression, adjusted for age and sex, was used to analyze the relationship between SB and PI.

Results: There was a trend for a positive association between SB and mean PI in the inferior ICA $(p=.052)$. When examined hemispherically, there was a significant association between SB and PI in the right inferior ICA $(p=.044)$ but not the left $(p=.241)$. No other cerebral vessels revealed significant associations between SB and PI ( $p>.234)$.

Conclusions: Findings from this study show that as SB increases, PI increases in the ICA, indicating increased vessel stiffness. Because increased vessel stiffness has been reported in persons with AD, this suggests that reductions in SB may promote vessel compliance, thereby protecting against cerebrovascular changes related to $\mathrm{AD}$.

Correspondence: Kaitlin M. Maxa, Undergraduate, Geriatrics, University of Wisconsin Madison, 20667 Hampshire Way, Lakeville, MN 55044, United States.E-mail:maxa2@wisc.edu

\section{E.C. MCINTOSH \& D.A. NATION. Untreated Hyperglycemia Accelerates Cognitive and Functional Decline in Older Adults.}

Objective: Type 2 diabetes mellitus (T2DM) is associated with increased risk for Alzheimer's dementia. Some studies have shown that glycemic control and medication use may attenuate the risk for dementia in diabetics. However, there is a paucity of studies comparing treated T2DM and untreated hyperglycemia on neuropsychological measures. The present study investigated differences in cognition between euglycemic older adults and those with treated T2DM versus untreated hyperglycemia over a two-year period.

Participants and Methods: Non-demented older adults from the Alzheimer's Disease Neuroimaging Initiative (N=901) were classified as euglycemic, treated T2DM or untreated hyperglycemic using fasting blood glucose and medication history. Analysis of covariance and linear mixed models were used to compare euglycemic older adults to those with treated T2DM or untreated hyperglycemia on neuropsychological test scores after controlling for age, gender, education, and apolipoprotein (APOE)-e4 carrier status.

Results: Findings showed a group-by-time interaction for dementia staging scores (Clinical Dementia Rating Sum of Boxes (CDR-SB)) wherein the untreated hyperglycemia group declined faster than the euglycemic group $(p=0.001)$. Both the treated T2DM and untreated hyperglycemia groups had worse CDR-SB scores compared to euglycemic adults at the two-year follow-up. On the MMSE, the untreated hyperglycemia group declined significantly faster compared to euglycemic adults $(p=0.041)$. There were no differences in rates of decline on any neuropsychological measures $(p>0.05)$.

Conclusions: The present study demonstrated the untreated hyperglycemia group declined faster on a dementia staging measure and global cognition measure compared to euglycemic older adults, however there were no findings on more specific cognitive measures. The results suggest that untreated hyperglycemia may increase risk of accelerated cognitive decline or dementia compared to adults with treated T2DM or who are euglycemic.

Correspondence: Elissa C. McIntosh, MA, Psychology, University of Southern California, 3620 McClintock Ave, Suite 501, Los Angeles, CA 90089,United States. E-mail: ecmcinto@usc.edu
M.E. MEINERDING, S. CHAPMAN, P. SUNDERARAMAN, L.E. COLVIN, B.L. DEFEIS, B. IDNAY, E. HUEY, D. WEISS \& S. COSENTINO. Retrospective Subjective Cognitive Decline relates to Essentialist Beliefs and Subjective Age.

Objective: Subjective Cognitive Decline (SCD) is hypothesized to be a promising early indicator of Alzheimer's disease (AD). SCD is a multidimensional construct; while it is known that mood may influence SCD, whether SCD also reflects one's attitudes about aging (AA) such as age stereotypes, subjective age (SA), essentialism (ES), and age identity is unknown. We examined the relationship between SCD and AA, testing whether the manner in which SCD is measured influences its association with AA.

Participants and Methods: 36 healthy older adults (61\% female, $80 \%$ Caucasian) with an average age of $70.03(\mathrm{SD}=8.07)$ and 16.86 $(\mathrm{SD}=2.52)$ years of education completed measures of $\mathrm{AA}$, mood, objective cognition, and SCD. SCD was assessed using a 20-item Likert scale questionnaire presented in three frameworks assessing cognitive complaints: 1) in general (standard); 2) compared to 5 years ago (retrospective); and 3) compared to others the same age (age-anchored). Correlations measured associations between SCD in each framework, AA, cognition, demographic variables, and mood. Automated general linear models were used to compare model fit.

Results: Standard and age-anchored SCD were unrelated to AA. Retrospective SCD (SCD-R) was associated with two AA: ES ( $\mathrm{r}=-.37$, $\mathrm{p}=.03)$ and $\mathrm{SA}(\mathrm{r}=-.35, \mathrm{p}=.04)$, and also episodic memory $(\mathrm{EM} ; \mathrm{r}=.36$, $\mathrm{p}=.03)$, but not demographics or mood. ES and SA were unrelated to each other or to EM. Inclusion of ES and SA as predictors of SCD_R improved model fit $\left(\mathrm{AIC}_{\mathrm{C}}=-10.08\right)$ compared to either alone $\left(\mathrm{AIC}_{\mathrm{C}}=-7.65, \mathrm{AIC}_{\mathrm{C}}=-8.02\right)$.

Conclusions: When SCD is measured in regard to perceived change over time, it is linked to attitudes about aging, such as the degree to which one believes aging is a fixed process (ES), and the age one feels (SA). ES and SA are not associated with objective memory, and considering both together significantly predicts SCD-R. While the directionality of associations is not yet clear, we argue against measuring SCD retrospectively when attempting to specify SCD for detection of pre-clinical AD. Correspondence: Maria E. Meinerding, MS, The Taub Institute for Research on Alzheimer's Disease and the Aging Brain, Columbia University, 630 West 168th Street, PH19-123, New York, NY 10032, United States.E-mail:mm4817@cumc.columbia.edu

A.K. MILLER, S.J. BANKS, J.B. MILLER \& A. BONNER-JACKSON. Relationship Between Cerebrospinal Fluid Biomarkers of Alzheimer's Disease and Neuropsychological Functioning.

Objective: Alzheimer's disease (AD) is the most common etiology of major neurocognitive disorder (i.e., dementia) and leads to increased cognitive and functional impairment over time. Biomarkers obtained through analysis of cerebrospinal fluid (CSF) can provide useful diagnostic information regarding underlying disease pathologies, often times before clinical symptoms are present. Prior literature has shown higher total tau (t-tau) and phosphorylated tau (p-tau) concentrations to be associated with cognitive decline and increased risk for the development of AD among healthy individuals. However, previous studies have included few neuropsychological measures and have not examined all domains of neuropsychological functioning. The present study aimed to examine the impact of CSF biomarkers on neuropsychological test performance among a memory clinic population.

Participants and Methods: 102 patients (54\% male; 88\% Caucasian; age: $M=66.8$ years; education: $M=15.1$ years) were administered a comprehensive battery of neuropsychological tests as part of their routine clinical care. Additionally, CSF biomarkers, including t-tau and p-tau, were collected on all patients.

Results: Regression analyses were used to evaluate the importance of CSF biomarkers in predicting neuropsychological functioning. T-tau and p-tau significantly predicted memory, executive functioning, and confrontation naming, with higher concentrations associated with worse performance. In contrast, neither t-tau nor p-tau significantly predicted 
other areas of cognitive functioning (i.e., visuospatial/visuoconstruction, attention, processing speed, or verbal fluency).

Conclusions: CSF biomarkers of AD (i.e., t-tau and p-tau) predicted neuropsychological test performance on memory, executive function, and confrontation naming measures, which corresponds with the earliest cognitive symptoms typically seen in $\mathrm{AD}$. These results have meaningful implications for treatment planning.

Correspondence: Ashley K. Miller, Ph.D., Cleveland Clinic, 2599 Church Ave., Unit C206, Cleveland, OH 44113, United States. E-mail: ashley. kay.miller@gmail.com

M.Z. NAKHLA, J. HERRERA, D. BANUELOS, K. GONZALEZ \&. J. RAZANI. The Differences Between Semantic and Episodic Memory Predicting Shopping Skills in Alzheimer's disease and Mild Cognitive Impairment.

Objective: The purpose of this study was to determine whether episodic memory, semantic memory, or executive functioning best predict shopping skills in Alzheimer's disease (AD) and Mild Cognitive Impairment (MCI).

Participants and Methods: Episodic memory which refers to rote memory was assessed with CVLT free delay recall, semantic memory which refers to contextual/meaningful memory was assessed with CVLT cued recall, and executive function which was assessed with WCST error were collected on $58 \mathrm{MCI}$ and $61 \mathrm{AD}$ participants. The Direct Assessment of Functional Status, an observational-based assessment of activities of daily living, was utilized to measure shopping skills.

Results: Stepwise regression was performed separately for AD and MCI, with neuropsychological measures as the predictor variables and subscales on the shopping task (shopping from free recall, shopping with recognition from a mock store, and shopping with a list) as the outcome variables. Episodic memory significantly predicted shopping free recall and shopping with recognition in $\mathrm{AD}$, whereas semantic memory significantly predicted the same subscales in MCI. In contrast, semantic memory significantly predicted shopping with a list in AD only. Conclusions: Generally speaking, the findings of this study suggest that the different types of memory (semantic and episodic) are more sensitive predictors of shopping abilities than executive functioning. These findings further suggest that MCI patients use cueing and contextual memory aids in shopping tasks, while AD patients use rote memory skills.

Correspondence: Marina Z. Nakhla, B.A., Psychology, California State University, Northridge, 18111 Nordhoff St, Northridge, CA 91330, United States.E-mail: marina.nakhla.304@my.csun.edu

\section{NANCE, A. RITTER, J.B. MILLER \& S.J. BANKS. Pathology of Rapid Cognitive Decline.}

Objective: Patients with Alzheimer's disease (AD) vary in their rate of cognitive decline. Previous research suggests the existence of a subgroup of patients with rapid cognitive decline (RCD). We used a large group of patients with clinical and pathological data to determine the distinguishing features of RCD.

Participants and Methods: National Alzheimer's Coordinating Center (NACC) data was used. Included subjects had completed autopsy, a clinical diagnosis of AD at their primary visit, and Mini Mental Status Examination (MMSE) and neuropsychological test data from their first three visits. RCD was defined as an average decline on the MMSE of $>3$ points per year over three years; all others were termed nRCD. We investigated differences in neuropathological data, neuropsychology scores, and additional patient health history.

Results: 60 individuals identified as RCD and 230 identified as nRCD. Groups did not differ on demographic factors (age, sex, race, ethnicity, or years of education) and there was no significant difference of the MMSE score at baseline. RCD had more severe AD pathology (Thal Phase, Braak Stage, and Neuritic Plaques), increased neocortical Lewy Bodies, and increased cerebral cortical atrophy. Neuropsychological assessments that differed between groups at baseline are as follows:
WMS Logical Memory Immediate Recall, Animal Naming, Trails B, WAIS Digit Symbol, and BNT. There was also a significant increase in the presence of thyroid disease in the RCD group, but no other observed significant variables in health history.

Conclusions: RCD can be differentiated from nRCD based on baseline presence of language and executive dysfunction despite similar baseline MMSE. These patients may be especially good targets for clinical trials and should be distinguished from patients with a more typical rate of decline. Subsequent research using biomarker evidence in vivo will elucidate whether more severe pathology is evident even in the early stages of RCD.

Correspondence: Christin Nance, Bachelors, Neuropsychology, Cleveland Clinic, 888 West Bonneville Avenue, Las Vegas, NV 89119, United States.E-mail:nancec@ccf.org

E.A. PUGH, E.C. KEMP, C.H. VAN DYCK, A.P. MECCA \& E.S. SHARP. Effects of Normative Adjustments on Test Characteristics of the Montreal Cognitive Assessment.

Objective: Objective: To investigate effects of various normative adjustments on the performance of the Montreal Cognitive Assessment (MoCA) as a screening instrument for Mild Cognitive Impairment (MCI) and Alzheimer's disease (AD-dementia).

Participants and Methods: Participants and Methods: 910 adults 48 to 91 years old who were enrolled in the Alzheimer's Disease Neuroimaging Initiative (ADNI) and were administered the MoCA during their baseline visit. Participants were classified as either cognitively normal (CN), MCI, or AD-dementia at baseline based on expert clinical assessment. Receiver operating characteristic (ROC) analyses were conducted to produce ROC curves for raw MoCA scores, MoCA scores adjusted using educational achievement level, as well as a regression based adjustment derived from the National Alzheimer's Coordinating Center data (NACG).

Results: Results: Areas under the curve (AUC) were similar for ROC curves constructed with raw MoCA scores, education adjustment, and NACC adjustment with a slight improvement when adjustments of increasing complexity were applied. When distinguishing CN from MCI the AUCs were $0.71,0.72$, and 0.73 respectively. When distinguishing $\mathrm{CN}$ from $\mathrm{AD}$ the AUCs were $0.95,0.97$, and 0.97 respectively. Characteristics of test performance were calculated for various cutoffs after each normative correction method.

Conclusions: Conclusions: This study adds to the understanding of how normative adjustments can affect the test performance of the MoCA. Specifically, only small improvements in AUC are gained with adjustments. Optimal cutoffs to detect MCI and $\mathrm{AD}$-dementia may be somewhat lower than previously described. However, the need to detect individuals who require further evaluation must be balanced with the desire to prevent over-diagnoses and costly workups for people who do not need them.

Correspondence: Erika A. Pugh, MA, Psychiatry, Vale University, 1 Church St., 8th Floor, NEW HAVEN, CT 06511-6910, United States. E-mail: erika.pugh@yale.edu

M. RINDGE, M. DANIEL \& G.G. MONCRIEF. Predictors of Conversion from Mild Cognitive Impairment to Alzheimer's Dementia Confirmed by Autopsy: A Pilot Study.

Objective: This study explores the extent to which neuropsychological measures, depression, and ratings of functional status predict conversion from mild cognitive impairment (MCI) to autopsy confirmed Alzheimer's dementia (AD).

Participants and Methods: 10 subjects ( 6 male, 4 female) were selected from the National Alzheimer's Coordinating Center that were diagnosed with MCI and later had autopsy evaluation. Five subjects had AD pathology 2 to 6 years after MCI diagnosis (mean=4.4, $\mathrm{SD}=1.82$ ) and five did not 1 to 6 years after MCI diagnosis (mean=3.6, SD=1.95). Because of the small sample size, the non-parametric Komogorov-Smirnov Hypothesis Test was used for this exploratory analysis. 
Dependent variables included Logical Memory II (LMII), Trails B, Geriatric Depression Scale (GDS), Clinical Dementia Rating (CDR) scale, Mini-Mental Status Exam (MMSE), and Boston Naming Test.

Results: There were no significant differences on any of the measures for subjects with and without primary AD pathology at autopsy.

Conclusions: We found no previous research examining neuropsychological, mood, and functional predictors of conversion from MCI to autopsy confirmed AD. Although no significant results were found, previous research indicates verbal memory, executive functioning, functional ability, and depression are significant predictors of conversion to a clinical diagnosis of AD (i.e., without autopsy confirmation). The small sample size of the present study and differences in tests/measures may account for the different findings in this small autopsy confirmed sample and studies using clinically defined groups.

Correspondence: Melissa Rindge, MA, School of Graduate Psychology, Pacific University, 18673 NW Cedar Falls Loop, Hillsboro, OR 97006, United States. E-mail: mrindge@pacificu.edu

J. SCHAFFERT, C. LOBUE, L.S. HYNAN, N. DIDEHBANI, L. LACRITZ, H.C. ROSSETTI \& M. CULLUM. Traumatic Brain Injury History and Survival After Dementia Onset in AutopsyConfirmed Alzheimer's Disease.

Objective: Traumatic brain injury (TBI) is a risk factor for an earlier onset of Alzheimer's disease (AD) in some individuals, but has not been studied in relation to survival time after AD onset. We evaluated whether a history of TBI with loss of consciousness (LOC) was associated with a shorter survival time after dementia onset.

Participants and Methods: Data from 1,422 participants with autopsy-confirmed AD (defined as Braak stages III to VI and CERAD scores - moderate to frequent) were obtained from the National Alzheimer's Coordinating Center. Subjects were categorized by presence/absence of a reported remote history of TBI with loss of consciousness (LOC) occurring $>1$ year prior to their first clinical visit (TBI+ or TBI-). A Cox proportional hazard model was used to assess whether TBI predicted time from clinician-estimated age of onset to death, adjusting for sex and stratified by age of onset. Analysis of covariance was used to assess group differences in age of dementia onset, adjusting for sex, race, and education.

Results: The overall median survival time from onset to death was 10 years $(\mathrm{IQR}=7-12)$ and the average age of death was 80.1 years old. TBI with LOC was not significantly associated with shorter survival time after onset $(p=.844 ; H R=1.02 ; 95 \% C I[0.85,1.22])$, whereas male sex was $(p=.005 ; H R=1.17 ; 95 \% C I[1.05,1.3])$. As expected, the mean age of dementia onset was 2.92 years earlier $(p=.015)$ in the TBI+ group $(N=1292 ; M=67.72 ; S D=11.96)$ compared to the TBI- group $(N=130 ; M=70.64 ; S D=10.53)$.

Conclusions: Although mechanism(s) remain unclear, a history of TBI with LOC was not associated with shorter survival time after clinician-estimated AD onset, but was associated with an earlier age of dementia onset. Future imaging, biomarker, and neuropathological studies are needed to explore the potential mechanisms of earlier dementia onset following TBI.

Correspondence: Jeff Schaffert, Psychiatry, UT Southwestern Medical Center, 5323 Harry Hines Blvd, Bass Building, Dallas, TX 75390,

United States.E-mail: jeffrey.schaffert@utsouthwestern.edu

H.L. SHOUEL, M. ZUELSDORFF, L.R. CLARK \& S.C. JOHNSON. Social Support and Cognition in White and African American Older Adults at Risk for Alzheimer's Disease.

Objective: Social support may mitigate cognitive decline through mental stimulation and by buffering stress among older adults. In a prior analysis, we investigated this relationship across a racially diverse cohort of older adults from the Washington Heights Inwood Columbia Aging Project (WHICAP) using two social support constructs-objective social support (network size, OSS) and subjective social support (perceived quality, SSS)-that may be differentially related to mental stimulation and stress buffering. We found that SSS was related to better cognition in Whites, whereas social support was not related to cognition in African Americans. The purpose of this study was to test the external validity of the prior findings by replicating the analysis in a cohort at risk for age-related cognitive decline and Alzheimer's disease (AD).

Participants and Methods: N=1,346 non-demented participants in the Wisconsin Registry for Alzheimer's Prevention (WRAP) completed a neuropsychological battery and questionnaires. We ran multiple regressions to determine cross-sectional, independent associations of OSS and SSS with cognition. The model included age, sex, race/ethnicity, education, family history of AD, partner status, and depressive symptoms. Group differences were examined with descriptive statistics and stratified analyses.

Results: OSS and SSS were correlated $(r=0.28)$. Whites reported the highest levels of OSS and SSS. SSS was only related to better cognition in Whites $(p=0.007)$, but this relationship was attenuated once depression was included in the model $(p=0.399)$. Social support was not related to cognition in African Americans.

Conclusions: Similar findings in the WRAP and WHICAP cohorts, despite differences in cohort design, methodology, and demographics, suggest that social support (i.e., SSS) is only beneficial for cognition in Whites. Additionally, depressive symptoms may mediate this relationship. Longitudinal analyses are needed to better understand these relationships and to further investigate this potential disparity.

Correspondence: Heather L. Shouel, BS, University of WisconsinMadison, 600 Highland Ave, J5/1 Mezzanine, Madison, WI 53705, United States.E-mail: hlshouel@medicine.wisc.edu

A. STASENKO, D. JACOBS, D. SALMON \&. T. GOLLAN. Sensitivity of the Multilingual Naming Test (MINT) to Level of Cognitive Impairment Across the Alzheimer's Disease (AD) Spectrum.

Objective: To investigate the sensitivity of the MINT, a picture naming test recently added to the National Alzheimer's Coordinating Center (NACG) Uniform Data Set (UDS) neuropsychological test battery, to level of cognitive impairment in AD.

Participants and Methods: Data from the initial administration of the MINT were obtained from the NACG. Participants diagnosed as cognitively normal ( $\mathrm{n}=1931)$, mildly cognitively impaired (MCI; $\mathrm{n}=547$ ) or demented $(\mathrm{n}=781)$ with presumed etiology of $\mathrm{AD}$ were included in these analyses. Dementia severity was rated by Clinical Dementia Rating (CDR) global score: 0.5 (very mild), 1 (mild), and 2 (moderate), and participants were divided into three age $(<65,65-75,75+)$ and three education $(\leq 12,13-16,17+)$ groups. NACC participants with co-morbid conditions contributing to their cognitive impairment and non-primary English speakers were excluded.

Results: A Group x Age $x$ Education ANOVA revealed main effects of group $(p<.001)$ and education $(p<.05)$, with lower scores associated with less education and increasing cognitive impairment, as well as an age $\mathrm{x}$ education interaction $(p=.001)$, with naming scores decreasing most dramatically in the oldest, least educated participants (this 2-way interaction was also significant in healthy controls, however the 3-way interaction was not significant, $p=.41$ ). Planned comparisons collapsing across age and education groups revealed the MINT to be sensitive to cognitive decline across each level of dementia severity.

Conclusions: The MINT successfully detects and tracks naming impairments across levels of severity of cognitive impairment in patients with AD. Comparison to age and education corrected norms to determine impairment is essential. The particular vulnerability to naming deficits in older individuals with low education may reflect limited cognitive reserve or impending cognitive decline in some individuals classified as normal. The NACC database is funded by NIA/NIH Grant U01 AG016976.

Correspondence: Alena Stasenko, Clinical Psychology, Psychiatry, University of California, San Diego, 723 West Lewis St, San Diego, CA 92103, United States. E-mail: astasenk@gmail.com 
M. STOJANOVIC, Y. JIN, A. FAGAN, T. BENZINGER, C. CRUCHAGA, J. MORRIS \& D. HEAD. Exercise Engagement and Longitudinal Change in Alzheimer's Disease Biomarkers.

Objective: Past research suggests that exercise engagement may play a protective role against cognitive decline and dementia. In addition, a previous study in humans that examined the association of exercise engagement with biomarkers of Alzheimer's disease (AD) neuropathology reported that individuals who engaged in more exercise evidenced lower amyloid deposition estimated with positron emission tomography (PET) and levels of cerebrospinal fluid (CSF) A $\beta_{42}$. Although the effect of exercise engagement on AD biomarkers has been studied cross-sectionally, the longer-term effect of exercise engagement has been understudied. The current study examined whether individuals with higher baseline exercise engagement exhibit less longitudinal change in $\mathrm{AD}$ biomarkers than individuals with lower baseline exercise engagement. Another goal was to examine whether $A P O E$ and/or BDNF genotype, genetic risk factors for $\mathrm{AD}$, moderate the effect of exercise on longitudinal changes.

Participants and Methods: Individuals who were clinically normal at baseline were administered a questionnaire on their physical exercise engagement over the prior 10 -year period. Ninety-five individuals had serial CSF samples collected to examine $\mathrm{Ab}_{42}$ and tau, and 181 individuals underwent multiple assessments of amyloid PET imaging with Pittsburgh Compound B (PIB).

Results: We observed significant change in AD biomarkers over time, with CSF tau and PET-PIB levels increasing and CSF A $\beta_{42}$ levels decreasing, consistent with the AD pattern. However, the level of baseline exercise engagement did not affect longitudinal change in any of these AD biomarkers. Neither APOE nor BDNF genotype moderated the effect of exercise on AD biomarker changes.

Conclusions: These results suggest that physical exercise engagement may not be a robust moderator of changes in $\mathrm{AD}$ biomarkers over time, regardless of $A P O E$ or $B D N F$ status.

Correspondence: Marta Stojanovic, Department of Psychological and Brain Sciences, Washington University in St. Louis, 5877 Nina Place, unit 3W, Saint Louis, MO 63112, United States. E-mail: martastojanovic@wustl.edu

\section{A. VANMETER \& J. SUHR. Cognitive Outcomes in Caregivers of Individuals with Dementia.}

Objective: Caring for individuals with dementia is consistently related to negative physical and psychological health outcomes, but few studies have examined caregiving's cognitive impact. In the present study, we compared caregivers to non-caregiver controls in cognitive, physical, and psychological health. We hypothesized that caregivers would perform worse on subjective and performance measures of cognition, as well as show worse physical and psychological health, and that physical and psychological variables would be related to cognitive performance in caregivers.

Participants and Methods: 29 dementia caregivers were compared to 48 non-caregiver controls on a battery of tests including measures of memory, psychomotor processing speed, physical health, and psychological health.

Results: As expected, caregivers performed worse than non-caregivers in memory, $p=.002$, but not psychomotor processing speed (ps ranging from .12 to .65). Caregivers reported more subjective cognitive decline, although differences were not significant, $p=.11$. Consistent with prior studies, caregivers also demonstrated worse physical (sleep, self-reported health, and systolic blood pressure) and psychological (depression, anxiety) health than non-caregivers. Correlates of poorer cognitive performance in caregivers included higher anxiety, more sleep disturbance, higher systolic blood pressure, higher caregiver burden, and more distress about neuropsychiatric symptoms in care recipients. Conclusions: Results confirm prior findings of worse physical and psychological health in dementia caregivers, and expand the negative outcomes of caregiving to the cognitive realm. Implications of our findings include the need to assess for cognitive performance in caregivers, given its potential contribution to the quality of caregiving, and to consider interventions and resources for caregivers that focus on their physical, psychological, and cognitive health.

Correspondence: Julie Suhr, Ph.D., Psychology, Ohio University, 200 Porter Hall, Department of Psychology, Athens, OH 45701, United States.E-mail:suhr@ohio.edu

E.E. SUNDERMANN, P.M. MAKI, L.H. RUBIN, M. MIELKE, M. MACHULDA \& M.W. BONDI. Sex-based norms in clinical tests of verbal memory might improve diagnostic accuracy in Alzheimer's disease.

Objective: Females show an advantage over males on tests of verbal memory, which are used to diagnose Alzheimer's disease (AD) and amnestic mild cognitive impairment (aMCI). This female advantage may delay AD diagnosis and contribute to more advanced pathology at aMCI diagnosis in women versus men. We examined whether the use of sex-adjusted cut-scores for verbal memory tests might improve diagnostic accuracy of MCI.

Participants and Methods: We first calculated sex-adjusted and non sex-adjusted (conventional) cut-scores for impairment on the Auditory Verbal Learning Test (AVLT) using data from the Mayo Study of Aging. Cut-scores were also age- and education-adjusted. We then applied those cut-scores to 772 women and 944 men from the Alzheimer's Disease Neuroimaging Initiative (aged 55-90). Next we compared sex differences in rates of MCI (Jak/Bondi criteria) for conventional versus sex-adjusted norms. Then, using sex-based cut-offs as the true condition and conventional cut-offs as the predicted condition, participants were labeled as True Positives (TPs), False Positives (FPs), True Negative (TNs), or False Negative (FNs). Lastly, we compared levels of CSF Ptau/ Amyloid- $\beta(\mathrm{A} \beta)$ and cortical A $\beta$ deposition ([18F]AV45 PET) between these four groups stratified by sex.

Results: The rate of MCI was higher in men only for conventional cut-off scores. There was a $9 \% \mathrm{FN}$ error rate in women and a $10 \%$ FP error rate in men. Biomarker analysis supported the sex-adjusted diagnostic groupings over the conventional groups; AD biomarkers were higher in the FNs compared to the TNs, similar in FNs compared to TPs, and lower in FPs compared to TPs.

Conclusions: Previous reports of a higher rate of MCI in men might reflect the use of conventional cut-offs. The application of sex-adjusted norms in clinical tests of verbal memory may improve diagnostic accuracy and identify women at an earlier disease stage when currently available treatments are the most beneficial.

Correspondence: Erin E. Sundermann, PhD, Neurology, Albert Einstein College of Medicine, 1207 Herschel Woods Ln., Cincinnati, OH 45208, United States.E-mail: erin.sundermann@einstein.yu.edu

\section{M.B. UNGRADY, J. HUNG, M. GROSSMAN \& J. REILLY. Predicting} Lexical Dropout in Progressive Aphasia via Eye Tracking.

Objective: A substantive challenge for language rehabilitation in progressive aphasia involves predicting which words will drop out of a patient's lexicon over time. We hypothesize that the stability and efficiency of visual gaze patterns to distinctive semantic features (e.g., faces) will predict language dropout in a longitudinal cohort of patients with progressive aphasia.

Participants and Methods: We tracked eye gaze patterns of patients with Alzheimer's Disease or PPA $(\mathrm{N}=5,1)$ during visual confrontation naming of the Boston Naming Test (Kaplan et al, 1983) at multiple time points over two years. Within each target picture, we delineated areas of interest (AOI) via an 8x8 AOI matrix generated by averaging the gaze patterns of neurotypical controls $(\mathrm{N}=12)$. We contrasted gaze to key AOIs at the item-level for three categories of words named over a 2-year span: known, unstable, and forgotten.

Results: Patients trended toward slower visual entry times to the key AOIs for known items $(p=.07)$, and entry time was further delayed for unstable words $(p=.03)$. No reliable gaze patterns were discerned 
for forgotten words, suggestive of an increasingly slowed and chaotic visual search.

Conclusions: Progressive anomia is characterized by inexorable loss of names for common objects and people. Using eye tracking, we repeatedly tested a cohort of patients with progressive aphasia as they named pictures over 2 years. Based on retrospective accuracy, we contrasted items that were consistently known versus those that were forgotten or unstable (inconsistently named). Patients with anomia secondary to progressive semantic impairment are slower to reach key semantic features prior to naming a word. These results demonstrate promise for eye tracking (specifically AOI entry time) as a sensitive measure of prediction for a word's status as either known or growing increasingly vulnerable to being forgotten.

Correspondence: Molly B. Ungrady, BA, Temple University, 4720 Pine Street, Apt D2, Philadelphia, PA 19143, United States. E-mail: ungradym@mail.med.upenn.edu

\section{A.M. VANDEBUNTE. The Effect of Depression on the Cognition of those with Alzheimer's Disease.}

Objective: This study sought to understand the particular features of depression associated with Alzheimer's disease (AD) and how these features interact with cognitive functioning.

Participants and Methods: 122 adults (62 women, $M$ age $=78.44$ years, $M$ education $=14.45$ years ) with $\mathrm{AD}$ pathology completed the BNT, COWAT, ROCF, HVLT-R, BVMT-R, and subtests of the WAISIV, WMS-IV, DKEFS, and Trailmaking. Features of depression were measured through the GDS.

Results: Minimal levels of state depression were endorsed by $58 \%$, mild depression by $22 \%$, and moderate-to-severe levels by $8 \%$. Significantly greater levels of the cognitive factor of depression were endorsed than all other factors, and significantly more symptoms of dysphoria were reported than hopelessness and withdrawal/apathy. There were significant negative associations between overall state depression and scores on WAIS-IV Coding and Trails A. Performance on WAIS-IV Arithmetic and Coding was negatively correlated with dysphoria, and hopelessness was significantly negatively associated with WAIS-IV Coding, WAIS-IV Arithmetic, and DKEFS Inhibition scores. Performances on HVLT-R Total Recall, BVMT-R Trials 1-3, BVMT-R Delayed Recall, and ROCF 3 ' were also significantly negatively correlated with hopelessness. All ps $<.05$.

Conclusions: The depression of those with AD appears to be disproportionately characterized by cognitive features and dysphoria. Increased state depression in $\mathrm{AD}$ seems to contribute to difficulties in graphomotor speed and visual scanning, and greater levels of hopelessness in particular seem related to worse visual processing speed, verbal list learning, nonverbal learning and memory, and working memory and response inhibition. Inferior visual processing speed and working memory also occur among those with greater dysphoria in their depression. These results suggest there are distinctive features to the depression in AD, and these features seem to be adding something unique to patients' cognitive profiles.

Correspondence: Anna M. VandeBunte, Psychology, Westmont College, PO Box 1553, Summerland, CA 93067, United States. E-mail: avandebunte@westmont.edu

U.M. VENKATESAN, E.K. FESTA, B.R. OTT \& W.C. HEINDEL. Disentangling Deficits in Semantic Integration and Retrieval in Aging and Alzheimer's Disease.

Objective: Semantic memory deficits in Alzheimer's disease (AD) have been attributed to both deficient retrieval processes and degraded semantic content. To better understand the nature of semantic loss in $\mathrm{AD}$, we examined retrieval of two types of semantic information, category exemplars and object feature attributes, while independently manipulating semantic association strength.

Participants and Methods: Patients with AD $(n=20)$ and elderly controls $(n=17)$ completed a semantic verification task requiring the user to decide whether an exemplar is a member of a particular category $(\mathrm{E} / \mathrm{C})$ or whether a feature is associated with a particular object $(\mathrm{F} / \mathrm{O})$. Trials contained either high or low dominance semantic associations. Response times and accuracy were recorded. Two summary scores reflecting the dominance effect on $\mathrm{E} / \mathrm{C}$ or $\mathrm{F} / \mathrm{O}$ retrieval were derived from participants' response times on correct trials in each condition. Results: All participants were slower on low dominance trials overall, with $\mathrm{AD}$ patients displaving marked slowing on low dominance $\mathrm{F} / \mathrm{O}$ trials. AD patients were significantly less accurate than controls during $\mathrm{F} / \mathrm{O}$ but not $\mathrm{E} / \mathrm{C}$ retrieval. A significant group by summary score interaction was observed, with controls showing a large dominance effect in the $\mathrm{E} / \mathrm{C}$ relative to $\mathrm{F} / \mathrm{O}$ condition, but $\mathrm{AD}$ patients displaying small dominance effects in both conditions.

Conclusions: Findings support the dissociation of exemplar and feature retrieval, which are differentially affected in healthy aging and AD. High dominance exemplars during category verification may confer a performance advantage in the context of deficient top-down attentional processing but preserved bottom-up semantic activation in healthy aging. The presence of both impaired top-down processing and degraded bottom-up semantic integration in AD may eliminate the benefit of high dominance association during both exemplar and feature retrieval. This phenomenon may be useful in differentiating semantic content loss from controlled retrieval difficulty in AD.

Correspondence: Umesh M. Venkatesan, Psychology, Pennsylvania State University, 500 Angell Street, Apartment 707, Providence, RI 02906, United States.E-mail: umi414@gmail.com

C. VILA-CASTELAR, J.J. LY, L. KAPLAN, K. VAN DYK, J. BERGER, L.O. MACINA, J. STEWART \& N.S. FOLDI. Attention predicts long-term cognitive, functional and behavioral response to donepezil in Alzheimer's disease.

Objective: Donepezil is the primary line of treatment for Alzheimer's disease (AD) although it has modest effect sizes and a heterogeneous, difficult-to-detect treatment response. We proposed that as donepezil increases available acetylcholine, and acetylcholine is directly associated to attention, short-term attention performance under high-load conditions should be sensitive and predict long-term cognitive, functional and behavioral treatment response.

Participants and Methods: We conducted a longitudinal, open-label donepezil trial assessing 23 participants newly diagnosed with AD at baseline (T1), 6 weeks (T2) and 6 months (T3) of treatment. Outcome measures: Attention (accuracy, variability and fatigue under high load using the Foreperiod Effect, Covert Orienting and Attentional Blink tasks); Memory (Hopkins Verbal Learning Test-Total Recall); Executive function (Delis-Kaplan Executive Functioning Scale-Trail Making Test Condition-4), Instrumental Activities of Daily Living (IADLs, LawtonBrody); Neuropsychiatric Inventory (NPI), and Cognitive Screen (ADAS-Cog). Linear and stepwise hierarchical regressions assessed whether change in attention from T1 to T2 predicted ADAS-Cog T3-T1 change score, and whether attention measures at T2 predicted ADAS-Cog T3-T1 change score, and IADL and NPI scores at T3.

Results: Preservation of attention accuracy from T1 to T2 predicted stable ADAS-Cog scores by T3 $(\beta=-.52, p=.04)$. Accuracy at T2 predicted ADAS-Cog change scores (T3-T1) $(\beta=-.63, p=.04)$ better than measures of memory or executive function. Moreover, higher accuracy at T2 predicted better IADLs at T3 $(\beta=-.48, p=.02)$, while higher accuracy $(\beta=-.60, p=.02)$ and lower variability $(\beta=.48, p=.02)$ at T2 also predicted better NPI scores at T3.

Conclusions: Findings show that performance on high-load attention tasks after only 6 weeks of donepezil predicted cognitive, functional, and behavioral response after 6 months of treatment. Thus, treatment response to donepezil should include high-load measures of attention. Correspondence: Clara Vila-Castelar, Ph.D. in Clinical Psychology, Psychology, Queens College and The Graduate Center, CUNY, 6530 Kissena Boulevard, Psychology Department, Queens, NY 11367, United States. E-mail: clara.vilacastelar@qc.cuny.edu 
A.A. WANK, J.J. BERCEL \& M.D. GRILLI. Disrupted Retrieval Fluency for Remote Autobiographical Events in Cognitively Healthy Apolipoprotein E e4 Positive Individuals.

Objective: Cognitively healthy middle-aged and older adult carriers of the apolipoprotein $\mathrm{E}$ ( $\mathrm{APOE}$ ) e4 allele, a population at increased risk of developing Alzheimer's disease, exhibit abnormalities in the medial temporal lobe-cortical network that supports episodic memory long before the onset of diagnosable memory impairment. However, the degree to which subtle, preclinical disruptions to memory can be detected in this population is less clear. Recently, we found that ef carriers retrieved less episodically rich autobiographical memories (AM) than non-carriers (Grilli et al., under review). The current study sought to target specific mnemonic processes that might contribute to this e4-related AM disruption and had the potential to be adapted for clinical assessment.

Participants and Methods: Thirty healthy middle-aged and older adults were recruited. Fifteen were carriers of at least one APOE e4 allele and fifteen were non-carriers. Participants completed a number of experimental tasks designed to assess particular AM cognitive mechanisms, including retrieval fluency, spatial memory, pattern separation, emotional memory, and recollection and familiarity retrieval processes. Results: An adapted AM fluency task, which required the participants to rapidly generate unique events from three time periods varying in remoteness, was the single experimental task to elicit significant results. Although there was not an overall group effect, the ef carriers generated significantly fewer events than the non-carriers for the most remote time period (i.e., childhood).

Conclusions: These results build on our previous finding that AM is disrupted in cognitively healthy ef carriers and suggest that reduced retrieval fluency for remote events is one feature of AM that can be disrupted in this population before the onset of diagnosable memory impairment. Future work should focus on adapting these findings into an assessment appropriate for clinical settings.

Correspondence: Aubrey A. Wank, University of Arizona, 1503 E University Blvd, P.O. Box 210068, Tucson, AZ 85721, United States. E-mail:aalw@email.arizona.edu

G. WEISSBERGER, C.P. NGUYEN, N. STRICKER, D.A. NATION \& D. HAN. The Impact of Education on Neuropsychological Differences Detected in Early Preclinical Alzheimer's Disease: A Meta-Analysis.

Objective: This meta-analysis examined the impact of differences in education level on neuropsychological differences observed between cognitively intact older adults who are Alzheimer's disease (AD) biomarker positive and cognitively intact older adults who are AD biomarker negative.

Participants and Methods: A search of Pubmed, PsychINFO, and other sources yielded 775 total sources with 61 studies utilized in the final meta-analysis. Search terms "amyloid and (control or normal) and (cognition or neuropsychology) and (PET or CSF or cerebrospinal fluid)" were applied. Neuropsychological measures were grouped into seven domains: global cognitive function, memory, language, visuospatial, processing speed, attention/working memory/executive functions, and premorbid function. As previously reported, amyloid positive persons scored poorer than amyloid negative persons across all domains. To investigate whether education differences explain differences in cognitive performance between groups, standardized differences in weighted means of years of education between groups were applied in meta-regression analyses for each domain.

Results: Examining the impact of education differences on cognition differences between $\mathrm{AD}$ biomarker positive and negative groups across the seven domains revealed that education differences explained $93 \%$ of the variance for processing speed $(n=12$ studies; Coef $=.93, \mathrm{SE}=.33$, $\mathrm{CI}=.27-1.58, z=2.79, p=.005)$. Results were non-significant for other cognitive domains (all $p \mathrm{~s} \geq .18$ ).

Conclusions: Education differences only accounted for variance in processing speed between groups that were positive and negative according to AD biomarker status. Differences by biomarker status in global cognitive function, memory, language, visuospatial, attention/ working memory/executive functions, and premorbid functions cannot be explained by education differences. More work is needed to determine the impact of education on cognitive function in preclinical Alzheimer's Disease.

Correspondence: Gali Weissberger, Ph.D., Geriatric Research Education and Clinical Centers, West Los Angeles Veteran's Administration, 15133 Dickens St, Apt. D, Sherman Oaks, CA 91403, United States. E-mail: gweissbe@gmail.com

\section{MCI (Mild Cognitive Impairment)}

G. WEISSBERGER, A. JIMENEZ, T.A. NARVAEZ \& R. MELROSE. Intra-individual Variability in Reaction Time Performance Predicts BOLD Activation During a Working Memory fMRI Task in Healthy Older Adults and Older Adults with Mild Cognitive Impairment.

Objective: Intra-individual variability (IIV) in reaction time (RT) may reflect subtle neural changes and serve as an early marker of cognitive decline. This study investigated whether IIV predicts average BOLD activation during a working memory fMRI task across three task-positive networks.

Participants and Methods: Participants with amnestic mild cognitive impairment (MCI; n=25) and healthy elderly controls (EC; n=19) completed an event-related working memory fMRI task. Average BOLD activity was extracted for three empirically derived task positive networks: dorsal attention (DA), ventral attention (VA), and frontoparietal (FP). Standard deviation of RT during the task was divided by mean RT to derive IIV. Hierarchical linear regression was employed to investigate the degree to which IIV (Model 2) independently predicts activity in task-positive regions after controlling for mean RT, \%correct, and diagnosis (Model 1).

Results: EC and MCI were not statistically different on age, education, task-related variables (\%correct, mean RT, and IIV), and activity in FP and VA regions. EC had significantly greater DA activation than MCI $(p=.04)$. Regression results revealed that Model 1 predicted $28 \%$ of variance in DA activity $(p<.01)$. Addition of IIV increased the explained variance to $35 \%$, a significant change $(p=.04)$. Model 1 explained $23 \%$ of VA activity $(p=.02)$. Adding IIV increased the explained variance to $29 \%$, a trend towards significance $(p=.08)$. Results were not significant for FP. Given differences in DA activity between EC and MCI, a post-hoc analysis investigated whether IIV differentially predicts DA activity by diagnosis. Addition of the interaction term explained $4 \%$ additional variance, a non-significant change $(p=.12)$.

Conclusions: Findings support IIV as a predictor of efficient attentional control in EC and MCI groups, beyond other indicators of task performance. Results suggest that IIV during a working memory task is most predictive of activity in DA regions important for focused attention and task maintenance.

Correspondence: Gali Weissberger, Ph.D., Geriatric Research Education and Clinical Centers, West Los Angeles Veteran's Administration, 15133 Dickens St, Apt. D, Sherman Oaks, CA 91403, United States. E-mail: gweissbe@gmail.com

R.K. MACAULAY, A. BOEVE, D.A. LOWE, D.M. SZELES, N. MILANO \& M.T. WAGNER. MCI and Depression Increases Fall Risk Through Slowed Gait.

Objective: Falls are the leading cause of morbidity and death in older adults. Slowed gait is associated with fall risk and is found in patients with depression as well as Mild Cognitive Impairment (MCI). The present study examined the interplay between depression and gait speed on fall risk and whether these effects differed between MCI and Normal Aging (NA) patients. 
Participants and Methods: 140 patients $\left(M_{\mathrm{age}}=66, S D=9.17\right)$ underwent comprehensive neuropsychological testing, clinical interview, and gait speed assessment at a memory clinic for cognitive complaints. 60 patients met criteria for MCI. Fall history within the past year was collected via clinical interview. A depression symptom scale was formed from converting Beck Depression Inventory- $2^{\text {nd }}$ edition and Geriatric Depression Scale scores into z-scores.

Results: One-way ANOVAs respectively found that fallers $(n=40)$ as compared to non-fallers $(n=100)$ had slower gait speed and higher depression scores, $p s<.05$. MCI patients had significantly slower gait speed than NA. PROCESS analyses found that depression positively associated with slower gait speed and that gait speed mediated the relationship between depression and fall history. Chi-square tests examined the frequency of falls and depression relative to cognitive status. Cognitive status overall did not associate with fall risk. Patients with MCI and depression were more likely to fall than MCI patients without depression. No interaction between NA and depression on fall risk was found. Conclusions: Our results indicate that depression and MCI increases fall risk through slowed gait. Deficits in attention/concentration and the ability to efficiently coordinate movements are posited common factors underlying the complex relationship between gait, depression, MCI, and fall risk. Fall risk screening may be improved by assessing both gait speed and depression. Multimodal interventions aimed at remediating depression and gait slowing may reduce fall risk.

Correspondence: Angelica Boeve, B.A., ME, United States. E-mail: angelboeve@gmail.com

T.P. BULL \& V.G. WADLEY. Investigating Driving Performance in Older Adults with Mild Cognitive Impairment Amnestic SingleDomain and Multiple-Domain.

Objective: Previous studies have suggested that Mild Cognitive Impairment (MCI) amnestic multiple-domain (AMD) is characteristic of further progression on the MCI-dementia continuum as compared with MCI amnestic single-domain (ASD). This study examines the differences in driving performance of older adults with MCI-ASD and MCI-AMD. Participants and Methods: 64 older adults (ages 55-88) with MCI were evaluated through a comprehensive neuropsychological battery, functional assessment questionnaire, and medical history, and diagnosed as either MCI-ASD or MCI-AMD. The subjects were administered an on-road driving test (ORT) by a Certified Driving Rehabilitation Specialist (CRDS) and a back-seat driving evaluator (DE). Driving behaviors including scanning, lane position, steering steadiness, speed, gap selection, braking, blinker use, road sign obedience, and overall performance were rated by the CDRS and DE. Driving behavior ratings were averaged between the CRDS and DE and analyzed through independent samples t-tests. A rating of driving safety (unsafe, with restrictions, and safe) was conducted by only the CDRS, and the relationship of MCI diagnosis and safety was analyzed through Chi-square analysis. Results: The independent samples t-tests demonstrated significantly lower ratings for older adults with MCI-AMD in lane position, steering steadiness, gap selection, braking, and overall performance than those with MCI-ASD. The Chi-square analysis indicated a significant relationship between MCI diagnosis and safety rating, $X^{2}(2, \mathrm{~N}=64)=8.89$, $p=.01$. Of the 27 MCI-ASD drivers, only 1 was rated as unsafe and 2 were rated as with restrictions, whereas of the 37 MCI-AMD drivers, 4 were rated as unsafe and 13 as with restrictions.

Conclusions: These findings suggest that those with MCI-AMD demonstrate worse driving performance and safety than those with MCI-ASD. While many older adults with MCI maintain driving fitness, further classification of MCI may be important for remaining vigilant to driving impairments in older adults.

Correspondence: Tyler P. Bull, Psychology, University of Alabama at Birmingham, 1101 11th Ave S., APT 505, Birmingham, AL 35205, United States.E-mail:tpbull@uab.edu
C. COHEN, J. NOVITSKI, C.P. WILLIAMS \& M. SEIDENBERG. Age of Acquisition and Typicality in Semantic Fluency and Mild Cognitive Impairment.

Objective: Semantic fluency tasks are commonly used in the clinical assessment of neuropsychological functioning of older adults. Fluency performance is typically examined by total words produced and the strategic use of clustering and switching. Recently, there has been increased interest in the linguistic features of words produced during different intervals in the time course of fluency tasks. We compared typicality (TY) and age of acquisition (AoA) for words produced by Mild Cognitive Impairment (MCI) and cognitively intact individuals (CI) over 60 seconds.

Participants and Methods: 27 CI (mean age 78 years) and 20 MCI (mean age 76 years) were administered semantic fluency tasks for animals and musical instruments. No group differences in age or education. Scoring was conducted at 30 second intervals over 60 seconds. AoA and TY were scored based on published guidelines and normative databases. Group X Time Interval $(2 \times 4)$ analysis of variance was conducted for AoA and TY. Cohen's $(d)$ effect sizes for group differences are reported.

Results: Overall, the MCI group produced more highly TY words and earlier AoA words. However, the MCI group produced more TY words than the CI, particularly during the final 30 seconds compared to the first 30 seconds for both animals (Cohen's $d=.61, d=.31$ ) and instruments $(d=.64, d=.45)$. The MCI group also produced earlier AoA words, particularly over the last 30 seconds compared to the first 30 seconds for animals $(d=.76, d=.57)$, but not instruments $(d=.25, d=.44)$.

Conclusions: These findings suggest that age of acquisition and word typicality during semantic fluency provide information about differences in semantic retrieval operations and semantic organization between MCI and CI. Additional examination of different semantic categories is also warranted.

Correspondence: Cari Cohen, Rosalind Franklin University of Medicine and Science, 60 Ferndale Road, Deerfield, IL 60015, United States. E-mail: cari.cohen@my.rfums.org

B.C. ALLRED. DALLEY, K.R. SUHRIE \& K. DUFF. Validating premorbid RBANS scores in older adults with amnestic MCI.

Objective: Formulae to estimate premorbid cognitive functioning on the Repeatable Battery for the Assessment of Neuropsychological Status (RBANS) were previously developed in a sample of cognitively intact older adults. However, validation in a clinical sample is needed. The current study sought to validate these premorbid RBANS models in a sample of patients with amnestic Mild Cognitive Impairment (MCI).

Participants and Methods: One hundred twenty-two patients with amnestic Mild Cognitive Impairment (MCI) were administered the RBANS and the Reading subtest of the Wide Range Achievement Test (WRAT). Predicted premorbid RBANS scores were calculated using formulas generated from an intact sample. Dependent t-tests were used to identify discrepancies between the premorbid and observed RBANS scores.

Results: When the formulae were applied to the MCI sample, there were large discrepancies between premorbid and current RBANS abilities, which were most noticeable for the Immediate Memory Index (discrepancy $=-28.12$, dependent $\mathrm{t}(121)=-18.06, \mathrm{p}<.001)$, Delayed Memory Index (discrepancy $=-32.03$, dependent $\mathrm{t}(121)=-16.65, \mathrm{p}<.001)$, and Total Scale score $($ discrepancy $=-24.24$, dependent $\mathrm{t}(121)=-20.22, \mathrm{p}<$ $.001)$.

Conclusions: For each RBANS score, predicted premorbid RBANS scores significantly exceeded current RBANS scores, which suggest decline in those abilities. Although further validation is needed, there is growing support for these estimates of premorbid memory abilities, which may aid clinicians and researchers in determining change across time in older patients. 
Correspondence: Bonnie C. Allred. Dalley, B.S., University of Utah, 650 Komas Drive \#106A, Salt Lake City, UT 84108, United States. E-mail: bonnie.dalley@hsc.utah.edu

\section{B.C. ALLRED. DALLEY, K.R. SUHRIE \& K. DUFF. Predicting} premorbid RBANS scores in older adults.

Objective: Assessing cognitive change during a single visit requires comparison between estimated premorbid abilities and current neuropsychological functioning. While premorbid intellect has been widely examined, methods to estimate other premorbid cognitive abilities have received less attention. The current study used demographic variables and an estimate of premorbid intellect to predict premorbid memory and other cognitive abilities on the Repeatable Battery for the Assessment of Neuropsychological Status (RBANS) in a sample of cognitively intact older adults.

Participants and Methods: One hundred forty-five community-dwelling, cognitively intact, older adults were administered the RBANS and the Reading subtest of the Wide Range Achievement Test (WRAT). A series of stepwise linear regressions was used to identify if demographic variables and WRAT score predicted current performances on the RBANS.

Results: Across the six main indexes of the RBANS, WRAT Reading was the best predictor of current cognitive functioning, with gender adding to one of the prediction models $\left(R^{2}=0.17-0.23, p<0.03\right)$. When these predicted premorbid RBANS scores were compared to observed RBANS scores, differences tended to be relatively small in this cognitively intact sample (mean discrepancy between observed and predicted scores $=-4.7$ standard score points).

Conclusions: If validated, then these prediction models can be used to assist clinicians and researchers in determining cognitive decline in older adults.

Correspondence: Bonnie C. Allred. Dalley, B.S., University of Utah, 650 Komas Drive \#106A, Salt Lake City, UT 84108, United States. E-mail: bonnie.dalley@hsc.utah.edu

L. NICCOLAI, V. DEL BENE, A. GERSTENECKER, K. TRIEBEL, R. MARTIN \& D. MARSON. Trail-Making Test Performances of Patients with Amnestic and Non-Amnestic MCI.

Objective: The Trail Making Test (TMT) has previously been shown to be sensitive in patients with dementia and mild cognitive impairment (MCI) relative to controls. These studies typically use the traditional TMT A and B portions. The aim of this study was to identify key differences in TMT performances for patients with amnestic versus non-amnestic forms of MCI (due to AD) relative to healthy controls, using TMT $\mathrm{A}$ and $\mathrm{B}$, and a custom designed TMT C portion.

Participants and Methods: Participants included cognitively normal controls $(n=71)$, persons with amnestic MCI (aMCI) $(n=51)$, and persons with non-amnestic MCI (naMCI) $(n=18)$. Participants completed TMT A, B, and C. TMT A is a measure of visual attention and processing speed in which participants sequence numbers. TMT B is a related measure of processing speed including set-shifting between numbers and letters in sequential order. TMT C is a more complex task with participants shifting between numbers, letters, and dots. We conducted a multivariate analysis of variance (MANOVA) on the three TMT versions, analyzing the aMCI and naMCI group differences in time to completion and number of errors across the three TMT tasks. Results: The MANOVA revealed a significant main effect of group $(p<.001)$. Pairwise comparisons revealed significant differences between both MCI groups and controls on time to complete TMT A and $\mathrm{B}$, but there was no time to completion difference between MCI groups. On TMT C, both MCI groups differed from controls on time to completion and number of errors, and aMCI patients showed a strong trend for completing TMT C more slowly than naMCI patients $(p=.056)$. Conclusions: Unlike TMT A and B, time to completion on TMT C may differ between patients with aMCI and naMCI due to AD. The increased complexity of set-shifting between three categories on TMT C increases the cognitive demand, which may be more difficult for patients with more classical entorhinal forms of aMCI due to AD.

Correspondence: Victor Del Bene, Ph.D., Psychiatry \& Behavioral Science, Johns Hopkins, 124 Crowne Reserve Drive, Hoover, AL 35244 , United States.E-mail: victor.delbene@gmail.com

V. DEL BENE, L. NICCOLAI, A. GERSTENECKER, K. TRIEBEL, R. MARTIN \& D. MARSON. Financial capacity deficits in patients with amnestic and non-amnestic forms of mild cognitive impairment.

Objective: Prior research has shown declines in financial capacity in both patients with Alzheimer's dementia and amnestic mild cognitive impairment (aMCI). It remains unclear if non-amnestic MCI patients (naMCI) also show financial capacity deficits and if their financial performance differs from aMCI patients. This study compared the financial capacity performance profiles of aMCI and naMCI groups.

Participants and Methods: Healthy controls $(n=71)$, aMCI $(n=51)$, and naMCI $(n=18)$ patients participated in a clinical study of financial capacity (COINS) that included the Financial Capacity Instrument (FCI). aMCI and naMCI patients had an AD etiology based on consensus conference diagnosis. Age and education did not differ between MCI groups, but sex was significantly different $\left(\chi^{2}=9.59, \mathrm{df}=2, p=.008\right)$ and retained as a covariate.

Results: MANCOVA revealed a main effect of group, $F(18,258)=3.99$, $p<.001$. Pairwise comparisons showed significant group differences of aMCI patients relative to controls on all FCI domains and total scores ( $p$-value ranges .021 to <.001). naMCI patients performed significantly worse than controls on domains of conceptual knowledge $(p=.001)$, cash transactions $(p=.001)$, bank statements $(p<.001)$, bills $(p=.009)$, and investment decision-making $(p=.034)$. Basic money skills, checkbook management, and financial judgment did not differ between naMCI patients and controls. Both MCI groups did not differ.

Conclusions: aMCI and naMCI patients with an underlying diagnosis of $\mathrm{AD}$ both showed impairments across a range of financial skill domains. The naMCI group displayed fewer domain level impairments compared to controls than did the aMCI group, which may reflect differential cognitive impairments across the two MCI groups. However, the aMCI and naMCI groups themselves did not differ on any FCI domains or global scores, suggesting that $\mathrm{AD}$ as an underlying disease causes equivalent levels of financial capacity loss irrespective of its prodromal cognitive presentation.

Correspondence: Victor Del Bene, Ph.D., Psychiatry \& Behavioral Science, Johns Hopkins, 124 Crowne Reserve Drive, Hoover, AL 35244 , United States.E-mail: victor.delbene@gmail.com

V. DEL BENE, L. NICCOLAI, A. GERSTENECKER, K. TRIEBEL, R. MARTIN \& D. MARSON. Verbal memory decline on the California Verbal Learning Test-II (CVLT-II) in APOE4 gene carriers: A replication study.

Objective: The apolipoprotein (APOE) gene, found on chromosome 19. has three major alleles. One of these alleles, APOE 4 , has been shown to be a risk factor for Alzheimer's disease. It has also previously been shown to be associated with cognitive decline in non-demented adults and with reduced hippocampal volumes. With the recent call to arms regarding a replication crisis in psychology and neuroscience, the aim of this study was to replicate a previously published finding (Bondi et al., 1995).

Participants and Methods: Non-demented APOE risk gene (E4) carriers $(n=75)$ and non-risk carriers $(n=52)$ completed the CVLT-II as part of a larger NIH study. APOE 4 carrier and non-carriers did not differ on demographic factors of age, sex, or education.

Results: Multivariate analysis of variance (MANOVA) revealed a main effect of group, $F(6,120)=2.29, p=.039$. Pairwise comparisons showed that APOE 4 allele carriers performed worse on CVLT-II five trial learning, $\left(M_{\text {Diff }}=-5.83, p=.008\right)$, Trial B $\left(M_{\text {Diff }}=-0.43, p=.036\right)$, Short Delay Free Recall $\left(M_{\text {Diff }}=-0.83, p<.001\right)$, Short Delay Cued Recall $\left(M_{\text {Diff }}=-0.71, p=.002\right)$, Long Delay Free Recall $\left(M_{\text {Diff }}=-0.77\right.$, 
$p=.001)$, and Long Delay Cued Recall $\left(M_{\text {Diff }}=-0.72, p=.002\right)$. No group differences were seen on recognition memory $\left(M_{\text {Diff }}=0.39\right.$, $p=.058)$. For each CVLT variable, excluding recognition memory, non-carriers performed better than carriers.

Conclusions: Consistent with prior work, we found that APOE 4 risk allele carriers show declines in memory relative to non-carriers on CVLT-II variables measuring encoding, storage, and retrieval of high load verbal information. Replication in this independent study showed the effects of the APOE risk factor on cognitive performance in a sample of older adults.

Correspondence: Victor Del Bene, Ph.D., Psychiatry \& Behavioral Science, Johns Hopkins, 124 Crowne Reserve Drive, Hoover, AL 35244 , United States.E-mail: victor.delbene@gmail.com

A.N. DEVITO \& M. CALAMIA. Examining Differences in Neuropsychiatric Symptom Prevalence by Empirically Derived Mild Cognitive Impairment Subtype.

Objective: To examine differences in the prevalence of neuropsychiatric symptoms (NPS) in empirically derived mild cognitive impairment (MCI) subtypes at baseline and one-year follow up.

Participants and Methods: Participants were 463 individuals recruited through the Alzheimer's Disease Neuroimaging Initiative (ADNI). Participants were $56.8 \%$ male and $86.8 \%$ Caucasian, with an average age of $73.9(\mathrm{SD}=7.4)$ and $15.8(\mathrm{SD}=2.8)$ years of education. Participants completed the Neuropsychiatric Inventory-Questionnaire (NPIQ), multiple self-report measures, neuroimaging, biomarker testing, and a neuropsychological battery that examined episodic memory, language, and executive functioning at baseline and are followed yearly thereafter. Results: Two hierarchical cluster analyses included participants with MCI based on ADNI criteria or those based on neuropsychological test scores. Clusters identified across analyses included: amnestic, dysexecutive/mixed, impaired language, and normal cognition (NC). No differences were observed in the number of NPS differed at baseline (all $p>.05$ ). However, at follow-up, individuals who were classified as MCI using neuropsychological criteria were more likely to experience higher rates of NPS than those who were identified as $\mathrm{CN}(40.2 \%$ vs. $18.4 \%, \chi=14.08, p<.01)$. Additionally, at follow-up, significant differences were found between amnestic $(19.6 \%)$, impaired language $(8.9 \%)$, and dysexecutive/mixed $(2.7 \%)$ subtypes $(\chi=10.86, p<.01)$.

Conclusions: Neuropsychological testing can aid in characterizing cognitive profiles and clinical correlates of MCI such as NPS. Prior research has suggested that empirically derived MCI subtypes aid in diagnostic precision (Bondi et al., 2014); these results extend previous findings by determining that empirically derived MCI subtypes are differentially associated with future elevations of NPS.

Correspondence: Alyssa N. DeVito, M.A., Clinical Psychology, Louisiana State University, 1005 Field House Drive, Room 217, Baton Rouge, LA 70809, United States. E-mail: and2129@tc.columbia.edu

\section{A. ECONOMOU, D. PAVLOU, I. BERATIS, G. YANNIS \& S.G. PAPAGEORGIOU. Predictors of Accidents in Patients with Mild Cognitive Impairment, Mild Alzheimer's Disease and Healthy Controls in Simulated Driving.}

Objective: To investigate predictors of accidents in healthy drivers, drivers with mild cognitive impairment (MCI) and mild Alzheimer's disease $(\mathrm{AD})$.

Participants and Methods: Participants were volunteers who were active drivers: 37 MCI patients, 16 mild AD patients and 21 controls over 52 years (age of the youngest patient). All participants underwent comprehensive neurological, neuropsychological, and ophthalmological assessments and a driving simulator assessment. Data from four rural driving conditions were analyzed: moderate traffic-no distraction (R1), high traffic-no distraction (R2), moderate traffic-conversation (R3), high traffic-conversation (R4). Two unexpected incidents occurred per driving condition.
Multiple regression analyses were performed for each driving condition. Predictors were average speed; headway average (distance from the vehicle ahead); average lateral position (distance from the right road border), average speed variation ( $S D$ of average speed), headway variation ( $S D$ of headway average); lateral position variation ( $S D$ of lateral position average); average reaction time (RT) at unexpected incidents; participant status (patient-control). Outcome was number of accidents at unexpected incidents.

Results: Speed variation and RT predicted accidents in all four conditions, with the addition of lateral position variation in R1 and R2. Larger RT, speed variation and lateral position variation were associated with more accidents. Predictors of RT were speed for R1, R2, R4 (R4 with patient status), headway variation and lateral position variation for R3. Slower speed, larger headway variation and lateral position variation were associated with larger RT.

Conclusions: Larger RT, higher speed variation and lateral position variation predicted accidents at unexpected incidents. Driving slower did not compensate for response time. Patient status was not associated with accident rate over and above the driving measures and is not by itself a sufficient indicator for the decision to drive at a given point in time.

Correspondence: Alexandra Economou, Ph.D, Psychology, National and Kapodistrian University of Athens, G. Blessa 40, Papagos, Athens 15669,Greece.E-mail: aecon@otenet.gr

E.C. EDMONDS, K.R. THOMAS, J. EPPIG, M.L. THOMAS, D. SALMON \& M.W. BONDI. Improved Staging of "Early" and "Late" MCI Using a Neuropsychological Approach.

Objective: The conventional diagnostic criteria for Mild Cognitive Impairment (MCI) define objective memory impairment based on a single episodic memory test. The Alzheimer's Disease Neuroimaging Initiative (ADNI) further separates "early" and "late" MCI subgroups based on level of impairment on this test. We compared ADNI's early/ late MCI classification to our neuropsychological approach which more broadly canvasses the breadth and depth of cognitive functioning.

Participants and Methods: Participants in the ADNI-2 MCI cohort were classified as "early" ( $n=178)$ or "late" $(n=158)$ MCI based on one story from the WMS-R Logical Memory II subscale. Cluster analysis was performed on baseline neuropsychological test data, including measures of memory, attention/executive function, and language. Participants were reclassified based on the cluster results. Survival analysis was used to examine progression to Alzheimer's disease (AD).

Results: Cluster analysis revealed: (1) "early MCI" with impaired memory and below average naming $(n=147)$; (2) "late MCI" with deficits in several cognitive domains $(n=49)$; and (3) consistent with our previous studies, a group that performed within normal limits on neuropsychological tests despite their MCI diagnosis, ("false-positives"; n=140). Thirty-seven percent of our "late" group was considered "early" by ADNI, while $29 \%$ of our "false-positives" were classified "late MCI" by ADNI. Our reclassified MCI groups had significantly steeper survival curves than ADNI's groups.

Conclusions: Our neuropsychological method of staging MCI better predicted progression to dementia, and produced early and late MCI groups largely devoid of false positive diagnostic errors. ADNI's MCI sample, particularly their "early" group, was contaminated by the inclusion of a large number of cognitively normal individuals. Examination of multiple neuropsychological measures and cognitive domains provides an improved method of staging MCI and predicting progression to AD. Correspondence: Emily C. Edmonds, Ph.D., University of California San Diego, 11784 Carmel Creek Rd. \#104, San Diego, CA 92130, United States.E-mail:ecedmonds@ucsd.edu 
S. EMRANI, V.J. WASSERMAN, S. HIGGINS, K.D. GARRETT, R. SWENSON \& D.J. LIBON. Visual versus Verbal Serial List Learning in Mild Cognitive Impairment: A Comparison Between the BVMT-R and the CVLT-MS.

Objective: To compare visual versus verbal serial learning in mild cognitive impairment (MCI) and non-MCI.

Participants and Methods: Memory clinic patients were assessed with the Brief Visuospatial Memory Test-R (BVMT-R) and the 9-word California Verbal Learning Test-Mental Status (CVLT-MS). Three groups were examined; patients with amnestic MCI (aMCI) and mixed/dysexecutive MCI (mx/dysMCI) using Jak/ Bondi (2009) criteria; and non-MCI where comprehensive neuropsychological performance were generally above 1sd. Groups (aMCI, $\mathrm{n}=15$; mx/dysMCI, $\mathrm{n}=18$; non-MCI $\mathrm{n}=33$ ) did not differ on age $(M=77.2 \pm 6.0)$, education $(M=14.2 \pm 2.9)$, or the MMSE $(\mathrm{M}=27.1 \pm 1.9)$. Performance was expressed as age-corrected $\mathrm{z}$-scores. Results: CVLT-MS total immediate free recall were better for non-MCI patients than aMCI and mx/dys MCI $(\mathrm{p}<.001)$. On delayed free recall, aMCI scored poorly $(\mathrm{p}<.040)$ and produced more delayed cued recall intrusions than non-MCI $(p<.024)$. Delayed recognition dissociated all three groups $(p<.058)$. Only aMCI scored $>-1$ sd on any CVLT-MS test parameter. Total immediate recall BVMT-R recall was better for non-MCI compared to aMCI $(\mathrm{p}<.015)$. Groups did not on delayed free recall. Delayed recognition hits were better for non-MCI compared to aMCI $(p<.001)$. There were no differences for BVMT-R recognition false positive responses. Remarkably, all groups generally scored $>-1$ sd on virtually all BVMT- $\mathrm{R}$ variables.

Conclusions: Both tests identified amnesia. However, norm-based BVMT-R scores were generally worse than analogous CVLT-MS scores, suggesting verbal and visual memory assessment is not interchangeable. Performance on visual serial list learning warrants further investigation as it may be a more sensitive measure for MCI than verbal serial list learning.

Correspondence: Sheina Emrani, Rowan University, 201 Mullica Hill Rd, Glassboro, NJ 08028, United States.E-mail: emranis8@students. rowan.edu

J. EPPIG, K.R. THOMAS, E.C. EDMONDS \& M.W. BONDI. Considerable Cognitive Heterogeneity in 'Amnesic Mild Cognitive Impairment' in ADNI: A Longitudinal Latent Profile Analysis.

Objective: Our past work has empirically identified neuropsychological subtypes of mild cognitive impairment (MCI) using latent profile analysis (LPA). The current study employed serial LPA to examine the stability of empirically-defined MCI subtypes over 2 years within ADNI. Participants and Methods: 868 subjects were diagnosed via ADNI's criteria as MCI at baseline (BL): 672 of these individuals were retested at 2 years. Performance on tests of language (Animals, BNT), attention/ executive (Trails A \& B), and memory (AVLT Recall \& Recognition) functions were adjusted for age, education, and gender using standardized regression coefficients based on the performance of "robust" normal controls (BL: $n=381$, Year 2: $n=347$ ). Z-scores for all 6 cognitive tests were subsequently used as indicators for LPAs at BL and year 2 .

Results: A 6-class LPA vielded the optimal solution at both BL and year 2. At BL, the 6 classes included single-domain amnesic MCI $(27.2 \%)$, dysexecutive MCI (5.4\%), and dysnomic MCI (13\%), and a mixed MCI subtype that was impaired in all 3 cognitive domains $(10.8 \%)$. Two classes demonstrated intact cognition: the first $(17.1 \%)$ had all scores within-normal limits (WNL), while the other $(26.5 \%)$ produced WNL scores but AVLT recall was low average. Similar classes were produced at year 2 , including single-domain amnesic MCI $(19.9 \%)$, mixed MCI $(15.2 \%)$, and the 2 groups with intact cognition (all scores WNL: $17.3 \%$; WNL but low average recall: $28.3 \%$ ). Moreover, the LPA at year 2 produced MCI subtypes with an amnesic/dysnomic $(9.7 \%)$ as well as a dysexecutive/dysnomic profile $(9.6 \%)$.

Conclusions: Results indicate relative stability of latent MCI classes from BL to vear 2 , with unique cognitive phenotypes bevond single-domain memory deficits. Additionally, more than $40 \%$ of subjects at BL and vear 2 were classified as cognitively normal using comprehensive neuropsychological testing, despite an MCI diagnosis by ADNI. Future work should use mixture modeling to examine the transition probabilities of each class between years.

Correspondence: Joel Eppig, B.A. in psychology, Clinical Psychology, SDSU/ UCSD, 1205 Colusa St, Apt \#3, San Diego, CA 92110, United States.E-mail: joel.eppig@temple.edu

S.A. EVANS, L. ZHAO, K. STEENLAND, A. COOK MAHER, F.C. GOLDSTEIN \& S.E. JOHN. Initial Visit Predictors of Diagnostic Discrepancy Between Clinician and Test-Based Diagnoses of Mild Cognitive Impairment.

Objective: We evaluated predictors of diagnostic discrepancy between clinician and test-based diagnoses of mild cognitive impairment (MCI). Criteria for a clinical diagnosis include subjective judgments by clinicians, which may be influenced by comorbid conditions. We hypothesized that medical and psychological comorbidities would predict diagnostic discrepancy.

Participants and Methods: Participant data at initial visit was obtained from the National Alzheimer's Coordinating Center for those diagnosed as cognitively normal (CN) or MCI. Participants were independently diagnosed as CN or MCI according to neuropsychological criteria. Participants were stratified by age, race, and education, and raw neuropsychological test scores were transformed into domain specific composite $z$-scores (amnestic, executive, mixed). Two subsamples were created, those with composite $z$-scores of -1.5 and -2 standard deviations (SD) below the mean. For each group, a logistic regression evaluated predictors of diagnostic discrepancy, including age, gender, race (Caucasian vs. African American), education, diabetes, depression, heart disease, hypertension, and vascular disease (stroke/TIA).

Results: Of the total sample in the -1.5 SD group $(\mathrm{N}=8708), 1951$ participants had a discrepant diagnosis $(\mathrm{p}<.0001)$. Of the total sample in the -2 SD group ( $N=9438), 2525$ had a discrepant diagnosis $(p<.0001)$. In both models, diagnostic discrepancy was predicted by older age, male gender, less education, greater self-reported depression, and history of stroke/TIA. Race was only a significant predictor of diagnostic discrepancy in the -1.5 SD group ( $\mathrm{p}=.024$ vs. $\mathrm{p}=.43$ ), with discrepancies occurring more frequently for African Americans than Caucasians.

Conclusions: The findings underscore the complex nature of an MCI diagnosis and warrant further evaluation to better understand the role these predictors play in neuropsychological performance/decline and clinician perceptions of cognitive functioning.

Correspondence: Sarah A. Evans, B.S., Neurology, Emory University School of Medicine, 12 Executive Park Dr. NE, Atlanta, GA 30329, United States.E-mail: saevan4@emory.edu

M. GONZALES, P. INSEL, C. NELSON, D. TOSUN, M. SCHOLL, N. MATTSSON, D. BICKFORD, M. WEINER, S. MACKIN \& A. NEUROIMAGING INITIATIVE. The Association Between Chronic Depressive Symptomatology and Cerebrospinal Fluid Biomarkers in Mild Cognitive Impairment.

Objective: To investigate the association between chronic subsyndromal symptoms of depression (SSD), cerebrospinal fluid (CSF) biomarkers, and neuropsychological performance in individuals with mild cognitive impairment (MCI).

Participants and Methods: Participants included 238 older adults with MCI from the Alzheimer's Disease Neuroimaging Initiative repository with cognitive and CSF amyloid beta $\left(\mathrm{A} \beta_{1-42}\right)$, total tau (t-tau), and phosphorylated tau (p-tau) data. The Neuropsychiatric Inventory identified individuals with stable endorsement (SSD group $\mathrm{N}=80$ ) or no endorsement (non-SSD group $\mathrm{N}=158$ ) of depressive symptoms. CSF biomarker and cognitive outcomes were evaluated with mixed effects models adjusting for age, education, gender, APOE genotype, global cognitive status, and SSD group.

Results: As compared to the non-SSD group, the SSD group displaved lower CSF $A \beta_{1-42}$ levels $(\beta=-24.293, S . E=6.345, p<0.001)$. No group differences were observed for CSF t-tau $(p=0.497)$ or $p$-tau levels 
$(p=0.392)$. Lower CSF $A \beta_{1-42}$ levels were associated with poorer performance on immediate $(\beta=0.041, \mathrm{~S} . \mathrm{E}=0.018, \mathrm{p}=0.021)$ and delayed memory $(\beta=-0.012, \mathrm{~S} . \mathrm{E}=0.005, p=0.031)$ measures, whereas higher CSF $t$-tau levels were associated with poorer performance on measures of global cognition $(\beta=0.022, \mathrm{~S} . \mathrm{E}=0.008, \mathrm{p}=0.007)$ and confrontation naming $(\beta=-0.010, S . E=0.004, p=0.019)$. SSD was independently associated with diminished global cognition, learning, memory, language, and executive function performance over and above the effects of CSF biomarkers (all $\mathrm{p}<0.05)$.

Conclusions: Chronic SSD was associated with lower CSF A $\beta_{1-42}$ levels, suggesting that SSD may interact with neuropathological proteins associated with incipient Alzheimer's disease. Additionally, CSF biomarkers and SSD independently accounted for variance in cognitive performance, indicating that SSD may confer cognitive risk through multiple pathophysiological pathways.

Correspondence: Mitzi Gonzales, Ph.D., Psychology, University of Texas Austin, 2908 Pearl St Unit D, Austin, TX 78705, United States. E-mail: mitzi.m.gonzales@gmail.com

Y. HSU, M. TU \& C. LO. Prospective Memory Performance and Microstructural Correlates in Subjective Cognitive Decline and Mild Cognitive Impairment.

Objective: Prospective memory (ProM) has been evidenced defective in early stages of Alzheimer's disease, indicating its sensitivity to subtle pathological change. Despite hypothetical reliance on a distributed neural network, limited research has explored its relationship with structural connectivity. The current study aimed to examine ProM performance and the role of structural integrity of major fiber tracts in high-risk groups of Alzheimer's disease.

Participants and Methods: Twenty-two adults diagnosed with subjective cognitive decline (SCD) and 17 with mild cognitive impairment (MCI) each received a comprehensive neuropsychological assessment, including the Cambridge Prospective Memory Test, and a diffusion tensor imaging (DTI) examination, from which fractional anisotropy (FA) and mean diffusivity (MD) values were measured. Group comparisons were conducted between diagnostic groups and between people with high and low scores on the ProM tasks. Spearman rank-order correlation coefficients between ProM measures and DTI parameters were also examined.

Results: When teasing apart the ProM process, the MCI group performed significantly worse than the SCD group on the prospective component of the time-based ProM tasks $(p=0.03)$. Correlational analyses revealed significant relationships of this component with both FA $(p<0.01)$ and MD $(p=0.04)$ values of the right anterior thalamic radiation, and the FA value of the right inferior longitudinal fasciculus $(p=0.02)$. When comparing participants with higher and lower successful rates on the component, significant differences were found on the FA values of the right anterior thalamic radiation $(p<0.01)$ and the right inferior longitudinal fasciculus $(p=0.04)$.

Conclusions: Elevated ProM failure rate, as manifesting in the prospective component of the specific task type with time as action cue, may reflect underlying microstructural alterations and serve as a behavioral indicator for dementia risk.

Correspondence: Yen-Hsuan Hsu, Ph.D., Department of Psychology, National Chung Cheng University, 168 University Rd., Minsiung Township, Chiayi County 621, Taiwan.E-mail: oliviayhh@gmail.com

\section{S. KIM, M. LOPEZ, D. CHAR \& G.J. LEE. Differences in Time to Conversion Between Subtypes of Amnestic Mild Cognitive Impairments.}

Objective: Mild cognitive impairment (MCI) is the transitional stage between normal age-related cognitive decline and dementia, and is associated with increased risk for dementia. Although not all MCI patients progress to dementia, for those that do, the time to conversion is highly variable. Amnestic multiple domain MCI (aMCI-md) is characterized by memory impairments and deficits in at least one other non-memory cognitive domain (i.e. executive, language, visuospatial or attention). The aim of the study is to determine which of these non-memory domains, is associated with faster conversion rates to dementia.

Participants and Methods: 859 aMCI-md subjects (456 males) from the NACC UDS dataset were examined. Subjects had at least 3 follow-up visits and ranged from 50 to 96 years old (mean=73.41, $\mathrm{sd}=8.63$ ). All subjects had impairment in only one non-memory domain, i.e., executive functioning (EF, 55.4\%), language (LAN, 31\%), visuospatial (VS, $7.3 \%$ ) and attention (ATT, 6.3\%). A survival analysis was conducted using Cox regression to examine which of the four non-memory domains was associated with faster conversion to dementia.

Results: After adjusting for baseline age and years of education, Cox regression analysis revealed that the aMCI-ATT group exhibited significantly $(\mathrm{p}<0.03)$ faster conversion rate to dementia $(\mathrm{HR}=1.54,95 \% \mathrm{CI}=$ $1.05-2.26)$ than the aMCI- EF, aMCI-LAN or aMCI-VS groups. There were no significant differences between the other groups.

Conclusions: Individuals with both memory and attention deficits were faster to convert to dementia compared to the other groups. Therefore, impairments in attention may act as a harbinger of future decline. This information can also help identify which patients are at highest risk for conversion.

Correspondence: Michael Lopez, CA, United States.E-mail:mlopez13@ alliant.edu

S. KIM, D. CHAR, M. LOPEZ \& G.J. LEE. Subtypes of Amnestic Mild Cognitive Impairments and Functional Status.

Objective: Mild cognitive impairment (MCI) is the intermediary phase between normal aging and early dementia. Individuals diagnosed with amnestic multiple domain mild cognitive impairment (aMCI-md) have deficits in memory and at least one other non-memory cognitive domains (i.e. language, visuospatial, attention or executive functioning). The aim of the current study is to compare differences in functional abilities between individuals with different aMCI-md subtypes.

Participants and Methods: 1,721 aMCI-md subjects from the National Alzheimer's Coordinating Center dataset who were $\geq 50$ years old were evaluated. All subjects had only one non-memory impairment, i.e., executive functioning (EF, 56.1\%), language (LAN, 29.1\%), attention (ATT, 7.4\%), and visuospatial functioning (VS, 7.4\%). A one-way ANCOVA was conducted to evaluate the differences between the four aMCI-md subtypes in functional impairment, measured through the Functional Activities Questionnaire (FAQ).

Results: Controlling for age, education, and Clinical Dementia Rating (CDR) score, analysis showed that FAQ scores significantly differed between groups, $F(3,1714)=2.87, \mathrm{p}<.04$. Pairwise comparisons showed that there were greater functional deficits among the aMCI-EF $(\mathrm{p}<.01)$ and aMCI-LAN $(\mathrm{p}<.05)$ groups compared to aMCI-ATT, though the aMCI-EF and aMCI-LAN groups did not differ significantly from each other. There were no significant differences between the aMCI-VS subjects and other groups.

Conclusions: This study shows that among those with aMCI-md, there are differences in functional abilities depending on which non-memory domain is impaired, namely, that those with executive or language deficits in addition to memory impairment may have more functional deficits than those with difficulties in attention. This can be helpful in informing patients and/or families of the possible need of additional assistance with more complex activities of daily living.

Correspondence: Derek Char, CA, United States.E-mail:dchar@llu.edu

W.S. KREMEN, E. VUOKSIMAA, M. LOGUE, M.S. PANIZZON, E. JEREMY, D. GUSTAVSON, S. HATTON, L. MCEVOY, X. TU, M.J. LYONS \& C.E. FRANZ. Improving the Diagnosis of MCI and the Potential for Early Identification of Alzheimer's Disease Risk. Objective: The Jak-Bondi approach has shown that diagnostic accuracy of mild cognitive impairment (MCI) is improved if multiple tests are employed and impairment is defined based on $>1$ test in a cognitive domain being below expectation given one's age and education. In the 
Alzheimer's Disease Neuroimaging Initiative (ADNI), we examined the effect of adding just one test on MCI diagnosis. In the Vietnam Era Twin Study of Aging (VETSA), we tested if an AD polygenic risk score (PRS) could differentiate MCI diagnosed by the Jak-Bondi approach from cognitively normal (CN) adults.

Participants and Methods: In ADNI, we assessed baseline CSF A $\beta$ and tau, hippocampal atrophy, and conversion to AD in 394 MCI and 230 $\mathrm{CN}$ adults (mean age $=75$ ). We divided MCI into AVLT+ and AVLTgroups based on normal vs. impaired Rey AVLT recall. In VETSA, we examined $1050 \mathrm{CN}, 83$ amnestic MCI (aMCI), and 43 non-amnestic MCI (naMCI) men. VETSA mean age $=56 ; 89 \%$ were only in their $50 \mathrm{~s}$. We tested 6 p-value thresholds for an AD PRS based on SNP data from the International Genomics of Alzheimer's Project. We adjusted for the first 3 principal components, age, depression, hypertension, diabetes, and head injury.

Results: In ADNI, the 3 -year conversion rate was $17 \%$ for AVLT+ vs. $51 \%$ for AVLT - groups (HR=4.21). The 3 groups differed significantly on all biomarkers. The AVLT- group also had steeper hippocampal volume declines. Even in a subgroup with baseline hippocampal volume $\geq$ the CN mean, the AVLT- group still had higher conversion rates and steeper neurodegeneration slopes. In VETSA, higher PRSs were associated with greater odds of being aMCI than CN (ORs=1.36-1.43); $\mathrm{OR}=3.22$ for upper vs. lower quartile of PRS distribution. ORs were slightly lower for naMCI. Diabetes was associated with increased odds of naMCI (ORs=3.10-3.41).

Conclusions: Adding just a single test can dramatically improve MCI diagnosis. The AD PRS results strongly support the validity of this diagnostic approach, its value for early identification, and the differentiation of aMCI and naMCI.

Correspondence: William S. Kremen, Psychiatry, UCSD, 9500 Gilman Dr.(MC 0738),La Jolla, CA 92093, United States.E-mail:wkremen@ ucsd.edu

S.K. LAGEMAN, T.V. CASH \& Q. WANG. Clinical Trial Results Comparing a Neurocognitive Intervention to Supportive Therapy in Individuals with Parkinson's Disease.

Objective: Parkinson's disease (PD) impacts non-motor symptoms, including mood, cognition, and quality of life (QOL); however, evidencebased treatments are lacking. This trial aimed to adapt empirically-supported memory and problem solving techniques for use in PD.

Participants and Methods: This randomized controlled trial evaluated the feasibility and preliminary efficacy of a novel neurocognitive intervention (COG; $n=16$ ) of memory enhancement and problem solving strategies, compared to nondirective supportive therapy (ST; n=15) in individuals with PD and mild cognitive impairment (MCI; $N=31$ ). Outcomes included neuropsychological tests of attention, processing speed, memory, and executive functions, and self-reported mood symptoms and QOL, given at baseline, immediate post, and 6-month post-treatment.

Results: Repeated measures analysis of variance revealed significant immediate post-treatment improvement in word list memory ( $p=.030$, $\left.\eta^{2}=10\right)$ for the total group, but no changes in mood or QOL and no changes by condition. Additional analyses identified that the groups were not equivalent on several cognitive tests on pre-trial clinical data. Exploratory analyses revealed that the cognitive data were more homogeneous for patients with Movement Disorder Society-Unified PD Rating Scale (MDS-UPDRS) total scores $\leq 30$ than those $>30$, and treatment response was observed only in the $>30$ group. If the MDS-UPDRS total score was $>30$ (indicating greater symptom severity), memory improved immediate post-treatment in the COG group $(n=8$; average improvement $=.8750$ ), but not in the ST group ( $\mathrm{n}=8$; average improvement $=.3125 ; p=.1304)$.

Conclusions: These results suggest that cognitive and disease-symptom variables may need to be examined to identify patients who best benefit from MCI interventions. Focused interventions targeting memory skills may help counteract aspects of neurocognitive symptoms experienced by individuals with PD. However, further research is needed to determine who benefits from treatment and how to maintain improvements. Correspondence: Sarah K. Lageman, Ph.D., Neurology, Virginia Commonwealth University, 11958 W. Broad Street, 4th Floor, Room 4023, Richmond, VA 23233, United States.E-mail: sarah.lageman@ vcuhealth.org.

B.K. LEBOWITZ, C. WEINSTEIN, Y. LIU, S. DEVINE, C. DECARLI, J. MOSSARO, E. ROSEMAN, T. PRESTON \& R. AU. White/ Gray Matter Correlates of Math Fluency and Error Analysis in Community-Based Older Adults.

Objective: The current study sought to identify whether neuroimaging biomarkers were related to information processing as measured by math fluency (MF) performance in older adults.

Participants and Methods: Between 2009-2015 the MF subtest of the Woodcock Johnson Test of Achievement-III and brain MRI scans were administered to 1361 subjects from the Framingham Heart Study Offspring cohort. After excluding subjects with stroke, dementia, age< 60, and no MRI scans within 60 days of Math assessment, the final sample was 727. The total score (TS) indicated the number of correct one-step addition, subtraction, and multiplication problems in three minutes (e.g., 8+3, 9X7). Error analysis included miscalculations, perseverations, and mis-read signs. MRI metrics included white matter (WM) and gray matter (GM) total and lobar volumes and white matter hyper-intensity (WMH) volumes.

Results: After accounting for age, sex, and educational attainment and correcting for multiple comparisons, TS was negatively associated with WMH $(\mathrm{p}<.001)$ but was positively associated with total and temporal WM volumes. Calculation errors were associated with larger frontal and total WM volumes $(\mathrm{p}<.01)$. Perseverative errors were associated with larger parietal GM and WM volumes $(\mathrm{p}<.01)$. Membership in the top $10 \%$ of subjects with mis-read sign errors was negatively associated with frontal GM volume.

Conclusions: Math Fluency performance appears to be sensitive to WMH burden. This finding supports previous research associating WM pathology with decrement in information processing. Qualitative analysis revealed differences in error types and brain regions highlighting how qualitative analysis can enhance understanding of brain-behavior relationships that are not revealed from single total scores.

Correspondence: Brian K. Lebowitz, PhD, Neurology, Stony Brook University Medical Center, 14 Technology Drive, Suite 12B, Setauket, NY11733,United States.E-mail:Brian.Lebowitz@stonybrookmedicine. edu

D.A. LOWE, R.K. MACAULAY, D.M. SZELES, N. MILANO \& M.T. WAGNER. Dual-Task Gait Assessment in a Clinical Sample: Implications for Improved Detection of Pre-Clinical Dementia.

Objective: Gait dysfunction under dual-task conditions (i.e., simultaneously walking and completing a cognitive task) has been linked to mild cognitive impairment (MCI) and dementia risk in research settings. Preliminary findings by our group in clinical settings have validated these findings and suggest that gait performance (e.g., walking speed) in dual-task conditions can help distinguish normal aging (NA) from MCI. The present study extended this investigation by examining additional variables (i.e., cognitive performance) under single- and dual-task conditions.

Participants and Methods: Two hundred nine patients ( $M$ age $=66.26)$ presented with memory concerns in an academic medical setting. Ninety-five were ultimately diagnosed with MCI and 114 as NA. Patients underwent neuropsychological testing and gait assessment (single- and dual-task conditions). Number of errors in spelling "WORLD" backward was compared between non-walking (single-task Mini Mental Status Examination performance) and added walking (dual-task) conditions.

Results: Similar to previous findings, patients with MCI walked significantly more slowly than those with NA under dual-task conditions after 
adjusting for age, sex, and education, $F(1,189)=8.96, p<.01$. A trend in the same direction was noted for single-task gait speed, $F(1,201)$ $=3.54, p=.06$. A repeated-measures ANOVA revealed a significant interaction between task condition and diagnostic group for cognitive performance, $F(1,197)=4.22, p<.05$. That is, patients with MCI made more errors in spelling "WORLD" backward in dual-task relative to single-task conditions than those diagnosed as NA.

Conclusions: This study replicates our prior research in a larger sample and demonstrates decreased cognitive efficiency during dual-task gait performance in patients with MCI. Findings suggest that dual-task gait and cognitive dysfunction may represent a marker of cognitive impairment. Likewise, gait assessment may represent a targeted screening tool for cognitive impairment in clinical samples.

Correspondence: Deborah A. Lowe, Ph.D., Psychiatry \& Behavioral Sciences, University of Oklahoma Health Sciences Center, Department of Psychiatry \& Behavioral Sciences, 920 Stanton L. Young Blvd. WP 3440 , Oklahoma City, OK 73104, United States. E-mail: deborah.a.lowe@ gmail.com

A. COOK MAHER, S.E. JOHN, S. EVANS, L. ZHAO, K. STEENLAND \& F.C. GOLDSTEIN. Racial Differences in Clinician-Test Diagnostic Discrepancy of Mild Cognitive Impairment with Executive Deficits.

Objective: The diagnosis of mild cognitive impairment (MCI) with executive dysfunction by either clinician judgment or neuropsychological performance criteria may be vulnerable to biases that contribute to inflated diagnosis in black versus white older adults. The present study examined clinician-test discrepancy in the diagnosis of MCI with executive deficits in black and white older adults as well as factors contributing to this discrepancy.

Participants and Methods: Data from 1,168 white and 221 black older adults with baseline clinical diagnosis of MCI with executive deficits were obtained from the National Alzheimer's Coordinating Center (NACC) database. Executive function composite $\mathrm{z}$-scores were created based on the proposed NACC battery factor structure. Participants with executive composite $\mathrm{z}$-scores $\geq 1$.5SD below the mean for their age, education, and race were classified with test-based MCI with executive deficits. The association between race and clinician-test diagnostic discrepancy was examined using a chi-squared test. Demographic and health factors (age, gender, education, family dementia history, heart disease, stroke, hypertension, diabetes, depression) that may influence diagnostic discrepancy were evaluated by logistic regression within each race.

Results: In older adults with a clinician diagnosis of MCI with executive deficits, clinician-test diagnostic discrepancy was greater for blacks $(88 \%)$ than whites $(75 \% ; p<0.001)$. Male gender, stroke, and depression were significant positive predictors of diagnostic discrepancy in whites while education was a significant positive predictor of discrepancy in blacks $(p ' s<0.05)$.

Conclusions: Diagnostic discrepancy was high among both black and white older adults with clinician diagnosed MCI with executive deficits, yet was significantly higher in blacks. Results suggest different demographic and health factors predict diagnostic discrepancy in the two races, although cardiovascular risk factors often associated with executive dysfunction were not among them.

Correspondence: Amanda Cook Maher, MA, MS, Cognitive Neurology \&. Alzheimer's Disease Center, Northwestern University, 320 E Superior St, Searle 11, Chicago, IL 60611, United States. E-mail: amandacook2017@u.northwestern.edu

R. MIS, K.N. DEVLIN, D. DRABICK \& T. GIOVANNETTI. Heterogeneity of Informant-Reported Functional Performance in Mild Cognitive Impairment: A Latent Class Analysis of the Functional Activities Questionnaire.

Objective: Individuals with mild cognitive impairment (MCI) show subtle functional difficulties, though little is known about the range of these difficulties and their relation to cognitive functioning and subsequent decline. We characterized patterns of informant-reports of functional abilities among participants with MCI.

Participants and Methods: Data from 4,273 MCI participants from the National Alzheimer's Coordinating Center (NACC) Uniform Data Set (UDS), contributed by the NIA-funded ADCs and funded by NIA/ NIH Grant U01AG016976, were included in latent class analyses (LCA) of informant-ratings of functional performance (i.e., Functional Activities Questionnaire; FAQ). Classes from the best fitting model were compared on demographic, clinical, and cognitive variables.

Results: The best fitting model supported three classes varying by level and type of difficulty: intact function (Intact; $n=3,299$ ), intermediate (IR; $n=769)$ and high ratings of difficulty (HR; $n=205)$. In the IR group, items related to finances, remembering dates, and travel were rated as most difficult, while the HR group included these items and meal preparation. IR and HR classes were significantly more likely to progress to dementia than the Intact class. Classes did not differ on type of MCI, sex or education. but the Intact class was younger and consisted of a higher proportion of minorities than the IR and HR classes. The Intact class showed the best performance and the IR class displayed better or equal performance to the HR class on tests of global cognitive function, episodic memory, executive function, and processing speed.

Conclusions: Informant ratings of function in MCI vary in terms of number and severity of task difficulties, and classes based on these indices differed on demographic and cognitive variables. Individuals with MCI whose informants report even intermediate-level functional difficulties are more likely to progress to dementia, suggesting that even subtle functional difficulties place individuals at higher risk for future decline.

Correspondence: Rachel Mis, Psychology, Temple University, 1701 North 13th Street, Philadelphia, PA 19122, United States. E-mail: rachel.mis@temple.edu

I.E. MOSCA, S. SORBI, C. MACCHI, F. VANNETTI, F. CECCHI, M.R. TIMPANO SPORTIELLO, S. DANTI \& S. TOCCHINI. Comparison Between Free and Cued Selective Reminding Test and Wechsler Memory Scale-IV Edition: A Descriptive Study on Amnesic Mild Cognitive Impairment.

Objective: The early detection of cognitive impairment is extremely important. Similarly, it is universally accepted the hypothesis that the impairment of episodic memory is one of the earliest and most prominent symptoms of cognitive decline, in particular in $\mathrm{AD}$. To contribute to the debate we carried out a comparative analysis between two neuropsychological instruments widely used in the clinical setting: the Free and Cued Selective Reminding Test (FCSRT) and verbal subtests of Wechsler Memory Scale - Fourth Edition (WMS-IV). Our aim is to investigate the accuracy of both in the early detection of cognitive impairment, the predictive value of risk and the progression of cognitive decline.

Participants and Methods: Our sample was formed by 23 patients $(\mathrm{F}=9)$ and 23 age-matched healthy controls. Patients have been diagnosed amnestic MCI according to Petersen's criteria. FCSRT and the verbal subtests of WMS-IV (LM I. II, recognition: VPA I, II, recognition. free recall) were administered. After 7 months we retested patients and controls.

Results: Performances in FCSRT showed an impaired free and total recall, with a low sensitivity to the cueing (respectively, IFR, ITR, DFR, DTR, ISC) and in WMS-IV patients showed a poor performances on LM II, VPA II, both in recognition and in word recall. These scores are significantly lower than controls scores $(\mathrm{p}<.005)$. Moreover, after 7 months in the verbal subtests of WMS, aMCI patients have scores significantly lower than the first assessment $(p<.005)$. The differences between the first assessment and retest aren't significant in FCRST indexes. Conclusions: Both tools are useful to assess the cognitive decline and in particular episodic memory impairment. The results suggest that verbal subtests of WMS are more sensitive to progression of cognitive decline and they can have an important predictive value of risk of cognitive decline. 
Correspondence: Marco R. Timpano Sportiello, Professor, of Psychology, University of Pisa-Italy, via P.Maroncelli, 73-Viareggio, Viareggio 55049, Italy.E-mail:viatene@alice.it

\section{A.L. NORMAN \& J.L. WOODARD. Mild Cognitive Impairment: 5-Year Growth Trajectories.}

Objective: The reversion rate of mild cognitive impairment (MCI) to normal cognition collapsed across samples is $24 \%$, suggesting a high potential for false positive MCI diagnoses. Limitations of prior longitudinal MCI research include using few data points ( $<$ three), using only the first and last data point, and using subjectively-defined cognitive trajectories.

Participants and Methods: The National Health and Aging Trends Study, consisting of Medicare beneficiaries aged 65 or older, was used to address these limitations. MCI status for a sample of 6,795 participants was determined independently for each of five years based on preserved functional abilities and impairment in one or more cognitive domains (orientation, executive/visuospatial abilities, verbal memory). Latent classes of MCI trajectories were statistically determined by latent class growth analysis.

Results: 2- and 3-class solutions provide the best fit; however, do not distinguish reversion from stable non-impaired cognition. 4- and 5- class solutions provide adequate fit, with statistical preference for 5 -classes of cognitive trajectories: Stable No-MCI $(80 \%)$, Progressing MCI $(13 \%)$, Stable MCI $(4 \%)$, MCI reversion $(2 \%)$, No-MCI to MCI $(1 \%)$.

Conclusions: The observed statistically-derived cognitive trajectories identify possible baseline factors associated with derived trajectories. Incorporating these baseline factors into the diagnostic process may enhance MCI diagnosis accuracy, thereby reducing false positive diagnoses.

Correspondence: Andria L. Norman, Psychology, Wayne State University, 5057 Woodward Ave, Floor 7, Detroit, MI 48202, United States.E-mail:andria.norman@wayne.edu

A.D. ROCHETTE, M. SPITZNAGEL, G. STRAIN, M. DEVLIN, R. CROSBY, J. MITCHELL \&. J. GUNSTAD. Mild Cognitive Impairment Category Predicts Weight Loss Following Bariatric Surgery.

Objective: Recent work has found that over half of persons with severe obesity (BMI >35) meet diagnostic criteria for Mild Cognitive Impairment (MCI), regardless of age, but no study has examined whether MCI also predicts weight loss success. The current study examined the relationship between pre-operative baseline MCI and percent weight loss (\%WL) at 12 months following bariatric surgery using two commonly-used criteria for MCI.

Participants and Methods: Data was extracted from the Longitudinal Assessment of Bariatric Surgery (LABS) project for those individuals with complete medical history and computerized Integneuro cognitive testing. This resulted in a sample of 97 bariatric surgery patients $(43.98 \pm 10.55$ years, range $=22-62 ; \mathrm{BMI}=46.41 \pm 5.86 ; 80.4 \%$ female). MCI was operationalized using two commonly-used definitions, including comprehensive criteria established by Jak and colleagues ( $\geq 1$ SD below the normative mean on $2+$ tasks in a cognitive domain) and conventional criteria outlined by Petersen and Morris ( $\geq 1.5$ SD below the normative mean on $1+$ tasks in the neuropsychological battery).

Results: After controlling for demographic and medical variables known to predict weight loss outcomes, individuals meeting comprehensive criteria for MCI showed lower \%WL 12 months post-surgery $\left(\mathrm{MCI}=53.6 \%\right.$, Total $\mathrm{R}^{2}=.11, \Delta \mathrm{R}^{2}=.40, \beta=-.20, p<.05, \%$ $\mathrm{WL}=32.09 \%$ vs $36.02 \%)$. However, when classifying MCI using conventional criteria, MCI was not associated with $\% \mathrm{WL}$ (MCI $=58.8 \%$, Total $\left.R^{2}=.07, \Delta \mathbf{R}^{2}=.00, \beta=-0.05, p=.63, \% \mathrm{WL}=33.43 \% \mathrm{vs} 34.51 \%\right)$. Conclusions: Findings indicate that bariatric surgery patients meeting comprehensive criteria for MCI demonstrate lower \%WL at 12 months post-surgery. This encourages cognitive screening to help identify persons at risk for reduced weight loss success. More work is needed to investigate possible mechanisms, particularly studies examining the role of adherence.

Correspondence: Amber D. Rochette, BA, Psychology, Kent State University, 3494 Bent Tree Lane, \#213, Stow, OH 44224, United States.E-mail:amber.rochette88@gmail.com

E.E. SMITH, L.S. HYNAN, L. LACRITZ, M. CULLUM, A. VAN WRIGHT, M. WEINER \& H.C. ROSSETTI. A Comparison of Three Neuropsychological Instruments in Detecting Mild Cognitive Impairment (MCI) in African Americans.

Objective: Compare three common measures of global cognition to evaluate sensitivity, specificity, and diagnostic accuracy in distinguishing mild cognitive impairment (MCI) in African Americans (AA) from those with normal cognition (NC).

Participants and Methods: Subjects included 130 AA participants from a larger study on aging diagnosed as NC $(n=45)$ or MCI $(n=90)$ based on consensus diagnosis using clinical history, Clinical Dementia Rating score, and comprehensive neuropsychological test results. Logistic regression models were used to predict MCI vs NC using sex, education, age at visit, and total scores from the Montreal Cognitive Assessment (MoCA), Mini Mental State Examination (MMSE), and Consortium to Establish a Registry for Alzheimer's Disease (CERAD; max 100 points). Receiver operating characteristic (ROC) curve analyses were used to compare sensitivity, specificity, and diagnostic accuracy among the three tests.

Results: The NG group was younger $\left[M_{N C}=62.33(6.76)\right.$, $\left.M_{M C I}=64.76(5.87) ; \mathrm{p}=0.033\right]$, more educated $\left[M_{N C}=14.36(2.51)\right.$, $\left.M_{M C l}=13.07(2.37) ; \mathrm{p}=0.004\right]$, and had higher scores across measures [MoCA: $M_{N C}=25.47(2.13), M_{M C l}=21.26(3.85)$; MMSE: $M_{N C}=28.58(1.22), M_{M C l}=26.94(1.96) ;$ CERAD : $M_{N C}=81.36(7.47)$, $M_{M C I}=73.04(9.64)$; all $\left.\mathrm{p}<0.01\right]$ than the MCI group. Logistic regression models resulted in non-significant demographic measures. The area under the curve (AUC) was significant for all measures $(\mathrm{MoCA}=0.826, \mathrm{MMSE}=0.747, \mathrm{CERAD}=0.759$; all $\mathrm{p}<0.01)$. Optimal cutoffs $($ MoCA $<23.5$; MMSE $<28.5$; CERAD $<77.5)$ were determined to maximize sensitivity and specificity: MoCA: $84 \%, 72 \%$; MMSE: $56 \%$, $79 \%$; CERAD: $68 \%, 73 \%$, resulting in slightly better diagnostic accuracy for the MoCA $(76 \%)$ than the MMSE (71\%) and CERAD $(71 \%)$. Conclusions: This is the first study to compare these three cognitive screening measures in African Americans with respect to diagnostic accuracy of MCI. All three tests performed adequately in detecting MCI subjects, although the MoCA showed the best results in this sample.

Correspondence: Emily E. Smith, B.S., Psychiatry, UT Southwestern Medical Center, 5323 Harry Hines Blvd. , Dallas, TX 75390-9044, United States. E-mail: emilye.smith@utsouthwestern.edu

\section{K. STEWARD, R. PERRY \& V.G. WADLEY. Relationship between BDNF Val66Met Polymorphism and Processing Speed in Older Adults with Amnestic MCI and Probable Dementia.}

Objective: The Brain Derived Neurotropic Factor (BDNF) Val66Met polymorphism is associated with reduced BDNF expression, decreased volume in the hippocampus and prefrontal cortex, and impaired cognition. Met allele carriers (Met+) have slower processing speed (PS) and more rapid decline in PS over time. However, this relationship has not been examined in older adults with Mild Cognitive Impairment (MCI) and dementia despite evidence that Met+ MCI/dementia patients are more impaired in other cognitive domains. The aim of this cross-sectional study was to examine whether this polymorphism is associated with slowed PS in this population, which is important given that impaired PS is a risk factor for future cognitive decline.

Participants and Methods: BDNF genotypes were determined from salivary samples of 86 individuals ( $47 \%$ male; 91\% white) aged 54-95 (M=74.6 years) with amnestic MCI $(\mathrm{n}=68)$ and probable dementia $(n=18)$ recruited primarily from university-affiliated clinics. 
Participants were administered a cognitive and functional test battery, which included 9 PS measures.

Results: The Met+ group ( $\mathrm{n}=28)$ did not differ from the Met- group $(n=58)$ on demographics, diagnosis, global cognition, or depressive symptoms. Multivariate analysis of covariance models revealed that BDNF genotype was unrelated to PS on either cognitive (Wilks' lambda $=.907, \mathrm{~F}(6,75)=1.275, \mathrm{p}=.279$ ) or functional tests (Wilks' lambda $=.992, \mathrm{~F}(3,68)=.186, \mathrm{p}=.905)$. Contrary to hypotheses, the Met+ group had a nonsignificant trend towards better performance on cognitive PS measures.

Conclusions: These findings suggest that the BDNF Val66Met polymorphism is not strongly related to PS in older adults with amnestic MCI and dementia. Interestingly, there was evidence that Met+ individuals may perform better on cognitive tests of PS, which is consistent with other studies that have found a protective effect of Met genotype for certain cognitive domains in the elderly. Future longitudinal studies should examine whether this polymorphism predicts rate of PS decline. Correspondence: Kayla Steward, B.S., Medical/Clinical Psychology, University of Alabama at Birmingham, 1620 12th St. S., Apt C, Birmingham, AL 35205, United States.E-mail: ksteward@uab.edu

D.M. SZELES, D.A. LOWE, R.K. MACAULAY, N. MILANO \& M.T. WAGNER. Dual-Task Gait Performance in Normal Aging and Mild Cognitive Impairment Subtypes.

Objective: Dual-task gait function (i.e., walking while engaged in a cognitive task like conversing) reflects a complex process. As balance may require greater cortical control with age to accommodate orthopedic change, dual-task functioning may also decline.

During the dual-task, older adults may 1) slow down to attend to the cognitive task or 2 ) focus more on walking at the expense of cognitive performance. Our group demonstrated that both may be more pronounced with Mild Cognitive Impairment (MCI) versus normal aging (NA). The current study aimed to identify specific patterns of compromise in MCI subtypes (vascular versus probable Alzheimer's disease etiologies).

Participants and Methods: Seventy-four patients (42 females) presented with memory concerns to a memory disorders clinic and completed neuropsychological testing; 9 were diagnosed with Vascular Cognitive Impairment (VCI, ages 66-87), 14 as amnestic MCI (aMCI, ages 66-82), and 51 normal aging (NA, ages 66-87). Gait assessment involved both single-task (walking alone) and dual-task (walking while spelling "ARROW" backwards) trials. Speed during the single- and dual-tasks, and number of correctly sequenced letters during the dualtask were compared across groups.

Results: A One-Way ANOVA revealed a significant effect of group on single-task gait speed $F(2,71)=4.91, p=.01$. Groups did not significantly differ in gait speed during the dual-task, though a significant difference was found for cognitive performance $F(2,70)=4.71, p=.01$. Post-hoc analyses with Bonferonni correction revealed that patients with VCI walked more slowly than those with both aMCI and NA under single-task conditions; patients with aMCI sequenced fewer correct letters on the dual-task than NA.

Conclusions: This study extends our prior research and suggest that while all individuals demonstrate a dual-task decrement in gait speed, individuals with VCI may be more physically limited at baseline, while those with aMCI may struggle more in terms of dual-task cognitive performance.

Correspondence: Dana M. Szeles, Ph.D., Neurology, Medical University of South Carolina, 96 Jonathan Lucas Street, Charleston, SC 29425, United States.E-mail:szeles@musc.edu
L. TOMMASINI, G. MANCIOPPI, S. DANTI, M.R. TIMPANO SPORTIELLO \& S. TOCCHINI. Visual Reproduction of the Wechsler Memory Scale-Fourth Edition as a Sensitive and Specific test for Amnestic Mild Cognitive Impairment Diagnosis.

Objective: The amnestic form of Mild Cognitive Impairment (aMCI) is widely considered to be a prodromal stage of Alzheimer disease (AD). Peterson criteria for aMCI requires an objective memory impairment, preferably on a delayed recall of a prose memory test. Similarly, in the Jack/Bondi actuarial neuropsychological criteria, a word list test is recommended for episodic memory assessment. Although measures of verbal episodic memory have usually been indicated as most sensitive to early changes in the course of $\mathrm{AD}$, visual memory has also been documented as having early involvement in the preclinical phase of the disease. The aim of this study is to evaluate sensibility and specificity of WMS-IV Visual Reproduction test (VR) on an aMCI group.

Participants and Methods: 75 aMCI out-patients and 45 non-amnestic MCI (na-MCI) out-patients, diagnosed according to the Petersen criteria, have been compared with a demographically matched sample of healthy individuals. We used a wide battery of neuropsychological tests, supported by neuroimaging techniques. Memory domain has been assessed using WMS-IV battery. Verbal memory tests have been used in order to do the diagnosis, while the VR-I and VR-II were used as supplement experimental test.

Results: Our research showed a very good accuracy (81\%) of the VR-II in discriminating between aMCI subjects and cognitive normal subjects. It also suggested a worse performance on visual reproduction test compared to verbal paired associated test in the aMCI group.

Conclusions: These findings suggest the importance to evaluating the nonverbal memory domain in the aMCI group both for diagnosis and prognosis. The only use of verbal memory test during the assessment of MCI subjects could bring to a higher prevalence of false negative errors. Additionally, some studies proved that aMCI with both memory predominant deficit (verbal and visual impairment) had higher progression rate to $\mathrm{AD}$

Correspondence: Marco R. Timpano Sportiello, Professor, of Psychology, University of Pisa-Italy, via P.Maroncelli, 73-Viareggio, Viareggio 55049,Italy.E-mail:viatene@alice.it

R. VAN PATTEN, K. BRITTON \& G. TREMONT. Enhanced clinical utility of the Modified Mini Mental State Examination compared to the MMSE in older adults with mild cognitive impairment.

Objective: To demonstrate enhanced clinical utility of the Modified Mini-Mental State Examination (3MS) as compared to the original MiniMental State Examination (MMSE) in older adults with mild cognitive impairment (MCI).

Participants and Methods: 247 older adults $(65.60 \%$ amnestic MCI and $34.40 \%$ nonamnestic MCI, aged 51-94 $(M$ age $=74.59$ [7.84]; $M$ years of education $=14.06[3.06])$, and $96.40 \%$ Caucasian. Participants completed the MMSE, 3MS, and a comprehensive neuropsychological battery at a hospital-based clinic in the northeastern U.S.

Results: As expected, the 3MS exhibited a greater range of scores (68-99) than the MMSE (24-30). Moreover, compared to age-corrected normative data, $45.7 \%$ of our MCI participants scored at the $16^{\text {th }}$ percentile or lower ( 1 SD below the mean) on the $3 \mathrm{MS}$, while only $13.1 \%$ of participants scored at the $16^{\text {th }}$ percentile or lower on the MMSE. Finally, we conducted partial correlations (controlling for age and education) between these two screening measures and composite metrics of the following neuropsychological domains: processing speed/ attention, language, visuospatial abilities, memory, and executive functioning. In every case, the 3MS explained more variance in neuropsychological performance than the MMSE. The largest difference occurred in the language composite, where the MMSE accounted for only $4.5 \%$ of the variance in language performance, while the $3 \mathrm{MS}$ accounted for $21.1 \%$ of variance. These results did not differ by MCI subtype.

Conclusions: The 3MS appears to be a significant improvement over the MMSE in an MCI population. It has a greater range of scores, it exhibits greater sensitivity to MCI, and it explains more variance in 
neuropsychological performance than the MMSE. While not a replacement for comprehensive neuropsychological examinations, the current data support the utility of the $3 \mathrm{MS}$ as an initial cognitive screening instrument. Future research should compare the performance of the 3MS against other cognitive screening tests such as the Montreal Cognitive Assessment.

Correspondence: Ryan Van Patten, B.S., Psychology, Saint Louis University, 1209 Covington Manor Lane, Apt 5, Saint Louis, MO 63125, United States.E-mail:rvanpatt@slu.edu

V.J. WASSERMAN, S. EMRANI, S. HIGGINS, K.D. GARRETT, R. SWENSON \& D.J. LIBON. Visual Serial List Learning in Mild Cognitive Impairment: An Analysis of Learning Curves and Errors of Commission.

Objective: To analyze visual serial list learning immediate free recall and commission errors in mild cognitive impairment (MCI).

Participants and Methods: Memory clinic patients were assessed with the Brief Visuospatial Memory Test-R (BVMT-R). Three groups were examined; patients with amnestic MCI (aMCI) and mixed/dysexecutive MCI (mx/dys MCI) using Jak/Bondi (2009) criteria; and non-MCI where comprehensive neuropsychological performance were generally above 1 sd. Groups (aMCI, $n=11 ; m x / d y s M C I, n=14$; non-MCI, $n=27$ ) did not differ on age $(M=77.2 \pm 6.0)$, education $(M=14.2 \pm 2.9)$, or MMSE $(\mathrm{M}=27.1 \pm 1.9)$.

Results: Using age-based norms mean scores on all trials for all three groups were $>-1$ sd. Examination of the immediate free recall trials with a $3 \times 3$ (group $\times$ test trials) repeated measures ANOVA yielded a significant effect for trials $(p<.001)$. A flat learning curve characterized non-MCI patients. Learning curves for both MCI groups demonstrated a negative slope (within group; $\mathrm{p}<.015$ ). A 3 x 3 (group $\mathrm{x}$ trial) repeated measures ANOVA for commission errors found that $\mathrm{mx} /$ dys patients generated more errors $(p<.001)$. A group $x$ trial interaction $(p<.047)$ found that non-MCI and $\mathrm{mx}$ /dys MCI patients made more errors on trial 3 than 1 ( $p<.008)$, while errors made by aMCI patients were unchanged as a function of trial.

Conclusions: Markedly reduced free recall performance on the BVMT-R was found in all three groups ( $>-1$ sd). A free recall negative slope coupled with increasingly commission errors as a function of test trials in non-MCI and $\mathrm{mx}$ /dys groups was noted. Interference effects mediated by dysexecutive impairment could underlie these profiles. Correspondence: Victor J. Wasserman, Psychology, Rowan University, 414 Chanticleer, Cherry Hill, NJ 08003, United States. E-mail: wasserman.victor@gmail.com

\section{A. WEAKLEY \& M. SCHMITTER-EDGECOMBE. Naturalistic Assessment of Task Interruption in Individuals with Mild Cognitive Impairment.}

Objective: Interruptions are ubiquitous in everyday life and require several cognitive processes working in tandem. In this study, we assessed the effects of an interruption on the performance of individuals with mild cognitive impairment (MCI) completing everyday tasks in a naturalistic apartment setting.

Participants and Methods: Thirty-two persons with MCI and 64 cognitively healthy older adults (HOA) completed two different sets of everyday activities, of which one received an interruption. One set of activities required participants to make a glass of lemonade and determine if sandwich bags needed replacing. The other set asked participants to make a bowl of lemonade and check the hydrogen peroxide expiration date. Each set of activities had 20 steps and equivalent opportunities for omission and substitution errors. The Participants also completed tests assessing cognitive constructs thought to be important in interruption recovery (i.e., retrospective memory, prospective memory, planning, working memory, executive function).

Results: As a consequence of an interruption MCI participants took longer to complete primary task demands, but did not make more omissions than during the no-interruption condition. In contrast, HOAs made more omissions, but their time on task was not affected. These findings suggest that MCI participants may have strategically prioritized step completion over time on task, whereas the opposite may be true for HOAs. Although MCI participants were able to complete task demands, they did make significantly more substitution errors when interrupted, potentially due to reduced executive control. The data also suggest that the more ecologically valid interruption task was more predictive of everyday functional status than the traditional neuropsychological measures.

Conclusions: Overall, a brief task interruption was detrimental to both MCI and HOAs in distinct ways and appeared to evoke speed-accuracy trade-off strategies to mitigate interrupting effects.

Correspondence: Alyssa Weakley, Psychiatry, University of California, Los Angeles, 10911 Rochester Apt 306, Los Angeles, WA 90024, United States.E-mail: aweakley@mednet.ucla.edu

K.C. HEWITT, C.M. MULLEN, J. LYLES \& C. MARKER. Application of Virtual Reality-Based Neuropsychological Assessments within Mild Cognitive Impairment.

Objective: To improve the ecological validity of neuropsychological measures, the field has developed various virtual reality-based neuropsychological assessments (VR-BNA) over the course of two decades. Neuropsychological assessment focuses on the evaluation of specific activities that are associated with observable behaviors; VR-BNA recreate a real environment and emphasize observational tasks. Empirical literature suggests VR-BNA are sensitive in detecting abnormal cognitive functioning to identify neurocognitive disorders. Thus, this current systematic review aimed to assess the application of these newly developed measures specifically within the mild cognitive impairment (MCI) population.

Participants and Methods: A search using GALILEO, which encompasses PsychINFO and PubMed, databases with the terms "virtual reality," "mild cognitive impairment," and "neuropsychological assessment" resulted in $\mathrm{N}=3,216$ total articles. After excluding irrelevant studies lacking virtual reality data and MCI, N=16 articles remained. Results: A review of relevant studies revealed expansion in the field of neuropsychology for VR-BNA when exploring the feasibility and validity for the diagnosis of MCI. Our analysis examined the application of VR-BNA for the diagnosis of MCI. Research demonstrated the use of VR-BNA provides insight on the performance of everyday functional tasks that allow for differentiating between age-associated cognitive decline and abnormal deterioration.

Conclusions: The results of this systematic review strongly reinforce the need for neuropsychology to expand and strengthen VR-BNA and utilize functional based assessments that are comparable to real-life experiences. Assessment of MCI should include measures that satisfy internal, external, and ecological validity. Future research should investigate the incremental validity of virtual reality measures in comparison to traditional paper-and-pencil assessments that may assist with earlier detection.

Correspondence: Kelsey C. Hewitt, Psy.D., Clinical Medical Psychology, Mercer University, 3695 Cumberland Blvd. Unit 1134, Atlanta, GA 30339, United States. E-mail: Kelsey.Caitlin.Hewitt@live.mercer.edu

\section{Dementia (Non-AD)}

\section{K.C. HEWITT \& D. LORING. A Neuropsychological Case Study of Dementia with Lewy Bodies.}

Objective: Dementia with Lewy bodies (DLB) is the second most common neurodegenerative disease after Alzheimer's disease. We present findings from a 77-year-old man referred from neurology after evaluation for fainting and cognitive difficulty. Based upon neuropsychological test findings combined with autonomic fluctuations, acting out dreams, and hallucinations, his impressions contributed to the early diagnosis of DLB. 
Participants and Methods: Following coronary bypass surgery (age 72 ), the patient developed dizziness, blood pressure fluctuation, and episodes of syncope. Cognitive difficulty involved memory, understanding complex tasks, and concentration, which started approximately one year prior to evaluation. Sleep disturbances, visual hallucinations, and occasional drooling were also reported. He obtained a MoCA $=25 / 30$ during his initial neurologic evaluation. MRI indicated normal age-related atrophy and hippocampal volume in the $20^{\text {th }}$ percentile.

Results: There was a meaningful discrepancy between verbal comprehension $(\mathrm{VCI}=110)$ and perceptual reasoning (PRI=84) abilities. Memory was generally intact for verbal information (AVLT; Logical Memory) and poor for visuospatial information (Visual Reproduction; Complex Figure). "Frontal lobe"/executive function performance was variable. The pattern of relatively preserved verbal memory and decreased higher order visual spatial constructional and problem solving skills are consistent with the expected neuropsychological profile associated with DLB.

Conclusions: DLB is typically associated with extrapyramidal motor symptoms, but this patient had no tremor and only mild cogwheel rigidity. Based upon the constellation of suggestive and supportive features, early diagnosis of DLB was made and his neurologist entered him in a clinical trial of intepirdine vs. placebo. Our case enriches the understanding of differential diagnosis with the onset of cognitive deficits relative to motor symptoms and emphasizes early diagnosis despite definite motor symptoms.

Correspondence: Kelsey C. Hewitt, Psy.D., Clinical Medical Psychology, Mercer University, 3695 Cumberland Blvd. Unit 1134, Atlanta, GA 30339,United States.E-mail: Kelsey.Caitlin.Hewitt@live.mercer.edu

\section{A.M. CARMICHAEL, Y. GLIKMANN-JOHNSTON, M. IRISH \&} J. STOUT. Autobiographical Memory in Huntington's Disease.

Objective: Huntington's disease (HD) is a progressive autosomal dominant neurodegenerative disorder characterised by motor, psychiatric and cognitive dysfunctions. Cognitive decline can be detected in group studies ten or more years before motor onset. Autobiographical memory, which refers to the memory of personally experienced events, has not been well-characterised in HD. The current study investigated episodic and semantic autobiographical memory recall across the lifespan in pre-manifest and early Huntington's disease.

Participants and Methods: Twenty-four participants genetically confirmed to have HD (pre-manifest and early HD) and 24 age-matched healthy control participants completed the Autobiographical Interview (Levine et al., 2002). Participants were required to recall a memory of an event from five different life periods. Interview details were scored according to a standard scoring protocol to determine the nature of information recalled (semantic, episodic) across free recall, general probe, and specific probe conditions.

Results: Participants with HD recalled significantly fewer episodic details, including happenings, locations, emotions, perceptions from a specific time and place. In addition, participants with HD recalled a lower proportion of episodic details and higher proportion of semantic details compared to control participants.

Conclusions: Our findings are evidence of a deficit in episodic autobiographical memory recall in $\mathrm{HD}$, with a relatively spared semantic autobiographical memory. These findings extend our knowledge of memory in $\mathrm{HD}$, and lend evidence to cognitive changes that occur in both manifest HD, as well as prior to clinical 'motor' diagnosis. Correspondence: Anna M. Carmichael, Monash University, 65 Bethanga St, Mt Eliza,VIC 3930,Australia.E-mail:anna.carmichael@ monash.edu
G. CHANEY, C. ONYIKE \& V. KAMATH. The Neuropsychological Profile of Abnormal Feeding Behavior in Behavioral Variant Frontotemporal Dementia.

Objective: Abnormal eating represents a core and distinguishing diagnostic feature of behavioral variant frontotemporal dementia (bvFTD), differentiating it from other neurodegenerative disorders and late-life psychiatric conditions. While early work linked hyperphagia and hyperorality to frontal dysfunction, the observation of increased sweet preferences and food foraging indicate that bvFTD is accompanied by fundamental dietary changes associated with hypothalamic and insular atrophy. In the current study, we examined whether neuropsychological deficits contribute to abnormal eating in bvFTD.

Participants and Methods: We analyzed first-visit eating and neuropsychological data from 7 Centers in the National Alzheimer's Coordinating Center database (December 2016 data freeze; $N=330$ ). Subjects were assigned to four groups based on informant-reported appetite changes (none vs. moderate/severe) and NHLBI body mass index category (lean vs. overweight/obese). We used general linear models to compare domains of attention, processing speed, language, memory, and executive functioning between these patient groups.

Results: Neuropsychological differences between lean and overweight/ obese bvFTD patients with and without disturbed eating were not statistically significant (all $p$ 's > .44).

Conclusions: These findings suggest that cognitive dysfunction does not explain abnormal eating behavior in bvFTD. Future clarification studies with finer definitions of abnormal feeding, and more comprehensive neuropsychological profiling and physiological/neuroimaging indices are warranted.

Correspondence: Grace-Anna Chaney, Master of Arts, Psychiatry and Behavioral Sciences, The Johns Hopkins University, 600 N. Wolfe Street, Meyer 218, Baltimore, MD 21287-7218, United States. E-mail: gchaney2@jhmi.edu

C. DOMINGUEZ-VIVERO, L. WU, S. LEE, G. CHERAN, M. MANOOCHEHRI, S. CINES, B. RIZVI, M. BUDGE, A. NARKHEDE, A.M. BRICKMAN, E. FALLON, T. LYNCH, J. HEIDEBRINK, H. PAULSON, E. HUEY \& S. COSENTINO. Structural Brain Changes in Preclinical Tauopathic BehavioralVariant Frontotemporal Dementia (bvFTD).

Objective: This study sought to characterize volumetric brain changes evident in preclinical behavioral-variant Frontotemporal Dementia (bvFTD). Characterizing changes at the earliest stages of disease can improve conceptualization of disease progression, and identify biomarkers useful for early diagnoses.

Participants and Methods: 56 subjects from five families carrying mutations in the Microtubule-Associated Protein Tau (MAPT) gene underwent comprehensive neurological and neuropsychological assessment, and Magnetic Resonance Imaging (MRI). Global and regional brain volume were derived from a T1-weighted MPRAGE sequence using Freesurfer. Clinical Dementia Rating (CDR) scores were assigned by the evaluating neurologist, and mutation status determined by genetic testing of DNA extracted from blood samples. 12 subjects were found to be mutation carriers, of which 6 were presymptomatic $(\mathrm{CDR}=0)$, and 6 were showing questionable early symptoms not meeting bvFTD diagnostic criteria and received $\mathrm{CDR}=0.5$.

Results: To enable unbiased assessment of global patterns of atrophy, values were compared without defining a priori regions of interest (ROIs). Carriers had significantly smaller volumes in 32 ROIs after multiple comparison corrections. To explore whether brain volume can distinguish carriers from non-carriers, we conducted a multivariate elastic-net regression with 500 iterations of bootstrapping. Five ROIs (amygdala, temporal pole, rostral anterior and posterior cingulate, and lingual gyrus) were consistently selected.

Conclusions: Relative to familial controls, MAPT carriers had decreased volume in the amygdala, temporal pole, rostral anterior cingulate and posterior cingulate cortices, and in the lingual gyrus. These findings in a sample of preclinical mutation carriers suggest that structural brain 
changes are detectable before clear clinical changes manifest in tauopathic bvFTD. Here, we explore the implications of specific findings. Correspondence: Gayathri Cheran, Bachelor of Science, Neurology, Columbia University Medical Center, 630 West 168th Street P\&S Box 16, New York, NY 10032, United States. E-mail: gc2646@cumc. columbia.edu

M. FRANCZAK, D.S. SABSEVITZ, S. SWANSON, E. GRANADILLO, A. KLEIN \& J. ULMER. Early Episodic Memory Loss in Semantic Dementia: A Phenotypic Variant?

Objective: Semantic dementia (SD) is a variant of primary progressive aphasia characterized by progressive loss of semantic knowledge. The core features include impaired confrontation naming and single-word comprehension. Speech remains fluent and grammatically correct and repetition is spared. Episodic memory is spared during the early stages of the disease, unlike in $\mathrm{AD}$ where memory loss is an early defining feature. The current case study examines a patient with early memory loss suggesive of AD but imaging biomarkers supporting a diagnosis of SD.

Participants and Methods: Patient PS is a 79-year-old female with a 2 -year history of forgetfulness for recent events and naming problems. Based on her clinical history, neurologic exam and cognitive screen she was diagnosed with possible AD by Neurology. Neuropsychological screening showed prominent impairments in new learning and delayed recall without benefit from recognition cueing, suggesting an encoding/ storage-based memory deficit, impaired object naming and semantic fluency, and borderline clock drawing, all thought consistent with AD. Results: Striking atrophy was seen on MRI within the anterior left temporal lobe with knifelike gyri. Volumetric MRI demonstrated volumes loss in the left anterior temporal lobe, anterior hippocampus (10th\%) and amygdala (14th\% compared to age matched controls). Control data was based on ADNI. Arterial spin labeling was down in the anterior, ventral and lateral temporal lobe, amygdala, hippocampus, all on the left. DTI showed increased diffusivity within the anterior left temporal lobe. Imaging was thought more consistent with SD than AD. Given the conflicting data amyloid PET scan was done, which showed no amyloid plaques, arguing against AD.

Conclusions: This case illustrates the value of integrating MRI biomarkers with neuropsychological data for elucidating types of dementia. The current case also suggests a potentially distinct phenotype of SD presenting with early episodic memory impairment.
Correspondence: David S. Sabsevitz, Ph.D., Neurology, Medical College of Wisconsin, 9200 W Wisconsin Ave, Milwaukee, WI 53226, United States.E-mail: david.sabsevitz@mcw.edu

K. WESBECHER \& L. PALMER. Postoperative Cognitive Dysfunction Following Total Knee Arthroplasty: A Case Study.

Objective: Cognitive impairment following a non-cardiac surgical procedure, termed postoperative cognitive dysfunction (POCD), occurs in approximately $41 \%$ of adults over the age of 60 (Monk et al., 2008). Although procedures often have the potential to dramatically improve one's quality of life, research suggests that declines in executive functioning and memory can occur. Here, we present the pre- and postoperative neuropsychological profile of an 85 year-old man who exhibited cognitive decline after total knee arthroplasty (TKA).

Participants and Methods: The patient was an 85 year-old, righthanded Caucasian man with a history of hypertension, arterial fibrillation and coronary artery disease. Testing occurred 5 years prior to TKA and 3 months after. The neuropsychological evaluation included review of medical records, clinical interview and neuropsychological testing consisting of the Wechsler Abbreviated Scales of Intelligence-II (WASI-II), the Dementia Rating Scale, Second Edition (DRS-2) as well as the Geriatric Depression Scale (GDS).

Results: Preoperative testing revealed high average premorbid intellectual ability and functioning within or exceeding normal limits across all neurocognitive domains. There was no indication of depression. Of note, immediately before surgery, no changes in cognition were reported. Postoperative testing was most notable for impairments in executive functioning and processing speed. Memory testing was variable and but generally characterized by poor encoding and retrieval.

Conclusions: This case expands findings by showcasing the potentially deleterious effects of orthopedic surgery on executive functioning in older adults. Previous research has suggested that preoperative neuroimaging evidence of white matter hyperintensities may account for some of the executive function decline observed (Price et al., 2014). Therefore, the need for baseline neuroimaging in patients with vascular risk factors will be discussed.

Correspondence: Kristen Wesbecher, MS, Professional Psychology and Family Therapy, Seton Hall University, 400 S Orange Avenue, South Orange, NJ 07079, United States. E-mail: kwesbecher@phhp.ufl.edu

\section{THURSDAY AFTERNOON, FEBRUARY 15, 2018}

\section{Lunch (On Own) \\ 1:15-2:15 p.m.}

\section{Benton / Mid-Career Awardee Presentation: Objects and Actions in the Healthy and Damaged Brain}

\section{Award Recipient: Laurel J. Buxbaum}

$$
\text { 2:15-3:15 p.m. }
$$

\section{L.J. BUXBAUM. Tools in the Mind: Objects and Actions in the Healthy and Damaged Brain.}

Our ability to functionally use manipulative objects rests on a distributed left hemisphere network. Lesions to portions of this network result in a type of limb apraxia characterized by deficits in object action knowledge, while lesions elsewhere in the network are associated with deficits in action selection. Fueled in part by these findings, our laboratory has developed and (over two decades, elaborated) a cognitive neuroanatomical model of the mechanisms and architecture of a distributed two-stream network critical to the representation and selection of object-related actions. Called the "Two Action Systems Plus (2AS+)" framework, the model posits a complementary role for stored object manipulation knowledge ("action semantics") and online computations, and specifies the neurocognitive substrates of task-relevant action selection. This presentation will provide background on current controversies in the domain of object use and semantic manipulation knowledge, explain the 2AS+ architecture in the context of prominent two stream models in the language domain, and provide an overview of how the $2 \mathrm{AS}+$ framework helps us to understand both healthy object use and apraxia.

Correspondence: Laurel J. Buxbaum, Moss Rehabilitation Research Institute, 50 Township Line Road, Elkins Park, PA 19027, United States.E-mail:lbuxbaum@einstein.edu 


\section{Symposium 4. Technological advances in pediatric neuropsychological assessment and intervention}

\author{
Chair \& Presenter: Danielle Shapiro
}

Discussant: H. Gerry Taylor

\author{
Presenters: Seth Warschausky, Shari L. Wade, \\ Andrew Hurwitz
}

$$
\text { 2:15-3:45 p.m. }
$$

D. SHAPIRO, S. WARSCHAUSKY, S.L. WADE, A. HURWITZ \& H. TAYLOR. Technological advances in pediatric neuropsychological assessment and intervention.

Rapid developments in technology have the potential to transform research and clinical care in the field of neuropsychology. This symposium is comprised of a multidisciplinary group of psychologists and policy researchers employing technologies in novel ways to address important questions in pediatric neuropsychology. The presentations will illustrate the ways in which technological advances have expanded the neuropsychological research questions that can be asked, the populations that can be accessed, and the clinical tools that can be employed. First, Dr. Warschausky discusses a set of studies on technological strategies to increase the accessibility of assessments for individuals with severe motor and speech impairments. The second talk (Shapiro and Johnson) considers the use of technology as an archival record of past behavior, exploring social media analysis as a naturalistic source of preand post-morbid neuropsychological data for individuals with acquired brain injuries. Third, Dr. Wade discusses technological strategies that increase the reach of intervention services. She presents findings from several randomized controlled trials suggesting that telehealth interventions may be beneficial for youth with moderate to severe brain injuries. Finally, the fourth talk (Hurwitz, Borradaile, and Reeves) presents qualitative data examining the experiences of schools that implemented $g$ force sensor measurement programs, highlighting how this measurement program influenced both concussion prevention and management at the schools and student athletes' behavior. Their findings suggest a role for neuropsychology in injury prevention and also raise concerns about the ecological validity of using sensors as measurement devices. Discussion led by Dr. Taylor will focus on the implications of these novel methods in particular, and the trend toward a more connected and technologically-oriented clinical environment in general, for research, policy, and clinical care.

Correspondence: Danielle Shapiro, PhD, Physical Medicine and Rehabilitation, University of Michigan, 325 E. Eisenhower Parkway, Suite 300, Ann Arbor, MI 48108, United States. E-mail: dnshap@med. umich.edu

\section{S. WARSCHAUSKY. Challenges in utilizing technology for accessible testing.}

Objective: To describe challenges, solutions and future directions in the programmatic study of technology-assisted accessible cognitive testing for persons with significant motor and speech impairments.

Participants and Methods: Participants in a series of studies (Alcaide-Aguirre et al., in press; Huggins et al., 2015; Kaufman et al., 2014; Warschausky et al., 2012) were children and young adults, ages 6-27, with cerebral palsy, and typically developing peers. Participants were administered standard and accommodated versions of forcedchoice format tests. Accommodated test responses were obtained via assistive technologies (ATs) that included Pressure Switch, head mouse, or Brain-computer Interface (BCI).

Results: Measurement stability for accommodated tests in which the stimuli were presented in dichotomous or quadrant forced-choice array was sufficient for the three types of AT. Measurement stability for a forced-choice format with a more complex array such as the Raven's CPM was variable across samples. In the course of study, challenges have included pretesting of choice-making capabilities, identifying the optimal AT interface for the individual and developing effective practice trials. In addition, investigators have had to address the high attentional demands of BCI-accommodated testing, provide ways to confirm an intended response and address special issues in the assessment of processing speed. Finally, there is concern with the differing cognitive loads of AT interfaces.

Conclusions: The results from this series of studies provide evidence that technological approaches to specific types of test administration, using Pressure Switch, Headmouse®, and Brain-computer Interface are feasible and reliable. There are important challenges to successful development and implementation of these approaches, but they have the potential to greatly increase the accessibility of neuropsychological assessments for persons with significant speech and motor impairments. Correspondence: Seth Warschausky, Ph.D., Physical Medicine \& Rehabilitation, University of Michigan, 325 E. Eisenhower Pkwy, Suite 300, Ann Arbor, MI 48108, United States.E-mail: sethaw@umich.edu

D. SHAPIRO \& A. JOHNSON. \#BrainInjury: Using social media analysis as a naturalistic measure of change associated with acquired brain injury.

Objective: This study uses social media analysis to address a critical problem in neuropsychological assessment with individuals with acquired brain injuries: it is very difficult to obtain valid premorbid data on psychological, cognitive, and social functioning. We use archives of participants' social media activity as a naturalistic measure of pre- and post-injury functioning.

Participants and Methods: Participants include 20 adolescents and young adults aged 15-23 with a history of acquired brain injury. The majority of the sample $(\mathrm{N}=14)$ sustained a moderate to severe traumatic brain injury and the remainder were diagnosed with hypoxic brain injury or encephalitis with discrete onset. Archives of social media activity, spanning from the date the accounts were created until the date of participation, were downloaded from Twitter and Facebook platforms. Text posts from before and after the injury were coded using a natural language processing program (LIWC) that assesses valence, linguistic complexity, and content of text.

Results: We demonstrate statistically significant step-wise changes at the time of brain injury in the linguistic complexity and content of social media posts. For some variables, slopes also differed pre- and post-injury, indicating the trajectory of recovery of these functions.

Conclusions: Social media analysis is a feasible and informative method for measuring changes related to brain injury and, more tentatively, charting the trajectory of recovery. This method has the clear advantage over traditional approaches of providing face-valid indicators of pre-morbid functioning and therefore may be a useful supplement to traditional, cross-sectional approaches to research and clinical assessments in this population.

Correspondence: Danielle Shapiro, PhD, Physical Medicine and Rehabilitation, University of Michigan, 325 E. Eisenhower Parkway, Suite 300, Ann Arbor, MI 48108, United States. E-mail: dnshap@med. umich.edu

S.L. WADE. Telehealth interventions for youth with acquired brain injury: Lessons learned.

Objective: This presentation distills findings from e-health problem-solving interventions with youth with traumatic brain injuries (TBI) by reviewing evidence across trials regarding efficacy, which individuals benefit, and advantages and challenges from patient, family and therapist perspectives.

Participants and Methods: Participants include 365 youth with moderate to severe TBI, ages 6-18, who participated in one of four randomized clinical trials of Online Problem Solving (OPS). Participants were enrolled within 24 months of their injury and randomly assigned to OPS or an internet resource comparison group (IRC). OPS consisted of 7-14 sessions delivered over 6 months of problem-solving, 
communication, and self-regulation training and included self-guided online modules and subsequent Skype sessions with a trained therapist. With the exception of a larger RCT $(\mathbf{n}=153)$ with a teen-only treatment arm, OPS was delivered to the family.

Results: Across trials, both children and parents rated the OPS interventions as easy to use and helpful in addressing TBI-related challenges. Improvements in child behavior and executive functioning in OPS versus IRC were greater in older children and adolescents as well as those with lower SES. The teen-only intervention was not more effective than the IRC suggesting the importance of family involvement. Therapists indicated that intellectual and attention impairments may reduce the effectiveness of telehealth interventions in this population relative to face to face treatments.

Conclusions: This series of studies provides evidence of the feasibility and efficacy of telehealth interventions for youth with TBI while raising some important considerations regarding implementation. Younger children and those with greater cognitive impairments may be more difficult to engage and may derive less benefit. Family involvement may be particularly beneficial in promoting successful implementation of skills into their everyday lives.

Correspondence: Shari L. Wade, Ph.D., Pediatrics, Cincinnati Children's Hospital Medical Center, 3333 Burnet Avenue, MLC 4009, Cincinnati, OH 45229-3039, United States.E-mail: shari.wade@cchmc.org

A. HURWITZ, K. BORRADAILE \& W. REEVES. Sensors as a social intervention: Understanding how schools utilize head impact sensors.

Objective: Head impact sensors are gaining popularity as a tool for schools to monitor the $g$ forces experienced by an athlete during contact in sport. The purpose of this qualitative investigation was to understand how schools and students who use such sensor technology describe their experiences and how these experiences affected head injury policy and practice.

Participants and Methods: Two schools were selected for the investigation, representing distinct student demographics. Twelve semi-structured interviews were conducted with coaches, parents, and administrators. Two focus groups were conducted with approximately eight to ten student athletes in each group. The typological framework, described by Hatch (2002) was used for qualitative analysis.

Results: Study results revealed three unique insights. First, both schools set a $\mathrm{g}$ force threshold for concussion, despite schools being instructed that these sensors are not diagnostic devices for concussion. Second, the investigation highlighted the logistical difficulties that emerge when schools implement a sensor program. Third, insights with regard to athlete behavior showed athletes who wore the sensor consciously decided to modify their own behavior such that when executing contact they did so using less deleterious techniques. Conclusions: Caution regarding the limits of these sensors are addressed; namely, these devices are not clinical diagnostics, which needs to be made transparent to stakeholders, including clinical neuropsychologists working with a concussed population. Useful guidelines for how schools can address the logistical hurdles when implementing a sensor program are provided. Finally, the notion that sensors are passive devices recording phenomena is challenged as evidence suggests these devices can modify participant behavior. Together, these insights provide suggestions for how practitioners can consider ways to help mitigate concussion risk in youth athletes

Correspondence: Andrew Hurwitz. E-mail: AHurwitz@mathematicampr.com

\section{H. TAYLOR. Discussion of issues raised and future directions.}

The technology applications illustrated in this symposium have the potential to dramatically shift the methods we use in neuropsychological research and practice. Careful analysis of these methods is critical in determining their benefits and limitations. The discussion will highlight the major benefits of these technologies to each subject area, including more sensitive assessment of individuals with neuromotor impairments, increased rigor in assessment of preinjury status and recovery of function in children with TBI, increased access to interventions following TBI, and assessment of blunt force in monitoring and treating concussion. Challenges inherent in the use of these and other technological advances in neuropsychology will also be addressed, and directions for future research considered. Because these newer technologies are not only here to stay but will rapidly evolve, it is important to critically evaluate their potential for broader-scale applications, cost-benefit ratio, and ethical and policy implications.

Correspondence: H. Gerry Taylor, Department of Pediatrics, Case Western Reserve University and Rainbow Babies \&. Children's Hospital, University Hospitals Cleveland Medical Center, W.O. Walker Building Suite 3150, 10524 Euclid Avenue, Cleveland, OH 44106, United States. E-mail:hgt2@case.edu

\section{Symposium 5. The Present and Future of Technology in Neuropsychological Assessment: What is Feasible (and Fundable) Today, Tomorrow and in Years to Come}

Chair: Melissa Lamar

Discussants: Richard Gershon, David Libon, Daniel A. Nation, Dana Penney, Jonathan King, Wendy Nilsen, Nina Silverberg, Molly Wagster

\section{Presenters: Catherine C. Price, Dorene Rentz, Rhoda Au}

2:15-3:45 p.m.

M. LAMAR. The Present and Future of Technology in Neuropsychological Assessment: What is Feasible (and Fundable) Today, Tomorrow and in Years to Come.

This symposium will discuss ways to bridge present-day neuropsychological assessment that relies heavily on paper and pencil techniques with current, evolving, and future advances in technology. Formal talks will highlight three ways to develop and integrate technology into neuropsychological assessment: 1-to facilitate assessments in fast-paced environments using select techniques for individual tests developed by clinicians, for clinicians; 2-through the use of a comprehensive set of measurements from the convenience of an iPad developed in conjunction with NIH; and 3-capturing real time, real world activity through 'smart home' technologies with international partners. More specifically, Dr. Catherine Price will discuss the utility of smart pen digital technology in fast-paced hospital settings where time for neuropsychological assessments may be limited but the need for highly sensitive data is not. Dr. Dorene Rentz will discuss her work developing, validating and implementing the iPad based NIH Toolbox (NIHTB) and Computerized Cognitive Composite (C3) in the clinic and home environments. Meanwhile, Dr. Rhoda Au will take us out of the clinic and far from the laboratory as she presents data on evolving advances in e-Home technologies that allow for moment-to-moment assessments of everyday functions through wearable activity monitors, clothes and other in-development assessment devices. During our Round Table Discussions, we will first hear from Clinical and Research Neuropsychologists who have been developing and using these and other methods of technological neuropsychological assessments. Then, stakeholders from research and government including Program Directors and Branch Chiefs across federally funded agencies will discuss future development of, strategic plans for and funding priorities surrounding the use of technology in clinical trials as well as 'bench-based' research endeavors. Audience participation in both Round Table Discussions will be encouraged. 
Correspondence: Melissa Lamar, PhD, Rush Alzheimer's Disease Center, Rush University Medical Center, 600 S Paulina St, Chicago, IL 60612, United States.E-mail:Melissa_Lamar@rush.edu

\section{C.C. PRICE, L. HIZEL, S. AMINI, D.J. LIBON, D. PENNEY \& R. DAVIS. Digital Pen Technology.}

Smart pen digital technology is being applied to rapid paced settings such as pre-surgical anesthesia centers, but the value of this technology needs investigation. For this presentation, Dr. Price will present data from two separate federally funded investigations assessing cognitive change over time with digital pen technology and clock drawing for older adults electing major surgery. The first half of the presentation will compare standard clock drawing scoring techniques (Montreal Cognitive Assessment, Mini-Cog, Libon error based criteria) to digital clock drawing features for older adults electing cardiothoracic $(n=280)$, abdominal $(\mathrm{n}=208)$, and orthopedic patients $(\mathrm{n}=180)$, and non-surgery peers $(n=120)$. The second half of the presentation will highlight three variables from digital clock drawing (post clock face latency, inter-digit latency, pre-second hand latency), their association with neuropsychological measures of memory and planning, and how patients electing total knee arthroplasty (surgery $n=65$, non-surgery $n=60$ ) change over the course of a year on digital clock drawing relative to other more standard neuropsychological measures. The significance of digital, relative to standard neuropsychological screening assessments in pre-operative settings will be discussed. R01NR014181(CP), NSF13-543(RD/CP); R01AG055337(CP)

Correspondence: Catherine C. Price, Ph.D., Clinical and Health Psychology, University of Florida, PO Box 100165, Gainesville, FL 32610, United States.E-mail: cep23@PHHP.UFL.EDU

D.M. RENTZ, K.V. PAPP, I. ORLOVSKY, R. SPERLING, D. PENNEY \& R. DAVIS. iPad and Digital Based Assessments in Clinic and Home Environments.

As prevention trials for Alzheimer's disease (AD) move into asymptomatic populations, identifying older individuals who manifest the earliest cognitive signs of AD is critical. Computerized testing provides a potential method for identifying those individuals with subtle cognitive impairment that may not be detectable using conventional paper and pencil tests. However, it is unclear whether computerized tasks can completely substitute for the diagnostic sensitivity of conventional neuropsychological measures or whether computerized tests can provide complementary or unique information. The first half of the presentation will highlight the validity and sensitivity of iPad assessments using Cogstate (C3) and NIH Toolbox Cognition Battery (NIHTB-CB) against conventional paper and pencil neuropsychological tests and the feasibility of home-based assessment. The second half of the talk will address the sensitivity of digital technology in identifying amyloid and tau burden at the stage of Preclinical Alzheimer's disease in a sample of 70 individuals from the Harvard Aging Brain Study.

Correspondence: Dorene M. Rentz, PsyD, Neurology, Harvard Medical School, 221 Longwood Avenue, Boston, MA 02115, United States. E-mail:DRENTZ@PARTNERS.ORG

R. AU, H. LIN, M. KOHLI, Y. ZHANG, A. WONG, J. YANG, N. AN \& V. MOK. e-Cog and e-Home Technologies: Wearable Activity Monitoring and Assessment Technology.

Despite the depth and breadth of Alzheimer's disease research, effective methods for treatment remain elusive. The emphasis on medical intervention has led to a more recent emphasis on identifying biomarkers decades before the threshold for clinical diagnosis is met. Predominate are studies of beta amyloid $(\mathrm{Ab})$ detected by PET imaging or measured from CSF. Aside from the intrusiveness and expense of these methods leading to biased studies, nearly 70 percent of cognitively normal individuals aged 70-79 are free of significant amyloid on PET imaging. Further, neuropathological studies report that tau, not amyloid is significantly related to cognition and that some people are clinically asymptomatic even when these hallmark pathologies are present in the brain. Given the insidious onset process coupled with reports suggesting that delaying onset of symptoms by five years can cut risk for disease by nearly $50 \%$, it is surprising that cognition is viewed as an outcome rather than a potential predictor of disease. Technology is changing what we can do and how we can do it, including preclinical AD symptoms. Digital technologies have translated into a plethora of wearable sensors and smart home devices that offer a solution for monitoring and detecting cognition well before changes meet the threshold for clinical diagnosis. Through a multi-site effort, we have launched the development of an e-Cognitive Health platform. The long-term significance of this effort could potentially lead to a transformative shift from the primary medical intervention model of "personalized medicine" to a more comprehensive focus on "personalized brain health," and reduce the likelihood of disease altogether.

Correspondence: Rhoda Au, PhD, Anatomy \& Neurobiology, Neurology and Epidemiology, Boston University Schools of Medicine and Public Health, 72 E Concord Street, B6, Boston, MA 02118, United States. E-mail: rhodaau@bu.edu

\section{R. GERSHON, D.J. LIBON, D.A. NATION \& D. PENNEY. Round} Table Discussion 1: Clinical \& Research Neuropsychologists.

Clinician and research neuropsychologists face increasing pressure to remain relevant in today's world of science; this will only continue as we move into the future of advanced technologies and blood biomarkers for interventions and investigations of lifespan development and degeneration. The first of our two Round Table Discussions will focus on the thought processes that initiate technological applications in neuropsychological assessments, the foundation work in validation and application needed to ensure these applications remain true to the field and the pitfalls that often go hand-in-hand with success. For example, Dr. David Libon, Dr. Dana Penney, and Dr. Daniel Nation will describe their experience incorporating digital technology into their work and/ or the larger corpus of formal neuropsychological tests available for free or for purchase. A brief discussion of taking technology to market will also ensue. In addition, Dr. Richard Gershon will provide his expertise on present and future advances for the use of technology for increasing the impact and reach of psychometrically robust health measurements as well as his experience working with NIH and other stakeholders to conduct with work. Moderator Dr. Melissa Lamar will press the panel as well as the audience to think about ways to extract granulated data from existing paper files to ensure that the wealth of information currently and previously collected - as well as neuropsychologist with less access to digitized tools - will continue to contribute to our knowledge of brain-behavior relationships. Thus, pitfalls and concerns as well as advice and methods sharing will be prominently on display during this portion of the symposium and the audience will be invited to share their views and experience with developing and using technology in neuropsychological assessment.

Correspondence: Richard Gershon, PhD, Medical Social Sciences, Northwestern University, 6545 North LeMai Avenue, Lincolnwood, IL 60712, United States. E-mail: gershon@northwestern.edu

\section{J. KING, W. NILSEN, N. SILVERBERG \& M. WAGSTER. Round Table Discussion 2: NIA/NIH and NSF Stakeholders.}

Funding stakeholders will discuss the current, evolving and in-development platforms for incorporating technology into neuropsychological assessment; both as it relates to the work presented by our invited speakers but also as it relates to the federal level and the strategic plans of the agencies they represent. The National Science Foundation (NSF) perspectives and initiatives will be represented by Program Director, Dr. Wendy Nilsen while the National Institute on Aging, National Institutes of Health (NIA/NIH) will be represented by Program Directors Dr. Jonathan King (Division of Behavioral and Social Research) and Dr. Nina Silverberg (Division of Neuroscience) and the Chief of the Behavioral \& Systems Neuroscience Branch Dr. Molly Wagster. All these individuals 
have been integrally involved in advancing the use of technology in assessments, cognitive and otherwise to allow for larger scale epidemiological studies to collect important behavioral data and for investigators to amass information on comparable datasets across the lifespan. Ongoing and upcoming initiatives including Program Announcements and Requests for Applications will be discussed as well as more longrange plans for future work in this area.

Correspondence: Jonathan King, 31 Center Drive, MSC 2292, Bethesda, MD 20892,United States.E-mail:kingjo@mail.nih.go

\section{Paper Session 4. Concussion and Traumatic Brain Injury}

\section{Moderator: Nyaz Didehbani}

\section{2:15-3:45 p.m.}

S.L. WADE, E. KAIZAR, H. ZANG, H. TAYLOR, B. KUROWSKI, K.O. YEATES, M. NARAD \& N. ZHANG. Behavior Problems following Childhood TBI: The Role of Sex, Age, and Time Since Injury.

Objective: To understand differences in the nature of behavioral challenges following pediatric traumatic brain injury (TBI) as a function of age, sex and time since injury, we examined the Child Behavior Checklist (CBCL) profiles of 600 children with moderate to severe TBI.

Participants and Methods: Participants included 600 caregivers of children ages $3-18$ years with moderate to severe TBI who were enrolled in randomized controlled trials of family-focused interventions. Prior to trial initiation, parents completed the CBCL.

Results: Approximately 30\% of parents rated their child as having borderline or clinically significant externalizing behaviors, and this did not differ by age. Internalizing symptoms were uncommon in children $<6$ years of age, whereas $1 / 3$ of children 6 and older were rated as having borderline or clinically significant levels of internalizing problems. Affective Problems and ADHD were also more prevalent in children $>6$ years. Although the rates of many problems were highest between 6-12 months post injury, rates of ADHD, Rule Breaking and Somatic Symptoms were highest among those more than 12 months post injury. For males, the odds of having borderline or clinically significant levels of deficits in the domains of conduct, activities, aggressive behavior, social problems subscales $(p<.05)$ were significantly lower in older participants; female participants had significantly higher odds of withdrawn/depression with increasing age $(\mathrm{p}<.05)$. Analyses revealed a curvilinear effect of time since injury on attention problems and school scales with the highest symptoms $2-4$ years post injury.

Conclusions: Behavioral impairments are common with greatest prevalence during the subacute phase of recovery, although some problems peak several years post injury. Adolescent girls may be at greater risk for affective problems. Findings suggest age and sex differences in post-TBI behavioral problems and provide impetus for timely screening and treatment.

Correspondence: Shari L. Wade, Ph.D., Pediatrics, Cincinnati Children's Hospital Medical Center, 3333 Burnet Avenue, MLC 4009, Cincinnati, OH 45229-3039, United States. E-mail: shari.wade@cchmc.org.

E.M. SCHORR, K. KOWALSKI, A. BACEVICE, H. TAYLOR, B. BANGERT, E.D. BIGLER, D. COHEN, L. MIHALOV, N. ZUMBERGE \& K.O. YEATES. The Relationship Between PreInjury Internalizing Symptoms and Post-Concussive Symptoms in Children with Mild Traumatic Brain Injury and Orthopedic Injury. Objective: This study examined the relationship of type of injury and pre-injury internalizing symptoms to post-concussive symptoms (PCS), acutely and at three months post-injury, in children with mild traumatic brain injury (mTBI) or orthopedic injury (OI).
Participants and Methods: 95 children with mTBI and 55 with OI 8 through 15 years of age were recruited from the Emergency Departments of two Ohio children's hospitals at the time of injury and assessed approximately ten days and three months post injury. Parents rated children's pre-injury behavioural adjustment retrospectively using the BASC-2 and cognitive and somatic PCS using the Health and Behaviour Inventory (HBI).

Results: After controlling for pre injury PCS, pre-injury internalizing symptoms and injury type were significant predictors of both somatic and cognitive PCS post acutely. However, the groups did not differ significantly in the association between internalizing symptoms and PCS. Although the injury groups did not differ in PCS at three months, pre-injury internalizing symptoms continued to be significantly related to both somatic and cognitive PCS at that follow-up.

Conclusions: Children with mTBI had higher parent-reported PCS than children with OI post acutely, but not at three months. Children with more pre-injury internalizing symptoms had more PCS both post acutely and at three months, regardless of injury type. Pre-injury internalizing symptoms did not moderate the effect of mTBI on PCS at either time. Consistent with previous research, the effect of mTBI on PCS appears to dissipate over time. The findings also raise the possibility that children with internalizing problems may be more prone to reporting PCS after a traumatic injury.

Correspondence: Emily M. Schorr, MSc, Psychology, University of Calgary, Alberta Children's Hospital 2888 Shaganappi Trail NW, Calgary, AB T3B 6A8, Canada. E-mail: emily.schorr1@ucalgary.ca

C. MILLER, B.G. ZUCCATO, A. SCAVONE, L.A. ERDODI, B.M. MERKER \& C. ABEARE. Detecting ADHD symptoms in young athletes using the ImPACT.

Objective: Attention-deficit/Hyperactivity Disorder (ADHD) is a common childhood psychiatric disorder. Parents often cope with child overactivity by enrolling them in sports. However, young athletes with ADHD are at higher risk for concussion, and their ADHD symptoms may subsequently interfere with recovery. Furthermore, the presence of ADHD may complicate the tracking of recovery. Because not all individuals with ADHD are diagnosed, a subset of athletes likely have undiagnosed ADHD, increasing their risk of concussion and complicated recovery. We examined the utility of ImPACT, a computerized battery commonly used in concussion management, for identifying individuals with self-reported ADHD.

Participants and Methods: In a large sample of youth athletes $(\mathrm{N}=7440 ; 50.1 \%$ female; mean age: 14.83 years) collected between 2010 and 2016 at a Midwestern urban medical center, 10.9\% reported a history of ADHD. Age, gender, visual-motor speed, reaction time, impulse control, and cognitive symptoms were entered into a logistic regression with previous $\mathrm{ADHD}$ diagnosis as the dependent variable.

Results: All variables except reaction time were significant predictors of ADHD status $(p<.001)$. A conservative cut-off $(>0.25)$ had low sensitivity (.15), but high specificity (.97). A more liberal cut-off $(>0.15)$ improved sensitivity (.39) at the expense of specificity (.86).

Conclusions: Acknowledging the limitations of using a dataset with uncertain diagnostic accuracy (ADHD status was determined based on self-report), our results indicate that the ImPACT has potential as a quick screening tool to identify young athletes with previously undiagnosed ADHD who might benefit from a comprehensive ADHD assessment prior to sports participation. Clinicians working with injured young athletes who use the ImPACT to monitor recovery should also be aware that the ImPACT is sensitive to both concussion and ADHD svmptoms and therefore, and therefore clinicians should take these findings into consideration when assessing youth diagnosed with ADHD. Correspondence: Carlin Miller, PhD, Psychology, University of Windsor, 401 Sunset,Windsor, ON N9B3P4, Canada.E-mail: cjmiller@uwindsor. $c a$ 
C.A. BUSSELL \& B.E. GAVETT. Effects of Media Sensationalization on Cognitive Performance and Post Concussive Symptoms.

Objective: The current study aims to examine if media that sensationalizes mild traumatic brain injury (mTBI) has a negative impact on cognitive functioning and persistent mTBI symptoms. More specifically, this study investigated if, as hypothesized, participants with a history of mTBI perform less well on a cognitive assessment and indicate more severe post-concussion symptoms after being exposed to a sensationalized media clip about mTBI as compared to a non-sensationalized media clip.

Participants and Methods: One-hundred and two participants (MAge $=37.15 ; \mathrm{SD}=22.61$ ) with a history of post-acute mTBI, recruited through a community research registry and an undergraduate recruitment system, were included in this study. Participants were assessed with a measure of health literacy, the S-TOFHLA, and randomized to watch either a sensationalized or non-sensationalized news clip focused on mTBI. They were then assessed with the Paced Auditory Serial Addition Test (PASAT). Rivermead Post Concussion Symptoms Questionnaire (RPQ), PROMIS Depression scale, and the PTSD Checklist (PCL-5).

Results: Bayesian analyses indicated that sensationalized media was not a strong predictor of PASAT score $(\beta=0.04)$ or post-concussion syndrome symptom severity $(\beta=-0.03)$. Age category (older vs. younger; $\beta=-0.63)$ was the strongest predictors of cognitive functioning. PTSD symptoms $(\beta=0.54)$ were the strongest predictors of post-concussion syndrome severity.

Conclusions: Despite concerns about possible iatrogenic effects of media reporting that sensationalizes the effects of concussion, the current results, when combined with prior information based on the nocebo effect and related literature, suggest that these effects are negligible. Individuals with more reported symptoms of PTSD are likely to report more post-concussion symptoms regardless of the media to which they are exposed.

Correspondence: Cara A. Bussell, B.A., Psychology, University of Colorado Colorado Springs, 737 W Buena Ventura St, Colorado Springs, CO 80907, United States.E-mail: cbussell@uccs.edu

K. WILMOTH, A. MASUCCI, T. TARKENTON, N. DIDEHBANI, L.S. HYNAN, S.C. BUNT, J. GARNER-ROBERTS, G. BLOOMGARDEN, A.J. ZYNDA, S. MILLER, K. BELL \& C.M. CULLUM. Psychological and Injury-Related Factors as Predictors of Prolonged Return to Play in Adolescents following Sports-Related Concussion.

Objective: Variability in adolescents' recovery from sports-related concussion (SRC) is poorly understood, and lingering symptoms can negatively impact academic, social, and emotional functioning. Though literature suggests injury and patient characteristics may be associated with a longer time to return-to-play (RTP), few studies have examined psychological factors. To this end, we explored the influence of postinjury mood and sleep ratings on prolonged RTP following SRC in adolescents.

Participants and Methods: 169 students age 12-18 who sustained SRC and underwent evaluation through clinics participating in the North Texas Concussion Registry (ConTex) within 28 days of injury. Measures at initial visit included General Anxiety Disorder-7 Item Scale (GAD-7), Patient Health Questionnaire-9 (PHQ-9), Pittsburgh Sleep Quality Index (PSQI), and Sports Concussion Assessment Tool, 3rd edition (SCAT-3) Symptom Scale. Medical record review at 3-months confirmed time to RTP. Logistic regression for shorter and longer time to RTP ( $\leq 21$ days $\& \geq 28$ days) was conducted based on 1 ) self-reported injury/medical factors: gender, prior history of concussion, loss of consciousness, amnesia, \& initial symptom log total score, and 2 ) psychological factors including GAD-7 total score, PHQ-9 total score, and PSQI overall sleep quality.

Results: $63 \%$ of the 169 participants had RTP $\leq 21$ days; $24 \%$ RTP $\geq 28$ days. For injury/medical factors alone, the overall model was significant, $\mathrm{p}=.003$; concussion symptom scores were the only reliable predictor of
RTP, $p=.004$. However, once psychological factors were added into the model, only GAD-7 scores were significantly predictive $(\mathrm{p}<.001)$ with a 1.5 times [95\% CI 1.2-1.8] greater chance of RTP $\geq 28$ days for every point increase in GAD-7 score.

Conclusions: Our findings demonstrate initial anxiety symptoms may have higher predictive power for prolonged RTP following SRC in adolescents, even considering injury and medical factors. These results suggest the importance of postconcussion mood assessment and management.

Correspondence: Kristin Wilmoth, UT Southwestern Medical Center, 5323 Harry Hines Blvd, Dallas, TX 75390-9044, United States. E-mail: Kristin.Wilmoth@UTSouthwestern.edu

S. RASKIN \& A. LEE. Time Perception and Prospective Memory in People with Brain Injury.

Objective: Time perception (TP) is an important aspect of PM performance, however, there is little research on the relationship. TP has been shown to be impaired in people with brain injury (BI) for time reproduction, time production and verbal estimation. This study aimed to measure the relationship between TP and PM.

Participants and Methods: 40 adult individuals with BI and 40 healthy adults ages 20-55 were included. In the BI group, all individuals will be at least one-year post injury, will have obtainable medical records. TP was measured via computer via two tasks. The prospective task required participants to hold a bar down for a prescribed time period (2 seconds, 10 seconds, 30 seconds, 1 minute) while performing a visual search task (press the bar if you see an $\mathrm{E}$ ); and the retrospective task required the same search task however, during the task, the screen woudl flash twice and the participant had to determine the time between the two flashes $(2,10,30$ seconds and 1 minute). PM was mesured via the Memory for Intentions Screening Test (MIST). All participants were also administered Brief Test of Attention, Stroop Color-Word Interference, and Hopkins Verbal Learning.

Results: One-way ANOVA and post-hoc testing revealed that the groups were significantly different for prospective TP at 10 seconds, 30 seconds and 1 minute and for retrospective TP for 30 seconds and 1 minute. In addition those with BI showed greater variability. MIST performance was significantly different between groups on time-based, event-based, and total score as well as PM errors. MIST total performance was significantly related to prospective time estimation but not retrospective time estimation. Of the neuropsychological measure only the Stroop was related to MIST total and to prospective TP.

Conclusions: TP was significantly related to PM functioning in inviduals with BI. This suggests that one strategy for improving PM performance might be time sense training, at least in the subset that shows this as a primary deficit.

Correspondence: Sarah Raskin, Ph.D., Psychology, Trinity College, 300 Summit Street, Hartford, CT 06119, United States. E-mail: sarah. raskin@trincoll.edu

\section{Paper Session 5. Parkinson Disease and Movement Disorders}

\section{Moderator: Dawn Schiehser}

$$
\text { 2:15-3:45 p.m. }
$$

L.E. COLVIN, S. CHAPMAN, M. MALGAROLI, E. LOUIS, E. HUEY, M. AZAR, T. CERSONSKY, S. KELLNER \& S. COSENTINO. Exploring the association between psychological characteristics and metacognition among adults with essential tremor.

Objective: Recent work has identified discrete personality/mood classes that relate to metacognition (MC) in a community-based cohort of older adults. The extent to which such classes explain metacognitive variability in older adults with neurologic illness is not known. 
Essential Tremor (ET) is among the most prevalent neurological diseases. In addition to its hallmark kinetic tremor, impaired cognition has emerged as an important feature in some individuals with ET, and recent work suggests such individuals have reduced metacognitive functioning. This study examined whether psychological characteristics are associated with MC among older adults with ET.

Participants and Methods: We performed a cross-sectional analysis in 218 non-demented ET cases (age $80.5 \pm 9.5$ years) enrolled in clinical-pathological study. Cases underwent a comprehensive battery of motor-free neuropsychological tests and a functional assessment to inform clinical diagnoses. Latent Class Analysis (LCA) examined whether discrete classes of depression and personality (NEO-FFI, GDS) emerged in our sample. Demographically-adjusted Generalized Linear Model was used to determine the association between these classes and metacognition for language, executive functioning, and memory.

Results: Two discrete classes emerged in the LCA: Class A was characterized by predominantly high neuroticism and high depression $(\mathrm{N}=39$; $17.9 \%$ ) and Class B characterized by predominantly low neuroticism and low depression $(\mathrm{N}=179 ; 82.1 \%)$. Class membership was unrelated to metacognitive functioning.

Conclusions: As opposed to the pattern seen in older adults, associations between latent classes of personality/depression did not relate to MC in ET. This may reflect the fact that anxiety was included in the LCA for older adults but was unavailable for ET. However, it may also speak to the likelihood that metacognitive variability in ET may in part reflect neurologic changes associated with the illness. These findings reinforce a multidimensional, biopsychosocial conceptualization of MC. Correspondence: Leigh E. Colvin, PhD, Clinical Psychology, Columbia University, 1185 park avenue, New York, NY 10128, United States. E-mail:lec2151@tc.columbia.edu

S.A. SPERLING, B.B. SHAH, A.E. BOND, D.S. HUSS, M.J. BARRETT \& W.J. ELIAS. Non-Motor Outcomes and Quality of Life AfterFocused Ultrasound Thalamotomy in Tremor-Dominant Parkinson Disease.

Objective: To examine non-motor outcomes and predictors of quality of life (QoL) at 3 and 12 months post-focused ultrasound (FUS) thalamotomy in tremor-dominant Parkinson disease (tdPD).

Participants and Methods: Participants were $27 \mathrm{tdPD}$ patients in a double-blinded, sham-controlled, randomized pilot clinical trial. Comprehensive neuropsychological evaluations, including assessment of mood (BAI; BDI-II), behavior (FrSBe), and QoL (PDQ-39) occurred at baseline, 3 months, 3 months post-crossover in the sham group, and 12 months post-active treatment. Mann-Whitney $\mathrm{U}$ tests were used to examine differences between the active $(n=20)$ and sham $(n=7)$ groups at 3 months. After unblinding, six patients crossed-over to active treatment. Wilcoxon signed-rank tests were used to examine within group changes post-FUS at 3 and 12 months. Kendall's tau-b tests were used to examine associations between disease variables and QoL. A regression was used to examine post-FUS predictors of QoL.

Results: There were no significant differences in cognition, mood, behavior, or QoL between the active and sham groups at 3 months. There were no significant within-group differences in cognition, mood, or behavior at 3 months post-FUS. At 12 months, letter fluency significantly declined $(p=.006)$. Significant improvements were seen in QoL post-FUS at 3 and 12 months ( $p=.003$ and $p=.031$, respectively). At 3 months post-FUS, the BAI, BDI-II, UPDRS Part III (all $p<.01$ ) and Total FrSBe $(p<.05)$ were significantly associated with QoL. Each remained significant at 12 months. Neither disease duration, MoCA, treated upper limb tremor score (CRST A\&B), nor change in tremor score were associated with QoL. Only BAI at 3 months $(\mathrm{p}=.004)$ and Total FrSBe at 12 months $(p=.043)$ predicted QoL.

Conclusions: In tdPD, FUS thalamotomy appears safe from a non-motor perspective. QoL post-FUS was associated with mood, behavior, and overall motor functioning, but not tremor or tremor change, disease duration, or cognition. Anxiety level and behavioral functioning predicted QoL post-FUS.
Correspondence: Scott A. Sperling, PsyD, Neurology, University of Virginia, PO BOX 800394, Charlottesville, VA 22908, United States. E-mail:sas7yr@virginia.edu

K. DHIMA, L.S. HYNAN, G. RODRIGUEZ-LARRAIN, S.M. MCCLINTOCK, R.B. DEWEY, D. GERMAN \& L. LACRITZ. A Preliminary Examination of Parkinson's Disease Subtypes and Associated Differences in Cognitive and Motor Symptom Trajectories.

Objective: Establishing distinct Parkinson's disease (PD) subtypes could inform physiological underpinnings related to PD's heterogeneous phenotype and progression. This study examined PD subtypes in recently diagnosed de novo patients based on multiple clinical variables and analyzed associated cognitive and motor symptom trajectories. Participants and Methods: Subjects included 384 PD patients from the Parkinson's Progression Markers Initiative, assessed at baseline (T1) and 4 years (T2). Variables included PD onset age and motor, cognitive, psychiatric, and behavioral measures (MDS-UPDRS, Animal Fluency, B-JLO, SDMT, WMS-III LNS, HVLT-R, MoCA, GDS-15, STAI, ESS, RBDQ, MS\&E-ADL, UPSIT, QUIP-RS, SCOPA-AUT). DaTscan SPECT (caudate/putamen) and CSF biomarkers ( $\alpha$-synuclein, A $\beta 1-42$, total tau, P-tau181P) were also included. T1 subtypes were extracted via hierarchical Ward's cluster analysis with squared Euclidean distances. Repeated measure ANCOVAs (covarying for levodopa dose at T2) were used to analyze differences in motor and cognitive function between subtypes across time.

Results: A 3-Cluster solution was found based on 1)low, 2)medium, and 3)high CSF biomarker concentrations with respective 1)high and 2)low anxiety scores. Repeated measure ANCOVAs found Cluster 1 performed significantly worse over time on visuospatial function (B-JLO; $p=.02$ ) and verbal memory [HVLT-R delay $(\mathrm{p}=.03)$ and recognition discrimination $(p=.03)]$ vs. Clusters 2 and 3 . No significant differences emerged for motor measures over time.

Conclusions: This study found that recently diagnosed de novo PD patients with higher anxiety and lower CSF concentrations of $\alpha$-synuclein, A $\beta 1-42$, total tau, and P-tau181P exhibited worse memory and visuospatial function over a 4-year period. The extent to which this is associated with distinct subtypes warrants further investigation. However, these findings suggest that anxiety and CSF biomarkers could be used to help predict risk for cognitive decline and inform the development of personalized medicine in PD.

Correspondence: Kaltra Dhima, Clinical Psychology, Psychiatry, University of Texas Southwestern Medical Center, University of Texas Southwestern Medical Center, 5323 Harry Hines Blvd., Dallas, TX 75390-9044, United States. E-mail: Kaltra.Dhima@UTSouthwestern. edu

D.S. BROWN, M.J. BARRETT \& S.A. SPERLING. Cognitive Correlates of Apathy in Parkinson's Disease.

Objective: To understand the relationship between apathy and cognition in Parkinson's disease (PD).

Participants and Methods: 111 participants with PD were administered the Apathy Scale [AS; $M=10.26$ (6.36)], Montreal Cognitive Assessment [MoCA; $M=24.20$ (2.88)], Trail Making Test (TMT), Wechsler Adult Intelligence Scale-IV Matrix Reasoning subtest (MR), letter (F-A-S) and category (animals) fluency, and Hopkins Verbal Learning Test-Revised (HVLT-R) as part of a larger prospective longitudinal investigation of neuropsychiatric symptoms in PD. Demographic information was also collected. A one-way multivariate analysis of variance was conducted to assess for significant differences between nonapathetic $(\mathrm{AS} \leq 13)$ and apathetic $(\mathrm{AS}>13)$ groups for cognitive variables. Then, a forward stepwise Wald logistic regression analysis was run with cognitive variables as predictors and apathy group as the outcome measure, controlling for demographic factors, including age $(M=68.53)$, education $(M=16.13$ years), disease duration ( $M=6.17$ years), sex ( $60.7 \%$ male), and use of dopaminergic medication $(29.5 \%)$. 
Results: There were significant differences $(p<.05)$ between the nonapathetic $(n=79)$ and apathetic $(n=32)$ group for MoCA, TMT Part B, MR, letter fluency, and HVLT-R Total Learning and Delayed Recall. TMT Part B $(p=.01 ; \mathrm{OR}=.93)$ was the only predictor retained in the final logistic regression model, with a correct classification rate of $76.5 \%$ (Nagelkerke $R^{2}=.34, p<.01$; Hosmer Lemeshow $p=.46$ ), controlling for age, education, and sex.

Conclusions: Global cognition, executive functioning, letter fluency, encoding, and recall, which all implicate frontal system involvement with the exception of global cognition, are associated with apathy in PD. Neither processing speed nor semantic fluency were significantly associated with apathy in this cohort of individuals with mildly impaired cognition overall. These results provide further evidence that apathy and executive dysfunction have similar underlying pathology.

Correspondence: Daniel S. Brown, PhD, Neurology, University of Virginia Health System, 269 Colonnade Dr., \#04, Charlottesville, VA 22903, United States. E-mail: brown.daniel@gmail.com

L. HIZEL, J. KLEIN, B. WALLACE, K. RODRIGUEZ, S.J. CROWLEY, M.E. WIGGINS, J.J. TANNER, M. DING \& C.C. PRICE. Digestive health in Parkinson's disease relates to fatigue, pain, and affective dysfunction but not cognition.

Objective: Non-motor symptoms of Parkinson's disease (PD) include dorsal stream fronto-striatal cognitive deficits (e.g., working memory, reasoning) and ventral fronto-striatal stream associated symptoms (e.g., depression, pain, fatigue). Less understood is gastrointestinal distress in PD. Here, we examined the hypotheses that gastrointestinal distress would be higher in PD than non-PD peers and gastrointestinal distress in PD would uniquely associate with ventral striatal non-motor symptoms (e.g., pain, fatigue) but not dorsal striatal cognitive symptoms (e.g., working memory, reasoning).

Participants and Methods: 77 participants (55 PD without dementia, 22 non-PD peers) completed measures of gastrointestinal symptoms (Digestive Health Questionnaire; DHQ), pain (Brief Pain Inventory; BPI), fatigue (Fatigue Severity Scale; FSS), and depression (Geriatric Depression Scale; GDS). An independent samples t-test evaluated PD/ Non-PD group differences. Partial correlations, correcting for disease duration and age, evaluated relationships between DHQ severity (Range: 0-80) and other non-motor symptoms.

Results: Groups were different in education, which was additionally controlled for in the following analyses. DHQ severity was significantly higher in $\mathrm{PD}(\mathrm{PD}=24.2 \pm 14.2$; Non- $\mathrm{PD}=17.7 \pm 8.0, \mathrm{p}<0.05)$. Within $\mathrm{PD}$, increasing DHQ positively associated with depressive symptoms $(\mathrm{r}(59)=0.276, \mathrm{p}<0.05)$, pain $(\mathrm{r}(59)=0.512, \mathrm{p}<.01)$, and fatigue $($ FSS $r(58)=0.551, p<.01)$. No relationships were found with working memory, reasoning, and other cognitive measures (all r's $<0.10$ ).

Conclusions: Digestive health symptoms are more severe in PD than non-PD peers and associate with ventral striatal symptoms but not dorsal striatal symptoms. Future research should validate these findings with ventral/dorsal stream structural and functional connectivity analyses. Funding: NIH R01 N5082386

Correspondence: Loren Hizel, M.S., Clinical and Health Psychology, University of Florida, $1810 \mathrm{NW} 23 \mathrm{rd}$ Blvd Unit 160, Gainesville, FL 32605, United States. E-mail: Ihizel@phhp.ufl.edu

E.E. MORAN, R. ORTEGA, C. PALMESE, D. RAYMOND, I. PERERA, I. MEIJER, L. NEIMAND, K. PETERS, M. SWAN, R. SACHDEV, L. OZELIUS, S. BRESSMAN, M.E. ZIMMERMAN \& R. SAUNDERSPULLMAN. Cognitive and Motor Functioning in Heterozygous Non-manifesting Glucocerbrosidaise (GBA) Carriers.

Objective: Homozygous and heterozygous mutations in the glucocerebrosidase (GBA) gene are a significant genetic risk factor for the development of Parkinson's disease and dementia with Lewy Bodies. GBA related PD is associated with more prominent cognitive impairment than idiopathic PD. However, the burden of harboring a GBA mutation among those currently unaffected by PD is unclear. The aim of the present study is to examine the relationship between mutation status and cognitive, motor, and psychiatric functioning in non-manifesting GBA carriers.

Participants and Methods: A total of 30 non-manifesting GBA carriers and 72 controls completed the motor section of the Unified Parkinson's Disease Rating Scale (UPDRS-III), neuropsychological assessment including the Montreal Cognitive Assessment (MoCA).

Results: In a linear regression model of genetic status, age, and gender predicting MoCA, the predictors account for a significant portion of the variance $\left(R^{2}=0.10, p<0.05\right)$ and the partial regression coefficients of genetic status and age were significant $(p<0.05)$. In a linear regression model of predicting UPDRS-III, the predictors account for a significant portion of the variance $\left(\mathrm{R}^{2}=0.15, p<0.05\right)$ and the partial regression coefficients of genetic status and age were statistically significant $(\mathrm{p}<0.05)$.

Conclusions: This study demonstrates that non-manifesting carriers of a GBA mutation may differ from controls in cognitive and motor functioning. Non-manifesting GBA carriers may demonstrate subclinical burden of mutation status and thus warrant further investigation. Correspondence: Eileen E. Moran, MSc, Psychology, Fordham University, 600 W. 138th St., Apt 33, New York, NY 10031, United States. E-mail: emoran@fordham.edu

T. Costabile, V. Capretti, F. ABATe, A. Liguori, F. PACIELlO, C. PANE, G. DE MICHELE, A. FILLA \& F. SACCÀ. Emotion Recognition and Psychological Comorbidity in Friedreich's Ataxia.

Objective: Friedreich's Ataxia (FRDA) is an autosomal recessive disease presenting with ataxia, corticospinal signs, peripheral neuropathy, and cardiac abnormalities. Little effort has been made to understand the psychological and emotional burden of the disease. Aim of our study was to measure patients' ability to recognize emotions using visual and non-verbal auditory hints, and to correlate this ability with psychological and neuropsychological variables.

Participants and Methods: We included 20 patients with FRDA, and 20 age, sex, and education matched healthy controls (HC). We measured emotion recognition using the Geneva Emotion Recognition Test (GERT). Neuropsychological status was assessed measuring global intelligence, memory, executive functions, and prosopoagnosia. Psychological tests were: Patient Health Questionnaire-9 (PHQ-9), State Trait Anxiety Inventory-state/-trait (STAI-S/-T), Structured Clinical Interview for DSM Disorders II.

Results: FRDA patients scored worse at the global assessment and showed impaired immediate visuospatial memory and executive functions. Patients presented lower STAI-S scores, and similar scores at the STAI-T, and PHQ-9 as compared to HC. Three patients were diagnosed with personality disorders. Emotion recognition was impaired in FRDA with $29 \%$ reduction at the total GERT score $(95 \%$ CI $-44.8 \%,-12.6 \%$; $\mathrm{p}<0.001$; Cohen's $d=1.2)$. Variables associated with poor GERT scores were the 10/36 spatial recall test, the Ray Auditory Verbal Learning Test, the Montreal Cognitive Assessment, and the STAI-T $\left(\mathbf{R}^{2}=0.906\right.$; $\mathrm{p}<0.001)$.

Conclusions: FRDA patients have impaired emotion recognition that may be secondary to a diffuse neuropsychological impairment. Depression and anxiety were not higher than in the general population and should not be considered as part of the disease.

Correspondence: Teresa Costabile, University of Naples "Federico II", Via S. Pansini, Naples 80131, Italy. E-mail: teresa.costabile@gmail.com 


\section{Poster Session 5. Child Medical and Neuropsychiatry}

\section{2:30-3:45 p.m.}

\section{ADHD/Attentional Functions}

WITHDRAWN. A. AIERBE, M. GONZÁLEZ, M. MORENO, M. MEJÍAS \& M. REDONDO. Flexibility Capacity: Differences Between Young and Older People in Nesplora Aquarium Test.

Objective: Ageing results incognitive changes, tsually in a dectine of the eognitive eapacities. This work aims to identify the age-related-e hange in the flexibility eapaeity, defined as the ability to change your behavior to a new sittration.

Partieipants and Hethrods: 1102 participants without cognitive impairment (age range: 16-90; average age: 44.50 ; female: $63 \%$ ) were assessed with the Nesplora Aquarimm test. This neuropsychologieal toot, based on virttral reality, assesses the attentional processex. The test is divided int three sub-tasks. 1) AX paradigm, 2) duad fot to sk, 3) duat No-GO taks with changed target stimuli in ore to sest the resistance to perseveranee. There are two meastres of fen pilty: 1) the ehange betweentask 1 and task 2, 2) He- of thetweentask 2 and task 3.

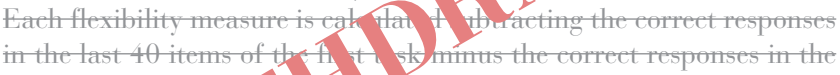
first 40 items of the ect ta, . The approximate length of the test is Results: . I I of 1 , He ealentated the age groups for the normative study ant te btained three different groups: 16-40 years-old, 41-60 years-old, $61+$ years-old. Then, we carriect ont nom parametrie andyses betweenthe 3 age gromps and separated formate and femate. We found that in both ment and women, the youngest group performed better in flexibility 1 but the oldest group outperformed in flexibility 2 .

Gomeltusions: Some cognitive apacities suffered a dectine with ageing but others can still be at the same level or even improve. The results of this work point out to different hypotheses: a) the older people benefit more than younger from the training; b) younger people show more imputsiveness, c) the sustained attention in yourger people falts throughout the test. More researeh is needed in order to clarify the outperformanee in some kind of flexibility of the older people in order to enthance and maintain this capacity as long as possible.

Gorrespontence. Amata Aierbe, Miketetegi, 58, Planta 0, Local \&, san Sebastián 20009, Spain. E-mail: aaierbe@nesplora.com

WITHDRAWN. A. AIERBE, M. REDONDO, M. GONZÁLEZ, M. MORENO \& M. MEJÍAS. Nesplora Aquarium: Utility of the Tool to Identify People with Attention-Deficit Hyperactivity Disorder.

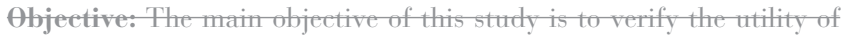
Eesplora AQUARHEM test to discern between people with ADH diag= nosis and without it.

Partieipants and Methods: The sample eonsists of 47 people, 25 with previons diagntosis of Attention-Defieit Hyperactivity Disorder (AD(W) (32\% female) according to DSM-V criteria, with a mean age of 32.8 , and 22 without any disease ( $54 \%$ female), with mrapd of 35 . The partieipants of the eontrol group were matelect a a age, gender and

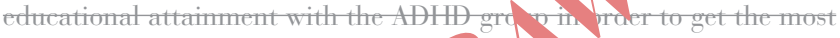
similar groups as possible.

In order to involve people with AI Hh Angrosis, we collaborated with an $\mathrm{ADH}$ association calt $1,9 \mathrm{~A}$ H, inteitentat.

All participants efre tmminstered the test Nesplora AQUARIUM. This tool, basere in th trat reality, has been ereated for the evaluation of attentioma put esses. After a trability task, the test is dividect int three sub-tasks ant each one has its training: one of them is an $\mathrm{AX}$ paradigm and the other two are dual No-GO tasks, which appear with ehanged target stimm li in order to assess the resistanee to perseverante.
Results: A non-parametric comparison of the principal scores' means of the test has been earried out between both groups.

The restlts show that the partieipants with a previous $\mathrm{ADH}$ diagnosis make more ontrission errors than participants without diagnosis int the 3 tasks.

The gromp with $\mathrm{ADH}$ shows more eommission errors in the first task than the other group. Differentes int the meant response time (RT) have not been observed, but they have been observed int the deviation of the RT, being higher for the ADIID group in the seeond task.

Conelusions: Differenees between groups have been observed in the seores obtained int the test related to inattention (omrissions and deviation of RT) and impulsivity (eommissions). It is necessary to delve into results with a larger sample.

Gorrespondenter. Antuia Alierbe, Miketetegi, 58, Ptanta 9, Local S, sam Sebastian 20099, Spain. E-mait. ative@nesplora.com

J. ALI, J.E. SCHREIBER, K. BERLIN, J. PORTER, J. HANKINS, S. ANDERSON \& F. ANDRASIK. Attention, Executive Function, and Transition to Adult Care Among Youth with Sickle Cell Disease. Objective: Rates of mortality and morbidity increase during young adulthood among those with sickle cell disease (SCD) and successful transition from pediatric to adult health care is considered a major factor in the health outcomes among this population. Executive functioning (EF) has been linked to successful adaptation and transition to adult care among youth with other chronic illnesses; however, the relationship between attention, EF, and successful transition to adult care among youth with SCD has not been studied.

Participants and Methods: Fifty-eight patients with SCD underwent a neurocognitive evaluation at the mean age of 16.98 years $(S D=.83)$ and additionally completed a structured transition to adult care program at a mid-south children's hospital. Exploratory Structural Equation Modeling (ESEM) was used to explore the ability of attention and EF to predict "successful" transition to adult care, defined as (1) time to complete first adult clinic visit and (2) completion of first adult clinic visit within 6 months of last pediatric appointment.

Results: Fifty patients (86\%) completed an adult visit within 6 months of leaving pediatric care. A post hoc multivariate regression analysis indicated that Omission errors from the Conners' Continuous Performance Test-II (CPT-II) successfully predicted attendance of the first adult health care visit within 6 months of the last pediatric health care appointment. As Omission errors increased, patients were less likely to attend the adult clinic within 6 months.

Conclusions: Our findings suggest important connections between inattention and engaging in adult healthcare. These findings highlight the importance of a greater focus on the role of attention dysfunction in the successful adaptation and transition to adult care among youth with SCD.

Correspondence: Jeanelle Ali, Psychology, The University of Memphis, 3720 Alumni Ave, Memphis, TN 38104, United States.E-mail: jsali@ memphis.edu

S. ALI \& S. MACOUN. Intra-Individual Variability in Children is Related to Parent Ratings of Attention, but not Teacher Ratings.

Objective: Intra-individual variability (IIV) refers to within-person variability on a single task over multiple trials on the same testing occasion. IIV is increasingly seen as an endophenotype of various neurodevelopmental disorders involving attention problems (e.g., ADHD). Previous research has noted that parent ratings of attention are related to IIV in children with ADHD (Gomez-Guerrero et al., 2011), suggesting that IIV may be a useful objective marker for clinical attention problems. The current study aimed to investigate whether this relationship holds true for parent and teacher ratings for children with attention difficulties. Participants and Methods: Participants were drawn from a broader cognitive intervention study and included children ages 5-12 years, identified by their classroom teachers as having attention and EF problems. Parents and teachers completed the attention composite of the Comprehensive Executive Function Inventory. IIV of reaction times 
was calculated from a computerized go/no-go task using the residual ISD method.

Results: Parent and teacher ratings of attention were not significantly correlated $r=0.23, p=$ n.s. Parent ratings of attention were significantly correlated with IIV $(r=-0.38, p<0.01)$ whereas teacher ratings were not $(r=-0.21, p=$ n.s. $)$ Using regression analysis, IIV significantly explained variance in parent ratings $(\mathrm{F}(1,63)=10.14, p<0.01)$ while no such relationship was found with teacher ratings $(\mathrm{F}(1,47)=2.06, p=$ n.s. $)$

Conclusions: The non-significant correlation between parent and teacher ratings of attention aligns with prior research supporting the value of using multiple informants for assessing attention problems. The significant relationship between parent ratings of attention and IIV is also consistent with prior research in children with ADHD, suggesting that IIV may be useful as an objective marker of attention problems. We will discuss the use of IIV as a clinical marker of attention problems and reasons for the nonsignificant relationship between IIV and teacher ratings. Correspondence: Sheliza Ali, Clinical Psychology, Psychology, University of Victoria, Department of Psychology, P. O. Box 1700 STN CSC, University of Victoria, Victoria, BC V8W 2Y2, Canada. E-mail: sheliza@uvic.ca

K. ANGERS, G.J. LEE, A.G. HARRISON \& J. SUHR. Indices of Self-Report Validity in Attention-Deficit/Hyperactivity Disorder (ADHD) Symptomatology and Its Relationship to Self-Reported Functional Impairment.

Objective: Diagnosing ADHD in adulthood is complicated by the fact that it relies on patients to accurately report symptoms and impairment. Faking ADHD may come with secondary gains such as access to stimulant medication or academic accommodations. As such, it is important for clinicians to assess the validity of self-report symptoms and impairment when making diagnostic decisions. The present study investigated whether the validity of self-reported ADHD symptoms was related to self-reported functional impairment in individuals concerned they may have ADHD.

Participants and Methods: Participants were from a larger database $(n=273)$ of adults being evaluated for potential ADHD for the first time; 108 participants who completed measures of interest for the present study were included for analysis, including the Weiss Functional Impairment Rating Scale (WFIRS), designed to assess ADHD-related functional impairment across 6 domains and the Conner's Adult Attention Deficit/Hyperactivity Rating Scale (CAARS), with two recently developed symptom validity measures as the focus of the present analyses: the CAARS Infrequency Index and the Dissimulation Index.

Results: Using the Infrequency Index, non-credible symptom responders (NSRs) reported significantly higher impairment on WFIRS total score, $\mathrm{p}<.001$, and on the risks, $\mathrm{p}<.001$, social, $\mathrm{p}=.02$, learning/work, $\mathrm{p}=.002$, and self-concept, $p=.03$, subscales than credible responders (CRs). Using the WFIRS cutoff for clinical impairment, a higher percentage of NSRs scored in the clinically impaired range in the learning/work domain. Using the Dissimulation Index, NSRs scored significantly higher than CRs on the WFIRS total, $\mathrm{p}<.001$, and also on the home, $\mathrm{p}=.03$, social, $\mathrm{p}=.003$, and risks, $\mathrm{p}<.001$, subscales; a significantly higher percentage of NSRs also scored in the clinically impaired range in the home, life skills, social, and risk taking domains.

Conclusions: Results demonstrate the importance of assessing for self-report validity when determining functional impairment in ADHD assessment.

Correspondence: Kaley Angers, B.S., Psychology, Ohio University, 22 Richland Ave, Athens, OH 45701, United States. E-mail:ka434415@ ohio.edu
B.A. BAILEY, A.M. BRENNER, A.M. SVINGOS, S.M. GREIF, E.M. TURNER, M.W. MOORE \& S.C. HEATON. The Contribution of Executive Dysfunction to Social Self-Concept in Youth with ADHD.

Objective: Children with ADHD exhibit a variety of functional impairments, including deficits in social functioning. Children with ADHD generally have fewer close friends and are more often rejected by classmates. However, these youth often underestimate the extent of their deficits. It has been hypothesized that this lack of awareness occurs in ADHD predominantly due to the executive dysfunction also characteristic of this disorder, but this has not been empirically examined. The current pilot study sought to examine the associations among measures of executive functioning, social functioning, and social self-concept in a sample of youth with ADHD.

Participants and Methods: Archival clinical data of youth with ADHD ages 10 to 17 years $(\mathrm{N}=17)$ were examined. Social functioning was examined using the Peer Relations subscale of the Conner's Parent Rating Scale. Social self-concept was examined using the Multidimensional Self Concept Scale, and executive functioning was measured using the Behavioral Rating Inventory of Executive Functioning and the Color-Word Identification subscale of the Delis-Kaplan Executive Functioning System.

Results: The association between social self-concept and parent-reported social functioning was non-significant, as was the association between social self-concept and parent-reported executive functioning. However, performance-based executive functioning was significantly associated with social self-concept such that youth who performed worse on this measure reported better self-concept $(b=-.43, p=.05, r-s q=.17)$. Conclusions: Results support the hypothesis that executive dysfunction may contribute to a lack of awareness of social deficits in youth with ADHD. However, these findings should be replicated in a larger sample. Additionally, further studies should examine which components of executive function (eg., self-monitoring) most robustly predict awareness of social deficits among youth with ADHD.

Correspondence: Brittany A. Bailey, Bachelor of Arts, Clinical Health Psychology, University of Florida, 1225 Center Drive, Rm 3158, Gainesville, FL 32508, United States. E-mail: bbailey92@phhp.ufl.edu

\section{J. BORRANI, A. GARCIA, C. RAMIREZ \& P. VALDEZ. Analysis of ADHD and Conduct Disorder Symptoms, Attention and Executive Functions in Juvenile Delinquents.}

Objective: The objective of this study was to analyze attention deficit and hyperactivity disorder (ADHD) and conduct disorder (CD) symptoms, as well as components of attention and two components of executive functions (cognitive inhibition and flexibility), in juvenile delinquents. Participants and Methods: Participants were 87 males $(17.87 \pm 1.54$ years old). A group of 29 juvenile delinquents (JDG) was compared to an age-paired group of 29 non-delinquents (APG), and to an ageand education-paired group of 29 non-delinquents (AEPG). ADHD and CD symptoms were evaluated with the Conner's Questionnaire. Components of attention (tonic alertness, phasic alertness, selective attention and sustained attention) were assessed through a Continuous Performance Task (CPT). Cognitive inhibition was assessed through a modified Stroop task, and cognitive flexibility through the Wisconsin Card Sorting Test (WCST).

Results: The JDG reported more ADHD and more CD symptoms than the non-delinquent groups. The JDG and the AEPG (low-education adolescents) had a lower percentage of correct responses on tonic alertness, selective attention, phasic alertness and sustained attention on the CPT, took more time to name the colors on the Stroop task, which suggests difficulties on cognitive inhibition, and had more perseverative responses on the WCST, which suggests difficulties on cognitive flexibility, compared to the APG. The JDG and the AEPG had no differences on task performance. All reported differences were significant at $\mathrm{p}<0.05$. Conclusions: Juvenile delinquents have more self-reported ADHD and CD symptoms than non-delinquent adolescents. On the other hand, difficulties on the components of attention, cognitive inhibition and 
cognitive flexibility appear on all low-education adolescents, delinquents and non-delinquents. ADHD and CD symptoms are related to criminality; nevertheless, low executive functions are related to academic, occupational and social problems, as well as criminality, in juvenile delinquents and in low education adolescents.

Correspondence: Jorge Borrani, Laboratory of Psychophysiology, School of Psychology, Universidad Autonoma de Nuevo Leon, Mutualismo 110, Mitras Centro, Monterrey64460, Mexico.E-mail: jorgeborrani@gmail. com

B. BROOKER, L. DESJARDINS, D. OSTOJIC \& C. MILLER. Differential Associations of Inattention and Impulsivity Across Modes of Neuroenhancement Behavior.

Objective: Neuroenhancement (neurotypical students' use of substances with intent to enhance cognition) has increasingly garnered research attention. Non-medical use of prescription stimulants (NMUPS) for this purpose is associated with inattention/impulsivity, suggesting that neuroenhancement may constitute "self-medication" for subclinical/ undiagnosed ADHD. However, it remains unclear whether these symptoms are shared across all classes of neuroenhancement (using legal substances, e.g., caffeine/nicotine; using illicit drugs) or uniquely drive NMUPS. This study sought to examine relations of attention and impulse control across three classes of neuroenhancement.

Participants and Methods: University students ( $=200)$ completed measures of attention and impulsivity, including self-report ratings (ADHD symptoms, trait impulsivity) and performance-based measures (delay discounting, Stroop, Academic Diligence Task). Students also reported lifetime history of three classes of neuroenhancement: (1) legal substances, (2) illicit substances, and (3) NMUPS.

Results: MANOVA models revealed a significant effect of NMUPS $(p<.01)$ and legal neuroenhancement $(p=.02)$ histories on the combined impulsivity and attention variables. A nonsignificant trend for illicit neuroenhancement $(\mathrm{p}=.06)$ was also identified. Post-hoc analyses demonstrated differential associations of self-report and performance-based variables across classes of neuroenhancement. Greater self-reported inattention and trait impulsivity were associated with NMUPS and legal neuroenhancement histories, whereas ratings of ADHD hyperactivity/impulsivity were only associated with the latter. Performance-based variables were associated only with NMUPS.

Conclusions: Students who report inattention/impulsivity may be at increased risk of neuroenhancement broadly; however, differential patterns of attention/impulse control across separate classes of neuroenhancement suggest discrete mechanisms for specific modes of neuroenhancement.

Correspondence: Brianne Brooker, University of Windsor, 401 Sunset Ave, Windsor, ON N9B 3P4, Canada.E-mail:brookerb@uwindsor.ca

C. COOK, G.J. LEE, A.G. HARRISON \& J. SUHR. Relationship of Performance Validity Test Failure to Self-Reported Functional Impairment in Attention-Deficit/Hyperactivity Disorder (ADHD). Objective: Evaluating adults for possible ADHD often involves the potential for secondary gain (i.e., access to stimulant medications or academic or work accommodations). Validity of both self-reported information and test performance must therefore be considered when conducting such evaluations; however, most self-report scales lack validity indices and are easily manipulated. The present study investigated whether performance validity test (PVT) failure was related to higher levels of self-reported functional impairment in individuals concerned they may have ADHD.

Participants and Methods: Participants were selected from a larger study ( $\mathrm{n}=273$ ) of adults undergoing ADHD evaluation for the first time. Mean participant age was $22.16(\mathrm{SD}=5.45)$, and $56 \%$ were males. Only participants who completed all measures of interest for the present study were included $(n=110)$. Measures of interest include the Weiss Functional Impairment Rating Scale (WFIRS), designed to assess ADHD-related functional impairment; the Medical Symptom Validity
Test; and the Symptom Exaggeration Index from the Test of Variables of Attention.

Results: Individuals who failed at least one PVT ( $n=34)$ reported significantly higher total impairment $(\mathrm{p}=.008)$ than individuals who passed both PVTs $(n=78)$. Using the WFIRS cutoff for clinical impairment, a higher percentage of non-credible performers reported clinically significant impairment in the learning and work domain $(p=.048)$. The life skills domain and total impairment also trended towards significance. Conclusions: Results demonstrate the importance of considering performance validity when assessing ADHD-related functional impairment. While self-report validity and performance validity are not always highly related, these results suggest that non-credible performance is associated with elevated symptom reporting, which may interfere with accurate diagnosis.

Correspondence: Carolyn Cook, M.S., Psychology, Ohio University, 22 Richland Ave, Athens, OH 45701, United States. E-mail: cc541611@ ohio.edu

G. ALANÍS, M. GUERRERO, C. RAMIREZ, A. GARCIA, P. VALDEZ \& V. DE LA GARZA. Analysis of Components of Attention Under low Stimulation.

Objective: The objective of this study was to determine the effects of a low stimulation condition on the components of attention.

Participants and Methods: Participants were 25 undergraduate students, age $=18.95 \pm 2.09$ years, 14 women and 11 men. One group of 11 students were recorded in a moderate stimulation condition (seated with lights on) and 16 students were recorded in a low stimulation condition (lying down in a bed in complete darkness). The participants responded an auditory continuous performance task (aCPT), designed to measure the components of attention: tonic alertness, selective attention, phasic alertness and sustained attention. Task duration was 11 minutes and 52 seconds.

Results: The low stimulation group showed a lower percentage of correct responses of tonic alertness (moderate stimulation $=98.11 \pm 2.20 \%$, low stimulation $=91.21 \pm 9.87 \%, \mathrm{~F}=4.47, \mathrm{p}<0.05)$ and a higher percentage of omissions (moderate stimulation $=0.57 \pm 0.71 \%$, low stimulation $=4.08 \pm 4.65 \%, \mathrm{~F}=6.43, \mathrm{p}<0.05)$, compared with the moderate stimulation group.

Conclusions: A low stimulation condition reduces tonic alertness without modifying others components of attention. This effect may contribute to produce errors and accidents in workers that perform activities under low stimulation conditions.

Correspondence: Vania De la Garza, Monterrey, Mexico. E-mail: vaniaa_94@hotmail.com

A. ELLIS, C. KINZEL, G. SALGARI \& S. LOO. Frontal Alpha Asymmetry Predicts Inhibitory Processing in Youth with Attention Deficit/Hyperactivity Disorder.

Objective: Atypical asymmetry in brain activity has been implicated in the behavioral and attentional dysregulation observed in ADHD. Specifically, asymmetry in neural activity in the right versus left frontal regions has been linked to ADHD, as well as to symptoms often associated with ADHD such as heightened approach behaviors, impulsivity and difficulties with inhibition. Clarifying the role of frontal asymmetry in ADHD-like traits, such as disinhibition, may provide information on the neurophysiological processes underlying these behaviors.

Participants and Methods: ADHD youth (ADHD: $\mathrm{n}=25$ ) and healthy, typically developing controls (TD: $\mathrm{n}=25$ ) underwent an electroencephalography (EEG) recording while completing a go/no-go task-a commonly used test measuring behavioral inhibition. In addition, advanced signal processing for source localization estimated the location of signal generators underlying frontal alpha asymmetry (FA) during correct and incorrect trials.

Results: This is the first study in ADHD to demonstrate that the dorsal-lateral prefrontal cortex (DLPFC) may be responsible for generating frontal alpha. During failed inhibition trials, ADHD 
youth displayed greater FA than TD youth. In addition, within the ADHD group, frontal asymmetry during later processing stages (i.e., 400-800ms after stimulus) predicted a higher number of commission errors throughout the task.

Conclusions: These results suggest that frontal alpha asymmetry may be a specific biomarker of cognitive disinhibition among youth with ADHD.

Correspondence: Alissa Ellis, PhD, Psychiatry, UCLA, 760 Westwood Plaza,Los Angeles, CA 90024, United States.E-mail: aellis@mednet. ucla.edu

C. Gallegos, A. Garcia, C. RAMirez, J. BorRani \& P. VALDEZ. Effects of practice on the attentional blink.

Objective: The aim of the present study was to determine the effects of practice in the attentional blink

Participants and Methods: Fourteen undergraduate students $(18.07 \pm 1.44$ years $)$ participated in a total of 24 sessions, during 3 different days, eight sessions per day. In each session, participants responded to a rapid serial visual presentation (RSVP) task, to measure the efficiency to identify two stimuli (T1 and T2) with lags within the attentional blink (200-500 ms), and after it (600-800 ms).

Results: An attentional blink appeared clearly in the first sessions, as manifested by a lower efficiency to respond to T2 at $200 \mathrm{~ms}(73.43 \%)$, than to $600(89.10 \%)$ and $800 \mathrm{~ms}(92.86 \%)$. After practice (last sessions), the attentional blink was still present, but efficiency was reduced to T2 at $200 \mathrm{~ms}(63.97 \%)$, at $600(79.10 \%)$ and $800 \mathrm{~ms}$ $(74.33 \%)$.

Conclusions: After practice, performance was better at 600 than at $800 \mathrm{~ms}$. These results suggest that when performance to a rapid serial visual presentation task is automatized with intensive practice, the attentional blink is still present and attentional resources are directed to a short interval after the blink. This changes in the attentional blink mean a reduction of attention resources after practice, which may increase errors and accidents in daily life activities which require automatized responses to several stimuli at the same time.

Correspondence: Carlos Gallegos, Universidad Autonoma de Nuevo Leon, School of Psychology, Dr. Carlos Canseco \#110, Col. Mitras Centro, San Nicolás de los Garza 66455, Mexico.E-mail: car.gallegos90@gmail.com

\section{S.K. HAMILTON. Experiential Avoidance and Attention Among Stressed Graduate Students.}

Objective: Although literature supports a negative association between stress and attention, this study focused on the relationship between a method of coping with stress (i.e., experiential avoidance [EA]) and attention abilities. Previous studies have examined the relationship between EA and other components of cognitive functioning (e.g., perceptual motor abilities, working memory, learning), but none have examined the relationship between EA and attention. Therefore, this study aimed to explore the relationship between EA and objective and self-reported attention difficulties in stressful situations.

Participants and Methods: Participants consisted of 79 graduate students from The Chicago School of Professional Psychology (Mean age $=27.7, \mathrm{SD}=5.4)$. Most participants were female $(77 \%)$, White/Caucasian $(69.6 \%)$, and enrolled in the PsyD program (84.8\%). A correlational design was used to examine the relationship between students' attention abilities and their tendency to engage in EA during periods of increased stress (i.e., midterms/finals). Participants completed the Acceptance and Actions Questionnaire - II (AAQ-II), the Wechsler Adult Intelligence Scale - IV Digit Span Forward subtest (WAIS-IV DSF), and the Barkley Adult ADHD Rating Scale - IV Inattention subscale (BAARS-IV).

Results: Bivariate correlation analyses revealed a small but nonsignificant relationship between EA (AAQ-II) and objective attention (WAIS-IV DSF; $r=-.11, p=.331$ ). Conversely, a significant, small-tomedium sized positive relationship was found between EA and self-reported attention difficulties (BAARS-IV; $r=.29, p=.011$ ).
Conclusions: EA is a common strategy used to cope with stress; however, these findings suggest that utilization of EA will negatively impact students' self-reported rating of their attention abilities, but not their ability to perform on one specific objective attention task. Correspondence: Stephanie K. Hamilton, PsyD, Clinical Psychology, The Chicago School of Professional Psychology, 3333 Harry Hines Blvd, APT 8105, Dallas, TX 75201, United States.E-mail: steph.hamilton3@ gmail.com

G.M. HIDALGO, S. O'NEILL, L. MCWILLIAMS, C. LIU, L. MACEIRA-VILLALOBOS \& J. HALPERIN. Preschool ADHD but not Neuropsychological Functioning Is Associated with Elevated School-Age BMI.

Objective: (i) Do ADHD and neuropsychological functioning at age 4 predict body mass index (BMI) at age 7? (ii) Do BMI and neuropsychological functioning at age 4 predict ADHD at age 7? We hypothesized: (i) higher preschool ADHD severity and weaker neuropsychological functioning would predict higher BMI at age 7; (ii) higher preschool BMI and weaker neuropsychological functioning would predict higher ADHD at age ?.

Participants and Methods: An ethnically (Hispanic=36.8\%) and racially diverse (non- White $=42.8 \%)$ sample of preschoolers $(\mathrm{N}=152$; $73.7 \%$ male) was recruited from the community. Participants were evaluated at Mean $(\mathrm{SD})=4.54(0.29)$ years and again at $7.62(0.31)$ years. At age 4 , processing speed was measured using the WPPSI-III Processing Speed Quotient and executive functioning (EF) was measured using NEPSY Visual Attention. At ages 4 and 7, teachers evaluated ADHD severity using the ADHDRS-IV and objectively measured height and weight was used to generate BMI percentiles adjusted for age and sex. BMI percentiles were converted to normal curve equivalents before conducting hierarchical linear regression analyses.

Results: Preschoolers' ADHD severity $(\beta=.21, p=.016)$, but not processing speed $(\beta=.11, p=.20)$ or $\mathrm{EF}(\beta=.09, p=.27)$ predicted age 7 BMI after controlling for age 4 BMI $(\beta=.58, p<.0001)$ and SES $(\beta=.06$, $p=.47)$. The final model accounted for $42.1 \%$ of variance. Preschoolers' processing speed $(\beta=-.21, p=.045)$, but neither BMI $(\beta=.01, p=.89)$ nor $\mathrm{EF}(\beta=-.02, p=.89)$ predicted age 7 ADHD severity after controlling for age 4 SES $(\beta=-.20, p=.05)$ and ADHD severity $(\beta=.34, p<.0001)$. The final model accounted for $21.0 \%$ of variance.

Conclusions: Early intervention for ADHD behaviors may lower risk for worsening ADHD and physical health outcomes over time. Early BMI did not predict age 7 ADHD. Early processing speed, but not EF predicted later ADHD while neither predicted later BMI, perhaps due to the developmental period in which children's cognition was assessed. Correspondence: Geneva M. Hidalgo, Psychology, The City College of New York, 70 E 115th St Apt 14J, New York, NY 10029-1119, United States.E-mail: ghidalg000@citymail.cuny.edu

L.N. IRWIN, A. MOLTISANTI, E. WELLS, E. SOTO, N. FERRETTI \& M.J. KOFLER. Is There a Functional Relationship Between Set Shifting Demands and Hyperactivity in Children With and Without Attention-Deficit/Hyperactivity Disorder?

Objective: Underlying deficits in executive functions (EF) may drive attention-deficit/hyperactivity disorder's (ADHD) phenotypic behavioral presentation (Rapport et al., 2009). Set shifting, the ability to shift between competing rule sets (Miyake et al., 2012), is a core EF that may be impaired in ADHD (Willcutt et al., 2005). However, the relationship between this impairment and ADHD behavioral symptoms is not well-established. The aim of this study was to experimentally examine the effect of imposing set shifting demands on objectively-measured activity level in children with and without ADHD.

Participants and Methods: The current study used the global-local task (Miyake et al., 2000) and two control variants (global-global, local-local) to impose set shifting demands in children ages 8-13 with ADHD (n=43) and without ADHD (n=30). Shift costs were calculated $\left(\mathrm{RT}_{\text {shift }}-\mathrm{RT}_{\mathrm{no}-\text {-shift }}\right)$ to confirm that the global-local task imposed set 
shifting demands. Activity level was sampled during each task using high-precision actigraphs. Total hyperactivity scores (THS) were calculated by summing activity level across three actigraph sites ( 2 ankle, 1 nondominant hand).

Results: Results of a 2x3 ANOVA for shift costs revealed that the experimental manipulation was successful (task main effect, $p<.001, \omega^{2}=.19$ ), such that the global-local task elicited greater shift costs than did the control conditions. Results of a 2x3 ANOVA for hyperactivity revealed a significant main effect of task $\left(p=.005 ; \omega^{2}=.02\right)$ where imposing set shifting demands significantly increased THS. However, this manipulation did not disproportionally increase hyperactivity in ADHD as demonstrated by non-significant group $(p=.09)$ and task $\mathrm{x}$ group interaction effects $(p=.56)$.

Conclusions: Results of this manipulation indicate that set shifting demands increase activity level in children, but do not differentially affect children with ADHD. These findings suggest that set shifting is unlikely to play an etiological role in eliciting/maintaining hyperactive behavior in ADHD.

Correspondence: Lauren N. Irwin, Psychology, Florida State University, 1107 W. Call Street, Tallahassee, FL 32304, United States. E-mail: irwin@psy.fsu.edu

L.A. KATZ, L. BALABAN, S.A. LANGENECKER \& A. PASSAROTTI. Inhibitory Control in Children with Pediatric Bipolar Disorder and Attention-Deficit/Hyperactivity Disorder.

Objective: The purpose of this study is to examine inhibitory control in children with Pediatric Bipolar Disorder (PBD) compared to children with Attention-Deficit/Hyperactivity Disorder (ADHD) and healthy controls (HC) using a Stop Signal Task (SST) and the Behavior Rating Inventory of Executive Function (BRIEF). The SST, a well-established measure of inhibitory control, has not been extensively studied in children with PBD. We hypothesized that the PBD group would demonstrate poorer performance on the SST compared to HC, but would perform similarly to children with ADHD, as both groups have inhibition deficits. Furthermore, BRIEF scores for the Emotion Regulation subscale would differentiate children with PBD from children with ADHD, but not other subscales. Finally, BRIEF scores on the Inhibit subscale would be inversely correlated with performance on the SST across groups.

Participants and Methods: 40 subjects ages 9 to 18 years $(\mathrm{PBD}=12$, $\mathrm{ADHD}=16$, Healthy Controls=12) completed the SST and the BRIEF. Results: Results from a one-way ANOVA indicate near significance between groups on "stop" trials of the SST, $[F(2,37)=3.14, p=.055]$. A Tukey post hoc test revealed that SST accuracy was significantly lower in the $\mathrm{ADHD}(\mathrm{M}=0.60, \mathrm{SD}=0.09)$ group compared to HC $(\mathrm{M}=0.69$, $\mathrm{SD}=0.07),[\mathrm{p}=0.051]$; however, the $\mathrm{PBD}(\mathrm{M}=0.66, \mathrm{SD}=0.13)$ group did not significantly differ from the ADHD group $[p=0.26]$ or $\mathrm{HC}[\mathrm{p}=0.71]$. Emotion Regulation scores on the BRIEF significantly differentiated the ADHD (M=51.25, $\mathrm{SD}=7.81)$ and $\mathrm{PBD}(\mathrm{M}=71.33, \mathrm{SD}=14.14)$ groups $[\mathrm{t}(11)=3.74, \mathrm{p}=.003]$. Scores on the Inhibit subscale of the BRIEF did not correlate with SST performance across groups; however, there were other notable BRIEF trends, particularly for the PBD group (i.e. Global Executive Composite $[\mathrm{p}=.065])$.

Conclusions: Future research should be conducted to better assess and understand the underlying nature of inhibition and executive function deficits in PBD and ADHD.

Correspondence: Lindsay A. Katz, M.A., Psychology, Roosevelt University, $630 \mathrm{~N}$ State St, \#1510, Chicago, IL 60654, United States. E-mail:lkatz1791@gmail.com

K. KIM, C.W. YEATMAN, S. DEBOARD-MARION, J. TURNBULL, A.A. TURK NOLTY \& Y. OH. Sluggish Cognitive Tempo and Executive Functioning in Children With Learning Difficulties.

Objective: Behavioral symptoms such as drowsiness, lethargy, and hypoactivity are characteristics of individuals with sluggish cognitive tempo (SCT), which is associated with dysfunction in attention and executive functioning. We hypothesized that for children with identified learning difficulties, levels of attention and executive functioning would be negatively correlated with SCT symptoms.

Participants and Methods: We recruited 50 youth (31 boys, 19 girls) between the ages of 5 and 14 from an Emerging Needs program of a private Christian school, obtaining SCT scores from 4 items on the parent Child Behavior Checklist (CBCL), and 5 items on the Teacher Report Form (TRF). We measured executive functioning from parent and teacher ratings on the Behavior Rating Inventory of Executive Function (BRIEF), and utilized attention markers from the Conners' Continuous Performance Test, Second Edition.

Results: The average SCT score derived from the CBCL was 0.29 $(S D=0.36)$, and from the TRF, $0.47(S D=0.49)$, where scores over 0.67 on the CBCL or 0.75 on the TRF were considered to indicate high SCT. Most of the BRIEF subscale scores were significantly and negatively correlated with SCT, with Working Memory problems reported by both parents' and teachers' ratings most strongly associated with the level of SCT. In addition, weak trends toward significant negative correlations were found between reaction-time-related attention markers and SCT.

Conclusions: Children with higher levels of SCT may take longer to react to stimuli than children without SCT, which may mean that they have a more careful approach to tests and somewhat slower, more measured reaction time. In everyday life, SCT seems to have a stronger association with the cognitive capacity for self-managing and self-monitoring tasks than with the ability to control emotions and behaviors. This study suggests that SCT may be an important consideration for some children with learning difficulties

Correspondence: Kyoungjun Kim, Ph.D., School of Psychology, Fuller Theological Seminary, 26 Alice st, Apt A, Arcadia, CA 91006, United States.E-mail:wisp3457@hotmail.com

N. KORHONEN, M. VIRTA, J. LAUNES, S. LEPPÄMÄKI, K. MICHELSSON \& L. HOKKANEN. Childhood ADHD Symptoms and Intellectual Functioning in Adulthood.

Objective: The aim of this study was to investigate intellectual functioning of adults with childhood ADHD symptoms in a group with perinatal risks. Association between lower IQ scores and ADHD in childhood has been substantiated but the results for adults are more varied. Also, evidence is lacking on whether differences in IQ in ADHD adults are due to attention deficits per se or display an initial association of ADHD and lower IQ.

Participants and Methods: The subjects are part of a Finnish longitudinal cohort with perinatal risks followed from birth. The participants were divided into three groups according to clinically assessed and informant-reported ADHD symptoms: non-ADHD (nADHD); subclinical ADHD (sADHD) and probable ADHD (ADHD). Estimated IQs were assessed with subtests from the Wechsler Intelligence Scale for Children (WISC) at 9 years and Wechsler Intelligence Scale -IV (WAIS-IV) at 40 years.

Results: Cognitive performance at 9 years significantly correlated with IQ estimates at 40 years in all groups [overall $r(341)=.690$, $p<.001]$. The ADHD group $(\mathrm{n}=50)$ performed significantly poorer than the $\mathrm{nADHD}(\mathrm{n}=190)$ and sADHD $(\mathrm{n}=91)$ groups even after controlling for childhood IQ $[F(2,331)=6.40, p=.002, \eta 2 \rho=.038]$. The mean adjusted difference between the ADHD and the nADHD groups in IQ scores was 7.466 [95\% CI 12.520, 2.413].

Conclusions: Adults with perinatal risks who have displayed symptoms of ADHD in childhood score lower on IQ estimates. Furthermore, even though IQ scores for the ADHD group were initially lower in childhood, IQ in adulthood was independently associated with ADHD symptoms observed in childhood. These results suggest childhood ADHD symptoms are associated with lower cognitive performance throughout the lifespan. 
Correspondence: Nella Korhonen, Master of Psychology, Department of Psychology, University of Helsinki, Munkkisaarenkatu 10 C 39, Helsinki 00150, Finland.E-mail: nella.korhonen@helsinki.fi

G.J. LEE \& J. SUHR. The Effects of Treatment Expectancy on Subjective and Neuropsychological Outcomes in Neurofeedback (NFB) for Attention-Deficit/Hyperactivity Disorder (ADHD): A Pilot Study.

Objective: Placebo-controlled trials of NFB for ADHD are inconclusive as to its effectiveness, yet recognize expectancy as a key mechanism of the placebo effect. Treatment expectancies are anticipatory cognitions regarding effects of treatment on health that can shape outcomes. However, there have been limited NFB studies measuring effects of these expectancies. We examined effects of positive and negative NFB expectations on self-reported ADHD symptoms and neuropsychological performance. Participants and Methods: Forty-six undergraduates concerned about ADHD expected to receive active NFB, but instead were randomly assigned to receive placebo with false feedback indicating they successfully reached an attentive state (positive false feedback group; $n=23$ ) or failed to reach an attentive state (negative false feedback group; $n=$ 23). Effects of expectancy manipulation were measured on an ADHD self-report scale and visual and auditory Continuous Performance Test (CPT) pre- and post-NFB. Expectancy effects were analyzed with mixed ANOVA and MANOVAs.

Results: As expected, groups did not differ in ADHD symptoms pre-NFB $(p=.52)$, but differed post-NFB $(p=.001)$. Those who received positive false feedback reported decreases in ADHD symptoms from pre- to post-NFB $(p<.001)$, while those who received negative false feedback reported increases in symptoms $(p=.01)$. There was a significant interaction for hit rates on visual CPT trials, such that performance declined pre- to post-NFB for individuals who received negative false feedback ( $p=.01)$, but did not change for those who received positive false feedback $(p=.47)$. Hit rates did not change on auditory CPT trials. No significant effects were found for false alarms.

Conclusions: Results suggest a large effect of treatment expectancies on symptom report, though neuropsychological performance yielded mixed evidence for expectancy effects. Placebo-controlled trials of NFB should consider outcomes according to treatment expectancies, especially when using self-report scales.

Correspondence: Grace J. Lee, B.S., Psychology, Ohio University, Ohio University, 200 Porter Hall, Athens, OH 45701, United States. E-mail: gl107015@ohio.edu

E.T. MARCELLE, E.B. OWENS \& S. HINSHAW. Hypertensive Pregnancy Predicts Improved Working Memory Functioning in ADHD.

Objective: A significant portion of the functional impairment associated with ADHD can be attributed to a reduction in working memory (WM) capacity, yet contributors to the variability of WM capacity within this population remain unknown. The aim of the present study was to examine the associations between prenatal risk factors and WM capacity, in adolescent females with ADHD.

Participants and Methods: The ethnically diverse sample included 140 girls carefully diagnosed with ADHD during childhood and followed with high retention into adolescence ( $\mathrm{M}$ age $=14.5$ years $)$. Prenatal risk factors included maternal hypertension during pregnancy and maternal smoking during pregnancy, both assessed via retrospective report. WM capacity was assessed by combining standardized Wechsler Abbreviated Scale of Intelligence Letter-Number Sequencing and Digit Span Backwards scores. Multiple regression analyses were used to explore the extent to which maternal hypertension and smoking predicted adolescent WM functioning, covarying for child IQ (Wechsler) and maternal age during pregnancy.

Results: Results disconfirmed our hypothesis that hypertension and smoking during pregnancy would be associated with lowered WM capacity. Hypertensive pregnancy status positively and significantly predicted WM capacity $\left(B=1.088, p=0.025, R^{2}\right.$ change $\left.=0.05\right)$, above and beyond maternal age and child IQ. Maternal smoking during pregnancy was not found to be a significant predictor of adolescent WM capacity $(\mathrm{p}=0.91)$.

Conclusions: These findings suggest that among children with ADHD, maternal hypertension during pregnancy may serve as a protective factor regarding WM capacity. Mechanisms may be that moderately increased blood pressure in the pregnant mother may enhance nutrient supply to the brain of the developing fetus at risk for ADHD, potentially mitigating heritable neurodevelopmental abnormalities otherwise producing impaired WM. Future research may seek to elucidate these mechanisms and this brain-heart relation.

Correspondence: Enitan T. Marcelle, Psychology, UC Berkeley, 2205 Tolman Hall, Berkeley, CA 94720, United States. E-mail: emarcelle@ berkeley.edu

C. MILLER, B. BROOKER \& M. CAIRNCROSS. Mindfulness-based Interventions as Complementary Therapy for Attention Problems. Objective: Mindfulness-based interventions (MBI) descend from Buddhist traditions, but have been co-opted as secular intervention practices for medical, neurodevelopmental, and mental health disorders. Mindfulness meditation is commonly used to learn to focus one's attention on the present moment, and to reduce maladaptive cognitive processes, such as rumination. There is significant research evidence to support how MBI improve focused/selective attention and reduce the impact of impulsivity, symptoms which can be particularly challenging for individuals with Attention-deficit/Hyperactivity Disorder (ADHD) and related difficulties. This paper will present evidence from three separate studies investigating the role of MBI in improving attention and self-control.

Participants and Methods: The first study was a meta-analysis investigating MBI for children and adults with ADHD, with results that indicate MBI improve attention and impulsivity in people with ADHD. The second study implemented a brief daily MBI in a university course with resulting improvements in self-reported mind-wandering and distractibility during class. The third study implemented a seven-session MBI for pre-adolescents with ADHD using a wait-list, case-control research design. Participants exhibited improvement on an objective measure of sustained attention/inhibitory control and parents reported improvements in their child's overall functioning during the MBI.

Results: Across studies, it is clear that MBI show significant promise in improving attention across the spectrum of ADHD symptoms.

Conclusions: Given its effectiveness for this purpose and numerous additional benefits (e.g., MBI are relatively low cost due to the use of small group programming, are easy generalized to novel settings, are well-tolerated by clients and their families, and do not necessarily require the services of specialist physicians), we assert that MBI may be viewed as a helpful adjunct/complement to traditional (pharmacological and psychosocial) therapies for ADHD.

Correspondence: Carlin Miller, PhD, Psychology, University of Windsor, 401 Sunset, Windsor, ON N9B3P4,Canada.E-mail: cjmiller@uwindsor.ca

A. MURO, J. BORRANI, A. GARCIA, C. RAMIREZ \& P. VALDEZ. Analysis of the components of attention in early and late adolescents.

Objective: The objective of this study was to compare the components of attention (tonic alertness, selective attention, phasic alertness and sustained attention) between early and late adolescents.

Participants and Methods: There were 153 participants, a group of 68 early adolescents ( 31 female, mean age: $10.40 \pm 0.49$ years, range $=$ $10-11$ years) and a group of 85 late adolescents ( 60 female, mean age: $18.35 \pm 1.53$ years, range $=17-19$ years $)$. All participants responded a modified continuous performance task, designed to assess each component of attention. In this version 540 numbers from $0-9$ were presented randomly on a computer screen. The participants had to answer to any number from $0-8$ by pressing the number 1 on the keyboard (tonic 
alertness index), they had to press the number 2 on the keyboard when a number 9 appeared (selective attention index) and they had to press the number 3 on the keyboard when a number 4 appeared after a number 9 (phasic alertness index). Task duration was 11 minutes and 42 seconds. Results: Early adolescents had a lower level of tonic alertness (early adolescents $78.44 \pm 11.08 \%$, late adolescents $94.48 \pm 9.53 \%, \mathrm{t}=-9.63$, $\mathrm{p}<0.001$ ), selective attention (early adolescents $48.73 \pm 14.56 \%$, late adolescents $72.49 \pm 18.53 \% ; \mathrm{t}=-8.65 \mathrm{p}<0.001)$, and phasic alertness (early adolescents $61.38 \pm 19.17 \%$, late adolescents $84.68 \pm 13.23 \%$; $\mathrm{t}=-8.87 \mathrm{p}<0.001)$. No differences were observed between age groups in the sustained attention index.

Conclusions: The results imply that early adolescents have a lower level of development in three components of attention: tonic alertness, selective attention and phasic alertness, compared to late adolescents. It is important to consider this pattern of development for the neuropsychological assessment of attention in adolescence.

Correspondence: Angelica Muro, School of Psychology, Universidad Autónoma de Nuevo León, Mutualismo \#110, Mitras Centro, Monterrey 64460,Mexico.E-mail: naiielymuro95@gmail.com

M.E. NITTA, D.E. MARRA, E. VOGT, K.A. RITCHIE, P. MARSHALL \&. J. HOELZLE. Performance and Symptom Validity does not Impact the two Dimensional Structures of Barkley's Adult ADHD Rating Scale-IV Self-Report Measures.

Objective: Symptom validity is important to consider during interpretation of self-report measures. Particularly within adult ADHD samples, individuals may exaggerate symptoms for secondary gain. This study evaluates the underlying factor structure of Barkley's Adult ADHD Rating Scale-IV (BAARS-IV) self-report measures before and after excluding individuals with possibly invalid profiles.

Participants and Methods: Retrospective chart review was conducted of adult $(N=400)$ ADHD evaluation results [age $M=26.34$ (7.6), education $M=14.45$ (1.7)]. Each adult completed a BAARS current and childhood symptoms self-report measures, in addition to other neuropsychological measures. Individual profiles $(n=106)$ were identified as possibly invalid based on symptom and performance validity tasks. An independent samples $t$-test was conducted to evaluate group differences in symptom report frequency. Exploratory factor analysis (EFA) was conducted to evaluate the BAARS factor structure. EFA was conducted twice, once making use of the full sample $(N=400)$ and then after invalid profiles were excluded $(n=294)$.

Results: Individuals presenting in a non-credible manner reported a greater number of symptoms (Inattentive: Current $d=0.75$; Childhood $d=0.65$; Hyperactive/Impulsive: Current $d=1.06$; Childhood $d=0.92)$. EFA identified clear dimensions of inattention and hyperactivity when including all participant data. The factor structure did not meaningfully change after excluding individuals with suspect effort.

Conclusions: An emerging literature makes clear that research findings may change dramatically after excluding individuals with suspect effort. Nevertheless, this research identified highly congruent, robust dimensions underlying BAARS self-report measures before and after excluding individuals responding in a non-credible manner. While this supports the BAARS construct validity, additional research warrants further investigation of how psychometric properties (e.g., predictive validity) may change depending on response validity.

Correspondence: Morgan E. Nitta, Psychology, Marquette University, 2212 N. Holton St, Milwaukee, WI 53212, United States. E-mail: morgan.nitta@marquette.edu

\section{OSTOJIC, B. BROOKER \& C. MILLER. Using Ecological Momentary Assessments to Evaluate Extant Measures of Mind Wandering.}

Objective: Mind wandering is a commonly experienced phenomenon, and considered a characteristic feature of ADHD. Although self-report measures have been developed to uniquely capture these attentional lapses, examination of their correspondence with the reported frequency of these episodes in daily life is warranted. Using ecological momentary assessments (EMA), the present study aimed to validate 3 mind wandering measures: Mind Wandering Questionnaire (MWQ; Mrazek et al., 2013) and the Mind Wandering-Spontaneous (MWS) and Deliberate measures (MWD; Carriere et al., 2013).

Participants and Methods: First, university students $(N=100)$ completed a series of questionnaires, including mind wandering measures, and were instructed on the EMA data collection procedure. Next, using time-based EMA sampling, participants received 6 prompts daily, delivered via text-message, for 7 days. Each prompt asked students to report if their current thoughts were about something other than what they were doing using a scale ranging from completely on-task to completely on unrelated concerns.

Results: When examining the relation of self-report data with all EMA responses received (irrespective of response latency), higher mind wandering reported during daily life was associated with higher MWQ and MWS scores, but not with the MWD score. When specific thresholds for response latency were applied (e.g., responses received after 5, 20, and 30 minutes following the EMA prompt were respectively excluded), findings remained consistent; however, the strength of the relationship varied based on the time threshold used.

Conclusions: Results highlight the utility of EMA in validating measures designed to capture mind wandering episodes and the importance of reporting latency thresholds to promote comparison across studies. Because individuals with ADHD are often poor reporters of their symptoms, EMA holds promise as another strategy for assessing the impact of distractibility in daily life.

Correspondence: Dragana Ostojic, M.A. , Psychology, University of Windsor, 801-299 Mill Rd., Toronto, ON M9C 4V9, Canada. E-mail: ostojicd@uwindsor.ca

C. PATROS, M. MARTINELLI, S. MOSTOFSKY, K. SEYMOUR \& K.S. ROSCH. Predicting Adolescent Risk-Taking in AttentionDeficit/Hyperactivity Disorder: A Longitudinal Study.

Objective: Adolescence is characterized by heightened risk-taking behavior with a host of deleterious outcomes. Risk-taking behavior is often exacerbated among individuals with attention-deficit/hyperactivity disorder (ADHD). There is a need for longitudinal research examining neurocognitive predictors of risk-taking among adolescents with ADHD to better understand the developmental trajectory of ADHD. Participants and Methods: Children were comprehensively assessed between the ages of 8-12 years (time point 1 ) and at least two years later between the ages of 12-17 years (time point 2 ). using a variety of rating scales, clinical interviews, and cognitive testing. A total of 19 children diagnosed with ADHD at time point 1 and a typically-developing control group $(n=14)$ were included in analyses. Measures of working memory, response control, and delay discounting obtained at time point 1 were examined as predictors of an experimental measure of risk taking (the balloon analogue risk task) at time point 2 .

Results: A series of linear regressions were conducted to investigate variance in adolescent risk taking accounted for by childhood working memory, response control, and delay discounting. Among children with ADHD, delay discounting at time point 1 was a statistically significant predictor of risk taking at time point $2, \beta=-.584, \mathrm{t}(10)=-2.28$, $\mathrm{R}^{2}=.34, p=.046$. Neither response control nor working memory assessed during childhood predicted risk taking in adolescence among children with ADHD ( $p s>.19)$ and there were no significant predictors among TD controls ( $p$ s > .49)

Conclusions: Findings compliment previous work suggesting that children with ADHD who show a stronger preference for immediate reward have a propensity to make risky decisions during adolescence. Treatments that address the preference for immediate, suboptimal rewards during childhood, or address deficient cognitive constructs that underlie maladaptive choice, might attenuate future risky behavior.

Correspondence: Connor Patros, PA, United States.E-mail:chopatros@ gmail.com 
R.J. RICHARDSON, A.M. O'BRIEN, S. DEASLEY, T.A. DUDA \& J.E. CASEY. Errors on a Graphomotor Tracking Task as a Predictor of Pediatric ADHD.

Objective: Historically, most neuropsychological tests and neuroimaging measures are unable to demonstrate adequate sensitivity and specificity for discriminating between those with and without ADHD. Deficits in cognitive control (or response inhibition) may be a key variable underlying symptoms of ADHD, including difficulties inhibiting prepotent motor responses. This is consistent with data documenting motor control problems in an estimated 30 to $50 \%$ of children with ADHD. The present study sought to investigate whether errors made during a motor tracking task requiring high cognitive control differentiated between those with and without ADHD.

Participants and Methods: Participants $(N=40)$ included a community sample of children aged $9-15$ ( $M_{\text {age }}=11.8$ years $)$, of whom 16 met diagnostic criteria for ADHD. Participants used a stylus to follow a cursor that moved in a wavy or linear pattern on a digitizing tablet. Errors on this task were one of two types: (1) cognitive control errors (touching the tablet too soon or moving the stylus past the cursor), and (2) invalid trial errors (prematurely terminating a trial, timing out on a trial, or tracking an incorrect pattern). Receiver operating characteristic (ROC) curve analysis was employed to determine the diagnostic utility of the task.

Results: ROC demonstrated that the area under the curve was 0.67 (SE $=0.093,95 \% \mathrm{CI}[0.487,0.852], p=.073)$. The optimal cut-off score to maximize sensitivity and specificity was 10.5 errors (sensitivity $=0.69$, specificity $=0.67, J=0.36)$. A cut-off of 10.5 errors on the task still incorrectly classifies over $30 \%$ of controls (false positive rate $=33 \%$ ) and ADHD participants (false negative rate $=31 \%$ ).

Conclusions: ROC curve analysis revealed that errors on the high cognitive control motor tracking tasks classified children with and without ADHD fairly accurately. Although the performance-based task shows potential as a diagnostic screener for ADHD, additional research with a larger sample is needed.

Correspondence: Robin J. Richardson, B.A. Hons., Psychology, University of Windsor, 401 Sunset Ave, Department of Psychology, Windsor, ON N9B 3P4, Canada.E-mail: richar1a@uwindsor.ca

\section{A.R. ROECKNER, M. EPPERSON, D. HUDDLESTON, E. PEDAPATI, S. WU, S. MOSTOFSKY \& D. GILBERT. Comparison of Motor Cortex Physiology During Response Inhibition in Children with ADHD vs. Controls.}

Objective: The main purpose of this study is to determine if motor cortex inhibitory physiology during response inhibition differs between children with ADHD ages 8-12 years and typically developing (TD) control children.

Participants and Methods: Using Transcranial Magnetic Stimulation (TMS), short interval cortical inhibition (SICI) was evaluated in dominant motor cortex at baseline (20 trials) and during two blocks (80 trials) of a stop signal reaction time (SSRT) task in 80 right-handed children, (42 ADHD: mean age 10.7 years, 30 boys, 20 Caucasian; 38 TD: mean age 10.4 years, 24 boys, 28 Caucasian). A TMS-compatible child-friendly, race-car "Slater-Hammel" task was used, with optimal GO responses from 700 to $800 \mathrm{msec}$ and, in $25 \%$ of trials, dynamically timed STOP signals (which shift based on success/failure). Single and 3 msec paired (inhibitory) TMS pulses were timed to occur within 150 msec prior to the GO response and 150 msec after the STOP signal. Responses and physiological data were averaged by trial type and compared using t-tests.

Results: In children with ADHD (Dupaul Parent ADHD-Rating Scale score mean=32 out of 54; SD=10), Stop Signal Reaction Times were not different (ADHD mean=248 msec, SD=73; Control mean=230 msec, $\mathrm{SD}=56, \mathrm{p}=.23)$. However, GO (finger lift) times during were significantly later $(\mathrm{ADHD}$ mean $=840 \mathrm{msec}, \mathrm{SD}=55$; Control mean $=819 \mathrm{msec}$, $\mathrm{SD}=32, \mathrm{p}=.04)$. Compared to TD children, ADHD children averaged $29 \%$ less SICI (motor cortex inhibition) at rest $(p=.04) ; 37 \%$ less SICI during GO trials $(p=.26)$; and $70 \%$ less SICI during successful STOP trials $(\mathrm{p}=.003)$.

Conclusions: ADHD children demonstrated significantly less motor cortex inhibition (SICI) while successfully stopping in this response inhibition task. This may indicate differences in underlying mechanisms for successful stopping in ADHD.

Correspondence: Alyssa R. Roeckner, BS, Neurology, Cincinnati Children's Hospital, 3333 Burnet Ave, Cincinnati, OH 45229, United States.E-mail: Alyssa.Roeckner@cchmc.org

\section{J. ROITSCH, L. PHALEN \& S. WATSON. Predictors of Comfort Level in Speech-Language Pathologists (SLPs) Working with Students with Attention Deficit Hyperactivity Disorder (ADHD).} Objective: Although the comorbidity of ADHD with speech/language disorders is well-documented in the literature (Mueller \& Tomblin, 2012; Westby \& Watson, 2003), little is known regarding the comfort levels in working with children with ADHD. The purpose of this research was to determine what variables could be used to predict SLP comfort level when working with children with ADHD.

Participants and Methods:

A survey was posted to the discussion boards of two of the American Speech-Language-Hearing Association (ASHA)'s Special Interest Groups (SIGs). Following 6 weeks of posting, 86 school SLPs whose clinical caseload included students diagnosed with ADHD participated in the survey. Data were anonymously collected and analyzed via SurveyMonkey and IBM SPSS statistical software, version 22.

Results: Results of a logistic regression revealed one significant predictor ... adequate training. With an odds ratio of 7.90 , this survey suggests that SLPs who felt they had adequate training were 7.9 times more likely to report they were very comfortable working with students with ADHD than those who answered "no" when queried if they felt they had received adequate training. Simply stated, SLPs who felt they had adequate training were about $690 \%$ more likely to say they felt very comfortable working with students with ADHD.

Conclusions: The importance of providing education and training to SLPs during their graduate schooling cannot be understated. Further, this research suggests the need to ensure that SLPs are provided with tools to assess and treat this unique and growing population of students. As students are increasingly being referred for SLP services, many SLPs suggest that they sought out professional development coursework to augment their skill sets upon graduation when presented with students with ADHD on their caseload. The importance of addressing this need in graduate school can save resources, reduce frustration and provide optimal student assessment and intervention.

Correspondence: Jane Roitsch, Doctoral Student, Communication Sciences and Special Education, Old Dominion University, 1915 A EscadrillePt, Norfolk,VA 23508,United States.E-mail:jroitsch@odu.edu

K.S. ROSCH, A.W. SALI, B. ANDERSON, S. YANTIS \& S. MOSTOFSKY. Reduced Value-Driven Attentional Capture Among Children with ADHD Compared to Typically Developing Controls.

Objective: The current study examined whether children with ADHD were more distracted by a stimulus previously associated with reward, but currently goal-irrelevant, than their typically-developing peers. In addition, we also probed the associated cognitive and motivational mechanisms by examining correlations with measures of working memory and immediate reward preference (delay discounting).

Participants and Methods: Participants included 8-12 year-old children with $\mathrm{ADHD}(\mathrm{n}=28)$ and typically developing (TD) controls $(\mathrm{n}=26)$. Children were instructed to visually search for color-defined targets and received monetary rewards for accurate responses. In a subsequent search task in which color was explicitly irrelevant, we manipulated whether a distractor item appeared in a previously reward-associated color. We examined whether children responded more slowly on trials on which the previously-rewarded was distractor present compared to 
trials without this distractor, a phenomenon referred to as value-driven attentional capture (VDAC), and whether ADHD and control children differed in the extent to which they displayed value-driven attentional capture. Correlations among working memory performance, delay discounting and attentional capture were also examined.

Results: Although children with ADHD and TD controls initially showed significant value-driven attentional capture, children with ADHD were significantly less affected by the presence of the previously rewarded distractor than were control participants, $(p<.001)$. Furthermore, within the ADHD group, greater value-driven attentional capture was associated with poorer working memory performance $(r=-.44$, $p=.045)$ but not with delay discounting $(r=-.04, p=.855)$.

Conclusions: Although both ADHD and control participants were initially distracted by stimuli previously associated with reward, the magnitude of distraction was larger and persisted longer among control participants.

Correspondence: Keri S. Rosch, Ph.D., Neurology, Kennedy Krieger Institute, 716 North Broadway, Baltimore, MD 21205, United States. E-mail:ksrosch@gmail.com

\section{E. SUllivan \& C. NEUMANN. Assessing the Relationship between PTSD Symptoms and Attention in a Trauma-Impacted Female Sample.}

Objective: Trauma and posttrauma symptoms, including those present in PTSD, may cause disruptions in normal neuropsychological functioning. Facets of attention, including response time, omissions, and commissions, may be particularly affected. The present study sought to examine trends in PTSD symptoms and performance on attention measures in women. It was predicted that a positive correlation would exist between PTSD symptom severity and self-reported concentration difficulty. A negative correlation was predicted between PTSD symptoms and attention task performance.

Participants and Methods: A total of 25 female participants (mean age $=21.48, \mathrm{SD}=4.44$ ) were recruited from the University of North Texas and the surrounding community. All participants had experienced a traumatic event meeting DSM-5 Criterion A for PTSD. Each participant completed the Beck Depression Inventory (BDI), PTSD Checklist (PCL), and the CAPS structured interview. The computerized Conners' Continuous Performance Task 2 (CPT-2) assessed attention.

Results: CAPS PTSD severity was positively correlated with self-report items assessing concentration difficulties on the PCL $(\mathrm{r}=.64, \mathrm{p}<.01)$ and BDI $(\mathrm{r}=.45, \mathrm{p}<.05)$. When age was controlled for, CAPS severity was positively correlated with CPT commission errors $(\mathrm{r}=.414, \mathrm{p}<.05)$ and negatively correlated with CPT response time $(r=-.50, \mathrm{p}<.05)$. Conclusions: Women who have experienced trauma and have high PTSD symptoms may notice more difficulty with concentration. Results from an attention task suggest those with greater PTSD symptom severity may demonstrate more impulsivity, evidenced by higher commission errors and faster response time. Hypervigilance may help explain this relationship.

Correspondence: Erin Sullivan, Clinical Psychology, University of North Texas, 2025 Lakepointe Drive, Apt 28D, Lewisville, TX 75057, United States.E-mail: ErinSullivan2@my.unt.edu

\section{K.L. SWEENEY, H. SCHNEIDER, M. RYAN, L. FERENC, M. DENCKLA \& E.M. MAHONE. Finger Tapping Speed in Preschool Predicts ADHD Status and Symptom Trajectory.}

Objective: Children with ADHD manifest nonlinear trajectories of core symptoms between ages 4 and 7 years, with greatest change occurring by age 6 . Those motor skills showing the greatest overlap in development with change in $\mathrm{ADHD}$ symptoms may represent critically sensitive early biomarkers of the condition.

Participants and Methods: Participants included 48 children (22 ADHD, 26 typically developing-TD) ages $4-7$ years (62\% male) who completed three visits (T1, T2, T3), each one year apart (T1=48-

$71, \mathrm{~T} 2=60-83, \mathrm{~T} 3=72-95$ months). Four motor variables included
Total Overflow, Finger Tapping Time, Finger Sequencing Time, and Total Axial score from the Revised Physical and Neurological Examination for Subtle Signs (PANESS). ADHD symptoms from the Conners' Parent Rating Scale-Revised were obtained at each visit. Stepwise linear discriminant function analysis was used to predict group membership, and included each of the 4 motor scores at each visit, as well as all change scores (T1-T2, T2-T3). For those motor variables contributing significantly to group discrimination, the associations with ADHD symptom trajectory were examined using Pearson correlations.

Results: The discriminant function produced a significant degree of dissimilarity between groups. The only score to enter significantly into the discriminant function was T1 Finger Tapping Time, correctly classifying $75 \%$ of the sample. Within the ADHD group, inattentive symptoms at T1 predicted rate of improvement (T1-T2) in Finger Tapping Speed $(p=.05)$. The strongest association between ADHD symptom change and improvement in motor skills was also between T1-T2-inattentive symptoms predicting Finger Tapping $\left(\mathrm{r}^{2}=.10\right)$.

Conclusions: By age 4 years, "basic" repetitive skeletomotor speed represents a highly sensitive motor marker of ADHD, while overflow, axial movements and finger sequencing appear insensitive markers at that age. Between ages 4 and 6 years, finger tapping speed improves in parallel with inattentive symptoms of ADHD, suggesting a shared neural substrate.

Correspondence: Kristie L. Sweeney, Master of Science, Neuropsychology, Kennedy Krieger Institute, 1750 East Fairmount Ave, Baltimore, MD 21231, United States. E-mail: Sweeney@kennedykrieger.org

S.K. TADROUS-FURNANZ, S. DEBOARD-MARION, S. AMANO, C.W. YEATMAN \& A.A. TURK NOLTY. Can Cogmed have FarTransfer Effects on Reading for some Children with Emerging Needs?

Objective: The scientific community has shed significant doubts on the effectiveness of Cogmed working memory training among typically developing individuals, especially with regard to far-transfer effects. However, some studies have shown promising results for children with attention difficulties and learning challenges. Our aim was to examine whether children with emerging needs would show improvements in reading comprehension or oral reading fluency along with gains in working memory following computerized working memory training.

Participants and Methods: 17 children between the ages of 7.5 and 15 with difficulties in learning and attention (but Pseudoword Decoding scores > 85) completed five half-hour sessions per week of Cogmed working memory training over a period of 5 weeks. The children underwent neuropsychological assessment before and after training.

Results: When compared to the 6 children whose WISC-IV Working Memory Index did not improve, the subset of 11 children with an improved Working Memory Index posttraining showed significantly more improvement in both Reading Comprehension, $t(15)=2.68, p=$ .020 , and Oral Reading Fluency, $t(14)=2.66, p=.019$.

Conclusions: For individuals with emerging needs, Cogmed seems to contribute to improvement in some children's working memory performance, with subsequent potential far-transfer effects to areas such as reading comprehension and oral reading fluency. Yet, results need to be considered in light of the small sample size and this sample's characteristics that limit the generalizability of our findings.

Correspondence: Sandy K. Tadrous-Furnanz, MA, Psychology, Fuller Theological Seminary, 670 N. Hill Avenue, Apt \# 6, Pasadena, CA 91106, United States.E-mail: sandytadrous@fuller.edu

S. TAMEZ, G. ALANÍS, C. AZEVEDO, A. BELISIO, C. RAMIREZ, A. GARCIA, J. BORRANI \& P. VALDEZ. Changes of Components of Attention in Children from 4 to 7 Years of age.

Objective: The objective of this study was to analyze changes in the components of attention of children $4-7$ years of age.

Participants and Methods: Participants were 125 children (59 male, 66 female, age $=6.38 \pm 1.10$ years; mean $\pm \mathrm{SD})$ that responded a 
modified continuous performance task designed to assess the components of attention in children; task duration was 4 min and $20 \mathrm{~s}$. In this task, four types of figures (a heart, a ball, an arrow or a star) were used. The responses were recorded with the numeric keypad of a keyboard, in which the keys 1, 2 and 3 were respectively covered with a heart, a ball and an star. Participants had to press the key with the figure that matched the figure on the screen. Responses to the heart (the most frequent stimulus) indicated tonic alertness, responses to the ball (an infrequent stimulus that requires a specific answer) indicated selective attention, responses to the star (a stimulus always preceded by an arrow) indicated phasic alertness and sustained attention was measured through changes during the whole task (linear regression). Results: Tonic alertness improved with age on correct responses (68.09 $\pm 12.83 \%, 91.13 \pm 7.20 \%$, averages for 4 and 7 years old, respectively, $\mathrm{F}=7.17, \mathrm{p}<0.001)$ and reaction time $(985.05 \pm 96.45 \mathrm{~ms}, 735.72$ $\pm 145.91 \mathrm{~ms}, \mathrm{~F}=7.20, \mathrm{p}<0.001)$. Selective attention also improved on correct responses $(65.36 \pm 14.33 \%, 87.93 \pm 12.28 \%, \mathrm{~F}=7.17$, $\mathrm{p}<0.001)$, and reaction time $(1070.93 \pm 86.31 \mathrm{~ms}, 795.06 \pm 144.22 \mathrm{~ms}$, $\mathrm{F}=10.76, \mathrm{p}<0.001)$. Phasic alertness improved on reaction time (893.10 $\pm 138.14 \mathrm{~ms}, 559.11 \pm 176.68 \mathrm{~ms}, \mathrm{~F}=9.44, \mathrm{p}<0.001)$. Sustained attention also improved, since the linear regression approached zero as age increased, which means that performance remains at the same level from the beginning to the end of the task $(\beta=-0.46 \pm 0.38, \beta=0.00 \pm$ $0.50, \mathrm{~F}=3.12, \mathrm{p}<0.05)$.

Conclusions: All components of attention improved with age in children from 4 to 7 years old. It is important to consider these age-related changes when evaluating attention in children.

Correspondence: Sergio Tamez, Laboratory of Psychophysiology, Universidad Autónoma de Nuevo León, School of Psychology, Mutualismo 110, Col. Mitras Centro, Monterrey 64460, Mexico. E-mail: stamez.98@gmail.com

J. VAN DESSEL, E. SONUGA-BARKE, M. MOERKERKE, J. LEMIERE, S. VAN DER OORD \& M. DANCKAERTS. The Amygdala in Attention-Deficit/Hyperactivity Disorder: Structural and Functional Correlates of Delay Aversion.

Objective: Recent magnetic resonance imaging (MRI) studies implicate structural alteration of the amygdala, a brain region responsible for processing and experiencing negative emotions, in attention-deficit hyperactivity disorder (ADHD). Using voxel-based morphometry we investigated the functional significance of this by examining the correlation between amygdala structure and behavioural and neural measures of delay aversion - a negative affective state associated with ADHD, hypothesized to motivate the escape or avoidance of delay.

Participants and Methods: Structural T1-weighted 3T MRI scans from 28 right-handed male adolescents with combined type ADHD and 32 age-matched (10-18years) controls were analysed using the Computational Anatomy Toolbox 12. Regional grey matter volume differences between ADHD and control participants (Family Wise Error-corrected $p<0.05)$ were correlated with delay aversion self-ratings and neural activity in response to delay-related cues on a Monetary Incentive Delay fMRI variant where cues signalled that delay would occur either; (i) on all trials irrespective of performance (CERTAIN DELAY), (ii) only when responses were too slow (CONDITIONAL DELAY) or (iii) on no trials (NO DELAY).

Results: ADHD was associated with significantly reduced volumes in bilateral amygdala, parahippocampal and medial temporal gyrus $(\mathrm{p}[\mathrm{FWE}]<0.05)$, greater amygdala activation to certain delay cues $(\mathrm{p}[\mathrm{FWE}]<0.05]$ and higher delay aversion self-ratings $(\mathrm{p}<0.05)$. Amygdala volumes were significantly correlated $(p<0.01)$ with delay-cuerelated amygdala activity and self-reported delay-aversion in ADHD. Conclusions: We provide some of the first evidence of the functional significance of reduced amygdala volumes in ADHD by highlighting its relation to delay-induced brain activity linked to delay aversion. Longitudinal studies are required to test whether structural alterations in amygdala are a cause or a consequence of the experience of delay as aversive.
Correspondence: Jeroen Van Dessel, MSc, Neuroscience, KU Leuven, Kapucijnenvoer 33, Leuven 3000, Belgium.E-mail: jeroen.vandessel@ kuleuven.be

E.R. WALLACE, N.E. GARCIA, B.D. WALLS, C.M. BOSCH, K. BALTHROP \& D.T.R. BERRY. Attention-Deficit/Hyperactivity Disorder Malingering Detection in College Students: A MetaAnalysis of Performance and Symptom Validity Tests.

Objective: This meta-analytic review compares stand-alone and embedded performance validity tests (PVTs) and symptom validity tests (SVTs) for ADHD malingering detection in college students. At present, there is little consensus regarding which ADHD malingering indices are most effective; this review aims to inform future ADHD malingering research endeavors.

Participants and Methods: Data were coded from 11 ADHD malingering simulation design studies utilizing college student samples. Random effects meta-analytic models were conducted to provide aggregated weighted effect sizes (Hedges' $g$ ), indicating the difference between genuine ADHD and simulation groups.

Results: Overall PVTs (all PVT indices) produced a large effect size $(g=0.73,95 \%$ CI $[0.64,0.82], p<.05)$, whereas overall SVTs (all SVT indices) produced a medium effect size $(g=0.49,95 \%$ CI $[0.43$, $0.54], p<.05)$. Stand-alone PVTs $(g=0.97,95 \%$ CI $[0.87,1.07]$, $p<.05)$ outperformed embedded PVTs $(g=0.68,95 \%$ CI $[0.50,0.87]$, $p<.05)$. However, the stand-alone SVT $(g=0.66, p<.05)$ performed similarly to embedded SVTs ( $g=0.54,95 \%$ CI $[0.43,0.65], p<.05)$. Conclusions: Stand-alone PVTs for ADHD malingering detection among college students are supported by these results, whereas embedded PVTs and SVTs appear less effective. Particularly, the results endorse measures demonstrating high specificity for ADHD and large effect sizes (e.g., Test of Memory Malingering). Results also indicate that certain embedded PVTs (e.g., Woodcock-Johnson Processing Speed Index) may be useful for ADHD malingering detection. Results suggest that performance-based measures should be included in ADHD evaluation batteries, which in some cases consist solely of symptom self-report measures.

Correspondence: Elizabeth R. Wallace, B.S., Psychology, University of Kentucky, 171 Funkhouser Drive, 111C, Lexington, KY 40506, United States.E-mail:liz.wallace@uky.edu

E.C. GONZALES, J. AVILA, L. TREVIZO \& S.P. VERNEY. Socioeconomic Status and Neuropsychological Performance in College Students with and without ADHD Symptoms.

Objective: Measures of attention, processing speed, and executive functioning (EF) are typically used to aid the diagnosis of Attention Deficit Hyperactivity Disorder (ADHD); however, performance may be influenced by socio-demographic factors including age, gender, education, and socioeconomic status (SES). College students who may have been diagnosed with ADHD in childhood may no longer take medications, yet still experience significant ADHD symptoms. We investigated the relationship between socio-demographic factors and neuropsychological performance in college students with and without self-reported ADHD. Participants and Methods: Students with ( $\mathrm{n}=18$; non-medicated) and without $(\mathrm{n}=28)$ self-reported ADHD completed demographic and symptom severity questionnaires, and measures of attention, processing speed, and EF. Groups were of similar age, education, and gender; however, the ADHD group reported higher childhood family income and higher current ADHD symptom severity compared to the non-ADHD group.

Results: ADHD symptom severity was not significantly associated with test performance. The ADHD group performed more poorly on processing speed, but the groups were similar on other tests. Bivariate correlations revealed different associations across groups between test performance and socio-demographic factors. For the ADHD group, higher individual and parental educational attainment was associated with better performance on measures of processing speed, set-shifting, 
working memory, and attentional functions. In the non-ADHD group, higher childhood family income was associated with better performance on visual scanning, working memory, and executive control.

Conclusions: Indices of SES were associated with test performance, while ADHD symptom severity was not: thus, SES may be an important factor of performance regardless of childhood ADHD diagnosis. Socio-demographic factors provide a context for performance, and may guide appropriate diagnosis and treatment in non-medicated college students with ADHD.

Correspondence: Elisa C. Gonzales, University of New Mexico, 109 Elm St SE Apt 2, Albuquerque, NM 87102, United States. E-mail: elgonzales@unm.edu

\section{Medical/Neurological Disorders/Other (Child)}

E.C. GONZALES, J. POMMY, R. RIEGER, E. RHOADES-CLARK, R. YEO, J. LOWE, J. PHILLIPS, R. CAMPBELL \& R. OHLS. Relationship Between Brain Structures and Working Memory in Preschoolers Born Preterm.

Objective: Working memory (WM) is an aspect of executive functioning necessary for holding task-relevant information in the mind to guide future actions. Children born preterm have been found to experience WM deficits. This may be associated with structural brain changes in grey matter (GM) starting from the neonatal period. Neuroimaging techniques such as voxel- and surface-based morphometry may help us better understand the effects of preterm birth on cortical structure and cognitive functioning.

Participants and Methods: Preschoolers born preterm $(n=59)$ and full-term $(n=21)$ were recruited from 2 sites. Gyrification (GI), sulci depth (SD), and cortical volume, thickness (CT), and complexity (CC) were assessed using an age appropriate template with Computational Anatomy Toolbox, a neuroimaging toolbox. All p-values are uncorrected less than .001. WM was measured using scaled scores on the Memory for Words (MFW) subtest of the Woodcock Johnson III.

Results: The preterm group had significantly lower MFW scores than the full-term group. In the preterm group, lower MFW scores were associated with smaller GM volume in the left hippocampus, increased GI in the right middle frontal gyri, increased CC in the right inferior frontal gyri, smaller SD in the right middle and superior frontal gyri, and increased CT in the left cerebellum. In the full-term group, higher MFW scores were associated with larger GM volume in bilateral temporal regions, reduced GI in the occipital cortex, increased and decreased CC in the occipital cortex, and increased CT in the left insula and middle frontal gyri, with widespread increases in CT in the right hemisphere: lingual and supramarginal gyrus, precuneus and paracentral lobule. Conclusions: WM appears to have different neural correlates in typically developing (TD) children than children born preterm. Further, WM appears to be related to greater GM thickness and volume across multiple brain regions in TD children, but seems to be related to changes in cortical folding among preterm children.

Correspondence: Elisa C. Gonzales, University of New Mexico, 109 Elm St SE Apt 2, Albuquerque, NM 87102, United States. E-mail: elgonzales@unm.edu

A.M. CUREWITZ, A. FOY, M. PIERPONT, S. BERRY, K. SOMMER, C. MOERTEL, M. SEMRUD-CLIKEMAN, R. HUDOCK \& R. PIERPONT. Emotional functioning in children with neurofibromatosis type 1 and Noonan syndrome.

Objective: Neurofibromatosis type 1 (NF1) and Noonan syndrome (NS) are genetic syndromes within the RAS-MAPK signaling cascade that have been associated with attentional and social impairments (e.g., Barton et al., 2004; Pierpont et al., 2015). Increased emotional and behavioral challenges have been observed in children with these disorders compared to unaffected siblings on parent-rated measures (e.g., Pierpont et al., 2015). The aim of the current study was to examine both parent and self-reports of emotional and behavioral functioning among children with NF1 and NS compared to their unaffected siblings. Participants and Methods: Parents and children with NS ( $\mathrm{n}=39$ ), NF1 $(n=39)$ and their unaffected siblings $(n=32)$ completed broadband scales of emotional and behavioral functioning (BASC-2). Children also completed a measure assessing social anxiety (SPAI-C). Sample size was reduced when self-report measures were included due to missing data. Results: Mean scores on BASC-2 parent forms were higher in both NF1 and NS groups across subscales measuring internalizing behaviors (i.e., anxiety, depression) and symptoms of ADHD (i.e., inattention, hyperactivity). Unaffected siblings were also rated by parents as having elevated anxiety. Child self-report ratings were generally correlated with parent ratings, but showed minimal symptom severity. Among the children with NS, girls reported higher levels of social anxiety than boys.

Conclusions: While ADHD symptoms have been previously identified as a significant behavioral feature among individuals with RASopathies, our data suggest that children with NS, NF1 and their unaffected siblings are also at heightened risk for internalizing disorders. Children, especially boys, may not always self-report acute psychopathology. Study findings may assist with guiding family-based interventions to address emotional challenges.

Correspondence: Alana M. Curewitz, MN, 420 Delaware St. SE, Mayo Mail Code 486, Minneapolis, MN 55455, United States. E-mail: curew001@umn.edu

A. HEITZER, J. PIERCY, A. MATTES, B. PETERS, J. KLARR \& S. RAZ. The Fetoplacental Ratio and Neuropsychological Functioning of Preschool-Age Children Born Prematurely.

Objective: The placenta is the fetus' sole source of oxygen and nutrients during pregnancy, and thus plays an essential role in neurodevelopment. The fetoplacental weight ratio (FPR), the ratio of birth weight to placental weight, has been proposed as an index of placental efficiency. Our goal was to examine the associations between the FPR and neuropsychological outcome of preterm-born preschoolers.

Participants and Methods: Eighty preterm (<34 weeks), singleton, preschoolers (3-4 years) were included. Information about trimmed placental weight was extracted from medical records. We excluded cases with moderate to severe intracranial pathology and cerebral palsy. Cognitive, language, and motor abilities were assessed using subtests from the Wechsler Preschool and Primary Scale of Intelligence (WPPSI -III/IV), Clinical Evaluation of Language Fundamentals-P2 (CELP-P2), and Peabody Developmental Motor Scales-2 (PDMS-2), respectively.

Results: We used simultaneous linear regression with FPR as predictor of interest. Adjustment was made for demographic (socioeconomic status and sex) and medical risk (gestational age, sum of perinatal complications, appropriateness of birth weight for gestational age) factors. The FPR was inversely related to measures of verbal-linguistic knowledge, accounting for a unique portion of the variance in WPPSI-III/IV Information subtest score $(p=.015)$ and CELF-P2 Expressive Language Index $(p=.018)$, but not receptive language or motor indices.

Conclusions: Our findings revealed that within a cohort of pretermborn preschoolers, the FPR accounted for $6 \%$ and $10 \%$ of the variance in verbal intellectual and expressive language performance, respectively. Because the FPR was inversely related to outcome in our preterm sample, we concur with the notion that increased placental efficiency also connotes less reserve capacity which, in turn, renders the fetus more vulnerable to antenatal stressors commonly observed in the preterm infant.

Correspondence: Andrew Heitzer, Wayne State University, 681 West Canfield Street, Detroit, MI 48201, United States.E-mail: aheitzer17@ gmail.com 
A. HOOD, A. NAMCHUK, D. WHITE, A. KING \& M. DEBAUN. Cognitive Developmental Trajectories in Children with Sickle Cell Disease.

Objective: Sickle cell disease (SCD) is a genetic disorder characterized by the production of abnormal hemoglobin. The sequelae of SCD include cognitive deficits; however, longitudinal studies are rare. Our goal was to characterize the cognitive developmental trajectories of children with SCD with and without infarct in comparison to healthy children.

Participants and Methods: This study used cognitive data from studies conducted at Washington University between 1999 and $2011(\mathrm{n}=133)$ and from a subset of children from the Silent Infarct Transfusion Trial (SITT). Inclusion criteria for the SITT were an age of 5 to 15 years, diagnosis of hemoglobin SS or hemoglobin S $\beta 0$ thalassemia, at least one SCI on the screening MRI, no history of a stroke, and normal transcranial Doppler measurement. Only participants in sites from the US sites were included in the analyses $(n=162)$. Children were randomly allocated to blood transfusion or observation over a median of 3 years. Data from healthy children came from the NIH MRI study $(\mathrm{n}=332)$. Children from all studies completed the 4-subtest Wechsler Abbreviated Scale of Intelligence (WASI) and a general questionnaire.

Results: We conducted a mixed-effects regression including age, gender, and group (SCD with and without infarct and controls) as predictors separately for each subtest of the WASI in children aged $6-21$ years. We found significant main effects of age and group for Vocabulary, a 2-way interaction between age and group for Matrix Reasoning and a critical 3-way interaction for both SCD groups for Similarities and for the SCD infarct group only for Block Design in comparison to controls $(p s<.05)$. Conclusions: Our findings demonstrate that the cognitive development of children with SCD does not increase in the same way as controls and that for some cognitive domains trajectories differ by gender. This is the first study to characterize the developmental trajectories of children with SCD and highlights the need for cognitive interventions in this underserved population.

Correspondence: Anna Hood, PhD, Psychology, Washington University in St. Louis, One Brookings Drive, St. Louis, MO 63130, United States. E-mail:annahood@wustl.edu

M. CLAPP, A. HOOD, D. LAGONI, D. WHITE \& A. KING. IQ and Grade Retention Predict Poor Executive Functioning for Children with Sickle Cell Disease.

Objective: Sickle cell disease (SCD) a genetic disorder characterized by the production of abnormal hemoglobin in red blood cells. Silent cerebral infarcts (SCI) are the most common neurologic injury and are associated with deficits in executive functioning. We tested the hypothesis that biologic, cognitive, and educational factors would predict poorer executive functioning in children with SCD.

Participants and Methods: This study used a subset of children from the Silent Infarct Transfusion Trial. Inclusion criteria were an age of 5 to 15 years, diagnosis of hemoglobin SS or hemoglobin S $\beta 0$ thalassemia, at least one SCI on the screening MRI, no history of a stroke, and normal transcranial Doppler measurement. Of the 29 sites participating in the trial, only participants in sites from the US sites were included in the analyses $(n=143)$. Data reported here was collected at trial entry, prior to children being randomly allocated to blood transfusion or observation. Participants were administered the WASI to estimate full scale IQ. Caregivers completed the Behavior Rating Inventory of Executive Function (BRIEF) and a general questionnaire. Caregivers completed a household socioeconomic survey.

Results: We conducted a linear regression and found that hemoglobin S percentage, oxygen saturation, IQ, and grade retention significantly predicted executive functioning (Global Executive Composite) in children with SCD, $F(1,145)=5.93, p<.0001, r^{2}=.17$. Our model demonstrated that for every 1.3-point decrease in IQ there is a predicted 10-point increase in t-scores on the BRIEF, which indicates poorer IQ predicts poorer executive functioning.

Conclusions: Our findings demonstrate that biologic, cognitive, and educational factors predict poorer caregiver-reported executive functioning in children with SCD. These data suggest that the BRIEF could be a screening tool and highlight the importance of determining interventions that may improve executive functioning in this understudied population.

Correspondence: Anna Hood, PhD, Psychology, Washington University in St. Louis, One Brookings Drive, St. Louis, MO 63130, United States. E-mail:annahood@wustl.edu

L. ISAAC, L. QUAST, J. BARAN, C. BRODSKY, P. PHILLIPS \& M. HOCKING. Sex-Specific Processing Speed Recovery in Pediatric Brain Tumor Survivors.

Objective: Robust findings in animal models, pediatric traumatic brain injury (TBI), and high functioning autism (HFA) have shown neuroprotective and neuro-regenerative advantages in females. However, the literature on sex differences in pediatric brain tumor survivors (PBTS) is sparse. The purpose of this investigation was to determine if female PBTS have a differential trajectory in processing speed compared to males following tumor-directed therapy.

Participants and Methods: Participants included 18 PBTS (50\% females, mean age $=10.83,94 \%$ Caucasian) who recently completed tumor-directed treatment and had an initial IQ of 80 or above. The participants completed three brief neurocognitive evaluations - four months post-treatment (T1) and then six (T2) and 12 months (T3) after the initial visit. Processing speed was measured by the Processing Speed Index (PSI) on the WISC-IV.

Results: Male and female PSI scores did not differ at T1 or T2 $(p=$ $.35, .20)$. At T3, females' PSI scores $(M=107.7, S D=15.0)$ were significantly greater than males $(M=92.4, S D=12.0), t(16)=2.4, p=.03$. These results remained significant despite controlling for treatment intensity and treatments received ( $p=.03-.04)$. In within-sex comparisons, males showed no change from T1 $(M=90.1, S D=12.8)$ to T3 $(M=92.4$, $S D=12.0), t(8)=-.73, p=.49$. However, females showed a significant improvement from T1 $(M=98.3, S D=17.7)$ to T3 $(M=107.7, \mathrm{SD}=15.0)$, $t(8)=-3.2, p=.01$.

Conclusions: These findings suggest that female PBTS show a differential processing speed trajectory that may be reflective of a hormonedriven neuro-regenerative effect, potentially reflecting remyelination of white matter. The interaction of sex, hormones, and neural recovery is not well understood, especially in children. Future work in PBTS should explore sex differences in other cognitive domains and potentially integrate hormonal assays and neuroimaging to brain structure domains, such as white matter integrity.

Correspondence: Lee Isaac, M.Ed, Psychology, La Salle University, 8816 Ridge Ave \#22, Philadelphia, PA 19128, United States. E-mail: lee.v.isaac@gmail.com

N.N. KAMATH, J.M. FLETCHER, A.J. HOUTROW \& A. TREBLEBARNA. Ethnicity, SES, and Adaptive Function as Predictors of Health-Related Quality of Life Among Children and Adolescents with Spina Bifida Myelomeningocele.

Objective: Not only are individuals of ethnic minorities and lower socioeconomic status (SES) at an increased risk for being born with spina bifida myelomeningocele (SBM), but recent research suggests that they may also have differential outcomes. The objective of the present study was to examine associations of adaptive function, ethnicity, and SES on health-related quality of life (HRQL) among children and adolescents with SBM.

Participants and Methods: Participants included Hispanic ( $\mathrm{N}=75)$ and non-Hispanic White $(\mathrm{N}=86)$ children and adolescents with SBM and hydrocephalus (age $M=13.13 ; \mathrm{SD}=3.36$ ). Adaptive function was measured using the Scales of Independent Behavior-Revised, a measure of everyday functioning. To minimize confounds of physical/urological problems, a composite score was created from the non-motor skill domains. Participants completed subjective ratings of SBM-specific HRQL. Hierarchical regression examined the associations of age, sex, and medical variables (lesion level, ambulatory status, seizure history; 
step 1), adaptive function (step 2), and ethnicity, SES, and their interaction (step 3) on HRQL.

Results: Demographic and medical variables were not significant and were trimmed from the model. Adaptive function was significantly associated with HRQL $\left(\mathrm{R}^{2}=.146, \mathrm{p}<.001\right)$. After accounting for adaptive function, a significant interaction of ethnicity by SES was revealed $\left(\mathrm{R}^{2}=.813, \mathrm{p}=.010\right)$. Simple main effects of ethnicity for each SES stratum revealed that non-Hispanic Whites reported higher HRQL with increasing SES, whereas Hispanics reported lower HRQL with increasing SES.

Conclusions: The interaction of ethnicity and SES accounted for additional variance in HRQL over and above adaptive function. Surprisingly, Hispanic individuals demonstrate an inverse relationship between SES and HRQL, possibly related to a complex relationship between acculturation and outcomes. These results highlight the need for further exploration of the effects of sociodemographic variables on outcomes in individuals with SBM.

Correspondence: Navya N. Kamath, PsyD, Psychology, Indiana University of Pennsylvania, 2145 Lazor St Apt 142, Indiana, PA 15701, United States.E-mail: n.kamath@iup.edu

A. MATTES, A. HEITZER, J. PIERCY, J.B. NEWMAN, B. PETERS, J. KLARR \& S. RAZ. Retinopathy of Prematurity and Neuropsychological Functioning at Preschool-Age.

Objective: Preterm children are at increased risk of neonatal complications compared to their term-born counterparts. Retinopathy of prematurity $(\mathrm{ROP})$ is a vasoproliferative disorder of the retina known to adversely affect vision. Because ROP is associated with the incidence of intermittent hypoxemic episodes occurring during stay in the Neonatal Intensive Care Unit (NICU), we reasoned that even after adjustment for general perinatal and demographic risk factors, grade of ROP will be inversely related to neuropsychological performance at preschool age. Participants and Methods: For this study we used a large data set from an earlier cohort that provided us with a sufficient number of severe ROP cases to test our "dose-response" hypotheses. ROP screening was conducted during NICU stay for children at-risk (N=124). The cognitive, language, and motor skills of our preterm preschoolers (3-6 years of age) were assessed with the Wechsler Preschool and Primary Scale of Intelligence (WPPSI-R), Preschool Language Scales (PLS-3), and the Peabody Developmental Motor Scales (PDMS-2), respectively. Cases with moderate and severe intracranial bleeds or cerebral palsy were excluded.

Results: Mixed model analyses revealed that grade of ROP (0 - 4) explained a significant portion of the variance in the WPPSI-R Full Scale IQ ( $\mathrm{Z}=-1.97 ; p=.049)$ and Verbal IQ $(\mathrm{Z}=-2.05 ; p=.040)$, after statistical adjustment for gestational age, days of mechanical ventilation, appropriateness of birth weight for gestational age, the sum of perinatal medical complications, socioeconomic status, and sex.

Conclusions: Grade of ROP was found to be linked to verbal intellectual performance at preschool age. This relationship cannot be easily explained by ROP-associated visual impairment. This finding is consistent with reports that have linked ROP to the incidence of intermittent hypoxemic episodes in the preterm infant.

Correspondence: Allyssa Mattes, MI, United States. E-mail: allyssamattes@wayne.edu

\section{T. NORRIS. Subtypes of Attention Deficits in Survivors of Acute} Lymphoblastic Leukemia.

Objective: Survivors of pediatric acute lymphoblastic leukemia (ALL) treated with chemotherapy are at risk for cognitive late effects from their treatment. Attention is one of the areas of cognitive functioning that has commonly been found to be affected in this population, with approximately one-fourth of survivors of ALL showing evidence of deficits in attention. Few studies have examined specific subdomains of attention. The purpose of this study was to identify specific subdomains of attention that are particularly vulnerable in this population.
Participants and Methods: As part of a larger study examining cognitive late effects among survivors of ALL, 20 survivors and 20 healthy control subjects (ages 8-15) completed subtests from the Test of Everyday Attention - Children's Version (TEA-Ch) designed to measure four subdomains of attention (selective, divided, sustained, and shifting). Independent samples t-tests were performed to see if the two groups differed significantly in these areas.

Results: Participants in the control group performed statistically significantly better than participants in the clinical group on tests of sustained $[\mathrm{t}(38)=2.22, \mathrm{p}=.032 ; 10.25(3.01)$ vs $8.00(3.39)]$ and shifting $[\mathrm{t}(38)$ $=2.644, \mathrm{p}=.012 ; 10.55(3.33)$ vs $8.20(3.22)]$ attention. They also performed better on tests of selective and divided attention, but these differences were not statistically significant.

Conclusions: This study contributes to the literature on neurocognitive late effects among survivors of pediatric ALL treated with chemotherapy alone. Survivors in the current sample performed significantly worse than healthy peers on measures of two subtypes of attention, sustained and shifting. This can help target interventions and accommodations implemented to support attention weaknesses among survivors. Correspondence: Thea Norris, Ph.D., Cincinnati Children's Hospital Medical Center, 5632 Viewpointe Dr., Apartment I, Cincinnati, OH 45213, United States.E-mail: thea.norris@cchmc.org

\section{J. PIERCY, A. HEITZER, A. MATTES, J. KLARR \& S. RAZ. Arterial Cord pH and Neuropsychological Functioning of Preschool-Age Children Born Prematurely.}

Objective: Low arterial cord $\mathrm{pH}$, reflecting acidemia, is associated with increased risk of neonatal mortality, hypoxic-ischemic encephalopathy, intraventricular cerebral hemorrhage, and cerebral palsy. Nonetheless, a meager body of research has been dedicated to the relationship between arterial cord $\mathrm{pH}$ and long-term outcome. Our goal was to examine the associations between this index of peripartum fetal physiology and cognitive, language, and motor functioning of pretermborn preschoolers.

Participants and Methods: We included 139 preterm (< 34 weeks gestation) preschoolers (3-4 years) whose acid-base status was evaluated immediately after birth via umbilical cord blood analysis. We excluded cases with congenital anomalies, moderate or severe intraventricular hemorrhage, and cerebral palsy. Cognitive, language, and motor abilities were assessed with the Wechsler Preschool and Primary Scale of Intelligence (WPPSI -III/IV), Clinical Evaluation of Language Fundamentals-P2 (CELF-P2), and Peabody Developmental Motor Scales-2 (PDMS-2) respectively.

Results: We used mixed model regression analyses with arterial cord $\mathrm{pH}$ value as our predictor of interest. We adjusted statistically for demographic (socioeconomic status, sex and multiple gestation) and medical risk (gestational age, total number of perinatal complications, and appropriateness of birth weight for gestational age) factors. Arterial cord $\mathrm{pH}$ was found to be linked to intellectual (WPPSI-III/IV FSIQ; $t(1,130.09)=2.16, p=.033)$, language (CELF-P2 Core Language Index; $t(1,122.09)=2.75, p=.007)$, and motor (PDMS-2 Total Motor Quotient; $t(1,118.84)=2.84, p=.005)$ performance.

Conclusions: In the current study, Lower $\mathrm{pH}$ values were associated with poorer performance in multiple neuropsychological outcome domains in preterm-born preschoolers. Whether lower $\mathrm{pH}$ reflects differential physiological vulnerability to peripartum stress in this at-risk population remains to be investigated.

Correspondence: Jamie Piercy. E-mail: Jamiepiercy@wayne.edu

\section{J.A. PLIEGO, S.L. KELLY \& K.R. WOLFE. Group Differences in Neurocognitive Profiles for Children Post Heart Transplant.}

Objective: Neurocognitive impairments are well-documented in children with critical congenital heart disease (CHD) and children who have undergone heart transplantation. Within the pediatric heart transplant population, the neurocognitive functioning of children with pre-existing cardiomyopathy $(\mathrm{CM})$ compared to pre-existing $\mathrm{CHD}$ is unexamined. 
We investigated neurocognitive functioning and adherence to immunosuppressant medication in children with history of heart transplantation with consideration for pre-existing cardiac condition.

Participants and Methods: Retrospective chart review was conducted for 27 children (19 CHD, 8 CM) status post heart transplant. The sample was predominantly Caucasian with a mean age of 9.75 years at the time of neuropsychological evaluation. Groups did not differ on demographic variables. Inclusion criteria were a post-heart transplantation neuropsychological evaluation conducted between age 4 to 18 years. Measures included the Wechsler Intelligence tests, WIAT-III, CVLT-C, D-KEFS, Tower of London, BRIEF, NICHQ Vanderbilt Checklist, and ABAS-2. Results: Comparison of $\mathrm{CM}$ and CHD groups yielded differences in overall IQ $(p=.05)$, working memory $(p=.04)$, word reading $(p=.02)$, math calculation $(p=.05)$, and overall adaptive functioning $(p=.03)$, with lower performance in the CHD group across domains. The CM and CHD groups did not significantly differ on measures of attention, executive functioning, verbal memory, verbal reasoning, processing speed, or mean immunosuppression medication adherence levels.

Conclusions: Results suggest decreased intellectual functioning, working memory, academic, and adaptive functioning skills in children with CHD compared to CM post heart transplant. No group differences in executive functioning skills were seen, suggesting the frequently-cited executive functioning deficits in the heart transplant population may be related to factors surrounding heart failure or the heart transplant process itself.

Correspondence: Jessica A. Pliego, Ph.D., University of Colorado School of Medicine, 13123 E 16th Ave, Aurora, CO 80013, United States. E-mail: jesspliego12@gmail.com

J. POMMY, E.C. GONZALES, R. YEO, J. LOWE, J. PHILliPS, R.E. RIEGER \& R. OHLS. Cortical Structure Associated with Executive Functions in Children Born Preterm.

Objective: Premature birth has been associated with impaired executive function (EF). The neural correlates associated with EF may differ among individuals born preterm. We examined the relationship between EF performance and cortical structure in 3.5-to-5 year-old children born preterm (PT) and fullterm (FT).

Participants and Methods: 59 individuals born PT and 21 individuals born FT were recruited from two sites. Cortical structure and surface was assessed using the neuroimaging toolbox, Computational Anatomy Toolbox. Gray matter volume (GMV), cortical thickness (CT), gyrification (GI), cortical complexity (CC), and sulci depth (SD) were examined utilizing age-appropriate template. EF was assessed using spatial working memory and gift delay tasks. An EF composite score was generated using a principle components analysis.

Results: Group comparisons revealed reduced EF in the PT compared to FT group ( $p=0.041)$. Correlations between EF and GMV revealed higher EF was associated with greater GMV in the right inferior temporal gyrus in the PT group, with greater GMV in the right occipital middle and superior gyri in the FT group (uncorrected $p<0.001$ ). Surface analyses showed higher EF was associated with reduced GI in the right parahippocampal gyrus and greater SD in the left superior parietal gyrus in the PT group. Within the FT group, greater EF was associated with greater SD in the right superior parietal lobule, reduced CC in bilateral inferior temporal gyri (uncorrected $p<0.001$ ), and increased $\mathrm{CC}$ in the right inferior frontal gyrus (corrected FWE $<0.05$ ). Lastly, within the PT group increased EF correlated with greater CT in the left orbital, precentral and middle temporal gyri (uncorrected $\mathrm{p}<0.001$ ).

Conclusions: EF appears to have different correlates in typically developing children compared to children born PT. In particular, it appears EF may be more associated with a more complex pattern of cortical folding changes in children born at term, while among preterm children. $\mathrm{EF}$ is associated with greater cortical thickness.

Correspondence: Jessica Pommy, Psychology, University of New Mexico , 1101 Yale Blvd NE, Albuquerque, NM 87106, United States. E-mail: jpommy@gmail.com
R.E. RIEGER, R. YEO, J. POMMY, E.C. GONZALES, R. CAMPBELL, J. LOWE, J. PHILLIPS \& R. OHLS. Socioeconomic Status, Family Stress, White Matter, and Language Abilities in Preschoolers Born Preterm.

Objective: Prematurity and family SES predict language and influence white matter (WM) microstructure. Current research aims: (1) What aspects of SES are most important among those born preterm? (2) How is WM microstructure in childhood related to language?

Participants and Methods: 59 preschoolers born preterm (24-31 weeks) and 21 full term were recruited. Fractional anisotropy (FA) of major tracts was calculated. Principal component analysis (PCA) identified one large factor (FAPC). PCA of demographic variables identified socioeconomic (SEC) and family stress composites (FSC). The geometric mean of birth weight and gestational age represented prematurity (PRE). Language was assessed with the General Language Composite (GLC) of the WPPSI-III.

Results: The preterm group had lower GLC and GLC correlated significantly with FAPC, SEC, FSC, and PRE. Among the preterm, PRE was correlated with FAPC and GLM analysis revealed a positive impact for SEC and a negative impact of FSC. Best predictors for SEC were income and education, and for FSC, number of family moves. Analysis including Group revealed no interactions with SEC or FSC. To examine effects of WM on GLC among preterm we conducted a hierarchical regression, with sex, SCE and FSC (step 1) and FA PC (step 2). Inclusion of FA PC did not increase proportion of explained variance. Follow up analyses examined four specific left WM tracts associated with language. Including these WM tracts did not significantly increase the proportion of explained variance.

Conclusions: Demographics had strong relationships with language that did not vary across groups. Although WM microstructure was influenced by prematurity, it did not predict language in models with demographics. These findings highlight (1) the significant impact of family composition and stress on children born preterm, and (2) that the specific anatomic substrates of language ability in the preterm may not include WM microstructure.

Correspondence: Rebecca E. Rieger, PhD, Psychology, University of New Mexico, 1510 A Gold Ave SE, Albuquerque, NM 87106, United States. E-mail: rerieger@unm.edu

K. SKILLICORN \& L.M. MORAN. Anti-NMDA Receptor Encephalitis: Serial Assessment of Bilingual 5 year old Female.

Objective: Anti-NMDAR encephalitis is a neurological syndrome associated with acute onset neurological and neuropsychological changes, with evidence of residual neuropsychological impact. This pediatric case study describes the role of serial neuropsychological assessment in planning appropriate rehabilitation and monitoring cognitive, social, and academic functioning.

Participants and Methods: Data reported are of a bilingual Spanish-English female diagnosed with anti-NMDAR encephalitis at age 5:0. Treatment was initiated at 2.5 weeks post-symptom onset, with gradual improvement in neurologic functioning. Time 1 assessment occurred at age 5:4, with Time 2 assessment at age 6:4. Reliable changes indices were calculated to assess for statistically significant change with 95 percent confidence. Measures of academic, language, attention/executive function, memory, and psychosocial function were administered in English without use of an interpreter.

Results: Time 1 results showed clear deficits in the areas of language, attention/executive function (with an impact on memory), academic achievement, and fine motor speed, as well as behavior regulation difficulties. Speech therapy, physical therapy, educational services, parentchild interaction therapy, and stimulant medication interventions were completed as part of her rehabilitation course. No significant declines were noted at Time 2 . Data show significant improvements in verbal reasoning, deductive reasoning, math problem solving, naming, verbal fluency, and recognition memory. Persistent areas of deficit included language comprehension, sustained attention, phonological working memory, reading, math, fine motor speed, and cognitive control. 
Conclusions: Evidence suggests aspects of neuropsychological recovery following medical stabilization and provision of intensive interventions. Yet, persistent neuropsychological deficits remain. Results support the need for long-term monitoring and rehabilitation services. Correspondence: Kimberly Skillicorn, Psy.D., Pediatric Neuropsychology, Advanced Neurobehavioral Health of Southern California, 15644 Pomerado Rd Suite 305, Poway, CA 92064, United States. E-mail: kskillicorn@anhsocal.com

A.M. SVINGOS, S.M. TURNER, S.M. GREIF, E.M. TURNER, M.W. MOORE, B.A. BAILEY \& S.C. HEATON. Pediatric Anti-NMethyl-D-Aspartate Receptor Encephalitis: Aftermath of a Brain on Fire.

Objective: Anti-N-Methyl-D-Aspartate Receptor (NMDAR) encephalitis is an autoimmune condition which results in the acute onset of psychiatric and neurological symptoms such as aggression, paranoia, seizures, and mutism. Despite increased awareness of the condition, very little is known about its acute and chronic neuropsychological effects. Participants and Methods: Three cases of anti-NMDAR encephalitis were examined. Patients varied in age at diagnosis $(5,8,9)$, gender, and race/ethnicity (Caucasian, African-American, Hispanic). Initial neuropsychological assessments were conducted 6-14 months after disease onset, with one patient undergoing two additional follow-up evaluations (3 and 6 years). Performance-based testing was used to assess intellectual, memory, attention, executive, visuoconstructional, and motor skills.

Results: All patients exhibited attentional difficulties within the first year of disease onset. Patient $\mathrm{C}$, who experienced the longest duration of acute symptoms, performed within the impaired range on executive functioning tasks whereas the other children did not. The older patients (B and $\mathrm{C}$ ) exhibited impaired working memory and low average to impaired information processing speed and visuoconstructional skills. Memory functioning was variable across cases. All patients demonstrated spared fine motor dexterity. Patient C exhibited improvements in nearly all cognitive domains assessed over time; however, complex attention and executive-switching abilities remained impaired six years after disease onset.

Conclusions: Though attentional impairments appeared central to all examined cases, this case series highlights the heterogeneity in cognitive deficits observed among children with a history of anti-NMDAR encephalitis. Findings also demonstrate the potential chronicity of disease effects, particularly in the domains of attention and executive functioning. Continued research is needed in order to identify risk and resiliency factors for cognitive dysfunction within this population.

Correspondence: Adrian M. Svingos, M.S., Clinical and Health Psychology, University of Florida, P.O. Box 100185, Gainesville, FL 32610, United States. E-mail: adrianmchambers@phhp.ufl.edu

\section{J. THOMPSON, B.D. ESCHLER \& S.D. GALE. Cognitive Outcome in Individuals with Childhood Extracorporeal Membrane Oxygenation: A Case Series.}

Objective: To examine cognitive outcome in adolescents and young adults who underwent extracorporeal membrane oxygenation (ECMO) as children due to acute illness.

Participants and Methods: Eight participants (three male, five female; ages $11-22)$ at least 1 year post-ECMO procedure $(\mathrm{M}=5.6$ years; $\mathrm{SD}=2.1$ ) for acute respiratory or cardiac illness were evaluated with the NIH Toolbox Cognition Battery. Scores were compared to normative data for age to investigate potential impairment in multiple cognitive domains.

Results: Performance was impaired (at least one SD below the mean) in at least half of the participants for multiple tasks. Scores on a task of inhibitory control showed impairment in $75 \%$ of participants; $63 \%$ were impaired on a task of visual memory; $50 \%$ were impaired on tasks of executive function and processing speed; and $38 \%$ were impaired on a task of oral reading. Overall, half of the participants showed broad impairment in tasks of fluid intelligence. In contrast, crystallized intelligence was generally intact.

Conclusions: Performance on the NIH Toolbox Cognitive battery was impaired in over half of the tested individuals who underwent ECMO as children. Attention, executive function, processing speed, and visual memory were well below the expected range for age in the majority of participants. Crystallized intelligence tasks, such as vocabulary, were in the average to above average range for most participants, likely indicating normal baseline functioning. Thus, the NIH Toolbox appears to be sensitive to the cognitive sequelae of ECMO. Further study including comparison to a group of healthy controls is warranted to evaluate potential long-term effects of childhood ECMO on cognition.

Correspondence: Juliann Thompson, M.S., Clinical Psychology, Brigham Young University, 14410 NE 35th ST, APT K3, Bellevue, WA 98007 , United States. E-mail: thompson.juliann@gmail.com

E.M. TURNER, S. GREIF, A.M. SVINGOS, J. WARNICK, R. RAO \&. S.C. HEATON. Dual Cases of Non-Verbal Learning Disorder Following Twin to Twin Transfusion Syndrome: Coincidence or Causality?

Objective: Twin to Twin transfusion syndrome (TTTS) is a rare disease of the placenta that occurs in pregnancy with identical twins resulting in uneven blood flow between fetuses. As a result, the donor twin receives too little amniotic fluid, whereas the recipient receives excess fluid. Both conditions can result in widespread medical complications, mortality, and neurodevelopmental impairment. However, due to the rarity of this condition, little is known about the neurocognitive outcomes in effected youth.

Participants and Methods: The examined cases are Caucasian 11-year-old females born at 34 weeks gestation to a pregnancy complicated by TTTS. The twins were born with low (Twin A; recipient twin) and very low (Twin B; donor twin) birth weight. They were referred for concerns related to development, social, and academic functioning and completed a comprehensive neuropsychological assessment battery. Results: Both twins exhibited a highly similar neurocognitive and behavioral profile. Performance was average for intellectual and academic functioning, and learning and memory. Verbal skills were a prominent strength. Weaknesses included processing speed, visuo-constructional abilities, mathematical fluency, and social pragmatic communication. Notable impairments were identified on tasks assessing fine motor dexterity and visual spatial/perceptual skills. Discrepancy in performance emerged for motor coordination skills (Twin B displayed impairment) and attentional functioning (Twin A displayed impairment).

Conclusions: Although not currently recognized in the DSM- $V$, the twins' presentation and pattern of neuropsychological performance is consistent with Nonverbal Learning Disorder (NVLD). This case study highlights the importance of future research to determine if NVLD is a symptom profile of twins born with this medical condition.

Correspondence: Elise M. Turner, PHD, Clinical and Health Psychology, University of Florida, P0 Box 100165, Gainesville, FL 32610-0165, United States. E-mail: eliseturner@ufl.edu

\section{M.M. THOMASON, C. PETRANOVICH \& A. SHERWOOD. Cognitive} Effects of Subdural Empyema in an Adolescent Female.

Objective: Subdural empyema (SE) is a rare complication following sinusitis. Only 2 existing case studies have reported cognitive outcomes following $\mathrm{SE}$, both in adolescent males. One of these case studies reported deficits in expressive language and processing speed, while both reported impairments in fine motor functioning. The following case study is the first known study to describe cognitive findings following $\mathrm{SE}$ in an adolescent female.

Participants and Methods: The patient was diagnosed with multiple, recurrent SE due to bacterial sinusitis at 14 years of age. She was hospitalized on a pediatric intensive care unit for 1 month and underwent 3 brain surgeries due to recurrent bilateral SEs. She subsequently received inpatient rehabilitation for 1 month and 
participated in occupational, speech, and physical therapies. She continued to receive therapies consistently for 4 months following discharge and sporadically up to the time of the second evaluation due to personal barriers. She completed an inpatient evaluation at 2 months post-admission and an outpatient evaluation at 13 months post-admission.

Results: In both evaluations, there were consistent impairments in auditory working memory, reading comprehension, and fine motor skills in her right hand. At the initial evaluation, there were impairments in semantic fluency, processing speed, memory for a complex figure, and fine motor skills bilaterally. Reliable change indices indicated significant improvements in these areas at the repeat evaluation.

Conclusions: Consistent with previously reported case studies, this patient demonstrated many areas of intact functioning and/or improvement over time. Also in line with prior case studies, this patient evidenced persistent fine motor impairment following SE. However, the current case also presented with impairments in auditory working memory and reading comprehension following SE that were not reported in previously published case studies.

Correspondence: Molly M. Thomason, Ph.D., Psychology, Texas Children's Hospital/Baylor College of Medicine, 6701 Fannin Street, Suite 1630,Houston, TX 77030,United States.E-mail:mmthomas@ texaschildrens.org

\section{Autism Spectrum Disorders}

M.M. THOMASON, R.P. GOIN-KOCHEL, L.R. DOWELL, J. MCCARTHY, C. SCHAAF \& L.N. BERRY. Neurocognitive Phenotyping Distinguishes Schaaf-Yang Syndrome from PraderWilli Syndrome.

Objective: Paternally-expressed genes on chromosome 15q11-q13 have been implicated in the development of Prader-Willi syndrome (PWS). Recent research on a truncating pathogenic variant of the MAGEL2 gene, within the PWS-critical region of chromosome 15q11-q13, has led to the discovery of Schaaf-Yang syndrome (SYS). PWS and SYS share several clinical phenotypic features, including: neonatal hypotonia, feeding difficulties, and hypogonadism. The current study sought to examine the distinctiveness of SYS from PWS by characterizing the neurobehavioral phenotype of this disorder.

Participants and Methods: A sample of 10 participants with SYS were recruited from a clinical database. Participants received a neurocognitive evaluation, including assessment of developmental or intellectual functioning, adaptive functioning, autism spectrum disorder (ASD) symptoms, and behavioral/emotional functioning.

Results: Ratio developmental/FSIQ scores ranged from less than 1 to 61.8 . The majority of patients $(5 / 9)$ for whom developmental or IQ testing could be completed fell in the range of Severe to Profound Intellectual Disability (ID). Global adaptive functioning ratings were significantly below age-expectations for all participants. All participants obtained scores within the "Autism" range on the ADOS-2. Results from ADI-R and SRS-2 further supported significant ASD symptomatology in the entire sample. Almost all (9/10) participants met criteria for a clinical diagnosis of ASD. The one participant who did not receive the diagnosis still presented with significant ASD symptomatology, though cognitive functioning was too impaired to render a valid ASD diagnosis. No significant symptoms were reported on the Y-BOCS-II for any participant.

Conclusions: Results from the current study suggest that SYS is distinguished from PWS due to more severe ID, lack of obsessions/compulsions (which are common in PWS), and a higher frequency of ASD symptomatology than reported in PWS.

Correspondence: Molly M. Thomason, Ph.D., Psychology, Texas Children's Hospital/Baylor College of Medicine, 6701 Fannin Street, Suite 1630, Houston, TX 77030, United States.E-mail:mmthomas@ texaschildrens.org
K.R. BRADBURY, L.V. IBANEEZ, D.L. ROBINS, M. BARTON, W.L. STONE, Z.E. WARREN \& D. FEIN. Behavioral Comparison of High-Risk and Low-Risk Toddlers Identified with the Modified Checklist for Autism in Toddlers, Revised with Follow-Up.

Objective: The Modified Checklist for Autism in Toddlers, Revised with Follow-Up (M-CHAT-R/F) is a parent-report screener for autism spectrum disorder (ASD). Presence of an older affected child impacts how parents report behaviors in later-born children. The study explores how screener performance and behavioral characteristics differ between high-risk infant siblings (HR) and low-risk (LR) toddlers identified with the M-CHAT-R/F

Participants and Methods: Toddlers (HR $n=56$; LR $n=205$ ) screened positive on the M-CHAT-R/F and completed developmental evaluation. Two-way Analysis of Variance was conducted to examine the impact of risk status (HR vs. LR) and diagnostic group (ASD vs. NonASD) on screener performance, ASD severity, cognitive ability and adaptive skills.

Results: Sixty-one percent of HR toddlers and $49 \%$ of LR toddlers were diagnosed with ASD. No significant risk status $\mathrm{x}$ diagnostic group interactions were found. On the M-CHAT-R, there was a main effect of diagnostic group on total score at initial screen and at Follow-Up (FU), where the ASD group endorsed more at-risk items ( $p$ 's<.001). No main effect of risk status was seen at initial screen, however parents of LR toddlers were more likely to change responses at FU (from fail to pass; $p<.001)$. As anticipated, there were significant main effects of diagnostic group, where the ASD group had higher severity, lower cognitive scores, and weaker adaptive skills $(p$ 's<.001). A main effect of risk status was only seen on cognitive scores, where HR toddlers had significantly higher scores $(p<.001)$.

Conclusions: Risk status impacts children identified with the M-CHAT-R/F. Parents of HR toddlers were more accurate in reporting of at-risk behaviors. HR and LR toddlers had comparable ASD severity and adaptive functioning. Higher cognitive scores in HR toddlers suggest that the M-CHAT-R/F can successfully detect less delayed children with ASD. Findings highlight the importance of screening in HR samples, as children with stronger cognitive skills may benefit more from intervention.

Correspondence: Kathryn R. Bradbury, M.A., University of Connecticut, 406 Babbidge Rd, Unit 1020, Storrs, CT 06269-1020, United States. E-mail:kathryn.bradbury@uconn.edu

L.E. BRADSTREET, F. ZHANG, P.J. MOBERG, M.E. CALKINS, B. DALY, M.T. SCHULTHEIS \& D.L. ROBINS. Relationships Between Autism Traits, Psychosis Traits, and Mentalizing in a Clinical-Community Sample.

Objective: Disruptions in social processes cause significant impairment across several neurological/psychiatric conditions, notably autism spectrum disorder (ASD) and psychotic spectrum disorders (PSD). The dimensional traits that characterize ASD and PSD may be useful for understanding patterns of social cognitive performance. The current pilot study examines relationships between autism traits, psychosis traits, and mentalizing in a clinical-community sample of young adults with and without ASD.

Participants and Methods: Twenty-eight adults $\left(M_{\text {age }}=23.54\right.$ years, $S D=4.75$ years; 11 women) completed the Wechsler Abbreviated Scale of Intelligence, $2^{\text {nd }}$ Edition (WASI-II), Autism Spectrum Quotient (AQ), Schizotypal Personality Questionnaire (SPQ), Community Assessment of Psychic Experiences-42 (CAPE-42), and Social Attribution Task-Multiple Choice (SAT-MC). Five individuals (2 women) in the sample had diagnoses of ASD. Pearson correlations examined relationships between autism traits, psychosis traits, and mentalizing in the entire sample.

Results: AQ total scores were positively related to total scores on the $\mathrm{SPQ}(r=.52, p=.005)$ and CAPE-42 $(r=.40, p=.03)$ and negatively related to SAT-MC scores $(r=-.55, p=.003)$. SAT-MC scores were also negatively related to scores on the SPQ $(r=-.48, p=.01)$ and CAPE-42 $(r=-.59, p=.001)$. 
Conclusions: Preliminary findings support and extend previous work showing a positive relationship between autism traits and psychosis traits. Each set of traits was related to poorer accuracy on a mentalizing task. Relative levels of these traits may be important for conceptualizing social difficulties among individuals with these clinical conditions. The results support the importance of a transdiagnostic and dimensional approach to understanding psychopathology and social functioning. Correspondence: Lauren E. Bradstreet, Psychology, Drexel University, 2110 Hassell Road, Apt. 201, Hoffman Estates, IL 60169, United States. E-mail:16684@drexel.edu

\section{J.E. CASEY \& S.E. SCOTT. Differentiating Nonverbal Learning Disorder and Higher Functioning Autism.}

Objective: The social functioning of children with Autism Spectrum Disorder (ASD) has been studied extensively, leading to the development of tests sensitive to the behavioral features of the disorder. To date, no study has examined how children with NLD perform on such tests despite some similarities in behavioral functioning between the disorders. The present study examined whether NLD and HFA could be differentiated on measures used to diagnose ASD.

Participants and Methods: The NLD group included 10 children $\left(M_{\text {age }}=12.8\right)$ who met neuropsychological test criteria for NLD (adapted from Casey et al., 1991), without consideration of their psychosocial functioning. The HFA group included 12 children $\left(M_{\text {age }}=12.3\right)$ previously diagnosed using DSM-IV-TR or DSM-5 criteria by a community psychologist or psychiatrist, without consideration of the child's neuropsychological test results. Logistic regression and receiver operating characteristic (ROC) curve analysis were employed to determine the diagnostic utility of the Autism Diagnostic Observation Scale-Second Edition (ADOS-2; total score) and the parent-completed Children's Communication Checklist-Second Edition (CCC-2; Social Interaction Difference Index or SIDI score) in differentiating children with NLD and children with HFA.

Results: For the ADOS-2 total score, the area under the curve (AUC) was .79, indicating acceptable (Hosmer \& Lemeshow, 2000) discrimination accuracy; the optimal cut-off score was 7, yielding a sensitivity of .75 and a specificity of .70. For the SIDI score, the AUC was .87 , indicating excellent discrimination accuracy; the optimal cut-off score was -8 , yielding a sensitivity of .70 and a specificity of .75 .

Conclusions: The ADOS-2 and CCC- 2 , both well-validated tests, are useful in differentiating NLD and HFA and should be utilized whenever both disorders are considered in the differential diagnosis. The findings also support the validity of NLD as a separate clinical condition from ASD. Correspondence: Joseph E. Casey, PhD, Psychology, University of Windsor, 401 Sunset Avenue, Windsor, ON N9B 3P4, Canada. E-mail: jecasey@uwindsor.ca

Y. DAI, L. BRENNAN, A. COMO, J. HUGHES-LIKA, T. DUMONTMATHIEU, I. RATHWELL, O. MINXHOZI, B. ALIAJ \& D. FEIN. Training Albanian Parents of Children with Autism Spectrum Disorder via Video.

Objective: Behavioral intervention with parent participation is effective in reducing symptoms of Autism Spectrum Disorder (ASD) (Rogers \& Vismara, 2008). Yet access to intervention is limited (Chiri \&. Warfield, 2012), especially in developing countries like the Republic of Albania. This study examined whether a behaviorally based, self-directed video parent-training program would (1) be comprehensible and acceptable to parents, (2) increase parental knowledge of behavioral strategies and (3) increase parental self-efficacy.

Participants and Methods: 29 parents (13 in the Intervention group (IG)) completed the Early Intervention Parenting Self-Efficacy Scale (EIPSES; Guimond et al., 2008) and a quiz on program content, preand post- intervention. IG parents received access to a DVD about improving skills and behavior in children with ASD and rated acceptability for each module with the Treatment Evaluation Inventory Short Form (TEI-SF; Kelly et al., 1989). Control Group (CG) parents were put on a 4-month waitlist for the program.
Results: Groups were matched on maternal education, child age ( $M=47$ months) and child gender. Parent acceptability was high $(\mathrm{M}=4.33 / 5)$. Controlling baseline scores, group membership trended towards predicting time-two quiz scores, $\beta=.28, p=.09$, and selfefficacy for two items (worry that I am not a good enough parent, $p<.05$, and see progress made in becoming a better parent, $p=.09$ ), but not overall self-efficacy, $\beta=.17, p=.45$.

Conclusions: Parents of children with ASD in Albania rated this selfdirected DVD training as enjoyable and comprehensible. It increased aspects of self-efficacy and parents' knowledge of optimal teaching strategies. CG knowledge remained stable; IG parents' scores increased an average of 9 percent (baseline $M=52$, post-intervention $M=61$ ). Future research will explore the effect of this training on parents and children with a larger sample in the U.S., using web-based training with greater flexibility and more video examples.

Correspondence: Yael Dai, B.A., Psychology, University of Connecticut , 406 Babbidge Road, Storrs, CT 06269, United States. E-mail: yael. dai@uconn.edu

B.J. DIAMOND, K. HAPPAWANA, A.S. BUJALSKI, W. TSANG \& M. AHMED. Fearful Faces, Processing Speed and Autism Spectrum Disorder: Indexing Mild Autism with EEG Spectral Correlates.

Objective: Measure the speed and accuracy with which college-age individuals with autism spectrum disorder (ASD) compared with healthy individuals in discriminating fearful from neutral faces and determine if EEG spectral power and topographic characteristics differentiate mild ASD from severe ASD and performance in healthy individuals.

Participants and Methods: MA, a 20-year-old female and two 21-year-old individuals with mild ASD ( $\mathrm{FJ}=$ male and $\mathrm{WA}=$ female) were compared to 19 healthy controls $(\mathrm{M}=22.5$, sd $=2.6)$ on speed and accuracy on a computer n-back task. The EEGs of MA and FJ were compared to six healthy controls. A Fearful Face discrimination task was administered and performance was monitored using forty EEG channels. All participants provided assent and/or informed consent.

Results: MA was slower in classifying fearful versus neutral faces and was significantly slower $(\mathrm{M}=1490.2$, sd $=989.6)$ than controls $(\mathrm{M}=733, \mathrm{sd}=183.1)$ in classifying fearful $\mathrm{t}(18)=-18.0, p=.001)$ $(\mathrm{n}=19)$ vs neutral faces $(\mathrm{M}=758, \mathrm{sd}=207.2), \mathrm{t}(18)=-6.5, p=.001$, $\mathrm{n}=19)$. FJ and WA's latencies did not differ $(\mathrm{M}=743$, sd $=79)$ from controls although both were slower in classifying neutral faces. Gamma spectral power which may reflect visual information processing and attentional perceptual mechanisms and is enhanced by higher arousal emotions (e.g.,fear) was greater in MA, as was delta power (associated with emotional processing), particularly in frontal regions. The EEG 2D spectral topographical image series of FJ showed more diffuse activation than controls but less diffuse than MA.

Conclusions: The disproportionately heightened gamma and delta activity, in response to fearful faces, and spectral images showing diffuse activation appear to reflect less efficient processing in ASD. Importantly, this case series demonstrates that diffuse activation appears to be a more sensitive index of mild autism than information processing speed alone which did not differ from healthy controls.

Correspondence: Bruce J. Diamond, Ph.D, Psychology, William Paterson University, 300 Pompton Rd, 300 Pompton Road, Wayne, NJ 07470, United States.E-mail:diamondb@wpunj.edu

L.M. DIAZ-ORTIZ, R.E. OLIVERAS-RENTAS, F. VÁZQUEZSANTIAGO, J. MONTEIRO \& A. TORRES-REVERÓN. Hippotherapy and Molecular Markers in a Sample of Male Children with High Functioning Autism Disorder.

Objective: Autism Spectrum Disorder (ASD) is a complex neurodevelopmental disorder characterized by activity-focused repetitive behavior and restricted social interactions (RSI). There is no effective pharmacotherapy for treating the core symptoms in ASD. Hippotherapy (HT) uses equine-assisted movements as an alternative intervention to treat RSI. We sought to investigate the effect of HT on SRI among Puerto Rican male children with high functioning ASD (HFASD) and evaluate 
OTX and DNA methylation, as molecular correlates of HT response. We specifically focus on changes in oxytocin (OXT) and DNA methylation. No research to date has evaluated whether molecular markers are helpful to determine a response to HT objectively.

Participants and Methods: We performed a longitudinal approach of five series of therapy sessions among HFASD male participants of 6-9 years of age, classified following the gold-standard Autism Diagnostic Observation Schedule. Groups: HFASD with HT (n=6), HFASD with play therapy (PT, n=4), and non-ASD (MI, n=3) with HT. We evaluated biomarkers using immunoreactive methods.

Results: There were no group differences in behavioral scores. However, the HFASD HT group exhibited greater ATEC score in the health subscale $(p<0.05)$, higher global DNA methylation levels $(p<0.05)$, and lower OXT levels than other groups. Stratified comparisons revealed that methylated DNA levels were greater among HFASD after HT ( $p$ $<0.05$ ), compared to HFASD receiving PT. After stratifying for other variables, biomarkers exhibited no differences by therapy sessions.

Conclusions: Due to small sample size our results are preliminary, providing experimental evidence that HT may provide molecular marks in HFASD in response to HT. Future studies should address epigenetic responses of non-pharmacological interventions, as potential markers of psychotherapeutic response in ASD.

Correspondence: Leslie M. Diaz-Ortiz, Ph.D., Neuropsychology, Nicklaus Childrens Hospital, La Mansion Abades St NB31, Toa Baja, PR 00949, United States.E-mail:Imdiazortiz@gmail.com

\section{EVANS, S. GILLESPIE \&. J. BRADSHAW. Neurological and Behavioral Functioning in Infants at High- and Low-Risk for Autism Spectrum Disorder.}

Objective: Children diagnosed with Autism Spectrum Disorder (ASD) often have marked difficulties in attention and self-regulation, with deficits emerging as early as one year of age (Gomez \& Baird, 2005; Sally et al., 2016). Our study aims to investigate differences in neurological and behavioral development between infants at high- (HR) and low-risk (LR) for ASD, specifically in self-regulation and attention, by using the NICU Network Neurobehavioral Scale (NNNS; Lester \& Tronick, 2004). The NNNS is an assessment of neonatal neurologic integrity and behavioral functioning that has previously been used to help predict later developmental and behavioral outcomes in children (Liu et al. 2010).

Participants and Methods: Participants included infants at LR $(\mathrm{N}=29)$ and HR $(\mathrm{N}=32)$ for ASD and were seen longitudinally from 1 -week to 6-months of age. Measures of self-regulation and attention were obtained from the NNNS and differences between LR and HR infants were explored.

Results: Mixed effect repeated measures ANOVAs were used to analyze trajectories of self-regulation and attention. Self-regulation: Results revealed a main effect trending toward significance $(p=.058)$ for riskstatus, where HR infants scored lower regardless of age. In addition, HR participants scored significantly lower than LR participants at 1-month $(p<.05)$. Attention: Results revealed a significant main effect for age $(p=.015)$ and risk-status $(p<.001)$; HR infants scored lower regardless of age, while all infants' scores were increasing over time. Significant differences between mean attention scores were seen at $2-(p=.001)$ and 3 -month $(p=.05)$ time-points; HR infants had lower scores compared to LR infants at both these time-points.

Conclusions: Results suggest HR infants may exhibit a disruption around 2-months of age, when an infant's subcortically-controlled behavior shifts to cortically-controlled, volitional behavior. Early identification of vulnerabilities may allow for better outcomes as attentional and self-regulation demands increase during childhood.

Correspondence: Lindsey Evans, BA, Marcus Autism Center, Children's Healthcare of Atlanta, 915 W Peachtree St NW, Apt. 12013, Atlanta, GA 30309, United States.E-mail: lindseym.evans@choa.org
A. FLORES, K. MCFARLANE, R. GOODCASE, T. ANDERSEN, C. SWICK, J. BRENNAN, I. KOVELMAN, S. BOWYER \& R. LAJINESS-O'NEILL. Relationship between Resting State Alpha Motor Network Synchrony and Restricted and Repetitive Behaviors in Children with ASD.

Objective: Autism Spectrum Disorder (ASD) is characterized by atypical social functioning and restricted and repetitive behaviors (RRBs). Brain oscillations in alpha-band $(8-13 \mathrm{~Hz})$ are associated with motor functioning, and aberrant neural synchrony between motor regions may contribute to motor dysfunction in ASD. This study explored relationships between motor network synchrony (connectivity) in alpha band and RRBs.

Participants and Methods: Twelve ASD (Age: $M=9.1 ; S D=1.0$ ) and 13 neurotypical (NT) children (Age: $M=9.3 ; S D=1.3$ ) underwent magnetoencephalography at rest. Synchrony was quantified via coherence between cortical sites in the alpha band. Kendall Tau correlations between coherence in motor regions and the Social Responsiveness Scale, $2^{\text {nd }}$ Edition RRB scale were done. The regions of interest were precental and postcentral gyri, inferior, middle and superior frontal gyri, superior parietal gyri, angular gyri, and caudate and putamen.

Results: Group comparisons revealed that the ASD group had significantly more RRBs $(p<.001)$. Increased left intrahemispheric dorsal striatum coherence, as well as interhemispheric coherence between left putamen and right pre/post central gyri, was associated with increased RRBs in ASD $(p<.05)$. In contrast, increased right intrahemispheric coherence between the angular and inferior frontal gyri was associated with increased RRBs in NT $(p<.05)$.

Conclusions: In both groups hyperconnectivity between motor regions appeared to confer risk for more RRBs. In ASD, hyperconnectivity within regions of motor planning, control, and execution, as well as somatosensory information integration, is associated with increased RRBs. Within the normal range of RRBs, the NT group demonstrated a distinct neurocorrelate of these behaviors, as hyperconnectivity between motor inhibition and sensory motor regions was associated with increased RRBs. Results suggest an altered alpha motor control network in ASD, which may play a critical role in motor performance within this population. Correspondence: Ana-Mercedes Flores, Psychology, Eastern Michigan University, 509 West Forest Ave. , Apt 319, Ypsilanti, MI 48197, United States.E-mail: aflore14@emich.edu

S. KOCH, J. LEBERSFELD, F. BIASINI \& M. HOPKINS. Measures of Social Perception as Predictors of Clinician-Observed and Parent-Rated Autism Symptomology.

Objective: In addition to current "gold-standard" instruments for comprehensive evaluation of autism spectrum disorder (ASD), measures assessing related and theorized underlying areas of difficulty have been developed for clinical use. These include measures of affect recognition (AR; identifying other's emotions) and theory of mind (TOM; understanding others' perspectives). The goal of this study was to examine how performance on standardized measures of social perception (SP) relates to actual ASD symptoms.

Participants and Methods: 22 children (ages 5-12) with ASD and average cognitive skills completed this study. SP skills were assessed by AR and TOM subtests from the Developmental Neuropsychological Assessment (NEPSY-II). ASD symptom severity was evaluated via the Autism Diagnostic Observation Schedule (ADOS-2), including social affect (SA) and restricted and repetitive behavior (RRB) algorithm domains, as well as the parent-rated Social Communication Questionnaire (SCQ).

Results: The relationship of AR and TOM scores to ASD symptom severity was examined separately for each ADOS-2 domain and SCQ overall total. Correlational analyses revealed both AR and TOM were inversely related to RRB, $r(20)=-.69, p<.001$ and $r(20)=-.65$, $p<.01$, and TOM was inversely related to SCQ, $r(20)=-.48, p<.05$. All other correlations were not significant. Regression analyses examined the combined contribution of AR and TOM to symptom severity within 
RRB and SCQ. SP measures accounted for $64.5 \%$ of the variance in RRB, $F(2,19)=17.29, p<.001$, but did not significantly predict SCQ. Conclusions: Results suggest decreased performance on SP measures is related to increased ASD symptom severity, particularly RRB. Incorporating these measures within comprehensive assessments may provide greater clarity about an individual's social-emotional skills and aid clinicians in making more informed diagnoses, referrals, and treatment recommendations.

Correspondence: Sarah Koch, Psychology, University of Alabama at Birmingham, 500 Crawford Street Apt 632, Houston, TX 77002, United States.E-mail:sakoch@uab.edu

C.M. LEE, A. ESLER \& R. HUDOCK. Autistic Characteristics and Developmental Trajectories among Individuals with Fragile X Syndrome and Non-Syndromic Autism Spectrum Disorder.

Objective: Fragile $\mathrm{X}$ syndrome (FXS) is the most common genetic cause of autism spectrum disorder (ASD). The high prevalence of autistic features among people with FXS raises the hypothesis that FXS and ASD may share similar underlying neurobiological impairments. However, recent studies suggested that males with FXS exhibit distinct ASD symptoms compared to those with non-syndromic ASD. The purpose of this study is to examine the differences of ASD symptoms and developmental trajectories among children with comorbid FXS and ASD and those with non-syndromic ASD.

Participants and Methods: Eighteen children with full-mutation FXS and eighteen children with ASD matched on age, gender, and cognitive level completed comprehensive diagnostic evaluations in an ASD specialty clinic. Eight of the children received follow-up evaluations approximately one year after their first visits. The Fisher Exact Test was used to examine the group differences of ASD symptomology using items of the Social Communication Questionnaire (SCQ). The changes of ASD symptoms, cognitive abilities, and adaptive functioning across two visits were also examined.

Results: Children with comorbid FXS and ASD exhibited less social impairments (sharing interests, joint attention, and facial expression) but poorer adaptability and motor skills compared to children with non-syndromic ASD. Among children with FXS, those with comorbid FXS and ASD displayed more repetitive motor movements than those without ASD. At follow-up, children with non-syndromic ASD demonstrated improvement in ASD symptoms, language, social skills, and daily living skills, whereas children with FXS displayed decline in expressive language, communication, social skills, daily living skills, and motor skills.

Conclusions: Children with comorbid FXS and ASD displayed different presentation compared to those with non-syndromic ASD. The differences in ASD symptoms and developmental trajectories may be critical to understanding the causes of ASD and in developing appropriate treatments.

Correspondence: Chimei M. Lee, Ph.D., Psychology, Eastern Michigan University, 2212 Aldrich Ave S, Apt 104, Minneapolis, MN 55405, United States. E-mail: mchimeilee@gmail.com

K. MEYERING, J. WEISSMAN, A. DURKIN, E. FELDMAN, E. ISENSTEIN, L. DONNELLY, D. HALPERN, M. GORENSTEIN, A. WANG, M. TRELLES, A. KOLEVZON, J. BUXBAUM, J. FOSSFEIG, S. BISHOP \& P. SIPER. Web-Based Cognitive Assessments in Individuals with Autism Spectrum Disorder and their Immediate Family Members.

Objective: Several single-gene causes of autism spectrum disorder (ASD) have been identified to date, however, existing databases often lack relevant clinical information (e.g., IQ), particularly from unaffected family members. Remote administration of clinical assessments will substantially enrich our ability to assess genotype-phenotype relationships, while providing greater access to research participation. The current study seeks to establish the feasibility and convergent validity between web-based measures of language and cognition and an examiner-administered abbreviated IQ test.

Participants and Methods: Fifty individuals participated in this study, including 13 children and adolescents with ASD (ages 6-19), 14 unaffected siblings (ages 6-27), and 23 biological parents (age 38-69). Web-based administrations of the Peabody Picture Vocabulary Test, $4^{\text {th }}$ Edition (PPVT-4) and a Visual Reasoning task (testmybrain.org) were completed on a laptop in the clinic. A trained examiner administered the Wechsler Abbreviated Scale of Intelligence, $2^{\text {nd }}$ Edition (WASI-II). Results: Three participants with ASD were unable to complete the battery. Web-based administration of the PPVT-4 correlated very strongly with the WASI-II FSIQ-4 subtests $(r=.86, p<.001)$, FSIQ-2 subtests $(\mathrm{r}=.84, p<.001)$, and the Verbal Comprehension Index $(\mathrm{r}=.85$, $p<.001)$. Web-based Visual Reasoning correlated strongly with WASI-II FSIQ-4 $(\mathrm{r}=.70, \mathrm{p}<.001)$, FSIQ-2 $(\mathrm{r}=.67, \mathrm{p}<.001)$ and the Perceptual Reasoning Index $(r=.67, \mathrm{p}<.001)$.

Conclusions: Our findings suggest web-based assessments may provide a reliable method to obtain gross estimates of cognitive functioning. While WASI-II correlations were stronger with the PPVT-4, results from larger samples will clarify optimal measures based on diagnosis and age. There was no significant difference between two vs. four WASI-II subtests, which could reduce resources. Findings have implications for large-scale data collection in the context of ongoing genetics studies in ASD.

Correspondence: Kristin Meyering, BA, Psychiatry, Seaver Autism Center at the Icahn School of Medicine at Mount Sinai, 52 Indian Head Road, Riverside, CT 06878, United States.E-mail: kristin.meyering@ mssm.edu

J. CHEN, M. BARTON, D. FEIN \& L.E. MILLER. The Relationship Between Socio-Emotional and Cognitive Development in Infants and Toddlers at Risk for a Developmental Disorder.

Objective: To describe the relationship between socioemotional development and cognitive development in children at risk for a developmental disorder. To assess if this relationship is moderated by diagnostic status, age, and gender.

Participants and Methods: Infants and toddlers ( $N=40 ; 13-28$ months-old; mean age $=18.3$ months) who screened positive on developmental screeners (the Infant Toddler Checklist at 12 months, First-Year Inventory, Lite at 12 or 15 months, or M-CHAT-R at 15, 18 , or 24 months) or whose pediatricians indicated concerns attended a diagnostic evaluation. The Mullen Scales of Early Learning (MSEL) assessed cognitive skills. The Infant-Toddler Social and Emotional Assessment (ITSEA) assessed socioemotional competence. Children were grouped by diagnostic status: Delay (autism spectrum disorder, developmental delay, or language disorder) and No Delay (typical development or sub-clinical symptoms). MSEL domain ratio IQ's were calculated to address data skew, and were regressed individually on ITSEA Competence T-scores. Diagnostic group, age, and gender were moderators.

Results: Socioemotional competence was associated with visual reception $(\beta=.42, p=.007)$, fine motor $(\beta=.48, p=.002)$, receptive language $(\beta=.56, p<.001)$, and expressive language skills $(\beta=.33$ $p=.040)$. Diagnostic group moderated the relationship between socioemotional competence and visual reception skills $(\beta=.60, p=.045)$, with only the Delay group showing a significant positive relationship between variables.

Conclusions: Socioemotional competence during infancy and toddlerhood was associated with multiple areas of cognitive development in children who screened positive for a development disorder. Lowered ability to attend to and interact with one's environment, and maintain motivation for novel tasks may impact the ability to acquire some early learning skills, particularly for children with a developmental disorder. Findings should be replicated with a larger sample that allows for analysis by type of disorder.

Correspondence: Lauren E. Miller, CT, United States. E-mail: lauren. miller@uconn.edu 
D.P. PANFILI, E.E. ELINE \& K. OSIPOWICZ. Using Machine Learning on Resting State and DTI to Classify ASD.

Objective: Autism spectrum disorder (ASD) is a common but heterogeneous spectrum of developmental disorders. The developmentally deleterious effects of ASD have been demonstrated on cognitive, functional, and structural levels. All of these previous studies support the view that ASD is a network disease, affecting function and structure of cortical and subcortical regions throughout the brain. Our prior work supports the belief that ASD causes wide ranging network disruptions, as shown through multi-voxel pattern analysis of fMRI resting state scans of ASD patients. Our previous work also supports the notion that network disruptions are a distinguishing feature of both ASD and of ASD severity level. This study aims to extend those findings by exploring how network disruptions manifest in the underlying white matter anatomy. Participants and Methods: Here we use machine learning methods on model-free diffusion tensor imaging and resting state data to predict cognitive and clinical outcomes in a sample of ASD subjects. We extracted data from 131 ASD patients and 105 age matched controls. Results: We found that a predictive model incorporating white matter and functional disruptions is superior in predicting both classification and outcomes.

Conclusions: These findings suggest that ASD disrupts both the function and structural organization of the brain, and that the imaging profile of these disruptions is predictive of disorder manifestation. Correspondence: Daniel P. Panfili, Psychology, Drexel University, 206 N 34 th St, Philadelphia, PA 19104, United States. E-mail: daniel.paul. panfili@gmail.com

M.D. POWERS, L. KENWORTHY, L. ANTHONY, A.D. VERBALIS, S. SEESE, J. SAFER-LICHTENSTEIN \& A. RATTO. Linguistic Diversity in Neurodevelopmental Disorders: Dual Language Exposure Relates to Cognitive Flexibility in Children with ASD but Not ADHD.

Objective: To substantiate recent findings that dual language exposure (DLE) may provide an executive function (EF) advantage for children with Autism Spectrum Disorder (ASD; Iarocci et al., 2017; Baldimtsi et al., 2016), but an EF disadvantage for children with Attention-Deficit/ Hyperactivity Disorder (ADHD; Bialystok et al., 2017; Mor et al., 2016). Participants and Methods: Participants included 126 ( $\mathrm{n}=51 \mathrm{DLE})$ children with ASD $(n=42)$ or ADHD $(n=84)$ ages 8-11 $(M=9.65$, $\mathrm{SD}=.85$ ), who were participants in a larger school-based treatment study. All participants had an FSIQ>70 (M=97.72, SD=13.96) when tested in English using the Wechsler Abbreviated Scales of Intelligence-II. Cognitive flexibility was measured using the Flexibility Interference Scale (FIS), an unpublished seven-item parent rating scale with a maximum score of 21 . On the FIS, a higher score indicates more cognitive rigidity.

Results: A simple linear regression was conducted to predict cognitive flexibility skills based on DLE. Demographic factors known to influence EF skills including socioeconomic status (income and parent education) and linguistic skills were included in the model. In the overall sample, DLE status was significantly associated with better parent-reported flexibility, $\beta=-0.23, p=.046$, compared to monolingual peers with neurodevelopmental disorders. When differentiating by diagnosis, this association strengthened among DLE children with ASD, $\beta=-0.25, p=.031$. However, when looking only at children with ADHD, there was no evidence that the DLE children performed significantly better on measures of flexibility compared to their monolingual peers, $\beta=-0.93, p=.358$.

Conclusions: These findings support prior research that while children with ASD may benefit from DLE, children with ADHD do not reap these same benefits. Given the limited research available to parents seeking guidance about their child's linguistic upbringing, more research is needed to understand the role of DLE in clinical, pediatric populations.
Correspondence: Meredith D. Powers, Master of Education (M.Ed.), Center for Autism Spectrum Disorders (CASD), Children's National Health System, 2810 Abilene Drive, Chevy Chase, MD 20815, United States.E-mail: meredith.d.powers@gmail.com

S. RAU, M.F. SKAPEK, K. TIPLADY, A.R. BURNS, S. SEESE, A. SHARBER \& L. KENWORTHY. Comparing the Diagnostic Discriminability of the ADHD Rating Scale and Achenbach Checklists to Capture Comorbid Attention-Deficit/ Hyperactivity Disorder Symptoms in Autism Spectrum Disorder.

Objective: Compare the discriminability of parent and teacher report on the ADHD-RS-IV (symptom counts [SC] and T-scores) and the Attention Problems and ADHD Problems subscales (T-scores) from the Child Behavior Checklist (CBCL) and Teacher Report Form (TRF). Participants and Methods: Participants included individuals with ASD without intellectual disability (ID; ages 6-18, mean FSIQ=95.95). Three diagnostic groups were compared: ASD only ( $\mathrm{N}=90)$, ASD+ Attention-Deficit/ Hyperactivity Disorder (ADHD)-Combined presentation (ADHD-C; $\mathrm{N}=159$ ), and ASD+ADHD-Inattentive presentation (ADHD-I; $\mathrm{N}=38$ ). Doctorate-level clinicians made ADHD diagnoses using data from parent and teacher questionnaires, clinical interview, and neuropsychological and behavioral assessments. Receiver operating curve analyses were used to identify diagnostic discriminability of measures.

Results: Consistent with previous research completed by this group, all of the measures provided acceptable and greater levels of diagnostic discriminability for identifying symptoms of ADHD-C relative to ADHD-I when comorbid with ASD without ID. Comparisons of AUCs across measures indicated parent report, particularly from the ADHDRS-IV, provided greater diagnostic accuracy than teacher report for ADHD-C diagnoses. The ADHD-RS-IV Inattentive SC (AUC=.679) and T-Score (AUC=.671) were the only measures that provided acceptable levels of diagnostic discriminability for symptoms of ADHD-I.

Conclusions: The ADHD-RS-IV and Achenbach subscales are more accurate at capturing symptoms of ADHD-C than ADHD-I when the diagnoses are comorbid with ASD. This finding underscores the needs for future work to develop measures to better identify ADHD-I symptoms in populations with ASD. Parent report is more heavily relied upon than teacher report when clinicians make ADHD diagnoses.

Correspondence: Srishti Rau, PhD, Pediatric Neuropsychology, Children's National Health System, 12 Crusader Ct., Germantown, MD 20874, United States. E-mail: srrau@childrensnational.org.

V. SEGHATOL-ESLAMI, C. AMMONS \& R. KANA. Functional Connectivity of the Fusiform Face Area with the Social Brain during Action Perception in Autism.

Objective: Impairments in social communication are a hallmark of Autism Spectrum Disorder (ASD). Compromise to large-scale neural networks (e.g. social brain) operating in synchrony may result in social deficits in ASD (Kana et al., 2009; 2015). The fusiform face area (FFA) on the lateral fusiform gyrus (LFG) specializes in face processing (Kanwisher et al., 1997) and shows altered response in ASD (Schultz, 2005). This functional MRI study investigates connectivity of FFA with social brain regions in individuals with ASD during an action perception task.

Participants and Methods: 21 ASD and 20 typically developing (TD) adults matched on age, gender, and IQ viewed images of a model interacting with an object and made judgments about the means and intent of the action (ordinary vs unusual). We defined social brain regions-of-interest (ROIs) using Neurosynth meta-analysis and subject-specific LFG ROIs using participants' mid-fusiform sulcus anatomy. ROI-to-ROI functional connectivity was measured in $\mathrm{CONNv} 17$ and compared across groups.

Results: As expected, left and right LFG had stronger connections with the social brain network and individual social brain regions during action perception [all tests $\mathrm{T}(40)>2.55, \mathrm{p}<.05 \mathrm{FDR}$ ]. When judging 
the intent of the actor, there was increased connectivity between left LFG and posterior cingulate cortex (PCC)/precuneus in TD participants $[\mathrm{T}(39)=3.37, \mathrm{p}<.05 \mathrm{FDR}]$. There were no areas of greater connectivity in ASD.

Conclusions: Increased connectivity of the left FFA and PCC in TD participants while judging actor's intent reflects greater involvement of social brain in this task. PCC is involved in diverse cognitive functions including autobiographical memory and attentional regulation (Leech \& Sharp, 2014). Thus, TD individuals may use more self-referential thinking or attentional resources directed to the face when inferring intent. Lack of FFA/PCC coupling may contribute to theory-of-mind deficits in ASD.

Correspondence: Victoria Seghatol-Eslami, Psychology, University of Alabama at Birmingham, 1071 Magnolia Run, Hoover, AL 35226, United States.E-mail:vs129@uab.edu

K. SHADA, M. CORDOVA, S. DUVALL, A. GRAHAM, D. FAIR, J. NIGG \& E. FOMBONNE. Executive Function in ASD, ADHD, Comorbid ASD/ADHD and Typically Developing Children Assessed Using Parent Rating Scales and Task-Based Performance Measures.

Objective: Executive functioning (EF) deficits are commonly seen in children with Autism Spectrum Disorder (ASD) and Attention-Deficit/ Hyperactivity Disorder (ADHD) when compared to typically developing (TD) children. The comorbidity of ASD+ADHD has been hypothesized to lead to an increase in functional impairment when compared to TD, ASD or ADHD only children. It is unknown whether EF strengths and deficits for children with ASD+ADHD are similar in children with just ADHD. Elucidating the extent and nature of these deficits may aide in differential diagnosis and treatment.

Participants and Methods: 209 subjects (TD; $\mathrm{n}=69$, ASD; $\mathrm{n}=28$, ADHD; $n=73$, ASD+ADHD; $n=39$ ) received consensus diagnoses using research reliable ADOS and ADI-R, K-SADS, records, and parent/ teacher questionnaires. Measures used included the Behavior Rating Inventory of Executive Function (BRIEF) and standardized EF tasks (DKEFs Color Word and Tower, WISC-IV Digit Span, and NEPSY Word Generation). A Kruskal Wallis H test was used to examine differences between all groups corrected for multiple comparisons.

Results: The ASD+ADHD group showed greater impairment on BRIEF scales of Plan/Organize, Initiate, and Working Memory (vs. ASD), and on Shift (vs. ADHD). Of the tasks, the ASD+ADHD group showed greater impairment only on the Tower (vs. ADHD). Overall, the ASD+ADHD showed greater functional impairment on the BRIEF compared to ASD or ADHD only groups; however, this pattern was only mirrored on the Tower task (construct planning).

Conclusions: The comorbid group's functional EF appeared to be the most impaired when considering parent-report, indicating that deficits may be observed in the environment that are not well captured on laboratory tasks. The results suggest that children with ASD+ADHD perform similarly to other groups on laboratory tasks, with the exception of planning, but they may be experiencing additional functional difficulties in their environment as reflected by parent report.

Correspondence: Kiryl Shada, OR, United States. E-mail: shad9079@ pacific.edu

M.F. SKAPEK, C. PUGLIESE, S. SEESE, M.D. POWERS, L. KENWORTHY \& J.F. STRANG. Anxiety Relates to Interference Associated with Planning and Flexibility Weaknesses in Youth with ASD.

Objective: Elevated rates of anxiety are prevalent in youth with Autism Spectrum Disorders (ASD). Evidence suggests an association between anxiety and inflexibility, but little is known about the relationship between anxiety and other executive functioning (EF) skills, including planning. This study evaluates the relationship between anxiety and planning, as well as flexibility, in ASD.
Participants and Methods: Baseline data was used for 40 youths with ASD without intellectual disability ( $9 \mathrm{f}$, ages 12.6-14.1 [M=13.5, $\mathrm{SD}=1.7]$ ) who participated in a randomized comparative effectiveness trial of two school-based interventions. Standardized EF measures included the Delis-Kaplan Executive Function System (DKEFS) and the Tower of London (TOL). Parent-report variables were the Planning and Shift scales from the Behavior Rating Inventory of Executive Function $2^{\text {nd }}$ Ed., 14 unpublished items assessing planning/flexibility interference (I-Plan; I-Flex), and the Parent Anxiety Checklist-ASD*. Pearson correlations were calculated between total anxiety, planning scores (Plan/Org T, TOL Avg. T, I-Plan), and flexibility scores (Shift T, DKEFS Correct Sort SS, I-Flex).

*In development; final version to be released soon (Bearss et al. 2016) Results: Anxiety symptoms correlated with parent-reported inflexibility (Shift T: r=.6, p<.01) but not parent-reported planning or lab-based planning/flexibility measures (Plan/Org T: r=.29, p>.05; TOL Avg. T: $r=.001, p>.05$; Correct Sort SS: $r=.307, p>.05$ ). In contrast, flexibility and planning interference scores were associated with anxiety (I-Flex $\mathrm{r}=.63$, $\mathrm{p}<.01$; I-Plan $\mathrm{r}=.457, \mathrm{p}<.01$ ).

Conclusions: Results corroborated findings that anxiety and parentreported inflexibility are correlated in ASD. While anxiety was unrelated to parent-reported or lab-based planning skills, it was significantly related to interference in daily life associated with planning weaknesses. These findings encourage further inquiry into the interrelatedness between anxiety and EF difficulties in autistic youth

Correspondence: Mary F. Skapek, B.S, Neuropsychology, Children's National Health System, 15245 Shady Grove Rd, Rockville, MD 20850, United States.E-mail:mfskapek@childrensnational.org

\section{Epilepsy/Seizures}

D.T. PULSIPHER, E.M. KRAPF \& L.D. STANFORD. The Contribution of Executing Functioning to Memory Performance in Pediatric Focal and Generalized Epilepsy.

Objective: Children with epilepsy often exhibit deficits in memory and executive functioning (EF). In order to better understand the memory profiles of children with epilepsy, we examined whether EF could explain memory deficits in children with focal epilepsy compared to children with generalized epilepsy. We hypothesized that, due to greater thalamocortical involvement, the generalized group would show greater executive dysfunction and impact memory more than in the focal group. Participants and Methods: Sixty-nine children with focal epilepsy and 73 children with generalized epilepsy matched on age (8-18 years), gender, and intellectual functioning completed measures of intelligence, EF (DKEFS Trail Making Test Number-Letter Switching, Verbal Fluency Category Switching-Total, and Color Word Inhibition), and memory (RAVLT Delayed Recall [DR], WRAML-2 Story Memory Recall, and BVMT-R DR). Regression analyses were used to determine the amount of variance accounted for by the three DKEFS scores in each of the memory measures.

Results: The focal group performed significantly more poorly on DKEFS Number-Letter Switching ( $p=0.007)$, but the two groups did not differ on other EF or memory measures. EF accounted for $<2 \%$ of the variance on RAVLT DR in each group; however, it accounted for $33 \%$ of the variance in the focal group and $12 \%$ of the variance in the generalized group on WRAML-2 Story Memory Recall. Similarly, EF predicted greater variance on the BVMT-R in the focal group than the generalized group ( $20 \%$ vs. $4 \%$, respectively).

Conclusions: Contrary to expectations, EF accounted for a much smaller portion of variance in the generalized group, while EF accounted for significant variance on tasks of story memory and visual memory but not list learning in the focal group. Thus, the contribution of EF to memory varies by epilepsy type, which may have important implications for clinical data interpretation and suggests differing impacts of epilepsy type on neural substrates in each group. 
Correspondence: Dalin T. Pulsipher, Ph.D., NeuroDevelopmental Science Center, Akron Children's Hospital, 215 W. Bowery St., Suite 4500,Akron,OH 44308,United States.E-mail:dpulsipher@chmca.org

\section{Learning Disabilities/Academic Skills}

T. ALLAIRE, J.L. RIGGS, R. LAJINESS-0'NEILL \& K. SAULES. A Cluster-Analytic Approach to Examining Links Between Psychodiagnostic Profiles and Neuropsychological Task Performance Among an Outpatient Clinical Sample.

Objective: This study aimed to clarify the link between neuropsychological task performance and adult neurodevelopmental disorder diagnosis (i.e., attention-deficit/hyperactivity disorder [ADHD], learning disorder $[\mathrm{LD}])$. It was expected that diagnostic profiles would differ related to task performance.

Participants and Methods: A chart review of 111 adults $(53.6 \%$ female; $74.1 \%$ white; $49.5 \% \geq$ some college education) who received psychological assessment services at a psychology training clinic was conducted. Data include intellectual (Wechsler Adult Intelligence Scale), achievement (Wide Range Achievement Test), and attention (Conners Continuous Performance Test; Conners Adult ADHD Rating Scale) measures.

Results: Clinical diagnoses were coded "presence/absence" for ADHD and LD. Comorbidity was allowed. Two-step categorical cluster analysis yielded a 4-profile solution: developmentally typical (54.1\%), ADHD without LD (13.5\%), LD without ADHD (26.1\%), and comorbid ADHD/LD (6.3\%). No demographic differences emerged. Significant differences did emerge in intellectual functioning $(F[3,96]=5.18$, $\left.p<.01, \eta^{2}=.12\right)$, math skills $\left(F[3,39]=4.80, p<.05, \eta^{2}=.27\right)$, and working memory $\left(F[3,98]=8.07, p<.01, \eta^{2}=.14\right)$. Specifically, the LD-only group performed worse than the ADHD-only group on measures of intellectual functioning and math skills and worse than the ADHD-only and developmentally-typical groups on working memory. Conclusions: In this study, individuals diagnosed with LD-only were more likely to exhibit deficits in intellectual functioning, math skills, and working memory. Neither the comorbid nor ADHD-only group performed worse than the LD-only group on any measure, including measures of inattention. These results contrast emerging literature suggesting that adults with comorbid ADHD/LD exhibit poorer working-memory, compared to either ADHD- or LD-only. Results underscore the importance of understanding the relationship between neuropsychological task performance and adult neurodevelopmental disorder diagnosis.

Correspondence: Ted Allaire, PhD, Psychology, Eastern Michigan University, 3956 Cloverlane Dr., Ypsilanti, MI 48197, United States. E-mail: allaire.ted@gmail.com

N. BECKER, L.D. PICCOLO, M. LIMA, A. JÚLIO-COSTA, J. SILVA, V.G. HAASE \& J.F. DE SALLES. Double Disadvantage in Reading Performance: Socioeconomic Status amplify the Effects of Learning Disabilities on Reading Performance.

Objective: Studies investigating the influence of socioeconomic status (SES) on child development suggest that the environment can either boost or limit language development. An extensive body of research has demonstrated that children with learning disabilities (LD) have poorer performance in reading when compared to typically developed (TD) children. However, it is unclear whether SES may amplify or ameliorate the effects of $L D$ in reading performance. We examined the moderating effect of SES (income and parental education) in the association between LD and reading performance (accuracy and fluency).

Participants and Methods: We assessed 155 children (LD = 35 and $\mathrm{TD}=120)$ from 4 th grade, ranging from 9 to 11 years old $(M=9$; $S D=0.55)$ from public schools from Brazil. Groups were split according to performance in Teste de Desempenho Escolar (TDE - spelling and arithmetic subtests) and word and nonword reading task. Reading performance was evaluated through a reading (words and nonwords) task and a reading fluency task.

Results: In general, LD children showed lower reading performance in regular and irregular words, nonwords, and in fluency speed. Interestingly, moderation analyses (controlling for age) indicated that the impact of SES (specially parental education) was not constant across the reading performance between LD and TD children. LD children from most disadvantaged socioeconomic backgrounds underperform LD children from higher parental education families and TD children from either high and low parental education families in reading accuracy measures (regular and irregular words and nonwords reading). There were no SES effects on any reading measures among TD children.

Conclusions: These results draw attention to the importance of public policy-making targeting families from low SES in order to stimulate the development of literacy skills, given that the effects of learning disabilities seem to be greater for children from disadvantaged backgrounds. Correspondence: Natalia Becker, PhD Candidate, Instituto de Psicologia, Universidade Federal do Rio Grande do Sul, Rua Augusto Pestana, 226 apto 91, Porto Alegre 90040200,Brazil.E-mail: natxib@ gmail.com

P.T. CIRINO, J. MICIAK, Y. AHMED, M.A. BARNES, P. TAYLOR \& E.H. GERST. Contributions of Executive Function to Reading. Objective: More is known about early reading predictors relative to later outcomes, but the Simple View (Gough \&. Tumner, 1986) has much support, positing that decoding and listening comprehension interact to produce reading comprehension. Executive function (EF) has been associated with reading (e.g., Cain et al., 2004), but its role across reading skills, and in the context of known predictors is unclear. We hypothesized that EF would contribute over all known predictors, and interact with Simple View variables.

Participants and Methods: Participants were 846 students in grades $3-5$; they were given 4 groups of measures: demographic/cognitive covariates; language measures; $27 \mathrm{EF}$ variables derived from a recent bifactor framework (Cirino et al., 2017); and reading measures (decoding, fluency, comprehension). Students were demographically diverse, and many were at-risk for reading difficulty. Analyses used general and specific EF factor scores from the bifactor framework to assess their impact via regression.

Results: The word reading model was significant $\left(p<.001, R^{2}=.58\right)$; unique predictors were 3 specific EF factors, phonological awareness, and rapid naming, and several covariates, but with substantial shared variance. Fluency produced a similar pattern $\left(p<.001, R^{2}=.57\right)$. The comprehension model $\left(p<.001, R^{2}=.69\right)$ showed unique contributions of general EF, 1 specific EF factor, Simple View variables, and others, but importantly, both general EF and specific EF factors interacted with Simple View variables, $p^{\prime} s=.010$ and .014 , with even larger shared variance.

Conclusions: EF contributed to all reading outcomes, even over known predictors. EF also interacted with Simple View variables when general EF was low, Simple View variables predominated, but with higher general EF, this effect was lessened. The degree of shared variance and the interactive effects add nuance to extant findings (e.g., Locascio et al., 2010), and contribute to understanding of the Simple View model (Georgiou et al., 2009; Savage, 2006).

Correspondence: Paul T. Cirino, PhD, Psychology, University of Houston, UH TMC Annex, 2151 W Holcombe Blvd, 224a, Houston, TX 77204-5053, United States. E-mail: pcirino@uh.edu

M. FERNANDES \& E. TONE. Exploring associations among verbal language knowledge, social anxiety, and nonverbal processing in children.

Objective: Successful social interactions demand language skill; inaccurate decoding of words and nonverbal cues can contribute to awkward interactions (Kavale \& Forness, 1996; Philippot \& Feldman, 1990) and to social anxiety and avoidance (Goldston et al., 2007; McClure 
\& Nowicki Jr., 2001). Traditionally, research on language and social anxiety has focused on either nonverbal or verbal language skill in isolation, and most studies that measure nonverbal decoding have targeted facial expression recognition alone (Staugaard, 2010). We know little about how skill at two facets of language comprehension-understanding word content and reading vocal prosody-may combine to predict social anxiety, especially in children, who are developing both language and social skills. We anticipated that these facets would interact, such that social anxiety would be highest in children with the lowest scores on both vocabulary and emotional prosody decoding measures, and lowest in children with the highest scores on both measures.

Participants and Methods: In a sample of 62 typically developing $3^{\text {rd }}-4^{\text {th }}$ graders, we tested the hypothesis that verbal language knowledge (K-BIT Vocabulary score) and emotional prosody decoding skill (DANVA-II Voices scores) would interact to predict self-reported social anxiety.

Results: Multiple regression analysis yielded a significant interaction between verbal language knowledge and prosody recognition, $R^{2}=.157$, $F(5,56)=3.271, p<0.05$, such that for children with high K-BIT scores $(M=126.64)$, less accurate recognition of vocal emotion was associated with high social anxiety. For children with low average to average K-BIT scores $(M=105.95)$, in contrast, accurate recognition of vocal emotion did not significantly relate to social anxiety. All main effects were non-significant ( $p$ 's $>0.05)$.

Conclusions: Our findings suggest that children's language skills in both verbal and nonverbal domains warrant examination in tandem as factors that may interact to contribute to social distress and anxiety. Correspondence: Mary Fernandes, Psychology, Georgia State University, 571 Densley Drive, Decatur, GA 30033, United States. E-mail:mfernandes2@student.gsu.edu

J.M. GUERIN, Q. MANO, K.E. JASTROWSKI MANO, R.C. GIBLER, S.P. BECKER, L. TAMM \& J. EPSTEIN. Direct and Indirect Investment of Fluid Intelligence in Word Reading in Children Evaluated for Attention-Deficit/Hyperactivity Disorder.

Objective: To date, reading research has largely focused on "crystallized" intelligence $(\mathrm{G} c)$, leaving much unknown about the role "fluid" intelligence $(\mathrm{G} f$ ) plays in reading across development. New lines of research challenge the prevailing view that visual word recognition relies exclusively on $\mathrm{G} c$ to become fully automatized, suggesting instead that word reading never fully sheds its novelty and that $\mathrm{G} f$ continues to play a role in word reading beyond early moments of literacy acquisition. Participants and Methods: We examined direct and indirect (through $\mathrm{Gc}$ ) effects of $\mathrm{G} f$ on word reading in a sample of 362 children (ages 6-13) being evaluated for attention-deficit/hyperactivity disorder (ADHD). Conditional process modeling was used to examine whether developmental stage (i.e., grade levels 1-6) moderated possible direct and/or indirect effects of $\mathrm{G} f$ on word reading. In each analysis, inattentive and hyperactive/impulsive symptoms were entered as covariates. Gf, Gc, and word reading were all measured with standardized tests.

Results: Mediation analysis revealed significant direct and indirect effects of $\mathrm{G} f$ on word reading. The direct effect of $\mathrm{G} f$ on word reading in the total effect model was weakened in the presence of $\mathrm{G} c$, suggesting partial mediation. Although the index of moderated mediation was not significant, visual inspection of path coefficients suggested that the direct effects of $\mathrm{G} f$ on word reading lessened across development while indirect effects of $\mathrm{G} f$ on word reading through $\mathrm{G} c$ increased across development. Conclusions: Results broadly support Cattell's Investment Theory by showing that $\mathrm{G} f$ makes direct and indirect investment into word reading. Moreover, results expand on the current perspective on word reading automaticity by suggesting that $\mathrm{G} f$ invests in word reading throughout childhood and not exclusively during early literacy acquisition. Implications for the role of executive functions in reading among children with ADHD are discussed.
Correspondence: Julia M. Guerin, M.S., Psychology, University of Cincinnati, 4150 Edwards One Center, University of Cincinnati, Cincinnati, OH 45221, United States. E-mail: guerinja@mail.uc.edu

J.M. GUERIN, A. SYLVIA, D.A. MEDLER \& Q. MANO. Connecting Statistical Learning with Reading: The Importance of Task-Driven Attention and Key Mediating Variables.

Objective: The mediator(s) through which statistical learning is connected to reading remains a topic of active neuropsychological inquiry. Recent theoretical work suggests that one such mediator is sensitivity to subword combinatorial orthographic regularity (SCORe), which itself emerges from dynamic interplay between task-driven attentional control and incidental statistical learning. Following this two-process framework, we investigated the association between attention-dependent visual statistical learning (VSL) and SCORe-sensitivity in the context of broad reading skills.

Participants and Methods: In a sample of 71 university students $\left(M_{\text {age }}=19.53 ; S D_{\text {age }}=1.80\right)$, we examined whether SCORe-sensitivity mediates attention-dependent VSL and reading outcomes (i.e., word reading efficiency, silent word reading fluency). Sensitivity to SCORe was measured with a visual wordlikeness judgment task in which participant's viewed non-pronounceable letter-string that varied incrementally in SCORe and were instructed to judge whether they looked like a word. VSL was assessed with an embedded triplet learning task, designed to measure statistical learning in both attended and unattended streams of non-linguistic visual stimuli (i.e., attention-dependent VSL, incidental VSL; respectively). Word reading efficiency and silent word reading fluency were measured with standardized tests.

Results: Mediation analyses revealed that SCORe-sensitivity mediated the relation between attention-dependent VSL and word reading efficiency. Moreover, SCORe-sensitivity and word reading efficiency jointly mediated the relation between attention-dependent VSL and silent word reading fluency. No such results were observed for incidental VSL.

Conclusions: In support of the two-process framework, these results suggest that task-driven attentional control and SCORe-sensitivity are important for connecting statistical learning with reading. Implications for reading disability are discussed.

Correspondence: Julia M. Guerin, M.S., Psychology, University of Cincinnati, 4150 Edwards One Center, University of Cincinnati, Cincinnati, OH 45221, United States. E-mail: guerinja@mail.uc.edu

L.M. HARDY, M. TOMB, Y. CHA \& A.E. MARGOLIS. Neuropsychological Correlates of Bilingualism in a Sample of Children with Neurodevelopmental Disorders.

Objective: Relative to monolingual individuals, bilinguals perform better on some executive functions (set-switching, response inhibition, integration) but worse on others (verbal fluency). Bilinguals also show enhanced visual-spatial skills. Enhanced abilities may derive from benefits of switching between two languages, and decreased verbal fluency from competition between two lexicons. Herein, we evaluated whether these same patterns would be found in mono- and bilingual children from low SES backgrounds with neurodevelopmental disorders.

Participants and Methods: General linear models evaluated group differences (monolingual, bilingual) in cognitive outcomes from 141 monolingual and 160 bilingual children, ages 6-17, seen for a neuropsychological assessment. Verbal fluency was measured with KTEA-3 Associational Fluency, set shifting and response inhibition with NEPSY II Response Set, Inhibition-Inhibition, and Inhibition-Switching, and visual-motor/visual-spatial skills with Beery Visual-Motor Integration, NEPSY-II Memory for Designs (MD), and Memory for Faces (MF). Models controlled for age, sex, and diagnosis of ADHD or Reading Disorder.

Results: Relative to bilinguals, monolinguals showed better verbal fluency $(p=0.001)$ and worse visual-motor integration $(p=0.015)$ and visual memory skills (MF, $p=0.023 ; \mathrm{MD}, p=0.005)$. 
Conclusions: Bilingual children with neurodevelopmental disorders showed better visual-motor integration and visual memory but not set-switching or response inhibition than monolingual peers, suggesting the benefits of bilingualism are detected in visual but not verbal domains in children with neurodevelopmental disorders. Monolingual children showed greater verbal fluency than bilinguals, possibly because lexical retrieval is faster when searching vocabulary in only one language. This study extends prior findings of advantages in visual-motor and visual-spatial skills associated with bilingualism and verbal fluency with monolingualism to children with neurodevelopmental disorders.

Correspondence: Lindsay M. Hardy, Psy.D., Psychiatry/Promise Program, Columbia University Medical Center/New York Presbyterian, 635 W. 165th St., \#636A, New York, NY 10032, United States. E-mail: hardyli@nyspi.columbia.edu

\section{L.R. KIVISTO \& J.E. CASEY. What Factors Affect Children's Perceptions of Their Assistive Technology and Assistive Technology Training?}

Objective: Assistive Technology (AT) enables children with Specific Learning Disorders (LDs) to adequately access school curriculum. There is a paucity of literature addressing the use, perception of use, and training of students who qualify for AT. The few studies completed suggest that children with AT like their devices and find them useful. The current study examined what children liked and disliked about their AT, by qualitatively coding responses for emergent themes. The current study also examined which factors would predict children's perceptions of their AT and AT training. It was predicted that grade level, type of AT device, and themes of liking and disliking would predict children's perception of their AT and AT training. The current study aimed to increase the understanding of current practices of the distribution and utilization of AT.

Participants and Methods: Analysed was the archival data collected by the Learning Disabilities Association of Windsor-Essex County from 656 school-aged children over the span of four school years.

Results: For "What do you like most about your AT?" (Liking) 8 themes emerged, with the response theme "Helpful" being the most common $(50.5 \%)$. For "What do you like least about your AT?" (Disliking) 15 themes emerged, with "Technical Problems" being the most common response theme $(30.8 \%)$. Multinomial logistic regressions revealed that children's perceptions of their AT and AT training were influenced by their grade, AT device, and Disliking, but not Liking.

Conclusions: The results of the current study add to the understanding of current practices of AT training and the utilization of AT by children. Those with LDs often do not make use of the AT provided to them. The findings of the current study should guide AT distributors (e.g. school boards) and trainers in deciding which AT hardware to provide to children with LDs and how to provide training. Additionally, these results can benefit consumers and practitioners in their selections and recommendations of AT hardware.

Correspondence: Lynette R. Kivisto, Honors BA Psychology, Psychology, University of Windsor, 401 Sunset Avenue, Windsor, ON N9B3P4, Canada.E-mail:kwistol@uwindsor.ca

\section{K. MACDONALD, A. CHILD \& P. CIRINO. Longitudinal overlap of reading and math achievement and their predictors.}

Objective: This study examined the relative roles of domain general and domain specific predictors measured in kindergarten for several reading and math outcomes measured at the end of $1^{\text {st }}$ grade. We examined three pairs of math/reading subskills (core, fluency, complex) and hypothesized that some predictors would be more strongly linked to reading than math and vice versa, and that a different array of predictors would account for the overlap between reading and math depending on the subskill.

Participants and Methods: Participants included 193 students who were tested at the end of kindergarten and again at the end of $1^{\text {st }}$ grade. In kindergarten, students were assessed on a range of domain-specific reading predictors, domain-specific math predictors, and domain-general predictors. In $1^{\text {st }}$ grade, students were assessed on three pairs of math/reading outcomes.

Results: Multivariate regressions demonstrated that language-related skills (PA, RAN) were more strongly predictive of overall reading vs. math outcomes (PA: $p=0.014$; RAN: $p<0.001$ ), whereas counting knowledge $(p=0.005)$ and working memory $(p=0.046)$ were more predictive of math relative to reading. Partial correlations demonstrated that PA and symbolic naming were the strongest contributors to the overlap between core skills (reduction in $R^{2}=21 \%$ and $29 \%$, respectively), whereas the strongest contributors for fluency were RAN and symbolic naming (reduction in $R^{2}=16 \%$ and $24 \%$, respectively). The same predictors that were related to the overlap in core skills and fluency also accounted for the overlap in complex skills.

Conclusions: The results suggest that a small set of predictors (PA, RAN, symbolic naming, symbolic comparison, and counting knowledge) might be useful in early screening for both reading and math difficulty. Moreover, results also suggest that early screening approaches should consider achievement in both domains simultaneously, and that students identified as at-risk for one domain should also be monitored for performance in the other domain.

Correspondence: Kelly Macdonald, Clinical Psychology, Psychology, University of Houston, 5736 Petty Street, Houston, TX 77007, United States.E-mail:kmacdonald@uh.edu

\section{A. OSSOWSKI, J.C. FRIJTERS \& R. MORRIS. Effects of ADHD Symptoms and Executive Function Impairments on Response to Reading Intervention.}

Objective: Reading disabilities (RD) show very high comorbidity with ADHD. Those with comorbid RD+ADHD show increased behavioral and emotional problems, other learning disorders, and poor academic outcomes, compared to those diagnosed with only RD or only ADHD. Executive function (EF) impairments have been suggested to be an important cognitive deficit underlying ADHD in many children. If $\mathrm{EF}$ impairments represent a specific cognitive deficit in many RD+ADHD children, it is possible that EF impairments may also influence response to reading intervention, further mediating the effects of more behavioral ADHD symptoms (inattention, inhibition, etc.). However, the extent to which EF impairments, or comorbid ADHD symptoms, in children with RD influence their response to reading interventions has not been determined. This study examined the role of both ADHD behavioral symptoms and EF impairments in children with RD in predicting their response to intervention.

Participants and Methods: Utilizing a sample of 55 children with reading impairments, ages 8-10, we assessed whether higher reported baseline levels of ADHD symptoms, behavioral and/or cognitive measures of executive function predicted baseline reading skill and reading skill growth rates over the course of a reading intervention.

Results: Results showed that behavioral ratings of EF, particularly the BRIEF Metacognition Index, were predictive of baseline reading skill, whereas cognitive measurements of EF were predictive of reading skill growth rate over the course of the intervention. ADHD inattentive or hyperactivity symptoms alone were not predictive of baseline reading skill or response to intervention.

Conclusions: Cognitively measured EF deficits associated with ADHD may predict response to reading skill intervention, rather than measurements of overall ADHD symptoms.

Correspondence: Alexandra Ossowski, Clinical Neuropsychology, Psychology, Georgia State University, 3309 Grayson Place, Decatur, GA 30030, United States. E-mail: aossowski1@student.gsu.edu 
E. RIGGALL, R. WINTER, L. BRANUM-MARTIN, R. SEVCIK, R. MORRIS \& S. FROST. Deficits in Implicit Sequence Learning in Children with Dyslexia with and without Specific Language Impairment and Response to Reading Intervention.

Objective: We examined implicit sequence (IS) learning, measured by the Serial Reaction Time Task (SRTT), in children with Developmental Dyslexia (DD) with or without Specific Language Impairment (SLI) and typically developing (TD) readers. We also explored the relation between IS learning abilities and response to an intensive 70 hour reading intervention. IS learning, a type of procedural learning, is believed to be deficient in DD and SLI (Lum et al., 2010; Menghini et al., 2006). Though such impairments, measured by the SRTT, have positive and negative support, IS learning has been linked to grammar abilities across SLI and TD (Lum, 2012). This link remains unexamined in children with DD. Whether IS learning is related to response to intervention also remains unexamined in these populations.

Participants and Methods: Using growth models of initial reaction time and learning slope across SRTT, we modeled IS learning among elementary school children with DD $(\mathrm{N}=76)$ or DD+SLI $(\mathrm{N}=27)$, and TD children $(\mathrm{N}=20)$. We then examined predictive contributions of grammar, vocabulary, phonological awareness, and diagnostic groups on IS learning performance. We also explored relation between IS learning and change in reading performance across a reading intervention.

Results: TD children exhibited significant IS learning on the SRTT, while those with DD or DD+SLI did not. The predictors of grammatical understanding, vocabulary ability, phonological awareness, or diagnostic group, did not relate to rates of IS learning. The relation between individuals' IS learning growth estimates and response to reading intervention was also explored.

Conclusions: Results are discussed in terms of the SRTT as an indicator of IS learning deficit in these clinical samples, especially in relation to the diagnostic categorical results and in relation to approaches to analyzing such SRTT learning task data. Further discussion includes implications for IS learning ability as a predictor of response to reading intervention. Correspondence: Emily Riggall, M.A., Psychology, Georgia State University, 755 North Avenue NE, 2518, Atlanta, GA 30306, United States.E-mail:emily.riggall@gmail.com

S. VADNAIS, S. DYER, S. LEE, J.M. KLAVER, J. CONSTANCE, L. BEYES \& M.Y. KIBBY. Right Inferior Frontal Gyrus Volume Predicts Math Calculation in Children with Reading Disability.

Objective: The right inferior frontal gyrus (RIFG) is implicated in mental rotation (Boyle et al., 2005) and inhibition/cognitive control (Aron, Robbins \& Poldrack, 2014), and the left inferior frontal gyrus (LIFG) is implicated in phonological and semantic processing (Moss et al., 2005). The LIFG may play a role in reading (Price, 2012) and reading disabilities (RD; Shaywitz et al., 2002). It also has been implicated in algebra (Friedrich \& Frederici, 2013). Thus, it was hypothesized that LIFG size would predict word identification and calculation skills in RD.

Participants and Methods: Participants included 62 children with RD or controls, ages 8-12 years, who completed a neuropsychological assessment and MRI scan as part of larger, NIH/NICHD funded projects (R03 HD048752, R15 HD065627). Children completed the WJ-III Letter Word Identification (LWI) and Calculation subtests, the WISC-IV, and other measures. Analyze 11.0 was used to measure volume. The IFG was manually traced in the sagittal plane after inter-rater and intra-rater reliabilities $(r \mathrm{~s}>.90)$ were attained.

Results: Four hierarchical multiple regressions were run to predict LWI and Calculation for RD and controls, separately, with total brain volume and FSIQ entered as covariates in the first step. RIFG, Beta $=.53, t(20)$ $=2.30, p=.03$, but not LIFG $(p=.94)$, predicted Calculation in RD. FSIQ and total brain volume were not significant ( $p$ s > 10). Neither RIFG $(p=.16)$ nor LIFG $(p=.99)$ predicted Calculation in controls. RIFG $(p=.80)$ and LIFG $(p=.99)$ did not predict LWI in RD. RIFG $(p=.08)$ and LIFG $(p=.40)$ did not predict LWI in controls also.
Conclusions: Contrary to hypotheses, LIFG did not predict word identification or calculation in either group. Given most IFG literature uses AMRI, it is plausible that LIFG size is less important than its neuronal functioning in predicting reading ability. The relationship between RIFG and math in RD may be related to cognitive inhibition (Cragg \& Gilmore, 2013) or mental manipulation (Boyle et al., 2005).

Correspondence: Sarah Vadnais, Psychology, Southern Illinois University, 218 S Kipling, K101, St. Paul, MN 55119, United States. E-mail:svadnais@siu.edu

R. WINTER, E. RIGGALL, L. BRANUM-MARTIN, J.C. FRIJTERS \& R. MORRIS. Working Memory Related to Decoding Gains Across Reading Intervention in Children with Developmental Dyslexia. Objective: This study aimed to understand the relation between working memory (WM) and reading score changes in children with Developmental Dyslexia (DD) over the course of a 70 hour reading intervention. Working memory has been shown to be a key factor for both children and adults with reading difficulties (Smith, Spark et al., 2003). However, the nature of this relationship remains unclear. It has been proposed that DD readers compensate for their deficits in phonological skills by overextending their WM system (Swanson, 2012). This hypothesis remains untested in an intervention study. Additionally, the differential contribution of varying modalities of WM to our understanding of intervention-based reading changes remains unexplored.

Participants and Methods: Participants were elementary school children classified as either 'typical readers' ( $\mathrm{N}=20$ ) or 'DD' ( $\mathrm{N}=99$ ). Using tasks representing the three domains of WM (Baddeley, 2012): 1. domain-general (Counting Span), 2. phonological-loop (non-word repetition) and 3. visual-spatial sketchpad (Corsi-blocks backwards), we modeled the differential contribution of WM to skill-gains on singleword reading at four time points during an intensive reading intervention for the DD group, and over the course of a school based reading program for the typical group.

Results: The phonological and spatial WM task performances were significantly correlated among typical readers $(p=0.01)$ but not among the DD readers. A multiple regression showed that better scores on the domain-general WM task (Counting Span) uniquely predicted reading gains in the DD group $(\mathrm{p}<0.05)$, but not the typical readers.

Conclusions: These results support the distinct contribution of each modality-specific WM task in understanding their role in reading development. Results indicated that domain-general WM ability was a significant predictor of single word reading gain scores in children with DD. but not in typically developing readers, giving further support to the compensatory mechanism theory of WM.

Correspondence: Rebecca Winter, MA, Psychology, Georgia State University, 1282 Bramble Road, Atlanta, GA 30329, United States. E-mail: rwinter3@student.gsu.edu

\section{Other}

E.F. JONES, C. STEPHAN, L. JACOBSON, A. PRITCHARD \& T.A. ZABEL. Improvements in Parent Self-Efficacy Ratings Following Pediatric Neuropsychological Assessment.

Objective: This quality improvement project examined the use of parent self-efficacy ratings to assess the potential impact of comprehensive psychological and neuropsychological evaluation of children. Parents' beliefs concerning ability to manage their children's needs have been linked to improved health outcomes in other fields. Parent-self efficacy ratings may be a means of measuring the impact of neuropsychological assessment.

Participants and Methods: Parents of children (mixed clinical population) seen in an outpatient clinic provided responses to a 13-item (5-point Likert scale) parent self-efficacy questionnaire 1.) prior to assessment and 2.) immediately following post-appointment feedback. Items queried parents' confidence in their ability to address their 
children's needs across a variety of domains (e.g.., "I am confident in my ability to help my child perform well in school"). A core set of items were modeled after Austin et al., 2017, and the remaining items were theoretically derived. Exploratory factor analysis of pre-assessment ratings $(n=261 ; 63.39 \%$ male, $M$ age of child=10.21) was used to identify factors and composite scales. Pre-assessment and post-appointment ratings for a smaller preliminary sample $(\mathrm{n}=61 ; 39 \%$ male; M age of child $=9.89$ ) were compared using paired t-tests to determine changes in parent self-efficacy following assessment.

Results: Three factors of parent self-efficacy were indentified relating to parents' self-perceived ability to 1.) Understand, 2.) Manage, and 3.) Advocate for their children. Significant improvements were noted on the Understand and Manage factors, and a trend $(p=.06)$ towards improvement was noted on the Advocate factor.

Conclusions: These preliminary results suggest improvement in parent-perceived ability to understand and manage the needs of their children following comprehensive assessment and feedback. Next steps include follow-up assessment of parent self-efficacy at 3-months and 1-year post-appointment to further investigate lasting impact of assessment.

Correspondence: Erin F. Jones, BA, Neuropsychology, Kennedy Krieger Institute, 1750 E Fairmount Ave, Department of Neuropsychology, Baltimore, MD 21231, United States.E-mail:joneseri@kennedykrieger. org

\section{S. KIM \& J. YANG. Gait Variability of Children According to Walking Path Width and Motor Development Level.}

Objective: The stability of gait pattern is closely related with the protection ability in the falling. The purpose of this study is to investigate the difference of gait variability and step error according to the children's level of motor development and width of walking path.

Participants and Methods: The 103 children participated ranging from 7 to 8 years old. The subjects were grouped by the level of Movement Assessment Battery of Children-2(MABC-2) score(high and low). Each participant performed a narrow path walk with $50 \%$ of their Anterior Superior Iliac Spine(ASIS) width, and general path walk with no limitation path width. A total of 8 Qualisys cameras, markers and QTM were used to analyze 3D motion for gait patterns. The ankle, knee, and hip were used to analyze the coordination pattern.

Results: The results indicated a significant difference between the highlevel group and low-level group in step and coordination variability. The difference of step length and step width between two groups was larger in the narrow path width walk than the general path width walk. The analysis of coordination variability through continuous relative phase(CRP) revealed that the variability in ankle-knee at mid stance and knee-hip at early stance with a high-level group was significantly smaller than the low-level group. The CRP variability according to the path width was significantly different from the ankle-knee and hip-knee at early stance in the narrow path. In the analysis of step error, low-level group showed higher step errors than the high-level group.

Conclusions: The stability of walking is explained in terms of gait variability which is quantifiable indicator of walk. It seems that the increased gait variability is a strong risk factor of falling in children with low levels of motor development.

Correspondence: Seonjin Kim, Ph.D., Physical Education, Seoul National University, 71-1 dong 514, Kwanakro 1, Seoul 08826, Korea (the Republic of).E-mail:seonkim@snu.ac.kr

H. SCHOFIELD. Tailoring Feedback Sessions for Young Adults with Cognitive Difficulties: Case Examples From Oncology.

Objective: Limited research has centered on methods of providing feedback. This case study proposes a method for providing neuropsychological feedback to older adolescents/young adults, with the goals of enhancing understanding of cognitive strengths/weaknesses and improving self-advocacy.
Participants and Methods: Two cases were compared with regards to need for accommodations and structure during feedback. Case \#1 (20 year old male) was diagnosed with medulloblastoma at age 3 years and treated with surgical resection, chemotherapy, and cranial radiation. Case \#2 (21 year old male) was diagnosed with medulloblastoma at age 7 years and treated with surgical resection, chemotherapy, and cranial radiation. Both patients received repeat outpatient neuropsychological evaluations and completed feedback with the clinician. Parents were then asked to join the session and the patients were tasked with presenting results to their parents.

Results: Case \#1 had intact cognitive scores in the majority of assessed areas, with mild weaknesses in aspects of executive functioning. Feedback was provided on a primarily 1-to-1 basis, with good comprehension of results with minimal supports. This patient was able to provide results to his parent with limited assistance. Case \#2 had difficulty on tests of processing speed, verbal fluency, working memory, and reading comprehension. Given this, test results were provided in smaller chunks with more frequent comprehension checks and visual aids to reduce working memory load. Brief notes were written provided to the patient for use during the parent meeting. All individuals participating in the feedback process expressed satisfaction with the structure of feedback. Conclusions: Despite similarities in tumor type, these cases highlight the variability in cognitive outcomes for brain tumor patients. By tailoring feedback to the cognitive needs of the patient, our broader goal was to enhance adherence to recommended interventions and facilitate improvements in self-advocacy.

Correspondence: Hannah-Lise Schofield, Ph.D., Children's Hospital of Philadelphia, Division of Oncology, 3501 Civic Center Blvd., 10207 CTRB, Philadelphia, PA 19104, United States.E-mail: htschofield@ gmail.com

A. YAMAMOTO, C. BARONE, C.G. RICHARDSON, R. ZIVANOVIC, D. LIN \& S. MATHIAS. Cognitive Impairment in a Sample of Precariously Housed Youth.

Objective: To examine the degree and pattern of cognitive impairment in precariously housed youth in Vancouver, Canada.

Participants and Methods: Individuals with suspected cognitive impairment were referred for neuropsychological testing from an outpatient health care program for precariously housed youth. Chi-squares were used to compare referred versus non-referred youth on socio-demographic factors and mental health diagnoses. Domain and Global Deficit Scores (DDS, GDS) were calculated to identify the extent of global cognitive impairment and which cognitive domains are most commonly impaired.

Results: There were no differences between referred $(n=43)$ and non-referred $(n=453)$ youth for demographic and mental health diagnoses variables. Forty youth had enough cognitive data to calculate GDS. Seventy-five percent had GDS $\geq 0.5$, reflecting cognitive impairment. Attention/Working Memory and Processing Speed were the most commonly impaired cognitive domains, with most individuals $(63 \%)$ having cognitive impairment in at least half of the 6 cognitive domains (DDS $\geq 1$ reflecting impairment).

Conclusions: Many precariously housed youth have significant cognitive impairments, particularly for attention and processing speed. Cognitive evaluations are an essential part of any homeless youth program in order to identify those with cognitive impairment and provide the supports needed to optimize outcomes.

Correspondence: Aiko Yamamoto, Ph.D., St. Paul's Hospital, 1081 Burrard Street, Mental Health Wellness Clinic, Vancouver, BC V6Z 1Y6, Canada.E-mail: ayamamoto@providencehealth.bc.ca 
PM Coffee Break

3:45-4:00 p.m.

\section{Early Career Awardee Presentation: Neurocognitive Mechanisms Underlying Development, Course and Treatment of Major Depressive Disorder}

\section{Award Recipient: Marie-Jose van Tol}

$$
\text { 4:00-5:00 p.m. }
$$

M. VAN TOL. Neurocognitive Mechanisms Underlying Development, Course and Treatment of Major Depressive Disorder.

Major depressive disorder (MDD) is the most prevalent psychiatric disorder, affecting between 10 and $20 \%$ of the world population at some point in their lives. MDD is characterized by a high risk for relapse after recovery ( $40 \%$ within 2 years). Therefore, understanding and changing the highly recurrent course of MDD is of high clinical and societal importance.

In this talk, I will review results from the Netherlands Study of Depression and Anxiety (NESDA) Neuroimaging study ( $\mathrm{n}=301$ ), in which we studied risk factors, associations, and consequences of an unfavorable course of major depressive disorders. We investigated functional Magnetic Resonance Imaging (fMRI) characteristics associated with emotional processing, executive functioning, brain connectivity, in addition to structural brain characteristics. Results indicate differential predictors and consequences of an unfavorable course. Implications of these results for neurocognitive models of depression will be discussed. Additionally, a neurocognitive model of relapse and relapse prevention will be presented, which formed the basis of the NEW-PRIDE (Neurocognitive Working Mechanisms of Preventing Relapse in Depression) study. In this Randomized Controlled Trial, fMRI, pupillometry and neuropsychological assessments are employed to 1) understand the working mechanisms of preventive cognitive therapy for preventing relapse and 2) to develop neurocognitive predictors of individual treatment success. This study aims to contribute to effective preventive-treatment allocation, lower relapse-rates, and ultimately lower conversion into chronic-MDD by taking a neurocognitive approach.

Acknowledgement: Many thanks to André Aleman, Rozemarijn van Kleef, Dick J. Veltman, Nic J.A. van der Wee, Jan-Bernard Marsman, Claudi Bockting, Esther Opmeer, and Hui Ai for collaborations on the work presented during this talk.

Correspondence: Marie-Jose van Tol, Department of Neuroscience, University Medical Center Groningen, University of Groningen, United States.E-mail:m.j.van.tol@umcg.nl
Symposium 6. Chronic Effects of Mild Traumatic Brain Injury in Veterans and Service Memvers on Cognition, Vestibular Function, Motor Performance, and Brain Imaging: A Biopsychological Model

Chair: Harvey S. Levin

Discussant: Tracy Nolen

Presenters: Heather Belanger, David F. Tate, William Walker, Elisabeth A. Wilde

$$
\text { 4:00-5:30 p.m. }
$$

H.S. LEVIN, H. BELANGER, D.F. TATE, W. WALKER, E.A. WILDE \& T. NOLEN. Chronic Effects of Mild Traumatic Brain Injury in Veterans and Service Members on Cognition, Vestibular Function, Motor Performance, and Brain Imaging: A Biopsychological Model.

We report data from the Chronic Effects of Neurotrauma Consortium (CENC), a prospective, longitudinal, multicenter project funded in 2012 by the Department of Veterans Affairs and the Department of Defense to address the long term outcomes of mTBI in veterans and service members who sustained a mild traumatic brain injury (mTBI) in the recent Iraq and Afghanistan conflict. Four presentations report outcome data at least one year post-injury for cognition, brain imaging, vestibular function, and visuomotor performance, comparing those who sustained one or more mTBIs (mTBI group) and the No TBI group. Dr. Belanger presents cognitive outcomes and their association with mTBI, including the results of paper and pencil tests and computerized tests such as the NIH Toolbox. Dr. Tate presents diffusion tensor imaging (DTI), comparing white matter integrity of the mTBI and no TBI groups. He will also present DTI data in relation to posttraumatic stress disorder (PTSD) as compared with the effects of mTBI. Dr. Tate will coordinate with Dr. Belanger in presenting the relation of DTI to the cognitive findings. Dr. Walker will present data on computerized posturography, comparing vestibular functioning of the mTBI group and the no TBI groups. Dr. Wilde will compare the visuomotor performance of the mTBI vs no TBI groups on the Grooved Pegboard, including dominant and nondominant hands. She will also integrate the motor speed data with the brain imaging. Dr. Nolen, Director of the Biostatistics Core, will discuss how the data from these presentations fit a biopsychological model of mTBI, including how the CENC investigators have conceptualized the mediators, moderators, covariates, and confounders. With Dr. Levin, she will also direct the question answer portion of the symposium. Correspondence: Harvey S. Levin, Ph.D., Cognitive Neuroscienc e Lab, Baylor College of Medicine, 1709 Dryden Rd ste 725, Houston, TX 77030, United States. E-mail: hlevin@bcm.tmc.edu

H. BELANGER, T. NOLEN, H.S. LEVIN, N. PUGH, W. WALKER, R.S. SCHEIBEL, M. TROYANSKAYA, C. JARAMILLO, B. EAPEN, S. MCDONALD, S. DIKMAN, N. HSU, D.F. TATE, R. WILLIAMS, S.R. HINDS \& D.X. CIFU. Chronic Effects of $m$ TBI in Veterans and Service Members on Cognition: A Biopsychological Model.

Objective: Assess whether mTBI has an adverse relationship on chronic cognitive outcomes.

Participants: 492 individuals were in the initial analysis cohort from the Chronic Effects of Neurotrauma Consortium (CENC) longitudinal study (414 mTBI; 78 no TBI). Data were excluded if unreliable or invalid or due to questionable effort. Analyses included between 370 to 430 individuals.

Methods: Covariate-adjusted regression analyses accounting for potential confounders, covariates and effect moderators were used. Structural equation modeling was also employed to assess potential mediators (i.e., conditions such as PTSD that potentially impact the causal pathway between TBI exposure and cognitive outcomes). Factor analysis 
was also performed to reduce the dimensionality of the data using a MINRES model with Promax transformation; the TBI exposure models were applied to the resulting factors. To date, 16 outcomes have been analyzed from the NIH TB, WAIS, CVLT-II, BTACT and Trail-Making Test assessments.

Results: Results are preliminary. Factors associated with $\geq 50 \%$ of cognitive outcomes include mediators of PTSD, depression, and sleep, and covariates of age and high blood pressure. There is no current evidence of a consistent adverse relationship between mTBI exposure and cognitive outcomes. For example, the estimated difference in NIH TB fluid composite was $<2$ points between the $\mathrm{mTBI}$ and No-TBI groups ( $p>.05)$. An exception was WAIS-IV coding where scores were, on average, 4 points lower in the mTBI exposed group $(p<0.05)$. Additionally, list sorting results suggest that assessing the continuous measure of number of mTBI exposures may be informative. For the factor analyses, five factors appeared to describe 1) list learning, 2) working memory, 3) cognitive control, 4) fluency and 5) memory.

Conclusions: No observed evidence of an adverse association of mTBI exposure with cognition; analyses should be revisited when data are available on the complete study cohort.

Correspondence: Heather Belanger, FL, United States. E-mail: Heather. Belanger@va.gov

D.F. TATE, B.S. WADE, C.S. VELEZ, E.D. BIGLER, T.J. ABILDSKOV, G.E. YORK, B.A. TAYLOR, C. JARAMILLO, B. EAPEN, R. SCHEIBEL, H. BELANGER, W. WALKER, R. AGARWAL, J. STEINBERG, M. LENNON, A. BETTS, H.S. LEVIN, N. TUSTISON, J. STONE \& E.A. WILDE. Predicting Concussion History Using Diffusion Imaging in Veterans: Results from the Chronic Effects of Neurotrauma Consortium (CENC).

The observational study on late neurologic effects of combat is one of the primary objectives of the Chronic Effects of Neurotrauma Consortium (CENC). Its purpose is to establish a cohort of Service Members and Veterans with a history of mild traumatic brain injury (mTBI) that will be followed prospectively using multivariate clinical, biological, and cognitive measures. The current study is an interim predictive analysis of diffusion imaging and symptom reporting data collected within this cohort. Participants included 302 men and women with prior military combat deployments from four sites participating in CENC. Each participant underwent symptom assessment (Neurobehavioral Symptom Inventory (NSI) and PTSD Check List (PCL-5)) and MRI, including diffusion tensor imaging (DTI). The DTI sequence was analyzed using the ENIGMA consortium's standardized processing pipeline resulting in 48 white matter ROI measurements. Five separate supervised classification methods were used to predict concussion history groupings using symptom and diffusion ROI measures, as well as determine the relative importance of each measure in predicting concussion history. Significant predictive models (AUC $67-74 \%$ ) were derived for Veterans with three or more concussions compared to no concussion history. No significant models were derived for Veterans with one and two concussions compared to no concussion history or three or more concussions. NSI and PCL-5 total scores as well as the average fractional anisotropy (FA) for the superior longitudinal fasciculus, corpus callosum, and posterior corona radiata were the five most important variables in predicting concussion history. These findings demonstrate the value of using symptom measures and imaging measures to predict concussion history. These findings warrant additional analyses which explore the relation to demographic, injury and outcome variables.

Correspondence: David F. Tate, Ph.D., Missouri Institute of Mental Health, University of Missouri-St. Louis, 4633 World Parkway Circle, Suite 135, Berkeley, MO 63134-3115, United States. E-mail: David. Tate@mimh.edu
W. WALKER, T. NOLEN, E.A. WILDE, S. HINDS, D.F. TATE, H.S. LEVIN, S. SPONHEIM, L. MANNING, J. HENRY, K.M. SKOP \& K. NOWAK. The Relationship Between Historical Mild Traumatic Brain Injury and Balance Performance: A Chronic Effects of Neurotrauma Consortium (Cenc) Multi-Center Observational Study Interim Analysis.

Objective: Mild Traumatic Brain Injury (mTBI), a known cause of postural instability acutely, has been implicated in chronic balance impairment. We hypothesized that historical mTBI status is associated with poorer computerized dynamic posturography (CDP) performance when statistically controlling for other candidate predictor variables.

Participants: The CENC Multicenter Observational Study enrolls post9/11 era Service Members and Veterans with combat exposure and a spectrum of historical mTBI, from none to many. These analyses $(\mathrm{n}=295)$ from the interim sample $(\mathrm{n}=492)$ included all participants completing CDP testing at the 3 performing sites and excluded symptom exaggerators and aphysiologic CDP profiles.

Methods: The design was cross-sectional using structural equation modeling. Comprehensive structured interviews were used to determine all lifetime mTBIs and when present categorize then by PTA/LOC, blast causality, and repetitive mTBI. Balance was measured on CDP dual-plate force platform using the Sensory Organization Test (SOT). Assessments for candidate variables included structured interviews, record review, and questionnaires.

Results: The overall mTBI classification, positive versus negative history, showed only an indirect effect on the composite equilibrium score that was mediated through pain, anxiety, and PTSD. Pain in turn had the only significant interaction with composite score. Repetitive mTBI, defined as 2 or more lifetime mTBIs, had a significant total (direct + indirect) effect on composite equilibrium, with a larger direct than indirect parameter estimate. In secondary analyses of the various SOT conditions, the largest parameter size for repetitive mTBI was with condition 5, eyes blinded with moving platform.

Conclusion:This finding suggests overreliance on the visual system due to deficits in vestibular feedback or integration after repeated mTBI. Further study of CDP outcomes within the future complete cohort is warranted.

Correspondence: William Walker.E-mail: william.walker@va.gov

E.A. WILDE, T. NOLEN, D.F. TATE, B.S. WADE, K. NOWAK, E.D. BIGLER, T.J. ABILDSKOV, C.S. VELEZ, R.S. SCHEIBEL, M. TROYANSKAYA, H. BELANGER, C. JARAMILLO, B. EAPEN, H.S. LEVIN, S.R. HINDS, D.X. CIFU, R. WILLIAMS \& W. WALKER. Preliminary Findings on Motor Functioning in the Chronic Effects of Neurotrauma Consortium.

Objective: Assess the relation between mild traumatic brain injury (mTBI) and motor functioning as assessed by the Grooved Pegboard (GPB) test in Veterans and Service Members enrolled in the Chronic Effects of Neurotrauma Consortium (CENC) longitudinal study.

Participants: 492 individuals were included in a preliminary analysis (414 mTBI; 78 no-TBI). Data were excluded if deemed unreliable; the final sample included 391 individuals. Neuroimaging was also performed.

Methods: Covariate-adjusted regression analyses accounting for potential confounders, covariates, and effect moderators were used. Additionally, results of the GPB were examined in relation to quantitative volumetric MRI analysis and diffusion tensor imaging.

Results: Preliminary results indicate that factors associated with GPB performance include mediators of PTSD, depression, anxiety and pain, and covariates of age and premorbid functioning as measured by the Test of Premorbid Functioning. Results examining subgroups of participants with varying degrees of exposure reflected a significant difference between those with blast-related TBI versus those without TBI for both dominant $(p=0.005)$ and non-dominant $(p=0.007)$ hands, where more severe exposure was related to longer time to complete. A subgroup of 
subjects with repetitive mild TBI was also significantly slower for the non-dominant hand $(p=0.034)$. Finally, examination of the relation between GPB performance and imaging in the basal ganglia suggested that the presence of post-traumatic amnesia at injury may alter the relation.

Conclusions: Although the relation between mTBI and motor performance in OIF/OEF/OND Veterans and Service Members is complex and influenced by potential confounders, covariates and moderators, examination of subgroups of participants with blast-exposure and more severe or repetitive mTBI may reveal subtle fine motor consequences of combat exposure. Additional analyses will be examined in a larger cohort as the study progresses.

Correspondence: Elisabeth A. Wilde, Ph.D., Physical Medicine and Rehabilitation, Neurology and Radiology, Baylor College of Medicine, One Baylor Plaza, BCM637, Houston, TX 77030, United States. E-mail: ewilde@bcm.edu

\section{Paper Session 6. Pediatric Neuropsychiatry}

\section{Moderator: Lana Harder}

$$
\text { 4:00-5:30 p.m. }
$$

L.E. MASH, A. LINKE, C. FONG, M. FALAHPOUR, T. LIU \& R. MÜLLER. Atypical Relationship Between Static and Dynamic Functional Connectivity in Autism Spectrum Disorders.

Objective: There is ample evidence of atypical static functional connectivity (FC) in autism spectrum disorders (ASDs). However, flexible relationships between neural networks are not easily captured by conventional FC magnetic resonance imaging (MRI) methods. A few recent studies have suggested atypical temporal FC dynamics in ASDs, but the relationship between static FC and dynamic FC variability remains unclear. We applied a sliding-window approach to examine these associations in ASD and typical development (TD).

Participants and Methods: Resting-state functional MRI data from $61 \mathrm{ASD}$ and 57 TD participants were included, group-matched for motion, age, sex, handedness, and nonverbal cognitive ability. Highorder group independent component analysis and dual-regression were used to generate maximally independent, functionally cohesive regions of interest (ROIs) at the individual level. Static FC was calculated using entire time series. Dynamic FC between ROI pairs was examined using tapered sliding windows, generating a temporally contiguous series of connectivity matrices. For each subject, the standard deviation of FC (sd-FC) was calculated across all time windows.

Results: One-sample t-tests determined that across all ROI pairs, group differences in static FC differed from zero and primarily reflected hyperconnectivity in ASD $(p<.001)$. Additionally, higher sd-FC in ASD vs. TD group predominated $(\mathrm{p}<.001)$. There was an inverse relationship between static FC and sd-FC in both the ASD $(r=-.24, p<.001)$ and TD groups $(\mathrm{r}=-.22, \mathrm{p}<.001)$. However, the strength of this association at the individual level was predicted by diagnosis in a general linear model. being weaker in individuals with ASD $(\beta=.14, \mathrm{p}=.004)$.

Conclusions: We find that higher static FC is associated with reduced variability over time. Despite predominant overconnectivity, this relationship was unexpectedly weaker in ASD participants. Findings suggest that networks in ASD may be both poorly differentiated and less stable across time.

Correspondence: Lisa E. Mash, M.S., Psychology, San Diego State University, 4040 Centre St, San Diego, CA 92103, United States. E-mail: lisa.e.mash@gmail.com
B. Castelluccio, A. CANField \& I. Eigsti. Neural Mechanisms of Generalization for Language Learning in Autism Spectrum Disorder.

Objective: Generalization, the process by which abstracted features of past experiences are extended to new instances, is an area of clinically observed weakness in autism spectrum disorder (ASD). The current study aimed to determine whether linguistic generalization is an area of weakness for people with ASD, to compare the neural resources engaged for linguistic generalization in people with and without ASD, and to determine the degree to which linguistic generalization is a domain-general process.

Participants and Methods: Seventeen young adults with ASD and 17 well-matched typically developing peers completed two experiments that tested their ability to abstract a principle and generalize it to new stimuli while undergoing functional magnetic resonance imaging. One experiment tapped linguistic generalization, and a nonlinguistic comparison experiment tapped visuospatial generalization.

Results: On visuospatial generalization, participants with ASD performed significantly worse than TD controls. On linguistic generalization, participants with ASD performed worse, though not to the level of statistical significance. Adults with ASD showed subthreshold hypoactivation relative to TD adults in left STG and right MTG. Performances on linguistic and visuospatial generalization tasks were highly correlated with each other, and there was substantial overlap in neural resources serving generalization across the two domains.

Conclusions: Highly verbal, high-functioning adults with ASD exhibit a weakness in generalization, which may contribute to clinical deficits in language and communication. Group differences in neural substrates of linguistic generalization related to previously documented differences in language processing in ASD. Our data support a theory of generalization as a domain general function. Clinical implications include the consideration of specific supports for generalization in interventions targeted to high-functioning, highly verbal adults with ASD.

Correspondence: Brian Castelluccio, Psychological Sciences, University of Connecticut, 406 Babbidge Road, Unit 1020, Storrs, CT 062691020,United States. E-mail: brian.castelluccio@uconn.edu

P.A. ADUEN, M.J. KOFLER, D.E. SARVER, L.N. IRWIN \& D.J. COX. Neurocognitive Predictors of Motor Vehicle Crashes: A Prospective Cohort Study.

Objective: The current study examined underlying neurocognitive mechanisms and overt ADHD symptoms as hypothesized causal mechanisms that underlie future risk for causing motor vehicle crashes and near-crashes.

Participants and Methods: The Strategic Highway Research Program (SHRP-2) Naturalistic Driving Study included 3,226 drivers selected through probability-based sampling. Upon study entry, drivers rated their ADHD symptoms and completed the Conners' Continuous Performance Test (CPT-II). Each driver's car was then outfitted with sophisticated data acquisition technologies to continuously capture all aspects of routine driving from "engine-on to engine-off' for 1-2 years. Crashes and near-crashes were identified via kinematic algorithms; driver fault was coded reliably based on objective evidence that participant's error caused crash/near-crash.

Four PCA-derived CPT-II components (Disinhibition, Response Variability, Arousal Decrements, Vigilance Decrements) were modeled to predict prospective at-fault crashes and near-crashes with ADHD symptoms as mediator. Miles driven, age, gender, education, and marital status were adjusted. Total, direct, and indirect effects were estimated using maximum likelihood SEM with 10,000 bootstrapped samples (MPLUS).

Results: Response Variability exerted total effects on both crashes $(b=0.06$; 95\%CI: $0.021-0.101)$ and near-crashes $(b=0.04 ; 95 \% \mathrm{CI}$ : 0.002-0.076). Indirect effects of Disinhibition and Response Variability through ADHD symptoms were detected for both crashes $(b=0.003$; 95\%CI: $0.001-0.007 ; b=0.004 ; 95 \%$ CI: $0.001-0.009)$ and near-crashes $(b=.0 .003 ; 95 \% \mathrm{CI}: 0.001-0.008 ; b=0.005 ; 95 \% \mathrm{CI}: 0.002-0.009)$. 
Direct effects of Response Variability remained significant even after controlling for its effect on ADHD symptoms for crashes $(b=0.06$; 95\%CI: 0.017-0.097) but not near-crashes.

Conclusions: The current results provide the first prospective evidence associating neurocognitive dysfunction with motor vehicle crashes, both in conjunction with and independent of its role in regulating ADHD symptoms.

Correspondence: Paula A. Aduen, Psychology, University of Virginia, 5941 South Maryland Avenue, MC 3077, Chicago, IL 60637, United States.E-mail:paa4nr@virginia.edu

T. NGUYEN, M. SPENCER-SMITH, D. ZANNINO, A. BURNETT, S.E. SCRATCH, L. PASCOE, R. ELLIS, J. CHEONG, D. THOMPSON, T.E. INDER, L.W. DOYLE \& P.J. ANDERSON. Language Functioning at 13 Years and its Developmental Trajectory from 2 Years in Very Preterm Children.

Objective: To describe language functioning at 13 years of age and examine the developmental trajectory of language from 2 to 13 years in very preterm (VP) children comapred with full term (FT) controls. Participants and Methods: A cohort of 224 VP children (born $<30$ weeks' gestation) and 77 FT controls, recruited during the neonatal period, had language skills assessed using parent-report measures and direct assessments at 2, 5, 7 and 13 years. Regression models were used to compare verbal memory, grammar, semantics, and pragmatic skills between the VP and FT groups. Linear mixed effects models were used to examine the developmental trajectory of language from 2 to 13 years in VP children compared with FT controls.

Results: Compared with FT controls, VP children at 13 years had poorer functioning across all language components (mean group differences ranged from -0.47 to $-1 \mathrm{SD}$, all $\mathrm{p}<0.05$ ). At each follow-up age, the VP children showed poorer language functioning than FT controls, with both VP and FT groups showing similar developmental trajectories (slope difference $=-0.01 \mathrm{SD}$ per year, $\mathrm{p}=0.5$ ).

Conclusions: VP children continue to display poorer language skills compared with FT controls at 13 years, with no evidence of developmental "catch-up" from early childhood. Given the functional implications associated with language difficulties, language based interventions should be considered for VP children.

Correspondence: Thi-Nhu-Ngoc Nguyen, BSc, School of Psychological Sciences, Monash University, Victorian Infant Brain Study (VIBeS), Level 4, West Building, Murdoch Childrens Research Institute, Royal Children's Hospital, 50 Flemington Road, Parkville, VС 3052, Australia. E-mail:ngoc.nguyen@mcri.edu.au

A. TAN, C. hague, P. PluMb, L.S. hyNan, J. Hughes, L. LACRITZ, B. GREENBERG \& L. HARDER. Neurocognitive and Psychosocial Profiles of Pediatric Multiple Sclerosis and Transverse Myelitis.

Objective: Research shows children with multiple sclerosis (MS) are at risk for neuropsychological deficits, but clear profiles remain difficult to establish due to heterogeneity and complexity of the disease. Emerging evidence also suggests the possibility of cerebral involvement and neuropsychological deficits in children with transverse myelitis (TM). The present objective is to further investigate neuropsychological sequelae associated with pediatric MS and TM by characterizing and comparing neurocognitive and psychosocial profiles.

Participants and Methods: Thirty-nine MS (mean age $=14.92$, range $=7-18)$ and 33 TM (mean age $=12.49$, range $=7-18$ ) participants were administered a neuropsychological screening battery during their clinic visit (CVLT, Digit Span, SDMT, FAS, Trail Making Test B, Beery VMI, Grooved Pegboard, BRIEF, BASC-2, Pediatric Quality of Life Inventory, PedsQL Multidimensional Fatigue Scale). Participants were at least 30 days from acute event or steroid use. Separate profile analyses were performed on task-based neurocognitive measures and parentrated psychosocial variables.
Results: A neurocognitive profile analysis revealed significantly $(p<.05)$ lower overall performance in MS compared to TM, with differences between profile patterns due to significantly $(p<.05)$ worse performance on TMT B and Beery VMI in MS, and difficulties with TMT B, Beery VMI, and Grooved Pegboard being the most significant $(p<.05)$ contributors to combined profiles. A psychosocial profile analysis revealed no significant differences between MS and TM profile patterns, with poorer PedsQL and MFS ratings being the most significant $(p<.05)$ contributors to combined profiles.

Conclusions: These findings elucidate the neurocognitive and psychosocial sequelae of pediatric MS and TM. While MS patients demonstrated lower overall neurocognitive functioning, primarily due to lower cognitive flexibility and visuomotor integration skills, similar levels of fatigue and quality of life difficulties were observed in both populations. Correspondence: Alexander Tan, Clinical Psychology, University of Texas Southwestern Medical Center, 4810 Cedar Springs Rd., Apt. \#3201, Dallas, TX 75219, United States.E-mail: Alexander.Tan@ utsouthwestern.edu

J. VAN DESSEL, M. MOERKERKE, E. SONUGA-BARKE, J. LEMIERE, S. VAN DER OORD \& M. DANCKAERTS. Distinctive Neural Response Towards Certain and Conditional Monetary Loss Anticipation in Adolescents.

Objective: The neural response towards cues signalling monetary gain and loss has been extensively studied through the Monetary Incentive Delay (MID) task. Anticipation of loss is often directly compared against anticipation of gain to represent the trade-off between positive and negative outcomes. In daily life situations, these events occur independently from each other and monetary loss can be escapable. We used an adaptation of the MID functional Magnetic Resonance Imaging (fMRI) task to assess the specific brain response pattern towards monetary loss, representing a common social penalty

Participants and Methods: Thirty-three typically developing righthanded male adolescents (9-18 years) performed a reaction time task under three conditions: (i) on Certain Loss trials, a monetary loss of $€ 0.20$, $€ 1$, or $€ 5$ occurred irrespective of response speed, (ii) on Conditional Loss trials, monetary loss could be avoided when responding in time, and (iii) on No Loss trials, no money could be lost. Participants started with $€ 150$ and were told that they could take home the remainder after 135 trials. The game was set that $4 / 5$ of the start amount was lost. fMRI BOLD responses were acquired to compare anticipatory brain activation following the different cue types. Additional behavioral data were collected.

Results: Prospect of certain monetary loss altered the neural response in emotion-related brain regions towards the end of the task, while conditional loss provoked an increased aversive state that remained constant throughout the task. A significant $(p<0.05$ FWE-corrected $)$ dose-response relationship was found for the bilateral anterior insula, anterior cingulate cortex and parietal inferior gyrus for the Conditional Loss > No Loss contrast.

Conclusions: We provide some of the first evidence of a distinctive brain pattern between conditional and certain monetary loss. The identification of such contingencies can provide more inside how adolescents respond to monetary loss in daily life.

Correspondence: Jeroen Van Dessel, MSc, Neuroscience, KU Leuven, Kapucijnenvoer 33, Leuven 3000, Belgium.E-mail: jeroen.vandessel@ kuleuven.be 


\section{Paper Session 7. Cognitive Reserve}

\author{
Moderator: Rosemary Fama
}

$$
\text { 4:00-5:30 p.m. }
$$

M. ALTENDAHL, A.M. STAFFARONI, A. EMERY, P. MUMFORD, L. RUOFF, K. WALKER, B.M. BETTCHER, E. FOX, M.Y. YOU, R. SALONER, J. STIVER, T. NEYLAN, J. KRAMER \& C. WALSH. REM Sleep Associated with White Matter Integrity in Cognitively Healthy, Older Adults.

Objective: There is increasing awareness of how sleep abnormalities affect brain structure and function. For example, white matter integrity is vulnerable to the effects of sleep apnea. Less is known, however, about how sleep itself is associated with white matter. We investigated how time spent in various stages of sleep relates to white matter microstructure in older adults.

Participants and Methods: 45 cognitively healthy, older adults (mean age $=74.5$ years; $60 \%$ female) underwent diffusion tensor imaging (DTI) and a sleep assessment within a 12 month period. Participants used an at-home sleep recorder (Zeo) for 3-10 nights (mean= 7.2) to measure total sleep time (TST), total wake time (TWT), light sleep (LS), deep sleep (DS), and REM sleep (RS). We used multiple regression to assess the association of TST, TWT, and average time in each sleep stage with fractional anisotropy (FA) and mean diffusivity (MD) of the corpus callosum (CC), controlling for age, sex, education, and sleep apnea risk (Berlin Questionnaire). CC was chosen as a ROI because it can be reliably measured and may be sensitive to diffuse processes in the brain. Results: The higher time (min) spent in RS was significantly associated with higher CC FA $(\beta=0.54, p<0.001)$ and lower MD $(\beta=-0.42$, $\mathrm{p}=0.002)$. Similarly, the percent of time in RS correlated with CC FA $(\beta=0.47, p=0.001)$ and MD $(\beta=-0.37, p=0.006)$. Neither TST, TWT, nor time spent in LS or DS were significantly associated with CC FA or MD ( $\mathrm{p}>$.222).

Conclusions: White matter microstructure was positively associated with both total time and percent time spent in REM sleep, but not quantity of light and deep sleep. This finding suggests a specific relationship between REM sleep and white matter microstructure, and supplements the current literature linking sleep and brain structure. Future research aimed at elucidating the physiological mechanisms mediating the relationship between REM sleep and white matter integrity may improve the treatment of poor sleep hygiene and associated neurocognitive outcomes. Correspondence: Marie Altendahl, BA, Neurology, University of California, San Francisco, 675 Nelson Rising Lane, Rm 235, San Francisco, CA 94158, United States. E-mail: marie.altendahl@ ucsf.edu

M. SANDERSON-CIMINO, C. FENNEMA-NOTESTINE, M.S. PANIZZON, K. CROSS, X. TU, E. JEREMY, D. GUSTAVSON, S. HATTON, C.E. FRANZ, M.J. LYONS \& W.S. KREMEN. Evaluation of Phenotypic and Genetic Indices of Cognitive Reserve in Middle Age.

Objective: The theory of Cognitive Reserve (CR) attempts to account for discrepancies between pathological burden and observed cognitive performance. In aging and Alzheimer's research, CR has most often been approximated by education because early cognitive scores are rarely available. No study has evaluated a genetically-based CR index. Most studies focus on CR in later life, but to understand protective factors, it is important to examine how early CR effects become manifest. We examined two phenotypic CR indices (cognitive ability at age 20 and lifetime education), and two novel genetic CR indices (childhood IQ polygenic score [CI-PGS] and educational attainment polygenic score [EA-PGS]).

Participants and Methods: Participants were 183 men (mean age 62.5, SD 2.57) from the Vietnam Era Twin Study of Aging. Pathological burden was quantified as abnormal white matter (AWM) based on
MRI-assessed white matter hyperintensities. PGSs were derived from independent genome-wide association studies. Six cognitive domain scores were formed by averaging $\mathrm{z}$ - scores for tests in each domain. Controlling for age, total white matter, and correlated observations, we predicted CR effects-defined as significant CR x AWM interactions-for processing speed and executive function. Other domains were considered exploratory

Results: The CI-PGS showed CR effects for Processing Speed ( $p=0.02$. beta $=0.19)$ and a trend for executive function $(p=0.066, b=0.14)$ such that those with higher childhood IQ PGSs were less affected by AWM burden. Of the 12 remaining domain scores, only 2 showed significant CR effects (one for the CI-PGS and one for education).

Conclusions: To our knowledge, this is the first study of genetically-based CR indices. We observed CR effects even at midlife. Other CR indices may confound cause and effect, but PGSs must be purely causal; they exist prior to age-related declines and are unaffected by life experiences or pathological burden. The results support further examination of genetically-based CR indices.

Correspondence: Mark Sanderson-Cimino, B.S. Psychology, VMRF Psychology, 4347 Hamilton street, San Diego, CA 92104, United States. E-mail:mesandci@gmail.com

L. CAVANAGH, Z. MAHMOOD, A. HAMMOND, T. KUHN, R.A. NUNEZ \& A. THAMES. The impact of current leisure activities on cognitive and brain reserve among community-dwelling adults. Objective: Current engagement in leisure activities (LAs) is thought to contribute to cognitive reserve during aging; however, the extent to which these activities contribute to cognitive/brain reserve beyond educational attainment remains understudied, and findings are mixed regarding which types of LAs lend greatest benefit. This study aims to identify empirically-derived domains of LAs associated with cognitive and brain reserve in a sample of community-dwelling adults.

Participants and Methods: Participants $(\mathrm{N}=65)$ completed a leisure activity questionnaire (LAQ) and neurocognitive testing. MRI-derived volumetric data was obtained for relevant regions of interest (ROIs).

Results: Exploratory factor analysis of LAQ identified five factors, labeled as social hobbies, individual hobbies, social activities, cognitive activities and physical activities. Individual $(r=.24)$, cognitive $(r=.26)$, and physical $(r=.28)$ LAs were associated with increased hippocampal volume. Physical LAs were associated with increased basal ganglia volume $(r=.29)$. Social LAs were associated with reduced volume in the fusiform gyrus $(r=-.30)$, thalamus $(r=-.32)$, caudate $(r=-.29)$ and putamen $(r=-.33)$. No LA domains were significantly associated with neurocognitive tests, though cognitive LAs trended towards higher memory performance $(r=.20, p=.06)$. In evaluating the unique contribution of LAs to brain volume after consideration of age and education, cognitive LAs predicted hippocampal volume $(\beta=.28, p=.03)$, resulting in significant increment $\left(\Delta \mathbf{R}^{2}=.07\right)$ and overall proportion of variance explained $\left(\mathbf{R}^{2}=.04, \mathrm{~F}(1,64)=5.05, p=.03\right)$.

Conclusions: Current engagement in LAs can lend unique influence to brain morphometry in areas commonly associated with neurodegenerative susceptibility. Cognitively-stimulating LAs may influence hippocampal morphometry above and beyond commonly-used proxies of reserve. These findings highlight the potential utility of LAs as modifiable risk and resilience factors in age-related brain changes.

Correspondence: Lucia Cavanagh, UCLA, C8-747 Semel Institute, 740 Westwood Plaza, Los Angeles, CA 90024, United States. E-mail: luciacavanagh@gmail.com

\section{E. RHODES \& T. GIOVANNETTI. Factor Structure of Grit in Older} Adults: Consistency and Perseverance in Successful Aging.

Objective: Grit, the perseverance and passion for long-term goals, is a noncognitive trait that predicts late-life cognitive performance and successful aging. Research in young adults suggests that grit is comprised of two factors: consistency of interest and perseverance of effort. It is unclear if this factor structure is reliable across the lifespan 
and if either factor more strongly predicts positive cognitive outcomes in aging.

Participants and Methods: Participants over 55-years-old ( $\mathrm{N}=166)$ completed self-report surveys assessing demographics, grit (12-item Grit Scale), physical and emotional functioning (SF-36), and age-related changes in everyday cognitive functioning $(\mathrm{ECog})$. Principle component analysis was used to assess the factor structure of the Grit Scale. Then, relations between grit factors and measures of successful aging were investigated with bivariate Pearson correlations.

Results: Analyses supported the two-factor model of grit in older adults. The total grit scale as well as both factors showed adequate internal consistency $\left(\alpha_{S}>.7\right)$. Both consistency of interest and perseverance of effort were positively associated with better physical functioning $(r=$ $0.19, p>0.05$ and $r=0.25, p>0.01$, respectively), emotional functioning $(r=0.29, p>0.01$ and $r=0.36, p>0.01$, respectively), social functioning $(r=0.23, p>0.01$ and $r=0.24, p>0.01$, respectively), and reduced fatigue $(r=0.22, p>0.01$ and $r=0.4, p>0.01$, respectively). For reduced fatigue the correlation was significantly stronger for the perseverance factor. Only consistency was associated with age-related changes in everyday cognitive functioning $(r=-0.26, p>0.01)$.

Conclusions: Both factors of grit are associated with various aspects of successful aging. However, consistency, rather than perseverance, may be more important for the maintenance of everyday cognitive functioning over time, suggesting the possibility that consistency in daily routines may be protective against age-associated everyday cognitive decline.

Correspondence: Emma Rhodes, MA, Psychology, Temple University, 1701 N 13th Street, Department of Psychology - Temple University, Philadelphia, PA 19122, United States.E-mail:emma.rhodes@temple. edu

R. MELROSE, M. ETTENHOFER, A. JIMENEZ, A. HASRATIAN \& J. VELIZ. Compensatory Scaffolding of Prefrontal Cortex in Cognitive Aging.

Objective: The scaffolding theory of cognitive aging (STAC) posits that compensatory reorganization of prefrontal cortex (PFC) works to offset neural challenges (e.g., gray matter loss) associated with aging to preserve cognition. One mechanism of scaffolding is increased recruitment of PFC regions contralateral to those primarily involved in a task. We tested this theory across the spectrum of aging.

Participants and Methods: The sample included 47 adults age $60+(21$ healthy \& 26 with Mild Cognitive Impairment). Neural challenges were defined as whole brain (WBV) and hippocampal (HC) volume. Scaffolding was investigated using two fMRI tasks: visuospatial reasoning $(\mathrm{VR})$ and maintenance working memory (WM). The number of active fMRI voxels within FreeSurfer defined PFC regions was tabulated; absolute difference between right and left hemispheres was obtained. Lower scores indicated more bilaterality, hypothesized to reflect greater scaffolding. Immediate recall (IR) from neuropsychological tests represented cognition. Correlations and step-wise regression were used to understand the relative contribution of bilaterality and atrophy on cognition. Results: For VR, greater bilaterality of rostral middle frontal gyrus (MFG) was associated with smaller WBV $(r=.29, p<.05)$, HC $(r=.38$, $\mathrm{p}<.01)$, and worse IR $(\mathrm{r}=.31, \mathrm{p}<.05)$. Regression showed that while IR was associated with MFG bilaterality $\left(\mathrm{R}^{2}=10\right)$, the model was improved when $\mathrm{HC}$ was included $\left(\mathrm{R}^{2}=.23, \mathrm{p}<.001\right)$ and only $\mathrm{HC}$ was significant. For WM, greater bilaterality of inferior frontal gyrus, pars triangularis (IFG) was associated with smaller WBV $(\mathrm{r}=.41, \mathrm{p}<.01)$, and worse IR $(\mathrm{r}=.32, \mathrm{p}<.05)$. Regression showed IR was best predicted by IFG bilaterality $\left(R^{2}=.10\right)$ and fit did not improve when WBV was included.

Conclusions: Across two fMRI tasks, we observed that bilateral activation of PFC is associated with atrophy and poorer memory. This suggests that reliance on alternative networks is greatest in those in most need of compensatory strategies.
Correspondence: Rebecca Melrose, PhD, Brain, Behavior, and Aging Research Center, VA Greater Los Angeles Healthcare System, 11301 Wilshire Blvd,Los Angeles, CA 90048, United States.E-mail: rjmelrose@ ucla.edu

S.E. MACPHERSON, S. GHAROONI, E. CHAN, C. HEALY, M. ALLERHAND, T. SHALLICE \& L. CIPOLOTTI. The Impact of Age, Aetiology and Cognitive Reserve on the Cognitive Performance of Frontal and Posterior Patients.

Objective: Our previous work demonstrates that age and literacy attainment predict the cognitive performance of frontal patients on frontal-executive measures. In contrast, lesion aetiology does not influence performance. The notion that the effects of brain damage are mitigated by the premorbid efficiency, capacity and flexibility of cognitive processing (i.e., cognitive reserve) has been associated with the frontal lobes. However, it remains unknown whether this preservation is indeed only found in patients with frontal lobe lesions. In the current study, we examined whether the impact of age and literacy attainment on cognition are specific to frontal lesions and not to nonfrontal, posterior brain areas.

Participants and Methods: Eighty-six patients with unilateral lesions in the frontal lobes and 67 patients with unilateral lesions in nonfrontal, posterior brain regions were recruited for the study. We investigated the influence of aetiology (stroke, tumour), age, lesion (frontal, posterior) and the independent effects of two cognitive reserve proxies, education and literacy attainment (NART IQ), on measures of executive function, processing speed, naming, and perception.

Results: We demonstrated that posterior patients with different aetiologies did not significantly differ in their performance on any of the cognitive measures. We then fitted multiple linear regression models for each of the cognitive measures and found that age and NART IQ predicted executive performance; lesion group did not. NART IQ also predicted naming abilities and age predicted processing speed. Only perceptual abilities were predicted by lesion group.

Conclusions: These results suggest that the grouping of frontal and posterior patients caused by different aetiologies is a justified methodological approach in neuropsychological group studies. Our latter findings suggest that while age and literacy attainment play independent roles in executive performance, this preservation is not specific to frontal lobe lesions.

Correspondence: Sarah E. MacPherson, PhD, Department of Psychology, University of Edinburgh, 7 George Square, Edinburgh, Edinburgh EHs 9JZ, United Kingdom. E-mail: sarah.macpherson@ed.ac.uk

\section{Paper Session 8. Neurocognitive Trajectory and Aging}

\author{
Moderator: Kamini Krishnan
}

$$
\text { 4:00-5:30 p.m. }
$$

L. KAMALYAN, A.M. BRICKMAN, N. SCHUPF \& J.J. MANLY. Effect of Lifetime Occupation Level on Cognitive Trajectory among Diverse Older Adults.

Objective: Prior studies show that more complex occupational demands are related to lower rates of global cognitive decline. We hypothesized that lifetime occupational attainment has an independent effect on neuropsychological trajectory over time, and that race/ethnicity would moderate this relationship.

Participants and Methods: Participants were 1633 non-Hispanic Blacks, 1370 non-Hispanic Whites, and 2362 Hispanics in the Washington/Hamilton Heights/Inwood Columbia Aging Project, age 65 years and older $(67.2 \%$ female $)$ and not demented at baseline. Lifetime occupation was grouped into low (housewife, unskilled/semiskilled, skilled trade or craft, and clerical/office worker) and high 
(manager business/government and professional/technical). Neuropsychological tests were administered at baseline and every 18-24 months. Linear mixed effects models tested the relationship between level of occupational attainment and cognitive trajectory, adjusting for age, sex, and years of education, with race/ethnicity as a potential moderator. Results: Independent of covariates, participants with higher occupational levels had better global cognitive function at baseline $(\mathrm{t}=3.4$; $p<.001)$ and less decline over time $(t=-2.7 ; p=.007)$. High occupation did not confer as much of an advantage on global cognition at baseline among African Americans as it did among non-Hispanic Whites $(t=-1.9 ; p=.05)$. The effect of occupation on global decline over time was not moderated by race/ethnicity, but when memory was used as an outcome, the impact of low occupation on decline among Hispanics was weaker than among Whites.

Conclusions: Older adults with low occupational attainment have lower baseline cognitive scores and more rapid cognitive decline and these effects differ across race/ethnicity. Future analysis will explore whether occupational characteristics such as cognitive or interpersonal engagement and physical demands, impact cognitive trajectory in later life. Correspondence: Lily Kamalyan, 630 West 168th St., P\&S Box 16, New York, NY 10032, United States.E-mail: lk2681@tc.columbia.edu

K.J. BANGEN, N. ARMSTRONG, R. AU \& A. GROSS. Midlife Metabolic Syndrome and Late-Life Cognitive Trajectories in the Framingham Heart Study.

Objective: Research linking metabolic syndrome (MetS) and cognitive decline is based primarily on cross-sectional data or studies with relatively short follow-up. Therefore, we sought to clarify whether MetS is associated with risk of cognitive decline by leveraging longitudinal prospective data from the Framingham Heart Study.

Participants and Methods: 2,894 Framingham Offspring Study participants underwent repeat health examinations and neuropsychological assessments. MetS was defined as three or more of the following: abdominal obesity, hypertriglyceridemia, low HDL cholesterol, high blood pressure, and elevated fasting plasma glucose. Three cognitive composite scores were constructed: general cognition, executive functioning, and memory. We estimated the association between onset of MetS during midlife with late-life cognitive decline using joint survival-growth models.

Results: Sixty-seven percent of participants $(\mathrm{N}=1,938)$ developed MetS (average age of onset=53 years). Rate of cognitive change after age 55 years was similar in those with and without incident MetS. However, level of cognitive performance differed between groups with those with MetS performing more poorly across all three cognitive domains. When individual components of MetS were examined separately, higher fasting blood glucose was associated with steeper rate of decline in general cognition and executive functioning. There were no significant differences in rate of change based on the other components.

Conclusions: Cognitive changes may predate development of full MetS, as its components develop over long periods of time before criteria for MetS are met. Midlife elevated blood glucose was associated with steeper rate of cognitive decline and may be a useful intervention target to prevent cognitive impairment. Further, those with MetS are at greater risk of cognitive impairment and dementia given their lower level of cognitive functioning, and thus may be an important group to identify for screening.

Correspondence: Katherine J. Bangen, VA San Diego Healthcare System/UC San Diego, Department of Psychiatry, UC San Diego, 9500 Gilman Drive, mail code 151B, La Jolla, CA 92093, United States. E-mail:kbangen@ucsd.edu
M.Y. YOU, K.B. CASALETTO, A.M. STAFFARONI, R. SALONER, P. MUMFORD, E. FOX, M. ALTENDAHL, J. STIVER \& J.H. KRAMER. Subjective Cognitive Symptoms Predict Cognitive and Structural Brain Aging Trajectories in Otherwise Healthy Older Adults.

Objective: Subjective cognitive symptoms (SCS) are common in cognitively healthy older adults despite normal performances on neuropsychological and neurological evaluations. Whether SCS predict subsequent cognitive aging trajectories, however, remains unclear. Our aim was to determine whether baseline SCS, both global and domain-specific, predict longitudinal change in cognitive performance and brain volume. Participants and Methods: 247 functionally intact adults (baseline age: $\mathrm{M}=69.5 \mathrm{y}, \mathrm{CDR}=0$ all visits) were followed longitudinally and determined to be neurologically normal at baseline and all follow-up visits (visit range $1-13, \mathrm{M}=3.0$ visits, $\mathrm{M}=1.6 \mathrm{y}$ between visits). Participants completed the Everyday Cognition Self Report, including global and domain-specific SCS (Memory, Language, Visual, Planning, Organization, and Attention) metrics at baseline, and a neuropsychological battery and structural brain MRI at baseline and follow-ups. Mixed-effects models examined the impact of baseline SCS, time, and their interaction on trajectories of cognition and total gray matter volume, adjusting for baseline age, sex, education, depressive symptoms, and total intracranial volume for MRI models.

Results: Greater baseline Global, Memory, and Language SCS were associated with declines in CVLT-II delayed free recall (SCS*time $b s=-0.06$ to $-0.10, p s<0.03)$. Greater baseline Language and Planning SCS predicted greater processing speed slowing (SCS *time $b s=0.16$ 0.26, $\mathrm{ps}<0.01)$. Greater baseline Global, Planning, Organization, and Attention SCS were associated with increased global gray matter atrophy rates (SCS*time $b s=-3.2$ to $-1.4, \mathrm{ps}<0.05$ ).

Conclusions: In functionally intact older adults, greater baseline SCS were associated with steeper rates of decline in cognition and brain volume. Memory, Language, and Planning SCS were the strongest predictors of longitudinal changes. In the absence of objective impairment, endorsement of SCS may be a marker of more negative brain health trajectories, even in the context of "normal" cognitive aging. Correspondence: Michelle Y. You, BA, Neurology, University of California, San Francisco, 675 Nelson Rising Lane, Suite 190, San Francisco, CA 94158, United States. E-mail: michelle.you@ucsf.edu

A. SOLdAN, C. PETTIGREW, Y. SHAO, M. GREGA, Y. ZHU, M. CARLSON \& M. ALBERT. Self-reported lifestyle activity and relationship to longitudinal cognitive change.

Objective: We examined if current self-reported engagement in lifestyle activities is associated with current cognitive performance, prior longitudinal cognitive trajectories, and baseline levels of cognitive reserve (CR). Participants and Methods: This study included 189 individuals from the BIOCARD cohort who were cognitively normal at baseline ( $\mathrm{M}$ baseline age $=57$ years) and have been followed prospectively for up to 20 years $(\mathrm{M}=12$ years $)$ with annual clinical and cognitive assessments; 27 participants have developed MCI over time. The CHAMPS activity questionnaire, which measures self-reported engagement in physical activities, social activities and cognitive activities, was administered starting in 2015. Cognitive reserve (CR) was measured at baseline with a composite score combining education, vocabulary, and reading measures. A composite score was used to assess cognitive performance. Results: Mean age at the CHAMPS study visit was 71 years. Cross-sectional linear regression analyses showed that among those who remained cognitively normal, better cognitive performance was associated with higher frequency and duration of cognitive activities $(p$ 's $<0.05)$, independent of social and physical activities and of level of baseline CR, which was also associated with better cognitive performance $(p=0.001)$. In longitudinal analyses, among those with MCI, higher energy expenditure in low $(p<0.0001)$ and high $(p=0.002)$ intensity physical activities, longer duration of cognitive activities $(p=0.004)$ and engagement in greater total number of all activities $(p=0.01)$, had a more positive rate of change (i.e., less decline) in cognitive performance in previous years. 
Conclusions: These results suggest that greater engagement in lifestyle activities may be associated with less cognitive decline among those who develop symptoms of MCI. Prospective longitudinal evaluations are needed to confirm these findings.

Correspondence: Anja Soldan, Cognitive Neuroscience Division, Johns Hopkins School of Medicine, 1620 McElderry Street, Baltimore, MD 21205, United States. E-mail: asoldan1@jhmi.edu

J.M. COLON, A.M. BRICKMAN, G. TOSTO, N. SCHUPF, C.M. ABINADER \& J.J. MANLY. Relationship of African Ancestry with Cognitive Trajectory among Older Latino/as.

Objective: The current study evaluated the relationship of a) racial self-identification and b) genetic African ancestry to cognitive trajectory among older Caribbean Latinos, and investigated mediators of these relationships.

Participants and Methods: Participants were self-identified Hispanic/ Latino older adults from a longitudinal community-based study. Participants were evaluated with neuropsychological tests of language, executive function, memory, and speed at baseline and 18-24 month follow-ups. Race was self-identified according to US Census criteria. Population ancestral components were derived through genetic analysis of DNA, calculating percent African ancestry. Self-identified race and ancestry was compared. Linear mixed models tested the relationship of racial self-identification and African ancestry to baseline cognitive test scores and change over time. Measures of educational experience and adult SES were examined as potential mediators.

Results: Independent of age, sex, and medical burden, Latinos who self-reported Black race had a higher degree of African ancestry $(\mathrm{M}=56.81 ; \mathrm{SD}=21.14)$ than those who reported White race $(\mathrm{M}=15.34$; $\mathrm{SD}=13.75)$ and "other" race $(\mathrm{M}=33.06 ; \mathrm{SD}=19.38)$. Self-reported race was not related to baseline or slope in any cognitive domain. However, participants with more African ancestry obtained lower scores on measures of memory, visuospatial skill, speed, and language $(p<.01$ for all). There was no effect of African ancestry on rate of decline. The effect of ancestry on memory was accounted for by years of school. The effect of ancestry on language scores at baseline was explained by quality of education (Spanish reading level). The effect of ancestry on baseline visuospatial skill and speed remained after adjustment for all educational and adult SES mediators.

Conclusions: African ancestry levels among Caribbean Latinos may reflect experiences of discrimination or lifetime social disadvantage that influence cognitive test performance.

Correspondence: Juliet M. Colon, G.H. Sergievsky Center, College of Physicians and Surgeons, Columbia University, 622 W 168th St, PH 18, New York, NY 10032, United States.E-mail:jmb2396@cumc.columbia. $e d u$

K. SOL \& L.B. ZAHODNE. Psychosocial Predictors of Memory Decline in a Diverse Longitudinal Sample of Older Adults.

Objective: Racial disparities in Alzheimer's disease exist, with Blacks and Hispanics experiencing higher risk than Whites. Psychological factors may be relevant to dementia risk among Blacks and Hispanics. This study sought to quantify racial/ethnic disparities in episodic memory trajectories and determine whether well-being, locus of control, depressive symptoms, and negative affect are differently associated with memory trajectories across race/ethnicity.

Participants and Methods: Participants were drawn from the National Health and Aging Trends Study (NHATS), a nationally representative, longitudinal study of adults $65+$ years old. Analyses included 9914 participants $(68.8 \%$ white, $20.5 \%$ black, $5.6 \%$ Hispanic) without dementia at baseline. Average age was 76.20 years. Data was collected over five annual waves. Outcome was a composite of immediate and delayed trials from a word list memory test. Data were analyzed using linear mixed models controlling for age, gender, education, income, disease burden, and marital status.
Results: Black race and Hispanic ethnicity were associated with lower initial memory level, but only Hispanic ethnicity was associated with faster memory decline. More depressive symptoms, less well-being, and less control were each independently associated with lower initial memory. Only depressive symptoms were associated with faster rate of memory decline. There were no significant interactions between race/ ethnicity and the psychosocial variables.

Conclusions: Results extend previous studies showing racial/ethnic disparities in episodic memory trajectories, and the enduring effects of depression on cognitive health. Future longitudinal studies should explore other psychosocial factors and immigrant status to better delineate pathways contributing to faster rate of cognitive decline among older Hispanics.

Correspondence: Ketlyne Sol, PhD, Psychology, University of Michigan, 530 Church Street, Ann Arbor, MI 48109,United States. E-mail: ksol@ umich.edu

\section{Poster Session 6. Acquired Brain Injury 4:00-5:15 p.m.}

\section{Acquired Brain Injury (TBI/ Cerebrovascular Injury \& Disease - Adult)}

I. BABAKHANYAN, N.E. CARLOZZI, B. MCKENNA, K.B. CASALETTO, A. HEINEMANN \& R.K. HEATON. NIH Toolbox Emotion Battery: Application of Summary Scores to Spinal Cord Injury, Traumatic Brain Injury, and Stroke.

Objective: The NIH Toolbox Emotion Battery (NIHTB-EB) was developed to measure the full spectrum of emotional functioning and provide a "common currency" across clinical and research settings. Factor analytically-based summary scores were developed using the NIHTB-EB normative sample and include Negative Affect, Social Satisfaction, and Psychological Well-Being. The purpose of this study was to evaluate the known-group validity of the summary scores in healthy adults and those with neurologic conditions.

Participants and Methods: The NIHTB-EB was administered to 1036 English-speaking adults from the NIHTB normative project and 604 adults with documented neurologic conditions including spinal cord injury (SCI, $\mathrm{N}=209$ ), traumatic brain injury (TBI, N=184), and stroke (CVA, N=211). Univariate analyses of summary scores across the four groups identified group differences. Base rates of elevated scores (defined as > 1 standard deviation towards the problematic direction) were also described.

Results: The normative group demonstrated significantly better emotional functioning characterized by greater Social Satisfaction (TBI $d=0.38$, SCI $d=0.37$, CVA,$d=0.31 ; p$ 's $<.0001)$ and Psychological Well-Being (SCI $d=0.50$, CVA $d=0.40$, TBI $d=0.32 ;$ p's<.0001) compared with each neurologic group. The normative group demonstrated significantly less Negative Affect composite compared with the clinical groups; however, the effect size indicates the difference was only marginal for TBI (TBI $d=0.26$, SCI $d=0.19$, CVA $d=0.16$ ). The neurologic groups had higher rates of problematic emotions on all 3 summary scores compared with the normative group (20-32\% verses $13-16 \%$ ). $27 \%$ of the normative group had one more composites within the problematic range versus $55 \%$ for the clinical groups, $\left.\mathrm{X}^{2}(1, \mathrm{~N}=1443)=49.47, \mathrm{p}<0.001\right)$.

Conclusions: The NIHTB-EB summary scores are sensitive to emotional sequelae in SCI, CVA, and TBI, and may be useful to screen and identify individuals in need of psychological support or treatment.

Correspondence: Ida Babakhanyan, Ph.D. , Defense and Veterans Brain Injury Center (DVBIC) and UCSD, 33 Area Camp Margarita Bldg 33305, Camp Pendleton, CA 92055, United States. E-mail: ibabakhanyan@gmail.com 
I. BABAKHANYAN, A.N. PAULLADA, V. RAWLEIGH, D. KIM, S.J. KENTON, S. GALLARDO, K. STUESSI, P. SARGENT \& J. BAILIE. Cognitive Changes Following Intensive Outpatient Program for Mild Traumatic Brain Injury Within a Military Cohort. Objective: Identification of subtle cognitive changes following a mild traumatic brain injury (mTBI) is a challenge for researchers. The Automated Neuropsychological Assessment Metrics version 4 (ANAM) is a computerized measure widely used within the Department of Defense for TBI. The ANAM is accessible and easy to administer, with strong reliability and validity; yet, it has not been empirically evaluated to track response to cognitive rehabilitation following mTBI.

Participants and Methods: Participants in a 5-week IOP program for cognitive rehabilitation were administered the ANAM at pre and post treatment. The cognitive module consists of reaction time $1 \&$ 2 , memory, delayed memory, processing speed, working memory, and visuospatial memory. Global Deficit Score (GDS) was computed from ANAM standard scores to detect subtle impairment across multiple measures. Participants consisted of 31 active duty service members with a history of mTBI. Mean age was 33 years $(S D=7.6), 78 \%$ were Marines. Paired sample t-tests were used to evaluate pre and post treatment scores; repeated measure analysis of variance was used for interactions with pre-treatment GDS.

Results: Performance improved after cognitive rehabilitation for reaction time $1(p=0.03, d=0.33)$, processing speed $(p=0.04, d=0.27)$ and learning $(p=0.002, d=0.62)$. GDS also improved following treatment $(p=0.02, d=0.24)$ and $55 \%$ of participants showed improvement on GDS. There was a significant interaction between pretreatment GDS and improvement in learning $(p=0.007)$, working memory $(p<0.001)$, simple reaction time $1 \& 2(p<0.001, p=0.023)$, and processing speed $(p=0.023)$. Consistently, those with cognitive impairment at pretreatment had greater improvement.

Conclusions: This study demonstrates the ANAM's utility in detecting changes following cognitive rehabilitation in mTBI. Cognitive rehabilitation in IOP for mTBI is beneficial and those who present with impairment showed most gains. Future efforts will examine convergent validity with other cognitive tasks in a larger sample.

Correspondence: Ida Babakhanyan, Ph.D., Defense and Veterans Brain Injury Center (DVBIC) and UCSD, 33 Area Camp Margarita Bldg 33305, Camp Pendleton, CA 92055, United States. E-mail: ibabakhanyan@gmail.com

M. BEDARD, J. STEFFENER \& V. TALER. Long-term prospective memory impairment following mild traumatic brain injury with loss of consciousness.

Objective: Little attention has been devoted to the long-term neuropsychological sequelae of mild traumatic brain injury (mTBI), as the majority of investigations have focused on more severe cases. Interestingly, mounting evidence from neuroimaging studies have revealed structural and functional alterations to frontal areas of the brain years after mTBI, which may underly persistent cognitive dysfunction. Prospective memory (ProM) comprises the ability to "remember to remember" to perform future tasks, and places considerable demands on executive processes subserved by the rostral prefrontal cortex, particularly in the completion of time- rather than event-based ProM. Despite ProM impairments being widely found in cases of moderate-severe traumatic brain injury (TBI), a paucity of data is available for ProM functioning among those with mTBI, generally, and particularly for post-acute (i.e., > 3 months) periods. Thus, it remains unclear whether impairments in time- or event-based functioning may persist more than a year after mTBI.

Participants and Methods: Analyses were run on data from the Canadian Longitudinal Study on Aging (CLSA), a nationwide study on health and aging involving individuals between the ages of 45 to 85. Analyses were run on the 1,937 participants who experienced mTBI with loss of consciousness more than 12 months prior, and the 13,525 cognitively healthy adults. Participants were administered the Miami
Prospective Memory Test, and tests of retrospective memory and executive functioning.

Results: Those with mTBI were more likely to be impaired in timebased ProM relative to people with no history of TBI. Time-based impairments were predicted by older age, and executive dysfunction among those who spent more than one minute unconscious.

Conclusions: Those in the post-acute phase following mTBI with loss of consciousness may experience impairments in ProM, particularly when greater demands are placed on executive processes (time-based ProM). Correspondence: Marc Bedard, Psychology, University of Ottawa, School of Psychology, Vanier Hall, 136 Jean-Jacques Lussier, Ottawa, ON K1N9A8, Canada. E-mail: mbeda103@uottawa.ca

\section{S. BELKONEN, K.T. RHYNER, M. BLACKWELL, N. CASSELL \& B. SHENAL. Symptom Report in Rural OEF/OIF/OND Veterans with TBI and PTSD.}

Objective: To examine symptom report of rural Veterans who were seen for a Comprehensive TBI evaluation at the Salem VA. Research in OEF/OIF/OND veterans with mTBI has suggested that PTSD symptoms impact post-concussive symptom reports. This study seeks to better understand the symptom report of veterans in rural areas and whether participants with PTSD report significantly higher symptoms.

Participants and Methods: Participants included 329 Veterans attending a Comprehensive TBI Evaluation in the Polytrauma Support Clinic. A retrospective chart review was conducted to compare symptom report of participants with TBI and PTSD on the Neurobehavioral Symptom Inventory (NSI). The 4-factor model using vestibular, somatic, and affective symptoms of the NSI was used (Vanderploeg, et. al, 2015). ANOVAs were completed to compare means.

Results: Participants who were diagnosed with either PTSD $(p<0.01)$ or TBI $(p=.012)$ reported significantly more cognitive symptoms. Participants who were diagnosed with PTSD reported significantly more vestibular symptoms $(\mathrm{p}<0.00)$. Participants with PTSD reported significantly more somatic symptoms $(\mathrm{p}=0.03)$. Participants with PTSD $(p<0.00)$ reported significantly more emotional symptoms $(p<0.01)$. No interaction effects were significant.

Conclusions: Participants who are diagnosed with PTSD, independent of TBI, report greater symptoms on the NSI. Participants with TBI do report a significant level of cognitive symptoms. This study suggests, that consistent with previous literature, rural $\mathrm{OEF} / \mathrm{OIF} / \mathrm{OND}$ veterans with PTSD report higher levels of post-concussive symptoms.

Correspondence: Stacy Belkonen, Ph.D., Salem VA Medical Center, 1970 Roanoke Blvd., 116B, Salem, VA 24153, United States. E-mail: stacy.belkonen@va.gov

A.S. BODAPATI, H.L. COMBS, A.M. GIAMMITTORIO, N.J. PASTOREK, M. TROYANSKAYA, J. ROMESSER, A. SIM \& J. LINCK. Detection of Symptom Over-Reporting on the Neurobehavioral Symptom Inventory in OEF/OIF/OND Veterans with Mild TBI.

Objective: As part of routine care at Veterans Affairs (VA) facilities, veterans with a history of a service-related traumatic brain injury (TBI) are administered a self-report post-concussive symptom measure, the Neurobehavioral Symptom Inventory (NSI). Interpreting symptom report on the NSI can be problematic given that symptom exaggeration on self-report measures is often found in both civilian and military patient populations. This study aims to further investigate possible embedded scales on the NSI that identify possible and probable symptom exaggeration.

Participants and Methods: In the main study, 183 veterans with mild TBI from several VA sites were administered the Minnesota Multiphasic Personality Inventory, 2nd edition (MMPI-2) and the NSI. The participants were divided into symptom validity testing (SVT) pass and fail groups based on their performance on the MMPI-2 symptom validity scales, using both lenient and stringent criteria. Cut scores on the NSI total and Validity-10 (VAL-10) scores were then established and 
applied to two additional veteran populations with similar inclusion/ exclusion criteria.

Results: Using ROC curve analyses, sensitivity and specificity values were derived for all NSI total and VAL-10 values. Optimal cut scores were determined based on specificity level of $\geq 95 \%$, to minimize the incorrect labeling of symptom exaggeration. The cut score for the NSI total scale was $\geq 67$ for probable and $\geq 57$ for possible symptom exaggeration. For the VAL-10 scale, $\geq 27$ was indicative of probable and $\geq 22$ reflected possible symptom exaggeration.

Conclusions: Data revealed that both the NSI total and VAL-10 cut scores were consistently able to identify potential symptom exaggeration across 3 mild TBI samples. Clinicians and researchers who use the NSI are encouraged to utilize either embedded validity measure in their practice. The breakdown of possible and probable symptom exaggeration may serve as an extension of the Slick criteria in identifying individuals who may require further scrutiny.

Correspondence: Anjuli S. Bodapati, PhD, Michael E DeBakey VA Medical Center, 5014 Jackson St, Houston, TX 77004, United States. E-mail: anjuli.bodapati@gmail.com

M.J. BRAY, E.A. BARANY, A. COLANTONIO, S. HWANG, M. BAYLEY \& R. GREEN. Homelessness and the Neuropsychiatric Sequelae of Traumatic Brain Injury: A Scoping Review.

Objective: Traumatic brain injury (TBI) is fast becoming understood as an important factor in the etiology of homelessness, given the high prevalence of TBIs, and their frequent occurrence prior to the onset of homelessness. Specifically, it has been proposed that TBI exerts its detrimental impact through acquired cognitive/psychiatric sequelae of injury (vs. motor sequelae or pre-morbid factors, for example). Thus, this scoping review sought to characterize the relationship between cognitive/psychiatric sequelae of injury and homelessness.

Participants and Methods: Scoping review (Medline Ovid [19662016], Embase Ovid [1947-2016], and PsycInfo [1887-2016]) of primary research on cognitive and mental health, TBI, and homelessness; fourteen articles identified and relevant data extracted.

Results: Lending support to the putative causal role of cognitive and psychiatric sequelae, studies revealed that in homeless populations, TBI was associated with poorer mental health, with a higher incidence of mood disorders and post-traumatic stress disorder, and with greater cognitive impairment, though some reports implicated other causal factors for cognitive dysfunction (e.g., socioeconomic). There was evidence of bidirectional relationships, with TBI causing psychiatric/ cognitive sequelae leading to homelessness, and homelessness leading to psychiatric disorders and to TBIs. Increased suicidality in homeless or at risk individuals was found to be mediated by TBI-induced depression. Drug and alcohol abuse represented important risk factors for future TBI in this population.

Conclusions: TBI appears to exert its influence on homelessness via cognitive and psychiatric effects, though bidirectional relationships exist, raising the possibility of a vicious cycle that may increase chronicity of homelessness. Greater awareness of TBI and its sequelae may enhance recognition and treatment in this historically underserved population. Correspondence: Michael J. Bray, Institute of Medical Sciences, University of Toronto, 550 University Avenue, Room 11-207, Toronto, ON M5G2A2, Canada.E-mail:michael.bray@mail.utoronto.ca

E.K. BRENNER, R.A. BERNIER, E.C. GROSSNER, J.S. HONG \& F.G. HILLARY. Cognitive Reserve is Associated with Connectivity in Moderate to Severe Traumatic Brain Injury.

Objective: Cognitive reserve (CR) has been proposed as a protective mechanism again neurologic disruption, but its influence on connectivity after brain injury remains unclear. We used graph theory, functional MRI, and measures of premorbid function to examine the relationship between network connectivity and CR in patients with moderate to severe traumatic brain injury (TBI), defined as having a GCS of 3-12 or positive neuroimaging findings.
Participants and Methods: A total of 29 individuals with TBI and 30 matched healthy controls (HC) underwent resting-state fMRI and neuropsychological testing. Graph theory was implemented to examine specific connectivity profiles; adjacency matrices were created across 264 functionally defined regions of interest (Power et al., 2011). Analyses focused on networks associated with dysfunction in individuals with TBI. CR was calculated by obtaining a z-score for participants on either the Shipley Abstraction or the Test of Premorbid Functioning. Results: Examining positive connectivity within the executive control network (ECN), salience network, and subcortical network, there was an interaction between CR and head injury status, such that greater CR was associated with higher connectivity in the TBI group, $p=0.094$, $p=0.063, p=0.042$. Results also showed interactions between CR and injury status, where CR predicted better performance on VSAT and Trails B in the TBI group, $p=0.028, p=0.057$. Based on analyses showing a relationship between ECN connectivity and Trails A, $p=0.017$, a model including CR, Trails A, and injury status significantly predicted within-network positive connectivity in the ECN, accounting for $15.7 \%$ of the variance, $p=0.025$.

Conclusions: The findings demonstrate that greater CR is associated with higher connectivity in individuals with TBIs and lower connectivity in HCs. As hyperconnectivity has been shown to be a response to neurologic disruption (Hillary et al., 2015), these results suggest that higher CR may be associated with maximized network connections after injury. Correspondence: Einat K. Brenner, Psychology, Pennsylvania State University, 419 Bruce V. Moore Building, University Park, PA 16802, United States.E-mail: ebb5161@psu.edu

S.T. BROWN, K.C. HEWITT \& L. NASH. The Role of Neuropsychology in the Relationship Between Acquired Brain and Spinal Cord Injury.

Objective: Neuropsychologists are often consulted to work with dual diagnosis patients especially those who have sustained acquired brain injury (ABI) and spinal cord injury (SCI). Injuries to the central nervous system often result in dual diagnosis. While the mechanisms that cause SCI often cause ABI, ABI commonly goes undiagnosed in SCI. ABI does not entail complete absence of connectivity between cortex, brain stem, and spinal motor cells, but there can be altered neurological characteristics. We present a systematic review of dual diagnosis and stress the importance of neuropsychological involvement in identification and treatment.

Participants and Methods: A search was conducted using GALILEO with the terms "acquired brain injury," "traumatic brain injury," "spinal cord injury," and "neuropsychology," which resulted in N=3,508 total articles. Exclusion criteria included irrelevant studies lacking dual diagnosis and/or non-adult groups.

Results: A review of relevant studies revealed a wide variation in prevalence of dual diagnosis. A few key articles address the complicating issues of dual diagnosis, but there was a dearth of research addressing the importance of neuropsychological involvement with potential consequences in patient care. Adjustment to life after SCI involves adaptation, rehabilitation, and health management. Overlooked ABI diagnoses potentially affect activities of daily living, health care costs, and lasting implications for family and caregivers. Data analysis emphasizes the need for neuropsychological involvement to ensure accurate assessment of patient functioning and provision of best patient care.

Conclusions: This systematic review established the relationship between ABI and SCI as well as the consequences of missed diagnoses. Clinical assessment should include measures that satisfy diagnosis, level of disability, decision-making capacity, goal setting, changes in status, and discharge with SCI patients. Future research needs to expand upon ABI model systems of care within the SCI population.

Correspondence: Stephen T. Brown, M.S., Psychology, Mercer University, 2925 N. Boulder Drive Unit 21, Wichita, KS 67226, United States.E-mail:tyler.brown1988@gmail.com 
N. CASTOR \& F. EL MASSIOUI. The influence of education level on cognitive recovery in people with brain damage.

Objective: Neurological pathologies generally generate irreversible deficits, which produce many difficulties in brain-damaged patients' life. Therefore patients must face up to their loss. Some studies revealed that educational level (EL) can preserve cognitive performances or slow down degenerative process in neurological diseases. As traumatic brain injuries (TBIs) and strokes are neurological pathologies with a recovery potential, in this context, we chose to investigate cognitive recovery in these. The propose study aimed was to examine the role of EL in cognitive functioning in patients with TBIs and strokes.

Participants and Methods: We assessed twice, with a mean interval of 6 months between both evaluations, the cognitive performances of 49 patients who suffered from TBIs and 42 patients who suffered from strokes. Cognitive capacities were rated using the French version of the Barrow Neurological Institute Scale. We also considered the EL of participants which divided into less than 12 years group and more than 12 years group. Others factors such as age and emotional states were also considered as co-variables. Consequently, we assessed twice, depression, anxiety, resilience and self-esteem degree.

Results: Participants show an identical degree of cognitive capacities at first, $t(89)=0.23, N . S$ and second evaluation, $t(89)=0.84, N . S$. An improvement in cognitive abilities was observed at the second assessment, $t(90)=-2.21, p=0.03$. A significant effect of EL appeared for some cognitive performances, $F(3,81)=3.34, p=0.02$.

Conclusions: In this research we wanted to examine the influence of EL impact on cognitive performances. The study outcomes reveal that EL appears to participate in the recovery, even considering age, diseases and emotional states. The more EL is high and the more cognitive performances are elevated. This study is part of longitudinal research that attempts to expose the various factors of recovery in head traumas. Correspondence: Naomie Castor, Ph.D. student, Psychologie, Université Paris 8, 2 rue de la Liberté, Saint-Denis 93526, France. E-mail: castor. naomie@gmail.com

N. CASTOR \& F. EL MASSIOUI. Resilience and education level in brain damaged people: does it impact on cognitive performances? Objective: Traumatic brain injuries (TBIs) and strokes generally generate several deficits, which produce many difficulties in braindamaged patients' life. Improving capacities may possibly occurs when some factors such as resilience and high education level (EL) are present in individuals. The propose study aimed was to examine interaction effect between resilience and education level and does it impact on cognitive functioning in patients with TBIs and strokes.

Participants and Methods: We assessed twice, with a mean interval of 6 months between both evaluations, the resilience degree and cognitive performances of 49 patients who suffered from TBIs and 42 patients who suffered from strokes. Cognitive capacities were rated using the French version of the Barrow Neurological Institute Scale and for resilience degree, we used the Connor and Davidson's resilience scale (CD-RISC 25). Patients were divided into less resilient group and high resilient group by using resilience median (72). We also considered the EL of participants which divided into less than 12 years group and more than 12 years group.

Results: Participants showed similar cognitive capacities at first and second evaluation. An improvement in cognitive abilities was observed $t(90)=-2.21, p=0.03$. No significant interaction effect between resilience and EL was found, $F(7,77)=1.015, N S$, while a main effect of EL appeared for some cognitive performances, $F(3,81)=3.34, p=0.02$. Conclusions: In this research we wanted to verify if resilience and EL considered could impact on cognitive performances. The study outcomes reveal there was no interaction effect between these variables. However education level appears to participate in the cognitive recovery, even considering resilience. This study is part of longitudinal research that attempts to expose the various factors of recovery in head traumas.
Correspondence: Naomie Castor, Ph.D. student, Psychologie, Université Paris 8, 2 rue de la Liberté, Saint-Denis 93526, France. E-mail: castor. naomie@gmail.com

E.J. CONNORS, T.M. WILDGOOSE, L.A. FRAZIER \& B.J. SCHROCK. The Influence of Executive Function on two Types of Verbal Learning Among Individuals with a Traumatic Brain Injury.

Objective: Research supports that executive functioning (EF) has a differential impact on story learning versus list learning among certain clinical populations, such as stroke patients, however, there is limited research conducted on individuals with a traumatic brain injury (TBI). The purpose of this study is to determine if EF performance, as measured by the Trailing Making Test, Wisconsin Card Sorting Test, and Verbal Fluency (FAS), impacts an individual's performance on a story learning task and a list learning task, as measured by the Logical Memory subtest of the Wechsler Memory Scale-Fourth Edition (WMS-IV) and the Hopkins Verbal Learning Test-Revised (HVLT-R), respectively.

Participants and Methods: This was a retrospective observational study that utilized data from a sample of adults that were diagnosed with a TBI and referred to a neurorehabilitation facility. These individuals were then divided into two groups, those with significant executive dysfunction and those without.

Results: There were no significant between-group differences when comparing age, level of education, ethnicity, cause of TBI, or acuity level. A One-Way ANOVA revealed that those with significant EF dysfunction, compared to those without, performed significantly worse on measures of immediate story learning and immediate list learning, $F(1,32)=9.29, p=.005, d=1.046 ; F(1,32)=5.40, p=0.027, d=.798$, respectively. Those with EF dysfunction also performed significantly worse on delayed story recall, $F(1,32)=12.106, p=.001, d=1.19$, but there was a non-significant between-group difference on delayed list recall, $F(1,32)=.54, p=.468, d=.251$.

Conclusions: The findings from this study highlight the role of EF on verbal learning performance, with both story learning and list learning being impacted by impaired EF. This study is unique because it highlights the impact of $\mathrm{EF}$ on both types of verbal learning in TBI patients, which is inconsistent with other clinical populations.

Correspondence: Eric J. Connors, MA, Psychology, Alliant International University, 2950 Broadway, Unit \#16, San Diego, CA 92102, United States.E-mail: connors4891@gmail.com

L.D. CROCKER, S.M. JURICK, K.R. THOMAS, A.V. KELLER, M. SANDERSON-CIMINO, B. BOYD, C. RODGERS, S. NORMAN, A. LANG, E.W. TWAMLEY \& A.J.JAK. Do TBI Injury Characteristics Affect PTSD Treatment in Veterans with PTSD and a History of TBI?

Objective: Providers often express hesitation about the appropriateness of PTSD treatment for Veterans with a history of traumatic brain injury (TBI), given concerns that TBI-related sequelae may negatively affect PTSD treatment and/or should be the focus of treatment instead. However, evidence suggests that those with a history of TBI can benefit from evidence-based PTSD treatment. We extend these findings to examine whether certain characteristics of TBI injuries are associated with PTSD treatment engagement and response.

Participants and Methods: 100 Iraq/Afghanistan-era Veterans with PTSD and a history of mild to moderate TBI all received Cognitive Processing Therapy (CPT; either standard CPT or modified CPT that also included cognitive rehabilitation strategies). PTSD symptoms were assessed at 15 time points (baseline, each of 12 treatment sessions, posttreatment, 3-month follow up). Analyses examined whether number of TBIs, presence of loss of consciousness [LOC], presence of posttraumatic amnesia [PTA], and mechanism of injury (blast vs. other) for any TBI experienced were associated with treatment completion or response. 
Results: Only presence of PTA was associated with a higher rate of treatment dropout, but this association did not remain after controlling for baseline severity of PTSD, depression, and postconcussive symptoms. Multilevel modeling analyses examining change in PTSD symptoms indicated that none of the four injury variables examined moderated treatment response (all interactions with time were nonsignificant).

Conclusions: There was no evidence that TBI injury characteristics were associated with PTSD treatment completion and response beyond initial symptom severity, despite having more than adequate power to detect possible effects. Thus, a history of TBI should not preclude individuals from receiving CPT. In fact, PTSD treatment should often be a first line of treatment for these Veterans, given evidence of a mental health etiology to persistent post-concussive symptoms.

Correspondence: Laura D. Crocker, Ph.D., Psychology, Veterans Affairs San Diego Healthcare System, 3350 La Jolla Village Dr., 151B, San Diego, CA 92161, United States. E-mail: crocker.laura@gmail.com

\section{N.S. DAILEY, S. BAJAJ, A. ALKOZEI, R. SMITH, S. KNIGHT \& W.D. KILLGORE. Neural Correlates of Aggression in the Chronic and Post-Acute Stages of Recovery from Mild Traumatic Brain Injury: A Diffusion Tensor Imaging Study.}

Objective: Aggression is a commonly reported symptom associated with mild traumatic brain injury (mTBI). However, the neural basis of mTBI-related aggression is poorly understood. Here we sought to characterize aggression in adults at different stages of recovery from mTBI, and its relation to axonal integrity, using diffusion tensor imaging (DTI). Participants and Methods: Participants included 37 age-matched adults, including 16 healthy controls, 11 adults with post-acute mTBI ( $\leq 1$-month post-injury), and 10 with chronic mTBI ( $\geq 6$-months post-injury). Overall aggression, physical aggression, verbal aggression, anger, and hostility were measured using the Buss-Perry Aggression Questionnaire. FMRIB Software Library (FSL) was used to preprocess DTI data and calculate fractional anisotropy (FA), mean diffusivity (MD), radial diffusivity (RD), and axial diffusivity (AD). Tracts of interest that are particularly susceptible to injury in mTBI included the corpus callosum (CC), anterior thalamic radiation (ATR), cingulum $(\mathrm{Cg})$, and uncinate fasciculus (UF).

Results: Significant group-differences were found for physical aggression $(F(2,32)=5.83, p<.05, \mathrm{~d}=1.22)$, anger $(F(2,32)=4.06, p<.05$, $d=1.00)$, and total aggression $(F(2,32)=5.52, p<.05, d=1.19)$, with the chronic mTBI group reporting higher levels of physical $(p<.01)$ and total aggression $(p<.01)$, compared to healthy controls. No significant differences were found for the post-acute mTBI group. In the chronic mTBI group, physical aggression was negatively correlated with AD in the left ATR $(p<.05)$, and total aggression was negatively correlated with $\mathrm{FA}$ and $\mathrm{AD}$ in the right ATR $(p<.05)$.

Conclusions: The present study provides preliminary evidence supporting an association between reduced white matter integrity in the ATR and persistent and elevated levels of self-reported aggression in adults with chronic mTBI. In addition, the inverse relationship between $\mathrm{AD}$ and aggression is consistent with axonal damage, a characteristic of mTBI.

Correspondence: Natalie S. Dailey, Ph.D., Psychiatry, University of Arizona, 1501 N. Campbell Ave, Tucson, AZ 85724, United States. E-mail:ndailey@email.arizona.edu

\section{L.M. DOCKLER. Effect of Cognitive Deficit on Emotional Functioning following ABI.}

Objective: Gain novel insight regarding distress following ABI and identify associations with identified cognitive impairment presence, quantity, and type.

Participants and Methods: 117 adults were referred for cognitive evaluation following ABI. Analysis of archival data gathered from the HRNB, WAIS, WCST, Bender Gestalt Test, and the MMPI Clinical Scales. MANOVAs determined the effect of identified cognitive impairment presence and quantity on emotional functioning. Alpha corrected t-tests were also conducted to find differences between those with and without processing speed impairment, executive functioning deficits, motor impairment, and aphasic deficits.

Results: Groups with and without an identified deficit had clinically elevated mean T-scores on the Hs, D, Hy, Pd, Pt, and Sc MMPI Clinical Scales; only those with an identified cognitive deficit had clinically elevated mean T-scores on the Pa MMPI Clinical Scale. Deficit presence was statistically significant $(\mathrm{F}[9,108]=3.653, \mathrm{p}=.001 ; \mathrm{W}$ ilks' $\wedge=.767$; partial $\eta 2=.233)$ with an increase in Pt T-scores. Deficit quantity was also statistically significant $\left(\mathrm{F}[18,214]=2.21, \mathrm{p}=.004 ;\right.$ Wilks' $^{\prime} \wedge=.711$; partial $\eta 2=.157$ ) with an increase in Pt T-scores. Additionally, those with an identified processing speed deficit had significantly higher $\mathrm{Pt}$ T-scores, higher D, Pt, and Si T-scores for those with an identified motor deficit, and no differences in clinical scale T-scores for those with and without executive functioning or aphasic deficits.

Conclusions: The present study revealed that a significant portion of ABI survivors experience clinically significant emotional distress. The present study found that individuals with an identified deficit, with multiple identified deficits, and those with a processing speed or motor deficit were more likely to experience depressive and/ or anxiety symptoms relative to the MMPI Clinical Scales. The study highlights the need for focused mental health screening of all ABI survivors, regardless of deficit presence, quantity, or type.

Correspondence: Leah M. Dockler, California School of Professional Psychology, 73 Raintree Island, Apt. 3, Tonawanda, NY 14150, United States.E-mail:ldockler@alliant.edu

E. EPSTEIN, S.L. MARTINDALE \& H.M. MISKEY. Differences between Male and Female Iraq and Afghanistan Veterans in Behavioral Outcomes with co-occurring Traumatic Brain Injury and Posttraumatic Stress Disorder.

Objective: Women are projected to be the United States military subpopulation with the greatest growth over the next few years. This has led to increased demand for specialized services for female veterans and made improved characterization of sex differences in military veterans a critical next-step. The purpose of this study was to determine behavioral outcome sex differences between veterans with co-occurring traumatic brain injury (TBI) and posttraumatic stress disorder (PTSD).

Participants and Methods: Participants were 393 Iraq and Afghanistan veterans $(13.9 \%$ women $)$ with co-occurring TBI and PTSD. Veterans completed a comprehensive clinical interview, a lifetime TBI assessment, and several behavioral health questionnaires assessing alcohol and drug use, depression, sleep quality, PTSD symptoms, and pain. An independent samples $t$-test was conducted to determine mean differences on outcomes between sexes. Moderation analysis determined whether deployment status affected the association between sex and behavioral outcomes.

Results: Differences were significant for alcohol use, $p<.001$, between men, $M=3.25, S D=4.01$, and women, $M=7.10, S D=7.50$, as well as drug use, $p=.004$, between men, $M=0.40, S D=1.27$, and women, $M=1.88, S D=3.71$. There were no significant differences on measures of depression, sleep quality, PTSD symptoms, and pain. Moderation analysis indicated no effects of deployment status on the associations between sex and outcomes.

Conclusions: Male veterans were more likely to engage in both alcohol and drug abuse behaviors when compared to female veterans. The lack of differences in PTSD symptoms conflicts with literature using non-military, TBI, or PTSD diagnosis samples. This suggests that co-occurring TBI and PTSD may yield different treatment concerns and characteristics for female veterans compared with women in the general population. Correspondence: Erica Epstein, W. G. "Bill" Hefner Veterans Affairs Medical Center, $417 w$ sth street, APT A, Charlotte, NC 28202, United States. E-mail: ekinne4000@gmail.com 
A.A. FEDIO, J. SEXTON, S. CUMMINGS, C.L. ROPER, V. BUONOPANE, A. LEBEDUN \& P. FEDIO. Importance of Health Professionals Informing Brain-Injured Patients about Functional Changes.

Objective: This study explored effects on individuals with TBI of having been informed by a health care professional to expect changes in physical, cognitive, and/or emotional functioning during their recovery from brain injury.

Participants and Methods: Twenty individuals with TBI $(17 \mathrm{mod} /$ sev, 3 mild; 13 M, 7 F; mean age 36 ; 4 yrs post) served as participants. Measures included the Neurobehavioral Functioning Inventory (NFI), which assesses problems in physical, cognitive, and emotional functioning as reported by patients and their family. Patients also completed the Tennessee Self-Concept Scale (TSCS:2) and the Motivation for Rehabilitation Treatment Questionnaire.

Results: Nineteen patients were told to expect partial, rather than full or no, recovery from the effects of TBI. More patients were told to expect physical and/or cognitive changes than emotional changes. NFI and TSCS: 2 results were within the average clinical range. Patients reported most problems in cognition $(p=.03)$, whereas families reported most patient problems in memory/attention and depression. Having been told to expect physical problems had no effects on patients' test results. However, expecting cognitive problems yielded higher personal self-concept $(\mathrm{t}[17]=1.75, p<.05)$ and expecting emotional problems yielded lower moral self-concept $(\mathrm{t}[17]=1.90, p<.04)$. Moderate/severe TBI patients told to expect more problems across functional areas endorsed more reliance on professional help $(\mathrm{r}=.52, p=.04)$.

Conclusions: Having been told to expect cognitive problems resulted in patients having more positive self-worth. Patients expecting emotional changes were more sensitive to their impulsive behavior, often seen with depression, and suggesting less defensiveness. Patients told to expect more functional problems were more receptive to professional help. Findings underscore the importance of health professionals informing TBI patients and their families about the possibility of functional changes following brain injury, particularly involving cognition and emotions. Correspondence: Alison A. Fedio, PhD, PsyD, American School of Proessional Psychology, Argosy University, 1550 Wilson Boulevard, Suite 600, Arlington, VA 22209, United States. E-mail: afedio @argosy.edu

K. FRODSHAM, J. FAIR, R. FROST, S. MAJERCIK, J. BLEDSOE, D. RYSER, J. MACDONALD, R. BARRETT, S. HORN, D. PISANI, E.D. BIGLER, M. STEVENS, R. HOPKINS \& M. LARSON. Predicting Rehabilitation Outcomes Using Day-of-Injury Computed Tomography (CT): A Comparison Between Marshall and Rotterdam CT Scoring Criteria.

Objective: Accurate prediction of rehabilitation outcomes for patients with moderate-severe traumatic brain injury (M/S TBI) is elusive and there are few objective measures shown to be good predictors. One possible predictor of functional outcomes is pathology on day-of-injury (DOI) CT scans. The Marshall and Rotterdam systems are two ways to classify DOI CT pathology. We compared the relative predictive value of Marshall and Rotterdam scores for functional outcomes.

Participants and Methods: The study included 88 participants (25 females, mean age $42(\mathrm{SD}=21)$ years) with $\mathrm{M} / \mathrm{S}$ TBI. Two trained coders rated DOI CT images using both Marshall and Rotterdam criteria. Functional outcomes included rehabilitation length of stay and the cognitive and motor subscales on the Functional Independence Measure (FIM) at discharge from rehabilitation and 9-months post-discharge. Data were analyzed using separate multiple regressions. Independent variables included age, Glasgow Coma Scale (GCS) score, days from injury to admission to rehabilitation, and Marshall or Rotterdam score. Results: Older age was a significant predictor of worse scores on both acute FIM motor and FIM cognitive scores. Lower GCS score and greater number of days since injury both predicted a longer stay in rehabilitation. Marshall and Rotterdam scores overall were not significant predictors of functional outcome. A score of 5 on the Rotterdam and 6 on the Marshall (indicating high injury severity) both predicted worse acute FIM cognitive scores as compared to respective scores of 2 (low injury severity). Scores of 3 (compressed cisterns) and 6 (high) on the Marshall predicted longer stay in rehabilitation as compared to a score of 2 (low). None of the independent variables predicted 9-month outcomes. Conclusions: Overall, although a few of the high scores on both Rotterdam and Marshall scales predicted worse acute cognitive outcome, neither Rotterdam nor Marshall consistently predicted acute or 9-month functional outcomes for individuals with M/S TBI.

Correspondence: Kayla Frodsham, Brigham Young University, $138 \mathrm{~N}$ 900 E, Provo, UT 84606, United States.E-mail: kayla.alder14@gmail. com

K. GARCIA, D. PHAM, Y. CHOU, J. DSURNEY \& L. CHAN. White Matter Integrity and Memory Impairment in TBI.

Objective: Recent literature suggests that those with a traumatic brain injury (TBI) may be at risk for developing early-onset Alzheimer's disease (AD). Investigators have successfully utilized Diffusor Tensor Imaging (DTI) in AD to explore tracts involved in memory performance on the California Verbal Learning Test (CVLT-II). The present study aims to examine the relationship between DTI and CVLT-II performance in TBI patients.

Participants and Methods: Subjects were selected from a longitudinal TBI study. 12 participants who completed an MRI, and had impaired scores on the CVLT-II and one other neuropsychology test at one year post injury were selected for the present study. Memory was measured using CVLT-II short and long delayed free recall.

Five tracts were selected based on the literature and their implication in memory impairment in AD. DTI measures of fractional anisotropy (FA) were computed from diffusion-weighted images.

SPSS was used to perform t-tests and Pearson bivariate and partial correlations.

Results: There was a significant difference between subjects (Mean age: $52.29 \pm 18.20)$ with mild versus severe impairment on the CVLT-II for short delayed free recall, $\mathrm{t}(8)=3.77, \mathrm{p}=.005$. The corpus callosum (CC) posterior displayed a significant correlation with overall CVLT-II impairment, $r=.753, p=.012$, and when controlling for depression, further significance was shown.

Conclusions: Similar to the AD literature, we found that the CC posterior may have implications in memory impairment not caused by depression, therefore presenting as a potential ROI in a TBI population. To further determine if CVLT-II memory impairment in early-onset AD relates to the $\mathrm{CC}$ posterior as strongly as it did for TBI, this study should be completed alongside a mild, early-onset $\mathrm{AD}$ cohort. If the CC posterior is determined as a key region for memory impairment, it may serve as a marker for naturally occurring and TBI-caused early-onset AD. Correspondence: Katelyn Garcia, MD, United States. E-mail: katelyn. garcia@nih.gov

E.C. GROSSNER, E.K. BRENNER, R.A. BERNIER, J.S. HONG \& F.G. HILLARY. Frontal Network Connectivity Predicts Metacognitive Accuracy Following Moderate to Severe Traumatic Brain Injury.

Objective: Metacognition (MC) is a domain impacted by traumatic brain injury (TBI). Previous structural analyses with this data found gray matter volume (GMV) in frontal areas predicts MC accuracy differently in TBI vs. healthy control (HC) groups. This study built upon that work by examining functional networks associated with these prefrontal areas and their relationship to MC accuracy.

Participants and Methods: 21 TBI and 23 matched HCs underwent the same procedure, including BOLD fMRI scan and neuropsychological testing. The test battery included modified Matrix Reasoning tasks with retrospective confidence judgments. There were two versions of the task, one with increasing stimulus difficulty (ordered) and one with 
randomized stimulus difficulty (random). MC accuracy was quantified using an AUROC-defined curve. Network analyses were conducted using graph theory metrics based on a functional 264 atlas (Power, 2011). Results: Statistically significant interactions were found between head injury status and connectivity on $\mathrm{MC}$ accuracy for the random list within the default mode network ( $p=0.056)$, salience network $(p=0.024)$, and frontoparietal control network $(p=0.008)$. When examining functional networks based on the previous structural findings, there was an interaction between head injury status and connectivity on MC accuracy within the dorsolateral frontal network $(p=0.029)$. For all findings, higher network strength was associated with higher MC accuracy within the TBI group, but the opposite was found in the HC group.

Conclusions: Frontal GMV has been shown to predict MC accuracy. This study advances those findings by showing that responses in functional networks differentially predict MC in TBI and HC samples. Findings show increased network strength between networks related to MC was associated with better MC accuracy in the TBI group, but lower network strength was related to better accuracy in the HC group. This indicates that increased connectivity operates to resume network functioning in a disrupted system.

Correspondence: Emily C. Grossner, Psychology, The Pennsylvania State University, 650 Toftrees Ave., Apt. 212, State College, PA 16803, United States. E-mail:epg5130@psu.edu

E.K. BRENNER, E.C. GROSSNER, R.A. BERNIER, J.A. SOTO \& F.G. HILLARY. Racial and Ethnic Differences in Discharge Destination for Individuals with Traumatic Brain Injury in Pennsylvania.

Objective: Studies show discrepancies between how people of color (POC) receive trauma services compared to Whites. We examined the relationship between race and ethnicity on discharge destination (DD) after traumatic brain injury (TBI).

Participants and Methods: PA Trauma Outcome Study (PTOS) trauma services data from 2010-2016 was used. PTOS-defined racial groups included White, Black, Asian, and Other (American Indian, Alaskan Native, Asian/Pacific Islander); ethnicity was coded as either Hispanic or non-Hispanic. The ICD-10-CM codes used were: S802 (skull fracture) and S06 (intracranial injury). GCS scores were used to define moderate-severe TBI $(3-12 ; \mathrm{N}=13193)$ and mild TBI (mTBI) (13-15; N=65764). Payor types included were Medicare, Medicaid, Commercial, Third Party, and Self-Pay. All analyses controlled for differences in number of people within each racial/ethnic group.

Results: When examining differences in DDs for severe injuries, Whites were about as likely to be discharged to a rehabilitation center (RC) as any other group. For those with mTBI, Whites were $32 \%$ more likely to be sent to RC than Blacks, 24\% more likely than Asians, and 92\% more likely than the Other group. In ethnicity, non-Hispanics with TBI were $7 \%$ more likely to be discharged to RC than Hispanics, but within mTBI, they were $65 \%$ more likely to be sent to RC. Within TBI, non-Hispanics were $40 \%$ more likely to be discharged to a skilled nursing facility than Hispanics. In the mTBI group, non-Hispanics were 157\% more likely to be discharged there than Hispanics. Even when controlling for GCS and insurance type, race remained a significant predictor of DD, $\mathrm{p}=0.005$. Conclusions: These results highlight disparities in TBI treatment that covary with race and ethnicity. In mTBI, there is a large discrepancy in DD for different racial and ethnic groups; this was not seen in more severe injuries. Even when controlling for injury severity and insurance type, racial group is a significant predictor of DD.

Correspondence: Emily C. Grossner, Psychology, The Pennsylvania State University, 650 Toftrees Ave., Apt. 212, State College, PA 16803, United States. E-mail: epg5130@psu.edu
J. HAMMOND, A.A. HOMAECHEVARRIA \& D. HENDERSON. Promoting Helmet Use on the Ski Hill: Trends in Helmet Use Among Skiers and Snowboarders After Implementation of a Policy to Mandate Employee Helmet Use.

Objective: Nearly half ( $44 \%)$ of traumatic brain injuries sustained while skiing or snowboarding, approximately 7,700 brain injuries annually, could be prevented by helmet use. Yet, there are no major institutional recommendations for ski helmet use in the United States. This study examined trends in helmet use in the 2015/16 and 2016/17 ski seasons, after a ski resort mandated helmet use for employees between these seasons. Based on the view of ski patrollers and instructors as role models on the slopes, it was hypothesized that more participants (visitors to the ski hill) would wear a helmet if all employees wear helmets. Participants and Methods: Using an innovative data collection method, data (demographic factors, helmet use, injury characteristics) were entered by ski patrol immediately after an injury. In 2015/2016 season, 22 incidents were recorded (aged 13 - 62 years; $63.6 \%$ male, $36.4 \%$ female). In 2016/2017 season, 21 incidents were recorded (aged 12 - 81 years; $61.9 \%$ male, $38.1 \%$ female).

Results: Of persons involved in an incident that required ski patrol involvement, 77.3 percent wore a helmet in 2015/2016 season and 95.2 percent wore a helmet in 2016/2017 season. In 2015/2016, the highest rate of incidents was persons aged 40 to 49 years (45.5\%), with $70 \%$ helmet use among this age group. In 2016/2017, the highest rate of incidents was persons aged 11 to 20 years $(33.3 \%$ ), with $100 \%$ helmet use among this age group.

Conclusions: After implementation of a policy that mandated helmet use for employees at a ski hill, there was a trend of increased helmet use among participants. This research extends our understanding of ski helmet use behaviors and brain injury prevention, and warrants further investigation into the role of interventions on the ski hill to promote helmet use (e.g., interventions to reinforce the role of wearing a helmet as a professional responsibility). Furthermore, this research highlights the importance of interdisciplinary, innovative research in the area of injury prevention research and practice.

Correspondence: Julia Hammond, PhD, VA Northern California Health Care System, 10535 Hospital Way, Mather, CA 95655, United States. E-mail: juliahammondmt@gmail.com

J. HAMMOND, S. HALL, M. MCCARVEL, T. MARTIN \& E. CARROLL. Using the Transtheoretical Model of Behavior Change to Promote Bicycle Helmet Use: An Analysis Based on Stage of Change and Other Features.

Objective: Traumatic brain injury is a serious public health problem, and cycling represents the largest category of sports-related head injuries. Helmets can significantly lower the risk of brain injury for cyclists, yet minimal research exists to support application of a theory-based intervention for bicycle helmet use. This innovative study used a multidimensional model to apply Prochaska and DiClemente's Transtheoretical Model (TTM) of behavior change (Stages of Change, Decisional Balance, Self-Efficacy, Processes of Change) to examine bicycle helmet use in college-aged individuals, a high-risk age group.

Participants and Methods: A survey of bicycle helmet use behaviors and attitudes was administered to undergraduate students at two universities in the northwestern United States in 2015 and 2016 ( N=547).

Results: Of the total participants, $25.8 \%$ consistently wear a helmet while riding a bicycle. Compared to helmet non-wearers, helmet wearers indicated increased values placed on Pros of helmet use $(p<.005)$, increased confidence in one's ability to wear a helmet $(p<.005)$, and greater use of cognitive and behavioral processes of change $(p<.005)$. Significant associations between helmet use and demographic and bicycle riding behavior factors (older age, longer bike riding distance, commuting, history of bike accident that required medical attention) were identified.

Conclusions: This research demonstrates low base rates of bicycle helmet use in the college-aged population, and supports the application of the TTM to conceptualize helmet use. As predicted by the TTM model 
when applied to other health behavior change, persons who wear a bike helmet weigh the pros of helmet use higher than the cons, report greater confidence to wear a helmet than temptation to not wear a helmet, and actively engage in cognitive and behavioral processes to implement helmet-related behavior change. In a novel contribution to this area of study, this research provides meaningful information to modify and improve future helmet promotion interventions.

Correspondence: Julia Hammond, PhD, VA Northern California Health Care System, 10535 Hospital Way, Mather, CA 95655, United States. E-mail: juliahammondmt@gmail.com

T. HAN, A.T. KIRTLEY, T.F. BERGQUIST \& A.W. BROWN. Relationship Between Cognitive Complaints, Mood, Life Satisfaction, and Participation in a Traumatic Brain Injury Sample Presenting for Outpatient Rehabilitation.

Objective: To examine the relationship between self-reported cognitive symptoms, depression, life satisfaction, and participation in life activities for individuals with traumatic brain injury (TBI) at the time of admission to outpatient rehabilitation.

Participants and Methods: Participants were 46 patients with TBI who were admitted to interdisciplinary outpatient rehabilitation at a large Midwestern tertiary care medical center between September 2014 and May 2017 and had completed all measures at admission and discharge. Participants were $34.8 \%$ male, with an average age of $46.52( \pm 14.04)$ years, and an average of $14.91( \pm 2.84)$ years of education. Using the Mayo Classification System of TBI Severity, 34.7\% were classified as Possible TBI, $23.9 \%$ as Probable TBI, and $41.3 \%$ as Definite TBI. Time since injury was less than 3 months for $41.3 \%$, between 3 months and 2 years for $34.8 \%$, and greater than 2 years for $23.9 \%$ of patients. Correlation analyses were performed to examine the relationship between cognitive symptoms endorsed on the Neurobehavioral Symptom Inventory, and total scores on the Patient Health Questionnaire, Satisfaction with Life Scale, and the Participation Index of the Mayo-Portland Adaptability Inventory.

Results: Higher self-reported cognitive symptoms were significantly associated with higher depression $(r=.675, p<.001)$, lower life satisfaction $(r=-.451, p<.005)$, and lower participation $(r=.433$, $p<.005)$ at admission to outpatient rehabilitation.

Conclusions: Patients' subjective cognitive symptoms are strongly related to their experiences of depression, life satisfaction, and participation in daily or valued activities. These results support the need for comprehensive assessment that evaluates cognitive symptoms, as well as mood and other factors that impact quality of life in persons presenting for treatment. Results also support the value of an interdisciplinary model of treatment for persons with TBI.

Correspondence: Thida Han, Psy.D., Psychiatry \& Psychology, Mayo Clinic, 722 W Center St, Apt 202, Rochester, MN 55902, United States. E-mail: han.thida@mayo.edu

R. HANKS, S. MILLIS, S.E. SCOTT, R. GATTU, N. O'HARA, M. HAACKE \& Z. KOU. Prediction of Cognitive Outcomes from Diffusion Tensor Imaging in Traumatic Brain Injury.

Objective: To investigate the predictive validity of global and regional white matter fractional anisotropy (FA) values following traumatic brain injury with regard to cognitive outcome.

Participants and Methods: Adults who were healthy controls $(\mathrm{n}=18)$ or had a traumatic brain injury $(\mathrm{n}=27)$ underwent diffusion tensor imaging using a Siemens VERIO 3T scanner and calculation of global and regional FA values were undertaken. FA values were correlated with neuropsychological test scores (Symbol Digit Modalities Test, Trailmaking Test, Wechsler Test of Adult Reading, California Verbal Learning Test 2nd Edition, Digit Vigilance Test, and Wisconsin Card Sorting Test) and injury severity variables. Logistic regression and ROC curve analysis were used to investigate discriminative ability of the $\mathrm{FA}$ values.
Results: Within the traumatic brain injury group, FA values were significantly lower across brain regions compared with controls; global FA and five brain regions were associated with Symbol Digit Modality scores. The FA value within the body of the corpus callosum yielded excellent discrimination between groups.

Conclusions: Convergent findings supported the predictive validity of the corpus callosum body FA value following traumatic brain injury and provides further evidence for the clinical utility of DTI in deep white matter structures after moderate to severe TBI.

Correspondence: Robin Hanks, Rehabilitation Institute of Michigan, 261 Mack Blvd, Suite 555, Rehabilitation Institute of Michigan, Detroit, MI 48201, United States.E-mail: rhanks@med.wayne.edu

A.L. HARMELL, M. AMAYA, M. GROSS, K. YAFFE \& A.R. KAUP. Subjective Cognitive Complaints among Older Veterans with Traumatic Brain Injury are Related to PTSD Symptoms.

Objective: Findings suggest traumatic brain injury (TBI) may have long-term cognitive effects in aging. However, it has also been suggested that cognitive difficulties among individuals with remote TBI may be related to psychiatric symptoms. In older Veterans with history of TBI and current cognitive concerns, we investigated correlates of subjective cognitive complaints including associations with neuropsychological performance and psychiatric symptoms.

Participants and Methods: Participants were 39 Veterans (ages 60-84, 95\% male) in a pilot cognitive training trial, the Brain Aging in Veterans (BRAVE) Training study. Eligibility criteria included history of multiple mild TBIs or $\geq 1$ moderate TBI and current cognitive concerns. At baseline, participants completed a self-report inventory assessing extent of subjective cognitive complaints (Neuro-QoL Cognitive Function, lower score reflects worse functioning), a neuropsychological battery, the PTSD Checklist (PCL-C), and Geriatric Depression Scale Short Form (GDS). Using t-test and correlation analyses, we investigated associations between Neuro-QoL score, age, TBI number and severity, neuropsychological performance (global and domain composite z-scores), and psychiatric symptoms.

Results: Among older Veterans with TBI and current cognitive concerns, Neuro-QoL scores were associated with PCL-C scores $(r=-.37$, $p=.02$ ), such that subjective cognitive complaints were greatest among those with higher PTSD symptoms. There were no associations between Neuro-QoL scores and age, TBI severity, or neuropsychological performance (all $p>.10$ ). Associations between Neuro-QoL scores and number of TBIs $(r=-.27, p=.10)$ and GDS scores $(r=-.30, p=.07)$ were non-significant though suggestive of possible trends.

Conclusions: Subjective cognitive complaints among older Veterans with TBI are associated with higher PTSD symptoms, suggesting PTSD symptoms may be driving, or exacerbating, cognitive complaints in such individuals.

Correspondence: Alexandrea L. Harmell, Ph.D., Mental Health Service, San Francisco VA Healthcare System, 4150 Clement Street, San Francisco, CA 94121, United States.E-mail: aharmell@ucsd.edu

K.A. HOLIDAY, A.L. CLARK, S.F. SORG, M.J. WALSH, J. STROM, L. DELANO-WOOD \& D. SCHIEHSER. Hippocampal Volume Independently Predicts Subjective Memory Complaints in Mild Traumatic Brain Injury.

Objective: Veterans with mild traumatic brain injury (mTBI) frequently report memory deficits, which are not always supported by objective neuropsychological testing and are often related to psychiatric symptoms, such as PTSD. Given that neuroimaging allows for examination of possible neural correlates of subjective memory complaints, the objective of our study was to examine the relationship between hippocampal volumes, objective memory performance, and PTSD symptoms on subjective memory complaints in Veterans with history of mTBI.

Participants and Methods: 64 Veterans with mTBI and optimal effort completed tests of objective verbal and visual memory (WMS-IV Logical 
Memory and Visual Reproduction), subjective memory (NSI-14), the PTSD Checklist, and structural MRI. FMRIB's Software (FSL) was used to obtain hippocampal as well as intracranial volume (ICV) estimates. Two hierarchical regression analyses were conducted to examine whether right or left hippocampal volume, visual or verbal memory performance, and/or PTSD symptoms predicted subjective memory complaints controlling for ICV and age.

Results: Regression analyses demonstrated that although objective memory performance was not associated with subjective memory complaints, left hippocampal volume was significantly and independently associated with subjective complaints $(p=.001)$, with smaller volumes relating to greater complaint severity; results revealed a similar trend for the right hippocampal volume $(\mathrm{p}=.057)$. As expected, PTSD strongly predicted subjective memory complaints $\left(p^{\prime} s<.001\right)$.

Conclusions: In mTBI Veterans, subjective memory complaints are best accounted for by PTSD symptoms and hippocampal volume, but not objective performance. Importantly, smaller hippocampal volumes are independently associated with greater subjective memory severity, suggesting a neural basis of memory complaints in mTBI not accounted for by PTSD or objective memory performance. Future research is needed to elucidate the behavioral manifestations of subjective memory complaints in mTBI.

Correspondence: Kelsey A. Holiday, M.S., Psychology, SDSU/UC San Diego Joint Doctoral Program in Clinical Psychology, 3350 La Jolla Village Dr., Mail Code: 151-B, San Diego, CA 92161, United States. E-mail:kelsholiday@gmail.com

G. HROMAS, M. JAFFEE, C. DEMMING, S.M. GREIF, A. HAUSMAN, A. KRAUS, K. PLATEK, H. SIMPSON, S. SUTTON, L. FALK \& R.M. BAUER. The effect of ocular convergence insufficiency following traumatic brain injury on performance during a computerized neuropsychological screener.

Objective: Traumatic brain injury (TBI) can result in a number of oculomotor sequelae. Abnormal near point conversion (NPC), or convergence insufficiency (CI), is one such problem that has been found to affect as many as $40 \%$ of patients with a history of TBI. CI is a binocular condition in which eyes become extrophoric when viewing near objects. Since CI has previously been linked to poorer performance on neuropsychological measures, particularly measures that rely on reaction time, it may represent a possible confound in cognitive evaluations. However, recent conceptualizations of concussion "subtypes" raise the possibility that CI and neuropsychological dysfunction may operate as at least partially independent symptoms of TBI. This study evaluated whether the presence of CI predicts performance on neuropsychological tests of reaction time and RT-dependent measures of simple and complex attention.

Participants and Methods: 41 patients (22 females) with a history of TBI were evaluated at a multidisciplinary clinic at the University of Florida. Of these patients, $16(39 \%)$ had CI, defined as NPC $>5 \mathrm{~cm}$. NPC was measured by multiple specialists. Patients were also assessed via a brief neurocognitive screener (CNS Vital Signs).

Results: MANOVA results do not support the hypothesis of interference of CI on neuropsychological performance. Patients with normal NPC did not perform significantly better on measures of reaction time, complex attention or simple attention $(p>0.1)$. When treated as a continuous variable, CI explained a non-significant amount of variance in overall neuropsychological performance $\left(r^{2}=0.036, p>0.1\right)$.

Conclusions: Results lend support for the conceptualization of concussion subtypes, as CI did not have a significant effect on neuropsychological variables. Thus, ocular and cognitive symptoms may be part of independent clusters of TBI symptoms. It is also possible that results were affected by the wide range of ages and varying time since injury in this patient population.

Correspondence: Gabrielle Hromas, University of Florida, $4341 \mathrm{Nw}$ 49th St. Unit 103, Gainesville, FL 32606, United States. E-mail: galtomare@ufl.edu
G.L. IVERSON, E. CLARK, D.P. TERRY, M. IACCARINO, E. BUI, R. ZAFONTE \& M. HARVEY. A Two-Week Intensive Clinical Program for Veterans with Traumatic Brain Injury and Psychological Health Problems.

Objective: Many post-9/11 veterans experienced prior traumatic brain injuries (TBI) and current posttraumatic stress disorder (PTSD), depression, cognitive concerns, bodily pain, headaches, and insomnia. The Intensive Clinical Program (ICP) at Home Base provides an innovative model of care for 2 weeks. The TBI track intervention integrates evidence-based behavioral health care, cognitive rehabilitation, rehabilitative medicine, vestibular therapy, mindfulness training, wellness, integrative therapies, and family education. This study examines treatment outcomes from the first 28 veterans through this new program. Participants and Methods: Most participants experienced multiple exposures to mild TBIs and had PTSD symptoms (100\%), depression $(57 \%)$, sleep difficulty $(89 \%)$, chronic bodily pain $(82 \%)$, chronic headaches $(81 \%)$, and vestibular problems $(81 \%)$. Before and after the treatment program, veterans completed measures of post-concussion symptoms (Neurobehavioral Symptom Inventory; NSI), traumatic stress (PTSD Checklist), depression (Patient Health Questionnaire-9), satisfaction with social functioning (PROMIS Satisfaction with Participation in Social Roles) and cognitive self-efficacy (Self-Efficacy for Cognitive Management Scale).

Results: There was a statistically significant reduction in NSI cognitive $(p<.001$, Cohen's $d=.93)$, affective $(p<.001, d=.78)$, and somatic symptoms $(p=.01, d=.45)$. Similarly, there were significant reductions in traumatic stress $(\mathrm{p}<.001, \mathrm{~d}=.64)$ and symptoms of depression $(\mathrm{p}<.001$, $\mathrm{d}=.64)$, and large improvements in ratings of cognitive self-efficacy $(\mathrm{p}<.001, \mathrm{~d}=1.00)$ and participation in social roles $(\mathrm{p}<.001, \mathrm{~d}=1.05)$. Program satisfaction ratings were high.

Conclusions: Preliminary findings indicate that veterans experienced statistically significant and clinically meaningful improvements in post-concussion symptoms, traumatic stress, and depression. The magnitude of the effect sizes illustrates the usefulness of an intensive multi-modal treatment in this population.

Correspondence: Grant L. Iverson, MA, United States.E-mail: giverson@ mgh.harvard.edu

P.A. JANULEWICZ, M. KRENGEL, T. HEEREN, E. SISSON, N. KLIMAS, S. LEA, R. DENSON, R. TOOMEY, R. KILLIANY, J. O'CALLAGHAN, J.A. AJAMA \& K. SULLIVAN. Self- Reported Mild Traumatic Brain Injury (mTBI) and Toxicant Exposures During the 1991 Gulf War and Gulf War Illness: The Boston Gulf War Illness Consortium.

Objective: The Gulf War Illness Consortium (GWIC) focuses on identifying objective biomarkers of Gulf War Illness (GWI) in 1991 GW veterans $(\mathrm{GWV})$. Symptoms of GWI include fatigue, chronic pain and cognitive problems. Exposures to pesticides, sarin and prophylactic pills during the war have been identified as likely contributors to GWI. Previous studies with other GWV cohorts suggest that a history of traumatic brain injury is associated with increased current self-reported health symptoms and GWI. The objective of this study was to examine the association between self-reported history of mTBI, toxicant exposures and GWI in the GWIC cohort.

Participants and Methods: Participants included 92 deployed GWV, $73 \mathrm{GWI}$ cases and 19 healthy controls that did not differ by age, sex, or education. The mean age was 50 years with 15 years of education. GWV self-reported occurrence of mTBI sustained before, during, and/or after the war in the GWIC surveys. GWI was defined using the Kansas Criteria.

Results: Cases and controls were similar in self-reported history of mTBIs that occurred before the war (cases $39.7 \%$, controls $36.8 \%$; $\mathrm{p}=0.82$ ). There was a statistically significant difference in self-reported mTBI between the cases and control that occurred during the war mTBIs (cases $42.5 \%$ yes, controls $0 \%$; $=0.0005$ ). Cases also self-reported slightly higher but non-significant percent of mTBI after the war (cases $28.8 \%$, controls $10.5 \%, p=0.10)$. Significantly more cases also reported 
exposures to chemical/ biological warfare agents and pesticides during the war.

Conclusions: In this GWV cohort, there was a higher percent of self-reported mTBIs and neurotoxicant exposures in GWI cases compared to controls. This was particularly striking for mTBIs sustained during this relatively brief war. These results suggest that mTBI should be examined further as a potential contributor to the development of GWI and the interaction between mTBI and neurotoxicant exposures should be evaluated further.

Correspondence: Patricia A. Janulewicz, DSc, Environmental Health, Boston University School of Public Health, 715 Albany Street, Boston, MA 02118, United States.E-mail: paj@bu.edu

R. KANSER, L.J. RAPPORT, B. VERMILION, M. DE IORIO, J. BASHEM \& R. HANKS. Utility of WAIS-IV Digit Span Indices as Measures of Performance Validity in Complicated-Mild to Severe Traumatic Brain Injury.

Objective: Reliable Digit Span (RDS) and Age-Corrected Scaled Scores (ACSS) derived from the WAIS-III and WAIS-R are among the most studied embedded measures of performance validity. The addition of Sequencing to WAIS-IV Digit Span (DS) led to creation of new RDS indices, RDS Alternate (RDS Forward + RDS Sequencing) and RDS Enhanced (RDS Forward + RDS Backward + RDS Sequencing), as well as ACSS that includes Sequencing trials. Evidence suggests the new ACSS and RDS indices outperform the traditional RDS at distinguishing adults simulating brain injury from healthy adults providing full-effort; however, the new indices have not been tested on adults with verified neurocognitive impairment. This study compared classification accuracies of DS indices in a design that included adults with verified TBI. Participants and Methods: Participants included 53 adults with complicated-mild to severe TBI (TBI), 46 healthy adults coached to simulate TBI (SIM), and 70 healthy adult comparisons (HC). Participants completed the WAIS-IV DS subtest in the context of a larger test battery

Results: Mixed-design ANOVA indicated that all DS indices (except RDS Backwards) differed significantly across the three groups, with medium to large effect sizes. Post hoc analyses revealed that only RDS Forward and the traditional RDS differed significantly between SIM and TBI. A significantly greater percentage of TBI scored RDS Sequencing $<1$ as compared to SIM and HC. ROC curve analyses indicated that the traditional RDS was the best predictor of group status (i.e., SIM vs. TBI and SIM vs. HC).

Conclusions: Caution should be used when using new DS performance validity measures with individuals with complicated-mild to severe TBI. The Greiffenstein et al. (1994) traditional RDS appears to be the best DS index of performance validity in this clinical context. Findings highlight the importance of including individuals with verified TBI when evaluating and developing performance validity measures.

Correspondence: Robert Kanser, MA, Wayne State University, 44342 nd ave, Detroit, MI 48201, United States.E-mail: robert.kanser@wayne. edu

\footnotetext{
A.R. KAUP, M. AMAYA, M.S. GROSS, D.E. BARNES \& K. YAFFE. Feasibility of Mobile Cognitive Intervention among Older Veterans with Traumatic Brain Injury: Preliminary Findings from the BRAVE Training Study.

Objective: Traumatic Brain Injury (TBI), common in military personnel, appears to be a dementia risk factor, suggesting need for intervention among older Veterans with TBI. We investigated feasibility of iPadbased mobile cognitive intervention in older Veterans with TBI.

Participants and Methods: The Brain Aging in Veterans (BRAVE) Training study is a pilot cognitive intervention trial for older Veterans (ages 60-85) with history of multiple mild TBIs or $\geq 1$ moderate TBI and current cognitive complaints. Target enrollment is 90 Veterans. After baseline assessment, participants are randomized to 1 of 3 groups for 4 weeks. Groups 1 and 2 play an iPad game at home, either a multitasking
}

game [Project:Evolution (EVO) Multi] or word game (EVO Words), for about 25 minutes a day, $\geq 5$ days a week. Group 3 is a "no game" control group. Outcome assessment is completed at post-test and 3 -month follow-up visits. We examined adherence to game play (\%) and acceptability ratings (calculated as \% rated agree/strongly agree on 5-point Likert items).

Results: To date, we have enrolled 39 Veterans [mean age $=70.6$ ( $\mathrm{SD}=6.6), 95 \%$ male]. Of 38 randomized, 9 are active, 24 fully completed, 3 completed the post-test visit but not follow-up visit (loss to follow-up / withdrawn), and 2 were withdrawn prior to post-test. Most participants evidenced strong adherence to game play $(75 \%$ of EVO Multi participants and $100 \%$ of EVO Words participants were $\geq$ $90 \%$ adherent). Acceptability rating results included that most participants enjoyed their game (82\% of participants in EVO Multi, $91 \%$ in EVO Words; no group difference: $p>.9$ ), while ease of use tended to be higher for EVO Words than EVO Multi (100\% vs. $64 \%$ of participants agreed that game/iPad were easy to use, $p=.09)$.

Conclusions: Mobile cognitive intervention on iPads appears feasible among older Veterans with TBI. Results suggest mobile technology tools can be utilized to deliver interventions even among complex older adult populations.

Correspondence: Allison R. Kaup, PhD, Research / Psychiatry, San Francisco VA Healthcare System / University of California San Francisco, 4150 Clement St, 116B, San Francisco, CA 94121, United States.E-mail: allison.kaup@ucsf.edu

WITHDRAWN. S. KEY-DELYRIA \& C.M. MOSTELLER. Using Cognitive Profiles in Individuals With and Without Mild ClosedHead Injury to Predict Sentence Comprehension Performance. Objective: This study examines the relationship between cognitive task measures and sentence comprehension measures in individuals with and without mild elosed-head injury (mCH) history. Analyses foeused on eognitive funetions theorized as neessary to eomplete a sentence emprehensiontask: short-term and working memory (Digit Span), exeeutive ftnetioning and eognitive control (Stroop task), as well as sustained and selective attention (Trails Haking Task). Tasks were used to eompite a cognitive profite of the individtual then compared with results from a sentence proeessing task. By comparing these findings, we expect seores to correlate with sentence comprehension response

Partieipants and Hethods. Preliminary data om 4 thdividtals with mCHH and 4 control participants were deo for eomparison. Partieipants completed a series of gnith tasks that have been elinieally used to meastre jurt th the hort-term and working mentry, exective funter om to, mut attention. A computerized sentence eomprehens on as was administered to examine processing and rom reh, nston of ambigttous, unambiguous, and controlledsento ces

Results. 9. Ill affferences in profite performances were noted between giv tips on the Stroop task, Digit Span, and Trails A and B. Sentenee comprehension results inelude comprehension aectraey, PT, and readling time.

Gonclusions: This study looks at eognitive performanee and sentence processing for individuals with a history of mCHH and a control group. Sentence processing ant eomprehension require a myriat of ecgnitive skills. Variability of these resourees ean be attributed to the differenees in eognition and processing for both those with mCHI and without. Using an individual's cognitive profite to prediet sentence processing performance coutd improve the way elinieally relevant communieation defieits can be identified.

Gorrespontence. Sarah Key-DeLyria, Ph.D., Speech and Hearitry Sciemes, Porthand State University, POB Box 751 (SPHII), Porthand State University,Portland, OR 97207, United States.E-mait:keydel@pdx.edu 
W.D. KILLGORE, B.R. SHANE, J.R. VANUK, M. MILLAN, S. KNIGHT \& S. BAJAJ. Blue Light Therapy Accelerates Brain and Cognitive Recovery from Mild Traumatic Brain Injury.

Objective: Mild traumatic brain injury (mTBI) is correlated with persistent problems with sleep and fatigue in up to $50 \%$ of patients. We hypothesized that daily morning blue light exposure therapy would re-entrain the circadian rhythm, leading to improved overall sleep quality, and this would facilitate brain repair and cognitive performance. Participants and Methods: Patients who experienced an mTBI during the preceding 18 months ( $\mathrm{n}=28,15$ female; aged 18-48 years) underwent a comprehensive neuropsychological assessment and multi-modal neuroimaging. In a double-blind design, participants were randomly assigned to complete daily morning exposure with a light device fitted with an array of either blue $(n=14)$ or amber (placebo; $n=14$ ) wavelength light emitting diodes. The device was used at home for 6-weeks (30-minutes per day, prior to 11:00am). Participants returned for follow-up assessment and imaging at the end of treatment.

Results: Blue light led to an earlier bedtime, rise time, reduced daytime sleepiness, and improved balance compared to placebo light $(\mathrm{p}<.05)$. Structural magnetic resonance imaging (MRI) revealed that active bluelight treatment was associated with increased volume of the pulvinar nucleus bilaterally ( $p<.05$, FWE corrected), but not for the amber placebo condition. Blue light increased functional connectivity and integrity of white matter axonal pathways connecting the pulvinar to parietal regions compared to placebo ( $\mathrm{p}<.05$, FWE corrected), which correlated with improved neurocognitive performance.

Conclusions: Regular morning exposure to blue-wavelength light for 6-weeks led to improved sleep and associated modifications of thalamo-cortical structure, connectivity, and function relative to amber placebo exposure. These preliminary findings suggest that blue-light may improve fatigue by regulating sleep-wake schedules and suggest that light therapy may provide a novel method for improving recovery from some aspects of mTBI.

Correspondence: William D. Killgore, PhD, Psychiatry, University of Arizona, 1501 N. Campbell Ave, 7303B, Tucson, AZ 85724, United States.E-mail:killgore@psychiatry.arizona.edu

V. LA CORTE, L. MAZZOLINI, M. ABRAM, M. LUNVEN, C. EMMANUEL, A. BELLEGARDE \& P. PIOLINO. Neuropsychological rehabilitation for prospective memory deficits in traumatic brain injury: a new ecological treatment in virtual reality.

Objective: Prospective memory (PM) refers to our ability to remember to fulfil intentions at some point in the future. PM is one of the most disabling forms of memory impairments experienced by people with traumatic brain injury (TBI).

A recent systematic review pointed out a variety of interventions focused on PM deficits including compensatory deficits and remediation strategies aimed at improving the self-monitoring of personal goals. (Mahan 2017).

Nevertheless generalization is often a major problem in rehabilitation effectiveness (Wilson et al. 1987). The use of Virtual reality (VR) is proposed to be a possible means to achieve this goal. VR is an interactive computer technology that can create the illusion of being in an artificial world. The efficiency of VR has been shown in cognitive assessment and in cognitive remediation (Rizzo et al. 2004).

The main objective of the present study was to develop a novel PM ecological program using VR.

Participants and Methods: The complete training included 15 separate sessions including two first sessions focused on planning and orientation capacities, 6 sessions focused on the learning of strategies to facilitate encoding of the intentions, 6 sessions centered on the retrieval strategies, and a last session was dedicated to the training of attentional processes allowing to execute a double task. Here we present a TBI patient who completed our ecological program. The patient DY was a 25 years old man who had a severe TBI in $2015($ GCS $=6)$ due to a car accident. Before the treatment patient showed a severe amnesia with executive impairment and a deep anosognosia.

Results: After the treatment neuropsychological assessment reveal a significant increase of episodic memory capacities as well as a reduction of anosognosia in particular for PM deficits.

Conclusions: These preliminary findings suggest that VR-based training may be well accepted by severe TBI patients as encouraging improvement has been shown. Large-scale study with this novel ecological program seems indicated.

Correspondence: Valentina La Corte, Ph.D, Université Paris Descartes, 1Institute of Psychology, University Paris Descartes, Sorbonne Paris Cite, France, 71 avenue Edouard Vaillant, 92774, Boulogne 92774, France.E-mail: valentina.lacorte@gmail.com

L.M. LANGAN, N. KATZ, K. COULEHAN, K. RAM JUNNAKAR, M. CORNWELL, A. DESOLLAR-HALE, S. MANDELBAUM, L. MARCUSE, S. GHATAN \& H.A. BENDER. Refining Test Batteries Appropriate for Patients with Craniocerebral Gunshot Wounds Undergoing Pre-Surgical Assesements.

Objective: Craniocerebral gunshot wounds (CGSW), can result in medically refractory epilepsy necessitating surgical intervention. Given the importance of neurocognitive metrics in such cases, identification of brief, comprehensive test batteries for this unique population is key. We present a case series of two patients and discuss the role test data played in surgical decision making.

Participants and Methods: Patient one (P1) had a right parietal entry and patient two (P2) a left frontal CGSW. Post-GSW complications for P1 included three month coma, motor deficits, and reduced ADLs; EEG and MRI findings revealed greater right hemisphere slowing and right frontal encephalomalacia. Post-GSW complications for P2 included two-week coma followed by left frontal seizure onset; neuroimaging revealed a left inferior frontal hemorrhage. Both patients underwent similar assessments of attention, executive functioning, visuospatial skills, language, learning, memory and motor functioning. They were easily fatigued, required frequent breaks and were emotionally labile. Results: P1's neuropsychological profile was indicative of global impairment with greatest deficits in visuospatial skills and processing speed. P2's data indicated isolated weaknesses in attention and executive functioning (EF).

Conclusions: Resection was contraindicated given P1's global neuropsychological dysfunction, absence of clear localization or lateralization on testing and bilateral seizure onset on EEG. A more conservative approach of responsive neurostimulation was completed. In contrast, due to P2's EF weaknesses upon testing and localization of seizure onset, a frontal lobe resection was performed, maximizing potential seizure freedom. Batteries that can be completed in a single, short testing session are needed given the attentional variability and poor vigilance often seen in populations with CGSWs. Tests with the strongest potential for localization and lateralization should be prioritized, as should assessments of executive dyscontrol pre-/post-CGSW.

Correspondence: Lawrence M. Langan, Icahn School of Medicine at Mount Sinai, 23 Joyce Lane, Staten Island, NY 10307, United States. E-mail: llangan1@jhu.edu

S.M. LIPPA, R. LANGE, T. BRICKELL, L.M. FRENCH \& J. GILL. The Relationship between Plasma Amyloid and Neurocognition Following Mild Traumatic Brain Injury: A Longitudinal Analysis. Objective: Amyloid-beta 42 (A $\beta 42$ ) plaques have previously been associated with cognitive impairment and reduced performance on cognitive tasks in a variety of patient samples, including patients with a history of mild traumatic brain injury (MTBI). However, it is unclear whether plasma $A \beta 42$ is related to objective neurocognitive performance. This study aimed to examine the relation between levels of $A \beta 42$ and neurocognitive functioning in military service members following MTBI.

Participants and Methods: Participants were 49 U.S. military service members (Age $\mathrm{M}=30.9$ vears, $\mathrm{SD}=9.0$; Education $\mathrm{M}=14.3$ vears. 
$\mathrm{SD}=2.1 ; 98.0 \%$ Male; $87.8 \%$ White) who sustained an MTBI and passed performance validity tests. Participants completed a baseline assessment at Walter Reed National Military Medical Center including 22 neurocognitive measures and a blood draw 2-12 months post-injury $(\mathrm{M}=5.0, \mathrm{SD}=3.3)$. In addition to investigating individual neurocognitive measures, composite scores were formed for six domains (Attention, Processing Speed, Immediate Memory, Delayed Memory, Executive Functioning, and Visual Spatial) by averaging standard scores on four individual measures (the Visual Spatial composite score was comprised of only two measures). Twenty-two participants also completed a follow-up evaluation an average of 12.3 months post-injury ( $\mathrm{SD}=2.9)$. Amyloid-beta 42 levels were obtained using an ultra-sensitive immuneassay. Given the number of comparisons, a cutoff of $p<.01$ was used. Results: At baseline, $A \beta 42$ levels were not significantly related to any neurocognitive measures or composite scores (all $\mathrm{R}^{2}<.199$, all ps>.01). In the longitudinal sub-group, baseline $A \beta 42$ was similarly not significantly predictive of any individual or composite neurocognitive measures at follow-up (all $\mathrm{R}^{2}<.229$, all ps>.01).

Conclusions: There does not appear to be an association between A $\beta 42$ levels and neurocognitive performance following MTBI. A larger sample size is needed to confirm this lack of association.

Correspondence: Sara M. Lippa, PhD, Walter Reed National Military Medical Center, Walter Reed National Military Medical Center, Bethesda, MD 20889, United States. E-mail: saralippa@gmail.com

S.M. LIPPA, J. GILL, T. BRICKELL, L.M. FRENCH, T.R. OAKES, P. YEH \& R. LANGE. The Relationship between Plasma Tau and Amyloid and Diffusion Tensor Imaging Following Mild Traumatic Brain Injury.

Objective: This study aimed to examine the relation between plasma levels of tau and amyloid-beta $42(\mathrm{~A} \beta 42)$ and white matter integrity in military service members following mild traumatic brain injury (MTBI). Participants and Methods: Participants were 45 U.S. military service members (Age $\mathrm{M}=32.2$ years, $\mathrm{SD}=8.9$; Education $\mathrm{M}=14.7$ years, $\mathrm{SD}=2.3 ; 88.9 \%$ Male) who sustained an MTBI. Participants completed a blood draw and Diffusion Tensor Imaging of the whole brain, an average of three years post-injury ( $M=37.4$ Months, $\mathrm{SD}=35.3)$. Measures of fractional anisotropy (FA) and mean diffusivity (MD) were calculated across 18 regions of interest (ROIs). Plasma tau and $A \beta 42$ levels were obtained using an ultra-sensitive immune-assay. Given the number of comparisons, a cutoff of $\mathrm{p}<.01$ was used.

Results: Pearson correlations were run to identify significant relationships between demographics (i.e., age, education, gender, time since injury) and DTI metrics. Some demographics were significant predictors of $\mathrm{FA}$ in five ROIs (i.e., left infracallosal bundle, right inferior longitudinal fasciculus, left superior longitudinal fasciculus (LF) parietal bundle, and left and right supracallosal bundles) and MD in five ROIs (i.e., left uncinate fasciculus, left infracallossal bundle, left and right supracallosal bundles, and right corticospinal tract). To determine whether plasma tau and/or $A \beta 42$ is related to FA or MD in each of the 18 ROIs, a series of hierarchical regression analyses were conducted with significant demographic predictors entered in the first step, and tau and A $\beta 42$ entered in the second step. After controlling for relevant covariates in the first step, tau and $A \beta 42$ levels were not significant predictors of $\mathrm{FA}$ and MD values for any of the $18 \mathrm{ROIs}$ (all $\Delta \mathrm{R}^{2}<.136$, all ps>.01). Conclusions: There does not appear to be an association between plasma tau or A $\beta 42$ levels and white matter integrity three years following MTBI. A larger sample size is needed to confirm this lack of association.

Correspondence: Sara M. Lippa, PhD, Walter Reed National Military Medical Center, Walter Reed National Military Medical Center, Bethesda, MD 20889, United States. E-mail: saralippa@gmail.com
W. LOPEZ, F. MARTINEZ, E. WAITE, A. BICHLMEIER, D.J. HARDY, E. WOO, C. WANG, R. SWERDLOFF, D.F. KELLY, K.M. GUSKIEWICZ, R. CANTU \& M.J. WRIGHT. Memory Process Deficits in Retired Professional Football Players.

Objective: To determine the profile of memory process deficits in retired processional American football players.

Participants and Methods: Twenty-nine retired, professional, American football players (RFB) with a history of sports concussion and 20 healthy control participants. All participants provided voluntary consent to an IRB approved study that included the administration of the California Verbal Learning Test, Second Edition (CVLT-II). All participants passed performance validity testing. Also, the Item Specific Deficit Approach was applied to the CVLT-II to obtain reliable and valid indices of encoding, consolidation, and retrieval deficits.

Results: While the groups were matched in terms of sex, education, and race, the RFB group was significantly older than the control group. T-tests revealed that the RFB group demonstrated poorer learning $(p=.009)$, short delay free recall $(p=.003)$, long delay free recall $(\mathrm{p}=.038)$, and recognition $(p=.026)$ in contrast to controls on demographically corrected scores on the CVLT-II. Also, the RFB group showed significant consolidation deficits $(p=.005)$ in contrast to controls on age corrected ISDA indices. A follow up regression revealed that consolidation deficits in the RFB group significantly predicted their delayed recall $[F(1,18)=5.30, p=.03 ; b=-.30, t(18)=-2.30, p=.03]$, accounting for $23 \%$ of the variance.

Conclusions: RFB with a history of sports concussion demonstrate significant memory deficits in contrast to healthy controls. Further, RFB's memory process deficit profile is dominated by consolidation problems. This contrasts with previous work with chronic complicated mild and moderate-to-severe traumatic brain injury survivors who show primary encoding deficits and secondary consolidation deficits. It is possible that the different pattern of memory deficits between RFBs and other TBI groups is due to varying pathologies (e.g.. chronic traumatic encephalopathy in the RFB group vs. typical acceleration-deceleration injuries in other TBI groups).

Correspondence: Walter Lopez, CA, United States. E-mail:wdlopez31@ gmail.com

D.E. MARRA, M.E. NITTA, E. VOGT, K.A. RITCHIE \& J. HOELZLE. Re-analyzation of Belanger and Colleagues' (2005) mTBI MetaAnalysis via Meta-Regression.

Objective: There is considerable variability in the neuropsychological recovery from a mild traumatic brain injury (mTBI). Belanger and colleagues' (2005) meta-analysis shed light on factors that modify recovery. The primary objective of this study was to further investigate Belanger's original data employing a contemporary meta-analytic technique, meta-regression, to hold constant and quantify the effects of modifiers.

Participants and Methods: Data were retrospectively extracted from Belanger and colleagues' (2005) meta-analysis. The primary outcome measure was mean overall effect size difference (Cohen's $d$ ) of neuropsychological functioning between healthy controls and individuals post-mTBI from each study $(k=39)$. Mean time since injury $\left(M_{\text {Time }}\right)$ and sample selection (SS; litigation vs. non-litigant) were entered into a random-effects meta-regression.

Results: Visual inspection of a funnel plot suggested "publication bias." Prior to excluding outliers, the meta-regression revealed that neither $M_{\text {Time }}$ or SS were significant moderators of the overall effect size $(p$ 's $>.05)$. Seven studies were identified as outliers and were excluded from subsequent analyses. After excluding outliers $(k=32$; overall $d=.62$ ), funnel plots suggested the publication bias was largely resolved. A subsequent meta-regression $\left[y=.729-.021\left(M_{\text {Time }}\right)+.006\right.$ (SS)] revealed that $M_{\text {Time }}$ was a significant effect modifier $(p<.01)$, but SS, after removing outlying studies, was not. However, much of the between-study variability remained unexplained, suggesting the presence of other effect modifiers. 
Conclusions: Only one prior study, analyzing athletes, utilized a meta-regression to control effect modifiers in mTBI. Consistent with Belanger and colleagues' original findings, the current study suggest that time post-mTBI modifies overall neuropsychological functioning. However, this does not explain all between-study variability, which may be accounted for by other variables, such as premorbid psychological comorbidities and pre-injury disposition.

Correspondence: David E. Marra, MS, Psychology, Marquette University, 615A East Pleasant Street, Milwaukee, WI53202, United States. E-mail: david.marra@marquette.edu

V.C. MERRITT, A.L. CLARK, S.F. SORG, N.D. EVANGELISTA, M.L. WERHANE, M.W. BONDI, D. SCHIEHSER \& L. DELANOWOOD. APOE- $\varepsilon 4$ Genotype Modifies the Relationship Between TBI History and Neuropsychological Performance in Military Veterans.

Objective: Although genetic differences may contribute to the varied cognitive outcomes often observed after traumatic brain injury (TBI), the influence of genetic factors on neuropsychological performance following TBI remains poorly understood. We therefore investigated the influence of APOE polymorphisms on neurocognitive functioning in Veterans with post-acute TBI.

Participants and Methods: 121 Veterans (mild TBI=59, moderate $\mathrm{TBI}=10$; military controls $[\mathrm{MC}]=52$ ) underwent neuropsychological assessment and APOE genotyping. Veterans were divided into $\varepsilon 4+$ $(\mathrm{TBI}=17, \mathrm{MC}=14)$ and $\varepsilon 4-(\mathrm{TBI}=52, \mathrm{MC}=38)$ groups. Two composite scores were computed from 21 norm-referenced variables-a memory index $(\alpha=.84)$ and a speed index $(\alpha=.88)$, and the total number of impaired scores (>1.5 SD below mean) for each participant was calculated. Two-way ANOVAs were conducted to examine the effect of group (TBI vs. MC) and $\varepsilon 4$ status ( $\varepsilon 4+$ vs. $\varepsilon 4-)$ on cognition.

Results: The main effect of $\varepsilon 4$ genotype was significant for both cognitive composites $(\varepsilon 4+<\varepsilon 4-; p=.02-.03)$ and the main effect of group approached significance (TBI $<\mathrm{MC} ; p=.05-.08)$. Compared to the TBI $\varepsilon 4$ - group, the TBI $\varepsilon 4+$ group performed more poorly on the memory composite $(p=.02)$, and there was a trend in the same direction for the speed index $(p=.05)$. There was no effect of $\varepsilon 4$ status across cognitive indices in the MC group $(p>.05)$. Moreover, within the TBI group, $\varepsilon 4+$ Veterans showed a greater number of impaired scores than $\varepsilon 4-$ Veterans $(p=.01)$, and $82 \%$ of the TBI $\varepsilon 4+$ group demonstrated $\geq 1$ impaired score compared to $44 \%$ of the TBI $\varepsilon 4$ - group $(p=.01)$.

Conclusions: Results suggest that APOE- $\varepsilon 4$ genotype contributes to poorer memory and processing speed in Veterans with a history of TBI. Our findings extend prior work by suggesting that APOE- $\varepsilon 4$ genotype is associated with cognitive impairment even well beyond the acute phase of injury. Future work is needed to elucidate the underlying brain-based mechanisms of $\varepsilon 4$ allelic effects on cognitive and clinical outcomes following TBI.

Correspondence: Victoria C. Merritt, M.S., Psychology Service, VA San Diego Healthcare System, 3350 La Jolla Village Drive, MC 151B, San Diego, CA 92161, United States. E-mail: vcabarnes@gmail.com

J. MEYER \& P. ARNETT. Prevalence of Subconcussive Head Trauma, Traumatic Brain Injury, and Hypoxic Brain Injury in Victims of Intimate Partner Violence and Potential Long Lasting Effects on Cognitive Functioning.

Objective: Injuries to the head neck and face are considered signature injuries of intimate partner violence (IPV), yet there is limited research on the effects of head trauma in this population. Victims of IPV are at high risk for traumatic brain injury (TBI) due to direct hits to the head and face and experiences of being shaken, and are additionally at risk for hypoxic brain injuries (HBI) due to strangulation. Additionally, victims of IPV may sustain repetitive subconcussive hits that may have cumulative effects on their cognitive functioning. The present study aimed to establish the prevalence of the above injuries and evaluate chronic cognitive difficulties related to these injuries in this population.
Participants and Methods: 30 female participants ranging in age from 19 to $55(M=28.47, S D=9.99)$ with a history of at least one relationship that included physical violence completed a structured interview assessing for subconcussive hits, TBI, and HBI. Participants completed a neuropsychological test battery that included traditional and ecologically valid measures of cognitive functioning.

Results: Participants reported an average of 84 impacts to the head (Median $=8.5 ; S D=212.18$; range: $0-852$ ). They reported experiencing being shaken an average of 27.17 times (Median $=3.50 ; S D=10.35)$. No participants reported injuries consistent with moderate or severe TBI, however, $43.3 \%$ of participants reported at least one mild TBI (range: $0-183$ ). $46.7 \%$ of participants reported experiencing choking or another form of violence that obstructed their breathing and $26.7 \%$ of participants reported symptoms consistent with mild HBI.

Conclusions: The present findings support previous research that has indicated that victims of intimate partner violence experience high levels of head trauma and injuries consistent with TBI and HBI. This talk will also report on the relationship between cumulative head trauma and cognitive, emotional, and functional abilities in these IPV victims. Correspondence: Jessica Meyer, Penn State, 372 Moore Building, University Park, PA 16801, United States.E-mail: jem508@psu.edu

C.M. MULLEN, S.L. SALINAS \& S. PENNA. Subjective Cognitive Complaints and Emotional Symptoms in Veterans with Polytrauma and Mild Traumatic Brain Injury.

Objective: This study examined the role of subjective cognitive complaints and emotional symptoms in veterans with polytrauma and mild traumatic brain injury (mTBI). It was predicted those with high symptoms of emotional distress and medical problems would endorse greater cognitive symptoms.

Participants and Methods: Participants consisted of 151 veterans referred for neuropsychological evaluation due to reported concerns of cognitive deficits post-mTBI. The Beck Depression Inventory-II (BDI) and Beck Anxiety Inventory (BAI) were used to assess emotional symptoms. The Neurocognitive Symptom Inventory (NSI) was used to determine subjective cognitive concerns and the PTSD Checklist (PCL-C) for symptomatology. Polytrauma was defined as either number of medical problems or cognitive altering medications. Comparative and regression analyses were conducted to assess potential contribution of polytrauma on complaints.

Results: Regression analyses revealed BDI and BAI total scores significantly contributed to NSI total score, $F(2,99)=22.23, p<.01$ and NSI cognitive cluster $F(2,99)=15.91, p<.01$. Veterans on cognitive altering medications endorsed more somatic symptoms on the BDI, $t(145)=-2.09, p=.04$, and overall BAI symptoms, $t(84)=1.83, p=.07$. Veterans on cognitive altering medications had more cognitive and vestibular complaints on the NSI, $F(5,133)=2.51, p=.03, F(5,133)=2.36$, $p=.04$; respectively. Cognitive altering medications and medical problems were related to somatic symptoms on the BDI, $F(4,81)=2.26$, $p=.07, F(3,143)=2.53, p=.06$; respectively. Number of medical problems had a significant effect on the PCL-C, $F(24,123)=1.86, p=.02$.

Conclusions: Veterans on cognitive altering medications had greater symptom endorsement on overall BAI, somatic symptoms on the BDI, and subjective cognitive and vestibular complaints on the NSI. Number of medical conditions was associated with high scores on the PCL-C and somatic symptoms on the BDI. Findings could assist clinicians in educating veterans about appropriate attributions of symptoms.

Correspondence: Christine M. Mullen, University of Alabama at Birmingham, 3001 Mercer University Drive, AACC Building, Suite 463, Atlanta, GA30341-4115, United States.E-mail: christinemariemullen@ gmail.com 
WITHDRAWN. R. PERNA. Case Study: Disorder of Consciousness to full Functional Recovery.

Objeetive: When patients are in a disorder of eonseiousness and even a minimally conscious state it is hightly metear what their prognosis may be. It may be benefieial for elinieians to know that some individuals have very positive outeomes. This ease study looks at the recovery course of a patient who progressed from a $D \Theta G$ to a fully funterional recovery. Partieipants and Methods. Ms. A is a 26 year oth, married, mother of two with 14 years of edtueation. She suffered a severe TBI (GCS of 3 and 7), DAI, and bifrontal SAH from a high speed motor vehicle collision. On CPSP testing she was minininally responsive for three weeks. She then had perios of agitation and need for restraints. Subsequent to becoming responsive and exiting PTA, Ms. A exhibited erely impaired

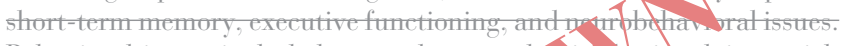

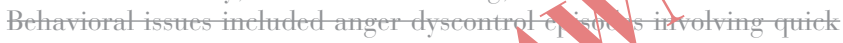
esealation in anger and very limited regll to that oeetrred. Results: Ms. A has made gradtual bo fo sittillg progress in her phys= ieal, cognitive, andemotional dit th yn recovery. At one year post TBI, at the time of adni vio to 40 st-acute netrorehabilitation, Ms. A exhibited 2nd prt mitit shent-term memory, depression, and anger aggression epig oo s. Fo bw-tup testing showed much improved eognitive

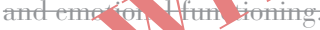

Conclusio s: Sixteen months after severe TBI and a disorder of eonseiousness, Ms. A is finishing treatment and getting ready for a retmm to work or volumtecr work. A follow-up netropsychological evaluation shows milth impaimments inverbalfluency, working memory, processing speed, and some aspeets of attention. It may be elinieally usefull to study individuals with these injuries who make excellent reeoveries and it may be helpful for clinicians andearegivers to understand that very positive outeomes are possible, although its still unclear which DOG behaviors prediet best outeomes.

Gorrespontence. Pobert Perna, Neuropsychology/Psychotogy, THR 9427 Greentwithou, Houston, TX 7.9970 , United States. E-mat: dr.perna@juno.com

E. Polejaeva, J. GRavano, Z. HOUCK \& W.M. PERLSTEIN. Relation between apathy, depression, and symptoms of Posttraumatic Stress Disorder in mild Traumatic Brain Injury.

Objective: Posttraumatic Stress Disorder (PTSD) and mild Traumatic Brain Injury (mTBI) are often co-morbid in veteran populations, containing overlapping symptoms which present challenges for disentangling the two. This study investigated how behavior associated with frontal lobe dysfunction (apathy, disinhibition, and dysexecutive function) is altered in individuals with symptoms of PTSD. Additionally, associated brain regions were explored via 8-minute resting-state functional magnetic resonance imaging (fMRI; 3T).

Participants and Methods: Veterans with mTBI and varying degrees of self-reported PTSD symptoms were administered the PTSD Checklist (PCL-M), Beck Depression Inventory (BDI-2), and the Frontal Systems Behavior Scale (FrSBe) $(n=14)$. Resting state analyses focused on Prefrontal Cortex (PFC), Anterior Cingulate Cortex (ACC), and Insula based on previous research associated with PTSD and mTBI.

Results: The study found a positive relation between individuals with symptoms of PTSD and amount of apathy symptoms reported on the FrSBe. Additionally, greater connectivity was present between default mode network (DMN), PFC, and insula during resting-state fMRI. However, when we controlled for symptoms of depression (BDI-2) these effects were no longer significant.

Conclusions: These pilot results suggest that depressive symptoms rather than symptoms of PTSD in individuals with TBI account for increased risk of developing apathy. Further investigation is needed to assess this relation and the pathwavs involved.

Correspondence: Elena Polejaeva, Clinical \& Health Psychology, University of Florida, PO Box 100165, Gainesville, FL 32610-0165,

United States. E-mail: polejaeva@ufl.edu
A.C. RAIKES, B.C. SATTERFIELD, S. KNIGHT \& W. KILLGORE. Grey Matter Volumetric Differences with Increasing Numbers of Previous Mild Traumatic Brain Injuries: A Voxel-Based Morphometric Study.

Objective: Mild traumatic brain injury (mTBI) is a functional injury without evidence of structural abnormality. While there is mounting evidence that mTBI may be associated with changes in grey matter (GM) volume, the direction, timing, and extent of these changes remain unclear. Few studies have investigated the relationship between the number of past mTBIs and GM volume changes. The purpose of this study was to quantify differences in GM volume with respect to the number of prior head injuries.

Participants and Methods: The T1 high-resolution structural scans of 39 right-handed individuals with a self-reported history of mTBI (14 males; mean age: $24.17 \pm 7.11 \mathrm{y}$ ) were used for volume-based morphometric analysis (CAT12). Images were segmented and normalized following an automated procedure in CAT12 and smoothed prior to analysis. GM volume was correlated with the total number of self-reported past mTBIs, after controlling for age, sex, total intracranial volume, and time since most recent mTBI. Volumetric data from the single surviving cluster were exported for additional analyses.

Results: GM volume in a single cluster encompassing areas of the left superior temporal and supramarginal gyri (proximal to Wernicke's Area) positively correlated with total number of mTBIs (FWE corrected, $p=0.035)$. GM volume in this cluster was additionally significantly positively correlated with Delis-Kaplan executive function system (DKEFS) tasks, including letter fluency $\left(R^{2}=0.102\right)$ and category switching $\left(\mathbf{R}^{2}=0.106\right)$.

Conclusions: In individuals with a history of mTBI, GM volume in the left superior temporal and supramarginal gyri was greater with increasing numbers of mTBIs. This increase in volume may reflect an adaptive neuroplastic response to increasing numbers of mTBIs that preserves aspects of language-based executive function. Longitudinal studies are needed to identify a causal relationship between mTBI and adaptive neuroplastic processes in the grey matter.

Correspondence: Adam C. Raikes, PhD, Psychiatry, University of Arizona, 1501 N Campbell Ave., Tucson, AZ 85724, United States. E-mail: raikes@psychiatry.arizona.edu

A.C. RAIKES, B.C. SATTERFIELD \& W. KILLGORE. Increased Cerebellar Grey Matter in the Presence of Decreased Subjective Sleep Quality Following Mild Traumatic Brain Injury.

Objective: Individuals with a history of mild traumatic brain injury (mTBI) often report insomnia, disturbed sleep, and lower general sleep quality than individuals with no history of concussion. These subjective complaints may persist long after injury, even without objective evidence of reduced sleep quality. To date, no neural correlates of such subjective complaints have been identified. In the present study, we correlated whole-brain grey matter with Pittsburgh Sleep Quality Index (PSQI) total scores in individuals within one year of an mTBI.

Participants and Methods: 39 right-handed individuals with a self-reported history of mTBI $(14$ males; mean age: $24.17 \pm 7.11 \mathrm{y})$ were administered the PSQI as part of a larger on-going study. Additionally, we obtained T1 high-resolution structural scans, which were segmented and normalized (CAT12) and smoothed (SPM12) prior to voxel-based morphometric analysis. Whole-brain grey matter volume (GMV) was correlated with total PSQI scores, after controlling for age, sex, total intracranial volume, and time since most recent mTBI. GMV in significant clusters was exported for further analysis.

Results: GMV in a cluster including portions of the left cerebellum's lobules 7 and 8 positively correlated with total PSQI score (FWE corrected, $p=0.019$ ), indicating worse sleep. GM volume in this cluster was additionally significantly negatively correlated with faster psychomotor vigilance task mean reaction time $\left(R^{2}=0.099\right)$ and positively with PVT reaction time coefficient of variation $\left(\mathrm{R}^{2}=0.137\right)$. PSQI total scores did not correlate with any PVT measures and prevented further mediation analysis. 
Conclusions: Individuals with mTBI who reported lower sleep quality had greater GMV in the left cerebellum. The lack of correlation between total PSQI and PVT performance metrics suggests that increased GMV in the cerebellum may be a compensatory mechanism for maintaining task performance in spite of perceived sleep decrement following mTBI. Correspondence: Adam C. Raikes, PhD, Psychiatry, University of Arizona, 1501 N Campbell Ave., Tucson, AZ 85724, United States. E-mail: raikes@psychiatry.arizona.edu

J. ROWLAND, J. STAPLETON-KOTLOSKI, D. DOBBINS, E. ROGERS, D. GODWIN \& K. TABER. Changes in RestingState Network Topology Associated with the Development of Posttraumatic Stress Disorder Following Deployment Acquired Traumatic Brain Injury.

Objective: A growing literature suggests traumatic brain injury (TBI) acquired during deployment represents an independent risk factor for developing posttraumatic stress disorder (PTSD). The current study will determine changes in whole-brain functional networks between individuals who do and do not develop PTSD following deployment acquired TBI. It was hypothesized that participants who developed PTSD would demonstrate higher levels of clustering coefficient, small worldness, and modularity.

Participants and Methods: Participants were post-deployment Iraq and Afghanistan war Veterans with a history of deployment acquired TBI determined by clinician administered interview. Seven participants were diagnosed with PTSD using the Clinician Administered PTSD Scale-5 (CAPS-5). Magnetoencephalography (MEG) was acquired in the resting state for 5 minutes. Active brain regions were identified using synthetic aperture magnetometry. Connectivity among all regions was calculated using the weighted phase lag index. Network metrics were calculated using the Brain Connectivity Toolbox. Univariate ANCOVA adjusting for age and estimated premorbid IQ were used to determine differences in network metrics between participants who did and did not develop PTSD following deployment acquired TBI.

Results: Participants who developed PTSD displayed higher levels of small-worldness, $\mathrm{F}(1,12)=5.364, \mathrm{p}<.039$, partial eta squared $=0.309$, and clustering coefficient, $\mathrm{F}(1,12)=12.204, \mathrm{p}<.004$, partial eta squared $=0.504$, than participants who did not develop PTSD. Modularity $(Q)$ and the number of modules present were not different between groups.

Conclusions: Results demonstrate significant differences in wholebrain resting-state networks between individuals who do and do not develop PTSD following deployment acquired TBI. This is the first study to identify neurobiological differences through which deployment acquired TBI may increase the risk of subsequently developing PTSD. Correspondence: Jared Rowland, Ph.D., Research \& Academic Affairs, W.G. "Bill" Hefner Veterans Affairs Medical Center, 1601 Brenner Ave - 11M-2, Salisbury, NC 28144, United States.E-mail:jared.rowland@ va.gov

S.L. SALINAS, C.M. MULLEN \& S. PENNA. The Effects of Polytrauma on Neuropsychological Performance: Veterans with Mild Traumatic Brain Injury.

Objective: This study examined the role of polytrauma in the expression of objective cognitive deficits with veterans who have sustained a mild traumatic brain injury (mTBI). We predicted that veterans with greater polytrauma would demonstrate more severe objective neurocognitive impairment on neuropsychological testing than those less affected by polytrauma.

Participants and Methods: All participants were veterans referred for neuropsychological evaluation due to reported concerns of cognitive deficits post-mTBI. Polytrauma was assessed and defined by examining two variables: veterans' list of medical problems and current medications he/she was taking in order to manage their various medical issues. Impact of cognitive altering medications was assessed on veteran neuropsychological performance.
Results: One-way ANOVAs revealed a significant effect of medication total on veteran performance on the Arithmetic, and Similarities subtests of the WAIS. Number of veteran medical problems had a significant effect on FAS, Animals, and Boston Naming scores. Cognitive altering medications negatively impacted psychomotor performance on Grooved Pegboard dominant and non-dominant hands. However, no differences were detected between the various levels of veteran polytrauma.

Conclusions: Prescription medication has been known to negatively impact speeded fluency tasks, as well as speeded psychomotor tasks (Stein \& Strickland, 1998). Other factors such as orthopedic injuries and peripheral nerve damage can also contribute to poor performance on speeded tasks. Attention and working memory may have also been negatively impacted by prescription medications and other polytrauma factors such as PTSD in this study (Nelson et al., 2011). Overall, these findings indicate that veteran polytrauma has a negative impact on neuropsychological measures relating to numerical reasoning and mental arithmetic, verbal and semantic fluency, confrontation naming, and psychomotor speed related to finger dexterity and manipulation. Correspondence: Stephanie L. Salinas, MS, MA, Department of Clinical Psychology, The University of Arkansas for Medical Sciences, $4301 \mathrm{~W}$ Markham, Slot 554, Little Rock, AR 72205, United States. E-mail: steph. salinas34@gmail.com

S.F. SORG, A.L. CLARK, L.M. CAMPBELL, M.L. WERHANE, K.A. HOLIDAY, V.C. MERRITT, M.J. WALSH, A.J. JAK, M.W. BONDI, D. SCHIEHSER \& L. DELANO-WOOD. The Relationship Between Subjective and Objective Memory Performance, PTSD Status and Injury Severity in Veterans with History of Mild Traumatic Brain Injury.

Objective: The validity of subjective memory complaints in mild traumatic brain injury (mTBI) remains an important clinical consideration, especially in the context of co-morbid psychiatric symptoms (PTSD). In this study, we assessed both prospective and retrospective memory complaints in Veterans with mTBI and investigated associations between memory complaints, PTSD, injury severity, and objective neuropsychological test performance.

Participants and Methods: 98 Veterans (mTBI=65, Military Controls $[\mathrm{MCs}]=26$ ) with optimal effort testing completed a structured TBI history interview, the Prospective-Retrospective Memory Questionnaire (PRMQ) with Prospective (PM) and Retrospective Memory (RM) subscales, the PTSD Checklist, and objective tests of memory (Logical Memory and CVLT-II) and executive functions (Wisconsin Card Sorting Test and D-KEFS Trails). All analyses were conducted using partial correlation analyses and ANCOVA.

Results: Compared to MCs, the mTBI group endorsed higher PM and RM complaints ( $p$ 's<.01). PM complaints were greater in TBI Veterans with loss of consciousness (LOC; $n=43$ ) relative to those reporting only alteration of consciousness (AOC; $\mathrm{n}=22, p<.05$ ), but there were no significant differences in RM ratings. In the mTBI group, objective memory test scores were associated with RM complaints ( $r=-.34--.40$, $p$ 's<.01), but not PM; executive function test scores were associated with both RM and PM (r=-.26 - -.40, p's<.05); and PTSD was associated with higher PM and RM complaints $(p$ 's<.01). All results remained significant after statistically adjusting for PTSD status.

Conclusions: Independent of psychiatric status, Veterans with mTBI report greater subjective memory complaints compared to those without mTBI history and greater injury severity (LOC $>$ AOC) was associated with worse subjective PM complaints. Findings suggest dissociable associations with object test performances between RM and PM ratings and underscore the importance of assessing both PM and RM complaints in Veterans with mTBI.

Correspondence: Scott F. Sorg, VA San Diego/UC San Diego, 3350 La Jolla Village Dr., San Diego, CA 92161, United States. E-mail:ssorg@ ucsd.edu 
J. STROM, S.F. SORG, K.A. HOLIDAY, A.L. CLARK, M.L. WERHANE, M.W. BONDI, D. SCHIEHSER \& L. DELANOWOOD. Associations Between Time Since Injury and Cognitive Recovery Across Mechanism of Traumatic Brain Injury: Preliminary Evidence for Protracted Cognitive Recovery in BlastRelated Neurotrauma.

Objective: Although recent studies have suggested that blast force traumatic brain injury (TBI) may cause damage to brain regions often unaffected by blunt force TBI, little is known about whether cognitive recovery differs across mechanism of injury (blast versus blunt force TBI). We therefore investigated associations between time since injury and TBI mechanism on executive function performance in a group of well-characterized Veterans with history of mild to moderate TBI.

Participants and Methods: 214 Veterans (blast-TBI [n=44], blunt-TBI [n=82], and non-TBI military controls [MCs: $\mathrm{n}=88$ ]) completed a clinical interview, neuropsychological testing, and psychiatric questionnaires. Groups were compared on the following executive function (EF) tests: D-KEFS Verbal Fluency (VF), Color Word Interference (CWI), Design Fluency (DF), and Trail Making Test (TMT).

Results: As expected, compared to MCs, both TBI groups performed significantly more poorly across EF tests (all $p$-values<.05). Regression analyses adjusting for age, severity and number of injury, and PTSD symptoms, showed that greater time since injury was significantly related to better EF performance within the blunt-TBI group [VF $(p=.011), D F(p=.036), C W I(p=.007)$, TMT $(p=.014)]$ In contrast, no such effects of time since injury on cognition were found in the blast TBI group (all $p$-values $>.05$ ).

Conclusions: Results showed that increased time since injury was related to better performance on tasks of executive functioning in Veterans with blunt force, but not blast-related TBI. Importantly, these findings were independent of PTSD symptomatology, and they were not explained by injury severity or total number of TBI events. Findings suggest that blast-exposed Veterans may evidence a more protracted recovery of cognitive functions in the aftermath of TBI. Future studies are needed in order to examine how brain regions may be differentially affected across mechanism of injury, and whether other variables (e.g., genetic differences) may modify any observed relationships.

Correspondence: John Strom, University of California, Riverside, 508 meadowmist ct., Encinitas, CA 92024, United States. E-mail: johnstrom2@gmail.com

P. SUNDERARAMAN, S. COSENTINo, A. JAMES \& M.T. SCHULTHEIS. The Influence of Impulsivity on Financial Capacity in Individuals with Chronic Acquired Brain Injury (CABI). Objective:

Impulsivity is a frequent consequence of acquired brain injury (ABI), and this deficit may contribute to the impaired ability to manage finances often seen in ABI. Indeed, previous work has suggested that in comparison to matched controls, individuals with ABI more often temporally discount (i.e., select smaller immediate financial rewards more often). However, few studies have directly examined the association between clinically characterized impulsivity, temporal discounting, and financial capacity in individuals with moderate to severe, chronic acquired brain injury (CABI). The current, prospective study sought to investigate these associations by using two measures of impulsivity, a clinical measure (Barratt Impulsivity Scale II; BISII) and a financial measure (Temporal Discounting task) as well as a measure of financial capacity, the Financial Competence Assessment Inventory (FCAI).

Participants and Methods: Twenty-five CABI participants (injury period, $M=17.61, S D=9.15$; age, $M=46.06, S D=8.26$; education, $M=13.28, S D=2.09 ; 32 \%$ females) were recruited from a day-treatment rehabilitation program.

Results: There was no relationship between temporal discounting and BISII $(r=-0.30, p=0.19)$. Only one out of five FCAI dimensions, Estate Management, was associated with a higher tendency to temporally discount $(r=-0.48, p<0.05)$. BIS II was associated with abstract reasoning $(r=0.47, p<0.05)$ and verbal fluency $(r=0.46, p<0.05)$.
Conclusions:

Overall, the results suggest that clinically characterized impulsivity is dissociable from financial impulsivity, and that contrary to expectations, neither construct is highly related to financial capacity in CABI. These results highlight the importance of understanding other predictors of FCAI such as cognitive and mood variables in individuals with CABI. Correspondence: Preeti Sunderaraman, PhD, Columbia University Medical Center, 630 West, 168th Street, New York, NY 10032, United States.E-mail:preetis.ps@gmail.com

D.P. TERRY, N. HUEBSCHMANN, N.E. COOK, B. MAXWELL, R. MANNIX, R. ZAFONTE, P.D. BERKNER \& G.L. IVERSON. PreInjury Migraine History as a Risk Factor for Prolonged Return to School and Sports Following Concussion.

Objective: Having a pre-existing migraine disorder might be a risk factor for worse outcome following a sport-related concussion. We examined whether having a migraine history was associated with a prolonged return to academics and athletics following a concussion.

Participants and Methods: High school and collegiate athletes ( $\mathrm{N}=1,265 ; 42 \%$ female) who sustained a sport-related concussion were monitored by athletic trainers using a web-based surveillance system that collects information about concussion recovery. Nonparametric Kolmogorov-Smirnov tests (KS) were used to compare days to return to academics/athletics across groups due to non-normally distributed outcome variables and unequal distributions of scores between groups. Chi-square tests examined the proportion of players who had not returned to academics/athletics at 7, 14, and 21 days post injury stratified by self-reported migraine history.

Results: There were 117 athletes $(9.2 \%)$ who reported a pre-injury migraine history. Athletes with a history of migraine took a median of 6 days to return to academics $(M=10.6, S D=14.2)$ and 15.5 days to return to athletics $(M=23.8, \mathrm{SD}=30.8)$, while those with no migraine history took a median of 5 days to return to academics $(M=7.5, S D=10.9)$ and 14 days to return to athletics $(M=19.4, S D=19.4)$. There were no statistically significant differences in days to return to school or athletics between the groups (KS $p s>.05$ ). Stratifying analyses by gender and setting (high school vs. college) did not alter the results. However, a lower percentage of athletes with a history of migraine had returned to school after 7 days $\left(57 \%\right.$ vs. $\left.68 \%, \chi^{2}=5.53, p=.02\right), 14$ days $(75 \%$ vs. $88 \%, \chi^{2}=14.21, p<.001$ ), and 21 days post injury ( $89 \%$ vs. $94 \%$, $\left.\chi^{2}=4.90, p=.03\right)$. There were no group differences in recovery rates when examining return to athletics.

Conclusions: Athletes with a pre-injury migraine history may be at an elevated risk for a protracted return to school after concussion.

Correspondence: Douglas P. Terry, PhD, Physical Medicine and Rehabilitation, Spaulding Rehabilitation Network, Massachusetts General Hospital, Harvard Medical School, 5 Wendell St Apt 7, Cambridge, MA 02138, United States.E-mail:_terry@mgh.harvard.edu

D.P. TERRY, N.D. SILVERBERG, J.E. KARR, W. PANENKA \& G.L. IVERSON. Perceived Injustice is Associated with Worse Outcome from Mild Traumatic Brain Injury.

Objective: Perceived injustice is a belief that one has been treated unfairly, disrespectfully, and is suffering unnecessarily as a result of another person's actions. Perceived injustice predicts chronic disability after musculoskeletal injury, but to our knowledge, has not been empirically studied in people with mild traumatic brain injuries (MTBIs). We examined perceived injustice and its correlates in patients who were slow to recover from MTBI.

Participants and Methods: Patients (N=102) were recruited from four concussion clinics. The sample was on average 41.2 years old ( $\mathrm{SD}=11.7$; range $=21-64), 46.1 \%$ men, and evaluated at 12 weeks post injury ( $\mathrm{SD}=6.3$; range 2-26). Patients completed measures assessing perceived injustice (Injustice Experience Questionnaire; IEQ), postconcussion symptoms, traumatic stress, depression, pain, disability, and neuropsychological performance validity. 
Results: Patients frequently endorsed items such as "I just want to have my life back" (85.2\%) and "people don't understand how severe my condition is" $(89.1 \%)$, with $23.5 \%$ of the sample scoring in the clinically significant range on the IEQ (Total Score>30). Internal consistency was very high (Cronbach's $\alpha=.91$ ). Patients who failed performance validity testing (Cohen's $\mathrm{d}=.48$ ) or were seeking/receiving compensation $(\dot{d}=0.92)$ reported greater perceived injustice. Greater perceived injustice was associated with greater post-concussion symptoms $(r=.48)$, traumatic stress $(r=.69)$, depression $(r=.60)$, bodily pain $(r=.32)$, and perceived disability $(r=.45$; all $p$ 's<.01).

Conclusions: Perceived injustice was common and it was associated with greater post-concussion symptoms, psychological distress, bodily pain, and perceived disability. Given that perceived injustice is a belief system that can influence health behaviors, it might be a viable target for psychological treatment.

Correspondence: Douglas P. Terry, PhD, Physical Medicine and Rehabilitation, Spaulding Rehabilitation Network, Massachusetts General Hospital, Harvard Medical School, 5 Wendell St Apt 7, Cambridge, MA 02138,United States.E-mail: dterry@mgh.harvard.edu

\section{J. TIBBS, M. CISSNE, J. SNELL \& K.S. CHIOU. Exploring the Role of Alcohol and Substance Use on Neuropsychological Status During Post-Acute Rehabilitation in Survivors of Traumatic Brain Injury.}

Objective: Research thus far has shown that individuals with alcohol use disorders are at increased risk for sustaining a traumatic brain injury (TBI). However, the effects of pre-injury patterns of alcohol use on recovery of function following TBI remains unclear. Thus, the purpose of the present study was to document neuropsychological function of individuals with TBI who had history of pre-injury alcohol/substance use patterns. We hypothesized that those with history of problematic alcohol use would show poorer neuropsychological scores compared to those who had a history of social (non-problematic) drinking or denied any history of prior alcohol use.

Participants and Methods: Medical record review was conducted on 60 individuals with moderate to severe TBI after admittance to a post-acute rehabilitation facility. Patient demographics, alcohol and substance use history (35\% of the sample denied any use, $13.3 \%$ reflected social use, $51.7 \%$ reflected problematic use), and neuropsychological data from the facility's routine intake assessment were extracted. Correlations and multiple linear regression models were used to test demographic and substance use variables as predictors in seven models of neuropsychological scores.

Results: Significant negative correlations were found between substance/alcohol use variables and age, and education $(p<.05)$, but not gender. Additionally, significant correlations were found between demographic variables and scores of semantic fluency, mental status, and perseverative errors. No significant linear relationships were found between alcohol/substance use history and performance on neuropsychological tasks. Similarly, each of the multiple regression models failed to reach significance.

Conclusions: In this particular sample, no significant differences were found between survivors of TBI with a history of no, social, or problematic alcohol use in predicting neuropsychological performance during post-acute rehabilitation. Limitations and future directions will be presented.

Correspondence: Jessie Tibbs, M.A., Psychology, University of Nebraska Lincoln, 2223 S 15th St, Lincoln, NE 68502, United States. E-mail: jessie.tibbs@gmail.com

A. TREBLE-BARNA, Y. CONLEY \& A.K. WAGNER. Epigenetic Changes in the Brain-Derived Neurotrophic Factor (BDNF) Gene Following Traumatic Brain Injury: Preliminary Findings.

Objective: Epigenetic factors are a promising but unexplored potential source of heterogeneity in neurobehavioral recovery following traumatic brain injury (TBI). During the last decade, the field of epigenetics has become central to studying the modulation of genetic phenotypes by environmental factors The best-characterized epigenetic modification to DNA is methylation. The density of methylation is low in transcriptionally active genes and high in silenced genes. BDNF is a protein important for neuroplasticity, neurogenesis, and neuronal survival and the gene for BDNF is regulated by DNA methylation. Genetic variation in BDNF is associated with TBI outcomes but epigenetic factors have not been explored in clinical samples. The objective of the present study was to examine the effect of TBI on the epigenetic profile of the BDNF gene in blood.

Participants and Methods: Blood samples were obtained from 19 adults with moderate to severe TBI (age 47.5 \pm 21.1 ) and from 13

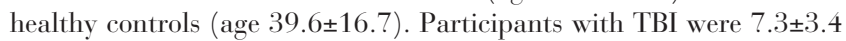
months post-injury at the time of sample collection. DNA was extracted using a salting out procedure and percent methylation of a $\mathrm{CpG}$ island within the BDNF promoter region was determined using EpiTect Methyl II PCR assays.

Results: A non-parametric Kruskal-Wallis test revealed significantly reduced BDNF methylation $(p=.035)$ in adults with TBI, M=22.52, $\mathrm{SD}=37.20$, relative to healthy controls, $\mathrm{M}=42.94, \mathrm{SD}=41.51$.

Conclusions: TBI is a significant biological event that may result in epigenetic changes in the BDNF gene that can be detected in peripheral blood. Epigenetic changes to the BDNF gene may impact neural recovery and plasticity following TBI through genetic regulation. Future studies should confirm these findings in larger samples and examine associations of epigenetic changes with clinical outcomes.

Correspondence: Amery Treble-Barna, PhD, Physical Medicine and Rehabilitation, University of Pittsburgh School of Medicine, 6816 Meade St \#2, Pittsburgh, PA 15208, United States. E-mail: amerytreblebarna@ gmail.com

Z. LIPMAN, J. MORGENLANDER \& L.A. TUPLER. Impairments in Crystallized and Fluid Intelligence in Veterans with History of Traumatic Brain Injury: Influence Through Effects of Severity and Multiplicity of Trauma on Depression and Posttraumatic Stress Disorder.

Objective: Heightened interest in cumulative head trauma has followed media attention to chronic traumatic encephalopathy from sports concussions and multiple traumatic brain injuries (TBIs) sustained in the Afghanistan and Iraq wars. TBI in veterans is associated with depression and posttraumatic stress disorder (PTSD), both of which can have confounding neurocognitive effects, requiring further differentiation.

Participants and Methods: We therefore examined 1,533 veterans (1,215 males, 318 females; $37.5 \pm 10.5$ years) mostly deployed to Iraq and Afghanistan, recruited from 4 Mid-Atlantic VA medical centers. TBI history was evaluated by clinicians using the McCormick TBI Interview, developed in house using American Congress of Rehabilitative Medicine criteria. Psychiatric diagnosis was assessed via an electronic version of the Structured Clinical Interview for DSM-IV Axis I Disorders (eSCID). Results: Of 810 veterans (53\%) positive for TBI, 464 (57\%) sustained multiple $(2.3 \pm 1.6)$ events. TBI was associated with both eSCID depression and PTSD $(p<.001)$. Compared with mild TBI, veterans with a single moderate/severe TBI were more likely to exhibit one or both disorders and had lower Abstraction $(p<.001)$ but not Vocabulary scores on the Shipley Institute of Living Scale (SILS). For veterans with multiple TBIs, depression and PTSD were associated with increasing numbers of TBIs and lower SILS Abstraction and Vocabulary scores $(p<.001)$. Further analyses showed that cognitive findings consistently related to the presence of each psychiatric disorder but not to TBI severity or multiplicity.

Conclusions: TBI severity and multiplicity are associated with depression and PTSD in veterans, which can govern declines in fluid intelligence (single TBI) and both crystallized and fluid intelligence (multiple TBIs). These findings highlight the importance of assessing for psychiatric disorder in veterans with TBI, which may account for neurocognitive impairment independently of neuronal injury.

We acknowledge the VA Mid-Atlantic MIRECC Workgroup 
Correspondence: Larry A. Tupler, Ph.D., Department of Psychiatry and Behavioral Sciences, Duke University Medical Center, Box 3018, Duke University Medical Center, Durham, NC 27710, United States. E-mail: ltupler@duke.edu

S.D. UCHANI, K.A. MILLEVILLE, A.K. WAGNER, Y. CONLEY \& A. TREBLE-BARNA. Adverse Childhood Experiences and Neuropsychological Outcomes Among Adults with Moderate to Severe Traumatic Brain Injury: Preliminary Results.

Objective: Recent research demonstrates a link between a higher number of retrospectively recalled adverse childhood experiences (ACEs) and a range of poor health outcomes in adulthood. The objective of the present study was to characterize ACEs among a pilot sample of adults with traumatic brain injury (TBI) and explore preliminary associations with neuropsychological outcomes.

Participants and Methods: Seventeen adults (mean age 44.3 \pm 20.4 ) with moderate to severe TBI completed the ACE questionnaire and a battery of standardized neuropsychological tests at $6.6 \pm 2.4$ months post-injury. The ACE includes 11 items asking about exposure to parental substance abuse, separation from a parent, domestic violence, and physical, emotional, and sexual abuse before the age of 18 . Neuropsychological composite scores were created by averaging $\mathrm{T}$ scores across measures within domains (executive functioning, attention and processing speed, memory, and verbal fluency) and by averaging domain composites for an overall composite score.

Results: $58.8 \%$ of participants reported no ACEs, $17.7 \%$ reported 1 ACE, 23.5\% reported 2 ACEs, and no participants reported 3 or more. Participants performed 1 to 1.25 standard deviations below the normative mean across neuropsychological domains and overall. Spearman's correlations (all $r<.2, p>0$.1) failed to show any significant associations between ACEs and neuropsychological performance.

Conclusions: Based on this small pilot sample, ACEs appear to be a relatively low frequency phenomenon among adults with TBI, especially at the cutoff level of 4 or more often associated with poor health outcomes in other populations. These preliminary results failed to show associations between ACEs and neuropsychological outcomes following TBI. A much larger sample will be required to better characterize ACEs among adults with TBI and their association with outcomes.

Correspondence: Srivatsan D. Uchani, Bachelor of Arts (BA), Physical Medicine \& Rehabilitation, University of Pittsburgh Medical Center (UPMC), 5525 Ellsworth Avenue, Pittsburgh, PA 15232, United States. E-mail: uchanisd@upmc.edu

D.M. UKUEBERUWA, D. THRASHER, C. YOU \& R. ASARNOW. Baseline Personality and Psychopathology Symptom Reporting of Veterans within a TBI/PTSD Program.

Objective: Empirical investigation of psychological functioning of Veterans with history of military service-related traumatic brain injury (TBI) and posttraumatic stress disorder (PTSD) is needed in a treatment-seeking context to better inform treatment decisions and understanding of treatment response. In the present study, we particularly sought to characterize symptom reports of Veterans who produced invalid psychological assessment profiles, historically considered overreporting of symptoms, within this context.

Participants and Methods: A sample of 62 Operation Iraqi Freedom/ Operation Enduring Freedom (OIF/OEF) Veterans referred for consideration within an intensive outpatient treatment program for history of mild to moderate TBI and PTSD were administered a neuropsychological evaluation that included the Minnesota Multiphasic Personality Inventory 2 Restructured Form (MMPI-2-RF). The profile validity cutoff was set at $\mathrm{f}-\mathrm{r}<99$. ANOVA was used to assess group differences in Restructured Clinical and Substantive Scale elevations between Veterans with valid and invalid profiles.

Results: 31 Veterans formed a group with Valid MMPI-2-RF profiles. 31 Veterans in the Invalid group reported significantly more symptoms $(p<.05$; Invalid group scale elevation T $>65)$ on RC1 Somatic, RC2 Low
Positive Emotions, RC7 Dysfunctional Negative Emotions, and RC8 Aberrant Experiences, in addition to the Helplessness/Hopelessness, Inefficacy, Cognitive Complaints, Anxiety, Aggression, and Disaffiliativeness Substantive Scales.

Conclusions: OIF/OEF Veterans frequently produced invalid MMPI2-RF symptom profiles within a diagnostic evaluation for a TBI/PTSD treatment program. Over-reporting occurred largely with internalizing dysfunctional emotion scales, but also occurred on somatic/cognitive and interpersonal functioning scales.

Correspondence: Dede M. Ukueberuwa, Ph.D., University of California Los Angeles, 760 Westwood Plaza C8-749, Los Angeles, CA 90095, United States.E-mail: dedemu@gmail.com

\section{E. VACCARIELLO, S. ROLIN \& J. DAVIS. Examining the Cognitive} Proficiency Index in Rehabilitation Patients.

Objective: This study examined the Cognitive Proficiency Index (CPI) of the Wechsler Adult Intelligence Scale-Fourth Edition (WAIS-IV) in relation to other WAIS-IV indices, overall test battery mean (OTBM), and test battery impairment (IMP) in an outpatient rehabilitation sample.

Participants and Methods: The sample $(N=329)$ was $35 \%$ female and $88 \%$ Caucasian with average age and education of $42.9(S D=13.5)$ and $13.6(S D=2.4)$ years, respectively. Participants were grouped by diagnosis and validity: traumatic brain injury (TBI; $n=187 ; 47 \%$ mild), stroke (CVA; $n=53$ ), other neurologic conditions (OTH; e.g.., anoxia: $n=47$ ), and questionable performance validity (QPV; $n=42$ ). OTBM was the average of up to 22 non-WAIS-IV tests; IMP was dichotomously defined as four or more test scores below cutoff $(\leq 35 \mathrm{~T})$.

Results: Significant group differences were observed on CPI and other WAIS-IV indices. OTBM was significantly different between groups, F $(3,325)=18.6, p<.001$. On post-hoc analysis (Sidak) the TBI group $(M=91.4, S D=10.0)$ scored higher than the CVA group $(M=84.7$, $S D=11.9)$; the OTH group $(M=87.9, S D=10.7)$ was not different from TBI or CVA groups; the QPV group $(M=78.9, S D=10.5)$ was lower than the other groups. IMP varied by group: TBI (44\%), CVA $(72 \%)$, OTH $(62 \%)$, and QVP $(90 \%), \chi^{2}(3)=36.5, p<.001$. Multiple linear regression with OTBM as outcome and education, GAI, and CPI as predictors was significant overall, $\mathrm{F}(3,283)=153.8, p<.001$, $R^{2}=.62$. CPI made a significant contribution to the model $(\beta=.40$, $p<.001)$. Nested logistic regression models with IMP as the outcome and GAI and CPI as predictors were fitted. The model with GAI classified $71 \%$ of cases with area under the curve (AUC) of .80. The model with GAI and CPI classified 74\% of cases with AUC of .88.

Conclusions: CPI showed incremental validity in predicting OTBM and IMP. CPI warrants further study as a clinically useful addition to other WAIS-IV indices.

Correspondence: Eleonora Vaccariello, BSc, Salt Lake City, UT, United States.E-mail: evaccariello12@gmail.com

R.S. VASUDEVAN, M.S. HERBERT, S.M. JURICK, N.E. DEFORD, A.V. KELLER, S.N. HOFFMAN, M. LEE, M. SANDERSON-CIMINO \& A.J. JAK. Examination of Symptom Over-Reporting and SelfReported Pain and Depression in Iraq and Afghanistan Veterans with Mild Traumatic Brain Injury.

Objective: Pain, depression, and mTBI are highly prevalent and comorbid in Veterans. The relationship between these conditions is unclear; TBI may be directly associated with pain or only when mental health conditions are present. Persistent symptoms following mTBI and chronic pain are associated with elevated somatic focus and symptom over-reporting, so it is important to understand how symptom over-reporting impacts the relationship between pain, mood, and mTBI.

Participants and Methods: 42 OEF/OIF Veterans with history of mTBI completed self-report measures of post-concussive symptoms, depression, and pain. Veterans were separated into possible symptom over-reporting (SE; $n=14$ ) or non-over-reporting ( $\mathrm{NE} ; \mathrm{n}=28$ ) groups using a cutoff score of 13 on the Validity-10 ( $\mathrm{V}-10)$, an embedded 
measure of symptom over-reporting in the Neurobehavioral Symptom Inventory. Group differences in pain and depression were examined via t-tests and linear regression.

Results: The SE group reported significantly greater depression symptoms, pain severity, pain catastrophizing, and pain interference (all $p ' s<0.05$ ) than the NE group despite no group differences on TBI injury variables. The relationship between depression and pain catastrophizing differed by group in a model containing pain severity. This relationship became non-significant in the NE group after controlling for pain severity $(r=0.219 ; p=0.292)$, but remained significant in the SE group $(r=0.778 ; p=0.002)$.

Conclusions: History of mTBI and elevated V-10 scores were associated with higher pain and depression symptoms, but in the SE group, the relationship between pain catastrophizing and depression was independent of pain intensity. Data suggest a general pattern of elevated symptom reporting in those with a history of mTBI and high V-10 scores where interpretation of symptoms may play a larger role in presentation and outcomes than objective markers of TBI or pain. Treatments for pain may need to be augmented to address pain catastrophizing in those with symptom over-reporting.

Correspondence: Rajiv S. Vasudevan, B.S. Physiology/Neuroscience, Psychiatry, University of California, San Diego, 3435 Lebon Drive \#1119, Palo Alto, CA 92122, United States. E-mail: rajüsvasudevan@ gmail.com

A. WALKER, E. WAITE, W. LOPEZ, D.J. HARDY, K.B. BOONE, D. PLURAD, P. VESPA, D. MCARTHUR, D. HOVDA, E. WOO, J. FUSTER \& M.J. WRIGHT. Use of Performance Validity Tests in Acute TBI.

Objective: To determine the utility of performance validity tests (PVT) for moderate-to-severe TBI participants at 6 months post-injury.

Participants and Methods: The study included 34 healthy controls, 21 moderate-to-severe TBI participants that were 6 months post-injury, and 27 chronic (12 months or more post-injury) moderate-to-severe TBI participants. All participants provided voluntary consent to this IRB approved study that included the administration of a brief neuropsychological battery. The PVTs that were used were the Dot Counting Test (DCT) and the Rey Fifteen Item Test plus a recognition trial (FIT+). Only participants with valid performances (PVT failure $<=1$ ) were included in this study.

Results: While participant groups were well matched in education, sex, ethnicity, and race, the chronic TBI group was significantly older than the control group $(p=.024)$, although no age difference between the acute TBI group and the other groups was detected. ANOVAs demonstrated that the controls showed better memory $(p=.001)$ and attention $(p=.003)$ performances on demographically adjusted domain T-scores in contrast to both TBI groups; the TBI groups did not differ from each other. With regard to the DCT and the FIT+ scores, ANCOVAs, correcting for age, showed that the groups did not differ $(p=.566 \&$ $p=.096$, respectively).

Conclusions: Our data provide preliminary support for the use of PVTs in TBI participants that are at least 6 months post injury.

Correspondence: Andrew Walker, CA, United States. E-mail: andrew. walker@pepperdine.edu

M.J. WALSH, S.F. SORG, A.L. CLARK, K.A. HOLIDAY, N.D. EVANGELISTA, L. DELANO-WOOD \& D. SCHIEHSER. Regional Gray Matter Volumetric Differences Predict Fatigue Symptoms in Veterans with Mild Traumatic Brain Injury.

Objective: Fatigue is a common complaint of military Veterans following mild traumatic brain injury (mTBI); however, the etiologic basis of fatigue remains poorly understood. Given that studies across other neurological disorders (e.g., multiple sclerosis, Parkinson's disease) have shown that greater levels of fatigue are associated with reductions in gray matter volume, we sought to clarify whether gray matter volumes are associated with self-reported fatigue in Veterans with history of mTBI

Participants and Methods: 106 Veterans $(\mathrm{mTBI}=$ 53, demographically-matched Veteran Controls $[\mathrm{VCs}]=53$ ) completed the Modified Fatigue Impact Scale (MFIS) and underwent structural MR imaging. Cortical and subcortical gray matter volumes were extracted from parcellated and segmented regions of interest (ROIs) using FreeSurfer. Analyses of Variance were conducted to evaluate group differences in MFIS scores and regional grey matter volumes. A hierarchical regression was conducted to determine whether there was a significant relationship between fatigue and significant regional gray matter volumes.

Results: Compared to VC, mTBI Veterans endorsed significantly higher levels of fatigue on the MFIS $(\mathrm{p}<.001)$ and evidenced reduced right nucleus accumbens (NA; $p<.001)$ volume as well as trend reduction of volume in the right rostral anterior cingulate (rACC; $p=.057)$. Reduced right NA volume $(p=.001)$ significantly predicted greater levels of fatigue, while the right rACC $(p=.064)$ showed a similar trend.

Conclusions: Our results demonstrated that higher levels of selfreported fatigue were predicted by reduced regional gray matter volumes in participants with a history of post-acute mTBI. These findings suggest that damage to specific brain regions may play a role in the manifestation of fatigue symptoms in the context of mild neurotrauma. Further studies must determine whether regional gray matter volumes are differentially associated with cognitive and physical fatigue, and whether mechanism of injury plays a role in outcomes.

Correspondence: Michael J. Walsh, Veterans Administration San Diego Healthcare System, 3350 La Jolla Village Dr., La Jolla, CA 92161, United States.E-mail:mjwalsh@ucsd.edu

A.L. WARE, B. BIEKMAN, R. HACHEY, M. MACLEOD, W. BIRD, E. CLARKE, A. BORRASSO, P. MORETTI, A. PUCCIO, K. GALVIN, K. POMIECKO, S.R. BEERS, H.S. LEVIN, J.M. FLETCHER, W. SCHNEIDER, D.O. OKONKWO \& E.A. WILDE. High-Definition Fiber Tracking Study of the Executive Control Network in BlastRelated Traumatic Brain Injury.

Objective: Blast-induced traumatic brain injury (bTBI) is common in Iraq and Afghanistan war veterans. However, subtle neural alterations and lack of a definitive biomarker impede clinical detection. The current study evaluated structure and functional correlates of executive control network (ECN) white matter tracts in veterans with and without bTBI to investigate the clinical utility of using High Definition Fiber Tracking (HDFT) to identify a biomarker of chronic bTBI.

Participants and Methods: Male veterans $(N=38)$ between 24 and 50 years old completed a standardized neuropsychological evaluation that included the Stop Signal Task and structural magnetic resonance imaging. HDFT was used to derive quantitative metrics of tracts between the dorsolateral prefrontal cortex (DLFPC) and the putamen, caudate, and thalamus.

Results: Relative to the comparison group, moderate to strong effects indicated that bTBI was associated with: elevated quantitative anisotropy $(\mathrm{QA})$ and reduced right hemisphere volume across tracts; reduced right DLFPC-putamen tract count and greater generalized fractional anisotropy (GFA); greater right DLPFC-thalamus tract count. A strong Group $\times$ Age interaction effect was observed on DLPFC-caudate tract count. Groups had similar task performance, which strongly correlated with density metrics across tracts in the comparison group; go and stop signal reaction time correlated positively with $\mathrm{QA}$ and negatively with tract volume and count; commissions correlated negatively with QA. Conclusions: Results support anomalous density and integrity of ECN tracts in bTBI, particularly in the right putamen and thalamus tracts. Veterans with bTBI showed worsening DLPFC-caudate density with older age. Although ECN dysfunction was not apparent via behavioral testing, better performance related to higher QA and lower tract count and volume in the comparison group. Spared ECN function, despite anomalous microstructure, could indicate functional compensation in bTBI, although alternate interpretations are being explored. 
Correspondence: Ashley L. Ware, M.A., Psychology, University of Houston, 2003 Southgate Blvd, Apt 2, Houston, TX 77030, United States.E-mail:aware2004@gmail.com

A. WEICHSELBAUM. Revealing Subtle Cognitive-Linguistic Differences in Adults with Mild Traumatic Brain Injury Through Discourse Analysis.

Objective: In mild cases of traumatic brain injury, cognitive deficits may not be detected by neuropsychological assessment measures. To diagnose a subtle cognitive disorder, it is necessary to elicit behaviors which require use of complex cognitive skills which require multiple skills such a linguistic, attentional, memory, and executive functioning domains. Discourse measures may be more sensitive. This study differs from previous work on discourse production after TBI because of the focus on mTBI with persisting symptoms, and yet demonstrate similar scores on neuropsychological assessments to controls. The purpose of this study is to examine whether macro/microlinguistic characteristics of discourse reveal cognitive deficits which are not apparent using neuropsychological assessment measures.

Participants and Methods: Participants were 6 adults without history of neurological damage, 5 adults with mild CHI, and 2 adults with severe TBI. Cognitive tests were administered to test working memory, cognitive control, processing speed, mood, and vocabulary. Additionally, participants performed a discourse task using an elicitation discourse production task, "Trip to New York".

Results: A one-way ANOVA was conducted to determine a difference of participant (TBI vs. non-TBI) on propositional density without fillers (PDWOF). The result showed a significant difference between TBI (M: .49, SD: .02) and control (M: .52, SD: .02), $F(1,9)=6.33, p=.03$. Results indicate a lower PDWOF on TBI group despite having no significant difference on cognitive assessments. Conclusions:

The findings of this study support the idea that discourse analysis may be a

useful tool for determining subtle, cognitive-communication deficits in the TBI population that common neuropsychological tests may not detect. Such information may be important clinically for validating persisting cognitive symptoms and guiding effective therapy goals. Linguistic disruptions may reveal cognitive impairments due to diffuse damage of the brain.

Correspondence: Amanda Weichselbaum, Clinical Fellowship , 1425 N Milwaukee Ave, Libertyville, IL 60048, United States. E-mail: alweichselbaum@gmail.com

WITHDRAWN. C. WENDEL, E. SCHENK ZU SCHWEINSBERG \& A. BATEMAN. LONG-Term Outcome After Stroke: What Contributes to Participation?

Objective: We followed a cohort of stroke patients over a period of four years to assess their partieipation (ICF), psychologieal resourees and correlation between these variables over time. We also set ext to provide a German version of a reliable, IGF-based instrumen of or of all nine life domains as defined by the ICF.

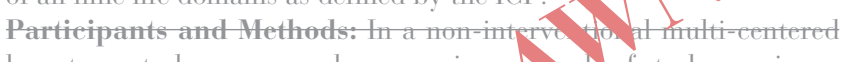

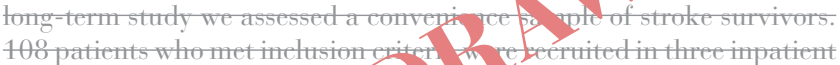
rehabilitation clinies. Postal th eys whe then conducted 4 - 6 months (twice, due to validation tा age $63,3 \pm 12,1$ × a rat $(c-2-90), 2$ years $(13, \mathrm{n}=66)$ and 4 years $(t 4, \mathrm{n}=56)$

For all 3 th tren nents, we used MPACT-S (ICF Meastre of Partieipation ant Neifivities questiomare - Sereener, Post et al. 2008 ). for 13 and 14 we also meastred Depression and Anxiety by HADS-D (Hermann-Lingen et al. 2011) and Resilienee by RS-13 (German short version of $\mathrm{PS}-25$. Koetret al. 2008 ).
Results: The German translation of IMPAGT-S proved to be a concep= tually and psychometrieally profound questiommaire (good internal eonsistency, test-retest reliability and eonvergent validity). Degree of participation was prectomninantly stable over time and medium to strongly interrelated to depression $(t 4, \mathrm{r}=-.71)$, anxiety $(\mathrm{t} 4, \mathrm{r}=-.52)$ and resilience $(t 4, p=.62)(p<0.01$ for all). One third of the cohort had ant unfavorable outeonte withe elintieally retevant depression and antiety at to with a tendener of worsenime at 14 .

Gonelusions: Most stroke patients approached a reasonable level of partieipation. At the same time, about one third of the cohort dealt with a chromic psychological burden which was strongly interconnected to partieipation-related outeome. There is a pressing need for strategies to identify and speeifieally treat affeeted patients. The late story is the true stomy and therefore deserves our stromgest level of comminiment to turn into a goots story.

Gorrespondence: Claudia Wendel, Psychology of Rehabilitation, University of Applied Seientes Magdeburg-Stendal, Osterburger Str. 25,

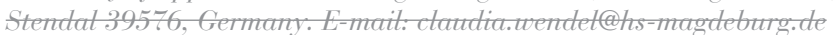

M.J. WRIGHT, W. LOPEZ, A. WALKER, F. MARTINEZ, D.J. HARDY, D. PLURAD, P. VESPA, D. MCARTHUR, D. HOVDA, E. WOO \& J. FUSTER. Semantic Clustering Partially Mediates Encoding Deficits in Traumatic Brain Injury.

Objective: Previous work has indicated that poor encoding drives recall deficits for verbal material in traumatic brain injury (TBI). We sought to determine if reduced semantic clustering might underlie TBI-related encoding deficits.

Participants and Methods: Fifty TBI and 25 matched control participants. All participants provided voluntary consent to this IRB approved study that included the administration of the California Verbal Learning Test, Second Edition (CVLT-II). All participants passed performance validity testing. The Item Specific Deficit Approach (ISDA) was applied to the CVLT-II to obtain indices of encoding, consolidation, and retrieval deficits.

Results: The TBI group indicated encoding, consolidation, and retrieval deficits in contrast to the controls. A hierarchal regression for the prediction of delayed recall by ISDA deficit indices was significant, $F(3,46)=20.38, p<.001, R^{2}=.57$. Similar to previous research, encoding deficits $(b=-.75, t(46)=-5.59, p<.001)$ and consolidation deficits predicted $(b=-.46, t(46)=-3.11, p=.003)$ delayed recall in the TBI group, and encoding deficits accounted for the majority of the variance in contrast to consolidation deficits $\left(R^{2}=.48 \mathrm{vs}\right.$. $\left.R^{2}=.09\right)$. In the mediation analysis, the overall model was significant, $F(1,48)=43.50, p<.001, R^{2}=.48$. Semantic clustering and encoding deficits were related, $F(1,48)=11.05, p=.002, R^{2}=.19$. A model containing semantic clustering and encoding deficits was significant, $F(2,47)=28.57, p<.001, R^{2}=.55$, and semantic clustering $(b=.17)$ reduced the effect of encoding deficits on delayed recall $(b=-.73$ vs. $b=-.90)$. This effect was confirmed with a Sobel test, $Z=-2.02$, $p=.04, R^{2}=.22$.

Conclusions: These data indicate that poor semantic clustering plays a role in encoding deficits in TBI. However, semantic clustering does not fully account for memory encoding deficits in TBI. It is likely that processing speed may also account for some of the variance in encoding deficits in TBI survivors.

Correspondence: Matthew J. Wright, Ph.D., Psychiatry, Harbor-UCLA Medical Center, 1124 W. Carson St., B-4 South, Rm. 111 (Box 490), Torrance, CA 90502, United States. E-mail: mwright@labiomed.org

K.L. ZANE, P. ROSKOS, T.W. BUCHANAN, R. BUCHOLZ \& J. GFELLER. An Exploration of Neuropsychological Status and Diffusion Tensor Imaging Findings in Adults with Mild to Severe Chronic Traumatic Brain Injury.

Objective: Traumatic brain injury (TBI) can produce complex effects on brain network function. Previous studies have examined relationships between cognitive impairment and white matter damage, such 
that greater white matter pathology predicts greater cognitive deficits. The present study further investigated the nature and strength of relationships between diffusion tensor imaging (DTI) findings and neurocognitive ability following chronic TBI.

Participants and Methods: Ninety participants were selected from a prior study sample that investigated attentional abilities in persons with TBI (Zane et al., 2016). Three groups of participants (i.e., healthy control, mild TBI, moderate or severe TBI) underwent DTI and neurocognitive testing (i.e., processing speed, attention, memory, and executive function). Composite cognitive domain scores were computed from individual test scores. Fractional anisotropy (FA) was the primary DTI measure for 15 a priori regions of interest (ROIs). Pearson's correlation coefficients between neurocognitive domains and DTI values were analyzed and confidence intervals were computed.

Results: Moderate to weak correlations were demonstrated between nearly all ROIs and the neurocognitive domains. Positive correlations were found between the processing speed composite and FA values for 14 of 15 ROIs, and between the memory composite and FA values for 12 of 15 ROIs. Positive, yet less robust correlations were found between the executive function composite and FA values.

Conclusions: The study findings add to the understanding of chronic TBI with regard to neurocognitive status and axonal integrity across the spectrum of brain injury severity. The study builds upon the foundation of findings that utilize contemporary neuroimaging techniques with well-established neuropsychological measures of processing speed, attention, memory, and executive function (National Institute of Neurological Disorders and Stroke: Common Data Elements, 2016).

Correspondence: Katherine L. Zane, Ph.D., Psychology, Saint Louis University, 1900 W Dickens Ave, Apt. 2R, Chicago, IL 60614, United States.E-mail: zanekl@slu.edu

K.L. ZANE, P. ROSKOS, T.W. BUCHANAN, R. BUCHOLZ \& J. GFELLER. An Examination of Neuropsychological Performance in Adults with Mild to Severe Chronic Traumatic Brain Injury.

Objective: Research has consistently found cognitive impairment in persons who sustain a traumatic brain injury (TBI). Some of the most commonly reported cognitive deficits include attention and processing speed, memory, and executive functioning. The present investigation examined these cognitive domains in adults with chronic TBI of varying injury severity.

Participants and Methods: Ninety participants (Zane et al., 2016) were selected from a larger study sample that investigated effort testing and neuropsychological functioning in persons with TBI (Gfeller \& Roskos, 2013). Three groups (i.e., healthy control, mild TBI, moderate or severe TBI) completed a neuropsychological evaluation including: California Verbal Learning Test-II; Conners' Continuous Performance Test-II; Rey Complex Figure Test; Stroop Color Word Test; Trail Making Test; Wechsler Adult Intelligence Test-III; Wisconsin Card Sorting Test, a verbal fluency task, and a cognitive screening measure. One-way multivariate analyses of variance and follow-up univariate comparisons were conducted to determine group performance differences.

Results: One-way MANOVAs yielded main effects on measures processing speed $[p=.004]$; attention $[p=.002]$; memory $[p=.002]$; and executive functions $[p=.000]$. Group differences were found on almost all neurocognitive measures, with the moderate or severe TBI group differing from the controls and to a lesser extent from the mild TBI group. The mild TBI group only differed from the controls on one test of mental processing speed.

Conclusions: These findings indicate that several neurocognitive abilities were compromised in persons with moderate to severe TBI but generally preserved in mild TBI. Specifically, measures of memory and executive function demonstrated the most notable group differences. The essentially non-significant findings in the mild TBI group are consistent with prior literature indicating most persons with mild TBI exhibit minimal impairment in neurocognitive abilities (McCrea et al., 2009).
Correspondence: Katherine L. Zane, Ph.D., Psychology, Saint Louis University, 1900 W Dickens Ave, Apt. 2R, Chicago, IL 60614, United States.E-mail: zanekl@slu.edu

\section{Acquired Brain Injury \\ (TBI/Cerebrovascular Injury \& Disease - Child)}

J.B. DYKSTRA, K. MAYS, K. MCNALLY, D. BODIN, K.E. PATRICK, K. KLAMAR \& J. SUHR. Adolescent maladaptive illness beliefs predict prolonged concussion recovery.

Objective: The aim of this study was to investigate the impact of maladaptive illness beliefs on subsequent recovery from concussion.

Participants and Methods: Participants were 67 adolescents (ages 12-18) and their parents who presented to a multidisciplinary concussion clinic for an initial visit. Participants completed adapted versions of the Illness Perception Questionnaire (IPQ), which assessed for negative expectations regarding concussion recovery. Adolescents also completed Trails A and B and Digit Span to screen for cognitive deficits. Demographic variables, injury factors, and recovery duration were obtained through chart review. Participants were dichotomized into favorable and prolonged recovery outcome groups with prolonged recovery defined by symptom persistence $>90$ days and/or physician determination that referral to a higher level of service was needed.

Results: Parent and adolescent IPQ scores were positively correlated on 4 of the 6 subscales, $r$ s between .33 and $.50, p s<.01$. Binary logistic regressions revealed that maladaptive adolescent beliefs on 4 subscales predicted whether the adolescent would experience a prolonged concussion recovery, even after controlling for demographic and injury variables, ORs between 1.3 and $1.4, p s<.02$. Belief that recovery will take a long time, that consequences of the concussion will be severe, that one is distressed by the concussion, and that engaging in cognitive efforts will compromise recovery (Cogniphobia) predicted prolonged recovery. Parent IPQ scores were not predictive. Adolescent Cogniphobia scores were negatively related to overall performance on Digit Span, $r=-.30$, $p<.05$, and Trails B, $r=-.29, p<.05$.

Conclusions: Negative expectations regarding recovery were predictive of prolonged recovery course and were related to poorer performance on neuropsychological screening in a sample of adolescents who sustained a concussion. These findings highlight the importance of identifying early non-injury factors that may complicate concussion recovery.

Correspondence: Jana B. Dykstra, Ph.D., Psychology, Nationwide Children's Hospital, 105 E, Columbus, OH 43206, United States. E-mail: jana.dykstra@gmail.com

B.D. ESCHLER, J.J. MIETCHEN \& S.D. GALE. Internalizing and Externalizing Symptomatology Following Pediatric Traumatic Brain Injury: A Meta-Analysis.

Objective: A meta-analysis was conducted to determine the average effect size of internalizing and externalizing symptomatology following pediatric traumatic brain injury across a range of injury severity.

Participants and Methods: 3721 titles and abstracts were collected from PubMed, PsycInfo, and Web of Science. Included studies were published before March 2017 in English, included a control group (orthopedic injury or typically developing peers), and a reliable and valid measure of internalizing and externalizing symptoms in children. Studies were excluded for non-accidental head injuries or if the sample recruited was outside the range of 2-17 years old at the time of injury. The analysis was based on 15 studies including 952 cases of mild TBI, 179 cases of moderate TBI, 162 cases of severe TBI, and 1536 control cases.

Results: Analyses revealed a large effect size for internalizing symptoms of children with mild TBI (Hedge's $g=-.720, p=.004$ ), a small effect size for moderate TBI (Hedge's $g=-.273, p=.019)$, and a moderate effect size for severe TBI (Hedge's $g=-.647, \mathrm{p}<.001$ ). These findings indicate that following a TBI children are more likely to exhibit internalizing 
problems such as anxiety and depression regardless of TBI severity. For externalizing symptoms, analysis demonstrated a moderate effect size for mild TBI (Hedge's $g=-.547, p=.005$ ), a small effect size for moderate TBI (Hedge's $g=-.278, p=.006$ ), and a large effect size for severe TBI (Hedge's g=-.969, $\mathrm{p}<.001)$. Thus, children who experienced a TBI demonstrated externalizing symptoms including hyperactivity and impulsivity with the largest effect size associated with severe TBI. Conclusions: Using meta-analytic methods, we found that children who experience traumatic brain injury are at greater risk for exhibiting internalizing and externalizing symptoms across levels of severity. However, for externalizing symptoms, the effect size was largest for the severe TBI group and this effect size was larger than the effect sizes found for internalizing symptoms.

Correspondence: Benjamin D. Eschler, M.S., Psychology, Brigham Young University, 907 E 820 N \#9, Provo, UT 84606, United States. E-mail:benjamineschler@gmail.com

N.J. GOODRICH-HUNSAKER, T.J. ABILDSKOV, K.O. YEATES, H. TAYLOR, A. BACEVICE, D. COHEN, L. MIHALOV, B. BANGERT, N. ZUMBERGE \& E.D. BIGLER. Integrity of the corpus callosum predicts outcomes of pediatric mild traumatic brain injury.

Objective: To determine if the integrity of the corpus callosum as measured by diffusion tensor imaging (DTI) is associated with post-concussive symptoms (PCS) in children with mild TBI.

Participants and Methods: As part of an ongoing study, participants were children 8-15 years of age with mTBI or mild orthopedic injuries (OI). DTI scans were obtained within 10 days of injury. We performed deterministic tractography using Automated Fiber Quantification (AFQ) to reconstruct the corpus callosum. The corpus callosum was segmented into 8 sections. Within each section, average fractional anisotropy (FA) across the entire fiber tract was extracted for each individual. Children and their parents rated PCS at 10 days post injury.

Results: Parent- and child-rated somatic symptoms were significantly higher in the mild TBI group. The mild TBI and OI groups did not differ in mean FA within any of the 8 corpus callosum segments. Within the orbital and superior frontal and superior and posterior parietal segments, lower FA was significantly related to child-rated somatic symptoms for the OI group, but not for the mild TBI group. There was also a statistically significant difference between the OI and mild TBI correlations.

Conclusions: These preliminary findings suggest that the integrity of the corpus callosum may be altered by mTBI. Although the groups did not differ significantly in FA, a significant association of lower FA with higher PCS in the OI group was not evident in the mTBI group, suggesting that mTBI disrupts typical brain-behavior relationships and raising the possibility of aberrant neural organization.

Correspondence: Naomi J. Goodrich-Hunsaker, PhD, Psychology, Brigham Young University, PO Box 971060, Orem, UT 84097, United States.E-mail:naomi.hunsaker@byu.edu

\section{A. HAIGHT, E. MCLEAN, H.M. GENOVA, A. OKUN-ALEXANDER \& J. LENGENFELDER. Exploring the relationship between social and empathetic performance in pediatric TBI.}

Objective: To examine the relationship between the Social Responsiveness Scale (SRS) and Empathizing (EQ-C) and Systemizing (SQ-C) Quotients in children with Traumatic Brain Injury (TBI) compared to typically developing children.

Participants and Methods: Participants consisted of thirteen children with a TBI, with a mean age of 12.54 (range 7-17), mean education 7.08 years (range $1-11$ ); $69 \%$ were male. Thirteen typically developing children were also included, with a mean age of 10.77 (range 7-16), mean education 5.15 (range 1-11); $54 \%$ were male. Upon approval of a background screen, participants and their guardian completed a battery from which the SRS, EQ-C and SQ-C were analyzed.

Results: Results comparing individuals with TBI and typically developing children indicate a significant main effect for the SRS Total Score,
$\mathrm{F}(1,24)=13.33, \mathrm{p}=.001$ indicating that children with TBI had greater social deficits than control children. Additionally, a main effect for EQ-C and SQ-C Total score, F $(1,24)=9.59, p=.005$ was found, indicating children with TBI had significantly poorer ratings of empathy compared to control children. Further, a significant correlation was found between SRS Total Score and EQ-C and SQ-C Total Score in pediatric TBI, $\mathrm{r}=-.602, \mathrm{p}=.030$, but not in typically developing children, $\mathrm{r}=-.401$, $\mathrm{p}=.174$.

Conclusions: Our results suggest that children with TBI display deficits in both social performance and empathy as compared to typically developing children. Previous research has established that children with TBI struggle socially and display problems with empathy. While the SRS, EQ-C and SQ-C have largely been investigated in Autism, the combination of these measures enables us to build upon the current literature by establishing a connection between empathy and social deficits. Understanding this relationship is critical for comprehending social functioning following TBI and determining potential treatments. Correspondence: Alison Haight, Kessler Foundation, 120 Eagle Rock Ave, Suite 100, East Hanover, NJ 07936, United States. E-mail: aph813@gmail.com

N. HASHEMIAN, P. FEDIO, E. SHEARIN \& G.A. GIOIA. Effects of Premorbid Attention-Deficit/Hyperactivity Disorder (ADHD) on Postconcussion Symptoms in Adolescents.

Objective: The combination of a concussion and a pre-existing neurological disorder amplifies the impairment of the residual level of concussion. The present study investigated the effects of an attention-deficit/ hyperactivity disorder (ADHD) in adolescents who subsequently sustained a concussion.

Participants and Methods: Archival data were selected from Children's National Medical Center Health System, Washington DC, and consisted of 351 adolescents (58.4\% male; ages 13 to 18 years; $M=15.46, S D=1.44)$ previously diagnosed with a concussion. The subjects completed a retrospective pre-injury baseline and post-injury PCSI test form. These scores were obtained with the Post-Concussion Symptom Inventory (PCSI-Child), at the SCORE clinic. The results were analyzed parametrically with a mixed model analysis of variance using repeated measures to examine within-subjects changes from pre to postconcussion periods (Concussion) and between-subjects group analyses for $\mathrm{ADHD}(n=87)$ vs. no ADHD (controls; $n=264$ ) and gender (Male vs. Female).

Results: Adolescents in the ADHD group reported a significantly higher number of pre- and post-injury symptoms than adolescents without ADHD. The effects for ADHD and Concussion $x$ Gender were both significant, $F(1,347)=6.767, p=.010$, and $F(1,347)=18.043, p<.001$, respectively. Concussed girls and boys with ADHD differed in their reported symptom expressions with girls reporting higher rating of symptoms postconcussion.

Conclusions: ADHD superimposed on a concussion during adolescence significantly adds to the resultant cognitive and emotional deficits. Adolescents with ADHD self-reported more pre- and post-injury symptoms overall. Females and males also differed in the level of symptoms: females reported higher post-concussion symptoms than male participants. Thus, females with combined $\mathrm{ADHD} /$ concussion were at a higher risk for emotional and cognitive dysfunctioning. Preconcussive effects of ADHD may impose an additive impairment on the cognitive and emotional deficits related to concussions.

Correspondence: Nicole Hashemian, Psy.D., Clinical Psychologyl Neuropsychology, American School of Professional Psychology, 8220 Crestwood Heights Dr, APT 1402, McLean, VA 22102, United States. E-mail:nnhashemian@gmail.com 
K. HASSARA, E.C. MAXWELL, T.J. BERNARD \& R. BOADA. Language Outcomes in Children with Arterial Ischemic Stroke. Objective: Language skills tend to be preserved in children with diffuse acquired brain injury, but language recovery in children with focal arterial ischemic stroke (AIS) has been less well characterized. We evaluated language outcomes in a large cohort of children with AIS, hypothesizing that left hemisphere strokes and the presence of acute aphasia will result in poorer language performance.

Participants and Methods: 83 children who suffered an AIS during childhood (mean age at AIS $=9.19$ years) were administered the Vocabulary subtest from the Wechsler Abbreviated Scale of Intelligence-II, Boston Naming Test, and the Sentence Repetition and Token Test subtests of the Multilingual Aphasia Examination. Testing was completed at least 10 months post-AIS. Analyses were conducted using ANOVA.

Results: Children with AIS had significantly lower performance on all language measures compared to the normative sample (all $p$-values $<0.01)$. Aphasia at the time of stroke significantly predicted poorer scores on WASI-II Vocabulary $(p=0.014)$, and trends for poorer performance on the Boston Naming Test $(p=0.066)$ and on the Token Test $(p=0.053)$. Left hemisphere involvement (left or bilateral strokes) significantly predicted lower performance on the Token Test, $F(1,72)$ $=4.725, p=0.033$, when compared to right hemisphere only strokes. Conclusions: Contrary to the plasticity hypothesis, poorer language outcomes were noted in children with focal AIS compared to the normative sample. Presence of aphasia at the time of stroke predicted worse vocabulary scores and a trend for worse confrontation naming and language comprehension. Unexpectedly, laterality did not reveal clear discrepancies in language performance; however, those with any left hemisphere involvement had poorer language comprehension. Possible mechanisms for these findings are discussed. These results support the need for speech therapy following childhood stroke, particularly for children with acute aphasia.

Correspondence: Kathleen Hassara, Children's Hospital Colorado, 1301 S.Wolff St., Denver, CO 80219, United States.E-mail: kecarlson7@ gmail.com

\section{W. HUANG. Neurocognitive Functions and Psychosocial Adaptions} In Survived Children With Posterior Fossa Tumor.

Objective: Medulloblastoma (MB) and Pilocytic Astrocytoma (PA) are the common cerebellar tumors in childhood. Survived children always confront various learning, interpersonal and vocational problems after medical treatment. The study investigates neuropsychological outcomes of survived children as foundation of intervention programs.

Participants and Methods: Survived MB/PA children were recruited by pediatric neurosurgery outpatient. Neurocognitive functions (i.e., intelligence, perceptual organization, memory, attention and executive function) and psychosocial adaptions (i.e., adaptive behavior and quality of life) were evaluated. We analyze results according to tumor types (malignant v.s. benign) and treatment strategies (dosage of radiation).

Results: The findings show that: (1) survivors have at least one specific cognitive deficit; (2) MB (malignant tumor) perform worse outcomes compared to PA (benign tumor), especially in processing speed, verbal/ visual memory and sustained attention; (3) longer follow-up duration would predict decreased FSIQ and QOL.

Conclusions: Cerebellar tumors implicate not only in movement and coordination, but also in cognitive and affective behaviors associated with numerous complications and difficulties in real life. More importantly, longtern follow-up and individualized intervention resulted from developmenatal age are necessary for survived children.

Correspondence: Wan-Ru Huang, Department of Physical medicine \& Rehabilitation, Taipei Veterans General Hospital, No.201, Sec. 2, Shipai Rd., Beitou District., Taipei City 11217, Taiwan. E-mail: ryouchibaby@hotmail.com
L.M. KOEHL \& D.Y. HAN. Irritability and Aggression as Indicators of Concussion Symptoms in a Pediatric Population.

Objective: Documented incidence rates of pediatric concussions have increased in recent years. Research has indicated that pediatric populations often display concussion symptoms longer than their older counterparts increasing the need for swift diagnosis and intervention. However, considerable heterogeneity in symptom presentations and psychosocial factors that hinder self-reports often make identification of concussion symptoms difficult. The current research examines irritability/aggression as a potential marker for ongoing concussion symptoms due to the observable nature of irritability that may signal continued difficulties to caregivers and healthcare providers.

Participants and Methods: Participants consisted of a pediatric group of 196 children between the ages of 6 and 17 (Mage $=14.08, \mathrm{SD}=2.65)$ who underwent neuropsychological evaluation following a concussion diagnosed by a physician. Participants were split into two groups: a control group that denied irritability/aggression (CONT) and a clinical group that endorsed irritability/aggression (AGG).

Results: No significant differences were found between groups in age, race, gender, or education. Additionally, endorsement of irritability/aggression did not differ by injury cause $\left(X^{2}=2.79 ; p=.593\right)$. Overall, AGG participants endorsed significantly higher rates of fatigue $\left(X^{2}=32.36 ; p=.000\right)$, disordered sleep $\left(X^{2}=27.10 ; p=.000\right)$, headache $\left(X^{2}=6.46 ; p=.001\right)$, and vertigo/dizziness $\left(X^{2}=23.72 ; p=.000\right)$. Conclusions: These findings suggest that increased aggression/irritability following a concussion may be indicative of ongoing symptomatology. Clinically, this indicates that mood monitoring is likely useful in identifying children who are at risk for persistent concussion symptoms and associated difficulties.

Correspondence: Lisa M. Koehl, Ph.D., Neurology, University of Kentucky, 4637 Honeycomb Trail, Lexington, KY 40509, United States. E-mail:lkoehl13@gmail.com

J.W. LACE, J. DORFLINGER, M.J. ROSS \& J. GFELLER. Convergent and Divergent Validity between ImPACT and CPT-3 in Adolescents with Mild Traumatic Brain Injury.

Objective: Research has reported construct validity for previous editions of Conners' Continous Performance Tests (CPTs; Conners, 2000) and the Immediate Post-Concussion Assessment and Cognitive Testing (ImPACT; Iverson et al., 2005) in separate samples. To date, no work has reported convergent and divergent validity between ImPACT and Conners' CPT, third edition (CPT-3; Conners, 2014) in a sample of adolescents with mTBIs.

Participants and Methods: Archival data from a Midwestern hospital included 83 adolescents seen for neuropsychological evaluation after mTBI. Sample demographics were: Mean age $=14.86 ; 53 \%$ male; $60 \%$ Caucasian. Measures were five scores from ImPACT (Verbal Memory, Visual Memory, Motor Control, Reaction Time, and Impulse Control) and four from CPT-3 (Omissions, Commissions, Hit Response Time, and Hit Rate Variability). Pearson's correlation coefficients were performed with significance level set at $p<.05$.

Results: Mean ImPACT (e.g., Visual Memory $M=49.77$ ) and CPT-3 (e.g., Commissions $M=50.05$ ) scores were within normal limits, suggesting that most participants did not demonstrate overt cognitive deficits associated with their injury. Selected CPT-3 variables significantly correlated with ImPACT Impulse Control ( $r$ s ranged from .30 to .47), and Motor Control ( $r$ s ranged from -.35 to -.26). CPT-3 Hit Response Time significantly correlated with ImPACT Reaction Time $(r=-.53)$. Nonsignificant correlation coefficients emerged between ImPACT memory composites and selected CPT-3 variables ( $r$ s ranged from -.15 to -.01$)$.

Conclusions: Significant correlations between measures of similar constructs (e.g.. ImPACT RT and CPT-3 HRT) and nonsignificant correlations between those of diverse constructs (e.g., ImPACT Verbal Memory and CPT-3 HRV) supported convergent and divergent validity for ImPACT and CPT-3. Future psychometric study on computerized 
measures of neurocognitive functioning in individuals with mTBIs should consider using ImPACT and CPT-3.

Correspondence: John W. Lace, MS, Psychology, Saint Louis University, 3700 Lindell Blvd, Suite 1200, St. Louis, MO 63103, United States. E-mail: lacejw@slu.edu

J.W. LACE, J. DORFLINGER, M.J. ROSS \& J. GFELLER. Relationships between Parent- and Self-Reported Executive Dysfunction in Adolescents with Mild Traumatic Brain Injury.

Objective: The Behavioral Rating Inventory of Executive Function (BRIEF; Gioia et al., 2000) completed by parents and its self-report equivalent (BRIEF-SR; Guy et al., 2004) have demonstrated significant correlations (Wilson et al., 2011). However, Byerley \& Donders (2013) found that parents reported greater BRIEF elevations on each domain, compared to adolescents self-report. The present study sought to replicate these findings in a sample of adolescents with mTBIs.

Participants and Methods: Archival data from a Midwestern medical center included 83 adolescents seen for neuropsychological evaluation after mTBI. Sample demographics included: Mean age $=14.86$; $53 \%$ male; $60 \%$ Caucasian. Measures included three indices from the BRIEF and BRIEF-SR (Behavioral Regulation Index [BRI], Metacognition Index [MI], and Global Executive Composite [GEC]). Pearson's correlation coefficients and paired-samples $t$-tests with Bonferroni stepdown corrections were performed.

Results: Results revealed significant correlations between bivariate pairs of BRIEF and BRIEF-SR indices ( $r$ s ranged from .35 to .52 , all $p$ s <.01). Parents' BRI $(M=47.43)$ and GEC scores $(M=49.13)$ were significantly greater $(p s<.05, d$ s ranged from .25 to .32$)$ than adolescents' self-reported BRI $(M=44.14)$ and GEC scores $(M=46.27)$. No significant differences were found between parents' MI scores $(M=$ $50.22)$ and adolescents' self-reported MI scores $(M=48.53)$.

Conclusions: Results from correlation coefficients suggested that as parents noted greater levels of executive dysfunction in their children, so did adolescents report greater levels in themselves. Results from paired $t$-tests indicated that parents reported higher levels of behavioral and overall executive dysfunction, but that parents and adolescents observed similar levels of metacognitive dysfunction. Factors that may account for these differences between parent and self-report of executive dysfunction symptoms are explored.

Correspondence: John W. Lace, MS, Psychology, Saint Louis University, 3700 Lindell Blvd, Suite 1200, St. Louis, MO 63103, United States. E-mail: lacejw@slu.edu

E. LEBLOND, J. SMITH-PAINE, J.J. RIEMERSMA, P.S. HORN, S.L. WADE \& B. KUROWSKI. Neurocognitive Outcomes of Methylphenidate Treatment for Secondary Attention Deficit Hyperactivity Disorder Following Pediatric Traumatic Brain Injury.

Objective: To investigate the effects of methylphenidate on processing speed and sustained attention in children with Secondary ADHD (SADHD) following pediatric traumatic brain injury (TBI). TBI is a leading cause of childhood morbidity and can have pervasive longterm neurobehavioral consequences. Approximately $16 \%$ of children meet diagnostic criteria for SADHD 6-months post injury, almost twice the prevalence in the general population. While stimulant medication is commonly prescribed, there is limited and inconsistent evidence regarding its efficacy for SADHD.

Participants and Methods: 26 participants aged 6-17 years who were hospitalized for moderate and severe TBI participated in a randomized, double-blinded, placebo-controlled cross-over design. Participants were enrolled if parents endorsed > 6/9 symptoms on the Vanderbilt Attention Deficit Hyperactivity Disorder Parent Diagnostic Rating Scale. The WISC-IV Processing Speed Index (PSI) and Continuous Performance Test II (CPT II) were administered at baseline and 4- and 8-week follow-ups following titration of medication or placebo.
Results: WISC-IV PSI: Children performed better on the PSI during the methylphenidate condition than placebo condition $(t(18)=-2.55$, $p=.020)$. This effect was driven by differences on the Coding subtest $(t(18)=.024)$. CPT II: No differences in overall accuracy were found between the treatment conditions $(t(15)=-0.88, p=.391)$. However, children had significantly lower coefficient of variation $(t(14)=2.29$, $p=.038)$, reaction time $(t(32)=2.51, p=.017)$, and standard deviation of reaction time $(t(32)=2.42, p=.021)$ during the methylphenidate compared to placebo condition.

Conclusions: Attention problems are common following pediatric TBI and negatively impact school performance, social relationships, emotional well-being, and quality of life. Methylphenidate significantly improved children's processing speed and sustained attention suggesting its potential utility.

Correspondence: Elizabeth LeBlond, Cincinnati Children's Hospital Medical Center, 2926 Vaughn Street, Cincinnati, OH 45219, United States.E-mail: elizabeth.leblond@cchmc.org.

J. LEDOCHOWSKI, K. MCDONALD, T. WILLIAMS, M. DESORCHER \& R. WESTMACOTT. Mental Health Outcomes in Children with Unilateral Basal Ganglia Arterial Ischemic Stroke and Secondary Dystonia.

Objective: Dystonia is a movement disorder in which excessive, involuntary muscle contractions result in twisting or repetitive movements, or abnormal posturing. Up to $21 \%$ of children who sustain a stroke affecting the basal ganglia and/or thalamus go on to develop secondary hemi-dystonia. The basal ganglia and the pathophysiology of dystonia have been implicated in mood regulation, yet little is currently known about the mental health outcomes of this population. The objective of this study was to explore mental health outcomes in children with a unilateral arterial ischemic stroke (AIS) involving the basal ganglia and/ or thalamus with and without and secondary dystonia.

Participants and Methods: Participants were 110 children, 35 with dystonia (18 females, Mean age $=10.45)$ and 75 without dystonia $(22$ females, Mean age $=10.58)$ who sustained a stroke at 16 years of age or less. We examined a parent-completed questionnaire assessing behavioral and emotional concerns (Behavior Assessment System for Children [BASC]) and coded clinical neuropsychological reports for the presence of concerns relating to anxiety and depression.

Results: Mann-Whitney U analyses revealed that children with secondary dystonia had significantly higher scores on the Depression and Anxiety scales, and the Internalizing Index from the BASC, with effect sizes ranging from $\eta^{2}=0.04-0.05$. Differences were also found in clinical reports, with a greater number of children with dystonia showing mild to moderate or severe mental health concerns compared to children without dystonia, with effect sizes $\eta^{2}=0.14$ for depression and $\eta^{2}=0.10$ for anxiety.

Conclusions: These results suggest that children who develop secondary dystonia after a stroke involving the basal ganglia and/or thalamus are at risk for poorer mental health outcomes, which has direct clinical implications for treatment and indicates the importance of further investigation of mental health outcomes in this population.

Correspondence: Justine Ledochowski, MA, Psychology, York University, Behavioural Science Building, Room 101, 4700 Keele Street, Toronto, ON M3J1P3, Canada.E-mail: jledoch@yorku.ca

J. LENGENFELDER, H.M. GENOVA, C. BOBER, A. HAIGHT, A. OKUN-ALEXANDER \& N. CHIARAVALLOTI. Attention After Pediatric Brain Injury: Objective Assessment Versus Parent Report.

Objective: To examine attentional abilities in pediatric TBI using both objective and subjective measures.

Participants and Methods: Participants consisted of 13 individuals with TBI, (age: $\mathrm{M}=10.77, \mathrm{SD}=3.37$ ) and 13 typically developing children with no neurological injury (age: $\mathrm{M}=12.53, \mathrm{SD}=3.83$ ). Participants completed the WISC-IV Cancellation and Symbol Search subtests as 
part of a larger battery and parents were asked to complete the Behavior Assessment System for Children-2 (BASC-2).

Results: A significant difference was noted between children with TBI and typically developing children on both the WISC Cancellation $(\mathrm{F}(1,26)=6.68, \mathrm{p}<.05)$ and Symbol Search $(\mathrm{F}(1,26)=8.98, \mathrm{p}<.01)$ subtests, indicating children with TBI perform at lower levels than typically developing children in regard to attention, selective attention, and vigilance. A significant difference was additionally found on parent ratings of attention problems on the BASC-2 for children with TBI as compared with typically developing children $(\mathrm{F}(1,26)=15.63$, $\mathrm{p}<.001)$. Parents of children with TBI rated their children as having significantly more attentional problems then typically developing children. Furthermore, parent ratings on the attentional subscale of the BASC-2 were significantly correlated with the children's performance on Cancellation $(r=-.65, p=.02)$ and Symbol Search $(r=-.66, p<.001)$ only for the individuals with TBI.

Conclusions: Findings demonstrate a relationship between attentional problems identified via objective neuropsychological measures and attentional problems captured by parent report. While objective performance may not always correspond to subjective reports, the deficits seen in children with TBI on neuropsychological measures of attention may also be evidenced in their everyday behaviors such that parents report problems in attention. This relationship should be further elucidated to determine if interventions targeting attentional abilities impact both objective measures of attention as well as parant ratings of such abilities. Correspondence: Jean Lengenfelder, Kessler Foundation, 300 Executive Dr., Suite 70, West Orange, NJ 07052, United States. E-mail: jlengenfelder@kesslerfoundation.org.

J.R.M. LIU, E. LIMA, A. PATEL, K. HARTLINE, N. PAWLENKO \& A. HERRERA-HAMILTON. Association Between Depressive Symptoms in Post-Concussional Children and Adolescents and Missed School Days Following Concussion.

Objective: Limited published data exists on the effects of post-concussion depressive symptoms on the quantity of school days missed following a concussion in children and adolescents. The current study aims to assess the impact of post-concussion depressive symptoms as a predictor of total number of missed school days.

Participants and Methods: Participants. Children and adolescents ( $\mathrm{N}=118$ ) (ages 4.3-19.6; $\mathrm{M}=13.68 ; \mathrm{SD}=3.5 ; 53 \%$ male; 47\% female). Participants were from a diverse population (58\% Caucasian, $28 \%$ Latino, 8\% African American, 4\% Asian American, 2\% Native American). All participants were diagnosed with concussion and were receiving post-concussion treatment at Children's Hospital Los Angeles (CHLA). All patients were examined for neuropsychological testing due to persistent symptoms (e.g., headache). Method. Initial exam (3 days - 3 months post-injury) BASC-2 Depression scores were compared to total number of missed school days following concussion. All analyses were conducted in SPSS 21.0 (IBM, 2012).

Results: Two Simple Linear Regressions were conducted. Results indicated that the level of endorsed depression explained a significant amount of the variance in the number of school days missed post-concussion $\left(R^{2}=.211\right)$. As reported level of depression increased by one point, number of missed school days increased by .460 days, 95\% CI [.505, 1.304], $(p<.001)$. Additionally, level of endorsed baseline depression explained a significant variance in post-concussion depression scores $\left(R^{2}=.34\right)$. As reported level of pre-injury depression increased by one point, level of post-concussion depression increased by .583 points, $95 \%$ CI $[.472,1.15],(p<.001)$.

Conclusions: The results of the study support our hypothesis that post-concussion depressive symptoms predict total missed school days due to concussion. Findings suggest that depressive symptoms may be a mediating factor in concussion recovery and may have implications for baseline testing, return protocols, and concussion management.
Correspondence: Jessica R.M. Liu, M.A., Neuropsychology, Children's Hospital Los Angeles, 4650 Sunset Blvd., Attn: Anita Herrera Hamilton, Ph.D., ABPP-CN, Los Angeles, CA 90027, United States. E-mail: jess. liu.robinson@gmail.com

E. MCLEAN, A. HAIGHT, H.M. GENOVA, A. OKUN-ALEXANDER \&. J. LENGENFELDER. Empathy in Pediatric Traumatic Brain Injury (TBI).

Objective: To examine the Empathizing (EQ-C) and Systemizing Quotient (SQ-C) in children with traumatic brain injury (TBI) in comparison to typically developing children.

Participants and Methods: Participants consisted of thirteen children with TBI, with a mean age of 12.54 (range 7-17), mean education 7.08 years (range $1-11$ ); $69 \%$ were male. Thirteen typically developing children were also included, with a mean age of 10.77 (range 7-16), mean education 5.15 (range 1-11); 54\% were male. Upon approval of a background screen, participants and their guardian completed a battery from which the EQ-C and SQ-C were analyzed.

Results: Results indicate a significant main effect was found for EQ-C, $\mathrm{F}(1,24)=10.03, \mathrm{p}=.004$, and Total Score, $\mathrm{F}(1,24)=9.59, \mathrm{p}=.005$. Children with TBI were rated as having greater deficits in empathy than typically developing children. Results comparing the individual with TBI and typically developing children on the SQ-C were not significant, although there was a trend toward significance, $\mathrm{F}(1,24)=3.34, \mathrm{p}=.08$. Conclusions: Our findings suggest that children with TBI displayed poorer performance on empathetic abilities as compared to typically developing children. The EQ-C and SQ-C have been primarily used with children with autism and has not been previously utilized in pediatric TBI. Research investigating empathy in pediatric TBI is limited, especially applying standardized measures that directly evaluate empathy, such as the EQ-C and SQ-C. The current study provides evidence that empathic abilities are inferior in pediatric TBI as compared to typically developing children. Additional work is need to examine the nature of empathy in children with TBI and the impact of deficits in empathy on social relationships.

Correspondence: Erin McLean, M.A., Neuroscience/Neuropsychology Lab, Kessler Foundation, 317 Old Mill Rd., Spring Lake Heights, NJ 07762, United States. E-mail: eamclean814@gmail.com

\section{A.A. ROY, C. KOTERBA \& J.P. LUNDINE. Use of the Cognitive and Linguistic Scale (CALS) and the Functional Independence Measure for Children (WeeFIM) in a Pediatric Brain Injury Population.}

Objective: The WeeFIM and CALS are clinician-rated assessments to monitor recovery following acquired brain injury. The WeeFIM assesses level of independent functioning in mobility, self-care, and cognition, while the CALS quantifies cognitive and linguistic functioning. Recently, WeeFIM Enhancement Items were added to more closely assess emotion regulation, inhibition, safety, self-monitoring, response time, learning, initiation, and attention. No research to date has documented the use of the WeeFim Enhancement Items. The aim of this study is to examine the validity of the WeeFim Enhancement Items compared to other outcome measures used on an inpatient rehabilitation unit (WeeFim and CALS). Participants and Methods: Participants: Twenty-one children/adolescents (5-19yrs; 13 males) who sustained a moderate to severe traumatic brain injury and were admitted to inpatient rehabilitation (length of admission 4-44 days).

Methods: The CALS and WeeFim were administered at admission and discharge. A change score was calculated based on the difference between admission and discharge total scores for each measure and WeeFim Enhancement Items. Pearson correlations examined relationships between measures.

Results: All scores were highly correlated at admission (.52-.86) and discharge (.45-.84). Change scores revealed significant correlations between CALS total and emotion regulation (.49), response time (.62), and safety (.64) scores from the WeeFIM Enhancement Items. The WeeFIM total change scores were significantly correlated with only the self-monitoring (.56) WeeFIM Enhancement Item. 
Conclusions: Three of the WeeFIM Enhancement Item change scores are significantly correlated with the CALS total change score, suggesting that the Enhancement Items and CALS measure similar cognitive constructs. Only one Enhancement Item change score correlates significantly with the WeeFim Total change score, suggesting that the Enhancement Items and the WeeFim measure unique constructs. More research is needed to further validate Enhancement Items.

Correspondence: Ashley A. Roy, Ph.D., Psychology, Nationwide Children's Hospital, 114 Frankfort Sq, Columbus, OH 43206, United States.E-mail: ashley.roy@nationwidechildrens.org.

A. MAFFETT, A. FEDER, S. COURREGE \& R. SKEEL. Survey of Professionals' Recommendations for Rest following Concussion. Objective: Previous research has provided mixed information on the effectiveness of various treatment practices for youth concussion particularly the recommendations of activity restriction and rest. There are a host of recommended best practices but it is less clear as to what treatment practices are common among various professionals treating youth concussion. This current pilot study aims to survey various health professionals on their practices in the treatment of youth post-concussion symptoms.

Participants and Methods: An online questionnaire, distributed via professional email listservs, was used to gather information on the current practices recommended for youth following concussion. The survey consisted of Likert scale and free response items, This pilot study examined the survey results of 18 professionals surveyed from June 2017 to July 2017.

Results: Findings from this preliminary study indicate that professionals most frequently recommended cognitive rest (66\%), physical rest $(62 \%)$, and psychotherapy $(61 \%)$ for post-concussion treatments. The majority of respondents $(67 \%)$ recommended strict prohibition of alcohol and recreational drugs (73\%). Only 33\% of respondents recommended strict prohibition for playing sports. Symptom profiles (i.e., vestibular symptoms, cognitive symptoms, increased emotionality, and sleep disturbance) and mental health history were the factors rated as most important when providing treatment.

Conclusions: The current findings provide preliminary support for the importance of investigating current concussion practices among various health professions.

Correspondence: Reid Skeel, Ph.D., Psychology, Central Michigan University, 136 Sloan Hall, Mt. Pleasant, MI 48859, United States. E-mail:reid.skeel@cmich.edu

H. WADSWORTH, N. DIDEHBANI \& M. CULLUM. Concussion Symptom Reporting in Male and Female Adolescent Ethnic Minority Athletes.

Objective: Data on recovery from sport related concussion suggest important gender differences, yet little is known about potential ethnicity effects on symptom reporting following concussion. The aim of this study was to examine differences in post-concussion symptom reporting among males and females of different ethnic groups.

Participants and Methods: Participants were recruited as a part of ConTex, a North Texas concussion registry. The sample included 301 adolescents between the ages of 13 and $18\left(\mathrm{M}_{\mathrm{age}}=15.15, \mathrm{SD}=1.45\right)$ who sustained a sports related concussion and were evaluated within 35 days of injury. Race/ethnicity groups included Black/African American ( $n=51,43.1 \%$ female), Hispanic/Latino ( $n=67,49.3 \%$ female), and White/Caucasian ( $\mathrm{n}=182,48.9 \%$ female). A one-way ANOVA was run to screen for group differences in age. Total post-injury symptom reports along with symptom clusters (physical, sleep, psychiatric, cognitive) were compared using MANOVA to examine for main effects of gender and race/ethnicity in addition to an interaction effect.

Results: No significant differences were found in age among groups. A significant main effect of gender was observed in terms of mean total symptoms $(\mathrm{F}(1,298)=5.70 ; p=<.05)$, physical symptoms $(\mathrm{F}(1,298)=7.94 ; p=<.001)$, and psychiatric symptoms $(\mathrm{F}(1,298)=3.90$; $p<.05$ ), with females endorsing items to a greater degree than males. There was not a significant main effect of race/ethnicity $(p>13)$ or a significant interaction effect ( $p>$.17).

Conclusions: Results support emerging literature indicating higher symptom reporting among adolescent females than males following sports related concussion, and this holds regardless of race/ethnicity. While preliminary, these findings further suggest that race/ethnicity may not influence concussion symptom reporting in similar adolescent sports concussion populations.

Correspondence: Hannah Wadsworth, BA, University of Texas Southwestern Medical Center, 6333 Forest Park Rd, 1st floor BLA 100, Dallas, TX 75390, United States. E-mail: hannah.wadsworth@ utsouthwestern.edu

C. WILSON, K. MCNALLY, J. SMITH-PAINE, M. NARAD, H. ZANG, N. ZHANG, H. TAYLOR, K.O. YEATES \& S.L. WADE. Associations of Attention Problems Following Early Childhood Pediatric Traumatic Brain Injury.

Objective: Previous research has established how children who have sustained a traumatic brain injury (TBI) are at increased risk of developing neurocognitive, social, and behavioral problems, but less is known about the types of attention problems for children who sustained a TBI in early childhood.

Participants and Methods: Children and adolescents [11.94 years $(\mathrm{SD}=1.13)]$ who sustained an early childhood TBI $(n=60,6.84$ years post injury), comprised of TBI severity groups including severe $(n=16)$, moderate $(n=15)$, and complicated mild ( $n=29)$, were compared with an orthopedic injury control group $(n=74)$. Measures of attention from the Attention Network Task (ANT) including alerting, orienting, conflict, and tau (a measure of positive skew and reflective of intermittent long reaction time) were correlated with cognitive tests, parent reported social/emotional behavior and executive functioning. Analysis of covariance and t-tests were used to test whether attention differed by TBI severity status, sex, and time since injury.

Results: Overall ANT scores (alerting, orienting, conflict) did not vary by injury group, but a significant effect of injury group on the ex-Gaussian parameter tau was noted. Tau was significantly higher in the TBI group compared to the OI group $[t(108)=-2.23, p=.03]$. Tau was significantly correlated with time since injury $(r=.21, p=.03)$, but not age of injury. Females were observed to have significantly higher tau $(p<.05)$. Greater tau also correlated with lower overall IQ, math calculation, writing skills, and parent reported executive functioning, internalizing and externalizing problems.

Conclusions: Children who sustain early TBI demonstrate a greater degree of lapses in attention compared to those with OI. Lapses in attention were more notable as time since injury increased, and for females. Finally, the attentional lapses were associated with poor cognitive, academic and behavioral functioning. Further study is needed to better understand the development and emergence of attention problems over time.

Correspondence: Camille Wilson, M.A., Psychology, Nationwide Children's Hospital, 700 Children's Dr., Colombus, OH 43205, United States.E-mail: camille.wilson@nationwidechildrens.org

K.L. WRIGHT, F. ROBERTSON, R. HOPKINS \& E.D. BIGLER. White Matter Hyperintensities in Pediatric TBI: Comparison of Imaging Methods.

Objective: Objective: MRI identified white matter hyperintensities (WMHs) are frequently observed following traumatic brain injury (TBI). WMHs provide insight into the severity and extent of neuronal injury and white matter pathology, important for understanding the neurocognitive and neurobehavioral effects from TBI. There are a number of methods to assess WMHs including clinical ratings and volumetric analyses along with newly introduced automated WMH detection and quantification. The purpose of this study was to compare new and existing clinical and volumetric methods of WMHs in neuropsychological research. 
Participants and Methods: Participants and Methods: Sixty participants $(65 \%$ male) from ages $8-13$, had a complicated-mild $(53 \%)$, moderate $(15 \%)$ or severe TBI $(32 \%)$ an average of 2.6 years prior to testing. T2-FLAIR MR images were used for WMH detection. LesionQuant ${ }^{\circledR}$, a promising new automated method, was compared to gold standard manually traced WMH volumes and Scheltens clinical ratings to assess associations between methods.

Pearson's correlations, intra-class correlations, and Bland-Altman plots were used to assess agreement between the methods.

Results: Results: Although positively related, there was no significant correlation or agreement between LesionQuant ${ }^{\circledR}$ and Scheltens clinical ratings $(r=.239, p=.082 ; I C C=.372)$, or between LesionQuant ${ }^{\circledR}$ and manually traced volumes $(r=.114, p=.433 ; I C C=.043)$. Manually traced WMH volumes and Scheltens ratings were significantly correlated $(r=.577, p=.000)$ with good agreement $(I C C=.719)$.

Conclusions: Conclusions: Manually traced WMH volumes, Scheltens clinical ratings and LesionQuant ${ }^{\circledR}$ varied in their relation to one another and ability to identify white matter pathology in this sample of pediatric TBI. Thus, automated analyses yield different findings than clinical ratings or manually traced WMH volumes. Limitations of this study include restricted range of injury severity, heterogeneity of lesions, and small sample sizes in regards to injury severity.

Correspondence: Kacie L. Wright, PhD, Psychology, Brigham Young University, 488 E. 100 S. , Orem, UT 84097, United States. E-mail: kacie.birtcher@hotmail.com

V. KWAN, V. PLOURDE, K.O. YEATES \& B. BROOKS. Headache Long After Pediatric Concussion: Severity and Relation to Subjective and Objective Cognitive Functioning.

Objective: Youth with concussion often report headache acutely. However, less is known about headache in the long-term. Thus, our aim was to examine the severity of headache several years after concussion, and the relation between headache, subjective cognitive ratings, and objective cognitive performance.

Participants and Methods: Participants $(N=78)$ were 8-19 years old (mean=14.2, $\mathrm{SD}=2.9$ ) in three groups: single concussion, multiple concussions, or an orthopedic injury (OI). They averaged 2.7 years ( $\mathrm{SD}=1.66)$ since their most recent injury. Parents and youth completed parent and self-report forms of the Postconcussion Symptom Inventory (PCSI), which includes a single-item rating of headache severity (from 0 to 6 ) and a composite cognitive symptom scale, based on the past two days. Participants also completed the Headache Rating Scale (on a scale from 0 to 10) at the time of the assessment (HRS-current) and based on the past week (HRS-week). Objective cognitive ability was assessed using CNS Vital Signs.

Results: The groups did not differ significantly in any of the headache ratings. However, self-rated headache from the PCSI, HRS-current, and HRS-week correlated with self-reported cognitive symptoms $(r=0.460$, $p<0.001, r=0.450, p=0.001, r=0.501, p<0.001$, respectively). Parents' reports of children's headaches on the PCSI also correlated with parents' ratings of children's cognitive symptoms $(r=0.434$, $p<0.001)$. Objective cognitive test scores did not differ across groups (means were within the average range), nor did they correlate with any of the headache ratings.

Conclusions: Long after injury, youth with and without concussion did not differ in headache ratings. Headache correlated with subjective cognitive ratings, but not objective cognitive performance. This suggests that youth with headache are likely to perceive cognitive difficulties, and that headache may be an important therapeutic target for the care of youth after concussion.

Correspondence: Keith O. Yeates, PhD, University of Calgary, 2500 UniversityDr NW, Calgary, AB T2N1N4,Canada.E-mail: KYeates@ ucalgary.ca

\section{Acquired Brain Injury (TBI/ Cerebrovascular Injury \& Disease - Adult)}

M. ETTENHOFER, J. HERSHAW \& J. ENGLE. Multimodal Neuropsychological Assessment in Chronic Traumatic Brain Injury: The Fusion Project.

A subset of traumatic brain injury (TBI) patients experience chronic symptoms or impairments after injury. However, many factors may impact recovery from TBI, and subjective symptom reports may be inconsistent with objective measurements of cognitive performance. Advanced forms of neuropsychological assessment may provide a more comprehensive, multi-dimensional perspective to facilitate diagnosis and treatment planning for these patients. With the use of sensors and algorithms embedded within a computerized assessment platform, providers could have access to a rich source of information about the functioning of multiple cognitive and motor systems.

This symposium will provide an overview of results from the Fusion Project, a Department of Defense-supported effort to develop and evaluate methods for multimodal assessment of TBI. Previously, our group has shown that saccadic eye movements can provide valuable information about cognitive processing and TBI-related impairment. Additionally, studies have shown that TBI patients are particularly susceptible to degraded motor function under conditions of high cognitive load. However, little is known about the combined impacts of TBI and cognitive load on oculomotor function. The Fusion Project was designed to address these issues and to advance technology for clinical assessment. Our first symposium speaker, Dr. Mark Ettenhofer, will provide an overview of Fusion Project methodology and share findings relating to saccadic and manual performance across levels of cognitive load and severity of TBI. Next, Ms. Hershaw will describe the relationship of the pupillary response to neural processing, and examine load and TBI effects on Fusion pupillary metrics. Our third speaker, Dr. James Engle, will examine effects of TBI, cognitive load, and motor performance on participants' subjective ratings of mental workload. Speakers will also discuss potential clinical applications for assessment of TBI and directions for future research.

Correspondence: Mark Ettenhofer, NMCSD, DVBIC, 34730 Bob Wilson Dr. Ste 201, ATTN: DVBIC, San Diego, CA 92134-3201, United States. E-mail: ettenhofer@gmail.com

M. ETTENHOFER, J. HERSHAW, J. ENGLE, A. SAFFORD, E. CORDERO, J. KEGEL \& M. ROY. Saccadic Evidence for Reduced Cognitive Efficiency in Chronic Traumatic Brain Injury.

Objective

Previous research has demonstrated that motor performance may be degraded under conditions of high cognitive load, and that this decrement may be magnified among those with traumatic brain injury (TBI). Saccadic eye movements have been shown to provide high sensitivity to TBI, however, the impact of cognitive load on saccadic performance is unknown. The current study was conducted to examine saccadic performance across multiple levels of cognitive load and TBI severity. Methods

Adult participants $(\mathrm{N}=61)$ with chronic TBI $(\mathrm{n}=20$ mild TBI; $\mathrm{n}=15$ moderate-to-severe TBI) and uninjured controls $(\mathrm{n}=26)$ completed a TBI interview (OSU TBI-ID) along with a battery of neuropsychological tests (WAIS-IV Digit Span, Symbol Search, and Digit Symbol Coding; HVLT-R; Trail Making Test A\&B) and the Fusion n-Back eye tracking test. On the Fusion Test, Saccadic and Manual response time (RT) were examined across simple RT, 0-back, and 1-back load conditions. Analyses were conducted using RMANCOVAs and partial correlations, each controlling for age.

\section{Results}

Saccadic RT demonstrated significant slowing associated with group $\left(\eta_{\mathrm{p}}{ }^{2}=.09, p=.02\right)$, load $\left(\eta_{\mathrm{p}}{ }^{2}=.11, p=.005\right)$, and the group*load interaction $\left(\eta_{\mathrm{p}}{ }^{2}=.08, \mathrm{p}=.01\right)$, with more pronounced effects of load within the TBI group. Follow-up partial correlations demonstrated a dose-response 
effect of TBI severity on saccadic RT $\left(r_{\mathrm{p}}=.33, p=.01\right)$. Manual RT and global neuropsychological performance did not differ by group. Conclusions

Greater saccadic impairment was found with increased severity of TBI. These TBI-related impairments were most prominent at high levels of cognitive load. This study provides additional evidence for the unique value of neurocognitive eye tracking in detecting TBI-related impairments. Results suggest that TBI may reduce cognitive efficiency in wavs that may be overlooked by conventional assessment methods. Further research is needed to elucidate the neural mechanisms of these findings. Correspondence: Mark Ettenhofer, NMCSD, DVBIC, 34730 Bob Wilson Dr. Ste 201, ATTN: DVBIC, San Diego, CA 92134-3201, United States. E-mail: ettenhofer@gmail.com

J. ENGLE, J. HERSHAW, A. SAFFORD, E. CORDERO, J. KEGEL \& M. ETTENHOFER. Subjective Mental Workload and Motor Impairment in Traumatic Brain Injury.

\section{Objective}

Individuals with traumatic brain injury (TBI) commonly report greater mental effort and frustration associated with cognitive activity. However, self-report ratings after TBI often diverge from objective performance. This study was conducted to examine patterns of subjective workload across increasing levels of cognitive task load, and to examine the influence of chronic TBI and objective performance.

Methods

Sixty-one adults with $(\mathrm{n}=35)$ and without $(\mathrm{n}=26)$ chronic TBI completed the OSU-TBI-ID diagnostic interview and the Fusion n-Back Test. Following each Fusion subtask (i.e., simple reaction time [RT], 0-back, 1-back), the 6-item NASA TLX was used to probe mental, physical, and temporal workload along with perceived effort, frustration, and performance. Effects of task load and TBI on TLX ratings were evaluated with RMANOVA. Associations among TLX ratings, saccadic and manual RT were evaluated with Pearson's correlations.

Results

A main effect of group demonstrated greater subjective workload within the TBI group $\left(\eta_{\mathrm{p}}{ }^{2}=.13, p=.005\right)$. The effect of TBI was most pronounced for mental $\left(\eta_{\mathrm{p}}{ }^{2}=.20\right)$ and performance $\left(\eta_{\mathrm{p}}{ }^{2}=.18\right)$ probes; TBI participants demonstrated elevated and flat subjective effort ratings across levels of task demand, whereas controls' workload ratings increased with task demand. TBI and non-TBI participants demonstrated divergent associations between subjective workload, manual RT, and saccadic RT. For those with a history of TBI, workload tended to correlate with manual, but not saccadic RT

Conclusions

Chronic TBI was associated with high levels of subjective mental demands, even at low levels of cognitive load. Self-reported ratings were generally related to manual, but not saccadic RT. While individuals with
TBI were aware of demands on manual performance, saccadic performance appears to fall outside of conscious awareness. Assessment of TBI may be enhanced through consideration of self-reported workload in addition to behavioral performance.

Correspondence: James Engle. E-mail: jrengall@gmail.com

J. HERSHAW, A. SAFFORD, E. CORDERO \& M. ETTENHOFER. Identification of Measurable Cognitive Processing Differences in Chronic TBI Using Pupillometry.

Objective

Self-reported cognitive symptoms in chronic traumatic brain injury (TBI) are inconsistently related to conventional performance metrics and neuropsychological outcomes. This warrants development of more sensitive measures. Given that pupil diameter has been shown to be a robust indicator of cognitive effort, the current research examines potential pupillary biomarkers for impaired cognition in chronic TBI that can be easily measured in clinical settings.

Methods

Adults with chronic TBI ( $\mathrm{n}=19$ mild TBI; $\mathrm{n}=12$ moderate-to-severe TBI) and uninjured controls $(\mathrm{n}=23)$ completed the computerized Fusion n-Back eye tracking task while pupil diameter was recorded. Manual and saccadic reaction time (RT), baseline pupil diameter (PD), and task-evoked pupillary responses (TEPRs) during three tiered cognitive load conditions were compared between the three groups. A brief neuropsychological battery was also administered. Analyses were conducted using RMANCOVAs controlling for age.

Results

Despite no group differences in manual RT or global neuropsychological performance, pupillary metrics were sensitive to mild TBI. A group*load interaction on baseline PD $\left(\eta_{p}^{2}=.11, p=.02\right)$ demonstrated that the mild TBI group had elevated baseline PD unaffected by load condition. while baseline PD in the control group was smaller and influenced by cognitive load. This effect persisted when controlling for saccadic RT. The moderate-to-severe TBI group had similar baseline PD to controls. TEPRs showed no group effects.

\section{Conclusions}

Relative to controls and the moderate-to-severe TBI group, the mild TBI sample had larger baseline PD regardless of cognitive load level, suggesting that they required additional neural resources to complete the Fusion task to the same level of manual performance as controls. Results support the development of clinically-feasible pupil-based measures of cognitive processing that may reveal cognitive processing differences in the absence of behavioral differences.

Correspondence: Jamie Hershaw, MS, Medical and Clinical Psychology, Uniformed Services University, 4301 Jones Bridge Road, Bethesda, MD 20814,United States.E-mail: jamie.hershaw.ctr@usuhs.edu

\section{THURSDAY EVENING, FEBRUARY 15, 2018}

\section{Plenary C. Linking Brains to Machines: From Basic Science to Neurological Neurorehabilitation}

\section{Presenter: Miguel Nicolelis 5:30-6:30 p.m.}

\footnotetext{
M. NICOLELIS. Linking Brains to Machines: From Basic Science to Neurological Neurorehabilitation.

In this talk, I will describe how state-of-the-art research on brain-machine interfaces makes it possible for the brains of primates to interact directly and in a bi-directional way with mechanical, computational and virtual devices without any interference of the body muscles or sensory organs.
}

I will review a series of recent experiments using real-time computational models to investigate how ensembles of neurons encode motor information. These experiments have revealed that brain-machine interfaces can be used not only to study fundamental aspects of neural ensemble physiology, but they can also serve as an experimental paradigm aimed at testing the design of novel neuroprosthetic devices. I will also describe evidence indicating that continuous operation of a closed-loop brain machine interface, which utilizes a robotic arm as its main actuator, can induce significant changes in the physiological properties of neural circuits in multiple motor and sensory cortical areas. This research raises the hypothesis that the properties of a robotic arm, or other neurally controlled tools, can be assimilated by brain representations as if they were extensions of the subject's own body. 
Learning Objectives

Attendees will be able to explain the concept of the brain-machine interface and neuroprosthetic devices.

Attendees will be able to describe how brain-machine interfaces have influenced the development of a new generation of neuroprosthetics for treating neurological and psychiatric disorders.

Attendees will learn about a new theory of brain function.

Attendees will learn how a brain-machine interface can control an exoskeleton.
Correspondence: Miguel Nicolelis, Duke University, Box 103905 Duke University, Durham, NC27710, United States.E-mail:nicoleli@neuro. duke.edu

\section{Student Social, Hosted by the INS Student Liaison Committee}

7:00-9:00 p.m.

FRIDAY MORNING, FEBRUARY 16, 2018

\section{CE Workshop 9. Vulnerability to Post Traumatic Distress Disorder After Traumatic Brain Injury; Chronic Stress and Accelerated Aging}

\author{
Presenter: John B. Williamson
}

7:20-8:50 a.m.

J.B. WILLIAMSON. Vulnerability to post traumatic distress disorder after traumatic brain injury; chronic stress and accelerated aging.

Patients with traumatic brain injuries (TBI) often develop post traumatic stress disorder (PTSD). This syndrome, defined and diagnosed by psychological and behavioral features, is associated with symptoms such as anxiety and anger with an increase of arousal and vigilance, as well as flashbacks and nightmares. It is unclear to what extent, if any, mild traumatic brain injury may influence the development or the course of PTSD. This course will review heterogeneity of brain imaging findings documented in mild TBI and PTSD, behavioral correlates including autonomic shifts associated with chronic stress, and, over time, associated brain and other physiological changes that contribute to deteriorating health. As a result of participation in this course, the learner will achieve the following objectives: 1) Be able to identify commonly impacted white matter structures associated with mild traumatic brain injury. 2) develop a deeper understanding of autonomic factors in PTSD and how limbic system dysregulation associated with mild TBI may impact these systems and 3 ) how chronic stress associated with PTSD may impact the aging process.

Correspondence: John B. Williamson, PhD, Neurology, University of Florida, 2775 NW 106 Way, Gainesville, FL 32606, United States. E-mail: john.williamson@ufl.edu

\section{CE Workshop 10. Cognitive and Brain Aging: Neurodegeneration, Cumulative Risk and Etiological Factors, or Epigenetic Phenomena?}

\section{Presenter: Ron A. Cohen}

$$
\text { 7:20-8:50 a.m. }
$$

R.A. COHEN. Cognitive and brain aging: Neurodegeneration, cumulative risk and etiological factors, or epigenetic phenomena? Cognitive and brain aging are the focus of this course. Following an introduction to the concepts of successful cognitive and brain aging, we will consider cognitive aging from epidemiological and developmental perspectives. Next will be a review of the biology of aging. Cognitive aging as a manifestation of three possible underlying mechanisms will be considered: Neurodegeneration, cumulative risk and etiological factors, and epigenetics. Age-associated cognitive and brain changes with and without neurodegenerative disease will be discussed. This is followed by a review of the research literature on age-associated cognitive decline in the context of medical risk factors and etiologies, including heart disease, diabetes, other metabolic disorders, and infectious disease (i.e., HIV). Psychosocial factors, including alcohol and drug use will also be considered. The second half of the course will focus on recent findings from studies that have employed neuroimaging and laboratory biomarkers in conjunction with neuropsychological assessment to achieve greater understanding of the structural, functional and metabolic brain changes that occur with advanced age and their influence on cognitive and functional abilities, quality of life, and the daily activities of older adults. The course concludes with a discussion of clinical interventions to optimize cognitive aging with discussion of pharmacological, neuromodulation. cognitive training and behavioral approaches. Through participation in this course, the learner will achieve (1) a deeper understanding of the manifestations and factors underlying cognitive and brain aging, (2) awareness of current interventions that are being investigated to enhance cognitive and functional abilities for successful aging in older adults; and 3) be familiar with clinical-translational research on cognitive aging, including findings from studies incorporating neuroimaging and laboratory biomarker approaches.

Correspondence: Ron A. Cohen, PhD, Center for Cognitive Aging and Memory, University of Florida, Center St, PHHP Bldg, Gainesville, FL 32611, United States.E-mail: roncohen@ufl.edu

\author{
INS Business Meeting (Business \& Bakeries) \\ INS President: Michael Kopelman \\ 8:15-9:00 a.m.
}

Plenary D. Where Neuromodulation and Neuropsychology Meet - Promoting Plasticity to Enhance Brain Health

Presenter: Sarah H. Lisanby

9:00-10:00 a.m.

S.H. LISANBY. Where Neuromodulation and Neuropsychology Meet - Promoting Plasticity to Enhance Brain Health.

The advent of neuromodulation tools, such as transcranial magnetic stimulation (TMS), accelerated scientific discovery of the functional role of neural circuits in the brain. Because tools like TMS can modulate brain function, not just measure it, their availability has ushered in a new era for intervention development in which knowledge of circuits underlying illness can be used to develop circuit-guided therapies. While much of the work with these tools has focused on applying brain stimulation as a monotherapy, the ability to induce plasticity in targeted regions of the brain represents exciting opportunities for multi-modal strategies 
that combine neuromodulation with cognitive behavioral interventions. Using focal interventions like TMS, neuromodulation-induced plasticity can be targeted to key brain regions to promote learning during a cognitive behavioral intervention. Using less focal tools like transcranial direct current stimulation (tDCS), the impact on circuit function could be 'functionally focalized' by having the individual engaged in a cognitive task during the stimulation. Multi-modal intervention development represents an opportunity for the fields of neuromodulation and neuropsychology to converge in innovative ways that may advance understanding of brain-based disorders and may accelerate discovery of more effective treatments. This presentation will review the latest work coupling simultaneous neuromodulation and cognitive intervention for the enhancement of function, including studies on working memory and treatment of depression. Learning Objectives include: 1) learners will be able to articulate the state-of-the-art of neuromodulation tools for clinical intervention, and 2) learners will be able to discuss the scientific rationale for coupling neuromodulation with cognitive behavioral interventions to promote circuit function.

Correspondence: Sarah H. Lisanby, M.D., Director, Noninvasive Neuromodulation Unit, National Institute of Mental Health, 6001 Executive Blvd, MSC 9632, Bethesda, MD 20892, United States. E-mail: sarah.lisanby@nih.gov

\section{AM Coffee Break}

10:00-10:15 a.m.

\section{Invited Symposium 3. Challenging the Status Quo: Future Directions in Cultural Neuropsychology}

\section{Chair: April Thames}

\section{Presenters: Monica Rivera Mindt, Desiree Byrd, Jennifer J. Manly, Tedd Judd}

\section{0:15-11:45 a.m.}

A. THAMES, M. RIVERA MINDT, D. BYRD, J.J. MANLY \& T. JUDD. Challenging the status quo: Future directions in Cultural Neuropsychology.

The field of Clinical Neuropsychology has grown considerably over the past few decades. Part of this growth includes acknowledging the extent of the cultural, linguistic, and cognitive diversity of the global population, and challenging many of the existing paradigms and theories that have permeated the field. Despite many successes, challenges still remain with linking international efforts, having clear guidelines for what constitutes "cultural competence training", a general consensus on the scientific study of diversity issues, and directions for advocacy and policy implementation. In order to continue our success, new benchmarks in the areas of research, education/training, advocacy and policy must be set with respect to issues of diversity and inclusion.

In a US response to this need, the Cultural Neuropsychology Summit, hosted by the Hispanic Neuropsychological Society, was held in June 2017 in Boston. The Summit brought together a diverse group of neuropsychologists to provide recommendations in the areas of research, education/training, and policy for US neuropsychology. We will present on the core recommendations that emerged from the Summit as well as progress updates. Dr. Monica Rivera-Mindt will present on the current state of training in cultural neuropsychology in the US as it pertains to cultural and linguistic competencies. Dr. Desiree Byrd will present on the latest recommendations for cultural neuropsychology research. Dr. Jennifer Manly will present on policy recommendations and advocacy training. Dr. Tedd Judd will present on neuropsychology efforts in other nations to address their diverse populations. Dr. April Thames will serve as Discussant. Consistent with the INS theme of "Connecting the present with the future," the goal of each presentation is to provide foundational steps for moving forward in cultural neuropsychology.

Correspondence: April Thames, Ph.D., Psychiatry, UCLA, 740 Westwood Plaza 28-263, Los Angeles, CA 90095, United States. E-mail: AThames@mednet.ucla.edu

M. RIVERA MINDT. Integration of Cultural and Linguistic Competency into Neuropsychology Training.

The U.S. population is becoming increasingly culturally and linguistically diverse, and cultural/linguistic minorities are disproportionately impacted by myriad conditions (e.g.., Alzheimer's, HIV/AIDS, cardiovascular disease) that make them particularly vulnerable to neuropsychological impairment. However, our current training models and workforce remain underprepared to ensure the provision of empirically-supported, culturally/linguistic competent research and practice for these populations. This presentation will provide a comprehensive analysis of pertinent sociocultural considerations that are germane to the training of neuropsychologists across the professional development spectrum (e.g.., predoctoral, early- and mid-career, etc.). Next, this presentation will review the current state of training in cultural neuropsychology as it pertains to cultural and linguistic competencies. Lastly, this presentation will provide the INS membership with an overview of the proceedings and recommendations of the 2017 Cultural Neuropsychology Summit to advance cultural and linguistic competence in neuropsychological training.

Learning Objectives:

1. Raise awareness of specific needs for increasing emphasis of cultural and linguistic diversity into neuropsychological training.

2. Demonstrate knowledge of approaches for implementing cultural and linguistic competencies into neuropsychological training.

3. Demonstrate awareness of the 2017 Cultural Neuropsychology Summit recommendations advancing cultural and linguistic competence in future neuropsychological training

Correspondence: Monica Rivera Mindt, Ph.D., ABPP-CN, NY, United States.E-mail: riveramindt@fordham.edu

\section{BYRD. Cross cultural neuropsychological research: Developments and forecasts.}

Research into cross cultural cognitive phenomena is necessary to expand the core neuropsychological knowledge base and guide competent practice. In recent years, the research platform for this subspecialty has realized tremendous growth despite the politicized history of scientific investigations of cultural variation in cognitive test performance. This presentation will review the significant advances in cross cultural neuropsychological research in topical areas such as: the nuanced application of race-specific normative data, psychometric methods for the evaluation of validity and cultural bias, the expansion of cross cultural investigations to previously under-studied ethnic groups and the application of neuroimaging tools to cross-cultural cognitive paradigms. Throughout the review, methodological challenges and strengths will be highlighted. This presentation will also summarize recent research developments from African and South American investigators and explore the contributions of this work to American conceptualizations of cross cultural neuropsychology. Finally, this talk will present the recommended priorities for the direction of future research in cross cultural neuropsychology as informed by participants in the 2017 Cultural Neuropsychology Summit sponsored by the Hispanic Neuropsychological Society. Correspondence: Desiree Byrd, Ph.D., NY, United States. E-mail: desiree.byrd@mssm.edu 
J.J.MANLY, R.CASAS, T.M.SCOTT, K.POSTAL, B.A. MARCOPULOS \& A.E. PUENTE. Advocacy in Cultural Neuropsychology.

Broad infusion of cultural neuropsychology into all aspects of theory, research, training, and practice is the next challenge for our field. In other words, we are ready to move on from mere justification of the need for cultural neuropsychology to a transformation of the field to better serve a diverse population, improving access to and benefit from neuropsychology. The 2008 Diversity Summit for cultural neuropsychology highlighted the importance of building organizational and inter-organizational infrastructure for change, but did not specify a potential mechanism for achieving this goal, and did not focus explicitly on advocacy or policy change as a professional skill set or competency. Widening disparities in brain health highlight the importance of assuring the relevance of neuropsychology across cultural, geographic, socioeconomic, and political domains. The 2017 Cultural Neuropsychology Summit specifically addressed advocacy and policy change by recommending inter-organizational infrastructure and support for diversity-focused initiatives. The recommendations acknowledge that advocacy efforts for cultural neuropsychology need to occur across contexts (e.g. educational institutions, within organizations) and levels (e.g. local, state, national). The group recommended an inter-organizational effort to compile and streamline advocacy efforts for cultural neuropsychology, to work within this organization to implement financial incentives for postdocs in underserved populations, to leverage opportunities to advocate for funds for cultural neuropsychology research, and to incorporate cultural neuropsychology advocacy training at the graduate and postdoctoral level.

Correspondence: Jennifer J. Manly, PhD, Neurology, Columbia University, 630 West 168th St., P\&S Box 16, New York, NY 10032, United States.E-mail:jjm71@cumc.columbia.edu

\section{T. JUDD. Global Neuropsychology Priorities.}

There is much more to cultural neuropsychology than finding the right norms.

Many subjects of global importance are under-researched and undertaught in Western neuropsychology, particularly involving linguistic, cultural, educational and cognitive diversity as well as epidemiological issues. To illustrate there are about 6000 languages in the world. There is a diversity of writing systems (abjads, alphabets, abugidas, syllabaries, ideograms) that have a major impact on how learning disorders, such as dyslexia are manifested. Moreover, there are approximately 750 million illiterates in the world, representing $17 \%$ of the global adult population. About half the world's population is bilingual. There are about 45 million involuntarily displaced people globally and untold hundreds of millions of voluntary internal and external migrants encountering mixes of language and culture. We have good reasons to believe that these factors significantly impact neuropsychological functioning, but we are poorly equipped to determine exactly how. Malnutrition, iodine deficiency, cerebral malaria, and various industrial toxicities are major global causes of neuropsychological dysfunction. However, regional work in such issues rarely receives much attention in global neuropsychology forums. This presentation will explore examples of regional work in such issues from Asia, Africa, and Latin America. I will then address possible ways that international neuropsychology might collaborate to prioritize global neuropsychological public health issues and address them effectively. I will further address ways in which working with cultural, linguistic, and cognitive diversity can strengthen clinical skills and neuropsychological theory. I will explore varied models of knowing, learning, and inference that may be pertinent to clinical and research global neuropsychology. These will include an emic-etic approach, dynamic assessment, collaborative assessment, and a valuedriven approach.

Correspondence: Tedd Judd. E-mail: teddjudd@gmail.com

\section{Symposium 7. Bringing Neuropsychology to the People: Transforming Our Knowledge of Executive Function (EF) Phenotypes into Effective Community-based Interventions for Children}

\author{
Chair \& Presenter: Lauren Kenworthy
}

Discussant: Hilde Geurts

\author{
Presenters: Kristina K. Hardy, Shari L. Wade, \\ John Strang
}

\author{
10:15-11:45 a.m.
}

L. KENWORTHY, K.K. HARDY, S.L. WADE, J. STRANG \& H. GEURTS. Bringing Neuropsychology to the People: Transforming Our Knowledge of Executive Function (EF) Phenotypes into Effective Community-Based Interventions for Children.

Executive functions (EF) rely on distributed brain networks with protracted developmental courses, making them highly vulnerable to disruption. The profound impact of EF on key outcomes has been established in many brain disorders. This symposium builds on past research defining dysexecutive phenotypes with presentations describing trials of four EF interventions for children with epilepsy, autism and ADHD. They are each novel, phenotype-driven treatments, allowing presenters to directly address questions of cross-diagnosis treatment efficacy posited by the Research Domain Criteria (RDoC) theory and methods. The interventions address economic, ethnic and racial disparities in access to care by delivering treatment in homes and schools. Moving treatment outside of the clinic is important when targeting EF, because EF deficits are influenced by economic disparities: the number of years of income insecurity in early childhood has a linear dose-response relationship to later EF abilities. Thus, treatment providers confront a double disparity expressed in increased EF difficulties and reduced access to clinical diagnosis/treatment. Furthermore, EF skills are notoriously difficult to generalize from the clinic to everyday settings. Dr. Wade presents data demonstrating the feasibility and preliminary efficacy of an innovative e-health intervention that teaches strategies to build EF skills in children with epilepsy. Drs. Kenworthy and Hardy describe an RCT of two school-based EF interventions, Unstuck and On Target and contingency behavioral management, that were adapted for low-income and Spanish speaking families and children with ADHD or autism. Dr. Strang reports on a successful RCT of a middle school cognitive-behavioral EF intervention for autism. Each presentation points toward a vital future in which our current neuropsychological knowledge is leveraged to empower teachers, parents, and children to improve EF in the everyday settings where it matters most.

Correspondence: Lauren Kenworthy, PhD, Neuropsychology, Children's National Medical Center, 15245 Shady Grove Road, Suite 350, Rockville, MD 20850,United States.E-mail:lkenwort@cnmc.org

L. KENWORTHY, C. PUGLIESE, K.K. HARDY, J. STRANG, A. RATTO, J. SAFER-LICHTENSTEIN, M. BIEL, S. SEESE, M. WILls, C. LUONG-TRAN, L. CANNON, A. SHARBER, B. ANTHONY \& L. ANTHONY. Evidence for Cross-Diagnosis Efficacy of School-Based Unstuck and On Target Executive Function Intervention in Low Income Communities.

Objective: Economic disparities in access to diagnosis and treatment are prominent in ASD and in ADHD, both of which are strongly associated with executive dysfunction. Unstuck and on Target (UOT) is a cognitive-behavioral executive function (flexibility, goal-setting and planning) intervention, which we previously found to be effective in middle-income children with ASD. Unlike other cognitive-behavioral treatments, UOT is implemented in schools instead of clinics, making it low cost, accessible and more likely to generalize to real world settings. 
This study investigates its effectiveness for ADHD and ASD in low-income communities.

Participants/Methods: UOT was delivered in elementary schools in which at least half of the students qualify for free/reduced lunch fees. All children: had IQ> 70; were in $3^{\text {rd }}-5^{\text {th }}$ grade; were identified by school staff as being inflexible and having characteristics of ADHD or ASD; and met diagnostic criteria for either ASD (ADOS-2) or ADHD (MINIKid). Approximately half the qualifying children had not received an ADHD or ASD diagnosis prior to the study. Only $18 \%$ were white/non-Hispanic. 77 children (ASD $n=22$; ADHD $n=45$ ) completed the trial. UOT was delivered by school staff to small groups of students in 20 sessions of 30-40 minutes each.

Results: Observer fidelity ratings of the groups indicated good fidelity overall. Significant improvement in both groups from pre- to post-intervention was observed on: classroom observations, classroom teacher ratings (SKAMP), parent ratings (BRIEF) and WASI Block Design and Executive Function Challenge Task scores (all p's $<.05$; range of Cohen's d's .42-.70).

Conclusions: Children with ASD and ADHD in low-income schools benefit from UOT, which can be delivered by school staff with fidelity, providing access to treatment for many children who are not accessing clinic services. UOT is a phenotype-specific intervention which can help children across diagnostic categories.

Correspondence: Lauren Kenworthy, PhD, Neuropsychology, Children's National Medical Center, 15245 Shady Grove Road, Suite 350, Rockville,MD20850,United States.E-mail: lkenwort@cnmc.org

K.K. HARDY, C. PUGLIESE, J.F. STRANG, A. RATTO, S. SEESE, J. SAFER-LICHTENSTEIN, A. SHARBER, C. LUONG-TRAN, L. CANNON, M. WILLS, M. BIEL, B. ANTHONY, L. ANTHONY \& L. KENWORTHY. Applying Contingency-Based Behavioral Management Strategies to Treat Cognitive Inflexibility in Children with ADHD and ASD: Development and Efficacy of a School-Based Intervention in Low-Income Communities.

Objective: Cognitive inflexibility is a hallmark impairment in children with autism spectrum disorder (ASD), and also occurs in a sizable subset of children with attention deficit/hyperactivity disorder (ADHD). Flexibility problems are associated with functional impairments across domains (home, school, social), and thus are a salient target for intervention. Methods: The PATSS (Parents and Teachers Supporting Students) intervention was developed using a community-based participatory research approach to treat $3^{\text {rd }}$ to $5^{\text {th }}$ graders with ADHD or ASD with cognitive and behavioral inflexibility in low-income schools. PATSS included parent and teacher trainings, 15 student groups, and 5 sessions of classroom monitoring by school-based interventionists trained by study staff. Parent and teacher content focused on teaching evidencebased behavioral management strategies, while student groups taught cognitive-behavioral techniques to set goals, improve communication, accept consequences, cope with stressors, and self-monitor. PATTS was evaluated in a randomized trial, with participants assigned to either PATSS or another school-based cognitive flexibility program (Unstuck and On Target). Results: Participants were children with ASD ( $\mathrm{n}=26$; mean age $=9.9 ; 100 \%$ male, $61 \%$ White $)$ and ADHD ( $n=43$; mean age $=9.7 ; 74 \%$ male, 35\% White), enrolled in Title I schools. Pre-post paired sample t-tests indicated improvement in children with ADHD on a performance-based cognitive flexibility task $(t=4.00, p<.01)$, blinded classroom observer ratings $(t=-3.32, p<.01)$ of cognitive flexibility, and parent-reported ADHD problems $(t=3.29, \mathrm{p}<.01)$. In contrast, children with ASD evidenced significant improvement only in parent-rated interference of inflexibility $(t=4.04, p<.01)$. Conclusion: The PATSS program can be effectively delivered in low-income communities by school personnel. Treatment is associated with improvements in flexibility for children with ADHD, but fewer changes for children with ASD.
Correspondence: Kristina K. Hardy, PhD, Neuropsychology, Children's National Health System, Children's National Health System, 111 Michigan Ave, NW, Washington, DC 20010, United States. E-mail: KKhardy@childrensnational.org.

S.L. WADE, M. SCHMIDT, A. SMITH, N. GLASER, L. TURNIER, A. COMBS, B. HATER \& A. MODI. Epilepsy Journey: An E-Health Intervention to Improve Executive Functioning in Adolescents with Epilepsy.

Objective: Executive function (EF) deficits are present in 1/3 of youth with epilepsy and contribute to academic, social, and functional impairments. Our aim was to describe the preliminary efficacy and feasibility of an e-health EF intervention.

Participants and Methods: Twenty-eight adolescents were recruited $\left(\mathrm{M}_{\mathrm{age}}=15.3 ; 68 \%\right.$ female $)$. Participants had to have at least two subclinical (>1 SD) or one clinically-elevated subscale on the parent-reported Behavior Rating Inventory of Executive Function (BRIEF) to be eligible for the open trial. The weekly program consisted of 10 self-directed online modules with synchronous therapist-guided Skype sessions. Content was organized around a journey theme in which different "lands" targeted EF domains. Parents completed the BRIEF and the Behavior Assessment Schedule for Children (BASC) pre- and post-intervention.

Results: Sixteen participants have completed Epilepsy Journey to date (94\% completed 10 modules). Significant improvements were found on the BRIEF Inhibition scale $(\mathrm{t}(15)=2.96, p<.01)$ and Behavioral Regulation Index $(\mathrm{t}(15)=2.15, p<.05)$. Of the participants with BRIEF elevations, improvements to normative levels were noted for $67 \%$ on Inhibition, $22 \%$ on Shifting, $33 \%$ on Emotional Regulation, $50 \%$ on the Behavioral Regulation Index, $40 \%$ on Initiation, $30 \%$ on Working Memory, $29 \%$ on Planning/Organization, $33 \%$ on Organization of Materials, $45 \%$ on Metacognitive Skills, and $38 \%$ on the Global Executive Functioning Composite. Significant improvements were found for the BASC Hyperactivity $(t(15)=2.52, p<.05)$ and Functional Communication $(\mathrm{t}(15)=-2.24, \mathrm{p}<.05)$ scales. Adolescents reported satisfaction with the program length, content, skills learned, and use of web-based and Skype delivery.

Conclusions: Findings suggest that implementing an online EF intervention for youth with epilepsy is highly feasible. Moreover, significant improvements in parent-reported EF and behavioral functioning provide preliminary evidence of efficacy.

Correspondence: Shari L. Wade, Ph.D., Pediatrics, Cincinnati Children's Hospital Medical Center, 3333 Burnet Avenue, MLC 4009, Cincinnati, OH 45229-3039, United States. E-mail: shari.wade@cchmc.org

J.F. STRANG, L. ANTHONY, C. PUGLIESE, L. CANNON, M. ADLERWERNER, S. SEESE, M.F. SKAPEK \& L. KENWORTHY. Improving Autistic Adolescent Executive Function Skills: Development and Evaluation of the On Target for Life School Curriculum.

Objective: Executive function (EF) challenges increase as autistic youth enter the teen years, and executive dysfunction is related to long-term adaptive outcomes. On Target for Life (OTL), an upward age-extension of the elementary school Unstuck and On Target curriculum, is an adolescent intervention designed to improve EF skills by employing the relative strengths of the EF profile common to autism - the ability to "get stuck" on specific useful details and routines.

Participants/Methods: OTL was developed through an iterative community-based participatory process, engaging key stakeholders in the objectives and design. A randomized comparative trial ( $\mathrm{n}=33$; mean age $=13.2$; mean verbal IQ $=96.7 ; 27.3 \%$ female) was conducted in nine public middle schools, five receiving OTL and four receiving PEERS, a research-proven social skills intervention. Pre-post assessments were conducted with youth and their families, as well as two masked classroom observations in the students' English classes conducted near the beginning and end of the school year.

Results: Pre-post paired sample t-tests indicated significant improvement for both intervention groups in social functioning as measured 
by masked classroom observations and standardized parent report (all p's<0.05). The OTL group made significant gains $\left(p^{\prime} s<0.05\right)$ on masked classroom observations of executive functioning, a masked behavioral assessment measure of flexibility and planning skills in the social context (Executive Function Challenge Task), as well as on a composite measure of planning skills (average of Tower of London DX key indices). Autism-specific anxiety symptoms decreased in both groups $(\mathrm{p}<.05)$.

Conclusion: OTL is associated with improvements in flexibility and planning skills measured across multiple real-world modalities. In accordance with growing evidence of linkages between EF and social functioning, OTL is also associated with gains in social functioning. Correspondence: John F. Strang, PsyD, Center for Autism Spectrum Disorders, Children's National Medical Center, 15245 Shady Grove Road Suite 350,Rockville, MD 20850, United States. E-mail: jstrang@ cnmc.org

\section{Symposium 8. Toward a biomarker-only diagnosis of Alzheimer's disease}

\section{Chair \& Presenter: Adam M. Brickman}

\section{Presenters: Clifford Jack Jr, Adam M. Brickman, Mark W. Bondi, Jennifer J. Manly}

\section{0:15-11:45 a.m.}

\section{A.M. BRICKMAN, C. JACK JR, M.W. BONDI \& J.J. MANLY. Toward a biomarker-only diagnosis of Alzheimer's disease.}

Alzheimer's disease (AD) was identified over 100 years ago when Dr. Alois Alzheimer used modern histology to identify insoluble protein aggregates in postmortem tissue obtained from a patient with presenile dementia. These protein aggregates were later identified as the beta amyloid plaques and tau-related neurofibrillary tangles that define the disease pathologically. In the 1980s, AD diagnostic criteria were codified as the presence of an amnestic neuropsychological syndrome, functional decline, and ruling out of other known causes of dementia; definite $\mathrm{AD}$ diagnosis could only be identified by direct examination of brain tissue. The early 2000 s have brought tremendous advancement in the development of biomarkers--for the first time plaques and tangles can be appreciated in vivo via neuroimaging and analysis of cerebrospinal fluid--and the 2011 research criteria for AD added biomarker status to complement neuropsychological diagnostic features. But biomarker studies reveal a disconnection between clinical presentation and biomarker status: up to $30 \%$ of older adults who are positive for AD biomarkers are asymptomatic and a up to $30 \%$ of individuals with the clinical symptoms of $\mathrm{AD}$ are biomarker negative. As a result, newly proposed research diagnostic criteria for AD require biomarker evidence of amyloid plaques and neurofibrillary tangles without consideration of any symptomatology. This symposium will focus on the development of new diagnostic criteria for AD. Dr. Jack will introduce the evolution and rationale behind a biomarker-based diagnosis. Dr. Brickman will discuss issues surrounding its validity and role of other pathologies. Dr. Bondi will review advances in neuropsychology in $\mathrm{AD}$ conceptualization. Dr. Manly will discuss implications of the new diagnostic approach for research on disparities, given evidence for multiple mechanistic pathways and the representativeness of the populations providing evidence for biomarker-based diagnoses.

Correspondence: Adam M. Brickman, PhD, Taub Institute for Research on Alzheimer's Disease and the Aging Brain, Department of Neurology, Columbia University, P \& S Box 16, 630 West 168th Street, New York, NY 10032, United States.E-mail: amb2139@columbia.edu
C. JACK JR. Implications of a biologically based definition of Alzheimer's disease.

In 1984, Alzheimer's disease (AD) was conceived of as a clinical-pathological entity. An individual with typical symptoms was expected to have AD pathology at autopsy and an individual without symptoms was expected to not have AD pathology. Both autopsy and more recently biomarker studies have shown that the clinical diagnosis of "AD" dementia is not due to AD in roughly $20 \%$ of cases. Moreover, up to $40 \%$ of cognitively normal individuals beyond the age of the mid $80 \mathrm{~s}$ have AD.

The NIA AA has established a work group whose charge is to update and harmonize a framework for $\mathrm{AD}$ research that is founded on a biological rather than a syndromal definition of $\mathrm{AD}$. The workgroup recognized that a syndrome is not an etiology; rather, it is the clinical consequence of an etiology or disease. A biological rather than a syndromal definition of $\mathrm{AD}$ is therefore a logical step toward greater understanding of the mechanisms underlying clinical expression of the disease.

The NIA AA update is labeled a "research framework" because its intended use is observational and interventional research, not routine clinical care. In the 2018 research framework AD is defined by pathologic changes, which can be documented by post-mortem examination or in vivo by biomarkers. Biomarkers are grouped into those of $\beta$-amyloid deposition, tau pathology, and neurodegeneration. Three cognitive staging schemes are described. Defining AD as a biological construct will enable a more precise understanding of the sequence of events that lead to cognitive impairment and the multi-factorial etiology of dementia. This approach will also enable a more precise approach to therapeutic intervention trials where specific pathways can be targeted at specific points in the disease process and to the appropriate people. Importantly, the validity of this construct should be determined in more diverse populations.

Correspondence: Clifford JackJr. E-mail: jack.clifford@mayo.edu

\section{A.M. BRICKMAN. Diagnosing Alzheimer's disease with biology not behavior: Are we there yet?}

The ability to quantitate in vivo amyloid and tau protein aggregates is among the most exciting scientific developments. Historically, clinical AD was a diagnosis of exclusion--in the context of an amnestic neuropsychological profile and functional decline known causes were ruled out to assign diagnosis. In 2011, biomarkers were introduced to add diagnostic confidence and today criteria are based solely on the presence of biomarkers without consideration of behavior. There is little debate about whether biomarkers can detect pathology, but it is unclear whether diagnosis should be determined exclusively by presence of current biomarkers. This presentation will focus on two issues.

First, there is weak agreement between biomarkers and clinical symptoms, with large numbers of unimpaired individuals having positive biomarkers and symptomatic individuals being biomarker negative. The issue highlights the poor sensitivity and specificity of using symptoms to detect amyloid and tau, but raises questions about the extent to which these pathologies play a causal role in the AD syndrome. Experimental evidence through phase 3 clinical trials shows no effect on behavior despite successful clearance of amyloid. Validation of diagnostic criteria needs to consider the causal link to symptoms. Second, amyloid and tau rarely occur in isolation. The vast majority of symptomatic individuals have other changes, particularly cerebrovascular disease, that predict severity and course, raising questions about whether proposed criteria fully capture the myriad biological changes in AD.

There is desperate need to identify viable treatment for AD. The introduction of biomarkers revolutionized the tools to understand AD biology and to identify individuals appropriate for treatment studies that target specific proteinopathies. But increased understanding of what drives clinical symptoms is necessary before replacing a behaviorally-based diagnosis with a purely biological one. 
Correspondence: Adam M. Brickman, PhD, Taub Institute for Research on Alzheimer's Disease and the Aging Brain, Department of Neurology, Columbia University, P \& S Box 16, 630 West 168th Street, New York, NY 10032, United States.E-mail: amb2139@columbia.edu

\section{M.W. BONDI. Improving neuropsychological characterization of} the preclinical period of Alzheimer's disease.

Despite increasing sophistication in biomarker models of Alzheimer's disease (AD) pathogenesis and diagnosis, concomitant sophistication in profiling cognition along the Preclinical-MCI-AD continuum has not kept pace. There are a host of possible reasons, including: (1) over-reliance on cognitive screens or composites that may lessen sensitivity to mild impairment, (2) the use of few measures that may diminish reliability, and (3) over-reliance on subjective report, rating scales, and screens that increase the potential for diagnostic errors.

In a series of studies we have compared two methods of diagnosing MCI: conventional Petersen/Winblad criteria as operationalized by large studies (e.g., ADNI, donepezil trial) and an actuarial neuropsychological method put forward by Jak and Bondi (Jak et al., 2009; Bondi et al., 2014) designed to balance sensitivity (i.e., defining impairment below $-1 \mathrm{SD}$ as opposed to $-1.5 \mathrm{SDs}$ ) and reliability (i.e., requiring two impaired scores within a domain as opposed to one). Results suggest that the conventional method, with its reliance on a single impaired score and subjective ratings, is susceptible to both false positive and false negative diagnostic errors, whereas MCI diagnosed via actuarial neuropsychological methods yield dissociable phenotypes (e.g., amnestic, dysnomic, dysexecutive), significant biomarker associations, more stable diagnoses, greater percentages who progress to dementia, and improvements in trial findings than conventional diagnostic criteria. Findings support refining diagnoses by incorporating more comprehensive neuropsychological methods. Such an approach will provide a more thorough sampling of cognitive domains and ultimately more complete accounting of the validity and reliability of diagnoses. Improving neuropsychological characterizations across the AD prodromal period can clarify and strengthen biomarker findings as well as lead to more efficient trials requiring considerably fewer participants.

Correspondence: Mark W. Bondi, PhD, CA, United States. E-mail: mbondi@ucsd.edu

\footnotetext{
J.J. MANLY. Will research on disparities in Alzheimer's Disease benefit from a biomarker-focused classification framework? Incorporation of PET and CSF biomarkers into research on Alzheimer's disease (AD) provides the opportunity to accurately detect and locate brain amyloid and tau in vivo. These methods can refine endpoints for treatment trials targeted at clearance of these proteins, and can be used to enrich samples for interventions or observational research with people who are at elevated risk for cognitive decline and dementia. Biomarkers within longitudinal epidemiological studies are necessary to clarify the causal pathways between social, genetic, and environmental risks, cerebrovascular, amyloid, and tau pathology, and cognitive and functional decline. Despite these advantages, a biomarker only diagnostic framework is premature, particularly because of its potential impact on AD disparities research. First, there is a severe lack of diversity in the evidence used to support a biomarker-led diagnostic research framework. The biomarkers proposed for use have been identified in clinical studies that for the most part, did not include racially and socioeconomically diverse people. Base rates of $\mathrm{AD}$ will be lower in community-based samples than in clinical samples, altering the sensitivity and specificity of biomarkers as predictors of subsequent a clinical course of AD. The influence of selection and survival bias on participation in biomarker studies, and on association of biomarkers with future clinical milestones, is not yet understood. Biomarker-only diagnostic approaches must not restrict $\mathrm{AD}$ research in low income settings and in developing countries. Imaging is expensive and requires specialized equipment, and is therefore unattainable in some locations. CSF is more difficult to obtain in disparities populations who have considerable mistrust of
}

research involving invasive techniques. Finally, a premature focus of $\mathrm{AD}$-specific biomarkers on amyloid and tau may limit the ability to assess multiple mechanistic pathways that contribute to disparities in the AD clinical syndrome.

Correspondence: Jennifer J. Manly, PhD, Neurology, Columbia University, 630 West 168th St., P\&S Box 16, New York, NY 10032, United States.E-mail:.jjm71@cumc.columbia.edu

\section{Paper Session 9. Alcohol and Substance Abuse}

\section{Moderator: Michael Kirkwood}

\section{0:15-11:45 a.m.}

\section{R. FAMA, S.A.SASSOON, A. LE BERRE, N.ZAHR, A. PFEFFERBAUM \&. E.V. SULLIVAN. Neural Correlates of Selective Cognitive and Motor Deficits in Alcoholism.}

Objective: Chronic heavy drinking is associated with selective cognitive and motor deficits, with heterogeneity observed in deficit severity and pattern among alcoholics with differential relations to brain structural volumes. Here, we predicted that less frontal gray matter volume would be related to poorer executive functions (EF) and motor (MOT) scores, whereas less medial temporal and posterior gray matter volume would be related to poorer episodic memory (MEM) and visuospatial (VS) scores.

Participants and Methods: Study groups comprised 96 alcoholics (ALC:21-69 yr) and 41 normal controls (NC:21-73 yr). Test scores were age- and education-corrected. Brain volumes were age- and head-size corrected. Data reduction with PCA yielded 2 behavioral factors, EF/ MOT and MEM/VS, and 3 brain volume factors, cortical gray (frontal, temporal, parietal, occipital, cingulate), thalamic and hippocampal, and white matter (corpus callosum, centrum semiovale).

Results: Frontal and cingulate volumes were unique predictors of EF/ MOT scores, whereas parietal gray matter volumes predicted MEM/ VS scores. Lower EF/MOT ( $p=.0037)$ and MEM/VS ( $p=.002)$ scores correlated with greater lifetime total alcohol consumption. Even after taking normal age into account, older age was associated with lower scores on both cognitive and motor factors $(p=.007, p=.041)$ in ALC. Older ALC had smaller hippocampal/thalamic $(p<.0001)$ and white matter volumes $(p=.002)$ than younger ALC, and lifetime alcohol consumption was not related to hippocampal/thalamic or white matter volumes.

Conclusions: Cognitive and motor deficits in ALC were associated with selective cortical volumes. These data support the relevance of frontal systems in executive functions and motor abilities and of parietal systems in episodic memory and visuospatial abilities in alcoholism. In addition, age and total lifetime alcohol consumption were measurable, moderating factors in cognitive and motor compromise in alcoholism. (Support: AA010723, AA017168, AA005965, AA013521)

Correspondence: Rosemary Fama, Ph.D., Department of Psychiatry and Behavioral Sciences, Stanford University School of Medicine, 401 Quarry Road, Stanford University School of Medicine, Stanford, CA 94305, United States. E-mail: rfama@stanford.edu

\section{R. GONZALEZ, I.M. PACHECO-COLON \& C. LÓPEZ-QUINTERO. Reward Sensitivity and Motivation among Adolescent Cannabis Users.}

Objective: Others have reported altered brain activation during reward sensitivity (RS) tasks that are thought to reflect poorer motivation among cannabis users (CU). Yet, the link between RS and motivation among CU is not well studied. We examined whether RS was associated with lower motivation among adolescents and whether the magnitude of this association varied with amount of cannabis use.

Participants and Methods: Participants were 347 healthy youth (ages 14-17) from a larger study on cannabis use and decision-making (DM). 
RS was assessed via the Cups Task, a measure of DM under specified risk, which varies the expected value of risky choices and includes conditions of possible gains or loss. We used the difference between $\%$ of risk advantageous and risk disadvantageous choices on gain trials as our index of RS, with higher scores indicating more RS. Motivation measures were the Apathy Evaluation Scale (AES) and the Motivation and Engagement Scale (MES).

Three groups were formed based on cannabis use history: no cannabis use (NCU, n=62); regular CU using about $3 \mathrm{x} /$ wk or more during the 6 mo prior to assessment (RCU, $\mathrm{n}=80$ ); light $\mathrm{CU}$ who reported using less frequently (LCU, n=205). Multiple linear regressions were conducted with group, RS, and their interaction as IVs, and each motivation measure as a DV. Between-group differences on potential confounds prompted covarying for age, estimated IQ, and frequency of alcohol and nicotine use in all analyses.

Results: RS was not significantly associated with any of the motivation measures. Two covariates were significantly associated with an MES index, such that lower estimated IQ $(p=.02)$ and more frequent alcohol use during the 6 mo prior to assessment $(p<.01)$ were associated with more amotivation.

Conclusions: Contrary to our hypotheses, RS was not associated with motivation. Our results suggest caution when interpreting altered RS as indicative of poor motivation among $\mathrm{CU}$ and indicate that IQ and alcohol use may be relevant contributors to levels of motivation. Grant\#R01DA031176

Correspondence: Raul Gonzalez, PhD, Psychology, Florida International University, 11200 SW 8th Street, AHC-4, Room 453, Miami, FL 33199, United States.E-mail: raul.gonzalezjr@fiu.edu

N.E. WADE, A.L. WALLANCE, A.M. SWARTZ \& K.M. LISDAHL. Aerobic Fitness and Marijuana Use Interact to Predict Neuropsychological Functioning in Emerging Adults.

Objective: Research suggests neurocognitive deficits associated with marijuana (MJ) use in emerging adults (Lisdahl \& Price, 2012). Regular MJ use downregulates endocannabinoid activity (Hirvonen et al., 2012), while aerobic exercise may upregulate cannabinoid receptor 1 activity (Ferreira-Vieira et al., 2014). Here we examine the influence of MJ use on a battery of neuropsychological tests, and whether aerobic exercise moderates these effects.

Participants and Methods: 88 subjects (38MJ, 50controls) aged 16-25 were balanced for gender (38F); $64 \%$ were Caucasian. Exclusion criteria: Left handed, comorbid Axis-I disorders, major medical or neurologic disorders, prenatal medical issues, or prenatal alcohol/ illicit drug exposure. Subjects completed a neurocognitive battery and a maximal exercise test. Multiple regressions predicted cognitive performance from past year $\mathrm{MJ}$ use, $\mathrm{VO}_{2}$ max, and $\mathrm{MJ} * \mathrm{VO}_{2}$ interactions, controlling for past year alcohol use and cotinine.

Results: Increased MJ use predicted decreased working memory (total score $\mathrm{p}=.02$; digit length $\mathrm{p}=.04$ ). Higher aerobic fitness predicted improved word reading on a Stroop task $(p=.02)$, and trails number $(p=.03)$ and letter $(p=.04)$ sequencing. MJ*VO2 predicted initial verbal memory $(p=.02)$, trails number $(p=.05)$ and letter $(p=.05)$ sequencing, switching ( $p=.04)$, and motor sequencing $(p=.002)$. Increased alcohol use predicted increased verbal fluency $(\mathrm{p}=.02)$ and fewer commission errors on an attention task $(\mathrm{p}=.01)$. Visual memory and processing, decision making, and executive functioning as measured by card sorting were not related to any predictors.

Conclusions: Increased MJ use significantly predicted poorer cognitive performance, especially in working memory. Improved aerobic fitness moderated the impact of MJ on initial learning, processing speed, and cognitive control, as more aerobically fit MJ users demonstrated improved performance relative to low-fit MJ users. Therefore, aerobic fitness may present a viable intervention for MJ users to improve cognitive functioning.
Correspondence: Natasha E. Wade, M.S., Psychology, University of Wisconsin-Milwaukee, 2441 E. Hartford Ave., Milwaukee, WI 53211, United States.E-mail:twright857@pointloma.edu

J. SCOTT, S. SLOMIAK, J.D. JONES, T.M. MOORE \& R.C. GUR. A Meta-Analysis of Cannabis and Cognitive Functioning in Adolescents and Young Adults.

Objective: Cannabis use in adolescents and young adults has been associated with cognitive dysfunction. Yet there is considerable controversy about the magnitude of deficits, especially after periods of abstinence. Here, we report a quantitative synthesis of the literature to date examining cognitive functioning in youth with heavy or frequent cannabis use.

Participants and Methods: Data from 78 cross-sectional studies was analyzed, totaling 8,332 participants, including 2,436 heavy/frequent cannabis users and 5,896 comparison participants with minimal cannabis exposure. Effect size estimates were calculated using mixed-effects models across thirteen cognitive domains: attention; immediate, delayed, and prospective memory; language; processing speed; visuospatial abilities; risky behavior; and components of executive functioning, including shifting/abstraction, inhibition, and working memory.

Results: There was a small overall effect size for cognitive functioning associated with problematic cannabis use (Cohen's $d=-.22$ ), with the largest effects in shifting/abstraction $(d=-.30)$ and immediate memory $(d=-.28)$. Effect size magnitudes were consistent across age group (i.e., adolescent, young adult, mixed), and use onset (i.e., early vs. late). However, studies requiring an abstinence period of 72 hours or greater had an overall effect size $(d=-.05)$ that was not significantly different from zero and was significantly less than studies with less stringent abstinence criteria $(p<.05)$.

Conclusions: Across the cross-sectional literature, associations between cannabis use in adolescents and young adults and cognitive functioning are likely small and of questionable clinical significance. Furthermore, abstinence of greater than 72 hours may further diminish the cognitive deficits associated with cannabis use. Studies of cannabis using youth may have overstated the extent and magnitude of cognitive deficits due to heavy or problematic use, and effects may be more associated with withdrawal or carryover effects from acute use.

Correspondence: J. Cobb Scott, Ph.D., Psychiatry, Perelman School of Medicine at the University of Pennsylvania, 3400 Spruce Street, 10th Floor, Gates Building, Philadelphia, PA 19104, United States. E-mail: scott1@upenn.edu

L.C. OBERMEIT, E.E. MORGAN, J.E. IUDICELLO, R.K. HEATON, S.P. WOODS, P. GILBERT, S. ROESCH \& I. GRANT. The Impact of Methamphetamine Dependence, HIV, and Frontal Systems Dysfunctions on the Relationship Between Sexual Risk Intentions and Behaviors.

Objective: Methamphetamine (MA) use and HIV preferentially impact the frontostriatal brain circuits and their dysfunction may increase risky behaviors, including sexual risk-taking. Risk interventions often target motivation, but intentions do not always translate to behaviors. Here we explored the contributions of HIV status, MA use, disinhibition, executive dysfunction (ED), and apathy to the link between intentions and behaviors. It was hypothesized that disinhibition, ED, HIV+, and MA+ would moderate the relationship between reported safe intentions and actual risk-taking behavior, and intentions would mediate the relationship between apathy and behavior.

Participants and Methods: The sample included 234 adults with/ without HIV (HIV+/-) and/or MA dependence (MA+/-), who had comprehensive cognitive and behavioral assessments. Composite variables represented disinhibition, ED, and apathy and self-report measures were used for intentions and risk behaviors. Interactions were evaluated through linear regressions and the mediated relationship was evaluated through bootstrapping. 
Results: As hypothesized disinhibition and MA+ were significant moderators $(\mathrm{ps}<.05)$ of links between intentions and behaviors, such that safer intentions predicted safer behaviors $(p s<.001)$, but only for MA- individuals or those with better inhibition. There also was a significant three-way interaction between intentions, ED, and HIV, such that only for HIV+ participants, better executive functioning resulted in a positive relationship between intentions and behaviors $(p=.005)$ and those with worse ED more often failed to implement their intentions $(p=.845)$. Finally, intentions mediated the relationship between apathy and behaviors $(\mathrm{CI}=[.006, .130])$ in the expected direction.

Conclusions: Disinhibition, ED, apathy, MA+, and HIV+ are important factors that may interact in complex ways to interfere with the implementation of safe intentions and may each be an important intervention target for reducing sexual risk-taking.

Correspondence: Lisa C. Obermeit, SDSU/UCSD Joint Doctoral Program in Clinical Psychology, 220 Dickinson Street, Suite B, San Diego, CA 92103, United States. E-mail: lisaobermeit@gmail.com

T.A. LEVINE \& L.J. WOODWARD. Early Inhibitory Control and Spatial Working Memory Abilities of Children Prenatally Exposed to Methadone.

Objective: Opioid use is increasing worldwide, with methadone maintenance being the most common method of treating opioid-dependence in pregnancy. However, little is known about the early neuropsychological development of methadone-exposed children. The aim of this study was to examine the early inhibitory control and spatial working memory abilities of a regional cohort of methadone-exposed and non-exposed comparison children.

Participants and Methods: Data were drawn from a prospective longitudinal study of 100 children born to women enrolled in methadone maintenance treatment during pregnancy who were studied alongside a randomly identified comparison group of 110 non-methadone-exposed children. At age 2 years, the Snack Delay Task, which measures inhibitory control, and the Three Boxes Task, which measures spatial working memory, were administered. At age 4.5 years, parents completed the Strengths and Difficulties Questionnaire.

Results: Methadone-exposed children had significantly poorer inhibitory control than non-exposed children. These differences were explained by family social risk at birth. On the working memory task, both groups performed similarly on the first trial set; however, on the second trial set, methadone-exposed children did not improve their task performance, while non-exposed children did. Finally, inhibitory control difficulties at age 2 years were predictive of behavioural adjustment at 4.5 years, including the extent of attention, conduct, and peer problems. Conclusions: Methadone-exposed children show early difficulties with inhibitory control and potentially poorer sustained attention or task learning, with these difficulties being predictive of later behavioural problems. These difficulties appear to reflect the effects of social contextual factors associated with maternal pregnancy drug use rather than direct drug effects. However, further follow-up is needed.

Correspondence: Terri A. Levine, PhD, Newborn Medicine, Harvard Medical School, 221 Longwood Avenue, Boston, MA 02115, United States.E-mail:terrialevine@gmail.com

\section{Panel Discussion, Presented by the INS Student Liaison Committee: Technology and New Approaches in Cognitive Rehabilitation}

\section{Presenters: Jonathan Evans, Bruce Luber,} Sarah S. Morimoto, Matthew Kurtz

$$
\text { 10:15-11:45 a.m. }
$$

\section{Poster Session 7. Assessment Across the Lifespan \\ 10:15-11:30 a.m.}

\section{Assessment/Psychometrics/Methods (Adult)}

A.P. AHRENS, W.R. COLE, J. ARRIEUX \& A. FINKEL. Deconstructing the Relationship Between Computerized Neurocognitive Assessment Tools (NCATs) and Mild Traumatic Brain Injury (mTBI) Symptomatology.

Objective: Mild traumatic brain injury (mTBI) engenders both physical (e.g. headache, vision, vestibular, sleep) and mental (e.g. cognitive, behavioural) symptoms. The ability of computerized neurocognitive assessment tools (NCATs) to identify sequelae specific to mTBI is essential for their use with populations exposed to higher rates of mTBI, e.g. military service members (SMs). Comparison to controls is the current standard for evaluating the validity of NCATs. We hypothesize that using symptom reports may be a methodological improvement for evaluation of NCATs.

Participants and Methods: SMs (272 controls, 232 acute mTBI) took two NCATs (ANAM4, CNS-VS, CogState, ImPACT), a traditional neuropsychological battery, and three validated symptom inventories (e.g. PHQ9, PCL, NSI). Multivariate analyses isolated the unique variance in NCAT scores attributable to symptom factors (identified by factor analysis) within the mTBI cohort, controlling for IQ.

Results: Self-reported symptoms loaded onto five main factors: somatic complaints, depression, Post-traumatic stress symptoms, cognitive issues, and sleep/fatigue. Aggregated, factor scores explained a significant proportion of variance in NCAT performance $\left(R^{2}\right.$ change $=.150$ to .307, $p<0.001)$, notably on the composite score, reaction-time and processing speed tests on ANAM4, verbal memory domain on CNS-VS, the composite score, reaction time and visual processing tests on CogState, and visual memory and motor speed indices on ImPACT. Predictive contributions varied, with somatic and cognitive issues explaining the most variance in tests of reaction time, visual processing and motor speed, and the affective symptoms together explaining the most variance in tests of learning and memory.

Conclusions: The use of symptom ratings may allow for more granular understanding of the role of NCATs in identifying sequelae after mTBI. This methodology appears to show more promise than categorical injury classifications and may be a more clinically relevant means of measuring the utility of NCATs.

Correspondence: Angelica P. Ahrens, MBA, Intrepid Spirit, Defense and Veterans Brain Injury Center, Womack Army Medical Center, Intrepid Spirit, Womack Army Medical Center, 3908 Longstreet Road, Ft. Bragg, NC20307,United States.E-mail: angelica.ahrens@alumni.duke.edu

S.L. AITA, S. TAYLOR, J. BEACH, J.S. HOLCOMBE, C.A. SOFKO, A. BOETTCHER, M. CALAMIA, E. ELLIOTT \& B.D. HILL. An Examination of the Construct Validity of CNS Vital Signs Using EFA.

Objective: The construct validity of the GNS Vital Signs (CNSVS) computerized neuropsychological test battery was evaluated using an exploratory factor analysis (EFA) approach.

Participants and Methods: 362 healthy participants $(M$ age $=21.26$, $S D=4.82 ;$ M education $=13.00, S D=0.98 ; 56.4 \%$ female; $67.7 \%$ White, 
22.7\% African American, 4.7\% Asian, 1.4\% Hispanic) completed a large neuropsychological battery that consisted of both paper and computerized experimental and clinical cognitive tasks. Data from 17 measures, which varied from verbal reasoning to executive functioning, was entered into an exploratory factor analysis. Using principle axis factoring and promax rotation, five factors were extracted based on the scree plot and used as latent cognitive variables. The five factors were labeled as followed based on cognitive task loadings: fluid reasoning. verbal comprehension, working memory, memory updating, and information processing speed. To examine the CNSVS's construct validity with various domains of cognitive functioning, Pearson correlation was used with CNSVS Composite indices (i.e., memory, psychomotor speed, complex attention, and cognitive flexibility) and the EFA generated factor scores.

Results: Overall memory was related to fluid reasoning, verbal comprehension, and general working memory ( $r=.19$ to $.27, p$ values<.05). Psychomotor speed was significantly related to all factors $(r=.23$ to $.42, p$ values<.01) while complex attention was only related to fluid reasoning $(r=.20, p<.05)$. Cognitive flexibility was related to all latent factors except for verbal comprehension $(r=.16$ to $.35, p$ values <.05). Conclusions: All CNSVS composite domains were significantly related to at least one of the latent extracted factors with correlation coefficients ranging from small to moderate strength. The CNSVS psychomotor speed domain had the strongest relation with the information processing speed factor. This study improves on efforts to establish the construct validity of the CNSVS with preexisting non-computerized cognitive tasks.

Correspondence: Stephen L. Aita, M.S., Psychology, University of South Alabama, 6451 Old Shell Rd. Unit 701, Mobile, AL 36608, United States.E-mail:sla1521@jagmail.southalabama.edu

G. ARMSTRONG, E.S. WANDINGER, J. HANSEN, M.E. CAMPBELL, A.F. PARKER, J. UPSHAW, H. MILLER, M. LIBBEN \& J. KOSS. A Computerized Version of the Color Trails Test.

Objective: Neuropsychological tests are performance-based measures used to assess cognitive functioning. The Color Trails Test (CTT) is a pencil and paper neuropsychological task that assesses executive functioning, sequencing skills and attention. We endeavoured to create a computerized version of CTT. We sought to demonstrate its equivalency with the standardized paper and pencil version. Additionally, we explored the validity of the second part of computerized CTT as a measure of executive functioning. We did this by comparing results from computerized CTT part 2 and a computerized version of the Wisconsin Card Sorting Test (WCST).

Participants and Methods: Participants consisted of undergraduate students who were attending the University of British Columbia - Okanagan $(N=40)$. Every participant completed all 3 neuropsychological tests during a 30-minute assessment.

Results: Significant relationships were found, using linear bivariate regression, between computerized and written versions of CTT part $1(r=.46, p<.001)$ and CTT part $2(r=.66, p<.001)$. Multiple Pearson product-moment correlation coefficients were computed to assess the relationship between the second part of the computerized CTT and the computerized WCST. Significant correlations were found, after a Bonferroni correction for multiple significance tests, between the computerized color trails test part 2 and categories completed $(r=.67$, $p<.001)$, conceptual level responses $(r=.56, p=.002)$, and perseverative responses $(r=.49, p=.001)$.

Conclusions: Our computerized version of CTT yielded similar results to the traditional paper and pencil version of the test and shows preliminary indications of equivalency. Additionally, the computerized CTT part 2 and WCST indicate that they measure a similar construct.

Correspondence: Graham Armstrong, Undergraduate, Pyschology, University of British Columbia - Okanagan, 3930 Patten Drive, Armstrong, BCVOE 1B2, Canada.E-mail:g_armstrong@live.com
G. ARMSTRONG, E.S. WANDINGER, D. LEITNER, J. UPSHAW, B. O'CONNOR, H. MILLER \& M. LIBBEN. Generating Neuropsychological Assessment Profiles Using Latent Class Analysis.

Objective: Neuropsychological tests are performance-based methods of measuring cognitive functioning. They are used to examine the cognitive consequences of brain damage, brain disease, and severe mental illness (Harvey, 2012). Latent class analysis (LCA) is a subset of structural equation modeling used to find groups or subtypes of cases in multivariate categorical data. The present study endeavoured to demonstrate the utility of LCA by identifying neuropsychological test profiles that correspond to various stroke types and locations. In doing so, the present study sought to lend evidence to the ecological validity of neuropsychological measures in a stroke population.

Participants and Methods: Participants who incurred a stroke and were receiving acute inpatient rehabilitation at Kelowna General Hospital were recruited to the study $(N=109)$. Participants were administered a comprehensive neuropsychological test battery during their rehabilitation.

Results: Latent class analysis revealed 3 neuropsychological test profiles based on scores across 8 neuropsychological tests. Additionally, 4 stroke profiles were identified among 4 stroke characteristics: 3 stroke locations ( $\mathrm{L}$ vs R, A vs P, and cortical vs subcortical) and 1 stroke type (ischemic vs hemorrhagic). A significant association was found between stroke type and 2 of the classes of neuropsychological test scores: Class 1 $(z=2.91, p<.05)$ and Class $2(z=-2.06, p<.05)$.

Conclusions: Results suggest that LCA could be useful for identifying neuropsychological test profiles that correspond to the location and type of stroke incurred. This demonstrates early evidence for the utility of LCA in a clinical neuropsychology setting. Additionally, our results provide evidence of ecological validity for the neuropsychological test battery.

Correspondence: Graham Armstrong, Undergraduate, Pyschology, University of British Columbia - Okanagan, 3930 Patten Drive, Armstrong, BCVOE 1B2, Canada.E-mail: g_armstrong@live.com

\section{J. AVILA \& S.P. VERNEY. Investigating Measurement Invariance of English and Spanish Versions of the National Alzheimer's Coordinating Centers Uniform Dataset Neuropsychological Battery in Hispanics.}

Objective: English to Spanish translations of neuropsychological batteries for dementia have the potential to better serve the Spanish speaking elderly Hispanic population in the U.S. However, previous research has inconsistently demonstrated construct equivalence between Spanish and English translations of the same measures. The purpose of this study was to examine construct comparability across the Spanish and English versions of the NACC UDS neuropsychological test battery in older Hispanics with varying levels of cognitive functioning.

Participants and Methods: The NACC UDS is a standardized evaluation conducted in a sample based on the U.S. population. The neuropsychological test battery is comprised of nine measures designed to assess cognitive domains affected early in the course of AD. NACC participants age 65 and older, who self-reported as Hispanic with normal cognition, MCI, or mild AD, were evaluated in either English (ENG; n=525) or Spanish (SPN; n=602). Groups were similar in age and gender; however, educational attainment was significantly higher for the ENG participants.

Results: Confirmatory factor analysis of the neuropsychological tests resulted in the four cognitive domain factors of memory, attention, executive functioning/processing speed and language. The model showed excellent fit for both groups. Invariance analyses indicated partial measurement invariance with the four-factor model demonstrating configural and metric invariance but not scalar invariance.

Conclusions: Findings suggest that neuropsychological test scores may measure equivalent cognitive traits across these two language-administration groups; however, intercept level differences indicate that absolute levels of performance cannot be meaningfully compared across 
groups. These findings demonstrate the need for group specific normative means for individual measures and factor scores. Failure to examine measurement invariance across different linguistic groups may lead to inaccurate classification of cognitive impairment.

Correspondence: Justina Avila, M.A., Psychology, University of New Mexico, 1820 Avenida Alturas NE, Albuquerque, NM 87110, United States.E-mail: jfavila@unm.edu

J. BEACH, S. TAYLOR, S.L. AITA, J.S. HOLCOMBE, J. EPKER, A. BOETTCHER, R. BURRELL, J. TAYLOR, M. ESPENAN \& B.D. HILL. Pain Catastrophizing Predicted by Emotional Distress and Cognitive Function in Chronic Pain Sample.

Objective: The purpose of the current study was to examine the relationship between emotional/internalizing dysfunction, cognitive function, and pain catastrophizing.

Participants and Methods: Two hundred sixty-two participants $(M$ age $=52.3, \mathrm{SD}=12.9 ; M$ education=13.5, $\mathrm{SD}=2.5 ; 60.5 \%$ Female, 89.9\% White, $10.1 \%$ African American) presented to a Southeastern pain clinic for pre-surgical evaluation. All participants completed the Emotional/Internalizing Dysfunction (EID) scale as part of the Minnesota Multiphasic Personality Inventory-2-Restructured Form (MMPI2-RF). Additionally, participants completed the Repeatable Battery for Assessment of Neuropsychological Status (RBANS), and the Pain Catastrophizing Scale (PCS) as part of a larger battery of tests.

Results: Pearson's product-moment correlations revealed that both the EID scores $\mathrm{r}(260)=.361, \mathrm{p}<.001$ and RBANS total scores $r(260)=-.233, p<.001$ were significantly correlated with PCS ratings. Additionally, a multiple linear regression using backwards elimination was calculated to predict scores on the PCS based on both the EID scale and the RBANS total score. A significant regression equation was found $(\mathrm{F}(2,260)=24.80, \mathrm{p}=<.001)$, with an $\mathrm{R}^{2}$ of .160 and both EID score $(\beta=.302, p<.001)$ and RBANS total score $(\beta=-.144, p=.003)$ significantly predicted pain catastrophizing scores.

Conclusions: The experience of pain is multidimensional and may depend on many intrapersonal factors including mood, affect, and cognitive ability. Higher scores on the emotional/internalizing scale as well lower scores on the RBANS significantly predicted the catastrophizing of pain. Further research should investigate the relationship between cognitive status, pain, and restructured clinical (RC) subscales of the MMPI-2-RF.

Correspondence: Jameson Beach, M.A., University of South Alabama, 162 April St, Mobile, AL 36608, United States. E-mail: jdb1623@ jagmail.southalabama.edu

\section{J. BERG, J. DURANT, S.J. BANKS \& J.B. MILLER. Comparing the Boston Naming Test and the Neuropsychological Assessment Battery Naming Subtest in an Outpatient Memory Disorders Clinic.}

Objective: The Boston Naming Test (BNT) and the Neuropsychological Assessment Battery Naming subtest (NAB-N) are two commonly used confrontation naming tests to evaluate word-finding ability in individuals suspected for neurodegenerative disease. Observations in practice suggest a consistent discrepancy between scores on the BNT and NAB-N in a dementia population. This study sought to explore this discrepancy in greater detail.

Participants and Methods: Records from 36 consecutive referrals seen for neuropsychological evaluation as part of routine care in an outpatient memory disorders clinic were reviewed. Discrepancy scores, concordance correlation coefficients (CCC) and root mean squared differences (RMSD) were calculated between demographically adjusted T scores on the BNT and NAB-N. Spearman's $p$ correlations between raw scores on the BNT, NAB-N, demographic variables, WRAT-4 Reading, WAIS-IV Similarities, Verbal Fluency and Animal Naming were calculated.

Results: Task performance differed by up to 27 points $(M=3.4$, $S D=10.3$ ) with $33 \%$ of the sample having a discrepancy score of greater than 1 standard deviation. The CCG was 0.37 , indicating a low level of agreement based on a consistency definition of agreement, and the RMSD was 10.29. BNT and NAB-N were significantly correlated ( $r=.58$; $p<.001)$. NAB-N was not significantly correlated with any other measure after accounting for multiple comparisons. BNT was significantly correlated with age $(\mathrm{r}=-.42 ; p<.01)$, education $(\mathrm{r}=.48 ; p<.01)$, WAIS-IV Similarities $(\mathrm{r}=.55 ; p<.001)$ and Animal Naming $(\mathrm{r}=.58 ; p<.001)$.

Conclusions: Despite similar task demands, individuals in a dementia population perform differently on the BNT versus NAB-N. Older adults and those with lower-levels of education perform worse on the BNT. Importantly, the BNT is much more strongly related to other measures of semantic knowledge than NAB-N, which showed no association. These findings suggest that the BNT may be more sensitive to language declines among those suspected for dementia than NAB-N.

Correspondence: Jody-Lynn Berg, M.S., Neuropsychology, Cleveland Clinic Lou Ruvo Center for Brain Health, $888 \mathrm{~W}$. Bonneville Ave., Las Vegas, NV 89106, United States. E-mail: bergj@ccf.org

J. BERNSTEIN, S. ROYE \& M. CALAMIA. Evaluating the Construct Validity of the King-Devick Test in a Psychological Outpatient Clinical Sample.

Objective: To examine the construct validity of the King-Devick test (K-D), a timed measure of rapid number-reading used to screen for symptoms after concussion.

Participants and Methods: Participants ( $\mathrm{n}=72,56.5 \%$ female) were individuals seeking psychoeducational evaluations at a university training clinic. Of the participants, $74.1 \%$ were Caucasian, $12.9 \%$ African-American, 3.5\% Hispanic, 3.5\% Asian, and 1.2\% Native American. Participants had a mean of $24.2(S D=7.9)$ years of age and mean of $14.4(S D=2.4)$ years of education. Participants completed measures examining domains indicative of convergent validity (e.g., processing speed, simple and sustained attention) as well as divergent validity (e.g., other aspects of intellectual functioning, inhibition, verbal fluency, conceptual flexibility).

Results: Slower total time to completion on the K-D was associated with worse processing speed (WAIS-IV Processing Speed Index: r $(70)=-.33$, $\mathrm{p}<.01)$ and poorer performance on a of basic reading speed (D-KEFS Word Reading: $\mathrm{r}(70)=-.26, \mathrm{p}<.05)$. Slower total K-D time was also associated with slower reaction time to target stimuli on a measure of sustained attention (Conners Continuous Performance Test $3^{\text {rd }}$ Edition Hit Reaction Time: $\mathrm{r}(70)=.275, \mathrm{p}<.05)$. K-D time was not associated with other domains of cognitive functioning (e.g., verbal comprehension, conceptual flexibility) (all $\mathrm{p}>.05$ ).

Conclusions: Consistent with prior literature, the K-D appears to be a valid measure of processing speed, attention and timed reading ability. Future work should examine these relationships in a more heterogeneous clinical sample that includes individuals with recent concussions.

Correspondence: John Bernstein, Louisiana State University, 217 Audubon Hall, Louisiana State University, Baton Rouge, LA 70803, United States. E-mail: jberns3@lsu.edu

A. BLOCH, S. MARIL, G. KAVE \& D. MARGALIT. A New Vision for Neuropsychological Assessment: Unlocking the Gate to Rehabilitation for Individuals with Brain Damage.

Objective: Brain damage is easily misdiagnosed or overlooked even when it has drastic effects on cognition and emotion. Health laws in many countries entitle citizens to neuropsychological assessment, but world health statistics reveal that few individuals are referred. We aimed to define when neuropsychological assessment is indicated and to propose a clear and feasible referral model. To do so, we examined referral policies in a number of health systems and conducted an initial survey of current knowledge regarding neuropsychological assessment among Israeli physicians.

Participants and Methods: 50 primary care physicians in Israel completed an anonymous online survey with 12 questions addressing their knowledge of and views on neuropsychological assessment, the extent to which they found it necessary, and the number of referrals 
they made annually, as well as information about relevant resources and policies in the academic and professional organizations with which they were affiliated.

Results: Most participants believed neuropsychological assessment was beneficial. However, one third reported that they had not referred a single individual to neuropsychological assessment in the year prior to the survey, despite having seen several clients who needed it. Nearly all doctors $(80-90 \%)$ said that they had not been exposed to formal guidelines for referral to neuropsychological assessment during their education and professional training as well as during their years of practice within the organizations at which they worked. Fewer than $20 \%$ of participants felt that they had clear guidelines on which to base referral decisions.

Conclusions: The findings emphasize the need to increase awareness of neuropsychological assessment among physicians, to define the target population for assessment, and to implement standardized and feasible referral policies. Implications and applicability of the proposed referral model within and beyond the Israeli health system are discussed. Correspondence: Ayala Bloch, Ph.D., The National Institute for the Rehabilitation of the Brain Injured, 89 Itzhak Sadeh St., Tel Aviv 6722820, Israel.E-mail: ayalabl@shikumil.org.il

E. BOLCEKOVA, T. NIKOLAI, G. VECHETOVA, P. DUSEK, R. RUSINA \& E. RUZICKA. Interference Scores In Trail Making Test And Prague Stroop Test Do Not Seem As Valid Discriminative Measures In Patients With Neurodegenerative Diseases.

Objective: Besides raw scores, some neuropsychological tests offer additional computed indices. In Trail Making Test (TMT) and Prague Stroop Test (PST), interference scores can be calculated. They should reflect the subject's executive functions, particularly the ability to cope with interfering information, corrected for psychomotor speed. The aim of this work was to evaluate discriminative ability of these scores in different neurodegenerative diseases. We hypothesized that the scores would be more impaired in patients with frontotemporal lobar degeneration (FTLD), and Parkinson's disease (PD), where the deficit of executive functions is expected, in comparison to patients with Alzheimer's disease (AD), where memory impairment is dominant.

Participants and Methods: The study includes 202 subjects (119 females and 83 males, mean age 74.5, SD 7.3, mean education 13.5 years, SD 3.2). There were 41 patients with $\mathrm{AD}, 25$ patients with PD, 19 patients with FTLD, and 117 healthy control subjects. All groups were matched for age and education, and the patients groups were also matched for MMSE score. All participants completed a comprehensive neuropsychological battery, including TMT and PST. Interference scores were calculated as TMT B / TMT A, and PST C / PST D. Only patients who were able to complete all parts of TMT and PST were included. Results: Neither of the interference scores correlates with age or education on the whole sample. Both correlate significantly with MMSE, FAB, and RBANS Total score $(p<.01)$. ROC analysis shows AUCs from 0.53 to 0.71 for TMT, and from 0.56 to 0.70 for PST, i.e. low discriminative abilities.

Conclusions: These results suggest that computed interference scores do not add much information to neuropsychological examination. Raw test scores seem a more appropriate tool to describe the patient's neuropsychological state. Further research could focus on finding more adequate computational procedures for interference effects.

This work is supported by Progres Q27/LF 1 (Charles University, Prague).

Correspondence: Eva Bolcekova, Ph.D., 1st Faculty of Medicine, Charles University, Katerinska 30, Praha 2128 21, Czechia. E-mail: eva. bolcekova@lf1.cuni.cz
A.M. BRUNETTE, M. CALAMIA, J. BLACK \& D. TRANEL. Is Episodic Future Thinking Important for Instrumental Activities of Daily Living? A Study in Neurological Patients and Healthy Older Adults.

Objective: Episodic future thinking is the ability to mentally project oneself into the future. This construct has been explored extensively in neuroscience and may be relevant for adaptive functioning. However, it has not been determined whether the measurement of episodic future thinking might be valuable in a clinical neuropsychological setting. The current study investigated: (1) the relationship between episodic future thinking and instrumental activities of daily living (IADLs); and (2) whether episodic future thinking is related to IADLs over and above standard measures of cognition.

Participants and Methods: 61 older adults with heterogeneous neurological conditions and 41 healthy older adults completed a future thinking task (the adapted Autobiographical Interview), a performance based measure of instrumental activities of daily living (the Independent Living Scales), and standard clinical measures of memory and executive functioning.

Results: Episodic future thinking significantly predicted IADLs after accounting for age, education, gender, and depression (increase in $\mathrm{R}^{2}=.050, \mathrm{p}=.010$ ). Episodic future thinking significantly predicted IADLs over and above executive functioning (increase in $\mathrm{R}^{2}=.025$, $\mathrm{p}=.030)$, but was not predictive of IADLs over and above memory $(\mathrm{p}=.157)$.

Conclusions: This study suggests that episodic future thinking is significantly associated with IADLs, beyond what can be accounted for by executive functioning. However, there is limited evidence for the clinical utility of episodic future thinking because episodic future thinking did not predict IADLs over and above memory. The findings suggest that an episodic future thinking task does not provide enough valuable information about IADLS to justify its inclusion in a clinical neuropsychological setting.

Correspondence: Amanda M. Brunette, Bachelor of Arts, Psychological and Brain Sciences, University of Iowa, 44 Washington Street Apt 1007, Brookline, MA 02445, United States. E-mail: amanda-mward@uiowa.edu

M.E. CAMPBELL, A.F. PARKER, D. LEITNER, J. UPSHAW, G. ARMSTRONG, H. MILLER \& M. LIBBEN. A Comparison of Canadian and American Normative Scores on the Wechsler Adult Intelligence Scale- Fourth Edition in a Traumatic Brain Injury Population.

Objective: The Wechsler Adult Intelligence Scale- Fourth Edition (WAIS-IV) is a commonly used measure of intellectual ability. Canadians were found to score two to five points higher than Americans on Wechsler scales when using American norms, therefore Canadian norms were developed. However, large differences have been noted when scores are calculated using American norms compared to Canadian norms, which can result in a different classification of impairment. This can impact decisions regarding competency and potential further care. The present study examined differences in results when Canadian vs. American norms were used for the WAIS-IV in a traumatic brain injury (TBI) population.

Participants and Methods: This study included $33(n=11$ female, $n=22$ male) Canadian individuals, who sustained a TBI and received a neuropsychological evaluation through a private practice. Participants were ages 18-64 $(M=38.52, S D=16.01)$, and had 9-19 years $(M=12.67, S D=2.30)$ of education. This study used archival data from private practice files of patients assessed between 2010 and 2016. Raw scores were converted to standardized scores with both the Canadian norms and American norms. Composite scores calculated with Canadian norms were compared to corresponding composite scores calculated with American norms for each scale (VCI, PRI, WMI, PSI, FSIQ, GAI). Results: Dependent t-tests revealed no significant difference between American and Canadian normed scores for the VCI after the Bonferroni adjustment $(p=.019)$. However, significant differences (all $p$ values 
$<.001)$ were found between composite scores calculated with Canadian vs. American norms for all other scales (PRI, WMI, PSI, FSIQ, GAI), with Canadian normed scores significantly worse than their American normed counterparts.

Conclusions: The findings of this study contribute to the existing literature on disparate results produced by the use of Canadian vs. American norms with the WAIS-IV and highlight the importance of consistency in norms chosen for clinical practice.

Correspondence: McKenzie E. Campbell, BSc. (Hons), University of British Columbia - Okanagan, 4890 Lakeshore Rd, Kelowna, BC V1W4H7, Canada.E-mail: mckenzie.campbell@alumni.ubc.ca

C.I. CARRIón, C. PERSAD, N. SCAPINI, E. PRINCE, R. CHIGURUPATI, S. KLEM \& B. GIORDANI. Alertness Maintenance Tasks Improve Driving Behaviors for Younger and Older Adults.

Objective: Alertness Maintenance Tasks (AMT) used for short durations led to reduced sleepiness and improved driving performance in drivers under simulated driving conditions (Song et al., 2017). In the present study, we extended the duration and added additional AMTs to determine whether younger and older adults would be differentially affected under the extended time conditions.

Participants and Methods: Thirty younger (18-34, $M=22.94$, $S D=3.26)$ and 18 older adults $(56-78, M=65.14, S D=6.27)$ were randomly assigned to one of three AMTs (tunes, comedy, trivia). Sleepiness/fatigue was induced through a 50 -minute simulated boring drive under dim light conditions. Participants then completed two driving conditions: 1) 10-minute normal driving (single task) and 2) 20-minute AMT driving (dual task). Driving behavior was measured by velocity variance and lane position variability (lane offset). Repeated measures ANCOVAs were used to determine effects of AMT and age on driving behaviors.

Results: Simple main effects showed lane position variability decreased for both younger $(F(1,27)=6.62, p<.05)$ and older $(F(1,15)=6.32$, $p<.05)$ drivers when exposed to the AMT. There were no significant differences between older and younger adults' velocity variability across driving conditions. There were no significant interactions between the effects of age group and driving condition on driving behaviors.

Conclusions: Our study demonstrated that both younger and older adults' driving behaviors improved after being exposed to an AMT irrespective of dual task content. In addition to assisted driver technologies (e.g. lane departure warning), vehicle manufacturers should explore the benefits of built-in AMTs in reducing driver fatigue and potentially decreasing the overall rate of motor vehicle collisions.

Correspondence: Carmen I. Carrión, PsyD, Psychiatry, University of Michigan, 2101 Commonwealth Blvd., Suite C, Ann Arbor, MI 48105, United States.E-mail: carrionneuropsy@gmail.com

M. CHAN, D. HARVEY, D. MUNGAS \& S. FARIAS. Associations Between the Everyday Cognition (ECog) Scales and Neuropsychological Performance Among Diverse Racial/Ethnic Groups.

Objective: The purpose of this study is to explore how the ECog, a measure of everyday functioning, is associated with cognitive performance across several ethnic groups including Caucasians, Hispanics, and African Americans. We also examined the association of ECog and change in cognition across these groups.

Participants and Methods: Cross-sectional data from a sample of 330 Caucasian, 156 African American, and 143 Hispanic participants at the University of California, Davis Alzheimer's Disease Center were used to assess if the ECog was similarly related to cognition (episodic memory and executive functioning) across these racial/ethnic groups. Longitudinal data from a subset (126 Caucasians, 51 African Americans, and 37 Hispanics) were used to examine if the ECog was similarly associated to cognitive change across the groups.
Results: The association between the ECog and both episodic memory and executive functioning was similar among Caucasians, African Americans, and Hispanics. The ECog was not associated with change in episodic memory in any of the groups, but higher baseline ECog (worse everyday function) was also associated with faster decline in executive abilities among all three ethnic groups. There were no detectable interactions in either cross-sectional or longitudinal analysis, suggesting the functional and cognitive relationships were similar across groups.

Conclusions: Previous work has shown that neuropsychological test performance can be affected by a variety of demographic and other factors such as years of education, quality of education, English as a second language status, etc. The current findings suggest that the relationship between functional abilities, as measured by the ECog, and aspects of cognition are similar across our three ethnic/racial groups. Results suggest that the ECog may be measuring everyday functioning similarly among these groups, although further study is still warranted. Correspondence: Michelle Chan, Ph.D., Neurology, University of California, Davis, 4860 Y Street, Suite 3700, Sacramento, CA 95817, United States.E-mail: mlchan@ucdavis.edu

J. DAVIS \& S. ROLIN. Estimating Premorbid Ability in Rehabilitation Patients Using the Test of Premorbid Functioning and Wide Range Achievement Test-4.

Objective: Berg, Durant, Banks, and Miller (2016) examined the concordance of premorbid ability estimates using Test of Premorbid Functioning (TOPF) and Wide Range Achievement Test-4 Reading (WRAT) performances. This study sought to replicate the analytic approach in a rehabilitation sample and extend the findings by including a current estimate of intellectual ability (FSIQ).

Participants and Methods: The sample $(N=145)$ was $28 \%$ female with average age and education of 40.6 and 13.2 years, respectively. Participants underwent outpatient neuropsychological evaluation in the context of traumatic brain injury $(n=96)$, stroke $(n=22)$, and other conditions ( $n=27$; e.g., anoxia). Participants completed the TOPF, WRAT, and Wechsler Adult Intelligence Scale-IV among other measures. Initial analyses compared TOPF and WRAT. Subsequent analyses compared TOPF and WRAT with FSIQ. Analyses included paired and one sample t-tests, pairwise correlations, concordance correlation coefficients (CCC), and root-mean-squared differences (RMSD).

Results: TOPF $(M=93.7, S D=14.8)$, WRAT $(M=93.4, S D=13.9)$, and FSIQ $(M=92.6, S D=13.9)$ scores were not significantly different using paired t-tests $(p=.18-.54)$. Pairwise correlations were strong: TOPF-WRAT $(r=.92)$, TOPF-FSIQ $(r=.76)$, and WRAT-FSIQ $(r=.73)$. TOPF and WRAT showed acceptable CCC $($ rho-c $=.92)$; RMSD was 5.9. The TOPF-WRAT difference $(M=0.30, S D=5.9)$ was not significantly different from $0(p=.54)$. Compared to FSIQ, TOPF and WRAT ability estimates were similar in CCC (rho-c $=.75$ and .72 , respectively) and RMSD (10.1 and 10.1, respectively). TOPF-FSIQ $(M=-1.12, S D=10.1)$ and WRAT-FSIQ $(M=-0.83, S D=10.1)$ differences were not significantly different from $0(p=.18-.33)$.

Conclusions: TOPF and WRAT ability estimates were comparable across a range of metrics and demonstrated similar relationships to FSIQ. Findings provide preliminary support for WRAT ability estimates in a rehabilitation sample.

Correspondence: Jeremy Davis, Psy.D., Physical Medicine and Rehabilitation, University of Utah, 30 N 1900 E, Salt Lake City, UT 84132, United States. E-mail: jeremy.davis@hsc.utah.edu

\section{J. DONDERS, K. DARLAND \& A. PENDERY. SCREENING FOR DEPRESSION AFTER TRAUMATIC BRAIN INJURY.}

Objective: The purpose of this study was to determine the predictive validity of the Patient Health Questionnaire-2 (PHQ-2) and Patient Heath Questionnaire-9 (PHQ-9) when screening for symptoms of depression following traumatic brain injury. 
Participants and Methods: This was a retrospective analysis of data collected as part of routine clinical outpatient care over a period of 30 months. Participants included168 persons with mild to severe traumatic brain injury who were referred for neuropsychological evaluation within 1-12 months. They all completed the short PHQ-2 and the full PHQ-9 as part of their evaluation. In addition, valid data from the Minnesota Multiphasic Personality Inventory, Second Edition, Restructured Form (MMPI-2-RF) were available for $137(82 \%)$ of these individuals.

Results: PHQ-2 scores $\geq 2$ had 90\% sensitivity in predicting PHQ-9 scores $\geq 10$, and $95 \%$ sensitivity in predicting endorsement of suicidal thoughts on the PHQ-9. PHQ-9 scores $\geq 10$ had a sensitivity of 91.7 and a specificity of 60.2 in predicting a final diagnosis of major depression. Correlations between PHQ-9 scores and MMPI-2-RF scales Demoralization (0.64) and Low Positive Emotions (0.48) ranged from large to medium. A premorbid history of personal abuse and/or outpatient psychiatric treatment was the most consistent predictor of PHQ-9 elevations as well as final diagnoses of major depression.

Conclusions: The PHQ-2 and PHQ-9 have adequate clinical utility as a screening instrument for depression in outpatients with traumatic brain injury. However, elevations on this instrument are affected by premorbid issues and cannot be automatically be attributed to neuropathology. Clinicians should conduct more thorough follow-up assessment when PHQ-9 scores are highly elevated.

Correspondence: Jacobus Donders, Psychology, Mary Free Bed Rehabilitation Hospital, 235 Wealthy, S.E., Grand Rapids, MI 49503, United States. E-mail: jacobus.donders@maryfreebed.com

N. FEIRSEN, B. FREILICH, T. RUBINSTEIN, C. FERNANDEZCARBONELL \& E. WELTON. The Attention, Memory, and Frontal Abilities Screening Test (AMFAST): A Normative Study in Children \& Adults.

Objective: The purpose of this study is to provide normative data for a novel cognitive screening measure designed to assess basic attention, memory, and executive functioning (EF); these domains are often impacted in many neurologic/psychiatric disorders in both children and adults (e.g., TBI, MS, lupus, HIV, Parkinson's disease, schizophrenia, unipolar/bipolar depression, ADHD). To date, no other screening measure specifically evaluates these domains exclusively. The AMFAST requires minimal training to administer and can be completed in less than 10 minutes. Ultimately, it can be used to determine if further neuropsychological testing is needed.

Participants and Methods: 136 healthy controls (ages 11-89) were recruited. Individuals were excluded if they presented with any cognitive complaints, medical conditions known to impact cognition, or if they were taking medications with known cognitive side effects. Participants represent a Bronx community sample with roughly equal numbers of males/females of various ethnic backgrounds and education levels. The AMFAST was administered at the time of recruitment.

Results: Scores for each of the 6 individual AMFAST subtests were calculated using a scoring system that was based on the distribution of the responses in the normative sample. Total AMFAST scores were then calculated by adding up the individual subtest scores. Total scores were normally distributed, and no significant differences were found between groups in terms of sex, age, or education. The mean total score was found to be $88.61 / 100(\mathrm{SD}=7.06)$.

Conclusions: The AMFAST is a novel cognitive screener that can be quickly administered to identify the presence of attention, memory, and EF deficits in children and adults. Given that there were no significant effects of age, sex, or education, total AMFAST scores do not require any adjustments for interpretation.

Correspondence: Nicole Feirsen, Ph.D., Psychiatry, Montefiore Medical Center, 3307 Bainbridge Avenue, Bronx, NY 10467, United States. E-mail: nfeirsen@montefiore.org
N. FEIRSEN, B. FREILICH, T. RUBINSTEIN \& E. WELTON. The Attention, Memory, and Frontal Abilities Screening Test (AMFAST): A Clinical Efficacy Study in Children and Adults.

Objective: The present study describes the effectiveness of a novel cognitive screening measure designed to assess impairments in processing speed, attention, memory, and executive functioning (EF) that are present in many neurologic and psychiatric disorders in both children and adults.

Participants and Methods: 94 individuals seeking neuropsychological evaluation were recruited for the study. They represent a diverse Bronx community sample from ages 11-89, with roughly equal numbers of males/females of various ethnic backgrounds and education levels. This mixed clinical sample included patients with a variety of neurological and psychiatric conditions such as Alzheimer's disease, vascular dementia, MCI, lupus, mood disorders, psychotic disorders, ADHD, sickle cell disease, cancer, and TBI. All participants received the AMFAST and comprehensive neuropsychological batteries, including testing in attention, memory, and EF domains. Impairment on the battery was defined as $1.5 \mathrm{SD}$ below the normative mean (i.e., < $<\%$ ile) on at least $25 \%$ of the administered attention, processing speed, EF, and memory tests. Impairment on the AMFAST was defined as scores $\leq 70 / 100$.

Results: An overall cutoff score of $\leq 70 / 100$ on the AMFAST had $95 \%$ sensitivity and $97 \%$ specificity (96\% classification rate) in terms of identifying cognitive impairment within the comprehensive neuropsychological battery. The AMFAST correctly classified $96 \%$ of the overall sample using this cutoff.

Conclusions: The AMFAST is a sensitive and specific measure in terms of its ability to identify the presence of cognitive impairment in children and adults. This screening measure can therefore be effectively utilized to identify attention, memory, and EF deficits and determine the need for further comprehensive neuropsychological testing.

Correspondence: Nicole Feirsen, Ph.D., Psychiatry, Montefiore Medical Center, 3307 Bainbridge Avenue, Bronx, NY 10467, United States. E-mail:nfeirsen@montefiore.org

R.A. FORRESTER \& J.A. MOSES. Independent Components of Visual-Spatial Perception are Factorially Related to Specific Dimensional Components of Intelligence and Spoken Language. Objective: Factorial relationships among visual spatial perception, nonverbal and verbal intelligence and spoken language were examined. Participants and Methods: The sample consisted of 112 ambulatory American Veteran patients with mixed neuropsychiatric diagnoses. All participants completed the Judgment of Line Orientation (JLO), WAIS-III, and Multilingual Aphasia Examination (MAE). There were no demographic or diagnostic exclusion criteria. All participants who completed these three tests were included in the analysis. Mean age of the sample was $49.54(\mathrm{SD}=14.03)$ and mean education level was 13.16 $(\mathrm{SD}=2.52)$. Principal component analysis of the $30 \mathrm{JLO}$ items (5 item groups) produced a two-factor solution (JLO-1_easy: items 1 - 10 and JLO-2_Difficult: items $21-30)$. JLO-1 and JLO-2 were refactored with the four WAIS-III factors to produce a new four-factor solution with JLO-2 grouping with POI and JLO-1 grouping with WMI. Factor scales were computed for these computed variables.

Results: Principal component analysis of the MAE Token Test (Auditory Comprehension), Sentence Repetition (SR), Verbal Fluency (VF), and Visual Naming (VN) subtests occurred with the four JLO_WAIS-III factors to produce a new four-factor solution which explained $70 \%$ of the variance. JLO_1 grouped with SR_WM, JLO-2_VCI grouped with VN, JLO-2_POI grouped with AC and JLO2fac.NoLoad_VF grouped with PSI.

Conclusions: Individual aspects of visual spatial ability appear to contain components that are dimensionally related to factorial components of spoken language and intelligence. Both verbal and nonverbal mediation occur during visual spatial processing. Grouping of JLO_1 with SR_WM suggests that early JLO items are moderated by verbal 
working memory. Grouping of JLO-2_VCI with VN and grouping of JLO-2_POI with AC suggests that successful response to difficult JLO items is mediated by verbal concept formation ability.

Correspondence: Robert A. Forrester, M.S., Clinical Psychology, Palo Alto University, 9130 Nolan Street Apt \#163, Elk Grove, CA 95758, United States.E-mail: rforrester@paloaltou.edu

E.B. GALLANTER, J. HONG, E. MULLER-OEHRING \& T. SCHULTE. Characterizing procedural learning and memory with perceptual-motor sequence tasks.

Objective: The process of procedural learning and memory is divided into three distinct phases: cognitive, associative, and autonomous. The first involves conscious learning and the second phase consists of rehearsal, while the third is a truly automatic phase, in which the task is independent from conscious thought.

Participants and Methods: The current study aims to present a FingerTyping Task (FTT) able to distinguish between the phases of procedural learning and therefore capable of confirming that a procedural memory has formed. Twenty-two healthy participants ( 15 women; $36.1 \pm 19.9$ years) were assessed using the FTT, in which they typed a repeating sequence (RS) of letters. The test was split by a rest period, and the RS trials were nested between two groups of non-repeating sequence (NRS) trials.

Results: We were able to statistically differentiate between the cognitive, associative, and autonomous phases of procedural learning using the FTT. An initial (cognitive phase) learning curve was identified at the beginning of the RS trials, followed by steady improvements through rehearsal (associative phase), ending with a sustained high level of performance (autonomous phase). Following a rest period, there was a second learning curve, which culminated in a new high level that was again sustained. Statistical analysis of the FTT dataset intercepts identified this period as a second cognitive phase, followed by a second autonomous phase.

Conclusions: Our study provides support to the three phases model, and presents a measure capable of detecting when a procedural memory has been formed. We propose conceptualizing the cognitive and associative phases as the learning process, and the autonomous phase as a true, functionally automatic, procedural memory. The FTT can be useful in assessing motor procedural learning and memory skills during healthy development and aging, and in individuals with motor impairment. The FTT also permits functional MRI applications. Support: AA023165 Correspondence: Eden B. Gallanter, Palo Alto University \& SRI International, 333 Ravenswood Avenue, Menlo Park, CA 94025-3493, United States.E-mail: eden.gallanter@sri.com

L. GAVRON, T.J. ARENTSEN, B. STEPHENS, E. CROUSE \& B.L. ROPER. Elevations on the MMPI-2-RF Neurological Complaints and Cognitive Complaints Scales and Performance on Effort Measures on Neuropsychological Testing in Veterans.

Objective: Bolinger, et al (2013) found that MMPI-2-RF Neurological Complaints (NUC) and Cognitive Complaints (COG) scales were elevated in undergraduate psychology students instructed to simulate head injury who also failed performance validity tests (PVTs), and these scales helped predict group membership when combined with traditional validity scales. The current study examined differences on NUC and COG responding between veterans who evidenced credible (C) vs. non-credible (NC) performance validity during concomitant neuropsychological testing.

Participants and Methods: Participants included veterans who completed clinical neuropsychological evaluation that included an MMPI-2 (converted to MMPI-2-RF for study), divided into C $(n=162)$ and NC $(n=79)$ groups. C group criteria included administration of $>3$ PVTs, all PVT scores WNL, and exclusion of Major Neurocognitive Disorder or Intellectual Disability. NC group criteria were failure of $>2$ of up to 5 PVTs or of $>3$ of up to 7 PVTs.
Results: NC group scored significantly higher than C group on both $\mathrm{NUC}, F(1,240)=6.33, p<.01, w=.15$, and COG, $F(1,240)=24.75$, $p<.001, w=.30$. Regardless of group, NUC and COG were highly correlated with other validity scales (all $p<.001$ ), with $r$ s ranging from .58 to .78 for all validity scales except $\mathrm{Fpr}(r=.48$ for COG; $r=.24$ for NUC). Logistic regressions used by Bolinger et al were reproduced to predict NC performance. The traditional validity scales (i.e., Fr and RBS) led to overall group classification accuracy of $71.4 \%\left(X^{2}(2)=30.35\right.$, $p<.001)$. The addition of NUC and COG slightly decreased or had no change on classification accuracy (to $69.3 \%$ and $71.4 \%$, respectively). The only significant variable in both final models was the RBS raw score. Conclusions: Although scores on the NUC and COG are related to concomitant PVT performance in a veteran population, the RBS score is a more robust predictor in differentiating $\mathrm{C}$ versus NC performance, which differs from previously documented research based on a simulator study.

Correspondence: Lauren Gavron, PhD, Neuropsychology, Memphis VA Medical Center, 1030 Jefferson Ave, Memphis, TN 38104, United States. E-mail:lauren.gavron2@va.gov

J.M. GRABYAN, T. NGUYEN, R. COLlins \& D.K. CHEN. Performance on the First Trial of the Word Memory Test Predicts Failure on Later Trials.

Objective: Performance validity testing is an increasingly vital component of neuropsychological evaluation, though administration of standalone performance validity tests (PVTs) can be time-consuming. This barrier is more salient in the context of busy inpatient evaluations, where time with the patient is at a premium. The Word Memory Test (WMT) is an often-used and well-researched PVT. However, its use in shorter evaluations may be limited by the presence of the 30-minute delay between trials, during which the types of measures (e.g., verbal memory) one can reasonably use are restricted. To test if elimination of the delay trials is feasible, it was hypothesized that performance on the first trial, Immediate Recall (IR), would predict failure on the subsequent Delayed Recall (DR) and Consistency Index (CN) with high sensitivity and specificity.

Participants and Methods: Subjects were 423 inpatient veterans referred for neuropsychological screening as part of a week-long inpatient observation on an epilepsy monitoring unit. Patients were administered the WMT's IR and DR (and CN was then derived) as part of a larger battery.

Results: Using standard cut scores of $\leq 82.5 \%$ [GJM1] correct as indicative of failing DR or CN (and thus failing the overall measure), $\leq 87.5 \%$ correct on IR yielded a sensitivity rate of .85 and specificity of .93 . Youden's Index of receiver operator characteristic (ROC) analysis also revealed this $\leq 87.5 \%$ cut-off as the optimal choice.

Conclusions: WMT IR shows excellent classification statistics in predicting future WMT trial performance. In populations where time with the patient is scarce, such as brief inpatient stays, WMT IR can potentially be used as a stand-in for the WMT. Using this early discontinue criteria could allow the examiner to use their time more effectively, especially as they would not need to plan around the 30-minute delay. Nevertheless, even if the WMT is shortened, providers should continue to utilize a robust combination of stand-alone and embedded PVTs. Correspondence: Jonathan M. Grabyan, Ph.D, Mental Health Care Line, Michael E. DeBakey VA Medical Center, 2120 Texas, Apt 2204, Houston, TX 77003, United States. E-mail: jorabyan@gmail.com

Z. GRETH, A. RENO, B. ABLITZ, E. RINEHARDT \& S. LEININGER. Abbreviated Validity Testing: Differential Performance Between Litigating and Non-Litigating Clinical Outpatients.

Objective: Recent research has examined abbreviated methods of performance validity testing (PVT) using the Test of Memory Malingering (TOMM). TOMM Trial 1 and the first ten items on Trial 1 (TOMMe10) have been suggested as two separate PVT measures. Initial findings were favorable for both in a forensic setting. Previous findings 
with this study's clinical sample indicated a significant difference in TOMM Trial 1 scores between litigating and non-litigating outpatients during neuropsychological evaluation. The purpose of this study was to further investigate the sample's TOMMe10 performance.

Participants and Methods: Archival data was collected from a sample of 54 outpatients. The sample included three groups: (1) mild, moderate, and severe traumatic brain injury (TBI; $\mathrm{n}=23$ ); (2) mild-severe TBI under litigation (TBI-L; $\mathbf{n}=11$ ); and (3) dementia with mixed etiologies and severities (D; $n=20)$. Raw scores for TOMMe10 were compared between the three groups using an analysis of variance (ANOVA) and post hoc testing.

Results: An ANOVA highlighted a significant TOMMe10 difference between groups. Tukey's test revealed a significant difference between TBI and TBI-L $(\mathrm{M}=9.5$ and $\mathrm{M}=7.5$, respectively); as well as TBI-L and $\mathrm{D}(\mathrm{M}=7.5$ and $\mathrm{M}=9.3$, respectively $)$. Scores among patients with TBI and D were not significantly different. Only the TBI-L average exceeded the suggested cutoff of $\geq 2$ errors. TOMMe10 performance was not adversely affected by gender, ethnicity, age, or education. A strong, positive correlation $(r=0.91, p<0.01)$ existed between TOMM Trial 1 and TOMMe10.

Conclusions: The results were consistent with preliminary research, and expounded on TOMM Trial 1 and TOMMe10 in an outpatient medical center. The TBI-L group scored significantly lower on TOMMe10 (i.e., more errors) than TBI and D. This supports the use of TOMMe10 as an abbreviated PVT measure. Continued investigation may further validate TOMMe10 as a sensitive screening tool to assess effort in clinical outpatients during neuropsychological assessment.

Correspondence: Zach Greth, Bachelor of Science: Psychology, Sociology, Neuropsychology \& Psychology, Carolinas HealthCare System, 9024 Lenox Pointe Dr, Apt 303, Charlotte, NC 28273, United States.E-mail: zach.greth027@gmail.com

L. HAM, S. LEHMAN, A. MATCHANOVA, R. DIVERS, T. GIOVANNETTI \& C.C. TAN. Using Wearables to Measure Pauses During Everyday Activities.

Objective: Everyday functional difficulties are associated with various negative outcomes in healthy older adults; however, methods for efficient detection and characterization of mild functional difficulties are lacking. To assess subtle disturbances in functioning, we conducted a study using a smartwatch to capture arm movements while completing an everyday task.

Participants and Methods: 14 community-dwelling older adults and 21 healthy young adults were asked to prepare a lunch using real objects while wearing a smartwatch that recorded their hand movements and pauses. Young adults also prepared the lunch while simultaneously performing a secondary task to induce resource limitations (Dual Task). Trained coders tallied time to completion and total errors from videos. Pause counts were obtained from the smartwatch based on the speed of hand movements $($ pause $=$ speed $<.01 \mathrm{~m} / \mathrm{s}$ ) within a window of about $0.4 \mathrm{~s}$. Cognitive tests of episodic memory, attention, and executive function also were administered.

Results: Older and young adults did not differ in number of pauses ( $p=.62)$ or completion time $(p=.87)$, though older adults made more total errors $(p=.03)$. Participants who made more pauses also performed more poorly on a test of executive function $(r=.48, p<.01)$. Comparing young adult performances on the lunch task with and without the Dual Task showed significantly more pauses in the Dual Task $(p=.04)$, but no difference in completion time $(p=.96)$, and only marginally more total errors $(p=.06)$. Additionally, young adults who made more pauses also made fewer errors in the Dual Task $(r=-.40, p=.09)$.

Conclusions: When cognitive resources are limited, young adults make strategic pauses to compensate for increased cognitive load. Older adults pause as frequently as young adults but make more errors, suggesting older adults may not be slowing their movements to promote deliberate error monitoring. Wearables may offer deeper insights into functional difficulties that are not possible through traditional error coding.
Correspondence: Lillian Ham, Psychology, Temple University, 1701 N. 13th Street, Weiss Hall, Sth floor, Philadelphia, PA 19122, United States.E-mail: lillian.ham@temple.edu

W.C. HOCHBERGER, M. MCDONNELL \& V. ZIZAK. Augmenting Brief Cognitive Screens with Assessment of Judgement: Integration of The Test of Practical Judgement (TOP-J) and the Montreal Cognitive Assessment (MoCA).

Objective: There has been a steady growth of the geriatric population in the United States, which has resulted in an increasing prevalence, and risk, of cognitive and functional decline secondary to neurodegeneration. Brief cognitive assessment (such as the Montreal Cognitive Assessment $[\mathrm{MoCA}]$ ) presents as an efficient tool for detecting impairment due to its high sensitivity and relative ease of use. However, the MoCA's conceptualization of certain higher-order cognitive domains, notably judgement, is relatively limited. Retrospective analyses were employed to determine if adding a quantitative measure of problem solving and judgement (the Test of Practical Judgement [TOP-J]) would provide incremental validity to the MoCA's prediction of global executive function (derived from measures of set shifting, problem solving, and abstract reasoning), and if this addition would thus improve diagnostic accuracy.

Participants and Methods: As part of a larger clinical battery, the MoCA and the TOP-J were administered to geriatric Veterans (mean age $=73.5)$. This retrospective study included patients with no cognitive impairment $(n=2)$, mild neurocognitive disorder $(n=12)$, and major neurocognitive disorder $(n=5)$, to examine the incremental validity of the TOP-J in predicting 1) a global executive function composite score, and 2) cognitive diagnosis.

Results: Two hierarchical regressions were run controlling for premorbid ability (WTAR) standard score) in the first step, and global cognitive ability (MoCA raw score) in the second step. Across analyses, TOP-J $\mathrm{z}$-score significantly improved prediction of global executive ability by $13 \%[F(3,11)=12.78, p<0.005]$, and significantly improved prediction of cognitive diagnosis by $13 \%[F(3,12)=13.78, p<0.005]$.

Conclusions: These data suggest that addition of the TOP-J to the MoCA significantly improves prediction of global executive ability and diagnostic accuracy. Limitations and future studies will be discussed. Correspondence: William C. Hochberger, Mental Illness, Research, Education, and Clinical Centers, University of California San Diego/ San Diego VA Health Care System, 5901 E. 7th Street, Long Beach, CA 90822, United States. E-mail: whochberger@gmail.com

J. HURTUBISE, S. SAGAR, K. SEKE, C. CHARRON, A. DUNN, T. SPASOJEVIC, A. MCDERMOTT, C. LESPERANCE \& L.A. ERDODI. Performance Validity Indicators Embedded Within the D-KEFS Trails.

Objective: Performance validity tests (PVTs) assess whether an examinee's presentation on a neuropsychological battery reflects their true ability. Embedded PVTs are derived from established measures of cognitive ability, are relatively insensitive to neurological dysfunction, and require no additional test material or administration time. The objective of the present study was to determine the utility of the Delis-Kaplan Executive Function System (D-KEFS) Trail Making Test as an embedded PVT.

Participants and Methods: Data was collected from a mixed clinical sample of adult outpatients physician referred for neuropsychological assessment in the Northeastern United States ( $\mathrm{N}=157 ; 49 \%$ male). Exclusion criteria were diagnoses of dementia, intellectual disability, and acute dominant hand injury. The credibility of neurocognitive profile validity was psychometrically defined using the Word Choice Test, and two validity composites created by aggregating several embedded PVTs. Sensitivity and specificity scores were computed for multiple D-KEFS Trails cutoffs.

Results: Optimal validity cutoffs hovered around the "Larrabee Limit" of .50 sensitivity and .90 specificity. Trial 4 ACSS $\leqq 4$, Trial 5 ACSS $\leqq 5$, and the sum of Trials 1-3 ACSS $\leqq 13$ had specificities above .85 
against all three reference PVTs. Sensitivity fluctuated between .27 and .71 .

Conclusions: To our knowledge, this is the first attempt at establishing the utility of D-KEFS Trails as a PVT. Results support the use of this instrument as an embedded validity indicator. The study also suggests that combining multiple validity measures is superior to relying upon a single cutoff.

Correspondence: Jessica Hurtubise, M.Sc, Psychology, University of Windsor, Chrysler Hall South, Room 173 - 401 Sunset Avenue, Windsor, ON N9B 3P4, Canada.E-mail: hurtubij@uwindsor.ca

G.M. JACKSON, F.C. GOLDSTEIN, D. LORING \& S.E. JOHN. Construct Validity of Montreal Cognitive Assessment Index Scores in Cognitively Normal Adults.

Objective: The Montreal Cognitive Assessment (MoCA) is a global measure often used to characterize cognitive status and track longitudinal changes. However, the construct validity of the MoCA indices has not yet been evaluated. Rigorous validation of cognitive screens is imperative given their use as initial measures of cognition. We examined the relationship between performance on the MoCA indices and other established neuropsychological tests to determine the construct validity of the indices.

Participants and Methods: Two hundred and twenty five cognitively normal $\left(M_{M o C A}=26.44, S D_{M o C A}=2.20\right)$ middle-aged and older adults $\left(M_{\text {age }}=62.03, S D_{\text {age }}=7.37\right)$ completed comprehensive cognitive testing for a study of preclinical Alzheimer's Disease. Correlation analyses were conducted to examine the construct validity of the MoCA indices of executive functioning, memory, visuospatial, attention, and language. Results: There was statistically significant evidence $\left(P_{\mathrm{S}}<.05\right)$ of construct validity for the executive functioning, attention, memory, and visuospatial indices. However, most of these correlations were weak and revealed limitations in the MoCA's ability to thoroughly describe certain domains of functioning. The memory index was weakly correlated to performance on the Rey Complex Figure Test immediate $(r=.15)$ and delayed $(r=.17)$ recall trials, $\left(P_{\mathrm{S}}<.05\right)$. The language index was significantly related to Phonemic Fluency (F-A-S) $(r=.42, p<.001)$, but not to Animal Fluency or the Multilingual Naming Test $\left(P_{\mathrm{s}}>.09\right)$. Conclusions: The MoCA indices do not adequately relate to performance on longer cognitive tests of the same domains. The memory index does not accurately represent visual memory, and the language index does not capture semantic fluency or naming abilities. Our research supports the notion that more comprehensive neuropsychological testing is needed to accurately assess the domains of cognitive functioning and subtleties of early cognitive decline.

Correspondence: Grace M. Jackson, B.A., Neurology, Emory University, 12 Executive Park Drive NE, Atlanta, GA 30329, United States. E-mail: gracejackson2317@gmail.com

K. JENNETTE, M. KANGISER, B. KNECHT \& K. LISDAHL. The Influence of Binge Drinking Behavior on Verbal Learning and Memory Strategy in Young Adults.

Objective: Assess the influence of past year binge drinking behavior on verbal learning and memory performance as well as differential strategy use to encode and recall verbal information in young adults.

Participants and Methods: 76 [50.0\% female, 61.8\% White] young adults aged 18-25 were recruited from the community and administered the California Verbal Learning Test $-2{ }^{\text {nd }}$ Edition (CVLT-II) as part of a larger neuropsychological battery. An organization strategy ratio (OSR) score was calculated by dividing semantic by serial clustering score for each trial. Linear regressions were run with number of past year binge episodes predicting CVLT-II performance as well as OSR scores while controlling for gender, marijuana use, nicotine use, alcohol use, and Wide Range Achievement Test (WRAT) Reading score. The same analysis was conducted with a gender by binge interaction, with the addition of past year binge episodes as a covariate.
Results: Past year binge episodes alone did not predict CVLT-II performance but did predict OSR for Trial $1(b=-0.565, p=.050)$. Gender by binge interaction predicted CVLT-II performance but did not predict OSR scores. Visual inspection of scatterplots revealed that the relationship between increased binge drinking episodes and poorer verbal memory was driven by the males.

Conclusions: These results support previously reported effects of binge drinking on verbal learning and memory. However, the use of "higher order" encoding strategy differed only in Trial 1, wherein more efficient strategy was employed by those with fewer past year binge episodes. The interaction of binge by gender predicted differential performance, but this difference was not accounted for by the learning strategy employed. Correspondence: Kyle Jennette, MA, Psychology, University of Wisconsin - Milwaukee, 2717 E. Hartford Ave, Milwaukee, WI 53211, United States.E-mail:kjtommasi@gmail.com

C. KEITH, D.A.S. KAUFMAN, L. SCHWARZ \& P. RUPPERT. Relationships Among Self-Reported Sleep, Somatic Complaints, and Cognitive Performance in a Mixed Clinical Sample of Older Adults.

Objective: Aging is accompanied by a multitude of changes, affecting sleep, somatic symptoms, and cognitive functioning. Previous research examining the relationships between these variables has revealed disparate results. Additionally, prior studies have been primarily conducted in community dwelling volunteers. For the current study, a mixed clinical sample was analyzed in a neuropsychology clinic setting in order to explore these relationships and generate results that are more clinically applicable.

Participants and Methods: Participants consisted of 65 older adult patients (aged 60+) who have undergone an outpatient neuropsychological evaluation within an academic medical center. A mixed clinical sample was utilized, with diagnoses of MCI $(\mathrm{n}=30)$, dementia $(\mathrm{n}=12)$, MS, TBI, PD, HIV, and psychiatric disorders. Measures included the Pittsburgh Sleep Quality Index, PHQ-15, Digit Span, Trails $A$ and $B$, verbal fluency, and Wisconsin Card Sorting Test.

Results: For participants as a whole, cognitive performance did not differ between good and poor sleepers (as classified by the PSQI). Additionally, self-reported sleep quality and somatic complaints were not significant predictors of cognitive performance across domains such as attention/processing speed, working memory, verbal fluency, executive functioning, and global cognition composites.

Conclusions: The current study does not support general relationships among self-reported sleep, somatic complaints, and cognitive functioning in a mixed clinical sample of older adults. These findings may suggest that neuropsychological performance is robust to the influence of these contextual factors. However, the heterogeneous clinical nature of the current sample may have interfered with our ability to detect effects that are specific to particular diagnoses (e.g., MCI, dementia). Given the importance of sleep and somatic symptoms in pathological aging, further investigation is needed.

Correspondence: Cierra Keith, M.S., Saint Louis University, 3700 Lindell Blvd, St Louis, MO 63108, United States.E-mail: keithc@slu.edu

K. MEgARI, G. KIOSSEOGLOU, K. ANASTASIADIS \& M.H. KOSMIDIS. Neuropsychological Outcome and Cognitive Reserve Following Cardiac Surgery Employing Cardiopulmonary Bypass.

Objective: Neuropsychological outcome of patients undergoing cardiopulmonary bypass is typically accompanied by significant postoperative cognitive dysfunction (POCD), including impaired memory, attention, orientation, judgment and overall social functioning. While cognitive reserve $(\mathrm{CR})$ has been suggested to play a protective role against the effects of neuropathology and decline with aging and/or trauma, it has not been studied adequately in cardiac surgery patients. We examined the impact of CR on POCD in patients undergoing cardiac surgery employing cardiopulmonary bypass. 
Participants and Methods: We conducted neuropsychological assessments of 101 patients before and four months after cardiopulmonary bypass surgery with the use of extracorporeal circulation. Assessment included measures of attention, verbal working memory, executive functioning, learning, short-term and long-term memory, visuospatial perception, anxiety and depression.

Results: We defined cognitive dysfunction as a postoperative $20 \%$ decrease, relative to pre-surgery performance, on $20 \%$ of neuropsychological tests. CR was estimated based on age, educational level, occupation, functional index and vocabulary measures. Each patient was placed in the high $(n=50)$ or low CR ( $n=51)$ group, based on median split. Chi-square tests showed that patients with low CR were more likely to demonstrate cognitive dysfunction in attention, memory, visuospatial perception and executive functions $(\mathrm{p}<.001)$ than patients with high $\mathrm{CR}$ upon post-surgery neuropsychological assessment.

Conclusions: Given the frequency and severity of POCD following cardiac surgery and its impact on patients' performance and quality of life, it is important to predict those with the greatest need for cognitive rehabilitation. Our findings suggest that CR can predict neuropsychological outcome. This information could aid in the design of intervention programs that can enhance patients' overall functional outcome after surgery.

Correspondence: Mary H. Kosmidis, Ph.D., School of Psycholgy, Aristotle University of Thessaloniki, University Campus, Thessaloniki 54124, Greece.E-mail: kosmidis@psy:auth.gr

\section{K. KRISHNAN, K. CUNNINGHAM, O. HOGUE, A. BONNER- JACKSON, D. FLODEN \& R.M. BUSCH. Computerized Assessment of Cognitive Function in Older Adults.}

Objective: We investigated the utility of a computer based screening tool in a sample of older adults undergoing a standard neurocognitive evaluation.

Participants and Methods: Adults ages 55 and older referred by neurologists at Cleveland Clinic ( $\mathrm{n=77}$ ) completed a comprehensive neurocognitive assessment and two memory subtests from the University of Pennsylvania Computerized Neuropsychological Battery (PennCNP Word Memory Test and Face Memory Test, Immediate and Delaved Trials). A neuropsychologist, blinded to PennCNP results, classified patients as normal (19), presence of cognitive problems but not MCI (12), and MCI (46) based on performance on paper-and-pencil neurocognitive tests. Results of ANOVA and LSD post-hoc comparisons are reported. Results: There were no demographic differences between patient groups; however, they demonstrated differential performance on PennCNP memory measures with medium to large effect sizes. Normal controls performed better than patients with MCI on both tasks immediately and following a delay $(\mathrm{p}<0.001)$. Patients with cognitive problems scored higher than MCI patients on immediate and delayed faces $(\mathrm{p}<0.05)$. Patients with normal neurocognitive performance scored higher than those with cognitive problems on delayed word recognition $(p<0.05)$ and approached significance on immediate face recognition $(p=0.054)$. Conclusions: The memory subtests of the PennCNP show promise as a screening tool to identify MCI and prompt a comprehensive neurocognitive evaluation.

Correspondence: Kamini Krishnan, PhD, Psychiatry and Psychology, Cleveland Clinic, 9500 Euclid Ave, P 57, Cleveland, OH 44195, United States.E-mail:krishnk3@ccf.org.

S.L. MARTINDALE, R.D. SHURA, T.W. BREARLY, J. ROWLAND \& H.M. MISKEY. Symptom Burden and Cognitive Outcomes in Iraq and Afghanistan Veterans: The Role of Validity.

Objective: Determine what aspects of symptom burden drive failure on performance and symptom validity measures, and if these associations account for cognitive test performance.

Participants and Methods:

Participants were 226 Iraq and Afghanistan veterans. Participants completed the Personality Assessment Inventory (PAI), stand-alone performance/symptom validity measures, the Frontal Systems Behavior Scale (FrSBe), and a comprehensive cognitive battery. Participants with psychotic symptoms, current substance use disorder, combat exposure before 1985, moderate/severe traumatic brain injury, posttraumatic stress disorder unrelated to combat, and/or with an uninterpretable PAI profile (INF, ICN) were considered ineligible. Path analysis was conducted using PLS-SEM. Exogenous variables were the PAI clinical scales. Word Memory Test (WMT), Miller Forensic Assessment of Symptoms Test (M-FAST), and PAI NIM and PIM scales were endogenous mediators. The FrSBe total score and cognitive tests (BDS-II, BVMT-R, COWA, CPT-II, CVLT-II, FTT, GPT, RCFT, Stroop, TMT, UPSIT, WAIS-III) formed endogenous outcomes.

Results: All PAI clinical scales were directly associated with cognitive outcomes. Validity mediators of WMT failure and PIM were not associated with cognitive outcomes, though MFAST failure (verbal fluency) and NIM (visual learning, complex attention, verbal fluency, working memory) were. SCZ was the only PAI clinical scale indirectly associated with cognitive outcomes (verbal recall, verbal fluency, and working memory), through validity measures (MFAST, NIM). MFAST failure, $R_{\text {adj }}^{2}=.22$, NIM, $R_{\text {adj }}^{2}=.69$, PIM, $R_{\text {adj }}^{2}=.66$, visual processing speed (WAIS-III subtests), $R_{\text {adj }}^{2}=.26$, visual learning (BVMT-R), $R_{\text {adj }}^{2}=.16$, and subjective frontal dysfunction $(\mathrm{FrSBe}), R_{\text {adj }}^{2}=.73$, were constructs best explained by the model.

Conclusions:

Psychiatric symptom burden was independently associated with lowerorder objective cognitive function (processing speed and learning) and subjective frontal dysfunction beyond validity measure performance. Correspondence: Sarah L. Martindale, Ph.D., W.G. "Bill" Hefner VA Medical Center, 4801 Asherton Pl NW, Concord, NC 28027, United States.E-mail: Sarah.Martindale-Supak@va.gov

F. MARTINEZ, E. WAITE, A. WALKER. D.J. HARDY, D. BUDDING, E. WOO, J. FUSTER \& M.J. WRIGHT. A Role for Neuropsychology in Preventive Healthcare: Executive Ability Predicts Physical Injuries in Healthy Young Adults.

Objective: To ascertain if cognitive function, as assessed by standard neuropsychological tests, could predict the risk of physical injury in healthy adults.

Participants and Methods: Thirty-one healthy adults [Mean Age $=28.35 y r s$ (9.35), Mean Education $=14.34 \mathrm{yrs}$ (1.59), Percent Male $=67.7 \%$, Percent White $=56.3 \%$ ] took part in the current study. All participants provided voluntary consent to this IRB approved study that included the administration of a brief neuropsychological battery and a questionnaire assessing participant's history of physical injuries over the last five years.

Results: T-scores for attention, memory, and executive ability were calculated. Memory and executive function correlated with injury frequency ( $r=-.40$ and -.57 , respectively). A stepwise regression revealed that executive function was the sole predictor of injury frequency ( $b=-.57, t=-3.77, p=.001$ ), accounting for $33 \%$ of the variance.

Conclusions: Poorer executive ability predicts physical injury in healthy young adults. Identification and remediation of lower executive abilities in healthy adults may reduce the incidence of physical injuries (e.g., traumatic brain injury, work related injuries, etc.).

Correspondence: Fernando Martinez, CA, United States. E-mail: fmartinezjr10@gmail.com

T. MBURU, K. GARCIA, J. DSURNEY \& L. CHAN. Underutilized Psychometric Correlates of Executive Function in TBI.

Objective: The goals of this study are to identify novel subcategories of Executive Function(EF) and investigate if subscales of other-domain neuropsychological(NP) assessments are differently related to subcategories of abstract(abstraction), applied(problem-solving), and regulatory(self-monitoring) EF in a TBI population. 
Participants and Methods: Participants included 69 adults with mild, moderate and severe TBI at one year post injury, selected from a larger cohort study.

Traditional NP measures of abstract and applied EF included: abstract reasoning and verbal fluency; problem-solving and set-shifting; regulatory measures were not available. Uncommon subscales were: abstract-Booklet Category Test (BCT) Spatial Positional Reasoning and Proportional Reasoning(PR); applied-BCT Set Loss Attention and True Perseverations, CVLT-2 Long Delay Free Recall, WAIS-IV Digit Span Sequencing; and regulatory-CVLT Intrusions(I) and Repetitions, COWA Phonemic and Category Perseverations (CP), Trail Making Test A and B-Errors(B-E).

Spearman-Brown Correlation was used to explore relationships between traditional EF measures and uncommon other-domain subscales.

Results: Subjects mean age $46.5 \pm 17.2$ and education $15.6 \pm 2.6$ years. All predicted EF correlations were significant, with strongest: $\mathrm{PR}-\mathrm{BCT}(r s=-.879)$. Most significant regulatory EF correlation: TMT-B-E-TMT-B $(r s=-.488)$

Conclusions: All abstract and applied subscales correlated with traditional abstract and applied EF measures, respectively. Several regulatory subscales correlated with traditional EF measures as well as other regulatory subscales, particularly CVLT-I, COWA-CP, and TMT-B-E. This study highlights potential utility of traditional psychometric measures of memory, learning, attention, verbal fluency and other domains to reflect distinct and collaborative abstract, applied, and regulatory executive functions.

Correspondence: Tracy Mburu, MA, MD, United States. E-mail: tracyann.mburu@nih.gov

E. GARDIZI, I. MESSA, H. SINGH, J. MCCARTHY \& C. ABEARE. Use of the WCST and WCST-64 in the Assessment of First Episode Psychosis.

Objective: The Wisconsin Card Sorting Test (WCST) was designed as a measure of executive functioning and is commonly used in the assessment of various disorders, including psychosis. The original WCST, which consists of 128 cards, has been criticized as being too lengthy and frustrating for patients experiencing significant distress. Consequently, a shortened version of the test, which consists of a 64-card deck (WCST64), was created. However, the validity of this test version has been questioned in certain clinical populations. The present study sought to examine the validity of the WCST-64 in a unique clinical sample.

Participants and Methods: The full WCST was administered to a sample of 99 patients with first-episode psychosis. Performance on the full test was scored according to scoring guidelines for the WCST, and the first 64 responses were then re-scored according to the guidelines for the WCST-64. Measures of perseverative responses (PR) and non-perseverative errors (NPE) were compared across test versions.

Results: Findings showed the WCST-64 yielded a mean $T$ score for PR that was 4.08 points lower than the corresponding PR variable from the WCST, with a correlation between them of $r=.65$. For NPE, however, the mean discrepancy between $\mathrm{T}$ scores was only 2.34 , and the correlation was also much stronger $(r=.82)$. Almost half of the sample (44\%) had a $T$ score discrepancy between the respective PR indexes that was greater than one standard deviation while this level of discrepancy was observed in only $4 \%$ of the sample for NPE. Based on a cut-off of $T<40$ to define impaired performance on PR, $80 \%$ of the sample received the same classification on both versions of the test.

Conclusions: Overall, these findings suggest that NPE scores may be comparable across both test versions. Conversely, while the PR score from the WCST-64 and WCST can be used as a gross measure of impairment, these tests should not be used interchangeably to describe the extent and severity of perseverative tendencies in patients with psychosis.

Correspondence: Isabelle Messa, BSc, MSc, Psychology, University of Windsor, 140 Meloche Rd, Amhersburg, ON N9V 2Y9, Canada. E-mail: messaha@uwindsor.ca
I. MESSA, E. GARDIZI, L.A. ERDODI, J. MCCARTHY, H. SINGH \& C. ABEARE. Base Rates of Invalid Responding in First-Episode Psychosis.

Objective: Performance validity tests (PVTs) are essential to determine the credibility of neuropsychological test scores. Embedded PVTs (E-PVT) are traditional neuropsychological tests that also function as validity indicators. They minimize administration time and are relatively robust to coaching. However, by design, they are more vulnerable to the circular issue of genuine impairment versus invalid performance. The interpretation of PVT failure in patients with schizophrenia is similarly controversial. This study was designed to examine the base rates of failure $\left(\mathrm{BR}_{\text {Fail }}\right)$ in patients with schizophrenia on various E-PVTs.

Participants and Methods: Data were collected from 66 in-patients $(72.7 \%$ male) with first-episode psychosis. Mean age was 23.1 $(S D=4.98) . B_{\text {Fail }}$ was computed on five E-PVTs: letter and category fluency, Trails B, reliable digit span (RDS), and Vocabulary minus Digit Span (VC-DS).

Results: At liberal cutoffs, $\mathrm{BR}_{\text {Fail }}$ ranged from $15 \%$ to $35 \%$ on individual PVTs. At conservative cutoffs, $\mathrm{BR}_{\text {Fail }}$ was predictably lower (5\%-29\%). A third of the sample failed $\geq 2$ PVTs at the liberal cutoff, and $12 \%$ failed $\geq 3$ PVTs. Only $20 \%$ of the sample failed $\geq 2$ PVTs at the conservative cutoff, and $6 \%$ failed 3 PVTs.

Conclusions: Validity cutoffs embedded within verbal fluency tasks and the VC - DS had notably higher $\mathrm{BR}_{\text {Fail }}$ than RDS or TMT-B at both levels of cutoffs. Between 20 and $35 \%$ of the sample met the common forensic standard for invalid performance ( $\geq 2$ PVT failure). Given the confluence of genuine impairment and suboptimal effort in embedded validity indicators and the absence of data on stand-alone PVTs, the clinical interpretation of these results is difficult. Until future research elucidates this, published cutoffs on RDS and TMT-B may provide reasonable measures of performance validity, with $\mathrm{BR}_{\text {Fail }}$ in the expected range (6-17\%). Failing validity cutoffs on verbal fluency tasks should be interpreted with caution, as they may be prone to false positive errors. Correspondence: Isabelle Messa, BSc, MSc, Psychology, University of Windsor, 140 Meloche Rd, Amhersburg, ON N9V 2Y9, Canada. E-mail: messaha@uwindsor.ca

K. MIDDLETON, K. GARCIA, B. MOORE, J. DSURNEY \& L. CHAN. Validation of a Modified Reitan-Wolfson Screening Test Using MRI Findings in a TBI Population.

Objective: The Halstead-Reitan (H-R) battery is a well validated assessment of brain dysfunction. A two phase screening test was built from three H-R battery tests (Trail Making Tests A\&B, Finger Tapping Test, and Tactile Form Recognition) to quickly assess brain injury and dysfunction. Phase I and II testing correlate to performance on the entire H-R battery at $88 \%$ and $96 \%$ respectively. The present study aims to assess the relationship between Phase I (TMT B), modified Phase II (TMT A\&B and FTT) testing and MRI findings.

Participants and Methods:

53 subjects were selected from a larger natural history study. Tests were scored using the NDS score for TMT A\&B and FTT. MRI scans were read for indication of positive findings regardless of pathology. Data was collected at the 1 year post-injury time point.

SPSS was used to calculate t-tests as well cross-tabulation between neuropsychological test results and MRI classification.

Results: 33 subjects were male, 20 were female. The mean age and education of all subjects were $48.0 \pm 18.3$ years and $16.0 \pm 2.6$ years, respectively.

A new cut score for impaired/unimpaired classification (NDS $\geq 5$ ) was used for modified Phase II testing. A cut score of 5 on modified Phase II identified $97.2 \%$ of true positives as compared to $75 \%$ at a score of 7. False positives also increased from $52.9 \%$ to $82.4 \%$ when cut scores were changed from 7 to 5 . Phase I testing was not a useful assessment of impairment when employed alone.

Conclusions:

Modified Phase II is most useful in determining whether it is worth the expense, difficulty, and potential risks to obtain an MRI. This screening 
tool could be easily implemented in both military action and athletic evaluations. Manipulation of sub-tests weights in total NDS score determination should be done to identify an ideal cut score for this testing. Further testing is needed to assess this screening method in acute populations and with other methods for determining structural brain changes. Correspondence: Kathryn Middleton, BS, Wake Forest University, 30 Southgate Drive, Annandale, NJ 08801, United States. E-mail: middkt14@wfu.edu

J.B. MILLER, J. BERG \& J. DURANT. Performance validity tests and associations with hippocampal volume in neurodegenerative disease.

Objective: Performance validity tests (PVTs) are standard components of most neuropsychological evaluations and are touted as immune to all but the most severe neurological dysfunction. However, test operating characteristics of PVTs among older populations are much less studied due to an increased prevalence of neurodegenerative disease, which could increase false positive errors. This study seeks to evaluate the classification accuracy of 2 commonly used PVTs and explore performance in relation to hippocampal volume (HCVOL) in a memory disordered sample.

Participants and Methods: Records from 42 consecutive referrals (Age: $M=70.2, S D=8.2$; Education: $M=14.9, S D=2.8 ; 59 \%$ female) seen as part of routine care in an outpatient dementia clinic were reviewed. Primary measures of interest included the Medical Symptom Validity Test (MSVT), Test of Memory Malingering (TOMM), and Montreal Cognitive Assessment, and automated HCVOLs from clinical MRI scans. PVT failure rates were calculated using published criteria. HCVOLs were correlated with PVT performance, and cognitive and imaging data were compared between pass/fail groups via ANOVA.

Results: Seven percent of patients failed the TOMM and 23.8\% failed the MSVT. HCVOLs did not differ between groups, but those failing the MSVT had significantly lower MoCA scores $(\mathrm{F}(1,40)=11.78, p=.001)$. Semi-parital correlations between primary MSVT indices (immediate and delayed recall, consistency) and HCVOL accounting for age were significant $(\mathrm{r}$ values $=0.39-0.43, p<.01)$; correlations with free recall, paired associates, and the TOMM were not.

Conclusions: TOMM failure was infrequent and those failing the MSVT were more cognitively impaired than those who scored above criterion. Although HCVOLs did not differ between groups, primary MSVT indices were moderately correlated with HCVOLs after accounting for age, suggesting that MSVT performance may be dependent on hippocampal integrity. These findings require replication with a larger sample. Correspondence: Justin B. Miller, Ph.D., Cleveland Clinic Lou Ruvo Center for Brain Health, 888 West Bonneville Ave., Las Vegas, NV 89106, United States. E-mail: millerj4@ccf.org

V. MONTGOMERY, K. TOLLE, R.J. SPENCER \& J. STELMOKAS.
Preliminary evaluation of demographic adjusted MoCA scores and
functional competence in post-acute rehabilitation.
Objective: Functional and cognitive status often predict rehabilitation
potential and discharge status in post-acute rehabilitation. Screening for
cognitive deficits using the Montreal Cognitive Assessment (MoCA) may
aid in treatment and discharge planning. However, relying on scores on
cognitive screening measures to assess functional capacity risks either
under-detection or indiscriminate inclusiveness, depending upon how
cut scores are selected. We examined how strongly various cut scores
on the MoCA detect functional problems, as defined by the Independent
Living Scale Health and Safety subtest (ILS-HS).
Participants and Methods: The ILS-HS and the MoCA were given to
79 male Veterans on admission to a post-acute inpatient rehabilitation
unit (Age, $M=68.47$; Education, $M=12.56)$.
Results: MoCA and ILS-HS scores were significantly correlated,
$r=.49, p<.01$. MoCA cut scores included (1) standard <26, $(2)$ educa-
tion-adjusted, (3) norms from older adult Veteran outpatients, and (4)
demographically-adjusted scores from ethnically diverse outpatients.
Chi-square tests indicated no significant differences between the proportion of individuals with impaired scores on the ILS-HS (1.5 SD below the mean) compared to MoCA total score across most suggested cut scores. However, the ILS-HS was significantly different from the MoCA- demographically corrected cut score using the ethnically diverse population.

Conclusions: The standard MoCA cutoff of $<26$ is overly sensitive when detecting functional limitations relating to health and safety in postacute rehabilitation. Our findings suggest that, while more accurate, using demographically corrected scores on the MoCA may not detect many individuals with functional impairments. Although the MoCA provides moderately accurate assessment of functional status, it does not replace direct assessment of functional abilities. Research is needed to examine the incremental benefit of including functional measures in predicting relevant rehabilitation outcomes.

Correspondence: Valencia Montgomery, M.A., Mental Health, VA Ann Arbor, 5733 1/2 S. Condon, Windsor Hills, CA 90056, United States. E-mail: valencia.montgomery@gmail.com

N. NDUKWE \& J. KING. Quantifying a Relationship Between Somatic Concerns and Frequency of Exercising.

Objective: To explore whether there is a relationship between less time spent exercising and high somatic complaint scores reported on the Personality Assessment Inventory (PAI).

Participants and Methods: The study reviewed archival data from patients referred for a comprehensive neuropsychological evaluation in a private practice, including the PAI and an exercise questionnaire. A total of 60 consecutive files were reviewed, with 10 excluded from the study due to having no PAI or exercise questionnaire administered. Inclusion criteria were met for 50 participants ( 26 males and 24 females; mean age $=54$ years, range 20 to 79 years; mean education $=15$ years, range 9 to 20 years). The exercise questionnaire consists of four questions which ask the participant to provide the number of minutes they spend each week on average engaging in various types of exercise (i.e., aerobic or cardiorespiratory, strength training, stretching, and walking). The exercise questionnaire produces five scores, one for the four exercise types and one total exercise score. The T-score of the Somatic Complaint Scale (SOM) of the PAI and the results of the exercise questionnaire were correlated using the Pearson correlation.

Results: The results indicate that there is a significant correlation between aerobic exercise and the somatic T-score $(p=0.024)$. The result presents a negative Pearson correlation $(-0.318)$, indicating that as the somatic scores increase there was a drop in the times spent exercising. Conclusions: Of the four types of exercise assessed, aerobic (cardio) exercise was significantly negatively correlated with the SOM scale of the $\mathrm{PAI}$ in this population, with patients who engage in less aerobic exercise tending to report higher levels of somatic complaints. The design of the study reveals a correlational relationship but does not permit inferences regarding causation. This information may eventually be helpful in generating clinical recommendations for patients with high degrees of somatization and somatic symptom disorders.

Correspondence: Ngozi Ndukwe, Georgia School of Professional Psychology, 280 Interstate North Circle, Suite 450, Atlanta, GA 30339, United States. E-mail:ngndukwe@gmail.com

R. DEL PINO, M. DÍEZ-CIRARDA, J. PEÑA, N. IBARRETXEBILBAO, D.J. SCHRETLEN \& N. OJEDA. Estimation of cognitive performance based on the premorbid intelligence in Parkinson's disease.

Objective: This study aims to estimate the cognitive performance through the following premorbid intelligence $(\mathrm{PI})$ variables: Word Accentuation Test (WAT), Pseudo-Words (PW) Reading subtest from the Battery for Reading Processes Assessment-Revised (PROLEC-R), age, and years of education in non-demented Parkinson's disease (PD) patients compared to healthy controls (HC); and to analyze the 
discrepancies between the current and the predicted cognitive performance based on the PI.

Participants and Methods: A sample of $162 \mathrm{HC}$ and 39 PD patients underwent a neuropsychological assessment. The cognitive performance was estimated using the following PI variables: WAT, PW, age and years of education. A linear regression equation was used based on the HC group. The discrepancies between the predicted cognitive performance and the current raw scores were calculated.

Results: No significant differences were found in sociodemographic characteristics, neither in the PI variables between groups. Significant differences were found in semantic fluency $(\mathrm{t}=6.07 ; p<.001)$, verbal memory $(\mathrm{t}=6.63 ; p<.001)$ and executive functions $(\mathrm{t}=2.57 ; p=.013)$ and marginally significant differences in visual memory $(\mathrm{t}=1.97 ; p=.055)$ in the raw scores. The discrepancies between the predicted cognitive performance and the raw scores were significantly higher in PD group compared to HC group in semantic fluency ( $\mathrm{AUC}=.780 ; p<.001)$, verbal memory $(\mathrm{AUC}=.778 ; p<.001)$, visual memory $(\mathrm{AUC}=.636 ; p=.008)$ and executive functions ( $\mathrm{AUC}=.611 ; p=.032$ ).

Conclusions: The magnitude of the discrepancies between the current and the predicted cognitive performance based on PI pointed out the degree of cognitive impairment in PD patients. These PI variables (WAT, PW, age, and years of education) could be used to obtain a more accurate estimated cognitive performance of the person assessed. Correspondence: Natalia Ojeda, Universidades 24, Bilbao 48007, Spain.E-mail:nojeda@deusto.es

J. Paulson, T.M. OSWAld, R. FAzio \& G. ANDREWS. Replication of the Embedded Performance Validity Index within the Memory Module of the Neuropsychological Assessment Battery (NAB).

Objective: The use of performance validity measures is essential in neuropsychological evaluations, particularly in populations where secondary gain factors may be present. Embedded performance validity tests (PVTs) allow for more sophisticated inferences about optimal effort within neuropsychological evaluations; many recognition memory measures have established embedded PVTs. Within the Neuropsychological Assessment Battery (NAB; Stern \& White, 2003) - Memory Module a combination score generated from List Learning and Shape Learning scores demonstrated optimal specificity and sensitivity at a cut score of 14 . The present study aims to replicate the clinical utility of the NAB - Memory Module combination score and corresponding cut score. Participants and Methods: The sample consisted of 81 veterans seen for outpatient clinical neuropsychological evaluation. The battery included established PVTs (e.g., DCT, RDS, TOMM), the NAB Memory Module, and other clinical measures. Subjects were classified utilizing the Slick et al. criteria; ROC analyses were conducted to evaluate the $\mathrm{NAB}$ - Memory Module combination score.

Results: The NAB Memory Module combination score yielded an AUC of .801. Applying the cut score of 14 resulted in a specificity of .885 and a sensitivity of .500 . However, a cut score of 13 yielded a specificity of .902 and a sensitivity of .400 .

Conclusions: Within this validation sample, a cut score of 14 produced acceptable specificity and sensitivity, while a cut score of 13 produced optimal specificity. In an outpatient clinical neuropsychology clinic the embedded NAB - Memory Module combination score correctly identified veterans with poor effort $(n=20)$ and those with adequate effort $(n=61)$. Thus, the NAB -Memory Module combination score was validated. Utilizing the embedded NAB - Memory Module combination score will continue to be clinically useful in evaluating performance validity with $\mathrm{NAB}$ based batteries.

Correspondence: Jennifer Paulson, Ph.D., Neuropsychology, Central Arkansas Veteran's Healthcare System, 2200 Fort Roots Dr., (116/ NLR), North Little Rock, AR 72114, United States. E-mail: jennifer. paulson3@va.gov
J. PETERSON, J. LOWE, C. BOLTON, D. BARNHART, C. KIBBLE, K. KUTNAR \& B. PYYKKONEN. Use of the Victoria Symptom Validity Test to evaluate performance validity in baseline concussion testing.

Objective: Research has encouraged the use of performance validity tests during baseline administrations of neurocognitive batteries in a sports concussion management protocol. However, individuals seeking to deceptively influence their baseline scores are unique in that they are not actually attempting to feign impairment per se. Rather, they tend to be neurologically intact individuals, many of them student-athletes, who merely attempt to produce sufficiently low scores. This study seeks to evaluate use of the Victoria Symptom Validity Test (VSVT) for identifying invalid performances in this unique context.

Participants and Methods: A sample of 51 undergraduate students were administered the VSVT as part of a computerized baseline battery. One group was instructed to perform with full effort, a second group was directed to perform as if concussed so as to lower the baseline score and enable quicker return to play, and a third group was provided the same instruct set as the second group but also warned that the tests were sensitive to gross exaggeration. Cut scores for Easy Items, Difficult Items, Total Items, Easy - Difficult Items, and Items correct per block were evaluated.

Results: Multiple cut scores were identified as possessing excellent classification accuracy (i.e., sensitivity and specificity above $90 \%$ ). However, $<22$ Difficult Items correct and $<46$ Total Items correct yielded were the most effective, each with sensitivity at $97 \%$ and specificity at $94 \%$. Conclusions: The VSVT appears to be an effective measure of performance validity even among neurologically intact individuals warned of a battery's sensitivity to exaggeration. Interestingly, the most effective cut scores for this sample were higher than those identified in previous research involving clinical populations.

Correspondence: Jesse Peterson, Wheaton College, 2302 Whitegate Dr., Apt. 3A, Columbia, MO 65202, United States. E-mail: jesse. peterson.12@my.wheaton.edu

C. QUINN, N. PARÉ, L.A. SCHAEFER, P. SUNDERARAMAN, M. GARLINGHOUSE, A. NELSON SHEESE, H. RABIN, M.J. BORGOS, L. RABIN \& S. OMOLLO. Development and Preliminary Evaluation of an Alternate form of the Test of Practical Judgment.

Objective: The Test of Practical Judgment (TOP-J) evaluates judgment related to safety, medical, social/ethical, and financial issues and is widely used by neuropsychologists. In response to numerous requests for the TOP-J, we surveyed users' experience $(\mathrm{n}=52)$. The TOP-J's ecological validity, brevity, comprehensiveness, and psychometric properties were noted as unique and clinically valuable. However, some respondents articulated a concern about certain items for those with limited education and/or lower socioeconomic backgrounds. This identified need, and the importance of having alternate forms of neuropsychological measures, prompted us to develop an alternate form (TOP-J-alt). Participants and Methods: We developed new questions mirroring the original content areas. Expert feedback and piloting in a memory clinical sample led to item refinement and scoring criteria. The measure consists of 15 open-ended items, which have been administered to 13 memory clinic patients thus far (target $\mathrm{N}=200)$. An expert panel rated each question for primary domain assignment. We also conducted a preliminary investigation of the differences between TOP-J-alt total scores based on level of cognitive decline: dementia (mixed etiology) vs. non-dementia (subjective cognitive decline, mild and/or isolated cognitive impairment).

Results: Psychologists and trainees $(\mathrm{n}=21)$ ranked the primary domains of each question and the following content areas emerged for the items: 4 medical, 3 financial, 4 safety, and 4 social/ethical. On average, subjects with dementia (mean $=23.14 ; \mathrm{SD}=7.08$ ) had lower TOP-J-alt total scores than subjects without dementia $($ mean $=31.67$; $\mathrm{SD}=2.42), t(11)=2.80, \mathrm{p}=.017, \mathrm{r}=.645$. 
Conclusions: Preliminary findings suggest that the TOP-J-alt may be clinically useful in distinguishing older adults with and without dementia. Ongoing research will further investigate the validity and reliability of the TOP-J-alt in diverse clinical samples, and its use as a parallel form of the TOP-J.

Correspondence: Crystal Quinn, PhD, Psychology, The Graduate Center, CUNY, 2900 Bedford Avenue, Department of Psychology, Brooklyn, NY 11210, United States. E-mail: cquinn@gradcenter.cuny.edu

K.T. RHYNER, S. BELKONEN, N. CASSELL \& B. SHENAL. Equivalence of HVLT-R Forms in a Veteran Population.

Objective: HVLT-R Forms 1 and 2 have been shown to be psychometrically equivalent. However, given the potential differential relevance for Veterans of categories on Form 2 (kitchen utensils, weapons, alcohol) compared to Form 1 (four-legged animals, precious stones, human dwellings) this study evaluated the equivalence of these two forms specifically in a Veteran population.

Participants and Methods: Participants included 200 Veterans attending an outpatient Memory Assessment Clinic for reported memory concerns. A retrospective chart review was conducted to compare the psychometric equivalence for HVLT-R Form 1 and 2 total scores for each recall time point. Differential item functioning between the two forms was also explored using item response theory (IRT) methods to determine if item difficulty varied.

Results: There was no significant difference found between forms for the total number of correct responses for learning trials 1 through 3 , delayed recall, or recognition. However, IRT results indicated a different pattern of item difficulty for $58 \%$ items on Trial 1, 17\% items on Trial 2 , and $17 \%$ items on Trial 3. Both items on Trial 3 showed the Veteran participants were more likely to remember the alcohol or weapon category word compared to the word in the same serial position on Form 1 (Pistol vs. Tent, $p=0.03$; Bourbon vs. Cave, $p=0.04$ ). Form 1 also showed a strong serial position effect for all trials, particularly trial 1 , while Form 2 did not show this pattern.

Conclusions: Total items recalled for all time points were equivalent between Forms 1 and 2, indicating that these forms are clinically equivalent in a Veteran population, despite possible face-valid difference between the categories. However, the patterns of items recalled correctly were different between the forms, indicating a preference for remembering alcohol or weapon-related words. These findings provide important psychometric information for a commonly used memory test in a Veteran population.

Correspondence: Kathleen T. Rhyner, Ph.D., Psychology, Salem VAMC, 3815 Sunbreeze Cir., Apt. 343, Roanoke, VA 24018, United States. E-mail:kate.rhyner@gmail.com

S. ROYE, P.J. Castagna \& M. Calamia. The Compensatory ADHD Behaviors Scale (CABS): Development and Initial Validation.

Objective: ADHD is associated with EF deficits that lead to functional impairments in daily life. While several self-report measures are available with assess the severity of these deficits, few examine the behaviors adults with ADHD utilize to compensate for these deficits (e.g., organizational strategies such as to-do lists or calendars). This study aimed to examine the factor structure and psychometric properties of a newly developed measure designed to fill this gap: the Compensatory ADHD Behaviors Scale (CABS).

Participants and Methods: 500 participants (Mean Age $=36.83$, $S D=11.57$ ) were recruited using Amazon's Mechanical Turk and completed self-report measures of ADHD symptomatology, executive functioning, functional impairment, anxious and depressive symptoms, and the CABS. The construct validity of the CABS was examined using an exploratory factor analysis and by examining the convergent and discriminant validity with other study measures.

Results: Analyses revealed that both the use and perceived effectiveness of compensatory behaviors subscales had similar factor structures, where both scales had items that were present- and future-oriented. The present-oriented use and effectiveness subscales demonstrated significant relationships with previously established measures of ADHD $(r=-.25, p<.001$ and $r=-.09, p<.05$, respectively $)$, executive functioning $(r=-.30, p<.001$ and $r=-.18, p<.01$, respectively), and functional impairment $(r=-.23, p<.001$ and $r=-.14, p<.01$, respectively), while future oriented use and effectiveness subscales were related to anxious symptoms $(r=.14, p<.05$ and $r=.10, p<.05$, respectively). Conclusions: Results provide evidence supporting the CABS as a useful measure of compensatory ADHD behaviors. The CABS has the potential to add to adult ADHD assessment, as well as provide a useful measure of treatment outcomes.

Correspondence: Scott Roye, M.A., Clinical Psychology, Louisiana State University, 10720 Linkwood Ct, Apt \# 1033, Baton Rouge, LA 70810, United States. E-mail: sroye1@lsu.edu

S. SAGAR, C. MACDONALD, T.J. ORNSTEIN \& L.A. ERDODI. The Development of the Hyperactivity/Inattention Trait Scale for the Detection of Feigned Attention-Deficit/Hyperactivity Disorder: A Preliminary Examination.

Objective: Attention-deficit/hyperactivity disorder (ADHD) is a neurodevelopmental condition characterized by a pattern of attentional deficits, hyperactivity, and/or impulsivity that tends to persist into adulthood for a subset of patients. With the high base rate of feigned ADHD in university settings (estimated $25-50 \%$ ), there is risk of misappropriation of educational and health care resources (including academic accommodations). In an attempt to address this issue, the Hyperactivity/ Inattention Trait Scale (HITS) was designed specifically to differentiate between feigned and genuine adult ADHD.

Participants and Methods: The measure was administered to a large, non-clinical sample $(\mathrm{N}=230)$ of undergraduate students, along with several established performance validity tests (PVTs). Exploratory factor analysis (EFA) was conducted to determine the underlying structure of the scale. The PVTs were used as criterion measures to evaluate the HITS' ability to discriminate between credible and non-credible performance.

Results: EFA revealed factors related to adult ADHD (i.e., inattentiveness, hyperactivity, and impulsivity), a factor related to invalid responding, and an additional factor related to somatic symptoms. Cut-off scores for the main validity subscale were developed by creating a multivariate criterion measure based on the PVTs, resulting in a good combination of sensitivity (.67) and specificity (.91) to feigned ADHD. Conclusions: The current study extended previous work on the clinical assessment of ADHD overall, and particularly in the detection of non-credible presentations. These preliminary results suggest that the HITS has the potential to provide a cost- and time-efficient measure of both genuine and feigned ADHD in adults. Ultimately, this work may contribute to the development of a more accurate diagnostic algorithm for adult ADHD.

Correspondence: Sanya Sagar, The University of Windsor, 1028 Lincoln Road, Windsor, ON N8Y2H4, Canada.E-mail: sanya.sagar@gmail.com

M.A. REZA ARROYO, C.E. GARCÍA GUERRERO, M. GÁNDARA MARTÍNEZ \& O.A. SANTOS. Investigating the Relationship of Word Reading Tests and Verbal/Nonverbal Intelligence Measures in Spanish in a Mexican Sample.

Objective: Premorbid intelligence (PI) estimation is feasible in Spanish by using demographic variables and correctly reading aloud previously known infrequently accented words, like on the several versions of the Word Accentuation Test (WAT). Given the limited research on the WAT, we examined the relationship of two WAT versions with commonly used verbal/nonverbal intelligence proxies in a Mexican sample.

Participants and Methods: Forty-six cognitively normal, native Spanish speakers ( $60 \%$ males; ages $27 \pm 8.2$; education $14.8 \pm 2.7$ ) were administered the WAT-Chicago and -Revised versions and the Test of Nonverbal Intelligence-Second Edition (TONI-2), as well as Vocabulary 
(VO) and Block Design (BD) from the Wechsler Adult Intelligence ScaleFourth Edition (WAIS-IV) as measures of verbal and nonverbal intelligence, respectively. Partial correlations, bivariate and multivariate analyses of variance, and hierarchical multiple regressions were conducted. Results: Gender, age, education, and test performance differences were not found except for females outperforming males on VO. After controlling for demographics, $\mathrm{VO}$ and $\mathrm{BD}$ were significantly correlated with both WAT versions $(r=.53-.58)$ and the TONI- $2(r=.33-.45)$, whereas the latter showed no significant correlations with either WAT version. The WAT-Chicago $(91.67 \pm 8.5, p<.0001)$ and -Revised $(94.11 \pm 6.36, p<.0001)$ as well as the TONI-2 $(108.15 \pm 16.23, p<.01)$ were statistically different from the normalized IQ distribution. After controlling for demographics, VO (Step 3: $\left.\Delta R^{2}=.01-.07, p<.05\right)$ and BD (Step 2: $\left.\Delta R^{2}=.03-.16, p<.0001\right)$ predicted performances on both WAT versions, while only $\mathrm{BD}\left(\Delta R^{2}=.19, p<.01\right)$ predicted TONI-2 performance.

Conclusions: Results indicated that the selected verbal/nonverbal WAIS-IV subtests, but not the TONI-2, were associated with and predicted performance on both WAT versions. However, the WAT versions produced different PI estimations, warranting caution during interpretation and further research.

Correspondence: Melaniee Gándara Martínez, Mexico. E-mail: melaniee.gandara@udem.edu

J. SOBER, J.L. WOODARD, L. MILLER, P. MARTIN, A. DAVEY \&. L.W. POON. Measurement Invariance of a Verbal Fluency Task for the Oldest Old.

Objective: Verbal fluency tasks are measures of semantic memory and have been shown to distinguish normal aging from cognitive decline (Forbes-McKay, Ellic, Shanks, Venneri, 2005). It is not known whether scores on verbal fluency tasks represent the same latent construct (i.e., semantic memory) for the oldest old (i.e. those over 80 years old), a group of older adults at a greater risk than younger cohorts to develop Alzheimer's disease (Stern et al., 1994). This study examined the configural structure of verbal fluency tasks from the Fuld Object Memory Evaluation (Fuld, 1981) using measurement invariance techniques. We hypothesized that the verbal fluency measures are invariant across age groups.

Participants and Methods: Participants consisted of 78 octogenarians $\left(\mathrm{M}_{\text {age }}=84.3, \mathrm{SD}_{\text {age }}=2.81, \mathrm{M}_{\text {edu }}=13.34, \mathrm{SD}_{\text {edu }}=2.74,13\right.$ African American, 52 female) and 232 centenarians $\left(M_{\text {age }}=100.57\right.$, $\mathrm{SD}_{\text {age }}=2.04, \mathrm{M}_{\text {edu }}=10.65, \mathrm{SD}_{\text {edu }}=3.82,50$ African American, 195 female). All participants were obtained from a population based sample from the Georgia Centenarian Study (Poon, et. al., 2007).

Results: Results indicate that the dimensional structure $\left(\chi^{2}\right.$ $(45)=1312.917, p<.05 ; \mathrm{CFI}=.983 ;$ TLI $=.933$, RMSEA $=.039)$ of the model was shared across groups. All measures were equally related to the latent variables $\left(\chi^{2}(49)=68.022, p<.05 ; \mathrm{CFI}=.985 ; \mathrm{TLI}=.986\right.$, RMSEA $=.035)$ across groups. Residual variances were also invariant across groups $\left(\chi^{2}(50)=68.09, p<.05 ; \mathrm{CFI}=.986 ; \mathrm{TLI}=.987\right.$, RMSEA $=.034)$.

Conclusions: A one-factor model was shown to have the best fit. Equivalent loadings suggest that test scores and latent variables can be used in the same way for each group. Additional invariance testing suggests equal residual variances across groups. Because there were significant differences in performance on each task, intercept differences were not modeled and normative data for each group are needed. Findings indicate that the latent construct of semantic verbal fluency is measured identically across octogenarians and centenarians.

Correspondence: Jonathan Sober, Psychology, Wayne State University, 5057 Woodward Ave., Detroit, MI 48202, United States. E-mail: jsober@wayne.edu
S. TAYLOR, J. BEACH, S.L. AITA, J.S. HOLCOMBE, J. EPKER, A. BOETTCHER, R. BURRELL, J. TAYLOR, M. ESPENAN \& B.D. HILL. Examination of the Repeatable Battery for the Assessment of Neuropsychological Status Performance Validity Index in a Chronic Pain Sample.

Objective: This study evaluated the clinical utility of the Repeatable Battery for the Assessment of Neuropsychological Status (RBANS) Performance Validity Index (PVI) in a chronic pain sample.

Participants and Methods: Four-hundred fifty-nine subjects $(M$ age $=53.32, S D=13.55 ; M$ education $=13.10, S D=2.66 ; 61 \%$ female; 81\% White, $12 \%$ African American, .6\% Other) presenting for a pre-surgical evaluation at a Southeastern pain clinic completed the RBANS, the Word Memory Test (WMT), the Pain Catastrophizing Scale (PCS), and the Wechsler Abbreviated Scale of Intelligence (WASI) as part of a larger neuropsychological battery. The RBANS PVI was calculated using Paulson, Horner, and Bachman's (2015) methodology.

Results: Pearson correlations between the RBANS PVI, WMT consistency, PCS, and WASI full scale intelligence quotient (FSIQ) yielded significant correlations ranging from .17 to $.56(p<.001)$. Next, a multiple stepwise regression was used to predict WASI FSIQ using the RBANS PVI, PCS, and the WMT immediate recognition, delayed recognition, and consistency percentages. The RBANS PVI, PCS, and WMT consistency accounted for significant variance in the WASI FSIQ $\left(F(3,455)=89.77, R^{2}=.37, p<.001\right)$. In the regression model, RBANS PVI, WMT consistency, and PCS were significant predictors of intellectual functioning $(b=.43, p<.001 ; b=.19, p<.001 ; b=-.16, p<.001$, respectively). WMT immediate and delayed recognition did not significantly predict intellectual functioning and therefore were removed from the model.

Conclusions: RBANS PVI explained a significant proportion of variance in intellectual functioning. This finding supports the validity of this newly established embedded performance validity test and possible clinical utility of this index for detecting suspect effort on cognitive tests. Future research should further evaluate the relationship between the RBANS PVI, cognitive functioning, and reported distress in clinical and non-clinical populations.

Correspondence: Sarah Taylor, Psychology, University of South Alabama, 75 South University Blvd, Mobile, AL 36688-0002, United States. E-mail: set1622@jagmail.southalabama.edu

\section{K.A. TOLLE, V. MONTGOMERY, R.J. SPENCER \& J. STELMOKAS. The Implications of Different Cutoff Scores for the Montreal Cognitive Assessment (MoCA).}

Objective: The Montreal Cognitive Assessment (MoCA) is a well-validated cognitive screening tool. However, selection of specific cut scores remains controversial, with the originally-suggested cut score of $<26$ leading to over-diagnosis, particularly among those with diverse backgrounds or lower levels of education. Modifications to the original cut score include 1) adding one point to the total score for 12 or fewer years of education, 2) lowering the cut score to $<21$, or 3 ) deriving $\mathrm{z}$-scores from demographically adjusted samples. The aim of this study was to examine the prevalence of cognitive impairment on the MoCA using different cut scores in a post-acute care (PAC) rehabilitation sample. We further identified an optimal MoCA cut score based on predicted dichotomous classification of cognitive impairment as determined by performance on neuropsychological tests.

Participants and Methods: Participants included 100 Veterans $(92 \%$ male) admitted to PAC (Age, $M=67.94$; Education, $M=12.63$ ) who completed the MoCA; 58 of these participants also completed the criterion neuropsychological measures (Trails B, Digit Span, Institute of Living Scale-Health and Safety, and Hopkins Verbal Learning TestDelayed Recall). Classification of impairment was defined as obtaining $\mathrm{z}$-scores of less than -1.5 on at least half of the criterion measures.

Results: Prevalence of cognitive impairment on the MoCA varied from $10 \%$ to $92 \%$, depending on the cut score. ROC analyses identified an optimal MoCA cut score of $<22[\mathrm{AUC}=.83$, sensitivity $=.84$, specificity $=.73]$. 
Conclusions: Rates of cognitive impairment on the MoCA vary widely depending on the cut score; the commonly used cut score $(<26)$ appears overly sensitive in PAC rehabilitation settings with $92 \%$ of individuals scoring below this threshold. A cut score of $<22$ is more appropriate to this setting.

Correspondence: Kathryn A. Tolle, M.A., Psychiatry, VA Ann Arbor Healthcare System, 2129 Glencoe Hills Drive, Apartment 11, Ann Arbor, MI 48108, United States.E-mail: tolleka@gmail.com

E. TRIFILIO, J.J. TANNER, L.C. BUTTERFIELD, P.C. MANGAL, J.E. MAYE, A. CHOI, C.C. MORENO, M. MARSISKE, C.C. PRICE \& D. BOWERS. Neuroanatomical Correlates of an Alternative Story Memory Test in Older Adults: The Left Trumps the Right.

Objective: Prior findings from our lab with older adults suggest that a new set of verbal memory stories (NS), created by Newcomer et al. (1994) have strong concurrent validity with Logical Memory (LM). The current study examined neuroanatomic correlates of these same stories. Based on well-known memory-hippocampal relationships, we predicted that delayed memory scores would relate more so to volumes of left vs right mesial temporal regions, that this relationship would be stronger for verbatim than thematic recall, and that NS and LM would have similar neuroanatomic relationships.

Participants and Methods: 190 healthy older adults. Each participant was administered two story memory tests (WMS-III LM \& NS). NS were scored using "verbatim" (word for word) as well as 'thematic' criteria similar to LM. Of this sample, 111 (ages 60-85; MoCA=25) had complete story and neuroimaging data (T1-weighted). Neuroanatomic regions (ROIs) were extracted using FreeSurfer automatic segmentation and included: hippocampal volume, entorhinal thickness, subiculum/ presubiculum volume. We ran multiple regressions with ROIs predicting delayed recall for both LM and NS raw scores (both scoring types), controlling for age, education, and gender.

Results: Delayed recall (especially NS verbatim) was significantly correlated with left (not right) hippocampal and subiculum/presubiculum volumes and at a trend level with left entorhinal thickness. Only the relationship with subiculum/presubiculum remained when controlling for demographic variables. There were no significant differences between the NS and LM stories.

Conclusions: Findings correspond to views about the differential role of the two temporal regions in mediating verbal memory. Together with earlier findings (concurrent validity), the NS may be a viable clinical option to LM when testing memory over multiple occasions. Next steps include using a larger MCI group to determine utility in using NS to classify MCI vs. healthy as well as including a more diverse sample. Correspondence: Erin Trifilio, BA, Clinical and Health Psychology, University of Florida, 1225 Center Drive, Gainesville, FL 32601, United States.E-mail:etrifor@ufl.edu

M.R. UDALA, J. CLARK, H. MILLER, D. LEITNER \& M. LIBBEN. Cognitive Functioning and Psychopathology in a Traumatic Brain Injury Sample.

Objective: Research on individuals with a traumatic brain injury (TBI) purports that psychopathology is related to specific neuropsychological constructs (Gass, 1996). Gass (1996) found that the cognitive functions including verbal and visual memory, attention, and learning, were influenced by scores on the Minnesota Multiphasic Personality Inventory $-2^{\text {nd }}$ Edition (MMPI-2; Butcher et al., 1989). More specifically, results suggested that for patients with a TBI, Psychasthenia, Schizophrenia, Obsessional Thoughts, and Bizarre Mentation, significantly predicted lower neuropsychological performance on attention span. Further, Fears and Bizarre Mentation predicted lower scores on verbal memory. To follow up this study, Ross et al. (2003) found MMPI-2 variables, specifically the basic scales of Depression, and subscales of Fears and Bizarre Mentation predicted in the negative direction, most notably related to attention and list learning factors. The present study is a replication and extension of Gass (1996) and Ross et al. (2003).
Participants and Methods: A sample of 165 traumatic brain injury (TBI) patients referred for evaluation at a private practice clinic were administered a battery of neuropsychological measures of list learning, attention span, verbal memory, visuographic memory, and executive functioning.

Results: A factor analysis replicated Gass (1996) and Ross et al. (2003)'s findings, with an added fifth factor, executive functioning. Regression analysis indicated that the overall model of MMPI- 2 indices, and specifically the basic scales 2 (Depression) and 7 (Psychasthenia) and the content scales Fear and Bizarre Mentation, predicted lower performance for attention and executive function.

Conclusions: Results indicate that psychopathology is related to performance on neuropsychological measures for patients who suffered a TBI and have important implications for clinical psychological judgment and assessment.

Correspondence: Megan R. Udala, MA, Psychology, University of Britsh Columbia, 1049 Fuller Ave, Kelowna, BC V1Y 6X6, Canada. E-mail: megan.udala@ubc.ca

T.R. VALENTINE \& L. BOXLEY. Clinical Predictors of Subjective Cognitive Functioning Among Medically Healthy Younger Adult Neuropsychology Patients: An MMPI-2-RF Study.

Objective: There exists significant heterogeneity across studies in the relationship between cognitive complaints and cognitive performance. Discrepant results are likely due in part to the specificity of this relationship to sample characteristics and measures. This study examined objective cognitive performance and MMPI-2-RF measures of emotional health as predictors of subjective cognitive functioning in younger adults without significant medical morbidity.

Participants and Methods: Patients $(N=37)$ aged 20 to 40 years referred to a neuropsychology clinic at an academic medical center were studied. Subjective cognitive functioning was assessed via the Cognitive Complaints Scale (COG) of the Minnesota Multiphasic Personality Inventory-2-Restructured Form (MMPI-2-RF). Emotional health was examined using the MMPI-2-RF's demoralization (RCd), somatic complaints (RC1), low positive emotions (RC2), and dysfunctional negative emotions (RC7) scales. Measures of cognitive performance included the California Verbal Learning Test-II, Trail Making Test, Wechsler Adult Intelligence Scale-IV: Digit Span subtest and Wechsler Memory Scale-III: Logical Memory subtest. Linear regression analyses examined emotional health and a general cognitive performance composite as predictors of subjective cognitive performance, controlling for demographic factors.

Results: Significant predictors of subjective cognitive functioning included RCd $(\beta=.64)$, RC1 $(\beta=.52)$, RC2 $(\beta=.45)$, and RC7 $(\beta=.49$; all $p \mathrm{~s}<.001)$. Objective cognitive performance did not emerge as a significant predictor of subjective cognitive functioning in any of the analyses (all $p s>.05$ ).

Conclusions: In medically healthy younger adult neuropsychology patients, MMPI-2-RF measures of emotional health are stronger predictors of subjective cognitive functioning than is objective cognitive performance. These results demonstrate the importance of assessing emotional health in neuropsychology practice, especially when medical morbidity and age are minimally contributing factors.

Correspondence: Thomas R. Valentine, MA, Psychology, The Ohio State University, 1220 Chambers Road, 411C, Columbus, OH 43212, United States.E-mail:valentine.277@osu.edu

B. VERMILION, L.J. RAPPORT, S. KROHNER, B. BILLINGS, C.G. WONG, B. STACH \& M. DE IORIO. See what I'm Saying: Speechreading Facilitates Accuracy on List-learning.

Objective: Quantify speechreading (lipreading) ease of words in a common list-learning task and assess the extent to which this property is associated with accuracy for word recognition and recall.

Participants and Methods: The data were 24 learning-trial words from two forms of the Hopkins Verbal Learning Test-Revised. We developed 
a system to code whole words comprised of visemes (visual units of speech) using a modified system by Jeffers (1971). Speechreading ease was assessed via a composite score of viseme visibility rankings. Spoken word recognition (Trial 1) and Recall were assessed via accuracy data from 111 older adults, using counterbalanced video presentations in optimal and degraded auditory conditions. Word frequency, syllables, neighborhood density, and positional probability were derived from the Irvine Phonotactic Online Dictionary.

Results: Interrater reliability for visemes coding of words was excellent: $\mathrm{ICC}=.975[.972, .977]$. In general, high-visibility visemes were positively related to Trial-1 accuracy, whereas low-visibility visemes were inversely related to accuracy. Viseme-visibility was inversely related to syllables, density, and positional probability $(r-.22$ to -.55$)$, with quadratic relation to lexical frequency (.44). Viseme-visibility was significantly related to Trial-1 accuracy after partial correlation accounting for syllables $\left(r_{p}=.55\right)$; the effect was strongest in the degraded $\left(r_{p}=.69\right)$ versus optimal $\left(r_{p}=.34\right)$ auditory condition. The pattern on word recognition persisted to Recall (overall $r_{p}=.40$; degraded $r_{p}=.59$; optimal $r_{p}=.07$ ).

Conclusions: Speechreading visibility facilitates word recognition and recall on list-learning tasks, especially under degraded auditory conditions. Differences in speechreading properties of words across tests may create differential validity for persons with hearing impairment. Testing conditions such as lighting and visual access to the examiner's face for speechreading should be monitored during testing.

Correspondence: Barret Vermilion, MI, United States. E-mail: barret. vermilion@wayne.edu

T.A. WEBBER \& J. SOBLE. Utility of Various WAIS-IV Digit Span Indices for Predicting Noncredible Performance in Older Veterans With and Without Cognitive Impairment.

Objective: Reliable Digit Span (RDS), age corrected scaled score (ACSS), and to a lesser extent RDS-revised (RDS-R), are well-validated and commonly used embedded performance validity tests (PVTs) in neuropsychological evaluation of veterans, yet existing validation studies have largely focused on younger veterans with lower rates of cognitive impairment. This cross-sectional study compared the classification accuracy and sensitivity/specificity of RDS, ACSS, and RDS-R in a mixed clinical sample of older veterans at greater risk for neurocognitive disorders.

Participants and Methods: 89 middle-aged to older $(M$ age $=54.90$, $S D=15.31)$ veterans $(84.3 \%$ male $)$ completed the Dot Counting Test (DCT), Word Memory Test (WMT), and Wechsler Adult Intelligence Scale-Fourth Edition (WAIS-IV) Digit Span subtest during a more extensive outpatient neuropsychological evaluation. Validity group membership was determined by passing (valid; $N=71$ ) or failing (noncredible; $N=18$ ) both the DCT and WMT. Of the participants with valid performance, $52 \%$ were cognitively impaired. Logistic regression determined classification accuracy, while receiver operating characteristic curve analyses provided sensitivity/specificity values for identifying noncredible performance.

Results: RDS, ACSS, and RDS-R all significantly predicted group membership with respective classification accuracies of $84.3 \%, 83.0 \%$, and $85.4 \%$, and areas under the curve of $.79, .87$, and .83 . Optimal cut scores were: RDS $\leq 6, \mathrm{ACSS} \leq 5$, and RDS-R $\leq 9$, which produced respective sensitivities of $.56, .61$, and .50 , and specificities of $.85, .90$, and .87 . Conclusions: Consistent with previous research in veteran samples, ACSS yielded comparable classification accuracy but greater sensitivity/ specificity than RDS and RDS-R. Thus, while all three indices have utility as embedded PVTs and are robust to cognitive impairment, results suggested that ACSS may best identify veterans with noncredible performance while minimizing false positives.

Correspondence: Troy A. Webber, M.A., Psychology, South Texas Veterans Healthcare System, 6974 Oak Dr., Apartment 6106, San Antonio, TX 78256, United States. E-mail:webbertr@gmail.com
D. WEITZNER, A. DEVITO \& M. CALAMIA. The Relationship Between Self-Reported Executive Dysfunction and Self-Reported Everyday Functioning in Older Adults.

Objective: Subjective measures of cognition and functional impairment are used in neuropsychological assessment to assess a patient's perception of his or her functioning in daily life. The objective of the current study was to examine the associations between self-reported executive functioning and cognitively relevant functioning domains.

Participants and Methods: 332 older adults (66\% female, mean age of 62.9 years $(S D=4.7)$, range: 55 -80 years) were recruited on Amazon Mechanical Turk. Participants completed the Barkley Deficits in Executive Functioning Scale (BDEFS) Short Form, a self-report measure used to assess executive functioning, and the Measurement of Everyday Cognition (ECog) scale, a self-report measure of decline in functional skills related to multiple domains of cognition (i.e., memory, language, visual spatial skills, planning, organization, and divided attention). Participants also completed the Geriatric Anxiety Inventory (GAI).

Results: Multiple linear regressions were conducted including demographic variables as predictors of ECog domains in addition to the BDEFS and the GAI. Results indicated that the BDEFS significantly predicted each domain of the ECog $(p$ 's $<.01)$. The associations ranged from Beta $=.54$ for the visual spatial subscale to Beta $=.69$ for the organization subscale. Anxiety was largely unassociated with the ECog except for the organization subscale $($ Beta $=.79, p<.01)$.

Conclusions: Although prior work has shown that objective neuropsychological measures show differential relationships with ECog domains, self-reported executive functioning is robustly associated with all domains. Consistent with prior work, there was an association of anxiety with subjective cognition, although results were limited to only one domain. Future research should continue to explore the relationships between subjective cognition and functional ability, and their associations with psychological symptoms and objective performance. Correspondence: Daniel Weitzner, M.S., Psychology, Louisiana State University, 8038 NW 66th Way, Parkland, FL 33067, United States. E-mail: dweitz1@lsu.edu

C.G. WONG, L.J. RAPPORT, B. BILLINGS, V. RAMACHANDRAN, B. STACH, M. DE IORIO \& B. VERMILION. Verbal Memory Performance Under Real and Simulated Hearing Loss Conditions. Objective: Hearing loss has been found to be related to cognitive decline; however, many neuropsychological tests involve the presentation of auditory stimuli. Previously, we reported that adults with hearing loss performed worse than adults without hearing loss on an auditory-verbal memory test presented at a normal speaking volume, but performance on a visual version of the verbal memory test was equivalent between groups (Wong et al. 2016). This study examined the effects of different presentation conditions on verbal memory performance, and examined the relationship between hearing loss severity and verbal memory.

Participants and Methods: Forty-one adults with moderate-to-severe hearing loss (HL) and 41 adults with normal hearing (NH) participated. They completed the Hopkins Verbal Learning Testing-Revised under optimal and non-optimal auditory conditions.

Results: The NH group under the non-optimal condition (hearing loss simulation) performed significantly worse than the HL group under the optimal condition (amplified volume, $p<.001$ ) with large effect sizes $(d=0.84$ to 1.52$)$, and was equivalent to HL group performance at normal speaking volume $(d=.01$ to $.28, p \mathrm{~s}>.19)$. Within the HL group, hearing loss severity was inversely correlated with non-optimal auditory-verbal memory $(r=-.47$ to -.59$)$, but not with optimal auditory-verbal memory.

Conclusions: Hearing loss interferes with performance on tests with a high auditory demand, which in turn falsely inflates estimates of cognitive impairment. Under conditions of simulated hearing loss, adults with normal hearing perform like adults with hearing loss. Thus, prior findings reporting cognitive deficits associated with hearing loss are likely contaminated, at least in part, by measurement artifact. Future 
research should expand clinical measures that assess cognitive functioning independent of auditory modality to facilitate valid cognitive assessment of adults with hearing loss.

Correspondence: Christina G. Wong, Wayne State University, 4469 Cleveland Ave, Apt B, San Diego, CA 92116, United States. E-mail: cgwong23@gmail.com

A.E. WOOD, G. ST. LOUIS, T. FASS \& L. ASHENDORF. ReyOsterrieth Figure Copy Size and Cognitive Functioning in Veterans. Objective: The purpose of this study was to analyze the relationship between the Rey-Osterrieth Complex Figure Test (RCFT) copy size and domains of cognitive and psychological functioning among veterans with mild traumatic brain injury, PTSD, and depression. It was hypothesized that larger RCFT copies would correlate with increased executive functioning, better memory, and psychological functioning, as measured by psychological screening measures of depression and PTSD, among veterans with mild traumatic brain injury, PTSD, and depression.

Participants and Methods: Archival data of 73 veterans referred by various sources, such as psychologists, neurologists, psychiatrists, primary care doctors, and social workers, to receive neuropsychological evaluations through the Polytrauma clinic at Edith Nourse Rogers Memorial Hospital were reviewed. Data on executive functioning, memory, and PTSD (PCL-5) and depression (BDI-II) symptoms were gathered in addition to the measurement of the RCFT copy. A Pearson product-moment correlation was conducted to analyze the relationship among these factors.

Results: RCFT copy size and executive functioning approached significant $(\mathrm{r}=-0.225, \mathrm{p}=0.055)$. In addition, the PCL-5 and the BDI-II $(r=0.715, p=0.000)$, PCL -5 and memory $(r=-0.373, p=0.012)$, and executive functioning and memory $(r=0.498, p=0.000)$ were significantly correlated.

Conclusions: The negative relationship between the RCFT copy size and executive functioning, which approaches significance, demonstrates the possibility that the RCFT copy size may be an indicator of executive functioning. More research is necessary with a larger sample to elucidate this relationship. In addition, the significant relationship between PTSD and depression symptoms, PTSD symptoms and memory, and executive functioning and memory highlights the level of interconnectivity between these factors.

Correspondence: Ashley E. Wood, MA, Clinical Psychology, William James College, 4411 Kinsey Dr, Apt 721, Tyler, TX 75703, United States.E-mail: ashley_wood@williamjames.edu

R. YESIAN, M. DANIEL, E. WATSON \& P. MICHAEL. Third Party Observers and the California Verbal Learning Test-2: A Preliminary Analysis.

Objective: Previous research has found third party observation (TPO) negatively affects performance on memory tests. However, these studies mostly used non-clinical populations and between-subject designs, which do not control for individual differences in cognitive functioning. Additionally, there have been no published studies examining TPO effects on the California Verbal Learning Test-2 (CVLT-2), one of the most widely used memory tests. The current study examined the effects of TPO on CVLT-2 performance in a clinical group using a within-subjects design.

Participants and Methods: Twenty-one participants (57\% women; mean $/$ sd: age $=36.1 / 14.6$; education $=14.5 / 1.8)$ referred for neuropsychological evaluation were administered both the standard and alternate forms of the CVLT-2, in that order. Length of time between administrations was 1-58 days. TPO presence was counterbalanced for two groups. Results: Analyses used mixed design repeated measures ANOVAs. Participants performed significantly better on Discriminability on the standard form compared to the alternate form when a TPO was absent. Participants performed significantly better on Trial B on the standard form compared to the alternate form when a TPO was present. Although results for most variables were not statistically significant, there was a general trend for participants to perform better on the standard form compared to the alternate form, regardless of TPO presence.

Conclusions: Overall, there was no consistent effect of TPOs on CVLT-II performance. Limitations of this current study include small sample size, lack of random assignment, and apparent modest comparability of alternate CVLT-II forms.

Correspondence: Rebecca Yesian, PsyD, Graduate School of Psychology , Pacific University, 335 SE 32nd Ave, Portland, OR 97214, United States.E-mail: corm8043@pacificu.edu

B. YOCHIM, K. SCHENDEL \& J. BALDO. Performance on the Verbal Naming Test in Individuals with Left Hemisphere Stroke. Objective: The Verbal Naming Test (VNT; Yochim et al., 2015) enables the assessment of word-finding problems by using a naming-to-definition paradigm. The stimuli are based on word frequency in everyday spoken language, thereby increasing the sensitivity of the VNT to mild word-finding difficulty. In the current study, we tested individuals with a history of left hemisphere (LH) stroke to evaluate the validity of the VNT in a clinical stroke sample.

Participants and Methods: Individuals with a history of chronic LH stroke ( $=18)$ and a wide range of language impairments were administered the VNT. Their performance was compared to that of 10 age- and education-matched healthy control participants. On the VNT, the examiner reads aloud a series of 55 definitions, and the examinee is asked to name the word being described. If the examinee cannot provide the correct response in 10 seconds, a phonemic cue is provided.

Results: The LH stroke group correctly named an average of 31.4 items out of a possible $55(\mathrm{SD}=19.6$, range $0-55)$. This average performance was well below the clinical cut-off score of 46.5 , and was also significantly lower than the average score of the healthy control group in the present study $(\mathrm{M}=53.9, \mathrm{SD}=1.2$, range $51-55)$. VNT performance correlated with aphasia severity: LH individuals with moderate-severe aphasia (Broca's, Wernicke's, Global) scored very poorly (0-35 items correct), while LH individuals with mild or no aphasia scored in the upper range ( 43 and above). The phonemic cues provided an average additional 3.7 correct responses (SD $=5.6$, range $0-17$ ) in LH individuals and an additional 0.8 correct responses $(\mathrm{SD}=0.9$, range $0-2)$ in the healthy control group.

Conclusions: These data indicate that the VNT is sensitive to wordfinding deficits caused by LH stroke. Advantages of this measure include its brief administration time, and appropriateness for patients with visual impairments and in tele-neuropsychological assessments.

Correspondence: Brian Yochim, Ph.D., Psychiatry, VA Palo Alto Health Care System, VA St. Louis Health Care System (116B/JB), 1 Jefferson Barracks Dr., St. Louis, MO 63125, United States. E-mail: Brian. Yochim@va.gov

B.G. ZUCCATO, C. CHARRON, A. DUNN, C. MAYTHAM, A. ENACHE, A. MCDERMOTT \& L.A. ERDODI. Early Bird Fails the PVT? The Effects of Timing Artifacts on Performance Validity Tests.

Objective: This study was designed to investigate the effects of time of day and administration sequence on the likelihood of failing the accuracy and time-to-completion cutoffs on the Recognition Memory Test - Words (RMT) and Word Choice Test (WCT).

Participants and Methods: Data were collected from a consecutive case series of 196 patients $\left(M_{\mathrm{Age}}=44.5,55.1 \%\right.$ female $)$ medically referred for neuropsychological evaluation. Most common diagnoses were psychiatric $(39.3 \%)$, traumatic brain injury $(38.3 \%)$, and epilepsy $(7.1 \%)$. The RMT and WCT were administered in counterbalanced order either at the beginning (Time 1) or at the end (Time 2) of a battery of neuropsychological tests, with an average of $5.1(S D=1.4)$ intervening hours.

Results: Patients were more likely to fail the accuracy score on both the RMT [relative risk (RR): 1.44-1.64] and the WCT (RR: 1.21-1.50) at Time 1 across a range of cutoffs. Likewise, they were more likely to fail 
the time-to-completion score at Time 1 on both the RMT (RR: 1.301.94) and the WCT (RR: 1.58-3.75). Established accuracy (RMT $\leq 43$ ) and time (RMT $\geq 192$ "; WCT $\geq 171$ ") cutoffs failed to clear minimum specificity standards at Time 1 . The published accuracy cutoff for the WCT (s47) performed well at both time points. More liberal accuracy and time cutoffs for the RMT $(\leq 44 ; \geq 165$ ") reached good specificity (.87-.91) at Time 2. Similarly, a more liberal time cutoff $(\geq 150 ")$ for the WCT achieved adequate specificity at Time 2 (.84-.92).

Conclusions: When administered early in the testing session, the RMT and WCT may be prone to false positive errors. Conversely, when administered at the end of the day, existing cutoffs may have lower than expected sensitivity. Time and sequence of administration should be considered during both test selection and the interpretation of RMT and WCT scores. Using conservative cutoffs for morning administrations and liberal cutoffs for afternoon administrations may be necessary to neutralize timing artifacts.

Correspondence: Brandon G. Zuccato, B.A., Psychology, University of Windsor, 401 Sunset Ave., Windsor, ON N9B 3P4, Canada. E-mail: zuccatob@uwindsor.ca

\section{Assessment/Psychometrics/Methods (Child)}

C.A. AUSTIN, M. GERSTLE, B. LEJEUNE, A. BRADLEY \& D.W. BEEBE. Multifactor Predictors of Parent Satisfaction with Neuropsychological Assessment.

Objective: To examine multidimensional predictors of general parent satisfaction for pediatric neuropsychological evaluation

Participants and Methods: 363 parents of children receiving outpatient neuropsychological evaluation participated in the study. Parents completed questionnaires at 4 time points assessing their understanding of evaluation, their experience with the assessment process, and the utility of evaluation. Predictors were derived from the Parent Experience of Assessment Scale (PEAS) or study-specific measures. General satisfaction was the primary outcome variable

Results: General satisfaction had large significant correlations with Parent Assessor Relationship (.77, p<.01). and New Understanding of Child $(.75, \mathrm{p}<.01)$. Child Assessor Relationship had a significant moderate correlation with general satisfaction $(.43, \mathrm{p}=.01)$. Negative Feelings had an inverse moderate correlation with general satisfaction $(-.413 ; p<.01)$. Access to Services had a small significant correlation $(.26, \mathrm{p}=.01)$.

Linear regression was significant $(\mathrm{p}<.001)$ and explained $69 \%$ of the variance in general satisfaction. Access and Negative Feelings were removed from the model due to lack of significance $(p>.4)$. The final model indicated New Understanding of Child was the strongest significant predictor $(\mathrm{p}=.007)$, followed by Parent Assessor Relationship $(\mathrm{p}=.03)$.

Conclusions: Parent satisfaction was most strongly associated with process variables during the neuropsychological assessment, including new understanding of child and parent assessor relationship. Results were consistent with the general satisfaction literature, indicating that interpersonal factors, rather than access/logistics, often have the strongest association with satisfaction. Results also echoed previous findings involving child psychological assessment indicating New Understanding of Child has the strongest direct effect on parent satisfaction.

Correspondence: Cynthia A. Austin, Ph.D, Behavioral Medicine and Clinical Psychology, Cincinnati Children's Hospital and Medical Center, 3333 Burnet Avenue, Cincinnati, OH 45229, United States. E-mail:cynthia.austin@cchmc.org
A.N. BARTLETT, A.M. O'BRIEN, N. FROST, T.A. DUDA \& J.E. CASEY. The Association of the Grooved Pegboard Test to Everyday Motor Skills.

Objective: The Grooved Pegboard Test (GPT) is used to assess speeded eye-hand coordination and fine finger dexterity. Although its relationship to brain damage is well established (Larrabee, Millis, \& Meyers, 2008), its ecological validity in predicting fine motor skills used in daily functioning is not. The Developmental Coordination Disorder Questionnaire (DCDQ) is a screening measure of motor skills involved in daily functioning. It comprises three subscales: control during movement (CDM), fine motor skills (FMS), and general coordination (GC), as well as a global summary score. The present study sought to investigate the association between GPT and a parent-report measure of childhood motor functioning (DCDQ). Based on previous research with other motor measures, it was hypothesized that GPT would be significantly associated with the FMS subscale, but not the other subscales or the summary score.

Participants and Methods: Children ( $N=40 ;$ Mage $=11.8$ years; $63 \%$ male; $40 \%$ with ADHD) completed the GPT and their parent completed the DCDQ as part of a larger study examining handwriting in childhood ADHD. Dominant (DOM) and non-dominant (ND) GPT time scores were used.

Results: Contrary to the hypothesis, DOM and ND GPT performance showed no correlation with the FMS subscale [DOM: $r(38)=0.06$, $p=0.72$; ND: $r(38)=0.13, p=0.43]$. ND GPT performance was positively correlated with all other DCDQ scores [CDM: $r(38)=0.48, p=0.002$; GC: $r(38)=0.41, p=0.008$; Total: $r(38)=0.40, p=0.011]$. DOM GPT performance was only correlated with the GC subscale $[r(38)=0.32, p=0.048]$. Conclusions: Although the GPT was not associated with everyday FMS as measured by the DCDQ, ND performance did correlate with motor skills more generally. As such, GPT may not be an ecologically valid measure of FMS in children. Instead, GPT performance appears to provide an indication of general impairment in motor functioning, supporting the sensitivity of the GPT to brain dysfunction in general. Correspondence: Alicia N. Bartlett, Clinical Psychology, Department of Psychology, University of Windsor, 401 Sunset Avenue, Windsor, ON N9B3P4,Canada.E-mail: bartl115@uwindsor.ca

S. DEASLEY, N. FROST, L.R. KIVISTO, T.A. DUDA \& J.E. CASEY. Screening and Identification of Developmental Coordination Disorder in Children With and Without ADHD.

Objective: Developmental Coordination Disorder (DCD) is characterized by a delay in the acquisition of appropriate motor skills. Motor difficulties associated with DCD relate to problems with handwriting, attention, reading, and social cognition. Individuals with ADHD often have motor skills deficits, and comorbidity with DCD is high (30-50\%). Despite this, motor problems and DCD remain under-recognized and, consequently, undertreated. This study examined whether children with ADHD have more parent reported DCD symptoms than children without ADHD, as well as the prevalence of undiagnosed DCD. It was predicted that children with ADHD would have more DCD symptoms than children without ADHD. As DCD is under-recognized generally, it was predicted that some children without a DCD diagnosis would screen positive for DCD.

Participants and Methods: Parents $(N=32)$ of children $\left(M_{\mathrm{age}}=11.6\right.$ years; $59 \%$ male; $50 \%$ ADHD) completed the Developmental Coordination Disorder Questionnaire 2007 (DCD-Q), which assesses Control During Movement (CDM), Fine Motor Skills (FMS), and General Coordination (GC) in children.

Results: Children with ADHD had more DCD symptoms overall, $F(1,30)=14.15, p=.001$, as well as on the CDM, $F(1,30)=7.72$, $p=.009, \mathrm{FMS}, F(1,30)=15.57, p<.001$, and GC subscales, $F(1,30)=9.85$, $p=.004$, compared to children without ADHD. Despite no previous DCD diagnosis, $34 \%$ of children screened positive for the disorder.

Conclusions: Children with ADHD had more problems in all rated areas of motor functioning compared with children without ADHD. 
Many children screened positively for DCD despite not having a previous diagnosis. Findings indicate the value of using screening measures to identify children at risk of DCD. Since these deficits can cause added difficulty in the daily functioning of children with ADHD, diagnosis and treatment are especially important for this population.

Correspondence: Shanna Deasley, University of Windsor, 401 Sunset Avenue,Windsor, ON N9B3P4, Canada.E-mail: deasley@uwindsor.ca

I. GONZALEZ, L. HERNANDEZ, C. SADURNI, S. TANNERWOODWARD, P. PEREZ, B. SCHNEIDER, G. SANTACRUZ \& C. SANTIAGo. Comprehensive Pediatric Neuropsychological Clinical Battery in Spanish: A Case Study.

Objective: This case study describes the measures used in the comprehensive neuropsychological testing of a Spanish-speaker patient assessed in a hospital-based pediatric neuropsychological outpatient clinic in Miami, Florida. The purpose of the study is to contribute to the limited literature available regarding available neuropsychological measures that can be used on a comprehensive clinical battery for pediatric Spanish-speakers.

Participants and Methods: Comprehensive neuropsychological testing was completed over the course of 4 testing sessions, by two fluent Spanish-speaker examiners. The patient is a 12 -year-old, Hispanic female who was referred for a neuropsychological evaluation by her pediatric neurosurgeon seven months following a Cerebellar Arteriovenous Malformation (AVM) bleed.

Results: A comprehensive neuropsychological clinical battery using a combination of standardized instruments in Spanish and some non-verbal instruments standardized in English, with a total of 23 different measures, including self and parent reports, was administered to assess the following domains: Intellectual Functioning; Achievement; Verbal Functioning (Receptive/Expressive Language, Auditory Processing); Perceptual Spatial; Processing Speed; Memory/Learning (Verbal/Visual/Associative Learning); Fine Motor; Attention/Concentration (Passive, Effortful, Sustained/Vigilance, Auditory Sustained Attention); Executive Functioning (Nonverbal, Verbal, Sequencing, Cognitive Flexibility, Response Inhibition).

Conclusions: Neuropsychologists are often challenged to provide culturally appropriate measures for the uniqueness of the Spanish-speaking population. This study attempts to suggest the potential combination of different neuropsychological measures that could be used in a clinical setting to obtain an estimate of cognitive functioning using valid and reliable instruments and procedures, adding to the limited literature on the assessment of this population.

Correspondence: Isabel Gonzalez, Doctorate, Psychology, Carlos Albizu University, 901 NE 191 Street, Miami, FL 33179, United States. E-mail: isagongar@gmail.com

R. GREEN, H. AL-HAKEEM, L. SCHNAAS, M. TELLEZ ROJO, E. OSORIO, H. HU \& C. TILL. Developmental Trends of Neuropsychological Function in Mexican Youth using The Cambridge Neuropsychological Test Automated Battery (CANTAB). Objective: CANTAB is a computerized tool used to measure cognitive function in diverse populations, and is sensitive for assessing developmental changes in children. Although CANTAB has been used in several countries, its applicability in a Mexican child population is unknown. This study examined developmental trends on CANTAB in urban Mexico City youth, and tested the hypothesis that their performance would be similar to the American normative sample.

Participants and Methods: As part of a birth cohort, Early Life Exposures in Mexico to Environmental Toxicants, 826 children, ages 5-15, completed CANTAB. Subtests measured planning (Stockings of Cambridge; SOC), short-term memory (Delayed Matching to Sample; DMS), attention/processing speed (Rapid Visual Information Processing; RVP, Match to Sample Visual Search; MTS), flexibility (Intra-extra Dimensional Set Shift; IED), and response inhibition (Stop Signal Task;
SST). Effects of age, maternal/child IQ, and gender were analyzed. Equivalence testing compared performance with existing norms.

Results: Performance improved with age on all measures. Tests of memory and attention had the steepest incline from ages 5-10, and plateaued thereafter, whereas processing speed continued to improve into adolescence. Child-IQ was mildly associated with measures of memory and attention but not executive functioning, consistent with American norms. Maternal-IQ was not associated with any outcomes, and males performed better on IED. Mexican norms were comparable to American norms on almost all outcomes, with the exception of certain age groups (ages 8-11) on a short-term memory task (DMS).

Conclusions: Our findings demonstrate the expected maturational effects of executive function in a large sample of youth. Specifically, cognitive shifting and inhibition are continuing to develop into adolescence. Results obtained in Mexican children confirmed the normative values reported in Americans as well as expected associations with child IQ, indicating high applicability of CANTAB for Mexican youth.

Correspondence: Rivka Green, BA, Psychology, York University, 7460 Bathurst St., 1109, Thornhill, ON L4J7K9, Canada.E-mail: rrgreen@ yorku.ca

S.M. GREIF, E.M. TURNER, A.M. SVINGOS, B. HERNANDEZ, M.W. MOORE, B.A. BAILEY \& S.C. HEATON. Criterion Validity of the Memory Validity Profile in a Diagnostically Heterogeneous Clinical Pediatric Sample.

Objective: Assessment of effort is critical for accurate interpretation of neuropsychological testing. Though many stand-alone effort measures are available for use in adult populations, the recently published Memory Validity Profile (MVP) is the first developed exclusively for pediatric populations. The current study examined the criterion validity of the MVP total, visual, and verbal scales against an embedded effort measure previously validated for use in pediatric populations (WISC-V Reliable Digit Span (RDS)).

Participants and Methods: Archival data was examined from 52 youth referred for neuropsychological evaluation (ages 6-16; $M_{\text {age }}=10.9$, $S D=3.2$ ). RDS performance was dichotomized into cut-off scores of valid effort (RDS $>7 ; n=45)$ and poor effort (RDS $<6 ; n=7)$. Correlational analyses examined convergent validity of RDS and MVP, with concurrent validity addressed via calculation of MVP positive and negative predictive validity (PPV \& NPV).

Results: Despite wide heterogeneity in age, diagnosis, and impairment level, all participants were able to be administered the MVP. Correlations examining convergent validity between the MVP and RDS suggested small, non-significant, positive correlations for MVP total $(r=.16)$, visual $(r=.12)$, and verbal $(r=.19)$ scores. Though 2 participants failed the MVP using published cutoffs, no participant failed both the MVP and RDS (sensitivity and PPV $=0$ ). Results were suggestive of relatively high specificity $(96.2 \%)$ and NPV $(86 \%)$.

Conclusions: This is the first known examination of criterion validity of the MVP in a mixed clinical pediatric sample. While this study demonstrates the feasibility of using the MVP across a wide range of pediatric patients, results raise concern that the MVP may have neither good convergent nor concurrent validity with the RDS. Future research should examine validity of the MVP against other established measures of effort. Alternative MVP cut-off scores and patient-level characteristics of those failing MVP should also be studied.

Correspondence: Sarah M. Greif, BS, Clinical and Health Psychology, University of Florida, 1035 SW 9th ST, APT F24, Gainesville, FL 32601, United States. E-mail: sgreif1021@phhp.ufl.edu 
H. HENNRICK, S. POWDHAR, M. GOLDSTEIN, K. BONEFF \& C. FREEDMAN-DOAN. Significant Discrepancy Between DigitSpan Forward and Backward Impacts Relationship Between Working Memory and Executive Functioning.

Objective: Interpretation of Digit Span (DS) as one score fails to provide information that explains the source of potential processing deficits within/related to working memory. It has been recommended that Digit Span Forward (DSF) and Digit Span Backward (DSB) be examined separately so that clinicians can compare performance on a retrieval task to performance on a manipulation task. The objective was to examine how significant discrepancy between DSF and DSB may affect the relationship between working memory and executive functioning.

Participants and Methods: The sample consisted of 90 children, aged 8-12 years $(\mathrm{M}=10.44, \mathrm{SD}=1.44) .53 .3 \%$ of the children were male. Digit Span (WISC-4) and D-KEFS TMT Letter Number Sequencing (TMT NLS) were used as measures of working memory and executive functioning, respectively.

Results: Regression analyses show that children's performances on DSF and DSB were significant predictors of their performance on TMT Number Letter Sequencing $(\beta=.29, t(88)=2.82, p<.01),(\beta=.25$, $t(88)=2.46, p<.05)$, and accounted for $8.3 \%\left(\mathrm{R}^{2}=.083\right)$, and $6.4 \%$ $\left(\mathrm{R}^{2}=.064\right)$ of the variance in measured EF, respectively. Thus the Digit Span subtest scaled score was also a significant predictor of TMT NLS performance $(\beta=.35, t(88)=3.52, p<.01)$. However, when selecting only cases for which the child had a significantly higher performance on DSF, this relationship became non-significant $(\beta=.34, t(9)=1.07$, $p>.05)$. When selecting only cases for which the child had a significantly higher performance on DSB, this relationship was marginally significant $(\beta=.50, t(13)=2.06, p=.06)$.

Conclusions: Significant discrepancies between DSF and DSB may indicate related deficits in executive functioning. We agree that interpretation of DS as one score fails to elucidate the source of processing deficits and how these deficits may be interconnected or predictive of deficits across neurocognitive domains.

Correspondence: Heather Hennrick, Eastern Michigan University, 410 Ferris Street, Ypsilanti, MI 48197, United States. E-mail: hhennric@ emich.edu

R. LAJINESS-O'NEILL, J. BROOKS, A. LUKOMSKI, S. WARSCHAUSKY, A. HUTH-BOCKS, S. SCHILLING, A. MERCEDES, C. SWICK, R. REED \& T. ANDERSEN. Development and Validation of PediaTrac: A Web-Based Tool to Screen and Track Developing Infants.

Objective: To develop and examine the preliminary psychometrics of a mobile application that engages caregivers in gathering longitudinal, multi-domain data on infants' development.

Participants and Methods: PediaTrac is a 363-item web-based tool, administered in modules of $\sim 40$-items per sampling period, newborn (NB), 2, 4, 6, 9 and 12 months. Expert Panel Reviews (EPR) and Cognitive Interviews (CI) were conducted to validate the item bank. There were 30 caregiver-infant dyads in a longitudinal cohort and 102 dyads in a cross-sectional cohort. The pooled sample was $52.7 \%$ male, with mean gestation 38.9 wks. and mean birthweight $6.8 \mathrm{lbs}$. Questions cover the sensorimotor, feeding/eating, sleep, speech/language, cognition, social-emotional, and attachment domains. Classical Test Theory (CTT) and Item Response Theory (IRT) were employed to examine psychometrics.

Results: Intraclass correlation coefficients (ICC) for EPR revealed moderate agreement at 6-months and good reliability at other sampling periods. ICC estimates for CI showed moderate reliability at NB and 4 months, good reliability at 2,9 and 12 months and excellent reliability at 6 months. CTT revealed good coefficient alpha estimates $(\alpha>77$, five of the six ages) for the Social- Emotional/Communication, Attachment ( $\alpha>89$, all ages), and Sensorimotor ( $\alpha>.75$ at 6 -months) domains. IRT revealed good reliability $(r=.85-.95)$ for three distinct domains (Feeding/Eating, Social-Emotional/Communication and Attachment) and four subdomains (Feeding Breast/Formula, Feeding Solid Food, Social-Emotional Information Processing, Communication/Cognition). Conclusions: CTT and IRT suggest the Sensorimotor, SocialEmotional/Communication, Feeding, and Attachment domains for the PediaTrac instrument have potential to produce efficient estimates of infant development. Initial results also suggest improvements for PediaTrac that will include a greater variety of items for each age and refining the pool to remove low value items.

Correspondence: Renee Lajiness-O'Neill, Ph.D., Psychology, Eastern Michigan University, 320 King Hall, Ypsilanti, MI 48197, United States. E-mail: rlajines@gmail.com

M.W. MOORE, B.A. BAILEY, S.M. GREIF, E.M. TURNER, A.M. SVINGOS, R. RANGAMANNAR, S.C. HEATON, A.M. BRENNER \& B. HERNANDEZ. Role of Socioeconomic Status in the Relationship Between Parent and Performance-based Measures of Executive Function in Children with ADHD.

Objective: Executive function (EF) deficits are common among children with Attention-Deficit/Hyperactivity Disorder (ADHD). Interestingly, performance-based and parent-report measures of EF in this population are often discrepant. Current research suggests that socioeconomic status (SES) plays a complex role in neuropsychological interpretation. Thus far SES has been demonstrated to predict both performance-based and parental report of EF dysfunction. However, the role of SES has yet to be examined in regards to the discrepancy between these two modalities

Participants and Methods: Archival data was used from 51 youth with a primary diagnosis of ADHD who were referred for neuropsychological evaluation (ages 8-15; $\mathrm{M}_{\text {age }}=11.98, \mathrm{SD}=2.05$ ). SES was calculated from primary caregiver education and occupation. Selected subtests from the Delis-Kaplan Executive Function System (D-KEFS; Trail Making Test and Color-Word Interference) and Behavior Rating Index of Executive Function (BRIEF; Shift and Inhibit) were used to measure EF.

Results: The SES composite was not found to contribute significantly to the relationship between parent and performance-based measures of EF. However, taken independently the parental education component of SES explained a significant amount of variance in parent-reported cognitive flexibility $(\mathrm{F}(3,39)=3.46, \mathrm{p}=.026)$, but not inhibitory control $(\mathrm{F}(3,39)=.620, \mathrm{p}=.607)$.

Conclusions: Our results confirm the need for further investigation in the role of familial SES in neuropsychological interpretation. Caregiver report is critical in diagnostic decision-making, but is often at odds with standardized performance-based measures. Our findings suggest that parental education play a significant part in parent-reported EF and may serve to better predict parent-reported child neurocognitive outcomes than parental occupation. This might suggest that current algorithms for SES require refinement.

Correspondence: Makeda W. Moore, M.S., Clinical \& Health Psychology, University of Florida, 10 NW 25th Street, Gainesville, FL 32607, United States. E-mail:mmoore0815@phhp.ufl.edu

N. NOONAN, M. BODZY, B.C. KAVANAUGH, J. STUDENY, C. CORDEAUX \& K.A. HOLLER. Children in an Inpatient Setting Perform with Significant Weakness on Several Common Neuropsychological Measures When Compared to an Outpatient Sample.

Objective: Children admitted to inpatient psychiatric hospitals (IPH) represent an understudied group. The literature lacks comparisons between their performance on neuropsychological (NP) measures to that of outpatient referred groups (ORG). Representative samples used to standardize individual performance implement stringent exclusion criteria such as IQ cut offs, diagnostic exclusions, and other characteristics; however, these are many of the factors that make IPH populations unique (e.g. SES, IQ range, diagnoses, history, and psychiatric severity). 
This study used broad inclusion criteria for IPH children in order to capture the full extent of variability unique to this group and compare mean performance on NP measures to an ORG.

Participants and Methods: Children ages 6-12 years old were separated based on inpatient ( $\mathrm{n}=358)$ or outpatient status $(\mathrm{n}=192)$. T-tests compared means of standardized scores between groups for IQ and several NP measures including verbal fluency, WRAML-II, Beery-VMI, Rey-O Complex Figure, Trail making A \& B, and the Stroop test.

Results: Without using IQ cutoffs, each group had a mean IQ in the Average range, although the IPH group was statistically significantly lower than the ORG. Findings indicated that mean scores on most measures examined (semantic fluency [animals], WRAML-II [4 subtests], Rey-O [copy], Trails A, and Stroop) were significantly lower $(\mathrm{p}<.05)$ for the IPH group than the ORG group (ranging from Borderline to Average).

Conclusions: These findings suggest that child IPH populations are likely to perform significantly worse on many NP measures than outpatients. Factors unique to an IPH setting or population may be lowering scores. ORG's are comprised of individuals more similar to the normative sample than IPH. Thus, using these norms for the IPH group may result in an exaggeration of deficits. This may suggest a need for developing inpatient norms to reduce exaggeration of deficits.

Correspondence: Natalie Noonan, PsyD, Neuropsycholoy, Brown Univeristy, 50 Woods Xing, Holliston, MA 01746, United States. E-mail: natalie_noonan@brown.edu

\section{A. WILLIAMS, S. ROLIN, S. SANTISTEVAN-ANDREWS \& J. KING. Utility of the Memory Validity Profile in Detecting Performance Invalidity in a Pediatric Sample.}

Objective: This study examined performance validity test (PVT) outcomes in a mixed pediatric sample.

Participants and Methods: Participants $(\mathrm{N}=35)$ were $46 \%$ female and $50 \%$ Caucasian with average age and education of $11.4(\mathrm{SD}=3.0)$ and $5.9(\mathrm{SD}=3.0)$ years, respectively. Participants were evaluated using a flexible battery with several embedded and freestanding PVTs including the Non-Verbal Medical Symptom Validity Test (NV-MSVT), Conners' Continuous Performance Test-Second Edition (CPT-2), Tasks of Executive Control (TEC), and Memory Validity Profile (MVP). Participants were categorized according to cutoffs on these measures into valid (VAL) and below-expectation (LOW) groups.

Results: The following rates of below-expectation performances were observed across PVTs: NV-MSVT (11\%), CPT (9\%), and TEC (19\%). No cases scored below cutoff on the MVP. Participant groups based on the three PVTs in which below-expectation scores were observed showed a significant difference in age: VAL $(M=12.1, \mathrm{SD}=2.8)$ and LOW $(\mathrm{M}=9.6, \mathrm{SD}=2.6), \mathrm{t}(33)=2.4, \mathrm{p}=.02$. Groups also differed in education: VAL $(\mathrm{M}=6.6, \mathrm{SD}=2.8)$ and $\mathrm{LOW}(\mathrm{M}=3.9, \mathrm{SD}=2.8)$, $\mathrm{t}(32)=2.5, \mathrm{p}<.02$. Groups were not significantly different in MVP Visual score: VAL $(15.9, \mathrm{SD}=0.4)$ and $\mathrm{LOW}(\mathrm{M}=15.9, \mathrm{SD}=0.3)$, $\mathrm{t}(33)=0.1, \mathrm{p}=.90$. Groups were significantly different in MVP Verbal score: $\operatorname{VAL}(\mathrm{M}=15.9, \mathrm{SD}=0.3)$ and $\mathrm{LOW}(\mathrm{M}=14.9, \mathrm{SD}=2.1)$, $\mathrm{t}(33)=2.4, \mathrm{p}=.02$. Groups approached significant differences in MVP Total score: $\operatorname{VAL}(\mathrm{M}=31.8, \mathrm{SD}=0.5)$ and $\mathrm{LOW}(\mathrm{M}=30.8, \mathrm{SD}=2.4)$, $\mathrm{t}(33)=2.0, \mathrm{p}=.05$.

Conclusions: Although all participants scored above cutoff on the MVP validity scale, participant groups based on three other PVTs showed differences on some MVP indices. The MVP warrant further study to demonstrate its utility as a PVT.

Correspondence: Summer Rolin, Psy.D., Physical Medicine \& Rehabilitation, University of Utah, 1155 East 2100 South, 832, Salt Lake City, UT 84106, United States. E-mail: sumrolin@gmail.com
WITHDRAWN. S. SEESE, M.F. SKAPEK, M. POWERS, D. GOHARI, D. LIMON, L. ANTHONY, L. KENWORTHY \& J.F. STRANG. A Multimodal Research Battery for Assessing Executive Function Skills in Autistic Teens.

Objective: Exeentive function (EF) skills are multi-faceted eapaeities, eritied to assess in intervention researeh. A partientar challenge has been identifying a multimodal EF battery with strong interrelationships aeross modalities. This study evaluates a multi-measture battery for evaltrating EF betravion in atolesecnts with an Antism Spectrum Đisorder (ASD).

Partieipants and Methods: A sample of middle sehool adoleseents with ASD $(\mathrm{N}=33$, age $M=13.2$ years, $27.3 \%$ female; VO a de $M=98.4)$

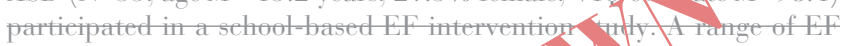
assessments were employed including: mas tw do ps. pont observations. an observational exeentive funetion eh llents ask (EFGT; similar to the ADOS), and elinie based new ot to obrgieal measures including

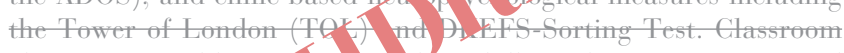
observations yield seons tro ve, 1 LF skills, steh as transitioning and attending to wort. A. EI T T rields seores for planning and flexibility. Pestrlts: Yuffirm tat, I medsures were interrelated. Observational measurn to Ssthom EF observations and EFGT) were strongly eorrelated $\mathbf{v}=-.625, \mathrm{p}<.001)$. Cross-modal comparisons were also observed. Performance on the TOL was signifieantly correlated with performante on the EFCT $(r=-390, p<.030)$ and classroom obser= vation seores $(r=.599, p<.001)$. Classroom observation seores were eorrelated with performance on the DKEFS- Sorting Test $(r=.453$, p<. 030 ).

Gonetusions: The agrecment between lab-based tasks of $\mathrm{EF}$ skills and real world EF observational methods provides initial validation of this new multimodal EF battery. This assessment method may provide a more compretrensive evaluation of the manifestation of EF for treatment studies.

Gorrespondenee: Sydney Seese, Bachelor's, Neuropsychology, Children's Aational Medieal Center, 198 Halpine Rd Apt 1466, Rockville, MD

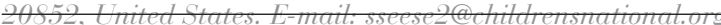

C. SWICK, A. FLORES, R. REED, T. ANDERSEN, K. MCFARLANE, J. BROOKS, A. LUKOMSKI, A. HUTH-BOCKS, S. SCHILLING, S. WARSCHAUSKY \& R. LAJINESS-O'NEILL. Criterion Validation of the Social-Emotional/Communication Domain of PediaTrac ${ }^{\mathrm{TM}}$, an Infant Screening Tool.

Objective:

There is significant need for a comprehensive infant screening tool to establish developmental trajectories. PediaTracTM is administered at newborn, 2, 4, 6, 9 and 12mo., assessing multiple domains of development including Social-Emotional/Communication (SEC). Criterion validity of the SEC domain was established by examining relationships between PediaTrac and the Infant Behavior Questionnaire-Revised (IBQ-R), Ages and Stages Questionnaire (ASQ-3), and Ages and Stages Questionnaire:Social-Emotional (ASQ:SE-2).

Participants and Methods: Data were collected from 132 infants in longitudinal and cross-sectional cohorts with mean gestational age of 38.48 ( $\mathrm{SD}=1.66$ ) and 39.1 weeks ( $\mathrm{SD}=2.19)$, respectively. The SEC domain was derived using item response theory. Pearson correlations were computed to examine relationships between the SEC domain and IBQ-R, ASQ-3, and ASQ:SE-2 from 2-12mo.

\section{Results:}

The SEC domain was related to the ASQ-3 Personal-Social domain at 2,4 , and 9mo. $(r=.48, p<.05 ; r=.41, p<.01 ; r=.38, p<.05)$ and ASQ-3 Communication domain at 9 mo. $(r=.56, p<.01)$. At 6 and 9 mo., higher SEC domain scores were related to more approach behavior and anticipation of pleasurable activities $(r=.57, p<.01 ; r=.48, p<.01)$ on the IBQ-R. Unexpectedly, at 6mo., higher SEC domain score was also related to low $\operatorname{mood}(r=.50, p<.05)$ on the IBQ-R.

Conclusions: The PediaTrac ${ }^{T M}$ SEC domain score was related to the ASQ-3 Personal-Social domain at 2, 4, and 9mo., and the Communication domain at $9 \mathrm{mo}$. The SEC domain score was related to domains 
of infant temperament at 6 and 9mo.. PediaTrac ${ }^{T M}$ measures aspects of SEC consistent with established measures of infant temperament and domains on the ASQ-3 but novel from the ASQ:SE-2.

Correspondence: Casey Swick, Psychology, Eastern Michigan University, 12317 Waterstone Ln. \#122, Perrysburg, OH 43551, United States. E-mail: cswick1@emich.edu

A.M. Teague, D.W. VAughn, J. WATSON, C. BROWN \& R. HIRST. Test of Memory Malingering (TOMM) performance and utility of trial 1 score in a pediatric sample.

Objective: Recent practice guidelines and recommendations encourage the use of performance validity tests (PVTs) in all pediatric neuropsychological assessments (e.g., Chafetz et al., 2015; Heilbronner et al., 2009). Unfortunately, there is relatively scant research on the use of PVTs with children. One consideration in using PVTs with kids is that they may require briefer PVT tests to accommodate short attention spans and poor tolerance of long testing sessions. Previous studies have suggested that the Test of Memory Malingering (TOMM) can be discontinued if an examinee scores 45 or higher on Trial 1, but more research is needed with children. The present study aims to examine the utility of the TOMM Trial 1 score in a pediatric sample.

Participants and Methods: The sample consisted of 72 youth athletes aged 8 to 16 years $(M=11.6, S D=2.2)$ who were recruited in Northern California as part of a longitudinal study examining sports-related concussion. Participants completed a comprehensive two and a half hour baseline neuropsychological evaluation that included the TOMM to assess performance validity.

Results: One child failed the TOMM according to published guidelines. Consistent with previous literature, all those who scored 45 and above on Trial $1(74 \%)$ went on to pass Trial 2 of the TOMM. In fact, all participants who scored above 36 on Trial 1 (99\%) ultimately passed Trial 2. Conclusions: Results provide support for the discontinuation of the TOMM in pediatric evaluations if the examinee scores above 44 on Trial 1. Findings also suggest that children who score greater than 36 on Trial 1 may not need to be administered Trial 2, but further research is needed to confirm this cut-off.

Correspondence: Anna M. Teague, M.S., Palo Alto University, 1610 Park Rd NW APT 417, Washington, DC 20010, United States. E-mail: ateague@paloaltou.edu

C. THOMAS, M. TAN \& E. GRIGORENKO. The effect of home location on performance on a phone-based memory task in rural Zambia.

Objective: Tablets and phones have been shown to have potential benefit for cognitive assessment and intervention in developing countries. We aimed to investigate which aspects of performance on a novel, phone-based working memory task were most affected by previous phone exposure associated with the child's home location, informing future use of technology for assessment.

Participants and Methods: The game, Memory King, modified to include only animals familiar to Zambian children, required children to flip 2 cards at a time to find animal matches. Children completed 2 learning trials of 2 pairs of cards, increasing to 4,8 , and 12 if they earned an accuracy of $40 \%$ or higher. 14 children (3-12 years; mean age 7.21 (sd=2.83); $50 \%$ boys) living in a small regional center in a rural area of Southern Zambia participated. 10 children were from a rural village area and 4 were living on a research institute campus where a parent was employed, giving them more phone access.

Results: There were no statistically significant differences in accuracy between children from the institute and village $(58.26 \% \mathrm{vs} .54 .02 \%)$. However, children from the institute completed the tasks significantly faster during the more difficult 8-pair trial $(\mathrm{F}=5.60, p=.023)$, and differences in speed approached significance during the first learning trial $(\mathrm{F}=3.63, p=.065)$. Regarding strategy, children from the village tended to flip open the same first card paired with a new one to find matches, while children from the institute would flip two new cards unless a previous card location was remembered.

Conclusions: Overall, children were able to learn the task regardless of phone experience, indicating comparable memory skills, but varied in strategies used and time to complete the task. Therefore, while the use of phones is promising for assessment in Zambia, the child's home location is important to consider in order to differentiate measurement of the skill of interest and skills associated with the familiarity of the tool, particularly in timed activities.

Correspondence: Christina Thomas, University of Houston, 3823 Buffalo Speedway, Apt 2202, Houston, TX 77098, United States. E-mail: tinathomas39@gmail.com

E.M. TURNER, B. HERNANDEZ, S.M. GREIF, A.M. SVINGOS, B.A. BAILEY, M.W. MOORE \& S.C. HEATON. Identification of Embedded Effort Measures Using the Children's Memory Scale.

Objective: Assessment of effort throughout pediatric neuropsychological assessment is a critical indicator of test validity. Research indicates that using embedded effort measures enhances assessment of effort throughout testing, with recognition tasks more accurately indicating effort. The current study examined several potential embedded effort indices within the Delayed Recognition trial of the Children's Memory Scale Word Pairs (CMS WP-DR) by examining concurrent validity with the WISC-V Reliable Digit Span (RDS).

Participants and Methods: Archival data for the CMS WP-DR and WISC-V RDS was extracted for a diagnostically heterogeneous sample of 77 youth ages $6-16$ years $\left(M_{\text {age }}=10.88, S D=3.11\right)$ undergoing clinical neuropsychological evaluation. Candidate embedded indicators included: CMS WP-DR total raw score, individual item recognition, and consistency in recognition of repeated word pairs.

Results: Thirty two percent of the participants failed the 'gold standard' effort measure (RDS; as defined by score <7). The CMS WP-DR total raw score was not useful in discriminating between poor and adequate effort (AUC=0.6-0.74). Examination of individual items on the CMS WP-DR scale revealed significant associations between RDS and five word pairs (items $5,6,8,9,18$; Chi-Square $p<0.05$ with sensitivity range $=80-100 \%$, specificity range $=72-74 \%$, positive predictive value $(\mathrm{PPV})$ range $=13-33 \%$, and negative predictive value $(\mathrm{NPV})$ range $=96-$ $100 \%$ ). Item 5 (melt-rough word pair) appears the most robust in discriminating effort similarly to the RDS (sensitivity $=100 \%$, specificity $=74 \%$, PPV range $=2-21 \%$, NPV range $=55-100 \%$ ). Last, consis tency in recognition of the ten repeated items was not associated with RDS performance.

Conclusions: Recognition of item 5 (melt-rough) of CMS WP-DR may have clinical utility as an embedded effort measure. Future research examining the utility of this item is needed with large sample sizes and use of additional confirmatory effort measures.

Correspondence: Elise M. Turner, PHD, Clinical and Health Psychology, University of Florida, P0 Box 100165, Gainesville, FL 32610-0165, United States.E-mail: eliseturner@ufl.edu

\section{Other}

A. KISELICA, K. VOLGENEAU \& H. BELANGER. What is the Clinical and Economic Value of a Neuropsychological Assessment? A Systematic Review.

Objective: Objective: In the healthcare field, clinical neuropsychology is increasingly asked to justify its existence. Researchers have yet to summarize empirical support for neuropsychology's impact on distal outcomes. This study consists of a systematic review of literature demonstrating an effect of neuropsychological evaluations on patient outcomes (e.g., symptoms), treatment variables (e.g., adherence), and/or service delivery (e.g., treatment costs). The intent is to synthesize and analyze existing evidence of clinical neuropsychology's impact on clinical and economic outcomes and provide guidance for future studies. 
Participants and Methods: Method: The review followed PRISMA guidelines. A literature search yielded 2201 abstracts. A review of abstracts resulted in exclusion of 1926 articles that did not involve neuropsychological assessment, only included non-human subjects, or did not measure an outcome of interest. The remaining 255 entries were submitted to full-text review, resulting in selection of 9 articles that met inclusion criteria.

Results: Results: Most studies lacked methodological rigor. The most rigorous studies revealed no statistically significant effects of neuropsychological assessment on patient outcomes, such as physical health $(d=.08)$, mental health $(d=.11)$, and concussion symptoms $(-.02 £$ $d £ .15)$. However, a pre-post study suggested that receiving a neuropsychological assessment may reduce the incidence of ER visits $(-4 \%)$, the number of hospitalizations $(d=.14)$, and the length of hospitalizations $(d=.17)$, resulting in hundreds of thousands of dollars in cost savings. Conclusions: Conclusions: There is a lack of empirical data regarding the utility of neuropsychological evaluations on patient outcomes; nonetheless, there is some evidence that neuropsychological evaluations may result in healthcare cost savings. Further randomized controlled trials and longitudinal studies are needed to demonstrate that neuropsychological evaluations yield long-term clinical and economic benefits.

Correspondence: Andrew Kiselica, MA, Neuropsychology, Bruce W. Carter VA Medical Center, 1201 NW 16th Street, Miami, FL 33136, United States.E-mail: akiselica@mail.usf.edu

\section{Plenary E. Neuropsychology and Neuroimaging of Alcohol Use Disorder With and Without Korsakoff Syndrome: A Better Understanding for a Better Treatment}

Presenter: Anne Lise Pitel

$$
\text { 11:45 a.m.-12:45 p.m. }
$$

A. PITEL. Neuropsychology and neuroimaging of Alcohol Use Disorder with and without Korsakoff syndrome: a better understanding for a better treatment.

Alcohol Use Disorder (AUD) has harmful effects on brain structure and function. The Papez circuit and the frontocerebellar circuits are especially affected, resulting in impairments of episodic memory, working memory and ataxia. The severity of impairments in AUD patients lies along a continuum from mild to moderate, when in the absence of specific neurological complications, to severe, as occurs in Korsakoff syndrome (KS). In AUD without KS, neuropsychological deficits are mainly observed early in abstinence, typically when alcohol treatment is sought, required, or enforced. Yet, cognitive-behavioral approaches, psychoeducation and motivation to change drinking behavior require efficient cognitive abilities. Thus even in absence of KS, all AUD patients may not be cognitively ready or able to benefit from alcohol treatment. Alcohol-related brain dysfunction should be systematically detected to optimize cognitive and brain recovery and to enhance the chances for successful alcohol treatment outcome. In addition, some AUD patients without clinically detectable KS are at risk of developing this severe and persistent neurological complication. They could be identified based on their profile of specific cognitive deficits, brain abnormalities and thiamine level. The neuropsychological and multimodal brain imaging comparison of AUD patients with and without KS is not only clinically relevant, but also provides insight into the brain mechanisms involved in the natural history and evolution of the disease, including impending amnesia.

As a result of participation in this course, the learner will be able: -to recognize alcohol-related brain dysfunction,

-to use relevant screening tools to detect alcohol-related neuropsychological impairments,

-to identify brain dysfunction observed in AUD patients without neurological complications from those reported in Korsakoff patients, -to choose alcohol treatment options adjusted to the cognitive profile. Correspondence: Anne Lise Pitel, Inserm, Centre Cyceron, Campus Jules Horowitz Boulevard Henri Becquerel, Caen 14074, France. E-mail: pitel@cyceron.fr

\section{FRIDAY AFTERNOON, FEBRUARY 16, 2018}

\section{Lunch (On Own) \\ 12:45-1:45 p.m.}

\section{Invited Symposium 4. From Genes to Behavior in ADHD - How is the Brain Involved?}

\section{Chair: Barbara Franke}

\section{Presenters: Marta Ribases, Janita Bralten, Stephen V Faraone, Barbara Franke}

$$
\text { 1:45-3:15 p.m. }
$$

B. FRANKE, M. RIBASES, J. BRALTEN \& S. FARAONE. Attentiondeficit/hyperactivity disorder (ADHD): emerging insights into a category at the extreme of a population continuum.

ADHD is characterized by sustained, age-inappropriate inattention and/ or hyperactivity/impulsivity. ADHD has a childhood onset and often persists into adulthood. The disorder is highly heritable; its genetic component is based on the contribution of multiple genetic variants of limited effect size. Genetic factors linked to ADHD are thought to change over the lifespan, with only limited overlap between factors involved in disease onset and persistence.

Recently, the first genetic risk variants for childhood ADHD have been identified through genome-wide association studies (GWAS). As part of the International Multicenter persistent ADHD collaboration (IMpACT), we have now also performed the first GWAS of adult ADHD, and will present molecular genetics data on the genetic overlap between ADHD in childhood and adulthood.

At the phenotypic level, multiple studies have shown that ADHD forms the extreme of a distribution of behavioural traits present in the population. At the molecular genetic level, this continuum has now also been shown to exist. Opportunities arising from such findings - for ADHD as well as for other neurodevelopmental disorders - will be discussed during this symposium.

Downstream of gene-identification, it is important to map the pathways leading from genetic risk factor to disease. In this way, genetics research can contribute to the development of novel treatment approaches. As we will showcase during the symposium, such mapping should include 
the identification of molecular/cellular processes altered in patients and effects on the brain substrates of the disorder.

An important current discussion in the field of ADHD is the existence of an adult-onset form of the disorder, following several publications suggesting the occurrence of the disorder in the absence of childhood symptoms. However, it is currently unclear whether such findings represent artefacts of the assessment methods used. During the symposium, we will discuss current evidence.

Correspondence: Barbara Franke, Radboud University Medical Center and Donders Institute for Brain, Cognition and Behaviour, Geert Grooteplein 10, Nijmegen 6525GA, Netherlands. E-mail: Barbara. franke@radboudumc.nl

\section{RIBASES. Genetics of ADHD across the lifespan.}

Attention-Deficit and Hyperactivity Disorder (ADHD) is among the most heritable psychiatric disorders. Given that around $65 \%$ of children diagnosed in childhood continue to suffer from ADHD during adulthood, we aim to identify the genetic influence on the stability of ADHD symptoms that may contribute to discern between ADHD subjects with and without symptomatic persistence using the genomic data from the International Multi-centre persistent ADHD CollaboraTion (IMpACT) and iPSYCH. In an attempt to unravel novel genes underlying persistent ADHD, we conducted a meta-analysis of GWAS for adult ADHD in a total sample of 5,981 cases and 15,180 controls. None of the findings exceeded the genome-wide threshold for significance $(\mathrm{P}<5 \mathrm{e}-08)$. Top hits included genes previously associated with learning, memory deficits, impaired long-term potentiation, bipolar disorder, suicidality or autism. The SNP heritability for adult ADHD was estimated as $h 2=0.15$ $(\mathrm{S} . \mathrm{E} .=0.03)$, which is in line with previous estimates in children. The partition of SNP heritability by functional category showed nominal significant enrichment in the ADHD heritability by SNPs located in conserved regions and on specific histone marks. We also found evidence for a strong overlap in genetic risk variants between adult and children ADHD ( $\mathrm{rg}=0.105$ ( $\mathrm{SE}=0.26)$; $\mathrm{P}$-value=4.2e-05), supporting a shared genetic background. These data suggest that common genetic variation is involved in the etiology of the persistent form of the disorder and that many risk loci exert shared effects on ADHD through lifespan. We also identified genetic correlation between adult ADHD and other psychiatric disorders and traits including Major Depressive Disorder, Schizophrenia, Neuroticism, Educational Attainment, or Subjective well-being. Prospective studies of children with ADHD may discern between individuals with and without symptomatic remission and will provide more insights into the genetic influence on the stability of ADHD symptoms.

Correspondence: Marta Ribases.E-mail:marta.ribases@vhir.org

J. BRALTEN. Categories at the extreme of population distribution of behaviour - the real nature of neurodevelopmental disorders? Gene-finding in complex disorders, like ADHD, using genome-wide association analyses requires enormous sample sizes (tens of thousands), due to phenotypic heterogeneity, polygenicity, and generally small effect sizes of individual (common) genetic variants. This has severely hampered progress in understanding disorder aetiology. Additionally, cross-disorder genetic overlap shows that diagnostic categories for those disorders do not follow the underlying biology well.

There is now substantial evidence from phenotypic studies that psychiatric disorders represent the extreme ends of normal distributions of disorder-like traits within the general population. At the molecular genetic level, this continuum has now also been shown to exist. This opens up new opportunities in the field of psychiatric genetics as in can provide an easy and elegant way to increase our sample sizes tremendously.

In our lab we investigate whether there is a shared genetic etiology as well as a shared biology between psychiatric like traits within the population and clinical disorders. Subsequently we use genome-wide genetic data of population based cohorts to indentify novel genes and molecular mechanisms involved in psychiatric disorders. Finally, we test the trait-associated genetic risk factors for their influence on brain structure and brain connectivity in the world-wide largest data-sets to investigate biological pathways.

In this symposium I will present the concept and the current status of the continuum idea in psychiatric genetics. I will focus on ADHD, but also include examples of other psychiatric disorders. Using population-based analysis of disease-like traits provides a unique opportunity to help us uncover novel underlying aetiological mechanisms related to complex psychiatric disorders and their overlap. Ultimately this approach can provide a starting point for alternative ways to classify (heterogeneous) psychiatric disorders.

Correspondence: Janita Bralten.E-mail: janita.bralten@radboudumc.nl

\section{S. FARAONE. Adult onset ADHD - what is the evidence really?}

Reports from longitudinal, population studies propose a paradigmatic shift in our understanding of attention deficit hyperactivity disorder (ADHD). They conclude, not only that ADHD can onset in adulthood, but that childhood onset and adult onset ADHD may be distinct syndromes. These studies stand in contrast to two large bodies of research: longitudinal studies of clinically referred children and cross-sectional studies of clinically referred adults. The existence of adult onset ADHD is a novel idea which, if true, would have dramatic implications for clinical practice. The idea that adult and child ADHD are etiologically distinct suggests that much prior thinking about the disorder's etiology is wrong. To clarify these findings in the context of other work, these studies are reviewed to assess the strength of the evidence supporting the existence of adult onset ADHD as are the clinical studies that suggest that adult ADHD is simply the persistence of the childhood syndrome. When taking all these studies into account, the concept of adult onset ADHD as originally articulated, is found wanting. Instead, a dimensional view of ADHD that recognizes the existence of subthreshold cases seems to fit the data better.

Correspondence: Stephen V Faraone. E-mail: sfaraone@ childpsychresearch.org

\section{B. FRANKE. From genes to behaviour in ADHD - how is the brain involved?}

Despite the fact that more and more genes for ADHD are being identified through large international collaborations, we still understand relatively little about the biology of ADHD. This is due to the fact that we do not understand the mechanistic pathways leading from gene to disorder. Mapping of such pathways requires interdisciplinary research. In our lab, we use several approaches and model systems for this purpose: for the identification of the molecular/biochemical processes altered by the malfunctioning of a gene, we make use of bioinformatics approaches, leveraging the in silico resources publicly available. The cellular processes involved are investigated using induced neurons based on induced pluripotent stem cell (iPSC) technology and a novel animal model - the fruit fly Drosophila melanogaster. At the level of the brain, we make use of magnetic resonance imaging (MRI) data from large local and international cohorts. This enables us to visualize the effects of genetic variants directly on the living human brain.

During the lecture, I will present examples of our research to map the biological pathways relevant to ADHD at the different levels of organismal complexity described above. We have identified neurite outgrowth as an important cellular process altered in ADHD, and show, how monoaminergic and circadian circuits are involved in ADHD. With sufficient genetic data available, such research can lead to novel druggable targets and may provide insights that can help in the prevention of the persistence of ADHD into adulthood.

Correspondence: Barbara Franke, Radboud University Medical Center and Donders Institute for Brain, Cognition and Behaviour, Geert Grooteplein 10, Nijmegen 6525GA, Netherlands. E-mail: Barbara. franke@radboudumc.nl 


\section{Symposium 9. Transcranial direct current stimulation in older adults}

\section{Chair \& Presenter: Benjamin M. Hampstead}

\author{
Presenters: Jaclyn Reckow, \\ Annalise Rahman-Filipiak, Adam Woods
}

\author{
1:45-3:15 p.m.
}

\section{B.M. HAMPSTEAD. Transcranial direct current stimulation in older adults.}

Cognitive decline due to age and neurodegenerative disease is increasingly recognized as a threat to the international healthcare community. Thus, there is an urgent need to identify interventions that not only enhance cognition but may also delay clinical progression. Neuromodulatory techniques are being increasingly investigated as one such treatment approach given their ability to directly target brain regions and associated neural networks. This symposium will focus on transcranial direct current stimulation (tDCS) due to its potential for widespread use, with an initial overview of the methodology provided by Drs. Hampstead and Woods. In order to perform high-quality clinical trials, two fundamental aspects must be established: tolerability and effective blinding. Dr. Jaclyn Reckow will first review published tolerability and blinding literature using traditional pad-based tDCS in older adults and will then summarize data from $100+$ older adults using the more focal High Definition (HD) tDCS approach. Next, Dr. Annalise Rahman-Filipiak will use the null results from a recent HD-tDCS study to highlight vital methodological factors such as dose, montage, timing of outcome measure acquisition, and participant characteristics. The explicit discussion of such factors is rarely provided but can obviously have a profound impact on the integrity and outcome of tDCS research. The use of tDCS relies on its ability to modulate brain functioning. Thus, the final two talks will review neurophysiological effects of pad-based (Dr. Woods) and HD-tDCS (Dr. Hampstead), using both task- and resting-state functional magnetic resonance imaging. Taken together, this series of talks will both orient and inform the membership to this promising non-pharmacologic intervention.

Correspondence: Benjamin M. Hampstead, University of Michigan; VA Ann Arbor, 2101 Commonwealth Blvd Suite C, Ann Arbor, MI 48105, United States. E-mail: bhampste@med.umich.edu

J. RECKOW, A.M. RAHMAN-FILIPIAK, S. GARCIA, S. SCHLAEFFLIN, O.C. CALHOUN, A. LESNOVSKYA \& B.M. HAMPSTEAD. Tolerability and blinding of $2 \mathrm{~mA}$ versus $3 \mathrm{~mA}$ in cognitively diverse older adults receiving HD-tDCS.

Objective: Transcranial direct current stimulation (tDCS) is a non-invasive brain stimulation technique with growing research supporting its therapeutic use. While generally regarded as well tolerated and safe, comparatively little work has examined tDCS in older adults and even less is reported on High Definition (HD) tDCS. Thus, we will first provide a review of tolerability, safety, and blinding data for traditional (pad-based) tDCS. Then, we will examine these factors using our growing dataset with HD-tDCS in older adults.

Participants and Methods: A sample of 100 older adults $\left(M_{\mathrm{age}}=69.88\right.$, $\mathrm{SD}=9.48$ ) of varying diagnosis (Healthy $=64$, Amnestic MCI $[\mathrm{aMCI}]$ $=32$, Non-Amnestic MCI $[$ naMCI $]=1$, Other $=3$ ) were recruited across four HD-tDCS studies. All participants received between one and thirty 20 - to 30-minute sessions of active or sham stimulation at either $2 \mathrm{~mA}$ or $3 \mathrm{~mA}$. All participants completed a standardized side effect questionnaire and were then asked whether they received real or fake (sham) stimulation.

Results: Fisher's Exact tests revealed that active and sham groups did not differ significantly in their ratings of any side effects. Intensity $(2 \mathrm{~mA}$ and $3 \mathrm{~mA}$ ) had no significant effect on side effect profile. Similarly, there were no significant group differences in the frequency of individuals correctly stating their stimulation condition. No differences were evident when the $2 \mathrm{~mA}$ and $3 \mathrm{~mA}$ groups were considered separately.

Conclusion: Our findings are among the first to demonstrate that HD-tDCS is well-tolerated and that blinding is effective in older adults, even at $3 \mathrm{~mA}$. These data are vital for supporting the use of HD-tDCS in randomized controlled trials that will allow for rigorous evaluation of its merits as a treatment for cognitive and emotional functioning in older adults.

Correspondence: Jaclyn Reckow. E-mail: jreckow@med.umich.edu

A.M. RAHMAN-FILIPIAK, J. RECKOW \& B.M. HAMPSTEAD. Why did a single-session of 3mA HD-tDCS have no effect on object location memory or working memory in older adults? A methodological evaluation.

Objective: Transcranial direct current stimulation (tDCS) has been variably effective in enhancing cognition in older adults in prior studies. Much of this variability may be accounted for by methodological factors. This presentation will utilize the null findings from a study of high definition tDCS (HD-tDCS) over the left lateral prefrontal cortex to discuss critical study design elements and how they may drive affect study outcome.

Methods: Forty-two older adults $\left(M_{\text {age }}=66.2, S D=9.71\right)$ were randomized to a single session of active or sham anodal HD-tDCS ( $3 \mathrm{~mA}$ for 20 minutes; center anode over F5). Participants completed an object location touchscreen task (OLTT) before HD-tDCS, then encoded an alternate version of the OLTT online (i.e., concurrent with active or sham stimulation). Finally, they completed the memory portions of the OLTT and an n-back task offline ( 10 minutes post-stimulation). Results: A two-way mixed design repeated measures MANOVA revealed no significant effect of time, nor a significant interaction between time and tDCS condition for any of the OLTT measures. Likewise, there were no between-group differences on the n-back task.

Discussion: This study, the first of its kind to use $3 \mathrm{~mA}$ intensity HD-tDCS to target associative and working memory in healthy older adults, demonstrated null findings; however, it provides a framework through which several aspects of tDCS research design can be examined. We will specifically discuss the use of HD- versus traditional pad-based tDCS, dose (duration, intensity, number of sessions), selection of montage and associated brain area(s), the timing of outcome measure acquisition (i.e., online versus offline), and participant characteristics. Explicit post-mortem discussion of these factors is needed to advance the larger field of tDCS research.

Correspondence: Annalise M. Rahman-Filipiak, Ph.D., Mental Health Service, VA Ann Arbor Healthcare System, 26596 Vassar Ave., Redford, MI 48240, United States. E-mail: rahmanaam@gmail.com

A.J. WOODS, N. NISSIM, A. O'SHEA, L. RICHARDS \& R. TELLES. Functional neural mechanisms of tDCS-related working memory improvement in older adults.

Background: Decline in working memory is a central facet of the cognitive aging process. Prior research has attempted to use tDCS to enhance working memory performance in older adults with and without neurodegenerative disease, often in the context of N-back cognitive training paradigms (e.g., the ACT study). While these interventions show promise for clinical application, the neural mechanism of tDCS improvement in working memory brain systems is not well understood. The current study investigated the functional neural mechanisms of tDCS-related working memory improvements using an fMRI-tDCS N-back working memory paradigm.

Participants and Methods: Sixteen healthy older adults (mean age $=72 \pm 7$ ) completed a 2 -session within-subjects fMRI-tDCS paradigm. Active tDCS was performed over the frontal lobes at $2 \mathrm{~mA}$ for 12 min with the anode electrode at F4 and the cathode at F3. Participants underwent a 12 min N-back fMRI paradigm before, during, and after stimulation, either receiving sham or active tDCS. Sessions were separated by two weeks and order counterbalanced. Functional connectivity 
was assessed using CONN software evaluating specific changes within the working memory network (FDR .05).

Results: Functional connectivity within the working memory network was not significantly different before stimulation during active and sham visits. Compared to Sham, Active frontal tDCS significantly enhanced functional connectivity from inferior parietal lobe to right medial posterior parietal, bilateral ventrolateral prefrontal cortex, and bilateral supplementary motor area $(\mathrm{FDR}<.05)$. Effects were maintained after stimulation as well.

Discussion: Our results suggest that tDCS-related enhancement in working memory is associated with enhanced connectivity between posterior and frontal nodes in the working memory network. While stimulation was delivered to frontal areas, effects on functional connectivity extended beyond stimulated brain regions.

Correspondence: Adam J. Woods, PhD, Department of Aging and Geriatric Research, Center for Cognitive Aging and Memory, McKnight Brain Institute, University of Florida, 2004 Mowry Rd, Office 3118, Gainesville,FL32610,United States.E-mail: ajwoods@ufl.edu

B.M. HAMPSTEAD, S. MA, S. PELTIER, A. LESNOVSKAYA, S. GARCIA, A. BHAUMIK \& F.G. HILLARY. Neurophysiological effects of high-definition transcranial direct current stimulation in older controls and patients with mild cognitive impairment.

Background: The neurophysiological effects of transcranial direct current stimulation (tDCS) are largely based on motor evoked potentials acquired during a passive (resting) state. Unlike the hardwired motor system, cognition has proven more challenging to modulate with tDCS, at least partially due to the sole reliance on behavioral change as opposed to direct neurophysiological measurement. We will use functional magnetic resonance imaging (fMRI) data from two separate double-blind, cross-over studies to evaluate the neurophysiological effects of High Definition (HD) tDCS.

Methods: 54 older adults (20 with mild cognitive impairment - MCI) completed two sessions wherein they were randomized to active or sham HD-tDCS ( $2 \mathrm{~mA}$ for 20 minutes) over the superior parietal lobule (SPL; center anode at $\mathrm{Pz}$ or P2). Participants underwent both resting-state and task-based fMRI immediately after stimulation. The spatial navigation task required participants to encode a series of allocentric and egocentric environments, memory for which was evaluated after the scan session. Results: Active HD-tDCS had relatively nominal effects on resting-state connectivity at the group level. However, individual differences were evident and partially driven by the volume of the targeted cortex such that there was an inverse relationship between connectivity change and SPL volume. Task-related changes were evident in the SPL of cognitively intact older adults, suggesting a local effect. MCI patients showed increased hippocampal activation (bilaterally) to levels comparable with the cognitively intact older adults after active stimulation. However, memory test performance was unchanged by active HD-tDCS.

Discussion: Our data demonstrate that HD-tDCS can, in fact, alter neurophysiology even in the absence of a significant behavioral change. Findings add to knowledge of individual differences in tDCS response and hold promise for more extensive neurorehabilitative approaches that target cognition.

Correspondence: Benjamin M. Hampstead, University of Michigan; VA Ann Arbor, 2101 Commonwealth Blvd Suite C, Ann Arbor, MI 48105, United States. E-mail: bhampste@med.umich.edu

\section{Paper Session 10. Bipolar Disorder and Schizophrenia}

\author{
Moderator: Jimmy Choi
}

1:45-3:15 p.m.

S. RYMAN, C.J. WERTZ, N.A. SHAFF, A.B. DODD, B. STEVENS, J. LING, J. CAVANAGH, R. YEO \& A.R. MAYER. Impaired Theta Power and Connectivity During Proactive Cognitive Control in Schizophrenia.

Objective: Disrupted proactive cognitive control, a form of early selection and active goal maintenance, is hypothesized to underlie the broad cognitive deficits observed in schizophrenia patients (SP). Current research suggests the disrupted activation within and connectivity between regions of the cognitive control network contribute to these deficits, however, no study has examined the electrophysiological mechanisms.

Participants and Methods: Twenty-six SP (17 males; $34.46 \pm 8.77$ y.o.) and 28 healthy controls (HC; 16 males; $31.43 \pm 7.23$ y.o.) underwent an electroencephalogram (EEG) while performing the AX Continuous Performance Test (AX-CPT). To examine the extent of activation and level of connectivity between regions within the cognitive control network, power and intersite phase clustering (ISPC) metrics were calculated. A series of $2 \times 2$ mixed-measures analyses of variance (ANOVAs) examined both behavioral and neural measures of cognitive control. Regression analyses examined whether differences in cognitive control conditions predicted functional outcomes.

Results: SP exhibited expected general decrements in behavioral performance relative to $\mathrm{HC}$ and a selective deficit in conditions requiring proactive cognitive control. Additionally, SP exhibited selective deficits in midline theta power and connectivity during proactive cognitive control trials. Specifically, HC exhibited a significantly greater theta power for B cues relative to A cues, whereas SP exhibited no significant differences between A and B cue theta power. Only behavioral measures of proactive cognitive control predicted functional outcomes.

Conclusions: We observed evidence that low frequency midline theta oscillatory activity is selectively disrupted during proactive cognitive control in SP. The disrupted midline theta activity may reflect a failure of SP to proactively recruit cognitive control processes

Correspondence: Sephira Ryman, M.S., Semel Institute for Neuroscience and Human Behavior, University of California Los Angeles, 3121 3rd St., Santa Monica, CA 90405, United States.E-mail:sephira.ryman@ gmail.com

J.L. CRAWFORD, D.Y. SULLIVAN, A. BUCHHOLZ, A. SAWA \& D.J. SCHRETLEN. Facial Affect Recognition and Memory Impairment in Schizophrenia.

Objective: Persons with schizophrenia show impairments of (1) facial discrimination, (2) facial affect recognition, and (3) visual memory, but the relationships among them are unclear. To further investigate this, we developed a test of facial affect recognition and memory (FARM).

Participants and Methods: We administered the FARM and Brief Visuospatial Memory Test (BVMT-R) to 89 adults with schizophrenia (72\% male; $25+9$ years old) and 89 controls (69\% male; $27+8$ years old). For the FARM, participants were shown 24 unique faces and asked to choose which emotion (happy, sad, angry, or neutral) each depicted. Later, participants were shown the same 24 pictures and 24 new faces in pseudorandom order and asked to decide whether each was old or new. We recorded accuracy and response times (RT) for both tasks. Two (group) x 4 (emotion) ANOVAs were conducted for both the affect recognition and facial memory tasks, and we added BVMT-R performance as a covariate for facial memory analyses.

Results: Significant main effects $(p<0.05)$ of group and emotion were found for facial affect recognition accuracy and RT, and for facial memory accuracy but not RT. Persons with schizophrenia performed more poorly on both tasks, and happy expressions were recognized 
faster and more accurately than expressions of other emotions by both groups. No interaction effects were found until BVMT-R performance was entered as a covariate. Covarying for BVMT-R performance eliminated group differences in facial memory accuracy but not RTs and revealed a group by emotion interaction in which patients showed greater sensitivity to emotion than controls in facial memory RTs.

Conclusions: Persons with schizophrenia show greater impairment of facial affect recognition than facial memory, and group differences in facial memory are largely attributable to deficits in visual memory more generally. Accounting for the latter might unmask subtle group differences in the effect of specific emotions on facial memory.

Correspondence: Jeffrey L. Crawford, Bachelor of Arts in Psychology, Medical Psychiatry, Johns Hopkins University, 3307 Pinole Valley Road, Baltimore, MD 21231, United States. E-mail: jcrawf29@jhmi.edu

T. KARPOUZIAN, J. SWEENEY, L. RUBIN, B. CLEMENTZ, E. GERSHON, M. KESHAVAN, G. PEARLSON, C. TAMMINGA \& J. REILLY. Reduced Task-Evoked Pupillary Pesponse on an Executive Control Task in Individuals across the Psychosis Spectrum and Relatives with Elevated Psychosis Spectrum Personality Traits.

Objective: Task-evoked pupillary response (TEPR) is a measure of physiological arousal in response to cognitively demanding tasks and is related to response preparation on the antisaccade task. In healthy controls, greater TEPR occurs prior to correct antisaccade trials vs. error trials. Inhibitory errors on the antisaccade task have been established in individuals with psychotic disorders and their relatives, although the relationship between pupillary response and antisaccade performance in these groups has not yet been investigated.

Participants and Methods: Probands with schizophrenia, schizoaffective disorder, psychotic bipolar disorder $(n=431)$, their first-degree relatives $(n=419)$, and healthy controls $(n=181)$ from the Bipolar Schizophrenia Network on Intermediate Phenotype (BSNIP) study completed an antisaccade task. Percent change in pupil size during the preparatory period prior to target appearance was measured for correctly and incorrectly performed trials. TEPR measures were compared across patient and control groups, as well as between relative and control groups.

Results: Linear mixed-effects models revealed that all patient groups showed reduced TEPR compared to controls $(p<.001)$; patient groups did not differ from each other $(p s>.35)$. Changes in TEPR were not related to medication or symptoms. Non-psychotic relatives with elevated psychosis spectrum personality traits also had reduced TERP while relatives without elevated traits did not.

Conclusions: Greater pupil size in preparation for intact antisaccade performance was demonstrated in healthy controls compared to individuals across the psychosis spectrum. These findings provide evidence that reduced top-down control during the response preparation period may contribute to deficits in executive control reflected in impaired inhibitory behavioral control. Additionally, non-psychotic first-degree relatives with elevated psychosis spectrum personality traits had reduced TEPR, suggesting that this abnormality may be related to clinical risk for psychosis.

Correspondence: Tatiana Karpouzian, M.S., Psychiatry and Behavioral Sciences, Northwestern University, 710 N Lake Shore Dr, Chicago, IL 60611, United States. E-mail: t-karpouzian@northwestern.edu

E.C. ALDEN, D.J. COBIA, J. REILLY, L. WANG, K. ALPERT, J.G. CSERNANSKY \& M.J. SMITH. Verbal working memory and hippocampal surface anatomy in high and low functioning schizophrenia.

Objective: Schizophrenia (SCZ) is a severe mental illness characterized by various positive and negative symptoms, impaired cognition, poor functional outcomes, and structural brain abnormalities. Changes in subcortical gray matter structures may be a key neurobiological deficit in SCZ, particularly in the hippocampus. The hippocampus is implicated in verbal working memory $(\mathrm{vWM})$ which is known to be impaired in SCZ. Previous work has shown that $\mathrm{WWM}$ differs between high and low functioning SCZ subgroups (HF-SCZ and LF-SCZ), and may in turn be related to differences in functional outcomes among these individuals. We evaluated the relationship between hippocampal shape and vWM in HF-SCZ, LF-SCZ, and healthy controls (CON).

Participants and Methods: HF-SCZ (n=23), LF-SCZ (n=18), and CON ( $n=45$ ) underwent $3 \mathrm{~T}$ structural Magnetic Resonance Imaging. Hippocampal surface features were derived through high-dimensional brain mapping and principal component analysis. Mixed effects modeling was used to compare groups on deformation characteristics. Relationships between overall hippocampal shape and vWM were examined within each group through correlations.

Results: Mixed effect analysis showed an effect of group ( $F=3.39$. $\mathrm{p}<.05$ ); post-hoc tests revealed group differences between HF-SCZ and CON only. Pearson correlations revealed a significant relationship between hippocampal shape and $\mathrm{vWM}$ in both HF-SCZ and LF-SCZ $(\mathrm{r}=-.43, \mathrm{p}<.05$ and $\mathrm{r}=-.53, \mathrm{p}<.05$; respectively $)$ but not controls $(\mathrm{r}=.03$, $\mathrm{p}=\mathrm{N} . \mathrm{S}$.).

Conclusions: The present study demonstrated significant differences in hippocampal shape between HF-SCZ and CON. In addition, hippocampal shape accounts for variation in $\mathrm{VWM}$ performance in HF-SCZ and LF-SCZ, but not CON. This study extends prior findings of hippocampal abnormalities in SCZ to demonstrate an association with cognitive deficits but not functional ability.

Correspondence: Eva C. Alden, M.S., Dept. of Psychiatry and Behavioral Sciences, Northwestern University, 7870 NW 37th Rd, \#246, Gainesville, FL 32606, United States.E-mail: eva.alden@u.northwestern.edu

\section{B.L. CALLAHAN. Early Signs of Dementia in Bipolar Disorder.}

Objective: Epidemiologic work suggests adults with bipolar disorder (BD) may have increased dementia risk, yet studies investigating dementia prodromes systematically exclude subjects with psychiatric conditions. Thus, little is known about early signs of neurodegeneration in older BD patients. This study documents the longitudinal cognitive profile of adults with BD who develop dementia, compared to those who do not.

Participants and Methods: Participants were 114 adults with BD from the National Alzheimer's Coordinating Center. All were nondemented on admission, and underwent annual assessment of memory, language and executive abilities (mean 4.4 visits). Raw scores were Z-transformed. Twenty-six participants (22.8\%) eventually developed dementia as per DSM criteria (incipient cases). Longitudinal cognitive features associated with dementia were determined with linear mixed-effects models. Yearly differences between incipient and no-dementia cases were examined using ANCOVA, covarying for differences in $\operatorname{sex}\left(\chi^{2}=15.0, p<.001\right)$ and lithium use $\left(\chi^{2}=4.1, p=.044\right)$. Groups were matched on age $(t=-1.6, p=.108)$, education $(t=1.9, p=.054)$ and vascular risk factors $\left(\chi^{2}=0.3, p=.603\right)$.

Results: Performance on all measures was associated with incipient dementia (all models $p<.007)$. Backwards digit span $(\mathrm{F}=5.0, \mathrm{p}=.029)$, semantic fluency $(\mathrm{F}=4.6, \mathrm{p}=.036)$, Trails A $(\mathrm{F}=6.3, \mathrm{p}=.014)$ and confrontation naming $(\mathrm{F}=12.6, \mathrm{p}=.001)$ were reduced in incipient cases up to 2 years before diagnosis. Recall of a short story differed between groups up to 4 years before diagnosis $(\mathrm{F}=4.8, \mathrm{p}=.035)$. Digit symbol substitution (DSS) showed the earliest between-group differences, up to 5 years before diagnosis $(\mathrm{F}=4.5, \mathrm{p}=.046)$. A DSS Z score $<-1.5 \mathrm{SD}$ at any time was associated with $96.6 \%$ sensitivity and $46.2 \%$ specificity of future dementia.

Conclusions: Cognitive testing reveals signs of dementia in BD patients up to 5 years before diagnosis. In this study, DSS was especially sensitive to early decline, and may potentially be a cost-effective clinical screening tool.

Correspondence: Brandy L. Callahan, Department of Psychology, University of Calgary, 2500 University Drive, AD 131C, Calgary, AB T2N 1N4, Canada.E-mail: brandy:callahan@ucalgary:ca 
T.T. NGUYEN, A.N. SUTHERLAND, T. SHEKHTMAN, J. KELSOE \& L.T. EYLER. Apolipoprotein E (APOE) Genotype is Associated with Poorer Executive Function in Bipolar Disorder.

Objective: The apolipoprotein E (APOE) $\varepsilon 4$ allele is a genetic risk factor for Alzheimer's disease and may influence cognition in healthy individuals and other populations, including affective disorders. The relevance of the APOE genotype in bipolar disorder (BD) is less clear. The present study investigated the association of APOE genotype and neurocognitive function in patients with $\mathrm{BD}$.

Participants and Methods: Outpatients with BD $(n=36)$ and healthy comparison subjects (HC; $n=73$; age range: $30-79$ ) provided blood samples for genotype analysis and completed neuropsychological tests of executive function (D-KEFS Trail-Making. Color-Word Interference). We examined the relationship between APOE genotype and demographic and clinical factors. ANOVA was used to assess the relationship of diagnostic group, APOE allele status, and their interaction to cognitive performance.

Results: BD and HC groups did not significantly differ on the frequency of APOE genotypes $(p=0.39)$ or $\varepsilon 4$ allele $(p=0.63)$. HC carriers of the $\varepsilon 4$ allele had higher systolic blood pressure $(p=0.03)$, while BD carriers had higher diastolic BP $(p=0.04)$. Presence of the $\varepsilon 4$ allele interacted with diagnostic group on measures of inhibition $(F=5.19, p=0.03)$ and inhibition-switching $(F=5.04, p=0.03)$. BD $\varepsilon 4$ carriers demonstrated worse inhibitory control and cognitive flexibility compared to $\varepsilon 4$ non-carriers; no $\varepsilon 4$ relationship was in seen in HC. BD patients with the $\varepsilon 2$ allele demonstrated significantly worse performance on measures of inhibitory control and cognitive set-shifting.

Conclusions: Results suggest that the APOE $\varepsilon 4$ allele may also be a risk factor for cognitive decline in BD, particularly in the domain of executive function. This is consistent with existing research associating the APOE $\varepsilon 4$ allele with frontal cortical size. However, the finding that the APOE $\varepsilon 2$ allele, typically considered protective, was associated with worse performance on executive tasks was contrary to expectations. Additional research is needed to further investigate its role in BD.

Correspondence: Tanya T. Nguyen, Ph.D., Psychiatry, University of California, San Diego, 3350 La Jolla Village Drive, San Diego, CA 92161, United States. E-mail: ttn050@ucsd.edu

\section{Paper Session 11. MCI and Dementia}

\section{Moderator: Heidi C. Rossetti}

$$
\text { 1:45-3:15 p.m. }
$$

\section{K.R. THOMAS, E.C. EDMONDS, J. EPPIG, D. SALMON \& M.W. BONDI. Using Process Scores to Identify Subtle Cognitive Decline and Predict Progression to MCI.}

Objective: We previously operationally defined subtle cognitive decline (SCD) in preclinical Alzheimer's disease (AD) using total scores on neuropsychological (NP) tests. We also showed that memory process scores predict progression to mild cognitive impairment (MCI), even after controlling for NP total scores and AD biomarkers. We aimed to integrate memory process scores into the SCD definition to identify stages of SCD and improve sensitivity to detect those at risk for decline. Participants and Methods: Alzheimer's Disease Neuroimaging Initiative participants who did not meet NP criteria for MCI at baseline (i.e., all were "cognitively normal") were classified as either "early" SCD (>1 SD below mean on 2 process scores OR on 1 process score plus $1 \mathrm{NP}$ total score; $\mathrm{n}=150$ ), "late" SCD (our published SCD criteria of $>1$ SD below norm-adjusted mean on $2 \mathrm{NP}$ total scores in different domains; $n=110)$, or "no SCD" $(n=494)$. Process scores considered in the SCD criteria were word-list intrusion errors, retroactive interference, and learning slope. Logistic regression and survival analysis examined sensitivity/specificity and rate of progression to MCI.

Results: "Late" SCD had faster initial progression to MCI within 5 years than "early" SCD $(p<.05)$. However, over 10 years, the "early" and "late" SCD groups did not differ in rate of progression to MCI; the survival curves converged at 7-8 years. Both "early" and "late" SCD progressed to MCI faster than the "no SCD" group $(p<.001)$. When the "early" and "late" SCD groups were combined, sensitivity to detect progression to MCI more than doubled relative to the "late" SCD group only.

Conclusions: "Early" SCD participants took longer to progress to MCI than "late" SCD in the short-term, though ultimately a comparable proportion met MCI criteria over 7-8 years. Results suggest that process scores can be integrated into the definition of SCD to allow for earlier identification of cognitively normal older adults at risk for decline prior to frank impairment on NP total scores.

Correspondence: Kelsey R. Thomas, PhD, VA San Diego Healthcare System, 3350 La Jolla Village Drive (116B), San Diego, CA 92161, United States.E-mail:krt006@ucsd.edu

A.J. WEIGAND, E.C. EDMONDS, L. DELANO-WOOD \& M.W. BONDI. Inaccuracy of Subjective Cognitive Complaints in the Diagnosis of Mild Cognitive Impairment.

Objective: Subjective cognitive complaints remain an integral component of the diagnostic criteria for mild cognitive impairment (MCI), despite findings that self-reports may not accurately reflect cognitive ability. Within the Alzheimer's Disease Neuroimaging Initiative (ADNI), we investigated longitudinal change in the discrepancy between selfand informant-reported cognition across empirically-derived subtypes of MCI and normal control (NC) participants.

Participants and Methods: Data were obtained for 448 ADNI participants with MCI and 142 "robust" NC participants who maintained cognitive normality throughout the duration of the study. Our work previously classified MCI participants into three subtypes via cluster analysis: Amnestic MCI, Mixed MCI, and a "cluster derived normal" (CDN) group whose neuropsychological scores did not differ from NC participants despite their MCI diagnosis. Subjective cognitive complaints were measured using the Everyday Cognition Questionnaire, and discrepancy scores were calculated as the difference between self- and informant-report. Analysis of change in discrepancy scores over a 2-year interval was conducted using $3 \times 4$ mixed ANCOVA.

Results: Amnestic and Mixed MCI subtypes followed a trajectory of increasing discrepancy scores over time. This disparity was driven by an increase in informant-reported cognitive complaints despite a stable self-report, indicating that MCI participants consistently underestimated their cognitive problems. These findings differ from the CDN and NC groups, who over-reported cognitive difficulty at all three time points. Conclusions: MCI participants show increasing discrepancy between self- and informant-reported cognitive problems over time, likely driven by lack of awareness of progressive cognitive decline. The consistent over-reporting in the cognitively normal groups indicates that subjective complaints may not predict impending cognitive decline. These results call into question the utility of subjective cognitive complaints in the diagnosis of MCI.

Correspondence: Alexandra J. Weigand, VA San Diego Healthcare System and University of Califonira, San Diego, Mailbox 151B, 3350 La Jolla Village Dr., San Diego, CA 92161, United States. E-mail: alexjweigand@gmail.com

D.A. NATION, A. TAN, S. DUTT, E.C. MCINTOSH, B. YEW, J.K. HO, A.E. BLANKEN, J.Y. JANG, K.E. RODGERS \& A. GAUBERT. Circulating Progenitor Cells Correlate with Memory, Posterior Cortical Thickness and Hippocampal Perfusion.

Objective: Bone marrow-derived progenitor cells survey the vasculature and home to sites of tissue injury where they can promote repair and regeneration. It has been hypothesized that these cells may a play protective role in neurodegenerative and vascular cognitive impairment. We evaluated progenitor cell levels in older adults with and without mild cognitive impairment (MCI), and related circulating levels to memory, brain volume, white matter lesion volume and cerebral perfusion. 
Participants and Methods: Thirty-two older adults, free of stroke and cardiovascular disease, were recruited from the community and evaluated for diagnosis of MCI versus cognitively normal (CN). Participants underwent brain MRI and blood samples were taken to quantify progenitor reserve using flow cytometry (CD34+, CD34+CD133+ and CD34+CD133+CD309+ cells).

Results: Participants with MCI $(n=10)$ exhibited depletion of all progenitor cell markers relative to those who were $\mathrm{CN}(\mathrm{n}=22)$, after controlling for age, sex and education. Post-hoc age, sex and education matched comparisons $(\mathrm{n}=10 \mathrm{MCI}, \mathrm{n}=10 \mathrm{CN})$ also revealed the same pattern of results. Depletion of CD34+ cells correlated with memory performance, left posterior cortical thickness and bilateral hippocampal perfusion. Participants exhibited low levels of vascular risk and white matter lesion burden that did not correlate with progenitor levels.

Conclusions: Circulating progenitor cells show protective associations with cognitive impairment, memory, cortical atrophy, and hippocampal perfusion, suggesting that progenitor depletion may contribute to, or may be triggered by, cognitive decline and cortical atrophy. Further study of progenitor cell depletion in older adults may open novel therapeutic targets to prevent or delay dementia.

Correspondence: Daniel A. Nation, Ph.D., Psychology, University of Southern California, 3620 South McClintock Ave., Los Angeles, CA 92008,United States.E-mail: danation@usc.edu

E. VAKIL, S. MCDONALD, N. VARDI-SHAPIRO \& S. ALLEN. Facial Expressions Yielding Context Memory Effect: The Additive Contribution of Eye Movements.

Objective: The dissociation between the two aspects of face recognition (identity \& expression) has been well documented. This study will test context effect on face recognition by viewing facial expressions as context and face identity as the target (information to be remembered). Participants and Methods: Three groups were defined - Neutral, Happy and Angry, reflecting the facial expressions of the faces presented at the study phase. At the study phase, participants were presented with 42 color photos of faces for five seconds each. At the test phase, participants were presented with 84 pictures of faces, half of which had been viewed beforehand (old). One-third of the old and new faces displayed the same facial expression shown at study (Repeat condition), and the remaining two-thirds had one of the other two expressions (Re-pair condition). In addition to the participants' behavioral data, eye movement data were collected as well.

Results: It was found that consistency of facial expressions between study and test facilitated face recognition. Eye-tracking results showed that lengthier focus on a face at the study phase forms stronger binding between facial identity and facial expression, which gives the participant an advantage only when that exact face is presented again at the test phase. Furthermore, it was found that angry expressions intensify binding more than happy or neutral expressions, resulting in higher costs when changing facial expression between study and test.

Conclusions: Therefore stronger binding is an advantage under the Repeat condition, but it is a disadvantage under the Re-pair condition in terms of facial identification. These results are further indications of the link between facial identity and facial expression systems.

Correspondence: Eli Vakil, PhD, Psychology, Bar Ilan University, Bar Ilan University Ramat Gan, Ramat Gan 52900, Israel.E-mail: vakile@ mail.biu.ac.il

K.E. OSBORN, D. LIU, K. GIFFORD, E. MOORE, F.E. CAMBRONERO, J.E. BOGNER, C. SEABOLT, J. THOMPSON, L. WALLJASPER, S.L. LAMBROS, S.P. BELL, L. ACOSTA, T.J. HOHMAN, K. BLENNOW, H. ZETTERBERG \& A.L. JEFFERSON. Verbal Memory Performance Across Preclinical Alzheimer's Disease (AD) and Suspected Non-AD Pathophysiology Cerebrospinal Fluid Biomarker Profiles.

Objective: Verbal episodic memory impairment is an early clinical marker of Alzheimer's disease (AD). Onset often coincides with cerebrospinal fluid (CSF) tau evidence of neurodegeneration (ND), but concomitant white matter pathology may exist. This study relates a CSF axonal injury marker (neurofilament light, NFL) to the California Verbal Learning Test-II (CVLT-II) across AD and suspected non-AD pathophysiology (SNAP) biomarker profiles.

Participants and Methods: Vanderbilt Memory \& Aging Project participants free of clinical dementia and stroke $(\mathrm{n}=155,72 \pm 6$ years) who completed a lumbar puncture and neuropsychological protocol were dichotomized by $\beta$-amyloidosis $\left(A \beta_{42}\right)$ and ND (tau). Least-squares regression related NFL $(\mathrm{pg} / \mathrm{mL}) \mathrm{x}$ biomarker category $(\mathrm{A} \beta$-/ND-, $\mathrm{A} \beta$-/ND-, $\mathrm{A} \beta$-/ND+, $\mathrm{A} \beta$-/ND+) to CVLT-II, adjusting for age, sex, education, race/ethnicity, cognitive diagnosis, and $A P O E-\varepsilon 4$ status. $A \beta_{42}$ and tau were also analyzed as continuous variables.

Results: NFL predicted worse Long Delay Free Recall (LDFR; $\beta=-0.001, p=0.03)$. Biomarker category interacted with NFL for Total Learning $(\beta=-0.01, p=0.005)$, LDFR $(\beta=-0.002, p=0.048)$, and Recognition $(\beta=-0.0008, p=0.03)$, with associations only in $\mathrm{A} \beta-/ \mathrm{ND}+$ (SNAP). Tau related to all variables and $A \beta_{42}$ with LDFR, but neither interacted with NFL on any measure ( $p$-values $>0.23$ for $A \beta_{42}$, $p$-values $>0.10$ for tau). Diagnostic interactions and stratified results suggested the mild cognitive impairment (MCI) group accounted for the $\mathrm{A} \beta_{42}$ and tau associations whereas the normal cognition group drove the NFL associations. Conclusions: NFL predicted worse verbal memory in SNAP, suggesting a non-AD axonal injury pathway for early impairment. Episodic memory changes typically regarded as early cognitive markers of AD pathology may also reflect non-AD-related axonal injury in preclinical SNAP. Axonal injury may affect memory processes in an asymptomatic phase that precedes clinical symptom onset (MCI) driven especially by tau pathology.

Correspondence: Katie E. Osborn, Psy.D., Neurology, Vanderbilt Memory \& Alzheimer's Center, 1207 17th Avenue South, 2nd Floor, Suite 204, Nashville, TN 37212, United States.E-mail:katie.osborn@ vanderbilt.edu

T. SHANY-UR, A. LEE, L. NGUYEN, P. POORZAND, S.N. GROSSMAN, B. MILLER \& K.P. RANKIN. Neuroanatomy of Recognizing Realistic versus Static Emotions in Neurodegenerative Diseases.

Objective: Neurodegenerative diseases, particularly those with right temporal and/or prefrontal involvement, often impair emotion recognition. While real-life emotions are expressed in a dynamic manner, emotion recognition is traditionally studied using static visual stimuli. Our goal was to delineate the neuroanatomical correlates of realistic versus static emotion recognition in patients with neurodegenerative diseases.

Participants and Methods: The study was conducted at UCSF's Memory and Aging Center. 213 patients with neurodegenerative diseases (AD, bvFTD, svPPA, nfvPPA and PSP) and 59 older controls performed a realistic emotion recognition (by naming) task (The Awareness of Social Inference Test [TASIT] Emotion Evaluation Test; McDonald et al., 2003), and a static emotion recognition task (Comprehensive Affect Testing System [CATS] Affect Naming Subtest; Froming et al., 2006). Participants underwent structural MRI. Brain-behavior relationships were analyzed using voxel-based morphometry (VBM) on SPM12, controlling for sex, age, non-emotional semantic knowledge, TIV, and magnet strength.

Results: Patients with neurodegenerative diseases performed poorly on both tasks compared with controls, though bvFTD and svPPA patients demonstrated greater impairments. Poor performance on a realistic emotion recognition test correlated with atrophy in temporal poles, insulae, temporal gyri, amygdalae, medial temporal structures, orbital gyri, medial PFC, basal ganglia, and thalami. Poor performance on a static emotion recognition test correlated with atrophy in medial and dorsal frontal regions.

Conclusions: VBM analysis revealed striking differences between the neuroanatomical correlates of emotion recognition using realistic 
versus static stimuli. Performance on a realistic test predicted atrophy in regions involved in emotional processes, while the static test performance predicted atrophy in regions mostly involved in executive processes. Clinical implications for bedside testing are discussed.

Correspondence: Tal Shany-Ur, PhD, Psychology, The Hebrew University of Jerusalem, 14 Refidim St. Entrance 4 Apt. 2, Tel-Aviv 6998221, Israel.E-mail:talshany@gmail.com

S. DEVAUGHN, K.B. CASALETTO, A.M. STAFFARONI, G. MARX \& J. KRAMER. The Differential Cognitive and Neural Substrates of Episodic Memory Performance in Semantic Variant Primary Progressive Aphasia and Alzheimer's Disease.

Objective: Semantic variant primary progressive aphasia (svPPA) and Alzheimer's disease (AD) demonstrate verbal episodic memory deficits, but the underlying cognitive and neural mechanisms may differ. We examined the relative contributions of semantic knowledge and episodic memory, and the neural substrates of verbal episodic memory performance in svPPA and AD.

Participants and Methods: $375 \mathrm{AD}$ and 50 svPPA patients completed the CVLT-SF, Benson Figure recall, and Peabody Picture Vocabulary Test (PPVT). $86 \mathrm{AD}$ and 36 svPPA patients completed brain MRI. Adjusting for demographics, visual recall (Benson recall) and semantic knowledge (PPVT) were entered simultaneously to predict CVLT-SF delayed recall (tertiles) and recognition (d') in two separate models. In MRI models, left temporal pole (semantic memory) and hippocampus (episodic memory) were simultaneously entered, controlling for intracranial volume.

Results: Verbal delayed recall and recognition scores did not differ between svPPA and AD. Visual recall, but not semantic knowledge significantly predicted delayed verbal recall and recognition in AD. Visual recall and semantic knowledge significantly predicted verbal delayed recall in svPPA, but only semantic knowledge significantly predicted verbal recognition. In AD, left hippocampal volume correlated with verbal recall, whereas left temporal pole volume correlated with verbal recognition. Entered together, hippocampus and temporal pole were not independently associated with verbal memory in svPPA. Separately, only left temporal pole correlated with verbal memory in svPPA. Conclusions: Cognitive and structural contributions to verbal memory performance differed between AD and svPPA. A double dissociation existed: visual episodic memory predicted verbal recognition memory in $\mathrm{AD}$, but semantic knowledge predicted verbal recognition in svPPA. Temporal pole integrity plays a role in verbal memory in AD and svPPA. Unique cognitive and neural processes may underlie verbal episodic memory performance depending on neurologic syndrome.

Correspondence: Saskia DeVaughn, MS, Palo Alto University, 1748 B Fell Street, San Francisco, CA 94117, United States. E-mail: s.devaughn@paloaltou.edu

\section{Poster Session 8. Adult Neurological}

$$
\text { 1:45-3:00 p.m. }
$$

\section{Behavioral Neurology/Cerebral Lateralization/Callosal Studies}

\footnotetext{
J.S. MILler, J.J. SU, L.K. PAUL \& W.S. BROWN. Adaptive Behavior in Agenesis of the Corpus Callosum: Self and Informant Reports.

Objective: Agenesis of the corpus callosum (ACC) is a congenital brain abnormality involving neuropsychological and behavioral deficits. There is limited research on adaptive behaviors in adults with ACC and normal intelligence. Miller (2016), using the self-report Adaptive Behavioral Rating System $2^{\text {nd }}$ edition (ABAS-II), showed that adults with ACC did not report themselves to have adaptive deficits. However, anecdotal
}

reports from family members indicate that persons with ACC may have deficiencies in adaptive functioning. The current study sought to clarify adaptive function in ACC by comparing self-reports with reports from informants who know the person with ACC well.

Participants and Methods: Adults with ACC $\left(\mathrm{N}=36 ; \mathrm{M}_{\text {age }}=33.25\right)$ with normal IQ $\left(M_{I Q}=99.11\right)$, as well as informants, completed the ABAS-II. Self-report and informant report scores were compared to age-standardized norms using one-way t-tests. Patient-informant comparisons were conducted using a multivariate analysis of variance (MANOVA) for the evaluation of skill areas, and independent t-tests for composite scores.

Results: ACC self-report scores did not differ significantly from age-adjusted norms, indicating they perceive their adaptive abilities to be similar to neurotypicals. Informant ratings were significantly below norms for all ABAS-II composites $(p<.01)$ and most skill-areas: communication, functional academics, home living, leisure, self-care, self-direction, and work $(p<.05)$. Patient-informant ratings were different in the following areas: health and safety, leisure, and social functioning $(p<.05)$, as well as the social composite $(p<.05)$.

Conclusions: Findings were consistent with prior research revealing that persons with ACC perceive their adaptive skills as similar to neurotypicals. Conversely, informant reports suggested that persons with ACC have deficits or inefficiencies in adaptive functioning of which they were generally unaware. Future item analyses may help parse out more specific domains of adaptive deficits.

Correspondence: Justin S. Miller, MA, Graduate Psychology, Fuller Theological Seminary, 765 E California Blvd, Pasadena, CA 91106, United States.E-mail: justin.s.miller@me.com

G. STEFANATOS, R.M. BORSCHELL \& R.M. HAJ. Fused Dichotic Word test: A comparison of standard vs computer-mediated adminstration.

Objective: The Fused Dichotic Word test (FDWT) is one of the most highly regarded dichotic listening tests used for the noninvasive assessment of cerebral lateralization of language. In its standard administration, listeners are presented with dichotically presented pairs of words and are asked to circle the word on a response form (4-Alternative Forced Choice) corresponding to their percept. In this study, we compared the results obtained with this traditional written administration to findings from a computer-mediated version we developed (FDWTc).

Participants and Methods: Fifty-eight right-handed, native Englishspeaking participants were administered the FDWT in two sessions separated by a week. Participants were split into 4 groups: groups 1 and 4 were administered the standard or the computerized protocols on both occassions (FDWT-FDWT and FDWTc-FDWTc, respectively). Groups 2 and 3 adminstered alternating forms (FDWT-FDWTc and FDWTc-FDWT, respectively)

Results: The results showed a marked reduction of the expected right ear advantage (REA) when using the automated version. In Group: 1, $85 \%$ of individuals showed a laterality index indicating left hemisphere dominance. Despite, comparable findings in behavioral adminstration in the remaing groups, the REA was substantially reduced in frequency in the computer-administered sessions in the same individuals.

Conclusions: These findings suggest that the standard and computerized forms of the FWDT are not equivalent. Caution is therefore warranted in using a computerized version of the FDWT in the assessment of cerebral dominance for language. Reasons .for these differences in the findings are discussed.

Correspondence: Gerry Stefanatos, D.Phil., Communication Sciences \& Disorders, Temple University, 1701 N. 13th St., Philadelphia, PA 19122, United States. E-mail: stefang@temple.edu 


\section{Epilepsy/Seizures}

W.A. ALVERSON, J.M. GRABYAN, C.B. DUNN, D.P. SHEPPARD, J.M. STINSON \& R. COLLINS. Interrelations Among Cognition, Symptom Validity, Depression, and Self-Reported Disability.

Objective: Disability is often assessed by self-report measures, such as the World Health Organization Disability Assessment Schedule 2.0 (WHODAS 2.0). Such self-report instruments are marginally related to objective cognitive functioning, and more strongly related to depression and psychiatric symptom severity. Studies examining these relationships have not accounted for symptom validity, an important consideration in disability evaluations.

Participants and Methods: Participants included 285 Veterans who underwent evaluation for seizure disorders on an epilepsy monitoring unit. Structural equation modeling approaches were used to examine the extent to which depression, cognition, and symptom validity predicted self-reported disability.

Results: Across models, depression was the strongest predictor of self-reported disability $(\beta$ range $=[.65, .71])$, accounting for almost all of the variance explained $\left(\mathrm{R}^{2}\right.$ range $\left.=[.45, .52]\right)$. There was no evidence of a moderating effect of symptom validity on the relationship between objective cognitive performance and self-reported disability. Models which excluded depression as a predictor explained significantly less variance $\left(R^{2}=.20\right)$, and revealed that symptom validity is moderately predictive of self-reported disability.

Conclusions: The study added to a growing literature on the utility of self-reported disability by providing further evidence that objective cognitive performance is not associated with perceived disability, whereas levels of depression, and to a lesser extent, the tendency to report infrequent symptoms, are more strongly related. Self-reported disability should therefore be interpreted with caution, and in the context of psychiatric factors.

Correspondence: William A. Alverson, Ph.D., Psychology, South Texas Veterans Health Care System, 14811 Huebner Rd, Apt 2129, San Antonio, TX 78231, United States. E-mail:waalverson@gmail.com

R. COLLINS, W.A. ALVERSON, J.M. GRABYAN, C.B. DUNN, D.P. SHEPPARD \& J.M. STINSON. WHODAS 2.0 and Symptom Validity in an Inpatient Seizure Disorder Sample.

Objective: The World Health Organization Disability Assessment Schedule 2.0 (WHODAS 2.0) is a self-report measure of functional limitations. Thus far, it has not been well-studied in patients with seizure disorders including epilepsy and psychogenic nonepileptic events (PNEE). The present study also aims to contribute to a better understanding of WHODAS 2.0 as it relates to symptom validity concerns. Participants and Methods: The present study included data collected from 285 Veterans residing within the catchment area of the Michael E. DeBakey VA Medical Center, who underwent evaluation on the longterm epilepsy monitoring unit (EMU). Patients completed a number of self-report measures including WHODAS 2.0, Minnesota Multiphasic Personality Inventory 2, Restructured Form (MMPI-2-RF), and measures of depression. Sample characteristics are reported. Group differences in item endorsement between those who scored above and below cutoffs on the Infrequent Somatic Complaints scale (Fs) of MMPI2-RF were investigated.

Results: Patients who scored above the recommended cutoff on the Fs scale endorsed significantly greater symptomatology across self-report measures, including reporting greater disability on WHODAS 2.0. Proportionally more subjects with PNEE scored above the recommended cutoff on Fs, compared to subjects with epilepsy. A diagnosis of PNEE was also associated with greater endorsement on self-report measures of depression, as well as disability.

Conclusions: Findings were generally consistent with expectations that apparent exaggeration of symptoms, as assessed by scores above recommended cutoff on the Fs scale, is associated with greater disability reporting on WHODAS 2.0. Significantly higher levels of disability reported by patients with PNEE compared to those with epilepsy is also consistent with previous studies. Results contribute to a growing literature on the relative influence of diagnosis and symptom exaggeration on disability endorsement.

Correspondence: William A. Alverson, Ph.D., Psychology, South Texas Veterans Health Care System, 14811 Huebner Rd, Apt 2129, San Antonio, TX 78231, United States. E-mail: waalverson@gmail.com

S. SAGHAFI, L. FERGUSON, O. HOGUE, J.M. GALES, R.A. PRAYSON \& R.M. BUSCH. ILAE Hippocampal Sclerosis Subtype is Related to Verbal Memory Performance in Temporal Lobe Epilepsy.

Objective: In 2013, the International League Against Epilepsy (ILAE) proposed a classification system for hippocampal sclerosis (HS) based on location and extent of hippocampal neuron loss (Blumcke et al., Epilepsia, 2013): Type1a=cell loss CA1>CA4, CA3 (sparing CA2), Type $1 \mathrm{~b}=$ substantial cell loss CA1-CA4, and Type $2=$ cell loss predominantly CA1. HS has long been associated with reduced episodic memory; however, little is known about the clinical relevance of the new ILAE classification system as it pertains to memory. This study examined differences in memory performance as a function of HS ILAE classification in a large sample of patients with temporal lobe epilepsy.

Participants and Methods: 213 patients with pharmacoresistant epilepsy underwent anterior temporal lobe resections and had histopathological evidence of HS (Type $1 \mathrm{a}=92$; Type $1 \mathrm{~b}=103$, Type $2=18$ ). ANOVAs and chi-squares examined group differences in demographic and disease characteristics. Differences in preoperative memory performance on the Auditory and Visual Indices of the Wechsler Memory Scale- $3^{\text {rd }}$ Edition were examined using simple linear regressions. Fisher's exact tests examined group differences in the proportion of patients with memory scores classified as intact (SS $>85)$, moderately impaired ( $\mathrm{SS}=71-85)$, or severely impaired $(\mathrm{SS} \leq 70)$.

Results: Group differences were seen on Auditory Immediate $(\mathrm{F}=3.4$, $\mathrm{p}=.037)$ and Delayed Indices $(\mathrm{F}=3.2, \mathrm{p}=.045)$; patients with HS Type 2 had higher scores than those with HS Type 1a (both indices) and HS Type $1 b$ (immediate only). There were no differences in visual memory performance as a function of HS type and no differences in the proportion of patients who demonstrated moderately or severely impaired performance.

Conclusions: Patients with hippocampal cell loss predominantly restricted to the CA1 region perform better on verbal memory tasks than those with more widespread cell loss. This is consistent with preliminary data in the literature and suggests HS ILAE classification may have clinical relevance for episodic memory.

Correspondence: Robyn M. Busch, Ph.D., Epilepsy Center, Cleveland Clinic, 9500 Euclid Avenue, P57, Cleveland, OH 44111, United States. E-mail:buschr@ccf.org

T.J. EHRLICH, V. UTTARWAR, S. HARTMAN, A. REYES, Y.A. CHANG, K. MATHUR, B. PAUL \& C. MCDONALD. The Role of Executive Functioning in Quality of Life for Adults with Treatment-Resistant Temporal Lobe Epilepsy.

Objective: This study sought to examine the contribution of depression, executive functioning (EF), and mesial temporal sclerosis (MTS) to quality of life (QoL) in patients with temporal lobe epilepsy (TLE). Participants and Methods: Forty-eight patients with treatment-resistant TLE (22 with MTS) were administered the Quality of Life in Epilepsy-31 (QOLIE-31), Beck Depression Inventory-II (BDI-II), the Trail Making Test-Part B (TMT-B) and the D-KEFS Color-Word Interference Test-Inhibition (CWIT). A composite score was calculated using the two EF measures. Hierarchical regression was used to evaluate the unique contributions to QOLIE-31 scores (total and subscales) of the BDI-II score, EF composite, MTS status, and the interaction between EF (composite score) and MTS.

Results: When only main effects were explored, higher BDI-II scores were associated with poorer total QoL $(=-.697, \mathrm{p}<.001)$, whereas neither MTS status $(=.157, \mathrm{p}>.05)$ nor EF $(=.082, \mathrm{p}>.05)$ 
significantly contributed to the model. When the interaction variable was added in a second block, there was a marginally significant increase in variance explained $\left(R^{2}\right.$ change $\left.=.054\right)$, with a significant EF by MTS interaction $(=.567, \mathrm{p}=.024)$. This interaction revealed that poorer $\mathrm{EF}$ was associated with poorer QoL, but only in patients with MTS. This pattern was most pronounced on the Social Functioning Subscale of the QOLIE-31. Neither age of seizure onset nor differences in EF explained the significance of the interaction between MTS and EF for QoL.

Conclusions: Depression is known to be a strong predictor of QoL in treatment-resistant TLE. However, our findings demonstrate that EF is also an important factor in QoL for patients with MTS, particularly with regard to social well-being. These findings suggest that a combination of biological and neuropsychological characteristics contribute to QoL in the syndrome of TLE with MTS that should be considered when planning psychosocial interventions for these patients.

Correspondence: Tobin J. Ehrlich, M.S., Palo Alto University, 1103 SB Street, San Mateo, CA 94401, United States. E-mail: tehrlich@ paloaltou.edu

K.H. ELVERMAN, Z. RESCH, E.E. QUASNEY, D. SABSEVITZ, J. BINDER \& S. SWANSON. A Cluster Analytic Approach to Exploring Cognitive Phenotypes in Temporal Lobe Epilepsy.

Objective: Neuropsychological assessment is critical for understanding the impact of seizures on cognition and informing treatment decisions. While focus is often placed on examining groups based on seizure type/ epilepsy syndrome, an alternate approach emphasizes empirically derived groups based solely on cognitive performance (Hermann et al., 2007). The current study sought to replicate prior work and identify cognitive phenotypes in a separate, larger cohort of temporal lobe epilepsy (TLE) patients.

Participants and Methods: Participants included 185 TLE patients (Mage=37.9, Medu=13.0, 57\% female, 88\% white). Baseline neuropsychological testing was used to compute Intelligence, Language, Visuoperception, Immediate Memory, Delayed Memory, Executive Function (EF), and Speed domains. Cluster analysis of the domains was conducted to identify groups, which were compared on demographic and clinical variables.

Results: Cluster analysis revealed 3- and 4-cluster solutions. The 3 -cluster solution produced groups with a graded level of performance across domains. The 4-cluster solution produced Globally Low (GL), Globally High (GH), Low EF/Speed (Low EF/S), and Low Language/ Memory (Low L/M) groups. The GH group had more education than the GL group and the Low EF/S group was older than the Low L/M group. The GL and Low L/M groups had an earlier age of recurrent seizures than the Low EF/S group $(p<.01 ; p=.04)$. A trend $(p=.06)$ toward earlier age of onset was seen in the GL versus Low EF/S group. The GL group was less left lateralized for language (Wada) than the Low EF/S $(p<.01)$ and GH $(p=.02)$ groups and not different from the Low L/M group $(p=.49)$.

Conclusions: Two clinically meaningful cluster solutions emerged, with a 4-cluster solution producing groups that varied by overall level of cognitive performance and specific domains of weakness (EF/Speed, Language/Memory). Current results vary somewhat from past findings (w/ a smaller $\mathrm{n}$ ), which favored a 3-cluster solution, but also revealed groups with memory and $\mathrm{EF} /$ speed weaknesses.

Correspondence: Kathleen H. Elverman, Ph.D., Neurology, Medical College of Wisconsin, $8701 \mathrm{~W}$ Watertown Plank Rd, Milwaukee, WI 53226, United States. E-mail: khelverman@gmail.com

E.J. FANTO, L. SEPETA, X. YOU, E.M. BOUTZOUKAS, W. GAILLARD \& M. BERL. The relationship of language and explict memory in pediatric epilepsy.

Objective: Memory and language are often at risk in pediatric epilepsy but findings are mixed. We investigated the relationship between explicit memory and language in children with epilepsy and controls using data-driven statistical methods applying model-based clustering to explore if different cognitive profiles emerge.

Participants and Methods: 276 children ages 6.1 to 17.5 were evaluated as part of ongoing studies. 149 patients with focal epilepsy (EPI) and 127 typically developing children (TD) completed language (EOWPVT, WASI VIQ) and memory (CVLT-C) measures. Six normative $z$-scores were used ( 4 from CVLT-C) for: (1) Pearson r correlations and (2) the model-based clustering procedure. For the latter, we applied Gaussian finite mixture model-based clustering on the 6 scores through $R$ package mclust using Bayes Information Criterion to determine if scores clustered into distinct profiles and how EPI and TD fell in each profile. Epilepsy variables were compared to ascertain if they related to group membership.

Results: Language scores were positively correlated with all memory scores $(r$ 's $>0.26, p$ 's $\leq 0.001)$. The optimal cluster number was 2 across TD and EPI; language and memory separated into high $(\mathrm{n}=206$, $\mathrm{Mz}=0.44, \mathrm{EPI}=46 \%, \mathrm{TD}=54 \%)$ and low performance groups $(\mathrm{n}=70$, $\mathrm{M} z=-1.04, \mathrm{EPI}=77 \%, \mathrm{TD}=23 \%$ ). The groups did not differ on age. Within EPI only, 2 was also the optimal number of clusters. Even though the majority of the low group was EPI, it represented only $1 / 3$ of all patients. Epilepsy variables were no different across groups.

Conclusions: Language and memory are associated and when included in model-based clustering two distinct groups were revealed. The method found heterogeneity within EPI that may explain prior mixed results. Parsing groups in a data driven manner may reveal subgroups that may have biomarkers for their cognitive profiles thus indicating a protective factor or a risk. Further studies will test for biomarkers (e.g. findings from structural or functional imaging) that predict these cognitive profiles.

Correspondence: Eleanor J. Fanto, B.A., Epilepsy and Neurophysiology, Children's National Medical Center, 2101 16th St. NW, Apartment 226, Washington, NY 20009, United States. E-mail: efanto@ childrensnational.org

\section{M.M. FIUMEDORA, M. FISCHER, L. TURKELSON, C. WILKISON \& P. SHEAR. Self-Report and Objective Memory Deficits in Temporal Lobe Epilepsy.}

Objective: In contrast to the left temporal lobe's well-documented role in verbal memory, the functions of the right temporal lobe are not consistently captured by neuropsychological tests or self-report measures. It is thought that the right hippocampus is involved in memory for spatial information, but it is unknown whether spatial memory loss coincides with a subjective experience of route-finding difficulty. The objective of this study is to compare subjective spatial memory complaints and performance on visuospatial memory tasks in patients with left and right temporal lobe epilepsy (TLE).

Participants and Methods: Neuropsychological data were examined from 37 presurgical patients with video-EEG-confirmed TLE (26 left TLE; 11 right TLE; mean age 42+12.41). Subjective cognitive complaints recorded during the clinical interview were coded and compared between left and right TLE patients, together with 3 objective measures of visuospatial memory (Brief Visuospatial Memory Test-Revised [BVMT-R], Total Score; and Wechsler Memory Scale-IV, Designs I Content and Spatial Indexes).

Results: Right TLE patients performed significantly more poorly than left TLE patients on the BVMT-R and Designs I Content ( $p<.025$ for all comparisons). Subjective difficulty with route finding was frequently endorsed among all patients (37.83\% of the sample), independent of seizure lateralization. Visuospatial memory test performance did not differ significantly between those who did and did not endorse routefinding difficulty in everyday life.

Conclusions: As expected, patients with right (versus left) TLE perform more poorly on visuospatial memory tasks. Although prior work has shown that right TLE patients are deficient on tests that simulate route-finding (e.g., mazes), subjective cognitive complaints related to route-finding were not found to be specific to right TLE and were not 
associated with any objective visuospatial memory score. Future work needs to clarify the ecologic implications of right temporal dysfunction. Correspondence: Marianne M. Fiumedora, University of Cincinnati, 2007 Freeland Ave, Cincinnati, OH 45208, United States. E-mail: fiumedmm@mail.uc.edu

\section{A. GRANT, C. STACHNIAK, L. HARKER \& G. ARAUJO. Memory and Executive Functions in Children with Frontal and Temporal Lobe Seizures.}

Objective: This study investigated whether children with epilepsy, with seizures originating in either the frontal or temporal lobe, show distinctive deficits in executive and memory functions. We hypothesized that children with frontal and temporal lobe seizures would show selective deficits in executive and memory skills, respectively.

Participants and Methods: Participants were 98 children with epilepsy ranging from 7-16 years of age. Forty three participants (44\%) had seizures originating in the frontal lobe and $55(56 \%)$ had seizures originating in the temporal lobe based on EEG. All participants completed standardized tests of cognitive ability as part of a clinical neuropsychological evaluation. Executive and memory functions were assessed using scores from the Behavior Rating Inventory of Executive Function, Delis-Kaplan Executive Function System, Wechsler Intelligence Scale for Children, Children's Memory Scale, California Verbal Learning Test-Children's Version, and Developmental Neuropsychological Assessment-Second Edition.

Results: T-tests for single sample were used to compare patients with epilepsy to normative data. Profile analyses were used to examine test scores between children with frontal and temporal lobe seizures. Multiple analyses were corrected for using the False Discovery Rate. Children with frontal and temporal lobe seizures both performed worse on tests of working memory, shifting, and visual memory $(p<.05)$ compared to normative data. However, children with frontal and temporal lobe seizures did not show any differences in scores on measures of executive or memory functions.

Conclusions: Our results are relatively consistent with prior research comparing executive and memory functions across children with frontal and temporal lobe epilepsies. Taken together, these findings have implications for clinical practice, and suggest that memory and executive skills should be thoroughly assessed in patients with epilepsy, regardless of the site of seizure origin.

Correspondence: Alexandra Grant, Saint Louis University, 2208 South 12th Street Apt A, Saint Louis, MO 63104, United States. E-mail: grantaf@slu.edu

\section{N. KATZ, K. COULEHAN, M. CORNWELL, S. MANDELBAUM, V. GUZMAN, L. LANGAN, K. RAM-JUNNARKAR, L. MARCUSE, S. GHATAN \& H.A. BENDER. Neurocognitive Functioning Pre and Post Surgery in a Male Adult with Epilepsy Secondary to Gun Shot Wound: A Case Study.}

Objective: Surgical resections are increasingly employed as treatment for intractable seizures. As such, surgical intervention for refractory post-traumatic epilepsy (PTE) is increasingly more common. However, minimal literature exists on post-surgical neurocognitive outcomes following resection in patients with refractory PTE secondary to penetrating cerebral gunshot wounds (GSW). We describe a serial assessment case study of PTE secondary to GSW in a police officer shot during the line of duty.

Participants and Methods: Initial surgeries following GSW included shrapnel removal and CSF leak repairs. Pre-surgical subjective cognitive complaints included reduced verbal fluency, anomia, short term memory difficulties, and diminished task vigilance. The patient underwent EEG, invasive monitoring, and brain imaging prior to surgical intervention. Serial neuropsychological assessments were conducted using the RBANS at eight months pre-surgical, two weeks post-surgical, and three weeks post-surgical. Cutoff for reliable change indices (RCIs) from pre to post assessment data was set at 1.96 .

Results: Significant declines in immediate memory, language and attention were seen from pre-surgery to three-weeks post-surgery. However, immediate and delayed memory significantly improved from two weeks to three weeks post-surgery. Remaining areas of cognition were stable. Qualitatively, the patient was more alert to tasks at three weeks compared to two weeks post-surgery.

Conclusions: We examined pre-post-surgery cognitive functioning in an adult with medically refractory PTE secondary to a cerebral GSW. Cognitive recovery was observed for memory in as little as three weeks post-surgical resection. Early serial assessment following surgical resection is needed to determine at what rate cognitive recovery to baseline occurs

Correspondence: Nicole Katz, M.S., Neurology, Mount Sinai, 38 hill lane,Roslyn heights, NY 11577, United States.E-mail:nicole.g.katz@ gmail.com

K. KHURSHID, P. RUPERT, N.L. MINNITI, M.A. CARSWELL, D. MECHANIC-HAMILTON, V. KAMATH, R.L. DOTY, P.J. MOBERG \& D.R. ROALF. Olfactory Dysfunction in Epilepsy. Objective: Epileptic patients often report olfactory abnormalities. Clinically, some patients with epilepsy report olfactory auras prior to seizures. While abnormalities in olfactory functioning in epilepsy are well documented, there is mixed evidence of the effects of epilepsy on specific domains of olfaction. Thus, we aimed to complete a comprehensive meta-analysis to investigate the influence of epilepsy on olfactory performance across several olfactory domains.

Participants and Methods: A meta-analytic study was completed by reviewing studies investigating epilepsy and olfaction. Twenty-two studies (117 effects) were identified, encompassing a total of 882 epilepsy patients and 760 healthy controls.

Results: Analysis of effect sizes across olfactory domains and demographic characteristics for the epilepsy sample indicated a large overall effect size $(\mathrm{k}=117, \mathrm{~d}=-1.01,95 \% \mathrm{CI}=-1.16<\delta<-0.87)$ that was significantly heterogeneous $\left(Q_{B}[116]=608.01, p<1.0 \times 10^{-4}\right)$. Individuals with epilepsy demonstrated the greatest deficits in odor identification $(\mathrm{d}=-1.37)$, followed by odor memory $(\mathrm{d}=-1.073)$, odor discrimination $(\mathrm{d}=-1.039)$ and odor detection threshold $(\mathrm{d}=-0.37)$. Patients with temporal lobe epilepsy (TLE) and mixed frontal epilepsy (M-F) demonstrated greater olfactory impairment compared to those with mixed epilepsy (MIX) ( $<<1.0 \times 10^{-4}$ and $p=0.024$, respectively). The effect of surgery status on olfaction was nominally significant with postsurgical patients having weaker performance than nonsurgical patients $(p=0.086)$. Additional moderator variables were also explored. Conclusions: These findings have important clinical implications, as neuropsvchological evaluations assessing olfactory functioning mav provide greater insight into the effects seizure disorder on patients' olfaction and cognition. Olfactory testing may be useful in localizing and/or lateralizing the epileptogenic area for presurgical purposes.

Correspondence: Kiran Khurshid, M.A., Psychology, St. John's University, 34 Park St, New Haven, CT 06519, United States. E-mail: kiran.khurshid@yale.edu

\section{H. LOBLEIN, R. MCLAUGHLIN \& J.B. TITUS. The Role of Stigma} in Depression and Anxiety in Youth with Epilepsy.

Objective: Youth with epilepsy experience anxiety and depression at higher rates than healthy controls. The etiology of anxiety and depression in pediatric epilepsy is multifactorial, but likely includes biological, psychosocial, and familial factors. While perceptions of stigma are a concern for youth with epilepsy, to date there is limited research that examines the role of parental perceptions of stigma on anxiety and depression.

Participants and Methods: The present study included 94 patients (45 male) with epilepsy (age 2-18 years). Epilepsy variables were collected 
via medical chart review. Emotional functioning was assessed with the BASC-2 or BASC-3. Parental perception of stigma was assessed with the Epilepsy Stigma Scale (Austin et al., 2004), and health-related quality of life was assessed with the QOLCE.

Results: A high rate of at-risk or clinically significant features of anxiety and depression were endorsed by parents $(28.7 \%$ and $24.5 \%$, respectively), and $13.8 \%$ of youth exhibited elevated features of both anxiety and depression. Separate linear regressions were calculated to predict anxiety and depressive features based on IQ, age, seizure frequency, and parent perception of stigma. Variance in depression features was found to be uniquely accounted for by stigma ratings $(F(4,66)=4.528$, $p<0.01)$. Anxiety features were also found to be uniquely accounted for by stigma ratings $(\mathrm{F}(4,66)=2.991, p<0.05)$. Features of anxiety and depression were also indirectly correlated with quality of life.

Conclusions: The current work demonstrates the significant role stigma plays on anxiety and depression features in youth with epilepsy. Beyond cognitive and seizure-specific factors, social stigma uniquely predicted anxiety and depression features on the BASC. Moreover, our findings indicated that stigma in epilepsy is indirectly related to health-related quality of life. The implications of these findings for treatment planning and identifying risk factors for psychopathology in youth with epilepsy will be discussed.

Correspondence: Hayley Loblein, The University of Texas at Austin, 1912 Speedway, Austin, TX 78712, United States. E-mail: hayley. loblein@gmail.com

\section{A. LUCCHETTI, T. OWENS, M. ZACCARIELLO, M. TRENERRY, E. WIRRELL \& J. FIELDS. Using Epilepsy Risk Factors to Predict IQ in Pediatric Patients.}

Objective: Childhood epilepsy is a common neurological condition with a range of cognitive outcomes. Risk factors for comorbid cognitive and psychiatric problems include age at seizure onset, status epilepticus, intractability, abnormal EEG, and abnormal neurology exam (Berg et al., 2012, Kim \& Ko, 2016). The purpose of this study was to investigate if and which epilepsy risk factors predict IQ in pediatric patients with epilepsy.

Participants and Methods: Participants included pediatric patients ( $\mathrm{N}$ $=51,2-18$ years) with a neurologist-confirmed diagnosis of epilepsy who were referred for a neuropsychological evaluation at a large multidisciplinary medical center. Epilepsy risk factors mentioned above were included. Outcome variables included Full Scale IQ (FSIQ) and composites on the Wechsler intelligence measures (Wechsler Intelligence Scale for Children, $4^{\text {th }}$ Edition [WISC-IV]; Wechsler Adult Intelligence Scale, 4th edition [WAIS-IV]; Verbal Comprehension [VCI], Perceptual Reasoning [PRI], Working Memory [WMI], and Processing Speed Indices [PSI]; FSIQ).

Results: FSIQ ranged from impaired to superior (range $=48-123)$. Abnormal neurology exam significantly predicted FSIQ $(\beta=-.64$, $p=.02)$, VCI $(\beta=-.62, p=.003)$, PRI $(\beta=-.52, p=.014)$, and PSI $(\beta=-.49, p=.008)$. EEG dysrhythmia grade added further predictive power to PSI $(\beta=-.42, p=.02)$. Intractability significantly predicted WMI $(\beta=-.56, p=.007)$.

Conclusions: Given limited access to a neuropsychologist in many areas, identifying epilepsy patients at highest risk for weaknesses in cognition is critical. Abnormal neurology exam during the clinic visit was the most predictive medical variable of those examined, yielding important clinical information for referrals. Abnormal EEG at the time of evaluation and history of intractability were also significant risk factors. Future directions include identifying protective factors in pediatric epilepsy.

Correspondence: Amanda Lucchetti, MA, Psychiatry and Behavioral Sciences, Children's Hospital Colorado, 5100 Leetsdale Drive, Unit 401, Denver, CO 80246, United States.E-mail:amandalucchetti@gmail.com
M. MAIMAN, K. COULEHAN, E. LEAFFER, M. VASSERMAN \& W.S. MACALLISTER. Does Executive Dysfunction Predict Behavioral Problems in Children with Focal Epilepsy?

Objective: Children with epilepsy frequently display executive functioning (EF) and behavioral problems. Prior work has shown that poor executive functioning is related to behavior problems within generalized pediatric epilepsy. However, few studies have examined this relationship in focal epilepsy, and none have used the Child Behavior Checklist (CBCL). This study aims to explore how parent-reported EF on the Behavioral Rating Inventory of Executive Function (BRIEF) is related to behavioral problems on the CBCL in children with focal epilepsy.

Participants and Methods: Parents of 98 children with focal epilepsy $(M=10.48$ years; $S D=3.63)$ completed the BRIEF and CBCL. Two-stage hierarchical regressions $(\alpha=0.01)$ were conducted with CBCL index scores as the dependent variables. Stage one controlled for epilepsy severity factors (age of seizure onset, number of anti-epileptic medications). BRIEF index scores were entered at stage two.

Results: Epilepsy severity variables did not contribute significantly to any of the indices on the CBCL. Adding BRIEF index scores contributed significantly to total problems $(F(5,92)=30.64, p<0.01)$, externalizing problems $(F(5,92)=26.94, p<0.01)$, and internalizing problems $(F(5,92)=12.70, p<0.01)$, with the global executive composite (GEC) being the only significant predictor in the model. However, GEC only predicted total problems $(\mathrm{t}=2.76, p<0.01)$ and externalizing problems $(\mathrm{t}=3.29, p<0.01)$, but not internalizing problems. Additionally, GEC only accounted for a small percentage of the variance in scores for both total problems $(3 \%)$ and $(5 \%)$ externalizing problems.

Conclusions: Poor global EF contributes to increased rates of psychopathology in children with focal epilepsy. However, in contrast to generalized epilepsy, it does not appear to play as strong of a role in focal epilepsy. Still, intervention efforts focused on improving behavioral problems in children with epilepsy may want to target executive difficulties in this population.

Correspondence: Moshe Maiman, B.A., Psychology, Drexel University, 2040 Market St, \#802, Philadelphia, PA 19103, United States. E-mail: mainman89@gmail.com

M. MAIMAN, K. COULEHAN, E. LEAFFER, M. VASSERMAN \& W.S. MACALLISTER. Does Working Memory Predict Math and Reading Skills in Children with Epilepsy?

Objective: Deficits in working memory (WM) have been associated with poorer academic achievement. However, this has not been examined in children with epilepsy. This study aims to explore whether objective WM and parent-reported WM is related to math and reading skills, controlling for intellectual functioning, in pediatric epilepsy.

Participants and Methods: Ninety-two children with epilepsy ( $M=9.34$ years; $S D=3.01$ ) completed the Wechsler Individual Achievement Test Third Edition (WIAT-III) and the backward digit span subtest of the Wechsler Intelligence Scale for Children - Fourth Edition (WISC-IV). Parents completed the WM scale on the Behavior Rating Inventory of Executive Functioning (BRIEF). Two-stage hierarchical regressions $(\alpha=0.01)$ were conducted with WIAT-III subtests (i.e., reading comprehension, numerical operations, and math problem-solving) as the dependent variables. Stage one controlled for intellectual functioning, as assessed by the general abilities index of the WISC-IV. Scores for the WM scale on the BRIEF and backward digit span were entered at stage two.

Results: Overall, intellectual functioning was a significant predictor for reading comprehension $(F(1,61)=53.22, p<0.01)$, numerical operations $(F(1,92)=73.80, p<0.01)$, and math problem-solving $(F(1,41)$ $=83.42, p<0.01)$, accounting for $42 \%$ to $47 \%$ of the variance in these WIAT-III subtests. Adding the BRIEF WM subscale and the WISC-IV backward digit span subtest did not contribute significantly to any of these WIAT-III subtests $(\mathrm{p}>0.01)$.

Conclusions: Preliminary results suggest that intellectual functioning, rather than WM, best explains performance for reading comprehension, numerical operations, and math problem-solving, in children with 
epilepsy. Future studies should re-examine the relationship between WM and reading and math performance, controlling for intellectual functioning, in other clinical populations to determine whether WM still predicts reading and math performance even after controlling for intellectual functioning.

Correspondence: Moshe Maiman, B.A., Psychology, Drexel University, 2040 Market St, \#802, Philadelphia, PA 19103, United States. E-mail: mainman89@gmail.com

M. MAIMAN, V. DEL BENE, E. FARRELL, W.S. MACALLISTER, L. VAURIO, B. LEMONDA, S. SHELDON, M. SLUGH, M. ARCE RENTERIA \& W. BARR. Observed sex differences with the Repeatable Battery for the Assessment of Neuropsychological Status (RBANS) in adult epilepsy patients.

Objective: The Repeatable Battery for the Assessment of Neuropsychological Status (RBANS) is a frequently used cognitive screener. Prior research has shown that RBANS performance can be impacted by demographic variables, including sex. However, to date, no study has investigated if sex impacts RBANS performance in adults with epilepsy, a population that often undergoes neurocognitive assessments. This study aims to explore if RBANS performance varies as a function of sex in adults with epilepsy.

Participants and Methods: Forty-five clinically referred males with epilepsy (Age: $M=42.07 ; S D=16.64$; IQ: $M=95.05 ; S D=19.16$ ) and 52 females with epilepsy (Age: $M=45.38 ; S D=16.56$; IQ: $M=92.35$; $S D=19.33$ ) completed the RBANS. Groups did not differ significantly for age, education, age of seizure onset, number of anti-epileptic drugs, disease duration, or IQ. A series of Analysis of Variance (ANOVAs) were computed for each of the indices on the RBANS to assess for group differences, with the significance level set at .05.

Results: A series of ANOVAs revealed significant group differences for Immediate Memory $(t=1.97, d f=95, p=.05, d=.40)$, such that females exhibited poorer performance. No other significant group differences were observed.

Conclusions: Consistent with studies that have found sex differences on the RBANS for other clinical populations, sex differences did emerge in adults with epilepsy. Specifically, females with epilepsy demonstrated greater deficits on the immediate memory index of the RBANS. However, unlike other groups studied (e.g. schizophrenia), sex does not have a widespread impact on RBANS performance in adults with epilepsy, and its impact is minimal. These findings are in line with the general neurocognitive profile found in adults with epilepsy, namely that sex does not play a large role.

Correspondence: Moshe Maiman, B.A., Psychology, Drexel University, 2040 Market St, \#802, Philadelphia, PA 19103, United States. E-mail: mainman89@gmail.com

K.R. MCCLEARY, J. BARRASH, M. GRANNER, K. MANZEL, A. GRIEDER \& R. JONES. The Safety and Efficacy of Propofol as a Replacement for Amobarbital in Intracarotid Wada Testing of Pre-surgical Epilepsy Patients.

Objective: The Wada test is the gold standard for language and memory lateralization, and an important part of pre-surgical work up for medically intractable epilepsy. Due to shortages in sodium amytal, providers have turned to less-studied alternatives like propofol. Our study uses the largest sample so far to directly compare the safety and efficacy of propofol versus sodium amytal in the Wada test.

Participants and Methods: A retrospective review was conducted of 97 Wada procedures performed at University of Iowa Hospitals and Clinics from 2007 through mid-2015. Success of language and memory lateralization was established based on the confidence of the neuropsychologist's recommendations. Adverse events (AEs) were ranked by relative severity. Patients were excluded only from analyses for which their records lacked relevant data; no patients were excluded from all analyses.
Results: There was no significant difference between rates of successful language or memory lateralization for propofol versus amobarbital, nor were there between-group differences in performance on any tested subscale. Patients had similar risk of experiencing an AE regardless of drug. None of the demographic variables evaluated were reliable predictors of failure to lateralize. Only diastolic blood pressure was significantly associated with experiencing a major AE in propofol patients.

Conclusions: Consistent with previous studies, anesthetic type appears to have no significant impact on success to lateralize memory or language. Demographic screening of patients beyond appropriateness for surgery is not recommended given their minimal association with risk of an $\mathrm{AE}$ or test failure. The clinical utility of diastolic pressure management may be of interest for future studies. This study provides comprehensive support that propofol is as safe and efficacious as amobarbital. It can continue to be used in Wada procedures with confidence, and subsequent research may consider combining data with low concern of drug type as a confounder.

Correspondence: Kaci R. McCleary, University of Iowa, 102 Hawkeye Ct. Apt 101, Iowa City, IA 52246, United States. E-mail: kacim@alumni. iastate.edu

K. ONO, D. BEARDEN, C. SIMS, K. HASS \& T. DEGRAUW. Psychosocial Comorbidities in Children and Adolescents with New Onset Seizures.

Objective: Seizures are a common neurological problem in pediatrics and are often associated with psychosocial comorbidities. Little is known about distribution of these comorbidities in children with new onset seizures (NOS). The current study compared psychosocial comorbidities in two pediatric NOS groups: patients with a history of $>1$ unprovoked seizure, and patients who have had only one seizure.

Participants and Methods: A sample of 76 participants (28 male, 48 female), ages 7-16 years old participated in the current study. Children were placed in one of two groups: history of $>1$ unprovoked seizure (i.e., epilepsy; $n=48)$ or history of a single seizure $(n=28)$. Parent ratings of children's psychosocial functioning using the Behavior Assessment System for Children, $3^{\text {rd }}$ Ed (BASC-3) were analyzed to assess group differences in hyperactivity, anxiety, depression, and inattention.

Results: No significant psychosocial differences were found between the groups on the BASC-3. However, compared to normative data, more children than expected in both groups had clinically significant ratings on BASC-3 indices. In the single-seizure group, $14 \%$ had anxiety, $25 \%$ had attention problems, $32 \%$ were hyperactive, and $18 \%$ had depression. In the epilepsy group, 15\% had anxiety, $23 \%$ had attention problems, $15 \%$ were hyperactive, and $15 \%$ had depression. Further, a MANOVA revealed an interaction between diagnostic group and age, $\mathrm{F}(2,70)=2.89, \mathrm{p}=.05$, such that in the epilepsy group, anxiety decreased with age, whereas in the control group, anxiety remained stable.

Conclusions: Results from this study revealed increased rates of psychosocial comorbidities in pediatric NOS patients compared to the general population. Comorbidities were not associated with number of seizures patients experienced. Findings suggest that these problems are a manifestation of atypical neurodevelopment as opposed to being a product of seizures themselves. Results also suggest that anxiety decreases with age in pediatric patients with epilepsy.

Correspondence: Kimie Ono, PhD, Neuropsychology, Children's Healthcare of Atlanta, 5455 Meridian Mark Road Suite 180, Atlanta, GA30342, United States.E-mail:kim.ono@choa.org

M. PINJALA, C.I. BERMUDEZ, K.F. MCINERNEY, M. SARNO, N.S. BANERJEE, A. BURE-REYES, S. GETZ, C. MILLAN, A. KANNER, R. RIBOT, E. SERRANO, L. TORNES, M. LOWE, N. VELEZ-RUIZ \&. G.J. REY. Relationship of Cognitive Functioning to Quality of Life in Patients with Epilepsy.

Objective: To examine the unique role of cognitive functioning on Quality of Life (QOL) in people with intractable epilepsy, in relation to other variables known to be associated with QOL in this population. 
Participants and Methods: Participants (n=29) with medically refractory epilepsy underwent pre-surgical neuropsychological evaluation at the University of Miami International Center for Epilepsy. Subjects were primarily male $(70 \%)$ and right handed $(83 \%)$, with a mean age of 38 $(S D=17.2)$ and an average of 13 years of education $(S D=2.0)$. Average duration of seizure disorder was 20.5 years $(S D=16)$.

The Personal Impact of Epilepsy Scale (PIES) scales measures adverse effects of seizures, medications, comorbidities and overall QOL. Tests of verbal memory, word retrieval and FSIQ were used to assess their influence on PIES scales beyond the contribution of factors known to influence QOL, including age of onset, duration of disorder and psychological well-being. The BDI-II and BAI were used as measures of emotional functioning.

Results: The Total and Comorbidities scales from the PIES had high negative correlations with the BDI-II, BAI and FSIQ and low correlations with cognitive variables. Hierarchical multiple regressions were conducted, with the PIES scales as the dependent variables. Clinical variables were entered first, then BDI-II and BAI scores, followed by the cognitive scores as predictors.

The BDI-II and BAI significantly predicted the PIES Total and Comorbidities score $(F(4,19)=4.92, p=.007 ; F(4,19)=9.43, p<.001)$, and accounted for $50 \%$ of the variance in Total and $65 \%$ in the Comorbidities score. The cognitive and clinical variables did not add significant variance to either score.

Conclusions: Results confirm large contribution of anxiety and depression on QOL in this population. Commonly reported cognitive complaints were not associated with QOL. Interestingly, higher FSIQ was associated with lower QOL on the PIES. Further research can aid in guiding specialized intervention to maximize QOL in this population. Correspondence: Maya Pinjala, M.S., Neurology, University of Miami, 2906 S. University Dr., Apt 10304, Davie, FL 33328, United States. E-mail:mp1835@mynsu.nova.edu

E.E. QUASNEY, K.H. ELVERMAN, D.S. SABSEVITZ, J.R. BINDER, L. GLASS, M. RAGHAVAN, W. MUELLER \& S. SWANSON. Wada Language Lateralization Index (LI) and Wada Memory Asymmetry (WMA) Scores as Predictors of Verbal Memory Decline After Left Anterior Temporal Lobectomy (L-ATL).

Objective: Baseline verbal memory performance and WMA scores historically have been considered the best predictors of memory decline after L-ATL. Recently, language lateralization as determined by fMRI was found to be a better predictor of memory outcome than an fMRI memory task. The present study examines the value of Wada language LI and WMA scores for predicting memory decline following L-ATL. Participants and Methods: L-ATL patients $(N=76)$ underwent Wada testing and pre- and post-operative neuropsychological evaluation. Wada language LI and WMA scores were calculated. Regression analyses were conducted entering baseline memory performance, Wada language LI, and WMA for predicting post-operative memory change on the following measures: Long Term Storage (LTS), Consistent Long Term Retrieval (CLTR), and Delayed Recall (SRT-DR) from the Selective Reminding Test and Delayed Recall (LM-DR) from WMS-IV Logical Memory.

Results: Across all measures, pre-op memory and Wada language LI predicted significant variance in memory change $(p s<.05)$. Pre-op memory explained 22 to $31 \%$ of the variance in memory decline for SRT and WMS-IV measures with Wada language laterality predicting 6 to $9 \%$ additional variance. WMA did not add to the prediction of memory change even when WMA was added to the model prior to Wada language LI $(p \mathrm{~s}>.05)$. Further, there were no group differences in memory decline when patient subgroups with left language dominance and either strong right or left WMAs were compared ( $p s>.05$ ).

Conclusions: Across numerous measures of verbal memory, baseline memory performance and Wada language LI are consistent predictors of verbal memory decline following L-ATL. WMA scores do not add to the prediction. Moreover, after selecting left language dominant patients, there were no group differences in degree of memory decline between subgroups with left versus right lateralized memory. The results support claims that the Wada memory test is not sufficiently material-specific to assess lateralization of verbal memory functions.

Correspondence: Erin E. Quasney, Neurology, Medical College of Wisconsin, 610 N 112th Street, Wauwatosa, WI 53226, United States. E-mail: equasney@gmail.com

A. REALE-CALDWELL, R. SEVER, R.S. RUM, S.L. PENA \& M. SCHOENBERG. Predictive Ability of Verbal Memory Tests in Post-Operative Epilepsy Patients.

Objective: Managed care and time constraints present significant concerns to the neuropsychological batteries currently employed in the medical field. As such, neuropsychologists must be judicious in the tests they decide to give during this limited time-frame. The aim of the current study is to determine the predictive utility of the Word Memory Test and Wechsler Memory Scale-IV Logical Memory in determining verbal naming and memory changes following surgery for medication refractory temporal lobe epilepsy (TLE). Results from this study can better inform the test selection process of a time-limited neuropsychological battery.

Participants and Methods: Fifty-four patients underwent pre- and post-surgical neuropsychological evaluations as part of their surgical workup for medication refractory temporal lobe epilepsy. The range of age was between 14 and 61 years $(M=34, S D=11.35)$. Gender difference ratio was $28(51.9 \%)$ male and $26(48.1 \%)$ female. Their average education was $13.32 \pm 2.63$ years.

Results: Multiple regressions were used to assess the ability of four control measures, Word Memory Test Multiple Choice (WMTMC), Paired Associates (WMTPA), Free Recall (WMTFR) and Wechsler Memory Scale-IV Logical Memory II to predict verbal memory change and confrontation naming change following surgery. The total variance of the model evaluating verbal memory decline was $20.8 \%$. $F(4,13)=.85, p=.52$. The total variance of the model evaluating confrontation naming decline was $61.7 \%, F(4,13)=5.23, p=.01$. Only WMTMC and WMTFR were statistically significant predictors of confrontation naming change with WMTMC recording a higher beta value $($ beta $=1.24, p=.005)$ than WMTFR $($ beta $=-.53, p=.044)$.

Conclusions: Preoperative WMT multiple choice and free recall subscales better predicted change in confrontation naming following surgery for TLE. Further research should be conducted to determine utility of preferential use of WMT over WMS LM in pre-operative neuropsychological assessment as an accurate and time-saving method.

Correspondence: AmberRose Reale-Caldwell, MA, University of South Florida, 5506 Fulmar Drive, Tampa, FL 33625, United States. E-mail: amberrose47@yahoo.com

K.D. SRNKA, Z. RESCH, D.A. KELLY, J. JONES, D. ALMANE, B. HERMANN \& M. SEIDENBERG. 5-year Longitudinal Change in Attentional Vigilance in Childhood Epilepsy.

Objective: Impairment in attentional vigilance is a core feature of cognitive functioning in childhood onset epilepsy. Previous research has identified disruption in multiple indices of attentional vigilance: Omission errors (O), Commission errors (C), Mean Response Time (RT), and Intraindividual Variability (IIV). Here we report the two and five year longitudinal change in performance for these indices of attentional vigilance. Participants and Methods: 65 children with recent-onset epilepsy (M age $=12$ years $)$ and 60 healthy children $(\mathrm{M}$ age $=12.22)$ were administered the Connors Continuous Performance Test (CPT) at time of initial diagnosis, 2 years and 5 years post diagnosis. Linear Mixed-Effects Modeling (LME) examined group differences for intercept (baseline) and slope (change) over the three assessments. T-tests were used to determine if groups were significantly different on CPT variables at 5 years. Additional LME analyses compared children with idiopathic generalized epilepsy (IGE) to children with localization related epilepsy (LRE). 
Results: The childhood epilepsy group had significantly higher baseline T-scores on O, RT, and IIV ( $p$ 's <.05), but not C ( $p>.05)$. No significant slope differences were found between groups $(p<.05)$, except for $\mathrm{C}$ $(\mathrm{p}<.01)$. Persisting significant group differences on IIV $(\mathrm{t}=-3.89$, $\mathrm{p}<.001)$ and $\mathrm{C}(\mathrm{t}=-1.43, \mathrm{p}<.05)$ were evident at the five year testing. Both IGE and LRE groups showed similar longitudinal findings.

Conclusions: Disruption on multiple indices of attentional vigilance are apparent at time of diagnosis with a similar trajectory over five years. These findings are consistent with the idea that the cognitive impact of childhood onset epilepsy is evident at time of diagnosis without significant differences in progression between groups over a 5 year period. Investigation of distinct childhood epilepsy groups is needed to refine these findings.

Correspondence: Zachary Resch, Neuropsychology, Medical College of Wisconsin, 1538 S 4th Street, Milwaukee, WI 53204, United States. E-mail: zachary.resch@my.rfums.org

A. RAICU, D.S. SABSEVITZ, S. SWANSON, E. QUASNEY, J.R. BINDER, M. RAGHAVAN \& W. MUELLER. Does Memory Decline Really Matter Following Epilepsy Surgery? Impact of Memory Decline on Perceived Cognitive Functioning in a Sample of Left Anterior Temporal Lobectomy (ATL) Patients With Well Controlled Seizures.

Objective: ATL is considered an effective treatment for temporal lobe epilepsy. Patients who undergo dominant ATL are at particular risk for verbal memory decline. Previous research has shown that seizure control is the strongest predictor of QOL and QOL remains stable or improves in patients with good seizure control following ATL despite memory decline. The degree to which patients perceive changes in memory in their daily life is not well understood. The current study examines the relationship between memory decline and perceived cognitive impact using cognitive specific items from the QOLIE-31 in patients with good seizure outcome following left ATL.

Participants and Methods: Participants in this study were 51 left ATL patients with Engel Classifications of I or II who completed pre- and post-operative neuropsychological testing. Memory measures included the Selective Reminding Test and WMS Logical Memory (LM). Memory change scores were computed by subtracting pre- from post-operative scores. The correlation between memory change scores and the overall QOL rating and the memory specific items on the QOLIE-31 were examined.

Results: There was no significant correlation between overall QOL rating and memory change in this sample ( $p>.05)$; however, significant correlations were observed between memory change and the QOLIE memory specific item for LM I $(\mathrm{r}=.298, \mathrm{p}=.047)$ and II $(\mathrm{r}=.368, \mathrm{p}$ $=.013)$ and SRT CLTR $(\mathrm{r}=.341, \mathrm{p}=.014)$ with the positive correlation suggesting greater declines in verbal memory were associated with poorer perceived memory. Depression ratings did not explain this relationship.

Conclusions: Consistent with previous reports, memory decline was not associated with overall QOL ratings. However, memory decline was associated with memory specific QOL ratings despite all patients having good seizure outcome, suggesting that memory decline can impact perceived cognitive functioning.

Correspondence: David S. Sabsevitz, Ph.D., Neurology, Medical College of Wisconsin, 9200 W Wisconsin Ave, Milwaukee, WI 53226, United States.E-mail: david.sabsevitz@mcw.edu

L. GIAMBRONE, S.L. SALINAS, A. KEMP, J. KLEINER, J. GESS \& L. LARSON-PRIOR. Gender Differences on a Test of Spatial Memory Among Patients with Epilepsy.

Objective: Neuropsychological testing is a valuable tool in evaluation of pre-surgical epilepsy patients, as it can be used to predict potential cognitive impairments following surgical intervention. The purposes of this study was to assess whether spatial or drawing accuracy on the Brief
Visuospatial Memory Test-Revised (BVMT-R) better predicts location of the hemisphere from which seizures are generated.

Participants and Methods: The sample included 59 patients (33 male, 26 female) ages 18-67 (M=37) who were previously diagnosed with epilepsy and had undergone comprehensive neuropsychological pre-surgical evaluations. Patients were divided into the lateralization of their seizure onset, with 26 right and 33 left hemisphere focus. The mean of participant BVMT-R subscores for drawing accuracy and spatial location were compared using SPSS. It was predicted that spatial subscores would be the most accurate in lateralizing memory location.

Results: Statistical comparisons revealed no significant differences between individuals with left or right lateralized epileptic foci on either the spatial or accuracy subscores on the BVMT-R. The spatial and accuracy subscores on both the immediate and delayed recall were not measurably different depending on the side of the epileptic foci. However, when comparing gender performance, females generated higher spatial subscores for both immediate and delayed recall trials when compared to males.

Conclusions: The results of the current investigation did not reveal any apparent differences in the spatial and accuracy scores in relation to right or left hemisphere epileptic foci, therefore suggesting that the BVMT does not predict seizure focus lateralization. Although the finding of superior performances by females on the spatial subscores was an unanticipated finding, it is nonetheless consistent with previous reports that there are sex differences in spatial memory functions.

Correspondence: Stephanie L. Salinas, MS, MA, Department of Clinical Psychology, The University of Arkansas for Medical Sciences, $4301 \mathrm{~W}$ Markham, Slot 554, Little Rock, AR 72205, United States. E-mail: steph. salinas34@gmail.com

W. SCHRAEGLE, J.B. TITUS \& N. NUSSBAUM. Neuropsychological Functioning in Youth with Temporal Lobe Epilepsy With or Without Hippocampal Sclerosis: An Unexamined Cognitive Phenotype?

Objective: Temporal lobe epilepsy (TLE) is associated with material specific deficits in adults, but similar neurocognitive impairments are less easily recognized and studied in youth. While youth with TLE often present with non-specific findings, no studies to date have compared neuropsychological profiles of children with TLE and those with TLE plus hippocampal sclerosis (TLE+HS).

Participants and Methods: The study included 62 patients (40 male) with TLE (HS, $n=17$ ) age 8-16 years. Epilepsy variables were collected via medical chart review. Neuropsychological battery included the WISC-IV, BNT (Total), D-KEFS (Verbal Fluency), WCST (Perseverative Errors), CVLT-C (List A Total, SDFR, LDFR), and the TOMAL-2 (MFS, MFSD, FM). Quality of life was assessed with the QOLCE.

Results: Group differences were not present for demographic and seizure variables $(p>0.05)$. For those youth with TLE+HS, independent samples $t$ tests revealed diminished neuropsychological performance on CVLT-C List A Total, SDFR, LDFR, and on WCST Perseverative Errors $(p<0.05)$. No effects of laterality were observed. Finally, youth with TLE+HS were associated with a threefold risk of diminished quality of life (odds ratio $=3.70, p<0.05)$.

Conclusions: The current work lends initial support for TLE+HS as a distinct cognitive phenotype in pediatric TLE. While the TLE group demonstrated a non-specific neuropsychological profile, youth with TLE+HS showed significant impairment in list-learning, short and delayed recall, as well as cognitive flexibility. These findings appear to underlie a temporo-frontal network dysfunction that may be more disrupted when epileptiform activity is localized to mesial temporal regions. Special attention to this population is merited given risk factors for diminished quality of life.

Correspondence: William Schraegle, The University of Texas, Austin, 1307 Kinney Ave \#114, Austin, TX 78704, United States. E-mail: waschraegle@gmail.com 
M. MAIMAN, V. DEL BENE, E. FARRELL, W.S. MACALLISTER, L. VAURIO, B. LEMONDA, S. SHELDON, M. SLUGH, M. ARCE RENTERIA \& W. BARR. Examining Cutoff Values of Elevated BDI and BAI Scores in Relation to Validity Scales on the MMPI-2-RF in Adults with Epilepsy.

Objective: Adults with epilepsy often have comorbid depression and anxiety. The BDI and BAI are self-report inventories widely used to screen for psychological functioning in adults with epilepsy. These instruments do not contain validity scales for detecting over-reporting. This study examines the sensitivity and specificity of different BDI and BAI cutoff scores in relation to invalid profiles on the MMPI-2-RF in adults with epilepsy.

Participants and Methods: Fifty-seven clinically referred adults with epilepsy (Age: Mean= 40.21; $\mathrm{SD}=15.16$ ) underwent neuropsychological assessment, which included the BDI, BAI, and MMPI-2-RF. Invalid profiles were defined by using a previously established cutoff score on one or more of the following scales: F-r $>120$, Fs $>100$, FBS-r $>100$, or RBS $>100$. Sensitivity and specificity rates were then calculated for different cutoff scores on the BDI and BAI, using invalid MMPI-2-RF profiles as the criteria for classifying over-reporting.

Results: In this sample, a score of BDI $\geq 26$ and $\mathrm{BAI} \geq 33$ could detect invalid profiles on the MMPI-2-RF with $80 \%$ sensitivity and $88 \%$ and $96 \%$ specificity, respectively. No other cutoff score on the BDI or BAI had more optimal combined sensitivity and specificity levels.

Conclusions: These results support the need for practitioners to ideally use self-report measures in conjunction with clinical interview and symptom validity tests. Self-report measures, such as the BDI and BAI, are likely to be affected by over-reporting in adults in epilepsy. Preliminary findings suggest that a score of $\mathrm{BDI} \geq 26$ and $\mathrm{BAI} \geq 33$ is likely to be associated with over-reporting in adults with epilepsy.

Correspondence: Mitch Slugh, NY, United States. E-mail:mbm95@ drexel.edu

K.D. SRNKA, J. JONES, D. ALMANE, B. HERMANN \& M. SEIDENBERG. Intraindividual Variability Mediates Five Year Longitudinal Relationship With Age of Diagnosis, IQ, and Academic Achievement.

Objective: Disruption in attentional vigilance is a common cognitive feature of childhood onset epilepsy. We recently reported that intraindividual variability (IIV) in sustained attention: discriminated between newly diagnosed children with epilepsy and matched controls, significantly predicted concurrent academic and IQ scores, and partially mediated the relationship between age of epilepsy diagnosis and concurrent academic achievement (AA) and IQ. IIV is measured as the variability of reaction time in a continuous performance task. Here we examined the long term ( 5 years) predictive value of baseline IIV in mediating the relationship of age of diagnosis with IQ and AA

Participants and Methods: 65 children (M age=12 years, M onset=11.6 years, $48 \%$ generalized epilepsy and $52 \%$ localization related epilepsy) were administered Connor's Continuous Performance Test-2 (CPT2), Wide Range Achievement Test-III (WRAT-III), and Wechsler Abbreviated Scales of Intelligence (WASI) at baseline and 5-years. We conducted mediation analysis with SPSS Process, an add-on package, to test the longitudinal ( 5 years) mediation of baseline IIV between age of diagnosis and the following cognitive variables: WASI Performance (PIQ) and Verbal (VIQ) and the WRAT-III (Word Recognition, Spelling, and Arithmetic). Percentile bootstrap confidence intervals (CI) were used to test for mediation effects.

Results: Baseline IIV showed partial mediation between age at diagnosis and functioning five years later for: PIQ (0098-.1032), VIQ (.0020.0878), Spelling (.0027-.0897), and Arithmetic (.0146-.1388), but not Word Recognition (-.0027-.0792).

Conclusions: Intraindividual Variability of attentional vigilance found soon after diagnosis of childhood epilepsy may be an early cognitive marker for IQ scores and AA 5 years later. In addition, IIV may also play a role in characterizing the cognitive mechanism by which early age of epilepsy diagnosis influences subsequent cognitive performance.
Correspondence: Kyle D. Srnka, M.S, Psychology, Rosalind Franklin University of Medicine and Science, 3503 Green Bay Rd, \#307, North Chicago,IL 60064, United States.E-mail:kyle.srnka@my.rfums.org

A.K. STEFANATOS, M. LIBSENSON, J.R. MADSEN \& K. BOYER. Neuropsychological Outcomes of Adolescents Following Hemisperectomy: A Multiple Case Study.

Objective: Functional reorganization has been documented in individuals who have undergone hemispherectomy in treatment of medically-refractory epilepsy. This effect has most clearly been demonstrated following resection of the left hemisphere in early childhood. Less is known about the process of reorganization in individuals who undergo resection in later adolescence. Consequently, this multiple case study retrospectively evaluated the neuropsychological and clinical outcomes after hemispherectomy in adolescents with refractory epilepsy.

Participants and Methods: This study examined three cases of patients who underwent hemispherectomy in adolescence (ages 13, 13 and 15). Etiology included diffuse polymicrogyria (P1) and perinatal stroke (P2 and P3). Seizure onset ranged from infancy (P1) to early childhood (P2 and P3). P3 underwent a left hemispherectomy, while P1 and P2 underwent a right hemispherectomy. All patients were administered a comprehensive neuropsychological assessment before and following surgical intervention.

Results: All three patients obtained seizure freedom following surgery. Performance across various measures including intellectual functioning and memory functioning were found to range from the Severely Impaired to Average range overall. RCI analyses demonstrated significant increases in nonverbal reasoning and memory in the two patients who had undergone resection of the right hemisphere, with a significant decrease in aspects of verbal reasoning/memory. The patient who had undergone a left hemispherectomy demonstrated some pattern of improvement on verbal tasks.

Conclusions: The observed changes in performance are consistent with classic "crowding" and "release of cortical inhibition" effects, which, interestingly, were more robust in the right hemispherectomy patients. This suggests that cerebral reorganization can occur following hemispherectomy in adolescence. The implication of these findings will be discussed.

Correspondence: Arianna K. Stefanatos, Ph.D., Boston Children's Hospital, 300 Longwood Avenue, BCH 3021, Boston, MA 02115, United States.E-mail: arianna.stefanatos@childrens.harvard.edu

\section{Movement and Movement Disorders}

N.S. BANERJEE, N. SUN-SUSLOW, S. GETZ, M. SARNO, A. BUREREYES, M. PINJALA, C. LUCA \& B.E. LEVIN. Age-related Differences in the Association Between Cardiometabolic Risk Factors and Cognition in Parkinson's Disease.

Objective: Cardiometabolic risk factors, such as hypertension (HTN) and diabetes mellitus (DM), are linked to cognitive dysfunction in the elderly. However, the impact of these factors on cognition in Parkinson's disease (PD) is less clear. This study examined relationship between two cardiometabolic factors and cognition in PD patients, as well as the potential moderating effect of age.

Participants and Methods: This study utilized an ethnically diverse sample of 152 patients with idiopathic PD $\left(M_{\text {age }}=62.0\right.$, range: 39-74; $\left.M_{\text {educ }}=13.5\right)$. Patients were divided into 3 groups: HTN $(n=43)$, HTN + DM $(n=14)$, and PD without HTN or DM $(n=95)$. ANCOVA was used to compare neuropsychological test performance among groups, controlling for sex, age, language, disease stage, and education. Next, a moderation analysis was performed to evaluate the influence of age on the relationship between HTN and cognition.

Results: The HTN, HTN+DM, and PD only groups did not differ on any neuropsychological measure. However, in a separate analysis, age significantly moderated the relationships between HTN and WAIS Digit 
Span $(b=.18, p=.03)$ and COWAT phonemic fluency $(b=.59, p=.04)$, such that they were significant only among patients younger than 63 and 53 , respectively.

Conclusions: Neither the HTN nor comorbid HTN + DM group exhibited worse cognitive performance compared to PD patients without these conditions. However, for individuals below the age of 65 , HTN was associated with poorer performance on tasks involving working memory and phonemic fluency. These findings suggest that the impact of HTN on cognition may differ across the age continuum in PD. Specifically, younger patients may be particularly vulnerable to the additive effects of HTN on cognitive impairments resulting from PD-related frontal-subcortical dysfunction. These data also highlight the importance of early assessment and aggressive treatment of HTN and cognitive dysfunction, especially among younger PD patients.

Correspondence: Nikhil S. Banerjee, Bachelor's, Psychology, University of Miami, 350 S Miami Ave, Apt. 1704, Miami, FL 33130, United States. E-mail:n.banerjee@umiami.edu

\section{G. CHANEY, L. MORRA, C.M. FIGUEROA \& V. KAMATH. Clinical Utility of Neuropsychological and Olfactory Measures in the Differential Diagnosis of Parkinson's Disease.}

Objective: Approximately $10 \%$ of patients who meet current diagnostic criteria for Parkinson's Disease (PD) lack evidence of a dopamine deficiency. Although participants with dopamine

transporter imaging scans without evidence of dopaminergic deficit (SWEDDs) have otherwise indistinguishable cardinal physical features of PD, the absence of a dopaminergic deficit may lead to misdiagnosis of PD. The potential for misdiagnosis has prompted interest in identifying clinical and cognitive indices that distinguish SWEDD from idiopathic PD (IPD) to facilitate accurate diagnosis and treatment. In the present study, we examined whether neurocognitive, neuropsychiatric, and olfactory indices could correctly distinguish these groups.

Participants and Methods: Baseline clinical and cognitive data from the Parkinson's Progression Marker's Initiative (PPMI) was examined in persons newly diagnosed with IPD $(n=413)$ and SWEDD participants $(n=61)$. A binary logistic regression analysis was employed to determine whether these measures could correctly predict group membership. Results: The logistic regression model significantly discriminated the groups $(p<.001)$, and showed good classification accuracy $(85.9 \%)$. Among 11 neurocognitive and neuropsychiatric indices, odor identification and semantic fluency were the only significant predictors of group membership ( $p$ 's <.03). Odds ratios revealed that a one unit increase in an individual's odor identification score decreased the likelihood of a PD diagnosis by $24 \%$. Conversely, a one unit increase in semantic fluency increased the likelihood of PD diagnosis by $64 \%$.

Conclusions: These findings indicate that measures of olfaction and semantic fluency are useful in the differential diagnosis of newly diagnosed PD. Further investigations utilizing longitudinal cohorts to examine the predictive accuracy of these measures in disease classification appears warranted.

Correspondence: Grace-Anna Chaney, Master of Arts, Psychiatry and Behavioral Sciences, The Johns Hopkins University, 600 N. Wolfe Street, Meyer 218, Baltimore, MD 21287-7218, United States. E-mail: gchaney2@jhmi.edu

\section{S.J. CROWLEY, L. HIZEL, J.J. TANNER, Y. CRUZ-ALMEIDA \& C.C. PRICE. Cognitive Phenotypes in Early Idiopathic Parkinson's Disease.}

Objective: Individuals with Parkinson's disease have dominant processing speed and working memory difficulties; however, other cognitive profiles have also been reported. This prospective investigation recruited individuals with tremor-dominant, idiopathic PD to statistically define cognitive phenotypes. We hypothesized that this analysis would result in four cognitive phenotypes: executive deficits, learning and memory deficits, mixed memory-executive deficits and cognitively well.
Participants and Methods: 153 participants (100 PD; 53 controls) completed neuropsychological testing as part of a federally funded investigation (NIH/NINDS R01-NS-082386; K23-NS060660). Inclusion criteria included no dementia, no stroke history, education greater than eight years, and no other neurodegenerative disorder. Seven cognitive variables measuring processing speed, executive functioning and memory were used in the cluster analysis. A principal component analysis identified two components with loadings in executive functioning and memory. Factor loadings for the two components were saved and used as variables in a k-means analysis of the PD participants with four forced clusters. Separate measures of memory and executive functioning were used to validate clusters.

Results: 149 participants (97 PD; 52 controls) met final inclusion. The final clusters contained individuals with executive deficits $(n=21)$, memory deficits $(n=34)$ mixed deficits $(n=15)$, and cognitively well $(\mathrm{n}=27)$. The groups did not differ in age or education, but motor severity was worse in the executive deficit and mixed groups $(\mathrm{p}<.01)$ with mixed having shorter disease duration than executive deficit $(p=0.04)$. Groups differed on validating external measures of memory and reasoning.

Conclusions: Parkinson's disease has at least four distinct cognitive categories. Participants with mixed deficits show more severe symptoms despite shorter disease duration. Future studies should explore possible additional biomarker validation of cognitive subtypes.

Correspondence: Samuel J. Crowley, Clinical and Health Psychology, University of Florida, 2220 SW 34th Street Apt 207, Gainesville, FL 32608, United States. E-mail: samjcrowley@ufl.edu

K. HARRIS-RAINS, L.H. LU, S.A. KVAAL \& M. HELFORD. Title: The Unique Contribution of Motor, Cognitive, and Mood Symptoms to Functional Impairment in Parkinson's Disease.

Objective: Objective: Although Parkinson's Disease (PD) is a neurodegenerative movement disorder, nonmotor symptoms such as cognitive and depressive symptoms are prevalent. Given the prominence of motoric symptoms, the emotional and cognitive symptoms of the disorder are not often prioritized in assessment and treatment, though these symptoms can significantly impact quality of life. The objective of the current study was to assess the extent to which cognitive and depressive symptoms impact functionality after controlling for the effect of motor symptoms, to better characterize patients' experience of PD. Participants and Methods: Participants and Methods: Data was from 937 PD participants in the National Alzheimer's Coordinating Center's Uniform Data Set (UDS; Weintraub et al., 2009). We used hierarchical regression to examine the extent to which cognitive measures (i.e., Trail Making Test, WAIS Digit Symbol Coding, WMS Logical Memory, and Semantic Fluency Test) and the Geriatric Depression Scale-15 predicted scores on the Clinician Dementia Rating Scale (CDR) and the informant-based Functional Assessment Questionnaire (FAQ) after controlling for demographic variables and motor symptoms (Unified Parkinson's Disease Rating Scale-Motor Examination).

Results: Results: Results indicated that cognitive but not depressive symptoms predicted $32.3 \%(\mathrm{~F}(7,276)=35.69, \mathrm{p}<.01)$ of the variance in FAQ, while both cognitive and depressive symptoms predicted $27.7 \%$ $(\mathrm{F}(7,507)=36.97, \mathrm{p}<.01)$ of the variance in CDR after controlling for motor symptoms. Semantic fluency was the most robust predictor of both outcome measures.

Conclusions: Conclusions: Results suggest cognitive symptoms influence activities of daily living in PD above and beyond motor symptoms' impact. Depressive symptoms have a similar impact, though the effect was only observed in the clinician rating of functionality. These findings indicate the importance of comprehensive assessment and holistic intervention for PD to better support patients' daily functioning.

Correspondence: Katie Harris-Rains, Roosevelt University, 2120 Bank Street, Baltimore, MD 21231, United States. E-mail: kharris24@mail. roosevelt.edu 
T. HENDERSHOTT, R. FAMA, E. MULLER-OEHRING, E.V. SUlLIVAN, A. PFEFFERBAUM, K. POSTON, H. BRONTËSTEWART \& T. SCHULTE. Cognitive and Motor Processes Differentially Affect Visuospatial Performance in HIV Infection and Parkinson's Disease.

Objective: Visuospatial (VS) abilities, which rely on component cognitive and motor processes, can be compromised in HIV infection and Parkinson's disease (PD). We examined whether supporting functions would differentially affect VS performance in HIV and PD, predicting that cognitive deficits would predict VS deficits in HIV, whereas motor deficits would predict VS deficits in PD.

Participants and Methods: Groups comprised $20 \mathrm{HIV}, 22 \mathrm{PD}$, and 20 normal controls (NC), examined on executive functioning, information processing, episodic memory, and upper motor speed and dexterity. Judgement of Line Orientation (JLO) and Hooper Visual Organization task (HVOT) assessed VS abilities. Test scores were age- and education-corrected based on NC; composite scores were theoretically derived. Principal components analyses yielded 2 factors: a cognitive factor (COG) comprising attention, working memory, information processing, and episodic memory; and a motor factor (MOT) comprising fine finger movement and finger tapping.

Results: HIV and PD scored lower than NC on JLO ( $p=.0017)$ but did not differ from each other. No group differences were observed on the HVOT. PD scored lower than HIV on MOT, whereas HIV scored modestly lower than PD on COG. JLO and HVOT correlated with COG in HIV, whereas only JLO correlated with MOT in PD. Multiple regression analyses revealed that in HIV COG accounted for $19.4 \%$ and MOT for $7 \%$ of the variance in JLO scores. By contrast, in PD MOT accounted for $28 \%$ and COG $0 \%$ of the variance in JLO scores. JLO was not related to any UPDRS score or levodopa dosage in PD. Even after controlling for normal age, older age in PD correlated with lower JLO score.

Conclusions: Despite similar levels of VS impairment in HIV and PD, the underlying component processes contributing to impairment differed. Although neurodegenerative processes in HIV and PD involve partially overlapping basal ganglia-thalamocortical networks, our findings highlight the unique aspects of each disease.

Correspondence: Taylor Hendershott, B.A., Neurology and Neurological Sciences, Stanford University School of Medicine, 1901 Emory Street, SanJose, CA 95160, United States.E-mail:trhendershott@gmail.com

\section{A. LARUM, R. SALAZAR \& A. CRONIN-GOLOMB. Non-Motor Symptoms in Men and Women with Parkinson's Disease: Impact on Quality of Life.}

Objective: Parkinson's disease (PD) is associated with numerous non-motor symptoms (NMS) in addition to its hallmark motor symptoms. This study assessed the impact of NMS on quality of life, and considered if the prevalence and severity of NMS differs between men and women.

Participants and Methods: The Non-Motor Symptom Questionnaire (NMS-Quest), a 30-item self-report measure of NMS in PD, was completed by 141 individuals (73 men, 68 women). NMS were rank ordered by prevalence for the entire group and separately for men and women. Chi-square analyses assessed group differences on NMS items with a rank-order discrepancy of $10^{+}$. A continuous severity scale was added to the 30 yes-no NMS-Quest items to assess the correlation between NMS severity and quality of life per the 39-item Parkinson's Disease Questionnaire (PDQ-39) and Neuro-QoL domains: physical health (fatigue), emotional health (anxiety, positive affect), cognitive health (executive function), and social health (social ability).

Results: Men and women reported comparable NMS prevalence (mean of 11) and severity. The most common NMS were sense of urgency to pass urine $(n=101)$, regularly awakening to pass urine $(n=94)$, and loss of smell $(n=89)$. Men reported more difficulty than women with apathy $(p<.002)$, drooling $(p<.024)$, and having sex $(p<.0001)$. NMS severity correlated with all PDQ-39 and Neuro-QoL subscales $(p<.005)$, most strongly for PDQ-39 emotional well-being, cognition, communication, bodily discomfort, and Neuro-QoL cognition ( $r=.51-.70)$. NMS rankings were similar by prevalence and severity, with drooling showing the largest difference (ranked 14 by prevalence, 24 by severity).

Conclusions: NMS severity related to poorer quality of life, especially in mood, cognition, communication, and bodily discomfort. Men and women with PD were differentially affected by apathy, drooling, and sexual function (NMS-Quest), suggesting potential treatment targets. Considering NMS severity and prevalence contributes to a comprehensive conceptualization of PD in men and women.

Correspondence: Amie Larum, 648 Beacon St, 2nd Fl, Boston, MA 02215, United States.E-mail: amie.larum@yahoo.com

F.V. LOPEZ, C. SUMIDA, E.J. VAN ETTEN, M. SPLIT, C.F. PLUIM, D. SCHIEHSER, E. PIROGOVSKY-TURK, R. MOORE, I. LITVAN, S. LESSIG, M. SCHMITTER-EDGECOMBE, P. GILBERT \& V. FILOTEO. Medication Management in Parkinson's Disease: Novel Error Analysis Reveals Those with MCI Indicated Taking Fewer Pills than Instructed on a Performance-Based Task.

Objective: Polypharmacy is common within the Parkinson's disease (PD) population, and poor medication management in PD leads to worse clinical outcomes and increases in health care costs. The current study sought to examine differences on a novel error analysis of the Medication Management Abilities Assessment (MMAA) between individuals diagnosed with PD with either normal cognition (PD-NC) or mild cognitive impairment (PD-MCI) and demographically similar healthy adults (HA).

Participants and Methods: The participants in the HA (n=71), PD-NC $(n=95)$, and PD-MCI ( $n=33)$ groups were administered the MMAA as a part of a comprehensive neuropsychological battery. In addition to the MMAA total score, two error scores were calculated to quantify how many pills participants indicated taking above or below the instructed amount.

Results: Separate Poisson regression analyses were performed on MMAA outcome measures to examine group differences, with gender and levels of depression as covariates. In comparison to the HA group, the PD-MCI group performed significantly worse on the MMAA total score $(p<.05)$ and indicated taking significantly fewer pills than instructed $(p<.05)$. No other significant relationships were found. Spearman correlations were performed to examine the relationship between MMAA performance and standardized neuropsychological measures of memory, executive functioning, processing speed, and attention. Memory was significantly associated with the PD-NC group's tendency to indicate taking more pills than instructed $(r=-.25 ; p<.05)$ and was related to HA group's MMAA total score $(r=.24 ; p<.05)$. No significant relationships were found for PD-MCI.

Conclusions: The PD-MCI group exhibited deficits in medication management characterized by a tendency to indicate taking fewer pills than instructed, when assessed with a performance-based task. These results suggest that the MMAA error variables capture more nuanced aspects of medication management abilities in PD-MCI.

Correspondence: Francesca V. Lopez, B.A., Clinical Health and Psychology, University of Florida, 1225 Center Dr, Gainesville, FL 32603, United States. E-mail: francesca.v.lopez@gmail.com

C.F. PLUIM, M. SPLIT, F.V. LOPEZ, N. WHITELEY, R. MOORE, S. LESSIG, I. LITVAN, V. FILOTEO \& D. SCHIEHSER. Are Apathy and Fatigue Distinct Symptoms in Parkinson's Disease?

Objective: Both apathy and fatigue are common debilitating non-motor symptoms of Parkinson's disease (PD). Previous research has suggested that apathy and fatigue share a common neurobiological origin and it is currently unclear if the two are in fact discernible constructs in PD. As the relationship between apathy and fatigue is not well understood and better understanding this relationship has important clinical implications, the current study sought to determine whether apathy and fatigue are distinguishable in PD.

Participants and Methods: Individuals with PD (n=174) completed the Starkstein Apathy Scale (AS) and the Modified Fatigue Impact Scale 
(MFIS). Reliability analyses and a principal component analysis with varimax rotation were conducted on items from both scales to assess item reliability and to identify underlying factors of the AS and MFIS. Results: Cronbach's alpha revealed high internal consistency $(\alpha=.948)$ of the items in both scales. Item-total scale correlational analysis resulted in one AS item ("concern about condition") being deleted. The remaining 34 items resulted in a 2 - and 4 -factor model, accounting for $49.5 \%$ and $59.8 \%$ of the total variance, respectively. The 2 -factor model clearly differentiated between fatigue and apathy, with all AS items except for one strongly loading onto one factor, while the MFIS items loaded onto the other. The 4-factor model corresponded to two MFIS factors related to cognitive fatigue and physical fatigue and two AS factors corresponding to indifference and amotivation.

Conclusions: Our results suggest that apathy and fatigue are discreet factors in PD. As such, clinicians should consider screening for both fatigue and apathy. Understanding the multidimensionality of apathy and fatigue may allow for the classification of symptom subtypes, which could inform future clinical assessment and targeted treatment. Future studies exploring the pathophysiology of apathy and fatigue in PD may afford insight into the most effective treatment approach for each respective symptom.

Correspondence: Celina F. Pluim, VA San Diego Healthcare System, 9739 Mesa Springs Way Unit 150, San Diego, CA 92126, United States. E-mail: celpluim@gmail.com

S. PUlASKI, F. PONCE, K. HANSON \& A. TROSTER. Cognitive Impairment and its Relationship to Perceived Quality of Life in Parkinson's Disease.

Objective: To evaluate the impact of specific cognitive changes on quality of life (QOL) after deep brain stimulation (DBS) for Parkinson's disease (PD).

Participants and Methods: 83 patients (Mean age 67.1 years; Mean education 14.8 years) underwent unilateral $(n=17)$ or bilateral $(n=66)$ subthalamic nucleus (STN) or globus pallidus (GPi) DBS for treatment of motor symptoms of PD. Patients completed neuropsychological testing pre- and post-DBS. ANCOVAs were conducted to identify if: 1 . Those with postoperative memory, verbal fluency, or confrontation naming declines $(\geq 0.5 \mathrm{SD})$ reported greater reduction in QOL (PDQ-39 Cognition or Communication, as appropriate) in comparison to those showing cognitive test score increments of $\geq 0.5 \mathrm{SD}$. Partial correlation analyses address whether depression moderates the relationships between postoperative verbal memory, verbal fluency, and naming impairments and perceived cognitive and communication aspects of QOL.

Results: Each ANCOVA yielded nonsignificant results $(p>.50)$. Partial correlations examining depression as a moderator for the relationship between postoperative memory and language impairments and cognitive QOL and communication QOL were nonsignificant $(p>30)$. Only the relationship between postoperative depression and cognitive QOL was significant $(p<.05)$.

Conclusions: Perceived QOL is not significantly impacted by objective changes in postoperative memory or language functioning following DBS surgery in patients with PD although significant cognitive changes are rare. Those with more depression symptoms report poorer QOL related to cognition. Findings support prior suggestions that, generally, cognitive alterations after DBS are well tolerated. Those with more depressive symptoms are more apt to be dissatisfied with cognitionrelated QOL, emphasizing importance of control of depression for optimal QOL outcomes after DBS.

Correspondence: Sarah Pulaski, Psy.D., Clinical Neuropsychology, Barrow Neurological Institute, 222 West Thomas Road, Suite 315, Phoenix, AZ 85013, United States. E-mail: sarah.pulaski@ dignityhealth.org
K.L. RAEIN, J. BENGE, T. HEEMSBERGEN, R.A. NELSON, D.O. KONESHECK, B. KONESHECK \& C.S. AARON. Patient and Care Partner's Priorities for Cognitive Treatment Outcomes in Parkinson's Disease.

Objective: Little is known about what constitutes a meaningful outcome for persons with Parkinson's Disease (pwPD) and their care partners (CP) who are considering cognitive treatments. Understanding such outcomes is critical to make cognitive outcomes research more patient centered. The current project reports on the relative importance and priorities of cognitive symptoms as treatment targets for pwPD and their CP.

Participants and Methods: PwPD/CP dyads were recruited to participate in separate focus group or questionnaire phases of a study. The pwPD were administered the Montreal Cognitive Assessment (MOCA) and were staged by study staff with the Hoehn \& Yahr scale. Focus group dyads ( $\mathrm{n}=22 \mathrm{pw} \mathrm{PD} / \mathrm{CP})$ generated potential cognitive endpoints. These endpoints were rated by a separate group of pwPD/CP dyads ( $n=50$ dyads). Separate ratings were obtained from pwPD and CP, allowing for paired comparisons of ratings.

Results: Generally, pwPD and CP ranked all cognitive symptoms as moderately important. Overall, pwPD prioritized memory and language outcomes, with $46 \%$ identifying improving name retrieval as a priority symptom. In contrast, CP emphasized executive outcomes, particularly decision-making $(50 \%)$.

Conclusions: A variety of cognitive domains are considered important potential treatment targets by pwPD and CP. Developing ecologically sensitive and valid measures to explore these phenomena and understand their impact on daily life in pwPD and CP will be important. In addition, this study highlights the importance and feasibility of partnering with pwPD and CP to improve the patient-centeredness of cognitive research projects.

Correspondence: Katrina L. Raein, PhD, Neurology, Baylor Scott \& White Health, 2401 S. 31st Street, Temple, TX 76508, United States. E-mail: katrina.raein@bswhealth.org.

S.A. ROSEN, J. BENGE \& R. PHENIS. The Verbal Series Attention Test (VSAT) as a Measure of Executive Functioning in Parkinson's Disease.

Objective: Objective: The current study seeks to examine the convergent validity, sensitivity, and specificity of performance on the Verbal Series Attention Test (VSAT) as a measure of executive functioning in patients with Parkinson's disease (PD).

Participants and Methods: Participants and Method: A retrospective chart review of 30 patients with PD who were administered the VSAT and at least two of the following: the Modified Wisconsin Card Sorting Test (MWCST) categories correct, Trail Making Test B (TMTB), and phonemic verbal fluency. The sum of the errors from the serial subtraction, months backwards, and letter/number sequencing items was utilized as a VSAT executive error score.

Results: Results: There were significant negative correlations between the VSAT executive error score and the demographically corrected scores on executive measures overall $\left(r_{\mathrm{s}}=-.51, \mathrm{p}=.004\right)$, ТМTB $\left(\mathrm{r}_{\mathrm{s}}=-.52, \mathrm{p}=.003\right)$, and MWCST $\left(\mathrm{r}_{\mathrm{s}}=-.47, \mathrm{p}=.019\right)$. A cut point of 3 errors on the VSAT executive tasks correctly classified $66.67 \%$ of PD patients as having executive dysfunction with adequate sensitivity $(63.16 \%)$ and specificity $(72.73 \%)$. A cut point of 6 errors resulted in $100 \%$ specificity.

Conclusions: Conclusion: Overall, this preliminary study demonstrates the utility of simple, brief, and motor fair tasks from the VSAT as screening measures for executive functioning in individuals with PD. Correspondence: Sabra A. Rosen, Psy.D., Neuropsychology, Baylor Scott \&. White Healthcare, 1201 Hidden Valley Drive, Apt 426, Round Rock, TX 78665, United States. E-mail: sabra.rosen@bswhealth.org. 
S. COCOZZA, T. COSTABILE, E. TEDESCHI, F. ABATE, C. RUSSO, A. LIGUORI, W. DEL VECCHIO, F. PACIELLO, M. QUARANTELLI, A. FILLA, A. BRUNETTI \& F. SACCÀ. Brain Functional Changes and Cognitive Dysfunction in Friedreich's Ataxia.

Objective: Non-motor symptoms, including depressive mood and impairment of visuospatial abilities, information processing speed and executive functions, have been described in Friedreich's Ataxia (FRDA). Aim of our study was to assess brain FC changes in FRDA by mean of resting-state functional MRI (RS-fMRI) analysis, and to test their possible correlations with neuropsychological performance.

Participants and Methods: Twenty-four FRDA patients (M/F:15/9, mean age $31.3 \pm 15.0$ ) and twenty-four healthy controls (HC; M/F:15/9, mean age $30.7 \pm 15.5)$ were enrolled in this cross-sectional study. All patients underwent a thorough neuropsychological battery, investigating different cognitive domains. FC to seeds relevant to executive and cognitive functions known to be compromised in FRDA was probed voxelwise throughout the brain. Correlations between functional and clinical data were tested by Spearman's correlation coefficient.

Results: Neuropsychological assessment showed overall worse scores in FRDA patients compared to HC in several domains, including global cognitive assessment, clear-thinking ability and non-verbal assessment of intelligence, spatial and verbal memory, visuo-perception and visuo-spatial functions, and executive functions.

Analysis of RS-fMRI data showed the presence of clusters of significant difference between FRDA patients and HC in the following areas: right medial frontal gyrus, bilateral angular gyri, cingulate gyrus and cerebellum. After Bonferroni correction, no correlation emerged between cluster of altered FC and neuropsychological tests.

Conclusions: In FRDA patients, widespread alterations of FC are present compared to HC. These results, in conjunction with clinical findings and neurofunctional tests, may shed new light on the pattern of supratentorial and infratentorial involvement and on dynamics of brain plasticity in this disease.

Correspondence: Francesco Saccà, MD, Neuroscience, Reproductive and Odontostomatological Sciences, University Federico II, Via Pansini, 5, Edificio 17, piano terra, Napoli 80131, Italy.E-mail: francesco.sacca@ unina.it

\section{R. SALAZAR, A. LARUM \& A. CRONIN-GOLOMB. Relation of Stigma Perception to Clinical and Demographic Characteristics of Parkinson's Disease.}

Objective: The stigmatizing burden of Parkinson's disease (PD) is understudied, with little known about demographic and clinical correlates or about which specific motor and non-motor symptoms contribute to stigma perception.

Participants and Methods: For 141 individuals with PD (73 men, 68 women; age range 40-91, mean 63.9), we administered an online study with self-report measures of stigma (Stigma Scale for Chronic Illness, SSCI) and PD symptoms: Non-Motor Symptom Questionnaire, MDS-Unified Parkinson's Disease Rating Scale (UPDRS), Part 1 (non-motor symptoms) and Part 2 (motor symptoms). We assessed the correlation between stigma and current age, age of PD onset, disease duration, UPDRS, and specific symptoms for the entire group and separately for men and women (Pearson r; alpha .001). T-tests compared men and women on stigma severity.

Results: Stigma perception (SSCI) was associated with younger current age, earlier age of disease onset, longer disease duration, and more severe motor and non-motor symptoms (all $\mathrm{p}<.001$ except age, $\mathrm{p}<.04$ ). Severity of individual non-motor symptoms correlated with stigma: memory, apathy, depression, falling, sleep, urinary control, constipation; the motor symptoms included, choking, handling food, dressing, bathing, doing hobbies, turning in bed, tremor, balance, and freezing gait (all p<.001). For women only, stigma also correlated with fatigue and speech problems. Men drove the PD correlations for memory, urinary control, dressing, bathing, turning in bed, and balance. Men and women did not differ for overall stigma severity.
Conclusions: PD stigma perception related to particular demographic, clinical, and symptom characteristics, which may be valuable in the conceptualization of PD treatment. Overall perceived-stigma severity was similar in men and women, but was correlated with different motor and non-motor symptoms. These findings raise the potential for interventions for PD stigma perception that may differ by age and for men and women.

Correspondence: Robert Salazar, M.A., Boston University, 648 Beacon St, 2nd Fl, Boston, MA 02215, United States.E-mail: rdsalaz@bu.edu

M. SARNO, A. BURE-REYES, N.S. BANERJEE, S. GETZ, K.F. MCINERNEY, N. SUN-SUSLOW, M. PINJALA, C. LUCA, H. MOORE, C. SINGER \& B.E. LEVIN. Is Age of Onset Related to Cognition and Mood in Essential Tremor?

Objective: Essential tremor (ET) is a common movement disorder associated with deficits in executive function, memory, and attention. Later age of onset in Parkinson's disease (PD) is well known to be linked to reduced cognition, but studies have been inconsistent regarding age of tremor onset in ET. Recent research found that early onset vs. late onset (EO vs. LO) ET cases share similar cerebellar postmortem features and do not differ significantly in terms of neuropathological changes. An examination of the influence of age of ET onset on cognition and mood is therefore warranted. This study examined whether LO ET was associated with poor cognition and mood relative to EO ET.

Participants and Methods: A neuropsychological battery was administered to 25 deep brain stimulation (DBS) ET candidates $\left(M_{\text {age }}=69 \pm 7.05\right.$; $M_{\text {education }}=13.96 \pm 3.9 ; 64 \%$ male), assessing learning/memory, visuospatial skill, executive function, working memory, and mood. EO was defined as $<50$ years $(n=15)$, and $L O$ as $\geq 50$ years $(n=10)$. LO patients were more likely to be male, older, and have a shorter symptom duration, but did not differ on education. ANCOVA, controlling for symptom duration and sex, was used to compare T-scores.

Results: The LO group performed worse than early onset participants on measures of verbal learning $F(1,21)=4.47, p=.04$ and delayed recall $F(1,21)=5.04, p=.04$, visual memory $F(1,20)=4.97, p=.04$, working memory $F(1,20)=6.46, p=.02$, and two measures of visual-perceptual ability $F(1,21)=7.93, p=.01$. The groups did not differ on measures of executive function, depression, or anxiety.

Conclusions: This study suggests that later emergence of ET symptoms is linked to neurocognitive decrements on visual and verbal learning/ memory, visual-perceptual abilities, and working memory. These findings add to current literature examining the influence of age of onset as a prognostic indicator of cognition in ET. Moreover, this study emphasizes the importance of careful test selection when assessing DBS candidacy for patients with ET.

Correspondence: Marina Sarno, Psy.D., Neurology, University of Miami, 9224 Emerson Avenue, Surfside, FL 33154, United States. E-mail: m.sarno@med.miami.edu

B.M. SCOTT, S. THOMPSON, K. WINSHIP, J.A. LAFO, M. MARSISKE \& D. BOWERS. Mixed Linear Modeling of Changes in Functional Independence in De Novo Parkinson's Disease Motor Subtypes.

Objective: To determine the relative contribution of disease variables $\&$ self-reported symptoms on perceived declines in functional independence (FI) in de novo Parkinson's disease (PD) patients in the Parkinson's Progressive Markers Initiative (PPMI). Aim 1: Identify baseline characteristics influencing mean changes in FI. Aim 2: Model the relationship between changes in FI \& self-reported symptoms over time. Based on the literature, we predict patients with greater baseline axial impairment \& disease severity will report lower baseline FI \& a steeper rate of decline. We also expect variability in both motor \& non-motor symptoms to correspond with changes in FI over time.

Participants and Methods: 244 PD patients \& 135 healthy controls (HC) enrolled in the PPMI for 3 years. PD motor phenotypes were identified via $\mathrm{K}$-means analysis with subscores derived from the 
clinician-administered Motor Exam (UPDRS-III: Baseline M=22.2). Disease severity (Hoehn \& Yahr), self-reported motor (UPDRS-II) \& non-motor (UPDRS-I) symptoms were compared via ANOVA \& Chi-square. MLM was used to model changes in FI (Schwab \& England). Results: Two PD clusters were found: Typical ( $T=172)$; Akinetic-Rigid $(A R=72)$. AR-PDs had greater disease severity \& lower FI at baseline than T-PDs. Rate of FI decline differed between groups ( $\mathrm{HC}=0.9 \%$; $\mathrm{T}=6.1 \% ; \mathrm{AR}=6.7 \%)$, but group \& baseline disease severity did not predict the average rate of change. At the group level, UPDRS-II scores were more strongly associated with FI decline than UPDRS-I scores. At the individual level, only UPDRS-I variability was significant, indicating a person-to-person difference in the influence of non-motor symptoms on FI over time. The final model explained $42-74 \%$ of the variance.

Conclusions: Average changes in self-rated FI within the first 3 years of PD onset are primarily accounted for by perceived changes in motor symptoms. However, clinicians should remain cognizant that the impact of perceived non-motor symptoms on self-reported FI varies substantially between individual patients.

Correspondence: Bonnie M. Scott, M.S., Clinical and Health Psychology, University of Florida, P.O. Box 100165, Gainesville, FL 32610, United States.E-mail:bonnie.m.scott@gmail.com

M. SPLIT, F.V. LOPEZ, C.F. PLUIM, A. TUAZON, P. GILBERT, R. MOORE, I. LITVAN, S. LESSIG, V. FILOTEO \& D. SCHIEHSER. Memory for Positive and Negative Faces and Words in Parkinson's Disease.

Objective: Parkinson's disease (PD) is a neurodegenerative disorder characterized by several neural circuit disruptions, including the basal ganglia-thalamocortical (limbic loop) pathway, which regulates emotion and memory. Consistent with the neuropathological circuitry, studies have demonstrated that emotion recognition is impaired in PD, with some evidence of greater impairment in the recognition of negative vs. positive emotions. This study sought to extend prior work by examining if a similar dissociation is observed in respect to memory for emotional verbal and facial stimuli.

Participants and Methods: Participants were PD (n=18) and ageand gender-matched normal controls $(\mathrm{NC} ; \mathrm{n}=24)$. All participants were administered tests of emotional (positive and negative) facial and word recognition memory. Statistical analyses were conducted with 2 Group (PD v. NC) $x 2$ Emotion (positive v. negative) mixed model ANCOVAs, with face and word recognition accuracy as dependent variables and education serving as a covariate.

Results: A significant main effect of emotion $(p=.035)$ revealed that both $\mathrm{PD}$ and $\mathrm{NC}$ participants remembered more positive compared to negative words; a trend interaction effect $(p=.09)$ indicated that PD participants were more accurate in their recognition of positive words, whereas controls were more accurate in recognizing negative words. There were no significant effects for face memory, although there was a trend $(p=.06)$ for both groups to be more accurate in recognizing negative compared to positive faces.

Conclusions: Results suggest that recognition memory for emotional words and faces is intact in PD. Both PD and healthy older adults recognize more positive compared to negative words and show a trend for recognizing more negative compared to positive faces. These findings have important clinical implications; positive verbal content and negative faces may be better remembered by those with PD. The development of tools for capitalizing on these relative strengths could be used in rehabilitation settings.

Correspondence: Molly Split, Veterans Affairs San Diego Healthcare System, 3350 La Jolla Village Drive, San Diego, CA 92161, United States. E-mail: molly.split11@gmail.com
J. THORN, F.C. GOLDSTEIN, D. BLIWISE \& S. FACTOR. Associations Between Hallucinations and Sleep Quality in Nondemented Patients with Parkinson's Disease.

Objective: Psychosis and sleep disturbances are common non-motor features of Parkinson's disease (PD) and are associated with decreased quality of life and development of dementia. The current study examined whether there are associations between components of sleep quality and hallucinations in nondemented PD patients.

Participants and Methods: Patients $(\mathrm{n}=126$, mean age $=65.2, \mathrm{SD}=8.7)$ with idiopathic PD were consecutively recruited from the Emory University Movement Disorders Clinics. Exclusion criteria were late stage dementia, history of cerebrovascular disease, atypical parkinsonism, and prior multiple head injuries. They were administered the Schedule for Assessment of Positive Symptoms (SAPS) to measure the global severity and types of daytime psychotic symptoms. The Neurodegenerative Disease Sleep Questionnaire evaluated the quality of sleep. Spearman Rank-Order correlations and analyses of covariance controlling for age, MMSE score, UPDRS motor score, and medications that cause hallucinations examined associations between hallucinations and sleep. Significance was set at $\mathrm{p}<.05$, two-tailed.

Results: Hallucinations were present in $33(26 \%)$ patients, with visual hallucinations the most common subtype. An increase in the severity and/or number of different types of hallucinations were significantly correlated with greater trouble falling asleep, greater frequency of nightmares, greater nocturnal urinary frequency, and poorer overall sleep quality. Patients who had hallucinations reported more difficulty falling asleep and a higher frequency of nocturnal urination. Patients with visual hallucinations reported receiving less hours of sleep per night.

Conclusions: The findings indicate that the presence and severity of daytime hallucinations are associated with the quality of sleep in nondemented PD patients, and that both comorbidities need to be worked up and treated early in the course of the disease.

Correspondence: Jakob Thorn, 12 Executive Park NE, Atlanta, GA 30329, United States.E-mail: jthorn@emory.edu

\section{A.M. VANDEBUNTE \& S. ROGERS. The Effect of Depression on} the Cognition of those with Parkinson's Disease.

Objective: Depression can be a common sequela to Parkinson's disease (PD), but it is unclear how certain features of depression affect the cognition of those with PD. This study aimed to better understand the particular profile of depression in PD and the corresponding cognitive correlates.

Participants and Methods: A total of 102 adults (31 women, $M$ age $=$ 73.66 years, $M$ education $=15.44$ years ) with parkinsonian pathology completed the BNT, COWAT, ROCF, HVLT-R, BVMT-R, and subtests of the WAIS-IV, WMS-IV, DKEFS, and Trailmaking. Features of depression were measured via the GDS.

Results: Minimal levels of state depression were endorsed by $53 \%$, mild depression by $30 \%$, and moderate-to-severe levels by $9 \%$. Significantly greater levels of the cognitive factor of depression were endorsed than the dysphoria and the withdrawal/apathy factors. There was a significant negative association between overall state depression and performance on ROCF 30' delay, as well as between the cognitive factor and scores on WAIS-IV Arithmetic, DKEFS Color Naming, Trails B, and DKEFS Inhibition. Performances on BVMT-R Delayed Recall and ROCF 3' and 30' delays were also significantly negatively correlated with the cognitive factor. Those with more of the hopelessness factor scored worse on DKEFS Color Naming, WAIS-IV Coding, DKEFS Inhibition, and HVLT-R Delayed Recall. All $p \mathrm{~s}<.05$.

Conclusions: Those with PD appear to experience more cognitive relative to other features of depression, and those with more cognitive symptoms seem to exhibit worse visual processing speed, nonverbal learning and memory, divided attention, and response inhibition. Lower visual processing speed, response inhibition, and delayed verbal recall are also characteristic of those who have greater symptoms of hopelessness. These findings highlight the distinctive symptoms of depression in 
PD and suggest that some of these features exacerbate the preexisting cognitive deficits associated with PD, whereas others alter the profile typical for PD.

Correspondence: Anna M. VandeBunte, Psychology, Westmont College, PO Box 1553, Summerland, CA 93067, United States. E-mail: avandebunte@westmont.edu

\section{Multiple Sclerosis/ALS/Demyelinating Disorders}

WITHDRAWN. D. ANAND, S. AWASTHY \& R. ANAND. Living under the Shadow of Chronic Illness: Coping and Conflicts in Multiple Sclerosis.

Objeetive: To highlight the ennotional and psyehologieal burden of MS from the perspective of patients and earegivers throught an int-depth inquiry into their experienees of living with the illness.

Partieipants and Methods: Using a qualitative approach, detailed personal interviews were conducted with 25 individuats comprising of patients and their earegivers. Themes streh as impaet on tersomal rela= tionships, eareer, edueation, fantily life, coping, alter oriffectrse and so on were touched upon in the interviews in at jiton to participants straring their own personal journey withthe the th vervien transeripts were subjected to analysis based on terph ind Phenomenologieal

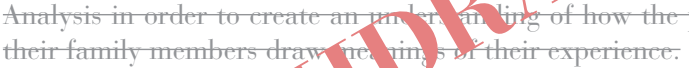

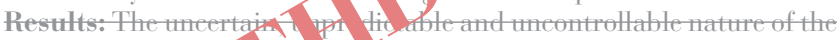
illness beeomes trec stan ontextual reality in the lives of each family member. Itis fho 1 th with a so of not freing in control of their lives and future at all and the patients teal with feelings of guilt about the change that the illness eatses in the life of their family members apart from their own pain. Gonelusions: Data obtained through the study draws out deep seated and muanced impact of MS int the life of fannily members and earegivers in addition to its impaet on patients. It was seen that living with MS weighs on the emotional equilibrium of patients and earegivers alike. Atso, different ways of making sense of the disease and its impaet was seen across and within families. Lastly, the need for integrative eare models for managing MS and other ehronic health eonditions was highlighted.

Gorrespontence. Dimita Anand, PhD, Psychotogy, Bharathiar University, 1292 Meera Bagh, india, New Delhi 110057 , India. E-mait: dimitidamand@momit.onm

E. BARLOW-KRELINA, E.A. YEH, J. O'MAHONY, R.E. GUR, R.C. GUR, K. RUPAREL, B. BANWELL \& C. TILL. Examining Executive Dysfunction as a Contributor to Verbal Reasoning Deficits in Youth and Young Adults with Pediatric-Onset Multiple Sclerosis.

Objective: Individuals with pediatric-onset multiple sclerosis (MS) frequently demonstrate deficits in core executive function (EF) skills. Given the reliance of verbal reasoning (VR) skills on intact EFs, we sought to examine the degree of concurrence between such deficits in MS patients to inform theory on processes contributing to VR impairment. Participants and Methods: 42 patients (mean age $=19.6 \pm 3.8 ; 30$ female; mean disease duration $=6.2 \pm 3.7)$ and 30 healthy controls $($ mean age $=17.7 \pm 5.2 ; 21$ female $)$ were recruited from nine children's hospitals across Canada and the USA. Participants completed the Penn Computerized Neurocognitive Battery. Accuracy scores on the Children's Verbal Reasoning Test, Go-No-Go task, Continuous Performance Test, Letter N-back task, and Conditional Exclusion Test were compared between patients and controls, with age and response time included as covariates. Accuracy on the VR task was then correlated with age at disease onset and accuracy on the impaired EF tasks in MS patients, with age at testing included as a covariate.
Results: MS patients demonstrated poorer performance than HCs on tasks of VR, inhibition, attention, and working memory, $\mathrm{p} \leq 0.010$. Groups did not differ on a measure of mental flexibility. VR performance correlated with measures of inhibition $(r=0.35)$, attention $(r=0.54)$, and working memory $(r=0.48), \mathrm{p} \leq 0.012$. Individuals with a younger age at onset demonstrated a trend toward lower VR scores $(r=0.25$, $p=0.055)$.

Conclusions: Youth and young adults with pediatric-onset MS demonstrate lower VR and EF scores than healthy peers. Although an early disease onset may lead to greater disruptions to the acquisition of knowledge and development of verbal skills, the current findings suggest that executive dysfunction in the domains of inhibition, sustained attention, and working memory may contribute to VR deficits. Longitudinal analysis of the role of executive dysfunction in moderating the development of reasoning skills is warranted.

Correspondence: Emily Barlow-Krelina, Clinical Developmental Psychology, Psychology, York University, 4700 Keele St., BSB 130, Toronto, ON M3J 1P3, Canada.E-mail: embarlow@yorku.ca

K. Chapman, D. Calvo, J. Petersen, B. POllock, H. GERHART, A. RIDGEL \& M. SPITZNAGEL. Fitness Outcomes After Starting a New Exercise Routine in Persons with Multiple Sclerosis: The Role of Cognition.

Objective: Exercise may help manage symptom severity in multiple sclerosis (MS), however, impaired cognitive functioning could reduce the learning and execution of new exercise routines in this population. The current work explored the role of cognitive function in predicting degree of benefit from a new exercise routine for persons with MS. We hypothesized that better baseline cognitive functioning would predict greater fitness outcomes.

Participants and Methods: 34 patients with MS were assigned to either an exercise program $(n=17)$ or a control group $(n=17)$. The exercisers began a new, daily aquatic group exercise routine while controls continued their typical routine. All participants completed the Paced Auditory Serial Addition Test (PASAT) at baseline, and the 2-Minute Step Test (2MST) at baseline and after one week.

Results: A linear regression to determine if baseline PASAT score moderated the relationship between intervention group and 2MST performance at post-intervention, controlling for pre-intervention 2MST performance showed the overall model $\left(R^{2}=.84, F(4,29)=38.1, p<.001\right)$ and interaction term were significant $(b=-.6196,95 \%$ CI [-1.14, -.101], $t=-2.44, p=.02$ ). Participants with lower baseline PASAT scores were more likely to show 2MST improvements after one week.

Conclusions: Contrary to expectations, individuals with better cognitive performance did not show greater fitness benefit from exercise. While possible this relationship is not present in MS, it is more likely that the PASAT instead served as a proxy for overall debilitation (i.e., those with poorer performance showed greater benefit due to higher baseline level of debilitation). Given the highly structured nature of our exercise intervention, future work should investigate if relationships differ in a more ecologically valid context.

Correspondence: Kimberly Chapman, BA, Psychological Sciences, Kent State University, 460 Munroe Falls Ave, \#20, Cuyahoga Falls, OH 44221, United States. E-mail: kchapm15@kent.edu

T. COSTABILE, A. CAROTENUTO, A. DE ROSA, R. LANZILLO, C. PANE, C. RUSSO, V. BRESCIA MORRA \& F. SACCÀ. Exploring Mental Imagery in Relapsing Remitting Multiple Sclerosis.

Objective: Motor Imagery (MI) is defined as a dynamic state during which a subject mentally simulates a given action. MI is usually studied using Parson's hand rotation test, in which participants are asked to judge the laterality of hands rotated in different angles. MI has become a promising tool in rehabilitation of motor impairments.

Our aim was to explore MI abilities in Relapsing Remitting Multiple Sclerosis (RRMS) patients, and their correlations with motor and cognitive features. 
Participants and Methods: We included 20 patients with RR-MS and 20 age, sex, and education matched healthy controls (HC). Mini-Mental State Examination, Frontal Assessment Battery and a computer-based test to assess Mental Imagery abilities were administered. EDSS, Fatigue Impact, Sleep quality and Depression were also investigated in the MS group.

Results: Significant difference in reaction times between RRMS and $\mathrm{HC}$ in general performance $\mathrm{F}(1,38)=4,91 \mathrm{p}=0.033$, task $\mathrm{F}(3,114)=9$ $p<0.001$ and orientation $F(3,114)=52,09 p<0.001$ were found. The interaction between task and orientation was also significant $\mathrm{F}(9,342)=2,68 \mathrm{p}=0.005$.

At the letter task test there was no specific difference between RRMS and HC.

At the hand rotation task we found significant differences with the right hand, while no differences between groups at all test of and rotation in front/back view of bodies.

Correlations between cognitive and different measures were observed for RRMS but not for HC. In RRMS, EDSS correlated with Mental Rotation of Hands in front view bodies $(\mathrm{R}=.471 \mathrm{p}<0.05)$; MOCA with Mental Rotation of hands $(\mathrm{R}=-.625 \mathrm{p}<0.001)$ and, finally, FAB with Mental Rotation of " $\mathrm{R}$ " ( $\mathrm{R}=-.841 \mathrm{p}<0.001)$, and Hands in both front and back view bodies $(\mathrm{R}=-.685 \mathrm{p}<0.001 ; \mathrm{R}=-.623 \mathrm{p}<0.001)$

Conclusions: RRMS patients are able to generate and manipulate MI, but require more time than HC. This impairment correlates with the degree of cognitive impairment, showing that Motor Imagery is a complex higher cognitive function that needs a deep assessment before using it as a rehabilitation tool.

Correspondence: Teresa Costabile, University of Naples "Federico II", Via S. Pansini, Naples 80131, Italy.E-mail:teresa.costabile@gmail.com

L. CRIVELLI, M.F. FAREZ, M.P. FIOL, M.C. YSRRAELIT, R.F. ALLEGRI \& J. CORREALE. Inhibitory Control Dysfunction in Early Multiple Sclerosis.

Objective: Executive dysfunction can be present early on in the course of MS. Inhibitory control, a key component of executive function, is defined as the capacity to suppress inappropriate behavior; promoting flexible responses to changing environments. The purpose of this study is to characterize inhibitory function in patients with early MS.

Participants and Methods: Patients were recruited from the outpatient MS clinic at the Raul Carrea Institute for Neurological Research (FLENI) by attending neurologists. Twenty-nine relapsing-remitting MS patients, fulfilling 2005 McDonald criteria, with less than 2 years disease duration and scores $\leq 2$ on EDSS, were included. Twenty-nine subjects matched for age, gender, and educational level, recruited from a local volunteer group, served as controls. Both groups underwent complete neuropsychological evaluation. Inhibitory control was assessed using a computerized test (STOP-IT).

Results: In line with previous reports, patients and controls differed significantly in scores obtained for the following tests: attention (Digit Symbol Test $p=0.003$ ); verbal memory (Selective Memory Test $p=0.004)$; language (Verbal Fluency $p=0.001$ ); and executive function (PASAT3 sec. $p=0.004$, WAIS III Digits backwards $p=0.03$ ). Furthermore, when tested with STOP-IT, patients presented a 33.37 msec delay in inhibition of response, compared to controls $(p=0.032)$. Although patients took longer to inhibit response, no differences were observed in likelihood of achieving response inhibition, between patients and controls $(\mathrm{p}=0.4)$.

Conclusions: Patients with recent MS diagnosis present specific inhibitory control deficits. Inhibitory control is an important component of executive functions and when altered, can have significant impact on patients' quality of life. Careful dissection of executive function into its basic components, combined with analysis of impaired domains can help individualize the design of cognitive rehabilitation strategies. Correspondence: Lucía Crivelli, Institute for Neurological Research Dr. Raúl Carrea (FLENI), Montañeses 2325, Buenos Aires C1428AQK, Argentina.E-mail: lcrivelli@fleni.org.ar
E. DE SOMMA, R. BROWN, M. SADEGHI, J. O'MAHONY, B. BROOKS, E.A. YEH, D. ARNOLD, L. COLLINS, B. AUBERTBROCHE, S. NARAYANAN, R. MARRIE, A. BAR-OR \& B. BANWELL. Disrupted cognitive development following acute demyelinating syndromes: A longitudinal study.

Objective: To determine whether monophasic acquired demyelinating syndromes (ADS) and multiple sclerosis (MS) during childhood disrupt maturing cognitive processes, specifically working memory and processing speed; and whether such failure to achieve age-expected cognitive functioning is associated with brain growth rate.

Participants and Methods: Participants included 27 youth with ADS (10 of whom were diagnosed with MS), who underwent two neuropsychological (NP) evaluations, at 6 and 24 mo post-onset. Serial MRI scans were obtained at incident demyelinating event, 3, 6, 12mo post-ADS, and yearly thereafter. Brain volume was transformed into a z-score (zbrain) using age- and sex-matched controls from the NIH pediatric dataset. Change in age-normed scores on the Symbol Digit Modalities Test (SDMT) and the WJ-III Auditory Working Memory (AWM) test were examined using repeated measures ANCOVA, controlling for IQ. General linear mixed model analyses were used to determine whether the rate of individual change in zbrain associated with change in cognitive performance.

Results: Children with MS were older at onset $(13.0 \pm 2.2 \mathrm{yrs})$ compared to monophasic ADS $(11.1 \pm 2.6 \mathrm{yrs} ; p=.063)$. Groups did not differ on NP outcomes at 6 or 24 mo (SDMT: $p=.91$; AWM: $p=.36$ ). Both groups showed a decline on SDMT ( $p=.032$; Cohen's $d=1.54)$ and AWM $(p=.009$; Cohen's $d=2.46)$ from 6 to $24 \mathrm{mo}$. A total of 122 MRI scans were analyzed (median 5 scans/patient). A negative rate of change in zbrain over a mean follow-up of $2.5 y$ rs associated with decline (from 6 to $24 \mathrm{mo})$ on SDMT $(\beta=.47, p=.023)$ and AWM $(\beta=.30, p=.010)$ in both groups.

Conclusions: Both monophasic ADS and MS confer a risk for declining age-expected performance on tests of working memory and processing speed over time that is predicted by brain growth rate. The biological processes underlying this negative impact, which occurs even in absence of chronic demyelination, has implications for all forms of ADS, and for the study of MS, in which progressive impairments have been attributed to disease chronicity.

Correspondence: Elisea De Somma, Clinical Developmental Psychology, Psychology, York University, 4700 Keele St, Toronto, ON M3J 1P3, Canada.E-mail: edesomma@yorku.ca

M.G. DI BENEDETTO \& L. STROBER. Can't Stress it Enough: Examining the Role of Personality, Coping, and Percieved Stress in Multiple Sclerosis.

Objective: Personality is presumed to play a role in how one copes with present stressors. More specifically, conscientiousness (C) and extraversion (E) have been linked with adaptive, problem-focused coping while openness $(\mathrm{O})$ is related to use of humor and positive reappraisals. Agreeableness (A) has been shown to be related to seeking support. Neuroticism $(\mathrm{N})$ has consistently been associated with emotional and avoidance coping. In the present study, we sought to examine the role of personality, coping, and perceived stress in multiple sclerosis (MS). Participants and Methods: 181 individuals with MS completed the NEO personality inventory, Perceived Stress Scale (PSS), and COPE measure. The COPE scales were divided into five domains of coping: Adaptive, Problem-focused, Avoidant, Negative emotion, and Social support.

Results: Avoidant and negative emotion coping was most associated with $\mathrm{N}$ and inversely with $\mathrm{C}$. Adaptive coping was associated with $\mathrm{O}$ and E. Problem focused coping was related to C, E, and O. Social support was related to $\mathrm{E}, \mathrm{O}$, and $\mathrm{A}$. When examining the relationship between coping and perceived stress, all were significant except a trend for problem-focused coping. However, when controlling for N, E, and A, some of these relationships were no longer significant, suggesting a major role. $\mathrm{C}$ and $\mathrm{O}$ did not have an effect on the relationship between coping and stress. 
Conclusions: At face value, coping is assumed to play a large part in how one deals with stress, such as MS. Personality is seldom taken into account when examining this relationship. Findings suggest that further attention be given to the role of personality on coping in MS and that assessment of such may be warranted.

Correspondence: Michael G. Di Benedetto, Neuropsychology and Neurology Laboratory, Kessler Foundation, 120 Eagle Rock ave, East Hanover, NJ 07936, United States.E-mail: mdibenedetto@ kesslerfoundation.org

C. HAGUE, A. TAN, K. CROCFER, P. PLUMB, B. GREENBERG \&. L. HARDER. Comparing fatigue in pediatric multiple sclerosis and transvers myelitis.

Objective: Demyelinating diseases are autoimmune, inflammatory disorders impacting the central nervous system (CNS): multiple sclerosis (MS) is a chronic condition affecting the CNS, whereas transverse myelitis (TM) is a monophasic disease of the spinal cord. Although existing research has investigated the prevalence of fatigue symptoms associated with pediatric MS and TM separately, differences in fatigue experienced by patients with these conditions has yet to be investigated. The present objective is to evaluate and compare fatigue symptoms between pediatric patients with MS and TM.

Participants and Methods: Forty-one participants with MS (mean age $=14.95)$ and 37 participants with TM $($ mean age $=11.92)$ and their caregivers completed the Pediatric Quality of Life Inventory Multidimensional Fatigue Scale (PedsQL MFS). Correlation analyses were conducted to assess the relationship between parent and self-report ratings of fatigue. A multivariate analysis of variance (MANOVA) was used to assess for differences between participants with MS and TM on self-reported symptoms of fatigue.

Results: Correlation analyses revealed significant correlations $(p<.05)$ between self- and parent-reported symptoms of fatigue. MANOVA results of self-report revealed that participants with MS experience significantly $(p<.008)$ greater cognitive fatigue than those with TM; however, groups did not differ in areas of sleep and general fatigue.

Conclusions: Results suggest that although pediatric MS patients are at greater risk for cognitively-related fatigue, consistent with having a chronic brain-based disease, the degree of fatigue endorsed by MS and TM groups in other domains is otherwise similar. This is the first study to demonstrate that pediatric patients with TM experience similar levels of fatigue as compared to individuals with pediatric MS. These findings support the need for consistent monitoring of fatigue, and other quality of life factors, in patients with both MS and TM.

Correspondence: Cole Hague, Ph.D., Psychology, University of Texas Southwestern Medical Center, 200 Treadway Plaza, Dallas, TX 75204, United States.E-mail: cdhague1@gmail.com

\section{P. HAKE, Y. GOVEROVER \& J. DELUCA. Sensory Processing in Multiple Sclerosis and its Relation to Processing Speed.}

Objective: While cognitive, motor and visual impairments have been studied in persons with multiple sclerosis (MS), other sensory processing impairments have not yet been extensively studied. The current study aims to first examine whether sensory processing impairments exist in MS, and then to examine if a relationship exists between sensory impairment and cognitive processes.

Participants and Methods: Participants, consisting of 36 individuals with MS and 21 healthy controls (HCs), were asked to complete the Adolescent/Adult Sensory Profile Questionnaire in order to measure several aspects of sensory processing. The BICAMS was used to measure cognitive processes due to its composite score being composed of tests involving processing speed (SDMT), verbal memory (CVLT-II), and visuospatial memory (BVMT-R).

Results: Participants with MS reported having significantly lower sensory registration and significantly higher sensory sensitivity compared to HCis. Lower processing speed was shown to be negatively correlated with low registration of sensory information. The total BICAMS score was not associated with sensory processing.

Conclusions: Sensory processing impairments were shown to exist in participants with MS. High sensory sensitivity causes individuals to be more easily distracted by irrelevant stimuli. Individuals with low registration tend to react more slowly, or not at all, to sensory stimuli, which may explain its correlation to low processing speed scores.

Correspondence: Patrick Hake, Neuropsychology and Neuroscience Laboratory, Kessler Foundation, 120 Eagle Rock Ave, Suite 100, East Hanover, NJ 07936, United States. E-mail:Phake@kesslerfoundation.org

\section{J. HOFFMEISTER, M.R. BASSO, L. LAU, D. COMBS \& D. WHITESIDE. Reliability and Validity of the Perceived Deficits Questionnaire in Multiple Sclerosis.}

Objective: Cognitive dysfunction affects approximately $66 \%$ of individuals diagnosed with multiple sclerosis (MS). Because of the relative inaccessibility of neuropsychological assessment services, self-report measures of cognitive dysfunction have been proposed as an inexpensive means of screening for impairment. The Perceived Deficits Questionnaire (PDQ) is a 20-item questionnaire referring to MS-related subjective complaints of cognitive dysfunction. Little research has evaluated its relationship with objective neuropsvchological function.

Participants and Methods: Participants were 97 individuals diagnosed with MS, and 43 healthy people. All participants were given a comprehensive neuropsychological battery which included domains measuring prospective and retrospective memory, attention, and planning. Participants completed the PDQ and the Chicago Multiscale Depression Inventory (CMDI).

Results: The PDQ achieved satisfactory reliability (alpha=.95). Nonetheless, after employing correction for Type I error, it achieved nonsignificant to weak relationships across neuropsychological domains, with correlations ranging from -.06 to -.31. In contrast, the CMDI achieved significant correlations with all PDQ measures, and these ranged from .27 to .63

Conclusions: Although the PDQ possessed excellent reliability, it failed to correlate with objective indicators of neuropsychological performance. Rather, the PDQ correlated significantly and consistently with self-reported emotional distress. This is consistent with extant research which shows the PDQ tends to correspond with malaise and distress (cf. Strober et al., 2016). Regardless, the PDQ is a poor substitute for neuropsychological assessment in determining cognitive status in people with MS.

Correspondence: Jordan Hoffmeister, B.S., Dept. of Psych/University of Tulsa, 800 South Tucker Drive, Tulsa, OK 74104, United States. E-mail:joh0515@utulsa.edu

A. LEBKUECHER, N. CHIARAVALLOTI \& L. STROBER. Say What?: The Role of Language and Articulatory Speed in Verbal Fluency of Patients with Progressive Multiple Sclerosis.

Objective: While cognitive deficits in memory and processing speed have been well-documented in patients with multiple sclerosis (MS), language processing is largely considered to be intact. Patient performance on verbal fluency tests varies between studies. Those that find evidence for verbal fluency deficits attribute the deficiency to impaired processing speed or executive dysfunction. This study evaluates the contribution of various cognitive factors to verbal fluency including language processing, articulatory speed, processing speed, and executive function.

Participants and Methods: 31 patients with progressive MS completed a battery of neuropsychological tests. Tests included Delis-Kaplan Executive Function System Verbal Fluency, Color-Word \& Trails, Wechsler Abbreviate Scales of Intelligence (WASI) Vocabulary, Wechsler Adult Intelligence Scales-IV Letter-Number Sequencing, and Symbol Digit Modalities Test (SDMT). We conducted two step-wise regression analyses using D-KEFS Letter and Category Fluency as outcome variables and the other listed tests as predictor variables. 
Results: WASI Vocabulary and D-KEFS Color Naming scores predicted D-KEFS Letter Fluency performance with each variable accounting for $33.5 \%$ and $14.8 \%$ of the variance respectively. Only D-KEFS Color Naming scores predicted performance on D-KEFS Category Fluency accounting for $24.7 \%$ of the variance.

Conclusions: Findings suggest a significant contribution of language processing to performance on letter fluency as evidenced by the relationship between vocabulary and letter fluency tests. Additionally, articulatory speed (Color Naming) significantly contributes to both letter and category fluency. Results also suggest that language impairment does occur in patients with MS and that letter fluency may be a more sensitive measure of this impairment than category fluency.

Correspondence: Amy Lebkuecher, Kessler Foundation, PO Box 185, Portland,PA 18351, United States.E-mail: amyllebkuecher@gmail.com

\section{L.D. MEDINA, B. WEDEMAN, E. ALVAREZ, K. NAIR \& B. HOYT. Disease Modifying Treatments in Multiple Sclerosis and Their Differential Impact on Cognition.}

Objective: Multiple sclerosis (MS) is an autoimmune disease associated with damage to myelin that leads to various pathology including cognitive changes. While the specific cause of the disease remains unknown, numerous disease modifying treatments (DMTs) have been designed to target aspects of MS pathology. However, little is known about the impact of DMTs on cognition in MS. The current study aimed to elucidate the relationship of an older injectable DMT (glatiramer acetate, or GLA) and newer oral DMT (fingolimod, or FIN) on cognition from baseline to one year follow-up.

Participants and Methods: A sample of 105 (GLA=52, FIN=53) MS patients were recruited from a large academic hospital. Patients were stable on treatment for at least two years before study enrollment. Groups did not differ in age, education level, race/ethnicity, gender, or baseline cognitive performance. All patients completed a battery assessing executive function, processing speed, memory, and verbal fluency. Patients also completed self-report quality of life outcomes measures related to physical symptoms, mental and cognitive health, and social abilities.

Results: Repeated measures analysis of covariance (ANCOVA) controlling for disease duration revealed a cognition by treatment group interaction. Although cognitive changes from baseline to follow-up were not significant between groups, FIN users demonstrated a rate of improved performances on aspects of memory and verbal fluency significantly $(p<0.05)$ better than GLA users. There were no significant between- or within-group differences on self-report measures.

Conclusions: Controlling for disease duration and compared to GLA, FIN may be associated with improved effects on aspects of memory and verbal fluency. These results suggest that more efficacious oral FIN may better mitigate, and possibly improve, the deleterious effects of MS on cognition.

Correspondence: Luis D. Medina, PhD, Neurosurgery, University of Colorado School of Medicine, 12631 E. 17th Ave., C307, Aurora, CO 80045, United States.E-mail:LUIS.MEDINA@ucdenver.edu

\section{R. NEUFELD, T. FABRI, L. SERGIO, A. WALDMAN, A. LAVERY, D. GORBET \& C. TILL. The Effect of Cognitive Load on Visuomotor Integration in Youth with Multiple Sclerosis.}

Objective: Fine motor speed and visuomotor integration are commonly impaired in patients with pediatric-onset multiple sclerosis (MS). Clinical tools used to assess visuomotor integration typically limit one's need to integrate both cognition and motor abilities concurrently. However, in daily life, visuomotor skills demand attentional resources, which can also be affected by MS. This study examined how visuomotor skills are impacted in youth with MS under conditions that place demands on attentional resources.
Participants and Methods: Participants were 10 patients with pediatric-onset MS (mean age $=15.9$ yrs, $\mathrm{SD}=2.7$ ) and 9 healthy age and sex-matched controls (mean age $=14.2$ yrs, $\mathrm{SD}=2.3$ ). A computerized Cognitive-Motor Integration (CMI) task required participants to move a cursor from a central to peripheral target appearing in one of four directions on a screen. Task complexity was varied by manipulating the congruence of the target and response planes (congruent vs. non-congruent) and by adding an inhibitory control component (same vs. reversed direction). Performance between groups for the low-load (congruent plane, same direction) and high-load (incongruent plane, reversed direction) conditions was compared using a 2x2 ANOVA.

Results: Accuracy was lower and overall reaction and movement times slower in the high-load relative to the low-load condition for both groups. Compared to controls, the MS group performed significantly worse on measures of overall reaction time and movement time in both the high and low-load conditions but did not differ in accuracy. A group by condition analysis did not suggest that findings were moderated by task difficulty. In other words, patients were not disproportionately slower in the high-load condition.

Conclusions: Findings suggest that increased cognitive demands do not create an additional burden on visuomotor integration in youth with MS. Reduced visuomotor speed appears to be an early manifestation of MS. The CMI task offers promise as a sensitive tool for visuomotor assessment in MS.

Correspondence: Raichel Neufeld, York University, 245 Palmerston Ave, Toronto,ON M6J 2J3, Canada.E-mail:raichel@my:yorku.ca

M. PITTERI, S. ZICCARDI, M. GUANDALINI, V. CESTARI \& M. CALABRESE. Switching And Clustering Performance In Verbal Fluency Tasks In A Group Of Multiple Sclerosis Patients Without Cognitive Impairment: Preliminary Investigation With Clinical Implications.

Objective: Verbal fluency tasks have been used to explore lexical and semantic strategies that can be affected in multiple sclerosis (MS) patients, showing an imbalance between "clustering" and "switching" phenomena (Troyer et al., 1998). Clustering refers to the strategy of generating words within a subcategory, whereas switching consists in jumping from one subcategory to another, requesting a semantic involvement (Mayr, 2002). The aim of this study was to verify whether qualitative measures of clustering and switching in semantic and phonemic verbal fluency tasks might reveal earlier signs of neurodegeneration related to MS.

Participants and Methods: We assessed 31 RRMS patients (Age: $36.26 \pm 7.6$ years; Education: $13.4 \pm 3.4$ years; $\mathrm{F}=24$ ) without cognitive impairment (no pathologic score on each subtest of the Brief Repeatable Battery and the Stroop test) and 39 matched healthy controls (HC). Phonemic verbal fluency was assessed with the letter "F", whereas semantic verbal fluency was assessed with the category "Animals".

Results: Considering the quantitative total score, results showed no difference between MS patients and HC with respect to total words generated both in the phonemic and semantic fluency tasks ( $p>.05)$. However, the qualitative analysis showed that MS patients produced higher number of switches $(p=.010)$ and lower clustered elements $(p=.016)$ than HC in semantic fluency, but not in the phonemic fluency task.

Conclusions: Our results indicate that semantic and phonemic fluencies involve different processes. More switches and smaller clusters in the semantic verbal fluency might be an indication of subclinical neurodegneration phenomena that can be prodromal of cognitive deficits (Sepulcre et al., 2006). The quantitative total score in fluency tasks is not sufficient to detect slight signs of cognitive deficits in MS patients and should be integrated with deeper qualitative analysis of clustering and switching phenomena.

Correspondence: Marco Pitteri, University of Verona, Piazzale L.A. Scuro 10,Verona37134, Italy.E-mail:marco.pitteri@univr.it 
J. RANDOLPH, J.S. RANDOLPH \& H.A. WISHART. Subgroup Analysis of Individuals with MS Showing Increased Resilience and Cognitive Reserve.

Objective: While many individuals with multiple sclerosis (MS) experience subjective and objective cognitive impairment, little is known about those who do not have these difficulties. A better understanding of cognitively unimpaired MS patients could provide insights into coping with this condition over time. The present study compared demographic and disease-related features of cognitively healthy MS participants with those who report or exhibit cognitive impairment to determine potentially unique characteristics of the intact group.

Participants and Methods: We compared three groups of non- or minimally depressed participants with MS: intact MS ( $N=22$; no objective cognitive deficits on standard neuropsychological testing or subjective impairment per self and informant reports); subjectively cognitively impaired (SCI) but objectively normal MS $(\mathrm{N}=25)$; and cognitively impaired (CI) MS ( $\mathrm{N}=32)$.

Results: The intact MS group was similar to other groups regarding age, gender, MS subtype, EDSS rating, symptom and disease duration, and daily hours of sleep. Intact MS participants were more educated than SCI and CI groups $(p<.01)$ and showed higher WRAT-Reading scores than the CI group $(p<.001)$. They also reported somewhat less fatigue, subclinical anxiety, and subclinical depressive symptoms (all $p<.05$ ), and endorsed slightly more compensatory cognitive strategy use than other groups, though not at a statistically significant level. Use of medications with potential cognitive side effects did not differ across groups. Conclusions: We found that a cognitively healthy MS group had fewer emotional symptoms and fatigue and higher cognitive reserve than other MS groups, despite having similar overall levels of disability and disease. These findings suggest that some individuals with MS have dispositional or other features associated with better clinical status despite otherwise similar disease characteristics.

Correspondence: John Randolph, Ph.D., Psychiatry, Geisel School of Medicine at Dartmouth, 20 W. Park Street, Suite 215, Lebanon, NH 03766, United States. E-mail: john.randolph@randolphnp.com

\section{A. RAPHAIL \& M.T. SCHULTHEIS. Examining the Impact of Cognitive and Motor Impairment on Visual Perception in Individuals with Multiple Sclerosis.}

Objective: Visual perception is an important aspect of daily life that requires intact oculomotor ability and cognition (i.e. processing speed). Multiple sclerosis (MS) affects both these domains and subsequently may lead to impaired visual search. The aim of this study was to examine the relationship between visual search, cognition, and motor in individuals with MS using reaction times and eye movements during three visual search tasks.

Participants and Methods: 19 individuals with MS and 21 age-andgender-matched healthy controls (HC) were administered neuropsychological testing and three eye-tracking tasks of varying cognitive difficulty. Task 1 required participants to look at a circle as quickly as possible. Task 2 was a simple visual search task that required them to find a green " 2 " in a matrix of different colored numbers. Task 3 was a complex visual search task in which they searched for an " $\mathrm{X}$ " hidden in real-world scenes. Saccade peak velocity (eye movement speed) and reaction times were measured.

Results: Using a $2 \times 2$ mixed ANOVA, it was found that the MS group had significantly slower reaction times than the HC group on the number and scene search tasks, $F(1,37)=7.24, p=.03$. There was no significant interaction between task and group, $F(1,37)=2.11, p=.48$. In terms of saccade peak velocity, there was no significant main effect of group, $F(1,27)=1.35, p=.78$, or task across the three tasks, $F(1.49$, $40.42)=.48, p=1$. There was no significant interaction between task and group, $F(1.49,40.42)=.030, p=1$.

Conclusions: These findings indicate that visual search is slower in individuals with MS, suggesting that they need more time to perceive and make decisions about visual information. Furthermore, given that there was no difference in saccade velocity, these slowed reaction times are likely driven by cognitive impairment more so than oculomotor deficit.

Correspondence: Ann-Marie Raphail, MS, Psychology, Drexel University, 136 N 21st St., 2R, Philadelphia, PA 19103, United States. E-mail:ar3223@drexel.edu

M. EDMUNDSON, O. SANCHEZ, P. THURAS \& G. LAMBERTY. Minnesota Multiphasic Personality Inventory-2 Restructured Form (MMPI-2 RF) Profiles in Veterans with Multiple Sclerosis.

Objective: Multiple sclerosis (MS) is a demyelinating condition that presents with diverse neurological symptoms. Given that individuals with MS may report vague, diffuse, and mood-related symptoms (e.g., pain, fatigue, depression), the diagnostic process for MS may include psychological evaluation to rule out somatic symptom disorders (SSDs) and other psychopathology. The present study examined MMPI-2 RF profiles for MS vs. SSD cases.

Participants and Methods: The study included veterans with MS $(\mathrm{n}=27)$ or SSDs $(\mathrm{n}=81)$ who had (1) completed an MMPI-2 RF or MMPI-2 since 2008, (2) diagnoses meeting McDonald (MS) or DSM-5 (SSD) criteria, and (3) neuroimaging to ensure the presence/absence of demyelinating lesions.

Results: As hypothesized, the MS group showed clinical elevations on RC1, MLS, NUC, and COG. The SSD group demonstrated more frequent and higher levels of validity scale elevation, especially for Fs-r ( $p \leq .001)$, FBS-r, and RBS ( $p \leq .05$ ), as well as a wider range of specific somatic and RC scale endorsements. However, the MS and SSD groups did not differ in their endorsement of MLS or NUC. A binary logistic regression indicated RC1 best predicted group membership.

Conclusions: Individuals with MS produce a more selective profile, whereas those with SSD show a greater level of questionable symptom reporting, more diverse somatic and psychiatric symptom endorsements, and higher RC1 elevations specifically.

Correspondence: Orlando Sanchez, Minneapolis, MN, United States. E-mail: orlando.sanchez@va.gov

S.T. SCHMIDT, J. LENGENFELDER, N. CHIARAVALLOTI \& H.M. GENOVA. Social Cognition Impairments in Multiple Sclerosis are Associated with Poor Community Integration.

Objective: Recent research has demonstrated that individuals with Multiple Sclerosis (MS) have measurable deficits in social cognition (such as impaired Theory of Mind; ToM) and can also exhibit poor community integration. Although social cognition deficits are likely associated with poor community integration, the relationship between these two variables has not yet been examined in MS. The current study examines this relationship.

Participants and Methods: 28 individuals with MS participated. Social cognition was assessed using a test of ToM ( $3^{\text {rd }}$ subtest of The Awareness of Social Inference Test; TASIT) wherein participants were asked questions about the beliefs, meaning, intentions, and feelings of actors in 16 video-taped vignettes portraying the usage of sarcasm and lying. Community integration was assessed using the Community Integration Questionnaire (CIQ), which assessed community integration across 4 domains: home, job/school, social, and participation in productive activities. Pearson correlations were calculated to determine the relationship between TASIT performance and self-reported scores on the CIQ.

Results: There was a significant positive correlation between performance on TASIT and scores on the CIQ, indicating that participants with lower scores on the TASIT reported lower integration into community. For example, reduced social integration was associated with an impaired ability to understand lying $(\mathrm{r}=.465, \mathrm{p}=.006)$, as well as sarcasm $(r=.376, p=.024)$.

Conclusions: Findings of the current study highlight the importance of social cognition on overall participation in society for individuals with MS. Our results indicate that identifying effective treatments for impairments in social cognition have the potential to positively impact 
community integration. Further research is therefore needed to determine potential treatments for deficits in social cognition, which may lead to greater community integration and better overall quality of life. Correspondence: Samantha T. Schmidt, Bachelor's of Art in Psychology , TBI, Kessler Foundation, 120 Eagle Rock Ave., \#100, East Hanover, NJ07936,United States. E-mail: sschmidt@kesslerfoundation.org

G. SPADONI, M. PRESENTI, M.R. TIMPANO SPORTIELlo \& S. TOCCHINI. Amyotrophic Lateral Sclerosis (ALS) and Primary Lateral Sclerosis (PLS): the Differences in Cognitive Functioning and in Emotional Recognition Giulia Spadoni ${ }^{2}$, Maria Luisa Presenti $^{2}$, Marco Timpano ${ }^{2}$ and Stefania Tocchini ${ }^{12}{ }^{1}$ Public Health Authority ASL Nordovest della Toscana ${ }^{2}$ University of Pisa.

Objective: Several studies show that patients with Amyotrophic Lateral Sclerosis (ASL) and with Primary Lateral Sclerosis (PLS) have a cognitive impairment, particularly in executive functions, and emotional recognition deficit; the research is still lacking in PLS. Our study aims to assess neuropsychological functioning, particulary emotional recognition in ASL and PLS patients.

Participants and Methods: 20 patients: 12 ALS (12 males), 8 PLS (5 females) and 20 (F 6) age-matched healthy controls.

We used standard a set of neuropsychological tools to asses all cognitive domains and Amyotrophic Lateral Sclerosis Functional Rating Scale (ALS-FRS) for screening physical variables. We examined the recognition of basic emotions (disgust, anger, fear, sadness and happiness) using two batteries for facial and prosody emotional recognition: Facial Emotion Recognition Battery (FERB) and the Emotional Prosody Recognition Battery (EPRB). Moreover we used a revised form of International Affective Picture System (IAPS-R) and International Affective Digitized Sounds (IADS-R) inquiring respectively the same basic emotions through complex scenes and linguistic and not linguistic sounds.

Results: The scores in executive tests were significantly lower in ALS and PLS patients than in healthy controls $(\mathrm{p}<.005)$ and within experimental group the PLS performances are better than ALS performances. Moreover, the recognition of disgust and fear facial expressions are impaired but not significantly. Impairments became statistically significant with complex stimuli (IAPS-R) in ALS patients.

Conclusions: Our results confirm an impairment in executive functions in SLA and PLS patients. Furthermore they suggest presence of dissociation in emotional recognition, between primary innate stimuli, with a direct path processing (EPRB and FERB) and complex stimuli requiring deep processing (IAPS-R and IADS-R). Finally, we hypothesized a continuum in cognitive impairment between those two disorders. Correspondence: Giulia Spadoni, , University of Pisa, Via della Libertà, 3/A, Aulla 54011, Italy. E-mail: spadonigiulia@libero.it

\section{A. SPIROU \& E. DOBRYAKOVA. Aversive Motivation is Associated with Greater Learning and Caudate Volume in MS.}

Objective: Gray \& McNaughton (2000) hypothesized that activation of the behavioral inhibition system (BIS; aversive motivational system) during novel but not overly threatening situations not only leads to heightened arousal and attention but also leads to a cautious approach towards new stimuli. The caudate has been shown to be involved in motivational tendencies, learning, and in the fast generation of strategies when introduced to novelty. Due to learning being an integral part of rehabilitative practices with clinical populations, we examined the effects of aversive motivation on learning and caudate volume in multiple sclerosis (MS).

Participants and Methods: Twenty participants with MS completed the Brief Visuospatial Memory Test-Revised (BVMT-R) to measure learning, and the Behavioral Inhibition/Behavioral Activation (BIS/BAS) scale to assess motivational tendencies. A median split was performed on the data from the BIS scale to create two groups: high (HBIS) and low (LBIS) scoring BIS MS participants. Volumetric analysis of sub-cortical structures from anatomical MRI scans was performed.
Results: Significant between-group differences were observed on each trial that measured learning of the BVMT-R (Trial 2: $\mathrm{p}=0.04$; Trial 3: $p=0.02$; Learning: $p=0.02)$ and the delayed recall trial $(p=0.01)$, with the HBIS group scoring higher than LBIS group. Volumetric analysis of sub-cortical structures showed significant between-group differences in caudate volume $(p=0.025)$, with the HBIS group having a larger caudate than the LBIS group. Significant positive correlations were found between caudate volume and BIS ( $r=.54, p=.01)$, BVMT-R Trial $2(\mathrm{r}=.46, \mathrm{p}=.04)$, and BVMT-R Trial $3(\mathrm{r}=.46, \mathrm{p}=.04)$ scores.

Conclusions: We found that individuals with MS who have higher aversive motivational tendencies also exhibit higher caudate volume and greater learning. Thus, the current study provides pioneering evidence of the positive effects of aversive motivational tendencies on learning in MS.

Correspondence: Angela Spirou, M.A., Traumatic Brain Injury Laboratory, Kessler Foundation, 9 Katharina Place, Washington Township, NJ 07676, United States. E-mail: aspirou@kesslerfoundation. org

D.J. TIRRI, J. LENGENFELDER, H.M. GENOVA \& N. CHIARAVALLOTI. Examining the Relationship between Resilience, Anxiety, Depression, and Social Integration in MS.

Objective: Examine the effect of resilience on social integration and mood in persons with multiple sclerosis (MS)

Participants and Methods: 37 individuals with clinically definite MS, (Age: $\mathrm{M}=49.8, \mathrm{SD}=6.6$; Education $\mathrm{M}=15.9, \mathrm{SD}=2.0$ ) and 21 healthy controls (HC: Age $\mathrm{M}=43.5$; $\mathrm{SD}=10.9$; Education $\mathrm{M}=16.9$, $\mathrm{SD}=1.9$ ) completed the Beck Depression Inventory-II (BDI-II), State Trait Anxiety Inventory-II (STAI-II), and the Community Integration Questionnaire (CIQ). Participants' significant others also completed questionnaires assessing social and interpersonal relationships. Resilience was measured using the 25 item Connor-Davidson Resilience Scale (CD-RISC). Items are scored on a 0-4 scale (range: 0-100), with higher scores indicating more resilience. Pearson correlations were run to determine the relationship between resilience, depression, anxiety, and social integration.

Results: The average CD-RISC score was 74.0 (SD=13.7) for the MS group and $76.1(\mathrm{SD}=13.5)$ for the HC group. There was a significant correlation between the BDI-II and CD-RISC within both the MS group $(\mathrm{r}=-.57, \mathrm{p}<.001)$ and the HC group $(\mathrm{r}=-.63, \mathrm{p}<.01$. The STAI-II was also significantly correlated with the CD-RISC within both groups (MS: $r=-.62, p<.001 ;$ HC: $r=-.84, p<.001)$. However, only the MS group demonstrated a significant correlation between the social integration subscale of the CIQ and CD-RISC $(r=.47, \mathrm{p}<.01)$.

Conclusions: Findings indicate a significant relationship between resilience and depression and anxiety, as well as social integration in individuals with MS. Additional work is needed to better understand the potentially protective role resilience may have in regard to the experience of anxiety and depression, as well as its potential role in promoting healthy socialization in persons with MS. Understanding the role of resiliency may allow us to identify those at risk for psychological distress or social difficulties. Additional work would allow the development of programs promoting resilience and increasing overall well-being in individuals with MS.

Correspondence: Dennis J. Tirri, Bachelor of Science, Kessler Foundation, 120 Eagle Rock Avenue, Suite 100, East Hanover, NJ 07936, United States.E-mail: dtirri@kesslerfoundation.org. 


\section{Stroke/Vascular Cognitive Impairment}

J. EVANS, S. BAYLAN, C. HAIG, M. MACDONALD, C. STILES, J. EASTO, M. MCGINLAY, B. CULLEN, T. QUINN, D. STOTT, S. MERCER, N. BROOMFIELD \& H. MURRAY. A Pilot SingleBlind Randomised Controlled Trial of Mindful Music Listening to Enhance Cognitive Recovery and Mood After Stroke: Feasibility, Acceptability and Effect Sizes.

Objective: Low mood and cognitive deficits are common post-stroke. Music listening is suggested to have beneficial effects on cognition, while mindfulness is effective in treating mood disorders. We hypothesised that combining these two approaches may enhance cognitive recovery and improve mood post-stroke. This study investigated the feasibility and acceptability of combining music listening with brief mindfulness exercises in a randomised controlled trial (RCT) early poststroke. Retention rates, and effect sizes for key outcome measures, were determined.

Participants and Methods: 72 ischemic stroke survivors were randomised to an 8-week intervention of: 1) mindful music listening $(\mathrm{n}=23), 2)$ music listening $(\mathrm{n}=24)$, or 3$)$ audiobook listening $(\mathrm{n}=25$, control). Measures of feasibility, fidelity, and retention were taken. Cognition and mood were assessed at baseline, 3-months and 6-months post-stroke by a blinded assessor.

Results: The interventions were acceptable and feasible to deliver ( $96.9 \%$ of treatment sessions were rated fully feasible) with excellent treatment fidelity (94.4\% fully consistent with protocol). Retention to endpoint was high $(83.3 \%)$ with $68.1 \%$ completing $\geq 6$ treatment visits. Consistent with predictions, the largest effect sizes were found for measures of verbal memory. Based on variance of change scores obtained for a key outcome measure of cognition (Delayed Story Recall), a fullscale three-arm trial would require randomisation of 312 participants to detect a clinically substantial difference in improvement (z-score difference $=0.66, p=0.017$, two-tailed, $80 \%$ power). This would also provide $87 \%$ power to detect a modest (two point) difference in mood measured using the Hospital Anxiety and Depression Scale (HADS).

Conclusions: The results support the feasibility and acceptability of mindful music listening post-stroke. A full scale trial to investigate efficacy is warranted.

Correspondence: Jonathan Evans, University of Glasgow, mental health \& wellbeing, Glasgow G12 OXH, United Kingdom. E-mail: Jonathan. Evans@glasgow.ac.uk

J. HANSEN, K. DONNELLY, J. UPSHAW, D. LEITNER, H. MILLER \& M. LIBBEN. In the Eye of the Beholder: Investigation of Attentional Patterns in Neglect through Eye Tracking.

Objective: Hemispatial neglect is defined as a failure to report, respond, or orient to stimuli on the contralesional side of space in patients who have suffered damage to the brain, and is commonly seen in stroke patients. While the consequences of neglect are well documented, few studies have investigated the specific visual attentional patterns that underlie this prevalent and debilitating syndrome. This study aimed to provide a qualitative description of neglect patients' eye movements and corresponding allocation of attention in order to enhance understanding of neglect and its subtypes.

Participants and Methods: 13 stroke patients completed a number of eye tracking tasks including a modified version of the Apples Test (to differentiate between allocentric and egocentric neglect), single and multiple line-bisection tasks, and a compound word task. Specific attentional patterns of neglect were investigated using eye tracking metrics representing location and duration of patients' eye movements. ANOVAs were conducted using Dwell Time and First Fixation Duration data to contrast early vs. late attention processes respectively.

Results: Across all tasks, probability of prolonged fixation across the page decreased from right to left in patients with egocentric neglect. Initial fixations, however were not significantly different from those without neglect. Both ego- and allocentric participants showed a processing benefit for compound words as opposed to non-words.
Finally, single word compounding errors were significantly more likely in allocentric neglect participants.

Conclusions: Results indicate a potential sparing of preattentive/early visual processing in neglect, while later, more controlled mechanisms are impaired. Additionally, there was an association between allocentric neglect and lexical errors typically associated with neglect dyslexia. Further investigation into specific patterns of spatial allocation of attention in neglect patients is vital to understanding the disorder and improving rehabilitative strategies.

Correspondence: Jessica Hansen, Psychology, University of British Columbia Okanagan, 11275 Darlene rd, Lake Country, BC V4V1Y4, Canada.E-mail:jessicahansen@mail.com

A. JAYWANT, J. TOGLIA, F. GUNNING \& M. O'DELL. The Clinical Utility of a 30-Minute Cognitive Screening Battery in Acute Inpatient Stroke Rehabilitation.

Objective: The National Institute of Neurological Disorders and Stroke (NINDS) and Canadian Stroke Network (CSN) have proposed a 30-minute standardized cognitive screening battery for use in vascular disease. The clinical utility of this battery is unknown in persons with stroke in acute inpatient rehabilitation. We evaluated the internal consistency, sensitivity to cognitive impairment, and predictive validity of a modified version of the NINDS-CSN battery, including analysis of individual tests within the battery.

Participants and Methods: A prospective cohort of 100 inpatients with mild-moderate stroke. The NINDS-CSN battery was administered within three days of admission and comprised the Symbol-Digit Modalities Test (SDMT), FAS, Animal naming, Trail Making Test (TMT) A \& B, and the Hopkins Verbal Learning Test-Revised (HVLT). We determined the percentage of our sample that was classified as impaired on each test, defined as a normative score $\leq 1.5$ standard deviations. Relative functional gain in rehabilitation was assessed using the Functional Independence Measure.

Results: The NINDS-CSN battery had strong internal consistency (Cronbach's $\alpha=.82$ ). All tests demonstrated sensitivity to cognitive impairment, with the SDMT, TMT-B, and HVLT-Delayed Recall being most frequently impaired $(64 \%, 62 \%$, and $63 \%$ respectively). The SDMT and HVLT-Delayed Recall were most frequently impaired in individuals who scored in the normal range on a brief cognitive screen. Mean performance on the battery predicted functional gain during rehabilitation $(p<.05)$, with the SDMT and Animal naming demonstrating the strongest association with rehabilitation gain.

Conclusions: The NINDS-CSN battery demonstrates strong internal consistency, sensitivity to cognitive impairment, and association with rehabilitation gain in persons with stroke. Individual tests comprising the battery have varying sensitivity and predictive validity, with the SDMT emerging as the most clinically useful.

Correspondence: Abhishek Jaywant, Weill Cornell Medicine, 713 Halsey St, \#2, Brooklyn, NY 11233, United States.E-mail: abj2006@med. cornell.edu

N. JOHNSON, R.K. HEATON, I. FLORES, A. UMLAUF, C. BAUM, A. WONG, A. YOUNG, J.J. MANLY, A. HEINEMANN \& M. MARQUINE. Racial differences in rates of return to work poststroke.

Objective: We investigated racial differences in rates of return to work among non-Hispanic (NH) Black and White stroke survivors, including differences in factors explaining these disparities (i.e., socio-demographic and stroke characteristics, and cognitive, motor and emotional functioning).

Participants and Methods: Participants were one-hundred-forty stroke survivors from two academic medical centers and one rehabilitation center, who self-identified as NH Black $(n=74)$ or White $(n=66)$, and reported being employed prior to stroke (For overall cohort: Age: $M=54.4, S D=11.4$; Education: $M=14.5, S D=2.5 ; 51 \%$ male; minimum 1 year post-stroke). Cognitive, motor, and emotional functioning were 
assessed via the NIH-Toolbox T-scores; cognitive and motor scores are adjusted for age, gender, education, language, and race/ethnicity; emotion scores are unadjusted but normalized and census weighted.

Results: Blacks were less likely to return to work (34\%) than Whites $(53 \% ; p=.021)$. Blacks also obtained lower scores on the NIH-Toolbox measures of Fluid Cognition $(p=.045)$ and motor functioning (i.e., locomotion, $p<.001$ ), and reported more negative emotions (i.e., negative affect, $p=.049$ ). A multivariable logistic model showed that racial differences in return to work were no longer significant after adjusting for cognitive, motor, and emotional factors. In the final multivariable model, fluid cognition and locomotion were independent significant predictors of return to work, while race and negative emotional functioning were not significant.

Conclusions: We provide evidence that Blacks are less likely to return to work post-stroke than Whites. This disparity was accounted for by cognitive and motor factors, but causal links remain unclear. Correspondence: Neco Johnson, Psychological and Brain Sciences, Washington University in St. Louis, 2632 Park Ave, Apt A, St. Louis, MO 63104, United States. E-mail: necoxjohnson@gmail.com

\section{R.P.C. KESSELS, S. GEURTS, V.I.H. KWA \& S.P. VAN DER WERF. Accelerated Long-Term Forgetting in Patients with TIA or Minor Stroke: A More Sensitive Measure for Detecting Vascular Cognitive} Impairment?

Objective: Cognitive changes after minor stroke or TIA occur. Studies described a 'vascular' cognitive profile with spared episodic memory, although many patients report memory complaints. It has been suggested that long-term forgetting rates are a more sensitive measure to detect subtle memory impairment. This study examined whether abnormal long-term forgetting is present in patients with minor stroke or TIA by comparing the one-week delayed recall and recognition performances with that of a healthy control group.

Participants and Methods: 30 patients (17 men) who had either experienced a TIA or a minor stroke 0.5-6 months prior to the assessment were included. Age- and education-adjusted performance on the Dutch version of the Rey Auditory Verbal Memory Test (RAVLT) had to be within the normal range. 28 healthy participants $(15$ men) were matched on sex, education level and age. All participants performed the standard RAVLT (immediate recall + 20-30-min delayed recall), followed by a 1-week long-term delayed test. Participants also completed the MetaMemory Questionnaire (MMQ).

Results: Results revealed equal performances on the standard delayed memory tests, but steeper forgetting rates on free recall for the patient group compared to the control group over the prolonged period $(F(1,56)=10.8, p=0.002)$, but not on recognition $(\mathrm{U}=438.5, \mathrm{Z}=0.29$, $\mathrm{p}=.77)$. However, patients did not report more memory worries than controls on the MMQ subscales Ability $(\mathrm{F}(1,53)=0.95, \mathrm{p}=0.34)$ and Contentment $(\mathrm{F}(1,53)=1.49, \mathrm{p}=0.23)$.

Conclusions: This study is the first to investigate long-term recall forgetting in patients with cerebrovascular disease, showing that accelerated forgetting rates may be a more sensitive measure in detecting memory difficulties after minor stroke or TIA than traditional memory assessments. Possibly, reduced effort, attention and mnemonic strategies contribute to subtle consolidation problems, although this does not necessarily result in daily complaints.

Correspondence: Roy P.C. Kessels, PhD, Donders Institute for Brain, Cognition and Behaviour, Radboud University, Montessorilaan 3, DCCNRP, Nijmegen 6525 HR, Netherlands.E-mail: r.kessels@donders.ru.nl

\section{K.A. LEHOCKEY \& W. GARMOE. Patients Participating in Inpatient Cardiac Rehabilitation and Stroke Programs are Similarly Impaired on a Brief Cognitive Screening.}

Objective: Although cardiac conditions and procedures have been associated with cognitive deficits in the literature, there is a risk of overlooking possible impairment in those without known brain injury. Given the multifaceted demands of inpatient rehabilitation, it is especially important to determine a patient's current level of cognitive functioning to allow for optimal recovery. The aim of the current study was to examine the possible presence of cognitive impairment in inpatients participating in cardiac rehabilitation. An additional aim was to compare this group's performance to a group of inpatients with known brain injury that is typically associated with cognitive impairment (i.e., stroke).

Participants and Methods: Data were obtained from 158 adult inpatients at the MedStar National Rehabilitation Hospital (NRH). Inpatients were grouped by primary diagnosis on admission: 113 Cardiac and 45 Stroke. Patients were administered the Orientation Log (O-Log) and Cognitive Log (C-Log) within one week of their admission to NRH as a part of their initial neuropsychological evaluation. Scores on these measures served as an estimate of overall gross cognition.

Results: Independent samples t-tests were conducted to compare O-Log and C-Log performance across two inpatient rehabilitation patient groups (i.e., Cardiac and Stroke). There was no significant difference in O-Log scores between Cardiac $(M=29.09, S D=1.29)$ and Stroke $(M=29.00, S D=1.55), t(156)=-.13, p=.896$. There was also no significant difference in C-Log scores between Cardiac Rehabilitation $(M=23.68, S D=3.56)$ and Stroke $(M=22.47, S D=3.84), t(156)=1.89$, $p=.06$.

Conclusions: The Cardiac group was not found to be significantly different from the Stroke group in orientation or gross cognition. Importantly, means for both groups were indicative of clinically impaired gross cognition. Findings highlight the importance of cognitive assessment for inpatients participating in cardiac rehabilitation services.

Correspondence: Katie A. Lehockey, Ph.D., Psychology, MedStar National Rehabilitation Hospital, 102 Irving St., NW, Washington, DC 20010,United States.E-mail:Katie.A.Lehockey@MedStar.net

\section{RAMÍREZ, B. SALDAÑA, F. GÓNGORA \& X. ORTIZ. Decline in Semantic Verbal Fluency is Associated with Cerebrovascular Risk Factors.}

Objective: The current study measures verbal fluency performance, both at baseline and after two years, in healthy old adults with cerebrovascular risk factors (CVR) and with no risks (NR).

Participants and Methods: Sixty old adults (38 women) with a mean age of $69 \pm 5$ years and $14 \pm 4$ years of education were studied, of which 30 had hypertension and/or diabetes (CVR group) and $30 \mathrm{did}$ not (NR group). No significant differences were found in sex, age and education between both groups ( $p>.05$ ). At baseline, all participants scored above the MMSE clinical cut-off, reported no depression or anxiety, had competence for their activities of daily living and had no prior history of Stroke. Semantic and Phonological Verbal Fluency tests were administered at baseline and after two years. Number of correct responses (CR), switches (SW), hard switches (HS), cluster switches (CS) and mean cluster size (MC) were analyzed. One tailed t-tests were used to analyze decreases in performance between both sessions, two tailed t-tests were used to analyze differences in performance between both groups.

Results: There were no differences between groups at baseline and after two years in any of the measures of the phonological task, nor in the number of CS and MC of the semantic task ( $p>.05)$. After two years, the CVR group showed a decrease in number of CR $(18.9 \pm 3.5$ vs $17.1 \pm 3.6$, $\mathrm{t}(29)=2.0601, \mathrm{p}=.02), \mathrm{SW}(8.3 \pm 2.2 \mathrm{vs} 7.4 \pm 2.3, \mathrm{t}(29)=2.0759, \mathrm{p}=.02)$ and HS $(5.3 \pm 2.8$ vs $4.3 \pm 1.9, \mathrm{t}(29)=1.8223, \mathrm{p}=.04)$ on the semantic task, compared to their baseline performance. And compared with the NR group, the CVR group had a lower number of CR $(17.1 \pm 3.6$ vs $19.7 \pm 3.9$, $\mathrm{t}(58)=2.6345, \mathrm{p}=.01), \mathrm{SW}(7.4 \pm 2.3$ vs $8.8 \pm 2.4 \mathrm{t}(58)=2.3959, \mathrm{p}=.02)$ and HS $(4.3 \pm 1.9$ vs $5.7 \pm 3.0, t(58)=2.0922, p=.04)$ on the semantic task at follow up, but not at baseline ( $\mathrm{p}>.05)$.

Conclusions: Cerebrovascular risk factors are associated with the decline of semantic verbal fluency, specifically to hard switches, its executive sub-component. This measure could be used to predict vascular dementia. 
Correspondence: Diego Ramírez, Bachelor, Psychology, Universidad Autónoma de Nuevo León, Guerrero 109, San Nicolás 66400, Mexico. E-mail: diegogz95@gmail.com

\section{Visuospatial Functions/Neglect/Agnosia}

L. HIZEL, A. MARION, S. AMINI, R. DAVIS, D. PENNEY, D.J. LIBON \& C.C. PRICE. Post clockface latency as an indicator of executive functioning in Parkinson's disease.

Objective: The command condition of the Clock Drawing Task requires numerous cognitive functions: declarative memory, language, inhibitory function, working memory, visuoconstruction, and planning. The copy condition is associated with fewer cognitive demands. We hypothesized drawing the face of a clock and continuing to the next stroke (termed Post Clock Face Latency; PCFL) requires individuals to utilize working memory, inhibitory, and memory abilities. We hypothesized these abilities are more involved in the command condition than the copy condition. This was examined within a sample of individuals with non-demented Parkinson's disease (PD) and non-PD peers.

Participants and Methods: A total of 120 participants (77 individuals with Parkinson's disease: 43 non-PD peers) completed clock drawing using a digital clock drawing tool that captures behavior 82 times per second. Inclusion criteria: no dementia or stroke, over seven years of education, and no other neurodegenerative disorder. Regression analyses evaluated hypothesized relationships between PCFL (recorded in seconds), inhibitory function (TMT B-TMT A), processing speed (WAIS-III PSI), and visual memory (ROCF) abilities for copy and command conditions.

Results: 113 participants (73 PD; 40 controls) met inclusion and were utilized in analyses. Following corrections for age and education, command PCFL related to mental flexibility $\left(\mathrm{R}^{2}=.06, F(1,108)=6.78\right.$, $p=.01)$ and visual memory $\left(\mathrm{R}^{2}=.14, F(1,79)=13.54, p<.01\right)$. No relationships were found with age, education, group membership, or remaining cognitive abilities for the command condition. No significant relationships with PCFL were found in the copy condition for the entire sample.

Conclusions: Findings suggest frontal lobe dysfunction (e.g. switching, cognitive slowing) and visuospatial memory contribute to longer PCF latency times. Additionally the greater precision of the dCDT supports utilization of technology in future neuropsychological investigations. Funding: NIH R01 N5082386. NSF13-543

Correspondence: Loren Hizel, M.S., Clinical and Health Psychology, University of Florida, $1810 \mathrm{NW} 23 \mathrm{rd}$ Blvd Unit 160, Gainesville, FL 32605, United States.E-mail: Ihizel@phhp.ufl.edu

\section{SAADATPOUR, H. GLAZER, L. DOTY \& K.M. HEILMAN. A Case} of Posterior Cortical Atrophy with Vertical Neglect.

Objective: Posterior cortical atrophy (PCA) is a neurodegenerative disease that presents with visuospatial deficits, dyslexia and features of Balint's svndrome. The most common underlying pathology is Alzheimer's disease. PCA has been associated with signs of horizontal neglect in the literature; however, the presence of vertical (altitudinal) neglect has not vet been reported in a patient with PCA or other forms of neurodegenerative diseases. Here we report a patient with PCA who showed signs of upper altitudinal spatial neglect on the vertical line bisection and cancellation tests.

Participants and Methods: Our case was a 70 yo man with progressive visuospatial problems since one year ago. His symptoms started with difficulty reading, judging the distances while driving as well as difficulty playing cards. On his neurological examination, he had optic ataxia, oculomotor apraxia and prosopagnosia. His vertical line bisection showed upward deviation in three attempts. When asked to mark open squares on a cancellation test, he did not mark squares on the upper half of the paper. On his Mini-Mental Status Exam (MMSE), he had a hard time copying the intersecting pentagons. His brain MRI showed mild bilateral temporal and occipital lobe atrophy.

Results: This is a reported case. Therefore, we do not present any results.

Conclusions: Inattention to the lower part of the space is often associated with parietal lobes lesions whereas upper visual field neglect is mostly due to ventral occipito-temporal lesions. The presence of vertical neglect may not only be important for localization of neuronal dysfunction in neurodegenerative diseases but also have functional implications in patient's daily life. Unawareness to a portion of space may be responsible for serious injuries Therefore, we suggest that line bisection and cancellation tests be given to patients being evaluated for neurodegenerative diseases.

Correspondence: Leila Saadatpour, MD, Neurology, University of Florida, 3230 SW Archer RD, APT\# B110, Gainesville, FL 32608, United States.E-mail: leila.saadatpour@neurology.ufl.edu

\section{PM Coffee Break}

$$
\text { 3:15-3:30 p.m. }
$$

\section{Symposium 10. Teleneuropsychology Coming of Age in Clinical and Research Applications}

\section{Chair: Munro Cullum}

\section{Discussant: Robert L. Kane}

Presenters: Lana Harder, Bert Vargas

$$
\text { 3:30-5:00 p.m. }
$$

M. CULLUM, L. HARDER, B. VARGAS \& R.L. KANE. Teleneuropsychology Coming of Age in Clinical and Research Applications.

Telehealth continues to grow, allowing the expansion of specialty services to rural and underserved areas. Given the growth of telehealth practices, the expansion of technology in test development, and initial research supporting its utility, teleneuropsychology has a bright future, though our field has been slow to embrace the exciting potential of this assessment medium. Through five related talks, this symposium will provide an overview of practical issues, clinical and research applications, current evidence base in adults and children, novel programs, and the future of teleneuropsychology.

1) Practical and Ethical Issues in Teleneuropsvchology (Harder and Cullum). Practical considerations of setting up a teleneuropsychology operation, along with adaptations to traditional assessment procedures, and a discussion of ethical considerations and practice guidelines will be covered.

2) Evidence Based Teleneuropsvchology in Adults (Munro Cullum). Following a review of the literature, results of our recent psychometric studies in the remote assessment of older individuals will be summarized, along with patient satisfaction data

3) Feasibility, Validity, and Patient Satisfaction of Teleneuropsychology in Pediatric Populations (Lana Harder). A home based pediatric teleneuropsychology study will be presented, showing similar results obtained between tele- and traditional assessment conditions and high satisfaction ratings.

4) Teleconcussion (Bert Vargas). A telemedicine based sideline assessment program utilizing a telemedicine robot allowing remote, real-time audiovisual interaction will be covered, in addition to an overview of a comprehensive sports concussion telemedicine program.

5) Future Directions (Robert Kane). A synopsis of the state of the art of teleneuropsychology procedures and paradigms related to assessment and rehabilitation will be provided, in addition to a glimpse 
into future technologies that will guide the further development of teleneuropsychology.

Correspondence: Munro Cullum, PhD, Psychiatry, Univ. of Texas Southwestern Medical Ctr., Neuropsychology Section, UTSW, 5323 Harry Hines Blvd, Dallas, TX 75390-8846, United States. E-mail: munro.cullum@utsouthwestern.edu

\section{CULLUM \& L. HARDER. Practical and Ethical Issues in Teleneuropsychology.}

This talk will provide attendees with an introduction to teleneuropsychology technology, including practical considerations of setting up a teleneuropsychology operation and a review of different models of teleneuropsychology practice. Adaptations to traditional assessment procedures are sometimes required, although patients readily adapt to the videoconference-based testing environment as demonstrated by a growing number of studies and reviews. Given that this is an emerging area, presenters will discuss key ethical considerations and initial practice guidelines will be presented. Questions that will be covered include: What does a teleneuropsychology setup look like and what are the key practical considerations in developing a teleneuropsychology program? What tests work best and what modifications need to be considered when doing remote assessment? What training is required to practice teleneuropsychology? What are the HIPAA issues? Issues regarding practicing across state lines, and reimbursement will also be addressed. Correspondence: Munro Cullum, PhD, Psychiatry, Univ. of Texas Southwestern Medical Ctr., Neuropsychology Section, UTSW, 5323 Harry Hines Blvd, Dallas, TX 75390-8846, United States. E-mail: munro.cullum@utsouthwestern.edu

\section{CULLUM. Evidence-Based Teleneuropsychology in Adults.}

A growing literature supports the feasibility, reliability, and validity of remote teleneuropsychology-based assessment, although the adoption of this technology in our field requires an evidence-based approach. Following a brief history and review of the literature, results of several of our recent studies using common neuropsychological tests in the evaluation of older individuals will be summarized. Results have shown highly significant $(\mathrm{P}<.001)$ correlations between test results obtained via in-person vs. remote video teleconference conditions, with a mean correlation of approximately .80 across measures of global cognition. attention, naming, verbal fluency, verbal learning/memory, and visuospatial function. We have shown similar results among urban, rural, and geropsychiatric populations, and we will present new data supporting the validity of teleneuropsychology in the assessment of older adults with and without cognitive impairment. Special considerations for remote assessment of older individuals will be discussed, along with data regarding patient satisfaction with teleneuropsychology procedures. Correspondence: Munro Cullum, PhD, Psychiatry, Univ. of Texas Southwestern Medical Ctr., Neuropsychology Section, UTSW, 5323 Harry Hines Blvd, Dallas, TX 75390-8846, United States. E-mail: munro.cullum@utsouthwestern.edu

\section{HARDER. Feasibility, Validity, and Patient Satisfaction of Teleneuropsychology in Pediatric Populations.}

Teleneuropsychology applications in children have been limited, but this approach to assessment offers the potential to expand services to outlying areas and promote access to specialty care. This presentation will include data from the first home-based pediatric teleneuropsychology study. Participants, ages 7 to 18 years, were recruited from a clinic serving pediatric patients with rare CNS demyelinating conditions who traveled long distances (mean=213 miles) to receive care. They completed a neuropsychological assessment at an in-person session and via videoconference from home. Results demonstrated no significant differences in results obtained between assessment conditions. Additionally, results indicated that participants and caregivers found the experience to be satisfactory, felt comfortable with the technology, and most indicated that they did not have a preference between the in person and videoconference sessions. Results support the use of teleneuropsychology in children and adolescents and offer promising findings for clinicians and researchers.

Correspondence: Lana Harder, PhD, Psychiatry and Neurology, Children's Medical Center Dallas/UT Southwestern, 1935 Medical District Drive, CH 9.01, Dallas, TX 75235, United States. E-mail: lana. harder@childrens.com

\section{B. VARGAS. Teleconcussion Assessment.}

Sports-related concussion (SRC) is a hot topic. Unfortunately, there are significant gaps in access to concussion evaluation and treatment expertise especially in rural and underserved communities. As many state concussion laws mandate evaluation and eventual return-to-play (RTP) by a provider with expertise in concussion, many individuals could benefit from remote telemedical access to neurobehavioral concussion specialists. This presentation will describe a telemedicine-based sideline assessment program utilizing a telemedicine robot allowing remote, realtime audiovisual interaction with Northern Arizona University college football players suspected of having a concussion. Results from the only feasibility study evaluating the accuracy of these assessments will be discussed. Additional data from other SRC telemedicine programs will also be presented. Using current evidence, a comprehensive SRC telemedicine program spanning acute injury evaluation, to rehabilitation and recovery, to eventual RTP will be discussed.

Correspondence: Bert Vargas, MD, Dept. of Neurology, UTSW 5323 Harry Hines Blvd, Dallas, TX 75390, United States. E-mail: Bert. vargas@utsw.edu

\section{Paper Session 12. Traumatic Brain Injury Moderator: Maria T. Schultheis}

$$
\text { 3:30-5:00 p.m. }
$$

R.A. BERNIER, E.C. GROSSNER, E.K. BRENNER, M. CADDEN \& F.G. HILLARY. Diminishing returns in network plasticity: thresholds for hyperconnectivity as a predictor of cognition after moderate and severe TBI.

Objective: The current literature examining the relationship between functional connectivity $(\mathrm{FC})$ and cognitive functioning remains equivocal, with both positive and negative associations between $\mathrm{FC}$ and performance reported in individuals with moderate and severe traumatic brain injury (TBI). Previous investigations of functional activation during episodic memory tasks revealed a quadratic relationship between activation and performance in TBI (e.g., Strangman et al., 2008). However, similar relationships have not been examined. This study seeks to determine the best model fits explaining the relationship between connectivity with the default mode network (DMN) and cognition.

Participants and Methods: To test whether a quadratic relationship better explained the relationship between DMN FC during a one-back task and cognition compared to a linear relationship, performance on cognitive measures was regressed on FC in the DMN, defined using a functionally defined atlas (Power et al., 2011), during task, and the square of DMN FC in 17 individuals with TBI (Glasgow Coma Scale 3-12 or positive neuroimaging) 1 year post injury.

Results: Within individuals with TBI, both a linear $\left(\mathrm{r}^{2}=.25, \mathrm{p}=.049\right)$ and a quadratic relationship $\left(r^{2}=.43 ; p=.006\right)$ between DMN FC during task and TMTB were significant, such that FC increases predicted higher performance on Trails B to a point, until this relationship plateaued $[\mathrm{F}(15,2)=14.09, \mathrm{p}<.001]$. A similar pattern was observed with two other cognitive measures (Trails A and Digit Span Forward).

Conclusions: After TBI, increased DMN FC during task is associated with better cognitive functioning to some threshold whereby this relationship plateaus. A broader implication of this relationship might be 
that any cost (e.g., metabolic cost) associated with maintaining increased FC is no longer accompanied by increased benefit. These findings are discussed in the context of heightened brain metabolism in TBI and increased risk for neurodegenerative disease associated with TBI. Correspondence: Rachel A. Bernier, B.A., Psychology, The Pennsylvania State University, 419 Bruce $V$. Moore Building, University Park, PA 16802, United States. E-mail: rub221@psu.edu

U.M. VENKATESAN \& F.G. HILLARY. Functional Connectivity within Lateral Posterior Parietal Cortex and its Alteration in Traumatic Brain Injury.

Objective: Spatially distributed functional brain networks show points of regional convergence ("hubs"), such as lateral posterior parietal cortex (PPC), a region critical to heteromodal processing. Traumatic brain injury (TBI) alters global connectivity patterns, but little is known about its effect on local hub environments. We examined PPC functioning in relation to distributed network connectivity and cognition in moderate-severe TBI.

Participants and Methods: Individuals with TBI $(\mathrm{n}=18)$ and healthy controls $(n=19)$ underwent resting-state functional MRI and neurocognitive testing. Seed-voxel and graph theoretical techniques were used to characterize global and local functional connectivity patterns of right/left PPC. Local functional organization of PPC was examined with modularity analyses. Graph metrics and cognitive measures were correlated to investigate behavioral relationships.

Results: Compared to controls, TBI showed increased connectivity from PPC to remote right default mode and frontoparietal control regions, and within right PPC itself. Across groups, there was right>left connection strength and right<left betweenness centrality, a measure of hubness, within PPC. In TBI, PPC strength negatively correlated with auditory attention span, while global efficiency, a measure of network integration, negatively correlated with attentional reaction time. Although magnitude of modularity was similar across groups, modular structure showed less interindividual variability in TBI.

Conclusions: Findings substantiate hyperconnectivity on both global and local levels after TBI, and propose a special role for local right hemispheric connectivity in facilitating large-scale information transfer. Greater consistency of local functional organization within TBI also suggests shared neurologic response mechanisms despite clinical heterogeneity. Importantly, local network organization in TBI has consequences for cognitive functioning, potentially reflecting a need to balance functional resource demand with network efficiency.

Correspondence: Umesh M. Venkatesan, Psychology, Pennsylvania State University, 500 Angell Street, Apartment 707, Providence, RI 02906, United States. E-mail: umi414@gmail.com

S. HAJ-HASSAN, R. DUNN, S. RESLAN \& T. GOLA. The RBANS Effort Scale and Effort Index: Base Rates in an Inpatient Rehabilitation Setting.

Objective: The Effort Scale (ES) and Effort Index (EI) of the RBANS were developed to assist clinicians in discriminating patients who demonstrate adequate effort from those who do not. No existing research has examined performances on these measures in persons with acquired brain injury (ABI) within an acute inpatient rehabilitation setting. Using a combined sample of individuals with stroke and traumatic brain injury (TBI), base rates of failure on the ES and EI were calculated.

Participants and Methods: Participants included 54 patients $(74.1 \%$ male, $51.9 \%$ TBI) who completed routine neuropsychological assessment during their inpatient rehabilitation stay. Patients with expressive or receptive language deficits were excluded. Traditional cut-scores for the ES and EI were calculated to determine the base rate of failure (i.e., a cut-off of $>12$ was used to determine poor effort on the ES, while a cut-off of $>3$ was used for the EI).

Results: Findings demonstrated that $24.1 \%$ of the total sample met criteria for poor effort on the ES, and $20.4 \%$ met criteria for poor effort on the EI. When examined by injury type, participants with TBI met criteria for poor effort at an increased rate on the ES $(21.43 \%)$ compared to performance on the EI. In contrast, participants with stroke met criteria for poor effort at an increased rate on the EI $(30.77 \%)$ compared to performance on the ES.

Conclusions: Findings suggest indices of effort on the RBANS in an acute inpatient setting may lead to individuals with ABI being disproportionately identified as giving suspect effort. Although feigned impairment cannot be ruled out, motivation to intentionally feign impairment was expected to be relatively low in this sample. Future research should examine the role of factors prevalent in an inpatient setting such as medication side effects, fatigue, pain, and insomnia to clarify their contribution to performance on the ES and EI. Additionally, future research is needed to determine if different cutoffs would lead to greater specificity in this population.

Correspondence: Shereen Haj-Hassan, Rehabilitation Institute of Michigan/Wayne State University, 261 Mack Blvd., Detroit, MI 48201, United States. E-mail: shereen.hassan@comcast.net

A.L. CLARK, S.F. SORG, V.C. MERRITT, K.J. BANGEN, K.A. HOLIDAY, N.D. EVANGELISTA, M.W. BONDI, D. SCHIEHSER \& L. DELANO-WOOD. Blast Exposure is Associated with Anterior Cortical Thinning in Veterans with Mild Traumatic Brain Injury. Objective: Many Veterans with mild traumatic brain injury (mTBI) are exposed to blast while on deployment. Blast exposure — even at low levels - has been linked to gray and white matter brain changes, yet few studies have examined associations with cortical thickness. Therefore, we sought to clarify whether regional differences in cortical thickness exist between those with mTBI who were and were not exposed to blast. We hypothesized that blast-exposure would be associated with increased cortical thinning, especially in vulnerable frontal regions where blast waves may concentrate.

Participants and Methods: 89 mTBI Veterans underwent neuroimaging, neuropsychological testing, and completed the post-traumatic stress (PTSD) inventory. Participants (mTBI+blast, $\mathrm{n}=60$; mTBI-blast, $\mathrm{n}=29$ ) were compared on cortical thickness values derived from the following regions of interest (ROIs) using FreeSurfer: middle frontal gyrus (MFG), inferior frontal gryus (IFG), orbitofrontal cortex (OFC), and anterior cingulate cortex (ACC).

Results: ANOVAs revealed the groups were comparable on basic demographics, psychiatric symptoms, and injury characteristics. ANCOVAs controlling for age, number of TBIs, and PTSD symptoms revealed that the mTBI+blast group showed thinner cortices of the bilateral MFG $(p$ 's $<.05)$ and right IFG $(p=.01)$ relative to the mTBI-blast group. No significant differences between groups were observed for the right and left $\mathrm{OFC}$ and ACC $(p ' s>.05)$. Across the sample, regression analyses adjusting for age and PTSD revealed that thinner MFG cortices were associated with poorer executive functioning ( $p$ 's $<.05)$.

Conclusions: Findings suggest that Veterans with mTBI and blast exposure may be at especially high risk for negative brain and behavioral changes. Future longitudinal studies are needed in order to determine whether blast exposure exacerbates mTBI-related brain changes or independently negatively influences brain structure.

Correspondence: Alexandra L. Clark, M.S., UCSD/SDSU Joint Doctoral Program in Clinical Psychology, 3350 La Jolla Village Dr. , San Diego, CA 92161, United States.E-mail: alexandra.leigh.cl@gmail.com

M.J. BRAY, J. COTTRELLE, B. COLELLA, M. BAYLEY \& R. GREEN. Shifting Perspectives on Post-Traumatic Brain Injury Psychosis: Evidence for a Novel Neurodegenerative Hypothesis.

Objective: The symptomatology of post-injury psychosis suggests an etiology associated with degenerative change in the hippocampi. Progressive hippocampal decline post-TBI may contribute to dysregulation of dopaminergic networks implicated in psychosis. This study examines the novel hypothesis that hippocampal degeneration may contribute to the etiology of post-TBI psychosis. 
Participants and Methods: Secondary analysis of data from a study in patients with moderate-severe TBI was undertaken. For $\mathrm{N}=50$ participants, quantitative MRI was performed 5 and 12 months post-TBI and a scale of psychotic symptom severity (PAI-SCZ-P) was completed at 12 months post-TBI.

Results: Volumetric decline in the right hippocampal head and tail between 5 and 12 month time points was found to correlate positively with the PAI-SCZ-P at 12 months, controlling for injury severity and family history of psychotic disorder $(r=-0.326, p<0.05$ and $r=-0.484$, $\mathrm{p}<0.01$ respectively).

Conclusions: Though relatively rare, psychosis is one of the most grievous psychiatric consequences of TBI. Results suggest progressive hippocampal atrophy decline may be a risk factor for psychosis post-TBI. Validation of this novel hypothesis may present an opportunity for preventative treatments, such as allocentric spatial navigation and environmental enrichment paradigms, which show promise for preventing post-TBI hippocampal decline.

Correspondence: Michael J. Bray, Institute of Medical Sciences, University of Toronto, 550 University Avenue, Room 11-207, Toronto, ON M5G2A2, Canada.E-mail:michael.bray@mail.utoronto.ca

J.R. VANUK, S. BAJAJ, S. BERRYHILL, A. ALKOZEI \& W.D. KILLGORE. Short-Wavelength Light Therapy as a Way of Improving Sleep, Cognition, and Amygdala-Prefrontal Connectivity Following a Mild Traumatic Brain Injury.

Objective: Disruptions in sleep, limbic network function, and increases in symptoms related to anxiety have been reported in association with the occurrence of a mild traumatic brain injury (mTBI). Evidence suggests that there is a relationship between improvements in neurobehavioral impairments and more regulated sleep architecture, but the relationship between recovery rate following a mTBI and the presence of anxiety symptoms is not well understood. Blue light therapy (BLT) has been shown to be an effective means of entraining and stabilizing the circadian rhythm and improving sleep. Here we explored the effect of a six-week course of BLT on the functional connectivity between the medial prefrontal cortex (mPFC), a region involved in regulating emotions, and the amygdala, a region well known to play a role in emotional responses.

Participants and Methods: Twenty-six adults with self-reported sleep disturbances subsequent to a documented mTBI occurring within the preceding 18 months were recruited to receive either BLT or a placebo amber light therapy (ALT) for 30-minutes each morning for a six-week period. Participants underwent resting state functional magnetic resonance imaging (fMRI) and neurocognitive testing at baseline and again at the conclusion of treatment.

Results: BLT was found to be associated with significant increases in functional connectivity between the left amygdala and $\mathrm{mPFC}(p=.021)$, whereas no change was observed for ALT. The increases in connectivity for BLT were associated with significant decreases in sleep onset latency $(p=.046)$, state anxiety $(p=.035)$, perceived invincibility $(p=.034)$ from baseline to post treatment.

Conclusions: Blue light therapy may provide an effective method for regulating the sleep-wake cycle and improving cognition and emotion among individuals recovering from mTBI. Better sleep may serve to strengthen the functional connectivity between the mPFC and amygdala, thereby improving emotional functioning.

Correspondence: John R. Vanuk, Bachlors of Science, Psychiatry, University of Arizona, 733 S. Herbert Ave., Tucson, AZ 85701, United States.E-mail:johnnyv@psychiatry.arizona.edu

\section{Paper Session 13. Alzheimer's Disease}

\author{
Moderator: Duke Han
}

3:30-5:00 p.m.

\section{HAN, C.P. NGUYEN, N. STRICKER \& D.A. NATION. Detectable Neuropsychological Differences in Early Preclinical Alzheimer's Disease: A Meta-Analysis.}

Objective: The development of methods for in vivo detection of cerebral beta amyloid retention and tau accumulation have been increasingly useful in characterizing preclinical Alzheimer's disease (AD). While the association between these biomarkers and eventual AD has been demonstrated among cognitively intact older adults, the link between biomarkers and neurocognitive ability remains unclear.

Participants and Methods: We conducted a meta-analysis to test the hypothesis that cognitively intact older adults would show statistically discernable differences in neuropsychological performance by amyloid status (amyloid negative $=\mathrm{A}-$, amyloid positive $=\mathrm{A}+$ ). We secondarily hypothesized a third group characterized by either CSF tau pathology or neurodegeneration, in addition to amyloidosis $(\mathrm{A}+\mathrm{N}+$ or Stage 2$)$, would show lower neuropsychology scores than the amyloid positive group $(\mathrm{A}+\mathrm{N}-$ or Stage 1$)$ when compared to the amyloid negative group. Pubmed, PsychINFO, and other sources were searched for relevant articles, yielding 775 total sources. After review for inclusion/ exclusion criteria, duplicates, and risk of bias, 61 studies were utilized in the final meta-analysis.

Results: Results showed A+ was associated with poorer performance in the domains of global cognitive function, memory, language, visuospatial ability, processing speed, and attention/working memory/executive functions when compared to A-. A+/N+ showed lower performances on memory measures when compared to $\mathrm{A}+\mathrm{N}$ - in secondary analyses based on a smaller subset of studies.

Conclusions: Results support the notion that neuropsychological measures are sensitive to different stages of preclinical AD among cognitively intact older adults. Further research is needed to determine what constitutes meaningful differences in neuropsychological performance among cognitively intact older adults.

Correspondence: Duke Han, PhD, ABPP-CN, Family Medicine, University of Southern California, 1000 S. Fremont Avenue, Unit 22, HSA Building A-6, 4th Floor, Room 6437A, Alhambra, CA 91803 , United States. E-mail: Duke.Han@med.usc.edu

V. LA CORTE, M. ABRAM, S. FERRIEUX, A. MICHON, M. TEICHMANN, B. DUBOIS \& P. PIOLINO. Patterns of cognitive prospection impairment in early Alzheimer's disease and semantic dementia.

Objective: In the last decade a consistent number of studies has shown cognitive prospection deficits in neurodegenerative disease in particular in Alzheimer's disease (AD) and in Semantic dementia (SD). Nevertheless there is a clean cut in the literature between studies focused on prospective memory (PM) (i.e remembering to perform a planned action in the future) and investigations of episodic prospection capacity (EP) (i.e the ability to foresee personal events in the personal future). The principal aim of the study is to investigate the cognitive mechanisms underlying PM and EP capacity as a function of different temporal distance in early AD and in SD.

Participants and Methods: We administered a virtual reality task to assess PM functions by asking to participants (both groups of patients and a group of healthy elderly subjects) to execute different types of actions in a virtual town and the Temporally Extended Episodic autobiographical memory task, in which participants they had to foresee personal specific events in episodic future (TEEAM task) (Abram et al. 2014) to assess EP capacities.

Results: Within the PM task AD patients were deeply impaired in time-based action, but they show significantly better performance in event-based action, when they are asked to directly act in the virtual 
environment. Conversely SD patients showed preserved performances in time-based action in the context of impaired performance in linked event-based PM. Furthermore, results in the TEEAM task show a double dissociation in function of the temporal distance: $\mathrm{AD}$ patients were impaired in near temporal context whereas SD patients were mostly impaired in distant future thoughts.

Conclusions: Altogether these finding suggest a possible dissociation within cognitive prospection between prospective memory and episodic future thoughts. We will discuss the findings in the light of a new theoretical account that predicts the increasing role of semantic memory as a function of temporal distance in prospection (TEDIFT model, La Corte \& Piolino, 2016).

Correspondence: Valentina La Corte, Ph.D, Université Paris Descartes, 1Institute of Psychology, University Paris Descartes, Sorbonne Paris Cite, France, 71 avenue Edouard Vaillant, 92774, Boulogne 92774, France.E-mail: valentina.lacorte@gmail.com

M.D. GRILLI, A.A. WANK, J.J. BERCEL \& L. RYAN. Evidence of Reduced Autobiographical Memory Richness in Individuals at Increased Genetic Risk of Alzheimer's Disease.

Objective: Alzheimer's disease (AD) can elude clinical detection for years, not noticeably impacting cognition or daily behavior until brain pathology is well advanced. The discovery of more sensitive, preclinical cognitive indicators of $\mathrm{AD}$ risk could lead to earlier intervention, before the disease has advanced beyond an optimal window for effective treatment. With this goal in mind, we sought to determine whether episodic autobiographical memory, which is a type of memory that taxes brain regions compromised early in $\mathrm{AD}$, might provide a previously undetected preclinical marker in otherwise healthy individuals at increased risk of $\mathrm{AD}$.

Participants and Methods: The study sample included 15 cognitively healthy middle-aged and older adults who were carriers of the ef allele of the apolipoprotein E gene and thus at increased risk of AD, and 15 matched non-carriers of e4. In addition to neuropsychological testing, participants completed an experimental task that required retrieving memories for unique life events from remote and recent time periods. In order to measure episodic autobiographical memory integrity, we then scored these memories for episodic details and semantic details using a well-validated protocol.

Results: All participants performed normally on standard neuropsychological assessment. Nevertheless, in comparison to the non-carriers, the e4 carriers generated autobiographical memories that contained significantly fewer episodic details. The e4-related memory weakness was not affected by memory remoteness or detail subtype (i.e., spatiotemporal context, perceptual, or emotion), and it was not alleviated by additional episodic cueing.

Conclusions: Our results suggest that reduced episodic richness of autobiographical memory may be an early warning sign of AD risk, possibly because this type of memory taxes the functionality of a neural network that is vulnerable to AD-related abnormalities.

Correspondence: Matthew D. Grilli, PhD, Psychology, University of Arizona, 1503 E. University Blvd., Tucson, AZ 85721-0068, United States.E-mail:mdgrilli@email.arizona.edu

V.A. GUZMAN, A. NARKHEDE, B. RIZVI, J.M. COLON, J.J. MANLY, N. SCHUPF, R. MAYEUX \& A.M. BRICKMAN. Is the Alzheimer's disease-associated Increase in Regional White Matter Hyperintensity Volume due to Wallerian Degeneration?

Objective: White matter hyperintensities (WMH), typically considered MRI markers of small vessel cerebrovascular disease, are elevated in individuals with and at risk for Alzheimer's disease (AD), particularly when distributed in the parietal lobes. Some have argued that this regional distribution points to a neurodegenerative etiology, such that parietal WMH reflect Wallerian-like degeneration secondary to tau pathology and not to small vessel disease. We tested this hypothesis by determining whether the relationship between cortical atrophy and parietal lobe WMH is different in individuals diagnosed with mild cognitive impairment (MCI) or AD versus unimpaired controls.

Participants and Methods: Five hundred sixteen older adults (mean age $=74.2+/-5.8 ; 56 \%$ women) classified as impaired (MCI, n=85/clinical AD, $n=10)$ or controls $(n=421)$ received 3T MRI scans. Regional WMH and cortical thickness in key $\mathrm{AD}$-related regions were quantified. We first determined whether WMH and cortical thickness differed between the two groups; next we examined the relationship between cortical thickness and parietal WMH, and whether this relationship varied as a function of diagnostic group.

Results: Impaired individuals had greater WMH burden, particularly in parietal lobes (Group $\mathrm{x}$ Region interaction, $\mathrm{F}=2.75, \mathrm{p}=0.04$ ), and lower cortical thickness in key AD-related areas (main effect of Group, $\mathrm{F}=15.35, \mathrm{p}<0.001)$. Lower cortical thickness was associated with higher parietal lobe WMH $(\beta=-2.49, p=0.002)$, but the interaction between cortical thickness and impairment status on parietal WMH was not significant $(\mathrm{p}=0.80)$.

Conclusions: We confirmed the increased regional WMH in individuals with and at risk for AD. Although this WMH increase was associated with cortical thinning, the relationship between the two was similar in individuals with impairment and those without. The findings speak against the hypothesis that parietal WMH reflects Wallerian degeneration secondary to cortical AD pathology.

Correspondence: Vanessa A. Guzman, Masters of Science, Psychology, Fordham University, 365 West End Ave, Apt 203, New York, NY 10024, United States.E-mail:vguzman4@fordham.edu

L.S. GAYNOR, R. CURIEL, A. PENATE, M. WICKLUND, D. LOEWENSTEIN \& R.M. BAUER. Translational object discrimination task and associated imaging variables improve prediction of early cognitive impairment related to $\mathrm{AD}$.

Objective: Early accumulation of Alzheimer's disease (AD) pathology in the medial temporal lobe results in episodic memory and perceptual deficits, including impaired object discrimination (OD). OD is supported by the perirhinal cortex, a component of the parahippocampal gyrus (PHG). This study sought to determine if performance on a translational OD task and structural measures of MTL atrophy improved detection of mild cognitive impairment (MCI) in an ADRC sample.

Participants and Methods: Seventy-two subjects from the 1Florida Alzheimer's Disease Research Center (Cognitively Normal [CN] $=17$; preMCI=8; amnestic MCI=33; $\mathrm{AD}=14$ ) completed the Object Recognition and Discrimination Task (ORDT), the Hopkins Verbal Learning Test, Revised (HVLT-R), and the NACC Delayed Paragraph Recall (NACG-DPR). Measures of volume and cortical thickness of memory-relevant structures (hippocampal cortex [HPC], inferior temporal gyrus [ITG], entorhinal cortex [ERC], and PHG) were derived from brain MRI using ADNI-specified FreeSurfer segmentation.

Results: ORDT, HVLT-R, and NACG-DPR performances all showed significant positive correlations with TICV corrected volumes of left and right HPC, left ERC, left and right PHG, and thickness of left and right ERC and left ITG (all $\mathrm{p}<0.05$ ). Binary logistic regressions were then conducted to determine whether inclusion of the ORDT or imaging variables improved discrimination of $\mathrm{CN}$ v. a mild impairment group (preMCI, aMCI) over the standard use of HVLT-R and NACC-DPR. In these regressions, Block 1 included one or both traditional measures, age, and education, and Block 2 included ORDT or structural MRI data added in a Forward Likelihood Ratio Method. Overall classification accuracy was increased by $9.1 \%$ with ORDT, and $14.3 \%$ with right PHG volume.

Conclusions: Inclusion of the ORDT and right PHG volume improved diagnostic discrimination of MCI v. CN patients, improving detection of individuals who may benefit from timely therapeutic intervention. Correspondence: Leslie S. Gaynor, Neuropsychology, Clinical and Health Psychology, University of Florida, 25 SW 5th Terrace, Apt 4331, Gainesville, FL 32601, United States.E-mail: lesliesg2@ufl.edu 
C.J. VESPERMAN, L. LAW, J. OH, R. DOUGHERTY, D. EDWARDS, R. KOSCIK, C. GALlaGHER, C. CARLSSON, H. ROWLEY, B. BENDLIN, S. ASTHANA, M. SAGER, B. HERMANN, S.C. JOHNSON, D. COOK \& O.C. OKONKWO. Cardiorespiratory Capacity Attenuates Age-Associated Aggregation of White Matter Hyperintensities: Findings from the Wisconsin Registry for Alzheimer's Prevention.

Objective: White matter hyperintensities (WMHs) are associated with cognitive decline and contribute to Alzheimer's disease (AD). WMHs are caused by cerebral small vessel disease, which is related to cardiovascular disease (CVD), and are more prevalent with increasing age. Habitual physical activity has been shown to lower the risk of CVD, promote cognitive health, and lessen the burden of AD-related brain alterations in older adults. Accordingly, the aim of this study was to determine whether habitual physical activity, as indexed by cardiorespiratory capacity, attenuates age-related accumulation of WMHs in middle-aged adults at risk for AD.

Participants and Methods: One hundred and seven cognitively healthy, late-middle aged adults (age $=64.18 \pm 5.85$ years, $65.4 \%$ women) from the Wisconsin Registry for Alzheimer's Prevention, participated in this study. Participants performed graded maximal treadmill exercise testing, and peak oxygen consumption (VO2peak, $\mathrm{ml} / \mathrm{kg} / \mathrm{min}$ ) was used as the measure for cardiorespiratory capacity. Participants also underwent 3D structural MRI scanning, including T1, T2, and FLAIR sequences. WMHs were quantified using Lesion Segmentation Toolbox. Linear regression, adjusted for relevant covariates, was used to examine relationships between age and WM lesion volume, as well as determine the extent to which the association was attenuated by VO2peak.

Results: There was a strong association between age and WMHs in the cohort $(p=<0.001)$. Importantly, there was a significant interaction between age and VO2peak $(p=0.017)$. Simple main effects analyses revealed that the effect of age on WMHs was significant in the Low VO2peak group $(p<.001)$ but not in the High VO2peak group ( $p=.358)$. Conclusions: In a cohort at risk for AD, increased cardiorespiratory capacity abates the adverse impact of age on WMHs. This suggests that participation in regular physical activity can improve cerebrovascular health, potentially decreasing future risk of $\mathrm{AD}$

Correspondence: Clayton J. Vesperman, Geriatrics, University of Wisconsin Madison, 8439 Stage Road, Lancaster, WI 53813, United States.E-mail:vesperman2@wisc.edu

\section{Paper Session 14. Neurophysiology and Neuroimaging Markers}

\section{Moderator: Scott A. Langenecker}

$$
\text { 3:30-5:00 p.m. }
$$

\section{R. GEVA. Electrophysiological Changes and Attention Correlates Following Preterm Birth.}

Objective: The effects of prenatal adversity and preterm birth on the integrity of early maturing neural networks are not well understood. We aimed to evaluate brainstem electrophysiological functions in infants born very preterm and explore its cognitive and socio-emotional correlates later on in development.

Participants and Methods: A large sample of low-risk infants born preterm ( $\mathrm{N}=183$, gestation age range: $29-36.1$ weeks) and birth weight $830-2644 \mathrm{~g})$. They were recruited and evaluated at the neonatal intensive care unit soon after birth (on average at gestation week 34.1) using a neurobehavioral evaluation and an auditory brainstem evoked response test $(75 \mathrm{~dB}$ hearing level, at a $10.1 \mathrm{~Hz}$ rate). They were then enrolled in a prospective follow up study. At 9 years-of-age a subsample ( $\mathrm{N}=44)$ was tested using EEG and their attention was evaluated using gaze tracking paradigms.
Results: Data showed that $37.5 \%$ of the evaluated cases had compromised brainstem functions at birth. At nine years of age changes in EEG were noted in alpha and in theta wave spectrums on EEG in the compromised group as compared with the typical group. Specifically higher EEG frontal alpha power $(p<0.003)$ and lower frontal theta power $(p<0.007)$ was seen along with particular attention regulation differences $(p<0.046)$ in the children that were diagnosed with compromised neonatal brainstem dysfunction.

Conclusions: Prematurity seems to double the risk for atypical electrophysiological activity in early maturing brainstem pathways at birth. These deficits are later related to long-term changes in brain electrical activity along with attention regulation changes, evident through the first decade of life and possibly there after.

Correspondence: Ronny Geva, PhD, Psychology, Bar Ilan University, Gonda BRain Research Center, Bar Ilan University, Ramat Gan 52900020, Israel.E-mail: Ronny.Geva@biu.ac.il

T.L. MCKINNEY, H. OKABE \& M. EULER. The Moderating role of Uncertainty in Fluid Intelligence and Neural Activity.

Objective: Fluid intelligence (Gf) bears on many neuropsychological tests, yet its theoretical and neural basis are poorly understood. This study tests a theoretical model of Gf, where it is proposed that Gf is the ability to respond adaptively despite uncertainty. In turn, specific neural processes should relate to Gf under different types of uncertainty. Using a choice decision time (DT) task with concurrent EEG, temporal uncertainty (TU) was manipulated by changing the target stimulus' time of onset, while spatial uncertainty (SU) was manipulated by varying its spatial location. It was hypothesized that pre-stimulus processes such as alpha phase $(\alpha)$ and pre-motor potentials (MP) may relate to TU, while consistency of post-stimulus neural processing (NCon) should relate to $\mathrm{SU}$

Participants and Methods: Fifty-six healthy undergraduates were administered the WAIS-IV followed by a choice-DT task with concurrent EEG recording. The Perceptual Reasoning Index was used to operationalize Gf. Multilevel modeling was used to examine effects of TU and SU on DT and the EEG indices, as well as whether they moderate task-Gf and brain-Gf relations.

Results: While overall DT was related to Gf $(p=.005)$, neither TU nor SU preferentially influenced that relationship. In contrast, both NCon $(p=.047)$ and $\alpha(p=.018)$ were more related to Gf under SU conditions. While the MP was affected by both SU $(p=.002)$ and TU $(p=.03)$, neither type of uncertainty influenced its relationship with Gf. Conclusions: Greater uncertainty increased the relations of $\alpha$ and NCon with Gf, supporting the current hypotheses. However, while overall DT was related to Gf, greater uncertainty did not strengthen this relationship. This overall pattern suggests dissociable effects of uncertainty on neural versus behavioral correlates of Gf. These results further inform the nature and neural basis of Gf, and its functional significance in neuropsychological assessment.

Correspondence: Ty L. McKinney, PhD of Clinical Psychology, Psychology, Univeristy of Utah, 975e 400s \#10, Salt Lake City, UT 84102, United States. E-mail: ty.mckinney.412@gmail.com

H. OKABE, T.L. MCKINNEY, E. VACCARIELLO \& M. EULER. EEG Correlates of Mental Exertion During Sustained Reaction Time Performance.

Objective: Valid cognitive assessment presumes sufficient examinee effort. However, as time on a task increases so does mind-wandering. making it more difficult to sustain good performance. EEG studies have implicated mid-frontal theta activity in mental exertion and posterior alpha in mind-wandering. However, their contributions to task performance as a function of task difficulty and duration are less well understood. This study sought to further clarify the influence of these factors on EEG indices of task engagement. 
Participants and Methods: Fifty-seven undergraduates underwent EEG recording while performing a choice decision time (DT) task with three difficulty levels, across eight blocks. Alpha-power $(\sim 8-12 \mathrm{~Hz})$ was calculated from pre-stimulus parieto-occipital sites and theta power $(\sim 4-8 \mathrm{~Hz})$ from fronto-central post-stimulus sites. Difficulty was manipulated by changing the number and location of possible response targets. Effects of difficulty and task duration on DT, DT variability (DTSD), and alpha/theta power were examined.

Results: DT generally decreased and became less variable across blocks (both $p s<.005)$, and was shortest $(p<.001)$ and most variable $(p<.001)$ in the simplest condition. Alpha power increased across blocks $(p=.028)$ and tended to relate inversely with change in DTSD $(r=-.243, p=.069)$. In contrast, theta power showed no effect of block $(p>.05)$ but did vary with task difficulty $(p=.001)$, being higher in easier conditions. Last, increased theta over time tended to predict sustained good DT performance $(r=-.25, p=.057)$.

Conclusions: The increase in alpha power across time is consistent with its role in mind-wandering and the latter's impact on performance. Surprisingly, theta findings suggested that the least difficult condition requires the most mental effort, potentially because of its simplicity. These findings collectively suggest a more nuanced view of how task characteristics (subjective difficulty, duration, etc.) relate to mental effort and in turn task performance.

Correspondence: Hidefusa Okabe, BA, GRECC, VA Boston Healthcare System, 150 South Huntington Street, Jamaica Plain, MA 02139, United States.E-mail: okabe@fas.harvard.edu

J.L. WOODARD, B.J. HANSEEUW, L. DRICOT, L. QUENON, R. LHOMMEL \& A. IVANOIU. Unique and Shared Contributions of Imaging Biomarkers to Prediction of Cognitive Functioning.

Objective: Relationships between imaging biomarkers of Alzheimer's disease (AD), which are differentially sensitive to structural and metabolic aspects of the disease process, and performance in different cognitive domains have recently started to be investigated. In this study, we evaluated the relative unique and collective contributions of three imaging biomarkers for predicting cognitive performance.

Participants and Methods: Participants were 31 controls, 72 persons with mild cognitive impairment, and 29 persons with subjective cognitive impairment. Biomarkers were fluorodeoxyglucose (FDG) positron emission tomography (PET), amyloid PET standardized uptake value ratio using ${ }^{18} \mathrm{~F}$-flutemetamol, and mean bilateral entorhinal cortex thickness (EC) using structural magnetic resonance imaging. Multiple regression analyses included age and biomarkers as predictors, and mean z-scores across measures of memory, executive, language, visuospatial, and global cognitive functioning as outcome measures. Squared semi-partial correlations assessed each biomarker's unique contribution. Results: All three biomarkers significantly $(p<.01)$ predicted memory performance (total $\mathrm{R}^{2}=0.38$ ), but age did not. EC uniquely accounted for $12 \%$ of the variance, followed by $5 \%$ for FDG, and $3.5 \%$ for Amyloid PET while shared variance between biomarkers explained the remaining $17.5 \%$. FDG and age predicted executive functioning (total $\mathrm{R}^{2}=0.19$ ), while FDG, EC, and age each predicted language (total $\mathrm{R}^{2}=0.30$ ), and global cognitive functioning (total $\mathrm{R}^{2}=0.40$ ). Where significant, FDG uniquely accounted for $8-10 \%$ of the variance, followed by $5-7 \%$ for EC, and $3-6 \%$ for age. None of the biomarkers nor age predicted visuospatial performance.

Conclusions: A combination of three imaging biomarkers sensitive to differing aspects of the disease process in $\mathrm{AD}$ uniquely predicted memory performance independently of age. EC accounted for the most unique variance in memory, while FDG accounted for the most unique variance in language, executive, and global cognitive functioning.

Correspondence: John L. Woodard, Ph.D., Psychology, Wayne State University, 5057 Woodward Ave, 7th Floor, Psychology Department, Northville, MI 48202,United States.E-mail:john.woodard@wayne.edu
E.D. BIGLER \& M.A. SKILES. The Impact of FreeSurfer Version on Volumetric Analysis.

Objective: Automated methods like FreeSurfer (https://surfer.nmr. mgh.harvard.edu/) have revolutionized quantitative neuroimaging analyses. With the release of a FreeSurfer version 6.0, it is crucial to assess its comparability to the previously widely used version 5.3.

Participants and Methods: The current study used data from 412 participants in the ongoing Chronic Effects of Neurotrauma Consortium (CENC) Observational Study to compare the volumetric outputs of versions 5.3 and 6.0 across various regions of interest (ROI).

Results: ROI volumes derived from FreeSurfer 6.0 resulted in increased total brain volume including both cortical gray and white matter volumes $(p<0.05)$. In contrast, subcortical gray matter ROI volumes were decreased with version 6.0 except for the volume of the globus pallidus, which was significantly increased $(p<0.05)$. It was anticipated that there would be absolute volumetric differences between 5.3 and 6.0 due to modifications in classification and segmentation algorithms; however, if the differences are systematic between the two versions, in relation to a "ground-truth" principle, like age-mediated volumetric decline the two should be comparable for correlational analyses. To test that hypothesis, correlation between age and ROI volumes were examined comparing version 5.3 with 6.0. In general, correlational analyses resulted in similar regression slopes with smaller structures displaying the greatest inconsistency between versions. Although correlations did not differ significantly for most ROIs, the globus pallidus did result in a significantly different regression slope from 5.3 to 6.0 with the slope of 6.0 being much less pronounced.

Conclusions: FreeSurfer versions demonstrated systematically different absolute volumes indicating that they cannot be used interchangeably. However, when performing correlational analyses, considerable agreement was observed with 5.3 and 6.0, suggesting that Freesurfer 5.3 and 6.0 can be used interchangeably for correlational analyses in neuropsychological studies.

Correspondence: Marc A. Skiles, Neuroscience, Brigham Young University, 20014 53rd st SE, Snohomish, WA 98290, United States. E-mail: skiles.marc@gmail.com

B. RIZVI, A. NARKHEDE, M. BUDGE, J.M. COLON, C. HALE, K. IGWE, J.J. MANLY, N. SCHUPF, R. MAYEUX \& A.M. BRICKMAN. Fiber Tract-Defined Regional White Matter Hyperintensities and Memory.

Objective: The distribution of white matter hyperintensities (WMH) and its relation to cognition has not been studied comprehensively. Although WMH have a propensity for frontal and parietal distribution, the extent to which certain classes of white matter fiber tracts are affected by WMH may be more relevant to cognitive outcomes in aging. The purpose of this study was to examine the relationship of WMH severity within association, projection, and commissural fiber bundles with memory, and to determine if these relationships are independent of hippocampal volume and cortical thickness.

Participants and Methods: T1-, T2-weighted, and neuropsychological evaluation were conducted among 485 community-dwelling older adults (mean age $=73.84$ ). Total WMH volume was computed and regional tract volumes were derived by segmentation with a white matter tract mask. WMH volumes were computed within fiber tracts defined $a$ priori as association, commissural, and projection, and adjusted for the total volume of each tract bundle. Global cortical thickness and hippocampal volume were also derived. Analyses controlled for age, sex, race/ethnicity, education, and intracranial volume.

Results: Increased relative WMH volumes in association $(\beta=-0.142$, $p=0.001)$, commissural $(\beta=-0.088, p=0.032)$, and projection $(\beta=-0.149, p<0.001)$ tracts were associated with poorer memory performance. However, only relative WMH volumes in association tracts $(\beta=-0.115, p=0.005)$ and in projection tracts $(\beta=-0.111, p=0.008)$ were associated with memory performance after controlling for global cortical thickness and hippocampal volume. 
Conclusions: These findings highlight the importance of the regional distribution of WMH in cognition among older adults. White matter hyperintensities in association and projection fibers may be more relevant for memory functioning than WMH in commissural tracts. Correspondence: Batool Rizvi, Taub Institute, Columbia University Medical Center, 630 W 168th Street, PH 18-301, New York, NY 10032, United States.E-mail:br2499@cumc.columbia.edu

V.J. WILLIAMS, A. KOENIG, C. WENNICK, D.H. SALAT \& S.E. ARNOLD. Increased Task-related fMRI Activation During a Hyperinsulinemic-Euglycemic Clamp Procedure.

Objective: A growing body of evidence suggests significant overlap in risk, co-morbidity, and putative pathophysiological mechanisms across Type 2 Diabetes and Alzheimer's Disease. Although the effect of systemic insulin resistance on peripheral tissue is well-studied, the degree to which insulin impacts brain tissue in vivo remains unclear. To address this knowledge gap, we utilized an insulin clamp technique to continuously increase plasma insulin concentrations while maintaining normal blood glucose levels during task fMRI, thereby permitting investigation into the real-time effects of hyperinsulinemia on brain function. Participants and Methods: Four healthy adults (aged 59 - 79) completed 3T functional MRI while simultaneously undergoing a hyperinsulinemic-euglycemic clamp procedure under two experimental conditions: infusion of single blinded insulin infusate and infusion of single blinded placebo saline. During each visit, participants performed two runs of an fMRI "N-back" task to investigate within- and between-subject differences in neural activation across experimental conditions.

Results: Behaviorally, reaction time was significantly slower on 2-back trials during the insulin compared to placebo condition $(p<0.05)$. Using FSL, we also observed a significant increase in average within-subject fMRI brain activation on the N-back task (2-back $>0$-back contrast) during the insulin clamp compared to placebo condition in bilateral middle/inferior frontal, occipito-parietal, and precuneus cortices, bilateral thalamus, and left parahippocampal regions (all $p<0.01$, corrected)

Conclusions: We found that experimentally-induced hyperinsulinemia in the setting of euglycemia in healthy participants leads to real-time differences in cognitive performance and fMRI-indexed brain activation. Findings of increased reaction time, coupled with a greater degree of task-related fMRI activation, suggest that abnormality elevated insulin levels may contribute to more effortful processing in the context of cognitively demanding tasks.

Correspondence: Victoria J. Williams, Ph.D., NeuroImaging Research for Veterans Center, VA Boston Healthcare System, 150 South Huntington Ave, 151A, Boston, MA 02130,United States.E-mail:tori85@gmail.com

\section{Poster Session 9. Adult Medical}

$$
\text { 3:30-4:45 p.m. }
$$

\section{Cancer}

A. AILION, T.Z. KING, S. ROBERTS, J.A. TURNER, C.M. CONWAY
\& B. CROSSON. Double Dissociation of Auditory Attention and
Visual Scanning in Long Term Survivors of Childhood Cerebellar
Tumor: A Deterministic Tractography Study of the Cerebellar-
Frontal and the Superior Longitudinal Fasciculus Pathways.
Objective: This study investigated the relationship between the white
matter integrity of the multisynaptic cerebellar-frontal pathway and
processing speed, attention span, and working memory. The right
Superior Longitudinal Fasciculus II (SLF II) and visual attention was
included as a control tract and task.
Participants and Methods: The sample included 29 survivors of
CB tumors (13 astrocytomas, 12 medulloblastomas, 4 other), and 29 matched controls (age: $\mathrm{M}=22$ years ( $\mathrm{SD}=5$ ), $45 \%$ female). All survivors were treated with neurosurgery, 14 survivors with radiation therapy (RT) and chemotherapy, 1 with RT only, and 1 chemotherapy only. Average age at diagnosis was 9 years $(\mathrm{SD}=5)$, and time since diagnosis was 13 years. MRI scans with a 3 -Tesla Siemens Trio system included T1-weighted MPRAGE sequence, diffusion-weighted sequence, T2-weighted image, fMRI sequence, and fMRI field map. Mixture of Wisharts signal attenuation model in TrackTools and PanTrack were used for network tractography. Processing speed was defined using a composite measure that averaged timed neuropsychological measures. The following measures defined the remaining constructs: auditory attention span- Wechsler Memory Scale, Digit Forward Span; working memory- Auditory Consonant Trigram, 36 second; visual attentionDelis Kaplan Executive Functioning Scale, Trail Making Test Visual Scanning.

Results: Results of the correlation analyses supported a double dissociation in the survivor group using partial correlations that accounted for treatment factors. Cerebellar frontal white matter integrity was associated with auditory attention span (FA: $\tau b=.42, p<.05$; RD: $\tau b=-.50$, $p<.05)$, and was not associated with visual attention (FA: $\tau \mathrm{b}=-.11$, $p>.05$; RD: $\tau \mathrm{b}=-.11, p>.05)$. SLF II FA was associated with visual attention (FA: $\tau b=.44, p<.05$; RD: $\tau \mathrm{b}=-.17, \mathrm{p}>.05$ ), and was not associated with auditory attention span (FA: $\tau \mathrm{b}=.24, p>.05$; RD: $\tau \mathrm{b}=-.10, p>.05)$. Conclusions: This study advances the current understanding of brain-behavior relationships following cerebellar tumor.

Correspondence: Alyssa Ailion, M.A., Psychology / Neuropsychology, Georgia State University / Children's Hospital Colorado, 13123 East 6th Ave, B130, Denver, CO 80045, United States.E-mail: alyssa.ailion@ childrenscolorado.org

B.E. CLARK, S. HALL, M. HOFFMAN, E. CARROLL, P. BEAN, M. MCCARVEL, J. MCFARLAND \& M. REYNOLDS. Cancer Patients' Mental Health Concerns, Cognitive Abilities, and Spiritual Well-Being After Chemotherapy.

Objective: Cancer patients often cite declines in cognitive functioning after receiving chemotherapy for the treatment of cancer. This study examined the relationships between self-reported cognitive functioning, mental health concerns, and spiritual well-being in patients who had completed chemotherapy for the treatment of cancer. Understanding the complex relationship between cognitive and emotional functioning in cancer patients is critical to shaping interventions that address the multidimensional nature of recovery.

Participants and Methods: Adult participants ( $\mathrm{n}=53$ ) who had completed chemotherapy treatment were recruited from an outpatient cancer center in the Northwest. Participants completed Functional Assessment of Cancer Therapy (FACIT) questionnaires about their cognition (FACT-Cog, Version 3) and other types of health-related functioning, including spiritual well-being (Spiritual-12).

Results: Participants who reported mental health concerns rated their cognitive abilities significantly lower than participants who did not endorse mental health concerns, $t(32.8)=-1.974, p=.028$. With regard to spiritual well-being, this subset of participants also had significantly lower mean 'Peace' subscale scores $t(52)=-3.31, p=.001$, (but not significantly lower scores on the 'Meaning' and 'Faith' subscales of the Spiritual-12) than those who did not report difficulties with mental health and cognition.

Conclusions: These findings illustrate that mental health problems can negatively impact reported cognitive function on the FACT-Cog, V3. In addition, while key aspects of spirituality remain unaffected, 'peace' is a feature affected by mental health problems. These findings illustrate the complex relationship between emotional and cognitive functioning, and underscore the need for a multidimensional approach to rehabilitation following chemotherapy for cancer.

Correspondence: Brook E. Clark, PhD, Psychology, University of Montana, 4924 Potter Park Loop Unit A, Missoula, MT 59808-5311, United States.E-mail: brook.clark@umontana.edu 
H.M. CONKLIN, J.M. ASHFORD, M.A. SWAIN, L. HARDER, B. CARLSON-GREEN, J. MILLER, J. WALLACE, R. KANER, C. BILLUPS, A. ONAR-THOMAS, T. MERCHANT \& A. GAJJAR. Predicting Cognitive Performance in Children Treated for Brain Tumors During Infancy: Preliminary Findings from a Prospective, Longitudinal Trial.

Objective: We investigated predictors of cognitive outcomes in infants treated for brain tumors on an on-going, prospective, longitudinal trial. Participants and Methods: Patients were assessed prior to adjuvant therapy (baseline), six months post, one year post and annually for five years. Patients included in analyses had at least two assessments $[\mathrm{N}=129$; age at diagnosis $1.7 \pm 1.0$ years; $59 \%$ infratentorial tumors; $71 \%$ gross total resection (GTR)]. All patients received risk-adapted chemotherapy and 53\% received cranial irradiation. Assessments included an IQ test, as well as parent report on the Behavior Rating Inventory of Executive Function [BRIEF- working memory (WM) and Global Executive Composite (GEC)] and Behavior Assessment System for Children [BASC2- Attention Problems (AP)].

Results: At baseline, chi-square revealed patients had lower IQ than normative expectations $(31 \%<85 ; \mathrm{p}<.001)$ and elevated BRIEF WM $(28 \% \mathrm{~T}>60 ; \mathrm{p}<.05)$. Baseline performance estimated by linear mixed models revealed age $<1$ year at diagnosis, GTR, and CSF shunting predicted worse IQ; GTR predicted elevated BRIEF WM; age $<1$ year at diagnosis predicted elevated BRIEF GEC; and supratentorial tumors predicted elevated BASC2 AP $(p<.05)$. Linear mixed models revealed IQ remained stable over time; whereas, BRIEF WM showed a trend for worsening $(\mathrm{p}=.09)$. BRIEF GEC and BASC2 AP worsened over time $(p<.01)$. Worse performance over time was predicted by supratentorial tumors for IQ $(\mathrm{p}<.001)$; less than a GTR and CSF shunting for BRIEF WM $(p<.05)$; and CSF shunting for BRIEF GEC ( $p<.01)$. No differences were detected in infants treated with chemotherapy only and those also receiving cranial irradiation.

Conclusions: Prior to adjuvant therapy, infants with brain tumors experience cognitive difficulties influenced by age at diagnosis, tumor location, surgical extent and CSF shunting. Following adjuvant therapy, there is worsening of attention and executive problems related to tumor location, surgical extent and CSF shunting. These findings may guide treatment planning.

Correspondence: Heather M. Conklin, Ph.D., Psychology, St. Jude Children's Research Hospital, 262 Danny Thomas Place, Mail Stop 740, Memphis, TN 38105-2794, United States.E-mail: heather.conklin@ stjude.org

N.A. DASHER, A. SYLVIA \& K. VOTRUBA. Neurocognitive Outcomes in Patients Undergoing Hematopoietic Cell Transplantation (HCT) 100 Days Post-Operatively.

Objective: Evidence indicates that hematopoietic cell transplantation (HCT) often results in neuropsychological deficits within the first several months after transplant. As time demands often limit comprehensive cognitive testing, identification of sensitive measures to detect changes is important. The present study examined the neurocognitive functioning of adults undergoing HCT and the utility of neuropsychological measures in detecting changes after transplant.

Participants and Methods: Sixty-Eight adults underwent neuropsychological testing prior to HCT [33 allogenic (mean age: 53.3 years) and 35 autologous (mean age: 56.9) transplant recipients]. Eleven autologous and 7 allogenic transplant recipients completed follow-up testing 100 days after transplant. Tests included the Repeatable Battery for Assessment of Neuropsychological Status (RBANS), Trail Making Tests, and the Wisconsin Card Sort Test (WCST), as well as sub-tests from a computerized measure, Cogstate (Detection, Identification, One Card Learning, One Back, and Groton Maze Learning and Recall).

Results: Significant correlations were observed between Cogstate and RBANS tasks $(\mathrm{p}<0.05)$. Repeated measures ANOVA compared preoperative and postoperative performances. Main effects were found for RBANS Figure Recall $(F(1,16)=6.28, p=0.024, \eta p 2=0.28)$ and Groton Maze Recall $(\mathrm{F}(1,16)=6.60, \mathrm{p}=0.02, \eta p 2=0.28)$, suggesting that patients recall at day 100 was significantly improved compared to baseline. An interaction was found in overall errors on the WCST $(\mathrm{F}(1,16)=4.85, \mathrm{p}=0.043, \eta p 2=0.23)$, showing that allogenic transplant patients made significantly more errors compared to baseline, whereas patients receiving autologous transplants improved slightly from baseline to day 100 post-transplant.

Conclusions: Results indicate that measures of visual memory and problem solving may be sensitive to detect subtle changes in cognitive functioning after HCT. Early detection of changes in memory and executive functioning may prove important in treatment planning.

Correspondence: Nickolas A. Dasher, Psychiatry, University of Michigan, 1323 Natalie Lane, Unit 203, Ann Arbor, MI 48105, United States. E-mail:ndasher@gmail.com

T.A. DUDA, K.P. RAGHUBAR, E.M. MAHONE, K.O. YEATES \& M. RIS. Reliable Change in Pediatric Brain Tumor.

Objective: Children treated for brain tumor evidence declines or developmental lags in cognitive skills over time (Gragert \& Ris, 2011) that can include overall intellectual functioning, working memory, verbal reasoning, processing speed, and/or nonverbal reasoning (Kahalley et al., 2016; Palmer et al., 2003; Ris et al., 2001). Although group-level data indicate subtle changes over time, no study has utilized a clinical criterion to identify and describe reliable change in patients with pediatric brain tumor (PBT). The present study sought to examine cognitive change in PBT patients using group-level analysis and reliable change index (RCI) methodology.

Participants and Methods: Twenty-two children treated for PBT completed baseline and follow-up assessment as part of a longitudinal outcomes project. Cognitive outcome data included index score performance on the WISC-IV. Two RCI Z-scores were calculated for each participant on each index score: one with and one without accounting for practice effects. A critical Z-score value of |1.645| (90\% confidence interval) was used to operationalize reliable change.

Results: Patients did not demonstrate a statistically significant change from baseline to follow-up on WISC-IV index scores as a group $(\alpha=.05)$, although change in WMI performance neared significance $(p=.057)$. Thirteen patients demonstrated a reliable change from baseline to follow-up on at least one index score when accounting for practice effects, whereas ten patients showed a reliable change when not accounting for practice effects.

Conclusions: Although patients with PBT did not demonstrate a statistically significant change from baseline to follow-up assessment using group-level analysis, a substantial proportion of PBT patients evidenced a reliable negative change over time - particularly when accounting for practice effects. Findings have implications for future research that may help identify clinical factors that predict reliable change in some patients with PBT but not others.

Correspondence: Thomas A. Duda, PhD, Psychology, University of Windsor, 6701 Fannin Street, Suite 1630, Houston, TX 77063, United States.E-mail:taduda@texaschildrens.org

\section{J.A. EASTMAN. Cancer-Related Cognitive Impairment and} Cognitive Function in Older Adults With a Pre-Cancer Baseline Examination.

Objective: Cognitive changes associated with cancer and cancer treatment represent a significant concern for patients; however, a thorough understanding of how severe these cognitive changes are compared to prior functioning, what cognitive domains are affected, and why some patients recover full function while others do not, remains elusive. To address this question, we conducted an analysis of cognitive function in a cohort of older adults with established normal baseline cognitive function prior to cancer diagnosis, to investigate what if any long-term changes occur.

Participants and Methods: We conducted a secondary analysis of data from 14 cognitively normal individuals with a documented cancer diagnosis after study enrollment (CN-CA), and 14 sex and age matched 
cognitively normal controls absent a study concurrent cancer diagnosis (CN). Participants were identified through a review of records in the Alzheimer Disease Neuroimaging Initiative (ADNI). Two-way Mixed ANOVA examined differences in performance on the Wechsler Memory Scale Revised- Logical Memory Delay (LM-I \& LM-II) task, the Rey Auditory Verbal Learning Test Delay (RAVLT-1 \& RAVLT-II) tasks, the Trail Making Test (TMT-A \& TMT-B), a category fluency task (Animals), and the Boston Naming Test (BNT) by group across three time points: Pre-Cancer Baseline, first available study visit following completion of cancer treatment, and second available study visit following completion of cancer treatment.

Results: There was no significant interaction between the diagnostic groups and study time point $(p>0.05)$ for all cognitive testing performances. Examination of the main effects revealed no significant differences in test performance between CN-CA and CN participants.

Conclusions: Results suggest that cognitive function in older adults who were cognitively normal prior to cancer diagnosis does not differ significantly after treatment and recovery. This suggests that CRCI findings in older adults may be the result of other non-cancer etiologies.

Correspondence: Jennifer A. Eastman, MA, Psychiatry, Northwestern University, 852 1/2 W Agatite Ave, Unit 1, Chicago, IL 60640, United States.E-mail: jeastman@u.northwestern.edu

K. EDElstein, G. POND, N. D'Agostino, A. MATTHEW, K.J. WAHL, S. AUBIN, A. GUPTA, P. KAVAN, M. CRUMP, P. BEDARD, D. HODGSON, G. BATIST, T. MUANZA \& L.J. BERNSTEIN. Cancer-Related Cognitive Dysfunction in Young Adults (YA): A Prospective, Longitudinal Trial.

Objective: Cancer treatment has been linked with neurocognitive sequelae in older adults, even if they do not receive central nervous system directed therapy. Whether YA (age 18-39) are also at risk is unknown. In YA, cancer disrupts education and occupational attainment and is associated with emotional distress. In this study, we examine neurocognitive functions and emotional distress in YA prior to treatment and about 6 and 12 months later.

Participants and Methods: YA with cancer (YAC, n=107; lymphoma, breast, testicular, sarcoma) completed a $2 \mathrm{~h}$ battery of standardized tests and questionnaires within 3 months of diagnosis (and prior to chemotherapy for those requiring it; $\mathrm{n}=69$ ) and about 6 and 12 months later. Healthy YA $(n=63)$ were tested at similar time points. Test scores were converted to T-scores based on published norms, and grouped into memory, attention, processing speed, and executive function domains. Frequency of cognitive decline on raw test scores was assessed using standardized regression-based change scores.

Results: At baseline, IQ scores were average and there were no group differences in cognitive domains, number of impaired tests, or neurocognitive complaints (Kruskal Wallis, all p-values >.4). About $70 \%$ of each group completed all 3 assessments. All cognitive domain scores improved over time (random effects models, all p-values <.01); there were no group or group $\mathrm{x}$ time effects, even after adjusting for emotional distress or fatigue. There was also no difference in rate of decline on any test. However, compared to healthy YA, YAC reported higher levels of distress and a greater proportion had scores above clinical cutoffs at each time point.

Conclusions: Within a vear of treatment, YAC have elevated distress but do not demonstrate cognitive decline seen in older adults, consistent with research suggesting that aging brains are more vulnerable to neurotoxic insult. Whether neurocognitive effects of cancer treatment emerge later in YAC, placing them at risk for accelerated aging, remains to be examined.

Correspondence: Kim Edelstein, PhD, Supportive Care, Princess Margaret Cancer Centre, Pencer Brain Tumor Centre, 610 University Ave 18-716, Toronto, ON M5G 2M9, Canada.E-mail:kim.edelstein@ uhn.ca
C.L. EVANS, D. Gansler, J. GRIECO \& M.B. PUlSifER. Executive Functioning Following Proton Radiation Therapy for Pediatric Brain Tumor.

Objective: Children treated with photon radiation for brain tumor (BT) are at risk for neurocognitive deficits, including executive dysfunction. Proton radiation (PRT) research to-date has primarily focused on intelligence (IQ) and there is little research on its effect on other cognitive domains, such as executive function (EF). This study examines EF at baseline (BL) and first follow-up (FU) in relation to 1) extent of radiation 2) lesion location and 3) age at BL.

Participants and Methods: 141 patients $\left(M_{\text {Age at Baseline }}=13.09 ; M_{\text {interval }}\right.$ $=2.01$ years) received PRT for BT. 59\% had supratentorial tumors and $37 \%$ received whole brain PRT. Patients were administered age-appropriate measures assessing intelligence (Wechsler FSIQ) and EF (COWAT, TMT-B, CMS Attention/Concentration, and CPT-II Clinical Index). An omnibus EF score was derived and validated through Principal Components Analysis.

Results: Both mean IQ and EF were stable across time. EF at BL was inversely correlated to age $(\beta=-.045, t(1,139)=-2.81, p<.01)$; for every year increase in age at BL, EF performance at BL $(M=.031, S D=.71)$ decreased by $.05 \mathrm{z}$-score points. A 2 -way ANCOVA, with age as the covariate, was performed to examine the effect of extent of radiation and tumor location on EF at both BL and FU. Neither extent of radiation [BL: $F(1,130)=2.86, p=.094 ; \mathrm{FU}: F(1,72)=1.80, p=.18$ ] nor tumor location [BL: $F(1,130)=1.06, p=.31$; FU: $F(1,72)=.001, p=.98$ ] were significantly associated with $\mathrm{EF}$ score at either time point.

Conclusions: At 2 years post-treatment, PRT did not negatively impact IQ or EF in a large cohort of pediatric BT patients. Results compare favorably to photon literature. Neither location of tumor nor extent of radiation was related to $\mathrm{BL}$ or FU. EF was lower at BL for older children but remained stable at FU, suggesting that older children are more vulnerable to disruption in EF functions even prior to PRT initiation. EF in older children should be monitored and appropriate supports provided following PRT to optimize academic success.

Correspondence: Casey L. Evans, M.S., Psychiatry, Massachusetts General Hospital, 259 Beacon Street, 71, Boston, MA 02116, United States.E-mail: cevans8@partners.org

M.E. FOX, A. KALIDINDI \& T.Z. KING. The Neurological Predictor Scale as a Predictive Measure of Motor Outcomes in Long-Term Survivors of Childhood Brain Tumors.

Objective: The Neurological Predictor Scale (NPS) has been shown to be predictive of long-term outcomes in survivors of childhood brain tumors, including intelligence, adaptive functioning, and core cognitive skills. The present study aimed to evaluate its utility in predicting motor functioning in long-term survivors of suptratentorial (ST) and posterior fossa (PF) brain tumors.

Participants and Methods: Ninety-four survivors of childhood brain tumors ( 52 female) at least five years past diagnosis completed measures of visual-motor integration (VMI) and fine motor dexterity (Grooved Pegboard; GP). Tumor location (61 PF; 33 ST) and NPS data (presence of radiation, chemotherapy, neurosurgery, hydrocephalus, hormone deficiency, and seizures) were collected through medical record review. Correlations and multiple regression models with each factor were run in SPSS.

Results: Across the PF and ST tumor groups, NPS scores were correlated with VMI $\left(\mathrm{R}^{2}=0.76, p<.001\right)$ and $G \mathrm{P}\left(\mathrm{R}^{2}=0.89, p<.001\right)$ performance such that more treatment and sequelae factors predicted poorer motor scores. In the ST tumor group, NPS predicted performance on both measures over and above the predictive power of any individual risk factor $\left(.000<p_{\mathrm{NPS}}<.040\right)$. In the PF tumor group, NPS predicted VMI and GP over and above most treatment or sequela $\left(.001<p_{\mathrm{NPS}}<.039\right)$ but not the presence of radiation. NPS added to the model with radiation predicting $\mathrm{VMI}$ performance at trend level $\left(\Delta \mathrm{F}_{\mathrm{NPS}}=2.77, p=0.10\right)$ but not to that predicting GP performance in this group $\left(\Delta \mathrm{F}_{\mathrm{NPS}}=0.07, p=0.79\right)$. Conclusions: This study suggests that beyond its role in accounting for other cognitive outcomes, NPS is an effective tool in predicting motor 
function in long-term survivors of childhood brain tumors, particularly ST brain tumors. PF tumor survivors' motor function appears to be particularly impacted by radiation to the tumor site; this may be due to the compound effects of tumor and deleterious treatment in the cerebellum increasing the risk of damage to an area that is critical for motor control.

Correspondence: Michelle E. Fox, MA, Psychology, Georgia State University, 1026 th St NW, Apt C1, Atlanta, GA 30309, United States. E-mail:mfox9@student.gsu.edu

R.J. KAUTIAINEN, S. NA \& T.Z. KING. Neurological Predictor Scale Predicts Academic Achievement Outcomes in Long-Term Survivors of Childhood Brain Tumors.

Objective: Research suggests that survivors of childhood brain tumors exhibit impairments in academic performance and have lower rates of educational attainment compared to healthy same-aged peers. Prior research has demonstrated the concurrent validity of the Neurological Predictor Scale (NPS), a measure that incorporates tumor-related treatments and neurological complications into one cumulative score, in predicting IQ, adaptive functioning, and core neurocognitive skills. The purpose of this study is to evaluate the relationships between the NPS and academic achievement in adult survivors.

Participants and Methods: Sixty-two adult survivors (M age $=24(4.5)$ years, $M$ years post diagnosis $=16(4.75))$ completed four measures of academic achievement from the Woodcock-Johnson III: reading (Letter Word-ID and Passage Comprehension subtests), spelling (Spelling subtest) and math (Calculation subtest). NPS scores were calculated using medical record data.

Results: NPS scores significantly predicted performance on all four academic measures: Letter Word ID $\left(\mathrm{M}=0.51(1.06), \mathrm{R}^{2}=-.454\right.$, $\mathrm{p}<.01)$, Calculation $\left(\mathrm{M}=0.87(1.24), \mathrm{R}^{2}=-.262, \mathrm{p}<.05\right)$, Spelling $\left(\mathrm{M}=0.45(1.21), \mathrm{R}^{2}=-.428, \mathrm{p}<.01\right)$ and Passage Comprehension $\left(\mathrm{M}=0.33(0.91), \mathrm{R}^{2}=-.447, \mathrm{p}<.01\right) .16 \%$ of survivors were impaired on the Letter Word ID, $23 \%$ on Calculations, 19\% on Spelling, and $11 \%$ on Reading Comprehension subtests (impairment $=\mathrm{z} \leq-1.5$ ).

Conclusions: The current study extends prior research by demonstrating that the NPS is significantly associated with academic achievement in survivors many years after initial diagnosis. As a cumulative measure that is fast and easy to calculate, the NPS may be especially helpful in clinical research when studies lack the statistical power to investigate how treatments and related neurological conditions individually may contribute to long-term outcomes.

Correspondence: Rella J. Kautiainen, Clinical Neuropsychology PhD, Clinical Psychology, Georgia State University, 391 17th Street NW, Unit 4044, Atlanta, GA 30363. United States.E-mail: rkautiainen1@ student.gsu.edu

\section{K. OSWALD \& J. BO. Motor functioning in pediatric Acute} Lymphoblastic Leukemia survivors.

Objective: Pediatric oncology survivors experience an array of neurocognitive late effects following medical treatment. Motor deficits, including fine-, gross-, and visual-motor, have been identified in the pediatric cancer survivors. The present study sought to replicate evidence of motor impairments in a sample of Ácute Lymphoblastic Leukemia (ALL) survivors. It was predicted participants in the ALL survivor group would display worse motor functioning across performance-based measures and self-report questionnaires as compared to participants in a control group.

Participants and Methods: Thirteen age-, and sex-matched pairs of controls and ALL survivors ( 5 to 17 years old) completed performance-based motor measures (MABC, VMI, Grooved Pegboard) as well as a self-report measure of physical abilities (PSDQ). Also, parents completed two questionnaires assessing their child's motor functioning (MABC Checklist and DCDQ). Independent samples $t$-tests with Bonferroni correction $(p=0.002)$ were conducted to assess mean differences between the groups across all motor measures.
Results: Results revealed that the ALL Survivor group performed significantly lower compared to the Control group with large effect sizes on Grooved Pegboard Dominant hand $(p<0.001)$, MABC Manual Dexterity $(p<0.001)$, MABC Aiming and Catching $(p=0.001)$, MABC Balance $(p<0.001)$, and MABC Total $(p<0.001)$. The ALL Survivor group reported significantly lower scores on the PSDQ Health subscale compared to the Control group $(p=0.001)$. No significant differences were observed on parent-report measures of motor functioning.

Conclusions: These results are consistent with previous literature highlighting impairments across domains of motor functioning in pediatric oncology survivors. In light of the present findings, future research is needed to further investigate ways to assess and intervene upon motor deficits in the survivor population, along with understanding the underlying causes, medical and neurocognitive, and consequences of motor impairments.

Correspondence: Kaitlin Oswald, Psychology, Eastern Michigan University, 1045 Flynt Dr. Apt. G1, Flowood, MS 39232, United States. E-mail: koswald1@emich.edu

Y. MZAYEK, M.B. DE RUITER \& S.B. SCHAGEN. Improved Processing of Diffusion Tensor Imaging Data Increases Sensitivity to Detect Brain White Matter Changes in Breast Cancer Patients. Objective: To assess side effects of chemotherapy on brain white matter integrity in breast cancer patients as a neural correlate of cognitive problems. We implemented an enhanced approach for processing brain magnetic resonance imaging (MRI) diffusion tensor imaging (DTI) data in a longitudinal setting. We used an improved registration algorithm and omitted white matter "skeletonization' within the widely used tractbased spatial statistics (TBSS) framework.

Participants and Methods: Twenty-six breast cancer patients scheduled to receive adjuvant chemotherapy with or without endocrine treatment $(\mathrm{Ch}+), 23$ breast cancer patients who did not require chemotherapy or endocrine treatment (Ch-), and 30 age-matched healthy controls (HC) received a T1-weighted scan and a DTI scan at two time points. Baseline data for $\mathrm{Ch}+$ was collected after surgery but before receiving chemotherapy (t0). A follow-up session took place 6 months after chemotherapy and at matched intervals for Ch- and HC (t1). After eddy current correction and diffusion tensor model fitting, a T1-weighted group-wise template was built to warp the fractional anisotropy (FA) maps. We used the ANTs Symmetric Normalization $(\mathrm{SyN})$ registration algorithm.

Results: Ch+ showed a significant decrease in mean FA from t0 to t1 compared to the HC group. Voxel-wise between group analysis showed that both $\mathrm{Ch}+$ and $\mathrm{Ch}$ - had significant decreases in FA compared to the HC group, with Ch+ showing more affected voxels than Ch- (5203 vs 230 ).

Conclusions: These findings suggest a decrease of brain white matter integrity in breast cancer patients, with a possible added effect of chemotherapy treatment. The improved processing pipeline within the TBSS framework increases sensitivity to detect brain white matter changes in breast cancer patients and demonstrates the feasibility of using this pipeline for DTI data in this population.

Correspondence: Sanne B. Schagen, PhD, Psychosocial Research and Epidemiology, Netherlands Cancer Institute, Bellamystraat 52, Amsterdam1053BN, Netherlands.E-mail:s.schagen@nki.nl

T. TARKENTON, M. NI'SHUILLEABHAIN, M. CLEM, E. LAMPSON, P.L. STAVINOHA \& A. HOLLAND. School Attitude Associates with Academic Peformance Better than Neurocognitive Variables in Pediatric Leukemia Survivors.

Objective: Survivors of pediatric acute lymphoblastic leukemia (ALL) are at risk for poor academic outcomes, but contributing factors are not well understood. Given motivation associates with academic performance in pediatric cancer survivors (Holland et al., 2016), further investigation of school motivation relative to neurocognitive functioning is warranted to better understand academic outcomes. We hypothesized 
that school attitude and neurocogntive functioning would significantly predict academic performance, with school attitude being more influential.

Participants and Methods: 107 ALL survivors ages 8-19 years at testing ( $\mathrm{M}=12.80$; $\mathrm{SD}=3.18)$ completed self-report BASC-2 questionnaire (Attitude Toward School [ATS]) and a brief battery including the Trail Making Test Part B (TMT-B), Symbol Digit Modalities Test (SDMT), and Wechsler Processing Speed Index (PSI). The sample was dichotomized according to parent-rated Math and English performances: average/above-average $(\mathrm{N}=87)$ and below-average/failing $(\mathrm{N}=20)$. Independent $t$-tests were conducted to examine group differences. TMT-B, SDMT, PSI, and ATS were entered into stepwise binary logistic regressions to examine predictors of academic performance.

Results: Overall means were average for all outcome variables. The below-average/failing group had lower ATS and lower neurocognitive scores (TMT-B, SDMT, PSI) compared to the average/above-average group (all $p$ 's<.05). Only ATS (not TMT-B, SDMT, or PSI) significantly predicted parent ratings of math performance $\left(x^{2}=20.6, O R=.93\right)$ or English performance $\left(\mathrm{x}^{2}=14.6, \mathrm{OR}=.94\right.$; all $p$ 's<.05).

Conclusions: ATS was a stronger predictor of parent-reported academic performance than neurocognitive variables. To understand whether school attitude has a causal role in academic performance, future research should examine changes in school attitudes and neurocognitive functioning as related to academic performance at diagnosis and over the course of treatment.

Correspondence: Tahnae Tarkenton, Masters in Clinical Rehabilitation Counseling, Neuropsychology, UT Southwestern Medical Center, 5940 Forest Park Road, Dallas, TX 75235, United States. E-mail: tahnae. tarkenton@utsouthwestern.edu

M.M. THOMASON, L.H. MUTHS, L.S. KAHALLEY, D.R. GROSSHANS, M. RIS \& M. GRAGERT. Circumscribed Memory Impairment Following Proton Beam Radiation Therapy for Hypothalamic Tumor in an Adolescent Female.

Objective: Proton beam radiation therapy (PBRT) is an advanced radiation therapy (RT) method involving minimal entrance dose, maximal tumor dose, and no exit dose. PBRT thus spares more healthy tissue than conventional photon beam RT, theoretically reducing cognitive late effects. However, results of available cognitive outcome studies are equivocal. This case study describes the cognitive effects of PBRT for hypothalamic tumor in an adolescent female.

Participants and Methods: The patient was diagnosed with hypothalamic pilomyxoid astrocytoma at 16 years old and received focal PBRT for 6 weeks. She completed neuropsychological evaluations at 5 time points following treatment as part of a larger study of the cognitive effects of PBRT in a pediatric sample.

Results: Scores over time revealed stable intelligence, visual memory, sustained attention, executive functioning skills, visuospatial and visual-motor skills, fine motor skills, and adaptive functioning. However, performance on a measure of rote verbal memory revealed significant decline ( $>2 S D$ s) from time 1 (8 days post-PBRT) to time 2 (1 year, 4 months post-PBRT). Verbal memory performance then remained stable from time 2 to time 5 ( 6 years, 4 months post-PBRT).

Conclusions: This case study highlights that PBRT targeted to a hypothalamic tumor impacted verbal memory function without evidence of other cognitive effects. This is consistent with the theory of generalized cognitive sparing related to reduced exposure of healthy tissue in PBRT relative to photon RT. However, the findings are also notable for a significant degree of memory decline despite PBRT delivery at an age that has been associated with reduced cognitive risk in the pediatric tumor literature. As PBRT is now a more widely applied treatment for pediatric brain tumor, further research is needed to examine if PBRT reduces long-term cognitive impact and identify individual risk factors for adverse outcomes.
Correspondence: Molly M. Thomason, Ph.D., Psychology, Texas Children's Hospital/Baylor College of Medicine, 6701 Fannin Street, Suite 1630,Houston, TX 77030, United States.E-mail:mmthomas@ texaschildrens.org

K. VAN DYK, L. PETERSEN, C.M. CRESPI \& P.A. GANZ. The Effects of Cancer Treatment on Cognitive Functioning in Early Breast Cancer Survivorship.

Objective: Chemotherapy is often blamed for concerning cognitive changes in breast cancer survivorship, but other treatments (surgery, radiation, endocrine therapy (ET)) may also influence well-being and cognition. Prior studies have wide post-treatment assessment time variability. The UCLA Mind Body Study (MBS) focused on assessing recently treated breast cancer survivors (BCS) shortly after primary treatment and before the start of ET, as a prelude to additional evaluation of ET on cognitive function. To examine early effects, we compared baseline neuropsychological functioning between BCS exposed to different primary cancer treatments, and explored other clinical correlates.

Participants and Methods: 189 BCS (mean age $=51.4 \pm 8.3$ ) within three months of completing primary cancer treatment underwent comprehensive psychosocial and neuropsychological assessment. Sample-based z-scores of neuropsychological tests were aggregated into standard cognitive domains. Using a 2x2 ANCOVA, we examined the effects of chemotherapy and radiation on cognitive domain scores. Exploratory multivariable linear regressions included treatment exposure and other variables emergent from preliminary partial correlations. All analyses controlled for age, IQ and time since treatment completion. Results: We found no main effects of treatment exposure or interactions for most domains ( $p$ 's $>.05$ ), except for a main effect of worse Executive Functioning in those with chemotherapy exposure $(F(1,180)=4.09$, $\mathrm{p}=.04)$. Follow-up comparisons showed lower scores specifically in the chemotherapy + radiation group $(n=77)$ compared to neither $(n=28)$. In the multivariable adjusted model, only physical fatigue predicted Executive Functioning (adj $\mathrm{R}^{2}=.33, \mathrm{p}<.01$ ).

Conclusions: In early survivorship chemotherapy may interfere with executive functioning, linked to physical fatigue. Effects are largest between those who also received radiation versus neither. Encouragingly, we saw no other differential effects in other domains.

Correspondence: Kathleen Van Dyk, PhD, Psychiatry, UCLA Semel Institute for Neuroscience and Human Behavior, 760 Westwood Plaza, 37-360B, Westwood, CA 90095, United States. E-mail: kathleen.van. dyk@gmail.com

M. BLACKWOOD, K. REDMOND, H. SAIR, L. KLEINBERG \& T.D. VANNORSDALL. The Safety Profile of a Genu Avoidance Approach to Whole-Brain Radiotherapy to Prevent Cognitive Decline.

Objective: While central to the management of brain metastases and used prophylactically to treat small cell lung cancer, whole brain radiation therapy (WBRT) is associated with long-term declines in cognition and quality of life (QOL). Newer techniques can limit the delivery of radiation to certain brain regions (e.g. hippocampal-sparing WBRT), but it remains unclear which areas should be spared. Given the genu of the corpus callosum's role as a relay hub for frontoparietal functioning, it may be an effective target for minimizing the adverse effects of WBRT. Before examining the potential benefit of limiting radiation dose to the genu, the risk of metastasis in the genu avoidance region must be established.

Participants and Methods: Data were extracted from the T1 post-gadolinium MRIs of 99 adult patients with $<25$ brain metastases from any primary tumor treated with WBRT at a single large academic center. The genu of the corpus callosum was delineated by a trained neuro-radiologist. The number of metastases in the genu and surrounding parenchyma $(<5 \mathrm{~mm}, 5-15 \mathrm{~mm},>15 \mathrm{~mm})$ were recorded, as were clinical prognostic factors. 
Results: A total of 611 metastases were identified across 99 scans, none of which fell within the bounds of the genu. Metastases within $5 \mathrm{~mm}$ of the genu were observed in 5.05\% (95\% CI=0.7 - 9.4\%). At $15 \mathrm{~mm}$ outside the genu, the incidence was $19.2 \%$ (CI=11.4 - 27.0\%). There was a relationship between total number of metastases and proximity of metastases to the genu, but no other demographic or clinical characteristics predicted metastases in the region surrounding the genu. Conclusions: The incidence of metastases $<5 \mathrm{~mm}$ from the genu was no greater than that seen in other cognitively sensitive areas of the brain such as the hippocampus, for which radiation-sparing studies are already underway. Given the small proportion of tumors occurring in the genu avoidance area, sparing this region from radiation appears relatively low risk and may have the potential to prevent radiation-induced declines in cognition and QOL.

Correspondence: Tracy D. Vannorsdall, Ph.D., Psychiatry, Johns Hopkins University School of Medicine, 600 N. Wolfe St., Meyer 218, Baltimore, MD 21224, United States. E-mail: TVannor1@jhmi.edu

T.D. VANNORSDALL, K. POWERS, M. BLACKWOOD, J. ZHANG, Z. WANG, S. CHENG \& M. CARLSON. Cognitive Functioning in Cancer Survivors: Analyses of the Women's Health and Aging Study II.

Objective: Cancer-related cognitive impairment (CRCI) is most frequently observed in the domains of memory, processing speed, and executive functioning during and directly following treatment. Longitudinal studies find a sizable proportion of patients continue to demonstrate cognitive changes decades after treatment. Concern that CRCI could be a harbinger of later dementia have been partially allayed by epidemiological data showing reduced risk of Alzheimer's disease in cancer survivors. However, it remains to be seen whether survivors show a subtle pattern of CRCI, or if their history of cancer is protective for individual cognitive abilities. Prior studies have combined survivors receiving different treatments, and questions remain as to the roles of disease and treatment variables in relation to later cognitive status.

Participants and Methods: Participants included 436 older women who were free of cancer diagnoses and dementia at entry into the Women's Health and Aging Study II (M age=73.9yrs, SD=2.8). Longitudinal cognitive assessment included tests of memory (HVLT-R DR), processing speed (Trails A), and executive functioning (Trails B). We compared women with a history of cancer who did not receive chemotherapy to those with no history of cancer.

Results: Of the 436 WHAS II participants, 51 had a history of cancer at baseline and 385 had no such history. In unadjusted models, survivors showed a non-significant trend towards better memory at baseline (M HVLT-R DR difference $=0.5 p t s, p=0.07, n=396$ ). However, groups did not differ in any domain once analyses adjusted for demographic and disease characteristics $(\mathrm{ps}>0.05, \mathrm{n}=396)$.

Conclusions: The cognition of older, non-demented community dwelling female cancer survivors with no history of chemotherapy is equivalent to that of non-affected peers across cognitive domains sensitive to CRCI. Longitudinal analyses are needed to clarify whether the cognitive trajectory of cancer survivors without a history of chemotherapy remains on par with that of their no-cancer peers.

Correspondence: Tracy D. Vannorsdall, Ph.D., Psychiatry, Johns Hopkins University School of Medicine, 600 N. Wolfe St., Meyer 218, Baltimore, MD 21224, United States. E-mail: TVannor1@jhmi.edu

R. WIER, H.A. ALEKSONIS, M.M. PEARSON, C.J. CANNISTRACI, A.W. ANDERSON, J.F. KUTTESCH, B.E. COMPAS \& K.R. HOSKINSON. White Matter Integrity in the Inferior FrontoOccipital Fasciculus Mediates Behavioral Regulation and Internalizing Symptoms in Pediatric Brain Tumor Survivors.

Objective: Despite completing treatment, pediatric brain tumor survivors (PBTS) face emotional and behavioral challenges. This may be due to tumor or treatment-related changes in brain structures involved in emotion regulation including the inferior fronto-occipital fasciculus (IFOF), a bilateral white matter tract with limbic connections. We hypothesized that PBTS would exhibit greater difficulties with behavior and emotional functioning, and display reduced mean fractional anisotropy $(\mathrm{mFA})$ of the IFOF relative to healthy controls (HC). We further predicted that $\mathrm{mFA}$ of the IFOF would mediate the relationship between group and these outcomes.

Participants and Methods: Eleven 8-16 year old PBTS ( $>2$ years post-treatment) and $14 \mathrm{HC}$ matched approximately by age and sex underwent MRI, including a diffusion tensor imaging sequence to assess white matter integrity. Tractography (DSI Studio) quantified $\mathrm{mFA}$ of the IFOF. Parents rated children's emotional and behavioral functioning using the Behavior Rating Inventory of Executive Function and Child Behavior Checklist.

Results: Compared to HC, PBTS had lower mFA in the right IFOF $(d=-1.03, p<.05)$. Caregivers of PBTS reported poorer behavioral regulation $(d=1.59, \mathrm{p}<.01)$ and greater internalizing $(d=0.86, p<.05)$ and externalizing symptoms $(d=1.17, \mathrm{p}<.01)$. Right IFOF mFA mediated the relationship between group and behavioral regulation $\left(\mathrm{R}^{2} \Delta=.07\right.$, bias-corrected $95 \%$ confidence interval: $[-5.50,-0.26])$, as well as group and internalizing symptoms $\left(\mathrm{R}^{2} \Delta=.23\right.$, bias-corrected $95 \%$ confidence interval: $[-6.71,-1.01])$.

Conclusions: Reduced white matter integrity in the IFOF may contribute to poor behavior and emotional outcomes following pediatric brain tumor. Clinicians should note that PBTS with treatment known to impact white matter may be especially vulnerable. Further research with a larger sample should clarify this relationship, allowing for multiple mediators and consideration of factors like tumor and treatment type. Correspondence: Ryan Wier, B.S., Biobehavioral Health, Research Institute at Nationwide Children's Hospital, 700 Children's Drive, FB Suite3B.2, Columbus, OH OH, United States.E-mail: ryan.wier@ nationwidechildrens.org

V.W. WILLARD, I. QADDOUMI, K. MARTIN-ELBAHESH, R. BRENNAN, M. WILSON, C. RODRIGUEZ-GALINDO \& S. PHIPPS. Executive Functioning in School-Aged Survivors of Retinoblastoma.

Objective: Retinoblastoma $(\mathrm{Rb})$ is an ocular cancer typically diagnosed in children less than 2 years old. Treatment often involves enucleation and occasionally adjuvant chemotherapy, radiation, or focal therapies. Historically, neurocognitive and psychosocial late effects were considered rare; however a recent study demonstrated declines in developmental and adaptive functioning in children from diagnosis through age 5. As such, further exploration of the potential long-term consequences of $\mathrm{Rb}$ is critical. The objective of the current study is to describe the executive functioning of school-aged survivors of Rb.

Participants and Methods: Children with Rb completed comprehensive assessments of psychological functioning $\left(n=31, M_{A g e}=11.1 \pm 1.02\right.$. $51.6 \%$ female, $58.1 \%$ Caucasian). One patient was blind and only completed verbal measures. A third of patients had bilateral disease, and 51.6\% were treated with enucleation only. Participants completed a measure of estimated IQ and 3 subtests from the DKEFS; parents completed the BRIEF.

Results: Estimated IQ was in the average range ( $M=93.0 \pm 15.37)$. Summary scores on the BRIEF were within normal limits (Behavioral Regulation $\mathrm{M}=50.3 \pm 12.47$, Metacognition M=51.4 \pm 11.21 , Composite $\mathrm{M}=51.0 \pm 12.15$ ). Mean scores on 3 DKEFS scales (Color-Word Inhibition, Trail Making, Verbal Fluency) were also within normal limits, range: $8.9 \pm 3.58$ (Letter Sequencing) to $11.1 \pm 3.47$ (Category Switching Total Switching). Patients treated with enucleation had lower IQs $(p=.05)$ than those who kept their eye, while those treated with adjuvant therapies had lower DKEFS scores than those treated with enucleation only.

Conclusions: Preliminary results suggest that objective and subjective reports of executive functioning in survivors of $\mathrm{Rb}$ are within normal limits. Future research should examine other domains, such as academic and psychosocial functioning. Additional longitudinal research is also 
necessary to examine whether prior declines in early childhood could be transient and followed by a return to baseline over time.

Correspondence: Victoria W. Willard, PhD, Psychology, St. Jude Children's Research Hospital, 262 Danny Thomas Place, Mail Stop 740, Memphis, TN38015, United States.E-mail:victoria.willard@stjude.org

\section{Genetics/Genetic Disorders}

R.J. FEE, J. STEWART \& V.J. HINTON. Dystrophin gene mutation location and academic achievement in the dystrophinopathies.

Objective: To examine academic performance in dystrophinopathy as a function of dystrophin gene mutation position.

Participants and Methods: In a cross-sectional study, boys with dystrophinopathy (ages 5-17;n=50) completed tests of academics (Woodcock-Johnson-III: spelling, reading, calculation and total scores), single word comprehension and nonverbal reasoning. Dystrophin gene mutation positions were dichotomized into groups (upstream versus downstream of exon 43, location of Central Nervous System (CNS) isoforms previously linked to intellectual impairment). A one-way (2-group) ANOVA (Welch's test) was conducted between mutation groups (downstream of exon 43: $n=32$; upstream of exon 43: $n=18$ ) for dependent measures of academic performance.

Results: Academic performance was slightly, yet significantly, lower than IQ and varied as a function of dystrophin gene position, wherein boys possessing the downstream mutation exhibited greater impairment than boys with the upstream mutation.

Conclusions: Weak academic performance is associated with dystrophinopathy and is more common in downstream mutations. Thus, mutations that disrupt CNS dystrophin isoforms (Dp140 and Dp 71) affect academic performance.

Correspondence: Robert J. Fee, Neurology, Columbia University/Queens College/Northwell Health, 264 81st St, Brooklyn, NY 11209, United States.E-mail:feerobe@gmail.com

A. FOY, A.M. CUREWITZ, M. PIERPONT, S. BERRY, K. SOMMER, C. MOERTEL, M. SEMRUD-CLIKEMAN, R. HUDOCK \& R. PIERPONT. Predictors of Social Skills in Children with RASopathies: A Comparison of Noonan Syndrome and Neurofibromatosis Type 1.

Objective: Gene mutations within the RAS-MAPK signaling cascade cause Noonan syndrome (NS), neurofibromatosis type 1 (NF1) and related disorders. Recent research has established an increased risk for social difficulties and characteristics of autism spectrum disorder in these "RASopathy" syndromes (Adviento et al., 2014; Lewis et al., 2016; Pierpont et al., 2015), vet the medical and behavioral characteristics that underlie these social challenges are not well understood, particularly in children with NS.

Participants and Methods: Parents of children with NS ( $n=39)$, NF1 $(\mathrm{n}=39)$ and unaffected siblings $(\mathrm{n}=32)$ were administered well-validated questionnaires assessing their child's social skills, social-pragmatic language and attention deficit hyperactivity disorder characteristics. Results: On a measure of social skills, average ratings of children with both RASopathy syndromes were impaired relative to the unaffected sibling group. Similar to previous work, children with RASopathies in this cohort also showed heightened risk for characteristics of autism spectrum disorder. Patterns of social challenges across subdomains were similar for the NS and NF1 groups. Regardless of whether a child was diagnosed with NS or NF1, high levels of ADHD symptoms and poor pragmatic language skills were predictive of less social competence. Conclusions: Interventions targeting social language and behavioral self-regulation may increase social competence among children and adolescents with RASopathies.

Correspondence: Allison Foy, PhD, Eductional Psychology, University of Wisconsin-Madison, 213 North Hamilton Street, Apartment 2N, Madison,MN 53703,United States.E-mail:afoy2@wisc.edu
M. GODFREY, T. TAPERA, F. ZHANG, D. FIDLER, S. HEPBURN, C. ROBINSON \& N. RAITANO LEE. Identifying Autism Diagnostic Interview- Revised Algorithm Items that Significantly Distinguish Autism Spectrum Disorder and Down Syndrome.

Objective: There is growing interest in comorbid autism spectrum disorder (ASD) and Down Syndrome (DS), as DS is associated with elevated rates of ASD relative to the typical population. However, it is not clear if this is due to an overlap in symptoms explained by intellectual disability (ID) or a true increased risk for ASD. This study examined the ability of the Autism Diagnostic Interview- Revised (ADI-R) algorithm items to distinguish among children with DS only, ASD (with ID), and comorbid DS and ASD.

Participants and Methods: This study included three groups of participants with ID with a mean age of 4.5 years: (1) DS only $(n=21,7$ females) (2) ASD only ( $\mathrm{n}=42,14$ females) and (3) comorbid DS and ASD (DS+ASD, n=11, 3 females). Logistic regressions were conducted to identify ADI-R algorithm items predictive of group membership for the DS versus ASD groups, and the DS versus DS+ASD groups.

Results: The first logistic regression yielded a model in which 5 of 27 algorithm items were significantly predictive of group membership when examining the DS and ASD only groups (Sensitivity=1.00; Specificity=0.78). These included Use of Conventional/Instrumental Gestures, Imaginative Play, Direct Gaze, Social Smiling, and Imitative Social Play. Individual logistic regressions were then conducted with these five items to predict group membership for DS and DS+ASD groups. Two of these items, Conventional/Instrumental Gestures and Imaginative Play, were predictive of group membership.

Conclusions: Our analyses indicate that ADI-R items examining social-communication and play skills are predictive of ASD group membership for youth with DS, consistent with past research. These results suggest that weaknesses in social-communication and play skills may be core aspects of ASD within the context of ID that distinguish it from other ID groups such as DS. Moreover, weaknesses in the use of gestures and imaginative play may suggest concern for comorbid ASD in DS.

Correspondence: Mary Godfrey, Psychology, Drexel University, $30 \mathrm{~S}$ 22nd Street, Apt 10, Philadelphia, PA 19103, United States. E-mail: maryelizabethoodfrey@gmail.com

T. KHANG \& M. SEMRUD-CLIKEMAN. Neuropsychological Profile of 4 Biological Siblings with Myotonic Dystrophy Type 1.

Objective: This case study presents data from 4 neuropsychological evaluations on child siblings who have all been diagnosed with myotonic dystrophy type 1 with a goal of disseminating known information on this unique set of data.

Participants and Methods: The siblings comprise of 3 females and 1 male. Among the females, patient A is 6-vears, 2-months old, patient B is 8-years, 9-months old, and patient $\mathrm{C}$ is 10-years, 10-months old. The brother, patient $\mathrm{D}$, is 12 -years, 10 -months old. All children have complicating medical conditions ranging from heart defects, hypothyroidism. gastrointestinal difficulties, and oral motor deficits. All 4 patients had recent sleep studies indicating mild sleep abnormalities and have begun using BiPAPs. Also of note is a family history of mental health disorders including attention-deficit/hyperactivity disorder and autism spectrum disorder. Previous neuropsychological evaluations were also reviewed. Results: Two of the four children had average intellectual functioning. One child showed extremely variable performance with stronger verbal and visual spatial skills with the fourth showing a similar pattern although lower. Verbal memory was assessed in 3 patients and 1 child demonstrated impaired functioning. All 4 children showed deficits in fine motor dexterity. Three of the patients showed significant problems with attention, working memory, and impulsivity.

Conclusions: Children with this disease have a tendency to show significant problems with attention, impulse control, and motor speed dexterity. These areas appear to be most impacted by this disease. Generally verbal skills are more strongly developed than visual-spatial skills. These findings strongly suggest the existence of select neurocognitive 
deficits that require serial neuropsychological assessments to provide appropriate interventions.

Correspondence: Tou Khang, Psy.D., Pediatric Neuropsychology, University of Minnesota, 2210 6th St. , \#2, Minneapolis, MN 55411, United States.E-mail:khang036@umn.edu

R. LAURENT, D. TRANEL \& R. JONES. Neuropsychological Impairment in a Patient with Metachromatic Leukodystrophy.

Objective: Metachromatic leukodystrophy (MLD) is a rare autosomal recessive disease, in which dementia is one feature. Previous studies of MLD have provided limited cognitive data, or combined data across a small group of subjects, thereby limiting exposition of potential nuances of the cognitive syndrome associated with MLD. We provide a description of detailed neuropsychological testing, neuroimaging, and genetic testing results in a patient with MLD, with the aim of providing insight into the nature of this atypical dementia syndrome.

Participants and Methods: BS is a 41-year-old, left-handed, college educated woman, referred pursuant to concerns about forgetfulness, inattention, and apathy. Medical and psychiatric histories were unremarkable. During clinical interview, she denied problems with cognition. In contrast, her father indicated that her cognitive problems had worsened over the previous four years. He also described her as apathetic, and noted that her lack of initiation and drive had led to significant discord between her and family members.

Results: Behavioral observations were notable for poor focused attention, disorganized thought processes, impulsivity, diminished task persistence, and anosodiaphoria. Estimated pre-morbid intellectual functioning was superior (single word reading standard score $=123$ ). Neuropsychological test findings revealed widespread cognitive impairment. Neuroimaging (not performed heretofore) was recommended in order to further explore a neurological etiology. Findings from magnetic resonance imaging (MRI) of the brain revealed white matter abnormalities consistent with leukodystrophy. A diagnosis of MLD was later confirmed through genetic testing.

Conclusions: Our findings represent a unique combination of genetic, radiologic, and neuropsychological information related to MLD. Our goal in presenting these findings is to contribute to the accumulated knowledge of MLD and to help establish a clearer picture of the clinical manifestations of this rare disease.

Correspondence: Richard Laurent, Ph.D., Neurology, University of Iowa, 420 5th Street, Apt 11, Coralville, IA 52241, United States. E-mail: richard-laurent@uiowa.edu

K. NAYLON \& M. LACY. Fibromuscular Dysplasia Linked to Adverse Neurological and Neuropsychological Outcomes.

Objective: Fibromuscular dysplasia (FMD) is a rare genetic disorder that causes abnormal cell development in the beds of artery walls, especially the carotid and renal arteries. This may result in hypertension and an increased risk for strokes (Mettinger \& Ericson, 1982). Froehlich and colleagues (2017) also recently documented an increased prevalence of intracranial aneurvsms in females. The main objective of the present study is to describe the clinical presentation of FMD and to demonstrte the effects it may have on neuropsychological functioning through a clinical case presentation.

Participants and Methods: A 46-year-old African American female presented with right-sided weakness, upper lip numbness, and disorientation. Imaging scan report, neuropsychology data, medical history and familial medical history were obtained through a chart review and clinical interview.

Results: Personal medical history was significant for TIA, stroke, longstanding hypertension, and vascular abnormalities (submental and cavernous tongue hemangiomas) that developed in her late 20 's. She reported that she had multiple female cousins that had strokes in their late 20 's-early 30 's and both of her sisters had strokes in their 30 's. Neuropsychological assessment documented moderately impaired executive functioning and right side grip strength. CTA documented cobblestone appearance of internal carotid arteries, a hallmark feature used in diagnosis of FMD. Therefore, CTA was indicative of FMD of internal carotid arteries along with multiple ischemic events thought secondary to FMD.

Conclusions: FMD is a vascular disorder that is poorly understood and often underdiagnosed. However, it has been associated with adverse neurological and neurocognitive outcomes. Awareness and understanding of the clinical presentation of FMD may lead to earlier diagnosis and psychoeducation. Accurate diagnosis may lead to prevention of future adverse outcomes.

Correspondence: Kara Naylon, Clinical Psychology, Adler University, 420 west belmont avenue, apartment 28c, Chicago, IL 60657, United States.E-mail: knaylon12@jcu.edu

A.A. POWELL, M. BLACKWELL \& V.P. CULOTTA. Neurodevelopmental Varaibility in Social Skills Associated with Phelan-McDermid Syndrome.

Objective:

Objective: This case study examined a neuropsychological profile associated with Phelan-McDermid Syndrome (PMS). PMS is a rare, underdiagnosed genetic syndrome marked by a deletion of the terminal end of chromosome 22 or mutation of the SHANK3 gene. Clinical studies suggest a heterogeneous phenotype, including speech disorder, hypotonia, intellectual disability, hyperactivity and inattention, mood instability, seizures, sleep disturbance, and Autism Spectrum Disorder (ASD)

Participants and Methods: Participant and Methods: A 10-year-old male was referred for neuropsychological evaluation at an outpatient clinic secondary to a diagnosis of PMS, and concerns regarding poor communication skills impacting academic performance and social relationships. Evaluation included medical record review, parent interview, observations, and administration of a neuropsychological test battery. Results: Results: Evaluation findings revealed a Specified Neurodevelopmental Disorder related to Phelan-McDermid syndrome and characterized by: Language Disorder, Social (Pragmatic) Communication Disorder, ADHD, Developmental Coordination Disorder, and Specific Learning Disorders, and an Anxiety Disorder. Cognitive assessment revealed verbal deficits. Neuropsychological deficits were evident on tasks requiring language, learning, and executive functioning. Mild to moderate social responsiveness difficulties were identified on one rating scale, while behavior checklists did not indicate significant social problems. Records and observations indicate strong social intent and reciprocity. He did not display RRB's to meet diagnostic criteria for ASD. Conclusions: Conclusions: This case study illustrates the variable phenotype of PMS. Consistent with the existing literature, the current case is marked by neurocognitive, pragmatic language, and attention deficits. However, the identified social difficulties did not meet the full criteria for ASD, highlighting the wide range in severity and breadth of autism spectrum features in those with PMS.

Correspondence: andrew a. powell, B.S., MD, United States. E-mail: andrewapowell23@gmail.com

WITHDRAWN. E.A. SCHARAGA \& A. CHANG. Cognitive Sequelae of Neurofibromatosis Type 1: A Progressive Look.

Objeetive: Netrofibromatosis type 1 (NF1), an autosomal dominant genetic disorder, is eharaeterized by netrofibromas, axillary and/or inguinal freckling. Wisch nodtes, and cafe-atr-tait spots. Majority of studies in NF1 have been condueted in pediatries due to eognitive aeademic diffientlies across development. Further, the restlts of nemropsychologieal performance int older actutts with $\mathrm{NF} 1$ is sparse

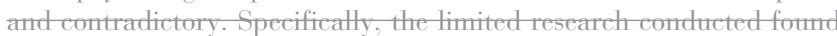
that defieits related to NF1 remain stable aeross the lifespan, with no evidence of progression. Thus, serial eognitive testing in an older adult

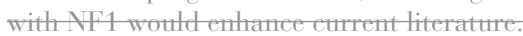

Participants and Methods: A case study of a 65-year-old mate who was diagnosed with $\mathrm{NF} 1$ as a teenager. He was referred for outpatient 
netropsychologieal evaluations in 2013 and 2017 due to eognitive tecline in 2008 .

Pesults: Gomparing the patient's 2017 performanter 2913 results, he evideneed further reduetions in nearly all aspe 15 ofeos itive funetion. He demonstrated declines in vistospatta fth tior, as expeeted

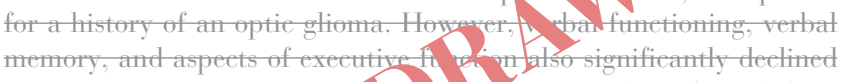
sinee 2013 . Moreover, mood rere ted tis, sess inereased signifieantly (i.e.) inereased vegetative and ter hy ymptoms), with limited insight into his eognitive definif

Gonelusions: If pat int's funetional and cognitive abilities deelined drastieak th fve Jar's, irrespeetive of his vistual deeline due to central

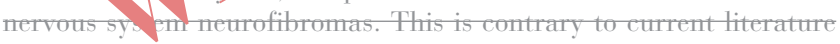
toeumenting stable cognition int olter atults with NF1. Our enrrent ease highlights the vulnerability of elderly NF1 patients for cognition decline, beyond the general aging poptlation. Speeifieally, vistospa= tial functioning, working memory, eognitive flexilbility, and lomg-term memory are impaired.

Gorrespondenee. Elyssa A. Seharaga, M.A., Clinieal Psychology, Keshiva University, 139 Altessa Blod., Melville, NT 11747, United States. E-mait: scharaga@gmatit.com

C. SEMERJIAN, K.S. WALSH, A. THOMAS \& J. MORRIS. Comparing Executive Functioning and Social Problems in Pediatric Neurofibromatosis Patients with and without comorbid Attention-Deficit/Hyperactivity Disorder.

Objective: Despite $30 \%-50 \%$ of children with neurofibromatosis type 1 (NF1) meeting criteria for attention deficit/hyperactivity disorder (ADHD), the differential profile of symptoms associated with NF1 children with comorbid ADHD is not well understood. We examined the executive profiles and social functioning of children with NF1, both with and without comorbid ADHD, and of healthy children with neurodevelopmental ADHD.

Participants and Methods: Parent-reports from the Behavior Rating Scale of Executive Function (BRIEF) and the Achenbach Child Behavior Checklist (CBCL, Social Problems Scale) were collected from forty-three children with NF1 only (Group 1), 39 children with NF1 and comorbid ADHD (Group 2), and 40 children with developmental ADHD (Group $3)$. An a priori paired comparison analysis was utilized to compare parent-reported symptoms of executive dysfunction and social competence in our comorbid group (NF1 +ADHD) with combined means of other groups.

Results: Planned contrasts revealed that significantly higher mean BRIEF MI ratings for Group 2 (NF1 + ADHD) compared to Groups 1 (NF1 only) and 3 (ADHD only) combined $t(111)=2.29, p=.024$. Additionally, there was a significantly higher BRIEF MI score for Group 3 (ADHD only) compared to Group 1 (NF1 only), $t(111)=-5.183$, $p=.000$. Further, mean CBCL Social Problems ratings for Group 2 (NF1+ADHD) were significantly higher than the CBCL Social Problems mean of Groups 1 (NF1 only) and 3 (ADHD only) combined $t(96)=2.18, p=.032$. However, comparison of Group 1 (NF1 only) to Group $3(\mathrm{ADHD})$ indicated no significant difference between mean CBCL Social Problems ratings $t(96)=.717, p=.475$.

Conclusions: NF1 children with comorbid ADHD appear to manifest different problems compared to NF1 children without ADHD and children with developmental ADHD. Elucidation of factors that differentiate this group is essential to understanding the pathophysiology of cognitive manifestations, and guiding treatment recommendations.

Correspondence: Claire Semerjian, M.S., Psychology, Universtiy of Florida, 206 E Chestnut Street, Apt 902, Chicago, IL 60611, United States.E-mail: csemer319@gmail.com
B.D. YUND, K. LEE, C. CASNAR \& B. KLEIN-TASMAN. Executive Functioning Profiles in Children with NF1 Using Lab-Based and Functional Measures: Influence of ADHD?

Objective: Research suggests executive function (EF) impairment occurs in children with neurofibromatosis type 1 (NF1), independent of attention-deficit/hyperactivity disorder (ADHD) diagnosis, suggesting that the presence of EF impairments is not uniquely associated with ADHD in NF1. However, functional questionnaires have not been consistently utilized to assess the influence of ADHD diagnosis on EF. Therefore, examination of EF in children with NF1 with and without ADHD using a multi-method approach is needed.

Participants and Methods: Participants were 37 children with NF1 (23 without ADHD; 14 with ADHD) ages 9-13 and their parent. Standardized lab-based measures (Differential Ability Scales-Second Edition: DAS-2, Recall of Sequential Order: RSO \& Recall of Digits-Backward: RDB), NIH Toolbox List Sorting Working Memory: LSWM, Dimensional Change Card Sort Test: DCCS) were administered to children and standardized functional measures (Behavior Rating Inventory of Executive Function: BRIEF; Conners-3 Parent Short Executive Function scale: Conners) were administered to parents.

Results: Children with and without ADHD did not differ significantly on lab-based measures of EF (RSO, $t(35)=1.40, p=.170$; RDB, $t(35)$ $=.304, p=.763 ;$ LSWM, $t(35)=1.27, p=.214 ;$ DCCS, $t(35)=1.03$, $p=.309)$; however, children with ADHD tended to perform in the low average range, while children without ADHD generally performed in the average range. Significant differences in performance between children with and without $\mathrm{ADHD}$ were apparent on functional parent-reported EF measures (BRIEF, $t(35)=-3.50, p=.001$; Conners, $t(35)=-2.08$, $p=.044)$.

Conclusions: Results indicate that children with NF1 demonstrate similar EF profiles on lab-based measures, independent of comorbid ADHD diagnosis. However, children with ADHD demonstrated significantly elevated levels of executive dysfunction based on functional parent-reported measures. Results highlight the importance of using functional measures when assessing EF in children with NF1.

Correspondence: Brianna D. Yund, Psychology, University of WisconsinMilwaukee, 4008 N Morris Blvd, Apt 3, Milwaukee, WI 53211, United States.E-mail:bdyund@uwm.edu

\section{HIV/AIDS/Infectious Disease}

K.N. DEVLIN, T. GIOVANNETTI, D.J. LIBON, W. DAMPIER, V. PIRRONE, M. NONNEMACHER, J. JACOBSON \& B. WIGDAHL. Functional Outcomes Among Latent Subtypes of HIV-Associated Neurocognitive Disorder (HAND).

Objective: Functional outcomes of HIV-associated neurocognitive disorders (HAND) are heterogeneous and may be associated with the profile of neuropsychological impairments. We examined everyday and occupational function among statistically identified neuropsychological subtypes in HIV+ adults, then examined whether these subtypes or conventional HAND criteria were better predictors of functioning.

Participants and Methods: $193 \mathrm{HIV}+$ adults enrolled in the Drexel Medicine CNS AIDS Research and Eradication Study (CARES) Cohort completed cognitive testing, reported their employment status, and rated their ability to perform complex daily tasks (Functional Activities Questionnaire; FAQ). Participants were classified into 1) subtypes of HAND based on the level and pattern of cognitive performance using latent class analysis and 2) conventional HAND categories based on level alone (i.e., major or mild; Frascati criteria). FAQ score and employment were examined as a function of latent classes and HAND categories.

Results: Among four latent classes, the moderate mixed impairment class and mild motor impairment class, but not the mild memory impairment class, demonstrated elevated functional difficulties relative to the cognitively intact class. Latent class membership accounted for $7 \%$ of the variance in FAQ score and 18\% of the variance in employment. 
HAND category accounted for $7 \%$ of the variance in FAQ score and $8 \%$ of the variance in employment.

Conclusions: $\mathrm{HIV}+$ persons with mild motor impairment demonstrate more functional difficulties than those with mild memory impairment despite similar levels of cognitive impairment. Conventional and statistical HAND categories perform similarly in predicting everyday functioning difficulties, but statistical criteria may better predict occupational impairment. Classification by neuropsychological profile, rather than severity alone, may clarify understanding of heterogeneous functional outcomes in $\mathrm{HIV}+$ persons and identify targets for cognitive rehabilitation.

Correspondence: Kathryn N. Devlin, M.A., Psychology, VA Maryland Healthcare System, 1018 N. Calvert St., Baltimore, MD 21202, United States.E-mail:kathryn.devlin@temple.edu

\section{D.J. HARDY \& N.A. MURPHY. Disentangling Measures of Information Processing Speed: A Meta-Analytic Illustration with HIV/AIDS.}

Objective: While decline in information processing speed is ubiquitous in persons with neurologic injury and disease, tests with wide ranging processing requirements are routinely grouped together under this rubric, often producing disparate results. A random-effects meta-analysis was conducted on HIV studies, where information processing speed decline is a classic symptom.

Participants and Methods: Data from two mostly non-overlapping meta-analyses were re-examined: the information processing speed domain in Reger et al. (2002), and Hardy and Hinkin (2002), which focused exclusively on reaction time studies.

Results: The mean weighted effect size estimate was $d=0.21(k=57$; $95 \% \mathrm{CI}=0.175,0.25)$. Because heterogeneity among effect sizes was evident via a $Q$ statistic, original outcome measures were grouped into two categories: (1) traditional neuropsychological tests (e.g., Symbol Digit), and (2) reaction time measures where all tests required a simple button-press and therefore minimal and uniform motor-response processing. The mean weighted effect size estimate for traditional neuropsychological tests was $d=0.26(k=28 ; 95 \% \mathrm{CI}=0.19,0.31)$, and for reaction time measures, $d=0.19(k=25 ; 95 \% \mathrm{CI}=0.13,0.25)$. Conclusions: Although results suggest, tentatively, a somewhat smaller effect size in reaction time measures, a direct comparison is difficult because of the non-independence across measures. Also, both distributions of effect sizes were heterogeneous (effect size differences within each distribution were larger than expected due to sampling error). These results represent an initial attempt at disentangling the diverse measures of information processing speed, and suggest the need to clarify and stratify such measures and this construct.

Correspondence: David J. Hardy, Ph.D., Psychology, Loyola Marymount University, 1 LMU Drive, Suite 4700, Los Angeles, CA 90045, United States. E-mail: david.hardy@lmu.edu

V.M. KORDOVSKI, S.M. TIERNEY \& S.P. WOODS. The Effects of Aging and HIV Disease on Alternating Semantic Verbal Fluency. Objective: Additive effects of aging and HIV disease are reliably observed on executive processes, including naturalistic switching during traditional phonemic and semantic verbal fluency tasks. The present study examined the combined effects of aging and HIV on alternating semantic verbal fluency, which may magnify generative switching deficits.

Participants and Methods: Participants ( $\mathrm{N}=346$ ) were classified by HIV serostatus and age (Younger < 41 years; Older $>49$ years), which yielded 114 Older HIV+; 77 Older HIV-; 86 Younger HIV+; and 69 Younger HIV-adults. The alternating semantic fluency task required switching between two semantic categories: animals and instruments. Participants also completed phonemic (i.e., letter "B") and semantic (i.e., clothing) trials, in addition to a comprehensive research neuropsychological battery.
Results: Multiple regressions adjusting for relevant covariates (e.g., education, race, mood, and hepatitis $\mathrm{C}$ ) revealed no significant main effects or interactions of older age or HIV serostatus on semantic or phonemic fluency ( $p$ s $>.05$ ). However, an age by HIV interaction was a significant predictor of alternating fluency $(\beta=-1.58, p=.03)$ such that Older HIV+ adults had fewer switches than the other groups (all $p s<.01$ ), but the three groups did not differ amongst each other ( $p s>.05)$. Among Older HIV+ adults, alternating fluency performance demonstrated medium associations with executive functions, attention/ working memory, episodic memory, and information processing speed, but not fine motor coordination. Switching performance was not related to disease variables or everyday functioning in Older HIV+ adults (all $p s>.05$ ).

Conclusions: Findings suggest that older age and HIV disease have additive adverse effects on alternating fluency performance, but not on overall phonemic or semantic fluency. Longitudinal studies are needed to examine the extent to which these deficits are associated with age or disease-related changes in fronto-striatal networks.

Correspondence: Victoria M. Kordovski, Psychology, University of Houston, 126 Heyne Building, Suite 204, University of Houston, Houston, TX 77021, United States.E-mail:vkordovski@uh.edu

C.F. LEVITCH, A. JAYWANT, C. LIN, C.B. GOPIN, F. GUNNING \& N. CHOWDHURY. Neuropsychological Assessment and Management of an Individual with Listeria Encephalitis in the Acute Inpatient Rehabilitation Setting.

Objective: Listeria encephalitis is an uncommon and often misdiagnosed CNS infection that has neurologic signs and symptoms, and lesion burden, that are distinct from other forms of encephalitis. Because Listeria preferentially affects the brainstem and cerebellum, a well-defined cognitive profile may exist that can inform rehabilitation; however, no previous research has determined the cognitive strengths and weaknesses or the clinical utility of neuropsychological assessment in this infection. We document the neuropsychological profile of a previously healthy, high functioning male with Listeria encephalitis, highlighting the role of neuropsychology in his successful inpatient rehabilitation. Participants and Methods: A 46-year-old, right-handed man with 18 years of education and no prior medical history was admitted to acute inpatient rehabilitation. Neuropsychological assessment was conducted at admission and discharge, and was adapted for his motor, visual, and neuropsychiatric clinical symptoms.

Results: Consistent with damage to the brainstem and cerebellum. and to associated structural and functional circuits, neuropsychological assessment revealed significant deficits in processing speed and executive functions, as well as visuospatial construction and verbal learning/ memory. These findings identified specific targets for the patient's successful cognitive remediation and functional rehabilitation in occupational, physical, and speech therapy. Re-evaluation prior to discharge demonstrated improvements across cognitive domains, though deficits in processing speed, executive functions, and verbal learning remained. Conclusions: Listeria encephalitis is associated with deficits in multiple cognitive domains, most prominently in executive functions. Neuropsychologists can play an integral role in the interdisciplinary rehabilitation of individuals with Listeria encephalitis by documenting the presence of, and change in, cognitive deficits and by guiding treatment.

Correspondence: Cara F. Levitch, BAS, Psychology, Fordham University, 240 E 46th St, Apt 2G, New York, NY 10017, United States. E-mail: clevitch@fordham.edu

Z. MAHMOOD, A. HAMMOND, R.A. NUNEZ, A. KARIMIAN \& A. THAMES. The Effects of Poor Sleep on Cognition Are Greater Among HIV+ versus HIV- individuals.

Objective: People living with HIV (PLWH) are more likely to report sleep difficulties and cognitive deficits. While cognitive impairment associated with sleep disturbance has been found in healthy and medical populations, less is known about whether poor sleep quality (SQ) confers 
greater cognitive compromise to PLWH. This study examined the effects of SQ on cognitive functioning in HIV+ and HIV- individuals.

Participants and Methods: Ninety-seven ( $=97$ ) adults recruited from the Greater Los Angeles community were administered a brief cognitive test battery and completed a questionnaire about sleep quality (SATED scale; Buysse, 2014). Two-step cluster analysis was used to classify participants into distinct HIV/SQ groups: [good sleep quality $(\mathrm{SQ}+)$ groups: $\mathrm{HIV}+/ \mathrm{SQ}+(\mathrm{n}=34)$ and $\mathrm{HIV}-/ \mathrm{SQ}+(\mathrm{n}=24)$; poor sleep quality (SQ-) groups: HIV-/SQ- $(\mathrm{n}=13)$ and HIV+/SQ- $(\mathrm{n}=26)$ ], based upon their responses to the SATED scale.

Results: $\mathrm{HIV}+$ participants reported significantly poorer SQ compared to controls $(p=0.04)$. SQ was positively correlated with global cognition, with the domains of executive functioning, learning and memory driving the overall relationship (all $p ' s<0.05$ ). MANOVA demonstrated that the HIV+/SQ- group $(M=34.00, S D=7.68)$ had poorer scores on learning compared to both $\mathrm{HIV}+/ \mathrm{SQ}+(M=40.51, S D=11.08)$ and HIV-/SQ+ groups $(M=48.86, S D=6.82)(p ' s<0.01)$. Further, $\mathrm{HIV+}$ SQ- individuals had lower memory scores compared to all comparison groups (all $p ' s<0.05$ ). No other statistically significant differences were found amongst the clustered groups.

Conclusions: The study findings coincide with prior investigations associating impairments in learning and memory with sleep disturbance. Moreover, our findings suggest that HIV+ individuals may be susceptible to greater cognitive impairment due to poor SQ, which underscores the scientific and clinical importance of monitoring sleep health to help identify individuals at greatest risk of poor cognitive outcomes.

Correspondence: Zanjbeel Mahmood, Psychiatry \& Biobehavioral Sciences, University of California, Los Angeles, 740 Westwood Plaza, 28-255, Los Angeles, CA 90095, United States. E-mail: zmahmood@ ucla.edu

S. MICHEL, R. DASHER \& J. BIRATH. Neurocognitive and Neuropsychiatric Change Due to HIV and Neurosyphilis May Worsen Personality Pathology: A Case Study.

Objective: The relationship between human immunodeficiency virus (HIV) and syphilis infections is complex, and coinfection can lead to both neurocognitive and neuropsychiatric symptoms. These symptoms may exacerbate premorbid personality traits, making it difficult to discern the etiology of a patient's mental health symptoms and provide effective treatment.

Participants and Methods: A 61-year-old Caucasian male was hospitalized with altered mental status, psychosis, mania, and suicidality and discovered to have untreated HIV and neurosyphilis. An initial neuropsychological evaluation was conducted three weeks post discharge. He underwent outpatient infectious disease and psychiatric treatment over the next 11 months, and he was then re-evaluated.

Results: Initial deficits included processing speed, verbal memory, executive functioning, and psychomotor abilities, consistent with HIV. Significant anxiety, somatization, and narcissistic personality traits were noted. Although he improved, it was unclear whether his ongoing functional and interpersonal difficulties were due to cognitive impairment, psychiatric symptoms, or personality pathology. Follow-up evaluation found improvement in verbal memory but continued weakness in processing speed, visuospatial memory, executive functioning, and psychomotor abilities. Evaluation of emotional and personality functioning indicated significant emotional dysregulation and other symptoms consistent with Borderline Personality Disorder. Collateral reports supported the diagnosis and indicated worsening of personality symptoms since the initial evaluation.

Conclusions: Neuropsychological evaluation of central nervous system infections should include careful examination of emotional functioning as well as personality traits. This case suggests that the neurocognitive impact of HIV and neurosyphilis may result in worsening of premorbid personality symptoms, such as increased emotional dysregulation. Such symptoms can have significant impact on treatment adherence and functional recovery.
Correspondence: Sandra Michel, MA, 14445 Olive View Drive, Department of Psychiatry, Sylmar, CA 91342, United States. E-mail: sandra.michel@pepperdine.edu

E. PAOlillo, E. PASipanodya, R.K. HeATON \& D. MOORE. Depressive Symptoms Are Associated with Cognitive Decline in HIV/AIDS.

Objective: Depression is common among persons living with HIV/AIDS (PLWHA), and is associated with neurological disturbances. The relationship between depression and cognition, however, is unclear. The current study explores associations between depressive symptoms (DS) and global cognitive functioning (GCF) over time.

Participants and Methods: Participants included 508 PLWHA enrolled in longitudinal studies at the HIV Neurobehavioral Research Program from 2002-2016. Participants had an average of 4.8 visits (range $=2-18$ ) over an average of 2.6 years (range $=0.5-12.4)$. DS were assessed via the Beck Depression Inventory (BDI-II) and categorized by clinical cutoffs: minimal (0-13), mild (14-19), and moderate-to-severe (20-63). To measure GCF, we used a practice effect-corrected (but not demographically-corrected) scaled score $(\mathrm{M}=10, \mathrm{SD}=3)$ comprised of all tests in a cognitive battery. Multilevel modeling was used to examine within- and between-person associations between DS and GCF over time (i.e., years). Covariates included baseline sex and education, and time-varying age and CD4 count.

Results: Within individuals: DS were associated with poorer GCF such that on visits when participants reported mild or moderate-to-severe DS, they exhibited worse GCF $(\mathrm{B}=-0.136, p=0.018$ and $\mathrm{B}=-0.160, p=0.013$, respectively) compared to visits with minimal DS. Between individuals: When averaging each participant's BDI-II scores across visits, higher mean DS severity was associated with greater declines in GCF from baseline to last visit. Specifically, those whose mean BDI-II score fell within the moderate-to-severe range exhibited a faster decline compared to those with minimal DS ( $\mathrm{B}=-0.079, p=0.005)$. Mild DS were also associated with greater GCF decline at trend level $(\mathrm{B}=-0.060, p=0.078)$. Conclusions: Among PLWHA, DS are related to worse GCF per visit as well as declines in GCF over time. Future research is needed to determine directionality of effects and to explore domain-specific cognitive deficits related to DS (e.g., psychomotor slowing).

Correspondence: Emily Paolillo, University of California, San Diego, 220 Dickinson Street, Suite B, San Diego, CA 92103, United States. E-mail: empaolillo@gmail.com

\section{J. SCOTT, T.M. MOORE, M. PERRIN, C. VOYTEK, D.S. METZGER,} D.L. EVANS \& R.C. GUR. Evaluation of a Brief Computerized Neurocognitive Battery for the Detection of HIV-Associated Neurocognitive Disorders (HAND).

Objective: Approximately 30-50\% of individuals with HIV evidence HIV-associated neurocognitive disorders (HAND). Currently, assessment of HAND is predominantly conducted with paper-and-pencil (P\&P) neuropsychological tests that are lengthy and involve extensive training in administration and scoring procedures. Modern computerized neurocognitive testing could improve this process by offering shorter testing times and sophisticated features unavailable in P\&P tests, such as adaptive testing and precise, automatic recording of response times and accuracy. Here, we tested the validity of the brief, wellestablished Penn Computerized Neurocognitive Battery (CNB) to detect HIV-associated cognitive impairment.

Participants and Methods: A traditional neuropsychological battery sensitive to HAND was administered in a counterbalanced order alongside an adapted CNB to 40 adults with HIV and 23 HIV seronegative individuals. Participants also received assessments of daily functioning and mood. Classification accuracy statistics were conducted to predict cognitive status.

Results: Of the individuals with HIV, 22 (55\%) were cognitively unimpaired and $18(45 \%)$ were diagnosed with HAND, as determined by the traditional neuropsychological battery and functional assessments. 
Of those with HAND, 13 (32.5\%) had asymptomatic neurocognitive impairment and $5(12.5 \%)$ had minor neurocognitive disorder. Using the traditional neuropsychological assessment as the gold standard, the CNB displayed adequate classification accuracy (77.5\%), with slightly lower sensitivity $(72.2 \%)$ than specificity $(81.8 \%)$, for detecting HAND. Total area under the ROC curve for predicting HAND status was 0.77 . Conclusions: Data from adults living with HIV support the feasibility and validity of the adapted CNB battery for assessing cognitive impairment associated with HIV. Future studies could deploy this battery in HIV clinics to gather additional cognitive performance data and valuable information on its practical usability and acceptability with patients and care providers.

Correspondence: J. Cobb Scott, Ph.D., Psychiatry, Perelman School of Medicine at the University of Pennsylvania, 3400 Spruce Street, 10th Floor, Gates Building, Philadelphia, PA 19104, United States. E-mail: scott1@upenn.edu

D.P. SHEPPARD, S.P. WOODS, R. HASBUN, L. SALAZAR, M.S. NOLAN \& K.O. MURRAY. Intraindividual Neurocognitive Variability is Associated with Lower Physical Quality of Life in West Nile Virus Infected Individuals.

Objective: West Nile Virus (WNV) is neuroinvasive and can produce mild-to-moderate neurocognitive impairments that persist after acute infection. Intra-individual variability (IIV) is an index of neuropsychological performance spread within an individual, which is an indicator of neurocognitive control and the integrity of prefrontal systems. The present study aimed to determine the extent to which IIV is related to health and well-being outcomes in the context of WNV infection.

Participants and Methods: Participants included 110 adults with a range of clinical WNV disease (27\% neuroinvasive disease; e.g., encephalitis, meningitis) who completed the RBANS. To compute covariance of variation $(\mathrm{CoV})$, intra-individual standard deviation across the 5 age-adjusted standard scores of the RBANS was generated and divided by the mean of the standard scores. Participants were assessed for health-related quality of life (QoL) using the RAND 36-item short form health survey (SF-36).

Results: Logistic regression controlling for age revealed a significant interaction between education and RBANS CoV in predicting neuroinvasive disease: in persons with higher (15+ years) education, neuroinvasive disease was associated with higher RBANS CoV (Cohen's $d=-1.15$, $p=.003)$. Mixed linear models controlling for estimated IQ, activities of daily living, depression, neuroinvasive disease, and fatigue showed that higher RBANS CoV was associated with lower physical $(p=.021)$, but not mental $(p>$.10) QoL on the SF-36.

Conclusions: Neuroinvasive disease was associated with RBANS CoV among WNV patients with high levels of education. Within-individual variability of neuropsychological performance on the RBANS was independently associated with poorer physical QoL, suggesting that even subtle changes in neuropsychological functioning may impact WNV survivors' recovery. Future studies should examine whether IIV predicts long-term health outcomes (e.g., mortality) in individuals infected with $\mathrm{WNV}$

Correspondence: David P. Sheppard, M.A., Psychology, University of Houston, University of Houston, 126 Heyne Building, Suite 204, Houston, TX 77004-5022, United States.E-mail: dpsheppard@uh.edu

\section{A. SUMMERS, D. BYRD, K. TURESON \& M. RIVERA MINDT. The Relationship Between Sociocultural Factors \& Decision-Making in HIV+ Latinx Adults.}

Objective: Sociocultural (SC) factors (e.g., acculturation, quality of education $[\mathrm{QoE}]$ ) are related to executive function. However, no study to date has examined how they impact the Iowa Gambling Task (IGT), a measure of decision-making. Given the high prevalence of executive dysfunction in HIV \& disproportionate impact of HIV on Latinx adults, this study aimed to determine how SC factors impact IGT performance.
Participants and Methods: This cross-sectional study included 64 $H I V+$ Latinx adults $(67 \%$ Male; $M$ Age $=45 \pm 8$ years; $M$ Education $=12 \pm 3$ years) who completed SC measures (Abbreviated Multidimensional Acculturation Scale [AMAS]; Brief Perceived Ethnic Discrimination Questionnaire [PEDQ Total]; Hollingshead Index [SES]; \& Wechsler Test of Adult Reading [WTAR; QoE]) \& the IGT (Total Draws from Decks A \& B = net loss; Decks C \& D = net gain).

Results: Deck A negatively correlated with the WTAR $\left(r_{s}=-.43\right.$, $p<.001)$. Deck B was not associated with any of the SC variables (all $p$ 's $>05$ ). Deck C was positively related to AMAS-Latinx Identity $\left(\mathrm{LI} ; r_{s}=.31, p<.02\right) \&$ negatively related to PEDQ $\left(r_{s}=-.28, p=.03\right)$. Deck D was positively correlated with WTAR \& Hollingshead $\left(r_{s}=.30-.36\right.$; all $p$ ' $<<.02$ ). Linear regressions revealed that the full model (AMASLI, Hollingshead, PEDQ, \& WTAR) significantly predicted Decks $\mathrm{A}\left(R^{2}=.17\right), \mathrm{C}\left(R^{2}=.17\right), \& \mathrm{D}\left(R^{2}=.24 ;\right.$ all $p$ ' $\left.<.05\right)$. Only WTAR uniquely predicted Deck A $(\beta=-.33, p=.02)$, AMAS-LI predicted Deck $\mathrm{C}$ at the trend level $(\beta=.23, p=.08)$, \& AMAS-LI $(\beta=-.25) \&$ WTAR $(\beta=.29)$ uniquely predicted Deck D (all $p$ ' $<.05)$.

Conclusions: This study indicates that greater Latinx acculturation, SES, \& QoE \& lower levels of perceived ethnic discrimination are all related to better decision-making in HIV+ Latinx adults. These findings are important given that decision-making has important implications for health behaviors (medication adherence) \& HIV transmission. Culturally tailoring interventions to incorporate the role of SC factors in decision-making may improve HIV education \& health outcomes for HIV+ Latinx adults.

Correspondence: Angela Summers, BA, Fordham University, 441 E Fordham Rd., DE 324, Bronx, NY 10458, United States. E-mail: ac.summers.09@gmail.com

S.M. TIERNEY, S.P. WOODS, D.P. SHEPPARD \& R.J. ELLIS. Additive Effects of HIV and Aging on the Unified Parkinson's Disease Rating Scale: Associations with Everyday Functioning and Quality of Life.

Objective: Age and HIV disease have additive effects on neural systems that support motor functioning. The current study examined the combined impact of aging and HIV on extrapyramidal motor functions in relation to activities of daily living (ADLs) and quality of life (QoL). Participants and Methods: Participants included 337 adults classified by HIV serostatus and age (older $\geq 50$ and younger $<40$ ): Older HIV+ $(\mathbf{n}=109)$; Older HIV $-(n=74)$; Younger HIV $+(n=84)$; and Younger HIV - $(n=70)$ individuals. A research nurse administered the Unified Parkinson's Disease Rating Scale (UPDRS), from which motor signs were characterized as "Present" (UPDRS $>0$ ) or "Absent" (UPDRS=0). Participants completed the Short Form Survey Instrument (SF-36) and the modified Lawton \& Brody Activities of Daily Living (ADL) questionnaire as part of a larger neuropsychological battery.

Results: A Cochran-Armitage Trend Test showed additive effects of HIV and aging $(p<.001)$, such that Older HIV+ adults had the highest rates of UPDRS elevations (51.4\%) compared to Older HIV- (44.6\%), Younger HIV + $(21.4 \%)$, and Younger HIV - (18.6\%) participants. These additive effects were independent of demographics, neurocognitive impairment, and medical/psychiatric comorbidities. Among Older HIV+ adults, a multiple regression controlling for disease severity, depression, and neurocognitive functions showed that presence of UPDRS motor signs was independently associated with basic and instrumental ADL declines, as well as lower physical $(p s<.05)$, but not mental QoL on the SF-36.

Conclusions: Age and HIV status have independent, additive effects on extrapyramidal motor signs and may modulate earlier observed associations of neuropsychological performance and function. When present in older HIV+ adults, motor signs confer an increased risk of daily functioning problems and lower health-related physical QoL. Longitudinal studies are needed to identify the trajectory of motor signs in HIV disease and potential moderators. 
Correspondence: Savanna M. Tierney, B.A., Psychology, University of Houston, 4807 Pin Oak Park \#1706, Houston, TX 77081, United States. E-mail:smtierney2@uh.edu

K. TURESON, D. BYRD, A. SUMMERS \& M. RIVERA MINDT. The Impact of Sociocultural Factors on Prospective Memory Function in HIV+ Latinx Adults.

Objective: US Latinx adults are disproportionately impacted by HIV. Sociocultural (SC) factors (e.g., acculturation, quality of education) are also related to neurocognitive performance in this group. However, the association between SC factors \& a key component of memory functioning in HIV, prospective memory, remains unknown. The aim of this study was to examine whether significant associations exist between $\mathrm{SC}$ factors \& prospective memory in HIV+ Latinx adults.

Participants and Methods: This cross-sectional study included 128 HIV+ adults (72\% Latinx \& 28\% non-Hispanic white [NHW]; 68\% Male; $M$ Age $=48 \pm 9$ years; $M$ Education $=13 \pm 3$ years) who completed measures of quality of education (QoE; Wechsler Test of Adult Reading [WTAR Standard Score]) \& prospective memory (PM; Memory for Intentions Screening Test [MIST; Summary Score, PM Errors, Recognition]). Latinx adults also completed a measure of acculturation (Abbreviated Multidimensional Acculturation Scale ([AMAS]; Latinx Identity [LI], US Identity [UI]).

Results: A series of $t$-tests showed that the Latinx group had more PM Errors \& worse PM Recognition than the NHW group (all $p$ 's <.05). Within-group analyses (Latinx group) using linear regressions revealed that our model of SC variables (AMAS-LI, AMAS-UI \& WTAR) predicted the MIST Summary Score $\left(R^{2}=.24, p<.01\right) \&$ PM Errors $\left(R^{2}=.13, p<.01\right)$, with AMAS-LI ( $\beta$ 's=-.25\&.22, respectively) \& WTAR ( $\beta$ ' $s=.36 \&$ -.24 , respectively) uniquely contributing to both models (all $p$ 's<.05). This model also significantly predicted PM Recognition $\left(R^{2}=.10, p<.05\right)$, with only WTAR providing a unique contribution $(\beta=.23, p<.05)$.

Conclusions: This study indicates that HIV+ Latinx adults demonstrate worse PM compared to HIV+ NHW adults. Acculturation \& QoE are important considerations for understanding PM in HIV+ Latinx adults. Thus, acculturation \& QoE should be evaluated when considering PM \& tasks that require PM, such as medication adherence. Future research should study the role of SC variables in PM within other underrepresented populations.

Correspondence: Kayla Tureson, BA, Fordham University, 503 West 148th St Apt 33, New York, NY 10031, United States.E-mail: ktureson@ fordham.edu

M.J. WILSON, T. LEE-WILK \& M. DUX. Methods for Determining Neurocognitive Risk Among Veterans with HIV/AIDS in an Interdisciplinary VA Infectious Disease Clinic.

Objective: The Baltimore VA treats $~ 700$ Veterans with HIV, with high rates of comorbid psychiatric $(76 \%)$ and substance use disorders (SUD, 81\%). Despite high viral suppression rates (90+\%), 78\% meet criteria for a neurocognitive disorder. Previous analyses show that medical comorbidities (e.g., vascular risk factors) account for significant variance in neurocognitive functioning when controlling for ID-specific markers (e.g., viral load), indicating that many factors, not just those directly associated with HIV, contribute to neurocognitive dysfunction. We evaluated a novel method for identifying Veterans with HIV at elevated risk for neurocognitive impairment.

Participants and Methods: The Veterans Aging Cohort Study (VACS) Index is a point system designed to quantify infectious disease markers (e.g., CD4 count) and related medical comorbidities (e.g., kidney/liver disease). To assign risk for neurocognitive dysfunction, we developed a modified VACS (mVACS) Index by adding known neurocognitive risk factors (e.g., vascular risk factors, SUD/psychiatric disorders). A provisional mVACS cutoff of $\geq 100$ was considered to indicate high risk for neurocognitive impairment. Patients identified as high risk were referred for neurocognitive assessments to inform treatment planning.
Results: Our sample consisted of 39 Veterans with HIV, mostly African American (80\%) and male (97\%), with an average age of $5 \dot{5}$ $(S D=9)$ and 12 years education $(S D=3)$. Mean CD4 count was 418 $(S D=262), 62 \%$ were HIV suppressed $(<40$ copies $/ \mathrm{ml})$, and $59 \%$ were HCV co-infected. The mVACS Index was associated with neurocognitive performance $\left(R^{2}=.63, F=4.9, p=.002\right)$ and demonstrated specific associations $(p<.05)$ with measures of executive functioning, language, and verbal/nonverbal memory. In contrast, the traditional VACS Index was not associated with neurocognitive functioning $(p=.12)$.

Conclusions: Results suggest that the mVACS may represent an effective method for identifying Veterans with HIV at increased risk of neurocognitive dysfunction.

Correspondence: Michael J. Wilson, Ph.D., Psychology, Minneapolis VAMC, 2915 W 50th St, Apt 401, Minneapolis, MN 55410, United States.E-mail: mjwilson14@gmail.com

\section{Medical/Neurological Disorders/Other} (Adult)

J. BELLONE, L. MILLER, W. WU, S. MORAN \& S. CORREIA. Feasibility and Initial Results of Inspiratory Muscle Training on Cognition and Functional Capacity in Older Veterans with Heart Failure.

Objective: To examine whether inspiratory muscle training (IMT) can enhance cognition and functional capacity in veterans with congestive heart failure (CHF). Inspiratory muscle weakness has been implicated as an underlying mechanism of exercise intolerance in CHF and may be associated with CHF-related cognitive impairment. IMT increases respiratory muscle strength through repeated inspiratory exercise with resistance, and has been associated with improved functional capacity in CHF at levels comparable to those seen with structured exercise programs.

Participants and Methods: Participants are 8 older male veterans (mean age $=75$ ) with clinically stable CHF and no known history of neurological conditions (e.g., stroke, TBI, dementia). They were randomized to active $(n=6)$ or pseudo-sham $(n=2)$ IMT. In active treatment, the IMT device is set to a resistance of $30 \%$ maximum inspiratory pressure (MIP; a measure of functional capacity); in pseudo-sham IMT, the device is set to $5 \%$ MIP or lowest setting. Participants completed up to 30 minutes of daily at-home breathing exercises for 6 weeks and attended weekly study visits to assess MIP and adjust the IMT device. Neuropsychological testing was completed at study baseline and end of study.

Results: The current small pilot sample size precludes formal statistical analyses, but a few trends have emerged. For example, the active-IMT group's MIP improved by 27.93 (SD=13.58) compared to 12.60 ( $\mathrm{SD}=12.16)$ for pseudo-sham from baseline to post-treatment. Cognition appears to be relatively stable within this limited sample. There were no unexpected study-related adverse events.

Conclusions: Initial findings indicate that a 6 -week IMT intervention is a feasible and generally well-tolerated at-home intervention for older veterans with $\mathrm{CHF}$.

Correspondence: John Bellone, Brown University, 57 Cypress St. Apt 2, Providence, RI 02906, United States.E-mail:john_bellone@brown.edu

J. BELSER-EHRLICH, P.C. MANGAL, J.A. LAFO, M. BRADLEY \& D. BOWERS. Neurocognitive-Affective Dysfunction in Dandy Walker Malformation Involving the Cerebellum: A Case Study.

Objective: Damage to the cerebellum has been associated with cognitive and emotional deficits, which have been linked in part to disrupted circuitry in the frontal and limbic regions. In this case report, we describe the neuropsychological profile of an adult with Dandy Walker Malformation, a congenital defect characterized by cerebellar dysgenesis, $4^{\text {th }}$ ventricular dilation, and enlarged posterior fossa. 
Participants and Methods: A 48-year-old single man, diagnosed at birth with DWM, underwent a neuropsychological evaluation that assessed intellect, executive, recent memory, language, visuospatial, and mood. Due to concerns raised by family regarding decision making and stability of employment, additional measures included: Iowa Gambling Test (IGT), Florida Affect battery, and measurement of startle eyeblink responses while viewing aversive, neutral, and pleasant pictures (IAPS). Results: Test results revealed overall high average intellect (FSIQ 114). Verbal skills and working memory were distinct strengths (superior), whereas other domains were average (recent memory, visuoperceptual-spatial, executive functions (NIH examiner). Processing speed was within normal limits but a weakness. He had no difficulty matching emotional faces. However, he demonstrated weak reward sensitivity decision making on the IGT, and failed to show significantly higher startle eyeblink responses while viewing aversive pictures, in the context of normal habituation MRI showed typical DWM findings, including alteration of the vermis (limbic cerebellum).

Conclusions: The findings indicate mild physiologic blunting to aversive pictures and insensitivity to reward contingencies in a patient with a congenital cerebellar defect. Cognition was intact. These findings possibly reflect alteration in cerebellar limbic circuitry as a result of his DWM. The obtained objective findings are consistent with functional concerns regarding his atypical employment and living arrangements, and decision-making skills.

Correspondence: Janna Belser-Ehrlich, PhD student, Ferkauf Graduate School of Psychology, 1165 Morris Park Ave, Bronx, NY 10461, United States.E-mail: jbelsere@gmail.com

\section{A. BLOCH, M. SHAHAM, G. ZEILIG \& E. VAKIL. Characterizing Implicit Sequence Learning Deficits Following Spinal Cord Injury Using an Oculomotor Serial Reaction Time Task among Individuals with Tetraplegia.}

Objective: A recent study on healthy participants suggests that an ocular-activated version of the serial reaction time (O-SRT) task might be a viable alternative for assessing implicit sequence learning in populations that are unable to perform motor tasks involving the spinal cord. The current study provided an initial exploration of this possibility by examining O-SRT task performance among individuals with tetraplegia following spinal cord injury (SCI). A further aim was to expand on previous work revealing a specific deficit in implicit motor sequence learning among individuals with paraplegia, as assessed using a manual serial reaction time task.

Participants and Methods: Thirteen participants with SCI in C.5-T1 were compared to 13 matched control participants on measures derived from the O-SRT task. Depression and additional measures of learning, memory, and intelligence were included to explore the source and specificity of potential learning deficits.

Results: The SCI group responded slower than the control group in each of the O-SRT task blocks. However, like controls, the SCI group did show a decline in reaction time and an incline in anticipation over the first six blocks of the task and an advantage for the initially presented sequence over a novel interference sequence, indicating intact implicit sequence learning. No between-group differences were found on additional measures.

Conclusions: The O-SRT task elicited a similar performance pattern to standard motor versions of the serial reaction time task, enabling participants with tetraplegia to demonstrate unimpaired sequence learning despite showing slower reaction times. This finding suggests that the previously reported implicit sequence learning deficit in SCI directly involved motor functioning, and did not reflect a general deficit in sequence learning per se, with important implications for rehabilitation and adjustment.

Correspondence: Ayala Bloch, Ph.D., The National Institute for the Rehabilitation of the Brain Injured, 89 Itzhak Sadeh St., Tel Aviv 6722820, Israel.E-mail: ayalabl@shikumil.org.il
S. CHOWDHRY, T. FERLAND, D.H. SALAT, W.P. MILBERG, R. MCGLINCHEY \& E. LERITZ. Reduced White Matter Volume in Metabolic Syndrome.

Objective: Cerebrovascular risk factors, such as hypertension, diabetes, and obesity, have been shown to negatively impact brain structure and cognitive performance. The presence of three or more co-occurring risk factors, called Metabolic Syndrome (MetS), has an additive effect on neural health. Associations between MetS and gray matter volume have been demonstrated; however, literature on the effects of MetS on white matter volume is limited. The present study examines white matter volumes in participants with MetS. We hypothesize reduced white matter volume underlying regions of gray matter found to be affected by MetS.

Participants and Methods: Fifty-nine participants were separated into MetS (n=18) and non-MetS (n=41) groups based on current diagnostic guidelines. All participants underwent magnetic resonance imaging (MRI) scanning. Volumetric analyses were conducted using FreeSurfer to identify regions of white matter volume affected by MetS. Participants also underwent neuropsychological testing. In the interest of reducing variables, a factor analysis of raw scores revealed three factors: memory, attention, and executive function.

Results: White matter volume differences between groups were found bilaterally in both the temporal gyrus and post-central gyrus, as well as the left isthmus cingulate cortex, right anterior cingulate cortex, right superior parietal cortex, and right supramarginal gyrus. Group differences were significant only on the executive function factor score.

Conclusions: These results provide preliminary evidence that MetS is associated with reduced white matter volume in frontal, temporal, and parietal regions. This is consistent with previous findings that MetS groups differ on measures of executive function. However, given the fact that no group differences were found with regard to memory or attention, it is possible that white matter volume differences in these regions reflect subclinical structural changes not yet apparent during cognitive testing.

Correspondence: Saba Chowdhry, MA, Behavioral Neuroscience, Boston University School of Medicine, 150 South Huntington Ave, Boston, MA 02130,United States.E-mail:sac89@bu.edu

A. ClaWSON, S. LALANI, T. DUFFIELD, E.D. BIGLER \& M. LARSON. Cognitive Profile and Rehabilitation in Susac's Syndrome: A Case Study.

Objective: Susac's Syndrome is an autoimmune disorder characterized by encephalopathy, visual disturbances, and hearing loss. Susac's Syndrome is associated with white matter lesions, particularly within the corpus callosum, deep gray matter lesions, and leptomeningeal enhancement. Due to the rarity of this condition, few reports have documented neurocognitive patterns and none have examined cognitive rehabilitation outcomes. This case study presents the neurocognitive profile of a patient with Susac's Syndrome and the impact of cognitive rehabilitation on subjective memory deficits.

Participants and Methods: The patient was a 54-year-old Caucasian woman who presented for neuropsychological evaluation five years following symptom onset. MRI of the brain revealed scattered white matter hyperintensities. At the time of evaluation, the patient was unable to ambulate and had a partial right visual field cut and bilateral partial hearing loss. Cognitively, she reported difficulty with short-term memory and problem solving. Following neuropsychological assessment, she participated in 20 sessions of Ecologically-Oriented Neurorehabilitation of Memory and provided subjective ratings of memory impairments and strategy use before and after treatment.

Results: Neuropsychological testing revealed intact expressive and receptive language, visuospatial processing, attention, and executive functioning. Deficits were observed in motor coordination, processing speed, and memory retrieval. Subjective ratings of cognitive rehabilitation indicated improvements across areas of memory and increased strategy use following treatment. 
Conclusions: Neuropsychological testing results indicated Susac's Syndrome may be associated with deficits in motor functioning, processing speed, and memory retrieval consistent with diffuse white matter dysfunction and cognitive variability noted in existing case reports. Further, cognitive rehabilitation may be effective in addressing memory deficits associated with Susac's Syndrome.

Correspondence: Ann Clawson, PhD, Neuropsychology, Kennedy Krieger Institute, 15 Charles Plaza, Apt. 1102, Baltimore, MD 21201, United States.E-mail: clawson.ann@gmail.com

\section{T.P. COTHRAN, A.R. CARLEW, K.L. ZANE \& S. MUSIL. Prediction of Cognitive Change in Normal Pressure Hydrocephalus Following External Lumbar Drain.}

Objective: The utility of neuropsychological evaluation in normal pressure hydrocephalus (NPH) for the prediction of cognitive improvement after shunt placement is a matter of debate (Farace \& Shaffrey, 2005). External lumbar drain (ELD) is a presurgical proxy test used to gauge potential benefits of shunt placement. Neuropsychological studies of pre/post ELD cognitive evaluations are mixed with both positive (Horton et al, 2014) and negative (Lendfeldt et al., 2012) findings. Prior studies have been limited by several factors (e.g., low statistical power and between-groups statistical methodologies) that make detecting predictors of cognitive improvement difficult. This study aimed to better document the role of neuropsychological testing in persons undergoing ELD for suspected NPH.

Participants and Methods: Fifty-eight consecutive patients with suspected NPH were administered the Repeatable Battery for the Assessment of Neuropsychological Status. Premorbid functioning was estimated using the updated Barona equation (Barona, Reynolds, \& Chastain, 1984; Helmes, 1996). A two-level, within-subjects hierarchical linear model (i.e., pre vs. post-drain evaluation) was performed and demographic variables were examined as predictors of change.

Results: On average, patients evidenced statistically significant improvement on the Immediate Memory Index $(\beta=5.24, p=0.002)$ and semantic fluency $(\beta=2.89, p<0.001)$. Planned post hoc analyses revealed premorbid functioning predicted improvement for immediate memory $(\beta=0.45, p=0.002)$, while age predicted improvement for semantic fluency $(\beta=-0.16, p<0.05)$.

Conclusions: Using a within-subjects design, we identified individual factors that may have practical utility in estimating the likelihood of improved cognition following ELD in NPH patients. This provides support for neuropsychology as a useful component of NPH treatment planning. Further research using a similar design may be helpful in identifying other individual variables that may be associated with outcome. Correspondence: Thomas P. Cothran, M.S., Behavioral Sciences, Rush University Medical Center, 1645 W. Jackson Blvd., Westgate Building, Suite 400, Chicago, IL 60612, United States.E-mail: tcothran@hawk. iit.edu

\section{DEMIAN, W.J. L. THORNTON \& R.J. SHAPIRO. Lower Health Literacy Levels are Associated with a Worse Clinical Profile in Renal Transplant Recipients.}

Objective: Health literacy (HL) is the ability to access and understand health information. HL is dependent on adequate cognitive abilities and is linked to disease outcomes. We sought to determine whether lower HL was associated with a worse clinical profile in renal transplant (TX) recipients after controlling for cognition.

Participants and Methods: 96 renal recipients $(M=53 \mathrm{y})$ completed tests of HL (Health Literacy Questionnaire) and cognition (Everyday Problem Solving (EPS) test and KBIT-Verbal) in Vancouver, Canada. Health variables were collected through a health questionnaire and patient charts, and included measures of renal functioning (CKD stage, creatinine levels), time on dialysis, diabetic history (HX), and depression (CES-D). Correlational and regression analyses were used to clarify the associations between patients' HL, cognition, and health.
Results: Lower HL was significantly $(p \leq .05)$ associated with poor renal functioning (later CKD stage; $r=-.21$ ) and a longer time on dialysis pre-TX $(r=-.23)$. Higher HL was associated with a HX of diabetes that successfully resolved $(r=.23, p \leq .05)$. Patients with lower HL had higher depressive levels $(r=-.47, p<.01)$ and lower EPS $(r=.22, p \leq .05)$ and verbal ability $(r=.23, p \leq .05)$. Lower HL was marginally associated with higher creatinine $(r=-.20, p=.06)$. Results of an ordinal logistic regression revealed that having lower HL increased the odds of being at a later CKD stage after controlling for cognition and depressive levels (OR=.96; $95 \% \mathrm{CI}=.93-.99 ; p=.02)$.

Conclusions: Renal recipients with lower HL had a worse clinical profile and higher depressive levels. Those with better HL were more likely to no longer have diabetes. Lower HL remained a risk factor for worse renal functioning after controlling for levels of depression and cognition. While causal relationships cannot be determined in this study, the findings suggest the importance of assessing HL as well as identifying and providing support for patients with lower HL to guard against potential poor clinical outcomes.

Correspondence: Maryam Demian, M.A., Psychology, Simon Fraser University, 8888 University Drive, Department of Psychology, Burnaby, BCV5A1S6, Canada.E-mail: demian@sfu.ca

E.L. DUCGa, B. COSTABILE, A. CRETE, A. PAGUYO, D. FOSSATTI, L. KEATING, S. SCHAFFER \& K.A. WALKER. Neuropsychological assessment following critical illness for the prediction of longterm functional outcomes.

Objective: Patients with non-neurological critical illness are at risk for developing long-term cognitive impairment and a loss of functional independence following discharge from the intensive care unit (ICU). The present study examined whether cognitive deficits measured at the time of ICU discharge, were associated reduced functional independence at follow-up.

Participants and Methods: Sixty-five participants without pre-existing neurocognitive impairment admitted to the ICU $>72$ hours and diagnosed with septic shock and/or respiratory failure were enrolled. The Mini Mental Status Exam (MMSE) and a list learning measure from the Repeatable Battery for the Assessment of Neuropsychological Status (RBANS) were administered within 3 days of ICU discharge. Functional independence and instrumental activities of daily living (IADLs) were assessed in 34 participants $(n=34)$ approximately eight months after ICU discharge using the Mayo-Portland Adaptability Inventory-4 (MPAI-4) and the Adaptive Behavior Assessment System (ABAS).

Results: After adjusting for age, sex, critical illness severity, medical comorbidity, and length of ICU stay, each standard deviation (SD) lower score on the list learning measure was associated with a $0.53 \mathrm{SD}$ lower overall adaptive functioning score on the MPAI (95\% confidence interval [CI]: $-1.05,-0.02 ; p=.044)$, a 0.75 SD lower home living score (CI: -1.33, -0.18; $p=.013$ ), a 0.82 SD lower home safety score (CI: -1.50, $-0.14 ; p=.021)$, and a 0.82 SD lower capacity for self-care score (CI: $-1.52,-0.12 ; p=.024)$ on the ABAS. MMSE score at ICU discharge was not associated with functional outcome.

Conclusions: A brief assessment of memory administered at the time of ICU discharge can be used to predict subsequent functional impairment within the critically ill population. Our findings highlight the utility of neuropsychological assessment in the critical care setting for risk stratification and outcome prediction.

Correspondence: Emma L. Ducca, St. John's University, 23-41 31st Drive, Astoria, NY 11106, United States.E-mail:ed752470@gmail.com

P. Figueroa, K.A. BURnette, A.H. Panos, L.K. PAUl \& W.S. BROWN. Self-Perception of Social Norms and Empathy in Adults After Childhood Right Hemispherectomy.

Objective: Previous research demonstrated that damage to the right hemisphere leads to deficits in social cognition, including social pragmatics, second-order meanings in social communication, and empathy. However, little is known about long-term outcomes for adults who 
underwent right hemispherectomies (HE) as children in order to treat intractable epilepsy. This study investigated understanding of social norms, and self-understanding of experiences of empathy in HE.

Participants and Methods: Four adults with right HE in childhood (age: 18-25) and to 21 neurotypical controls (age: 19-64) were administered the Social Norms Questionnaire (SNQ22), a self-report measure of adherence and over-adherence to mainstream U.S. socio-cultural norms, and the Interpersonal Reactivity Index (IRI; Davis, 1980), a self-report measure of cognitive (Perspective Taking and Fantasy) and emotional (Empathic Concern and Personal Distress) empathy.

Results: A one-way ANOVA of SNQ22 Total Score revealed that individuals with HE scored worse than controls $\left(p<.001, \eta^{2}=.56\right)$. Further analyses revealed significantly higher scores on Overadherence $\left(p<.001, \eta^{2}=.74\right)$ for individuals with HE. A MANOVA of IRI scores revealed no differences between persons with $\mathrm{HE}$ and controls across the four subscales of Perspective Taking $\left(\eta_{\mathrm{p}}{ }^{2}=.00\right)$, Fantasy $\left(\eta_{\mathrm{p}}{ }^{2}=.04\right)$, Empathic Concern $\left(\eta_{\mathrm{p}}{ }^{2}=.11\right)$, and Personal Distress $\left(\eta_{\mathrm{p}}{ }^{2}=.11\right)$.

Conclusions: As hypothesized, individuals with right HE were less likely to correctly identify what is socially acceptable and appropriate. Particularly, they were more likely to over-adhere to social norms, suggesting an inflexible, strict application of norms for social behavior. When asked to report their own levels of empathy, their reports were entirely normal. This result may suggest a lack of accurate self-perception of deficiencies in empathy in individuals with right HE, but further study will be necessary to confirm this conclusion.

Correspondence: Paul Figueroa, M.A., Fuller Grad Sch of Psych, 180 N. Oakland Ave., Pasadena, CA 91101, United States. E-mail: paulfigueroa@fuller.edu

C. HARRISON, P. FigueroA, A.H. PANOS, L.K. PAUL \& W.S. BROWN. Adult Behavior and Executive Functioning Following Childhood Hemispherectomy: Self-Report.

Objective: Hemispherectomy (HE) is an effective treatment for young children with intractable epilepsy caused by congenital or acquired cortical lesions. Collectively, research suggests a general trend toward functional independence (Moosa et al., 2013). While understanding of the more specific neuropsychological outcome of HE is essential, there has been a dearth of studies regarding the long-term neurocognitive and behavioral sequelae of childhood HE. The current study assessed the impact of childhood HE on behavioral and executive functioning in adults using a self-report survey (Behavior Rating Inventory of Executive Functioning, Adult Version, BRIEF-A).

Participants and Methods: Adults with childhood hemispherectomy ( $\mathrm{N}=10 ; \mathrm{M}_{\text {age }}=24.2 ; 7$ right hemispherectomy), completed the BRIEF-A. Scores for the Behavioral Regulation Index (BRI) and Metacognition Index (MI) composite scales, and the Global Executive Composite were derived from skill areas. One-sample t-tests were used to compare group scores against a T-score of 50 .

Results: There were no significant differences from norms for the BRI or MI, nor for the overall GEC, suggesting that individuals with childhood HE perceived their executive abilities to be similar to neurotypical controls. However, within the BRI, results revealed significantly worse outcomes in both the Self-Monitor $(p<.001)$ and Emotional Control $(p=.023)$ subscales. Additionally, there was a trend toward lower functioning for the Shift subscale $(p=.068)$.

Conclusions: The findings revealed that adults who had undergone childhood HE perceived their behaviors and executive functioning to be largely commensurate with neurotypical persons. However, within the domain of behavioral regulation they recognized mild to moderate deficits in their functioning. Given the possibility of limited self-insight in HE patients, future research should compare self-reports with reports from informants knowledgeable about their behavior in order to better understand executive functioning in adults with childhood HE.

Correspondence: Cory Harrison, Fuller Grad Sch of Psychology, 180 N. Oakland Ave., Pasadena, CA 91101, United States. E-mail: coryharrison@fuller.edu
R.M. ISLAM, A.D. BONO, K. SCORPIO, R.J. STAFFORD, J.T. TWAITE, E. MURRAY, M. HALFACRE, K. ALTERESCU, A. HALPERN, J. SPIELMAN, L. RAMIG \& J.C. BOROD. Unilateral Motor-Symptom Onset in Parkinson's Disease related to Facial Emotional Expression: Right-Hemisphere, Valence, and Motoric Direction Hypotheses.

Objective: Parkinson's disease (PD) mainly involves motoric features (e.g., tremor \& rigidity), but nonmotor symptoms (i.e., cognitive \& affective deficits) can also occur. Of note, motor-symptom onset in PD can present primarily on the right (RPD) or left side (LPD) of the body, reflecting contralateral hemisphere pathology. Despite work in this area on cognition and affect perception and some on posed emotional expression, very little has been done on spontaneous emotional expression and the facial channel, per se. Further, dimensions of emotion (valence \& motoric direction) have not been directly addressed. This study examined spontaneous facial emotional expression in RPDs and LPDs, using a range of emotions (positive/negative \& approach/withdrawal), and tested theories regarding hemispheric specialization for emotion.

Participants and Methods: Participants were 15 RPDs (53\% male) and 20 LPDs (75\% male). Groups were compared on demographic and clinical variables. PDs were videotaped while producing emotional monologues (Happy, Sad, Angry), using New York Emotion Battery procedures (Borod et al., 1992). Monologue segments were rated by 18 individuals for social engagement; emotional frequency, variability, and intensity; and facial mobility on 7-point scales (minimal to maximal). Results: A 3-way repeated-measures ANCOVA was performed for Group (RPD \& LPD), Gender (2), and Emotion (3), covarying for depression and illness stage. Overall, LPDs had lower scores than RPDs for 4 facial expression variables, consistent with the Right Hemisphere Hypothesis. However, interactions involving Emotion provided some support for the Valence Hypothesis, with more impairment for Sad and Angry than for Happy. Of note, these results occurred for female, but not for male, PDs.

Conclusions: LPDs displayed less facial emotional expressivity and mobility than did RPDs. When emotion dimensions were examined, there was some support for the Valence Hypothesis. The fact that findings were more pronounced for women than for men requires exploration. Correspondence: Rubyat M. Islam, M.A Behavioral Neuroscience, Psychology, Queens College, 9151 111th Street, Richmond Hill, NY 11418-3006, United States. E-mail:Rubyatislam1@gmail.com

E. KALSCHEUR, J. RESTREPO \& T. DEER. Subjective Cognitive Complaints in Patients with Fibromyalgia: Objective Results Compared to Those of a Neurological Sample.

Objective: Patients with fibromyalgia (FM) often report subjective cognitive complaints that interfere with their work and daily life. However, findings from studies examining subjective complaints and objective impairment have been mixed. The current study examined neuropsychological test results obtained from a group of individuals with FM referred by their rheumatologist for memory difficulties and compared the results to a separate medical population group also referred for memory difficulties by a medical specialist.

Participants and Methods: 19 FM patients referred by their rheumatologist and 27 patients without rheumatic disease referred by their neurologist underwent comprehensive neuropsychological testing to evaluate memory concerns. After excluding those with inconsistent effort, group differences on several neuropsychological measures were examined factoring in age and education where available.

Results: Independent samples t-tests revealed no significant differences between groups on age, education or word reading standard scores. While no significant differences existed between groups on a self-report measure of anxiety, significant between-group differences in self-reported depression symptoms were seen on one of two depression scales $(p=.014)$ with FM group reporting more symptoms than the neurological group. Significant differences between groups were seen on effortful verbal learning (total and delayed recall, $p=.049$ and $p=.007$, respectively), verbal fluency $(p=.026)$, category fluency $(p=.021)$ and visual 
attention/task switching $(p=.027)$ such that the neurological patient group scored lower than the FM patient group.

Conclusions: As expected, patients with FM report significantly more mood symptoms compared to a medical population group. However, despite mood disturbance and pain associated with the condition, FM patients perform better than the neurological patient group on tasks of memory and executive functions. Implications for future research and clinical utility are discussed.

Correspondence: Emily Kalscheur, Psychology, Rosalind Franklin University of Medicine \& Science, 87 E Haines Dr, Hainesville, IL 60030,

United States.E-mail: emily.kalscheur@my.rfums.org

S. MOZA, G.M. HADJIGEORGIOU, N. SCARMEAS, E. DARDIOTIS, M. YANNAKOULIA, P. SAKKA \& M.H. KOSMIDIS. Neuropsychological Effects of Pesticide Use Among Elderly Farmers.

Objective: The excessive use of pesticides has aroused considerable concern in the scientific community regarding the long-term effects of pesticide exposure on public health. Data are still limited, however, with respect to the elderly. We explored the attitude of elderly Greek farmers towards pesticide use and adherence to safety practices, and investigated the long-term effects of pesticide use on cognitive functioning.

Participants and Methods: Participants were 1943 elderly individuals, 276 of whom reported long-term exposure to pesticides through spraying their garden, open fields, and/or greenhouse. Assessment included neurological examination and clinical interview, neuropsychological evaluation, and a questionnaire regarding the level of pesticide exposure. Neuropsychological test results were categorized into six main cognitive domains: visual and verbal memory, inhibition, mental flexibility, language and visuospatial perception.

Results: A large percentage of older adults reported using prohibited pesticides, often without protective equipment (56.6\%). A smaller percentage admitted to smoking $(10.0 \%)$ or/and consuming food during spraying $(8.4 \%)$, exceeded the recommended frequency of use per year, and failed to consult an agronomist $(16.9 \%)$ and/or the instructions on the pesticide label $(25.2 \%)$. Controlling for covariates, exposure to pesticides was associated with impaired language ability (OR, .96; 95\% CI, 0.93-0.99) only. This association remained significant after excluding participants with mild cognitive impairment and dementia from the analyses.

Conclusions: Conclusions

Elderly farmers in Greece hold high risk attitudes towards pesticide use and exhibit poor compliance with safety rules. These practices appear to have significant negative association with their language abilities, but not memory, inhibition, mental flexibility or visuospatial perception. Correspondence: Mary H. Kosmidis, Ph.D., School of Psycholgy, Aristotle University of Thessaloniki, University Campus, Thessaloniki 54124,Greece.E-mail:kosmidis@psy.auth.gr

T.V. CASH, C. KILBOURN \& S.K. LAGEMAN. Training Leaders of Neurodegenerative Disease Support Groups: Development of a Support Group Well-Being Measure.

Objective: Community support groups provide resources and emotional support to families coping with neurodegenerative illnesses. Support group leaders play a pivotal role in maintaining healthy support groups, but have their own personal need for support and also typically lack formal training in managing complex behaviors commonly observed in neurodegenerative disorders. Two neurodegenerative disorder clinics and a local community agency collaborated to provide training for support group leaders of various disease-specific support groups and pilot a support group well-being survey.

Participants and Methods: Twenty-seven support group leaders attended the training (Parkinson's disease $(\mathrm{PD})=12$, Multiple Sclerosis $(\mathrm{MS})=8$, Huntington's disease $(\mathrm{HD})=4$, and Essential Tremor $(\mathrm{ET})=3$ ). The training provided educational materials designed to improve support group leaders' knowledge of neurodegenerative disorders and access to resources. Twenty-four attendees completed the Support Group Functioning Survey pre-training. Primary analyses were performed only on responses from support group leaders with prior experience leading groups $(n=15 ; \mathrm{MS}=7 ; \mathrm{PD}=4, \mathrm{HD}=2 ; \mathrm{ET}=2)$.

Results: An exploratory factor analysis evaluated the psychometric properties and internal consistency of the survey. The analysis identified a single factor five-item solution with good internal reliability (Cronbach's Alpha=.882), which was titled the Support Group Functioning Scale. Descriptive statistics were evaluated and preliminary interpretation guidelines were proposed.

Conclusions: Development of this scale is a first step in identifying areas of need for support group leaders. This tool can help improve the training and ongoing support of support groups leaders as they provide front-line assistance to caregivers and patients with neurodegenerative illnesses in communities. We encourage support group leaders, community agencies, and clinics to use and further assess the Support Group Functioning Scale to identify areas of need for support group leaders. Correspondence: Sarah K. Lageman, Ph.D., Neurology, Virginia Commonwealth University, 11958 W. Broad Street, 4th Floor, Room 4023, Richmond, VA 23233, United States. E-mail: sarah.lageman@ vcuhealth.org.

A. MATCHANOVA, A. HAGERTY, D. DRABICK, T.F. FLOYD, C.C. PRICE \& T. GIOVANNETTI. Post-Operative Cognitive Function after Cardiac Surgery: A Meta-Analytic Review.

Objective: Millions of cardiac procedures are performed in the US each year. Although Postoperative Cognitive Dysfunction (POCD) has been frequently studied after cardiac surgery, findings regarding these cognitive sequelae are mixed. We conducted a meta-analysis to examine the effect of cardiac surgery on cognition in the relative short-term. Moderators thought to be associated with POCD also were investigated. Participants and Methods: Major search engines with access to expansive databases of the scientific literature (e.g., PubMed, Google Scholar) were explored for articles reporting neuropsychological outcomes within 12 weeks following cardiac surgery. Pre- and post-cognitive scores $(M, S D)$, sample sizes, and information on moderators (i.e., neuropsychological domain, surgery type, age, and publication year) were collected.

Results: Thirty-four studies were identified that included 2,599 cardiac surgery participants. Analyses revealed no overall difference between pre-surgical versus post-surgical cognitive performance, $\mathrm{d}=0.02,95 \%$ $\mathrm{CI}=0.09-0.05$. However, there was significant heterogeneity among study effect sizes, Q [33] = 184.47, $p<.01$, and moderator analyses revealed a significant effect of neuropsychological domain $(p<.05)$. The effect of surgery was significant for delayed episodic memory, $\mathrm{d}=-0.30,95 \% \mathrm{CI}=-0.47-0.14, p<.01$. Effects were nonsignificant for basic attention, $\mathrm{d}=0.09,95 \% \mathrm{CI}=-0.01-0.19, p=.06$; immediate memory, $\mathrm{d}=0.15,95 \% \mathrm{CI}=-0.31-0.01, p=.07$; and executive functioning, $\mathrm{d}=0.07,95 \% \mathrm{CI}=-0.01-0.14, p=.09$. All other moderators, including surgery type, age, and year of publication, were not statistically significant.

Conclusions: Delayed episodic memory, which is typically associated with frontal and temporal lobe brain regions, may be more vulnerable to acute neurological consequences of cardiac surgery than other cognitive domains. Implications for pre-surgical screening and post-surgical cognitive remediation will be discussed.

Correspondence: Asal Matchanova, Psychology, Temple University, 1701 N. 13th Street Room 857, Philadelphia, PA 19122, United States. E-mail:matchanova.asal@gmail.com

Z. MERZ, N. HURLESS \& J. WRIGHT. Examination of the Construct Validity of the Repeatable Battery for the Assessment of Neuropsychological Status Language Index in a Mixed Neurological Sample.

Objective: The current study sought to examine the construct validity of the Language Index of the Repeatable Battery for the Assessment of Neuropsychological Status (RBANS) in a diverse clinical sample. 
Participants and Methods: Archival data was collected, composing of RBANS Language Index performance and related stand-alone measures, within the context of a comprehensive neuropsychological evaluation for 2,068 individuals of diverse neurological etiologies ranging in severity. Results: Strong correlations were seen between the RBANS Language Index and its respective indices (Semantic Fluency $\mathrm{r}=.726$; Picture Naming $r=.787$ ). Low to moderate correlations were seen between Semantic Fluency and both Category Fluency $(r=.375)$ and the Controlled Oral Word Association test $(\mathrm{r}=.373)$. Moderate correlations were seen between Picture Naming and both Visual Naming from the Multilingual Aphasia Examination $(r=.444)$ and the Boston Naming Test $(r=.520)$. Finally, Picture Naming was shown to significantly predict performance on both Visual Naming $[\mathrm{F}(1.495)=121.31$, $\mathrm{p}<$ $.000]$ and the Boston Naming Test $[\mathrm{F}(1,262)=97.12, \mathrm{p}<.000]$.

Conclusions: Given these results, support for the interpretation of RBANS Language Index appears warranted in a diverse clinical sample. Correspondence: Zachary Merz, Saint Louis University, 9906 heritage drive, apt c, Saint Louis, MO 63123, United States.E-mail: merzz@ slu.edu

\section{A.H. PANOS, P. FIGUeroa, J.S. MILler, L.K. PAUL \& W.S. BROWN. Adult Adaptive Functioning Following Childhood Hemispherectomy.}

Objective: Intractable epilepsy is related to a variety of neuropsychological and behavioral impairments. In many cases, surgical hemispherectomy (HE) is the only viable treatment for intractable seizures. However, little is known about the adaptive functioning outcomes for adults who underwent hemispherectomy during childhood. The current study assessed the impact of childhood hemispherectomy on adaptive functioning outcomes in adults using a self-report survey (Adaptive Behavior Assessment System, Second Edition: ABAS-II).

Participants and Methods: Ten individuals who sustained a hemispherectomy in childhood completed the ABAS-II (ages 19-32, $M=24.2$, $S D=4.6 ; 5$ females; 7 right HE). Scores for three composites of the ABAS-II (i.e., Conceptual, Social, and Practical skills) and the General Adaptive Composite (GAC) were derived from 10 self-reported skill areas. One-sample t-tests were used to compare group means to a T-score of 50 .

Results: The HE group rated themselves above the normative mean for the Conceptual $(p=.005)$ and Social $(p=.01)$ composites. No statistical differences were observed for the Practical Composite or for the GAC. Within the Conceptual Composite scores were significantly better than norms for the Communication $(p=.003)$ and Self-Direction $(p=.000)$ skill areas, and for the Social Composite scores were significantly higher for Leisure $(p=.001)$ and Social Interaction $(p=.005)$.

Conclusions: These results indicate that individuals with HE rate themselves as functioning at better than norms in the conceptual (Communication and Self-Direction) and social (Leisure and Social Interaction) domains of daily living. Since observations of these individuals do not suggest enhanced functioning in these domains, these self-ratings suggest deficient insight into their daily living skills. Thus, further research involving reports of adaptive skills from informants will help to clarify the current results.

Correspondence: Amanda H. Panos, MA, Fuller Graduate School of Psychology, 395 S Highland St., Apt. 418, Memphis, TN 38111, United States.E-mail: amandapanos@fuller.edu

\section{J.S. PRICE, S.C. ZANOWSKI \& J.C. HONG. Neuropsychological Assessment Informed Intervention during Liver Transplantation Evaluation: A Case Report.}

Objective: For patients in liver failure due to alcohol cirrhosis, pre-transplant non-adherence is associated with higher morbidity and mortality post-transplant. Comorbid cognitive deficits in attention, executive function and memory can negatively impact adherence. While there is evidence of improved neurocognition following liver transplant, non-adherence can limit access to transplant. We report use of a brief neuropsychological assessment to inform treatment planning for a critically-ill patient in liver failure.

Participants and Methods: Patient was a 25 vear-old Hispanic male with 8 years of education, daily alcohol use ages 16 to 24 and 6 months of abstinence. He had not completed AODA treatment. Following listing for transplant while hospitalized with portal hypertension and ascites, he engaged in non-adherent behaviors that compromised his health. including sneaking food and leaving AMA. Providers noted poor insight into consequences for behavior. A brain MRI was unremarkable.

Results: Results estimated borderline to impaired intellectual functioning. Simple attention and story memory were low average; complex attention, processing speed, unstructured verbal and nonverbal memory and executive ability were borderline to impaired. There was no memory degradation over time. Etiological considerations included premorbid intellectual dysfunction, longstanding severe alcohol use disorder, and liver failure with prior hepatic encephalopathy.

Conclusions: Deficits in higher order cognition were consistent with observed challenges with impulse control and adherence. Intervention focused on relative strengths in simple attention and memory for structured material: psychoeducation, reinforcement of daily compliance behaviors, use of visual cues and frequent feedback regarding progress. Patient demonstrated improved compliance but died 3 months later while awaiting transplant. This case highlights the utliity of neuropsychological assessment, feedback and focused intervention in optimizing candidacy for organ transplantation.

Correspondence: Jenessa S. Price, PhD, Surgery, Division of Transplant Surgery, Medical College of Wisconsin, $9200 \mathrm{~W}$ Wisconsin Ave, Milwaukee, WI 53226, United States.E-mail: jeprice@mcw.edu

\section{K.D. MAHDAVI \& S. ROGERS. Poor Punctuality Reflects Cognitive Impairment in Neurology Patients.}

Objective: Punctuality may be a behavioral indicator of global cognition. This study explores the relationship between punctuality and cognitive performance among geriatric neurology patients.

Participants and Methods: 308 patients ( 140 women, $M$ age $=73.19$. $M$ education $=14.74$ ) participated in comprehensive neuropsychological assessment as part of outpatient neurology evaluations. Timeliness to appointment was documented in five minute intervals, and measures completed included the MoCA, HVLT-R, BVMT-R, Trailmaking, BNT, RCFT, and subtests from WAIS-IV, WMS-IV, and DKEFS.

Results: Independent samples $t$-test analyses showed that those who arrived late to their testing appointments performed significantly worse than those who were early or on time on the MoCA, BNT, WAIS-IV Digit Span, WAIS-IV Picture Completion, and BVMT-R Total Trials 1-3 and Delayed Recall, $p \mathrm{~s}<.05$. According to chi-square analyses, those who arrived late were also significantly more likely to be experiencing problems in their instrumental activities of daily living compared to those who arrived early or on time, $p<.05$.

Conclusions: These findings suggest that patient punctuality may be a predictor of cognitive functioning. Those who arrive late to testing appointments appear to exhibit worse general mental status, simple auditory attention, visual perception, and nonverbal learning and delayed recall when under the pressure of time. Although punctuality may correspond to specific cognitive and anatomical processes, like frontal and parietal functions, it appears more likely associated with gross or global cognitive functions, since deficits or declines in the cognitive abilities affected in this study are strongly related to global cognitive impairment. These findings suggest that degree of punctuality may be an ecologically valid indicator of cognitive decline, which may be particularly useful when clinicians are unable to complete a comprehensive neuropsychological battery.

Correspondence: Steve Rogers, Ph.D., Santa Barbara, CA 93108, United States.E-mail: strogers@westmont.edu 
M. SНАН. 'Brain on Fire'- Autoimmune Encephalitis: A Neuropsychological Case Study.

Objective: The case study described the neuropsychological consequences of this rare autoimmune encephalitis. Brain on Fire as known as Limbic Encephalitis, is an immunological condition, includes symptoms of temporal lobe seizures, amnestic syndrome, and mood/behavioral changes including anxiety, depression, sleep disturbances, and hallucinations/delusions. Neurocognitive functioning is typically associated with impairments in memory, executive function, working memory, and processing speed.

Participants and Methods: The patient is a 55-year-old, Caucasian, right-handed female who was referred by her neurologist due to significant behavioral, psychological, and cognitive changes. Her history included: Limbic Encephalitis, seizure disorder, depression, anxiety, and sleep problems. A comprehensive neuropsychological examination was completed. Common psychometric neuropsychological tests were administered using the Meyers Neuropsychological System.

Results: Her pattern of findings were consistent with diffuse and lateralized ( $\mathrm{LCH}>\mathrm{RCH})$ cognitive deficits in verbal and visual memory, complex-incidental attention, verbal fluency, and naming. Contrary to recent literature, problem-solving, processing speed, abstraction, and working memory abilities were relatively spared.

Psychologically, she endorsed severe reactive derpression, anxiety, sleep disruptions, and hallucinations/delusions in the context of a normal psychiatric history.

Conclusions: Brain on Fire is a complex autoimmune condition that mirrors many diagnoses, including but not limited to dementias, seizures, and/or psychiatric conditions. Neuropsychological examination provides objective data to aid in multidisciplinary care and treatment planning. Accurate diagnoses and medical management is critical, as the neurological consequences are severe if left untreated.

Lastly, future research is needed from a neuropsychological point of view to identify associated cognitive profile.

Correspondence: Mihir Shah, NeuroRehabilitation Services-Life Span, 2004 Princess Ct, Woodbridge, NJ 07095, United States. E-mail: dr.mshah15@gmail.com

S. SUNDARAM, E. MULLER-OEHRING, R. FAMA, E.V. SULLIVAN, A. PFEFFERBAUM, K. POSTON, H. BRONTË-STEWART \& T. SCHULTE. Impaired Speed of Information Processing in Older Adults with HIV: A Comparison with Parkinson's Disease.

Objective: Individuals with human immunodeficiency virus (HIV) are now aging, making them susceptible to combined disease effects and aging on cognitive and motor functions. We hypothesized that information processing (IP) deficits occur in aging HIV similar to those in Parkinson's disease (PD), an age-related neurodegenerative disease, both of which affect frontostriatal pathways.

Participants and Methods: Groups comprised 29 HIV participants, age 47-71, 25 mild to moderate PD patients on dopaminergic medication, age 49-79, and 21 healthy controls (HC), age 45-77. Cognitive and motor testing included the Dementia Rating Scale (DRS-2), Unified Parkinson's Disease Rating Scale (UPDRS), speed of IP assessed with the Symbol Digit Modalities Test (SDMT), and the color naming condition of the Golden Stroop Task. The HIV group was younger and had lower education levels than HC and PD. IP scores were standardized on HC and corrected for age and education.

Results: HIV, but not PD, was impaired on speed of IP compared with HC on the SDMT and Stroop color naming. Even after controlling for normal aging effects, older age in HIV correlated with poorer performance at the outset of the task, rho=-.40, $p=0.032$. IP speed of SDMT and color naming were highly correlated in all 3 groups, and accounted for $42 \%$ of SDMT verbal speed variance in PD. Greater impairment in speed of IP in HIV was associated with lower DRS scores, rho=.51, $p<.005$ and with higher bradykinesia, $r h o=-.44, p=.026$, and UPDRS motor scores, $r h o=-.47, p=0.015$.
Conclusions: Individuals who are now aging with HIV-infection have notable impairments in speed of IP compared with mild-moderate PD patients. Age in HIV contributed to IP slowing over and above effects of healthy aging. Accelerated IP slowing may herald cognitive decline and motor impairment in aging HIV individuals.

Support: AA023165, AA017347, AA017168, NS075097, AG047366, Michael J. Fox Foundation for Parkinson's research

Correspondence: Saranya Sundaram, Master's in Rehabilitation Counseling, Clinical Psychology, Palo Alto University, 707 Continental Circle, Apt\# 2014, Mountain View, CA 94040, United States. E-mail: ssundaram1341@gmail.com

K.L. VICKERS, L.E. BRADSTREET, L. BRENNAN, S. JIN HONG, A.H. NORRIS \& P.J. MOBERG. A Quantitative Review of Neuropsychological Function in Lyme Disease: Current Status and Future Directions.

Objective: Lyme disease (LD) is a multisystem disorder that is among the best known tick-borne illnesses. Although musculoskeletal and neuropathic symptoms of the disorder have been well documented, the neuropsychological profile of LD is controversial because of observed variability across patients. The present meta-analysis aimed to classify the neuropsychological profile of LD.

Participants and Methods: Nine publications ( $k=125$ effects) were identified from PubMed and MEDLINE databases. Included articles reported neuropsychological testing for patients with LD and age-matched healthy controls. Moderators included neuropsychological domain, country of study (US vs. Europe), age, and sex.

Results: Analyses revealed mild to moderate cognitive impairment in individuals with $\mathrm{LD}$ compared to healthy participants, $d=-0.37,95 \%$ $\mathrm{CI}=-0.43<\delta<-0.30$, that was significantly heterogeneous, $Q_{B}[124]$ $=248.83, \mathrm{p}<.001$. Moderator analyses revealed a significant effect of neuropsychological domain, $Q_{B}[9]=18.83, \mathrm{p}<.03$. While nominally larger impairments were observed in verbal memory $(d=-0.51, \mathrm{CI}=-.63$ $<\delta<-0.39)$, verbal abilities $(d=-0.48$, CI $=-0.75<\delta<-0.21)$ and processing speed $(d=-0.43, \mathrm{CI}=-0.60<\delta<-0.27)$, the contrasts between most neuropsychological domains were not significant. Adult samples were more impaired than pediatric samples and impairment was greater in US samples. Meta-regression analyses identified greater cognitive deficits for women, $\mathrm{Z}[101]=6.29, \mathrm{p}<.001$, and with increased age, $\mathrm{Z}[85]=-4.06, \mathrm{p}<0.001$.

Conclusions: Meta-analytic results suggest mild to moderate cognitive impairment in LD across all domains, which are more significant in adults, women, and in studies conducted in the US. Results of this analysis also identify a need to address inconsistencies in diagnostic criteria of LD and the lack of measurement of key moderators, including mood and effort testing, in the future.

Correspondence: Kayci L. Vickers, MS, Psychology, Drexel University, 421 S. 15th Street, Apt 3F, Philadelphia, PA 19146, United States. E-mail:kayci.vickers@gmail.com

E. WEBER, J. WECHT, C. KATZELNICK, T. DYSON-HUDSON \& N. CHIARAVALLOTI. Learning and Memory Profile of Individuals with Spinal Cord Injury.

Objective: To describe the pattern of learning and memory deficits in individuals with spinal cord injury (SCI) and explore its everyday life impact.

Participants and Methods: Participants included 60 individuals with history of traumatic SCI (without comorbid diagnosis of TBI), who were at least 1 year post-injury, in addition to an age-/sex-matched healthy control group $(n=30)$. Learning and memory abilities were assessed using the CVLT-II, and quality of life (QOL) was assessed with the Spinal Cord Injury-Quality of Life (SCI-QOL).

Results: T-tests revealed significant group differences on measures of one trial learning (CVLT-II List A Trial 1; $p=0.001$; Hedge's $g=0.79$ ), total learning (Trials $1-5$ Total; $p=0.004 ; g=0.66$ ), delayed recall (Long 
Delay $[\mathrm{LD}]$ Free Recall Total; $p=0.023 ; g=0.51$ ), and delayed cued recall (LD Cued Recall Total; $p=0.011 ; g=0.58$ ). No significant differences were noted between groups on recognition memory (LD Total Recognition Discriminability; $p=0.141 ; g=0.33$ ), source memory (LD Total Source Discriminability; $p=0.157 ; g=0.31$ ), or intrusive errors (Recall Total Intrusions; $p=0.510 ; g=0.15)$. Despite the significant difference in recall, there was no difference in memory savings (LDFR vs. Trial $5 ; p=0.432 ; g=0.17$ ). In SCI, indices of memory (LDFR, recognition discriminability) were correlated with multiple facets of QOL (SCI-QOL Cognition, Fatigue, Positive Affect and Well-Being, Resilience subscales; all $p s<0.05$; Spearman's rhos 0.323-0.461); indices of learning (Trial 1, Trials 1-5 Total) did not significantly correlate with QOL.

Conclusions: These results suggest that individuals with SCI demonstrate impaired learning, with relatively spared retention. This pattern of deficits is consistent with that which has been observed in other neurological populations marked by reduced white matter integrity and hippocampal pathway damage, such as TBI and MS. Significant relationships between indices of memory and QOL underscore the importance of these cognitive processes to daily living in individuals with SCI. Correspondence: Erica Weber, Kessler Foundation, 120 Eagle Rock Avenue, Suite 100, East Hanover, NJ 07936, United States. E-mail: eweber@kesslerfoundation.org

K.A. WYMAN-CHICK, M.J. BARRETT, P. MARTIN \&. C.A. MANNING. Relationship Between Verbal Fluency Performance and Side of Motor Symptoms in Older Adults with Early-Stage De-Novo Parkinson Disease.

Objective: Verbal fluency (VF) is thought to lateralize to the left side of the brain in most individuals, particularly right-handed individuals. VF is often reduced in individuals with Parkinson disease (PD). We hypothesized that PD participants with right-sided onset of motor symptoms would demonstrate worse VF than individuals with left-sided onset of symptoms.

Participants and Methods: Baseline data were obtained from the Parkinson's Progression Marker Initiative (PPMI) database in April 2017. Participants with PD age $\geq 60$ were included. Individuals with symmetric motor symptoms were excluded. A total of 245 participants were included in the current analysis (Age= 67.5 \pm 5.2 ; Education=15.6 \pm 3.3$)$. Participants were administered letter fluency (F) and category fluency (Animals). Raw scores were converted to z-scores utilizing age-based norms (Ravdin et al., 2003). Independent samples $t$-tests were performed to compare VF between participants with right-sided $(n=150)$ vs. left-sided $(n=95)$ onset of motor symptoms. An additional analysis included only right-hand dominant individuals $(n=215)$. This was also repeated with only tremor-dominant individuals $(\mathrm{n}=203)$.

Results: There was no significant difference between participants with right-sided and left-sided motor symptoms participants in terms of letter fluency; $t(243)=-0.34, p=.732$ or category fluency performance; $t(243)=-1.35, p=.177$. In right-hand dominant individuals, there were no significant differences between letter fluency; $t(213)=5.15 . p=.607$ or category fluency; $t(213)=-0.97 . p=.334$. For tremor-dominant participants there were no significant differences between letter fluency; $t(201)=-0.55 . p=.585$ or category fluency; $t(201)=-1.12, p=.258$.

Conclusions: In this sample of older patients with PD, we did not find evidence of differences in VF performance based on side of onset of motor symptoms; however, the participants were in relatively early stages of the disease. Future research should examine more advanced disease stages and/or younger participants.

Correspondence: Kathryn A. Wyman-Chick, PsyD, Department of Neurology, University of Virginia, PO Box 800394, Charlottesville, VA 22908-0394, United States.E-mail: kateawyman@gmail.com
C.G. ZUNDEL, S.S. LAD, M.K. YEE, C.M. GRASSO, P.A. JANULEWICZ, K. SULLIVAN \& M. KRENGEL. Rates of Medical Conditions: Do Gulf War Veterans Differ from the General Population?

Objective: More than 25\% of Gulf War veterans (GWV) report chronic health concerns that do not fit into one medical diagnostic category. Their symptoms include fatigue, musculoskeletal problems, and cognitive and mood deficits that are commonly referred to as Chronic Multisymptom Illness (CMI). CMI criteria involves multiple body systems, including the central nervous system, and may present as many other medical conditions. Prior research has found that rates of medical conditions are significantly higher in the GWV population than in the general public. Specifically, rates of amyotrophic lateral sclerosis (ALS), multiple sclerosis (MS), and brain cancer have been reported. In our study, we expand on prior research by comparing the rates of GWV medical conditions to those of the general population.

Participants and Methods: 446 (mean age at time of reporting $=54$ ) GWV from the Fort Devens, Massachusetts cohort responded to a survey nearly 22-25 years post-deployment. Rates of 37 medical conditions were compared to the Center of Disease Control's (CDC) rates for their age matched peer group in the general population.

Results: It was found that GWV have a higher rate of hypertension, chronic pain, depression, chronic eczema and skin conditions, irritable bowel syndrome, chronic fatigue syndrome, and mild cognitive impairment compared to the general population. In the current sample of the Ft. Devens population, higher rates of ALS, MS, or any cancer was not observed.

Conclusions: Nearly 25 years after deployment GWV are reporting rates of medical conditions well above the general population. Future studies may need to include these diagnostic categories in the criteria for Gulf War CMI.

Correspondence: Clara G. Zundel, Behavioral Neuroscience, Boston University, 1910 Dorchester Ave., Apt. 509, Boston, MA 02124, United States.E-mail:cgzundel@bu.edu

\section{Other}

A. ALKOZEI, R. SMITH, N.S. DAILEY, S. BAJAJ, M. HAACK \& W.D. KILLGORE. Men, but not Women, Show a Decrease in Implicit Preferences for Low-Calorie Food After 3 Weeks of Chronic Sleep Restriction.

Objective: The high prevalence of obesity and chronic sleep deprivation are serious public health concerns in the United States. Importantly, obesity and sleep deprivation may be influencing each other in significant ways. However, no study to date has shown how sleep restriction may affect implicit food preferences. The aim of this study was to assess changes in implicit attitudes towards high- and low-calorie food after 3 weeks of chronic sleep restriction versus 3 weeks of control sleep.

Participants and Methods: Thirteen healthy adults ( 7 females, mean age $=24.5, \mathrm{SD}=4.2$ ) underwent two 25-day in-hospital stays (restricted sleep condition and sleep control condition). During the rested sleep condition, participants slept for 8 hours per night for 3 weeks. During the sleep-restricted condition, participants were allowed to sleep for 4 hours for 5 nights followed by 2 nights of recovery sleep of 8 hours, repeated over 3 weeks. On Day 21 of each visit, participants completed the Food Implicit Association Test (IAT), which measures implicit preference for low- and high-calorie foods.

Results: Changes in IAT-scores between the two sleep conditions were analyzed using a within-subjects repeated-measures analysis of covariance controlling for the effects of age, sex and body mass index. There was a significant main effect of sleep condition $(F(1,9)=5.35, p=.05$, $\mathrm{d}=1.54)$; however inspection of the interaction effects also revealed a significant sleep condition $\mathrm{x}$ sex interaction $(\mathrm{F}(1,9)=5.97, \mathrm{p}=.04$, $\mathrm{d}=1.62$ ). Compared to being fully rested, men showed a decrease in implicit preferences for low (versus high) calorie foods, whereas women showed an increase. 
Conclusions: These preliminary results suggest that men may be at increased risk of weight gain when sleep deprived due to a reduced preference for low-calorie foods. However, these results need to be replicated in larger sample sizes. Our findings have important implications regarding the behavioral health risks of sleep deprivation.

Correspondence: Anna Alkozei, Ph.D., Department of Psychiatry, University of Arizona Medical Center, University of Arizona Medical Center, Department of Psychiatry, 1501 N Campbell Ave, Tucson, AZ 85724, United States.E-mail: aalkozei@psychiatry.arizona.edu

V. SANBORN, M. SPITZNAGEL, R. CROSBY, K. STEFFEN, J. MITCHELL \& J. GUNSTAD. Cognitive Impairment is Associated with Poorer Quality of Life in Persons with Severe Obesity.

Objective: Both cognitive impairment and poor quality of life (QoL) are common in persons with severe obesity. No study has examined their possible association, despite past work showing that greater impairment is associated with poor QoL in other medically-ill populations. We anticipated that cognitive dysfunction would be associated with poorer overall and work-related QoL in this sample of persons with severe obesity.

Participants and Methods: Participants included 61 bariatric surgery candidates $\left(\mathrm{M}_{\text {age }}=43.59 \pm 10.39 ; \mathrm{M}_{\mathrm{BMI}}=45.90 \pm 5.27\right)$ enrolled in the Longitudinal Assessment of Bariatric Surgery (LABS) project. Participants completed computerized cognitive testing (IntegNeuro), a self-report instrument of subjective cognitive complaints, and a commonly-used measure of QoL (i.e. Influence of Weight on Quality of Life; IWQoL) at pre-operative baseline.

Results: After accounting for medical covariates, logistic stepwise regression showed that better Recognition Memory predicted poorer reported Total QoL $\left[R^{2}=0.15, R^{2}\right.$ change $\left.=.07, \beta=0.28, p=0.03\right]$. Similar analyses revealed that poorer Digit Span Backward was associated with worse Work-related QoL $\left[\mathrm{R}^{2}=.13, \mathrm{R}^{2}\right.$ change $\left.=.10, \beta=-0.31, p=0.01\right]$. Greater subjective cognitive complaints were correlated to poorer Total QoL $(\mathrm{r}=0.38, \mathrm{p}=.003)$ and work-related QoL $(\mathrm{r}=0.41, \mathrm{p}=.001)$. Conclusions: Objective and subjective cognitive function are independently associated with QoL in bariatric surgery candidates. This pattern is consistent with other medical populations and encourages future studies to develop interventions to improve cognitive function in this high risk population.
Correspondence: Victoria Sanborn, B.A., Psychology, Kent State University, 634 Walter Street, Kent, OH 44240, United States. E-mail: vsanborn@kent.edu

J. SKALAMERA, A. ALKOZEI, M. HAACK \& W.D. KILLGORE. Chronic Sleep Restriction Increases Implicit Racial Biases and Affects Actual Decision-Making About People.

Objective: Chronic sleep restriction is widespread in modern society and can have significant effects on emotion and decision-making. We recently showed that chronic sleep restriction increases negative implicit cognitive biases toward individuals from a minority group, but no research has investigated whether this can predict explicit behavior. Here we investigated whether increases in implicit biases as a result of sleep restriction can predict behavior within an emotional decision-making task.

Participants and Methods: Nine participants underwent two 25-day in-hospital stays during which they were either sleep-restricted ( 4 hours every weekday, 8 hours on the weekend) or able to sleep for a full 8 hours. On day 21 of each visit, participants completed the Arab Muslim Names Implicit Association Test (IAT) and a computerized task in which they had to take on the role of an airline security guard during a credible terrorist threat. Participants viewed a series of facial photographs of passengers (white men and women) and decided whom to detain. The photographs were previously rated for a set of character traits (e.g. caring) by independent judges.

Results: Following sleep-restriction, greater changes in negative implicit bias scores on the IAT were associated with the selection of passengers to be detained $(r=.75, p<.05)$. Controlling for participants' scores when rested, the association becomes marginally significant $(r=.57, p=.06)$. In other words, the greater the increase in implicit bias scores from rested to sleep-deprived, the more likely the participant was to detain more passengers.

Conclusions: This finding suggests that as sleep restriction reduces prefrontal control over negative implicit biases, individuals become more likely to apply ever more stringent standards when deciding whom to trust based on facial features indicative of dispositional traits. Sleep restriction affects not only unconscious biases, but also overt real-life decisions with potentially life-altering consequences.

Correspondence: Anna Alkozei, Ph.D., Department of Psychiatry, University of Arizona Medical Center, University of Arizona Medical Center, Department of Psychiatry, 1501 N Campbell Ave, Tucson, AZ 85724, United States.E-mail: aalkozei@psychiatry.arizona.edu

FRIDAY EVENING, FEBRUARY 16, 2018

\section{Plenary F (Birch Memorial Lecture). Medial Temporal Lobe Amnesia: Past, Present, and Future}

\section{Presenter: Mieke Verfaellie}

5:00-6:00 p.m.

\section{VERFAELLIE. Medial Temporal Lobe Amnesia: Past, Present, and Future.}

Since the seminal reports of HM's profound memory impairment following surgical resection of the medial temporal lobes (MTL) bilaterally, detailed investigations of patients with amnesia have been invaluable in characterizing the impairment as well as the preservation of aspects of memory following MTL lesions. More recently, such studies have also demonstrated that patients with MTL lesions have cognitive impairments that extend beyond the domain of memory. This presentation will highlight insights gleaned from the study of MTL amnesia about the nature of the processes and representations mediated by the
MTL. In addition, it will demonstrate how neuropsychological studies of amnesia have simultaneously sharpened distinctions between discrete aspects of memory and blurred distinctions between episodic memory and other cognitive functions.

Following this lecture, the listener should be able to (1) recognize which aspects of memory depend on the MTL; (2) describe functional specialization within the MTL memory system; and (3) appreciate non-mnemonic impairments associated with MTL lesions.

Correspondence: Mieke Verfaellie, PhD, Memory Disorders Research Center, VA Boston Healthcare System and Boston University School of Medicine, 150 S Huntington Ave (151A), Boston, MA 02130, United States.E-mail:verf@bu.edu 
SATURDAY MORNING, FEBRUARY 17, 2018

\section{CE Workshop 11. Neuropsychological Research in Hispanics/Latinos Living in the United States: The} Case of HIV

\section{Presenter: Maria Marquine}

$$
\text { 7:20-8:50 a.m. }
$$

M. MARQUINE. Neuropsychological research in Hispanics/ Latinos living in the United States: The case of HIV.

Hispanic/Latinos/as, hereafter referred to as Hispanics, are the largest ethnic/racial minority group in the United States. Hispanics are highly heterogeneous, comprising multiple national origins, patterns of immigration, and linguistic and educational backgrounds, and are also known to possess genetic backgrounds shaped by admixture from several source continental populations. As a group, Hispanics are disproportionally impacted by human immunodeficiency virus (HIV). They are at increased risk for $\mathrm{HIV}$-infection compared to non-Hispanic Whites and tend to be diagnosed and treated later in the course of the disease. Despite advances in the treatment of $\mathrm{HIV}$, neurocognitive impairment (NCI) continues to be prevalent and impactful among people living with HIV, particularly Hispanics. This workshop will review the science on differences in NCI between HIV-infected Hispanics and non-Hispanics, as well as among subgroups of Hispanics. Current research will also be discussed regarding biomedical and socio-cultural factors underlying these disparities, and the role of genetically-defined ancestry on ethnic group differences in HIV-associated NCI. The workshop will also discuss implications for neuropsychology research among Hispanics more generally, and for clinical practice with Hispanic patients. Thus, this workshop will help participants:

1 - Identify biomedical and socio-cultural factors underlying ethnic disparities in NCI, particularly between HIV-infected Hispanics and non-Hispanics.

2- Gain a deeper understanding of rates and pattern of NCI among diverse groups of HIV-infected Hispanics.

Correspondence: Maria Marquine, PhD, Psychiatry, University of California San Diego, 9500 Gilman Drive \#0664, San Diego, CA 92093,

United States. E-mail:mmarquine@ucsd.edu

\section{CE Workshop 12. Cross-Cultural} Neuropsychological Assessment: Challenges and Solutions

\section{Presenters: Alberto L. Fernandez, Jonathan Evans}

$$
\text { 7:20-8:50 a.m. }
$$

\section{J. EVANS \& A.L. FERNANDEZ. Cross-cultural neuropsychological} assessment: challenges and solutions.

In an increasingly globalised world all neuropsychologists need to consider the effects of language and culture on neuropsychological test performance. This is important if you are a neuropsychologist who assesses patients coming from a different linguistic and cultural background to that for which the tests you typically use were designed; if you work in a country where you have few locally developed tests but need to conduct neuropsychological assessments; or if you are a test developer and want to support others around the world to use your test. The American Academy of Clinical Neuropsychology has recognised this issue with the launch of its Relevance 2050 initiative, noting that by $205060 \%$ of the US population will not be testable on our current tests. In other parts of the world, particularly in low and middle-income countries, there are few neuropsychological tests available and so tests developed elsewhere (typically in the US or Europe) are sometimes used without adequate cultural adaptation and without appropriate normative samples. In this workshop we will explore the issues of language, culture and neuropsychological assessment. We will discuss how we can address these issues, including practical strategies in relation to use of interpreters in test sessions, the development and use of culture-fair tests and the procedures that should be followed when a test developed in one context is adapted for use in another. As a result of participation in this course, the learner will achieve the following objectives: (1) have a deeper understanding of the impact of culture on neuropsychological test performance; (2) be familiar with strategies for assessing patients from different cultural backgrounds including culture-fair options and how to adapt tests developed in one cultural context for use in another. Correspondence: Alberto L. Fernandez, PhD, Neuropsychology, Universidad Católica de Córdoba, Chile 279 PB, Córdoba 5000, Argentina.E-mail: albertolfernan@gmail.com

\section{Invited Symposium 5. Neuropolitics: Getting 'Under the Hood' of Political Attitudes, Identities and} Behaviours in a Turbulent World

\section{Chair: Laura Cram}

\section{Presenters: Jay Van Bavel, Ingrid Haas, Molly Crocket, Laura Cram}

$$
\text { 9:00-10:30 a.m. }
$$

L. CRAM, J. VAN BAVEL, M. CROCKET \& I. HAAS. Neuropolitics: Getting 'Under the Hood' of Political Attitudes, Identities and Behaviours in a Turbulent World.

Kannheman (2009:525) argued that we are "well on our way to the day when theoretical concepts about decision-making are shaped at least in part by findings from neuroscience'. Those at the forefront of developments in cognitive neuroscience have also argued that there is a need for greater integration between political theory and neuroscientific research (Damasio, 1994). The nascent field of neuropolitics (Jost et al., 2014) offers real potential to transform traditional approaches to the study of political attitudes, identities and behaviours and also raises new questions to be tackled by the cognitive neurosciences. In a turbulent international political environment, the contributors to this panel make a timely contribution to current political debates. Jav Van Bavel discusses the impact of political partisanship on information processing and the prospects for de-biasing information processes. Molly Crocket analyses responses to moral outrage in modern digital communications. Ingrid Haas examines the effect of incongruent political behaviour by politicians. Laura Cram concludes with a discussion of social belonging. the dynamics of trust and implications for political legitimacy. Correspondence: Laura Cram, PhD, NRlabs, University of Edinburgh, NRlabs, University of Edinburgh, Flat 2F2, 18 Buccleugh Place, Edinburgh EH89LN, United Kingdom.E-mail: laura.cram@ed.ac.uk

\section{J. VAN BAVEL. The Partisan Brain: A Value-Based Model of Political Belief.}

Democracies assume accurate knowledge by the populace, but the human attraction to fake and untrustworthy news poses a serious problem for healthy democratic functioning. This is exacerbated by the fact that Republicans and Democrats are more divided along ideological lines than at any point in decades. Although partisan antipathy accounts for deep rifts on a number of important issues, scientists still know very little about the neurobiological roots of polarization. In this talk. I will articulate why and how identification with political parties-known as partisanship-can bias information processing in the human brain. 
My research suggest that the roots of political partisanship are basic representations of group membership - grounding political differences in basic tribal distinctions between us and them. I will introduce a value-based model of belief for understanding the influence of partisanship on these cognitive processes. This model bridges politics, psychology \& neuroscience to help explain why people place party loyalty over policy, and even truth. Specifically, the model explains how partisanship has cognitive consequences that extend well beyond motivated political reasoning, to memory, implicit evaluation, and even perceptual judgments. In the final section, I will discuss strategies for de-biasing information processing to help create a shared reality across partisan divides.

Correspondence: Jay Van Bavel, NY, United States. E-mail: jay. vanbavel@gmail.com

\section{HAAS. Political Ideology Moderates Neural Evaluation of Incongruent Policy Positions.}

Political polarization at the elite level is a major concern in many contemporary democracies, which is argued to alienate large swaths of the electorate and prevent meaningful social change from occurring, yet little is known about how individuals respond to political candidates who deviate from the party line and express policy positions incongruent with their party affiliations. This experiment examines the neural underpinnings of such evaluations using functional MRI (fMRI). During fMRI, participants completed an experimental task where they evaluated policy positions attributed to hypothetical political candidates. Each block of trials focused on one candidate (Democrat or Republican), but all participants saw two candidates from each party in a randomized order. On each trial, participants received information about whether the candidate supported or opposed a specific policy issue, along with information about the certainty with which these positions were held. These issue positions varied in terms of congruence between issue position and candidate party affiliation. We modeled neural activity as a function of incongruence and whether participants were viewing ingroup or outgroup party candidates. Results suggest that neural activity in brain regions previously implicated in both evaluative processing and work on ideological differences (insula and anterior cingulate cortex) differed as a function of the interaction between incongruence, candidate type (ingroup versus outgroup), and political ideology. More liberal participants showed greater activation to incongruent versus congruent trials in insula and ACC, primarily when viewing ingroup candidates. We also find differences in neural processing as a function of the interaction between incongruent and uncertain information. Implications for the study of democratic representation and linkages between citizens' calls for social change and policy implementation are discussed.

Correspondence: Ingrid Haas, NE, United States. E-mail: ingrid.haas@ gmail.com

\section{CROCKET. Moral Outrage in the Digital Age.}

Humans reliably respond to insult and injury with moral outrage. Judging and punishing others for wrongdoing can deter future harms and promote group cooperation, but can also exacerbate social divides and escalate into destructive cycles of retaliation. This behaviour evolved in the context of small foraging groups, but is now widespread in massive online communities. In this talk I will explore how digital media changes the costs and benefits of moral outrage and its implications for social cohesion.

Correspondence: Molly Crocket, Oxford, United Kingdom. E-mail: molly. crockett@psy.ox.ac.uk

\section{CRAM. Trust, Fairness and Social Belonging: The Neuropolitics of Legitimacy.}

In what is a turbulent time for politics internationally, scholars, policymakers and commentators have struggled to make sense of domestic and global developments and to formulate effective public policies in response to these developments. In this paper I argue that insights from cognitive neuroscience and political psychology might help address the challenges to legitimacy that underlie the current turbulence. Legitimacy as a concept is linked to one of the oldest and most fundamental of political concepts - that of political obligation, a willingness to consent to being governed and to endow a system or an individual person with authority. Perceptions of fairness and social trust, of 'bottom up' acquiescence, are considered by some to be central to this process, and loss of these may provoke a legitimation crisis. I argue that understanding the neurobiological mechanisms that underpin fairness and trust calibrations and willingness to cooperate provides useful insights into the legitimation process and provides some support for these theories. I examine how perceptions of social belonging and exclusion modulate the processing of fairness and trust and discuss the implications of this for current discussions on legitimacy crises and the maintenance of authority.

Correspondence: Laura Cram, PhD, NRlabs, University of Edinburgh, NRlabs, University of Edinburgh, Flat 2F2, 18 Buccleugh Place, Edinburgh EH8 9LN, United Kingdom. E-mail: laura.cram@ed.ac.uk

\section{Symposium 11. Investigating Temporal Lobe Epilepsy with High Resolution Magnetic Resonance Imaging and Connectome Approaches}

\section{Chairs \& Presenters: Mary Meyerand, Bruce Hermann \\ Presenters: Veena A. Nair, Vivek Prabhakaran \\ 9:00-10:30 a.m.}

\section{B. HERMANN. Investigating Temporal Lobe Epilepsy with High Resolution Magnetic Resonance Imaging and Connectome Approaches.}

We propose a symposium presenting cutting edge brain connectomic research focused on adult Temporal lobe epilepsy (TLE). High resolution functional Magnetic Resonance Imaging acquired via multi-band acquisition has now made it possible to build a connectome - i.e. structural and functional connections between brain regions that make up epileptic networks, providing a promising new approach for understanding, predicting, and treating refractory epilepsy. TLE is the most common form of epilepsy in adults and the largest group among those with medically refractory seizures. As part of the Epilepsy Connectome Project (ECP) we are collecting detailed connectivity measurements in 200 people with idiopathic TLE using diffusion-weighted magnetic resonance imaging of structural connections, functional magnetic resonance imaging of dynamic network interactions, and magnetoencephalography to measure these interactions with millisecond time resolution. The methods will closely mirror those currently used by the Human Connectome Project (HCP) to study network connectivity in healthy participants, and the HCP data will provide a critical baseline against which to compare the ECP connectome data. In this symposium we will present details regarding rationale for the study, study goals, acquisition methods, and data sharing for the future. We will also present new findings regarding compromised network connections in TLE patients, neurobiology underlying processing speed impairment in these patients, and gender differences in structural and functional measurements in patients and healthy controls.

Correspondence: Bruce Hermann, PhD, Department of Neurology, University of Wisconsin-Madison, 600 N Highland Ave, Madison, WI 53792, United States. E-mail: hermann@neurology.wisc.edu 
M. MEYERAND, J. BINDER, D. ALMANE, R. BIRN, L. CONANT, E. DEYOE, E. FELTON, L. FERNANDINO, W. GROSS, B. HERMANN, C. HUMPHRIES, K. KOCH, P. KRAEGEL, R. MAGANTI, J. MATHIS, T. MUFTULER, T.M. MCMILLAN, V.A. NAIR, A. NENCKA, V. PRABHAKARAN, M. RAGHAVAN, M. ROZMAN, A. STRUCK, G. ZHAO \& C. USTINE. The Epilepsy Connectome Project.

Objective

The NIH-sponsored Epilepsy Connectome Project (ECP) aims to characterize brain connectivity changes in adults with temporal lobe epilepsy (TLE); correlate these changes with disease duration and severity, cognitive and behavioral deficits, and medication resistance; and measure longitudinal changes in connectivity and their relationship with seizure frequency and cognitive decline.

Participants and Methods

200 people with non-lesional TLE will be recruited. MRI protocols follow those used in the Human Connectome Project (HCP), and include 40 minutes of resting fMRI, 4 task fMRI scans examining language, semantic memory, emotion processing, and social cognition; high-resolution diffusion tensor imaging, and T1- and T2-weighted structural scans. Additionally, connectivity will be examined using magnetoencephalography (MEG) obtained during rest and 6 tasks (semantic decision, picture naming, story comprehension, and 3 control conditions). Participants undergo a 6-hour assessment that includes a medical history interview, cognitive and sensory-motor tests from the Epilepsy Common Data Elements and NIH Toolbox batteries; and self-report inventories covering psychiatric state and quality-of-life. Participants return after 1 year for one visit that includes resting fMRI and DTI, medical interview. and a sub-set of the cognitive tests.

Results

HCP multi-band protocols were implemented for the first time on GE $3 \mathrm{~T}$ scanners at the two participating sites. To-date, 58 TLE and 44 control participants have completed the MR and neuropsych protocol, and 28 MEG patients. MRI signal-noise ratio and head motion quality control metrics are well within $90 \%$ confidence intervals established by the HCP.

Conclusions

The ECP will provide the research community with a large and rich dataset for studying brain connectivity at the network level in TLE. De-identified data will be uploaded at yearly intervals to the Connectome Coordinating Facility for sharing worldwide.

Correspondence: Mary Meyerand.E-mail:memeyerand@wisc.edu

G. HWANG, J. MATHIS, V.A. NAIR, F. KAWSAR, O. NWOKE, N. TELLAPRAGADA, G. ZHAO, R. MOHANTY, M. ROZMAN, T.M. MCMILLAN, D. ALMANE, A. NENCKA, E. FELTON, A. STRUCK, R. BIRN, R. MAGANTI, L. CONANT, C. HUMPHRIES, B. HERMANN, M. RAGHAVAN, E. DEYOE, J. BINDER, M. MEYERAND \& V. PRABHAKARAN. Application of Machine Learning to Identify and Characterize Temporal Lobe Epilepsy. Objective

The NIH-sponsored Epilepsy Connectome Project (ECP) aims to reveal reliable biomarkers for temporal lobe epilepsy (TLE) by analyzing data of variety types. Here we used machine learning (ML) to classify between TLE patients and healthy controls using 3 types of data: brain structure, resting state connectivity, and cognitive test scores.

Participants and Methods

MRI data from 35 TLE patients (32.3 vears, 19 females) and 35 healthy controls (34.7 years, 19 females) were analyzed. Structural T1w was acquired with 3T GE 750 scanners $(0.8 \mathrm{~mm}$ isotropic). FMRI was acquired using whole-brain simultaneous multi-slice imaging (8 bands, 72 slices, TR $802 \mathrm{~ms}, 2 \mathrm{~mm}$ isotropic). Data were processed using the FreeSurfer and FSL-based Human Connectome Project (HCP) processing pipeline. HCP's Glasser parcellation scheme was used to create resting state connectivity matrices.

The sample consisted of 41 TLE patients ( 41.5 years, 24 females) and 55 healthy controls (33.8 years, 31 females), who completed 10 conventional cognitive tests and 4 NIH Toolbox tests. Age-corrected standard scores were used.

We used 2 ML binary classification methods using MATLAB: support vector machine (SVM) and linear discriminant analysis (LDA). For cross validation, leave-one-out-cross-validation (LOOCV) was used. For feature selection, we applied a t-test filter.

Results

Machine learning reached its highest LOOCV accuracy of $88.6 \%$ $(\mathrm{AUC}=0.90)$, when structural and functional features were combined. Among top discriminating features were left/right cerebellum cortex and connections between right area 46 and retrosplenial cortices. Using cognitive test scores, it reached $82.3 \% \quad(\mathrm{AUC}=0.83)$ using Pattern Comparison Processing Speed, Grooved Pegboard and Rey Auditory Verbal Learning scores.

Conclusion

Results seem to suggest that structural and functional features found in extra-temporal regions reflect most significant group difference between TLE patients and healthy controls.

Correspondence: Vivek Prabhakaran. E-mail: VPrabhakaran@ uwhealth.org

K. DABBS, T.M. MCMILLAN, G. HWANG, J. MATHIS, V.A. NAIR, F. KAWSAR, O. NWOKE, N. TELLAPRAGADA, G. ZHAO, M. ROZMAN, D. ALMANE, A. NENCKA, E. FELTON, A. STRUCK. R. BIRN, R. MAGANTI, L. CONANT, C. HUMPHRIES, J. BINDER, B. HERMANN \& M. MEYERAND. Neurobiological and Cognitive Correlates of Processing Speed in Temporal Lobe Epilepsy.

Objective: Psychomotor slowing is a prominent cognitive morbidity of temporal lobe epilepsy (TLE). Here we examined the neurobiological correlates of processing speed and its implications for memory and executive functions.

Participants and Methods: 41 TLE $\left(M_{\mathrm{age}}=41.6\right)$ and 55 healthy control $\left(M_{\mathrm{age}}=33.7\right)$ adult participants from the Epilepsy Connectome Project were administered the Rey Auditory Verbal Learning Test (AVLT) and NIH Toolbox Pattern Comparison Processing Speed, Flanker, Dimensional Change Card Sort and List Sorting Tests.

Relationships between processing speed (PS) and cortical and subcortical metrics from high resolution 3T T1 and T2-weighted MRI scans were examined using Freesurfer-v5.3-HCP, with age and intracranial volume (for volume measures) as covariates. Associations of PS with memory and executive tests, adjusted for IQ, were examined.

Results: PS was slower in TLE versus controls $(p<.01)$. In TLE. vertex analyses showed associations between faster PS and increased thickness in the left superior frontal $(p=0.0001)$, inferior temporal ( $p=0.0001)$, pars orbitalis $(p=0.0001)$, superior parietal $(p=0.0001)$, and rostral middle frontal $(p=0.038)$ regions; and right inferior temporal $(p=0.0001)$ and superior parietal $(p=0.0062)$ regions; as well as larger volumes of left $(p=0.04)$ and right thalamus $(p=0.05)$, mid-anterior corpus callosum $(p=0.03)$, and left amygdala $(p=0.025)$. In controls, there were no significant associations. PS was a significant predictor of verbal learning and memory (RAVLT total $p=0.04$; RAVLT delayed: $p=0.05$ ) and executive function (Dimensional Card Sort: $p=0.03$; Flanker: $p=0.01$; List Sorting: $p=0.01)$ in TLE but not controls.

Conclusions: Processing speed, a major cognitive complication in TLE is associated with a distributed neurobiological network including frontal, parietal, and temporal as well as subcortical (thalamus, corpus callosum) regions, with important implications for memory and executive function efficiency.

Correspondence: Bruce Hermann, PhD, Department of Neurology, University of Wisconsin-Madison, 600 N Highland Ave, Madison, WI 53792, United States.E-mail: hermann@neurology.wisc.edu 
O. NWOKE, G. HWANG, J. MATHIS, V.A. NAIR, F. KAWSAR, N. TELLAPRAGADA, G. ZHAO, M. ROZMAN, T.M. MCMILLAN, D. ALMANE, A. NENCKA, A. STRUCK, R. BIRN, R. MAGANTI, L. CONANT, C. HUMPHRIES, B. HERMANN, M. RAGHAVAN, E. DEYOE, J. BINDER, M. MEYERAND, V. PRABHAKARAN \& E. FELTON. Epilepsy Connectome Project (ECP) - Gender Differences in Temporal Lobe Epilepsy Patients.

Objective: Gender differences have been observed in the structure and connectivity of the human brain. These differences translate into pathophysiological variances in neurological disorders such as epilepsy. Women with epilepsy often have increased seizure severity and frequency during menarche, certain phases of the menstrual cycle, pregnancy, and menopause. We studied gender differences in temporal lobe epilepsy (TLE) patients and compared them to controls.

Participants and Methods: Subjects included 38 TLE patients (21 females, mean age 35.7 years) and 36 controls (20 females, mean age 32 years). High resolution structural T1 and T2-weighted $(0.8 \mathrm{~mm}$ isotropic) and resting state functional magnetic resonance imaging (fMRI) $(2 \mathrm{~mm}$ isotropic, repetition time $=802 \mathrm{~ms}$ ) were acquired on a 3T 750 General Electric scanner using Human Connectome Project (HCP) protocols. Data were processed using FMRI of the Brain Software Library (FSL) and FreeSurferbased processing pipeline. Resting state connectivity matrices were generated using HCP's Glasser Parcellation scheme.

Results: Decreased bi-hemispheric cortical surface area in female patients $(\mathrm{FP})$ when compared to male patients $(\mathrm{MP})(\mathrm{p}<0.005)$ of several key regions damaged in TLE, such as the middle and inferior temporal areas and insular lobes. Bilateral white matter surface area and estimated total intra-cranial volume are generally smaller in women than men, but the difference was markedly pronounced in epileptic women $(p \leq 0.005)$. Resting state functional connectivity data reveal that MPs showed stronger connections than FPs from the left hippocampus to right fusiform face area $(p<0.001)$. FPs in contrast showed stronger connections between the left superior temporal gyrus to the right premotor eye field $(\mathrm{p}<0.0001)$.

Conclusion: Distinct gender differences are observed in brain structure and connectivity of male and female TLE patients.

Correspondence: Veena A. Nair, PhD, Radiology, University of Wisconsin-Madison, 1111 HIGHLAND AVE, WIMR 1314, Madison, WI 53705,United States.E-mail:VNair@uwhealth.org

\section{Paper Session 15. Aging}

\section{Moderator: Vonetta M. Dotson}

\section{9:00-10:30 a.m.}

K. JAPARDI, S. BOOKHEIMER, K. KNUDSEN \& R.M. BILDER. The Big-C Project: Distinct Language Processes Responsible for Divergent and Convergent Thinking in Big-C Individuals.

Objective: Divergent thinking (DT) and convergent thinking (CT) are widely considered to be critical to creative thinking; however, the specific mechanisms underlying these traits are unknown. This study examined whether "Big-C" (exceptionally creative) and "little-c" (everyday creative) individuals: (1) show different functional activation during DT- and CT-related tasks and (2) exhibit functional activation unique to either task.

Participants and Methods: We analyzed 87 participants including 29 Big-C Visual Artists ( $48.3 \%$ f, $43 \pm 7.3$ yrs old), 28 Big-C Scientists $(50 \% \mathrm{f}, 45.5 \pm 8.2 \mathrm{yrs}$ old $)$, and 30 Comparison Group individuals $(50 \%$ f, $42 \pm 9.4$ yrs old) matched on age, sex, and full-scale IQ. During an fMRI session, participants completed an Alternate Uses Task (AUT) and Remote Associates Task (RAT), to measure DT and CT respectively. We conducted functional analyses using two main contrasts of interest:
AUT $>$ RAT and RAT $>$ AUT. Associations between the fluency and originality of AUT responses and correct recalled associations were examined to assess both cognitive processes.

Results: In the AUT>RAT contrast, we found significantly higher supramarginal gyrus activation within each group. Within this contrast, the comparison group also showed significantly higher left middle frontal gyrus (MFG) activation than Big-C Scientists. The RAT $>$ AUT contrast revealed significantly higher left MFG and inferior frontal gyrus within each group. We also found a positive correlation between AUT fluency and RAT correct associations $(\mathrm{r}=0.36, \mathrm{p}=0.003)$.

Conclusions: During a DT task, we see significant activation in regions associated with language production; while during a CT task, we see significant activation in regions associated with language comprehension. We also found a strong positive correlation between DT and CT performance. Overall, our research suggests a link between DT and CT ability, but clearly distinct language processing mechanisms.

Correspondence: Kevin Japardi, B.A. Molecular and Cellular Biology: Neurobiology, Psychiatry and Biobehavioral Sciences, UCLA, 760 Westwood Plaza, Semel B8-169, Los Angeles, CA 90025, United States. E-mail:kjapardi@mednet.ucla.edu

K. KNUDSEN, K.C. BARRETT, P. JIRADEJVONG, K. JAPARDI, S. BOOKHEIMER, C. LIMB, R.M. BILDER \& D. GHAHREMANI. The Big-C Music Project: Positive Psychopathology and Task Positive Brain Networks.

Objective: The Big C Music Project studies (1) whether "Big C" (exceptional) creativity in music is linked with schizotypal, autistic, and temperamental traits, (2) how Big C Music psychopathology and personality profiles compare with other domains, and (3) neural mechanisms underlying Big $\mathrm{C}$ jazz improvisation.

Participants and Methods: We examined 17 Big C Musicians (MUS; $88.89 \%$ male; ages $21-42$ ) with expertise in world-class jazz improvisation, as part of a broader study of $35 \mathrm{Big} \mathrm{C}$ Visual Artists (VA; $51.42 \%$ male; ages 29-57), 41 Big C Scientists (SCI; $51.21 \%$ male; ages 29-59) and 31 Smart Comparison Group individuals (SCG; $48.38 \%$ male; ages 22-59) matched on age, sex, ethnicity, and parental education. We directly compared across the four groups with the Creative Achievement Questionnaire (CAQ), the Schizotypal Personality Questionnaire (SPQ), Social Responsiveness Scale, and the International Personality Item Pool Representation of the NEO PI-R. MUS also completed a functional MRI task adapted from Limb et al.'s (2008) work that involves mentally generating jazz improvisations vs. mentally rehearsing music. Two fMRI paradigms represented distinct musical complexity: (1) scale improv vs. scale rehearsal and (2) melody improv vs. melody rehearsal. Results: MUS had markedly higher CAQ scores within their domain. MUS had significantly greater Cognitive/Perception and Ideas of Reference SPQ Factors than SCI (VA, MUS > SCI), with nominally greater scores than SCG. Regardless of task complexity, MUS showed greater striatal and DLPFC activation during jazz improv than during music rehearsal. We found deactivation in the anterior cingulate during improv relative to rehearsal, and greater activation in the temporal lobes and precuneus during melody vs. scale rehearsal.

Conclusions: These results validate the process of identifying Big C musicians, complement and extend past findings of SPQ traits associated with Big $\mathrm{C}$ visual artists, and delineate task positive networks by which exceptional musicians perform jazz improvisation.

Correspondence: Kendra Knudsen, UCLA, 760 Westwood Plaza, Semel Institute, Los Angeles, CA 90024, United States. E-mail: KKnudsen@ mednet.ucla.edu 
E. MOORE, F.S. BADAMI, D. LIU, K.R. PECHMAN, J.G. TERRY, S. NAIR, H.A. KRESGE, T.M. SHONE, F.E. CAMBRONERO, J.E. BOGNER, S.L. LAMBROS, C. SEABOLT, J. THOMPSON, L. WALLJASPER, S.P. BELL, K. GIFFORD, A.W. ANDERSON, T.J. HOHMAN, J.J. CARR \& A.L. JEFFERSON. Increased Left Ventricular Mass Index is Associated with Compromised White Matter Microstructure Among Older Adults: The Vanderbilt Memory \& Aging Project.

Objective: Left ventricular (LV) hypertrophy $(\mathrm{LVH})$ is associated with cerebrovascular disease and cognitive decline. Increased LV mass index (LVMI) is a subclinical imaging biomarker that precedes LVH. This study relates LVMI to white matter microstructure, white matter lesions, and cognition among older adults.

Participants and Methods: Vanderbilt Memory \& Aging Project participants free of clinical stroke, dementia, and heart failure $(n=313$, $73 \pm 7$ years, $58 \%$ male) underwent brain MRI, cardiac MRI to assess LVMI $\left(\mathrm{g} / \mathrm{m}^{2}\right)$, and neuropsychological assessment. Diffusion tensor imaging (DTI) was analyzed using tract-based spatial statistics. Voxelwise analyses relating LVMI to DTI metrics were generated using non-parametric permutations correcting for multiple comparisons. Models adjusted for age, sex, education, race/ethnicity, Framingham Stroke Risk Profile, cognitive diagnosis, and $A P O E-\varepsilon 4$ status. Adjusting for identical covariates, linear regressions related LVMI to neuropsychological performances and white matter hyperintensities (WMHs) with additional adjustment for intracranial volume. Secondary analyses included an LVMI $\mathrm{x}$ diagnosis interaction term with follow-up models stratified by diagnosis

Results: Increased LVMI related to global altered white matter microstructure $(p$-values $<0.05)$ and modestly related to worse California Verbal Learning Test-II Long Delayed Free Recall $(\beta=-0.04, p=0.047)$ but not WMHs $(p=0.57)$. There was also a LVMI $x$ diagnosis interaction ( $\mathrm{p}$-values<0.05) whereby associations between LVMI and DTI were present among mild cognitive impairment ( $p$-values $<0.05)$ but not cognitively normal participants ( $p$-values $>0.15$ ).

Conclusions: In the absence of heart failure, increased LVMI corresponds to altered white matter microstructure, particularly among those older adults with clinical symptoms of prodromal dementia. These results highlight the potential link between subclinical LV remodeling and white matter microstructure.

Funding: IIRG-08-88733, R01-AG034962, K24-AG046373, T32-GM007347, UL1-TR000445

Correspondence: Elizabeth Moore, TN, United States. E-mail: elizabeth.e.moore@vanderbilt.edu

L.C. WALZAK \& W.J. L. THORNTON. Theory of Mind in Aging: A Pilot Study Investigating Associations with Risky Decision-Making and Fraud Susceptibility.

Objective: Theory of Mind ( $\mathrm{ToM})$ allows us to reason about others mental states to better understand and predict behavior. Past research has identified predictors of reliable declines in ToM including age, pulse pressure and neurocognition. However, it is unclear how reduced performance on laboratory ToM tasks may translate into real world vulnerability to reduced decision-making and fraud susceptibility. Using a contemporary model of ToM with both cognitive and affective components, we examined risky decision-making and piloted a novel measure of fraud susceptibility, providing the first examination of links between ToM and everyday outcomes in adulthood.

Participants and Methods: 16 younger (12 females; $M=21$ years) and 11 older adults ( 7 females; $M=67$ years) completed standardized measures of neurocognition, cognitive ToM (Strange Stories), affective ToM (Reading the Eyes in the Mind), risky decision-making (Iowa Gambling Task; IGT), and fraud susceptibility (Everyday Decisions Task; EDT). We used correlational analysis and hierarchical regression to identify predictors of fraud susceptibility in both age groups.

Results: Cognitive ToM was associated with risky decision-making (IGT) in both age groups ( $r=.39, p=.05)$, and older adults were marginally more likely to participate in a fraudulent offer $(t=1.61, p=.06)$.
Stronger cognitive ToM performance was associated with better ability to discern genuine advertisements from fraudulent ones on the EDT $(\beta=.83,95 \% \mathrm{CI}=[.58,3.92], p=.01)$, while poorer affective ToM increased participants' likelihood to participate in a scam $(\beta=.84,95 \%$ $\mathrm{CI}=[.46,2.18], p=.01)$.

Conclusions: These preliminary findings from an ongoing study highlight the importance of ToM in predicting crucial everyday outcomes, such as risky decision-making and fraud susceptibility. Given that older adults are at increased risk for cognitive dysfunction and social deficits, a better understanding of the role of ToM in real-world functioning is clinically relevant for developing effective interventions in later life. Correspondence: Laura C. Walzak, B.A. (Hons), Clinical Psychology, Simon Fraser University, Department of Psychology, 8888 University Drive, Burnaby, BCV5A 1S6, Canada.E-mail: lwalzak@sfu.ca

A. SEELYE, N. MATTEK, J. AUSTIN, A. ALIOTO, S.A. MOSELEY, C. ANDERSON, W. VANVOORST, C.L. CLASON, B. DOANE, T. YAMADA, P. WITTER IV \& J. KAYE. Cognitive Correlates of Unobtrusively Monitored Medication Adherence in Older Adults. Objective: Medication taking is a complex cognitive task that is often affected early in the transition from healthy aging to MCI. Although lower cognition has been linked to poorer medication adherence in older adults, the specific cognitive processes involved are largely unknown and most research is limited by episodic clinic-based testing. This study objectively assessed older adults' routine medication taking using passive sensor monitoring and investigated associations with traditional neuropsychological tests.

Participants and Methods: Participants were 41 community-dwelling older adults ( $M$ age $=87.9, S D=6.9 ; 73 \%$ female; $2 \%$ MCI $)$ who took medications once per day from an instrumented pillbox ( $M$ duration= 555 days). Medication data were aggregated into two-month windows; in each window, two medication metrics were generated: variability in the timing medications were taken (IQR) and percent of days medications were missed (\%Missed). Hierarchical multiple regressions determined associations between memory and executive functioning tests and medication adherence, controlling for age and using each participant's last 2-months of medication data.

Results: Digit Span Backward (DSB) and Stroop Interference accounted for a significant amount of variance in IQR, $\mathrm{R}^{2}=.23, p=.03$. Better Stroop Interference score was associated with less IQR, $\beta=-.33, p=.05$. DSB and Logical Memory II Delayed Recall accounted for a significant amount of variance in $\%$ Missed, $\mathrm{R}^{2}=.54, p=.005$. Better DSB score was associated with fewer missed medications, $\beta=-.38, p=.01$.

Conclusions: Aspects of executive functioning such as complex attention and cognitive control were the strongest predictors of medication adherence. Results extend previous clinic-based studies while also adding a more precise understanding of the cognitive abilities involved in older adults' routine medication taking in natural environments. Future research will examine longitudinal trajectories of medication adherence in healthy aging and MCI.

Correspondence: Adriana Seelye, Minneapolis VA Healthcare System, VA Medical Center, One Veterans Drive, Minneapolis, MN 55446, United States.E-mail:seelyea@ohsu.edu

B.M. BETTCHER, J. NEUHAUS, M. WYNN, R. SALONER, R. FITCH, A. KARYDAS \& J. KRAMER. Increases in a Pro-Inflammatory Chemokine Are Related to Decreases In Episodic Memory Over Time.

Objective: Recent evidence suggests that chemokines may play a critical role in communication between the central nervous system and periphery, and that blood-borne monocyte chemotactic protein-1 (MCP-1) may detrimentally impact memory function. Little is known, however about the longitudinal relationship between MCP-1 and memory function in older adults. We hypothesized that increases in MCP-1 levels would be associated with decreases in verbal memory. 
Participants and Methods: We examined longitudinal plasma MCP-1 levels and a verbal memory measure (CVLT-II 20' free recall) in a sample of 395 asymptomatic older adults (mean age $=71.2$ ). Total visits ranged from 1 to 8 , with an average time of 2.1 years between visits ( $\mathrm{SD}=1.2$ ), yielding 922 total observations. Linear mixed effect models were conducted, with age at baseline, gender, and education entered as covariates and verbal recall as the longitudinal outcome. The predictor of interest was MCP-1 levels; in order to isolate change over time, we decomposed MCP-1 into subject-specific means (i.e. between subject factor) and longitudinal deviations from the subject-specific mean (i.e. within subject factor). In secondary analyses, we controlled for global cognition (MMSE) and APOE genotype.

Results: Controlling for baseline demographics, within-subject increases in MCP-1 levels were associated with decreases in free recall $(\mathrm{t}=-2.57$; $\mathrm{p}=.01$ ) over time. Results were independent of global cognitive function and $A P O E$ status $(\mathrm{t}=-2.18, \mathrm{p}=.03)$. Moreover, effect sizes for change in MCP-1 were larger than effect sizes for baseline MCP-1 levels $(p=.26)$ on longitudinal memory outcomes.

Conclusions: In an asymptomatic aging adult cohort, increases in MCP-1 levels were associated with modest longitudinal decline in episodic memory. Results add to a growing body of literature suggesting that 'healthy aging' is typified by remodeling of the immune system, and that the blood-borne chemokine, MCP-1, may be associated with negative cognitive outcomes.

Correspondence: Brianne M. Bettcher, PhD, Neurosurgery and Neurology, University of Colorado Anschutz Medical Campus, University of Colorado School of Medicine, 12631 E. 17th Avenue, C307, Aurora, CO 80045, United States. E-mail: brianne.bettcher@ucdenver.edu

\section{Paper Session 16. Sleep, Stress, and Exercise Moderator: Justin E. Karr \\ 9:00-10:30 a.m.}

D.M. O' SHEA, R. FIEO, A.J. WOODS, J.B. WILLIAMSON, E. PORGES \& R.A. COHEN. Discrepancies between crystalized and fluid ability are associated with frequency of social and cognitive engagement in community dwelling adults.

Objective: Discrepancies between crystalized and fluid ability (Gc-Gf) are associated with Alzheimer's disease biomarkers. However, individuals with higher cognitive reserve or crystalized ability may compensate for declines in fluid ability. Thus, the aim of the present study was to examine the association between $\mathrm{G} c$-G $f$ discrepancy and leisure activities.

Participants and Methods: Data from 96 older adults, (mean age $=$ $75, \mathrm{SD}=5.3$ ) were included in the present study. Measures from the NIH toolbox, were used to form the discrepancy scores. Physical, cognitive and social activities were identified using the Community Healthy Activities Model Program for Seniors activity questionnaire. A multivariate general linear model, controlling for age, education, gender and depressive symptoms was used to examine the association between $\mathrm{G} c-\mathrm{G} f$ on leisure activities (outcome variables).

Results: Significant main effects were followed up with multiple linear regression analyses. Results showed that greater discrepancies between crystalized and fluid ability was significantly and negatively associated with social $(\beta=-.320, p>.001)$ and cognitive activity $(\beta=-0.225, p>.05)$ but not physical activity.

Conclusions: Discrepancies between crystalized and fluid ability may be a sensitive marker of decline in advanced activities of daily living in highly educated individuals. Additionally, our results provide support for the adjustment of additional reserve metrics (i.e., leisure activities), in assessments of cognitive status.
Correspondence: Deirdre M. O' Shea, MS, Clinical and health psychology , University of Florida, 639 NE 6th street, Gainesville, Gainesville, FL 32601, United States. E-mail: dmo2123@ufl.edu

E. FOX, A.M. STAFFARONI, K.B. CASALETTO, R. SALONER, P. MUMFORD, L. RUOFF, A. EMERY, S. DUTT, M. WYNN, B.M. BETTCHER, M. ALTENDAHL, M.Y. YOU, T. NEYLAN, J. KRAMER, C. WALSH, K. WALKER \& J. STIVER. Baseline Sleep Efficiency Predicts rate of Parahippocampal Volume Loss in Cognitively Normal Older Adults.

Objective: Poor sleep may be a modifiable risk factor for age-associated cognitive decline and Alzheimer's-related (AD) pathology. Little is known, however, about whether poor sleep is associated with volume loss in AD-vulnerable regions in cognitively healthy older adults. Our goal was to determine the relationship between baseline sleep efficiency (SE) and longitudinal volume change in subregions of the medial temporal lobe (MTL) in this population.

Participants and Methods: 54 cognitively healthy older adults (mean baseline age $=74.6$ years) wore an at-home, mobile sleep electroencephalograph device (Zeo, Inc.) for 3-10 nights to gather objective sleep measures. Average SE was calculated across the observational period. All subjects completed a baseline 3T MRI scan, and 27 subjects had follow-up MRI 6 months to 4 years later. Total grey matter (TGM), parahippocampus (PH), entorhinal (EC), and hippocampus (HPC) volumes were extracted using the Desikan-Killiany atlas. We conducted linear mixed effects models with random intercepts, specifying an interaction of time and baseline SE to predict changes in brain volume.

Results: Lower baseline SE predicted greater rate of PH volume loss bilaterally (left: $b=.52, p=.017$; right: $b=.41, p=.006$ ) controlling for baseline age, sex, total intracranial volume, and APOE status. This relationship was specific and did not generalize across TGM volume $(\mathrm{b}=<.001, \mathrm{p}=.71)$ or occur within the HPC (left: $\mathrm{b}=.22, \mathrm{p}=.535$; right: $\mathrm{b}=.45, \mathrm{p}=.257$ ) or EC (left: $\mathrm{b}=.05, \mathrm{p}=.844$; right: $\mathrm{b}=.27, \mathrm{p}=.254$ ).

Conclusions: Poor baseline SE predicts faster volume loss in the $\mathrm{PH}$, an area known to be integral for contextual, associative learning. While this relationship was fairly specific to the PH, other MTL regions showed same-direction associations that did not reach significance with our sample size. This finding puts forth possibility that interventions to improve sleep hygiene in earlier stages of aging could ameliorate neuronal loss in the PH over time, tempering deficits in associative memory decline.

Correspondence: Emily Fox, B.A., Neurology, UCSF, 675 Nelson Rising Lane, Suite 190, San Francisco, CA 94158-1207, United States. E-mail: emily:fox2@ucsf.edu

Z. TAIWO, J.E. NEAL, D. LIU, K.E. OSBORN, K.R. PECHMAN, F.S. BADAMI, H.A. KRESGE, T.M. SHONE, J.E. BOGNER, S.L. LAMBROS, C. SEABOLT, J. THOMPSON, L. WALLJASPER, S.P. BELL, L. ACOSTA, K. GIFFORD, T.J. HOHMAN, K. BLENNOW, H. ZETTERBERG \& A.L. JEFFERSON. Sleep Fragmentation is Related to Altered Structural Brain Volume among Older Adults Free of Dementia: The Vanderbilt Memory \& Aging Project.

Objective: Sleep fragmentation (SF), reflecting disruption of sleep continuity, is associated with cognitive impairment and Alzheimer's disease (AD), possibly due to underlying structural brain changes. This study cross-sectionally relates SF to grey and white matter structural integrity in older adults.

Participants and Methods: Vanderbilt Memory \& Aging Project participants free of clinical stroke or dementia $(n=214,74 \pm 7$ years, $65 \%$ male) underwent $3 \mathrm{~T}$ brain MRI, 10 days of wrist actigraphy, neuropsychological assessment and an optional lumbar puncture ( $\mathrm{n}=97$ ) to determine $\mathrm{Ab}_{42}$ cerebrospinal fluid levels. Linear regressions related $\mathrm{SF}$ to MRI markers and cognition, adjusting for age, education, race/ethnicity, body mass index, Framingham Stroke Risk Profile, cognitive diagnosis, and APOE- $\varepsilon 4$ status. Follow-up models stratified by amyloid status. 
Results: Increased SF related to lower California Verbal Learning Test-II $(\beta=-0.14, p=0.03)$ and Biber Figure Learning Test Total Learning $(\beta=-0.50, p=0.045)$ and reduced right hemisphere $(\beta=-423.3, p=0.02)$, left hemisphere $(\beta=-519.4, p=0.008)$, right frontal $(\beta=-194.2, p=0.03)$, left frontal $(\beta=-244.2, p=0.02)$, right occipital $(\beta=-53.4, p=0.03)$, left occipital $(\beta=-61.1, p=0.01)$, left temporal $(\beta=-76.7, p=0.046)$ and increased left inferior lateral ventricle volumes $(\beta=10.05, p=0.04)$. When participants with prevalent cardiovascular disease (CVD) and atrial fibrillation (AFIB) were excluded, associations were modestly attenuated. SF was unrelated to white matter hyperintensities $(p=0.59)$. In stratified models, increased SF related to smaller left hippocampal volume $(\beta=-19.9, p=0.007)$ in amyloid-positive participants, even after excluding participants with CVD and $\mathrm{AFIB}(p=0.02)$.

Conclusions: Findings suggest SF is associated with neuroanatomical markers of accelerated brain aging and AD. Early hippocampal changes may occur in the presence of amyloid burden, further supporting the sleep-amyloid hypothesis.

Funding: IIRG-08-88733, R01-AG034962, R01-HL111516, K24-AG046373, UL1-TR000445

Correspondence: Zinat Taiwo, Georgia State University, Department of Psychology, 140 Decatur Street, Atlanta, GA 30303, United States. E-mail: ztaiwo1@student.gsu.edu

K.A. CARBINE, J. DAVIES, E. MODERSITZKI, L. TUCKER, B. BAILEY, J. LECHEMINANT \& M. LARSON. Neural Indices of Food-Related Inhibitory Control: The Influence of a Morning or Evening Exercise Program.

Objective: Food-related inhibitory control (withholding dominant responses to eat palatable food) may be improved with aerobic exercise. We tested how a morning or evening exercise program influenced neural indices of inhibitory control (measured by the N2 event-related potential) and if inhibitory control related to changes in diet and weight. We hypothesized that 1 ) there would be larger N2 difference amplitudes (greater inhibitory control) after the evening than morning program; 2 ) there would be greater reductions in caloric intake and weight in the evening than morning program; and 3) after the program, individuals with larger $\mathrm{N} 2$ difference amplitudes would show greater reductions in weight and energy intake, particularly for evening exercisers.

Participants and Methods: 38 healthy female participants were randomly assigned to eight weeks of morning or evening moderate treadmill exercise. Caloric intake, weight, and EEG data were obtained at baseline and after the program. EEG data were collected during two Go/No-Go tasks (one inhibiting to high-calorie and one inhibiting to low-calorie foods). ANOVAs were used to assess differences in caloric intake, weight, and $\mathrm{N} 2$ amplitude from pre- to post-program visits. Regression models were used to predict changes in weight and caloric intake.

Results: Inhibiting to high-calorie foods elicited a greater inhibitory response than low-calorie foods $(p=.03)$; exercise did not influence N2 difference amplitudes $(p=.37)$ or caloric intake $(p=.72)$. Individuals in the evening compared to morning group lost more weight $(p=.04)$. After eight weeks, individuals with larger N2 difference amplitudes showed greater reductions in weight $(p=.05)$ but not caloric intake $(p=.91)$, regardless of exercise time.

Conclusions: Increased recruitment of inhibitory control resources could potentially help individuals manage weight and should be further examined. As exercise seemed to not significantly impact inhibitory control, research is needed to test why evening exercise may be effective in managing weight.

Correspondence: Kaylie A. Carbine, B.S., Psychology, Brigham Young University, 6605 Amber Lane, Pleasanton, CA 94566, United States. E-mail:kaylie.carbine@gmail.com
K.B. CASALETTO, A.M. STAFFARONI, F. ELAHI, J. NEUHAUS, E. FOX, M.Y. YOU, R. SALONER \& J. KRAMER. Perceived stress potentiates age-related cytokine markers of macrophage inflammation in cognitively normal older adults.

Objective: Chronic stress leads to poorer cognitive aging but the mechanisms of this relationship are not well understood. Aging increases expression of primed and activated macrophages, leading to an exacerbated immune response to stressors. We aimed to determine the impact of stress and aging on macrophage-related inflammation and cognition in healthy older adults.

Participants and Methods: 386 neurologically-normal adults were followed longitudinally (baseline: age $\mathrm{M}=73 \mathrm{y}$, range $55-99 \mathrm{y}$; visit range $1-8, \mathrm{M}=2.5$ visits, $\mathrm{M}=1.5 \mathrm{y}$ between visits). Participants completed the Perceived Stress Scale, a cognitive battery, and blood draws. Plasma was analyzed for cytokines related to macrophage function: IL-6, TNF $\alpha$, MIP1 $\alpha$, MIP1 $\beta$. A macrophage cytokine composite was calculated. Mixed-effects modeling examined the effects of baseline age, baseline stress, time, and their interactions predicting the macrophage composite and cognition, adjusting for sex, education, and depressive symptoms. Results: Baseline perceived stress demonstrated a significant interaction with baseline age, but not time, predicting macrophage inflammation, and delayed recall, executive functions, and fluency performances $(p s<0.05)$. As age increased, the deleterious effects of stress on inflammation (stress*age $b=-0.01$ ) and cognition (atress*age $b$ 's $=0.01-0.06$ ) decreased. Models examining processing speed or visuospatial skills were not significant ( $\mathrm{ps}>0.05)$. Baseline stress was not associated with baseline age $(r=0.01)$ or aging $(p=0.98)$.

Conclusions: Stress demonstrates nonlinear aging effects on inflammation and cognition. Greater baseline stress evidenced a disproportionately negative impact on macrophage-related inflammation and cognition in earlier stages of aging that attenuated with increasing age. Stress did not simply increase with age and did not predict changes in inflammation or cognition. An exacerbated macrophage response to stress may be most relevant in earlier aging processes and supports early stress interventions to optimize cognitive aging.

Correspondence: Kaitlin B. Casaletto, PhD, Neurology, University of California, San Francisco, 675 Nelson Rising Ln, San Francisco, CA 94158, United States.E-mail: kaitlin.casaletto@ucsf.edu

\section{Poster Session 10. Cognitive Neuroscience, Neuromodulation, and Rehabilitation}

\section{9:00-10:15 a.m.}

\section{Cognitive Intervention/Rehabilitation}

K. CHIPMAN, G. ESKES, P. EBERT \& S. MUNK. Using Goal Management to Support Transfer of Memory Training to Everyday Life in Mild Cognitive Impairment.

Objective: Research shows that individuals with Mild Cognitive Impairment (MCI) may benefit from learning memory compensatory strategies; however, generalization of strategies to everyday memory tasks remains a challenge. This pilot project investigated the feasibility and effectiveness of combining two evidence-based cognitive intervention techniques aimed at optimizing transfer of learning to real-life. To this end, a modified goal management approach was used to teach individuals with MCI how to apply memory strategies to everyday tasks.

Participants and Methods: Twenty-six participants (11 men, 15 women, mean $(\mathrm{M})$ age 76 years) with MCI (Petersen criteria) and partners were randomly assigned to intervention or wait-list control. Participants were taught a 3-step goal management approach to help them apply memory strategies to everyday tasks during 10 weekly, 2-hour group sessions. Outcomes included standardized memory tests and memory questionnaires (Memory Strategy Toolbox (MST), Multifactorial Memory Questionnaire (MMQ)). 
Results: Group sessions had $95 \%$ attendance and were rated as easy to understand, helpful and applicable (M ratings=4.78, 4.74, and 4.69/5 respectively). Post-training ratings of memory strategy awareness (MST, p=.01) and self-reported memory strategy use (MMQ, p=0.024) were improved. Satisfaction and confidence in dealing with common memory tasks also significantly improved on a study-specific evaluation ( $p$ 's<0.002), as did contentment with memory ability (MMQ, p=.016). These changes remained significant at a long-term follow-up assessment $(n=16)$, despite continued memory difficulties on standardized memory tests.

Conclusions: Overall, this pilot project suggested that teaching a goal management approach to support the training and application of memory strategies to real-world settings is feasible, acceptable and helpful in increasing awareness, strategy use and confidence. Further research to confirm these preliminary findings is warranted.

Correspondence: Karen Chipman, Nova Scotia Health Authority, Nova Scotia Hospital, Rm 0035 Mount Hope Bldg, 300 Pleasant Street, Dartmouth, NS B2Y 3Z9, Canada.E-mail:karen.chipman@nshealth.ca

WITHDRAWN. K. CORNETT. Efficacy of Interdisciplinary PostAcute Rehabilitation for Impairments in Attention and Processing Speed in Traumatic Brain Injury.

Objective: The purpose of this study was to elarify how post-aente interdisciplinary retrabilitation informs cogntitive recovery following tratumatic brain injury.

Partieipants and Methods: This study compared neuropsychologieat test seores administered prion to and following treatment (i.e., singlegrottp, pre-and post-test design). Participants were workers' compent= sation claimants referred for elinieal evaluation to a private, multidiseiplinary, outpatient post-aeute brain injury rehabilitation program from September 2915 to December 2016.28 adthts 20 mild TBY and 16 moderate/severe TBI) were administered the De 1 J atabte Battery for the Assessment of Netropsychologieal Stans ob vs form A prior to beginning the program and RBA NS Formt at discharge. Fowr were

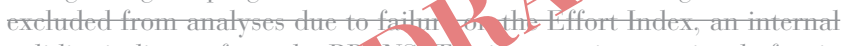
validity indieator from the PB VS. A, intervention consisted of a six to eight week, post-agk iin ro, elplinaty treatment program with a physiatrist, physida sas stât, psychologist, and nemopsychologist

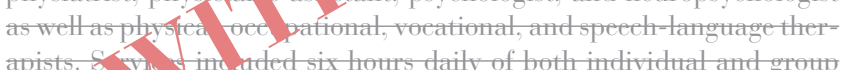
apists. \&
therapies. $1 \mathrm{w}$ ins hypothesized that scores on a meastre of attention (Pigit Spanstrbtest from PBANS) and proeessing speed (Goting subtest from RBANS) would improve following treatment.

Results: Paired t-tests demonstrated signifieant improvements in seores for Digit Span $(\mathrm{p}=.000)$ and Goding $(\mathrm{p}=.001)$ subtests from baseline to tischarge. While there was nocontrol gromp int his study, a recent stuty of 36 healthy adtut controls administered the RBA NS at six-week interval revealed only a $0.9 \%$ inerease in seores from baseline to discharge on Đigit Span (compared to $29 \%$ inerease int the etrrent stuty) and a $1.16 \%$ on Goding (compared to $26 \%$ increase in the current study). Gonelusions: This struetured post-aette, interdiseiplinary eognitive rehabilitation program improved performanee on generalized meastres of attention and processing speet.

Gorrespondence: KELLY CORNETT, Psy.D., Thehabilitation Institute of Hashington, 8228234TH STREET SH, EDMONDS, HA 98026, United states. E-mait. akitokelloe@gmait.com

L. DE WIT, D.M. O' SHEA, M. CHANDLER, J. FIELDS, D. LOCKE, V. PHATAK \& G. SMITH. Cognitive Functioning and Self-Efficacy as Predictors of Memory Support System Training Responsiveness in Mild Cognitive Impairment.

Objective: The Memory Support System training (MSS) was developed for patients with amnestic mild cognitive impairment (MCI) to support functional independence. MSS focuses on training the consistent use of a calendar throughout the day. The current study extends previous research by investigating factors that predict which individuals may most efficiently master MSS.
Participants and Methods: Data was selected from the Behavioral Interventions to Prevent or Delay Dementia study. Baseline Dementia Rating Scale-2 (DRS-2) and memory Self-Efficacy (memSE) scores, and end-of-treatment MSS data was available for 114 persons with MCI (age 53-95). After two weeks of MSS (10 sessions), 26.3\% of our sample was still in the acquisition phase, 28.9 reached the application phase, and $44.7 \%$ mastered the most advanced, adaptation phase of MSS. An ordinal logistic regression analysis was employed to examine whether self-efficacy in memory, cognitive functioning, age, and education were associated with the MSS phase after 2 weeks.

Results: Our model was a better fit than the null model $(p<.001)$ and had a small-to-medium pseudo r-squared (Nagelkerke=.244). The proportional odds assumption held $(\mathrm{p}=.826)$. Higher DRS-2 scores $(p<.001)$ and lower memSE scores $(p=.001)$ were associated with increased odds of advanced (or greater) mastery of MSS; the conditional odds were 1.38 and 0.96, respectively. Age and education were not significant in this model. The classification accuracy of our model was more than $25 \%$ better than chance.

Conclusions: Higher cognitive functioning was associated with more progress in MSS, highlighting the importance to intervene before progression to dementia. Lower memory self-efficacy was associated with more progress in MSS, possibly reflecting more insight and more motivation for training. The current results may help determine for which individuals MSS may be the most helpful.

Correspondence: Liselotte De Wit, M.S., Clinical and Health Psychology, University of Florida, 805 NE 2nd street \#2, Gainesville Fl, FL 32601, United States.E-mail: ldewit@phhp.ufl.edu

C. DIGIOVANNI \& B. LIANG. Traumatic Brain Injury (TBI) Facebook Support Groups: How Online Supportive Exchanges Relate to Subjective Well-Being as a Function of TBI Sequelae.

Objective: TBI survivors face complex physical and emotional challenges following their injury. This study determined how four types of supportive exchanges (i.e., emotional support provided, emotional support received, informational support provided, and informational support received) between users of TBI Facebook support groups are associated with positive and negative feelings. Given the variability in post-injury sequelae, this study also examined whether cognitive ability problems (e.g., attention) and adjustment problems (e.g., irritability) tied to a TBI moderated the relationship between each type of supportive exchange and positive and negative feelings.

Participants and Methods: Participants were 191 individuals (mean age $=44.7 ; \mathrm{SD}=12.0 ; 74 \%$ female) across 14 TBI-related Facebook support groups. Participants completed an online survey assessing demographics, cognitive ability problems and adjustment problems due to their TBI (MPAI-4), perceived frequency of online supportive exchanges (i.e., emotional and informational support provided and received), level of extraversion, and positive and negative feelings (SPANE).

Results: Analyses used multiple regression controlling for extraversion. There was a significant interaction between emotional support provided and adjustment problems $(p<.05)$, such that those with less severe adjustment problems experienced more positive feelings when providing high levels of emotional support compared to those with more severe adjustment problems. There was also an interaction between informational support received and cognitive abilities $(p<.05)$, such that those with more severe cognitive ability problems experienced more negative feelings when receiving high levels of informational support compared to those with less severe cognitive ability problems.

Conclusions: Results suggest that participation in TBI Facebook support groups is associated with greater subjective well-being for those with less severe cognitive ability and adjustment problems related to their TBI.

Correspondence: Craig DiGiovanni, Lynch School of Education, Boston College, 916 Hoya Avenue, Harrisburg, PA 17112, United States. E-mail: digiovcr@bc.edu 
P. FARRIS, K. SOL, A. KRAAL \& L.B. ZAHODNE. Work-Family Conflict and Cognition Among Older Adults Working Full-Time.

Objective: Human and animal work demonstrates that stress negatively affects cognitive health, but it is unclear which stressors are most likely to impair older adults' cognition and whether effects differ by age or gender. Work-family conflict (WFC) is an organizational stress perceived when the divide between work and family is blurred. Given that more older adults are working longer, the specific aims of this cross-sectional study were to: (1) test whether WFC is negatively associated with older adults' cognition; and (2) examine age and gender differences in associations.

Participants and Methods: Data were obtained from 484 participants over age 55 in the first follow up of the National Survey of Midlife in the United States (MIDUSII). Only participants who reported currently working full- time (over 35 hours a week) were included. Multiple aspects of WFC were measured via self-report questionnaires, and cognition was measured over the phone with tests of episodic memory and executive functioning. Linear regressions examined associations between WFC and cognitive composite scores, controlling for age, gender, education, occupation, income, marital status, family size, caregiving, physical health, and depressive symptoms.

Results: Negative family-to-work spillover was associated with worse episodic memory, but not executive functioning. Older age was associated with less family-to-work spillover. Men and women reported similar levels of WFC. Neither age nor gender moderated associations between WFC and cognition.

Conclusions: Negative family-to-work spillover may negatively affect episodic memory among older adults. Longitudinal studies are needed to clarify the direction of effects. Among older adults, aging may protect against the experience of WFC. Future work should identify potential intervention targets to reduce WFC and improve cognitive health among older adults

Correspondence: Penelope Farris, University of Michigan, 530 Church ST., Ann Arbor, MI 48109, United States.E-mail: pcfarris@umich.edu

E.L. FISHER, T.Z. KING, C. GHILAIN, D. MURDAUGH \& K. O'TOOLE. Improvements in Adaptive Functioning among Children with Medical Disorders Following Cognitive Remediation Program: An item-level analysis.

Objective: Children with medical disorders often display deficits in adaptive functioning. The purpose of this study was to assess the efficacy of an individualized cognitive remediation program (CRP) in increasing patient independence by examining item level data from three scales of an adaptive functioning measure (ABAS-2).

Participants and Methods: The participants were 34 children (ages 9 to 17) recruited from an outpatient neuropsychology clinic. Inclusion criteria were documented adaptive and/or executive functioning deficits per a parent or teacher, Full Scale IQ score greater than 70, and English as the primary language. Medical diagnoses included congenital heart disease, epilepsy. TBI, preterm birth, and others.

The CRP consisted of 8 individual treatment sessions delivered over the course of 4 weeks. Key elements of the program included a home reward system and individualized goal setting. Teaching strategies included sequencing of steps needed to complete a task, external cues for promotion of task sequencing, and behavioral management using contingent rewards. Parents completed the ABAS-2 prior to and following intervention.

Results: Paired samples t-tests indicated statistically significant pre to post-intervention increases on all three scales (Home Living $\mathrm{t}=3.6$, $p=.001 ;$ Self-care $t=4.4 p<.001$; Self-direction $t=4.9 p<.001)$. Items in which the sample showed the highest proportion of increases included specific self-care and household tasks (e.g. "brushes or combs hair"), anger management (e.g. "controls temper"), and general skills tasks that require executive functioning (e.g. "plans ahead for projects"). Conclusions: These findings highlight specific improvements in adaptive functioning following the CRP. Patterns in the item level data indicated that, in addition to expected improvements on specific tasks that were often individualized goals, the program may provide general benefits to anger management and executive functioning.

Correspondence: Evelyn L. Fisher, M.A., Psychology, Georgia State University, 2062 Brian Way, Decatur, GA 30033, United States. E-mail: evelyn.lisa.fisher@gmail.com

K. JEAN, C. MEWBORN, M. GOGNIAT, T. ROBINSON, C.A. LINDBERGH \& L. MILLER. The Effect of a Dual Driving/ Distraction Task Game on Visuospatial Skills in Older Adults.

Objective: Visuospatial skills play an important role in functional independence and in particular, driving ability, especially for older adults. The purpose of this study was to test whether advancement in a highly challenging and visually demanding dual driving/distraction task would also lead to improved visuospatial skills in older adults.

Participants and Methods: There were 16 older adult participants in the study $(\mathrm{M}=72.8$ years; $\mathrm{SD}=5.73)$. Participants played a computer-based dual-task driving /distraction video game three times a week for four weeks. Improvement on the driving/distraction intervention was assessed by the number of levels the participant advanced. Improvement in visuospatial ability was assessed using pre- to post-intervention change scores on a judgment of line orientation (JLO) task where participants had to correctly identify angles that did or did not match. A bivariate correlation was used to determine the relationship between improvement on the driving/distraction task and improvement on the JLO task.

Results: The correlation between overall improvement on the driving task was significantly related to overall JLO improvement. $p=.04$. The relationship between overall task improvement and overall improvement on the JLO task was characterized by a large effect size, $r=.52$.

Conclusions: Our findings suggest that a highly challenging, visually rich and attention demanding driving intervention may improve visuospatial abilities in older adults. This could serve as an intervention that may be useful in prolonging an older adult's ability to remain capable of driving.

Correspondence: Kharine Jean, Psychology, University of Georgia, 975 Baxter St, Apt 2, Athens, GA 30606, United States. E-mail: kjean@ uga.edu

P.C. MANGAL, J.E. MAYE, E. TRIFILIO, L.C. BUTTERFIELD, K.R. THOMAS, D. BOWERS \& M. MARSISKE. Mindfulness and Cognitive Training Interventions in Older Adults Improve Performance in Cognitive Control.

Objective: Increasing evidence shows that cognitive training and mindfulness interventions may improve attentional processing, executive functions (inhibition and set-shifting), and working memory. The current study investigated whether Mindfulness Based Stress Reduction (MBSR) and computer-based cognitive training (Posit Science Brain HQ) improved in executive functions in independently living older adults.

Participants and Methods: Participants ( $\mathrm{N}=53$; Age $=76.8 \pm 7.8$ ) were cognitively normal $(\mathrm{MMSE}=28.9 \pm 2$ ) and inexperienced in meditation and cognitive training. Individuals were assigned to one of four groups: no contact control (NC; $n=9)$, mindfulness only (MBSR; $n=13$ ), cognitive training only (CT; $n=15$ ), and combined mindfulness and cognitive training (MBSR-CT; n=16). The NIH Examiner, a computerized battery of executive functions, was used to compute cognitive control and working memory composites. Multilevel model (MLM) analyses were used to assess group differences in cognitive control and working memory over three time points: baseline, post-intervention. and 3-month follow-up.

Results: Controlling for age, MLM analyses revealed significant main effects of age and a significant Group $\mathrm{x}$ Occasion interaction for both cognitive control and working memory. Post hoc tests showed that compared to baseline, the MBSR and combined MBSR-CT groups demonstrated improvement on the cognitive control composite post-intervention, with the MBSR-CT group showing the best performance most 
across groups. The MBSR group and the NC group showed improvement in working memory performance.

Conclusions: Mindfulness meditation, when completed alone or combined with cognitive training may improve cognitive control (e.g., inhibition, set shifting, number of errors). While mindfulness training may also be associated with improved working memory, these results may be explained by practice effects given the NC group also improved. Future research will investigate group differences in the sub-domains of each composite.

Correspondence: Paul C. Mangal, M.S., Clinical Health Psychology, University of Florida, P.O. Box 100165, Gainesville, FL 32610-0165, United States. E-mail: pmangal@phhp.ufl.edu

T. NOVAKOVIC-AGOPIAN, E. KORNBLITH, G. ABRAMS \& J. BURCIAGA. Training in Attention Regulation Applied to Participant Defined Goals for Veterans with PTSD and mild TBI.

Objective: Difficulties in executive control functions are common sequelae of both TBI and PTSD. Goal-Oriented Attentional Self-Regulation (GOALS) training was designed to target these deficits with attention regulation training applied to participant-defined goals. In previous studies both Veteran and civilian participants with chronic TBI significantly improved post GOALS, but not control training, on measures of attention/executive function, functional task performance, and self-report measures of emotional regulation (Novakovic-Agopian et al 2011, Novakovic-Agopian et al 2015), and on goal-directed control over neural processing on fMRI (Chen, et al 2011). The objective of this study is to assess effectiveness of GOALS training in Veterans with comorbid PTSD and mTBI.

Participants and Methods: 33 Veterans with diagnosis of PTSD, history of chronic mTBI and mild-moderate executive dysfunction were randomized to start with either 5 week of GOALS or matched control psychoeducation (EDU) training. Assessments at baseline and post training included neuropsychological, functional task performance, and self-report measures

Results: Post GOALS but not post EDU training, participants significantly improved on measures of: attention /executive function (overall performance, sustained attention, and inhibition); complex functional task performance (overall performance, attentional maintenance, switching, execution, and memory); and emotional regulation (significant symptom decrease on BDI-II, POMS and PCL-M)

Conclusions: GOALS training may be promising in Veterans with concurrent PTSD and chronic mTBI. Training self-regulatory cognitive and emotional control strategies applied to actual situations in participant lives and personally relevant goals may provide meaningful improvements in cognitive, emotional, and occupational functioning. The challenges and importance of: a) using participant-defined goals applied to relevant training, and b) assessing change in functioning at different levels, will be discussed

Correspondence: Tatjana Novakovic-Agopian, Ph.D, Neurology, UCSF, SFVA, 661 Myra way, San Francisco, CA 94127, United States. E-mail: tna@cns-site.com

N. OJEDA, M. DÍEZ-CIRARDA, G. MONTOYA-MURILLO, O. RILO, O. LUCAS-JIMÉNEZ, A. GÓMEZ-GASTIASORO, J. PEÑA \& N. IBARRETXE-BILBAO. Social Cognition and Functional Outcome after Cognitive Rehabilitation in Neuropsychiatric Pathology, Neurodegenerative Diseases and Healthy Elderly Population.

Objective: Cognitive impairment is common in neuropsychiatric pathologies, neurodegenerative diseases, and is part of the natural process in the elderly. Cognitive decline has been related to alterations in social cognition (SC) and functionality. The efficacy of cognitive rehabilitation on improving diverse cognitive functions has been demonstrated in these populations. However, little is known about SC and functional disability changes associated with cognitive rehabilitation. The aim of this project was to assess the role of SC and functional outcome after cognitive rehabilitation in Schizophrenia (SZ), Parkinson's disease (PD), Multiple Sclerosis (MS) and the elderly population.

Participants and Methods: One hundred and eleven patients with SZ. 44 PD patients, 42 MS patients and 105 elderly people were recruited for assessment. Participants underwent a neuropsychological battery including measures of SC and functional outcome at pre- and post-treatment. Participants were randomized into the experimental (3-month REHACOP program) or the control group. SC and functional changes in each population were assessed with repeated measures ANOVA 2x2 (groupXtime).

Results: Results showed that SC improved significantly in SZ (theory of mind, emotional and social perception and attributional style) and PD (theory of mind). In addition, functional disability was also improved in SZ, PD, and in the elderly. MS patients showed a trend to significant improvement in SC and functional disability after rehabilitation.

Conclusions: Findings suggest that cognitive rehabilitation can improve SC and functional disability in patients with various brain disorders. Transfer effects to real life functionality have also been found following cognitive intervention. These changes add evidence to the efficacy of cognitive rehabilitation programs on the therapeutic approach for neuropsychiatric pathologies, neurodegenerative diseases and elderly population.

Correspondence: Natalia Ojeda, Universidades 24, Bilbao 48007, Spain.E-mail:nojeda@deusto.es

M. SADEGHI, T. MCAULEY \& S. SANDBERG. Understanding Engagement and Experience with Computerized Cognitive Training in Youth with ADHD.

Objective: Working memory (WM), the ability to temporarily store and manipulate information, is a common deficit in children with attention deficit hyperactivity disorder (ADHD). Cogmed, a computerized training program, appears to yield WM improvements; however, its effectiveness for alleviation of ADHD symptoms and associated impairments is not widely supported. Our preliminary study examined whether individual differences in motivation of ADHD youth predicted engagement in a 10-week course of Cogmed.

Participants and Methods: 40 youth with ADHD (age $M=11.6$ years, $S D=2.2$ years; 8 female) completed 30 sessions of Cogmed, including embedded motivation questions administered pre-training. 10 of these youth (age $M=10.4 ; S D=1.7 ; 1$ female) were interviewed at 3 -month follow up. Performance changes were based on program-generated Max $(M=102.3 ; S D=14)$ and Start $(M=75.3, S D=9.1)$ index scores. NVivo 11 was used for thematic analyses of interviews.

Results: All youth completed and showed improvements on training tasks per the Improvement Index $(M=27 ; S D=8.1)$. Multiple regression was used to determine whether engagement with Cogmed, measured by an aggregate of the embedded motivation questions, predicted the Max Index after the Start Index was controlled. $76 \%$ of variance in the Max Index was explained by the model, with $4.5 \%$ of variance uniquely attributable to motivation $(F(1,18)=45.4, p<.001)$. Youth expressing more motivation demonstrated somewhat less improvement in training $(B=-.5)$. When interviews were examined qualitatively, however, $60 \%$ of youth reported improved focus and memory post-training and $60 \%$ also reported that they completed Cogmed based on intrinsic motivations (e.g. "I got motivated to change my habits").

Conclusions: Motivation was inversely related to training improvement yet many youth indicated intrinsic reasons for training and demonstrated both training and subjective gains. Further study of motivational styles in cognitive training is warranted to shed insight into variations in treatment outcomes.

Correspondence: Mahsa Sadeghi, Psychology, University of Waterloo, 8 Springbrook Drive, Richmond Hill, ON L4B3R9, Canada. E-mail: mahsasadeghi94@gmail.com 
K. SHOJI \& J. MARCEAUX. Application of Cognitive Stimulation Therapy for Dementia in a Veteran Population.

Objective: Cognitive Stimulation Therapy (CST) is a comprehensive, readily-implemented, cost-effective non-pharmacological intervention for dementia. The CST program developed by Spector and colleagues has proven effective in improving quality of life (QoL) and cognition in older adults with dementia. The present chart review study was innovative in that it was a preliminary examination of the utility of CST in a veteran population.

Participants and Methods: Clinical data for veterans referred for participation in a CST group from 2012-2017 was examined. Among 39 referrals, 24 met inclusion criteria (i.e., dementia diagnosis and availability of pre- and post-group assessment data). Participants completed 5.8 out of 7 CST sessions on average. Fourteen participants and 16 collaterals provided qualitative feedback regarding group participation. Paired-samples t-tests were used to determine if group participation was associated with changes in mood, QoL, cognitive, and functional scores. Results: Demographically, the all-male sample was largely Caucasian (71\%), married (71\%), and English language dominant (79\%), with 13 years of education. Twenty percent were on a cognition-enhancing medication. Regarding etiology, a majority were classified as either vascular $(21 \%)$ or Alzheimer's disease $(33 \%)$. Paired-samples t-tests revealed improvements in patient-reported QoL $(p<0.02)$, but not mood, cognitive, or functional scores. Feedback from veterans and caregivers was positive, noting improvements in communication, cooperation with care, and mood.

Conclusions: The CST program was found to be an easily transferrable method of cognitive stimulation for use in a veteran population. Consistent with previous studies, QoL significantly improved. Although there were no significant changes in mood, cognitive, or functional scores, qualitative feedback noted benefit in these areas. These preliminary findings are promising and warrant ongoing evaluation of CST among veterans.

Correspondence: Kristy Shoji, Ph.D., Psychology, South Texas Veterans Health Care System, 6309 Attucks Lane, San Antonio, TX 78238, United States.E-mail:kdouglas@crimson.ua.edu

A. TAUB, S.S. SIMON, F. LOSCHIAVO, E.C. MIOTTO \& B.A. WILSON. Special Interest Group - Brazil (SIG-BRA) on Neuropsychological Rehabilitation: Who works with NR in Brazil? Towards to professionals profile.

Objective: The Brazilian Special Interest Group (SIG-BRA) on Neuropsychological Rehabilitation (NR) is affiliated to the World Federation of NeuroRehabilitation (WFNR), whose objectives are promote evidence based practice on NR in Brazil, through various actions. The objective of the present work was to investigate the profile and main needs of professionals working with NR in Brazil.

Participants and Methods: Fifthy-four professionals from different regions of Brazil answered an online survey created with googledocs platform. The survey included questions on socio-demographics and regarding their work on NR. The survey was anonymous and required $\sim 10$ minutes for completion.

Results: Professionals working on NR field present a mean age of 39.1 years (25-74 years), and $85.2 \%$ are females. Most part of the sample $(85.2 \%)$ is original from southeast. The majority of professionals $(75.9 \%)$ has a background in Psychology and works in private office $(83.3 \%)$. In addition, the main needs reported by the professions are: 1$)$ more knowledge of techniques \& strategies; 2) more clinical meetings; and 3 ) better instruments to assess the efficacy of their interventions. In terms of community resources that can complement their work, the professionals elected three main aspects to be better developed: 1) public policy addressed to people with cognitive impairment; 2 ) institutions that offer rehabilitation programs; and 3) companies that promote inclusion programs to people with cognitive impairment.

Conclusions: In our perspective, theory and clinical practice are inseparable parts of NR and need to be consistent. This survey identified the profile and main needs of the professionals working with NR in Brazil, and its results can determine more efficient actions for the solidification of NR in our country.

Correspondence: Sharon S. Simon, Psychiatry, University of São Paulo, Rua Ovídio Pires de Campos, 785, São Paulo 05403010, Brazil. E-mail: sharon.sanzsimon@gmail.com

\section{Connectomics}

L. PASCOE, D. THOMPSON, M. SPENCER-SMITH, R. BEARE, C. ADAMSON, K. LEE, C. KELLY, N. GEORGIOU-KARISTIANIS, C. NOSARTI, E. JOSEV, G. ROBERTS, L. DOYLE, M. SEAL \& P.J. ANDERSON. Whole-Brain Structural Connectivity Relates to Intrinsic Motivation in Children born Extremely Preterm: Insights Using Graph Theory.

Objective: Intrinsic motivation is essential for academic success, as well as cognitive growth and organization. However, there is limited understanding of the neuroanatomical underpinnings of intrinsic motivation, particularly in childhood. This study applied graph theory to investigate whole-brain structural connectivity networks associated with intrinsic motivation in an at-risk group of children at age 7 years.

Participants and Methods: Fifty-one extremely preterm $(<28$ weeks' gestational age) or extremely low birthweight children $(<1000 \mathrm{~g})$ underwent $\mathrm{T} 1$ and diffusion weighted imaging at age 7 years $(\mathrm{M}=7.8$ years, $\mathrm{SD}=.43)$. Connectivity graphs comprising of 162 cortical and subcortical nodes were created using constrained spherical deconvolution-based tractography and Freesurfer parcellation based on the Destrieux atlas scheme. Networks were thresholded to a uniform $30 \%$ edge density to allow comparison between individuals. Connectivity measures were analysed in association with concurrent self-reported aspects of intrinsic motivation for school learning (mastery, challenge and curiosity) using linear regression.

Results: Reduced average path length and increased global efficiency (measuring network integration) was associated with a greater desire to master school learning $(p<.001$ and $p=.008$ respectively). There was also evidence that reduced average path length and increased small-worldness were associated with a greater desire for school challenge $(p=.02$ and $p=.03$ respectively). There was little evidence that connectivity measures were associated with curiosity.

Conclusions: Greater network integration may be important for reinforcing a desire for mastery, while a small-world network topology (high clustering with short paths) may facilitate a desire for challenge in extremely preterm children at early school-age.

Correspondence: Leona Pascoe, PhD, School of Psychological Sciences, Monash University, 5/248 Gordon Street, Footscray, VIC 3011, Australia.E-mail: lsin4@student.monash.edu

\section{Cognitive Neuroscience}

J. GRAVANO, J.E. LETZEN, K. MOFFETT, Z.M. HOUCK, E. POLEJAEVA \& W.M. PERLSTEIN. Associations Between Resting-State Connectivity and Cognition in Mild Traumatic Brain Injury and Posttraumatic Stress Disorder.

Objective: Individuals with PTSD and mTBI often share similar cognitive concerns of memory and attention. This study investigated how alterations in large-scale neural networks differentially relate to cognitive and affective symptomatology in a heterogeneous group of combat Veterans $(n=20)$. Parsing out the unique and overlapping neural bases of these often comorbid conditions can potentially inform appropriate treatments.

Participants and Methods: Veterans with mTBI $(n=6)$, PTSD $(n=2)$, combined mTBI and PTSD ( $n=8)$, and combat controls $(n=4)$ underwent 8 -minute resting-state functional magnetic resonance imaging (fMRI; 3T) and neuropsychological assessment emphasizing tests of 
cognitive speed, working memory, and executive skills. Resting state analyses focused on nodes of the Default Mode Network (DMN), task positive networks (TPN) and limbic/paralimbic sites identified in previous research as important in the acquisition, pathology, or maintenance of these disorders. Functional connectivity was assessed using the CONN toolbox. Relationships between connectivity, cognition, and affective symptoms were assessed using Pearson correlations.

Results: The study found that PTSD-symptom levels (PTSD Checklist - Military) were uniquely associated with DMN-Task positive and DMN-Limbic connectivity (medial prefrontal cortex-anterior cingulate cortex and posterior cingulate cortex-right amygdala, connectivity, respectively). In contrast, history of mTBI did not uniquely predict connectivity patterns. Among all participants, performance on cognitive measures was uniquely predicted by aberrant within-DMN and DMN-Task positive connectivity.

Conclusions: These pilot results suggest that connectivity among large scale neural networks at rest is associated with aspects of cognitive functioning in this heterogeneous population, warranting further investigation of common and unique mechanisms of cognitive symptoms, as well as pathways of treatment.

Correspondence: Jason Gravano, Ph.D., Neurology, University of California at Davis, 1043 39th Street, Unit 5, Sacramento, CA 95816, United States.E-mail: jtgravano@gmail.com

B.E. HAWKSHEAD, E.S. HALLOWELL, S.W. LIEBEL \& L.H. SWEET. Hormonal Effects on Cognition in Oral Contraceptive Users and Naturally-Cycling Women.

Objective: The combined oral contraceptive pill (COCP) is the most common method of hormonal birth control for U.S. women. A growing body of literature investigating hormone-related effects on cognition exists and general patterns have begun to emerge. Broadly, it appears that verbal memory (VM) and visuospatial functioning (VSF) tend to improve in women using COCPs in comparison to women during their natural menstrual cycle $(\mathrm{MC})$. This is believed to be related to overall reductions in sex hormones seen with COCPs. Hormonal variations during the natural MC have also been shown to impact cognition, with periods of high hormones (i.e., luteal phase) linked to improved VM and periods of low hormones (i.e., follicular phase) linked to improved VSF. We predicted that COCP-users would outperform naturally-cycling women on both VM and VSF tasks regardless of MC phase, but that luteal-phase women would outscore follicular-phase women for VM and that follicular-phase women would outscore luteal-phase women for VSF.

Participants and Methods: Using data from the Human Connectome Project, we examined group differences in cognition via two designs. The first analysis examined task performance differences in 58 COCP-discordant sibling pairs. The second analysis examined differences across three matched groups of 45 in the follicular phase, the luteal phase, and in COCPs.

Results: Two MANOVAs and relevant planned comparisons were conducted to examine the impact of hormone status on VM and VSF. No significant differences were observed among discordant siblings. For the matched groups design, follicular-phase women significantly outperformed luteal-phase women on the VM task, $t(88)=3.018$, $p=.019$. There were no differences in VSF.

Conclusions: Contrary to our predictions, VM scores were higher for women during the (low-hormone) follicular phase than during the (high-hormone) luteal phase. VSF did not differ as function of COCP use or MC phase. COCPs did not seem to have a significant effect on cognition in either of the present studies.

Correspondence: Brittany E. Hawkshead, Psychology, University of Georgia, Psychology Department, University of Georgia, Athens, GA 30602, United States.E-mail: bhawks@uga.edu
W.D. KILLGORE. Default Mode Activation and the Ability to Resist Sleep Deprivation.

Objective: People show consistent trait-like individual differences in vulnerability to the cognitive impairing effects of sleep deprivation (SD), but the neural mechanisms underlying this trait remain to be delineated. Moreover, this resistance capacity also differs across domains (e.g., objective vigilance versus subjective sleepiness) for each individual. The Default Mode Network (DMN) has been shown to be important in attentional lapses, so we hypothesized that individuals showing greater activation of DMN during baseline (i.e., non-SD) neuroimaging might show trait vulnerability to SD even when tested on a later date.

Participants and Methods: Forty-five healthy adults (23 male; Ages 20-43) completed $3 \mathrm{~T}$ functional magnetic resonance imaging (fMRI) during a cognitive conflict task. Between 1 to 4 days later, participants were sleep deprived for one night. Hourly assessments of psychomotor vigilance (PVT), visual analog mood scale (VAMS), and Karolinska Sleepiness Scale (KSS) were collected and converted into standardized metrics and then combined as a global index of vulnerability. This global vulnerability index was then correlated with fMRI within the medial DMN using SPM12, controlling for sex.

Results: Global vulnerability was predicted by two regions of activation, including anterior (ACC; $\mathrm{r}=-.59$ ) and posterior (PCC; r=-.63) cingulate cortex. Stepwise multiple regression showed that these two regions each contributed unique variance to the prediction of global vulnerability $\left(\mathrm{R}^{2}=.51\right)$, as well as the individual domains of sleepiness $\left(\mathrm{R}^{2}=.47\right)$ and $\operatorname{mood}\left(R^{2}=.39\right)$, while only PCC predicted vigilance $\left(R^{2}=.10\right)$.

Conclusions: Failure to suppress DMN activation during a difficult cognitive task when well rested predicted greater subsequent global vulnerability to sleep deprivation. This was most predictive of degradation of subjective sleepiness and worsening mood, but only modestly predictive of objective PVT performance. The medial DMN may be an important functional biomarker of subjective vulnerability to SD.

Correspondence: William D. Killgore, PhD, Psychiatry, University of Arizona, 1501 N. Campbell Ave, 7303B, Tucson, AZ 85724, United States.E-mail: killgore@psychiatry.arizona.edu

W.D. KILLGORE, V. CAPALDI, T. BALKIN \& G. KAMIMORI. Personality Traits Predict the Ability to Sustain Executive Function Abilities During Sleep Deprivation.

Objective: It is well established that there are trait-like inter-individual differences in the ability to sustain performance during periods of sleep deprivation. These have focused primarily on simple vigilance tasks or subjective sleepiness ratings. Interestingly, it has proven difficult to identify reliable cognitive, biological, or demographic variables that predict this trait. We have previously shown that the personality trait of introversion-extraversion is related to resistance capacity when measured by the psychomotor vigilance test. Here we explore whether this trait also predicts the ability to sustain higher-level executive functions on the Tower of London test. 
Participants and Methods: 17 males and 5 females $\left(\mathrm{M}_{\text {age }}=25.05\right.$, $\mathrm{SD}=3.98)$ completed baseline measures of personality traits and then underwent a double-blind drug study (placebo $\mathrm{N}=11$; caffeine $200 \mathrm{mg}$ every two hours between 0100 and $0700, \mathrm{~N}=11$ ) during 3 nights of sleep deprivation. Participants completed the Tower of London (TOL), a measure of planning and sequencing, after 4, 52, and 76 hours of total sleep deprivation. Stepwise linear regression was used to predict performance on the TOL from personality traits.

Results: At 52 hours of sleep deprivation, lower Extraversion (higher introversion; $\beta=-.41, p=.048)$ predicted better sustained TOL throughput above and beyond the effects of caffeine $(\beta=.32, p=.12)$. At 76 hours of wakefulness, lower Extraversion (higher introversion; $\beta=-.50, p=.01)$ again predicted higher TOL throughput, but the model was also significant when higher Conscientiousness $(\beta=.40, p=.04)$ was added, and this was above and beyond the non-significant effect of caffeine $(\beta=.16, p=.38)$.

Conclusions: The trait of introversion-extraversion was predictive of the ability to sustain the executive function capacities of after sleep deprivation. This effect was above and beyond the effects of caffeine. This is consistent with the notion that greater baseline prefrontal functioning is associated with greater resilience to sleep loss.

Correspondence: William D. Killgore, PhD, Psychiatry, University of Arizona, 1501 N. Campbell Ave, 7303B, Tucson, AZ 85724, United States.E-mail:killgore@psychiatry.arizona.edu

\section{KING, K. MANZEL, J. BRUSS \& D. TRANEL. Neural Correlates of Improvements in Personality and Behavior Following a Neurological Event: A Neuropsychological Approach.}

Objective: While research on changes in personality and behavior following brain damage has focused largely on negative outcomes (e.g., increased irritability, moodiness, and social inappropriateness), clinical observations suggest that some patients may actually show positive changes following a neurological event. In the current work, we investigated neuroanatomical correlates of positive personality and behavioral changes following a discrete neurological event (e.g., stroke, benign tumor resection).

Participants and Methods: Patients (N=97) were rated by a close family member or friend on five domains of personality and behavior: social behavior, irascibility, hypo-emotionality, distress, and executive functioning. We identified patients who showed positive changes in one or more domains of functioning and used proportional subtraction analytical methods to investigate areas of brain damage associated with improvement.

Results: 22 participants improved in personality and behavior. Lesion analyses indicated that positive changes in personality and behavior were most consistently related to damage to the bilateral frontal polar regions and the right anterior dorsolateral prefrontal region. In three illustrative cases of patient improvement, family members reported changes in a strikingly positive direction in all 5 domains of personality and behavior. All three patients had lesions in anterior frontal regions. Conclusions: These findings support the conclusion that improvements in personality and behavior can occur after a neurological event, and that such changes have systematic neuroanatomical correlates. Illustrative patient examples provide evidence that, in some cases, improvements can be notable. These findings support the intriguing proposal that, particularly in cases in which premorbid functioning is more extreme (e.g., more extreme expressions of emotions), altering neural circuits can lead to improved psychological outcomes.

Correspondence: Marcie King, MA, Psychological and Brain Sciences, University of Iowa, 1258 Shannon Drive, Iowa City, IA 52246, United States.E-mail:marcieking123@gmail.com
M.E. MCLAREN, A. O'SHEA, E. PORGES, R.A. COHEN \& A.J. WOODS. Frontal Structural Neural Correlates of Processing Speed Performance in Older Adults.

Objective: Aging is associated with declines in processing speed and changes in frontal brain structures. While processing speed performance differences are associated with changes in frontal brain regions, questions remain regarding the relationship between specific subregions of the frontal cortex and processing speed performance in older adults. This study investigated the impact of frontal subregion surface area and cortical thickness in older adults' performance on NIH toolbox processing speed measures.

Participants and Methods: Seventy-three healthy older adults completed NIH Toolbox measures of processing speed and underwent $3 \mathrm{~T}$ magnetic resonance imaging. Area and thickness data for frontal regions were extracted using FreeSurfer Imaging Software. Multiple linear regressions were run to examine if surface area or thickness predicted performance on either the Pattern Comparison Processing Speed $(n=67)$ or Oral Symbol Digit $(n=67)$ tests, while controlling for sex, education, and estimated total intracranial volume. A FDR $p=0.05$ was used to correct for multiple comparisons.

Results: Bilateral rostral middle frontal (left $p=0.018$, right $p=0.000$ ), right rostral anterior cingulate $(p=0.027)$, right superior frontal $(p=0.036)$, and left frontal pole $(p=0.000)$ surface areas were positively associated with performance on the Oral Symbol Digit Test. No finding survived FDR correction for the Pattern Comparison Processing Speed Test, and there were no significant findings for cortical thickness. Conclusions: Our results suggest that gray matter structural integrity of specific subregions in the frontal lobes contribute to processing speed performance in older adults. Consistent with prior work in healthy aging, surface area (structural integrity) was more strongly related to performance than cortical thickness (neuron density). Results also suggest Oral Symbol Digit Test performance may be particularly sensitive to differences in frontal brain structures.

Correspondence: Molly E. McLaren, PhD, Clinical and Health Psychology, University of Florida, Department of Clinical and Health Psychology, P.O. Box 100165, Gainesville, FL 32610-0165, United States.E-mail:memclaren@phhp.ufl.edu

N. MUSTAFA, T.L. MCAULEY, A. STATHIS \& L. BUCHANAN. Cognitive Profile Prior to Deep Brain Stimulation Surgery: A Case Study on Adult-Onset Leukodystrophy with Essential Tremor.

Objective: We provide a cognitive profile of an extremely rare case of Adult-Onset Leukodystrophy (ADLD) with essential tremor (ET). Essential tremors and concomitant neurodegenerative diseases are associated with emotional dysfunction (Li et al., 2011) as well as cognitive impairment (Higginson et al., 2008). These cognitive changes present with a similar profile, although there is some heterogeneity depending on the associated neurodegenerative disease (Kim et al., 2009). Due to the rarity of ADLD, there is a paucity of research examining the cognitive impairments associated with it.

Participants and Methods: We administered a neuropsychology battery (WAIS-IV, MoCA, WRAT-4, FTT, BRIEF, BDI-III, BAI, VABS, CPT-3, WMS-IV, WCST, GPB, CVLT-II, CIMbdae, DKEFs, and ACT) to 46-year-old VP. She was recently diagnosed with ADLD with ET and is awaiting Deep Brain Stimulation (DBS) surgery.

Results: As expected, motor and processing speed tasks dependent on motor skills fell within the impaired range. Similarly, her performance was impaired on self-report and executive functioning measures. In addition, she demonstrated inattentiveness on a vigilance task and experienced difficulty across tasks measuring various domains of memory. Despite these cognitive weaknesses, her verbal abilities were superior and she scored within the average range for intellectual and academic measures.

Conclusions: VP shows impairments relating to motor, attention, memory, and executive function abilities, with intact verbal, intellectual, 
and academic skills. Her cognitive profile is consistent with that observed in others with ET, with some differences that are likely due to her unique concomitant neurological disorder. We will compare post DBS surgery scores to examine its' impact on VP's neuropsychological functioning. Correspondence: Nawal Mustafa, Clinical Neuropsychology, Psychology , University of Windsor, 146 Askin Avenue, Windsor, ON N9B 2W9, Canada.E-mail: mustafan@uwindsor.ca

\section{N.A. LUEKE \& D. RUBINOW. Working Memory of Highly Self- Critical Men is Impaired by Failure.}

Objective: Heightened self-criticism (SC) has been conceptualized as a maladaptive self-orientation that entails relentless self-scrutiny, negative self-evaluation, and excessive concern with personal failure. Self-criticism has been linked to various psychopathologies, health vulnerabilities, as well as diminished goal progress. Due to high self-critics' sensitivity to failure, the effect of failure and success on working memory (WM) performance of females, as influenced by varying levels trait self-criticism, has previously been examined (Lueke \&. Skeel, 2017). Since Lueke \& Skeel (2017)'s findings cannot be readily generalized to males, the present study replicated the female-only study, by assessing the effect of feedback on the WM of males, across levels of self-criticism. Participants and Methods: 80 participants with varying levels of trait SC (low, moderate, and high) were randomly assigned to either a success or a failure feedback condition. Participants completed a baseline WM task, followed by a success/failure manipulation on a separate task, and then completed a second WM task.

Results: Results show that failure differentially impacts the WM of highly self-critical men and women. While high female self-critics were reported to display an increase in WM post-failure and a decrease in WM post-success, highly self-critical men maintain consistency in WM test-retest performance following success, however experience a significant drop in WM performance post-failure.

Conclusions: The present study not only introduces trait SC as an individual difference factor that affects men's WM performance following a negative feedback, it also shows that maladaptive SC has a different impact on WM performance, depending on one's sex. Findings have important implications for factors that impact the negative association between goal-directed behavior and SC in men.

Correspondence: David Rubinow, NY, United States. E-mail: dar259@ cornell.edu

H.A. SMITH, D.H. WEISSMAN, P.E. CLAYSON, B. KIRWAN \& M. LARSON. An assessment of cognitive control differences among individuals with psychopathology: Congruency sequence effects in depression, generalized anxiety, and OCD.

Objective: Psychopathology is often associated with altered cognitive control, measured in part through congruency sequence effect (CSE). The CSE is a smaller congruency effect with an incongruent rather than congruent previous trial. Prior research suggests the CSE is linked to altered brain activity in patients with depression and anxiety relative to controls, but with intact behavioral measures. Comparisons of CSE magnitude in patients with varying psychopathology are scarce, as well as research thus far confounded with feature integration and/or contingency learning in the CSE. We investigated whether the confound-reduced CSE varies among psychopathology groups (OCD, depression, and anxiety) and healthy controls.

Participants and Methods: Individuals with diagnosed major depression $(n=52)$, generalized anxiety $(n=45), \operatorname{OCD}(n=36)$, and healthy controls $(n=38)$ performed a computerized prime-probe task that measures the confound-reduced CSE. We conducted Group x Previous-trial congruency x Current-trial congruency repeated measures ANOVAs on mean response time (RT) and mean error rate (ER) to determine whether and how CSE magnitude varied between groups. Results: The congruency effect was present with slower RTs $(p<.001)$ and less accuracy $(p<.001)$ in incongruent relative to congruent trials. We also observed a previous-trial congruency x current-trial congruency interaction (i.e., a CSE) in both mean RT $(p<.001)$ and mean ER $(p<.001)$. The presence of psychopathology predicted the size of the congruency effect in ER $(p=.02)$ but not in RT $(p=.45)$, with more errors in anxiety than depression $(p=.02)$. Additionally, a trend toward larger CSE in OCD relative to the other three groups $(p=.08)$ was observed. No other group differences were observed

Conclusions: The present findings indicate that the "confound-minimized" CSE is present but does not significantly vary across any of these groups. However, it is possible that CSE brain activity (and, hence, to cognitive control) varies among these groups.

Correspondence: Hilary A. Smith, MS, Clinical Psychology, Brigham Young University, 12973 S. Harvest Field Cir., Riverton, UT 84065, United States.E-mail: hilary:anne.smith@gmail.com

M. TAGHDIMI, E. KALON, T. SCHULTE \& E. MULLER-OEHRING. Schizotypy, Attachment Styles, and Attentional Biases Toward Substance Related Stimuli in Young Adults.

Objective: Elevated cannabis and alcohol use has been reported in schizotypal individuals. Further, individuals with substance use appeared to have attentional biases toward substance related cues. This study investigated the relations between cannabis and alcohol use, schizotypy, attachment styles, and attentional biases to substances related words in healthy individuals.

Participants and Methods: Eighteen young adults (11 female) were tested on performance in a computerized Stroop match-to-sample task and completed attachment style, anxiety, and the perceptual aberration scales. All participants had used alcohol in the past and 6 had engaged in recreational marijuana use. Stroop color-word interference was measured with incongruent (Word RED printed in blue font) in contrast to congruent color-word content (RED printed in red). Interference from irrelevant reward-related information was measured using alcohol- (WINE printed in red) and marijuana-related content (WEED printed in green) relative to congruent color words (non-interference condition).

Results: Repeated-measures ANOVA showed longer RTs to incongruent than congruent color words, i.e., response inhibition, but faster RTs to alcohol and marijuana words, i.e., response facilitation. Regression analyses revealed that an avoidant attachment style, anxiety, and schizotypy scores explained over $50 \%$ of the variance of alcohol- and marijuana-Stroop facilitation.

Conclusions: These preliminary findings suggest that individual differences in psychosocial attachment, anxiety, and schizotypal personality traits are associated with attentional biases toward substance related stimuli in young adults, which may be a risk factor for increased substance use.

Correspondence: MARYAM TAGHDIMI, MS, MA, Psychology, Palo Alto University, 600 Rainbow Dr. Apt.218, Mountain View, CA 94041 , United States. E-mail: mtaghdimi@paloaltou.edu

M. TAGHDIMI, R. GOMEZ \& A. ETKIN. Relationship between PTSD Symptom Severity and Cognitive Functioning.

Objective: Previous research on the neuropsychology of PTSD has identified changes to cognitive processes. However, the association between cognitive function and specific PTSD symptom clusters is not well researched. This study explored the relationships between cognition (attention, learning and memory) with severity of different PTSD symptoms (re-experiencing, avoidance, numbing, and hypervigilance) in persons with PTSD. We hypothesized that cognitive function will be associated with some but not all PTSD symptoms.

Participants and Methods: As part of a larger study, 116 adults ages 19 to $61(\mathrm{M}=36)$ diagnosed with PTSD were included in this study. Diagnosis and PTSD symptom severity were assessed using the Structural Clinical Interview for Diagnosis and the Clinician Administered PTSD Scale. The California Verbal Learning Test II was used to measure verbal learning and recall. The Digit Span Subtest from WMS-III and the 
Continuous Performance Task were used to measure working memory and sustained attention.

Results: Results indicated that emotional numbing and avoidance (but not re-experiencing nor hypervigilance) were associated with cognitive function. Specifically, regressions revealed that numbing was associated with deficits on short delay recall $(\beta=.02, p<.02)$. Numbing was also positively correlated with errors of intrusion $(r=.29, p=.01)$. Further, greater avoidance significantly predicted poorer sustain attention [response accuracy $(\beta=.02, p<.01)$, commission errors $(\beta=.02, p<.04)$, and total errors $(\beta=.02, p=.02)$. Avoidance was also associated with deficits on working memory (i.e., capacity to encode information) $(r=.26$, $\mathrm{p}=.03)$.

Conclusions: Greater numbing was related to poorer short-term memory, and greater avoidance was related to poorer sustained attention, and working memory. These findings support the researchers' hypothesis, and suggest that some (numbing and avoidance) but not all (re-experience and hypervigilance) PTSD symptoms clusters were associated with cognitive function.

Correspondence: MARYAM TAGHDIMI, MS, MA, Psychology, Palo Alto University, 600 Rainbow Dr. Apt.218, Mountain View, CA 94041 , United States.E-mail: mtaghdimi@paloaltou.edu

C. URGESI, C. CORTI, M. MASSIMINO, R. BORGATTI \& G. POGGI. Dissociated Alterations of Self-Body Schema and Others' Body Holistic Perception in Children with Supratentorial and Infratentorial Tumor.

Objective: Alterations of cognitive and social functioning are common long-term consequences of pediatric brain tumors. However, it has not been yet clarified the impact of pediatric brain tumors on the development of self-body representation.

Participants and Methods: We compared the abilities of 15 patients with a supratentorial tumor (ST; 6 females, 8-16 years), 15 patients with an infratentorial tumor (IT; 6 females, $8-13$ years), and 30 gender- and age-matched children with typical development (TD) in two different tasks of body representation. In particular, we tested: 1) egocentric and object-based mental spatial transformations by requiring left-right judgments, respectively, on a visually presented human figure or on an external object; 2) configural and holistic processing of the body by using, respectively, the inversion effect and composite illusion paradigms. The body inversion effect refers to the striking disruption of recognition abilities when social stimuli (i.e., faces and bodies), but not other objects, are presented upside-down; the composite illusion refers to the difficulty in perceiving the match between two identical top halves of a body (or of a face) when they are combined with different bottom halves.

Results: Independently from tumor site, patients were impaired, as compared to TD children, in both egocentric and object-based mental spatial transformations as well as in the holistic perception of others bodies; configural body processing was instead spared. Importantly, however, ST children had specific impairments in egocentric mental spatial transformations. Conversely, IT children showed specific alterations in the holistic processing of others' bodies.

Conclusions: The dissociation between different aspects of body representation in ST and IT patients may reflect damage to temporo-parietal and basal temporal areas, respectively, and may help the neuropsychological evaluation and treatment of pediatric brain tumor survivors. Correspondence: Cosimo Urgesi, Scientific Institute (IRCCS) Eugenio Medea, Via Margreth,3, Udine 33100, Italy.E-mail: cosimo.urgesi@ uniud.it

E.M. WATSON, A.J. STEPHENSON, K.A. LEHOCKEY \& D.E. EVERHART. Poor Sleepers Have Delayed Behavioral Responses to Positive Sleep Imagery.

Objective: Sleeplessness poses a public health issue with approximately 50-70 million US adults experiencing some form of a sleep disorder. Impaired daily functioning, increased risk for chronic health conditions, and greater morbidity are but few of the consequences of disordered sleep. A growing literature has explored the role of attentional biases and mental imagery in specific psychological disorders, including sleep disorders. The current study employed an emotional variant of a Go/ No-Go paradigm task to provide further clarity in understanding cognitive biases related to disordered sleep.

Participants and Methods: Seventy-five undergraduate students were assessed on measures of emotional distress (Patient Health Questionnaire - 4) and subjective sleep quality (Pittsburgh Sleep Quality Index). Additionally, response latencies were recorded for an emotional variant of a Go/No-Go paradigm task using sleep imagery.

Results: Mann-Whitney U tests were employed to determine if there were differences in response times for positively or negatively-valenced sleep imagery between good and poor sleepers. Results showed response times for negatively-valenced imagery were similar and not statistically different between $\operatorname{good}(M d n=1225.89, S D=831.46)$ and poor sleepers $(M d n=1320.05, S D=574.58)$. In contrast, distributions of the response times for positively-valenced imagery were statistically significantly different, such that good sleepers $(M d n=867.34, S D=230.92)$ responded significantly faster than their poor sleeping counterparts $(M d n=1076.53, S D=344.70), U=256, z=-2.73, p=.006$. Further, a logistic regression was performed to determine the unique contribution of age, gender, emotional distress, and behavioral performance on the likelihood of disordered sleep among participants.

Conclusions: These findings suggest poor sleepers might have an attentional bias requiring the need for additional time to process positively-valenced visual material. Implications of these findings are discussed. Correspondence: Eric M. Watson, PhD, Department of Rehabilitation Medicine, Icahn School of Medicine at Mount Sinai, 112 Brownlea Drive, APT 14, Greenville, NC 27858, United States.E-mail:Watsone11@ students.ecu.edu

A. TSAPANOU, S. COSENTINO, Y. STERN \& N. SCARMEAS. Subjective cognitive complaints and sleep problems in the elderly. Objective: Sleep problems play a crucial role in general quality of life, and cognitive performance, especially in the elderly. Subjective cognitive complaints (SCC) is the self-perception of cognitive changes in comparison to a previously normal status. Although the link between sleep and cognitive performance has been explored, the association between sleep and SCC needs further investigation.

Participants and Methods: Cross-sectional design in the Hellenic Longitudinal Investigation of Aging and Diet (HELIAD), a population representative study of Greek elderly. Data from 1484 participants, all without MCI or dementia. Sleep quality/duration was estimated with the Sleep Scale from the Medical Outcomes Study. SCG were assessed using a structured complaint questionnaire designed to reveal subjective memory and other cognitive domain symptoms. An extensive neurological and neuropsychological assessment examining objective cognitive performance was administered to each participant. Linear regression analyses were used to examine whether sleep quality (higher score, poorer quality) and sleep duration were associated with SCG. Age, sex, education, sleep medication, anxiety and depression were used as covariates.

Results: Poor sleep quality ( $\beta=0.128, p \leq 0.0001)$, and shorter sleep duration $(\beta=-0.067, p=0.009)$ were associated with more total SCC. Poor sleep quality was also linked to more specific SCG in: memory, handling money, naming, orientation, and non-memory domains. Shorter sleep duration was related to more SCC in: memory, non-memory and money-handling function domains. Specific sleep disturbances associated with total SCC included snoring, awaken short of breath/with a headache, sleep inadequacy, and daytime sleepiness.

Conclusions: Poor sleep quality and shorter sleep duration were associated with more SCC in a large group of older adults. Future research should focus on training or treatment programs improving the sleep quality and lessen SCG, in order to help older adults in risk of developing cognitive decline. 
Correspondence: Angeliki Tsapanou, Taub Institute, Columbia University Medical Center, 630 West 168th Street, New York, NY 10032, United States.E-mail:at2859@cumc.columbia.edu

\section{Neuroimaging}

\section{A. TSAPANOU \& Y. STERN. Sleep medication and brain morphology in the elderly.}

Objective: To examine the association between frequency of sleep medication use and brain morohology in cognitively healthy older adults. Sleep medication use has been associated with quality of life. Sleep medication also acts directly on brain regions. Examining the association between sleep medication and any particularities of brain morphology is of a paramount importance

Participants and Methods: Cross-sectional design in 97 participants, 65 y.o. or older, with sleep and brain volumetric data. All free of dementia or any major psychiatric or neurological condition. Participants were drawn from two -cognitive normal individuals- studies. Sleep medication frequency was assessed with the following item from the Pittsburg Sleep Quality Index: 'During the past month, how often have you taken medicine (prescribed or 'over the counter') to help you sleep. Answers had a range of $0-3$ with higher score indicating greater frequency. T-weighted MRI images were acquired, and regional brain volumes were computed with FreeSurfer- human brain imaging software. Volumes of: total cortex, subcortical gray matter, total gray matter, cortical white matter, brain segmentation, and supratentorium were computed for each participant. All the brain measures were adjusted for intracranial volume. Age, sex, education, and body mass index were used as covariates. Linear regression models were computed, with sleep medication frequency as the predictor and each of the brain volumes as the dependent variable

Results: More frequent use of sleep medication was significantly associated with low: subcortical gray matter $(\beta=-0.361, p=0.028)$, total gray matter $(\beta=-0.347, p=0.040)$, as well as supratentorial $(\beta=-0.380$, $p=0.031)$ volumes. The associations remained significant even after adjusting for depression

Conclusions: This is the first study to examine the association between frequency of sleep medication use and brain morphology in a healthy group of older adults. Metabolic effects or a possible neurotoxicity could play a role to the observed association

Correspondence: Angeliki Tsapanou, Taub Institute, Columbia University Medical Center, 630 West 168th Street, New York, NY 10032, United States.E-mail:at2859@cumc.columbia.edu

H.A. ALEKSONIS, R. WIER, M.M. PEARSON, C.J. CANNISTRACI, A.W. ANDERSON, J.F. KUTTESCH, B.E. COMPAS \& K.R. HOSKINSON. Using Diffusion Tensor Imaging to Assess Associations of White Matter Integrity and Memory in Pediatric Brain Tumor Survivors.

Objective: Pediatric brain tumor survivors (PBTS) are at increased risk for memory deficits and decreases in white matter integrity after diagnosis and treatment. Two prominent white matter pathways include the inferior fronto-occipital fasiculus (IFOF), which connects frontal and occipital regions of the brain, and the uncinate fasciculus (UF), which connects frontal and temporal regions. The contribution of these pathways to visual and verbal memory deficits in PBTS remains unclear. Thus, we examined relationships among white matter integrity in the IFOF and UF and memory in PBTS.
Participants and Methods: Eleven PBTS (ages 8-16; >2 years post-treatment) and 14 healthy controls (HC) underwent MRI; diffusion tensor imaging tractography (DSI Studio) was used to assess white matter integrity in the IFOF and UF. Participants also completed the Children's Memory Scale Dot Locations and Stories subtests.

Results: Relative to HC, PBTS had significantly lower right IFOF $(d=1.05)$ and left UF mean fractional anisotropy ( $\mathrm{mFA} ; d=1.16)$. PBTS performed more poorly on Dot Locations Learning $(d=0.89)$ and Total Score $(d=1.10)$, and Stories Delayed Recognition $(d=0.86)$. Performance on Dot Locations was unrelated to white matter integrity; on the Stories subtest, higher mFA in the right IFOF $(r=.47)$ and left UF $(r=.41)$ were associated with better immediate recall. Higher $\mathrm{mFA}$ in the right and left IFOF $(r=.55-.57)$ and the right and left UF $(r=.45-.49)$ were associated with better delayed recall. Higher $\mathrm{mFA}$ in the right and left UF ( $r=.46-.47)$ were associated with better delayed recognition.

Conclusions: PBTS show reduced white matter integrity in the IFOF and UF. Based on correlations, the IFOF and UF may be especially important for verbal memory. The lack of associations in the IFOF and UF with Dot Locations could suggest other brain regions are involved in visual memory, particularly those with parietal projections. Clinical significance of memory deficits should be assessed in future research to determine effect on day to day memory.

Correspondence: Holly A. Aleksonis, B.S., Center for Biobehavioral Health, Research Institute at Nationwide Children's Hospital, 700 Children's Dr, FB Suite 3B.2, Columbus, OH 43205, United States. E-mail: holly.aleksonis@nationwidechildrens.org.

A.J. ALFINI, L.R. WEISS, J. WON, C. MICHELSON, C. SIMON, D. CALLOW \& J. SMITH. Effects of Acute Exercise on ConflictDependent Functional Activation in the Aging Brain.

Objective: Objective: Several neuroimaging studies have demonstrated that aerobic exercise training alters brain function and enhances cognitive performance. However, the effects of acute exercise on these outcomes remain unclear. Using functional MRI, we determined whether a single session of exercise elicited changes in functional activation and performance during an executive function task in a group of healthy physically active older adults.

Participants and Methods: Participants and Methods: Using a crossover repeated measures design, 21 participants (age 55-85) underwent two experimental visits. At each visit, participants engaged in 30 minutes of moderate intensity exercise (stationary bicycle) or seated rest, immediately followed by a task-based MRI scan. During the scan participants performed the Erikson Flanker Task as a measure of inhibitory control. Neuroimaging analyses were performed using AFNI and were restricted to regions of interest (ROIs) involved in conflict monitoring and inhibitory control.

Results: Results: While task behavioral performance did not significantly differ by condition, five ROIs exhibited exercise-induced changes in functional activation, including decreased activation in the $(\mathrm{L})$ middle temporal gyrus, (R) anterior cingulate cortex, and (L) putamen; and increased activation in the (L) inferior temporal gyrus, and (L) inferior parietal lobule (False Discovery Rate (FDR) corrected $P \leq 0.036$ ).

Conclusions: Conclusions: Thirty minutes of moderate intensity aerobic exercise significantly altered functional activation in brain regions known to be involved in inhibitory control, including a notable shift in activation from the anterior cingulate cortex to the parietal lobule. Interestingly, similar activation changes have been observed after aerobic exercise training, suggesting that the short-term effects of acute exercise, when accumulated over time, may facilitate cognitive improvements resulting from long-term exercise adherence.

Correspondence: Alfonso J. Alfini, Mental Health, Johns Hopkins University, 3050 Milltowne Drive, Apt. 306, Ellicott City, MD 21043, United States. E-mail: aalfini1@jhu.edu 
B.C. SATTERFIELD, A.C. RAIKES, W.D. KILLGORE \& A. ALKOZEI. A Voxel-Based Morphometric Analysis of Resilience to Vigilant Attention Impairment during Sleep Deprivation.

Objective: There are considerable inter-individual differences in vulnerability to cognitive impairment due to sleep loss. Resilient individuals maintain stable performance throughout sleep deprivation (SD), while vulnerable individuals show a profound degradation in performance. Previous studies have demonstrated that inter-individual differences may be predictable by genetic markers. However, it remains unclear how brain anatomy may predict resilience to cognitive impairments during SD. Here we assessed grey matter volume (GMV) as an indicator of resilience to SD

Participants and Methods: 41 healthy adults $(24.9 \pm 5.0$ yrs; 21 females) completed an overnight SD session. The psychomotor vigilance test (PVT) was administered hourly. Individual mean response speed on the PVT, normalized to baseline performance, was used as an index of subject-specific resilience to SD. Resilience scores were divided into tertiles, with the highest scores categorized as Resilient $(n=14)$ and the lowest scores categorized as Vulnerable $(\mathrm{n}=14)$. Subjects with intermediate scores were not included in analysis.

High-resolution MRI T1 structural scans were obtained up to four days prior to the SD session. Images were processed and segmented into GM images using the CAT12 toolbox for SPM12. Voxel based morphometric (VBM) analysis was used to identify differences in voxel-wise GMV between the Resilient and Vulnerable groups.

Results: In SPM12, a t-test controlling for intra-cranial volume, age, and gender showed a significant difference in GMV between the two groups. Subjects in the Vulnerable group had significantly less GMV $(p<0.011$, FDR corrected on cluster level $)$ in the right inferior frontal gyrus (IFG) compared to those in the Resilient group.

Conclusions: Reduced GMV in the right IFG is associated with vulnerability to cognitive impairment during SD. This region is critical for cognitive control and re-orienting attention, both of which are important for maintaining cognitive performance during SD.

Correspondence: Anna Alkozei, Ph.D., Department of Psychiatry, University of Arizona Medical Center, University of Arizona Medical Center, Department of Psychiatry, 1501 N Campbell Ave, Tucson, AZ 85724, United States. E-mail: aalkozei@psychiatry.arizona.edu

C. ARRINGTON, J.G. MALINS, R. WINTER, J.C. FRIJTERS, S. FROST, W. MENCL, K. PUGH \& R. MORRIS. Examining Individual Differences in Reading and Attention Networks Using an Oddball Paradigm fMRI Task.

Objective: Currently, no fMRI paradigms simultaneously assess reading and attention. Our aim was to develop a child-appropriate fMRI task capable of characterizing individual differences in both domains.

Participants and Methods: Forty-nine elementary students (age $=9.44)$ were presented with tetrads of auditory and visual stimuli in an event-related fMRI task in which a button press response was required only when the last two stimuli were identical (oddball trials). Reading network activation was assessed by contrasting printed words with false font standard trial stimuli. Attention network activation was assessed by contrasting standard versus oddball trials. Following preprocessing, single-subject statistical maps were entered into a groupwise analysis testing for main effects of stimulus and trial type. Effects of interest were calculated for each subject by subtracting beta weights between conditions; individual differences in neural responses were assessed via correlations with standardized behavioral reading and cognitive attention measures.

Results: Task performance was correlated with word reading accuracy $(r=-0.36, p<0.01)$, reading fluency $(r=-0.37, p<0.01)$ and attention $(r=-0.23$ to $-0.34, p<0.05)$. Left inferior frontal gyrus (IFG), superior/ middle temporal gyrus (MTG) showed a main effect of stimulus type with greater activation for words versus false font. The magnitude of the difference in activation between words and false font was correlated with word reading for both regions $(r=0.29$ to $0.39, p<0.05)$ and fluency for MTG $(r=-0.36, p<0.01)$. Regions including bilateral middle cingulate, insula and right IFG showed a main effect of trial type, with stronger activation for oddball trials. Right IFG $(r=-0.30, p<0.05)$ and left middle cingulate $(r=0.30, p<0.05)$ correlated with attention measures. Conclusions: Results indicate the task activated both reading and attention networks. Furthermore, activation of certain brain regions within these networks scaled with reading and attentional performance. Correspondence: C. Nikki Arrington, PhD, Psychology, Georgia State University, 831 Marietta St NW, Atlanta, GA 30318, United States. E-mail: carrington3@gsu.edu

H. BEDNARZ \& R. KANA. Relationship between cerebellar functional connectivity and symptoms of attention-deficit/ hyperactivity disorder.

Objective: The cerebellum has been implicated in neurodevelopmental disorders such as autism spectrum disorder (ASD), attention-deficit/ hyperactivity disorder (ADHD), and dyslexia, with altered connectivity of specific cerebro-cerebellar loops reported in each disorder. The aim of this study is to examine cerebellar connectivity within an ADHD population and relate it to ADHD symptoms.

Participants and Methods: Resting-state functional MRI (rs-fMRI) data were obtained from the ADHD-200 Sample (ADHD $N=60$, TD $N=$ 60, ages 7-18 years). Twenty-eight cerebellar regions of interest (ROIs) were created from the SUIT atlas. ROI-to-ROI functional connectivity was calculated using semi-partial correlations. Group differences were examined, and regression analyses were performed to examine relationships between cerebellar connectivity and inattentive and hyperactive symptoms of ADHD.

Results: Among all subjects, higher inattentiveness predicted increased connectivity between left lobule VIIIA and right Crus II, as well as decreased connectivity between left lobule VIIIA and right lobule VIIIB. When statistically comparing this relationship between ADHD and TD groups, there was a stronger positive association in ADHD between inattentiveness and vermis Crus II - left lobule IX connectivity. Among all subjects, greater hyperactivity was associated with increased connectivity of vermis $\mathrm{X}$ with left lobule VI and with left lobule $\mathrm{V}$; and decreased connectivity between vermis $X$ and vermis VI. No significant group differences were observed.

Conclusions: Results from this study indicate that cerebellar functional connectivity is related to inattentive and hyperactive symptoms. Several of these regions (lobule IX, lobule X) have been found to have decreased grey matter volume in ADHD. Additionally, these regions contribute to the dorsal attention (lobule VIIIB) and default mode networks (Crus II). This study has implications for elucidating the role of specific cerebro-cerebellar loops in ADHD.

Correspondence: Haley Bednarz, The University of Alabama at Birmingham, 1610 Valley Avenue, Apt G, Birmingham, AL 35209, United States.E-mail: hbednarz@uab.edu

E.M. BOUTZOUKAS, L. SEPETA, X. YOU, W. GAILLARD \& M. BERL. Verbal Working Memory Contributes to Atypical Language Dominance in Pediatric Epilepsy As Shown by fMRI.

Objective: FMRI WM tasks show bilateral activation. Atypical language dominance is more common in pediatric epilepsy compared to typically developing (TD) children. We explore whether WM demands are related to atypical language dominance.

Participants and Methods: 19 children with left hemisphere epilepsy (LHE) and 19 TD children, ages 8-17, completed a verbal fluency language fMRI task (auditory category decision (AUDCAT)), and a fMRI Nback WM task (2-back vs 0-back) that was adapted to use the same words from the AUDCAT task as targets. With this design, WM demands were increased while keeping language demands comparable. Imaging processing was conducted in SPM12. Language dominance was determined for frontal (IFG) and temporal regions using a lateralization index (LI). A change score examined LI as WM demands increased (AUDCAT LI - Nback LI). LI change was correlated with WM measures: DKEFS Verbal Fluency (VF), Trail Making (TM), and Color-Word 
(CW) Interference Switching Conditions, and WISC-IV Digits Span Backwards (DSB).

Results: As expected, LHE and TD children displayed left-lateralized activation for AUDCAT and groups were not different for language dominance (LHE Left=15, TD Left=18). For WM fMRI, both groups had an increase in right IFG activation, but LHE children displayed greater atypical dominance than TD children $(p=0.04)$. Performance on DKEFS CW and TM were positively correlated with IFG LI change $(p$ 's<.05), with a trend for $\mathrm{VF}(\mathrm{p}=0.09)$. For $\mathrm{WA}$, there were no group differences in language dominance for either task.

Conclusions: Recruitment of right IFG as WM demands increase on language tasks occurs for both LHE and TD children but there is greater atypical dominance for patients. Moreover, this increased right IFG activation correlates with better WM performance suggesting that bilateral IFG language activation is beneficial. Perhaps, the increased atypical dominance seen in epilepsy populations is not necessarily language reorganization, but may represent adaptive brain engagement to manage WM demands.

Correspondence: Emanuel M. Boutzoukas, B.S., Pediatric Neuropsychology, Children's National Medical Center, 1829 E St NE, Washington, DC 20002, United States.E-mail: emboutzouk@ childrensnational.org

C.T. EBRAHIMI, T. KUHN, K. JAPARDI, R.M. BILDER \& S. BOOKHEIMER. An Investigation of the Various Domains of Gifted Children Intelligence: Emotional vs. Exceptional Intellectual Intelligence.

Objective: Some studies have shown that high intelligence is a protective factor for many possible emotional issues in highly gifted kids (GK), while others have found that loneliness is a key characteristic. This study aims to explore the possible neurostructural associations between emotional intelligence, defined by the empathy Interpersonal Reactivity Index (IRI), and heightened intellectual intelligence.

Participants and Methods: Structural MRI/3D morphometry methods were used to compare the shape of bilateral amygdala between GK $(\mathrm{n}=10)$ and typically developing (TD) controls $(\mathrm{n}=7)$; average IQ among $\mathrm{GK}=142$. We examined empathy scores on the IRI. Paired-samples t-tests were conducted to evaluate whether empathy scores on the IRI differed in the GK group. Finally, we correlated amygdala shape with each empathy domain across the entire sample.

Results: Significant between-group differences were found in all four subtests of the IRI suggesting that gifted children demonstrated higher scores on the four empathy domains: perspective taking (PT), emphatic concern (EC), fantasy scale (FS), and personal distress (PD). Only for the PT domain, a significant within-group negative correlation was found in the left amygdala suggesting that gifted kids with higher PT empathy scores have smaller amygdala regions than controls. No significant associations were found for other domains. No significant difference was found for the right amygdala in any domain. No significant betweengroup differences were found in amygdala shape between the gifted kids and the TD controls irrespective of scores on the empathy index.

Conclusions: These results are consistent with the knowledge that intellectual giftedness is associated with higher emphatic emotional intelligence. Higher empathy was associated with smaller amygdala regions, suggesting that increased emotional intelligence may be related to amygdala function including emotional regulation. Future analysis will define the relationship between heightened emotional and intellectual intelligence.

Correspondence: Chantel T. Ebrahimi, B.S., Psychiatry \&. Biobehavioral Sciences, University of California, Los Angeles, 760 Westwood Plaza, B8-169, Los Angeles, CA 90095, United States. E-mail: cebrahimi@ ucla.edu
P.M. GARCIA-EGAN. Cerebellar morphometry abnormalities associated with early life stress.

Objective: The present study examined the relationship between reported exposure to early life stress (ELS) and cerebellar morphology. Participants and Methods: The sample included 233 healthy adults (52\% male) with an average age of 39 (18-80) and 14 years of education. Exclusionary criteria included neurologic disorder, major psychiatric illness, or substance use disorder. Participants completed the Early Life Stress Questionnaire (ELSQ) and were classified as ELS- $(n=82)$ if they reported 0-1 ELS events before age 12 and ELS+ $(n=151)$ if they reported 2 or more ELS events before age 12. Magnetic resonance imaging (MRI) was completed to determine volumes of the anterior and posterior cerebellar lobes. The cerebellar posterior lobe was further sub-divided into superior and inferior regions.

Results: Groups were similar in age, education and sex. Multivariate analyses revealed lower posterior lobe volume in the ELS+ group compared to the ELS- group $(F(1,231)=4.92, p=0.028)$. Item-level analyses revealed a select, but modest relationship between history of family conflict and witnessing of domestic violence and reduced volume of the superior-posterior lobe $(R=.03, F(2,230)=3.06, p=.05)$. No other multivariate or item-level associations were observed.

Conclusions: Self-reported ELS corresponds to smaller posterior cerebellar volume among otherwise healthy adults. The mechanisms associated with select morphometric differences may reflect an interaction between ELS exposure and regional differences in neurodevelopmental processes in the cerebellum. Future studies should address the effects of genetic variations and the interaction of stressors and stress response on the cerebellum.

Correspondence: Paola M. Garcia-Egan, PhD, Psychology, University of Missouri Saint Louis, One University Boulevard, 325 Stadler Hall, SAINT LOUIS, MO 63121-4499, United States.E-mail:pmob7b@mail. umsl.edu

M. NICHOLS, T. GATES, J. SOARES, K. MOFFAT, C. RAE, B. BREW $\&$ L. CYSIQUE. What is the Magnitude of Structural Brain Changes in Virally-Suppressed HIV-Infected Persons?

Objective: To robustly determine the magnitude of brain volume change in virally suppressed HIV+ individuals.

Participants and Methods: $92 \mathrm{HIV}$-positive (HIV+) and $50 \mathrm{HIV}$-negative $(\mathrm{HIV}-)$ participants (Med age $=60)$ underwent high-resolution anatomical Magnetic Resonance Image (MRI) scanning, neuropsychological evaluation (assessing 7 cognitive domains), and HIV laboratory tests. MRIs were processed via the automated segmentation software, FreeSurfer. Total cortical volume, total white matter (WM) volume, basal ganglia, lateral ventricles, fronto-striatal and fronto-parietal WM volumes were measured with reference to total intracranial volume. Neurocognitive performance was summarized using Global Deficit Score (GDS) method and impairment (NCI) was defined dichotomously as GDS $\geq 0.5$. History of HIV -related NCI (yes/no) was also obtained. HIV status group differences and the effects of age and cognitive performance on volumes of interest were assessed using multivariate analyses of variance, while multiple linear regression was used to assess the effects of HIV biomarkers.

Results: The HIV+ group demonstrated predominant subcortical grey $(d=0.50-0.60)$ and $\mathrm{WM}(\mathrm{d}=0.43-0.69)$ atrophy, while the cortex was relatively spared $(d=0.23)$. Historical and current NCI were separately associated with caudal-middle-frontal and superior-frontal WM atrophy. Caudal-middle-frontal WM volumes were smaller in those with both historical and current NCI than when only one or neither were present. The inferior-parietal WM was smaller in the HIV+ group compared to controls as a function of age. Finally, lower CD4/CD8 ratio was associated with volume loss in the $\mathrm{HIV}+$ group across several subcortical grey and WM regions.

Conclusions: Virally suppressed HIV+ individuals show moderate subcortical grey and WM atrophy and mild cortical atrophy, which is the product of historical and ongoing brain damage. Virally suppressed 
HIV neuropathogenesis may be primarily driven by chronic immune activation.

Correspondence: Thomas Gates, Master of Clinical Neuropsychology, Neurology, St Vincent's Hospital, Level 4, Xavier Building, 390 Victoria Street,Darlinghurst,NSW2010,Australia.E-mail:thomas.gates@svha. org.au

J.M. GULLETT, J. VELIZ, H. RISKIN-JONES, G. WEISSBERGER, D.L. SULTZER \& R. MELROSE. White Matter Disease in Amnestic Mild Cognitive Impairment: Contributions to White Matter Integrity.

Objective: Use T2-weighted magnetic resonance imaging (MRI)derived white matter hyperintensities (WMH) and diffusion tensor imaging (DTI)-derived fractional anisotropy (FA) values to determine their potential contribution to diagnosis status in amnestic Mild Cognitive Impairment (MCI-A) and Elderly Control (EC) groups.

Participants and Methods: Sixteen elderly patients with MCI-A and fourteen matched Elderly Control (EC) patients were recruited from VA clinics for a total sample of 28 participants. White matter FA was compared between groups, and significant results were compared to WMH Regions of Interest (ROI) in FA space. The two ROI's were overlaid and the contribution of WMH burden to differences in FA, age, and diagnosis were determined with SPSS.

Results: Significantly lower FA was found in the MCI-A group compared to controls after correction for multiple comparisons $(p=.01)$. Compared to EC participants, MCI-A patients had greater overlap between FA and WMH regions; a difference that was trending for statistical significance $(t[27]=-1.80, p=.08)$. The volume of the overlapping regions was positively associated with age $(\mathrm{r}[27]=0.49, \mathrm{p}<.001)$, and this positive association was higher within the MCI-A group.

Conclusions: This study identifies a network of decreased white matter integrity within MCI-A patients when compared to their healthy, wellmatched counterparts. There appeared to be a modest contribution of white matter disease burden to differences in white matter integrity; a finding likely limited by small sample size. When examining this overlap in more detail, we see a strong association with age and diagnosis status, where the positive association is greater among older individuals with MCI-A compared to EC participants. Thus, the present study brings to light several factors that suggest white matter disturbance in MCI is, at least in part, due to cerebrovascular disease pathology.

Correspondence: Joseph M. Gullett, Ph.D., Clinical and Health Psychology, University of Florida, Health Science Center, PO Box 100165, Gainesville, FL 32610, United States. E-mail: gullettj@php. ufl.edu

\section{A. JOSEPH, K. GARCIA, A. POLITIS, J. DSURNEY \& L. CHAN. Functional Connectivity in TBI Patients with Comorbid PTSD or Depression.}

Objective: Studies of those with traumatic brain injury (TBI) alone and those with comorbid PTSD or depression have found differences in MRI between groups. Differences have been seen in the hippocampus-striatum pathway in those with TBI and PTSD. In addition, relative increases in amygdala connectivity in depressed TBI patients have been identified. The goal of this study is to identify functional brain networks in TBI affected by PTSD and depression using resting-state fMRI.

Participants and Methods: 18 subjects within five years post injury were selected from a larger cohort study. Inclusion in the current study was based on having a usable rs-fMRI and completion of the BDI-II, BSI-18 and PCL-C. Subjects in the depression group $(n=7)$ had a score of $>20$ on the BDI-II and no significant levels of anxiety or PTSD. Subjects within the PTSD group $(n=3)$ had a score of $>44$ on the PCL-C with no significant anxiety or depression. Controls $(n=8)$ did not have significant symptoms on any of the three measurements.

72 seed correlations (including left and right structures) were selected based on previous literature. A between-group analysis was performed on functional connectivity differences between the TBI only and TBI with depression and PTSD.

T-tests were completed using R. P-values were then adjusted using FDR. Results: Uncorrected t-tests demonstrated multiple significant correlations for the PTSD and depression groups at $\mathrm{p}<.05$, however when adjusted for multiple comparison there were no significant findings. The following results for uncorrected correlations were found: For PTSD, Amygdala (Left)-Thalamus (Left), $\mathrm{t}(9)=3.5, \mathrm{p}=.002$. For depression, Hippocampus (Right)-Caudal Anterior Cingulate (Left), t $(13)=2.48$, $\mathrm{p}=.024$.

Conclusions: Our study did not find any significant functional brain networks affected by PTSD or depression in TBI subjects. However, this exploratory study may serve as a guide for ROI selection in the future. Future rs-fMRI studies in TBI with comorbid PTSD and depression may benefit from larger sample size.

Correspondence: Annie-Lori Joseph, BA, MD, United States. E-mail: annie.joseph3@nih.gov

J. KIM, S. WEISENBACH, E. AVERY, J. HEFFERNAN, M. PECIÑA, J. ZUBIETA \& B.J. MICKEY. Neurocognitive Function is Predicted by Striatal Dopamine D2/D3 Receptor Availability in MedicationFree Major Depressive Disorder Patients.

Objective: The striatum is known to mediate hedonic responses and behavior. However, its role in cognition is relatively unknown. In the present study we explore the association between striatal D2/D3 receptor availability and cognitive function in MDD.

Participants and Methods: 27 right-handed medication-free participants diagnosed with DSM-IV Major Depressive Disorder (MDD; 18 females, ages $18-59$, mean \pm SD: $37.3 \pm 14.1$ ) performed the Trail Making Test Parts A \& B; Stroop Color-Word Interference Task (Stroop), the Wisconsin Card Sorting Task (WCST), and the California Verbal Learning Test-second edition (CVLT-II). Subsequently, study participants were scanned with positron emission tomography (PET) using $\left[{ }^{11} \mathrm{C}\right]$ raclopride. D2/D3 receptor binding potential (BP) was extracted for three striatal regions bilaterally: caudate, putamen, and nucleus accumbens (NAcc). Partial correlation analyses were conducted using striatal BP data as a predictor, age, sex, and smoking status as covariates, and cognitive performance as outcome variables.

Results: Lower D2/D3 receptor availability in the putamen predicted worse performance on abstract-reasoning (WCST overall error, $r=.53$, $\mathrm{p}<.05$; perseverative error, $\mathrm{r}=.53, \mathrm{p}<.01)$ as well as worse verbal episodic memory learning and recall performance (learning-slope, $r=.46, p<.05$; long-delay-free-recall, r=.57, p<.005). On the other hand, lower D2/D3 receptor availability in the NAcc predicted relatively better set-shifting performance (Trails-B - Trails-A, $\mathrm{r}=-.50, \mathrm{p}<.05)$.

Conclusions: Lower D2/D3 receptor availability in the putamen and higher D2/D3 receptor availability in the NAcc is associated with worse executive function and episodic verbal memory in medication-free MDD patients. These results suggest a potential treatment target for MDD patients experiencing decrements in cognitive functioning.

Correspondence: Joseph Kim, Ph.D., Psychiatry, University of Utah, 159 West Lady Bank Way, Salt Lake City, UT 84115, United States. E-mail:dwqkim@gmail.com

L.R. KLING, K.L. PHAN, N. CRANE, S. GORKA, J.J. WEAFER, M. YOUNG, H. DE WIT \& S.A. LANGENECKER. Recent Binge Drinking Dampens Response to Anticipation of Potential Monetary Loss.

Objective: Binge drinking is usually defined as drinking $5+$ drinks for men, and drinking 4+ drinks for women in a two-hour time period. Binge drinking is linked to many serious health issues and risky behaviors. Despite this, individuals may persist in this behavior. The aim was to determine if individuals with recent history of binge drinking have disrupted neural activation in the face of potential loss.

Participants and Methods: 71 participants were involved, divided into those who had an episode of binge drinking in the last 28 days 
(17 female, 18 male, mean age $=24.72)$ and those who did not $(19$ female, 17 male mean age $=24.23)$. The mean number of binges was 1.59 for the binge group. Participants underwent fMRI at 3T while performing a Monetary Incentive Delay Task. The contrast of interest was loss-minus-neutral during anticipation of $\$ 1.20$ or $\$ 5.00$ potential loss. T-tests were used to determine group differences. Whole brain analysis was conducted at $\mathrm{p}<0.005, \mathrm{k}>55$.

Results: Non-binge drinkers had greater activation in a number of regions for anticipation of potential loss compared to binge drinkers including inferior frontal, anterior cingulate, mid-cingulate, precentral, postcentral, superior temporal, middle occipital, lingual, parahippocampal gyri, and precuneus thalamus, and caudate. There were no areas of greater activation for loss for binge drinkers relative to non-binge drinkers.

Conclusions: The results suggest that there is a widespread dampening effect in the neural response to loss in those who have had at least one binge-drinking episode in the past month. This lack of response to potential loss may provide insight into the reasons why individuals persist in behaviors like binge drinking that have a potential for negative ramifications.

Correspondence: Leah R. Kling, B.S. in Psychology, Psychiatry, University of Illinois at Chicago, $1601 \mathrm{~W}$ Taylor Street, Chicago, IL 60612, United States. E-mail: leahrkling@gmail.com

C.A. LINDBERGH, S. CORREIA, S. VAN HUIDEN, E.R. TUMINELLO. HARTMAN, S. SALLOWAY, P. MALLOY \& S. DEONI. White Matter Hyperintensities Relate to Functional Ability in Individuals with Mild Cognitive Impairment and Dementia.

Objective: Previous studies show white matter hyperintensities (WMH) correlate with cognitive function, though their relation to functional ability (FxA) is less well characterized. The present study sought to fill this gap in the literature, hypothesizing a negative association between WMH and FxA in individuals with varying levels of cognitive impairment.

Participants and Methods: Magnetic resonance imaging was acquired in 32 volunteers (mean age $=77 ; 63 \%$ female) with mild cognitive impairment $(n=18)$, mild dementia $(n=10)$, or moderate to severe dementia $(n=4)$. Data processing was implemented in FSL. Intensity thresholded FLAIR images were used to quantify WMH volume, which was in turn corrected for intracranial volume. Fx $\dot{A}$ was assessed via caregiver interview using the Disability Assessment for Dementia (DAD). Zero-order and partial correlations adjusted for age and education were calculated between WMH and DAD total and subscale scores.

Results: Zero-order bivariate correlations yielded significant associations between WMH and DAD Total Score $(r=-.443)$ as well as the Initiation $(r=-.561)$ and Planning/Organization $(r=-.416)$ subscales $(p$ s all $<.05)$. These associations remained significant upon adjusting for age and education (partial $r \mathrm{~s}=-.385,-.526$, and -.379 , respectively). The relation between WMH and the DAD Effective Performance subscale was non-significant before and after demographic adjustment. Conclusions: The present findings suggest the relation of WMH to cognition extends to everyday functioning in individuals at varying stages of decline, even when controlling for age and educational attainment. The observed correlation with initiation, planning, and organizational aspects of $\mathrm{FxA}_{\mathrm{xA}}$ is consistent with previous literature showing a relation between WMH and executive dysfunction. Future research would benefit from larger samples that permit mediation analyses to probe underlying mechanisms.

Correspondence: Cutter A. Lindbergh, M.A., Department of Psychiatry and Human Behavior, Brown University, Clinical Psychology Training Consortium, Alpert Medical School of Brown University, Box G-BH, Providence, RI 02912, United States.E-mail: cutter.lindbergh@gmail.com
S.L. SALINAS, J. KLEINER, J. GESS \& A. JAMES. Dissociable Patterns of Neural Activity for Semantically and Arbitrarily Related Word Pairs.

Objective: Verbal memory testing is of particular interest for neuropsychologists, as verbal memory impairment is often observed in many neurological conditions. Resting-state fMRI allows mapping of intrinsic functional connectivity (i.e. patterns of correlated activity among brain regions). This study seeks to further reveal how brain functional connectivity varies with individual differences in performance across word memory tasks.

Participants and Methods: These data were collected through the Cognitive Connectome project, an initiative to map neural representations of cognition in a normative sample of healthy adults $(n=79$, age 18-50). A total of 33 participants completed the WMS-IV Paired Word Associates Task and underwent resting-state fMRI data. Two regions of interest (ROIs) were selected a priori from PET imaging work on word pair encoding (Weintrob et al., 2002): the left inferior temporal gyrus (associated with semantic encoding) and left perirhinal cortex (associated with arbitrary encoding). ROIs' mean activity timeseries were regressed against overall brain activity to generate resting-state seed maps for each participant.

Results: Greater recall of semantically related word pairs was associated with greater connectivity of left inferior temporal cortex with right hippocampus and parahippocampus. Conversely, greater recall of arbitrarily related word pairs was associated with greater connectivity of left perirhinal cortex with multiple frontal regions including medial frontal gyrus, left superior frontal gyrus, and right ventrolateral prefrontal cortex.

Conclusions: Bilateral hippocampal/parahippocampal/inferior temporal connectivity for semantically related word pairs may reflect drawing upon existing mnemonic associations, whereas perirhinal to frontal connectivity for arbitrarily related word pairs may reflect the generation/development of new associations. These results indicate that resting state fMRI data may be a beneficial diagnostic tool for mapping brain regions associated with verbal memory encoding.

Correspondence: Stephanie L. Salinas, MS, MA, Department of Clinical Psychology, The University of Arkansas for Medical Sciences, $4301 \mathrm{~W}$ Markham, Slot 554, Little Rock, AR 72205, United States. E-mail: steph. salinas34@gmail.com

N. SIDEMAN, S. HALBERT, X. HE, G. DOUCET, G. CHAITANYA, S. ALWETHINANI, N. KIM, M. LEESE, A. LYON, M.K. BAJAJ, M. SPERLING, A. SHARAN \& J.I. TRACY. Task Specific Network Refinement: Multiple Cognitive Tasks Prune from a Broader Resting State Network.

Objective: The relationship between intrinsic resting state networks (RSNs) and task specific networks is unclear. Before, we evidenced task specific networks selected and pruned from a broader domain specific RSN in an expressive language task. To further test this "pruning" hypothesis, we investigated whether (1) a receptive language task also demonstrated selective pruning from intrinsic language RSNs, (2) comparable effects are seen in other cognitive domains such as executive function. We hypothesize that task-specific operations in each domain will select from the broader network captured by intrinsic functional connectivity (FC) emanating from the regional cores of each relevant domain.

Participants and Methods: We collected fMRI data in a sample of left language-dominant healthy controls during (1) a 5-min resting-state scan, (2) a Sentence Completion task ( $\mathrm{N}=38$, age $=33 \pm 11$; educ=17), (3) an adaptation of Wisconsin Card Sorting Task $(\mathrm{N}=19$, age $=38 \pm 11$, educ=17). Both tasks used a block design with interleaved ON (task-active) and OFF (task-inactive) conditions. Goodness of fit (GOF) between the task activation network (ON minus $\mathrm{OFF}$ as template) and $3 \mathrm{FC}$ networks (during task $\mathrm{ON}$, task $\mathrm{OFF}$ and resting-state) were generated with domain-relevant unilateral and bilateral seeds. Higher GOF reflects the degree to which the network matches the template. 
Results: Analyses demonstrated that the GOF's were significantly higher in both ON and OFF conditions compared to RSN ( $>5$, p $<0.001$, corrected), for all domain specific core (seed) locations.

Conclusions: Results show that across the two domains, the FC maps during task-related conditions were more restrained and closer to the full task activation map compared to their respective RSNs. These effects occurred despite differing domain-relevant cognitive cores. The data provides support for the pruning hypothesis, demonstrating it applies to different tasks within the same cognitive domain (language), and has applicability across other cognitive domains (executive function). Correspondence: Noah Sideman, Philadelphia College of Osteopathic Medicine, 725 Tasker St., Philadelphia, PA 19148, United States. E-mail: noah.sideman@jefferson.edu

J.J. TANNER, N.A. SCHWAB \& C.C. PRICE. Reliability and Validity of White Matter Cerebrovascular Disease Segmentation from T1. Objective: Leukoaraiosis (LA) is a term for hyperintense white matter regions on T2 MRI. Greater LA is associated with aging and declines in self-monitoring, mental flexibility, and working memory. Traditionally, measuring LA is based on T2 fluid-attenuated inversion recovery (FLAIR) MRI. FLAIR is not included in all scanning protocols, however. An acceptable alternative to quantification of LA might be available in FreeSurfer 6.0. We designed the current study to investigate the construct and criterion validity of FreeSurfer's hypointense segmentation relative to traditional FLAIR LA segmentation.

Participants and Methods: Participants included non-demented individuals $(n=175$; age: $68.88 \pm 6.42$; education: $15.88 \pm 2.72)$. We assessed relationships between LA, WMHypo, and raw scores from the Stroop test (Word, Color, and Color-Word) and the Hopkins Verbal Learning Test (HVLT total, HVLT delay). We manually quantified LA volumes from FLAIR (3T Siemens Verio). T1 white matter (WM) hypointensity (WMHypo), WM, and total intracranial (TICV) volumes were extracted from FreeSurfer with WM and TICV as correcting variables.

Results: LA and WMHypo volumes correlated but had low spatial overlap $(r=0.86, p<0.01$, intra-class correlation $=0.82$, Dice similarity coefficient $=0.39)$. LA and WMHypo volumes similarly correlated with Stroop Color and Stroop Color-Word scores ( $\mathrm{r}$ values $=-0.16$ to -0.27 , $\mathrm{p}<0.05$; Fisher's r-to-z $\mathrm{p}$ values $>0.27)$. WMHypo volumes negatively associated with HVLT total and delay scores $(p$ values $<0.05)$. LA associated with HVLT delay ( $\mathrm{p}$ values $<0.05$ ). Fisher r-to-z scores did not differ between LA and WMHypo for HVLT subtests ( $p$ values > 0.32). Conclusions: Manual LA from FLAIR and T1 WMHypo from FreeSurfer 6.0 produce similar estimates of LA volume in older adult brains. Associations with cognition and memory are also similar. FLAIR and T1 segmentations had poor spatial overlap, however, indicating limitations in using WMHypo for localization.

Funding: R01NR014181, R01NS082386, K23NS060660

Correspondence: Jared J. Tanner, Ph.D., Clinical and Health Psychology, University of Florida, $4916 \mathrm{NW}$ 80th RD, Gainesville, FL 32653,United States. E-mail: jjtanner@phhp.ufl.edu

\section{A. TART-ZELVIN, B. MAKWANA, K. OSIPOWICZ, R. ECHON, A.M. KORELL, J. WILLIAMS, K.J. TURLEY-AMES \& X. XU. Cognitive Strategy Use Reveals Neural Efficiency on a Working Memory Task.}

Objective: Strategy training positively influences working memory (WM) ability and predicts higher cognitive functioning (HCF) (TurleyAmes \& Whitfield, 2003). We examined neural activity during a WM task with and without the use of rehearsal strategy (RS). We hypothesized significantly different neural activity between the control (CG) and experimental (EG) groups. We also hypothesized that the EG would outperform the CG measures of HCF as measured by the Nelson-Denny (ND) and the matrix reasoning (MR) subtest of the WAIS-IV.

Participants and Methods: Participants included 40 healthy participants who did not significantly differ on age, gender, or years of education. All participants completed a modified operation span task in an MRI scanner (M-OSPAN; Turner \& Engle, 1989), the ND, and MR. The EG $(n=20)$ completed the M-OSPAN after one session of RS training.

Results: Direct contrast between the two groups was null. However, the $\mathrm{CG}(\mathrm{n}=20)$ displayed diffuse activation spanning the frontal lobes, while the EG demonstrated a significantly more parsimonious activation pattern $(p<.001)$. ROI analyses revealed the EG used significantly more of the left medial frontal gyrus and dorsal frontal gyrus. The EG used significantly more of the opercular part of the inferior frontal gyrus $(p<.001)$. The EG outperformed the CG on the WM free recall $(\mathrm{t}(38)=-2.94, \mathrm{p}=.006)$. Average free recall was positively correlated with MR, $r=0.37, n=40, p<.05$, and NDV, $r=0.28, n=40, p<.05$. Average forced-choice also positively correlated with MR, $\mathrm{r}=0.32$, $\mathrm{n}=40, \mathrm{p}<.05$ and average free recall, $\mathrm{r}=0.48, \mathrm{n}=40, \mathrm{p}<.01 . \mathrm{MR}$ positively correlated with ND vocabulary $(\mathrm{NDV}), \mathrm{r}=0.50, \mathrm{n}=40, \mathrm{p}$ $<.01$. NDV was also positively correlated with the composite score on the ND, $r=0.73, n=40, p<.01$, and ND comprehension (NDC), $\mathrm{r}=0.47, \mathrm{n}=40, \mathrm{p}<.01$.

Conclusions: Effective cognitive strategies are reflected in neural efficiency. These findings can help to inform early detection of cognitive decline and early cognitive interventions.

Correspondence: Ariana Tart-Zelvin, M.S., Psychology, University of Washington School of Medicine, 1551 Minor Avenue, Apt. 301, Seattle, WA 98101, United States.E-mail: atartz@gmail.com

S. TRAN, K.M. MCGREGOR, A. JAMES, G. KAUNDINYA, V. KRISHNAMURTHY, L.C. KRISHNAMURTHY \& B. CROSSON. Functional Connectivity of Attention Nodes at Midlife.

Objective: Task-negative networks and task-positive networks demonstrate an anticorrelated relationship in functional connectivity studies. This network dynamic is disrupted in aging and characterized by decreased functional connectivity. Specifically, the anticorrelation observed in younger adults becomes weaker in older adults as networks lose their oppositional coherence. However, relatively few studies have explored how anticorrelated network dynamics change at midlife. Two approaches to functional connectivity were used to examine changes between task-positive and task-negative attention regions at midlife. Resting-state and task-residual functional connectivity approaches offer unique information based on functional interactions that are either unrelated to a specific cognitive state or task-dependent, respectively.

Participants and Methods: Thirty-four young and midlife adult participants completed a verbal fluency task in the scanner and a resting state scan. The time series from both data sets were correlated between left-hemisphere pre-supplementary motor area and posterior cingulate/ precuneus nodes.

Results: In task-residual data, functional connectivity between the pre-supplementary motor area and posterior cingulate/precuneus was significantly weaker at midlife compared that of young adults. In contrast, resting-state functional connectivity revealed no significant difference between the two cohorts in the same regions.

Conclusions: Decreases in functional connectivity between anticorrelated regions involved in attention are present at midlife. However, differences were only observable when measuring functional connectivity during task engagement. It is possible that subtle changes to functional connectivity at midlife require context-dependent approaches in order to detect them.

Correspondence: Stella Tran, Psychology, Georgia State University, 140 Decatur St, Atlanta, GA 30303,United States.E-mail:stran12@ student.gsu.edu

\section{J. YUAN \& R. HOLTZER. $\mathrm{HbO}_{2}$ Variability and Gait Performance in Older Adults.}

Objective: Robust evidence has demonstrated the critical interdependence of gait and cognition in aging. Functional neuroimaging studies have shown that the prefrontal cortex (PFC) and related neural circuitry 
sub-serve gait, notably under dual-task conditions. We previously demonstrated that variability of oxygenated hemoglobin $\left(\mathrm{HbO}_{2}\right)$ in the PFC is higher during dual-task compared to single-task gait. The objective of this study was to determine the association between $\mathrm{PFC} \mathrm{HbO}_{2}$ variability and gait performance under single-task (NW) and dual-task (WWT) conditions in older adults.

Participants and Methods: Functional near-infrared-spectroscopy (fNIRS) was used to assess PFC $\mathrm{HbO}_{2}$ during $\mathrm{NW}$ and WWT in 274 older adults (mean age $=75.88$ years; female $=55.80 \%$ ). $\mathrm{HbO}_{2}$ variability was operationalized as the standard deviation of $\mathrm{HbO}_{2}$ values across six continuous trial epochs. Reliability and validity for this walking paradigm have been established.

Results: Separate linear regression models were conducted to examine the relationship between $\mathrm{PFC} \mathrm{HbO}_{2}$ variability and gait performance (gait velocity, stride length variability) during NW and WWT. Higher $\mathrm{PFC}_{\mathrm{HbO}}$ variability was associated with slower gait velocity (estimate $=-10.69 ; 95 \% \mathrm{CI}=-18.91$ to $2.48 ; \mathrm{p}=.011)$ and higher stride length variability (estimate $=4.11 ; 95 \% \mathrm{CI}=1.66$ to $6.56 ; \mathrm{p}=.001$ ) during WWT. The analyses controlled for gender, age, education, disease comorbidity, and mean $\mathrm{HbO}_{2}$ values.

Conclusions: Few prior studies have examined relationships between neural variability and task performance. Our findings are consistent with neural inefficacy models, whereby higher neural activation indexes deterioration in the efficiency of cognitive processes. The current study provides first evidence of the association between $\mathrm{PFC} \mathrm{HbO}_{2}$ variability and gait performance; however, important challenges remain in understanding the functional benefits and detriments of neural variability in older adults.

Correspondence: Jennifer Yuan, Yeshiva University, 720 Greenwich Street \#8F, New York, NY 10014, United States.E-mail:jenniferdyuan@ gmail.com

K.L. ZANE, P. ROSKOS, T.W. BUCHANAN, R. BUCHOLZ \& J. GFELLER. Diffusion Tensor Imaging Findings in Adults with Mild to Severe Chronic Traumatic Brain Injury.

Objective: Diffusion tensor imaging (DTI) assesses deficits in axonal integrity, which have been associated with dysfunction in brain connectivity. Traumatic brain injury (TBI) might best be conceptualized as primarily a disorder of white matter integrity given the heterogeneity of cognitive sequelae. The present study characterized white matter integrity for 15 a priori regions of interest (ROIs) utilizing DTI measurements in adults with chronic TBI across the spectrum of severity.

Participants and Methods: Ninety participants were selected from a prior study sample that investigated attentional abilities in persons with TBI (Zane et al., 2016). Three groups of participants (i.e., healthy control, mild TBI, \& moderate or severe TBI) underwent diffusion tensor imaging as a component of a complex study that included both neurocognitve and neuroimaging measures. Primary measures and analysis included fractional anisotropy (FA) and region of interest (ROI) for 15 a priori selected areas. Correlations and one-way multivariate analyses of variance were used to compare group's FA values and Cohen's d effect sizes were computed.

Results: Moderate correlations were found between TBI severity and DTI values in 14 of 15 ROIs, most notably for the anterior corona radiata, external capsule, and the right superior longitudinal fasciculus. Decreased FA was found in 12 of 15 ROIs between the moderate or severe TBI group and control group, and to a lesser extent the mild TBI group. However, the mild TBI participants did not differ from the controls.

Conclusions: The current findings add to our understanding of chronic TBI as a disorder of neural connectivity. Specifically, DTI findings differentiated patients with and without TBI of varying severity. Additional research, particularly longitudinal studies using standardized imaging and analysis approaches, will help understand the utility of DTI as a diagnostic and prognostic tool in TBI.
Correspondence: Katherine L. Zane, Ph.D., Psychology, Saint Louis University, 1900 W Dickens Ave, Apt. 2R, Chicago, IL 60614, United States.E-mail: zanekl@slu.edu

\section{Neurophysiology/EEG/ERP}

A.K. HOLLAND, J. REYNOLDS, A. ROSA, D.E. COX, P.K. HARRISON \& D.W. HARRISON. Evidence for Reduced Left Frontal Inhibitory Control in the Absorption Phase Relative to the Cephalic Phase of Food Intake.

Objective: Changes in parasympathetic activation have been demonstrated to be under control of the left hemisphere. With respect to food intake, anticipatory cephalic phase responses have been demonstrated to be primarily under parasympathetic control, with regions of the pancreatic islets receiving input from the vagal nerve. For this research, food intake was conceptualized as a left hemisphere stressor. We predicted greater beta magnitude across the left temporoparietal sites relative to the right temporoparietal sites.

Participants and Methods: Right handed, neurologically unimpaired undergraduate men $(\mathrm{n}=23)$ underwent two 1 -minute baseline EEG recordings taken prior to eating. Two 1-minute EEG recordings were taken 5 minutes after ingesting a sandwich containing around 45 grams of carbohydrates.

Results: A Hemisphere $\mathrm{x}$ Condition interaction was found $(F(1,22)=4.64$, $p=.04$ ), indicating an increase in beta magnitude across all left hemisphere sites during absorption. The right hemisphere electrode sites showed no significant increase. For the frontal electrode sites, a main effect for Hemisphere was found $(F(1,22)=39.51, p<.0001)$, indicating greater left frontal activation relative to the right frontal region. A Hemisphere $\mathrm{x}$ Condition interaction was present $(F(1,22)=4.58$, $p=.04)$, indicating increased activation across the left temporoparietal electrode sites compared to the right.

Conclusions: Beta magnitude was significantly higher across the left temporoparietal sites relative to the right temporoparietal sites, indicating a reduction in inhibitory control from the left frontal region. Interestingly, beta magnitude was higher for the left frontal electrode sites across conditions; although no significant Hemisphere $\mathrm{x}$ Condition interaction was observed. The left frontal region, while evidencing increased activation in the absorption phase, appears to show diminished capacity to regulate the left temporoparietal regions relative to right frontal sites, and this was especially evident in the absorption phase.

Correspondence: Alissa K. Holland, Ph.D., Psychology, University of South Carolina Lancaster, 476 Hubbard Drive, Lancaster, SC 29721, United States.E-mail: akhollan@mailbox.sc.edu

R. JUAN, M. LENERT, R.C. NICKS, R.R. SWIFT, T.J. SWIFT, S. RATLIFF \& L. AGUERREVERE. Relationship Between TaskSpecific QEEG Peak Frequency Values and Rey-Osterrieth Complex Figure Performance.

Objective: Quantitative Electroencephalography (QEEG) is a brain mapping technique useful in detecting electrical brain activity. Previous studies have shown that QEEG's Theta, Beta and High Beta frequency bands are related to attentional processes (Aguerrevere, 2015; Gola, Magnuski, Szumska, \& Wrobel, 2013; Loo \& Barkley, 2005). The present study examined how task-specific peak frequency (PK) QEEG values in the prefrontal lobes correlate with the ability to immediately remember an abstract figure.

Participants and Methods: Forty college students from a Southwestern University underwent QEEG recordings using the International 10-20 system at the F3 and F4 locations while scanning the Rey-Osterrieth Complex Figure (ROCF). We measured PK for EEG frequency bands: Theta, Alpha, Beta and High Beta at locations F3 and F4 referencing $\mathrm{Cz}$. The ROCF is a neuropsychological test of visuoconstructive learning 
ability that requires the reproduction of detailed abstract drawing (Osterrieth, 1944; Rey 1941). ROCF performance was based on immediate delay recall scores using the Mayo Clinic scoring criteria.

Results: Results demonstrated significant correlations between F3 PK Beta and ROCF performance $[r(39)=.343, p<.05]$ indicating that higher ROCF scores were positively related to a high peak in High Beta frequency at the left prefrontal lobe during stimulus presentation. Additionally, significant positive correlations were obtained for High Beta PK asymmetry between F4 - F3 and ROCF performance $[r(39)=.343$, $p<.05]$, suggesting that individuals who demonstrate a higher High Beta PK at F4 than F3 tend to perform better on the ROCF.

Conclusions: These results suggest that task-specific visual learning could be represented by PK values for Beta and High Beta on the prefrontal lobes. Therefore, QEEG indicators can potentially be used to discriminate between task-specific learning abilities, which may be related to visual attentive skills. Clinical implications are discussed. Correspondence: Roselia Juan, Stephen F. Austin State Unviversity, 2100 N Raguet,HSTC Bldg 105D, Nacogdoches, TX 75965, United States.E-mail:juanr@jacks.sfasu.edu

\section{K.A. LEHOCKEY, E.M. WATSON, K. BICKEL, A.J. STEPHENSON \& D.E. EVERHART. The Relationship Between Electrophysiology, Behavioral Activation, and Continuous Positive Airway Pressure Nonadherence in a Sample of Adult Patients with Obstructive Sleep Apnea.}

Objective: Obstructive sleep apnea (OSA) has been associated with cognitive dysfunction due to chronic nocturnal hypoxemia and sleep deprivation. Fortunately, OSA treatment via nightly continuous positive airway pressure (CPAP) sometimes results in cognitive improvement. However, nonadherence to CPAP is common. The aim of the current study was to investigate potential predictors for CPAP nonadherence, including frontal alpha asymmetry measured during polysomnography and behavioral tendencies such as those comprised within the Behavioral Inhibition and Behavioral Activation Systems (BIS, BAS).

Participants and Methods: Seventy-six adult patients with OSA were recruited from the Vidant Sleep Center in Greenville, NC. They completed self-report inventories prior to a scheduled polysomnography. CPAP adherence was tracked for 90 days after initiation of treatment. Results: At 90 days, only $46.8 \%$ of the participants were adherent. Multiple regression analysis predicting total hours of CPAP use at 60 days indicated that age $(M=55.71, S D=11.75)$, race $(M=1.68, S D=.50)$, and BAS-Reward Responsiveness $(M=16.21, S D=4.33)$ were significant predictors, $F(3,36)=6.67, p=.001, R^{2}=0.36,95 \%$ CI $[0.08,0.56]$. Additionally, a positive correlation was observed between high alpha values $(M=0.08$ microvolts, $S D=0.33)$ and BAS-Fun Seeking $(M=10.11$, $S D=2.98), r=.269, n=54, p=.05,95 \%$ CI $[0.002,0.50]$.

Conclusions: Findings from the current study indicated that poorer adherence was associated with younger age, minority status, and higher levels of BAS-Reward Responsiveness, which is thought to assess anticipation of reward. Correlation analyses between frontal alpha asymmetry and self-reported BAS-Fun Seeking indicated that patients with higher levels of this trait also had relatively greater left hemisphere baseline activity during polysomnography. Results highlight potential ways to tailor treatment approaches to those at risk of CPAP nonadherence and associated cognitive dysfunction.

Correspondence: Katie A. Lehockey, Ph.D., Psychology, MedStar National Rehabilitation Hospital, 102 Irving St., NW, Washington, DC 20010,United States. E-mail: Katie.A.Lehockey@MedStar.net

\section{E. MODERSITZKI, K.A. CARBINE, E. CHRISTENSON, L. TUCKER, B. BAILEY, J. LECHEMINANT \& M. LARSON. Neural Indices of Food-related Inhibitory Control and Attention: Accurate Prediction of Dietary Intake? \\ Objective: Since dietary decision-making is important for overall phys-} ical and mental health, we tested how cognitive neurophysiological responses to high-calorie foods differed from low-calorie foods and if neural indices of food-related cognition was associated with everyday dietary intake. We specifically tested the N2 event-related potential (ERP), which reflects inhibitory control (the ability to withhold a dominant response to accomplish a goal) and the LPP ERP, which reflects motivational attention.

Participants and Methods: EEG data were collected from 64 healthy adults while passively viewing pictures of food and non-food items. EEG data were also collected during two go/no-go tasks, one with high- and one with low-calorie foods as no-go stimuli. Task order was counterbalanced. Participants also recorded their food intake using the Automated Self-administered 24-hour Dietary Recall system for three randomized days.

Results: A 2-Task x 2-Trial interaction for the N2 showed there was a larger inhibitory response to high- than low-calorie foods $(p<.001)$. A 3-Trial one-way ANOVA for the LPP showed there was a greater attentional response to low- compared to calorie foods $(p=.01)$. When directly comparing standardized N2 and LPP values, a Food $\mathrm{x}$ ERP interaction $(p<.001)$ confirmed the $\mathrm{N} 2$ was larger to high-calorie foods and the LPP was larger to low-calorie foods. Regression models showed LPP and N2 amplitude did not predict caloric intake ( $p \mathrm{~s}>.15$ ). However, N2 amplitude to high-calorie foods predicted carbohydrate intake; the larger the inhibitory response was to high-calorie foods, the less carbohydrates participants consumed $(\beta=-.28, p=.02)$.

Conclusions: Inhibiting responses to high- compared to low-calorie foods requires increased recruitment of inhibitory control. There was greater motivational attention to low-calorie foods, possibly due to the motivation to eat foods lower in calories despite the draw of high-calorie foods. Data have implications for possible health and diet interventions. Correspondence: Erin Modersitzki, Psychology, Brigham Young University, 244 TLRB, 1109 North 900 East Provo, Provo, UT 84602, United States.E-mail: erinmod@hotmail.com

L.F. PIMENTEL-RODRÍGUEZ, A. CHAPA, L. RODRÍGUEZGÓMEZ, V. DE LA GARZA, C. RAMIREZ, A. GARCIA, J. BORRANI \& P. VALDEZ. Effects of Sleep Deprivation on Attention and EEG Delta Power.

Objective: To analyze changes on the components of attention (tonic alertness, phasic alertness, selective attention and sustained attention) and EEG delta power during the sleep onset process (SOP).

Participants and Methods: Nine healthy students of $18.56 \pm 1.24$ years of age participated. After a night of regular sleep, they were recorded at 13:00 h in the laboratory as a control session, and after 24 $\mathrm{h}$ of sleep deprivation at 10:00, 12:00, 14:00 and 16:00 h, according to a modified Multiple Sleep Latency Test (MSLT) protocol. On each session they responded the Continuous Performance Task to measure attention, while their EEG activity was recorded for spectral analysis. The sleep latency was determined by scoring a MSLT with polysomnographic criteria. The task performance and the delta power of C3-REF were compared between the last minute before the sleep onset and the last minute of the control session task. The 10:00 and 12:00 and 14:00 and 16:00 $\mathrm{h}$ values were averaged to obtain morning and afternoon session scores.

Results: During the last minute before the sleep onset, the percentage of correct responses decreased on the morning sessions on tonic alertness (control: $83.12 \pm 13.34 \%$, deprived: $36.56 \pm 27.28 \%$, T=1.00, $\mathrm{p}<0.05$ ), selective attention (control: $46.87 \pm 23.85 \%$, deprived: $14.06 \pm 12.83 \%$, $\mathrm{T}=1.50, \mathrm{p}<0.05$ ) and phasic alertness (control: $65.62 \pm 37.64 \%$, deprived: $15.62 \pm 12.93 \%, \mathrm{~T}=0.00, \mathrm{p}<0.05)$ compared to the control session. An increase of delta power appeared in the last minute of the morning session (control: 112.04 $\pm 56.09 \mathrm{~Hz}$, deprived: $270.55 \pm 66.90$ $\mathrm{Hz}, \mathrm{T}=0.00, \mathrm{p}<0.05)$. When comparing the afternoon sessions with the control session, only tonic alertness decreased (control: $83.12 \pm 13.34 \%$, deprived: $33.75 \pm 25.73 \%, T=1.00, \mathrm{p}<0.05)$. No differences were found on the delta power of the afternoon sessions.

Conclusions: After sleep deprivation, attention decreases and delta power increases during the SOP. This decrease on the capacity to 
respond and in brain activity could lead to accidents when people drive or operate machinery.

Correspondence: Luisa F. Pimentel-Rodríguez, Laboratory of Psychophysiology, School of Psychology, Universidad Autonoma de Nuevo Leon, Mutualismo 110, Mitras Centro, Monterrey 64460, Mexico. E-mail: pimentelr0260@gmail.com

J.REYNOLDS, A.K. HOLLAND, A. ROSA, D.E.COX, P.K. HARRISON $\&$ D.W. HARRISON. Changes in Cerebral Laterality in the Cephalic and Absorption Phases of Food Ingestion as a Function of BMI Classification: Neurophysiological Evidence of Reduced Cognitive Control in Overweight Men.

Objective: Changes in relative activation of regions in the left prefrontal cortex have been observed in overweight and obese individuals (Ochner et al. 2009) during all phases of food ingestion. For the current research, increased left hemisphere activation during the cephalic and absorption phase are conceptualized as neurophysiological indicators of reduced cognitive control. We predicted that overweight men would evidence greater beta magnitude before and after food ingestion relative to normal weight men.

Participants and Methods: Following a medical screening process, 12 normal weight $(\mathrm{BMI}<24.9)$, and 11 overweight/obese $(\mathrm{BMI}<25)$ men had two 1-minute baseline EEG recordings taken. Five minutes after eating a sandwich, two final 1-minute EEG recordings were taken. Results: A BMI $\mathrm{x}$ Hemisphere interaction was found $(F(1,22)=3.98$, $p=.05)$, indicating changes in beta magnitude across all left and right hemisphere electrode sites as a function of BMI classification. A BMI $\mathrm{x}$ Condition $\mathrm{x}$ Hemisphere interaction was found $(F(1,22)=4.82$, $p=.03$ ), indicating increased left hemisphere beta magnitude for overweight men in the absorption phase relative to normal weight men. For the left temporoparietal sites, a similar BMI $\mathrm{x}$ Hemisphere $\mathrm{x}$ Condition interaction was found $(F(1,22)=7.90, p=.01)$, indicating relative increases in beta magnitude for the overweight men in the absorption phase. This increase in beta magnitude was not observed for the right temporoparietal sites.

Conclusions: The results provide evidence of reduced cognitive control during the cephalic and absorption phase of food ingestion. Overweight men showed increased beta magnitude across all left hemisphere electrode sites relative to normal weight men, and this was especially evident in the absorption phase. Moreover, the same pattern of activation was observed across left temporoparietal sites. These results are consistent with previous research examining cerebral asymmetry as a function of BMI classification during the food ingestion process.

Correspondence: Jessica Reynolds, University of South Carolina Lancaster, 3100 Windsor Trace Drive, Indian Land, SC 29707, United States.E-mail:jer4@email.sc.edu

\section{KADZIOLKA, A. SCAVONE \& C. MILLER. Mindfulness Induction and Parasympathetic Tone.}

Objective: Research on mindfulness training suggests that there is increased parasympathetic activity during and following training, where elevated parasympathetic tone indicates better emotion regulation and decreased anxiety (Scott \& Weems, 2014; Tang et al., 2009). Moreover, mindfulness training is associated with left insula activity, which is predominantly responsible for parasympathetic effects (Oppenheimer et al., 1996). Among adults, meditation is associated with increased parasympathetic activity (Kubota et al., 2001). It is believed that amygdala modulation underlies increased vagal tone activity, a marker of parasympathetic nervous system activation and relaxation, during mindfulness training (Davidson et al., 2003). The current study examined whether mindfulness induction is associated with parasympathetic activity in a non-clinical sample.

Participants and Methods: Participants were 55 undergraduate students. They completed baseline physiological recordings, an emotional stressor task, a mindful breathing exercise, and a recovery phase. Participants completed the Five Facet Mindfulness Questionnaire (self-report). Bivariate correlations were carried out between the describe subscale of mindfulness and parasympathetic activation, across several experimental phases.

Results: Mindfulness was positively correlated with parasympathetic tone (the proportion of parasympathetic frequency to combined sympathetic and parasympathetic functioning) across baseline, the emotional recall task, calming phase, and the recovery phase $(r=0.27-0.34)$.

Conclusions: The results suggest that an increase in mindfulness contributes to parasympathetic tone. As parasympathetic tone was also increased during the emotional recall task, the current results further support the notion that amygdala modulation during mindfulness contributes to parasympathetic activation. Mindfulness exercises are likely beneficial in enhancing parasympathetic tone, which allows for greater emotion regulation and decreased anxiety.

Correspondence: Antonette Scavone, Psychology, University of Windsor, 805-166 University Ave W, Windsor, ON N9A5N9, Canada. E-mail: scavone@uwindsor.ca

\section{E. VACCARIELLO, T.L. MCKINNEY, H. OKABE, I.N. ROGERS \& M. EULER. Behavioral and EEG Correlates of Mental Effort and Mind-wandering.}

Objective: Valid cognitive assessment depends crucially on consistent examinee effort. Yet, the ways in which effort varies as a function of task difficulty and duration is not fully understood. EEG studies have implicated theta $(\sim 4-8 \mathrm{~Hz})$ and alpha $(\sim 8-12 \mathrm{~Hz})$ power in mental exertion and mind-wandering, respectively, suggesting that they might ultimately provide indices of mental effort during cognitive testing.

Participants and Methods: Thirty-nine healthy undergraduates underwent EEG recording while performing 100+ trials of a complex motor sequence. The sequence was presented visually, and difficulty was manipulated by pseudo-randomly presenting the sequence in novel contexts. Periodic novel sequences helped to ensure vigilance. The latency between stimulus onset and the first movement was used to calculate median response time (RT) and RT variability. Theta-activation was measured as fronto-central power change, pre-to-post stimulus. Alpha power was obtained from pre-stimulus activity at parieto-occipital sites. Repeated measures ANOVAs tested the effects of task novelty and duration on behavioral and neural variables.

Results: Greater novelty resulted in longer and more variable RT (both $p s<.001)$, as well as greater theta activation $(p=.041)$. RT also became more variable $(p<.001)$, and alpha power increased $(p=.001)$ as the task went on. Novelty and duration interacted for RT and RT variability (both $p s<.002$ ), but not for theta or alpha power (both $p s>.35$ ). Last, in the easiest condition of the final task block, greater theta activation predicted faster RT $(r=-.318, p=.048)$.

Conclusions: RT was more variable and alpha power increased with longer task length, corroborating alpha's role in mind-wandering or fatigue. Higher theta was linked to higher task demands, and better performance under less-arousing conditions, suggesting it may serve as a marker of mental exertion. Overall, the results support further investigation of alpha and theta power as indices of effortful engagement in cognitive assessment.

Correspondence: Eleonora Vaccariello, BSc, Salt Lake City, UT, United States.E-mail: evaccariello12@gmail.com

\section{A.R. WINTERS, A. NICOLETTA, E. WATSON \& D.E. EVERHART. Behavioral Inhibition Subscales Relate to Resting Frontal Asymmetry and Sleep.}

Objective: Poor sleep has been linked to neurocognitive dysfunction. risk for comorbidity, and exacerbated healthcare costs. The Reinforcement Sensitivity Theory (RST) has previously been utilized to examine the individual differences associated with sleep-related disorders. RST consists of distinct neurophysiological systems that relate to approach and withdrawal behaviors, including Behavioral Activation (BAS), and Behavioral Inhibition (BIS) Systems. BAS is associated with left frontal alpha activity (measured through EEG) and approach behavior, while 
BIS is associated with right frontal activity and withdrawal behavior. The BIS/BAS scales can be divided into 5 subscales, including BAS-Drive, BAS-Reward Responsiveness, BAS-Fun Seeking, BIS-Anxiety, and BIS-Fear. Research has linked elevated BIS with emotional dysregulation and poor sleep. Moving forward, the purpose of this study was to examine the relationships among the subscales of BIS, frontal baseline EEG asymmetry, sleep and quality of life (QOL). It was hypothesized that BIS-Fear and BIS-Anxiety would be related to greater right than left anterior activation at baseline. It was also hypothesized that the BIS subscales would be related to poorer sleep and QOL.

Participants and Methods: 75 undergraduate students completed EEG baseline data collection, BIS/BAS questionnaire, Pittsburgh Sleep Quality Index (PSQI), Insomnia Severity Index (ISI), and the Short Form Health Survey (SF12-v2).

Results: BIS-Fear was weakly associated with greater right baseline activity at electrode FP2, $r(52)=.233, p<.05)$. The MCS was negatively related with BIS-Anxiety, $r(75)=-.400, p<.01)$ and BIS-Fear, $r(75)=-.327, p<.01)$. The ISI was positively correlated with BIS-Anxiety, $r(75)=.457, p<.01)$ and BIS-Fear, $r(75)=.358, p<.01)$. The PSQI was also found to be positively correlated with BIS-Anxiety, $r(75)$ $=.280, p<.01)$, and BIS-Fear, $r(75)=.251, p<.05)$.

Conclusions: These results suggest that BIS subscales relate to sleep, QOL, and frontal EEG asymmetry.

Correspondence: Andrea R. Winters, Psychology, East Carolina University, 1001 East Fifth Street, Greenville, NC 27858, United States. E-mail:wintersa16@students.ecu.edu

\section{Neurostimulation/Neuromodulation}

\section{A.M. BRYANT, L. BOXLEY, K. EVERSOLE \& J. SUHR. Does Surgery Side Matter? Verbal Fluency Decrements Following Deep Brain Stimulation for Treatment of Parkinson's Disease and Essential Tremor.}

Objective: Deep brain stimulation (DBS) is a safe and effective treatment for Parkinson's disease (PD) and essential tremor (ET) with minimal adverse effects and substantial reductions in symptomatology. Depending on symptoms, DBS electrodes can be placed in right $(\mathrm{R})$, left (L) or bilateral (B) hemisphere(s). Cognitive sequelae of DBS are minimal with decrements in verbal fluency (VF) most commonly noted. We examined the impact of electrode laterality on VF performance.

Participants and Methods: Participants were 42 PD, 24 ET and 15 Controls (C) (12PD/3ET w/no hx of surgery) who presented for neuropsychological evaluation to determine candidacy for surgery. Follow-up evaluations were conducted post-surgery $(\mathrm{M}=207$ days $)$ or for $\mathrm{C}$ at subsequent evaluation ( $\mathrm{M}=380$ days). 13 had $\mathrm{R}$ electrode placement, 31 had L, 22 had B. DBS targeted subthalamic nucleus (n=41); additional targets were ventral intermediate nucleus of thalamus $(n=21)$ and globus pallidus $(n=4)$. Repeated measure general linear model was conducted to compare the effect of surgery side on average VF pre- and post-surgery.

Results: Groups did not differ with respect to age, sex, education or baseline ability (MMSE) (all $p$ 's $>.05$ ). There was a significant effect of surgery side on average $\mathrm{VF}[\mathrm{F}(1,3)=4.81, p=.004]$. Within subjects analysis indicated no changes in VF for $\mathrm{R}$ and $\mathrm{C}$ (all $p$ 's $>.05$ ); however, $\mathrm{L}$ and $\mathrm{B}$ demonstrated a significant decrease in average $\mathrm{VF}$ (all $p$ 's<.03) Post hoc comparisons using Bonferroni indicated that R DBS patients outperformed L and B DBS patients on VF post-surgery (all $p^{\prime} s<.05$ ). Conclusions: Findings demonstrate that laterality of electrode placement impacts the degree to which VF deficits are noted, regardless of disease type. Findings are consistent with previous literature demonstrating long-lasting decline in VF; however, few studies have delineated R/L/B electrode placement. Future studies may examine the impact of electrode target site (thalamus vs. globus pallidus) and hemisphere on cognitive changes post-surgery.
Correspondence: Andrew M. Bryant, M.A., Psychology, Ohio University, 269 Porter Hall, Athens, OH 45701, United States. E-mail: ab859013@ ohio.edu

M. EDWARDS, J. BELLONE, S. CORREIA, J. BARREDO, L. CARPENTER \& N. PHILIP. White Matter Integrity Before and After Repetitive Transcranial Magnetic Stimulation in Individuals with Comorbid PTSD and Depression.

Objective: Posttraumatic stress disorder (PTSD) and major depressive disorder (MDD) are associated with changes in white matter integrity (WMI). It is unclear how this relationship might affect treatment response to repetitive transcranial magnetic stimulation (rTMS). We examined the association of baseline WMI with pre- and post-rTMS symptoms in patients with comorbid PTSD and MDD.

Participants and Methods: Participants ( $\mathrm{n}=22$, subset of larger cohort; mean age $=53$ ) underwent pre-rTMS MRI with diffusion imaging and completed self-report measures of PTSD (PCL-5) and MDD (IDS-SR) pre- and post-rTMS. Participants received up to 40 unblinded rTMS sessions delivered at $5 \mathrm{~Hz}$ to left dorsolateral prefrontal cortex. We used the TRACULA toolbox to evaluate WMI (fractional anisotropy, FA; mean diffusivity, MD; radial diffusivity, RD) in fronto-temporo-limbic (FTL) pathways associated with PTSD and MDD: the forceps minor, bilateral cingulum, and uncinate fasciculi. We chose the bilateral angular cingulum and corticospinal tract as pseudo-control pathways. The association between WMI and pre-rTMS symptom severity, and post-rTMS percent symptom change in PTSD and MDD outcomes, were examined using linear regression after covarying for age and gender.

Results: Pre-rTMS: WMI was not significantly associated with PTSD or MDD symptom severity (FTL and non-FTL tracts). Post-rTMS: Forceps minor and left uncinate fasciculus were significantly associated with percent symptom change in both PTSD $(p<.03)$ and MDD $(p<.04)$. One pseudo-control tract (angular cingulum) was significantly associated with percent symptom change in both PTSD $(\mathrm{p}<.02)$ and MDD $(\mathrm{p}<.04)$. Conclusions: Lower diffusivity, but not higher anisotropy, in FTL pathways is related to greater symptom improvement in PTSD and MDD following rTMS. This suggests that the relationship may not be driven by axonal integrity but rather by other factors that impact white matter diffusion.

Correspondence: Melissa Edwards, PhD, Neuro-Oncology, UT MD Anderson Cancer Center, The University of Texas MD Anderson Cancer Center P.O. Box 301402, Unit 0431, Houston, TX 77230-1402, United States.E-mail: ml.edwards@yahoo.com

S.L. GARCIA, S. MA, S. SCHLAEFFLIN, A. BHAUMIK \& B.M. HAMPSTEAD. Variable behavioral and neurophysiological response to HD-tDCS in Primary Progressive Aphasia: Preliminary data from a case series.

Objective: Primary progressive aphasia (PPA) results in marked and progressively worsening language impairment for which there is no clear treatment. However, previous research suggests that transcranial direct current stimulation (tDCS) (alone or combined with cognitive training) can improve confrontation naming and word-list recall in PPA patients. However, no past work has investigated whether tDCS enhances fluency. As such, we targeted the left lateral PFC using high definition tDCS (HD-tDCS) in a case series of patients with PPA to examine the effects on free-style speech.

Participants and Methods: Four older adults with PPA (3 diagnosed with nonfluent PPA and 1 diagnosed with semantic PPA) underwent resting-state fMRI before and after a single HD-tDCS session. HD-tDCS was administered at $3 \mathrm{~mA}$ for 20 minutes (center anode electrode at FT7). Participants described a series of pictures before, during, and after HD-tDCS.

Results: HD-tDCS was well tolerated by all participants; however. the effects varied considerably for each patient. For the nonfluent PPA patients - one showed increased word production and network 
connectivity, another showed reduced word production and connectivity, and a third showed stable production but increased connectivity. Word production increased in the patient with semantic PPA but connectivity of the left lateral PCF remained unchanged.

Conclusions: The current study demonstrates that $3 \mathrm{~mA} \mathrm{HD-tDCS}$ is well-tolerated in those with PPA but that the behavioral and neurophysiological effects were heterogeneous. Future work should examine participant characteristics that affect stimulation as well as key methodological factors like dose (intensity, duration, number of sessions), montage, and the timing of outcome measures.

Correspondence: Sarah L. Garcia, Ph.D., Psychology, Stetson University, 232 N Leavitt Ave, Orange City, FL 32763, United States. E-mail:SLGarcia@Stetson.edu

D. MECHANIC-HAMILTON, L. SAAD, D. SACCHETTI \& R. HAMILTON. Pilot M.I.N.D.S. Study: Modulating Intellect with Noninvasive DC Stimulation.

Objective: This pilot study investigated the effects of concurrent transcranial direct current stimulation (tDCS) and computer-based cognitive training (CBCT) on cognition in cognitively healthy older adults. Participants and Methods: Participants included 16 older adults (56\% female; age $=74 \pm 7.2$, years of education $=16 \pm 2.7 ; 13$ white and 3 African American) with normal cognition who are participants in the Penn Alzheimer's Disease Center Cohort. The study included a double-blind design and participants were randomized to receive either sham or active tDCS stimulation (left prefrontal anode and right supraorbital cathode) for 20 minutes per day for five consecutive days while completing the CBCT (n-back task). Cognitive performance was collected on the training task, near- and far-transfer tasks, and included subtests from the Delis-Kaplan Executive Function System (D-KEFS) and the Repeatable Battery for the Assessment of Neuropsychological status (RBANS). Assessments were administered at four time-points: at baseline, after day 5 of sham/tDCS, 2 weeks post-, and 2 months post-intervention. Results: Overall, participants improved on the RBANS [ $\mathrm{t}(15)=-3.05$, $p=0.008]$, but there was no group effect of tDCS on far-transfer tasks. Near transfer tasks, within the broad domain of executive function, showed different effects for verbal and visual fluency. There was a trend toward greater improvement in letter fluency $[F(1,9)=2.442, p=0.153]$ in the active tDCS group and greater improvement in design fluency $[F(1,8)=6.49, p=0.034]$ in the sham group.

Conclusions: There were modest effects of tDCS and CBCT on cognition in this cohort of healthy older adults. The results suggest a possible trade-off of improvement in left-hemisphere mediated verbal fluency at the expense of right-hemisphere mediated visual fluency. Future studies should consider the importance of evaluating and monitoring a wide range of domains of cognition due to the differential effects of tDCS on targeted and non-targeted cognitive skills.

Correspondence: Dawn Mechanic-Hamilton, PhD, Neurology, University of Pennsylvania, 3400 Civic Center Blvd., PCAM - 2 South, Philadelphia, PA 19104,United States.E-mail:Dawn.Mechanic@uphs. upenn.edu

\section{Cognitive Neuroscience}

K. OSIPOWICZ, M. WILLIAMS, A. TART-ZELVIN, B. MAKWANA, E.E. ELINE \& T. MEREDITH-DULIBA. New Methods to study Cognitive Development using Diffusion Tensor Imaging.

The proposed symposium includes four presentations that examined Diffusion Tensor Imaging (DTI) and measures of cognitive abilities over the course of brain development. In order to provide a normative standard for development, the NIH sponsored a longitudinal neuroimaging study that enrolled 550 healthy children and adolescents, ages newborn to 18 years (See http://pediatricmri.nih.gov/nihpd/info/). The imaging methods included structural MRI with Diffusion Tensor Imaging. A comprehensive battery of Neuropsychological tests and rating scales were administered; for these studies we used measures of language and behavioral control. This Symposium will include four presentations covering the relationship of DTI Fractional Anisotropy (FA) and Mean Diffusivity (MD) to cognitive and neuropsychological abilities. The first will introduce a unique DTI method for combining FA and MD to reduce the noise in the relationship of DTI variables to cognitive and developmental measures. The next presentation will cover the relationship of patterns of brain development revealed by DTI to the patterns of development of language and behavior control. The following presentation will examine sex differences in brain development and changes in language. The final presentation will apply the methods derived from studying developmental changes using DTI to study ASD and cognitive performance. We used curvilinear regression analyses that modeled the relationships of neuropsychological test scores and DTI measures for 48 white matter regions of the brain that mediate language, executive control, and other functions.

Each presentation will cover the specific methods applied and results for each major analysis. In general, the new combined DTI measure has incrementally better predictive power relative to FA and MD alone. Correspondence: Michael Williams, PhD Neuropsychology, PA, United States.E-mail:jmicha5059@aol.com

\section{WILLIAMS \& K. OSIPOWICZ. Reducing Error in DTI: Methods} to Combine Fractional Anisotropy and Mean Diffusion.

This presentation will review neuroimaging methods used to assess cognitive development and brain maturation in sets of children between the ages of newborn to young adulthood (total $\mathrm{N}=450$ ). The general imaging methods used were gray and white matter volumetric studies and Diffusion Tensor Imaging (DTI). The scans were collected as part of the NIH MRI Study of Normal Development (http://pediatricmri.nih. gov/nihpd/info/). The ten research centers followed a standard protocol based on the MRI scanner used by each facility such that the data could be combined. For the T1 scans, this included a conventional method with a TR of $22 \mathrm{~ms}$ and a TE of $10-11 \mathrm{~ms}$. The centers were instructed to use 160-180 slices of 1-1.5mm thickness over the entire head. The DTI scans used diffusion encoded spin echo EPI with a minimum TR of $3 \mathrm{sec}$. The scan included 48-60 contiguous slices and 6 diffusion sensitization directions.

Following preprocessing, which involved registering to the JHU-DTI template, the average Fractional Anisotropy (FA) and Mean Diffusivity (MD) were extracted per each white matter region. Higher FA values indicate areas of the brain with greater structural complexity. Higher MD values indicate greater diffusion and less structural organization. Though FA and MD are often considered indicators of reciprocal white matter organizational processes (as FA goes up, MD goes Down), they are not perfectly inverse, with each contributing unique variance. We captured the combined variance of FA and MD (per region) by using principal components factor analysis; this procedure generated a set of factor scores, representing the combined variance of FA and MD. This factor optimizes the predictive power of DTI, allowing us to render a more accurate and reliable measure of brain development.

Correspondence: Michael Williams, PhD Neuropsychology, PA, United States.E-mail:jmicha5059@aol.com

M. WILLIAMS, A. TART-ZELVIN, B. MAKWANA \& K. OSIPOWICZ. Brain Development and Language Mediation of Behavior Control.

As the left hemisphere and language systems mature, parents and teachers rank their children as better controlled in behavior assessments, such as the Behavioral Rating Inventory of Executive Function (BRIEF). This study examined the hypothesis that language mediates behavior control over the course of brain development. Subjects $(\mathrm{N}=310)$ with DTI and BRIEF scores were selected from the NIH MRI Study of Normal Development. Children in this study were given the language measure consistent with their age (PLS, NEPSY, WASI); this resulted in many in the sample not receiving the same language test. From our analysis 
of published studies of the PLS and NEPSY, we generated a regression model estimating the WASI vocabulary score for children who lacked these scores, resulting in all of the subjects having either a true or an estimated WASI vocabulary score. Results indicated a major effect for language regions, specifically in the left hemisphere being associated with both language and behavior control.

Correspondence: Ariana Tart-Zelvin, M.S., Psychology, University of Washington School of Medicine, 1551 Minor Avenue, Apt. 301, Seattle, WA 98101, United States.E-mail: atartz@gmail.com

K. OSIPOWICZ, T. MEREDITH-DULIBA \& M. WILLIAMS. Examining Sex Differences in Language and Brain Development. Cerebral growth and maturation in infancy through late adolescence is an important aspect of development. We examined sex differences in brain development in a healthy population of children. This study sample consisted of 437 normal, healthy male and female children, ranging from infancy (3 months) through adolescence (18 years). We employed Diffusion Tensor Imaging (DTI). DTI measures such as Fractional Anisotrophy (FA) and Mean Diffusivity (MD) are usually interpreted as an indication of the development and complexity of white matter tracts. These were combined using a method described in the first section of the symposium. Utilizing such techniques aids in the understanding of white matter development and change over time. Relationships between DTI measures and cognitive measures were examined using curvilinear regression. Results of DTI analysis revealed a positive curvilinear trend early on in development. This pattern was notable in both sexes and presents a more pronounced slope in females across infancy into approximately 5 years of age. As development continues, this trend normalizes and similar white matter development patterns are present in both males and females. This unique acute trend in development observed so early on is a strong indicator and may be a useful tool in elucidating developmental and learning disparities between boys and girls. Correspondence: Karol Osipowicz, Ph.D., Psychology, Drexel University, 3141 Chestnut St, 204 Stratton Hall, Philadelphia, PA 19104, United States.E-mail:kzo22@drexel.edu

\section{E.E. ELINE, M. WILLIAMS \& K. OSIPOWICZ. Applying New DTI} Measures to Autism Spectrum Disorder.

Autism spectrum disorder (ASD) is a common but heterogeneous spectrum of developmental disorders. The deleterious effects of ASD have been demonstrated on cognitive, functional, and structural levels. Though the white matter hypothesis of ASD has been tentatively supported, to date, no study has demonstrated strong DTI support for the hypothesis. We suggest that this lack of support is caused by the susceptibility of DTI to movement induced artefact increasing the error term of the DTI derived measures of FA and MD. Here, we utilize the methods described in the previous symposium to investigate DTI differences between subjects with ASD and developmentally normal controls; and to investigate the curvilinear relationships between developmental brain organization and measures of cognitive and behavioral performance. We find that the new combined DTI measure supports stronger group differences and relationships with cognitive variables in ASD than both FA and MD. These findings show the utility of our method to a clinical population, and provide support of pronounced white matter disruption in the context of ASD.

Correspondence: Ellen E. Eline, Drexel University, 30 South Keesey Street,York,PA 17402, United States.E-mail: eee34@drexel.edu

H.M. GENOVA, A. RIZZO, V.A. ANDERSON, S. MCDONALD \& K.O. YEATES. Social Cognition: Innovative Approaches to its Examination and Assessment.

Social Cognition, the set of cognitive abilities which allow for processing of information critical for social interaction, is impaired in many clinical populations. Impairments in this domain may be a result of a developmental disorder, in which skills do not develop normally, or may be the result of acquired neuropathology or brain damage. This symposium will explore innovative approaches to the examination and assessment of social cognition in clinical populations, utilizing methods such as neuroimaging techniques, virtual reality, an iPAD application, and a dynamic measure of emotion perception. First, Dr. Genova will describe how neuroimaging, specifically functional magnetic resonance imaging (fMRI) and diffusion tensor imaging (DTI) may offer us additional insight beyond typical measures to examine social cognition. Specifically, how individuals with Traumatic Brain Injury may process emotional information differently in the brain compared to healthy controls. The next presentation by Dr. Rizzo will consider ways in which virtual reality can be used as a tool for examining the impact of social cognition factors on the assessment of individuals with Posttraumatic Stress Disorder using virtual human interviewers. Dr. Anderson will present her research on the development of a theory-driven, iPAD delivered social assessment app, "PEERS" (Pediatric evaluation of emotions relationships and socialization), which is currently designed to evaluate aspects of social cognition in children, with future research extending this work into adults. Dr. McDonald will present her research on a new tool to assess emotion perception following Traumatic Brain Injury: the Complex Audio Visual Emotion Assessment Task (CAVEAT). Finally, Dr. Yeates will provide a discussion of how the techniques described in the symposium provide a deeper understanding of social cognition, and will present future directions of social cognition research.

Correspondence: Helen M. Genova, Ph.D., Kessler Foundation, 300 Executive Drive, West Orange, NJ 07052, United States. E-mail: hgenova@kesslerfoundation.org

A. RIZZO. Can Automated Conversational Virtual Human Objective: A significant scientific literature has evolved investigating the use of Virtual Reality (VR) for a wide range of clinical health conditions (Dascal et al., 2017; Freeman et al., 2017; Hoffman et al., 2011; Howard, 2017; Maples-Keller et al., 2017; Rizzo et al., 2017). Recent advances in VR technology has now allowed for the creation of artificially intelligent, conversational Virtual Human (VH) agents that can engage in credible interactions with real humans (Rizzo et al., 2016; Talbot et al., 2012). Our VH research and development has studied their use as virtual patients for clinical training, as online wellness support coaches, and now in the role of clinical interviewers. We will present findings from a social cognition study that examines differences in self-report of mental health symptoms on a standard screening checklist vs. responding to the same questions when asked by a $\mathrm{VH}$ interviewer.

Methods: 24 military service members (SMs) returning from a combat deployment in Afghanistan were administered the standard Post Deployment Health Assessment (PDHA) questionnaire under three conditions: 1) Standard official PDHA self-report checklist; 2) Anonymous PDHA checklist; 3) PDHA questions delivered in a conversational mode by a VH. Results: SMs were more willing to endorse PTSD symptoms to a VH-interviewer than on either the standard or anonymized version of the PDHA $[\mathrm{F}(2,23)=4.29, p=.02]$.

Conclusions: VH agents may provide a useful method for conducting clinically-oriented interviews resulting in more honest symptom disclosure. These findings extend previous social cognition-relevant results with civilians who disclosed more personal information and recent "sad" events to a VH agent compared to users interacting with a VH avatar that they believed was operated by a real human-in-the-loop "Wizard of $\mathrm{Oz}$ " interviewer (Lucas et al., 2014). The VH agent evoked less concern about impression management and reduced the perceived risk about being evaluated.

Correspondence: Albert "Skip" Rizzo.E-mail: rizzo@ict.usc.edu 
V.A. ANDERSON, S. HEARPS, L. CROSSLEY, M. GREENHAM, D. DARBY, S. MCDONALD, J. DOOLEY, S. HEARPS \& M.H. BEAUCHAMP. Evaluating social competency using PEERS with paediatric clinical populations.

Background: Mature social skills are important for developing and sustaining rewarding relationships and are essential to quality of life across the lifespan. Social deficits are hallmark outcomes after child acquired brain injury and neurodevelopmental disorders. However, there are currently no comprehensive, ecologically valid assessment tools of children's social skills supported by adequate normative data sets, thus limiting the ability to accurately assess the social domain or to provide tailored remediation for specific social weaknesses. The Paediatric Evaluation of Emotions Relationships and Socialization (PEERS) is an app-delivered assessment tool designed to address these gaps in paediatric social skills assessment.

Methods: 60 children aged 4-18 years with acquired brain injury and neurodevelopmental disorders completed PEERS along with an age appropriate assessment of IQ between June 2016 and June 2017. Parent report of medical and developmental history, and social behaviour was also collected. Age and sex based standard scores were derived using data collected from typically developing controls (TDC: $n=700$ ) in a large-scale standardisation study.

Results: Compared to TDC, the clinical groups displayed relatively weaknesses in specific social domains. Specifically, the acquired injury group demonstrated reduced social cognition and social communication, with the exception of ADHD group which was characterised by attention/executive function deficits.

Conclusions: Preliminary data suggest that PEERS is a useful aid in the characterisation of social strengths and weaknesses in paediatric populations with neurological conditions, helping guide clinical decision-making and tailored interventions. Further investigations using PEERS in larger sample sizes, and additional clinical paediatric populations is warranted.

Correspondence: Vicki A. Anderson, PhD, Child Neuropsychology, Level 4 East, Murdoch Childrens Research Institute, Flemington Road, Parkville,VIC 3052, Australia.E-mail:vicki.anderson@rch.org.au

S. MCDONALD, H. ROSENBERG, J. ROSENBERG \& F. WESTBROOK. Development of the Complex Assessment of Audio-Visual Emotions (CAVEAT).

Objective

There are many tests available for assessing the six so-called 'basic' emotions. There are, however, much fewer that provide assessment of the wider array of emotions typically encountered in everyday life. This study reports on the development of such an assessment measure. The Complex Assessment of Audio-Visual Emotions (CAVEAT) comprises 88 audio-visual vignettes depicting 22 distinct emotions (11 positive, 11 negative) enacted by professional actors using ambiguous scripts. In this study, the CAVEAT was given to adults with traumatic brain injury (TBI) and demographically matched healthy participants to determine its reliability and validity.

Participants and Methods

Thirty-two adults with moderate-severe traumatic brain injury (TBI) and 32 matched controls (study 1 ) and 16 participants with TBI and 12 matched controls (study 2) participated in this study. They were assessed using the CAVEAT and a number of neuropsychological and independent social cognitive tasks.

Results

People with TBI performed more poorly on the CAVEAT than those without TBI even when controlling for other cognitive impairments and mood disorders. Internal reliability for the CAVEAT was good to excellent. CAVEAT performance correlated significantly with other measures of emotion perception, social cognition, empathy and alexithymia. Performance on the subgroup of "basic" six emotions was largely similar to the rates reported in the literature.
Conclusions

The CAVEAT has demonstrated reliability and construct validity and is sensitive to deficits in emotion perception following moderate-severe TBI. Its broader focus of types of emotions should provide a useful adjunct to existing measures of emotion.

Correspondence: Skye McDonald, PhD, Psychology, University of NSW, School of Psychology, Unversity of NSW, Sydney, NSW 2052, Australia. E-mail:s.mcdonald@unsw.edu.au

H.M. GENOVA, J. LENGENFELDER, E. DOBRYAKOVA, J. DELUCA \& N. CHIARAVALLOTI. Utilization of Neuroimaging to Examine Social Cognition in Traumatic Brain Injury.

Social cognition impairments represent a significant issue in individuals with Traumatic Brain Injury (TBI). While there are a number of behavioural measures which enable us to examine social cognition deficits, these measures provide limited information about what is happening at the level of the brain. By using neuroimaging measures such as functional Magnetic Resonance Imaging (fMRI) and Diffusion Tensor Imaging (DTI), we can begin to understand how neuropathology may contribute to social cognition deficits, as well as how the brains of individuals with TBI who have social cognition impairments may function differently from healthy adults. This presentation will review several studies which have utilized fMRI and DTI to examine social cognition impairments in individuals with TBI, including a study performed by the authors of the current presentation in which 42 individuals with moderate to severe TBI and 23 healthy controls performed a task of facial affect recognition and underwent diffusion tensor imaging. Correlational analyses were performed to determine where in the brain WM damage was associated with performance on the facial affect recognition task. DTI findings suggested that damage to several key tracts including the inferior longitudinal fasciculus and inferior-fronto-occipital fasciculus were associated with reduced performance on a task of social cognition task. By utilizing neuroimaging techniques, we have a better understanding of how TBI affects neural systems underlying social cognition. These techniques provide additional information not detected by traditional measures of social cognition.

Correspondence: Helen M. Genova, Ph.D., Kessler Foundation, 300 Executive Drive, West Orange, NJ 07052, United States. E-mail: hgenova@kesslerfoundation.org

\section{Executive Functions/Frontal Lobes}

K.S. CHIOU, S. COSENTINO, T. GIOVANNETTI, M. LARSON \& P. DOCKREE. Dynamic Evaluations of Self: New Insights to Understanding the Mutli-Dimensionality of Self-Awareness.

Researchers across disciplines have long-acknowledged the multidimensional nature of self-awareness. However, neuropsychological research has historically focused on the study of one's stable, foundational knowledge of self. Recently, the field has witnessed an increased interest in understanding complementary dimensions of self-awareness that include dynamic, online, "in-the-moment" evaluations of the self during task completion (e.g., metacognitive experience, error-monitoring). The accumulating evidence implicating online self-awareness in the modulation of successful every-day living prompts a need to improve our understanding of these constructs and their clinical implications. The goal of this symposium is to highlight new directions and applications of dynamic self-awareness research that are pertinent to both healthy and clinical populations. To begin, Dr. Stephanie Cosentino will show how factors influencing an individual's perception of self and metacognitive abilities are imperative to determining the accuracy of subjective cognitive decline reported by the elderly. Next, Dr. Tania Giovannetti will identify differences found in error-monitoring abilities during completion of everyday tasks between young and older adults. Following this, Dr. Michael Larson will review how modern 
neuroimaging methodologies have been applied in self-awareness research to promote identification of underlying neural substrates. Dr. Paul Dockree will then address the clinical relevance of multi-dimensional self-awareness research, as he characterizes metacognition and error awareness in 3 different neurological samples. Finally, Dr. Kathy Chiou will show that metacognitive efficiency is positively correlated to work-related outcome after traumatic brain injury, before closing with a summarizing discussion. Together, the presentations in this symposium reflect the innovative, inter-disciplinary ideas and methodologies that are transforming the future of self-awareness research.

Correspondence: Kathy S. Chiou, PhD, Psychology, University of Nebraska, 218 Burnett Hall, Lincoln, NE 68588, United States. E-mail: kchiou2@unl.edu

R. DIVERS, E. ROLL, S.S. RYCROFT, J. HULSWIT, A. MATCHANOVA, L. HAM, K. HOWARD \& T. GIOVANNETTI. Everyday Error Monitoring in Older Adults.

Everyday error monitoring is impaired in dementia and contributes to functional disability, but relatively little is known about error monitoring in non-demented older adults. We investigated whether older vs. younger adults showed disrupted everyday error monitoring due to slowing, reduced general resources, executive difficulties, and/or poor episodic memory. 21 healthy young adults and 14 non-demented older adults completed cognitive tests of episodic memory and executive function and two everyday tasks (breakfast, lunch) while video-recorded. Young adults also completed the everyday tasks under a Dual Task condition to examine monitoring under resource limitations. Videos were reviewed for a range of errors that were classified according to the speed of monitoring (i.e., mis-reaching only error = rapid error detection vs. overt error - actual incorrect movement or use of object $=$ slower error monitoring). Correlations between errors and cognitive tests also were analyzed. Older adults made more errors than young adults when performing everyday tasks $(\mathrm{p}<.01)$. Error patterns showed older adults made significantly more errors that were detected late or never detected at all (overt errors; $p=.04$ ), whereas young adults caught most of their errors before even making contact with the incorrect object (mis-reaching). Young adults made more errors in the Dual Task than the Standard Condition, but error patterns did not differ, indicating that error monitoring did not suffer due to resource limitations imposed by the dual task. Rapid error detection was significantly correlated with stronger episodic memory $(\mathrm{r}=.55)$ but not with executive function $(\mathrm{r}$ $=.04$ ). In sum. older adults made more errors and demonstrated more failures in error monitoring than younger adults. Slow and deficient error monitoring was associated with weak episodic memory. Strategies to improve error monitoring should target the decay of task goals over time.

Correspondence: Tania Giovannetti, PhD, Psychology, Temple University, Weiss Hall, Psych Dept, 1701 N 13th St, Philadelphia, PA 19121, United States. E-mail: tgio@temple.edu

S. COSENTINO, S. CHAPMAN, P. SUNDERARAMAN, M. AZAR, L.E. COLVIN, M.E. MEINERDING, B.L. DEFEIS, E. HUEY \& B. IDNAY. Measuring Metacognition to Improve the Utility of Subjective Cognitive Decline.

Subjective Cognitive Decline (SCD) has received increasing interest as a potential marker of pre-clinical Alzheimer's disease (AD); however, SCD's inconsistent link with objective cognition challenges this idea. Variability in self-awareness, or metacognitive functioning, is likely to play a key role in determining the extent to which SCD reflects an individual's true level of cognition. We hypothesized that considering the integrity of an individual's metamemory would provide information regarding the association between SCD and objective memory. We tested our hypothesis in a sample of 36 healthy older adults who performed within normal limits on standard neuropsychological testing (i.e., all scores >- 1.5 SD). The sample was $61 \%$ female and $80 \%$ Caucasian; average age $70.03(\mathrm{SD}=8.07)$ with $16.86(\mathrm{SD}=2.52)$ years of education.
All participants completed a 20-item, "age-anchored" SCD questionnaire asking whether they had specific cognitive difficulties in relation to others their same age. Participants also completed an experimental cognitive battery including a computerized short-term memory (STM) binding task requiring them to encode new associations between colors and shapes. This task has been shown to be sensitive to pre-symptomatic $\mathrm{AD}$ in carriers of a genetic mutation for the disease, even when performance on standard diagnostic neuropsychological measures is intact. Correlations and an automated linear regression were run to examine the extent to which SCD was associated with STM binding, and whether inclusion of metamemory as a predictor improved model fit. As hypothesized, higher age-anchored SCD was associated with lower STM binding $(\mathrm{r}=-.44 ; p=.007)$. Inclusion of SCD and metamemory improved model fit $(\mathrm{AICc}=-17.00)$ as compared to SCD alone (AICc=-15.50). Thus, while age-anchored SCD is sensitive to subtle changes in cognition, objective assessment of metacognition provides important clinical information about self-awareness and may improve the utility of SCD. Correspondence: Stephanie Cosentino, PhD, Neurology, Columbia University Medical Center, 630 West 168th, New York, NY 10804 , United States. E-mail:sc2460@cumc.columbia.edu

K.S. CHIOU, H.M. GENOVA, J. LENGENFELDER \& N. CHIARAVALLOTI. Investigation of Retrospective Monitoring and Functional Living Skills After Traumatic Brain Injury.

Objective: Recent research has shown that persons with traumatic brain injury (TBI) make less accurate metacognitive confidence judgments than healthy peers. However, it remains unclear whether reduced metacognitive accuracy translates to "real" life functional deficits. Thus, the goal of this study was to determine how metacognitive processes are related to functional living skills in adults with TBI. Participants \& Methods: 14 adults with moderate to severe TBI and 16 healthy peers completed a non-verbal learning task that included a computerized, delayed recognition trial. Retrospective confidence judgments (RCJs) and reaction times (RT) of the RCJ were collected after each item of the recognition trial. Participants also completed the Community Integration Questionnaire (CIQ) which assessed levels of functioning in everyday living. Independent samples t-tests were used to determine between group differences in RCJ gamma coefficients as well as RCJ RTs. Correlation analyses were used to examine the relationship between metacognitive performance and CIQ subscales. Results: RCJ gamma coefficients between adults with TBI and healthy peers did not differ significantly; however, adults with TBI had longer RCJ RTs compared to the healthy sample $(t[28]=2.3, p=0.03)$. For adults with TBI, no significant associations were found between RCJ gamma coefficients and subscales of the CIQ, but slower RCJ RTs were significantly correlated with lower levels of job re-integration $(r=-0.57, p=0.03)$. Conclusions: The results suggest that adults with TBI are at a temporal disadvantage when making metacognitive judgments. Furthermore, the speed of making a metacognitive judgment was significantly related to the ability to successfully return to work after injury. These findings suggest that assessments of the temporal aspects of metacognitive processes in particular, can provide important prognostic information about the likelihood of successful functional independence after TBI.

Correspondence: Kathy S. Chiou, PhD, Psychology, University of Nebraska, 218 Burnett Hall, Lincoln, NE 68588, United States. E-mail: kchiou2@unl.edu 
M. LARSON. Neuroimaging Approaches to Understanding Deficits in Awareness and Relationships with Illness Severity and Daily Functioning.

Objective: Individuals with traumatic brain injury (TBI) and neurodegenerative diseases, and some psychiatric disorders (e.g., OCD, schizophrenia), can show alterations in awareness of deficits. Lack of awareness can have a detrimental effect on rehabilitation, therapy, and medical adherence. Advances in neuroimaging techniques, including electroencephalogram [EEG] and event-related potentials [ERPs] and functional MRI, may provide insight into the neural substrates of awareness and risk factors for poor prognosis in rehabilitation and course of medical illness/recovery. The purpose of this study was to outline the broad contribution of neuroimaging studies to error awareness and provide details on specific relationships between awareness and illness severity and functional abilities. Methods: Neuroimaging studies using the Error Awareness Task (EAT) or other cognitive control tasks were reviewed, including data from participants in our own lab with moderate-to-severe TBI and OCD. Neural substrates of awareness and associations between awareness, illness severity, and functional impairment were tested using correlational methods. Results: Inferior parietal and insular cortices are largely implicated in a larger network that is sensitive to error awareness, while data are conflicting on the dorsal anterior cingulate (ACC) and prefrontal cortex activity. Individuals with TBI showed deficits in behavioral and day-to-day awareness that were related to injury severity but inconstantly related to ERP indices associated with awareness (e.g., the Pe component of the ERP). Individuals with OCD and poor awareness showed increased OCD severity, increased functional impairment, and poorer inhibition abilities. Conclusions: Awareness is associated with a complex network that includes insular (likely due to autonomic responses) and parietal cortices and some ambiguity in the role of the ACC. Error awareness is worse with increased illness severity and impacts functioning. Correspondence: Michael Larson, UT, United States. E-mail: michael_ larson@byu.edu

P. DOCKREE, L. FITZGERALD, E. LACEY, B. LAWLOR \& I. ROBERTSON. Assays of Metacognition Across Different Patient Populations: Implications for Understanding Impaired SelfAwareness.

Laboratory paradigms that assay meta-level judgments of confidence or error awareness provide a means to probe the neurocognitive processes that contribute to self-awareness and their breakdown in different patient populations. I will first present evidence to show that error awareness declines with natural aging and that its electrophysiological signature (error positivity: $\mathrm{Pe}$ ) is reduced in amplitude and slope build-up rate compared to young adults. Second, we show that further decrements in error awareness are seen in mild Alzheimer's Dementia (AD) patients relative to healthy older adults who were both matched in terms of task accuracy achieved through a staircase procedure. Error awareness was also correlated with a common screening tool, the Montreal Cognitive Assessment (MoCA) in the AD patients. These findings are discussed in the context of neuropsychological models of cognitive decline. Finally, the question of whether self-awareness operates as a domain general process or as a set of independent domain specific processes was investigated in both patients with traumatic brain injury (TBI) and stroke. Here, we captured "online" metacognitive processes, as measured by decision confidence judgments in the domains of perception and memory. Neuropsychological and electrophysiological findings from these brain injury patients suggest dissociable metacognitive systems may exist. The implications for the refinement of metacognitive assessment in clinical populations is also discussed.

Correspondence: Paul Dockree. E-mail: dockreep@tcd.ie
AM Coffee Break

10:30-10:45 a.m.

Symposium 12. Health-Promoting Activities as
Protective Factors Underlying Disease Outcomes in
MS

Chair \& Discussant: Peter Arnett

Presenters: Margaret Cadden, Brian Sandroff, Robert Motl, Cristina A.F. Roman

10:45 a.m.-12:15 p.m.

P. ARNETT, M. CADDEN, B. SANDROFF, R. MOTL \& C.A.F. ROMAN. Health-Promoting Activities as Protective Factors Underlying Disease Outcomes in MS.

Engagement in health-promoting activities such as exercise, cognitive stimulation, and socializing has seen increased study in neurological populations in recent years. Multiple Sclerosis (MS) has been an appealing disorder to study, because those with MS are often diagnosed in early to mid-adulthood, and usually live for many years following diagnosis. Thus, interventions involving the promotion of health-promoting activities could potentially have long-term positive functional consequences. With these considerations in mind, this symposium will examine health-promoting activities in MS as they relate to reducing depression and fatigue, improving cognitive functioning and muscle strength, and being associated with adaptive brain network changes in MS. Peter Arnett will open the symposium by providing an overview of these issues. Then Margaret Cadden will show that higher levels of Cognitive Reserve (CR) operationalized using traditional (i.e., vocabulary, education) and malleable (i.e., engagement in exercise, cognitive stimulation, and socializing) methods are protective against disease-related disability in relation to depression in MS. Brian Sandroff will then discuss a series of studies showing that aerobic, progressive treadmill walking exercise training is an optimal intervention for improving cognitive processing speed in fully-ambulatory persons with MS. Robert Motl will demonstrate that exercise training in MS reduces fatigue and depression, in addition to improving lower-extremity muscle strength and cardiorespiratory fitness. Finally, Cristina Roman will show that individuals with MS who walk more frequently each week have more efficient structural brain networks. She will also demonstrate that engagement in exercise is associated with less depression in individuals with less efficient structural networks. Subsequent to these talks, Peter Arnett will serve as a discussant regarding the clinical and theoretical implications of these findings.

Correspondence: Peter Arnett, Ph.D., Psychology, Penn State University, 352 Moore Building, University Park, PA 16802, United States. E-mail: paa6@psu.edu

M. CADDEN, C.A.F. ROMAN, M. LONG \& P. ARNETT. Cognitive Reserve, Depression, and Cognition in Multiple Sclerosis.

Objective. The goal of the current study was to explore the relationship between measures of cognitive reserve and measures of disease burden on depression and cognition in a sample of individuals in which brain pathology is thought to contribute to cognitive difficulties and depression (Multiple Sclerosis - MS).

Participants and Methods. Fifty-four individuals with MS participating in a neuropsychological and neuroimaging research study were examined. Cognitive reserve (CR) was operationalized in two ways: Crystalized CR (sum of Shipley Vocabulary and years of education), and malleable CR (composite consisting of measures of physical exercise, engagement in intellectually stimulating activities, and socializing from the Cognitive Health Questionnaire). Regressions on depression (BDI-FS) examining CR, EDSS, and their interactions were explored. 
Regressions were then repeated with thalamic volume as the measure of disease burden in lieu of EDSS. Traditional models of CR were investigated using cognition (SDMT) as the dependent variable.

Results. In regards to predicting depression, the interaction between EDSS and both CR measures were significant, $\mathrm{F}(4,50)=6.7, \mathrm{p}=.013$, $\mathrm{F}(4,47)=4.1, \mathrm{p}=.049$. Interactions between $\mathrm{CR}$ and thalamic volume were not significant. In regards to predicting cognition, crystalized CR significantly interacted with EDSS and there was a trend for an interaction with thalamic volume, $\mathrm{F}(5,48)=4.7, \mathrm{p}=.035, \mathrm{~F}(6,48)=3.96$, $\mathrm{p}=.053$. Interactions between malleable CR and measures of disease burden were not significant.

Conclusions. Both crystalized and malleable operationalizations of CR moderated the relationship between EDSS and depression in MS. Higher levels of both conceptualizations of CR appear to be protective against disease-related depression. Crystalized CR moderated the relationship between measure of disease burden and cognition. Higher levels of education and verbal IQ appear to be protective against disease-related cognitive impairment in MS.

Correspondence: Margaret Cadden, Psychology, The Pennsylvania State University, 372 Moore Building, University Park, State College, PA16802, United States. E-mail: mhc147@psu.edu

\section{B. SANDROFF. Exercise and Cognition in Persons with Multiple Sclerosis.}

Objective: Exercise training has been identified as a highly-promising approach for managing the pervasive and burdensome cognitive consequences of multiple sclerosis (MS). Using the literature in the general population as a backdrop, this presentation will discuss emerging data on the effects of objectively-measured exercise, physical activity, and physical fitness on cognition and brain structure/function in persons with MS.

Participants and Methods: The present line of research involved a novel framework for optimizing future exercise interventions for improving cognition in MS. This involved the systematic development of high quality exercise training interventions (i.e., randomized controlled trials) based on cross-sectional examinations of physical fitness and cognition, as well as the effects of single bouts of exercise (i.e., acute exercise) on cognition in MS. This line of research included 4 cross-sectional studies of physical fitness ( $\mathrm{N}=185$ persons with MS), 3 studies of physical activity ( $\mathrm{N}=366$ persons with MS), 2 studies of acute exercise ( $\mathbf{N}=48$ persons with MS), and 2 studies of exercise training ( $\mathrm{N}=93$ persons with MS) effects on cognition in persons with MS.

Results: This systematic line of research suggests that such an optimal exercise training intervention might involve aerobic, progressive treadmill walking exercise training for improving cognitive processing speed in fully-ambulatory persons with MS. Indeed, such an intervention was recently performed as a pilot randomized controlled trial and resulted in large improvements in cognitive processing speed $(d=0.95)$ and thalamocortical functional connectivity $(d>1.70)$.

Conclusions: Such a systematic approach has the promise to ultimately improve and optimize exercise training interventions on cognition in MS and further highlights select neural networks as potential targets for rehabilitation interventions for improving cognitive processing speed in cognitively-impaired persons with MS.

Correspondence: Brian Sandroff. E-mail: sandroff@uab.edu

\section{R. MOTL. Exercise and Its Benefits in Persons with Multiple Sclerosis.}

Objective: Exercise training represents an efficacious approach for managing the pervasive and burdensome consequences of multiple sclerosis (MS). This presentation will provide an overview of data regarding the effects of exercise training on aerobic and muscle fitness, fatigue and depression, and mobility in persons with MS.

Methods: Papers for this presentation were identified by searches of PubMed, Google Scholar, Scopus, and CINAHL between January, 2012 and July, 2017. The search terms were 'multiple sclerosis', 'exercise', 'physical activity', 'physical fitness', and 'rehabilitation.' We focused on meta-analyses, Cochrane reviews, and narrative, systematic reviews to capture the broad scope and overall effect of exercise on various consequences of MS.

Results: To date, there are over 60 clinical trials of exercise training in persons with MS, with over 50 studies published in the last 10 years alone. There is evidence for small improvements in lower-extremity muscle strength $(d=0.27)$ following resistance exercise training, and moderate-sized improvements in cardiorespiratory fitness $(d=0.63)$ following aerobic exercise training. Quantitative syntheses have reported overall statistically significant and moderate-sized reductions in fatigue $(d=0.57)$ following exercise training in persons with MS. The meta-analyses reported small $(d=0.37)$, but consistent beneficial effects of exercise on depressive symptoms. The studies demonstrated small $(d=0.25)$, but beneficial effects of exercise training on walking speed and walking endurance.

Conclusions: Collectively, exercise is one of the best rehabilitation approaches for managing symptoms, restoring function, promoting wellness, and boosting participation in activities of daily living in MS. Future research should improve upon the quality and scope of existing evidence regarding the benefits of exercise training for better informing clinical care of people with MS.

Correspondence: Robert Motl. E-mail:robmotl@uab.edu

C.A.F. ROMAN, M. CADDEN \& P. ARNETT. Exercise and Structural Connectivity in Multiple Sclerosis: A Graph Theoretical Perspective.

Objective. Exercise has been shown to be beneficial for brain health across numerous capacities (i.e., mood, cognition) and populations, MS included. Yet, many studies in MS have taken a behavioral perspective when examining the positive impact of exercise on functioning, leaving the relationship between exercise and structural brain networks unclear. The current study utilizes graph theory to investigate the relationship between exercise and structural connectivity and their relationship to depression.

Participants and Methods. Fifty-four individuals with MS were scanned during a DTI protocol and evaluated for depression (BDI-FS) and cognitive functioning. DSI Studio was used to implement a whole brain deterministic tractography algorithm. Graph theory metrics were calculated to examine white matter network properties. Exercise was quantified as the 'number of days walked per week.' Sedentary behavior was measured by 'hours per day sitting.'

Results. After controlling for age and physical disability (EDSS), "number of days walked per week' was found to be significantly related to clustering, small worldness (trend), and local efficiency, such that more efficient structural networks were present in those individuals who engaged in more physical activity per week. Alternatively, more hours sitting per day predicted less efficient structural connectivity. Multiple regression interaction analyses were also conducted to examine the predictive power of structural connectivity $\mathrm{x}$ exercise on depression. Results showed that for those individuals with less efficient structural networks, engagement in exercise significantly decreased depression. Conclusions. To the authors' knowledge, this is the first study to examine the relationship between exercise and sedentariness, structural brain networks, and depression in MS using a graph theoretical framework, pointing to possible brain altering benefits of physical activity in MS patients.

Correspondence: Cristina A.F. Roman, B.S. , Psychology, The Pennsylvania State University, 939 Southgate Drive, State College, PA 16801, United States. E-mail: cristina.a.roman@gmail.com 
Paper Session 17. Cancer Across the Lifespan

\author{
Moderator: Jeffrey S. Wefel \\ 10:45 a.m.-12:15 p.m.
}

C.M. SHARKEY, A. GIOIA, G. DOME, K.S. WALSH \& K.K. HARDY. Relations Between Observer- and Performance-Based Outcomes in Pediatric Cancer Survivors: Differences as a Function of Disease and Informant.

Objective: Concordance between questionnaire and performance-based measures is sometimes low in neuropsychological evaluations of pediatric cancer survivors. Knowledge of when, why, and how discrepancies occur can potentially improve clinical decision-making and recommendations. Thus, we aimed to examine relations between informant-reported and performance-based cognitive difficulties.

Participants and Methods: Clinical data from 197 survivors of leukemia (ALL; 57\%) and brain tumor (BT, 43\%) aged 6-18 (M=11.6, $S D=3.66,54 \%$ male) were abstracted. Parents and teachers rated cognitive and psychosocial functioning (BRIEF, CBCL). Children completed age-appropriate Wechsler scales and other measures.

Results: Parents reported global executive functioning (GEC) problems and working memory (WM) problems ( $\mathrm{T} \geq 65$ ) among $21 \%$ and $38 \%$ of the sample, respectively, and approximately one-quarter exhibited Wechsler FSIQ $(25 \%)$ and WMI (28\%) scores at least 1SD below the mean. Across diagnostic groups, greater teacher-reported executive functioning problems were associated with significantly lower FSIQ $(p<.02)$. BT survivors with parent-reported WM and/or GEC problems had lower WMI and FSIQ $(p<.05)$. Pearson's correlations between parent- and teacher-report and FSIQ were moderate for survivors of BT ( $r=-.41$ and -.42 , respectively). In contrast, ALL survivors with parent-reported WM and/or GEC problems had higher WMI and FSIQ scores than those without $(p<.05)$. Of interest, only teacher reported GEC, not parent-report, was associated with FSIQ ( $r=-.25$ vs. .13) for survivors of ALL.

Conclusions: Parents' and teachers' observations of executive dysfunction aligned more closely with performance-based measures for survivors of BT than for ALL. It may be that parents of ALL survivors are observing subtle or emerging EF impairments in the context of an otherwise intact cognitive profile. Results underscore the importance of interpreting data from multiple informants to optimize benefit of neuropsychological surveillance for BT and ALL survivors.

Correspondence: Christina M. Sharkey, M.S., Psychology, Oklahoma State University, $4599 \mathrm{~N}$ Washington St. Apt 6L, Stillwater, OK 74075, United States.E-mail: christina.sharkey@okstate.edu

K. VAN DER WILLIK, R. RUITER, F. WOLTERS, K. IKRAM, B. STRICKER, M. HAUPTMANN, A. COMPTER, S.B. SCHAGEN \& A. IKRAM. Mild Cognitive Impairment and Dementia Show Contrasting Associations with Risk of Cancer.

Objective: Multiple studies have shown a decreased risk of cancer in patients with dementia. Various biological mechanisms have been proposed for this link, but methodological bias has not been satisfactorily ruled out. Mild cognitive impairment (MCI) represents an earlier clinical manifestation of the disease process underlying dementia. We investigated the relation between MCI and cancer and contrasted that with the association between dementia and cancer.

Participants and Methods: 13,207 persons from the Rotterdam Study were followed between 1990 and 2013 for the onset of dementia and cancer. Between 2002 and 2005, a subset of 5,181 persons underwent extensive cognitive testing for MCI and subsequently were followed up for cancer until 2013. We used Cox proportional hazard models to determine the association between dementia and cancer and MCI and cancer. We repeated analyses lagged by two and five years to minimize potential effects of reverse causality.

Results: In total 1,404 patients were diagnosed with dementia, and 2,316 developed cancer (63 among dementia cases). Dementia was associated with a decreased risk of cancer (hazard ratio (HR) 0.53; 95\% CI 0.41-0.68). 513 persons were diagnosed with MCI and during follow-up 670 persons developed cancer (81 among MCI cases). In contrast to individuals with dementia, those with MCI tended to have an increased risk of cancer (HR 1.25; 95\% CI 0.99-1.58). Using a lag interval of two and five years did not affect the difference between dementia and MCI with respect to cancer risk.

Conclusions: We found that persons with MCI might be at an increased risk of cancer, whereas those with dementia have a decreased risk. These findings call into question a biological explanation for the inverse link between dementia and cancer, instead suggesting methodological bias. Research efforts need to be redirected towards understanding shared mechanisms.

Correspondence: Kimberly van der Willik, Drs, Netherlands Cancer Institute/ Erasmus Medical Center, Puccinistraat 2, Barendrecht 2992RK, Netherlands. E-mail: kimberlyvanderwillik@gmail.com

I. TONNING OLSSON, T.M. BRINKMAN, G. HYUN, P. BANERJEE, D.A. MULROONEY, I. HUANG, D. GREEN, D. SRIVASTAVA, L.L. ROBISON, M.M. HUDSON \& K.R. KRULL. Neurocognitive Outcomes in Long-Term Survivors of Childhood Wilms Tumor.

Objective: To examine neurocognitive outcomes, social attainment, emotional distress and Health Related Quality of Life (HRQOL) in long-term survivors of Wilms Tumor (WT) who are at risk for cardiac, pulmonary and endocrine disorders.

Participants and Methods: 148 survivors of WT (58.8\% female; mean[SD] age 33[9.1] years; time since diagnosis 29 [9.1] years) and 354 community controls (55.6\% female; $35[10.2]$ years) completed comprehensive neuropsychological testing, echocardiography/electrocardiography, pulmonary function testing, endocrine evaluation, and physical examination. Self-reports of emotional distress, HRQOL and social attainment were collected. Impairment was defined in relation to both controls and test norms (neurocognitive function) or to just controls (all other variables). T-tests were used for continuous variables and chi-square for categorical variables, adjusting for age and race. Adjusted generalized linear models were developed to examine impact of treatment and chronic health conditions (graded per the Common Terminology Criteria for Adverse Events).

Results: Survivors demonstrated cognitive impairment (z-scores; mean[sd]; verbal reasoning $-0.39[1.12]$, sustained attention $-0.47[1.35]$ ), as well as higher emotional distress (somatization $-0.15[0.97]$ ) and lower HRQOL (social functioning $-0.10[1.05]$ ). Survivors were less likely to graduate college (OR=2.23 95\%CI 1.46-3.41) and had more moderate to severe neurologic conditions $(18.9 \% \mathrm{v} 8.2 \%$, $p=0.001)$, which were associated with poor memory $(\beta=-0.61, p=0.02)$, attention $(\beta=-0.59, p=0.04)$, and multiple HRQOL indices compared to controls. Cardiopulmonary morbidity (higher in survivors) and treatment variables were not associated with impairment on any outcomes. Conclusions: Survivors of WT during childhood show impairment in cognition and HRQOL, lower social attainment and higher emotional distress during adulthood. Poorer cognitive performance and HRQOL were associated with neurologic conditions.

Correspondence: Ingrid Tonning Olsson, Ph.D, Epidemiology and Cancer Control, St Jude Children's Research Hospital, 262, Danny Thomas Place, MS-735, Memphis, TN 38105, United States. E-mail: ingrid.tonningolsson@stjude.org.

P. BANERJEE, M. ROSSI, D. ANGHELESCU, A. MAYHEW, K. REGER, N. PHILLIPS, W.E. REDDICK, J. GLASS, L.M. JACOLA, L.L. ROBISON, C. PUI, M.M. HUDSON \& K.R. KRULL. Anesthesia Exposure, Neurocognitive Function, and Neuroimaging Outcomes in Long-Term Survivors of Childhood Acute Lymphoblastic Leukemia.

Objective: To examine anesthesia variables in association with neurocognitive function and regional brain volumes in long-term survivors of childhood acute lymphoblastic leukemia (ALL). 
Participants and Methods: 5,698 anesthetic procedures were performed in 212 ALL survivors (mean[SD] age 14.4[4.8] years; 7.7[1.7] years post-diagnosis) who were treated with chemotherapy only. Data were abstracted for cumulative dose of anesthetic agents and number, type, and duration of anesthetic procedures. At $>5$ years post-treatment, neurocognitive testing and structural brain MRI were conducted. Multivariable analyses were used to examine anesthesia variables in association with neurocognitive function (age-adjusted Z-scores) and regional brain volumes, adjusting for age, sex, and chemotherapy. Results: Higher frequency of anesthetic procedures (mean[range] $27[12-48])$ was associated with smaller volumes of pallidum $(p=0.02)$ and entorhinal white matter ( $\mathrm{WM} ; \mathrm{p}=0.02)$. Longer anesthesia duration was associated with worse performance on Block Design $(p=0.02)$, Digit Symbol $(p<0.01)$, Trail Making $(p=0.02)$, and Rey Figure $(p=0.05)$, and smaller volumes of pallidum $(p=0.03)$, entorhinal $\mathrm{WM}(\mathrm{p}=0.02)$, and rostral middle frontal WM $(p=0.02)$. Higher cumulative dose of fentanyl was correlated with lower Rey Figure $(p=0.01)$ and Digit Symbol $(p=0.02)$ scores, and smaller supramarginal gyrus volume $(\mathrm{p}<0.01)$. Higher cumulative dose of propofol was associated with worse performance on Trail Making ( $p=0.03)$, Block Design ( $p=0.05)$, CPT-2 Omissions $(p=0.02)$, Spatial Span Forward $(p=0.01)$, and Stroop Inhibition $(p=0.01)$, and smaller supramarginal gyrus volume $(p=0.02)$. Conclusions: In long-term ALL survivors, longer anesthesia duration and higher cumulative doses of fentanyl and propofol were associated with impaired neurocognitive function and smaller volumes in related brain regions, adjusting for age, sex and chemotherapy intensity. Greater anesthesia exposure combined with neurotoxicity from chemotherapy may result in greater adverse impact in ALL survivors.

Correspondence: Pia Banerjee, PhD, Epidemiology and Cancer Control, St. Jude Children's Research Hospital, 262 Danny Thomas Place, MS 735, Room S6010, Memphis, TN 38105, United States. E-mail: piabanerjee@gmail.com

\section{Paper Session 18. Memory Function}

\section{Moderator: Amanda Gooding}

\section{0:45 a.m.-12:15 p.m.}

\section{K.L. SULLIVAN, S.P. WOODS, R.S. BUCKS, S. LOFT \& M. WEINBORN. Intraindividual Variability in Neuropsychological Test Performance Is Associated With Time-Based Prospective Memory in Older Adults.}

Objective: Older adults demonstrate poorer prospective memory (PM) performance compared to younger adults, particularly for time-based PM cues and other strategically demanding PM tasks. Intraindividual variability (IIV) in neuropsychological test performance is an index of cognitive control that may play an important role in the execution of strategically demanding PM tasks.

Participants and Methods: Participants included 194 Australian older adults (age 50 to 88) who completed the Memory for Intentions Screening Test (MIST), the Prospective and Retrospective Memory Questionnaire (PRMQ), and clinical measures of executive functions. A measure of naturalistic time-based PM was also administered in which participants were asked to call the examiner 24 hours after their appointment to report how many hours they slept. IIV was calculated as the mean-adjusted coefficient of variation $(\mathrm{CoV})$ across subtests of the Repeatable Battery for the Assessment of Neuropsychological Status (RBANS).

Results: Higher IIV was associated with poorer time-based laboratory PM $(r=-.22, p<.01)$ and poorer naturalistic time-based PM performance, $F(2,189)=3.39, p=.04$. Of particular interest, IIV was a significant predictor of time-based laboratory PM independent of demographics and other executive functions $(p \mathrm{~s}<.05)$. However, IIV was not related to event-based laboratory PM $(r=-.07, p=.33)$ or self-reported PM symptoms $(r=-0.02, p=.78)$.
Conclusions: These results indicate that cognitive control as indexed by IIV in neuropsychological test performance plays a unique role in naturalistic PM, as well as in highly strategic, but not automatic, laboratory-based PM among older adults. In particular, IIV may provide incremental value in predicting risk for PM failures in daily life above standard clinical measures of executive functions.

Correspondence: Kelli L. Sullivan, Psychology, University of Houston, University of Houston, 126 Heyne Building, Houston, TX 77004-5022, United States.E-mail:klsullivan3@uh.edu

L. HOKKANEN, E. LEHTO, J. LAUNES, M. VIRTA \& K. MICHELSSON. Prospective Memory - an Evaluation of the PROPS Test and the Impact of Low Birth-Weight and Other Perinatal Risks in Adulthood.

Objective: Low birth-weight (LBW) and other perinatal risks have been found to associate with cognitive deficits. Their impact on prospective memory functions in adulthood have not been studied before. The aims of the study were to evaluate the structure of the new Finnish Proper Prospective Memory Test (PROPS) and to explore the impact of LBW and other perinatal risks on prospective memory performance in adulthood.

Participants and Methods: Perinatal Adverse Events and Special Trends in Cognitive Trajectory (PLASTICITY)-project prospectively follows a cohort of subjects from birth. Participants were part of the risks cohort born in 1971-1974. The latest follow-up study took place at the age of 40 . The sample consisted of a LBW (2000 g) group $(\mathrm{n}=72)$, other perinatal risks group $(\mathrm{n}=303)$ and a control group with no early risks $(n=83)$. Prospective memory performance was assessed using the PROPS test both in laboratory (lab) and naturalistic (nat) setting. The statistical methods included confirmative factor analysis with Tucker-Lewis (TLI) and Comparative Fit -indices (CFI), and ANCOVA with Holm's Bonferroni correction.

Results: After fitting the model based on the modification indices by adding the covariance between the two nat subtests, the time-based (TB) and event-based (EB) prospective memory factors were confirmed in PROPS $($ TLI $=1.0$, CFI $=1.0)$. Adjusted for age, sex and education, the groups differed in PROPS-total score $(p=.02)$ and the EB-tasks $(\mathrm{p}=.03)$. LBW group showed impaired prospective memory performance compared to the control group in the lab tasks $(p<.01)$ but not in the nat tasks. The other perinatal risks group did not differ from the control group in PROPS performance.

Conclusions: PROPS test appears a valid tool in clinical assessment of the multifaceted prospective memory performance. Low birth-weight is associated with impaired prospective memory in adulthood.

Correspondence: Laura Hokkanen, PhD, Department of Psychology, University of Helsinki, P.O.Box 21, Helsinki 00014, Finland. E-mail: laura.hokkanen@helsinki.fi

A. ALKOZEI, R. SMITH, N.S. DAILEY, S. BAJAJ, S. KNIGHT \& W.D. KILLGORE. Exposure to Blue Wavelength Light During Memory Consolidation Improves Long-Delay Verbal Memory Performance.

Objective: Exposure to blue wavelength light has been shown to lead to increased alertness and improved performance on reaction time tasks, possibly due to noradrenergic influences. However, whether higher-order aspects of cognition can also be improved remains to be determined. The aim of this study was to investigate the effects of thirty minutes of blue versus placebo wavelength light exposure during memory consolidation on verbal memory performance.

Participants and Methods: Thirty healthy men and women (17 female; Mean age $=21.9 \pm 3.7$ ) completed the five learning trials of the California Verbal Learning Test (CVLT-II). Sixty minutes later, participants were randomized to receive either 30 minutes of blue $(n=12)$ or amber $(n=18)$ wavelength light. Fifteen minutes after light cessation, participants completed the long-delay verbal recall portion of the CVLT-II. Participants also completed the Wechsler Abbreviated 
Scale of Intelligence as a measure of general intelligence (IQ) and the Beck Depression Inventory as a measure of depressive symptoms.

Results: Blue light was associated with significantly better long-delay verbal recall compared to amber placebo, while controlling for the effects of depressive symptoms and IQ $(\mathrm{F}(1,26)=5.23, \mathrm{p}=.03)$. While participants in the blue light group forgot an average of 0.23 words, participants in the amber light group forgot an average of 1.81 words from short-delay to long-delay memory recall.

Conclusions: Exposure to blue light during consolidation enhanced memory recall. Memory consolidation is facilitated by increased norepinephrine release, which has been shown to be stimulated by exposure to blue light. This has important implications for the potential use of blue light as a safe way to improve memory in healthy and clinical populations with memory deficits. Future research will be necessary to extend these findings to clinical populations, and to identify the optimal timing and dose of blue light exposure in this context.

Correspondence: Anna Alkozei, Ph.D., Department of Psychiatry, University of Arizona Medical Center, University of Arizona Medical Center, Department of Psychiatry, 1501 N Campbell Ave, Tucson, AZ 85724, United States. E-mail: aalkozei@psychiatry.arizona.edu

J.F. BEATTIE, R. MARTIN, R. KANA, E.W.COOK, M.D. THOMPSON \& L. VER HOEF. Hippocampal Dentation in Healthy Children and Adolescents: Associations with Age and Verbal Episodic Memory. Objective: Surface convolutions of the hippocampal CA1 subfield ("dentation") are an under-studied morphological feature of the human brain. Recently, Fleming Beattie and colleagues demonstrated a positive association between dentation and aspects of verbal and visual episodic memory performance (EM) in healthy adults (2017). The present study investigates development of dentation as well as its association with verbal EM in healthy children and adolescents.

Participants and Methods: Structural MRI and neuropsychological data were obtained from the Cincinnati MR Imaging of NeuroDevelopment (C-MIND) database, supported by the Eunice Kennedy Shriver National Institute of Child Health and Human Development (HHSN275200900018C). We examined the relationship between age and number of dentes in 91 participants ( 9 months to 18 years). We also examined dentation and verbal EM (i.e., WRAML-2 Story Memory, Verbal Learning) in 75 participants ( 5 to 18 years).

Results: We found a positive association between age and total number of dentes $(\mathrm{r}=0.36, p=.001)$. Variability in dente count also increased with age, such that dentes ranged from absent to numerous among older children and adolescents. Number of total dentes was associated with the amount of narrative information recalled after a delay (\% Recall) $(\mathrm{r}=0.20, p=.05)$, but when age-adjusted scaled scores were used this relationship was not significant. No association was observed with listlearning $(p>.05)$.

Conclusions: Dentation tends to be positively associated with age during development; however, importantly, not all individuals develop visible dentation. We observed prominent variability in number of dentes among adolescents. Dentation was associated with semantic verbal EM, but not rote list-learning. Future directions include longitudinal analyses as well as examining dentation in relation to other EM modalities (e.g., visuospatial), in older adults, and in both children and adults with memory impairment.

Correspondence: Julia F. Beattie, M.A., Medical/Clinical Psychology, University of Alabama at Birmingham, 807 Lakeshore Ridge, Birmingham, AL 35211, United States. E-mail: flemingj@uab.edu

T. KUHN, K. JAPARDI, C.T. EBRAHIMI, D. GHAHREMANI, L. MARTIN-HARRIS, R.M. BILDER \& S. BOOKHEIMER. Early Intellectual Giftedness is Associated with White Matter Integrity in Regions Subserving Episodic Memory.

Objective: Studying the neuro-structural markers of intellectual giftedness (IG) will inform the scientific understanding of the processes that help children excel academically and creatively. While unique cognitive profiles and increased cortical thickness have been associated with IG, little is known about the relationship between IG and white matter integrity. Therefore, this study sought to determine whether (1) IG children evidence increased white matter integrity (2) white matter integrity is associated with IQ.

Participants and Methods: IG was defined as an IQ, measured using the Stanford-Binet, greater than 145. Tract Based Spatial Statistics (TBSS) was conducted on IG $(\mathrm{n}=16)$ and well-matched, normally developing (IQ 95-115; n = 11) children. Voxelwise two-tailed t-tests were conducted comparing fractional anisotropy (FA) and mean diffusivity (MD) between group. These analyses were conducted in nine regions of interest (ROIs): eight bilateral white matter tracts as well as forceps major and minor. TBSS was then used to correlate FA and MD with IQ within each ROI across the entire sample.

Results: FA in the white matter of the right anterior thalamic radiation (ATR) and right hippocampus was significantly higher $(0.001<\mathrm{p}<0.05)$ in the IG group compared to controls. MD was lower in bilateral ATR, hippocampus and uncinate fasciculus (UF) in the IG group compared to controls. Across the entire sample, FA was positively correlated with IQ in bilateral ATR and hippocampus. MD was negatively correlated with IQ in bilateral ATR, left hippocampus and left UF.

Conclusions: IG children evidenced increased integrity of white matter in regions associated with episodic memory relative to controls. Higher IQ was associated with higher white matter integrity in these same regions. This may relate to the propensity for gifted children to show higher levels of intrinsic motivation to read, think and spend time alone and may inform scientific and clinical advancements based on neurologic principles of exceptional intelligence.

Correspondence: Taylor Kuhn, PhD, Psychiatry \& Biobehavioral Sciences, University of California, Los Angeles, 760 Westwood Plaza, C8-749, Los Angeles, CA 90095, United States.E-mail:tkuhn@mednet. ucla.edu

L.J. WOODWARD, S. BORA, J. HORWOOD, A.R. MORRIS, T.A. LEVINE \& B. DARLOW. Visuospatial Working Memory Impairments in Very Preterm Survivors Persist from Childhood to Adulthood.

Objective: Between 40-50\% of children born very preterm (VPT) are subject to global cognitive delay. However, less is known about the specific nature of their neuropsychological difficulties and whether or not these improve with age. This study examined the visuospatial working memory performance of two cohorts of preterm survivors: a regional cohort assessed at age 12 and a national cohort assessed at age 26-28 years.

Participants and Methods: Data were drawn from two New Zealand cohorts studied prospectively from birth to age 12 (cohort 1) and 26-28 years (cohort 2 ). Cohort 1 consisted of a regionally representative sample of 110 VPT (23-33 weeks) and 106 full term (FT: 38-41 weeks) born children (97\% retention). Cohort 2 consisted of a national sample of all very low birth weight (VLBW; $<1500 \mathrm{~g}$ ) survivors born during 1986 $(\mathrm{n}=325,77 \%$ retention) and a FT comparison group $(\mathrm{n}=100)$. Both cohorts completed a visuospatial working memory task based on the Sternberg paradigm. Subjects were presented with an object display to memorize and then asked to recall whether one of the objects was in the same location or not following a 5-second delay. There were 60 trials divided into 5 blocks with 4 difficulty levels randomized across trials and blocks (1-4).

Results: An almost identical pattern of results was found in both cohorts. At level 1 (1 object), the accuracy of both groups was similar. However, as task difficulty increased, performance in the two VPT groups became increasingly impaired relative to their FT peers $(p<.007)$. VPT/VLBW subjects responded slower than FT subjects across all difficulty levels $(p<.02)$. These between group differences were reflected in task efficiency scores, with the VPT/VLBW groups being less efficient than FT groups on levels $1-4(p<.05)$.

Conclusions: These findings suggest that visuospatial working memory impairments persist from middle childhood into adulthood and are 
likely to impact long-term everyday functioning, quality of life, and academic and occupational achievement.

Correspondence: Lianne J. Woodward, PhD, Pediatric Newborn Medicine, Harvard Medical School, Brigham and Women's Hospital, 75 Francis Street, Boston, MA 02115, United States. E-mail: Liannejwoodward@gmail.com

\section{Paper Session 19. Cerebrovascular Issues and Epilepsy}

\section{Moderator: Michael Williams}

$$
\text { 10:45 a.m.-12:15 p.m. }
$$

S. MAHON, R. KRISHNAMURTHI, K. JONES, E. WITT, P. PARMAR \&. V. FEIGIN. Long-Term neuropsychological impairment in 4 year stroke survivors.

Objective:

Stroke has the potential to erase a lifetime of experiences or abilities in an instant. There is evidence that stroke should be considered a longterm condition, with many stroke survivors still experiencing adverse outcomes years after their stroke. Cognitive impairment is one of the most frequently reported consequences of stroke, affecting up to $70 \%$ of stroke survivors. Existing longitudinal data $>1$ year after stroke on cognitive outcomes is limited. This study examined prevalance and predictors of cognitive impairment in four year stroke survivors Participants and Methods:

This population-based follow-up study examined cognitive impairment in a cohort drawn from the fourth Auckland Stroke Regional Stroke Outcomes (ARCOS-IV) study, 4 years following stroke. Participants who agreed to further follow-up, completed face to face cognitive, psychological and health-related outcome measures

Results:

The greatest proportion $(84 \%)$ of 257 participants exhibited below average cognition as indicated by a MOCA score of $<26(\mathrm{~m}=20)$. Regression analysis found age, employment status and stroke type to be significant predictors of long-term impairment. Specific regression models for the Neuropsychological Assessment Battery (NAB) showed females more likely to have spatial impairment than males $(p=0.0178)$, and those who had a PACI $(p=0.0448)$ compared to LACI. Younger patients were more likely to have language impairment compared to older $(\mathrm{p}=3.57$ $\mathrm{x} 10-6)$. Retired/unemployed were more likely to have executive function impairment than those who were employed $(\mathrm{p}=0.0061)$

Conclusions: A considerable proportion of 4-year stroke survivors experienced cognitive impairment. Early identification of individual cognitive domains that contribute to outcomes post- stroke may inform the development of specific cognitive rehbalitation interventions to manage and/or cope with such deficits. Future research should focus on preventing/managing the long-term consequences of stroke, improving overall outcomes for stroke survivors and their families

Correspondence: Susan Mahon, PhD (final year), National Institute for Stroke and Applied Neurosciences, AUT University, AUT University, 90 Akoranga Drive, Auckland, Auckland 0627, New Zealand. E-mail: smahon@aut.ac.nz

E.B. LEAFFER, V.J. HINTON, K. ENGELSTAD, Y. GU, D. SHUNGU, S. DIMAURO \& D. DE VIVO. Distinct Neuropsychological Profile and Associated Neurochemical Changes in Patients with Mitochondrial Encephalomyopathy, Lactic Acidosis, and StrokeLike Episodes (MELAS).

Objective: Mitochondrial encephalomyopathy, lactic acidosis, and stroke-like episodes (MELAS) is a maternally inherited progressive multisystemic disorder. Occurrence of focal seizures, stroke-like episodes, and neuroimaging biomarkers (cerebral lactic acidosis, depleted N-acetylaspartate (NAA)) has been linked to cognitive deficits and trigger the developmental cascade of neurodegeneration. Regional brain vulnerability appears to begin posteriorly and migrate anteriorly, and a pattern of global cognitive deterioration has been reported. We examined this pattern in the largest MELAS cohort to date. We hypothesized that MELAS patients would perform worse overall and show a distinct profile of decline based on localization of seizures/ stroke-like episodes and lactate/NAA ratio, such that posterior-localized visually-mediated functions will decline faster than anterior-localized verbally-mediated functions.

Participants and Methods: Cognition was examined over time in 35 MELAS, 78 carriers, and 28 controls (aged 16-80) using composite $\mathrm{z}$-scores for mental status, attention, speed, mental flexibility, reasoning, language, memory, and visual-spatial skills, and for visually-mediated and verbally-mediated tasks. Chi-square, t-tests, ANOVA, and GEE were run $(\alpha=.05)$.

Results: Compared to carriers/controls, MELAS performed worse in all cognitive domains $(\mathrm{p}<.001)$ and performance negatively correlated with lactate/NAA ratio $(p<.05)$ at baseline. MELAS declined faster over time in visual-spatial skills $(p<.001)$, speed $(p=.045)$, and mental status $(\mathrm{p}=.02)$. Importantly, visually-mediated tasks declined faster in MELAS $(p=.02)$, while verbally-mediated tasks did not show this pattern $(\mathrm{p}=.77)$

Conclusions: MELAS show worse cognition at baseline and faster decline in mental status, speed, and visual-spatial skills compared to carriers/controls. Faster decline in visual, but not verbal, tasks supports our hypothesis that a distinct neurodegenerative profile exists affecting posterior brain regions and associated cognitive functions, in particular. Correspondence: Emily B. Leaffer, MA, MPH, Children's Hospital of Philadelphia, 630 West 168th Street, PH 18, New York, NY 10019, United States.E-mail: ebl2121@cumc.columbia.edu

B. YEW, S. DUTT, J.K. HO, A. GAUBERT, A.E. BLANKEN, J.Y.JANG, E.C. MCINTOSH \& D.A. NATION. Increased Cerebrovascular Resistance Correlates With Worse Cognitive Performance in Older Adults Exhibiting Normal Cerebral Blood Flow.

Objective: Cerebrovascular dysfunction has been increasingly implicated in Alzheimer's disease (AD), with elevated cerebrovascular resistance posited as a mechanism through which amyloid clearance is disrupted. The ratio of blood pressure (BP) to cerebral blood flow (CBF) represents a non-invasive index of cerebrovascular resistance (CVRi) that has been shown to predict cognitive decline and AD. Prior CVRi study, however, has relied upon seated BP, and CBF obtained on a separate occasion. We therefore sought to develop a more accurate CVRi measure, using multiple supine BP measurements collected immediately before neuroimaging.

Participants and Methods: Non-demented older adults $(N=40)$ underwent cognitive testing and arterial spin labeling MRI. Two supine brachial BP measurements were manually obtained prior to MRI onset. These were then averaged and used to calculate mean arterial pressure, which was in turn divided by regional CBF to determine CVRi in regions implicated in $\mathrm{AD}$. Linear regression was employed to evaluate associations between regional CVRi and cognition, controlling for age, sex, body mass index, and education.

Results: Higher left and right inferior parietal CVRi but not CBF was associated with poorer verbal memory, executive function, and language function. Elevated right inferior parietal CVRi but not CBF was associated with worse visual memory. Higher left and right posterior cingulate CVRi but not CBF was associated with poorer performance on visuospatial tasks.

Conclusions: Elevated CVRi derived from supine BP measured at the time of neuroimaging, is associated with poorer performance on memory, executive function, visuospatial, and language tasks, even in non-demented older adults. Notably, CBF in the same regions did not predict cognitive performance, suggesting that CVRi may predate and be more sensitive to preclinical cognitive decline than CBF changes alone. More broadly, our findings add to growing evidence implicating increased cerebrovascular resistance in cognitive decline and dementia. 
Correspondence: Belinda Yew, M.A., University of Southern California, Dept. of Psychology, SGM 501, 3620 South McClintock Ave, Los Angeles, CA 90089, United States.E-mail:belinday@usc.edu

A. REYES, G. WAGNER, A. MARSHALL, Y.A. CHANG, N. BAHRAMI, B. PAUL, T. GOLLAN \& C. MCDONALD. Does bilingualism increase cognitive reserve in patients with temporal lobe epilepsy? Objective: Bilingual healthy adults have been shown to exhibit an advantage in executive functioning (EF) that is associated with microstructural changes in white matter (WM) networks. Patients with temporal lobe epilepsy (TLE) often show deficits in EF that are associated with WM compromise. In this study, we investigate if bilingualism can increase cognitive reserve in bilingual patients with TLE, mitigating EF impairment and WM compromise.

Participants and Methods: Diffusion tensor imaging was obtained in 29 bilingual TLE, 32 monolingual TLE, 20 bilingual controls, and 26 monolingual controls. Fractional anisotropy (FA) was calculated for the uncinate fasciculus (Unc) and cingulum (Cing). Measures of EF included Trail Making Test-B (TMT-B) and D-KEFS Color-Word Inhibition/Switching. ANCOVA was conducted to compare FA of the Unc and Cing across groups. Correlations were used to investigate the relationship between $\mathrm{FA}$ and $\mathrm{EF}$.

Results: In bilingual TLE, FA was lower in the ipsilateral Unc compared to the other three groups and in the ipsilateral Cing compared to both control groups $(p$-values $<0.01)$. FA was also lower in the contralateral Cing in bilingual TLE compared to monolingual TLE $(p=0.032)$. However, bilingual TLE performed similarly to monolingual TLE on EF measures with comparable rates of impairment (32 \% bilingual vs $28 \%$ monolingual impaired). Conversely, bilingual controls showed a slight advantage in EF relatively to monolingual controls ( $75 \%$ bilingual vs $54 \%$ monolingual scored within the High Average range). In bilingual TLE, lower FA of left Cing $(p=0.03)$ and right Unc $(p=0.044)$ was associated with poorer TMT-B performance.

Conclusions: Despite poorer integrity of regional frontal lobe WM, bilingual TLE performed similarly to monolingual TLE on EF measures. These findings align with studies suggesting that bilingualism provides a protective factor for individuals with neurological disease, potentially through reorganization of EF networks that promote greater cognitive reserve.

Correspondence: Anny Reyes, MA, Psychiatry, University of California, San Diego, 6549 Jaffe Ct, San Diego, CA 92119, United States. E-mail: anr086@ucsd.edu

K. BLACKMON, W. BARR, C. MORRISON, W.S. MACALLISTER, M. KRUSE, X. WANG, P. DUGAN, A.A. LIU, O. DEVINSKY, R. KUZNIECKY \& T. THESEN. Temporal lobe gray-white blurring and Wada memory impairment in MRI-negative temporal lobe epilepsy.

Objective: MRI-negative temporal lobe epilepsy (TLE) may be a distinct syndrome from TLE with mesial temporal sclerosis (MTS). Common imaging and neuropsychological features of TLE with MTS are well known; yet, distinguishing features in MRI-negative TLE are only beginning to be described. This study aims to identify quantitative MRI markers of memory impairment in MRI-negative TLE.

Participants and Methods: Gray and white matter blurring (GWB) from 34 cortical regions and hippocampal volumes were quantified and compared across 28 people with MRI-negative treatment resistant TLE (18 left; 10 right) and 51 healthy controls (HCs). Regions with abnormally elevated GWB were correlated with memory scores obtained from the Intracarotid Amobarbital Procedure (IAP; i.e., Wada test).

Results: There were no hippocampal volume abnormalities in patients with MRI-negative TLE. GWB was elevated across several temporal lobe regions ipsilateral to the seizure onset zone (superior temporal sulcus: $p=0.0003$; temporal pole: $p=0.0001$; parahippocampal: $p=0.001$; entorhinal: $\mathrm{p}=0.002)$. There was no relationship between hippocampal volume and IAP memory scores in left or right MRI-negative TLE; however, decreased ipsilateral IAP memory scores were correlated with elevated GWB in the ipsilateral superior temporal sulcus of people with left MRI-negative TLE.

Conclusions: Localization of GWB abnormalities to the temporal lobe in people with MRI-negative TLE validates this metric as a measure of structural integrity in TLE. Extramesial temporal lobe GWB abnormalities were associated with decreased memory function. Thus, although hippocampal abnormalities might drive memory impairment in TLE with MTS, a loss of structural integrity in extramesial temporal lobe regions might be a marker for memory dysfunction in TLE without MTS. This suggests that reduced IAP memory scores do not necessarily implicate hippocampal pathology in MRI-negative TLE.

Correspondence: Karen Blackmon, Ph.D., Neurology, New York University School of Medicine, 190 E 7th St., Apt. 518, New York, NY 10009, United States. E-mail: karen.blackmon@nyumc.org

K. RAM-JUNNARKAR, K. COULEHAN, L.M. LANGAN, N. KATZ, J. SPAT, A.M.K. MILEY-AKERSTEDT, S. MANDELBAUM, F. VIQAR, L. MARCUSE, M. FIELDS \& H.A. BENDER. Decline in Activities of Daily Living in Epilepsy and MND: Risk Factor for Future Dementia?

Objective: Epilepsy and mild neurocognitive disorder (MND) both increase risk of developing dementia. The presence of poor independence in activities of daily living (ADL) is associated with progression to dementia among MND patients; however, little is known of how ADL decline contributes to the development of dementia in epilepsy. This study examined progression to dementia related to ADLs and cognitive decline among older adult epilepsy and MND patients.

Participants and Methods: Twenty-one patients (age > 65 years) underwent neuropsychological assessment; eleven patients diagnosed with epilepsy and ten patients diagnosed with MND, formerly "Mild Cognitive Impairment (MCI)." Chart review of epilepsy patients was conducted to determine indicators of ADL decline. Medical record review was conducted five to seven years post-assessment to determine progression to dementia.

Results: Six epilepsy patients had ADL decline (E-ADL $\downarrow$ ) at baseline, and all six of these epilepsy patients progressed to a dementia diagnosis. Five epilepsy patients without ADL decline (E-ADL $\uparrow)$ did not convert to dementia. All patients with MCI at baseline progressed to dementia. E-ADL $\downarrow$ performed significantly worse than E-ADL $\uparrow$ on naming and long-delay verbal free recall (Boston Naming Test $[t(7)=-3.41$, $p=0.01]$ and CVLT-II Delayed Recall $[t(3)=-2.15, p<.01])$. MCI patients performed significantly worse than E-ADL $\uparrow$ on naming (Boston Naming Test $[t(13)=-2.29, p=0.02])$. There were no significantly different language or memory performances between MCI and E-ADL $\downarrow$. Conclusions: Older adult patients with epilepsy and ADL decline are at increased risk for dementia, as compared to similarly aged epilepsy patients without ADL decline. Epilepsy patients with ADL decline performed similarly to patients with MCI in areas of language and memory. Functional impairment of epilepsy patients should be carefully assessed at baseline and serially assessed by neurologists/ neuropsychologists.

Correspondence: Kelly Coulehan, PhD, New York, NY 10029, United States.E-mail:kelly.coulehan@mssm.edu 


\section{Poster Session 11. Executive Function, Language, and Memory}

10:45 a.m.-12:00 p.m.

\section{Executive Functions/Frontal Lobes}

T.J. AINGER, L. PICK \& S. BINKS. Enhancing The Understanding of Executive Functioning of Individuals with Schizophrenia.

Objective: The purpose of this project is to demonstrate that there is a discriminate and valid range of executive functioning in individuals diagnosed with schizophrenia statistically comparable to the current normative standards, and to examine the utility of said range in gauging the functioning level of inpatient individuals. The goal is to assist clinicians and caregivers in providing more individualized and appropriate care to individuals being treated in an inpatient setting.

Participants and Methods: An adult-only population of inpatient individuals with schizophrenia was examined, between the ages of 18-69 $(N=83)$. The sample was age-banded into two subsets $(18-50$, 51-69). Scores on the first four subtests of the Delis-Kaplan Executive Function System (D-KEFS; Trail Making, Verbal Fluency, Color-Word Interference, Design Fluency) were compared to published normative values. Analyses were run to establish demographic statistics as well as between-group differences (sample vs. normative population) for each subtest trial. The distribution was scrutinized for spread differences.

Results: Overall, the scores in the sample population were found to be weaker, yet followed a statistically similar pattern as the normative sample. New group means and standard deviations were identified for the purpose of establishing a potential standard for comparison within similar clinical populations.

Conclusions: The pattern that emerged suggests that although executive functioning decreases in schizophrenia, it may do so along a trajectory. The findings herein, while not exhaustive and generalizable to the population at large, do lend credence to the postulate that schizophrenia has a profound and general impact on executive functioning abilities to individuals diagnosed, therein lowering their abilities significantly, but still parallels the normal population in dispersion. This may help contribute to an improved functional understanding of this population, and may serve to better inform clinical decisions made in care facilities. Correspondence: Timothy J. Ainger, Ph.D., Neurology, University of Kentucky, 2720 Burnt Mill Rd, Lexington, KY 40511, United States. E-mail: timothy.ainger@gmail.com

H.T. AL-KHARAFI, K.R. BRYANT, L.E. MILLER, M. HAUT \& J.W. HARTZELL. The Neurobehavioral Examination: Exploring the Differences Between Controls, Alzheimer's Disease and Parkinson's Disease Groups.

Objective: To assess the Neurobehavioral Examination's (NBE) ability to differentiate Alzheimer's (AD), Parkinson's disease (PD), and healthy Controls (HC). The NBE is a novel cognitive screening measure designed to elicit signs of frontal lobe/executive dysfunction while examining other aspects of cognition and motor, sensory, and visual functions.

Participants and Methods: Participants, aged 60-80, were administered the NBE as part of a comprehensive neuropsychological battery. Patient groups included: Alzheimer's disease (AD; $\mathrm{n}=25)$, Parkinson's disease (PD; $n=21$ ), and healthy controls (HC; $n=39$ ). Group differences on the 3 main NBE indices (total score, frontal score, and corrected score (total - frontal)) and specific NBE variables were analyzed.

Results: MANCOVA (age and education = covariates) showed a significant main effect for the 3 main NBE indices (Wilks' Lambda, $F=9.87$, $\mathrm{p}=.000)$. HC performed significantly better across indices. There were no significant differences between the $\mathrm{AD}$ and PD groups on the 3 indices; however, chi-square analyses showed significant group differences on many specific variables within the NBE. Eight NBE variables sampling reflexes, motor functioning, cognitive abilities, and frontal signs were included in a Multinomial Logistic Regression analysis. The resulting model had high classification rates: AD (100\%), PD (81\%), and HC $(92 \%)$ meaning that most participants were correctly matched to group. Conclusions: Exploratory research on the NBE found group differences between $\mathrm{AD}, \mathrm{PD}$, and HC, and combinations of specific variables within the NBE yielded high classification rates, thus supporting the measure's clinical utility and ability to differentiate neurologic patient groups.

Correspondence: Hussah T. Al-Kharafi, Behavioral Medicine and Psychiatry, West Virginia University, Neuropsychology Clinic, Behavioral Medicine and Psychiatry, 930 Chestnut Ridge Road, Morgantown, WV 26505, United States. E-mail: hussah.alkharafi@gmail.com

A.D. BAIRD, J. MCCARTHY, J. GLASGOW, A. KANDASAMY \& K. AN. Getting Things Done: Coaching Undergraduates with Attentional Complaints.

Objective: To explore benefits of brief assessment and feedback in collaborative and standardized conditions for undergraduates reporting attentional problems.

Participants and Methods: Nineteen undergraduates reporting attentional problems and interest in coaching were randomly assigned to standardized or collaborative $(n=8)$ conditions and completed assessment (BRIEF-A, DASS, NIH TB executive function tests, and letter fluency) and feedback administered by clinical graduate students or a faculty member in clinical psychology/neuropsychology. All received a written list with diverse suggestions as well as individual results and suggestions.

Results: Most students reported significant attentional and/or emotional concerns. On the BRIEF-A 8/19 students had at least one elevation >= $65 T$; on the DASS 11/19 had at least one elevation $>=1.5 z$. Age-adjusted executive function scores ranged from low average to above average or better. Self-report did not correlate with executive test performance. There were associations among direct performance, BRIEF-A subscale, and DASS scale scores.

A standardized questionnaire about assessment and feedback experiences disclosed positive changes in overall satisfaction from the first session to the second, which on average is neutral (the person is neither dissatisfied nor satisfied) after assessment and positive after feedback. This favorable shift was seen in three of four sections, especially in self-understanding. Attention ratings and grades the following semester were available for 12 and 18 participants, respectively. No significant change was noted in the sample as a whole nor was there an interaction with condition.

Conclusions: This pilot demonstrates the potential for brief neuropsychological assessment and feedback to be helpful to undergraduates reporting difficulties with attention.

Correspondence: Anne D. Baird, PhD, Psychology, University of Windsor, 917 Woodcrest Drive, 917 Woodcrest Drive, Royal Oak, MI 48067, United States.E-mail: abaird@uwindsor.ca

M. BERL, X. YOU, E.M. BOUTZOUKAS, C. VAIDYA \& L. KENWORTHY. Data Driven Profiles of Executive Function Distinguish Psychiatric Behaviors in Pediatric Populations.

Objective: Children with medical disorders are at increased risk for executive functioning (EF) and psychiatric problems. We used novel data-driven methods that apply graph theory to categorize EF profiles within this at-risk population; then, determined if profiles differ with respect to psychiatric behaviors.

Participants and Methods: 543 children ages 5-18 were included if they had a pediatric medical disorder and completed parent ratings for the Behavior Rating Inventory of Executive Functioning (BRIEF), DuPaul ADHD Rating Scale (ADHD-RS), and Child Behavior Checklist (CBCL). Most children also had IQ. EF profiles were determined using $10 \mathrm{z}$ transformed scores (8 subscales of the BRIEF; 2 subscales of the ADHD-RS) to create correlation matrices between subjects. Subsequent community detection was applied using the Louvain algorithm, a weight-conserving modularity algorithm not dependent on thresholds. We ran the algorithm 100 times and used the community structure that 
maximized the modularity quality score $(Q)$ indicating stronger separation. Next, we compared Internalizing and Externalizing Problems from the CBCL using ANOVA.

Results: Three EF profiles with strong segregation $(Q=.47)$ included: 1) peaks at Working Memory and Inattentive; 2) peak at Shift; and 3) peaks at Inhibit and Hyperactivity/impulsivity. Profile2 had significantly more internalizing behaviors, while Profile3 had significantly more externalizing behaviors and were younger. No IQ differences across profiles.

Conclusions: Delineating EF profiles among a large pediatric cohort is valuable for identifying common patterns of impairment-Poor W orking Memory/Inattention; Poor Flexibility; Poor Inhibitory Control—regardless of underlying etiology. Psychiatric behaviors were different among EF profiles suggesting that the EF profile may inform risk for comorbid psychopathology and the underlying EF deficit may be a target for treatment. Applying this novel approach may improve risk assessment and inform treatment plans regardless of medical etiology.

Correspondence: Madison Berl, PhD, Neuropsychology, Children's National Medical Center, 111 Michigan Avenue NW, Washington, DC 20010, United States. E-mail: mberl@childrensnational.org.

M.A. BLAIR, A. MOYETT, A. BATO, P. DEROSSE \& K. KARLSGODT. Neuropsychological Correlates of Adaptive Risk-Taking in Adolescence.

Objective: Adolescence is a critical developmental stage of decision making brain networks, often marked by increased risk-taking as neural systems mature. Traditional perspectives emphasize the maladaptive nature of risk-taking but it can also be adaptive as young people gain independence. Response variability, an index of behavioral control, is considered a key component of adaptive adolescent risk taking. This study evaluates the role of executive control function (ECF) in response variability during a risky decision making, in order to elucidate mechanisms of adaptive risk taking during adolescence.

Participants and Methods: A sample of 107 healthy individuals between ages 8-25 were administered the Balloon Analogue Risk Task (BART), a computerized measure of risk-propensity and the MATRICS Consensus Cognitive Battery to measure executive functioning. We used the BART's coefficient of variation $(\mathrm{CV})$ in adjusted pumps to measure response variability.

Results: Multiple regressions were performed to examine the relationship between ECF capacity and CV. We also conducted mediation analyses to assess if ECF explained the relationship between age and CV. Results indicated that stronger EF capacity predicted lower response variability, and mediated the relationship between age and improved inhibitory control. Results indicated that stronger EF capacity predicted lower response variability, and mediated the relationship between age and improved inhibitory control.

Conclusions: Our findings suggest that stronger ECF is related to greater behavioral control during risky decision making and may allow individuals to learn differences in outcome probability and apply this information to optimize performance. These findings contribute to a more comprehensive understanding of the neuropsychological correlates of adaptive risk taking, which could inform the development of cognitive interventions in risk-vulnerable populations.

Correspondence: Melanie A. Blair, Ph.D., Clinical Psychology, CUNY Graduate Center, 214 NORTH 11TH, APT. 3W, BROOKLYN, NY 11211, United States. E-mail: melanieablair@gmail.com

WITHDRAWN. L. CIPOLOTTI, E. CHAN, S. GHAROONI, S.E. MACPHERSON, B. SPANO, T. SHALLICE, J. DUNCAN \& P. NACHEV. Inhibition, Estimation and Response Generation: Evidence from Patients with Prefrontal Cortex Lesions.

Objective: The Stroop, the Hayling, the Gognitive Estimates (CET) and verbal and nom-terbal flueney are well-known nemropsychologieat tests developed to assess prefrontal cortex (PFG) executive functions streh inthibition, response and generation estimation. We investigated the performanee of $\mathrm{PFG}_{\mathrm{i}}$ patients on these tests to address the relationship with fluid intelligence since authors stgigested that redueed fluid intelligente account for exectitive impairments, at least for some tests (e.g. Roea et al, 2009). We also evaluated whether distinet PFG regions are differentially involved.

Partieipants and Methods: Patients with unilateral, foeal tesions eonfined to the fromtal tobes interment cognitive and remroimaging investigations.

Results: PFG patients were signifieantly impaite A wpaced with HG on

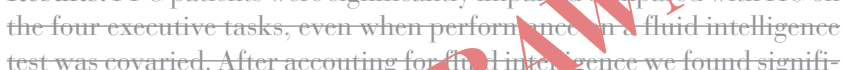
test was covaried. After aceouting for thy dinfer, gence we found signifi=

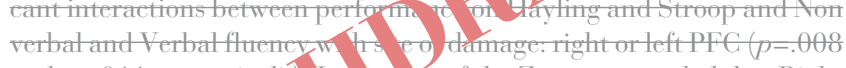

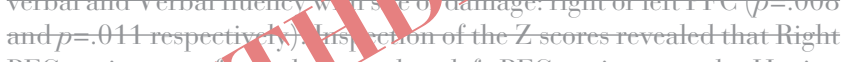
PFG patients pe IfIn d hrse than left PFG patients on the Haying and Nonve al fl, ney. In contrast, left PFG patients performed worse than righ of patients on the Stroop and verbal flueney. Pelatively eiremmscribut left PFG areas were involved in Stroop and verbal fluency whilst eiremeribed right PFG areas in Hayling and nom verbal flueney. Conclusions: Our results stggest that impairments in inhibition, response generation antestimation cannot be explained by fluid interligence. Instead they appear to assess dissociable eomponents of exeeutive funetions, related to anatomieally lateralized PFG eirenits. This has eliniealimplieations for exeentive intpaimments highlightimg the need to use a variety of tasks for their assessment and to develop different types of treatments.

Gorrespondence: Lisa Cipolotti, Institiute of Neurology, University Goltege Lonton, Department of Neuropsychotogy, National Hospitat for Neurology and Neurosurgery, Queen Square, London WC1N 3BG, United Kingdom.E-mait:l.cipototti@ucl.ac.uk

\section{S.V. CLARK. Cerebellar Contributions to Inhibitory Control in the Stop Signal Task: A Systematic Review.}

Objective: Inhibitory control is an executive function (EF) important for suppressing inappropriate actions and guiding flexible, goal-directed behavior. The stop signal task (SST) measures response inhibition, activating a right prefrontal-basal ganglia network. Meta-analyses have shown that the cerebellum is also active during EFs, but it is unclear how how it participates in specific EFs and is rarely investigated regarding inhibitory control. This systematic review aims to clarify the cerebellum's role in inhibitory control specifically, by qualitatively reviewing the available literature utilizing the SST in fMRI experiments.

Participants and Methods: A literature search was performed including the terms "stop signal AND fMRI". Articles were included if they utilized event-related fMRI with the SST, reported whole-brain activation results for healthy adults, included the cerebellum in imaging and analysis, and demonstrated cerebellar results. Cerebellar activations were investigated and reviewed regarding location and behavioral effects.

Results: Most cerebellar activations were located in the left posterior cerebellum. More activity was associated with monitoring than inhibition alone, with inferior regions (lobule 8) more associated with inhibition and posterior regions (crus II) more associated with monitoring. Some cerebellar activity was correlated with stopping efficiency. Connectivity studies revealed functional connections between the right inferior frontal cortex and posterior cerebellar regions during the SST.

Conclusions: Posterior cerebellar activations suggest a cognitive role in inhibitory control; left hemisphere activations suggest communication with the right-lateralized prefrontal inhibitory control network. It appears that the cerebellum works with the prefrontal cortex to predict and control behavior, and likely plays a monitoring role using internal models. Future studies and models of inhibitory control should consider the cerebellum, and compare cerebellar function among EFs.

Correspondence: Sarah V. Clark, PhD, Psychology, Georgia State University, 800 Peachtree St. NE, \#8513, Atlanta, GA 30308, United States.E-mail:sclark55@student.gsu.edu 
C. CORDEAUX, B.C. KAVANAUGH, M. BODZY, N. NOONAN, J. STUDENY \& K.A. HOLLER. Trauma and Executive Functioning: Predictors of Length of Stay in Inpatient Children.

Objective: Trauma and post-traumatic stress disorder (PTSD) have been implicated as a predictor of length of stay (LOS) for children and adolescents at inpatient hospitals. However, the role of neurocognitive factors such as executive functioning $(\mathrm{EF})$ is less well understood, especially in child populations. Neurocognitive factors are associated with LOS in adult inpatient populations, but fewer studies examine the role of EF in child inpatient LOS. Children admitted to inpatient psychiatric hospitals represent an understudied group. This study offers a unique opportunity to examine the contribution of both trauma and EF to LOS in a child inpatient population.

Participants and Methods: Hierarchical regression was used to examine variation in LOS in inpatient children ( $N=345)$ aged 5-12. The initial model examined contributions of trauma variables (history of maltreatment, $\mathrm{N}=206$; diagnosis of PTSD, $\mathrm{N}=61$ ) after controlling for demographic variables. Separate models examined contributions of EF constructs (trail making test, Stroop test, and continuous performance test) beyond demographic and trauma variables.

Results: Diagnosis of PTSD accounted for a portion of variance in LOS after controlling for demographic variables $(p<.05)$. The Stroop test showed a trend $(p<.09)$ towards accounting for additional variance when added to the model.

Conclusions: In summary, a diagnosis of PTSD accounted for the most variance in LOSfor inpatient children. In addition, model results including the Stroop test showed a trend toward significance and suggest a potential role for EF in understanding LOS for child inpatients.

Correspondence: Cara Cordeaux, Doctoral, Psychology, University of Connecticut, 406 Babbidge Road, Unit 1020, Storrs, MA 06269, United States.E-mail: cara.cordeaux@uconn.edu

K.A. COLBY, D.W. VAUGHN, C. BROWN, C. CONABOY, N. KASWAN \& R. HIRST. Executive Dysfunction in Eating Disorders: A MetaAnalytic Comparison of Anorexia Nervosa and Bulimia Nervosa.

Objective: Research estimates that 30 million people have a diagnosed eating disorder (ED) in the United States (Wade et al., 2011). While research on the cognitive implications of anorexia nervosa (AN) and bulimia nervosa (BN) has previously identified deficits in executive functioning (EF) skills, the impact of an ED diagnosis on the composite EF domain remains unknown (Weider et al., 2014). Thus, there is a compelling need to clarify the extent of EF disruption in individuals with $\mathrm{AN}$ and $\mathrm{BN}$, as no meta-analyses to date have analyzed the effects of both diagnoses on global EF abilities.

Participants and Methods: The current findings were based on a total of 36 studies, with 1,577 participants with either AN (27 studies) or BN (9 studies) and 1,780 healthy controls. Individual and grand mean effect sizes were calculated for the effects of both $\mathrm{AN}$ and $\mathrm{BN}$ on $\mathrm{EF}$ measures of decision making, problem-solving, cognitive flexibility and organization/planning skills.

Results: Individual effects of AN on EF ranged from small (e.g., Wisconsin Card Sorting Test, WCST, Failure to Maintain Set: $g=-0.28$ ) to medium-large (e.g., Iowa Gambling Task Net Score: $g=-0.74$ ). In contrast, the individual effects of BN on EF ranged from medium (e.g., Trail Making Test-Part B: $g=-0.57$ ) to large (e.g., WCST Perseverative Errors: $g=-0.80)$. The grand mean effect size of AN on EF was in the small range $(g=-0.39,95 \% \mathrm{CI}:[-.46,-.31])$, whereas the grand mean effect size of BN was medium $(g=-0.47)$.

Conclusions: Results suggest that individuals with EDs exhibit greater deficits in EF skills relative to healthy controls. In comparing the two diagnoses, there was a larger overall effect of $\mathrm{BN}$ on EF skills relative to AN. Implications will be discussed.

Correspondence: Katrina A. Colby, M.S., Palo Alto University, 1791 Arastradero Road, Palo Alto, CA 94304,United States.E-mail: kcolby@ paloaltou.edu
K.A. COLBY, C. BROWN, D.W. VAUGHN, N.R. KASWAN, C. CONABOY \& R. HIRST. Executive Functioning Deficits in Children and Adults with Anorexia Nervosa: A Meta-Analysis.

Objective: Anorexia Nervosa (AN) is the third most common chronic disease among individuals younger than 20 years old (Favaro et al., 2009). While an increasing amount of research has identified that individuals with $\mathrm{AN}$ often exhibit deficits in executive functioning (EF) skills (Crow \& Eckert, 2016), the relationship between age and development of EF is often unaccounted for. Since EF abilities emerge according to frontal lobe development, which typically concludes around age 26, there is an indisputable need to examine differences in EF abilities between children and adults with $\mathrm{AN}$.

Participants and Methods: The current meta-analysis was based on 1,278 participants with AN and 1,328 healthy controls in 27 studies. Individual and grand mean effects of AN on EF were derived based on reliable, validated measures of EF. A subgroup analysis based on age compared participants age $<25$ years to participants aged $>26$ for the effects of AN on EF. Random-models were applied where heterogeneity was significant; otherwise, outcomes were reported using fixed-effects estimators.

Results: Overall, results demonstrated a negative grand mean effect size of AN on EF $(g=-0.39, z=-9.60)$ and individual small-medium effect sizes in both adults $(g=-.40, z=-.861)$ and youth $(g=-.25$, $\mathrm{z}=-2.48$ ). When comparing age groups (i.e., $>26$ years and $<25$ ) using a mixed-effects analysis, there was a non-significant between-subgroup difference in total effect size $\left(\chi^{2}(1)=0.971, p>.05\right)$.

Conclusions: Individuals with AN exhibit impairments in global EF skills compared to healthy controls. The effect of AN on EF was larger in adults than children; however, subgroup age differences were not statistically significant. The non-significant group difference in EF between children and adults refutes the neurodevelopmental theory of EF; however, the larger effect in adults suggests that older participants exhibit greater effects of $\mathrm{AN}$ on $\mathrm{EF}$ because of the cumulative impact of ED pathology over time.

Correspondence: Katrina A. Colby, M.S., Palo Alto University, 1791 Arastradero Road, Palo Alto, CA 94304,United States.E-mail: kcolby@ paloaltou.edu

E. CRAUN, S. KAPLAN, M.M. WONG, S. LYNCH \& N. AUBUCHONENDSLEY. PTSD symptoms, traumatic experiences, and their relationship with neurocognitive functioning among inmates.

Objective: Incarcerated men and women have higher rates of trauma exposure than the general public (Gibson et al., 1999, Green et al., 2005). Previous research has found an association between the diagnosis of Post-Traumatic Stress Disorder (PTSD) and neurocognitive deficits (Horner \& Hamner, 2002). However, it is still unclear if these deficits are associated with the exposure to traumatic events or with other aspects of PTSD. Therefore, we assessed the relationships between (i) PTSD symptoms and (ii) total number of traumatic experiences with neurocognitive functioning in an incarcerated sample.

Participants and Methods: Two hundred and fifty inmates were randomly selected from two U.S. Northwestern jails (58.4\% women, aged 18-71) and completed diagnostic screening interviews and a brief neurocognitive battery. The PTSD Checklist for the DSM-5 (PCL-5) and Lifetime Stressor Checklist-Revised (LSC-R) were used to quantify the total number of PTSD symptoms and total number of traumatic experiences. Results: Multiple regression models revealed the total number of PTSD symptoms significantly predicted total errors $(b=-0.13(0.37), p<.05)$ and perseverative errors on the Wisconsin Card Sorting Test (WCST; $b=-0.15(0.06), p<.05)$, such that more PTSD symptomatology was associated with more perseverative errors (e.g., inability to change mental sets).

Conclusions: Number of endorsed PTSD symptoms was not associated with non-perseverative errors (i.e., all other incorrect responses on the WCST). The total number of traumatic experiences on the LSC-R was not associated with WCST performance. This suggests that PSTD 
symptomatology, not exposure to traumatic events, is associated with deficits in neurocognitive functioning. Clinical implications are discussed. Correspondence: Elizabeth Craun, Master's of Science, Idaho State University, 921 S 8th Ave, Pocatello, ID 83209, United States. E-mail: craueliz@isu.edu

M. DOWNES, M. DE HAAN, P. TELFER \& F. KIRKHAM. Executive Development in Preschool Children with Sickle Cell Disease: Shifting the Focus from Disease to Environment in Future Research.

Objective:

Executive functions are compromised in school-age children and adults with sickle cell disease even when there is no evidence of stroke. There is limited research on the development of executive functions in preschool-age children with sickle cell disease and the factors that contribute to executive dysfunction. This is partly due to the lack of age appropriate assessments that were previously available to measure executive functions in this age range, however a number of newly developed measures and techniques have more recently allowed for the extensive assessment of executive function at this early stage. This study assesses the development of executive function in preschool-age children with sickle cell disease and investigates the impact of environmental and disease factors on executive development.

Participants and Methods: Twenty-two preschool-age children with sickle cell disease (mean age 4.8, $\mathrm{SD}=.94$; mean $\mathrm{IQ}=98.6, \mathrm{SD}=11.4$ ) were compared with an ethnicity, age, gender, socioeconomic status, and IQ-matched control group on a battery of executive tasks, including lab based measures, ecologically-valid measures and an event-related potential paradigm. Hemoglobin levels on day of testing and clinical note review which included variables such as cerebral blood flow velocity were used to establish disease severity. Parent questionnaire was used to obtain information on sleep and environmental factors. The relation between executive function and disease severity, sleep, and environmental factors, including family function, was explored.

Results: Preschool children with sickle cell disease are already showing executive delays despite no evidence of stroke. Environmental factors, particularly family function, are more predictive of executive performance than disease severity.

Conclusions: Family function should be further investigated as a potential target for intervention with the aim to promote early executive development in this at-risk population and reduce the frequently reported achievement gap.

Correspondence: Michelle Downes, University College Dublin, Belfield, Dublin 4,Dublin0000,Ireland.E-mail:michelle.downes@ucd.ie

L. DOYLE, L. GLASS, C. COLES, J. KABLE, E. SOWELL, J. WOZNIAK, E.P. RILEY \& S. MATTSON. Impact of Comorbid Oppositional Behavior on Executive Function Among Youth with Histories of Heavy Prenatal Alcohol Exposure.

Objective: Youth with heavy prenatal alcohol exposure have a number of comorbid conditions, including oppositional behavior, which may exacerbate executive function (EF) deficits. We assessed the interaction of oppositional behaviors, as measured by standardized clinical interview, and alcohol exposure on direct assessment and parent-report EF. Participants and Methods: Subjects ( $N=268)$ ages 10-17y $(\mathrm{M}=13.2)$ comprised 3 groups: alcohol-exposed with oppositional behaviors (AE+, $n=102$ ), alcohol-exposed without oppositional behaviors (AE-, $n=45$ ), and controls (CON, $n=121$ ). Group differences on direct assessment (D-KEFS subtests) and parent-report (BRIEF) EF were tested with MANCOVAs (controlling for appropriate demographic variables and presence of ADHD). Significant differences $(\alpha=.05)$ were followed up with univariate ANOVAs and pairwise comparisons.

Results: On direct assessment (D-KEFS), there was a group main effect, $F(14,318)=2.586, p=.002,=.102$. Follow-up tests indicated significant group main effects on 3 of 6 variables $(p s<.001)$. The CON group performed significantly better on these tests than both exposed groups $(p s<.001)$, which did not differ $(p s>.251)$. On parent report (BRIEF), there was a significant group main effect, $F(16,508)=22.878, p<.001$, $=.419$. Follow-ups indicated significant group main effects on all BRIEF scales $(p s<.001)$, with the CON group having significantly better EF than $\mathrm{AE}-(p s<.001)$ which was significantly better than $\mathrm{AE}+(p s<.009)$. Conclusions: Oppositional behavior is common among youth affected by prenatal alcohol exposure. In this study, the presence of oppositional behavior exacerbated EF impairment based on parent report even when the presence of ADHD was statistically controlled. In contrast, deficits on direct measures of EF were not affected by the presence of oppositional behavior. Greater understanding of the impact of concurrent psychopathology and other factors on long-term outcomes for alcohol-exposed youth is needed.

Research supported by NIAAA grant U01 AA014834 and the CIFASD. Correspondence: Lauren Doyle, M.S., Psychology, SDSU/UCSD Joint Doctoral Program in Clinical Psychology, 6300 Alvarado Rd, Suite 100, San Diego, CA 92120, United States.E-mail: ldoyle-w@sdsu.edu

K. ENEVA, J. ARLT, S. MURRAY, A. YIU \& E. CHEN. Executive Functioning in Women with Binge-eating Disorder Across Weight Groups.

Objective: Objective: Executive functioning (EF) problems may serve as vulnerability or maintenance factors for Binge-Eating Disorder (BED). However, it is unclear if EF problems observed in BED are related to overweight status or BED status. The current study extends this literature by examining EF in overweight and normal-weight BED compared to weight-matched controls.

Participants and Methods: Participants and Methods: Participants were women with normal-weight BED $(n=23)$, overweight BED $(n=32)$, overweight healthy controls $(n=48$ ), and normal-weight healthy controls $(n=29)$. The EF battery utilized tests from the National Institutes of Health (NIH) toolkit and Delis-Kaplan Executive Function System (DKEFS).

Results: Results: After controlling for years of education and minority status, overweight individuals performed more poorly than normalweight individuals on a task of cognitive flexibility $(p<0.01)$ requiring generativity and speed on psychomotor performance tasks $(p=0.01)$. Normal-weight and overweight BED performed worse on working memory tasks compared to controls $(p=0.04)$. Unexpectedly, normalweight BED individuals out-performed all other groups on an inhibitory control task $(p<<0.01)$. No significant differences were found between the four groups on tasks of planning.

Conclusions: Conclusions: Regardless of weight status, BED is associated with working memory problems. Replication of the finding that normal-weight BED is associated with enhanced inhibitory control is needed.

Correspondence: Kalina Eneva, Psychology, Temple University, 1327 Fitzwater St., Philadelphia, PA 19147, United States. E-mail: kalina. eneva@temple.edu

L.S. EVERETT, I. HASSAN, A. OSBORN, M. TAN, C. SCHROTH, A. KENNEDY, J.M. BABIONE, E. PROESCHER, J. GREENSTEIN, D.M. AASE \& K.L. PHAN. Examining the Influence of PostDeployment Family Support on Executive Functioning in Post9/11 African-American Veterans.

Objective: Prior studies have demonstrated an impact of family support on executive functioning in African-American civilians (e.g., Sims et al., 2011). However, such impact is not well understood in post-9/11 African-American veterans, who also are at high risk for PTSD and related cognitive dysfunction. The present study evaluated the impact of post-deployment family support on indicators of executive functioning in post-9/11 African-American veterans while taking into account the presence of PTSD

Participants and Methods: Subjects were 44 African-American (8 females, 36 males) post-9/11 veterans who were assessed using a brief neuropsychological battery, the Clinician Administered PTSD Scale (CAPS-IV), and the Deployment Risk and Resilience Inventory-2 (DRRI-2). 
Results: Both PTSD diagnosis and post-deployment family support significantly predicted loss-of-set errors on the Wisconsin Card Sorting Test (partial $\eta^{2}=.13$ and .15 , respectively). An interaction effect was observed such that in the presence of a PTSD diagnosis, post-deployment family support did not predict loss-of set errors, but in the absence of a PTSD diagnosis, reduced family support was associated with greater loss-of-set errors (partial $\eta^{2}=.14$ ). Post-deployment family support did not significantly predict other indicators of executive functioning.

Conclusions: Findings are partially consistent with prior studies observing an influence of family support on executive functioning in African-Americans in non-veteran samples. In post-9/11 veterans with and without PTSD, post-deployment family support may be of clinical significance.

Correspondence: Llashe-Kaye S. Everett, MA, Illinois School of Professional Psychology, 1000 S Clark Street, Apt. 904, Chicago, IL 60605, United States. E-mail: sabrinaeverett1@gmail.com

M. FISCHER, M.M. FIUMEDORA, L. TURKELSON, C. WILKISON \& P. SHEAR. Self-Reported Executive Functioning Deficits in TLE Relate to Both Working and Episodic Memory.

Objective: Memory deficits are the most common subjective complaint and objective neuropsychological finding in patients with temporal lobe epilepsy (TLE). In addition to long-term memory (LTM) dysfunction, these patients often exhibit executive deficits. We have previously reported that TLE patients endorse disproportionate working memory (WM) deficits on a self-report measure of EF. The present study seeks to address whether self-reported WM deficits in everyday functioning are attributable primarily to objective WM deficits or if they also encompass LTM impairment.

Participants and Methods: Participants were 39 presurgical patients with refractory temporal lobe epilepsy (24 left; 12 right; 3 bilateral) with a mean age of $40.5(S D=12.3)$ and 13.2 years of education $(S D=2.6)$. All participants completed a self-report measure of EF (BRIEF-A) and laboratory measures of verbal and nonverbal learning and memory (CVLT-II, BVMT-R), working memory (WAIS-IV Digit Span), handedness (Crovitz), and confrontation naming (BNT).

Results: On the BRIEF-A, $72 \%$ of the sample endorsed clinically elevated WM concerns, and this elevation was significantly higher than any of the other BRIEF indices ( $p<.01$ for all comparisons). BRIEF-A WM deficits were associated with performance decrements on WAIS-IV Digit Span and CVLT-II Total Learning, but not BVMT-R. Multiple regression analysis indicated that Digit Span and CVLT-II Total Learning made significant $(p<.005)$ independent contributions to the prediction of BRIEF-A WM. The results were not affected by confrontation naming, lateralization of focus, or handedness.

Conclusions: TLE patients report clinically elevated WM deficits in everyday function. Contemporary cognitive neuroscience posits both WM and LTM correlates of mesial temporal dysfunction. Thus, it is both clinically and theoretically relevant that the present study indicates independent and additive contributions of objective WM and LTM ability to prediction of BRIEF-A WM scores.

Correspondence: Mark Fischer, Psychology, University of Cincinnati, 2828 CORTELYOU PL, Cincinnati, OH 45213-1202, United States. E-mail: fischemk@mail.uc.edu

B. GRAVES, R.J. RICHARDSON \& J.E. CASEY. Another Ineffective Performance-Based Task of Executive Functioning: Where Do We Go From Here?

Objective: Performance-based tests of executive function (EF) are increasingly criticized for being ineffective in predicting everyday EF. In an effort to improve predictive utility, several recommendations have been put forth. The Functional Assessment of Verbal Reasoning and Executive Strategies (FAVRES) test developers have embodied these suggestions, and the task appeared promising. The current investigation assessed the utility of the FAVRES in predicting everyday EF. It was hypothesized that the FAVRES would be a better predictor of everyday $\mathrm{EF}$ than an intelligence estimate.

Participants and Methods: University students $(N=78 ; 63 \%$ female; $M_{\text {age }}=22$ years) initially completed a questionnaire that included history of psychopathology, current stress levels, and other demographic information. They also completed the FAVRES, the Wechsler Test of Adult Reading (WTAR; an estimate of intelligence) and the Behavior Rating Inventory of Executive Function - Adult (BRIEF-A; a self-report measure of everyday EF behaviour).

Results: Neither FAVRES nor WTAR scores predicted BRIEF scores, $F(1,75)=0.34, p=0.37$. Post-hoc regression analyses, however, found that FAVRES performance was predicted by WTAR scores, $F(1,75)=11.17, p<0.01$. Additionally, BRIEF scores were predicted by psychopathology and current stress levels, $F(1,73)=10.14, p<0.01$. Conclusions: These findings further demonstrate that EF is difficult to measure with performance-based tests. The FAVRES was specifically designed to address criticisms of existing EF tasks, but predictive utility was not demonstrated. Importantly, the findings indicate a stronger relation between psychopathology/stress and everyday EF than between intelligence or performance-based testing and everyday EF. Thus, placing a greater conceptual emphasis on the effect of emotion on behaviour may improve performance-based measurement of EF. Correspondence: Bryanna Graves, Clinical Neuropsychology, Psychology, University of Windsor, 466 Campbell Avenue, Windsor, ON N9B2H4, Canada.E-mail: graves.bryanna@gmail.com

R.S. HUBER, D.G. KONDO, X. SHI, P. SUBRAMANIAM, P.F. RENSHAW \& D. YURGELUN-TODD. Executive Functioning and Anterior Cingulate Volume in Adolescent Bipolar Disorder.

Objective: Cognitive deficits are experienced by more than $60 \%$ of individuals with bipolar disorder (BD), although our understanding of these impairments at a mechanistic level remains limited. Abnormalities in the anterior cingulate cortex (ACC), which is known to regulate emotion and mediate executive function, have been implicated in BD. The aim of this study was to investigate the relationship between structural morphometry of the ACC and executive functioning in BD youth compared to healthy controls.

Participants and Methods: Twenty depressed participants with $\mathrm{BD}$ and ten age-and sex-matched control participants, ages 13 to 21, completed a diagnostic interview, mood, and executive function measures. Participants were administered the Wisconsin Card Sorting Task (WCST), Trail Making Test (TMT), and Color-Word Interference Test (CWIT) from which a measure of overall executive function was created. Additionally, participants underwent an MRI scan on a 3T Siemens Verio magnet. Morphometric analysis of images was performed using FreeSurfer.

Results: Between-group analysis of variance (ANOVA) indicated that BD participants performed significantly worse than controls on executive functioning tasks. No group differences in volume were observed in the ACC or any subregions, however in BD subjects Pearson's coefficients showed significant positive correlations between ACC rostral subregion volumes and executive function performance $(p<0.01)$ and total ACC volumes and executive function performance $(p<0.05)$. Specifically, BD youth with reduced ACC rostral and total ACG volumes had lower performance on the TMT and CWIT tasks.

Conclusions: Study results replicate previous observations of executive function deficits in youth with BD and extend findings in BD adults that have demonstrated a relationship between ACC volume and executive function performance. The present findings raise questions regarding the stage of development at which cingulate anomalies emerge in BD. Correspondence: Rebekah S. Huber, PhD, Department of Psychiatry, University of Utah, 383 Colorow Drive, Salt Lake City, UT 84108, United States.E-mail: Rebekah.Huber@utah.edu 
L.A. KAIS, C. LEE, M.J. KMIECIK \& R. SILTON. The Influence of Affect on Interference Processing in Blocked and Mixed Presentations of a Stroop Color-Word Task.

Objective: Planning and execution of goal-directed performance is dependent upon intertwined cognitive and emotional processes. While studies have examined differences in cognitive control abilities across categorical classifications of abnormal affective function, less is known regarding the dimensional influence of positive/negative (PA/NA) mood states. The present study evaluated the hypothesis that state PA improves interference processing as task difficulty increases during the Stroop CW-Task.

Participants and Methods: 47 participants (31 females) ages 18 to 24 $(\mathrm{M}=19.80, \mathrm{SD}=1.10)$ were recruited for the study. 23 participants met DSM-IV-TR criteria for lifetime history of depression; 24 participants were healthy controls. A multi-method approach was utilized, involving diagnostic interview (MINI), self-report of state affect (PANAS), and experimental assessment of a Stroop CW-Task that included a pure condition (incongruent OR congruent) and a mixed condition (incongruent AND congruent).

Results: Pure and mixed conditions differed in difficulty evidenced by reduced accuracy on the mixed task $(p<.05)$. The Stroop effect was observed in both tasks $(\mathrm{p}<.05)$ and there was a significant task $\mathrm{x}$ congruency interaction $(\mathrm{p}<.05)$. Regression results showed that for the more difficult task, PA was negatively associated with the Stroop effect $\mathrm{B}=-3.24, \mathrm{t},=-2.26, \mathrm{p}<.05$, such that high $\mathrm{PA}$ was associated with less of a Stroop effect. NA and depression history were not significant predictors of task performance, and PA was not associated with performance on the easier Stroop task.

Conclusions: Unlike pure conditions, the nature of mixed presentations elicits uncertainty between the stimulus-response relationship (mixing costs) as well as increases the possibility that previous trials aid or impede subsequent trial performance (switching costs). Building upon previous findings suggesting that PA increases cognitive flexibility, the results from this study show that PA influences cognitive function as tasks demands increase.

Correspondence: Lorri A. Kais, MA, Clinical Psychology, Loyola University Chicago, 2337 N Spaulding Ave, Apt 1, Chicago, IL 60647, United States.E-mail: lorrikais@gmail.com

V.M. LABODE, S.C. HADEN, B.A. SAUNDERS \& N. CAIN. Impulsivity as a Moderator of the Relationship Between Stressful Life Events and Binge Eating.

Objective: Binge eating disorder (BED) is characterized by recurrent episodes of eating large quantities of food, while feeling a lack of control and inability to stop (APA, 2013). Binge eating in subclinical populations, however, is ten times more common (NIMH, 2014). Research has shown a positive relationship between past trauma, current stress, binge eating symptomatology (BES), and impulsivity. This study proposed that impulsivity would moderate the relationship between stressful life events and BES, such that those with greater impulsivity would demonstrate higher BES than those with lower levels of impulsivity.

Participants and Methods: An ethnically diverse sample of undergraduate females (18-23 years old) was collected $(\mathrm{N}=140)$. Participants completed self-report measures assessing their levels of impulsivity and BES, as well as their experience of stressful life events (i.e., childhood trauma, objective and perceived stress, and discriminatory stress).

Results: Hierarchal regressions were used to test for moderating effects of impulsivity on the relationship between stressful life events and BES. Results revealed that childhood trauma, objective stress, and perceived stress were all significantly and positively related to BES. Impulsivity did not moderate any of these relationships. While discriminatory stress did not have a significant relationship with BES, impulsivity was a moderator in the

relationship between discriminatory stress and BES, such that the relationship between discriminatory stress and BES severity was positive for those individuals with high impulsivity and was negative for those individuals with low impulsivity.
Conclusions: There is a unique relationship between discriminatory stress and impulsivity on BES that differs from those relationships between other types of stress and impulsivity. Greater understanding of how discrimination interacts with cognition may lead to better appreciation for why at-risk populations continue to suffer.

Correspondence: Vanessa M. LaBode, PhD, Neurology, Columbia University, 93 Teak Road, Dumont, NJ 07628, United States. E-mail: labode.richman@gmail.com

S.G. MASSONI, M. TIMPANO SPORTIELlo, S. DANTI \& S. TOCCHINI. Exposure to family violence and executive functions: a pilot study.

Objective: According to literacture exposure to family violence not only affect children's health and safety but also may affect their psychological adjustment, social relations, academic achievement and neuropsychological functioning; children who have beeen exposed to chronic and severe trauma are vulnerable to lifelong neurobiological implications. This study evaluates the association of young children's exposure to violence events and executive functions. We also study the link between exposure to family violence and emotion recognition and comprehension. Participants and Methods: We studied 15 children attending primary school with a history of family violence.

We assessed the capacity of working memory using digit span forward, listening span test and Corsi Test backward. We assessed attention using Trail Making Test and inhibitory processes with Stroop Test. At last we used a Test of Emotions Comprehension to assess the ability to recognize emotions.

Results: Subjects exposed to family violence totalized Digit span FW, Listening Span Test and Corsi Test FW scores significantly low $(\mathrm{p}<0.05)$.

Trail Making Test and Stroop Test scores were significantly poor $(p<0.05)$. Emotion recognition was biased, expecially the perception of sadness and fear were impaired.

Conclusions: The developing brain is vulnerable to the negative effects of trauma and chronic stress. Witnessing violence has a negative impact on working memory, attention and inihbitory processes. Also emotions recognition is impaired in children exposed to family violence. More studies are necessary to better prevent neuropsychological consequences and impairment of exposure to violence.

Correspondence: Sonia G. Massoni, Public Health Autority ASL Nord Ovest, Via Vecchia di Vorno 7, Badia di Cantignano 55060, Italy. E-mail:soniamassoni@gmail.com

E. MCGLADE, E. BUELER, J. DIMUZIO, M. LEGARRETA \& D. YURGELUN-TODD. Impulsivity and Aggression: The Relationship to Suicidal Behaviors in US Veterans.

Objective: Suicide is among the leading causes of death for US veterans, although there remains a limited understanding of the underlying risk factors. Impulsivity and aggression related to reduced inhibitory function have been implicated in suicidal behavior. The current study extends prior research to examine the association between types of impulsivity and aggression in suicidal behavior in a sample of US veterans.

Participants and Methods: Participants included 79 veterans ( 25 with no history of suicidal behavior and 54 with a history of suicidal behavior). Participants completed the Columbia Suicide Severity Rating Scale (CSSRS), Barratt Impulsivity Scale (BIS), and Buss-Perry Aggression Questionnaire (BPAQ).

Results: Participants with a history of suicidal behaviors (SB+) reported significantly more impulsivity and aggression across the BIS and BPAQ subscales. Specifically, SB+ participants reported increased Total Impulsivity $(\mathrm{p}<0.001)$ with the following significant subscales (all $\mathrm{p}<0.002)$ : Motor, Attention, and Nonplanning. SB+ also evidenced increased scores on Verbal Aggression, Anger, Hostility, and Total Aggression (all $\mathrm{p}<0.05)$. In the $\mathrm{SB}+$ group, lifetime suicide behaviors correlated with BIS Nonplanning, BPAQ Anger, BPAQ Hostility, and BPAQ Total $($ all $\mathrm{p}<0.04)$. 
Conclusions: These findings are among the first to examine both impulsivity and aggression in veterans with suicidal behavior and to highlight the importance of understanding inhibitory processing as a cognitive feature in suicide risk in veterans. Individuals who are not strong planners, are more focused on the present than on the future, get angry easily, and feel as though life has treated them unfairly may be at increased risk for suicidal behaviors. Further research should examine executive function in combination with inhibitory processing and other intervening variables, including sex differences and the difference between individuals with suicidal ideation and suicide attempts. Correspondence: Erin McGlade, PhD, Diagnostic Neuroimaging Lab, University of Utah, 383 Colorow Dr, Salt Lake City, UT 84108, United States.E-mail:erin.mcglade@hsc.utah.edu

J.J. MIETCHEN, D. HEDGES \& S.D. GALE. Moderating Variables of Sleep Disordered Breathing and Executive Functioning: A MetaAnalysis.

Objective: Determine if age, sex, and apnea-hypopnea index (an index of apnea severity; $\mathrm{AHI}$ ) moderate executive functioning (EF) in children with sleep disordered breathing (SDB).

Participants and Methods: A meta-analysis on EF in pediatric SDB found a medium effect size for decreased generativity on objective neuropsychological tests. In this study we further investigated this association using meta-regression. Data were obtained from thirteen studies that met criteria with a total sample of 1697 children from 5 to 17 years of age $(\mathrm{M}=9.81 ; \mathrm{SD}=0.34)$. Females made up $45.75 \%$ of the sample. Performance on objective measures of EF was extracted from each source study along with demographic variables, including AHI, age, and sex. A series of meta-regressions were performed with these demographic variables acting as moderators.

Results: After controlling for SDB severity, age did not reach statistical significance as a moderator for effect size between studies $(B=0.08$; $\left.p=0.08 ; R^{2}=0.48\right)$. This model, including age and AHI accounted for $48 \%$ of the variability in effect sizes of EF between studies. Studies using tests specifically measuring working memory were examined because it was the only EF domain with enough studies to conduct a domain specific meta-regression. When examining effect sizes of working memory tasks, AHI was not a significant moderator of effect size between studies ( $\left.B=-0.03 ; p=0.06 ; R^{2}=0.72\right)$, although AHI accounted for $72 \%$ of the variability in effect sizes of working memory between studies. Sex was not a significant moderator of EF in children with SDB $\left(B=0.001 ; p=0.00 ; R^{2}=0.00\right)$.

Conclusions: Age, AHI, and sex were not significant moderators for EF in children with SDB. However, given the lack of a sufficient number of studies to conduct meta-regressions across all five EF domains for which we extracted data, it is probable that our meta-regression analyses were under-powered. Thus, to determine if there are moderators for EF in pediatric SDB additional studies will need to be conducted.

Correspondence: Jonathan J. Mietchen, MS Psychology, Department of Psychology, Brigham Young University, 1001 SWKT, Provo, UT 84602, United States.E-mail: jonathan.mietchen@gmail.com

\section{B. MORGAN \& J. SUHR. The Relationship of Craving and Negative Affect to Impulsive Decision-Making During Heightened mood Conditions.}

Objective: Problematic alcohol use among college students continues to be a substantial concern. Craving is one of the defining features of problematic alcohol use and has been recently added to the DSM-5 alcohol use disorder criteria. Negative affect is also consistently related to problematic alcohol use and greater craving. Increased negative affect and craving are both related to greater impulsivity on behavioral measures that strongly relate to problematic alcohol use, such as the Iowa Gambling Task (IGT). The present study examined the relationship of craving and IGT decision-making when in a manipulated mood state among high-risk alcohol users.

Participants and Methods: Participants were 144 undergraduate students (mean age 19.31; 108 female) who endorsed hazardous alcohol use on the Alcohol Use Disorder Identification Test (AUDIT) as part of a larger ongoing study. Participants completed demographic information and self-report measures including the Alcohol Craving Questionnaire-Short Form-Revised (ACQ-SF-R). Participants viewed 30 images selected from the International Affective Picture System (IAPS) depending on assigned mood condition (positive, negative, or neutral) and completed the ACQ-SF-R again. Participants then completed the IGT task while IAPS images continued to be displayed.

Results: Consistent with prior research, greater negative affect related to more risky decision-making performance on the IGT, $r=.30$, regardless of assigned mood condition. In the positive mood condition, greater craving was significantly associated with more risky IGT performance, $r=.38$., but not in the neutral, $r=-.09$, or negative, $r=.18$, mood conditions.

Conclusions: Results suggest that, although both negative affect and craving are related to more risky IGT performance, they show differential relationships depending upon assigned mood conditions. Future studies should examine the role of craving, negative affect, and their interaction in particular mood states on behavioral outcomes.

Correspondence: Brittni Morgan, Ph.D., Psychology, Ohio University, PorterHall 200, Athens, OH 45701, United States.E-mail:bm569811@ ohio.edu

C.M. NGUYEN, C. COPELAND \& J. LINCK. The Contribution of Executive Functioning to Performance on the Texas Functional Living Scale.

Objective: The Texas Functional Living Scale (TFLS) is a performance-based measure of instrumental activities of daily living (IADL). A number of cognitive variables, particularly memory and perceptual reasoning abilities, have been shown to contribute to performance on the TFLS. The contribution of executive functioning was investigated in the current study.

Participants and Methods: One hundred and twenty patients completed the TFLS as part of a comprehensive test battery at an outpatient neuropsychology clinic ( $M$ age $=70.1+/-12.8$ years; $57 \%$ women $)$. Demographic factors (i.e., age, sex, and education), measures of verbal and nonverbal abstraction (i.e., WASI-II Vocabulary and Matrix Reasoning subtests), domain-specific neurocognitive functioning (i.e., RBANS Indexes), and measures of executive functioning (i.e., Trails Making Test and Stroop Color and Word Test) predictor variables were included in exploratory hierarchical regression models with TFLS Total index score as the criterion variable.

Results: Demographic characteristics (Step 1: $R^{2} \Delta=.07 ; p=.116$ ), abstraction (Step 2: $R^{2} \Delta=.22 ; p=.000$ ), neurocognitive functioning across domains (Step $3: R^{2} \Delta=.11 ; p=.021$ ), and executive functioning (Step 4: $R^{2} \Delta=.11 ; p=.001$ ) variables collectively accounted for a statistically significant amount of adjusted variance in TFLS Total scores (adjusted $\left.R^{2}=.44, p<0.001\right)$. After accounting for these variables in the prediction model, RBANS Memory Index $(B=0.24, t=1.99$, $p=0.05)$ and Trail Making Test Part B $(\dot{B}=-0.45, t=-3.63, p=.001)$ scores significantly predicted TFLS Total scores. Stroop Color and Word Test scores did not significantly predict TFLS Total scores $(B=0.15$, $t=1.27, p=.210)$.

Conclusions: Results confirm prior findings of a relationship between general functional and cognitive abilities and further specify that executive functioning significantly predicts functional ability beyond demographic factors, neurocognitive domains, and abstraction.

Correspondence: Christopher M. Nguyen, Ph.D., Psychiatry and Behavioral Sciences, University of Oklahoma Health Sciences Center, 601 Robert S Kerr Ave, Apt 108, Oklahoma City, OK 73102, United States.E-mail: chris.minh@gmail.com

A. NICHOLS, C.M. MULLEN \& A.Y. STRINGER. A Cluster Analytic Approach to Defining Executive Dysfunction Subtypes.

Objective: Cluster analysis (CA) was used to develop subtypes of executive dysfunction based on the Emory Short Form Wisconsin Card Sorting Test (EWCST), which were then externally validated on a larger battery of executive function (EF) measures. 
Participants and Methods: Participants were 285 mixed neurological patients (31.6\% stroke, $23.9 \%$ epilepsy, $16.5 \%$ brain injury, $28 \%$ neurodegenerative, tumor, endocrine, or metabolic disorder), with a mean age 45.38 (SD 15.34). Participants completed the 48-card EWCST, which eliminates stimuli that match key cards on more than one dimension. Additional EF tests measured cognitive fluency, categorical reasoning, verbal and visual abstract thinking, motor regulation, and error monitoring during memory retrieval. CA of EWCST scores used the average linkage within groups method and squared Euclidean distance as the error measure to create homogeneous clusters that were then examined to develop rules for subtyping executive dysfunction. Subtypes were externally validated by contrasting performance on the larger battery of tests with Analysis of Covariance, controlling for differences in age and education.

Results: CA yielded 4 clusters: (1) intact EF group (IntG), (2) perseverative group (PG), (3) impulsive group (ImpG), and (4) ideational constriction (i.e., inability to conceptualize alternatives) group (IcG). The 4 groups differed statistically on the EWCST; IntG completed more sorts, IcG repeated the same ineffective strategy, and PG made more perseverative errors. Partial external validation of the subtypes was seen; IntG performed better across EF measures, IcG and PG showed poorer cognitive fluency and visual abstract thinking, and IcG additionally had poorer motor sequencing and verbal abstract reasoning.

Conclusions: CA led to executive dysfunction subtypes that performed consistent with expectations on both the EWCST and external EF measures. Results are consistent with an EF macrostructure model that incorporates concept formation and impulse and perseverative control. Correspondence: Anastacia Nichols, 1441 Clifton Rd, Suite 170, Atlanta, GA 30322, United States.E-mail: anastacia88@gmail.com

K.H. NIXON, D. BOSCH, S. AMANO, A. DREYER \& A.A. TURK NOLTY. Learning and Executive Functioning in Humanitarian Aid Workers During Times of Overwhelming Stress.

Objective: Objectives. Extreme or chronic stress can diminish executive functioning, leading to inefficient use of learning strategies and poorer performance on memory tasks (Brewin et al., 2007; McEwen 2010; Stuss \& Alexander, 2000) and lead to cognitive overload that can reduce problem-solving and memory efficiency. The aim of this study was to investigate learning difficulties in humanitarian aid workers during times of stress.

Participants and Methods: Participants and Methods. Twenty-four aid workers completed alternate forms of the Rey Complex Figure Test (RCFT) and the California Verbal Learning Test-II (CVLT-II) on the first and last days of a training workshop that involved a simulated kidnapping scenario.

Results: Results. Aid workers recalled significantly more total words from the CVLT-II on the first day $(M=49.1, S D=14.3)$ than when under stress $(M=43.1, S D=13.5), t(23)=2.26, p<.05$, as was the case for List B $(M=5.7, S D=2.1$ vs. $M=4.9, S D=1.3), t(23)=2.33$, $p<.05$, and the short delay recall $(M=11.1, S D=4.2$ vs. $M=9.9$, $S D=4.8), t(23)=2.16, p<.05$. Copy and immediate recall of the alternate form of the RCFT when under stress was the same as when relaxed, and delayed recall was stronger under stress $(M=24.1$, $S D=6.3$ vs. $M=21.1, S D=6.0), t(23)=2.45, p<.05$.

Conclusions: Conclusions. Verbal memory was much poorer under stress, whereas visual memory remained unchanged, except for RCFT Delayed Recall, which significantly improved. While participants in this study did not have a diagnosis of PTSD, performances of participants under stress emulated those individuals with disorders associated with traumatic stress. These findings support a differentiation in verbal learning against visual learning under stress (Scott et al., 2015; Brewin et al., 2007) such that stress negatively impacts verbal learning more than visual learning. For aid workers, bolstering executive functioning may be an important consideration.
Correspondence: Kyle H. Nixon, M.A., Fuller Graduate School of Psychology, 135 N. Oakland Ave., Pasadena, CA 91182, United States. E-mail: kylenixon@fuller.edu

\section{B.C. ORR, J. XU, C. HASS \& L. ALTMANN. Is Verbal Fluency a Language Measure?}

Objective: Neuropsychologists frequently use verbal fluency to assess language in pathological populations. The literature suggests that Semantic and Letter Fluency, the two components that make up verbal fluency, may dissociate. It has also been suggested that verbal fluency is more closely related to executive function than language ability. This study hopes to clarify the relationship between verbal fluency measures and language ability.

Participants and Methods: A sequence of cognitive tasks plus a language measure derived from sentence production were used to assess 54 non-demented older adults (mean age: 72). A principle component analysis revealed 4 underlying factors: working memory (WM), cognitive speed, inhibition, and language. These were used to predict performance on Letter and Semantic Verbal Fluency tasks.

Results: The best-fitting model predicting Letter Fluency accounted for $49.4 \%$ of the total variance with WM, age, and cognitive speed, explaining $25.8 \%, 15.8 \%$, and $7.9 \%$ of significant variance respectively, while the language factor did not account for any additional variance. The model accounting for the most variance in Semantic Fluency explained $19.7 \%$ of the total variance with cognitive speed explaining $12.6 \%$ and WM explaining $7.1 \%$ of the significant variance. Neither age nor language accounted for additional significant variance in Semantic Fluency performance.

Conclusions: Results support a dissociation between verbal fluency measures evidenced by the role of age in predicting Letter but not Semantic Fluency. Additionally, cognitive speed and WM played a role in both verbal fluency measures; however, WM played a greater role in Letter Fluency while cognitive speed was more important to Semantic Fluency. Language did not predict performance on either verbal fluency task. Future analyses will include additional language measures to provide a more comprehensive evaluation of the relationship between language ability and verbal fluency performance.

Correspondence: Brian C. Orr, M.A., Speech Language and Hearing Sciences, University of Florida, 1225 Center Drive, Box 100174, Gainesville, FL 32610-0174, United States.E-mail: brianorr@ufl.edu

\section{C.R. PAgan, K. KAUZOR \& J. RAZANI. Culture and English Fluency on Measures of Executive Functioning in Ethnically Diverse Groups.}

Objective: Objective: Cultural factors such as acculturation and years of education outside of the U.S. has previously been found to play a role in neuropsychological test performance in ethnically diverse (ED) individuals. This study will expand upon previous research to examine the extent to which cultural and English fluency factors account for performance in frequently used executive functioning (EF) measures in ED individuals.

Participants and Methods: Participants: A total of 91 ED healthy individuals were included in this study (Hispanic: $\mathrm{n}=13$; Asian: $\mathrm{n}=55$; Middle Eastern: n=23) and recruited from the greater Los Angeles area. Methods: Participants were administered the reading (WC-7) and writing (WC-13) subtests from the Woodcock Language Proficiency Battery-Revised (WLPB-R), the Trail Making Test (TMT-A and TMT-B) and the Color Trails Test (CTT-A and CTT-B). The amount of years in education spent outside of the U.S. was also gathered. Finally, the Acculturation Rating Scale for Mexican Americans (ARSMA) was adapted and administered to each ED group.

Results: Results: A multiple regression analysis was conducted to evaluate if cultural factors and English fluency significantly predicted participants scores on EF measures. Acculturation was significantly related to TMT-A $\left(F(1,67)=6.37, p<.01, R^{2}=.09\right)$. WC-13 was 
significantly related to TMT-B $\left(F(1,67)=6.26, p<.05, R^{2}=.09\right)$ and CTT-2 $\left(F(1,67)=4.76, p<.05, R^{2}=.07\right)$. Years of education outside of the U.S. did not predict performance on EF measures.

Conclusions: Conclusion: The results revealed that acculturation and writing fluency were significant predictors in performance on EF measures, even for test with very little verbal mediation and believed to be "culture-free". Acculturation and English proficiency should be considered when interpreting results of ED individuals on EF tasks in neuropsychological test batteries.

Correspondence: Carolyn R. Pagan, Master's in Clinical Psychology, Psychology, California State University, Northridge, 837 N. West Knoll Dr., unit 118, West Hollywood, CA 90069, United States. E-mail: carolyn.pagan@csun.edu

K.L. POLNASZEK, I. KAHRILAS, C. BATES, A. BOHNERT, K. HELLMAN, F. TU \& R. SILTON. Inhibitory Control and Psychological Symptoms Influence Pain Symptomatology in Emerging Adults.

Objective: Emerging adulthood is a critical window to optimize positive health behaviors in order to prevent long-term trajectories of severe pain and disability. It is also a distinct developmental period characterized by ongoing maturation of executive functions $(\mathrm{EFs})$ that enable individuals to navigate daily experiences. Previous research suggests that pain symptoms are associated with EF impairments. Co-occurring psychological symptoms, including depression, worry, and anxious arousal, or somatic symptoms, may also exacerbate pain symptoms, as well as EF impairments. The present study examined the associations between EFs (inhibition and shifting), psychological, and pain symptoms in order to identify psychological and cognitive symptoms associated with pain outcomes.

Participants and Methods: Emerging adults $(\mathrm{N}=1295)$ completed a series of questionnaires. Participants $(n=648)$ who reported experiencing pain symptoms for at least the past month were included in the present analyses. Self-report measures of pain, anxious arousal (Mood and Anxiety Symptom Questionnaire), depression (PHQ-9), worry (Penn State Worry Questionnaire), and EF (BRIEF) were used.

Results: Analyses revealed significant correlations between depression, worry, anxious arousal and most pain variables (i.e., pain frequency, intensity, and impairment) with correlations ranging from $r=.09$ to $r=.22$. A series of hierarchical regression analyses showed that inhibition impairments predicted pain frequency $(p<.05)$. Anxious arousal and depression symptoms significantly predicted pain intensity as well as limitations in daily life activities (ps <.05). Worry and depression symptoms predicted the amount to which pain was perceived to bother participants (ps <.05).

Conclusions: Specific profiles of of EF and psychological symptoms distinctly contributed to the expression of perceived pain frequency, intensity, and impairment in emerging adults, all of which are related to negative long-term health outcomes.

Correspondence: Kelly L. Polnaszek, MA, Psychology, Loyola University Chicago, $550 \mathrm{~W}$ Roscoe St, Apt 2B, Chicago, IL 60657, United States. E-mail: kellypolnaszek@gmail.com

J. POMMY, B. FINK \& M. FROST. Executive Function in the Context of Intimate Partner Violence.

Objective: Executive function (EF) plays an important role in social functioning. Impaired EF has been observed among individuals that commit and experience intimate partner violence (IPV). While there is some evidence to support the matching of EF may contribute to partner's relationship satisfaction in typical couples, the association between $\mathrm{EF}$ within couples experiencing IPV is unknown. The present analysis examined EF in the context of IPV.
Participants and Methods: 46 distressed, heterosexual couples were recruited: 28 distressed violent (DV) couples, and 18 distressed non-violent (DNV) couples. Self-reported EF was assessed using the Behavior Rating Inventory of Executive Functions (BRIEF). Group differences in the BRIEF scales (Global Executive Composite (GEC); Behavioral Regulation Index (BRI), Metacognitive Index (MI), and nine clinical scales) were examined using a MANOVA. Correlations between partners' EF was then examined within DV and DNV couples, separately.

Results: No group differences were observed in EF. When examining correlations between partners' higher-order EF scales, within the DV group, significant correlations were observed between female partner's MI and male partners' GEC and BRI $(r=0.384, p=0.044 ; r=0.483$, $p=0.009$, respectively). Within the NDV couples, no significant correlations were observed. Further, in the DV group, positive correlations were observed between female partner's MI subscales and male partner's BRI subscales. In the DNV group, positive correlations were observed between female and male partners' MI subscales; and female partner's BRI subscales were correlated with male partners' BRI and MI subscales. Conclusions: Results, though preliminary, suggest while no group differences in self-reported EF were observed between DV and DNV couples, differences in conflict escalation witnessed between these couple types may, in part, be reflected by the mismatch between partners' EF difficulties in the DV couples.

Correspondence: Jessica Pommy, Psychology, University of New Mexico , 1101 Yale Blvd NE, Albuquerque, NM 87106, United States. E-mail: jpommy@gmail.com

B.W. REYNOLDS \& M.R. BASSO. Gambler's Ruin: Negative Affect Guides Pursuit of Punishing Choices on the IGT.

Objective: The Iowa Gambling Task (IGT; Bechara et al., 1994) is a measure of risky decision-making. It has been utilized among multiple clinical populations. Despite its popularity, the IGT's traditional scoring metrics have been criticized. For example, the IGT has evidenced questionable validity, particularly among healthy adults. Authors have speculated that alternative metrics, such as dividing the task into two separate indices (i.e., early vs. later trials), may clarify constructs captured by the measure. Similarly, investigators have considered individual decks rather than composite scores in exploring decision-making constructs. Collectively, these analyses have explored relationships with affect, impulsivity, and executive function. The present study sought to clarify which personality and cognitive variables might be associated with each deck.

Participants and Methods: The IGT was administered to 54 healthy undergraduate students. Individual deck preference was calculated across task halves (i.e., Trials 51 to 100 - Trials 1 to 50). Affect was assessed using the PANAS, while trait impulsivity was explored with the UPPS-P. Concept formation was evaluated using the Wisconsin Card Sorting Test. The WAIS-IV Digit Span was also provided to assess working memory.

Results: Multiple regression analyses were conducted evaluating IGT deck preference. All variables were entered simultaneously. Tolerances were $>.65$. Across all four decks, only Deck A was significant. Higher negative affect $(s r=.49)$ and lower working memory $(s r=-.39)$ accounted for substantial variance in Deck A preference over task halves.

Conclusions: The current findings reemphasize the convoluted nature of traditional IGT metrics (i.e., individual decks). Specifically, we observed that IGT decks are not equivalent when compared across theoretically-associated constructs. Negative emotionality, in particular, appears to be uniquely linked with persistence in choosing from a frequent punishing alternative (Deck A).

Correspondence: Bradley W. Reynolds, PhD, Psychology, University of Tulsa, 800 S Tucker Road, Lorton Hall 308, Tulsa, OK 74104, United States.E-mail: bradley-reynolds@utulsa.edu 
K. RYU, Y. KIM, Y. CHUNG \& Y. CHEY. Relationship Between Naming Ability And Other Neuropsychological Functions.

Objective: Naming ability is critical for detecting cognitive decline. This study aims to examine the relationship among naming ability, verbal/visual memory, verbal fluency, inhibitory control function and vocabulary.

Participants and Methods: Fifty-two healthy Korean adults (28 females, 24 males), aged from 18 to 43 years $(\dot{M}=29.90, \mathrm{SD}=7.25)$, participated in the study. Their years of education ranged from 11 to 18 years $(\mathrm{M}=15.21, \mathrm{SD}=1.76)$. All participants provided written informed consents after procedures were fully explained. Participants with previous illness that could affect cognitive function were excluded. The Korean-Boston Naming Test (K-BNT) was used for naming ability test. Seoul Verbal Learning Test (SVLT) for verbal memory test, Rey Complex Figure Test (RCFT) for visual memory test, Controlled Oral Word Association Test (COWAT) for verbal fluency, Stoop test for inhibitory control, and vocabulary subtest of Korean Wechsler Adult Intelligence Scale-IV (K-WAIS-IV) for vocabulary test were used.

Results: Correlation analyses showed that naming ability was significantly correlated with verbal immediate memory (SVLT immediate recall: $\mathrm{r}=.35, p<.05$ ), with visual memory (RCFT immediate recall: $\mathrm{r}=.34, p<.05$, RCFT delayed recall: $\mathrm{r}=.38, p<.05$, RCFT recognition score: $\mathrm{r}=.30, p<.05$ ), with phonemic verbal fluency (COWAT phonemic: $\mathrm{r}=.42, p<.05$ ), and vocabulary (Vocabulary subtest of K-WAIS-IV: $\mathrm{r}=.40, p<.05)$.

Conclusions: This study found the naming ability is correlated to many cognitive functions such as verbal/visual memory, verbal fluency and vocabulary, suggesting that that the naming ability is closely associated with other cognitive functions. Our finding is consistent with the observations in clinical field that the low performance of naming test is very sensitive to cognitive dysfunction.

Correspondence: Kyoung Hi Ryu, Ph. D., Human Brain Research \& Consulting Co., 2nd Floor, 97, Seochojungang-ro 22-gil, Seocho-gu, Seoul 06632, Korea (the Republic of).E-mail:khryu1003@gmail.com

O.A. SANTOS, J. SOBLE \& J. MARCEAUX. Investigating the Effect of Bilingualism and Nonverbal Intelligence on Executive Functions.

Objective: While some studies have found variable support for a bilingual advantage in the development of executive functions (EFs), Roselli et al. (2016) found that nonverbal intelligence, rather than bilingualism, was robustly related to EFs in cognitively normal, young bilinguals. The present study sought to replicate these findings in a mixed clinical sample of bilingual and monolingual veterans.

Participants and Methods: Participants included 152 veterans (42 bilinguals and 110 monolinguals) who underwent neuropsychological evaluation and were administered the Trail Making Test (TMT), Stroop Test, Wisconsin Card Sorting Test-64 Card Version (WCST-64), and the Wechsler Adult Intelligence Scale-Fourth Edition (WAIS-IV) as part of a larger clinical battery. Executive variables included TMT-Part B, Stroop Color-Word (SCW) condition, and WCST-64 Perseverative Errors. WAIS-IV Block Design (BD) was included as a measure of nonverbal intelligence. Multivariate analysis of covariance (MANCOVA) and hierachical regression analysis were conducted.

Results: MANCOVA showed no significant bilingual group differences on the TMT-B, SCW, WCST-64, and BD after controlling for cognitive impairment (CI) status (Wilks's $\wedge=.97, F[4,146]=1.11, p=.36$, $\left.\eta^{2} \mathrm{p}=.03\right)$. Hierarchical regression demonstrated that nonverbal intelligence (Step 3: $\Delta R^{2}=.16-.20[p>.0001]$, adjusted $\left.R^{2}=.14-.19\right)$, but not bilingualism (Step 2: $\Delta R^{2}=.001-.04[p=.94]$, adjusted $R^{2}=-.01-.03$ ), significantly predicted performances on the three executive measures after controlling for CI status.

Conclusions: Results replicated and extended previous findings by Roselli et al., suggesting that nonverbal intelligence is a better predictor of performance on diverse EFs than bilingualism in a mixed clinical population, and that the bilingual advantage hypothesis may be potentially mediated by other cognitive processes.
Correspondence: Octavio A. Santos, PhD, Psychiatry \& Psychology, Mayo Clinic Florida, 4500 San Pablo Road, Jacksonville, FL 32224 , United States. E-mail: osantossolano@gmail.com

C.R. PENNINGTON, C.E. HENDERSON, C.N. BRYSON, B.E. RIDGE, K.K. MARSHALL, K.E. MCCALLUM \& A.T. SCHMIDT. Exploratory Factor Analysis of Neuropsychological Test Data Suggests a Four Factor Model of Executive Functioning in an Undergraduate Sample.

Objective: This study will conduct a factor analysis of executive functioning (EF) measures, rather than using a theory driven method to decide what EF measures represent a specific factor. Further, this study employs a greater number of EF tests, so each factor will potentially be comprised of more than one measure, again enhancing construct validity.

Participants and Methods: The sample consisted of 186 undergraduate students. Participants ranged in age from 18 to $34(M=20.0$, $S D=2.1), 40.9 \%$ of participants were male and $59.1 \%$ of participants were female. The sample group was ethnically diverse. Participants completed a demographics questionnaire along with the NEO-PI-3, three D-KEFS tests, the WASI-II, and the WCST.

Results: An exploratory factor analysis (EFA) was conducted using EF variables deemed appropriate from initial factor analyses. Principal component analysis was used to identify and compute composite scores for the factors that underlie EF tests. Both eigenvalues and the scree plot indicated that a four-factor model was the best fit. Overall, the four-factor solution explained $71 \%$ of the total variance.

Conclusions: This study provides an examination of the factor structure of EF based on a selection of commonly used neuropsychological tests. Four measures of EF were used in an EFA to discern if the commonly used three-factor model of EF (i.e., updating, shifting, inhibition) was supported. Results instead yielded a four-factor model. While the results of the current study are inconsistent with a three-factor model of EF, they provide further evidence as to the level of complexity and overlap among such measures. The present study expands upon previous literature by including tests that have been excluded from similar studies. Another contribution is the implication that these commonly used measures of EF are correlated with one another and appear to form distinct clusters.

Correspondence: Adam T. Schmidt, TX, United States. E-mail: adam.t.schmidt@ttu.edu

B.J. SHIELDS \& A. HEFFELFINGER. Impact of Early Neurologic Insult on Executive Functioning Development in Preschool Children.

Objective: Children who experience early neurologic insult (e.g.., stroke, hydrocephalus, seizures) are at risk for numerous long-term cognitive impacts, particularly impacts on executive functioning (EF; Max et al., 2003; Yeates et al., 2002). Less is known about the risk for disruptions in EF development in children under 5-years-old. As this is a time of peak neural development, insults may result in greater damage to functional systems responsible for EF than other times in development (Johnson, 2003). As such, we examined EF differences between a sample of children with and without early neurologic insult.

Participants and Methods: Participants were 27 children between 19-58 months old who completed an EF task. Twelve children $(58.3 \%$ male, $M=40.42+10.60$ months, $83.3 \%$ Caucasian) had been diagnosed with a significant neurological insult. Fifteen typically developing children (53.3\% male, $M=37.47+13.16$ months, $86.7 \%$ Caucasian) served as controls. Assessments were completed as part of a larger study examining attention and EF in children with early neurologic injuries. Children completed the Delayed Alternation task (DA; Espy et al., 2001), a developmentally appropriate test of EF measuring working memory. Mann Whitney U tests were conducted to examine between-group differences in DA performance. 
Results: Groups did not differ in total number correct (Neuro $M d n=12$, Control $M d n=11, U=89.50, p=.98$ ) or consecutive items correct (Neuro $M d n=2$, Control $M d n=2, U=87.00, p=.88$ ), despite significant differences in cognition (Mullen ELC, Neuro Mdn=84.50, Control Mdn=107.00, $U=49.50, p=.04)$.

Conclusions: Results were unexpected given the likely impacts on the development of neural systems responsible for EF due to early neurologic insult. Findings suggest that children may be at risk for EF difficulties that are not apparent at a young age. Future research will be important to assess EF development among patients with early insult longitudinally, as well as determine whether patterns of EF deficits differ depending on the type of insult.

Correspondence: Brian J. Shields, Ph.D., Medical College of Wisconsin , 2556 N. 69th St., Milwaukee, WI 53213, United States. E-mail: brianjshields@gmail.com

\section{K. BORESS, R. SKEEL, Y. KAHSAY \& A. FEDER. Understanding the Relationship Between Working Memory, Impulsivity, and Aggression.}

Objective: Objective: This study investigated the relationship between impulsivity, working memory, and aggression to address the role these constructs may play in individuals with impulsive and/or aggressive tendencies. A significant positive relationship between impulsivity and aggression, a significant negative relationship between working memory and aggression, and a significant interaction between impulsivity and working memory in predicting aggressive behaviors were hypothesized. Participants and Methods: Participants and Method: Male participants were recruited from psychology classes at a large Midwestern University. After screening for invalid data, data analysis was completed with 70 participants (87\% were Caucasian, $97 \%$ had English as a first language, and mean age $=19.8, \mathrm{SD}=2.02)$. Impulsivity was assessed through a self-report measure (the Barratt Impulsiveness Scale) and a behavioral measure (the Immediate Memory Task). Working memory was assessed through three behavioral measures (the Digit-Span Recall Task, and N-back Task, and the Dot Counting Task) and aggression was assessed through a self-report questionnaire (the Buss-Perry Aggression Questionnaire).

Results: Results: The results revealed a positive correlation between impulsivity and aggression $(r=.25, p=.03)$, a negative correlation between working memory and aggression $(r=-.26, p=.03)$, and trend toward a negative correlation between impulsivity and working memory $(r=-.23, p=.055)$. Regression analyses suggested impulsivity and working memory both predict aggression $\left(R^{2}=.11, F(2,67)=3.67\right.$, $p=.02, f^{2}=.08$. $)$. There was no significant interaction between impulsivity and working memory.

Conclusions: Conclusion: These results provide information about the relationship between impulsivity, working memory, and aggression that may aid in the future development of interventions for individuals with disorders characterized by impulsive and/or aggressive behavior. Correspondence: Reid Skeel, Ph.D., Psychology, Central Michigan University, 136 Sloan Hall, Mt. Pleasant, MI 48859, United States. E-mail:reid.skeel@cmich.edu

J. TESSIER, E. WHIPPLE, K. ROBINSON \& M.T. SCHULTHEIS. Sleep and Executive Functioning in Post-9/11 Combat Veterans With and Without Posttraumatic Stress Disorder.

Objective: To examine the relationship between sleep and executive functioning in post-9/11 combat Veterans with/without posttraumatic stress disorder (PTSD).

Participants and Methods: Thirty-eight post-9/11 combat Veterans were enrolled. Twenty were diagnosed with PTSD (measured by the PTSD Checklist-Military Version [PCL-M]) and 18 were controls. Executive functioning (EF) was measured using Trail Making Test B and D-KEFS Color-Word Interference (CWI) subtests. Hours of sleep the night before testing was reported.
Results: An independent samples t-test revealed that Veterans with PTSD reported significantly fewer hours of sleep the night before testing than controls, $t(36)=3.55, p=.001$. Hours of sleep were significantly correlated with total number of uncorrected errors on CWI subtests, $r(36)=-.37, p=.02$, and were not related to EF measures. PCL-M score was significantly correlated with CWI Inhibition, $r(36)=-.37$, $p=.02$, and CWI Inhibition/Switching, $r(36)=-.35, p=.03$, and was not related to other EF measures. A linear regression between PCL-M score and CWI Inhibition was significant, $R^{2}=.12, b=-.27$, S.E. $=.12$, $\beta=-.34, t(36)=-2.21, p=.03$. A linear regression between PCL-M score and CWI Inhibition/Switching was significant, $R^{2}=.11, b=-.24$, S.E. $=.11, \beta=-.33, t(36)=-2.09, p=.04$.

Conclusions: Executive functioning is a complex construct comprised of multiple domains, including inhibition, switching, and self-monitoring. Results of this study suggest that PTSD severity predicts poorer inhibition and switching. Furthermore, Veterans with PTSD endorsed significantly fewer hours of sleep the night before testing. However, hours of sleep were not associated with performance on executive functioning tasks. This suggests that severity of PTSD symptomatology is related to quick inhibition and switching, rather than poor sleep. Instead, fewer hours of sleep was related to more uncorrected errors, indicating potential contributions to impaired self-monitoring.

Correspondence: Jillian Tessier, Psychology, Drexel University, 30 S 22nd St, Apt 10, Philadelphia, PA 19103, United States. E-mail: jtess1018@gmail.com

A. VANDEN BUSSCHE, C.PADULA, K. GRISANZIO, A. GOLDSTEINPIEKARSKI, M. CORDOVA, N. HAUG \& L. WILLIAMS. Cognitive Underpinnings of Frontolimbic Dysfunction in PTSD and Impact on Quality of Life.

Objective: Posttraumatic stress disorder (PTSD) negatively impacts cognition via alterations in connections between the frontal lobes (executive functioning; EF) and limbic system (emotion recognition; ER). We examined: 1) EF and ER differences between PTSD and non-PTSD groups; 2) the relationship between EF and ER in those with PTSD; and 3) moderation of EF and ER on the relationship between PTSD severity and quality of life (QoL).

Participants and Methods: Participants recruited from the community ( $\mathrm{n}=373 ; 46.1 \%$ female) endorsed depressive or anxiety symptoms. They completed in-person demographic, diagnostic (International Neuropsychiatric Interview), self-report (PTSD Checklist, World Health Organization QoL), and computerized neuropsychological assessments (WebNeuro). EF tasks included inhibition, shifting, and planning. ER tests included explicit emotion identification and implicit emotion recognition. Independent samples t-tests, point-biserial correlations, and linear regression analysis were interpreted at $p<0.05$.

Results: The PTSD group ( $\mathrm{n}=48$ ) differed from the non-PTSD group $(n=325)$; those with PTSD performed faster on independent EF measures of inhibition, shifting, and planning (all $p$-values $<0.01$ ). PTSD group performed worse with accuracy of identifying disgusted faces $(p=0.04)$, but better on sad faces $(p=0.02)$. PTSD participants performed worse on recognizing previously seen fearful $(p=0.02)$ and neutral $(p=0.04)$ faces, but better with recognition of disgusted faces $(p<0.01)$. The PTSD also had faster reaction time recognizing fearful faces $(p=<0.01)$. We observed positive correlations between EF and ER in those with PTSD; poorer EF was associated with poorer ER. Linear regression revealed that poor $\mathrm{EF}(p=0.00)$ moderated the relationship between PTSD symptom severity and QoL $(p=<0.05)$.

Conclusions: Frontolimbic alterations in PTSD may be detectable using neuropsychological measures. Cognitive rehabilitation focused on EF and ER may improve treatment efficacy and overall QoL.

Correspondence: Alicia Vanden Bussche, Palo Alto University, 140 Lacrosse Street Avon \#2, Pittsburgh, PA 15218, United States. E-mail: avandenbussche@paloaltou.edu 
M. WELSH \& E. PETERSON. Child Maltreatment and Hot Executive Function as Predictors of College GPA.

Objective: Child maltreatment (CM) affects neurological systems underlying executive functions (EF) and emotion processing. "Hot" EF refers to inhibition, flexibility, etc. in contexts of heightened emotion, arousal, and motivation. CM is also associated with poorer college adaptation in emerging adulthood. The current study examined the degree to which $\mathrm{CM}$ and performance on a hot EF task predicts academic achievement. Participants and Methods: 166 college students (76\% female; $\mathrm{M}=19.19 \mathrm{yrs}, \mathrm{SD}=2.1$ ) participating in a longitudinal study of childhood stress were administered the Childhood Trauma Checklist (CTQ), a self-report measure of Emotional Abuse/Neglect, Physical Abuse/Neglect, and Sexual Abuse, and the WAIS-Vocabulary Subtest. We also gave the Iowa Gambling Task (IGT), a Hot EF task that measures adaptive (less risky) responding in an arousing context. Gradepoint average (GPA) the semester of testing was recorded.

Results: Correlational analyses demonstrated that all CTQ scales, except Sexual Abuse, predicted lower GPA. Controlling for Vocabulary score, adaptive responding on the IGT, particularly Block 4 (of 5 blocks), positively correlated with GPA. A hierarchical regression found that Vocabulary did not predict GPA at step 1, but entering CTQ Emotional Neglect $($ beta $=-.28)$ and IGT Block 4 (beta $=.19)$ yielded a significant model, predicting $13 \%$ of the variance in GPA.

Conclusions: As a multifactorial phenomenon, college GPA is difficult to predict from psychological variables. Consistent with the literature reporting poor college adaptation following CM, we demonstrated that self-reported CM history predicts GPA. Additionally, increased time to achieve adaptive responding on the IGT significantly predicted GPA. Controlling for vocabulary, history of emotional neglect and EF performance in an arousing context predict a significant proportion of individual differences in GPA. CM and Hot EF may represent potential intervention targets to improve college achievement and persistence. Correspondence: Marilyn Welsh, PhD, School of Psychological Sciences, University of Northern Colorado, Box 94, University of Northern Colorado, Greeley, CO 80639, United States.E-mail: marilyn.welsh@ unco.edu

E. PETERSON \& M. WELSH. Trauma-specific Symptoms and General Mental Health Status each Predicts Hot Executive Function Performance in a College Sample.

Objective: Traumatic stress in development affects neural systems underlying executive functions (EF) and emotion processing. Early traumatic stress is also associated with trauma-specific and overall mental health symptoms in emerging adulthood. We examined relationships between self-report on the Trauma Symptoms Checklist (TSC-40), general mental health (Symptoms Checklist-90, SCL-90), and performance on an EF task that varied in the degree to which it elicits "hot" EF (i.e., EF processes in contexts of heightened emotion or arousal). Participants and Methods: In a college sample, 165 students $(24 \%$ male; $\mathrm{M}=19.19 \mathrm{yrs}, \mathrm{SD}=2.1$ ) completed both the self-report measures and a modified Go-No-Go task. Across three blocks, we manipulated the Go-No-Go stimuli to compare performance across three conditions: cool (colors, go on blue); warm (neutral faces, go on male); hot (anger and fear emotion faces, go on male). Reaction time (RT), Go and No-Go accuracy were measured in each condition.

Results: The TSC total score negatively correlated with neutral and emotion face RT. TSC total and scale scores correlated negatively with neutral and emotion face No-Go accuracy, but not Go accuracy. Similarly, the SCL-90 subscale and composite scores negatively correlated with neutral and emotion face No-Go accuracy, but not Go accuracy. Importantly, no such relationships were observed for either self-report measure in the cool color condition.

Conclusions: Current trauma and mental health symptoms exhibited specific relationships with performance on an EF inhibition task in the "hotter" contexts of neutral and emotion face stimuli. No such relationship was observed the cool color condition. Mental health correlated with No-Go accuracy trials only; students with higher levels of symptoms were less able to inhibit responses to the No-Go stimuli involving both neutral and emotion (particularly fearful) faces. The findings suggest that tasks requiring EF in more arousing contexts will be more difficult for college students higher in symptomatology.

Correspondence: Marilyn Welsh, PhD, School of Psychological Sciences, University of Northern Colorado, Box 94, University of Northern Colorado, Greeley, CO 80639, United States.E-mail: marilyn.welsh@ unco.edu

J. XU, B.C. ORR, C. HASS \& L.J. ALTMANN. Predicting Performance in the Alternate Uses Task.

Objective: Neuropsychologists typically use the Alternate Uses Task (AUT) to assess divergent thinking and creativity. The AUT requires oral generation of multiple uses for common objects (e.g., a brick), but the cognitive foundations of the task are unknown. The sparse evidence suggests that the AUT taps vocabulary and verbal fluency, but these findings have not been replicated. This study investigates to what extent cognitive and language measures predict AUT performance.

Participants and Methods: Fifty-one non-demented older adults (Mean age 72.3) completed a battery of cognitive and language tasks. Eleven cognitive measures were entered into a principal components analysis which identified three underlying factors accounting for $63.6 \%$ of the variance. The three factors identified were processing speed (Trails A, verbal fluency, digit symbol substitution), working memory (digit span forward and back, operation span, visual memory), and inhibition (Stroop interference, Stroop colors, Trails Interference). Language measures comprised three scores derived from a picture description task. Cognitive factors, language measures, and age were used to predict AUT performance.

Results: The only predictor for the number of alternative uses produced in the AUT was the processing speed factor, accounting for $30.1 \%$ of the total variance. The completeness of picture descriptions, a language measure, accounted for an additional $7 \%$ of variance; however, better completeness scores predicted lower AUT performance. No other measures accounted for significant variance in AUT performance.

Conclusions: Processing speed was the best predictor of AUT performance, demonstrating that AUT is more of a cognitive task than a language task. Interestingly, higher completeness scores, which required convergent thinking, were associated with poorer AUT performance. Thus, being very good at convergent thinking may restrict responses on the AUT. Correspondence: Jingchen Xu, M.A., Speech Lanuage and Hearing Sciences, University of Florida, 1225 Center Drive, Box 100174, Gainesville, FL 32610-0174, United States.E-mail: jcxu@ufl.edu

R.E. ZIEMNIK, M. GIBBS, A.R. NILSSEN, S. LOPEZ, J.L. TAGGARTNERIA, M.A. NIERMEYER \& Y. SUCHY. Office-based measures of medication management as predictors of real-world medication management.

Objective: Developing tests that accurately predict real-world functioning (e.g., medication management: $\mathbf{M M}$ ) is an important goal in neuropsychology research. MM is a complex task, with potentially serious consequences if error(s) are made. The Pillbox Test (PT; Zartman, Hilsabeck, Guarnaccia, \& Houtz, 2013) is a measure of executive functioning purported to offer information about patients' MM capacity (Logue, Marceaux, Balldin, \& Hilsabeck, 2015), as it requires that examinees independently organize a week's worth of fake medications in a pillbox. Timed instrumental activities of daily living (TIADL; Owsley, Sloane, McGwin, \& Ball, 2002) is a comprehensive office-based measure of IADLs (Kraybill \& Suchy, 2011), but its MM item (TIADL-MM) only consists of simple reading of instructions on medicine containers. The current study compared PT and the TIADL-MM as predictors of real-world MM abilities.

Participants and Methods: Community-dwelling older adults $(n=50$, age 60-85) completed the PT and the TIADL-MM. Speed and accuracy were recorded. To assess MM, participants' actual pills were counted weekly for eight weeks. Extra and missing pills were documented as errors. 
Results: A series of hierarchical linear regressions with MM as the criterion variable and age, $\mathrm{PT}$, and TIADL-MM as predictors indicated that (a) PT speed accounted for $18 \%$ of variance beyond age $(p=.001)$, while PT errors failed to reach significance $(p=.32)$, (b) TIADL-MM speed accounted for $14 \%$ of variance beyond age $(p=.005)$, while TIADL-MM errors failed to reach significance $(p=.50)$, and (c) PT speed accounted for $8 \%$ of unique variance beyond both age and TIADL-MM ( $p=.027)$, whereas TIADL-MM failed to account for additional unique variance $(p=.09)$.

Conclusions: Speed was a better MM predictor than accuracy. Complex visual processing (both TIADL-MM and PT) appears to predict MM. However, independently following complex instructions (PT only) appears to add incremental utility beyond simple instruction reading. Correspondence: Rosemary E. Ziemnik, BS, Psychology Department, University of Utah, 614 Medical Plaza, Salt Lake City, UT 84112, United States. E-mail: rziemnik@gmail.com

\section{Language and Speech Functions/Aphasia}

C.L. CROOK, E.E. MORAN, J. WEATHERS, D. TOTO \& M.E. ZIMMERMAN. Sleep and Language Functioning in Young Adulthood.

Objective: It is important to understand the effects of sleep loss in young adulthood as environmental pressures that hinder biologically appropriate sleep patterns increase during this time. While it is well established that sleep loss negatively impacts cognition, the research on sleep and language is limited. This study examined whether sleep influences conversational and expository speech.

Participants and Methods: Forty-one undergraduate students (age mean $(\mathrm{SD})=19.95(1.82)$; education mean $(\mathrm{SD})=13.73(1.10) ; 66 \%$ Female; $63 \%$ Caucasian) wore an actigraphic monitoring device for two weeks and completed the Cookie Theft picture description task from the Boston Diagnostic Aphasia Examination. Responses were recorded, and later transcribed using Praat software. A Praat script calculated the speech variables of interest. Pearson's correlations were conducted to examine the relationship between sleep variables of interest (Average Sleep Onset Latency [SOL], Variability in SOL, Average Sleep Efficiency [SE], Variability in SE) and language (Total Duration [TD], Speech Rate [SR], Articulation Rate [AR], Average Syllable Duration [ASD]).

Results: Poorer average SE was associated with increased TD ( $r=-.47$, $p=.003)$, and increased variability in SE was associated with increased TD $(r=.46, p=.004)$; however, neither were significantly associated with $\mathrm{SR}, \mathrm{AR}$, or ASD. There were no significant correlations between average SOL or variability in SOL and the language variables.

Conclusions: Preliminary results showed that two markers of increased sleep disruption are linked to increased total duration of conversational and expository speech. Future research will determine if these associations are moderated by number of pauses, filler words, and elongated pronunciations. Overall, these findings underscore the importance of healthy sleep patterns in young adulthood, as language functioning constitutes a prominent area of intellectual functioning, and is a strong predictor of educational and occupational achievement.

Correspondence: Cara L. Crook, Clinical Psychology, Fordham University, 441 East Fordham Road, Fordham University, Dealy 318A, Bronx, NY 10458, United States. E-mail: cara.crook@gmail.com

M. DEKHTYAR, C. PEÑALOZA, S. KIRAN \& T. GRAY. Cognitive Control and its Relationship with Cognition and Language in Monolingual and Bilingual Aphasia.

Objective: It has been proposed that bilingualism may contribute to better cognitive control. In aphasia, language impairment might be associated with deficits in cognitive control. Here we examined whether monolinguals (MA) and bilinguals with aphasia (BA) show impaired cognitive control relative to healthy monolingual (MC) and bilingual controls (BC). We also examined whether BA have better cognitive control relative to MA, and if there is a relationship between cognitive control and language and cognitive ability in BA.

Participants and Methods: We examined 10 MA and 10 BA who were roughly matched by age, education, chronicity, and aphasia severity ( $p>.05$ in all cases). We also examined $10 \mathrm{MC}$ and $10 \mathrm{BC}$ for betweengroup comparisons. All participants underwent a computerized non-linguistic triad task (NLTT) tapping cognitive control [reaction times (RT) and accuracy]. The MA and BA also underwent language and cognitive testing including the BNT, Pyramid and Palm Trees (PAPT) and a non-verbal CLQT.

Results: The MC and $\mathrm{BC}$ did not differ in their performance on the NLTT (p>.05). Both the MA (higher RT t(18)=2.19, p=.04), and the BA (lower accuracy $(t(18)=-3.37, p<.01)$ exhibited poorer performance compared to their corresponding control groups. There were no group differences between the MA and the BA on either the NLTT, nonverbal CLQT, BNT or PAPT. Performance on the NLTT was significantly correlated with scores on the BNT $(\mathrm{r}(10)=.73, \mathrm{p}=.01)$ and marginally correlated with scores on the non-verbal CLQT $(\mathrm{r}(10)=.63$, $\mathrm{p}=.05)$ in the BA but not in the MA ( $p>.05$ in all cases).

Conclusions: Our findings show that both MA and BA present impaired cognitive control relative to healthy individuals. As observed in the healthy controls, BA do not seem to show an advantage in cognitive control relative to MA, but the integrity of their cognitive control resources is associated with better lexical retrieval and possibly executive function.

Correspondence: Maria Dekhtyar, B.A., Boston University, 635 Commonwealth Ave., Boston, MA 02115, United States. E-mail: mardek2@bu.edu

\section{J. MCCULLAGH \& S.B. PALMER. Relationships Between Cognitive, Language, and Central Auditory Processing Assessments in Children.}

Objective: Researchers have debated whether cognitive, language, and central auditory processing (CAP) assessments evaluate independent or related processes. Current professional guidelines for CAP recommend using caution when evaluating children with cognitive or language impairments. The purpose of the current study was to investigate the relationships between commonly used tests of cognition, language, and CAP in children.

Participants and Methods: This study was a retrospective review of 103 clinical patients aged 7 to 17 years who were evaluated for CAP between 2012 - 2016. Pearson correlations were determined for each of the CAP tests (Dichotic Digits, Compressed Speech, and Words-inNoise) and the core, receptive, and expressive language scores on the CELF and the full-scale IQ and four index scores on the WISC-IV. Not all subjects had results for all the tests of interest, so the final $\mathrm{n}$ for each correlation varied.

Results: Significant positive correlations between cognitive (FSIQ, Verbal Comprehension, and Perceptual Reasoning) and language (CELF Core) scores existed. Significant positive correlations existed between CAP scores (Dichotic Digits-Left Ear) and cognitive (Perceptual Reasoning and Working Memory) as well as language (CELF Core, Receptive, and Expressive) scores. Significant positive correlations also existed between CAP scores (Dichotic Digits-Right Ear) and cognitive (Working Memory) as well as language (CELF Core, Receptive, and Expressive) scores. Few correlations existed between other measures of CAP and cognition and language.

Conclusions: Findings suggest relationships between cognition, language, and CAP assessments. The Dichotic Digits test was correlated with language skills in this sample. However, compressed speech, which is a more linguistically loaded test, was not correlated with language scores. Implications for clinical evaluation of these constructs (cognition, language, and CAP) will be discussed. 
Correspondence: Jennifer McCullagh, Communication Disorders, Southern Connecticut State University, 501 Crescent St, Davis Hall 012I, New Haven, CT 06515, United States. E-mail: mccullaghj1@ southernct.edu

T.W. OLIVIER, J. BASS, J.M. ASHFORD, R. BEAULIEU, S. SCOTT, J. SCHREIBER, S.L. PALMER, D.J. MABBOTT, M. SWAIN, M.J. BONNER, R. FRANKS, K. EVANKOVICH, C.L. ARMSTRONG, S. KNIGHT, S. WU, A. ONAR-THOMAS, A. GAJJAR \& H.M. CONKLIN. Impact of Ototoxicity on Reading-Related Skills in Patients Treated for Pediatric Medulloblastoma.

Objective: Sensorineural hearing loss (SNHL) is associated with intellectual and academic declines in patients treated for pediatric medulloblastoma (MB). This study expands on existing research by examining neurocognitive processes that may result in reading difficulties in children with treatment-related ototoxicity/SNHL.

Participants and Methods: Serial neuropsychological and audiology data for 260 children/young adults (ages 3-21 yrs.; $M$ age $=9.15$ yrs.) enrolled on a multi-site $\mathrm{MB}$ research/treatment protocol, which included surgery, risk-adapted craniospinal irradiation (CSI; $n=147$ average risk; $n=49$ high risk), and chemotherapy, were analyzed using mixed models. Children were assessed at baseline and up to 5 years post-diagnosis and grouped according to degree of SNHL. Included were 196 children with intact hearing or mild/moderate SNHL (Chang grades 0 , 1a, 1b, 2a) and 64 children with severe SNHL (Chang grade $>2 b$ ). Seven Woodcock-Johnson Third Edition variables were analyzed (Letter-Word Identification, Reading Fluency, Passage Comprehension, Sound Awareness, Phonemic Awareness, Processing Speed, and Working Memory). Samples included 180-257 patients per measure.

Results: Children with severe SNHL performed significantly worse on all variables but Sound Awareness and Working Memory in comparison to children with intact hearing or mild/moderate SNHL $(p<.05)$. Controlling for age at diagnosis and risk-adapted CSI dose, performance on three variables remained significantly lower for children with severe SNHL (Phonemic Awareness, Passage Comprehension, Processing Speed; $p<.02)$.

Conclusions: We previously demonstrated that children with SNHL exhibit greater deficits in language skills as hearing loss increases. The present study suggests that after controlling for age at diagnosis and treatment exposure, children with severe SNHL may struggle most with phonological skills and processing speed, which impact higher-level language such as reading comprehension.

Correspondence: Traci W. Olivier, Psy.D., 262 Danny Thomas Place, M.S.740, Memphis, TN 38104, United States. E-mail:traci.olivier@ stjude.org

A.M. RAYMER, S. RINGLEB, H. SANDBERG, K. SCHWARTZ \&. G.S. WATSON. Influence of Noise and Visual Information on Auditory Processing in Aphasia.

Objective: Persons with aphasia (PWA) have difficulty in background speech noise where the visual modality is essential to support auditory processing. We examined the influence of competing speech noise during auditory (AUD) and auditory+visual (AV) conditions in PWA to examine the interplay of adverse listening conditions with visual information.

Participants and Methods: We tested 12 persons who passed hearing screening, 7 with chronic aphasia and 5 age-matched controls. We used Quick Speech in Noise (Killion et al., 2004), a sentence repetition test in the presence of background speech noise presented in auditory (AUD) and auditory+visual (AV)(face) conditions as signal-to-noise ratio (SNR) varied from 20 to 0 per block. The examiner coded repetition accuracy for 5 key words per sentence. Results were analyzed using ANOVAs with group (aphasia, healthy) as between subjects factor, and listening modality (AUD, AV) and visual advantage across SNR levels as within subjects factors.
Results: The aphasia group performed significantly below controls in AUD and AV conditions ( $\mathrm{F}=8.37, \mathrm{p}=.016$; partial $\left.\mathrm{h}^{2}=.46\right)$. Both groups were significantly better in AV than AUD $(\mathrm{F}=66.92, \mathrm{p}=.000$; partial $\mathrm{h}^{2}=.87$ ), with no group by modality interaction $(\mathrm{F}=1.86$, n.s.). Visual advantage across SNRs showed no group effect $(\mathrm{F}=3.60, \mathrm{p}=.087)$, and a significant within-subjects effect of SNR $(\mathrm{F}=22.03, \mathrm{p}=.001$; partial $\left.\mathrm{h}^{2}=.69\right)$. At the hardest level there was a significant group difference (SNR 0: $\mathrm{t}=2.36, \mathrm{p}=.04)$. PWA received less listening advantage at the most difficult SNR (2.6 words) than the controls ( 10.6 words).

Conclusions: Although PWAs were impaired in auditory processing, visual information provided an advantage in listening as in healthy listeners, contrary to earlier proposals. At SNR 0, PWA did not obtain the visual benefit to the extent seen in healthy listeners, suggesting that PWA may need intervention to maximize visual processing to support auditory comprehension.

Correspondence: Anastasia M. Raymer, Ph.D., Dept of Communication Disorders \& Special Education, Old Dominion University, 110 Child Study Center, Norfolk, VA 23529-0136, United States. E-mail: sraymer@odu.edu

\section{Memory Functions}

J.R. ANDERSON, J. GUNSTAD \& M. SPITZNAGEL. Variability in Actigraphic Sleep Duration Predicts Word List Recall Performance: A Bayesian Variability Analysis.

Objective: Prior work shows a link between mean nightly total sleep duration and recall memory. However, studies considering the role of intraindividual variability in nightly total sleep duration are sparse, and have two common limitations: 1) sleep duration variability is estimated using indices of low reliability (e.g., standard deviation), and 2) studies do not adjust for mean sleep duration, which is often correlated with sleep duration variability. The current study examined the relationship between sleep duration variability and word list recall in healthy young adults using a multilevel Bayesian variability model designed to address these limitations. We hypothesized that greater variability would predict poorer recall.

Participants and Methods: One hundred thirty-six young adults (average age $=19.76$ years; $77 \%$ female) underwent seven nights of wrist actigraphy to assess sleep duration and completed the CVLT-II. WRAT-IV word reading and the Depression Anxiety Stress Scale-21 were administered to examine potential confounding variables.

Results: After adjusting for covariates, sleep duration variability was quadratically associated with CVLT-II SDFR: as variability increased, scores improved $(b=13.91,95 \%$ CI: $[-3.62,29.25])$ at a steadily decreasing rate $(b=-4.39,95 \%$ CI:[-9.65, -1.09]). A similar relationship was found for CVLT-II LDFR: as variability increased, scores improved ( $b=12.62,95 \%$ CI: $[4.32,26.58])$ at a steadily decreasing rate $(b=-4.09,95 \%$ CI:[-8.75, -1.34]). No significant relationships were found involving mean sleep duration.

Conclusions: Slight variability in sleep duration may facilitate better word list recall independent of mean sleep duration, while a large degree of variability may be detrimental. Results suggest that sleep duration variability was an unmeasured confound in past work. Promoting consistent sleep may impact recall memory independent of increasing sleep duration. Future work should account for sleep duration variability. Correspondence: Jason R. Anderson, Clinical Psychology, Kent State University, 602 Wine Street, Vermilion, OH 44089, United States. E-mail:jasreidan@gmail.com 
O. BEZDICEK, A. FENDRYCH MAZANCOVÁ, H. BUSCHKE, J. ROTH, E. RUZICKA \& R. JECH. The learning/encoding versus retrieval deficit hypothesis in Parkinson disease revisited.

Objective: Parkinson disease (PD) patients suffer from the cognitive dysfunction that occurs even at the time of PD diagnosis and is heterogeneous. Typically, the cognitive dysfunction in PD afflicts the explicit memory. The traditional retrieval deficit hypothesis of memory impairment suggests that PD compromises access to stored memories, the frontal hypothesis proposes that the strategies required to initiate efficient retrieval are impaired in comparison to recent findings also suggesting a hippocampal memory disorder. As an efficient retrieval measure we took the Rey Auditory Verbal Learning Test (RAVLT) sum of trials 1-5; and as a hippocampal memory measure, the both 1st list and 2 nd list items recalled together by their shared category cue from Memory Binding Test (MBT) were chosen.

Participants and Methods: 19 PD without cognitive impairment (PD-NC) according to IPMDS criteria at Level II (mean age: $63.6 \pm$ 6.7, education: $14.8 \pm 2.6$ ), 18 PD mild cognitive impairment (PD-MCI; mean age: $65.1 \pm 7.3$, education: $13.1 \pm 2.9$ ) and 38 controls (NC; mean age: $63.0 \pm 7.5$, education: $14.8 \pm 3.3$ ) .

Results: There were significant between-groups differences in MBT (cued recall) $p=.002$, not in RAVLT (1-5) $p=.147$ (Kruskal-Wallis test). Post-hoc comparisons showed in MBT: PD-MCI=PD-NC ( $p=.164)$, $\mathrm{PD}-\mathrm{NC}<\mathrm{NC}(p=.023)$, and $\mathrm{PD}-\mathrm{MCI}<\mathrm{NC}(p<.001)$, however, in RAVLT (1-5): PD-MCI=PD-NC $(p=.233), \mathrm{PD}-\mathrm{NC}=\mathrm{NC}(p=.401)$, and even PD-MCI=NC $(p=.063)$.

Conclusions: The present results indicate a differential impairment of cued recall, i.e. hippocampal impairment of explicit memory in PD-MCI and $\mathrm{PD}-\mathrm{NC}$ in comparison to controls rather than retrieval deficit. Correspondence: Ondrej Bezdicek, PhD., Department of Neurology and Centre of Clinical Neuroscience, Charles University in Prague 1st Faculty of Medicine, and General University Hospital in Prague, Katerinska 30, Prague 12821, Czechia.E-mail: ondrej.bezdicek@gmail.com

\section{J.O. CARVALHO \& B. SPRINGATE. Does Perceived Control over Memory Affect Performance?}

Objective: Studies investigating general control beliefs, or the idea one has control over certain life circumstances, have been associated with memory performance in older adults (e.g., Windsor \& Anstey, 2008). We explored specific control beliefs in memory and cognitive performance.

Participants and Methods: Data were obtained from 3282 participants completing Wave II of the Midlife in US Study (MIDUS) surveys, a nationally representative sample of adults $(M$ age $=63.64, S D=11.35$; $M$ education $=7.51, S D=2.51 ; 53.3 \%$ female $)$. Variables included a composite memory score (immediate and delayed list recall) and response to the question "there's not much I can do to keep my memory from going downhill" on a Likert scale (agree strongly; somewhat; a little; neutral; disagree strongly; somewhat; a little).

Results: An ANCOVA controlled for participant age and education. Those who reported decreased perception of control over their memory deficits (feeling there is nothing they can do to control their memory) performed significantly worse than those who did not, after controlling for participant age and education $(F(6,2491)=3.552, p=.002)$. Significant differences in memory $(p s<.05)$ were observed between those who disagree and agree strongly $(p=.04)$, agree somewhat and disagree a little $(p=.009)$, somewhat $(p<.001)$ and strongly $(p<.001)$, and agree a little and disagree strongly $(p=.02)$. No group differences were observed for the neutral item.

Conclusions: Participants who endorsed less control over memory had worse memory performance than those who reported greater control. While it is unclear whether perception over control contributes to poorer cognitive performance or responses reflect perceived memory changes, this finding remains important. There is indication those with greater control beliefs can improve on cognitive tests (Caplan \& Schooler,
2003). Thus, focusing interventions and psychoeducation on control beliefs over memory may result in cognitive improvements.

Correspondence: Janessa O. Carvalho, Ph.D., Psychology, Bridgewater State University, 131 Summer Street, Bridgewater, MA 02325, United States.E-mail: janessacarvalho@gmail.com

C. DION, E.A. BOOTS, L. ZHAN, A. KARSTENS, J. COHEN, O. AJILORE, P.M. MAKI, D.X. MARQUEZ \& M. LAMAR. SeX Differences in the Contribution of Physical Activity to Verbal Learning and Memory in Older Adults.

Objective: Moderate-to-vigorous physical activity (MVPA; e.g., jogging, swimming) is associated with better verbal learning and memory (verbal-L\&M). Although men report more MVPA than women, women outperform men on verbal-L\&M measures independent of hippocampal volume. We investigated the separate and interactive cross-sectional associations of sex and MVPA on verbal-L\&M.

Participants and Methods: Ninety-nine non-demented/non-depressed older adults ( age $=68+6.7 ; 51 \%$ female $)$ completed the CHAMPS Activities Questionnaire assessing self-reported MVPA and the California Verbal Learning Test-II (CVLT-II) assessing initial learning (Trial 1), total learning (Trials 1-5), and memory (long delay free recall). Separate multivariate linear regression models per CVLT-II variable incorporated age, sex, education and a SEX*MVPA interaction.

Results: As expected, women out performed men across all verbal-L\&M variables ( $p$-values $<0.002)$ and higher MVPA was associated with higher initial and total learning $(p$-values<0.03). Despite equivalent self-reports of MVPA by sex, there was an interactive association of SEX*MVPA for initial learning only: higher MVPA in men associated with better performance $[\beta=-2.25, t(84)=-2.05, p=.04]$; this pattern was not seen in women.

Conclusions: Men showed positive associations between MVPA and initial verbal-L\&M while women did not. MVPA may confer differential initial verbal learning benefits for men compared to women, although men and women appear to benefit equally from MVPA in subsequent learning and delayed memory trials of the CVLT-II. Sex differences in the effect of MVPA on initial learning may reflect underlying effects on attentional networks which we are currently exploring.

Correspondence: Catherine Dion, BA, Rush Alzheimer's Disease Center, Rush University Medical Center, 1750 W Harrison, Suite 1000, Chicago, IL 60612, United States.E-mail: cdion2@ufl.edu

M.D. GRILLI, J.J. BERCEL, A.A. WANK \& S.Z. RAPCSAK. The Fractionation of Personal Semantic Memory: Evidence from Two Individuals with Anterior Lateral Temporal Lobe Lesions.

Objective: Although personal semantic memory contributes to various forms of autobiographical memory retrieval and prospection, the neural bases of this type of memory remain underspecified. Based on the idea that personal semantic contents can be devoid of any reference to spatiotemporal context and thus "experience-far," we sought to shed light on the contribution of the anterior lateral temporal lobe, a brain region implicated in the representation of abstract semantic associates of unique entities, to the storage and retrieval of personal semantic memory. Participants and Methods: We assessed two types of experience-far personal semantic memory, namely autobiographical facts and personality traits, in two individuals with extensive anterior lateral temporal lobe lesions. One of these participants had left lateralized lesions (patient MK), and the other had right lateralized lesions (patient DW). We also investigated general semantic memory for unique entities (i.e., names and facts about famous people and places), and we compared the lateral temporal lobe participants to a group of controls.

Results: Relative to the controls, MK exhibited impaired retrieval of experience-far autobiographical fact knowledge and general semantic memory, but spared retrieval of personal trait knowledge. In contrast, both types of experience-far personal semantic content were spared in DW, whereas general semantic memory was impaired. 
Conclusions: These findings support the idea that experience-far autobiographical fact knowledge depends on the left anterior lateral temporal lobe for storage and retrieval, indicating that this type of personal semantic memory shares neural mechanisms with general semantic memory. They also are consistent with the viewpoint that personal semantic memory is not a unitary construct, but rather consists of subtypes of content with distinct neural bases.

Correspondence: Matthew D. Grilli, PhD, Psychology, University of Arizona, 1503 E. University Blvd., Tucson, AZ 85721-0068, United States.E-mail:mdgrilli@email.arizona.edu

B. JACOBSON, C. IRANI, N.M. GATTO \& G.J. LEE. The Effect of Subsyndromal Symptomatic Depression on Verbal Learning and Memory.

Objective: The objective of this study is to examine whether Subsyndromal Symptomatic Depression (SSD) has a significant impact on verbal learning and memory in healthy, older adults.

Participants and Methods: Healthy, community-dwelling adults $(n=49 ; 47 \%$ Female; $53 \%$ Male) age 65 and older were recruited through the Adventist Health Study-2 at Loma Linda University. Participants underwent a 2 -hour comprehensive neuropsychological battery, including 2 tests of verbal learning and memory - the Rey Auditory Verbal Learning Test (RAVLT) and Logical Memory (WMS-IV LM). The Geriatric Depression Scale (GDS) was administered as a measure of depression symptoms. Participants were classified as either non-depressed (GDS $=0 ; n=10$ ) or with SSD (GDS=1-9;n=39) based on their GDS scores. Participants who reported clinically significant levels of depression $($ GDS $>=10)$ were excluded. Both groups were similar in age, education, and Mini-Mental State Examination scores. Independent samples t-tests were conducted to evaluate whether there were significant differences in memory performance between the two groups. Cognitive test scores were reported as age-adjusted z-scores.

Results: On the RAVLT, there was a significant difference in the immediate recall over 5 learning trials (List A, Trials 1-5) between the non-depressed group $(\mathrm{M}=0.97, \mathrm{SD}=0.88)$ and the $\mathrm{SSD}$ group $(\mathrm{M}=-0.24$, $\mathrm{SD}=1.04, \mathrm{t}(47)=3.38, \mathrm{p}=0.001)$, but no significant difference on their delayed recall scores. On the WMS-IV LM, there were no significant differences between the two groups on either immediate (LM I) or delayed recall (LM II).

Conclusions: Individuals with SSD performed significantly worse on a word list learning task than individuals who did not endorse any symptoms of depression. This suggests that even at the subthreshold level, depression has a significant impact on verbal learning and encoding. Correspondence: Bryce Jacobson, Psychology, Loma Linda University, 11833 St Andrews Pl, Loma Linda, CA 92354, United States. E-mail: bsjacobson@llu.edu

E. KALSCHEUR, M. ABRAHAM, C. KANDAH, J.L. WOODARD \& M. SEIDENBERG. Duration of Fame and Extent of Semantic Knowledge of Famous Names in Cognitively Intact Older Adults. Objective: Ribot's (1906) proposal of better survival for older memories compared to recent memories remains a topic of debate. Mixed findings have been reported and suggestions to reconcile differences include memory type, and type of dementia. Here, we present findings from cognitively intact older adults (CI) for recognition and semantic knowledge of remote famous names which varied by duration of fame and extent of biographical knowledge.

Participants and Methods: 19 (CI) individuals (mean age $=72$ ) answered forced-choice probes for occupation, associated name, and specific attribute of 60 famous names (20 Recent, 20 Remote Transitory, 20 Remote Enduring) and 60 non-famous names. Recent names included people well-known within the last 10 years. Transitory and Enduring remote famous names became popular 30+ years ago, but only Enduring names continue to be well recognized based on collected norms. Response time (RT) was examined to eliminate the typical ceiling effect found for accuracy performance. A Time $\mathrm{x}$ Knowledge $(3 \mathrm{x} 4)$ repeated measures ANOVA was conducted.

Results: A significant Time $\mathrm{x}$ Knowledge interaction $(F(2,17)=6.08$, $\left.p=.003, \eta_{\mathrm{p}}{ }^{2}=.74\right)$ revealed remote famous names, both Transitory and Enduring, were responded to faster than Recent names at all semantic levels $(p$ 's $<.05)$. Additionally, Enduring names were responded to faster than Transitory names at all semantic levels $(p$ 's $<.05)$. RT increased in a step-wise pattern from Recognition to Categorization to Associative knowledge, but remained the same between Associative and Attribute knowledge at all time points.

Conclusions: Factors other than memory age appear to influence the speed of retrieval of remote famous names in cognitively intact older adults. Here we found that both duration of fame and extent of biographical knowledge were significant factors in the speed of response. Examination of these factors in clinical groups such as Mild Cognitive Impairment and Alzheimer's Dementia is ongoing.

Correspondence: Emily Kalscheur, Psychology, Rosalind Franklin University of Medicine \& Science, 87 E Haines Dr, Hainesville, IL 60030, United States.E-mail: emily.kalscheur@my.rfums.org.

M. PRIMOSCH, J. EMMINGER, K. IVES, M. SNYDER, S. KAVANAUGH \& C.P. MCFARLAND. An investigation of the effects of depressive-rumination on prospective memory.

Objective: Depression has been found to negatively affect several neuropsychological domains including prospective memory (PM). However, research on depression-related PM impairment remains equivocal. No study to date has taken into account the possible effects of rumination, which is known to impair executive functions underlying PM. The current study addresses this gap.

Participants and Methods: Participants were grouped according to their scores on the Beck Depression Inventory-Second Edition (BDIII). Fifty-five individuals with low (BDI-II $<9)$, 17 individuals with moderate (BDI-II 9-18), and 16 individuals with high (BDI-II > 18) symptoms of depression were included in the study. Participants within each group were randomly assigned to either (a) actively ruminate about, or (b) distract their thoughts from, their current mood. Following this manipulation, participants completed a modified version of the Memory for Intentions Test.

Results: Regardless of depression severity, no relations emerged between state rumination and PM performance. Depression severity was unrelated to time- and event-based PM trials, and overall PM performance. Interestingly, trait rumination was negatively correlated with overall PM performance in the low depressive symptom group compared to the moderate group, wherein trait rumination was positively correlated with overall PM performance. Trait rumination was not correlated with overall PM performance in the high depressive symptom group. No significant deficits were found between groups[LR11] on ongoing task performance or clock monitoring checks.

Conclusions: The current study failed to demonstrate depression-related PM impairment as a function of depressive-rumination. Furthermore, depression severity had no effect on overall PM performance. Results from the current study should be interpreted with caution given the small sample size and low statistical power. Further research is needed to elucidate the effects of depressive-rumination on PM.

Correspondence: Mark Primosch, University of Montana, 2308 Mount Ave, Apt. A, Missoula, MT 59801, United States. E-mail: mark. primosch@umontana.edu

M. PRIMOSCH, B.T. STEWART, C. MAXSON, M. BRYNDZIAR, B. GORTER \& C.P. MCFARLAND. What if time isn't on my side?: Depressive symptoms and psychological distance from past and future events.

Objective: Objective: Research has revealed that equally distant personally experienced episodes feel close or remote, depending on whether they have favorable or damaging implications for one's current sense of self. Healthy individuals have a tendency to 'experience' positive events as 
occurring more recently as a means of bolstering one's current sense of self. Given the frequency of ruminative thinking in depression, it is possible that depression results in the 'experience' of a positive past event as more distant relative to less positive events that occurred at a similar time. The same may be true for imagining positive or negative future events. Participants and Methods: Participants and Method: Sixty Psych 100 students took part for course credit. Participants recalled and typed a detailed description of one "happy" and one "sad" event from the past 3 years and indicated on a 9 -pt. scale how near or far that event seemed. The same was done for happy and sad imagined events that could occur in the next 3 years. Participants completed the BDI-II, Trait Hope Scale, and the Ruminative Responses Scale.

Results: Results: BDI-II scores were positively correlated with perceived distance to a future happy event. The brooding subscale of the RRS was positively correlated with distance to future happy events and negatively correlated with distance to sad events. Total hope scores were negatively correlated with distance from both recalled and imagined happy events. Conclusions: Conclusions: This study revealed that depressive symptoms are related to happy future events feeling more remote than sad future events. Rumination may exacerbate this relation, by making sad future events feel as though they will occur in the near future, while happy events feel more remote. In contrast, hope appears to play a protective role by "pulling" past and future happy events closer to the present. Future work will investigate the relations between these variables and the potential clinical implications of the present findings. Correspondence: Craig P. McFarland, Psychology, University of Montana, Skaggs Bldg Room 202, Missoula, MT 59812, United States. E-mail: craig.mcfarland@umontana.edu

M.B. MEMEL \& L. RYAN. Visual Integration of Objects and Scenes Results in Age-Specific Changes in Hippocampal and Parahippocampal Contributions to Successful Memory Retrieval. Objective: Aging is associated with a decline in associative memory. However, encoding strategies, including verbal unitization and visual integration, improve memory performance in older adults. Visual integration is achieved by presenting an object and scene as one coherent representation, rather than separated as distinct entities on a white background. In a recent study, Memel and Ryan (2017) identified the neural correlates of visual integration at encoding, but no study has investigated age-related differences in the neural correlates of visual integration during recognition.

Participants and Methods: Twenty-four cognitively normal older adults and eighteen young adults studied photographs of objects and scenes. In the Combined condition, an object was positioned naturally in a scene. In the Separated condition, an object was presented on a white background adjacent to and visually distinct from a scene. An associative recognition task immediately followed. An analysis of fMRI activation during recognition was conducted through a $2 \times 2 \times 2$ ANOVA with factors of age group, condition, and response type. This analysis was limited to our medial temporal and prefrontal regions of interest. Results: Visual integration improved performance equivalently across age groups, and reduced activation in right lateral hippocampus (HC) and left perirhinal cortex (PRC). However, left middle HC activation increased for visually integrated pairs in older adults and decreased for young adults, as indicated by an age by condition interaction. Finally, visual integration increased the correlation between HC and parahippocampal (PHC) activation and correct rejections in older adults, but not young adults.

Conclusions: Visual integration is an encoding strategy that improves memory performance across age groups. While age invariant neural correlates of visual integration include a reduction in lateral HC activation, age-specific changes in medial $\mathrm{HC}$ and $\mathrm{PHC}$ activation occur in older adults.

Correspondence: Molly B. Memel, Master of Arts, Psychology, University of Arizona, 1503 E. University Avenue, Rm 219G, Tucson, AZ 85721, United States.E-mail:memel@email.arizona.edu
P. PIOLINO. Does Virtual Reality Have a Future for the Study of Episodic Memory in Clinical Neuropsychology?

Objective: Episodic memory (EM) is the memory of personal experiences located in specific spatiotemporal contexts, and contributes to the development and maintenance of a sense of identity. Despite its crucial role in daily life, most of the standard tools used in its neuropsychological assessment remain incomplete as they rarely test self-relevant experience and they do not consider all the different EM components (what, where, when, how).

Participants and Methods: We proposed a new naturalistic virtual reality (VR) EM paradigm to assess multifaceted EM components, especially binding, in groups of children, young and old adults, and patients with neurodegenerative diseases. The VR abilities were compared to those obtained with a standard EM task.

Results: The results showed that the VR assessment of the main aspects of episodic memory-what, where, and when-and of feature binding (e.g., events with perceptual details, spatial and temporal contexts) was more sensitive to assess EM performances in healthy subjects whatever their age and in patients than a standard EM task.

Conclusions: Virtual reality, therefore, appears to be an appropriate technique to assess crucial aspects of EM in the future practice of clinical neuropsychology as it should provide helpful tools for the detection of subtle memory deficits in daily life. Furthermore, it opens up the possibiliy of training and rehabilitation of the episodic memory.

Correspondence: PAscale Piolino, University Paris Descartes, 2 TER rue D'Alésia, Paris 75014, France. E-mail: pascale.piolino@parisdescartes. fi

K. REITER, M. LOMAN, PHD, S. SWANSON, S. COHEN, M. EARING, S. GINDE, P. BARTZ \& L. GLASS. Developmental amnesia: A case series of adults with congenital heart disease.

Objective: Developmental amnesia (DA) can result from early hypoxic/ anoxic events and is characterized by impaired episodic memory and intact semantic knowledge. This is the first study to report a case series of adults with congenital heart disease (CHD) diagnosed with DA. We present cases with previously diagnosed or undiagnosed DA who completed neuropsychological testing in early adulthood.

Participants and Methods: Three adults with DA were identified following completion of neuropsychological testing. Medical, psychosocial histories, and cognitive profiles were summarized.

Results: Consistent with DA, all 3 patients had deficits in memory encoding, storage, and retrieval while semantic memory was within expectation.

DA1 is a 20-year-old woman with hypoplastic left heart syndrome (HLHS). DA was identified during an evaluation when she was 11 . Developmental milestones were age-appropriate and she received academic accommodations in school. She has anxiety and emotion dysregulation. FSIQ was 98 with additional deficits in domains including visuospatial skills.

DA2 is an 18-year-old man with HLHS who was not previously identified as having DA. He had motor delays and required special education. He had difficulty forming friendships. He was previously diagnosed with anxiety and OCD. FSIQ was 70, and he had other cognitive deficits (e.g., visual sequencing and planning).

DA3 is a 20-year-old man with tetralogy of Fallot and, like DA2, was diagnosed with DA at the time of his evaluation. He required special education. Oppositional behaviors were noted. FSIQ was 65 along with impaired visuospatial abilities and other cognitive deficits.

Conclusions: This study shows that DA occurring in association with CHD has varied cognitive and psychosocial outcomes. In addition, some individuals with CHD have DA that is not identified until adulthood. Early cognitive testing and intervention in this population is recommend. Correspondence: Katherine Reiter, M.S. working toward Ph.D., Psychology, Marquette University, 3909 N. Murray Ave Apt 804, Milwaukee, WI 53211, United States.E-mail: kereiter@gmail.com 
B.T. STEWART. An Investigation of the Relations Between Stress and Prospective Memory.

Objective: Prospective memory (PM) is a future-oriented memory system that entails remembering to perform actions in the future. People spend much of their day-to-day lives forming and acting on intentions, and the ability to carry out intentions is vital for daily functioning. Another common human experience is stress. Despite the significance and ubiquity of both PM and stress, research linking the two is limited. This study is the first empirical study investigating how stress relates with non-focal event-based PM.

Participants and Methods: A total of 86 participants completed the study, with a participant mean age of 21.23 years. Those in the experimental group underwent the Trier Social Stress Test, while participants in the control group completed a word search activity. After these tasks, all participants completed a computerized PM task. This task entailed pressing a key on a keyboard when the target word "can" appeared in the context of another word (e.g., Canada), and pressing a key after every 4 minutes.

Results: Stress-induction procedures did not produce significant differences in non-focal event-based PM performance, $t(78)=-.650, p=.518$, or time-based PM performance, $t(78)=-.425, p=.672$. However, among a smaller subset of those in the experimental group who reported the highest levels of stress, increasing levels of acute stress correlated significantly with higher non-focal event-based PM performance, $r(6)=.840, \mathrm{p}=.009$. Further, higher levels of stress at the outset of the study correlated significantly with enhanced time-based PM performance, $r(78)=.293, \mathrm{p}=.011$.

Conclusions: These novel results suggest that the ability to carry out intentions may remain preserved even under conditions of stress. Further, the correlation between high levels of acute stress and non-focal event-based PM might point towards a dose-response relation. Future research will investigate whether under certain conditions, stress may enhance one's ability to monitor and carry out intentions based on time. Correspondence: Brandon T. Stewart, Master of Arts, Psychology, University of Montana, 32 Campus Drive, Missoula, MT 59812, United States.E-mail:brandon.stewart@umontana.edu

D. TANG, C. SCHWIMMER, C. MILLS, E. AUCONE, A. SEKHON \& J. STRANG. May I Have Your Keys? Prospective Memory and Remembering to Remember.

Objective: Prospective memory (PM) is not routinely assessed in neuropsychological evaluations. We examined its performance in a sample of U.S. Veterans diagnosed with psychiatric and/or neurological syndromes and sought to clarify its interrelationships with episodic memory (EM), executive functioning (EF), and ADL/IADL functioning with hierarchical regression and mediation analysis.

Participants and Methods: Retrospective sample of U.S. Veterans ( $\mathrm{N}=102)$ referred for outpatient neuropsychological evaluation at an urban VA medical center in the mid-Atlantic region. Measures included LM II, HVLT Delayed Recall and BVMTR Delay Recall for EM; Trails A/B and Letter Fluency for EF; Prospective Memory Test (PMT) for PM; and Lawton ADL/IADL Questionnaire.

Results: In hierarchical regression, EF initially predicted PM $(b=.235$, $p=.025,95 \%$ CI $[.034, .489])$. This effect became non-significant after controlling for EM, which predicted PM $(b=.426, p=.004,95 \%$ CI $\left.[.140, .712], \mathrm{R}^{2}=.12\right)$. When ADL/IADL impairment was regressed on PM, EM, and EF as predictors, only EF had a significant partial effect $\left(b=-.181, p<.001,95 \% \mathrm{CI}[-.279,-.084], \mathrm{R}^{2}=.19\right)$. Mediation analysis of the relationship between PM and ADL/IADL with EM and EF as its parallel sub-domain mediators indicated an indirect effect from PM to ADL/IADL through EF ( $a b=-.034$, bootstrap 95\% CI [-.070, -.005]). Conclusions: Prior literature on PM has argued that this ability is crucial for independent living. There has been debate about whether PM is more related to EM (i.e., remembering to remember) or to $\mathrm{EF}$ (i.e., intention formation, ongoing monitoring, and retrieval at the appropriate time). We found that performance on the PMT was more strongly related to EM performance than EF. While there was no direct effect of PM performance on ADL/IADLs, there was an indirect relationship between PM and ADL/IADLs mediated by EF. Our findings provide initial evidence for clinical utility of a brief PM task; further validity comparison with other PM measures is warranted.

Correspondence: Jennifer Strang, PhD, Mental Health, Washington DC VA Medical Center, 400 Commonwealth Ave., \#303, Alexandria, VA 22301, United States.E-mail: jmkstrang@gmail.com

C. WILKISON, P. CHIU, F.R. KARDES, L. TURKELSON, Q. MANO \& P. SHEAR. Omission Neglect as Insensitivity to Missing Information: Effect of Episodic Simulation and Relationship with Neuropsychological Measures.

Objective: The present study reports data on omission neglect, a bias in decision-making that reflects our tendency to under-weigh decision-relevant attribute information not currently presented to us (i.e., omission) and over-weigh the importance of presented attributes. We hypothesize that the magnitude of omission neglect may be related to executive functioning and memory abilities and can be reduced by episodic simulations.

Participants and Methods: Healthy young college students (21M/38F; age $=19.2 \mathrm{M} \pm 1.9$ years SD) were assessed on neuropsychological measures including executive functioning and episodic memory. To assess omission neglect, they were presented with positive descriptions of core-set attributes (e.g., style, speed, cost, turn radius) associated with the products (e.g., bicycle) or services in question, and they were asked to (a) rate their preference for such products / services, and (b) rate how important each attribute in the core set, and any attributes not yet presented, was in affecting their decision.

Results: When negative information on 1 or more important attribute (e.g., weight $=100 \mathrm{lbs}$ ) was omitted at time1, participants over-weighed the importance of the core set than when such omitted attribute information was presented with the core set at time 2 . Relative to baseline condition, engagement in episodic simulation at time 1 modestly reduced the omission neglect rate (from $26.9 \%$ to $21.7 \%, p=0.05$ ). Executive functioning measures including DKEFS color-word, Trails, working memory, and BRIEF did not correlate with omission neglect rates. In contrast, measures of episodic memory, including Verbal Pair Associates $(\mathrm{r}(56)=-.26, \mathrm{p}=0.05)$ and especially Brief Visuospatial Memory Test $(\mathrm{r}(56)=-.44, \mathrm{p}=0.001)$, correlated negatively with omission neglect rates.

Conclusions: These data suggest that omission neglect as a bias in decision making may be more prevalent in individuals worse in memory abilities, and that engaging in episodic simulation may be effective in reducing such bias in certain cases.

Correspondence: Claire Wilkison, B.S., University of Cincinnati, Edwards I, Room 4130F, Cincinnati, OH 45221-0376, United States. E-mail:wilkiscn@mail.uc.edu

\section{R.C. YEUNG \& M.A. FERNANDES. Enhanced Memory for Threat Distractors in High Social Anxiety.}

Objective: Past research is mixed regarding the conditions under which memory biases emerge in individuals with high levels of social anxiety (SA). The current study examined whether high SA would be associated with a memory bias for threatening, but task-irrelevant information, or whether they have a memory bias for all distractors.

Participants and Methods: 60 undergraduate students were recruited in each of Experiment 1 and 2 (E1, E2), with half in both experiments classified as high SA and half classified as low SA using the Social Phobia Inventory. Participants memorized a series of target words (E1) or faces (E2) that were either all neutral or all socially-threatening (e.g. the word 'embarrassed' in E1, or an angry face in E2). Simultaneously during encoding, participants also saw distractor words or faces that were neutral or socially-threatening. Memory for targets and distractors was then measured using recall (E1) and recognition (E1 and E2).

Results: There were no group differences in memory for threat versus neutral targets. However, recognition of socially-threatening distractors 
was enhanced in high relative to low SA, but only when targets were also socially-threatening $(p=.004)$. Further, this enhanced recognition of threat distractors was only observed for word stimuli, and vanished in E2 with the face stimuli $(p=.5)$.

Conclusions: Memory biases in high SA were shown to be specific for threat-related distractors rather than general for all distractors. This specific bias for threat also only emerged when the target to-be-remembered information was also threatening. When SA is primed during target processing, attention to irrelevant but socially-threatening information may be heightened. The modality and salience of the stimuli may be important predictors for when this memory bias will emerge. Correspondence: Ryan C. Yeung, Psychology, University of Waterloo, 200 University Ave West, Waterloo, ON N2L 3G1, Canada. E-mail: rcyeung@uwaterloo.ca

\section{Assessment/Psychometrics/Methods (Child)}

T.L. OTERO, D. SODEN, T. DUFFIELD, S. MASTEL, T.D. PARSONS, J. PIANTINO \& T. HALL. Virtual Reality as a Method of Phenotyping Neurocognitive Function in Children and Youth.

Objective: The main objective is to investigate the psychometric properties of traditional assessment modalities (i.e., paper-and-pencil and analogous computerized assessments) compared to a virtual reality (VR) testing environment amongst individuals with neurodevelopmental disabilities or traumatic brain injury (TBI). The long-term goal is to advance assessment practices for individuals by using VR administration.
Participants and Methods: Participants with neurodevelopmental disabilities ( $M=8.92$ years) were predominantly male $(62.5 \%)$ and were identified as having Autism Spectrum Disorder (37.5\%), Attention Deficit Hyperactivity Disorder (25\%), TBI (12.5\%), or comorbid disorders $(37.5 \%)$. Children were recruited from assessment and treatment clinics at the Oregon Health and Science University, Child Development and Rehabilitation Center. Participants received a counterbalanced battery: Delis-Kaplan Executive Function System Color-Word Interference Task (D-KEFS CWI), Automated Neuropsychological Assessment Metrics (ANAM) Stroop Task, and the Virtual Reality Classroom-Based Stroop Task (VRST).

Results: A 3-way repeated measures analysis of variance (ANOVA) evaluated global performance via differences in participant performance across instruments yielding a main effect for condition: $\mathrm{F}(2,14)=$ 48.504, $\mathrm{p}<.001$, partial $\mathrm{g} 2=.874$. The pairwise comparison of colornaming $(\mathrm{M}=60.443, \mathrm{SD}=3.981)$ and interference $(\mathrm{M}=29.985$; $\mathrm{SD}$ $=2.653)$ was significant, $p<.001$. The pairwise comparison of wordreading $(M=58.887, \mathrm{SD}=3.908$ and interference $(\mathrm{M}=29.985 ; \mathrm{SD}=$ 2.653) was significant, $\mathrm{p}<.001$.

Conclusions: Participants demonstrated degraded performance in the interference condition relative to both the color naming and word reading conditions, indicating that the VRST elicits a performance consistent with other testing modalities. Clinical implications and future research directions for the use of VR as a novel testing modality are discussed. Correspondence: Tiffany L. Otero, Ph.D., University of New Mexico, 1151 Crestview Dr, Los Lunas, NM 87031, United States. E-mail: oteroti@gmail.com

\section{SATURDAY AFTERNOON, FEBRUARY 17, 2018}

\section{Plenary G (Kaplan Memorial Lecture). Disembodied Minds and Embodied Brains}

\section{Presenter: Vilayanur Ramachandran}

$$
\text { 12:15-1:15 p.m. }
$$

\section{RAMACHANDRAN. Disembodied Minds and Embodied Brains.} Our goal is to revive the Golden age of neurology when Broca, Wernicke, Jackson, Goldstein Korsakoff, and others showed that we could draw conclusions about how the mind works by studying patients with focal brain lesions. Although single unit physiology, brain imaging, and other sophisticated tools are promising a revolution, old-fashioned case studies continue to yield new insights into how the brain works. The tradition was continued in the USA by Geschwind and many of his distinguished students including Edith Kaplan, whose 'Boston Process' approach, like my own, places less emphasis on detailed quantitative batteries. We begin with xenomelia- the desire to amputate oneself. Phantom limbs and phantom pain; RSD (CRPS), long regarded as intractable can be treated with mirrors. Even physical signs subside in a matter of minutes of visual feedback, which is a striking example of mind-body interactions. The mirror neuron system (MNS) allowed us to predict that an arm amputee watching the experimenter touch a volunteer hand will feel the touch on his own hand. This may be useful in the clinic as a phantom itch can be cured by watching a volunteer's body being scratched. Also if intersubjective synesthete (the inborn version) watches the examiner tickle the volunteer then she herself laughs uncontrollably. We also demonstrate in normal volunteers using convex mirrors that free will can appear delayed and/ or alienation of self can occur. We then switch focus to synesthesia, letters evoking colors, demonstrate its veracity and origin, and cross activation between grapheme and color neurons. Lastly we raise the question of how we use an internal mental calendar to keep track of appointments. 1 to 2 percent of people see the calendar like a circular hoolahoop around them. We used reaction time to demonstrate the effect was real and suggested that it may be mediated by connections from left angular gyrus with the brain's GPS in the hippocampus via the inferior longitudinal fascicles. We conclude by describing disturbances in self-awareness in Capers and xenomelia and point out that both are examples of Geschwind-Kaplan style disconnection syndromes.

Learning Objectives:

1) To revisit the modular versus holistic view of brain function and emphasize the striking interactions between "specialized" brain modules. For example visual (mirror feedback) affecting pain.

2) To learn about the astonishing degree of plasticity in the adult human brain which can be exploited therapeutically.

3) To learn about the extent to which "high-level" cognition is embodied-i.e. anchored in a framework provided by the physical flesh of the body.

Correspondence: Vilayanur Ramachandran, UCSD, Mandler Hall UCSD, 9500 Gilman Dr, San Diego, CA 92093, United States. E-mail: vramachandran@ucsd.edu

\section{Kaplan Lecture Luncheon}

\author{
1:15-2:15 p.m.
}




\section{Author Index \\ Forty Sixth Annual Meeting \\ International Neuropsychological Society}

\section{February 14-17, 2018 \\ Washington DC, USA}

\author{
Aaron, C.S. 206 \\ Aase, D.M. 32, 284 \\ Abate, F. 96, 207 \\ Abbott, K. 48 \\ Abdallah, C.G. 63 \\ Abeare, C. 52, 54, 55, 56, 57, 61, \\ 93, 175 \\ Abildskov, T.J. 124, 151 \\ Abinader, C.M. 130 \\ Ablitz, B. 171 \\ Abraham. M. 8, 37. 296 \\ Abraham, R.E. 15, 66 \\ Abram, M. 140, 220 \\ Abrams, G. 254 \\ Acosta, L. 67, 194, 250 \\ Adams Costa, E.B. 29 \\ Adamson, C. 255 \\ Adler-Werner, M. 161 \\ Aduen, P.A. 125 \\ Agarwal, R. 124 \\ Aghvinian, M. 37 \\ Aguerrevere, L. 266 \\ Ahmed, M. 113 \\ Ahmed, Y. 118 \\ Ahrens, A.P. 165 \\ Aierbe, A. 97 \\ Ailion, A. 224 \\ Ainger, T.J. 281 \\ Aita, S.L. 165, 167, 179 \\ Ajama, J.A. 138 \\ Ajilore, O. 3, 295 \\ Al-Hakeem, H. 184 \\ Al-Kharafi, H.T. 281 \\ Ala'i, S. 50 \\ Alanís, G. 99, 105 \\ Albert. M. 129 \\ Alden. E.C. 192 \\ Aleksonis, H.A. 229, 260 \\ Alexander, J. 48 \\ Alexander, U. 10 \\ Alfini, A.J. 260
}

\author{
Ali, J. 97 \\ Ali, S. 97 \\ Aliaj, B. 113 \\ Alioto, A. 13, 249 \\ Alioto, A.G. 43 \\ Alkozei, A. 3, 24, 134, 220, 243, 244. \\ 261,277 \\ Allaire, T. 118 \\ Allegri, R.F. 67, 69, 210 \\ Allen. M.D. 48 \\ Allen, S. 194 \\ Allerhand, M. 128 \\ Allison, S. 64 \\ Almane, D. 201, 203, 247, 248 \\ Alpert, K. 192 \\ Altendahl, M. 43, 127, 129, 250 \\ Alterescu, K. 239 \\ Altmann, L. 288 \\ Altmann, L.J. 292 \\ Alvarez, E. 212 \\ Alverson, W.A. 196 \\ Alwethinani, S. 264 \\ Amano, S. 36, 105, 288 \\ Amaya, M. 137, 139 \\ Amini, S. 92, 217 \\ Ammons, C. 116 \\ An, E. 36 \\ An, K. 281 \\ An, N. 92 \\ Anand, D. 209 \\ Anand, R. 209 \\ Anastasiadis, K. 173 \\ Andersen, T. 37, 114, 185, 186 \\ Anderson- Hanley, C. 17 \\ Anderson, A.W. 229, 249, 260 \\ Anderson. B. 104 \\ Anderson, C. 249 \\ Anderson, J.R. 294 \\ Anderson, P.J. 126, 255 \\ Anderson, P.L. 64 \\ anderson, s. 97
}

Anderson, S.L. 64

Anderson, V.A. 271, 272

Andrasik, F. 97

Andrews, G. 177

Ang, T. 35

Angers, K. 35, 98

Anghelescu, D. 276

Anthony, B. 160, 161

Anthony, L. 30, 116, 160, 161, 186

Apostolova, L. 7, 18

Araujo, G. 198

Arbuckle, A. 4

Arce Renteria, M. 200, 203

Ardolf, B. 26

Arentsen, T.J. 14, 171

Arias, F. 22

Arlt, J. 284

Armstrong, C.L. 294

Armstrong, G. 166, 168

Armstrong, N. 129

Arnett, P. 49, 53, 142, 274, 275

Arnold, D. 210

Arnold, S.E. 224

Arnsten, J. 22

Arrieux, J. 165

Arrington, C. 261

Arvanitis, N. 39

Asarnow, R. 147

Ashendorf, L. 182

Ashford, J.M. 225, 294

Asken, B.M. 45, 50, 52, 56

Assari, S. 25

Asthana, S. 71, 222

Au, R. 35, 83, 92, 129

Aubert-Broche, B. 210

Aubin, S. 226

Aubuchon-Endsley, N. 23, 283

Aucone, E. 298

Austiff, M.B. 6

Austin, C.A. 183

Austin, J. 249 
Avery, E. 263

Avila, J. 59, 60, 106, 166

AWASTHY, S. 209

Axelrod, J. 37

Azar, M. 15, 65, 66, 94, 273

Azevedo, C. 105

Babakhanyan, I. 130, 131

Babione, J.M. 32, 284

Babu, P. 25, 35

Bacevice, A. 93, 151

Badami, F.S. 249, 250

Bahrami, N. 280

Bailey, B. 251, 267

Bailey, B.A. 98, 111, 184, 185, 187

Bailie, J. 131

Bain, L. 62

Baird, A.D. 281

Baitz, H. 23

Bajaj, M.K. 264

Bajaj, S. 3, 134, 140, 220, 243, 277

Baker, D.A. 46

Baker, L.D. 5

Balaban, L. 101

Baldo, J. 182

Balkin, T. 256

Balsis, S. 67

Balthrop, K. 106

Banerjee, N.S. 5, 15, 200, 203, 207

Banerjee, P. 276

Bangen, K.J. 5, 68, 129, 219

Bangert, B. 93, 151

Banks, S.J. 72, 73, 167

Banuelos, D. 7, 73

Banwell, B. 209, 210

Bar-Or, A. 210

Baran, J. 108

Barany, E.A. 132

Barbosa, F. 19

Barcelos, N. 17

Barlow-Krelina, E. 209

Barnes, D.E. 139

Barnes, M.A. 118

Barnhart, D. 53, 177

Barone, C. 122

Barr, A. 23

Barr, W. 56, 200, 203, 280

Barrash, J. 200

Barredo, J. 269

Barrett, K.C. 248

Barrett, M.J. 95, 243

Barrett, R. 135

Barron, C. 10

Bartlett, A.N. 183

Barton, M. 112, 115

Bartz, P. 297
Bashem, J. 139

Bass, C. 55

Bass, J. 294

Basso, M.R. 26, 27, 28, 34, 211, 289

Bateman, A. 149

Bates, C. 289

Batist, G. 226

Bato, A. 282

Bauer, R.M. 45, 50, 52, 56, 138, 221

Baum, C. 215

Baxter, K. 46

Baylan, S. 215

Bayley, M. 41, 132, 219

Beach, J. 165, 167, 179

Bean, P. 54, 224

Bearden, D. 200

Beare, R. 255

Beattie, J.F. 278

Beauchamp, M.H. 272

Beaulieu, R. 294

Becker, N. 118

Becker, S.P. 119

Bedard, M. 131

Bedard, P. 226

Bednarz, H. 261

Beebe, D.W. 183

Beers, S.R. 148

Belanger, H. 123, 124, 187

Belisio, A. 105

Belkonen, S. 131, 178

Bell, K. 94

Bell, S.P. 61, 67, 194, 249, 250

Bellegarde, A. 140

Bellgowan, P. 50

Bellone, J. 236, 269

Belser-Ehrlich, J. 236

Bender, H.A. 140, 198, 280

Bendlin, B. 71, 222

Benge, J. 67, 206

Benzinger, T. 75

Beratis, I. 80

Bercel, J.J. 77, 221, 295

Berg, J. 167, 176

Berger, J. 76

Bergquist, T.F. 137

Berkner, P.D. 18, 47, 50, 145

Berl, M. 197, 261, 281

Berlin. K. 97

Berman, S. 71

Bermudez, C.I. 200

Bernard, T.J. 152

Bernier, R.A. 132, 135, 136, 218

Bernstein, J. 167

Bernstein, L.J. 226

Berry, D.T.R. 106
Berry, L.N. 112

Berry, S. 107, 230

Berryhill, S. 220

Bertrand, E. 65

Bettcher, B.M. 127, 249, 250

Betts, A. 124

Beyes, L. 121

Bezdek, M. 25

Bezdicek, O. 295

Bhangu, J. 38

Bhattacharjee, K. 10

Bhaumik, A. 191, 269

Biasini, F. 114

Bichlmeier, A. 141

Bickel, K. 267

Bickford. D. 81

Biddle, C. 26

Biekman, B. 148

Biel, M. 160, 161

Bigler, E.D. 93, 124, 135, 151, 155, 223, 237

Bilder, R.M. 248, 262, 278

Billings, B. 180, 181

Billups, C. 225

Binder, J. 197, 247, 248

Binder, J.R. 201, 202

Binks, S. 281

Birath, J. 234

Bird, W. 148

Birn, R. 247, 248

Bishop, S. 115

Bitan, Z. 45

Black, J. 168

Blackmon, K. 280

Blackwell, M. 131, 231

Blackwood, M. 228, 229

Blair, M.A. 282

Blanken, A.E. 68, 193, 279

Bledsoe, J. 135

Blennow, K. 67, 194, 250

Bliwise, D. 208

Bloch, A. 167, 237

Bloomgarden, G. 94

Bo, J. 227

Boada, R. 152

Bober, C. 153

Bodapati, A.S. 131

Bodin, D. 150

Bodt, B. 56

Bodzy, M. 32, 185, 283

Boege, M. 15

Boettcher, A. 165, 167, 179

Boeve, A. 77

Bogner, J.E. 61, 194, 249, 250

Bohnert, A. 289 
Bolcekova, E. 168

Bolton, C. 53, 177

Bond, A.E. 95

Bondi, M.W. 4, 5, 57, 69, 75, 80, 81, 142, 144, 145, 162, 163, 193 . 219

Boneff, K. 185

Bonetto, M. 67

Bonner-Jackson, A. 72, 174

Bonner, M.J. 294

Bono. A.D. 239

Bookheimer, S. 248, 262, 278

Boone, K.B. 148

Boots, E.A. 3, 295

Bora, S. 278

Boress, K. 291

Borgatti, R. 259

Borgos, M.J. 177

Borod, J.C. 239

Borradaile, K. 91

Borrani, J. 98, 100, 102, 105, 267

Borrasso, A. 148

Borschell, R.M. 195

Bosch, C.M. 106

Bosch. D. 288

Boutros, N. 37

Boutzoukas, E.M. 197, 261, 281

Bower, E. 16

Bowers, D. 180, 207, 236, 253

Bowyer, S. 37, 114

Boxley, L. 180, 269

Boyd, B. 51, 133

Boyer, K. 203

Bradbury, K.R. 112

Bradley, A. 183

Bradley, M. 236

Bradshaw, J. 114

Bradstreet, L.E. 112, 242

Brady, K. 55

Bralten, J. 188, 189

Branum-Martin, L. 121

Bray, M.J. 132, 219

Brearly, T.W. 174

Breiter, H. 48

Brennan, J. 114

Brennan, L. 113, 242

Brennan, R. 229

Brenner, A.M. 98, 185

Brenner, E.K. 132, 135, 136, 218

Brescia Morra, V. 209

Bressman. S. 96

Brew, B. 262

Brickell, T. 140, 141

Brickman, A.M. 12, 60, 70, 88, 128. 130, 162, 221, 223

Bridgett, T. 29
Briggs, J. 61

Brinkman, T.M. 276

Britton, K. 86

Brodsky, C. 108

Brontë-Stewart, H. 205, 242

Brooker, B. 99, 102, 103

Brooks, B. 156, 210

Brooks, J. 185, 186

Broomfield, N. 215

Brown, A.W. 137

Brown, B. 57

Brown. C. 187. 283

Brown. D.S. 95

Brown, G.G. 22

Brown, J.A. 14

Brown, J.S. 6

Brown, R. 210

Brown, S.T. 132

Brown, W.S. 195, 238, 239, 241

Brumback, B. 18

Brunette, A.M. 168

Brunette, M.F. 38

Brunetti, A. 207

Bruss, J. 257

Bryant, A.M. 269

Bryant, K.R. 281

Bryant, R. 63

Bryndziar, M. 296

Bryson, C.N. 290

Buchanan, L. 38, 257

Buchanan, T.W. 149, 150, 266

Buchholz, A. 37, 191

Bucholz, R. 149, 150, 266

Bucker, J. 33

Bucks, R.S. 277

Buckwalter, J. 36

Budding, D. 174

Budge, M. 70, 88, 223

Budson, A. 71

Bueler, E. 286

Bui. E. 138

Bujalski, A.S. 113

Bull, T.P. 78

Bunt, S.C. 94

Buonopane, V. 135

Burciaga, J. 254

Bure-Reyes, A. 5, 15, 200, 203, 207

Burghardt, K. 39

Burke, A. 63

Burke, S.N. 17

Burnett, A. 126

Burnette, K.A. 238

Burns, A.R. 45, 46, 51, 116

Burns, T. 49, 61

Burrell, R. 167, 179

Burton, J. 1
Busch, R.M. 174, 196

Buschke, H. 295

Bussell, C.A. 94

Butterfield, L.C. 180, 253

Buxbaum, J. 115

Buxbaum, L.J. 89

Byrd, D. 159, 235, 236

Cadden, M. 218, 274, 275

Caesar, E.E. 69

Cain, N. 286

Cairncross, M. 102

Calabrese, M. 212

Calamia, M. 80, 165, 167. 168. 178. 181

Calandri, I. 69

Calhoun, O.C. 190

Calkins, M.E. 112

Callahan, B.L. 192

Callahan, C. 16

Callow, D. 260

Calvo, D. 209

Cambronero, F.E. 61, 194, 249

Campbell, L.M. 4, 11, 144

Campbell, M.E. 166, 168

Campbell, R. 107, 110

Campos, J. 67, 69

Canfield, A. 125

Cannistraci, C.J. 229, 260

Cannon, L. 160, 161

Cantu, R. 141

Capaldi, V. 256

Capozzoli, M.C. 46

Capretti, V. 96

Caraher, K.J. 28

Carbine, K.A. 251, 267

Carlew, A.R. 238

Carlozzi, N.E. 130

Carlson-Green, B. 225

Carlson, M. 129, 229

Carlsson, C. 67, 71, 222

Carmichael, A.M. 88

carotenuto, a. 209

Carpenter, L. 269

Carr, J.J. 61, 249

Carrión, C.I. 169

Carroll, E. 54, 136, 224

Carswell, M.A. 198

Carvalho, J.O. 295

Casaletto, K.B. 14, 43, 129, 130, 195 , 250,251

Casas, R. 160

Casey, J.E. 104, 113, 120, 183, 285

Cash, T.V. 83, 240

Casnar, C. 232

Cassell, N. 131, 178

Castagna, P.J. 178 
Castelluccio, B. 125

Castor, N. 133

Castro, N. 22

Cavanagh, J. 191

Cavanagh, L. 127

Cecchi, F. 84

Cerbone, B. 65

Cerino, E. 70

Cerny, B.M. 32

Cersonsky, T. 94

Cervantes-Manzo, Y. 65

Cestari, V. 212

Cha, Y. 119

Chaitanya, G. 264

Chalmers, J.C. 5

Champagne, J. 6

Chan, E. 128, 282

Chan, J. 62

Chan, L. 135, 174, 175, 263

Chan, M. 169

Chan, S. 66

Chandler, M. 252

Chaney, G. 88, 204

Chang, A. 231

Chang, F. 18

Chang, Y.A. 196, 280

Chapa, A. 267

Chapman, K. 209

Chapman, S. 15, 65, 66, 72, 94, 273

Char, D. 82

Charron, C. 172, 182

Chen, D.K. 171

Chen, E. 284

Chen, J. 115

Cheng, S. 229

Cheong, J. 126

Cheran, G. 88

Cherner, M. 22

Chey, Y. 290

Chiaravalloti, N. 153, 211, 213, 214. $242,272,273$

Chigurupati, R. 169

Child, A. 120

Child, I. 56

Childs, A. 56

Chiou, K.S. 146, 272, 273

Chipman, K. 251

Chirles, T. 67

Chiu, P. 298

Choi, A. 180

Chou, C. 62

Chou, Y. 135

Choudhury, T.K. 67

Chowdhry, S. 237

Chowdhury, N. 233

Chrem Méndez, P. 67
Chrem, P. 69

Christenson, E. 267

Christian, H. 17

Chudoba, L. 15

Chung, Y. 290

Cifu, D.X. 123, 124

Cines, S. 88

Cipolotti, L. 128, 282

Cirino, P. 120

Cirino, P.T. 118

Cissne, M. 146

Clair, R. 48

Clapp, M. 108

Clarens, M. 69

Clark, A.L. 5, 57, 137, 142, 144, 145 , 148,219

Clark, B.E. 54, 224

Clark, D. 16

Clark, E. 138

Clark, J. 180

Clark, L.R. 67, 74

Clark, S.V. 282

Clarke, E. 148

Clason, C.L. 13, 249

Clawson, A. 237

Clay, O.J. 11, 53

Clayson, P.E. 258

Clem, M. 227

Clementz, B. 192

Clugston, J.R. 45, 52, 56

Cobia, D.J. 33, 192

Cocchini, G. 66

Cocozza, S. 207

Cohen, B. 17

Cohen, C. 78

Cohen, D. 93, 151

Cohen, G. 67

Cohen, J. 3, 57, 295

Cohen, R.A. 158, 250, 257

Cohen, S. 297

Colantonio, A. 132

Colberg, R.E. 53

Colbert, A.M. 46

Colby, K.A. 283

Cole, W.R. 165

Colella, B. 41, 219

Coles, C. 284

Collins, L. 210

Collins, R. 171, 196

Colon-Perez, L.M. 17

Colon, J.M. 130, 221, 223

Colvin, L.E. 66, 72, 94, 273

Combs, A. 161

Combs, D. 26, 27, 211

Combs, H.L. 131

Como, A. 113
Compas, B.E. 229, 260

Compter, A. 276

Conaboy, C. 283

Conant, L. 247, 248

Conklin, H.M. 225, 294

Conley, J. 66

Conley, Y. 146, 147

Connery, A.K. 46

Connors, E.J. 133

Considine, C.M. 61

Constance, J. 121

Constatinou, M. 19

Conway, C.M. 224

Cook Maher, A. 81, 84

Cook, C. 99

Cook, D. 71, 222

Cook, E.W. 278

Cook, N.E. 47, 50, 145

Copeland, C. 287

Coppola, G. 13

Cordeaux, C. 32, 185, 283

Cordero, E. 156, 157

Cordova, M. 117, 291

Corey-Bloom, J. 69

CORNETT, K. 252

Cornwell, M. 140, 198

Correale, J. 210

Correia, S. 236, 264, 269

Corti, C. 259

Cosentino, S. 15, 65, 66, 70, 72, 88 , 94, 145, 259, 272, 273

Costabile, B. 238

Costabile, T. 96, 207, 209

Cote, A. 38

Cothran, T.P. 238

Cottrelle, J. 219

Coulehan, K. 140, 198, 199, 280

Coulombe, P. 31

Courrege, S. 155

Cox, D.E. 266, 268

Cox, D.J. 125

Craft, S. 5

Cram, L. 245, 246

Crane, N. 263

Craun, E. 23, 283

Crawford, J.L. 38, 191

Crespi, C.M. 228

Crete, A. 238

Crivelli, L. 67, 69, 210

Crocfer, K. 211

Crocker, L.D. 49, 51, 133

Crocket, M. 245, 246

Cronin-Golomb, A. 31, 205, 207

Crook, C.L. 293

Crosby, R. 85, 244

Cross, K. 127 
Crossland, M.M. 47

Crossley, L. 272

Crosson, B. 64, 224, 265

Crouse, E. 14, 171

Crowe, M. 11

Crowley, J. 56

Crowley, S.J. 96, 204

Cruchaga, C. 75

Crump, M. 226

Cruz-Almeida, Y. 204

Csernansky, J.G. 33, 192

Cullen, B. 215

Cullum. C.M. 94

Cullum, M. 48, 55, 74, 85, 155 , 217. 218

culotta, v.p. 231

Cummings, S. 135

Cuneo, M. 50

Cunningham, K. 174

Curewitz, A.M. 107, 230

Curiel, R. 221

Cysique, L. 262

Czepielewski, L.S. 33

D'Agostino, N. 226

D'Errico. L. 68

Dabbs, K. 247

DaCosta, A. 15

Dai, Y. 113

Daiello, L.A. 9

Dailey, N.S. 3, 134, 243, 277

Dalley, B.C. Allred. 78, 79

Daly, B. 112

Dampier, W. 232

Danckaerts, M. 106, 126

Daniel, M. 32, 64, 66, 73, 182

Danti, S. 84, 86, 286

Darby, D. 272

Dardiotis, E. 240

Darland, K. 169

Darlow, B. 278

Dasher, N.A. 225

Dasher, R. 234

Davey, A. 179

Davidson, R.J. 25

Davies, J. 251

Davis, J. 147, 169

Davis, R. 92, 217

Day, L. 29

de Haan, M. 284

De Iorio, M. 139, 180, 181

De la Garza, V. 99, 267

De Michele, G. 96

De Rosa. A. 209

de Ruiter, M.B. 227

de Salles, J.F. 118

De Somma, E. 210
De Vivo, D. 279

de Wit, H. 263

De Wit, L. 252

Deasley, S. 104, 183

Debaun, M. 108

Deboard-Marion, S. 101, 105

DeCarli, C. 83

Deer, T. 239

DeFeis, B.L. 15, 72, 273

DeFord, N.E. 49, 14 ?

Degrauw, T. 200

Dekhtyar, M. 293

Del Bene, V. 79, 200, 203

del Castillo, A. 46

Del Pino, R. 176

Del Vecchio, W. 207

Delano-Wood, L. 5, 57, 69, 137, 142. 144, 145, 148, 193, 219

Delis, D. 69

DeLuca, J. 211, 272

Delucchi, K. 62

Demian, M. 238

Demming, C. 138

Denby, C.B. 8

Denckla, M. 105

Deng, J. 14

Dennison, K. 66

Deno, M. 24

Denson, R. 138

Deoni, S. 264

DeRosse, P. 282

Desjardins, L. 99

DeSollar-Hale, A. 140

Desorcher, M. 153

Dev, S.I. 34

DeVaughn, S. 195

Devine, S. 83

Devinsky, O. 280

DeVito, A. 181

DeVito, A.N. 80

Devlin, K.N. 84, 232

Devlin, M. 85

Dewey, R.B. 95

DeYoe, E. 247, 248

Dhima, K. 95

Di Benedetto, M.G. 210

Diamond, B.J. 113

Diaz-Ortiz, L.M. 113

Dickson, D. 25

Didehbani, N. 48, 55, 74, 94, 155

Díez-Cirarda, M. 176, 254

DiGiovanni, C. 252

Dikman, S. 123

DiMauro, S. 279

DiMuzio, J. 286

Ding, M. 96
Dion, C. 3, 295

Disner, S.G. 63

Divers, R. 4, 6, 8, 172, 273

Doane, B. 13, 249

Dobbins, D. 144

Dobryakova, E. 214, 272

Dockler, L.M. 134

Dockree, P. 272, 274

Dodd, A.B. 191

Dome, G. 276

Dominguez-Vivero, C. 88

Donders, J. 169

Donnelly, K. 215

Donnelly, L. 115

Doody, R.S. 67

Dooley, J. 272

Dorflinger, J. 152, 153

Dotson, V.M. 61

Doty, 1. 217

Doty, R.L. 198

Doucet, G. 264

Dougherty, R. 39, 71, 222

Dourado, M.C. N. 65

Dowell, L.R. 112

Downes, M. 284

Doyle, L. 255, 284

Doyle, L.W. 126

Drabick, D. 84, 240

Dreer, L.E. 53

Dreyer, A. 288

Dricot, L. 223

Dromerick, A. 55

Drzewiecki, M.A. 68

Dsurney, J. 135, 174, 175, 263

Du Plessis, S. 63

Dubois, B. 220

DuBois, S. 38

Ducca, E.L. 238

Duda, B. 8

Duda, T.A. 104, 183, 225

Duff, K. 78, 79

Duffield. T. 237, 299

Dugan, P. 280

Dumas, K. 23

Dumont-Mathieu, T. 113

Duncan, B. 47

Duncan, J. 282

Dunn, A. 172, 182

Dunn, C.B. 196

Dunn, R. 219

Dupee, B. 38

Dupont-Frechette, J. 32

Duque, C. 62

Durant, J. 167, 176

Durgerian, S. 8

Durkin, A. 115 
Dusek, P. 168

Dutt, S. 68, 193, 250, 279

Duvall, S. 117

Dux, M. 236

Dyer, S. 121

Dykstra, J.B. 150

Dyson-Hudson, T. 242

Eapen, B. 123, 124

Earing, M. 297

Easter, R.E. 25, 35

Eastman, J.A. 225

Easto, J. 215

Ebert, P. 251

Ebina, K. 39

Ebner, M. 70

Ebrahimi, C.T. 262, 278

Ebrahimi, E. 70

Echon, R. 265

Eckfield, M. 62

Economou, A. 80

Edelman, P. 6

Edelstein, K. 226

Edmonds, E.C. 4, 80, 81, 193

Edmonds, L. 2

Edmundson, M. 213

Edwards, D. 71, 222

Edwards, K. 15

Edwards, M. 269

Ehrlich, T.J. 196

Eigsti, I. 125

El Massioui, F. 133

Elahi, F. 14, 251

Elias, W.J. 95

Eline, E.E. 116, 270, 271

Ellingrod, V. 39

Elliott, E. 165

Ellis, A. 99

Ellis, R. 126

Ellis, R.J. 235

Elverman, K.H. 197, 201

Emery, A. 127, 250

Emmanuel, C. 140

Emmert, N.A. 4

Emminger, J. 296

Emrani, S. 81, 87

Enache, A. 182

Eneva, K. 284

Engelstad, K. 279

Engle, J. 156, 157

Epker, J. 167, 179

Epperson, M. 104

Eppig, J. 4, 80, 81, 193

Epstein, E. 134

Epstein, J. 119

Ercoli. L. ?
Erdodi, L.A. 55, 93, 172, 175, 178 , 182

Erickson, K.I. 57

Erickson, S. 31

Erlhoff, S. 43

Eschler, B.D. 111, 150

Eskes, G. 251

Esler, A. 115

Espenan, M. 167, 179

Esterlitz, J. 50

Estevis, E. 34

Etkin, A. 258

Ettenhofer, M. 128, 156. 157

Euler, M. 222, 268

Evangelista, N.D. 5, 57, 142, 148, 219

Evankovich, K. 294

Evans, C.L. 226

Evans, D.L. 234

Evans, J. 215, 245

Evans, L. 114

Evans, M.K. 13

Evans, S. 84

Evans, S.A. 81

Everett, L. 32

Everett. L.S. 284

Everhart, D.E. 259, 267, 268

Eversole, K. 269

Eyler, L.T. 34, 35, 193

Fabri, T. 212

Factor, S. 208

Fagan, A. 64, 75

Fair, D. 117

Fair, J. 135

Falahpour, M. 125

Falk, L. 138

Fallon, E. 88

Fama, R. 163, 205, 242

Fanto, E.J. 19 ?

Faraone, S. 188, 189

Farez, M.F. 67, 210

Farias, S. 44, 169

Farrell, E. 200, 203

Farris, P. 7, 253

Fass, T. 182

Fazio, R. 26, 177

Febo, M. 17

Feder, A. 155, 291

Fedio, A.A. 39, 135

Fedio, P. 39, 135, 151

Fee, R.J. 230

Feigin, V. 279

Fein, D. 112, 113, 115

Feirsen, N. 170

Feldman, E. 115

Felton, E. 247, 248

Fendrych Mazancová, A. 295
Fennema-Notestine, C. 127

Ferenc, L. 105

Ferguson, D. 53

Ferguson, L. 196

Ferland, T. 237

Fernandes, M. 118

Fernandes, M.A. 298

Fernandes, P. 34

Fernandez-Carbonell, C. 170

Fernandez, A.L. 245

fernandino, 1. 247

Ferretti, N. 100

Ferrieux, S. 220

Festa, E.K. 71, 76

Fidler, D. 230

Fields, J. 199, 252

Fields, L. 48

Fields, M. 280

Fieo, r. 250

Figueroa, C.M. 204

Figueroa, P. 238, 239, 241

Filla, A. 96, 207

Filoteo, V. 205, 208

Filshtein, T. 44

Fink, B. 289

Finkel, A. 165

Fiol, M.P. 210

Fischer, A.S. 38

Fischer, E.M. 5

Fischer, M. 197, 285

Fisher, E.L. 253

Fitch, R. 249

Fitzgerald, L. 274

Fiumedora, M.M. 197, 285

Flashman, L. 38

Fletcher, J.M. 1, 108, 148

Floden, D. 174

Flores, A. 114, 186

Flores, I. 215

Flowers, A.T. 7, 69

Floyd, T.F. 240

Foldi, N.S. 76

Foltz, R. 68

Fombonne, E. 117

Fong, A.K. 48

Fong, C. 125

Forrester, R.A. 170

Forte, M. 30

Foss-Feig, J. 115

Fossatti, D. 238

Fox, E. 13, 43, 127, 129, 250, 251

Fox, M.E. 226

Foy, A. 107, 230

Franczak, M. 89

Franke, B. 188, 189

Franklin, J. 62 
Franks, R. 294

Franz, C.E. 62, 82, 127

Frazier, L.A. 133

Freedman-Doan, C. 185

Freilich, B. 170

French, L.M. 140, 141

French, S. 65

Frijters, J.C. 120, 121, 261

Frodsham, K. 135

Frost, M. 289

Frost, N. 183

Frost, R. 135

Frost, S. 121, 261

Fujisawa, K.K. 24

Fuster, J. 148, 149, 174

Gaasedelen, O. 26

Gaillard, W. 197, 261

Gajjar, A. 225, 294

Galasko, D. 71

Gale, S.D. 111, 150, 287

Gales, J.M. 196

Gallagher, C. 71, 222

Gallagher, V. 48

Gallanter, E.B. 171

Gallardo, S. 131

Gallegos, C. 100

Galvin, K. 148

Gama, C. 33

Gándara Martínez, M. 178

Gansler, D. 226

Ganz, P.A. 228

Ganzer, C.A. 69

García Guerrero, C.E. 178

Garcia-Barrera, M.A. 44

Garcia-Egan, P.M. 262

Garcia, A. 98, 99, 100, 102, 105, 26 ?

Garcia, K. 135, 174, 175, 263

Garcia. N.E. 106

Garcia, S. 190, 191

Garcia, S.L. 269

Gardizi, E. 175

Garlinghouse, M. 177

Garmoe, W. 55, 216

Garner-Roberts, J. 94

Garner, C. 62

Garrett, K.D. 81, 87

Gates, T. 262

Gatto, N.M. 296

Gattu, R. 137

Gaubert, A. 68, 193, 279

Gause, M. 62

Gavett, B.E. 71, 94

Gavron, L. 14, 171

Gav, K. 50

Gaynor, L.S. 221
Genova, H.M. 151, 153, 154, 213, 214 , 271, 272, 273

Georgiou-Karistianis, N. 255

Geraci, L. 67

Gerhart, H. 209

German, D. 95

Gerner, G.J. 1

Gerrald, L. 48

Gershon, E. 192

Gershon, R. 92

Gerst, E.H. 118

Gerstenecker, A. 79

Gerstle, M. 183

Gess, J. 202, 264

Getz, S. 5, 15, 200, 203, 207

Geurts, H. 160

Geurts, S. 216

Geuze, E. 63

Geva, R. 222

Geyer, M.A. 22

Gfeller, J. 4, 149, 150, 152, 153, 266

Ghahremani, D. 248, 278

Gharooni, S. 128, 282

Ghatan, S. 140, 198

Ghilain, C. 49, 253

Giambrone, L. 202

Giammittorio, A.M. 131

Gibbs, M. 292

Gibler, R.C. 119

Gicas, K. 23

Giesbrecht, C. 23

Gifford, K. 61, 67, 194, 249, 250

Gilbert, D. 104

Gilbert, P. 17, 69, 164, 205, 208

Gill, J. 140, 141

Gillespie, A. 10

Gillespie, S. 114

Gilmore, M.M. 8

Ginde, S. 297

Gioia, A. 276

Gioia, G.A. 45, 46, 51, 151

Giordani, B. 169

Giovannetti, T. 4, 6, 8, 69, 84, 127. $172,232,240,272,273$

Girard, T.A. 41

Glaser, N. 161

Glasgow, J. 281

Glass, J. 276

Glass, L. 201, 284, 297

Glazer, H. 217

Glazer, J. 41

Glenn, M.A. 68

Glikmann-Johnston, Y. 88

Glisky, E.L. 9

Godek, T. 70

Godfrey, M. 230
Godwin, D. 144

Gogniat, M. 6, 12, 253

Gohari, D. 186

Goin-Kochel, R.P. 112

Gola, T. 219

Goldman, A. 9

Goldstein-Piekarski, A. 291

Goldstein, D.S. 27, 29

Goldstein, F.C. 81, 84, 173, 208

Goldstein, M. 185

Gollan, T. 74, 280

Gómez-Gastiasoro, A. 254

Gomez, R. 258

Góngora, F. 216

Gonzales, E.C. 106, 107, 110

Gonzales, M. 81

Gonzalez, I. 184

Gonzalez, J.S. 9

Gonzalez, K. 7, 73

González, M. 97

Gonzalez, R. 22, 163

Goodcase, R. 114

Goodman, A. 48

Goodrich-Hunsaker, N.J. 151

Gopin, C.B. 233

Gorbet. D. 212

Gorenstein, M. 115

Gorka, S. 263

Gorlyn, M. 63

Gorno-Tempini, M. 43

Gorter, B. 296

Gould, S. 53

Goverover, Y. 211

Grabyan, J.M. 171, 196

Gracian, E.I. 6

Gragert, M. 228

Graham, A. 117

Graham, D. 10

Granadillo, E. 89

Granner, M. 200

Grant, A. 198

Grant, I. 22, 164

Grasso, C.M. 243

Gravano, J. 143, 255

Graves, B. 285

Graves, L.V. 17, 69

Gray, T. 293

Green, A.I. 38

Green, D. 276

Green, R. 41, 132, 184, 219

Greenberg, B. 126, 211

Greenberg, L. 53

Greenham, M. 272

Greenstein, J. 32, 284

Grega, M. 129

Greif, S. 111 
Greif, S.M. 98, 111, 138, 184, 185, 187

Greth. Z. 171

Grewal, K. 70

Grieco, J. 226

Grieder, A. 200

Grigorenko, E. 187

Grilli, M.D. 77, 221, 295

Grisanzio, K. 291

Gross, A. 129

Gross, M. 137

Gross, M.S. 139

Gross. W. 24 ?

Grosshans, D.R. 228

Grossman, M. 75

Grossman, S.N. 194

Grossner, E.C. 132, 135, 136, 218

Grove, G. 57

Grove, T. 39

Gruber, S.A. 63

Gu, Y. 65, 279

Guandalini, M. 212

Guerin, J.M. 119

Guerrero, M. 99

Gullett, J.M. 263

Gunning, F. 215, 233

Gunstad, J. 52, 85, 244, 294

Gupta, A. 226

Gur, R.C. 21, 164, 209, 234

Gur, R.E. 209

Guskiewicz, K.M. 141

Gustavson, D. 82, 127

Guttmann, C.R. 14

Guty, E. 49, 53

Guzman, D. 26

Guzman, V. 198

Guzman, V.A. 221

Haack, M. 243, 244

Haacke, M. 137

Haaland, K.Y. 19

Haas, I. 245, 246

Haase, V.G. 118

Hachey, R. 148

Hackett, A. 45

Hackett, K. 69

Haden, S.C. 286

Hadjigeorgiou, G.M. 240

Hagerty, A. 240

Hague, C. 126, 211

Haig, C. 215

Haight, A. 151, 153, 154

Haj-Hassan, S. 219

Haj, R.M. 195

Hake, P. 211

Halbert, S. 264
Hale, C. 15, 60, 70, 223

Hale, M.H. 53

Halfacre, M. 239

Hall, S. 54, 136, 224

Hall, T. 299

Halliday, D. 70

Hallowell, E.S. 256

Halperin, J. 100

Halpern, A. 239

Halpern, D. 115

Halpin, A. 6

Ham, L. 4, 6, 8, 172, 273

Hamilton. R. 270

Hamilton, S.K. 26, 100

Hammond, A. 127, 233

Hammond, B. 6

Hammond, J. 136

Hampstead, B.M. 190, 191, 269

Hamza, M. 39

Han, D. 77, 220

Han, D.Y. 152

Han, T. 137

Hanita, K. 24

Hankins, J. 97

Hanks, R. 137, 139

Hannula, D.E. 44

Hanseeuw, B.J. 223

Hansen, J. 166, 215

Hanson, K. 206

Happawana, K. 113

Harder, L. 42, 126, 211, 217, 218, 225

Hardy, D.J. 141, 148, 149, 174, 233

Hardy, K.K. 160, 161, 276

Hardy, L.M. 119

Harker, L. 198

Harmell, A.L. 137

Harpaz-Rotem, I. 63

Harper, L. 20

Harris-Rains, K. 204

Harrison, A.G. 98, 99

Harrison, C. 239

Harrison, C.E. 7

Harrison, D.W. 266, 268

Harrison, P.K. 266, 268

Hart, J. 48, 55

Hartline, K. 51, 154

Hartman, E.R. Tuminello. 264

Hartman. S. 196

Hartzell, J.W. 281

Harvey, D. 169

Harvey, M. 138

Hasbun, R. 235

Hashemian, N. 151

Hasratian, A. 128

Hass, C. 288, 292
Hass, K. 200

Hassan, I. 32, 284

Hassara, K. 152

Haswell, C. 63

Hater, B. 161

Hatton, S. 82, 127

Haug, N. 291

Hauptmann, M. 276

Hausman, A. 138

Haut, M. 281

Hawes, S.W. 22

Hawkshead, B.E. 256

Hays, C.C. 11

He, X. 264

Head, D. 64, 75

Healy, C. 128

Hearps, S. 272

Heaton, R.K. 130, 164, 215, 234

Heaton, S.C. 56, 98, 111, 184, 185,187

Hedges, D. 287

Heemsbergen, T. 206

Heeren, T. 138

Heffelfinger, A. 29, 290

Heffernan, J. 263

Heidebrink, J. 88

Heilman, K.M. 217

Heindel, W.C. 71, 76

Heinemann, A. 130, 215

Heitzer, A. 107, 109

Helford, M. 204

Hellman, K. 289

Hence, A.C. $?$

Hendershott, T. 205

Henderson, C.E. 290

Henderson, D. 136

Hennig, N.M. 36

Hennrick, H. 185

Henricks, A.M. 38

Henry, B. 22

Henry, J. 124

Hepburn, S. 230

Herbert, M.S. 49, 147

Hermann, B. 71, 201, 203, 222, 246. 247,248

Hernandez, B. 184, 185, 187

Hernandez, L. 184

Herrera-Hamilton, A. 51, 154

Herrera, J. 73

Herrold, A. 48

Hershaw. J. 156, 157

Hessen, E. 19

Hewitt, K.C. 87, 132

Heyrend, R. 10

Heyworth, N. 71 
Hidalgo, G.M. 100

Higgins, S. 81, 87

Higuchi, Y. 39

Hill, B.D. 165, 167, 179

Hill, S. 37

Hill, S.B. 63

Hillary, F.G. 132, 135, 136, 191 , 218, 219

Hills, G. 51

Hinds, S. 124

Hinds, S.R. 123, 124

Hinshaw, S. 102

Hinton. V.J. 230, 279

Hirst, R. 187, 283

Hizel, L. 92, 96, 204, 217

Ho, J.K. 68, 193, 279

Hochberger, W.C. 172

Hocking, M. 108

Hodgson, D. 226

Hoelzle, J. 44, 55, 103, 141

Hoffman. M. 224

Hoffman, S.N. 49, 51, 147

Hoffmeister, J. 211

Hogue, O. 174, 196

Hohman. T.J. 61, 194, 249, 250

Hokkanen. L. 19, 20, 101, 277

Holcombe, J.S. 165, 167, 179

Holden, H.M. 17, 69

Holdnack, J.A. 44

Holiday, K.A. 137, 144, 145, 148, 219

Holland, A. 227

Holland, A.K. 266, 268

Holler, K.A. 32, 36, 185, 283

Hollified, M. 63

Holtzer, R. 265

Homaechevarria, A.A. 136

Honer, W.G. 23

Hong, J. 171

Hong, J.C. 241

Hong, J.S. 132, 135

Honig, L. 70

Hood, A. 108

Hoogendoorn, C. 9

Hope, D.A. 46

Hopkins, M. 114

Hopkins, R. 135, 155

Hopyan, T. 36

Horgas, A. 12

Horn, P.S. 153

Horn. S. 135

Horwood, J. 278

Hoskinson, K.R. 229, 260

Houck, Z. 143

Houck, Z.M. 45, 50, 52, 56, 255

Houtrow, A.J. 108

Hovda, D. 148, 149
Howard, K. 4, 6, 8, 273

Howard, V.J. 11

Howarth, R.A. 42

Howell, G. 62

Hoyt, B. 212

Hromas, G. 45, 52, 56, 138

Hsu, N. 123

Hsu, Y. 82

Нu, Н. 184

Huang, I. 276

Huang, W. 152

Huber, R.S. 285

Huddleston. D. 104

Hudock, R. 107, 115, 230

Hudson, M.M. 276

Huebschmann, N. 145

Huey, E. 15, 66, 72, 88, 94, 273

Hughes-Lika, J. 113

Hughes, J. 126

Hughes, T.M. 5

Hulswit, J. 6, 8, 273

Humphries, C. 247, 248

Hung, J. 75

Hurless, N. 240

Hurtubise, J. 172

Hurwitz, A. 90, 91

Huss, D.S. 95

Huth-Bocks, A. 185, 186

Hwang, G. 247, 248

Hwang, S. 132

Hynan, L.S. 74, 85, 94, 95, 126

Hyun, G. 276

Iaccarino, M. 138

Ibañez, L.V. 112

Ibarretxe-Bilbao, N. 176, 254

Idnay, B. 15, 66, 72, 273

Igwe, K. 223

Ikram, A. 276

Ikram, K. 276

Inada, Y. 39

Inder, T.E. 126

Insel, P. 81

Ipser, J. 63

Irani, C. 296

Irish, M. 88

Irwin, L.N. 100, 125

Isaac, L. 108

Isaacson, R.S. 69

Isenstein, E. 115

Islam, R.M. 239

Iudicello, J.E. 164

Ivanoiu, A. 223

Iverson, G.L. 18, 44, 47, 50, 138, 145

Ives, K. 296

Izawa, S. 24

Jack Jr, C. 162
Jackson, A. 38

Jackson, G.M. 173

Jacobs, D. 74

Jacobson, B. 296

Jacobson, J. 14, 232

Jacobson, L. 3, 121

Jacola, L.M. 276

Jaffee, M. 138

Jahanshad, N. 63

Jak, A.J. 2, 49, 51, 133, 144, 147

James, A. 145, 264, 265

Jang, J.Y. 68, 193, 279

Janulewicz, P.A. 138, 243

Japardi, K. 248, 262, 278

Jaramillo, C. 123, 124

Jastrowski Mano, K.E. 119

Jayakar, R. 64

Jaywant, A. 215, 233

Jean, K. 6, 12, 253

Jech, R. 295

Jefferson, A.L. 61, 67, 194, 249, 250

Jenkins, L.M. 62

Jennette, K. 173

Jeremy, E. 82, 127

Jimenez, A. 77, 128

Jin Hong, S. 242

Jin. Y. 75

Jiradejvong, P. 248

John, S.E. 81, 84, 173

Johnson, A. 90

Johnson, K. 43

Johnson, M. 48

Johnson, N. 215

Johnson, S.C. 67, 71, 74, 222

Johnston, J.M. 53

Johnston, M.V. 1

Jones, A.A. 23

Jones, E.F. 121

Jones, J. 201, 203

Jones, J.D. 164

Jones, K. 279

Jones, P. 55

Jones, R. 200, 231

Joseph, A. 263

Joseph, K.R. 50

Josev, E. 255

Jovanovic, T. 63

Joyce, J.L. 66

Juan, R. 266

Judd, T. 159, 160

Júlio-Costa. A. 118

Jurick, S.M. 49, 50, 51, 133, 147

Kable, J. 284

Kadomoto, E. 39

Kadziolka, M. 268

Kahalley, L.S. 228 
Kahrilas, I. 289

Kahrilas, I.J. 25

Kahsay, Y. 291

Kais, L.A. 286

Kaizar, E. 93

Kalidindi, A. 226

Kalon, E. 258

Kalra- Lall, A. 17

Kalscheur, E. 239, 296

Kamalyan, L. 128

Kamath, N.N. 108

Kamath, V. 38, 88, 198, 204

Kamimori, G. 256

Kana, R. 116, 261, 278

Kandah, C. 296

Kandasamy, A. 281

Kane, R.L. 58, 217

Kaner, R. 225

Kangiser, M. 173

Kanner, A. 200

Kanser, R. 139

Kaplan, L. 76

Kaplan, R. 14

Kaplan, S. 23, 283

Kardes, F.R. 298

Karimian, A. 233

Karlsgodt, K. 282

Karpouzian, T. 192

Karr, J.E. 18, 44, 145

Karstens, A. 3, 295

Karydas, A. 13, 249

Kasten, E. 19

Kaswan, N. 283

Kaswan, N.R. 283

Katz, J. 46

Katz, L.A. 101

Katz, N. 140, 198, 280

Katzelnick, C. 242

Kaufman, D.A.S. 173

Kaufman, M.L. 63

Kaufmann, C. 35

Kaundinya, G. 265

Kaup, A.R. 137, 139

Kautiainen, R.J. 227

Kauzor, K. 7, 69, 288

Kavan, P. 226

Kavanaugh, B.C. 32, 185, 283

Kavanaugh, S. 296

Kave, G. 167

Kawsar, F. 247, 248

Kay, G. 58

Kaye, J. 249

Kazakov, D. 6

Keating, L. 238

Keedy, S. 37
Kegel, J. 156, 157

Keilp, J.G. 63

Keith, C. 173

Keller, A.V. 49, 51, 133, 147

Kellner, S. 94

Kelly, C. 255

Kelly, D.A. 8, 201

Kelly, D.F. 141

Kelly, S.L. 109

Kelsoe, J. 193

Kemp, A. 202

Kemp, E.C. 70, 73

Kennedy, A. 32, 284

Kennedy, R.E. 11

Kenton, S.J. 131

Kenworthy, L. 30, 116, 117, 160, 161, 186, 281

Keshavan, M. 192

Kessels, R.P.C. 216

Key-DeLyria, S. 139

Khang, T. 230

Khurshid, K. 198

Kibble, C. 53, 177

Kibby, M.Y. 121

Kiefel, J. 47

Kilbourn, C. 240

Killgore, W. 143

Killgore, W.D. 3, 24, 134, 140, 220, 243, 244, 256, 261, 277

Killiany, R. 138

Kim, B.M. 60

Kim, D. 131

Kim, J. 263

Kim, K. 101

Kim, N. 264

Kim, S. 34, 82, 122

Kim, Y. 290

King, A. 63, 108

King, J. 28, 92, 176, 186

King, M. 257

King, T.Z. 61, 224, 226, 227, 253

Kinzel, C. 99

Kiosseoglou, G. 173

Kiran, S. 293

Kirkham, F. 284

Kirkwood, M. 46, 47

Kirtley, A.T. 137

Kirwan, B. 258

Kiselica, A. 187

Kivisto, L.R. 120, 183

Klamar, K. 150

Klarr, J. 107, 109

Klaver, J.M. 121

Klein-Tasman, B. 232

Klein, A. 89
Klein, J. 96

Kleinberg, L. 228

Kleiner, J. 202, 264

Klem, S. 169

Klimas, N. 138

Kling, L.R. 32, 263

Klinge, P. 60

Klipfel, K.M. 27, 29

Klumpp, H. 64

Kmiecik, M.J. 286

Knecht, B. 173

Knight, S. 134, 140, 143, 277, 294

Knudsen. K. 248

Koch, K. 247

Koch, S. 63, 114

Koehl, L.M. 152

Koenig, A. 224

Kofler, M.J. 100, 125

Kohli, M. 92

Kolevzon, A. 115

Komaiko, K. 62

Kondo, D.G. 285

Konesheck, B. 206

Konesheck, D.O. 206

Koop, J. 29, 44

Koopowitz, S. 63

Kopelman, M. 20

Kordovski, V.M. 233

Korell, A.M. 265

Korgaonkar, M. 63

Korhonen, N. 101

Kornblith, E. 254

Korthauer, L.E. 71

Koscik, R. 71, 222

Kosmidis, M.H. 173, 240

Koss, J. 166

Koterba, C. 154

Kou, Z. 137

Kovelman, I. 114

Kowalski, K. 93

Kraal, A. 253

Kraal, A.Z. 7, 8, 39

Kraegel, P. 247

Kramer, J. 13, 14, 43, 127, 195, 249 , 250,251

Kramer, J.H. 129

Kramer, N. 48

Krapf, E.M. 117

Kraus, A. 138

Kravitz, A. 46, 51

Kreitner, D. 22

Kremen, W.S. 62, 82, 127

Krengel, M. 138, 243

Kresge, H.A. 61, 249, 250

Krikorian, R. 69 
Krishnamurthi, R. 279

Krishnamurthy, L.C. 265

Krishnamurthy, V. 265

Krishnan, K. 174

Krohner, S. 180

Krull, K.R. 276

Kruse, M. 280

Kuhn, T. 127, 262, 278

Kunz, M. 33

Kurian, S. 62

Kurowski, B. 93, 153

Kusmiesz, C.J. 59

Kutnar, K. 53, 177

Kuttesch, J.F. 229, 260

Kuwabara, H. 30

Kuzniecky, R. 280

Kvaal, S.A. 204

Kwa, V.I.H. 216

Kwan, V. 156

La Corte, V. 140, 220

LaBode, V.M. 286

Lace, J.W. 152, 153

Lacey, E. 274

Lacritz, L. 74, 85, 95, 126

Lacy, M. 231

Lad, S.S. 243

Lafo, J.A. 207, 236

Lageman, S.K. 83, 240

Lagoni, D. 108

Lajiness-O'Neill, R. 37, 114, 118, 185 , 186

Laks, J. 65

Lalani, S. 237

Lamar, M. 3, 91, 295

Lambert, A. 43

Lamberty, G. 213

Lambros, S.L. 61, 194, 249, 250

Lampson, E. 227

Landeira-Fernandez, J. 65

Landon, L. 59

Lang, A. 133

Lang, D. 23

Langan, L. 198

Langan, L.M. 140, 280

Lange, R. 140, 141

Langenecker, S.A. 25, 32, 35, 101, 263

Lantigua, R.A. 12

Lanzillo, R. 209

Lapira, K. 57

Larsen. I. 34

Larson-Prior, L. 202

Larson, E.R. 61

Larson, K. 55
Larson, M. 135, 237, 251, 258, 267 , 272,274

Larum, A. 205, 207

Last, B.S. 70

Lau, L. 26, 27, 211

Launes, J. 101, 277

Laurent, R. 231

Lavery, A. 212

Law, L. 71, 222

Lawless, S. 65

Lawlor, B. 274

Le Berre, A. 163

Lea, S. 138

Leaffer, E. 199

Leaffer, E.B. 279

Lebedun, A. 135

Lebersfeld, J. 114

Lebkuecher, A. 211

LeBlond, E. 153

Lebois, L.A. 63

Lebowitz, B.K. 83

LeCheminant, J. 251, 267

Ledochowski, J. 153

Lee-Wilk, T. 236

Lee, A. 60, 94, 194

Lee, C. 25, 286

Lee, C.M. 115

Lee, G.J. 82, 98, 99, 102, 296

Lee, H. 18

Lee, K. 232, 255

Lee, M. 49, 147

Lee, S. 88, 121

Lee, Y. 56

Leese, M. 264

Legarreta, M. 286

Lehman, S. 172

Lehockey, K.A. 216, 259, 267

Lehto, E. 277

Leibel, D.K. 13

Leininger, S. 171

Leitner, D. 166, 168, 180, 215

LeJeune, B. 183

Lemiere, J. 106, 126

LeMonda, B. 200, 203

Lenert, M. 266

Lengenfelder, J. 151, 153, 154, 213 , 214, 272, 273

Lennon, M. 124

Leonova, O. 23

Leppämäki, S. 101

Leritz, E. 237

Lesnovskaya, A. 191

Lesnovskya, A. 190

Lesperance, C. 172

Lessig, S. 205, 208
Lettner, S. 19

Letzen, J.E. 255

Levan, A.J. 51

Levin Allen, S. 48

Levin, B.E. 5, 15, 203, 207

Levin, H.S. 123, 124, 148

Levine, T.A. 165, 278

Levitch, C.F. 233

Levy, I. 63

Lhommel, R. 223

Liang, B. 252

Libben, M. 166, 168, 180, 215

Liberzon. I. 63

Libon, D.J. 81, 87, 92, 217, 232

Libsenson, M. 203

Liebel, S.W. 256

Light, S.N. 25

Liguori, A. 96, 207

Lima, E. 51, 154

Lima, F. 33

Lima, M. 118

Limb, C. 248

Limon, D. 186

Lin, C. 233

Lin, D. 122

Lin. H. 92

Lin, S.S.-H. 71

Linck, J. 131, 287

Lindbergh, C. 6

Lindbergh, C.A. 253, 264

Lindley, T. 48

Lindo, J. 10

Ling, J. 191

Linke, A. 125

Lipman, Z. 146

Lippa, S.M. 140, 141

Lisanby, S.H. 158

Lisdahl, K. 173

Lisdahl, K.M. 164

Litvan, I. 205, 208

Liu, A.A. 280

Liu. C. 100

Liu, D. 61, 67, 194, 249, 250

Liu, H. 13

Liu, J.R.M. 51, 154

Liu, T. 125

Liu, Y. 35, 83

Llamas, V. 65

Lo, C. 82

Loblein, H. 198

LoBue, C. 74

Locke, D. 252

Loewenstein, D. 221

Loft, S. 277

Logue, M. 82 
Loman, PhD, M. 297

Long, M. 274

Loo, S. 99

López-Quintero, C. 163

Lopez, F.V. 17, 205, 208

Lopez, M. 82

Lopez, S. 292

Lopez, W. 141, 148, 149

Loring, D. 87, 173

Loschiavo, F. 255

Louis, E. 94

Love, C.E. 23

Løvstad. M. 34

Lowe, D.A. 67, 77, 83, 86

Lowe, J. 31, 53, 107, 110, 177

Lowe, M. 200

Lu, L.H. 204

Lubke, K.N. 17

Luca, C. 203, 207

Lucas-Jiménez, O. 254

Lucchetti, A. 199

Lueke, N.A. 258

Lukomski, A. 185, 186

Lum, P. 55

Luna, B. 2

Lundine. J.P. 154

Lunven, M. 140

Luong-Tran, C. 160, 161

Ly, J.J. 76

Lyles, J. 87

Lynch, S. 283

Lynch, T. 88

Lyon, A. 264

Lyons, M.J. 62, 82, 127

Ma, S. 191, 269

Mabbott, D.J. 294

MacAllister, W.S. 56, 199, 200, 203, 280

MacAulay, R.K. 6, 77, 83, 86

Macchi, C. 84

MacDonald, B. 59

MacDonald, C. 178

MacDonald, J. 135

Macdonald, K. 120

MacDonald, M. 215

MacDonald, S. 70

Maceira-Villalobos, L. 100

MacEwan, G.W. 23

Machulda, M. 75

Macina, L.O. 76

Mackin, S. 62, 81

MacLeod, M. 148

Macoun, S. 97

MacPherson, S.E. 128, 282

Madore, M. 59

Madsen, J.R. 203
Maffett, A. 155

Maganti, R. 247, 248

Mahdavi, K.D. 241

Mahle, W. 61

Mahmood, Z. 127, 233

Mahon, S. 279

Mahone, E.M. 105, 225

Mahoney, E.J. 44

Maiman, M. 199, 200, 203

Majercik, S. 135

Maki, P.M. 75, 295

Makwana, B. 265, 270

Malgaroli, M. 94

Malins, J.G. 261

Malleck, M. 52. 54

Malloy, P. 60, 264

Malone, C. 71

Maloney, M. 17

Mancioppi, G. 86

Mandelbaum, S. 140, 198, 280

Manderino, L.M. 52

Mangal, P.C. 180, 236, 253

Mangalonzo, A. 22

Manieri, M. 31

Manly, J.J. 12, 128, 130, 159, 160, $162,163,215,221,223$

Mann, J.J. 63

Manning, C.A. 243

Manning, K.J. 14, 17

Manning, L. 124

Mannix, R. 18, 47, 50, 145

Mano, Q. 119, 298

Manoochehri, M. 88

Månum, G. 34

Manzel, K. 200, 257

Marceaux, J. 255, 290

Marcelle, E.T. 102

Marcopulos, B.A. 160

Marcuse, L. 140, 198, 280

Margalit, D. 167

Margolis, A.E. 119

Margolis, S.A. 8, 9

Maril, S. 167

Marion, A. 217

Marker, C. 87

Marquez, D.X. 295

Marquine, M. 215, 245

Marra, D.E. 44, 103, 141

Marrie, R. 210

Marshall, A. 280

Marshall, D. 25, 35

Marshall, K.K. 290

Marshall, P. 103

Marsiske, M. 9, 12, 180, 207, 253

Marson, D. 79

Martin-Elbahesh, K. 229
Martin-Harris, L. 278

Martin, M. 69

Martin, P. 179, 243

Martin, R. 79, 278

Martin, T. 136

Martindale, S.L. 134, 174

Martinelli, M. 103

Martinez, F. 141, 149, 174

Martinez, J. 43

Marx, G. 195

Mash, L.E. 125

Mason, D.S. 47

Massimino, M. 259

Massman, P.J. 65

Massoni, S.G. 286

Mastel, S. 299

Masucci, A. 94

Matchanova, A. 4, 6, 8, 172, 240, 273

Mathews, C.A. 62

Mathias, S. 23, 122

Mathis, J. 247, 248

Mathur, K. 196

Matsui, M. 39

Matsuzawa, Y. 56

Mattek, N. 249

Mattes, A. 107. 109

Matthew, A. 226

Mattson, S. 284

Mattsson, N. 81

Maxa, K.M. 71

Maxfield, M. 10

Maxson, C. 296

Maxwell, B. 18, 50, 145

Maxwell, E.C. 152

Maye, J.E. 9, 180, 253

Mayer, A.R. 191

Mayeux, R. 12, 221, 223

Mayhew, A. 276

Mays, K. 150

Maytham, C. 182

Mazzolini, L. 140

Mburu, T. 174

McAndrews, M. 40

McArthur, D. 148, 149

McAuley, T. 254

McAuley, T.L. 57, 257

McCallum, K.E. 290

McCarthy, J. 112, 175, 281

Mccarvel, M. 136, 224

McCleary, K.R. 200

McClintock, S.M. 95

McCord, E. 43

McCrea, M.A. 55

McCullagh, J. 293

McDermott, A. 172, 182

McDonald, C. 196, 280 
McDonald, J. 70

McDonald, K. 153

McDonald, S. 123, 194, 271, 272

McDonnell, M. 172

McEvoy, L. 82

McFarland, C.P. 296

Mcfarland, J. 224

McFarlane, K. 37, 114, 186

McGinlay, M. 215

McGlade, E. 286

McGlinchey, R. 237

McGregor, K.M. 265

McInerney, K.F. 5, 15, 200, 207

McInnis, M. 25, 35

McIntosh, E.C. 68, 72, 193, 279

Mckenna, B. 130

McKenzie, R. 62

McKinney, T.L. 222, 268

McLaren, M.E. 257

McLaughlin, K.A. 63

McLaughlin, R. 198

McLean, E. 151, 154

McMillan, T.M. 247, 248

McNally, K. 150, 155

McVeigh, K.S. 9

McWilliams, L. 100

Mecca, A.P. 70, 73

Mechanic-Hamilton, D. 68, 198, 270

Medina, L.D. 212

Medler, D.A. 119

Megari, K. 173

Meijer, I. 96

Meinerding, M.E. 65, 66, 72, 273

Mejia, A. 30

Mejías, M. 97

Melchiorre, A. 10

Meléndez-Cabrero, J. 69

Melikyan, Z. 30

Melrose, R. 77, 128, 263

Memel. M.B. 297

Mencl, W. 261

Mendez-Lozano, N. 38

Mendoza, L. 10

Mercedes, A. 185

Mercer, S. 215

Merchant, T. 225

Meredith-Duliba, T. 270, 271

Merker, B.M. 53, 55, 56, 57, 93

Merritt. V.C. 5. 51, 53, 142, 144, 219

Merz. Z. 240

Messa, I. 175

Metcalfe, J. 66

Metzger, D.S. 234

Mewborn. C. 6. 12, 253

Meyer, A. 15

Meyer, J. 49, 53, 142
Meyerand, M. 247, 248

Meyering, K. 115

Michael, P. 182

Michel, M. 17

Michel, S. 69, 234

Michelson, C. 260

Michelsson, K. 101, 277

Michon, A. 220

Miciak, J. 118

Mickey, B.J. 263

Middleton, K. 175

Mielke, M. 75

Mietchen, J.J. 150, 287

Mihalov, L. 93, 151

Milano, N. 77, 83, 86

Milberg, W.P. 237

Miley-Akerstedt, A.M.K. 280

Millan, C. 200

Millan, M. 140

Miller, A.K. 72

Miller, B. 194

Miller, C. 55, 93, 99, 102, 103, 268

Miller. D. 16

Miller, E.K. 58

Miller. H. 166, 168, 180, 215

Miller, J. 225

Miller, J.B. 72, 73, 167, 176

Miller. J.S. 195, 241

Miller, K.J. 7

Miller, L. 6, 12, 179, 236, 253

Miller, L.E. 115, 281

Miller, S. 94

Milleville, K.A. 147

Millis, S. 137

Mills, C. 298

Milne, K. 52

Minassian, A. 22

Ming, S. 29

Minkina. I. 2

Minniti, N.L. 198

Minxhozi, O. 113

Miotto, E.C. 255

Mirabito, G. 25

Mis, R. 69, 84

Miskey, H.M. 134, 174

Mitchell, C. 71

Mitchell, J. 85, 244

Mjaanes, J. 48

Moberg, P.J. 112, 198, 242

Modersitzki. E. 251, 267

Modi, A. 161

Moelter, S.T. 68

Moerkerke, M. 106, 126

Moertel, C. 107, 230

Moffat, K. 262

Moffett, K. 255
Mograbi, D.C. 65

Mohanty, R. 247

Mok, V. 92

Molden, J. 10

Moll, A. 39

Moltisanti, A. 100

Moncrief, G.G. 73

Mondini, S. 20

Monteiro, J. 113

Montgomery, V. 176, 179

Montoya-Murillo, G. 254

Moore, B. 175

Moore, D. 234

Moore, E. 61, 194, 249

Moore, H. 207

Moore, M.W. 98, 111, 184, 185, 187

Moore, R. 205, 208

Moore, T.M. 164, 234

Morales, K. 10

Moran, E.E. 96, 293

Moran. J. 37

Moran, L.M. 110

Moran, S. 236

Moreno, C.C. 45, 52, 56, 180

Moreno, M. 97

Moretti. P. 148

Morey, R.A. 63

Morgan, B. 287

Morgan, E.E. 164

Morgenlander, J. 146

Morra, L. 204

Morris, A.R. 278

Morris, J. 64, 75, 232

Morris, R. 120, 121, 261

Morris, R.G. 65

Morrison, C. 280

Mosack, K.E. 6

Mosca, I.E. 84

Mosconi. L. 69

Moscovitch, M. 40

Moscufo. N. 14

Moseley, S.A. 9, 11, 13, 249

Moser, L. 16

Moses, J.A. 170

Moss, N.C. 31

Mossaro, J. 83

Mosteller, C.M. 139

Mostofsky, S. 103, 104

Motl, R. 274, 275

Moy, C. 50

Moyett, A. 282

MOZA, S. 240

Muanza, T. 226

Mueller, W. 201, 202

Muftuler, T. 247

Mullen, C.M. 87, 142, 144, 287 
Muller-Oehring, E. 171, 205, 242, 258

Müller, R. 125

Mulligan, R. 27

Mulrooney, D.A. 276

Mumford, P. 14, 43, 127, 129, 250

Mungas, D. 44, 169

Muniz, M.C. 43

Munk, S. 251

Munson, B. 25

Murdaugh, D. 49, 253

Muro, A. 102

Murphy, C. 16

Murphy, N.A. 233

Murray, E. 239

Murray, H. 215

Murray, K.O. 235

Murray, S. 284

Musil, S. 238

Mustafa, N. 257

Muths, L.H. 228

Mzayek, Y. 227

Na, S. 227

Nachev, P. 282

Nadeau, S.E. 2

Nadel, L. 40

Nagorskaya, I. 30

Nair, K. 212

Nair, S. 61, 249

Nair, V.A. 247, 248

Nakada, S. 39

Nakhla, M.Z. 35, 73

Nakhutina, L. 9

Namchuk, A. 108

Nance, C. 73

Narad, M. 93, 155

Narayanan, S. 210

Nardi, J. 31

Narkhede, A. 70, 88, 221, 223

Narvaez, T.A. 77

Nash, L. 132

Nation, D.A. 68, 72, 77, 92, 193, 220,279

Naylon, K. 231

Ndukwe, N. 176

Neal, J.E. 250

Neimand, L. 96

Nelson Sheese, A. 177

Nelson, C. 81

Nelson, L.D. 55

Nelson, R.A. 206

Nencka, A. 247, 248

Nestor, P. 30

Neuberger, K. 48

Neufeld, R. 212

Neuhaus, J. 14, 249, 251

Neumann, C. 105
Neuroimaging Initiative, A. 81

Newman, J.B. 51, 109

Neylan, T. 127, 250

Nguyen-Louie, T. 22

Nguyen, G.M. 287

Nguyen, C.P. 77, 220

Nguyen, D.V. 60

Nguyen, L. 194

Nguyen, T. 126, 171

Nguyen, T.T. 193

Ni'Shuilleabhain, M. 227

Niccolai, L. 79

Nichols, A. 287

Nichols, M. 262

Nickel, A.E. 44

Nicks, R.C. 266

Nicolelis, M. 157

Nicoletta, A. 268

Nielson, K.A. 8

Niermeyer, M.A. 10, 292

Nigg, J. 117

Nikolai, T. 168

Nilsen, W. 92

Nilssen, A.R. 292

Nissim, N. 190

Nitsch, K.P. 27

Nitta, M.E. 44, 103, 141

Nixon, K.H. 288

Nolan, M.S. 235

Nolen, T. 123, 124

Nomura, Y. 24

Nonnemacher, M. 232

Noonan, N. 32, 185, 283

Norman, A.L. 85

Norman, S. 133

Norris, A.H. 242

Norris, T. 109

Nosarti, C. 255

Novack, T.A. 53

Novakovic-Agopian, T. 254

Novitski, J. 78

Nowak, K. 124

Nunez, R.A. 127, 233

Nussbaum, N. 202

Nwoke, O. 247, 248

O' Shea, D.M. 250, 252

O'Brien, A.M. 104, 183

O'Callaghan, J. 138

O'Connor, B. 166

O'Connor, T.A. 23

O’Dell, M. 215

O'Donovan, K. 30

O'Hara, N. 137

O’Mahony, J. 209, 210

O’Neill, J.A. 53

O’Neill, S. 100
O'Shea, A. 190, 257

O'Toole, K. 253

Oakes, T.R. 141

Obermeit, L.C. 164

Oh, J. 222

Oh, Y. 101

Ohls, R. 107, 110

Ojeda, N. 176, 254

Okabe, H. 222, 268

Okonkwo, D.O. 148

Okonkwo, O.C. 71, 222

Okun-Alexander, A. 151, 153, 154

Olaru, M. 25

Olff, M. 63

Oliveras-Rentas, R.E. 113

Olivier, T.W. 294

Olsen, P. 22

Omollo, S. 177

Onar-Thomas, A. 225, 294

Ono, K. 47, 49, 200

Onyike, C. 88

Oquendo, M.A. 63

Ord, A.S. 10

Orlovsky, I. 92

Ornstein, T.J. 178

Orr, B.C. 288, 292

Ortega, J. 53

Ortega, R. 96

Ortiz, X. 216

Osborn, A. 32, 284

Osborn, K.E. 61, 194, 250

Osipowicz, K. 116, 265, 270, 271

Osmon, D.C. 6

Osorio, E. 184

Ossowski, A. 120

Ostojic, D. 99, 103

Osuna, J.R. 11

Oswald, K. 227

Oswald, T.M. 177

Otero, T.L. 299

Ott. B. 8

Ott, B.R. 76

Owens, E.B. 102

Owens, T. 199

Ozelius, L. 96

Pacheco-Colon, I.M. 22, 163

Paciello, F. 96, 207

Padula, C. 291

Pagan, C.R. 288

Paguyo, A. 238

Palmer, L. 89

Palmer, S.B. 293

Palmer, S.L. 294

Palmese, C. 96

Pane, C. 96, 209

Panenka, W. 23, 145 
Panfili, D.P. 116

Panizzon, M.S. 62, 82, 127

Panos, A.H. 238, 239, 241

Paolillo, E. 234

Papageorgiou, S.G. 80

Papp, K.V. 43, 92

Pardej, S. 29

Paré, N. 177

Parker, A.F. 166, 168

Parker, H.A. 61

Parmar, P. 279

Parsons, T.D. 299

Pascoe, L. 126, 255

Pasipanodya, E. 234

Passarotti, A. 101

Passler, J.S. 11

Pastorek, N.J. 131

Patel, A. 51, 154

Patrick, K.E. 150

Patros, C. 103

Patt, V.M. 22

Patton, H. 37

Paul, B. 196, 280

Paul, L.K. 195, 238, 239, 241

Paullada, A.N. 131

Paulson. H. 88

Paulson, J. 177

Pavlou, D. 80

Pawlenko, N. 51, 154

Pearlson, G. 192

Pearson, M.M. 229, 260

Pechman, K.R. 61, 249, 250

Peciña, M. 263

Peck, C.P. 29

Pedapati, E. 104

Peltier, S. 191

Peña, J. 176, 254

Pena, S.L. 201

Peñaloza, C. 293

Penate, A. 221

Pendery, A. 169

Penna, S. 142, 144

Penney, D. 92, 217

Pennington, C.R. 290

Perera, I. 96

Perez, P. 184

Perlstein, W.M. 143, 255

Perna, R. 143

Perrin, M. 234

Perry, R. 85

Perry, W. 22

Persad, C. 169

Persson. B. 19

Peterman, J. 25, 35

Peters, B. 107. 109
Peters, K. 96

Petersen, J. 209

Petersen, L. 228

Peterson, E. 292

Peterson, J. 53, 177

Peterson, R.L. 46, 47

Petranovich, C. 111

Petrauskas, V.M. 53

Petrick, J.D. 28, 54

Petry, C. 33

Pettigrew, C. 129

Peverill, M. 63

Pfefferbaum, A. 163, 205, 242

Phalen, L. 104

Pham. D. 135

Phan, K.L. 32, 64, 263, 284

Phatak, V. 252

Phenis, R. 206

Philip, N. 269

Phillips, J. 10, 107, 110

Phillips, N. 276

Phillips, P. 108

Phipps, S. 229

Piantino, J. 299

Piccolo, L.d. 118

Pick. L. 281

Piercy, J. 107, 109

Pierpont, M. 107, 230

Pierpont, R. 107, 230

Piers, R.J. 35

Pimentel-Rodríguez, L.F. 267

Pineault, L. 38

Pinjala, M. 200, 203, 207

Piolino, P. 140, 220, 297

Piper, L.E. 28

Pirogovsky-Turk, E. 205

Pirrone, V. 232

Piryatinsky, I. 60

Pisani, D. 135

Pitel, A. 188

Pitteri, M. 212

Platek, K. 138

Pliego, J.A. 109

Plourde, V. 156

Pluim, C.F. 205, 208

Plumadore, J. 62

Plumb, P. 126, 211

Plurad, D. 148, 149

Poggi, G. 259

Polejaeva, E. 143, 255

Politis, A. 263

Poll, K.M. 8

Pollock. B. 209

Polnaszek, K.L. 289

polsinelli, a. 11
Polsinelli, A. 9

Pomiecko, K. 148

Pommy, J. 107, 110, 289

Ponce, F. 206

Pond, G. 226

Ponsford, J.L. 19

Poon, L.W. 179

Poorzand, P. 194

Porges, E. 250, 257

Porter, J. 97

Possin, K.L. 43

Postal, K. 160

Poston, K. 205, 242

Potter, G. 17

Powdhar, S. 185

powell, a.a. 231

Powers, K. 229

Powers, M. 186

Powers, M.D. 30, 116, 117

Prabhakaran, V. 247, 248

Prado, C. 67

Prayson, R.A. 196

Presenti, M. 214

Preston, T. 83

Price, C.C. 18, 92, 96, 180, 204, 217. 240,265

Price, J.S. 241

Priluck, J.L. 39

Primosch, M. 296

Prince, E. 169

Pritchard, A. 3, 121

Pritchett, A. 24

Procyshyn, R.R. 23

Proescher, E. 32, 284

Puccio, A. 148

Puente, A.E. 30, 160

Pugh, E.A. 70, 73

Pugh, K. 261

Pugh, N. 123

Pugliese, C. 117, 160, 161

Pui. C. 276

Pulaski, S. 206

Pulsifer, M.B. 226

Pulsipher, D.T. 117

Purdie, R. 34

Pyykkonen, B. 53, 177

Qaddoumi, I. 229

Qiu, W.Q. 35

Quarantelli, M. 207

Quasney, E. 61, 202

Quasney, E.E. 197, 201

Quast, L. 108

Quenon, L. 223

Quinn, C. 177

Quinn, T. 215 
Quiroz, Y.T. 43

Rabelo da Ponte, F.D. 33

Rabin, H. 177

Rabin, L. 177

Rabinowitz, A.R. 53

Rae, C. 262

Ræder, J. 34

Raein, K.L. 206

Raghavan, M. 201, 202, 247, 248

Raghubar, K.P. 225

Rahman-Filipiak, A.M. 190

Raicu, A. 202

Raikes, A.C. 143, 261

Raitano Lee, N. 230

Rajendran, N. 3

Ram Junnakar, K. 140

Ram-Junnarkar, K. 198, 280

Ramachandran, V. 181, 299

Ramanan, S. 70

Ramig, L. 239

Ramirez, C. 98, 99, 100, 102 , 105, 267

Ramírez, D. 216

Randolph, J. 213

Randolph, J.S. 213

Rangamannar, R. 36, 185

Rankin, K.H. 43

Rankin, K.P. 194

Rao, R. 111

Rao, S.M. 8, 21

Rapcsak, S.Z. 295

Raphail, A. 213

Rapp, A. 7

Rapport, L.J. 139, 180, 181

Raskin, S. 45, 94

Rathwell, I. 113

Ratliff, S. 266

Ratto, A. 30, 116, 160, 161

Rau, S. 116

Rawleigh, V. 131

Ray, A. 10

Raymer, A.M. 294

Raymond, D. 96

Raz, S. 107, 109

Razani, J. 7, 69, 73, 288

Reale-Caldwell, A. 201

Ream, D. 34

Reckow, J. 190

Reddick, W.E. 276

Redmond, K. 228

Redondo, M. 97

Reed, R. 185, 186

Reeves, W. 91

Reger, K. 276

Reginella, V.M. 28, 54

Reilly, J. 48, 75, 192
Reiter, K. 297

Reno, A. 171

Rensberger, J. 36

Renshaw, P.F. 285

Rentz, D.M. 43, 92

Renzi-Hammond, L. 6

Reott, A. 48

Resch, Z. 6, 197, 201

Reslan, S. 219

Ressler, K.J. 63

Restrepo, J. 239

Rey, G.J. 200

Reyes, A. 23, 196, 280

Reynolds, B.W. 289

Reynolds, G.O. 8

Reynolds, J. 6, 266, 268

Reynolds, M. 54, 224

Reza Arroyo, M.A. 178

Rhoades-Clark, E. 107

Rhoads, T. 37

Rhodes, E. 127

Rhyner, K.T. 131, 178

Ribases, M. 188, 189

Ribot, R. 200

Richards, L. 190

Richardson, C.G. 122

Richardson, M. 31

Richardson, R.J. 54, 57, 104, 285

Ricker, J. 56

Ridge, B.E. 290

Ridgel, A. 209

Rieger, R. 107

Rieger, R.E. 31, 110

Riemersma, J.J. 153

Riggall, E. 121

Riggs, J.L. 118

Riley, E.P. 284

Rilo, O. 254

Rindge, M. 73

Rinehardt, E. 171

Ringleb, S. 294

Ris, M. 225, 228

Riskin-Jones, H. 263

Riss, R.H. 46

Ritchie, K.A. 44, 55, 103, 141

Ritter, A. 73

Rivera Mindt, M. 22, 159, 235, 236

Rivera-Rivera, L. 71

Rizvi, B. 70, 88, 221, 223

Rizzo, A. 271

Roalf, D.R. 198

roberts, G. 255

Roberts, S. 46, 224

Robertson, F. 155

Robertson, I. 274

Robins, D.L. 112
Robinson, C. 230

Robinson, K. 291

Robinson, T. 6, 253

Robinson, T.L. 12

Robison, L.L. 276

Rochette, A.D. 85

Rodebaugh, T. 64

Rodgers, C. 51, 133

Rodgers, K.E. 193

Rodriguez-Galindo, C. 229

Rodríguez-Gómez, L. 267

Rodriguez-Larrain, G. 95

Rodriguez, K. 96

Rodriguez, R.A. 12

Roeckner, A.R. 104

Roesch, S. 164

Rogers, E. 144

Rogers, I.N. 268

Rogers, S. 208, 241

Roitsch, J. 104

Rolin, S. 28, 147, 169, 186

Roll, E. 6, 8, 273

Roman, C.A.F. 274, 275

Romesser, J. 131

Roper, B.L. 14, 171

Roper, C.L. 135

Rosa, A. 33, 266, 268

Rosch, K.S. 103, 104

Roseman, E. 83

Rosen, H. 14

Rosen, S.A. 206

Rosenberg, H. 272

Rosenberg, J. 272

Roskos, P. 149, 150, 266

Ross, M.J. 152, 153

Rossetti, H.C. 74, 85

Rossetti, M. 34

Rossi, M. 276

Rotblatt, L.J. 12

Roth, J. 295

Roth, R. 38

Roth, R.M. 38

Rowland, J. 144, 174

Rowley, H. 222

Roy, A.A. 154

Roy, M. 156

Roye, S. 167, 178

Rozman, M. 247, 248

Rubin, L. 192

Rubin, L.H. 75

Rubino, S. 30

Rubinow, D. 258

Rubinstein, T. 170

Ruiter, R. 276

Rum, R.S. 201

Ruoff, L. 127, 250 
Ruparel, K. 209

Rupert, P. 198

Ruppert, P. 173

Rusina, R. 168

Russo, C. 207, 209

Russo, J. 67, 69

Ruzicka, E. 168, 295

Ryan, K. 25, 35

Ryan, L. 15, 221, 297

Ryan, M. 105

Rycroft, S.S. 4, 6, 8, 273

Ryman, S. 191

Ryser, D. 135

Ryu, K. 290

Saad, L. 270

Saadatpour, L. 217

Sabbah, L.E. 12

Sabe, L.R. 67

Sabsevitz, D. 197

Sabsevitz, D.S. 89, 201, 202

Saccà, F. 96, 207, 209

Sacchetti, D. 270

Sachdev, R. 96

Sachs, B. 5

Sadeghi, M. 210, 254

Sadurni, C. 184

Saemann, P. 63

Safer-Lichtenstein, J. 30, 116, 160, 161

Safford, A. 156, 157

Sagar, S. 172, 178

Sager, M. 71, 222

Saghafi, S. 196

Sair, H. 228

Sakka, P. 240

Salat, D.H. 224, 237

Salazar, L. 235

Salazar, M. 62

Salazar, R. 31, 205, 207

Saldaña, B. 216

Salgari, G. 99

Sali, A.W. 104

Salinas, C. 59, 60

Salinas, S.L. 142, 144, 202, 264

Salloway, S. 60, 264

Salminen, L. 63

Salmon, D. 69, 71, 74, 80, 193

Saloner, R. 13, 14, 43, 127, 129, 249. 250,251

Salyer, R.E. 12

Sanborn, V. 244

Sanchez. O. 213

Sandberg, H. 294

Sandberg, S. 254

Sanderson-Cimino, M. 49, 51, 127. 133. 147

Sandroff, B. 274, 275
Sanguinetti Czepielewski, L. 33

Santacruz, G. 184

Santiago, C. 184

Santistevan-Andrews, S. 28, 186

Santos Martins, D. 33

Santos, O.A. 23, 178, 290

Sargent, P. 131

Sarno, M. 5, 15, 200, 203, 207

Sarrett, L. 53

Sarver, D.E. 125

Sassoon. S.A. 163

Satterfield, B.C. 143, 261

Saules, K. 118

Saunders-Pullman, R. 96

Saunders, B.A. 286

Sautter, S. 10

Sawa, A. 38, 191

Scapini, N. 169

Scarmeas, N. 240, 259

Scavone, A. 55, 93, 268

Schaaf, C. 112

Schaberg, T. 51

Schaefer, L.A. 177

Schaffer, S. 238

Schaffert. J. 74

Schagen. S.B. 227, 276

Schanke, A. 34

Scharaga, E.A. 231

schechter, a. 60

Scheibel, R. 124

Scheibel, R.S. 123, 124

Schendel, K. 182

Schenk zu Schweinsberg, E. 149

Schiehser, D. 57, 137, 142, 144, 145 , 148, 205, 208, 219

Schillaci, A. 17

Schilling, S. 185, 186

Schlaefflin. S. 190, 269

Schleicher-Dilks, S. 55

Schmidt, A.T. 290

Schmidt, M. 161

Schmidt, S.T. 213

Schmitter-Edgecombe, M. 15, 87, 205

Schnaas, L. 184

Schneider, B. 184

Schneider, H. 105

Schneider, W. 148

Schneiderman, J. 59

Schoenberg, M. 201

Schofield, H. 122

Scholl. M. 81

Schorr, E.M. 93

Schraegle, W. 202

Schraufnagel, C. 55

Schreiber, J. 294

Schreiber, J.E. 97
Schretlen, D.J. 38, 176, 191

Schrock, B.J. 133

Schroth, C. 32, 284

Schulte, T. 171, 205, 242, 258

Schultheis, M.T. 112, 145, 213, 291

Schupf, N. 12, 128, 130, 221, 223

Schwab, N.A. 18, 265

Schwartz, K. 294

Schwarz, L. 4, 173

Schwebel, D.C. 53

Schwimmer, C. 298

Scorpio, K. 239

Scott, B.M. 207

Scott, J. 164, 234

Scott, S. 294

Scott, S.E. 113, 137

Scott, T.M. 22, 23, 160

Scratch, S.E. 126

Seabolt, C. 61, 194, 249, 250

Seal, M. 255

Sears, M.Z. 9

Seaton, K. 58, 59

Seedat, S. 63

Seeley, W.W. 14

Seelye, A. 13, 249

Seese, S. 30, 116, 117, 160, 161, 186

Seghatol-Eslami, V. 116

Seidenberg, M. 8, 78, 201, 203, 296

Seifan, A. 69

Seke, K. 172

Sekhon, A. 298

Semerjian, C. 232

Semmel, E. 61

Semrud-Clikeman, M. 107, 230

Sepeta, L. 197, 261

Sergi, M. 37

Sergio, L. 212

Serrano, E. 200

Setnik, B. 36

Sevcik, R. 121

Sever, R. 201

Sexton, J. 135

seymour, k. 103

Shaafi Kabiri, N. 38

Shada, K. 117

Shaff, N.A. 191

Shah, B.B. 95

Shah, M. 242

Shaham, M. 237

Shaked, D. 13

Shallice, T. 128, 282

Shane, B.R. 140

Shany-Ur, T. 194

Shao, Y. 129

Shapiro, D. 90

Shapiro, E. 55 
Shapiro, R.J. 238

Sharan, A. 264

Sharber, A. 116, 160, 161

Sharkey, C.M. 276

Sharp, E.S. 73

Shear, P. 197, 285, 298

Shearin, E. 151

Shekhtman, T. 193

Sheldon, S. 200, 203

Shenal, B. 131, 178

Sheppard, D.P. 196, 235

Sherwood, A. 111

Shi. X. 285

Shields, B.J. 290

Shoji, K. 255

Shollenbarger, S. 61

Shone, T.M. 61, 249, 250

Shouel, H.L. 74

Shull, E. 10

Shungu, D. 279

Shura, R.D. 174

Shvartsur, A. 18

Siddarth, P. 7

Sideman, N. 264

Silton, R. 25, 286, 289

Silva, J. 118

Silverberg, N. 92

Silverberg, N.D. 145

Sim, A. 131

Simon, C. 260

Simon, S.S. 255

Simpson, H. 138

Sims, C. 200

Singer, C. 207

Singh, H. 175

Sink, K. 5

Siper, P. 115

Sisson, E. 138

Skalamera, J. 244

Skapek, M.F. 116, 117, 161, 186

Skeel, R. 155, 291

Skiles, M.A. 223

Skillicorn, K. 110

Skop, K.M. 124

Slomiak, S. 164

Slugh, M. 200, 203

Small, G. ?

Smith-Paine, J. 153, 155

Smith, A. 161

Smith, E.E. 85

Smith, G. 252

Smith, G.N. 23

Smith, H. 61

Smith, H.A. 258

Smith. J. 8, 67, 260
Smith, L.C. 62

Smith, M.J. 33, 192

Smith, R. 24, 134, 243, 277

Snedden, T.R. 45

Snell, J. 146

Snyder, M. 296

So, R.P. 36

Soares, J. 262

Sober, J. 179

Soble, J. 181, 290

Soden, D. 299

Sofko, C.A. 165

Soichiro, N. 63

Sol, K. 7, 8, 130, 253

Soldan, A. 129

Solomito, M. 45

Solon, C. 9

Sommer, k. 107, 230

Sonuga-Barke, E. 106, 126

Sorbi, S. 84

Sorg, S.F. 57, 137, 142, 144, 145, 148,219

Sorond, F. 62

Soto, E. 100

Soto, J.A. 136

Sousa, M. 33

Sowell, E. 284

Spadoni, G. 214

Spano, B. 282

Spasojevic, T. 172

Spat, J. 280

Spencer-Smith, M. 126, 255

Spencer, R.J. 176, 179

Sperling, M. 264

Sperling, R. 43, 92

Sperling, S.A. 95

Spielman, J. 239

Spirou, A. 214

Spitznagel, M. 85, 209, 244, 294

Split, M. 205, 208

Sponheim, S. 124

Springate, B. 295

Srivastava, D. 276

Srnka, K.D. 201, 203

St. Louis, G. 182

Stach, B. 180, 181

Stachniak, C. 198

Staffaroni, A.M. 14, 43, 127, 129, 195. 250,251

Stafford, R.J. 239

Stanford, L.D. 117

Stange, J.P. 32

Stanghelle, J. 34

Stanley, B. 63

Stanley, R. 59
Stapleton-Kotloski, J. 144

Stark, J. 17

Stark, S.J. 62

Stasenko, A. 74

Stathis, A. 257

Stavinoha, P.L. 227

Stawski, R. 70

Steenland, K. 81, 84

Stefanatos, A.K. 203

Stefanatos, G. 195

Steffen-Allen, F. 14

Steffen, K. 244

Steffener, J. 131

Steffens, D.C. 17

Stein, D.J. 63

Steinberg, J. 124

Stelmokas, J. 176, 179

Stephan, C. 121

Stephens, B. 14, 171

Stephenson, A.J. 259, 267

Stern, Y. 65, 259, 260

Stevens, B. 191

Stevens, J.S. 63

Stevens, M. 135

Steward, K. 85

Stewart, B.T. 296, 298

Stewart, J. 76, 230

Stickel, A. 15

Stika, M.M. 27, 29

Stiles, C. 215

Stinson, J.M. 196

Stiver, J. 43, 127, 129, 250

Stojanovic, M. 75

Stone, J. 124

Stone, W.L. 112

Stott, D. 215

Stout, J. 88

Strain, G. 85

Strang, J. 160, 298

Strang, J.F. 117, 161, 186

Stricker, B. 276

Stricker, N. 77, 220

Stringer, A.Y. 287

Strober, L. 210, 211

Strom, J. 137, 145

Struck, A. 247, 248

Studeny, J. 32, 185, 283

Stuessi, K. 131

Su, J.J. 195

Subramaniam, P. 285

Suchy, Y. 10, 292

Suhr, J. 75, 98, 99, 102, 150, 269, 287

Suhrie, K.R. 78, 79

Sullivan, D.Y. 191

Sullivan, E. 105 
Sullivan, E.V. 163, 205, 242

Sullivan, K. 138, 243

Sullivan, K.L. 277

Sultzer, D.L. 263

Sumida, C. 15, 17, 205

Summers, A. 23, 235, 236

Sun-Suslow, N. 5, 15, 203, 207

Sundaram, S. 242

Sunderaraman, P. 15, 66, 72, 145, 177. 273

Sundermann, E.E. 75

Sutherland-Owens, A.N. 35

Sutherland, A.N. 34, 193

Sutton, S. 138

Suvak. M. 12

Suzuki, M. 39

Svingos, A.M. 45, 52, 56, 98, 111, 184 , 185, 187

Swain, M. 294

Swain, M.A. 225

Swan, M. 96

Swanson, S. 89, 197, 201, 202, 297

Swartz, A.M. 164

Sweeney, J. 192

Sweeney, K.L. 105

Sweet, L.H. 256

Swenson, R. 81, 87

Swerdloff, R. 141

Swick, C. 114, 185, 186

Swift, R.R. 266

Swift, T.J. 266

Sylvia, A. 119, 225

Szajer, J. 16

Szeles, D.M. 77, 83, 86

Taber, K. 144

Tachikawa, T. 24

Tadrous-Furnanz, S.K. 105

Taggart-Neria, J.L. 10, 292

TAGHDIMI, M. 258

Taiwo, Z. 25, 250

Taler, V. 131

Tam. J.W. 16

Tamez, S. 105

Tamm, L. 119

Tamminga, C. 192

Tan, A. 126, 193, 211

Tan, C.C. 172

Tan, M. 32, 187, 284

Tanaka, M. 24

Tang, D. 298

Tanner-Woodward, S. 184

Tanner, J.J. 18, 96, 180, 204, 265

Tapera, T. 230

Tapert, S. 22

Taraben, S. 37

Tarkenton, T. 94, 227
Tart-Zelvin, A. 265, 270

Tat, M. 71

Tate, D.F. 123, 124

Taub, A. 255

Taylor, B. 56

Taylor, B.A. 124

Taylor, H. 90, 91, 93, 151, 155

Taylor, J. 167, 179

Taylor, L. 16

Taylor, P. 118

Taylor, S. 165, 167, 179

Teague, A.M. 187

Tedeschi. E. 207

Teichmann, M. 220

Telfer, P. 284

Tellapragada, N. 247, 248

Telles, R. 190

Tellez Rojo, M. 184

Tepley, N. 37

Terpstra, A. 41

Terry, D.P. 18, 47, 50, 138, 145

Terry, J.G. 61, 249

Terry, S.C. 53

Terryberry-Spohr, L.S. 46

Tessier, J. 291

Thames, A. 127, 159, 233

Thesen, T. 280

Thomas, A. 232

Thomas, C. 187

Thomas, K. 38

Thomas, K.R. 4, 9, 51, 80, 81, 133, 193, 253

Thomas, M.L. 80

Thomason, M.M. 111, 112, 228

Thompson, D. 126, 255

Thompson, J. 61, 111, 194, 249, 250

Thompson, M.D. 278

Thompson, P.M. 63

Thompson, S. 207

Thorn, J. 208

Thornton, A.E. 23

Thornton, W.J. L. 238, 249

Thrasher, D. 147

Thuras, P. 213

Tibbs, J. 146

Tierney, S.M. 233, 235

Till, C. 184, 209, 212

Timpano Sportiello, M. 286

Timpano Sportiello, M.R. 84, 86, 214

Tiplady, K. 116

Tirri, D.J. 214

Tirso, R. 67

Tisson, J. 59

Titus, J.B. 198, 202

Tocchini, S. 84, 86, 214, 286

Toglia, J. 215
Tolle, K. 176

Tolle, K.A. 179

Tomasino, D.F. 17

Tomaszczyk, J. 41

Tomb, M. 119

Tommasini, L. 86

Tone, E. 64, 118

Tonning Olsson, I. 276

Toomey, R. 62, 138

Tornes, L. 200

Torres-Reverón, A. 113

Torres, C.X. 12

Tosto, G. 130

Tosun, D. 81

Toto, D. 293

Tracy, J.I. 264

Tran, M. 5

Tran, S. 265

Tranel, D. 168, 231, 257

Treble-Barna, A. 108, 146, 147

Trelles, M. 115

Tremont, G. 8, 86

Trenerry, M. 199

Trevizo, L. 106

Triebel, K. 79

Trifilio, E. 180, 253

Troster, A. 206

Troyanskaya, M. 123, 124, 131

Tsang, W. 113

Tsapanou, A. 259, 260

Tsoh, J.Y. 62

Tu, F. 289

Tu, M. 82

Tu, X. 82, 127

Tuazon, A. 208

Tucker, L. 251, 267

Tupler, L.A. 146

Tureson, K. 23, 235, 236

Turk Nolty, A.A. 36, 101, 105, 288

Turk, K. 71

Turkelson, L. 197, 285, 298

Turley-Ames, K.J. 265

Turnbull, J. 101

Turner, E.M. 98, 111, 184, 185, 187

Turner, J.A. 64, 224

Turner, S.M. 17, 111

Turnier, L. 161

Tustison, N. 124

Twaite, J.T. 239

Twamley, E.W. 51, 133

Uchani, S.D. 147

Udala, M.R. 180

Uhm, S.Y. 62

Ukueberuwa, D.M. 147

Ulmer, J. 89

Umlauf, A. 215 
Ungrady, M.B. 75

Unverzagt, F. 16

Upshaw, J. 166, 168, 215

Urgesi, C. 259

Urosevic, S. 13

Ustine, C. 247

Uttarwar, V. 196

Uy, P.J. 56

Vaccariello, E. 147, 222, 268

Vadnais, S. 121

Vaidya, C. 281

Vaillancourt, A. 16

Vakil, E. 194, 237

Valdez, P. 98, 99, 100, 102, 105, 267

Valentine, T.R. 180

Van Bavel, J. 245

Van Den Heuvel, L. 63

Van der Oord, S. 106, 126

van der Werf, S.P. 216

van der Werff, S.J. 63

van der Willik, K. 276

Van Dessel, J. 106, 126

Van Dyck, C.H. 70, 73

Van Dyk, K. 76, 228

van Erp, T.G. 63

Van Etten, E.J. 17, 69, 205

van Huiden, S. 264

Van Patten, R. 86

van Rooij, S.J. 63

van Tol, M. 123

Van Wright, A. 85

Van Zandvoort, M. 19

van Zuiden, M. 63

VanBrakle, M. 17

VandeBunte, A.M. 76, 208

Vanden Bussche, A. 291

Vander Wal, J.S. 4

VanMeter, A. 75

Vannetti, F. 84

Vannorsdall, T.D. 228, 229

Vanuk, J.R. 3, 140, 220

VanVoorst, W. 13, 249

Varanko, N. 20

Vardi-Shapiro, N. 194

Varga, C.M. 29

Vargas, B. 217, 218

Vargas, G. 56

Vasquez, B. 41

Vasserman, M. 199

Vasudevan, R.S. 49, 147

Vaughan, C.G. 46, 51

Vaughn, D.W. 187, 283

Vaurio, L. 200, 203

Vázquez-Santiago, F. 113

Vechetova, G. 168

Vega, E. 62
Velez-Ruiz, N. 200

Velez, C.S. 124

Veliz, J. 128, 263

Veltman, D. 63

Venkatesan, U.M. 76, 219

Ver Hoef, L. 278

Verbalis, A.D. 30, 116

Verfaellie, M. 244

Vermilion, B. 139, 180, 181

Verney, S.P. 59, 60, 106, 166

Vespa, P. 148, 149

Vesperman, C.J. 222

Vessey, W.B. 59

Vickers, K.L. 242

Vigil, O.R. 62

Vila-Castelar, C. 76

Vila-Rodriguez, F. 23

Vincent, A. 58

Viqar, F. 280

Virta, M. 101, 277

Vogel, R. 23

Vogt, E. 29, 44, 103, 141

Volgeneau, K. 187

Votruba, K. 225

Voytek, C. 234

Vuoksimaa, E. 82

Vuorre, M. 66

Waclawik, K. 23

Wade, B.S. 124

Wade, N.E. 164

Wade, S.L. 90, 93, 153, 155, 160, 161

Wadley, V.G. 11, 78, 85

Wadsworth, H. 155

Wagener, M.A. 61

Wagner, A.K. 146, 147

Wagner, G. 280

Wagner, M.T. 77, 83, 86

Wagster, M. 92

Wahl, K.J. 226

Waite, E. 141, 148, 174

Wakefield, D. 14

Waldman, A. 212

Waldstein, S.R. 13

Walker, A. 148, 149, 174

Walker, K. 127, 250

Walker, K.A. 238

Walker, W. 123, 124

Wall, K.M. 17

Wallace, B. 96

Wallace, E.R. 106

Wallace, J. 225

Wallance, A.L. 164

Walljasper, L. 61, 194, 249, 250

Walls, B.D. 106

Walsh, C. 127, 250

Walsh, K.S. 232, 276
Walsh, M.J. 137, 144, 148

Walters, S. 43

Walzak, L.C. 249

Wandinger, E.S. 166

Wang, A. 115

Wang, C. 141

Wang, L. 33, 62, 192

Wang, N.Y. 23

Wang, Q. 83

Wang, T.J. 61

Wang, X. 63, 280

Wang, Z. 229

Wank, A.A. 77, 221, 295

Ware, A.L. 148

Warnick, J. 111

Warren, Z.E. 112

Warschausky, S. 90, 185, 186

Wasserman, V.J. 81, 87

Watorek, V. 29

Watson, E. 182, 268

Watson, E.M. 259, 267

Watson, G.S. 294

Watson, J. 187

Watson, S. 104

Watt, J.C. 57

Watts, A. 19

Weafer, J.J. 263

Weakley, A. 15, 87

Weathers, J. 293

Webber, T.A. 181

Weber, E. 22, 242

Wecht, J. 242

Wedeman, B. 212

Weichselbaum, A. 149

Weigand, A.J. 193

Weiland, B. 3 ?

Weinborn, M. 277

Weiner, M. 81, 85

Weinstein, C. 83

Weisenbach, S. 263

Weiss, D. 15, 72

Weiss, L.R. 260

Weissberger, G. 77, 263

Weissman, D.H. 258

Weissman, J. 115

Weitzner, D. 181

Weizenbaum, E. 31

Wells, E. 100

Welsh, M. 292

Welton, E. 170

Wendel, C. 149

Weng, N.P. 13

Wennick, C. 224

Werhane, M.L. 5, 57, 142, 144, 145

Werry, A.E. 32

Wertz, C.J. 191 
Wesbecher, K. 89

Westbrook, C. 25

Westbrook, F. 272

Westhafer, J. 26

Westmacott, R. 153

Whipple, E. 291

White, D. 108

Whiteley, N. 205

Whiteside, D. 26, 27, 28, 211

Wicklund, M. 221

Wieben, O. 71

Wier, R. 229, 260

Wierenga, C.E. 11

Wigdahl, B. 232

Wiggins, M.E. 18, 96

Wilde, E.A. 123, 124, 148

Wildgoose, T.M. 133

Wilkinson-Smith, A. 42

Wilkison, C. 197, 285, 298

Willard, V.W. 229

Williams, A. 28, 186

Williams, C.P. 78

Williams, J. 265

Williams, L. 291

Williams, M. 270, 271

Williams, R. 123, 124

Williams, T. 58, 59, 153

Williams, V.J. 224

Williamson, J.B. 158, 250

Wills, M. 160, 161

Wilmoth, K. 94

Wilson, B.A. 255

Wilson, C. 155

Wilson, M. 229

Wilson, M.J. 236

Winocur, G. 40

Winship, K. 207

Winter, R. 121, 261

Winters, A.R. 268

Wirrell, E. 199

Wirtz. M.R. 51

Wishart, H.A. 213

Wisløff-Aase, K. 34

witt, e. 279

Witter IV, P. 249

Wolfe, K.R. 109

Wolff, J.D. 63
Wolfson, L. 14

Wolk, D.A. 68

Wollam, M.E. 57

wolters, f. 276

Wolterstorff, T. 10

Won, J. 260

Wong, A. 92, 215

Wong, C.G. 180, 181

Wong, M.M. 23, 283

Woo, E. 7, 18, 141, 148, 149, 174

Wood, A.E. 182

Woodard, J.L. 8, 85, 179, 223, 296

Woodhull, A. 30

Woods, A.J. 190, 250, 257

Woods, S.P. 164, 233, 235, 277

Woodward, L.J. 165, 278

Wozniak, J. 284

Wright, J. 240

Wright, K.L. 155

Wright, M.J. 18, 141, 148, 149, 174

Wrocklage, K.M. 63

Wu, L. 88

Wu, S. 104, 294

Wu, W. 236

Wyman-Chick, K.A. 243

Wynn, M. 249, 250

Xie, X. 57

Xu, H. 16

Xu, J. 288, 292

Xu, X. 265

Yaffe, K. 137, 139

Yamada, T. 249

Yamaguchi, T. 6

Yamamoto, A. 23, 122

Yamout, K. 26

Yang, B. 6

Yang, J. 92, 122

Yannakoulia, M. 240

Yannis, G. 80

Yantis, S. 104

Yeates, K.O. 93, 151, 155, 156, 225, 271

Yeatman, C.W. 101, 105

Yee, M.K. 243

Yeh, E.A. 209, 210

Yeh, P. 141

Yeo, R. 107, 110, 191
Yesian, R. 182

Yeung, R.C. 298

Yew, B. 68, 193, 279

Yiu, A. 284

Yochim, B. 182

Yokoyama, J. 13

Yoon, H. 35

York, G.E. 124

You, C. 147

You, M.Y. 13, 43, 127, 129, 250, 251

You, X. 197, 261, 281

Young, A. 215

Young, M. 263

Ysrraelit, M.C. 210

Yuan. J. 265

Yund, B.D. 232

Yurgelun-Todd, D. 285, 286

Zabel, T.A. 3, 121

Zaccariello, M. 199

Zafonte, R. 18, 47, 50, 138, 145

Zahodne, L.B. 7, 8, 130, 253

Zahr, N. 163

Zakrzewski, J.J. 62

Zane, K.L. 149, 150, 238, 266

Zang, H. 93, 155

Zannino. D. 126

Zanowski, S.C. 241

Zartman, A. 26

Zeilig, G. 237

Zetterberg, H. 67, 194, 250

Zhan, L. 295

Zhang, F. 112, 230

Zhang, J. 229

Zhang, N. 93, 155

Zhang, Y. 92

Zhao, G. 247, 248

Zhao, L. 81, 84

Zhu, Y. 129

Ziccardi, S. 212

Ziemnik, R.E. 10, 292

Zimmerman, M.E. 60, 96, 293

Zivanovic, R. 122

Zizak, V. 16, 172

Zlatar, Z.Z. 11

Zonderman, A.B. 13

Zubieta, J. 263

Zuccato, B.G. 54, 55, 56, 57, 93, 182 
Zuelsdorff, M. 74

Zumberge, N. 93, 151

Zundel, C.G. 243

Zynda, A.J. 94 


\title{
Keyword Index \\ Forty Sixth Annual Meeting \\ International Neuropsychological Society
}

\author{
February 14-17, 2018 \\ Washington DC, USA
}

academic achievement 46, 105, 119, 120, 200, 227, 228, 230, 278, 281, 292

acculturation 10, 30, 31, 235, 236, 245

activities of daily living / adaptive functioning $5,6,7,13,15$, 17, 73, 79, 82, 84, 87, 109, 168, 176, 204, 205, 208 ,

233, 238, 253, 264, 273, 275, 280, 287, 298

adolescence $3,22,25,52,53,94,98,99,101,103,112,122$. 155, 164, 184, 228, 253, 282

affective processing disorders 96, 106, 195, 258, 282

affective processing, normal 25, 26, 126, 262, 268, 286

aggression 134, 152, 287, 289, 291

aging disorders $3,4,5,7,9,11,14,15,16,17,18,66,67$,

$68,70,71,72,77,78,81,82,85,86,95,139,162,163$,

$173,182,190,193,194,195,226,236,240,255,270$,

$273,280,297$

aging, normal 4, 5, 6, 10, 11, 12, 13, 14, 15, 17, 18, 32, 43,

$61,66,68,72,80,85,92,93,127,128,129,130,158$,

$179,180,190,191,217,223,224,233,236,249,250$,

$251,253,254,257,260,265,266,270,273,277,293$,

295, 296, 297

alcohol 11, 22, 24, 146, 163, 173, 188, 241, 264

amyotrophic lateral sclerosis 214

anomia 76, 182

anosognosia 67

anterior cingulate 285

antisocial behavior 99, 291

anxiety 24, 64, 107, 117, 119, 199, 200, 214, 242, 289, 299

apathy $96,143,165,206,231$

apnea 267,287

apolipoprotein E 8, 57, 77, 80, 142, 193, 221

apraxia 89

assessment 10, 13, 21, 26, 29, 36, 45, 46, 49, 53, 54, 56, 68,

70, 84, 85, 98, 101, 107, 115, 130, 131, 133, 166, 168,

$170,171,172,174,175,176,178,181,182,183,184$,

185, 186, 187, 189, 200, 216, 235, 236, 241, 245, 270 .

281, 289

asymmetry 100, 243, 269

ataxia 96 attention 25, 32, 35, 76, 77, 78, 97, 98, 99, 100, 101, 102 , 103, 105, 106, 109, 114, 126, 154, 155, 156, 157, 169. 202, 203, 215, 222, 252, 256, 258, 261, 265, 268, 281, 299

attention deficit hyperactivity disorder 31, 47, 55, 93, 97, 99 , 100, 101, 102, 103, 104, 105, 106, 116, 117, 119, 120 , $126,151,153,161,178,184,185,189,200,232,254$, 261

auditory processing disorder 294

auditory processing, normal 181, 195

autism spectrum disorders 31, 112, 113, 114, 115, 116, 117. $125,161,162,186,230,231,271$

autoimmune disorders 42, 43, 111

awareness $157,273,274$

basal ganglia 33, 149, 153, 214

bilingualism 31, 111, 116, 120, 280, 290, 293

bipolar disorder 13, 25, 33, 34, 35, 101, 192, 193, 285

bone marrow transplant 225

brain damage 133, 156, 233

brain development 1, 3, 114, 189, 260, 270, 271, 291

brain disorder 210, 238

brain function 15, 37, 67, 106, 199, 222, 224, 264, 265, 266,299

brain injury 42, 53, 56, 94, 124, 131, 132, 133, 134, 136, 137, 139, 140, 144, 145, 154, 176, 219, 254, 291

brain plasticity $1,57,140,158,159,219$

brain structure $3,6,64,83,89,106,148,271$

brain tumor 108, 122, 152, 224, 225, 227, 228, 229, 259 , 260,276

breast cancer 227,228

cancer 109, 224, 225, 226, 227, 229, 230, 276, 277

cannabis $22,24,38,164$

cardiovascular disease 7, 12, 15, 39, 61, 62, 89, 110, 174 , 216, 240,249

caregiver stress/burden 14, 69, 75, 209

cerebellum 61, 207, 224, 237, 261, 262, 282

cerebral blood flow 5, 34, 68, 222, 231, 280

cerebrevascular accident/stroke 108, 130, 182, 215, 216, 231, 279

cerebrovascular disease 108, 163, 221, 224, 263, 265, 280

chemotherapy $226,227,228,276$ 
child brain injury 46, 51, 52, 57, 90, 91, 93, 152, 153 , 154,155

child development disorders 1, 100, 105, 113, 115, 118, 120 , 160, 189, 284, 297, 299

child development, normal 106, 122, 126, 184, 185, 187 childhood maltreatment 36, 286, 292

chronic pain $50,167,179$

chronic stress 251, 253, 298

clinical trials $36,76,139,161$

cognitive 9, 25, 36, 61, 71, 73, 74, 123, 124, 128, 149, 163 , 204, 207, 217, 219, 260, 268, 294

cognitive control 3, 13, 63, 104, 119, 191, 192, 223, 235, 254, 258, 266, 268, 286, 293

cognitive course 9, 95, 111, 129, 192, 225, 232

cognitive functioning $5,8,9,11,12,14,15,16,18,26,30$, $32,33,34,38,39,46,57,58,62,63,64,66,68,70,72$. 76, 84, 88, 94, 96, 101, 103, 107, 108, 110, 111, 112. 115, 118, 119, 122, 128, 131, 132, 133, 136, 137, 142 , $150,164,167,168,169,173,174,176,180,182,185$, 190, 196, 198, 200, 201, 202, 203, 204, 206, 209, 210 , $211,219,224,225,226,228,229,231,234,237,238$, $241,242,244,250,251,252,253,258,259,261,263$, $265,275,276,279,285,290,294,296,297$

cognitive neuroscience 40, 42, 76, 89, 113, 116, 157 ,

190, 192, 215, 222, 223, 245, 246, 259, 268 . 270, 271, 296

cognitive processing 25, 37, 99, 100, 139, 147, 211, 221 , 248. 267

cognitive rehabilitation 2, 41, 42, 131, 215, 233, 238, 252 , 253, 254, 255, 270

cognitive reserve 4, 12, 127, 128, 130, 132, 133, 213, 274, 275,280

cognitive screening $7,58,59,64,73,82,85,86,87,92,170$, $172,174,176,180,200,211,215,216,220,241,281$ cognitive style 24,259

computerized neuropsychological testing 21, 39, 44, 50, 53, $55,58,59,90,92,93,97,99,100,105,106,138,153$, $165,166,177,235,297,299$

concussion $2,18,28,41,45,46,47,48,49,50,51,52,53$, $54,55,56,57,94,132,139,141,145,147,150,151$, $152,153,154,155,167,177,218$

confabulation 20

congential disorders 195

corpus callosum 151, 195, 229

creativity 248

cross-cultural issues $7,12,23,30,31,74,129,130,159$, $160,163,179,187,245,286,290$

decision-making $3,16,22,103,145,178,235,244,245$, 282, 287, 289, 298

deep brain stimulation 206, 207, 258, 269

dementia with Lewy bodies 65, 88

dementia, Alzheimer's disease 4, 5, 8, 18, 21, 43, 44, 60, 64 , $65,66,67,68,69,71,72,73,74,75,76,80,82,83,85$, 92, 93, 162, 163, 167, 168, 176, 192, 193, 194, 195. 217, 220, 221, 222, 223, 224, 229, 264, 276, 280, 281 dementia, other cortical 88, 195, 231 demential, subcortical 238

demographic effects on test performance 10, 14, 17, 31, 79, 107, 185, 186

depression $24,25,28,33,34,36,54,63,64,68,76,78$, $82,93,123,143,147,148,149,154,159,170,197$. 199, 200, 209, 211, 214, 234, 238, 263, 269, 274 . $275,296,297$

diabetes $9,72,129,224$

disinhibition 165

driving 68, 78, 80, 126, 169, 253

dyslexia 1, 120, 121

eating disorders 283, 284, 286

ecological validity 97, 103, 166, 249

education 59, 60, 77, 118, 133, 255

effort 26, 27, 28, 29, 34, 45, 46, 47, 53, 54, 55, 139. $171,172,175,176,177,181,184,186,187$. 219, 223, 268

electroencephalography 100, 113, 191, 222, 266, 267. 268,274

ethnicity 8, 14, 136, 169, 285, 289

event related potentials 251, 267

everyday functioning $4,7,8,9,16,44,56,84,87,92,145$, $169,172,178,181,191,195,196,199,240,241,249$. $253,254,285,286,293$

executive abilities, abnormal 24, 32, 36, 40, 49, 61, 63, 71, 84, 87, 89, 97, 98, 103, 108, 117, 118, 128, 133, 145, $149,160,161,162,165,172,175,181,185,186,193$, 197, 198, 199, 200, 206, 209, 210, 217, 232, 237, 239. 254, 279, 281, 282, 283, 284, 285, 286, 287, 288, 289 . 291, 298

executive abilities, normal 6, 10, 11, 12, 14, 33, 44, 62, 71, 110, 118, 120, 153, 174, 175, 184, 257, 260, 265, 277. 282, 285, 287, 288, 289, 290, 292, 298

face processing 192, 259

facial affect 115, 192, 194, 214, 239

fatigue 148, 206, 211, 244, 261, 275

fluency 119, 233

fluid intelligence 119, 250

focal lesion 282

forensic neuropsychology 29, 177, 183

fragile $\mathrm{X}$ syndrome 115

frontal lobes 58, 121, 128, 226, 257, 267, 283, 291

genetic neuropsychology 13, 56, 83, 86, 96, 112, 127, 146, 189, 230, 231, 232, 279

geriatric depression 17

head injury, closed 48, 93

heroin 22

hippocampus 17, 40, 63, 68, 192, 196, 202, 220, 278

HIV/AIDS 23, 205, 233, 234, 235, 236, 242, 245, 263

hormones 18, 24, 36, 114, 256

Huntington's disease 69, 88

hydrocephalus 60,238

hypertension 204

hypoxia 109,142

Imaging (Functional) 17, 67, 72, 89, 158, 207, 245, 246, 260,264 
information processing speed 14, 65, 83, 86, 113, 147, 210, 242,257

informed consent 3

inhibitory control 22, 99, 101, 104, 165, 251, 282, 287

intellecutal functioning 33, 102, 109, 118, 119, 169, 171, $199,200,230$

intelligence 27, 29, 102, 179, 222, 262, 278, 290

language 104, 139, 143, 149, 152, 167, 197, 212, 241, 248, $262,265,288,292,293,294$

language: aphasia 2, 76, 270, 293, 294

language: development 107, 110, 121, 126, 270, 271

language: second/foreign 167, 184

laterality $195,200,201,239,262,266,268$

learning $32,80,119,125,133,171,173,214,237,243$, 288, 295

learning disabilities 1, 49, 104, 118, 120, 121

leukemia 227, 228

liver diesease 241

lyme disease 242

magnetoencephalography 144

malingering 26, 27, 28, 29, 44, 98, 99, 106, 132, 148, 173, $175,178,183$

mathematics disability 120

medical illness 62, 111, 112, 174, 225, 235, 236, 238, 240, 243. 279, 282

memory complaints $7,17,34,35,46,66,67,70,72,75,87$, 129, 138, 144, 180, 193, 198, 207, 216, 242, 295, 299

memory disorders 7, 20, 40, 69, 75, 79, 80, 81, 86, 88, 130 ,

$135,141,149,167,178,188,194,195,196,221,241$, 243, 244, 280, 295, 296, 297

memory training 83, 252, 254, 278

memory, false 71

memory, implicit 121, 171, 194, 237, 246

memory, normal 4, 5, 6, 14, 16, 17, 33, 34, 40, 180 ,

187, 194, 197, 208, 250, 264, 278, 290, 294, 295, 297. 298

memory, prospective 82, 94, 131, 140, 221, 236, 277 , 296. 298

metacognition 66, 72, 95, 273, 274

metamemory 18

methamphetamine 23

mild cognitive impairment 7, 18, 69, 71, 73, 74, 75, 77, 78, $79,80,81,82,83,84,85,86,87,130,174,180,191$, 193, 194, 205, 221, 223, 249, 252, 263, 276, 295

mild traumatic brain injury 18, 45, 48, 51, 52, 54, 55, 56,

$57,93,94,123,124,132,138,139,140,142,144,146$. $148,150,151,154,165,182,219,256$

minority issues $23,30,59,60,85,110,130,134,155$

motivation 22, 105, 164, 214, 246, 254, 255, 258

motor function 10, 15, 78, 95, 96, 104, 105, 114, 122, 171 .

183, 184, 216, 227, 236

motor speed 84, 125, 233

multiple sclerosis 21, 42, 126, 209, 210, 211, 212, 213, 214, 274, 275

muscular dystrophy 230, 231

naming 2, 43, 65, 74, 290 neglect 215,217

neural circuitry 62

neurocognition 22, 23, 38, 41, 50, 62, 63, 81, 112, 124 ,

$141,152,156,158,165,170,216,245,249,256$.

$263,268,277$

neurofeedback 102

neurofibromatosis 107, 230, 232

neuroimaging, functional 21, 26, 44, 48, 57, 77, 125, 126 , $128,190,191,246,248,256,260,261,265,266,270$, 272, 274, 297

neuroimaging, functional connectivity 14, 117, 125, 132, 136, $144,191,219,256,261,263,264,265$

neuroimaging, structural 6, 33, 41, 57, 61, 63, 64, 71, 89, $107,110,121,125,127,134,135,137,138,141,156$, 158, 163, 188, 192, 219, 221, 223, 224, 227, 229, 250, $251,257,262,263,264,265,270,271,277,278,280$

neuroimaging, structural connectivity 44, 82, 114, 116, 127 , 140, 149, 150, 237, 249, 255, 260, 266, 272, 275, 278

neuropsychological assessment 1, 3, 10, 17, 24, 28, 31, 39, $41,44,50,51,57,62,68,69,70,71,77,80,81,82,85$, 87, 88, 92, 106, 109, 122, 134, 139, 140, 144, 163, 164, 166, 167, 168, 171, 173, 174, 176, 177, 178, 180, 181. 182, 183, 184, 185, 186, 187, 188, 197, 198, 200, 202. 206, 208, 210, 212, 214, 218, 220, 225, 231, 238, 241, 248, 258, 282, 284, 285, 287, 288, 290, 292

neuropsychological outcome 39, 41, 60, 88, 92, 95, 108 , $111,112,122,137,142,143,147,149,150,174$. 188, 201, 203, 204, 225, 231, 233, 236, 238, 242 , $257,279,283,294$

neurotoxicity 240,243

neurotransmitter systems 13

normative data $18,74,75,170,281$

olfaction $16,38,198,204$

other $66,136,137,142,162,255$

outcome 47, 54, 66, 132, 134, 135, 145, 151, 227

Parkinson's disease 83, 95, 96, 158, 177, 204, 205, 206, 207. $208,217,239,242,243,269,281,295$

pediatric neuropsychology 29, 31, 42, 43, 45, 46, 47, 48, 49 , $90,91,98,104,108,109,111,115,118,120,126,151$, 152, 153, 154, 183, 184, 186, 187, 199, 203, 209, 212, 225, 226, 229, 230, 231, 260, 276, 283, 284, 294, 299

perinatal factors 102, 107, 109, 277

perseveration 97

personality 95, 167, 171, 176, 211, 213, 234, 242, 257, 269

positron emission tomography $43,75,263$

post traumatic stress disorder $32,35,36,51,56,63,94,105$, $131,134,137,138,144,147,158,182,256,259,269$. 271, 284, 291, 292

prematurity 31, 107, 109, 110, 126, 222, 255

premorbid functioning 43, 52, 79, 169, 177, 179

prosody 30

psychometric constructs 28, 44, 55, 67, 103, 153, 166, 167 , $173,178,179,187,233$

psychopathy 96, 203, 258

psychopharmacology $9,34,36,256$

psychosis 36, 37, 113, 122, 192, 208, 220 
quality of life 9, 95, 109, 128, 137, 152, 197, 201, 202, 205, $206,209,211,235,240,244,252,255,289$

radiotherapy 228,229

reaction time 39, 105, 213

reading disorders $118,120,121$

reading, normal 169,261

schizophrenia 33, 37, 38, 39, 175, 192, 258, 281

seizure disorders / epilepsy 118, 161, 171, 196, 197, 198,

199, 201, 202, 203, 246, 247, 248, 280

seizure disorders / epilepsy, surgical treatment 196, 200, 201, 203, 239, 241, 280

seizures, psychogenic 171, 196

self-esteem 25, 98, 135

self-monitoring $67,273,274$

self-report 46, 47, 53, 54, 64, 70, 99, 103, 107, 131,

137, 145, 156, 174, 193, 195, 196, 203, 208, 224. $239,241,285$

semantic processing 38, 65, 76, 149, 212, 296

sensory integration $6,123,124,211$

sickle cell disease 97, 108, 284

sleep 34, 61, 127, 173, 208, 220, 234, 244, 250, 251, 256.

$257,259,260,268,269,291,293,294$

sleep disorders $61,62,267,287$

social cognition 16, 30, 35, 37, 74, 113, 151, 195, 214, 239, $254,259,271,272,289$

spina bifida 109

spinal cord injury 132, 158, 237, 243

sports-related neuropsychology 45, 48, 49, 50, 52, 53, 54, 55, $56,57,93,94,145,155$ stroke recovery 149, 152, 153, 215, 216, 294

substance abuse 11, 23, 99, 122, 146, 165, 258, 287

temporal lobes 198, 201, 202, 285

test $86,90,169,186,289$

test development 43, 58, 59, 92, 140, 156, 157, 166, 170 , 178, 179, 185, 187, 277

test reliability 53

test validity $26,27,28,29,30,44,46,54,155,168,173$,

174, 178, 179, 180, 181, 182, 183, 203, 293

theory of mind 115, 154, 214, 249

training $6,19,20,59,60,140$

transdisciplinary research 93, 114

traumatic brain injury 18, 40, 41, 50, 51, 74, 91, 124 , $125,130,131,132,133,134,135,137,138,139$, $140,141,142,143,144,145,146,147,149,150$, $151,155,156,157,158,170,175,180,198,220$, $252,263,266,272,273$

treatment outcome 51, 62, 91, 102, 113, 134, 135, 138 , 143, 153, 160, 162, 178, 188, 190, 198, 212, 220, 227. 255

vascular dementia $4,72,83$

verbal abilities 10, 40, 201, 212, 217, 243, 269

visual imagery 87

visuoconstruction 217, 267

visuospatial 28, 64, 171, 202, 205, 213, 253, 279, 288

working memory $23,26,32,35,37,38,51,58,71,102,105$, $107,118,121,159,165,185,192,262,265,276,284$, $285,286,291$ 